\title{
The Weatherization Assistant User's Manual (Version 8.9)
}

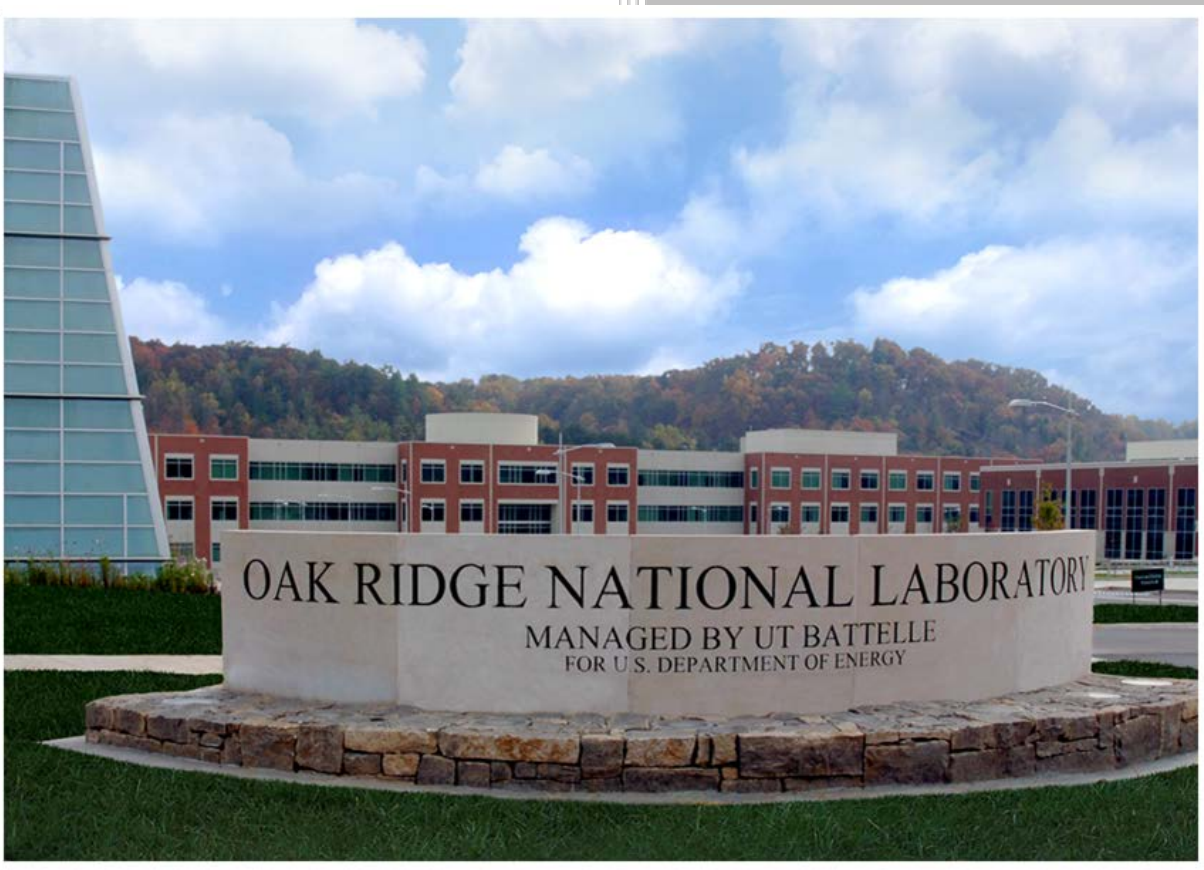

Approved for public release.

Distribution is unlimited.

Michael B. Gettings Mini Malhotra Mark P. Ternes

January 2015 


\title{
DOCUMENT AVAILABILITY
}

Reports produced after January 1, 1996, are generally available free via US Department of Energy (DOE) SciTech Connect.

Website http://www.osti.gov/scitech/

Reports produced before January 1, 1996, may be purchased by members of the public from the following source:

\author{
National Technical Information Service \\ 5285 Port Royal Road \\ Springfield, VA 22161 \\ Telephone 703-605-6000 (1-800-553-6847) \\ TDD 703-487-4639 \\ Fax 703-605-6900 \\ E-mail info@ntis.gov \\ Website http://www.ntis.gov/help/ordermethods.aspx
}

Reports are available to DOE employees, DOE contractors, Energy Technology Data Exchange representatives, and International Nuclear Information System representatives from the following source:

Office of Scientific and Technical Information

PO Box 62

Oak Ridge, TN 37831

Telephone 865-576-8401

Fax 865-576-5728

E-mail reports@osti.gov

Website http://www.osti.gov/contact.html

This report was prepared as an account of work sponsored by an agency of the United States Government. Neither the United States Government nor any agency thereof, nor any of their employees, makes any warranty, express or implied, or assumes any legal liability or responsibility for the accuracy, completeness, or usefulness of any information, apparatus, product, or process disclosed, or represents that its use would not infringe privately owned rights. Reference herein to any specific commercial product, process, or service by trade name, trademark, manufacturer, or otherwise, does not necessarily constitute or imply its endorsement, recommendation, or favoring by the United States Government or any agency thereof. The views and opinions of authors expressed herein do not necessarily state or reflect those of the United States Government or any agency thereof. 
Energy and Transportation Science Division

\title{
THE WEATHERIZATION ASSISTANT USER'S MANUAL (VERSION 8.9)
}

\author{
Michael B. Gettings \\ Mini Malhotra \\ Mark P. Ternes
}

Date Published: January 2015

Prepared by

OAK RIDGE NATIONAL LABORATORY

Oak Ridge, Tennessee 37831-6283

managed by

UT-BATTELLE, LLC

for the

US DEPARTMENT OF ENERGY

under contract DE-AC05-00OR22725 



\section{How to Use This User's Manual}

This manual is intended to be a training manual for new users of the Weatherization Assistant and as a reference manual for experienced users.

New users should read Chapters 1-4 to familiarize themselves with the Weatherization Assistant and its basic operation. Chapter 1 presents an overview of all the features and capabilities offered by the Weatherization Assistant. Chapters 2 and 3 step new users through the process of installing the Weatherization Assistant and setting up the software for the first time. Chapter 4 presents a quick-start tutorial on how to enter a new client and initiate an energy audit for this client, which is the primary purpose of the Weatherization Assistant.

Once new users have installed the software and understand the basic steps needed to run an audit, they will then need to read other chapters of the user's manual to learn about the details of performing an audit. Helpful information on how to navigate through the Weatherization Assistant's multiple forms and enter information into its data fields is presented in Chapter 5. The quick-start tutorial in Chapter 4 will emphasize the need for a new user to make sure accurate information is entered into the Setup and Supply Libraries before running an actual audit on a home for the first time; therefore, a new user will need to read Chapters 14 and 15, which discuss these libraries. A new user will also need to read Chapters 8-11, which discuss the details of how to enter information into the audits, and Chapter 12, which discusses the output report generated from running an audit that provides details concerning the recommended weatherization measures.

Experienced users can use the manual as a reference guide to obtain information on operational details of features and details of every data input field found in the Weatherization Assistant. Five Chapters (Chapters 8-12) discuss the details associated with the audits themselves. Separate chapters are provided for all the remaining key elements of the Weatherization Assistant: Agency, Clients, Work Orders, Setup Library, Supply Library, Preferences, and Data Link. A final chapter (Chapter 18) provides details on several features that have input fields found on multiple forms within the Weatherization Assistant. Although the input fields themselves are described as appropriate in the chapters in which they are encountered, an overall discussion of each feature is presented in Chapter 18. 
How to Use This User's Manual 


\section{Table of Contents}

How to Use This User's Manual....................................................................................... iii

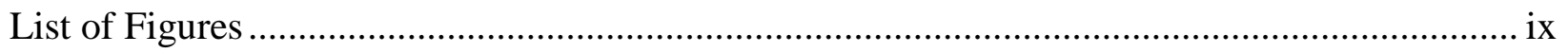

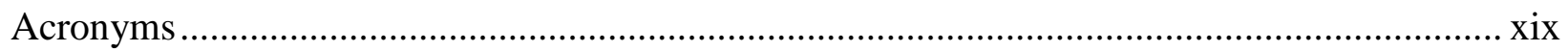

Chapter 1. Overview of the Weatherization Assistant...................................................... 1-1

1.1 Overview of NEAT and MHEA …...................................................................... 1-2

1.2 Software Overview ...................................................................................... 1-4

1.3 Program Overview .......................................................................................... 1-5

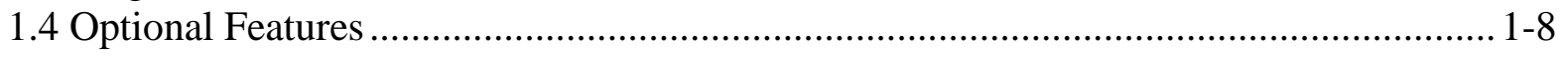

Chapter 2. Installation and Start-up ............................................................................ 2-1

2.1 Hardware and Software Requirements ................................................................... 2-1

2.2 Obtaining the Weatherization Assistant Software ...................................................... 2-1

2.3 Installing the Weatherization Assistant ............................................................... 2-3

2.4 Network Installation......................................................................................... 2-6

2.5 Obtaining and Installing the GIS Files................................................................ 2-7

2.6 Obtaining Manuals for the Weatherization Assistant .................................................. 2-8

2.7 Accessing Web-Based Training for the Weatherization Assistant ............................... 2-9

2.8 Starting the Weatherization Assistant ....................................................................... 2-10

2.9 The User Log On Form.................................................................................. 2-11

Chapter 3. Setting Up the Weatherization Assistant........................................................... 3-1

3.1 Initial Start-up of the Weatherization Assistant........................................................ 3-1

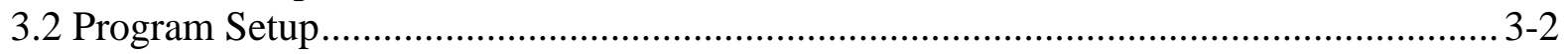

3.3 Importing Information from a Previous Version .................................................... 3-8

Chapter 4. Quick-Start Tutorial .............................................................................. 4-1

4.1 Creating a New Client................................................................................... $4-1$

4.2 Starting a New Audit ................................................................................... 4-4

4.3 Running a New Audit ........................................................................................ $4-8$

Chapter 5. Navigation and Data Entry ......................................................................... 5-1

5.1 Form and Record Navigation............................................................................... 5-1

5.2 Entering Data into the Weatherization Assistant .................................................... 5-3

5.3 Navigating Between Controls ......................................................................... 5-5

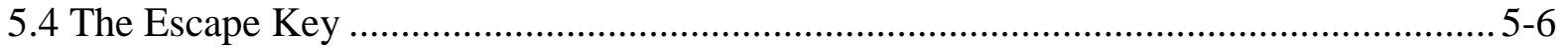

5.5 Required Versus Optional Textboxes and Drop-down Lists ...................................... 5-7

5.6 Field Defaults.................................................................................................. 5-7

5.7 Field Range Checking .......................................................................................... 5-8

5.8 Data Sheet and Form Views of Records ................................................................... 5-9 


\section{Table of Contents}

5.9 Copying and Pasting Data........................................................................................ 5-12

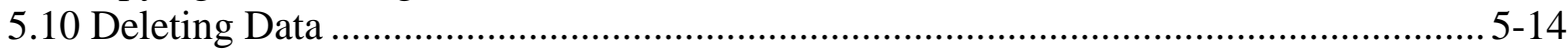

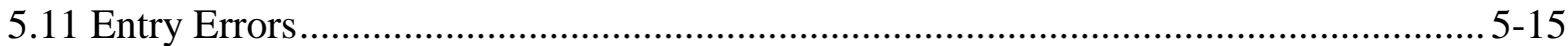

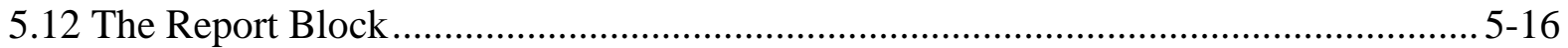

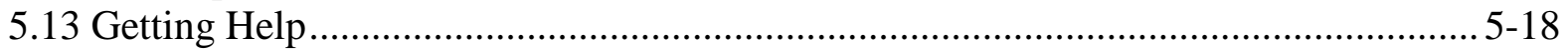

5.14 The References Button........................................................................................ 5-19

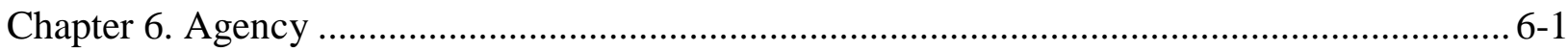

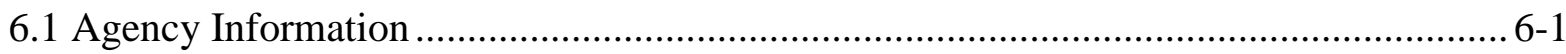

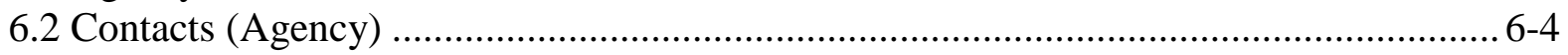

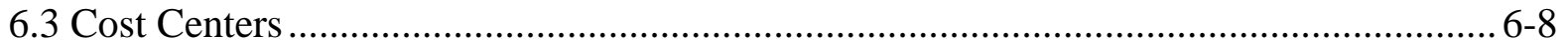

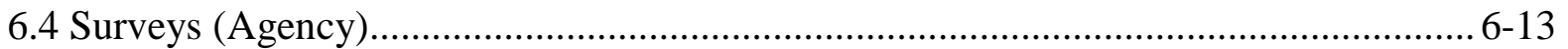

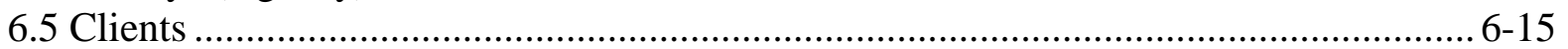

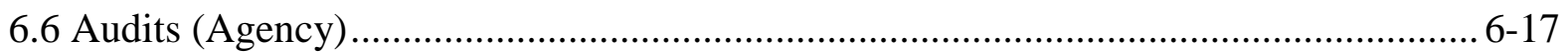

6.7 Work Orders (Agency) ..................................................................................... 6-19

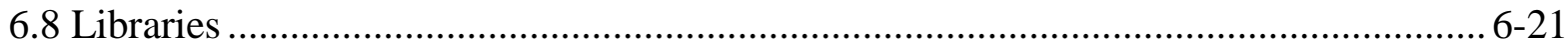

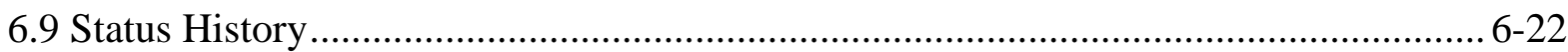

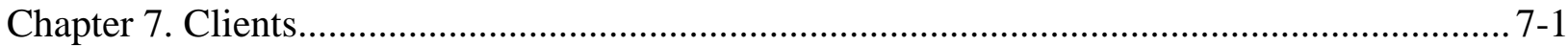

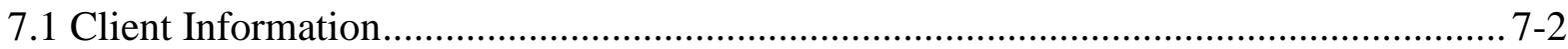

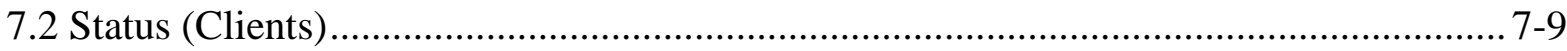

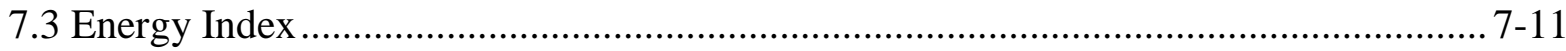

7.4 Contacts (Client) ………………………………….............................................. 7-14

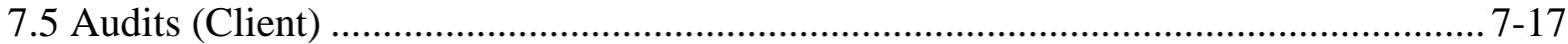

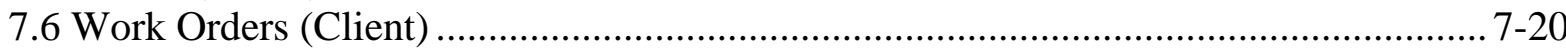

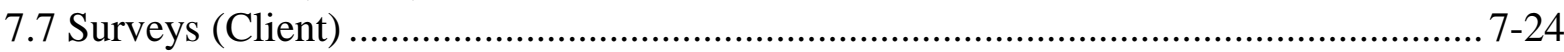

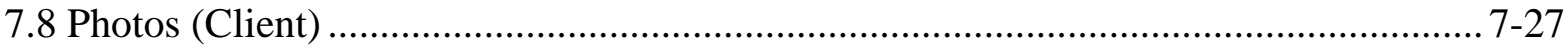

Chapter 8. NEAT and MHEA Introduction.........................................................................

8.1 Starting a New Audit and Accessing Previous Audits....................................................... 8-1

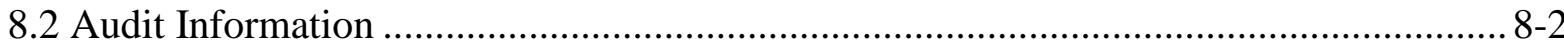

Chapter 9. NEAT Building Description............................................................................... 9-1

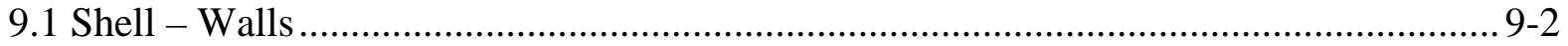

9.2 Shell - Windows.................................................................................................... 9-7

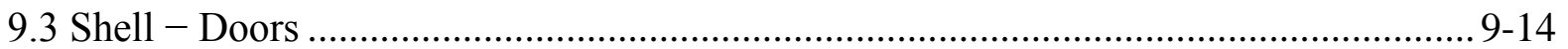

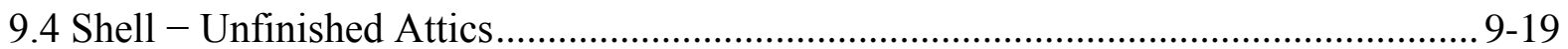

9.5 Shell - Finished Attics.......................................................................... 9-24

9.6 Shell - Foundations.................................................................................................. 9-26

9.7 Heating - General Description Data...................................................................... 9-34

9.8 Required Heating System Details Sub-Form ........................................................... 9-39

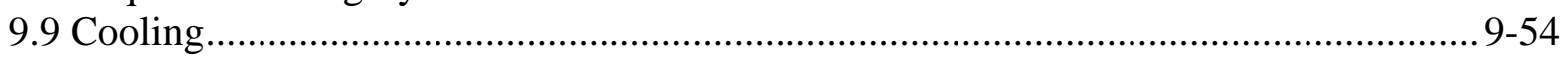




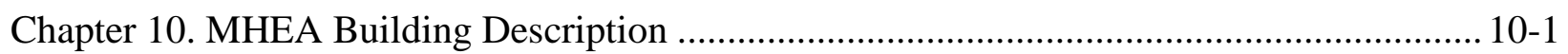

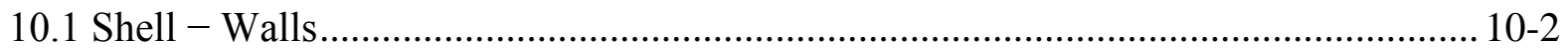

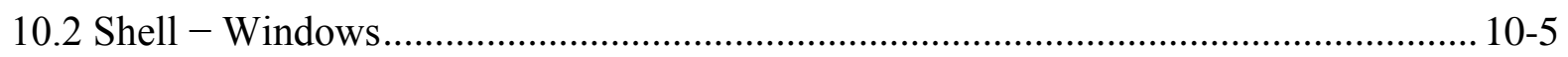

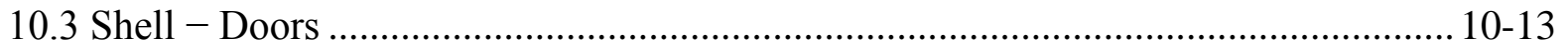

10.4 Shell - Ceiling …………………………………......................................... 10-16

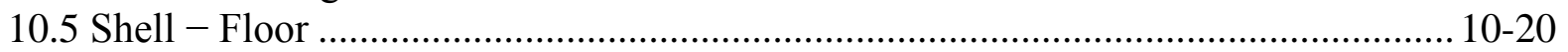

10.6 Addition ............................................................................................................

10.7 Heating - Primary, Secondary, and Replacement .................................................... 10-37

10.8 Cooling - Primary, Secondary, and Replacement ................................................... 10-43

Chapter 11. NEAT and MHEA Common Elements ..........................................................11-1

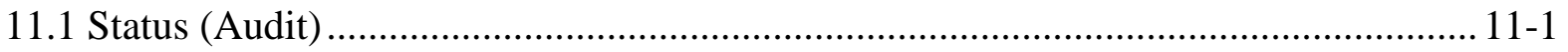

11.2 Ducts/Infiltration - Air and Duct Leakages ..............................................................11-3

11.3 Duct/Infiltration - Optional Forms ............................................................................11-18

11.4 Base Loads - Water Heating.................................................................................... 11-24

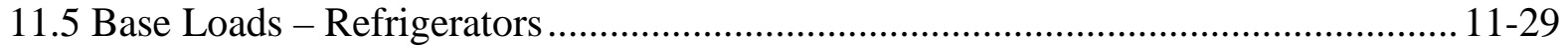

11.6 Base Loads - Lighting Systems ...........................................................................11-35

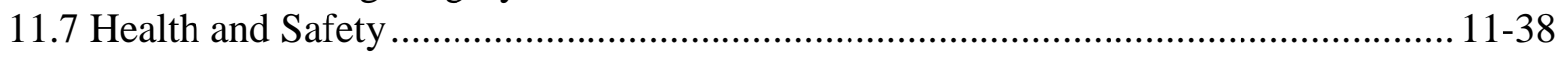

11.8 Itemized Costs................................................................................................. 11-41

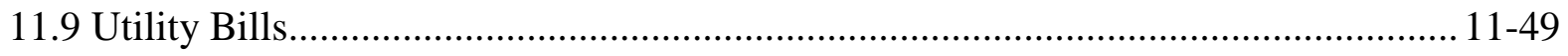

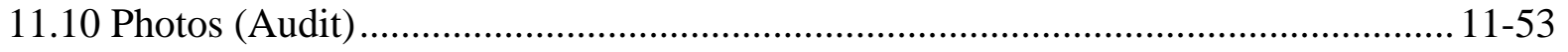

11.11 Measures (Audit) .............................................................................................11-54

11.12 Optional Heating System Details.................................................................11-59

11.13 Optional Water Heater Details ......................................................................................11-63

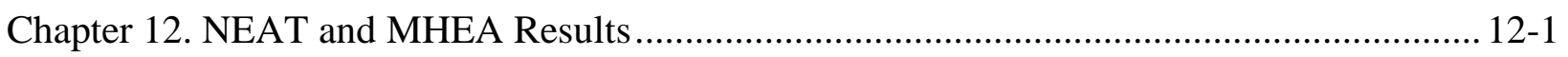

12.1 Running an Audit and Viewing the Results............................................................. 12-1

12.2 Recommended Measures Report ............................................................................ 12-2

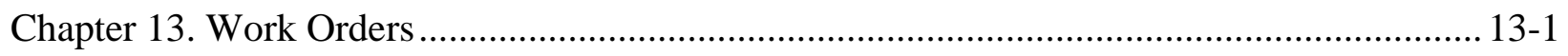

13.1 Work Order Information ................................................................................... 13-2

13.2 Status (Work Order)............................................................................................13-7

13.3 Measures (Work Order) ........................................................................................ 13-9

13.4 Photos (Work Order)............................................................................................ 13-24

Chapter 14. Setup Library ............................................................................................. 14-1

14.1 Setup Library Information ................................................................................... 14-2

14.2 Key Parameters ............................................................................................... 14-5

14.3 Fuel Costs................................................................................................... 14-21

14.4 Fuel Price Indices........................................................................................ 14-24

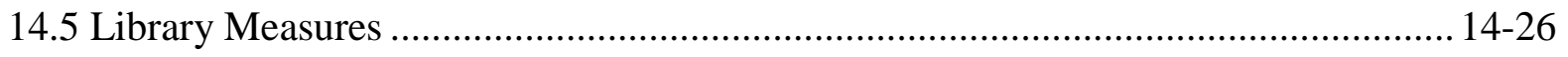

14.6 User Defined Measures....................................................................................... 14-31

14.7 NEAT Insulation Types ...................................................................................... 14-42 


\section{Table of Contents}

Chapter 15. Supply Library........................................................................................... 15-1

15.1 General Information Form ............................................................................ 15-2

15.2 Materials/Labor Forms........................................................................................

15.3 Energy Details Sub-Form .............................................................................. 15-10

Chapter 16. Preferences ..................................................................................................... 16-1

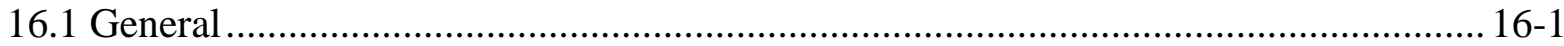

16.2 Range Check and Default Values ..........................................................................16-4

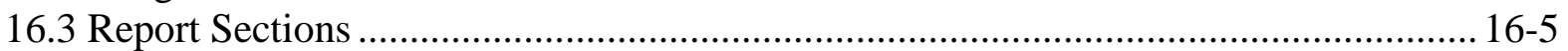

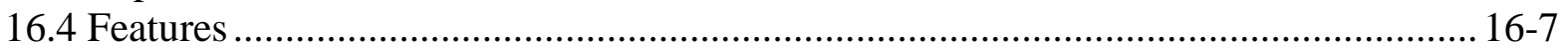

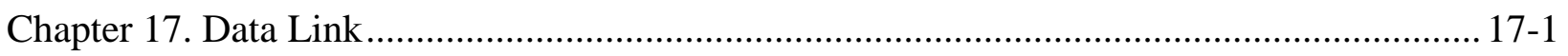

17.1 Currently Linked Backend Data File ………........................................................ 17-3

17.2 Currently Linked Reporting File............................................................................ 17-6

17.3 Data Transfer - Import/Export With Another MSAccess Database.............................. 17-7

17.4 Data Transfer - Import/Export Indirectly with Text Files..........................................17-15

17.5 Selecting Client Records .......................................................................................... 17-24

Chapter 18. Auxiliary Features ....................................................................................... 18-1

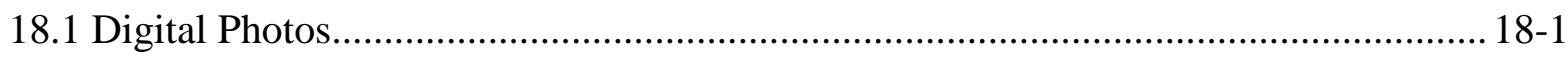

18.2 Geographic Information System (GIS) ………................................................... 18-6

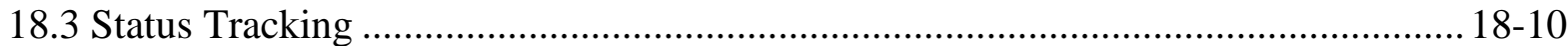

18.4 E-Mailing from Within Weatherization Assistant ..................................................... 18-15

Appendix A. NEAT Weatherization Measures ………………………………………........ A-1

Appendix B. MHEA Weatherization Measures ………………………………………….... B-1

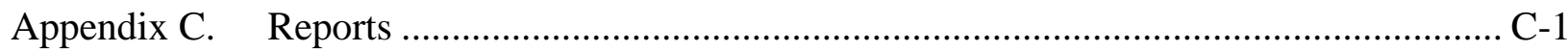

Appendix D. Development of Customized Reports …………………………………... D-1

Appendix E. Window Leakiness .................................................................................. 


\section{List of Figures}

Figure

Page

1.1. Design of the Weatherization Assistant................................................................... 1-1

1.2. The Weatherization Assistant's Main Menu........................................................... 1-6

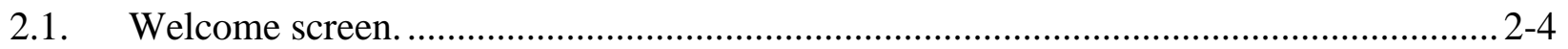

2.2. Disclaimer Statement screen............................................................................. 2-4

2.3. Destination Folder screen for a "New Installation." .................................................2-5

2.4. Destination Folder screen for an "Update." ............................................................... 2-5

2.5. Start Installation screen.......................................................................... 2-6

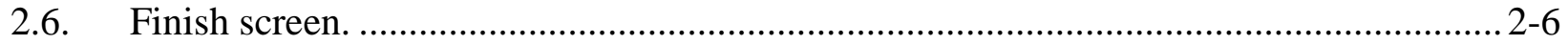

2.7. The Weatherization Assistant's icon for Version 8.9................................................ 2-6

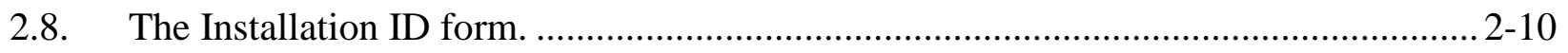

2.9. The Weatherization Assistant's Main Menu.......................................................... 2-12

2.10. The Weatherization Assistant Log On form..................................................... 2-12

3.1. The Weatherization Assistant's Main Menu................................................................ 3-2

3.2. The Agency Information form under the Main Menu’s “Agency” button..................... 3-3

3.3. The Contacts form under the Main Menu's "Agency” button..................................... 3-4

3.4. The General Information form under the Main Menu's "Supply Library” button.......... 3-5

3.5. The Setup Library Information form under the Main Menu’s “Setup Library”

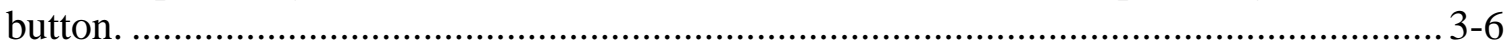

3.6. The Data Link form (Version 8.6) .......................................................................... 3-8

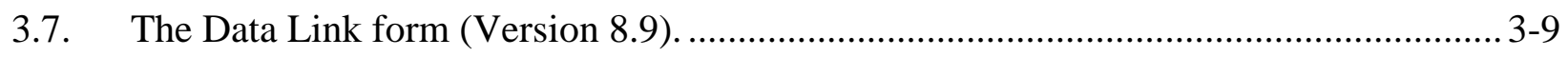

3.8. A blank Transfer CLIENTS Between Databases form............................................. 3-10

3.9. An example of the Transfer CLIENTS Between Databases form with client

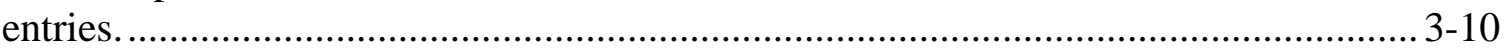

3.10. The Pick External Database for Import/Export form............................................. 3-11

3.11. The Client Information form under the Main Menu’s “Clients” button....................... 3-13

4.1. The Weatherization Assistant's Main Menu............................................................... 4-1

4.2. The Client Information form under the Main Menu's "Clients” button........................ 4-2

4.3. The Contacts form under the Main Menu's "Clients” button....................................... 4-3

4.4. The Audits form under the Main Menu’s "Clients" button. ......................................... 4-5 


\section{List of Figures}

4.5. The Audit Information form under the Main Menu's "NEAT" button. ......................... 4-5

4.6. The Audit Information form under the Main Menu's "MHEA” button. ........................ 4-6

5.1. The Audit record navigation block found on the Audit Information form under the Main Menu’s “NEAT Audit” and "MHEA Audit” buttons......................................... 5-1

5.2. The Clients form under the Main Menu’s “Agency” button. ...................................... 5-3

5.3. Two textboxes, one requiring a numeric entry. .................................................. 5-3

5.4. A drop-down list with its item list visible........................................................... 5-4

5.5. Two checkboxes, the top one being selected and with a "Yes" response......................5-5

5.6. Two textboxes, the first is a required field and the second is not...............................5-7

5.7. A status bar showing the reasonable range and default value for the Number of Conditioned Stories field. ......................................................................................... 5-8

5.8. Error indicating that a numeric entry is outside the acceptable range. ......................... 5-8

5.9. Warning indicating that a numeric entry is outside the reasonable range. ....................5-9

5.10. The Report block from the Client Information form. ............................................ 5-16

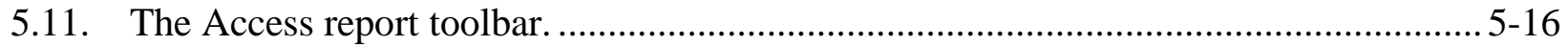

5.12. The "References to Setup Library:" window........................................................... 5-20

6.1. The Agency Information form under the Main Menu’s “Agency” button..................... 6-2

6.2. The Contacts form under the Main Menu's "Agency" button.......................................6-5

6.3. The User Group and Password sub-form.............................................................. 6-8

6.4. Cost Centers form under the Main Menu’s “Agency” button. ..................................... 6-9

6.5. The Show Fund Transactions (or Cost Center Funds) sub-form............................... 6-11

6.6. The Work Order Costs table under the Agency/Cost Centers tab. ............................. 6-12

6.7. The Surveys form under the Main Menu’s “Agency” button.................................... 6-13

6.8. The Clients form under the Main Menu’s “Agency” button. ..................................... 6-15

6.9. The Audits form under the Main Menu’s “Agency” button...................................... 6-17

6.10. The Work Orders form under the Main Menu’s “Agency” button............................. 6-19

6.11. The Libraries form under the Main Menu’s “Agency” button. .................................. 6-21

6.12. The Status History form under the Main Menu’s “Agency” button............................ 6-23

7.1. The Client Information form under the Main Menu's “Clients” button........................ 7-2

7.2. The Status form under the Main Menu’s “Clients” button....................................... 7-10

7.3. The Energy Index form under the Main Menu’s “Clients” button............................. 7-12 
7.4. The Contacts form under the Main Menu’s "Clients” button...................................... 7-15

7.5. The Audits form under the Main Menu's "Clients” button. ............................................. 7-18

7.6. The Work Orders form under the Main Menu’s “Clients” button.............................. 7-21

7.7. The Surveys form under the Main Menu’s “Clients” button...................................... 7-24

7.8. The Photos form under the Main Menu's “Clients” button....................................... 7-27

8.1. The Audit Information form under the Main Menu's "NEAT" button. ......................... 8-3

8.2. The Audit Information form under the Main Menu's "MHEA” button........................ 8-3

8.3. Dimensions associated with a manufactured home. ................................................. 8-8

9.1. The Walls form under the Main Menu’s "NEAT” button.......................................... 9-2

9.2. The Windows form under the Main Menu’s “NEAT” button. .....................................9-7

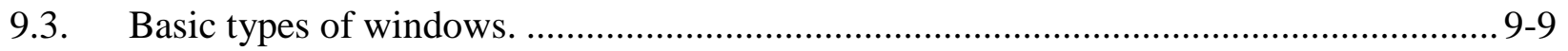

9.4. The Doors form under the Main Menu’s “NEAT”, button......................................... 9-15

9.5. The Unfinished Attics form under the Main Menu's “NEAT” button........................ 9-19

9.6. The Finished Attics form under the Main Menu's "NEAT" button. ............................ 9-25

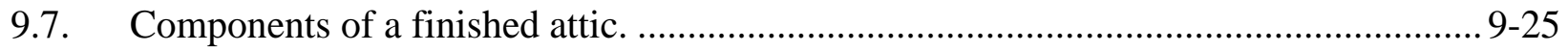

9.8. The Foundations form under the Main Menu's “NEAT” button................................. 9-27

9.9. The Heating form under the Main Menu's "NEAT" button...................................... 9-34

9.10. The Uninsulated Supply Ducts for Heating System sub-form.................................. 9-38

9.11. The Gas Furnace Details sub-form under the Main Menu's "NEAT” button............... 9-40

9.12. The Oil/Kerosene Furnace/Boiler Details sub-form under the Main Menu’s

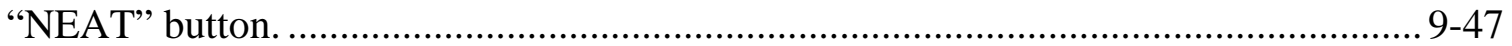

9.13. The Electric Resistance Heating System Details sub-form under the Main Menu’s "NEAT" button. ......................................................................................................... 9-48

9.14. The Heat Pump Details sub-form under the Main Menu’s “NEAT” button. ............... 9-51

9.15. The Space Heater Details sub-form under the Main Menu’s “NEAT” button............. 9-53

9.16. The Cooling form under the Main Menu's "NEAT" button...................................... 9-55

10.1. The Shell - Walls form under the Main Menu's "MHEA" button.............................. 10-2

10.2. Carport/Porch Roof Dimensions .............................................................................. 10-4

10.3. The Shell - Windows form under the Main Menu's "MHEA" button......................... 10-6

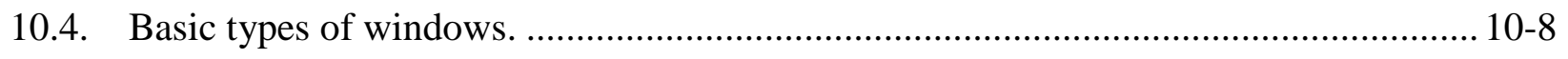

10.5. The Shell - Doors form under the Main Menu's "NEAT" button. ............................ 10-13 


\section{List of Figures}

10.6. The Shell - Ceiling form under the Main Menu's "NEAT" button. ......................... 10-16

10.7. Typical Manufactured Home Roof Configuration.................................................. 10-17

10.8. Mobile Home Cathedral Ceiling with Step Wall................................................... 10-19

10.9. The Shell - Floor form under the Main Menu's "NEAT" button. ............................ 10-20

10.10. Section of a Typical Mobile Home Floor. .......................................................... 10-21

10.11. Lengthwise and Widthwise Floor Joists. ......................................................... 10-22

10.12. Batt/Blanket Insulation Location...................................................................... 10-23

10.13. Typical Mobile Home Belly Configurations. ....................................................... 10-24

10.14. Addition of a Mobile Home.............................................................................. 10-26

10.15. The Addition - Walls form under the Main Menu's "MHEA” button....................... 10-27

10.16. The Addition - Windows form under the Main Menu's "MHEA" button.................. 10-30

10.17. The Addition - Doors form under the Main Menu's "MHEA" button. ..................... 10-31

10.18. The Addition - Ceiling form under the Main Menu's "MHEA" button.................... 10-32

10.19. The Addition - Floor form under the Main Menu’s "MHEA” button. ...................... 10-34

10.20. Dimensions of Mobile Home Addition................................................................ 10-35

10.21. Batt/Blanket Insulation Location......................................................................... 10-35

10.22. The Primary Heating System form under the Main Menu’s “MHEA” button........... 10-37

10.23. The Secondary Heating System form under the Main Menu's “MHEA” button........ 10-38

10.24. The Replacement Heating System form under the Main Menu’s “MHEA” button.... 10-38

10.25. The Primary Cooling System form under the Main Menu’s “MHEA” button............ 10-44

10.26. The Secondary Cooling System form under the Main Menu’s "MHEA” button........ 10-44

10.27. The Replacement Cooling System form under the Main Menu’s “MHEA” button.... 10-45

11.1. The Status form under the Main Menu’s "NEAT" button........................................... 11-2

11.2. The Air and Duct Leakages form under the Main Menu’s “NEAT” button. ................ 11-4

11.3. The Whole House Blower Door Measurements for Air Leakage sub-form................. 11-6

11.4. The Whole House Blower Door Measurements for Duct Leakage sub-form. .............. 11-9

11.5. The Blower Door Subtraction Measurements for Duct Leakage sub-form................ 11-11

11.6. The Duct Blower (Photo courtesy of The Energy Conservatory)............................. 11-13

11.7. The Duct Blower Measurements for Duct Leakage sub-form................................. 11-14

11.8. The Pressure Pan Measurements for Duct Leakage sub-form (MHEA only)............. 11-17 
11.9. The Optional Blower Door and Zonal Pressures form under the Main Menu’s "NEAT" button. $11-19$

11.10. The Zonal Pressures sub-form. $11-20$

11.11. The Pressure Pans sub-form. $11-21$

11.12. The Optional Pressure Balance form under the Main Menu's "NEAT" button. $11-22$

11.13. The Optional Pressure Pans form. $11-23$

11.14. The Water Heating form under the Main Menu's "NEAT" button. $11-24$

11.15. The Refrigerator form under the Main Menu's "NEAT" button. $11-29$

11.16. The Lighting Systems form under the Main Menu's "NEAT" button. $11-36$

11.17. The Health \& Safety - Whole House form under the Main Menu's "NEAT" button. 11-38

11.18. The Health \& Safety - Equipment form under the Main Menu's "NEAT” button...... 11-39

11.19. The Worst Case Draft Test for Heating System(s) sub-form. $11-40$

11.20. The Health \& Safety - Building Shell form under the Main Menu's "NEAT" button. $11-40$

11.21. Two checkboxes, one indicating a hazard. $11-41$

11.22. The Itemized Costs form under the Main Menu’s "NEAT” button..............................11-43

11.23. The Itemized Costs form under the Main Menu's "NEAT” button...............................11-43

11.24. The Itemized Costs form under the Main Menu's "NEAT" button...............................11-48

11.25. Data sheet view of the Itemized Costs form............................................................. 11-48

11.26. The Utility Bills form under the Main Menu's "NEAT” button. ................................. 11-50

11.27. The Photos form under the Main Menu's "NEAT” button.............................................. 11-54

11.28. The Measures form under the Main Menu's "NEAT” button....................................... 11-55

11.29. The Create Work Order sub-form..............................................................................11-58

11.30. The Create Work Order window..........................................................................11-58

11.31. The Optional Heating System Details buttons on the Heating form of the Main Menu's "NEAT" button (above) and Heating [Primary] form of the Main Menu's "MHEA" button (below).

11.32. The Operational Tests for Heating System sub-form. ................................................ 11-61

11.33. The Vent Tests for Heating System sub-form. ...........................................................11-61

11.34. The Other Components for Heating System sub-form. ............................................... 11-62

11.35. The Other Boiler Components for Heating System sub-form (NEAT only)................ 11-62 


\section{List of Figures}

11.36. The Inspections for Heating System sub-form.

$11-62$

11.37. The Thermostat Details for Heating System sub-form......................................... 11-62

11.38. The Optional Water Heater Details buttons......................................................... 11-63

11.39. The Operational Tests for Water Heater sub-form. ................................................ 11-64

11.40. The Vent Tests for Water Heater sub-form. ........................................................... 11-64

11.41. The Inspections for Water Heater sub-form. ......................................................... 11-64

12.1. The Introductory section of the Recommended Measures report.............................. 12-2

12.2. The Annual Energy and Cost Savings section of the Recommended Measures report.

12.3. The Energy Saving Measure Economics section of the Recommended Measures report

13.1. The Work Order Information form under the Main Menu’s "Work Orders” button..... 13-2

13.2. The Status form under the Work Order main Menu Item............................................ 13-7

13.3. The Measures form under the Main Menu’s "Work Orders” button (form view)......... 13-9

13.4. Message when copying a measure from Library Measures to a work order.

13.5. The "Materials Detail" sub-form displayed by selecting the "Show Audit Material Detail” button on the Work Order Measures form.

13.6. The Energy Savings for Measure: <Measure Name> sub-form.............................. 13-16

13.7. The Materials/Labor Details sub-form (form view) ............................................ 13-18

13.8. The Materials/Labor Details sub-form (data sheet view). .................................... 13-18

13.9. The Measures form under the Main Menu’s “Work Orders” button (data sheet view).

13.10. The Photos form under the Main Menu’s "Work Orders” button.

14.1. The Setup Library Information form under the Main Menu’s "Setup Library" button.

14.2. The NEAT Key Parameters - Economics form under the Main Menu's “Setup Library” button.

14.3. The NEAT Key Parameters - Set Points form under the Main Menu’s “Setup Library” button.

14.4. The NEAT Key Parameters - Insulation form under the Main Menu's "Setup Library” button 14-8

14.5. The NEAT Key Parameters - Equipment form under the Main Menu’s “Setup Library” button 
14.6. The NEAT Key Parameters - Windows form under the Main Menu’s "Setup Library” button. $14-11$

14.7. The MHEA Key Parameters - Economics form under the Main Menu’s "Setup Library” button. $14-13$

14.8. The MHEA Key Parameters - Set Points form under the Main Menu’s “Setup Library” button.

14.9. The MHEA Key Parameters - Insulation form under the Main Menu’s “Setup Library" button $14-15$

14.10. The MHEA Key Parameters - Heat Transfer form under the Main Menu's "Setup Library” button. $14-16$

14.11. The MHEA Key Parameters - Doors form under the Main Menu’s “Setup Library” button. 14-19

14.12. The MHEA Key Parameters - Windows form under the Main Menu’s "Setup Library” button..... 14-19

14.13. The MHEA Key Parameters - Base Loads form under the Main Menu's "Setup Library” button... $14-20$

14.14. The Fuel Costs form under the Main Menu’s "Setup Library” button. $14-21$

14.15. The Fuel Price Indices form under the Main Menu’s "Setup Library” button. $14-25$

14.16. The Library Measures form under the Main Menu’s “Setup Library” button. 14-26

14.17. The Unit Costs of Measure sub-form for the Attic Insulation R-30 measure. 14-29

14.18. The User Defined Measures form under the Main Menu's “Setup Library” button (with energy savings, Materials/Labor Details sub-form in form view)

14.19. The User Defined Measures form under the Main Menu’s “Setup Library” button (Health and Safety measure, Materials/Labor sub-form in data sheet view).

14.20. The User Defined Measures form (data sheet view, All Measures) (left-most columns). 14-33

14.21. The All User Measures Cost sub-form ................................................................... 14-38

14.22. The Materials/Labor Details sub-form (form view) ........................................... 14-39

14.23. The Materials/Labor Details sub-form (data sheet view) ....................................... 14-40

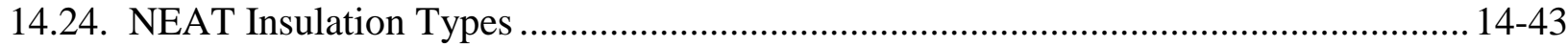

15.1. The General Information form under the Main Menu’s “Supply Library” button ........ 15-2

15.2. A typical form under Main Menu’s "Supply Library” button (form view).................. 15-5

15.3. The Construction Materials/Hardware Materials form (data sheet view) .................... 15-6

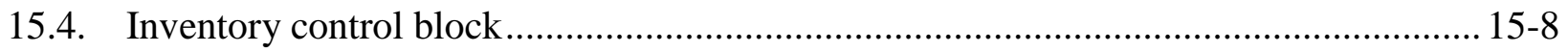




\section{List of Figures}

15.5. Inventory Purchase/Removal Summary ………........................................................ 15-8

15.6. Inventory Used Summary …………………....................................................... 15-8

15.7. The additional entries on the Hot Water Equipment and Refrigerator forms...............15-10

15.8. The Energy Details sub-form for Replacement Cooling Equipment .............................15-11

15.9. The Energy Details sub-form for Replacement Doors …………………………......... 15-12

15.10. The Energy Details sub-form for Replacement Heating Equipment ............................. 15-13

15.11. The Energy Details sub-form for Replacement Hot Water Equipment ......................... 15-14

15.12. The Energy Details sub-form for Insulation ................................................................ 15-14

15.13. The Energy Details sub-form for Replacement Lamps ………………………............ 15-15

15.14. The Energy Details sub-form for Replacement Refrigerators ...................................... 15-16

15.15. The Energy Details sub-form for Replacement Windows.........................................15-17

16.1. The General form under the Main Menu's "Preferences” button....................................16-2

16.2. Report displaying optional logo and header text ........................................................ 16-4

16.3. Range Check and Default Values form under the Main Menu's "Preferences"

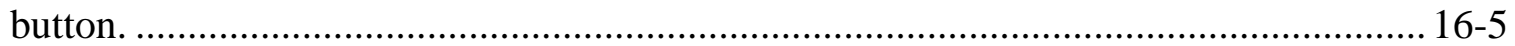

16.4. The Report Sections form under the Main Menu's "Preferences" button...................... 16-6

16.5. The Features form under the Main Menu's "Preferences" button................................... 16-7

16.6. Photo Folder field for third party photo browser ..........................................................16-8

16.7. Program Error History form.................................................................................... 16-10

17.1. The Data Link form .......................................................................................... 17-1

17.2. The "Currently Linked Backend Database" block..........................................................17-3

17.3. The Compact and Repair window.......................................................................... 17-4

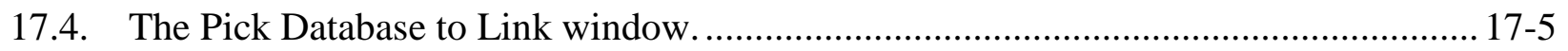

17.5. Import/Export via MSAccess Database (Transfer CLIENTS between databases) Check-in/Check-out feature activated (two clients selected for import). ........................ 17-7

17.6. The Pick External Database for Import/Export window................................................... 17-9

17.7. The WARNING: Overwrite with older data? window............................................... 17-12

17.8. The Transfer between databases form (via Text Files)................................................. 17-16

17.9. Browse window for selecting files to import via text files........................................ 17-17

17.10. Example "CSV" file for heating system records. ........................................................ 17-18

17.11. Exporting Clients (via text files) ............................................................................... 17-21 


\section{List of Figures}

17.12. The Client Selector form (GIS feature on) ...................................................... 17-25

17.13. The Sort/Filter menu window. .......................................................................... 17-26

18.1. Tab for pathname method of photo storage. ........................................................ 18-1

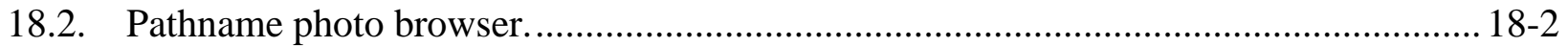

18.3. Controls on Information tab for third party photo browser. ....................................... 18-3

18.4. Third party photo browser navigation window.................................................... 18-4

18.5. Editor of third party photo browser. ..................................................................... 18-5

18.6. GIS Map for Single Client Location..................................................................... 18-7

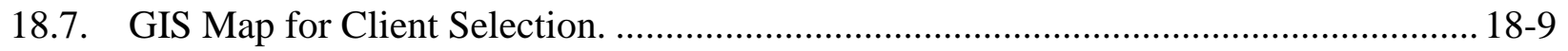

18.8. The Status Editor sub-form............................................................................ 18-13

18.9. E-mail specification form for reporting error message......................................... 18-15

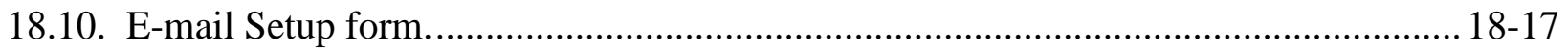




\section{Acronyms}

AFUE annual fuel utilization efficiency

ASHRAE American Society of Heating, Refrigerating, and Air- Conditioning Engineers

Btu British thermal unit

COP coefficient of performance

csv comma separated values (file format)

DOE Department of Energy

GIS Geographic Information System

GUI graphical user interface

HDD heating degree days

HSPF heating seasonal performance factor

MB megabytes

MHEA Manufactured Home Energy Audit

MMBtu million British thermal units

NEAT National Energy Audit Tool

NFRC National Fenestration Rating Council

NOAA National Oceanic and Atmospheric Administration

RAM random access memory

SHGC solar heat gain coefficient

SIR savings-to-investment ratio

SP2 service pack 2 (Microsoft)

UPW uniform present worth 


\section{Chapter 1. Overview of the Weatherization Assistant}

The Weatherization Assistant is a Windows-based energy audit software tool that was developed by Oak Ridge National Laboratory (ORNL) to help states and their local weatherization agencies implement the U.S. Department of Energy (DOE) Weatherization Assistance Program. It applies engineering and economic calculations to assist states and agencies select energy efficient retrofit measures that meet government criteria for cost-effectiveness and that can be installed in homes of low-income families enrolled in the Program. The Weatherization Assistant can be used to select and rank measures for individual houses, or to establish a priority list of weatherization measures for nearly identical housing types.

The Weatherization Assistant is actually an umbrella program for two individual energy audits or measure selection programs (see Figure 1.1): the National Energy Audit Tool (NEAT) and the Manufactured Home Energy Audit (MHEA). NEAT is designed specifically for site-built single-family homes, although it can be used with some limitations for small multifamily residences. MHEA is designed specifically for manufactured (or mobile) homes. The unique construction characteristics of mobile homes require evaluating and installing measures specifically for such homes in order to obtain effective weatherization with high energy and dollar savings.

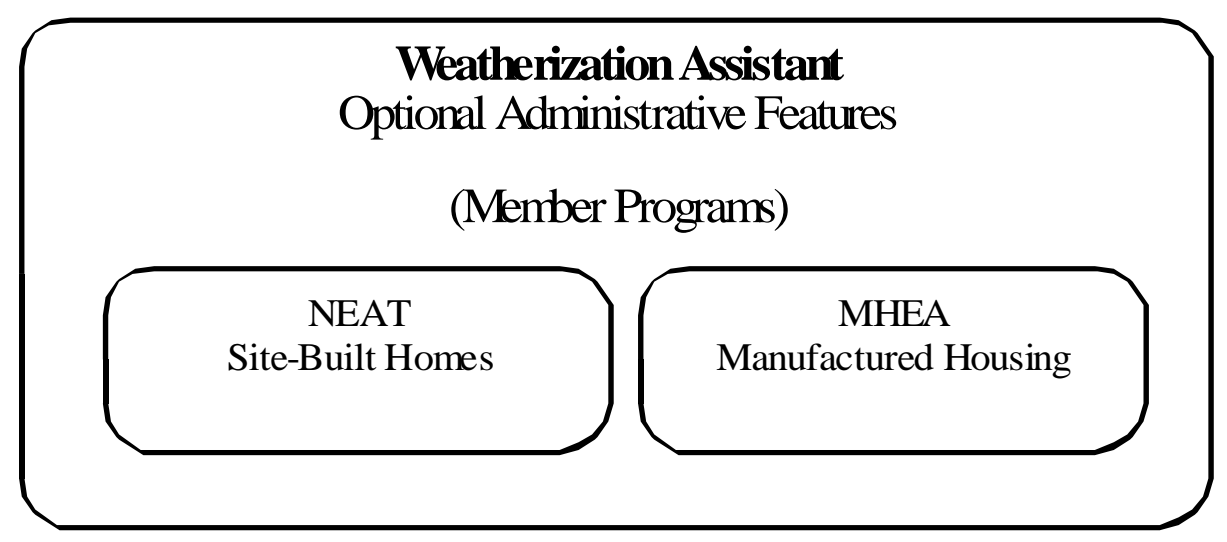

Figure 1.1. Design of the Weatherization Assistant.

In addition to serving as the umbrella program for NEAT and MHEA, the Weatherization Assistant provides optional features that are useful in implementing and administering weatherization programs. These optional features include recording an extensive amount of agency-related contact information and client 


\section{Chapter 1: Overview of the Weatherization Assistant}

intake data, developing work orders, recording diagnostic measurements and health and safety issues, storing digital photos and documents, using an internal map to locate or select clients, tracking the status of clients and jobs, tracking available funds and total expenditures, tracking inventory, and generating summary and analysis reports.

Installation of the Weatherization Assistant provides users with both of the measure selection programs as well as the optional administrative features. Installation procedures are provided in Chapter 2, Installation and Start-up.

\subsection{Overview of NEAT and MHEA}

NEAT was developed by ORNL. It was formally introduced in the summer of 1993 and has been modified and upgraded many times since. MHEA, on the other hand, was originally developed by the National Renewable Energy Laboratory (NREL). ORNL converted this original version of MHEA to a Windows-based program, packaged it with NEAT under the Weatherization Assistant umbrella, and now maintains and supports the software.

NEAT and MHEA evaluate each home individually after taking into account local weather conditions, weatherization measure costs, fuel costs, and specific construction details of the home. After describing envelope components, heating and cooling systems, and base load equipment (e.g., refrigerators, water heaters, lighting), NEAT and MHEA produce a prioritized list of cost-effective weatherization measures customized for the dwelling being evaluated. The output includes estimates of the dollar value for the projected energy savings, savings-toinvestment ratios (SIRs), installation costs, a list of the quantities of the major materials necessary to perform the recommended weatherization retrofits, and design heating and cooling loads needed to size any replacement equipment.

MHEA stands apart from other building energy analysis tools in many ways. Input and calculations incorporated into the software address constructions unique to mobile homes such as bellied floors and bowstring roofs. The retrofit measures evaluated by MHEA are all applicable to mobile homes.

NEAT and MHEA follow eight steps to select the energy efficiency measures for a particular home that meet a designated level of cost-effectiveness (i.e., measures that have an SIR greater than a specified value): 
1. NEAT and MHEA guide the user through the process of entering data on the home that describe the characteristics of the home, its mechanical systems, and other energy-related information. NEAT and MHEA also allow health and safety deficiencies and repair items to be noted. Both audits use blower door measurements, duct leakage measurements, and steady-state efficiency measurements of space-heating equipment, if available. Data may be collected in the field using data input forms and transferred to NEAT or MHEA later in the office, or data may be entered directly into NEAT or MHEA using a portable computer while auditing the home.

2. NEAT and MHEA use engineering calculations and weather data from over 200 weather cities in the continental United States and bordering provinces of Canada to compute the annual heat loss and heat gain of the home, and the annual space-heating and space-cooling energy consumption required to keep the home at a specific thermostat set-point. Both audits calculate heat loss and heat gain on a monthly basis using a variable-base degree-day method and tenyear average weather data for the selected city. They also consider the amount of solar energy absorbed by a home and the typical amount of heat generated inside a home by people and their refrigerators, water heater, other appliances, and lights. NEAT and MHEA estimate the heat gain and loss due to duct leakage if specified. NEAT and MHEA assume a home is maintained at average conditions regardless of specific occupants because efficiency measures typically remain after occupants move, and this follows the intent of the Weatherization Assistance Program that finances energy efficiency measures that generate savings over the life of the measures.

3. NEAT and MHEA compute the energy consumption of selected base load uses (water heating, refrigerators, and lighting in need of retrofit) if desired. Refrigerator and water heater descriptions use an extensive database of manufacturers and models, which eliminates the need to input detailed equipment descriptions by the user.

4. NEAT checks the applicability of 38 building envelope, space-heating and space-cooling system, and base load energy efficiency measures to the specific home being audited, while MHEA checks the applicability of 32 measures. These measures include air and duct leakage reduction, envelope insulation, window replacements and other treatments, space-heating and space-cooling equipment replacement and tune-up, replacement refrigerators, water heater 


\section{Chapter 1: Overview of the Weatherization Assistant}

tank and pipe insulation, replacement lighting, and more. Both audits then calculate an energy savings and discounted SIR for each applicable measure applied individually to the home. User-defined energy efficiency measures can also be entered and evaluated. The SIRs are calculated using fuel costs and installation costs representative of the home and agency as input by the user, as well as measure lifetimes appropriate for each measure.

5. NEAT and MHEA evaluate the interaction between efficiency measures (e.g., since insulation reduces the amount of energy needed for space heating, it also reduces the energy savings from a space-heating system replacement). Beginning with the measure with the highest SIR, NEAT and MHEA apply each energy efficiency measure to the home and then compute a new SIR for the remaining measures, taking into account savings gained by preceding measures.

6. NEAT and MHEA identify a final list of recommended energy efficiency measures by selecting those with an SIR greater than the cut-off value selected by the user once interactions between measures have been accounted for.

7. NEAT and MHEA generate a report of recommended energy efficiency measures that identifies, both individually and cumulatively, the energy savings, installation cost, and SIR of the recommended measures. The report also identifies those health, safety, and repair items selected by the user that need to be performed. In addition, an essential materials list is developed, and design heating and cooling loads needed to size replacement equipment are provided.

8. NEAT and MHEA can adjust their estimated energy savings based on actual utility consumption data and develop a second list of recommend energy efficiency measures if the user desires.

\subsection{Software Overview}

The Weatherization Assistant utilizes a commercially produced database software, Microsoft Access, to provide a user interface for data input and to store the information you provide and the results of running the program. The analysis engines (NEAT and MHEA) are written in the programming language "C." The Microsoft Access portions of the program are linked to the analysis engines by 


\section{Chapter 1: Overview of the Weatherization Assistant}

$\mathrm{C}++$ and Visual Basic programs. However, the Weatherization Assistant requires no software associated with any of these languages or applications for normal use.

The Weatherization Assistant features a Windows graphical user interface. Data input is provided to the program through Microsoft Access forms, which can be used in either "form" view (data are displayed on forms that are filled in) or "data sheet" view (as would be seen in a spreadsheet application).

The Weatherization Assistant stores all of its input and output information in a single database file (often called the "mdb database file" or just the "mdb file" because the file's extension is ".mdb"). Within this file, information is stored in linked tables, each table composed of as many "records" as is required to store the information required. For example, when you enter information on a new client by filling in data on a form, you are adding a new record to the client table. Information is retrieved simply by requesting to view different records in the database. You may already be familiar with the concept of tables, records, and forms if you have used other software products based on Microsoft Access.

With data stored in Access tables, persons experienced in Access can retrieve the information and import it to other Access applications without having to re-enter data. In addition, the Weatherization Assistant can produce and read comma separate value (csv) files containing data, which provides an additional mechanism for data transfer between computer applications. This data transfer capability provides the potential for collecting or storing all weatherization program data into state-wide or nationwide databases that could then be used in program evaluation or lead to better implementation processes.

\subsection{Program Overview}

The Weatherization Assistant divides its tasks into several main activities. These activities are accessed using buttons found on the initial form, called the Main Menu, which appears when opening the program (see Figure 1.2). Throughout this manual, these main activities are referenced by the names given to these buttons as seen on this Main Menu. 


\section{Chapter 1: Overview of the Weatherization Assistant}

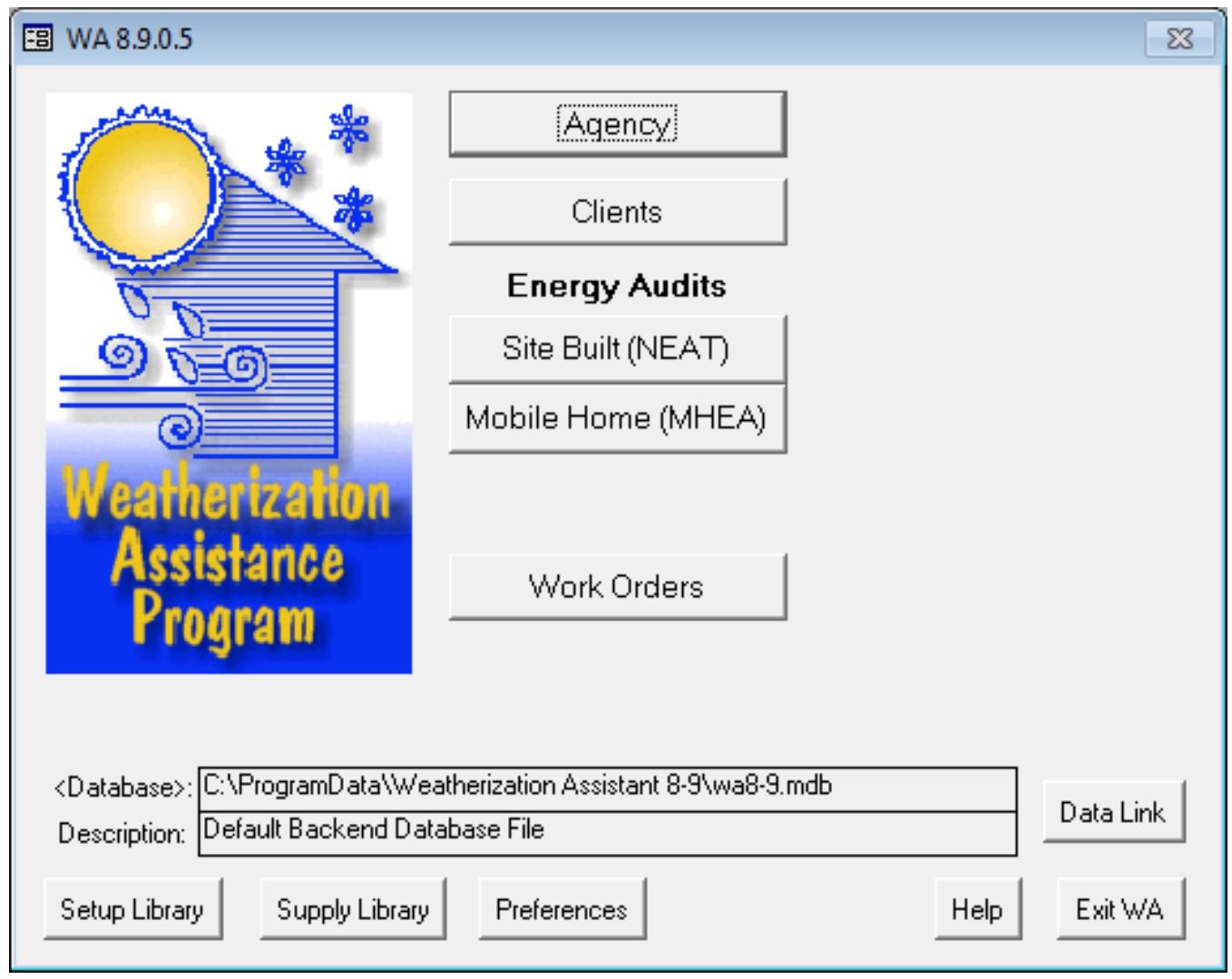

Figure 1.2. The Weatherization Assistant's Main Menu.

Forms under the Main Menu’s “Agency” button (see Chapter 6, Agency) allow you to enter information about your agency, your staff and their functions, and the contractors and suppliers used by your agency. You can list the sources of funding available for your work with balances of funds available in each, and you can also see and navigate to all the clients, audits, work orders, setup libraries, and supply libraries that are associated with your agency.

Forms under the Main Menu's “Clients” button allow you to enter information about the clients that your agency serves. The Weatherization Assistant keeps a listing of your clients separate from the descriptions of the homes of those clients. Minimal information is required to identify a client, but there is a considerable amount of optional data that you can enter if you desire. You must supply at least the minimal data on a client before you can continue and perform an audit on the client's home or continue entering data for other clients. Under the Main Menu's "Clients" button, you can also see and navigate to all the audits and work orders that are associated with that client. A detailed discussion of data entered under the Main Menu's “Clients” button is given in Chapter 7, Clients. 
Forms under the Main Menu's "Site Built (NEAT)" and "Mobile Home (MHEA)" buttons allow you to describe a client's home and enter other audit information once a client has been entered into the Weatherization Assistant. It is this home description that allows the Weatherization Assistant to make retrofit recommendations for improving the energy efficiency of the home. After all the house information has been entered and the audit has been run, you can view and print the audit recommendations, as well as prepare for the optional step of initiating work orders to install the recommendations. Data entered under the Main Menu's “NEAT” and "MHEA” buttons are fully described in Chapters 8-12.

Forms under the Main Menu's “Work Orders” button provide an optional feature that allows you to develop work orders needed for weatherization measures to be installed in a home. Work orders may be automatically generated by the execution of either NEAT or MHEA, developed manually (e.g., if the measures to be installed were recommended based on a predefined priority list rather than running NEAT or MHEA), or created by a combination of the two. The level of detail included in the work orders is determined by the user. Material descriptions and associated costs pre-entered in the Supply Library can be used to make the development of the work orders easier, faster, and more consistent. The use of work orders combined with the use of the Weatherization Assistant's optional inventory feature can help you track and maintain inventory. A detailed description of the optional work order feature is provided in Chapter 13, Work Orders.

Forms under the Main Menu's “Setup Library” button allow you to set up the Weatherization Assistant to reflect conditions in your agency. The parameters entered in the Setup Library affect the recommendations of the audits, although they are not a part of the individual home descriptions. Parameters that are entered in the Setup Library include fuel costs; identification of the weatherization measures predefined in NEAT and MHEA that your agency performs; material and labor costs associated with these weatherization measures; and values of selected key parameters associated with materials, equipment, thermostat set-points, etc. The Setup Library also allows you to enter your own weatherization measures and insulation materials as alternatives to those already programmed into NEAT and MHEA. Detailed discussions of the forms under the Main Menu's "Setup Library" button are provided in Chapter 14, Setup Library.

Forms under the Main Menu's "Supply Library” button allow you to identify or select materials and enter details on each material such as cost, R-value for 


\section{Chapter 1: Overview of the Weatherization Assistant}

insulation materials, etc. Entering materials into the Supply Library is most useful when you are going to develop work orders, as described under the Main Menu's "Work Orders" button. In this case, having a library of materials to choose from makes it easier to create a work order. If you are not going to use the work order feature, then the only materials that you may want to enter in the Supply Library are new refrigerators and water heaters that you use to replace existing units (if you perform these measures). The forms under the Main Menu's "Supply Library" button are discussed in Chapter 15, Supply Library.

Forms under the Main Menu's "Preferences” button allow you to choose various options affecting the appearance of the Weatherization Assistant or the operation of its interface. These options do not affect the calculations or the recommendations of NEAT or MHEA themselves. These options include the appearance of headers that are printed on the first page of all reports, the values used to perform range checks on every numerical data input field, and the ability to turn on or off optional features in the Weatherization Assistant. All of the options that can be controlled under the Main Menu's "Preferences" button are discussed in Chapter 16, Preferences.

Forms under the Main Menu's “Data Link” button allow you to transfer data with other users of the Weatherization Assistant or even with other database or analysis programs. The Data Link feature also allows you to choose which database you are connected to, although most users will only use the one database provided as part of the initial installation of the Weatherization Assistant. Note that on the Main Menu, the database file to which you are currently connected is displayed. In Figure 1.2, this file is "wa8-9.mdb" which is located in the folder "C:IProgram DatalWeatherization Assistant 8-9.” The use of the Data Link feature is presented in Chapter 17, Data Link.

\subsection{Optional Features}

The optional features in the Weatherization Assistant were incorporated into the program in such a way as to allow you to use the measure selection programs (NEAT and MHEA) almost as stand-alone programs, if you are not interested in using the optional features. This is why most of the optional features are accessed from their own tabs under the various Main Menu buttons (with the exception of work orders, which has its own Main Menu button and was described previously), and why there are separate buttons on the Main Menu of the program to take you 
directly to either NEAT or MHEA. In addition, the optional features are, for the most part, independent of one another. This allows you to pick and choose which optional features to use in a phased approach.

The Weatherization Assistant allows an extensive amount of diagnostic measurements and health and safety issues to be recorded within the NEAT and MHEA audits. NEAT and MHEA do not use most of these measurements and observations directly in their calculations. However, by recording these measurements and observations within the Weatherization Assistant, they become a permanent part of the electronic record for a house. Also, repairs can be automatically recommended to fix any identified health and safety issues if you set the Weatherization Assistant up to do so. Chapter 11, NEAT and MHEA Common Elements, discusses how to record diagnostic measurements and health and safety issues.

The Weatherization Assistant provides the capability of linking digital photos taken at any stage of the weatherization process or digital scans of additional documents to a client, audit, and/or work order. This can be useful to more clearly identify weatherization or repair items that need to be performed, or to document pre-existing conditions that will not be addressed during weatherization. This capability is discussed in Section 18.1, Digital Photos.

A Geographic Information System (GIS) can be installed with the Weatherization Assistant that allows mapping of a single client's location or any group of clients at once. This allows auditors, weatherization crews, inspectors, and others to find the location of a client's home, and could assist in scheduling efforts or in creating summary reports by geographic location. The GIS is described in Section 18.2, Geographic Information System (GIS).

A relatively extensive client status tracking system is incorporated in the Weatherization Assistant. This system allows an agency to track the overall status of a client as well the client's application, audit, work orders, inspections, and payments if desired. Section 18.3, Status Tracking, discusses how to use this system.

Although the Weatherization Assistant already provides many data fields under the Main Menu's “Agency" and "Clients" buttons for entering a wide range of information on clients and their houses, agencies and states often collect even more 


\section{Chapter 1: Overview of the Weatherization Assistant}

information. The Weatherization Assistant provides the capability of including this additional information in its database. User-generated survey or data forms can be developed under the Main Menu's “Agency” button (see Section 6.4, Surveys (Agency)), with the responses or data for each individual client stored and retrieved in reports under the Main Menu's “Clients” button (see Section 7.7, Surveys (Client)).

The Weatherization Assistant provides a tool to assist weatherization staff in tracking available funding provided that the work order feature is used. You can create cost centers (funding sources) and record the receiving of funds under the Main Menu's “Agency” button (see Section 6.3, Cost Centers). The funding balances of each of the cost centers will then be automatically updated as work orders are completed and actual costs are recorded under the Main Menu's "Work Orders” button (see Section 13.3, Measures (Work Order)).

The Weatherization Assistant provides predefined reports that summarize and analyze the information you have entered. Different reports are available and accessed under each of the Main Menu buttons. The primary report used in the Weatherization Assistant is the Recommended Measures report that summarizes the recommendations of a NEAT and MHEA audit (see Chapter 12, NEAT and MHEA Results). Many other reports are available. For example, reports are also available that document the data and information you entered into each NEAT or MHEA audit, list the cost of measures you entered in the Supply Library, summarize the work performed on a house, or identify all the houses weatherized between selected dates along with their final costs. If you are an experienced user of Microsoft Access, you can even develop your own reports as outlined in Appendix D, Development of Customized Reports. 


\section{Chapter 2. Installation and Start-up}

The Weatherization Assistant includes the two measure selection programs, NEAT for site-built homes and MHEA for mobile homes, as well as an umbrella of optional features addressing other tasks performed by agency personnel as part of the weatherization process. The instructions provided in this chapter will install all of these tools onto your computer. Instructions are also provided in this chapter on how to install the separate files needed to use the Weatherization Assistant's GIS to map the location of client homes, how to obtain the Weatherization Assistant User's Manual, and how to start the program.

\subsection{Hardware and Software Requirements}

The Weatherization Assistant must be installed on a Windows-based personal computer that has at least 100 megabytes (MB) of free random-access memory (RAM). The installation will require at least $150 \mathrm{MB}$ of available hard disk space. Your graphic card and monitor should be able to produce a display with a minimum resolution of $1024 \times 768$ pixels in order to allow the Weatherization Assistant's windows to be seen without scrolling.

The Weatherization Assistant needs only a Windows operating system (NT4.0, Win2000, XP, Vista, Windows 7 or Windows 8), which normally comes with the purchase of a computer. NT4.0 and Win2000 must have at least the service pack 2 (SP2) upgrades installed in order to ensure proper operation of the Weatherization Assistant. The upgrades are available through Microsoft, typically by using their Windows Update feature.

The Weatherization Assistant uses your existing Windows printer setup to both display and print results and reports. Thus, even though you may not have a printer physically attached to your computer, you must have an acceptable printer driver loaded and selected as the default in order to display the results. Output produced by the Weatherization Assistant is stored in its database file and displayed in richtext format from within the program.

\subsection{Obtaining the Weatherization Assistant Software}

The Weatherization Assistant software is copyrighted and cannot be altered in any way beyond the bounds set within the program. The latest version of the 


\section{Chapter 2: Installation and Start-up}

Weatherization Assistant is Version 8.9.0.5. All releases of Version 8.9 of the Weatherization Assistant are designated by four digits (i.e., 8.9.x.x), where the third digit indicates the update number and the fourth digit indicates the maintenance number.

The installation file for the latest release of Version 8.9 of the Weatherization Assistant (WA 8-9-0-5.exe) can be downloaded over the Internet from either the Weatherization Assistance Program Technical Assistance Center (WAPTAC) or ORNL web sites:

- http://waptac.org/Energy-Audits/Weatherization-Assistant.aspx and then select the "Weatherization Assistant 8.9" hyperlink, or

- $\quad$ http://eber.ed.ornl.gov/pub/weatherization and then select the "Weatherization Assistant 8.9” folder.

Right click your mouse on the "WA 8.9.0.5" hyperlink on the WAPTAC site or the "WA 8-9-0-5.exe" file from the ORNL site. Select "Save target as..." from the menu displayed. You will be presented with a "Save As" window asking where on your computer you wish to save the "WA 8-9-0-5.exe" file. Choose an existing or new location (folder) on your computer to save the file in. You might select your "Desktop" to allow easy access. You are encouraged to leave the "File name" as the default automatically entered in this field (i.e., "WA 8-9-0-5.exe"). Once the folder has been selected or created, select the "Save" button to begin the download, which may take several minutes if you are not using a high-speed Internet connection. After completion of the download, you may exit your Internet browser or download the GIS files and/or the manuals for the Weatherization Assistant (see Section 2.5, Obtaining and Installing the GIS Files).

The "WA 8-9-0-5.exe" file will not be needed for normal operation once it has been executed to install the Weatherization Assistant. However, you would need the file if you ever needed to re-install the program. Thus, it is recommended that you eventually save the file in a safe location, possibly in the folder created during

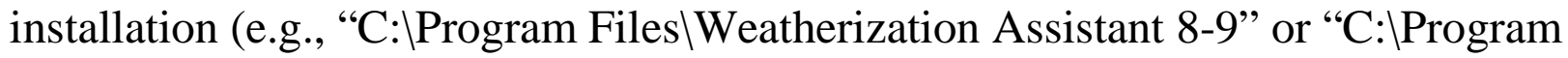
DatalWeatherization Assistant 8-9”). 


\subsection{Installing the Weatherization Assistant}

If your computer is configured to allow only certain users, normally a system administrator, to install software, then this user will have to be contacted in order to install the Weatherization Assistant. If you should attempt installation under such circumstances without proper authorization, you will receive messages indicating either the need for "administrative privileges" or that you do not have "write access" to certain locations on your computer.

If this is the first installation of the Weatherization Assistant program on your computer or you are upgrading from a previous version other than Version 8.9, then the Weatherization Assistant's installation procedure will automatically install Version 8.9 as a "new installation." A "new installation" will install Version 8.9 of the Weatherization Assistant in its own folder on your computer (the default folder is "C: Program Files $\backslash$ Weatherization Assistant 8-9" for a computer with an XP operating system or "C: ProgramDatalWeatherization Assistant 8-9" for a computer with a later operating system), provide you with a unique icon on your desktop with which to start Version 8.9 of the program, and give you a database file to store all your agency, client, audit, and other information. If earlier versions were previously installed, they will remain on your computer and will be fully functional.

If a previous release of Version 8.9 is already installed on your computer, then it is recommended that you install the latest release of Version 8.9 as an "update," although you have the option to install the latest release as a "new installation" if desired. Installation of the latest release of Version 8.9 as an "update" will install the latest release in the same folder as your previous release of Version 8.9, use the same desktop icon to start the Weatherization Assistant, and use the same database file as the previous release of Version 8.9. All the information entered in your previous release of Version 8.9 on your agency, clients, and audits and in your Setup and Supply Libraries will remain unchanged. ${ }^{1}$

\footnotetext{
${ }^{1}$ A change in only the fourth digit of the latest release indicates a change in the program that should not affect the program's output or recommendations (e.g., a new report was added). However, if the third digit in the latest release is different than your previous release, then a modification has been made to the program that could affect the program's output (e.g., savings or cost of a measure). Therefore, once the latest release is installed, rerunning an audit that was previously run under the previous release could result in changes in the Recommended Measures Report (e.g., a different list of recommended measures; different energy savings, costs, and/or SIR for a recommended measure). Once the latest release is installed, the Recommended Measures Report generated under the
} 


\section{Chapter 2: Installation and Start-up}

Before installing Version 8.9 of the Weatherization Assistant on your computer, close all Windows programs that are running. Then, to initiate the installation process, use Windows Explorer or My Computer to locate the "WA 8-9-0-5.exe" file that you downloaded to your computer and then double click on it. If the "WA 8-9-0-5.exe" file was downloaded to your desktop, simply double clicking on the file's icon on your desktop will initiate the process.

A welcome screen for the Weatherization Assistant Install program appears (see Figure 2.1). Select "Next," and then select "I Agree" after reading the Disclaimer Statement (see Figure 2.2).

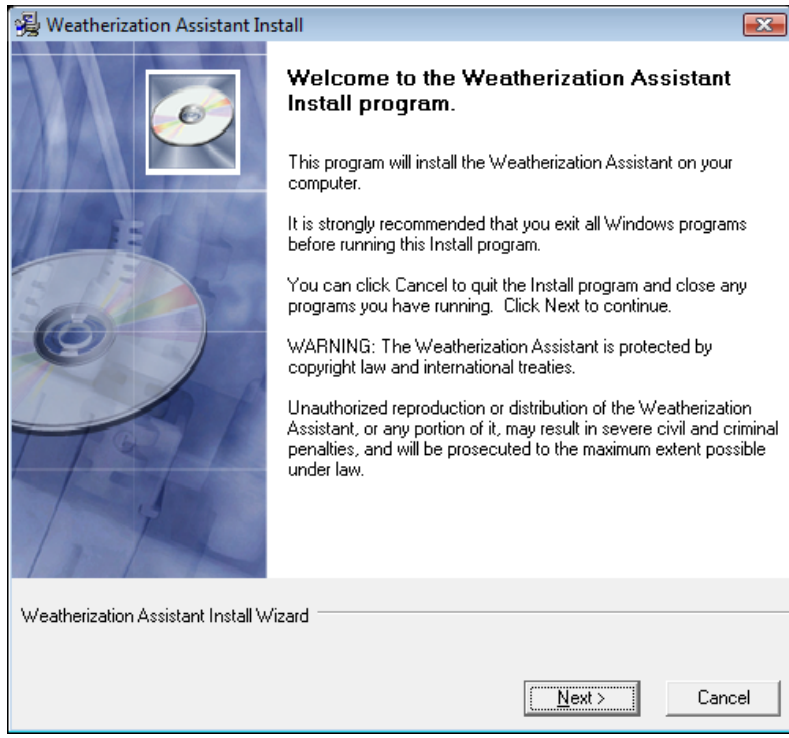

Figure 2.1. Welcome screen.

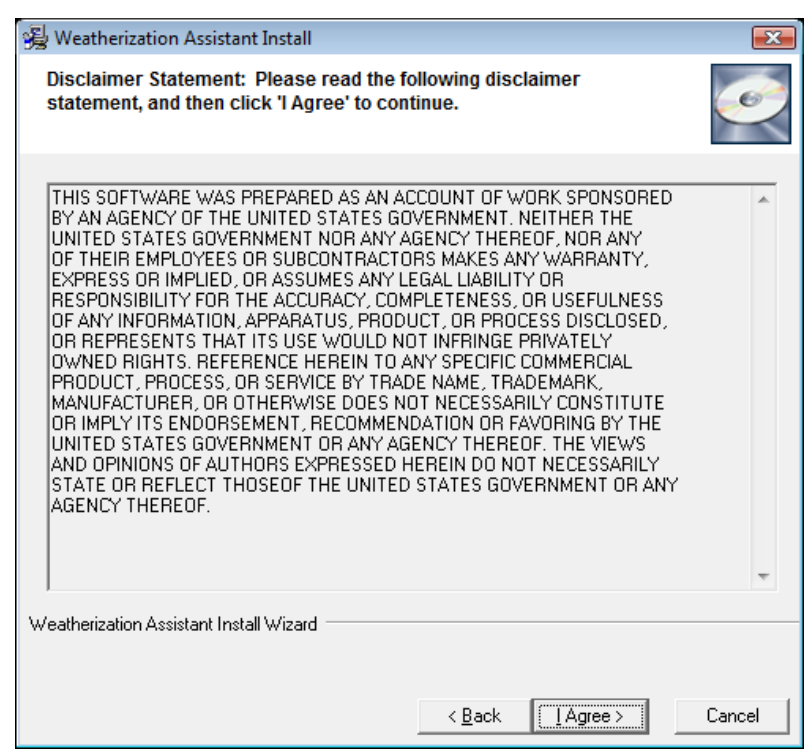

Figure 2.2. Disclaimer Statement screen.

The Destination Folder screen that appears identifies if a previous Version 8.9 was found on your computer and where the new release of Version 8.9 will be installed (see Figure 2.3 and Figure 2.4). It is recommended that you install the new release in the folder selected by the installer: "C: $\mathrm{P}$ Program Files\Weatherization Assistant 8-9” or "C: ProgramDatalWeatherization Assistant 8-9" for a "new installation," or the folder where the previous release of Version 8.9 is installed in the case of an "update.” However, the installation routine does allow you to select another folder if you desire. If you do choose a folder other than the default, make sure it is

previous release can be viewed without rerunning the audit by selecting the Recommended Measures report in the Report block in the lower right corner of the NEAT or MHEA Audit Information forms. If you want to rerun the audit using the latest release but keep the recommendations generated under the previous release, you should make a copy of the audit and then run the audit on the copied version (do not run the audit on the original version of the audit because this will write over the recommendations generated under the previous release). 


\section{Chapter 2: Installation and Start-up}

different from the folder of any other existing version of Weatherization Assistant. This will ensure that any building descriptions entered with a previous version will remain undisturbed.

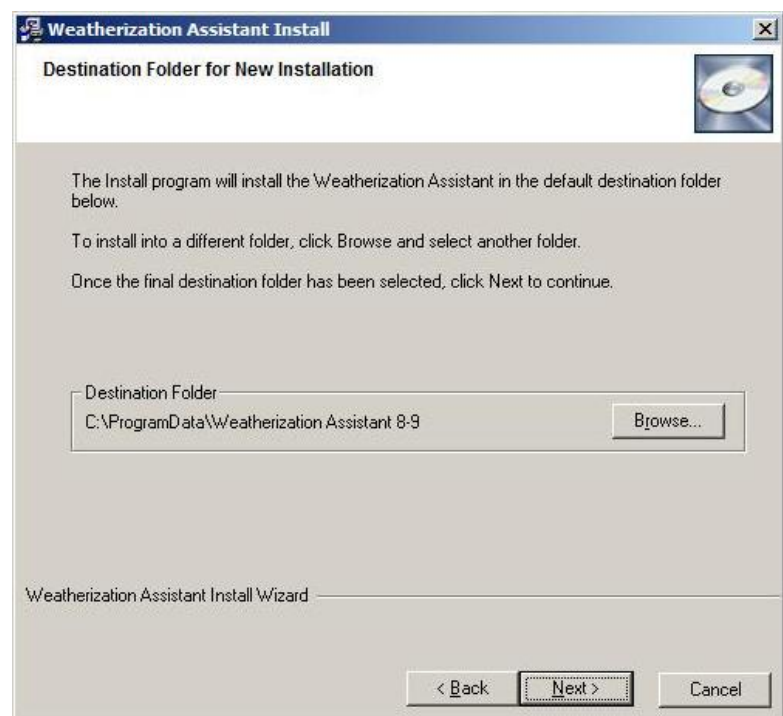

Figure 2.3. Destination Folder screen for a "New Installation."

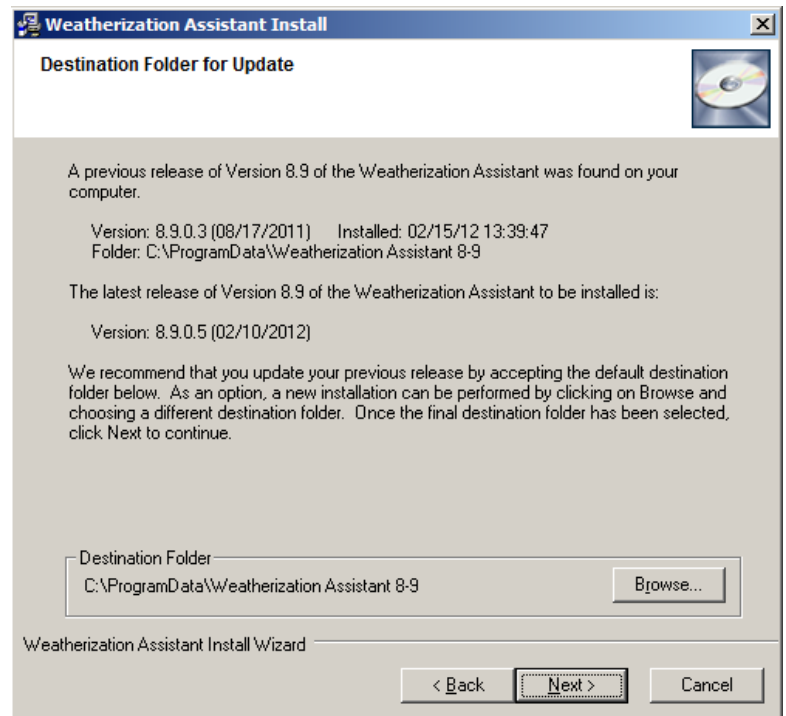

Figure 2.4. Destination Folder screen for an "Update."

Once the destination folder has been selected, click "Next" to obtain the Start Installation screen (see Figure 2.5). This screen confirms the release of Version 8.9 that will be installed. Click "Next" to start the installation of the actual Weatherization Assistant files.

The installation may take a minute or two. During the installation, several progress screens will appear that display the installation status. If the installation was successful, a final screen appears stating that the latest release was installed successfully (see Figure 2.6). Select "Finish" to complete the installation and exit the install program. 


\section{Chapter 2: Installation and Start-up}

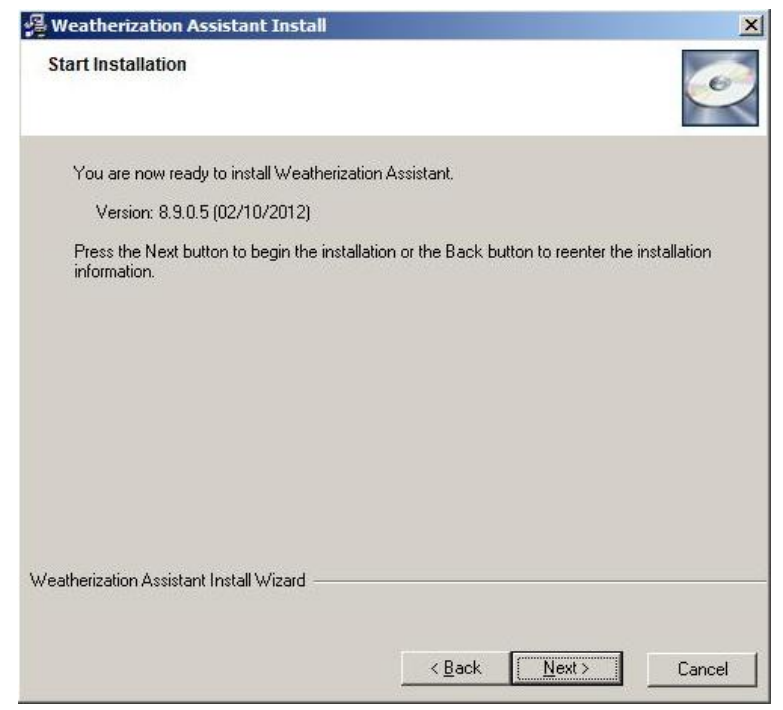

Figure 2.5. Start Installation screen.

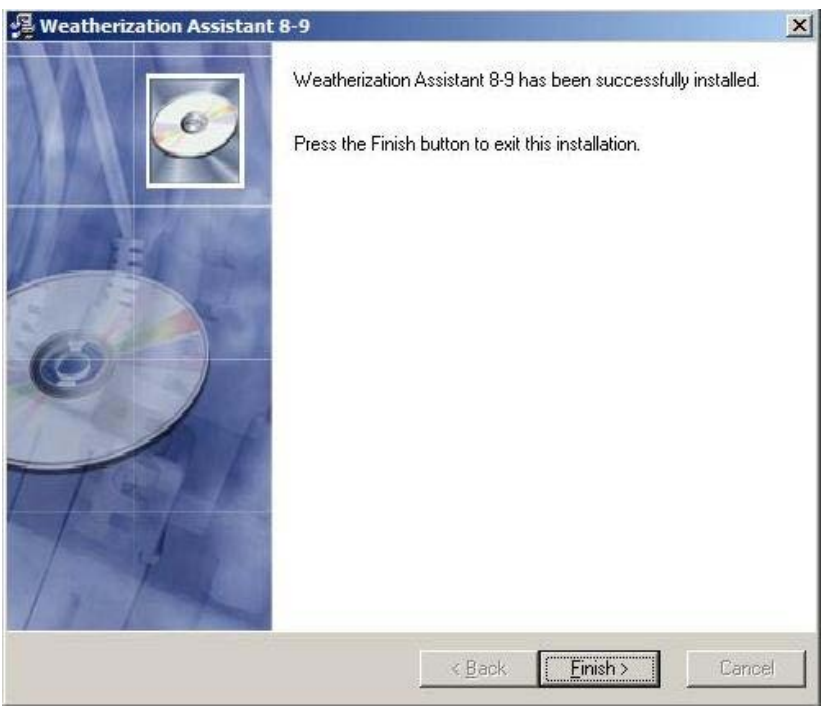

Figure 2.6. Finish screen.

If a "New Installation" was performed, the installation process will have placed a new icon on your desktop (see Figure 2.7). This icon will be called "WA 8.9" if no previous versions of Version 8.9 were on your computer and "WA 8.9.x.x" if there was. To run Version 8.9 of the Weatherization Assistant, just double click on this new icon. However, you should consult Chapter 3, Setting Up the Weatherization Assistant, to properly set up the program before proceeding with its

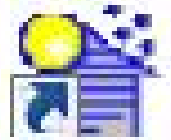

WA 8.9 use.

If an "Update" was performed, the installation process will have linked the icon used to run the previous release to the latest release. As before, just double click on this same icon to run the latest release of Version 8.9.

\subsection{Network Installation}

If you are installing the Weatherization Assistant on a network, you need to:

- Install the Weatherization Assistant on each of the client computers. The program will then be run individually from each client computer. 
- Copy one of the "wa8-9.mdb" files from a client computer to a shared folder on the network computer. Make sure that the shared folder has READ, WRITE, and CREATE permissions for all users, and that the "wa8-9.mdb" file in the shared folder has READ and WRITE permissions. The "mdb" files may be renamed, in order to prevent the confusion resulting from files of the same name, as long as each retains the "mdb" extension.

- Run the Weatherization Assistant on each client computer and use the Data Link feature on the Main Menu to link each client computer to the shared "wa89.mdb" file on the network computer (see Section 17.1, Currently Linked Backend Data File).

An alternate method of sharing information among multiple computers is to use the Import/Export feature of the program. See Section 17.3, Data Transfer Import/Export with Another MSAccess Database, for more details.

\subsection{Obtaining and Installing the GIS Files}

The GIS feature in Version 8.9 of the Weatherization Assistant allows the location of client homes to be mapped (see Section 18.2, Geographic Information System (GIS)). Before this optional feature can be used, you must first download and then unzip two files that are available only over the Internet (due to copyright restrictions) from the same two web sites used to obtain the program files for the Weatherization Assistant:

- http://waptac.org/Energy-Audits/Weatherization-Assistant.aspx and then select the "GIS Mapping" hyperlink, or

- http://eber.ed.ornl.gov/pub/weatherization and then select the "GIS Files" folder.

Download the "base.zip" file and the zip file for your state (i.e., "xx.zip" where xx is the two letter abbreviation for your state) following the same steps used to download the latest release of the Weatherization Assistant (see Section 2.2, Obtaining the Weatherization Assistant Software). These downloads may take several minutes if you are not using a high-speed Internet connection. 


\section{Chapter 2: Installation and Start-up}

After downloading the two files to your computer, right click on each zip file and choose "Extract All..." Select the destination for the extracted files to be the GIS folder under the location where you installed the Weatherization Assistant (e.g., "C: Program Files $\backslash$ Weatherization Assistant 8-9\gis" or "C: Program DatalWeatherization Assistant 8-9/gis"). After each file has been unzipped, the zip files are no longer needed and can be deleted. The GIS files are used under a license agreement and cannot be duplicated or further distributed.

After both zip files have been unzipped, turn the GIS feature on within the Weatherization Assistant by selecting the "Preferences" button from the Weatherization Assistant's Main Menu, click on the Features tab, check the box next to Feature 3, "Enable geographic information system (mapping)," and then click on the "Restart" button. See Sections 16.4, Features, and 18.2, Geographic Information System (GIS), for more details.

\subsection{Obtaining Manuals for the Weatherization Assistant}

Two manuals are available for the Weatherization Assistant:

- The Weatherization Assistant Users Manual (Version 8.9) - (This manual) The primary manual describing Version 8.9 of the Weatherization Assistant, including detailed descriptions of NEAT, MHEA, and all the associated administrative features offered in Version 8.9. This manual is provided in PDF format.

- The National Energy Audit (NEAT) Engineering Manual (Version 7) This engineering manual delineates the algorithms and assumptions used by NEAT in arriving at its measure recommendations based on the user's input of the building characteristics. This manual was published in September 2003 and, thus, does not include changes made in the Weatherization Assistant since that time. This manual is provided in PDF format. An update to the manual is forthcoming. No equivalent manual for MHEA is currently available.

Both manuals can be downloaded over the Internet from the same two web sites used to obtain the program files for the latest release of the Weatherization Assistant: 


\section{Chapter 2: Installation and Start-up}

- http://www.waptac.org/Weatherization-Assistant/Weatherization-AssistantManuals.aspx, or

- http://eber.ed.ornl.gov/pub/weatherization and then select the "Manuals" folder.

Selecting the files for the manuals from the WAPTAC or ORNL web sites will either:

- Automatically open the files with the PDF software because the software is already installed on your computer. In this case, you may use that software's "Print" button to immediately print the manuals, or you may use that software's "Save" button to save the files to a folder on your computer for later viewing and printing.

- Cause your Internet browser to display the same "Save As" window as seen when downloading the program files for the latest release of the Weatherization Assistant, because the PDF software is not installed on your computer. In this case, you may download the manuals following the same steps used to download the latest release of the Weatherization Assistant (see Section 2.2, Obtaining the Weatherization Assistant Software). However, you will not be able to view or print the manuals until you install the PDF software or a PDF viewer on your computer.

In both cases, it may take several minutes to open or download the files if you are not using a high-speed Internet connection. It is recommended that you download or save the manuals in the same folder or a subfolder where you installed the Weatherization Assistant (e.g., "C:IProgram Files\Weatherization Assistant 8-9” or “C: ProgramDatalWeatherization Assistant 8-9”).

\subsection{Accessing Web-Based Training for the Weatherization Assistant}

A web-based training course on the Weatherization Assistant has been developed and made available over the Internet. The course covers all of the information presented in three days of on-site training provided to new users of the Weatherization Assistant. Also provided is a module that presents information on the optional advanced features of the program. To access the training, go to:

http://www.weatherizationassistanttraining.org. 
The first time you visit the website, select the "REGISTER" hyperlink near the bottom of the page. This will lead you through a registration process. Once registered, use the LOGIN hyperlink to visit the training site as often as needed. There is no charge associated with use of this site.

\subsection{Starting the Weatherization Assistant}

Click on the Weatherization Assistant icon displayed on your desktop to start the program. Any other means of attempting to open the program will likely fail. The icon has specific instructions regarding the opening of the Weatherization Assistant that are needed for its successful operation.

The first time you start the Weatherization Assistant after installation, you will be asked to enter an "Installation ID” (see Figure 2.8). The entry requested is a brief name (up to 10 characters) identifying this specific installation of the software. If this will likely be the only installation of the Weatherization Assistant in your agency, this entry is not vital and may be something such as the acronym of your agency. However, if you plan to have multiple installations of the Weatherization Assistant on different computers within your agency and you plan to share data between them, the Installation ID should uniquely identify this specific installation. For example, you might enter "Inst 1," or the initials or last name of the person who is responsible for this specific computer.

The Installation ID is appended to new records (such as setup or supply libraries) when they are created (see Sections 14.1, Setup Library Information, and 15.1, General Information Form). For example, if the Installation ID you entered was 
"Smith," and you copied the library "Your Setup Library," then the copy of the Setup Library would be named "Your Setup Library [Smith]." If necessary for uniqueness, an integer will also be appended. If the Installation ID field on the Installation ID form is left blank, a default installation ID consisting of the time the record was created will be assigned. For example, under these circumstances, the copied setup library would be named "Your Setup Library [09:51:13]." Of course, you are free to rename the record, as long as the name remains unique.

The Installation ID is also used by default when exporting from an installation using text files in order to identify which computer the files are from (see Section 17.4, Data Transfer - Import/Export Indirectly with Text Files).

You can change the Installation ID at any time by selecting the "Preferences" button on the Weatherization Assistant's Main Menu (see Section 16.1, General).

After you have entered the Installation ID, select the "Done" button. If during installation you chose to install the Weatherization Assistant in a folder on your computer other than the default folder, the Weatherization Assistant may tell you that it must connect to the "back-end database." Click on the "OK" button to continue. You will then be asked to confirm that the linking was successful. A similar process will then be performed to link to a reporting module.

The Weatherization Assistant will now take you briefly to an introductory banner screen that indicates the version number and release date. Subsequent start-ups of the Weatherization Assistant will also take you directly to this introductory screen. This screen will automatically disappear once the program has fully loaded and be replaced by the Main Menu for the Weatherization Assistant (see Figure 2.9). It is from this Main Menu that you will access all of the functions included in the Weatherization Assistant. An overview of the Main Menu is provided in Section 1.3, Program Overview.

\subsection{The User Log On Form}

If you institute use of the User Logon feature of the Weatherization Assistant, you will be presented with the Weatherization Assistant Log On form each time you start the program (see Figure 2.10). This feature requires users of the program to be registered and have passwords assigned before they can access the program. A list of currently registered users is displayed by clicking on the downward pointing 


\section{Chapter 2: Installation and Start-up}

arrow in the "User Name" drop-down list. The following is displayed for each registered user: the User Name, the Full Name of the user, and the user's Group (indicating privileges), Agency, and State (of agency). Choose a user from the list and enter the password for that user. Only after successfully logging on will the Weatherization Assistant's Main Menu (see Figure 2.9) become available. See Feature 4, "Enable user logons with user names and passwords from the Agency/ Contacts tab" in Section 16.4, Features, and "Change Logon Group and Password" in Section 6.2, Contacts (Agency), for more information on the use of the User Logon feature.

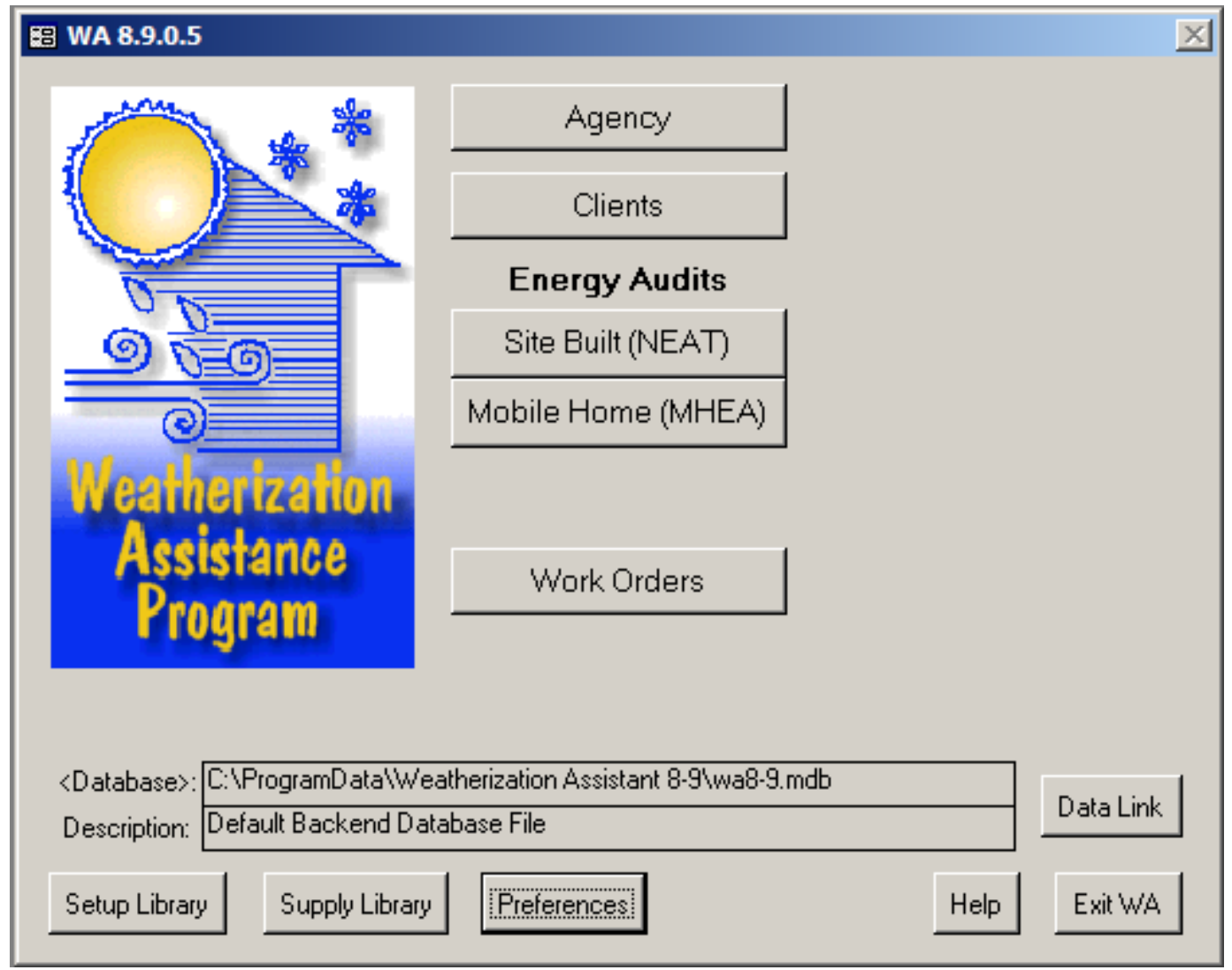

Figure 2.9. The Weatherization Assistant's Main Menu.

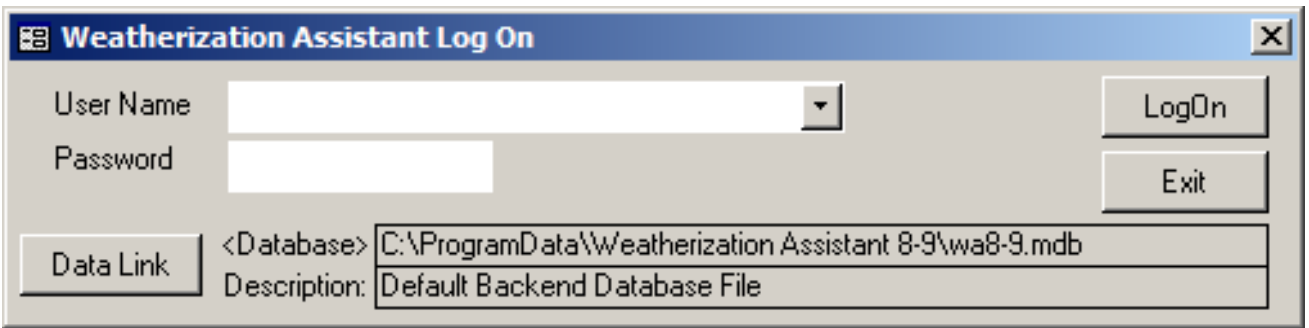

Figure 2.10. The Weatherization Assistant Log On form. 


\section{Chapter 3: Setting Up the Weatherization Assistant}

\section{Chapter 3. Setting Up the Weatherization Assistant}

A new installation of Weatherization Assistant requires the program to be properly set up for your specific agency before it can be used to select cost-effective weatherization measures for homes. This involves the simple, administrative tasks of initializing your agency information and renaming the default Setup and Supply Libraries to better identify them to yourself. It also involves the important steps of tailoring the Setup Library and, to a lesser extent, the Supply Library to represent your local costs, conditions, etc. These latter steps cannot be ignored.

If your installation of Version 8.9 is an upgrade from an earlier Version 8 of the Weatherization Assistant (e.g., Version 8.6), you can set up your new installation by importing agency information and the Setup and Supply Libraries from the previous version, as outlined in Section 3.3, Importing Information from a Previous Version. Otherwise, you must follow the steps outlined in Section 3.2, Program Setup, to set up the Weatherization Assistant manually. You must decide which method you will use before implementing it because confusion may result if both approaches are used.

This chapter assumes that you are familiar with navigation and data entry techniques common to programs using a Windows user interface. If you are not, you should consult Chapter 5, Navigation and Data Entry, to learn how to navigate through the Weatherization Assistant's multiple forms and enter information into its data fields.

\subsection{Initial Start-up of the Weatherization Assistant}

Click on the Weatherization Assistant icon displayed on your desktop to start the program. The first time you start the Weatherization Assistant after a new installation, an Installation ID form will be displayed (see Figure 2.8). If this is the first time the Weatherization Assistant has been started since it was installed, follow the steps presented in Section 2.8, Starting the Weatherization Assistant, to correctly set up the Installation ID. 


\section{Chapter 3: Setting Up the Weatherization Assistant}

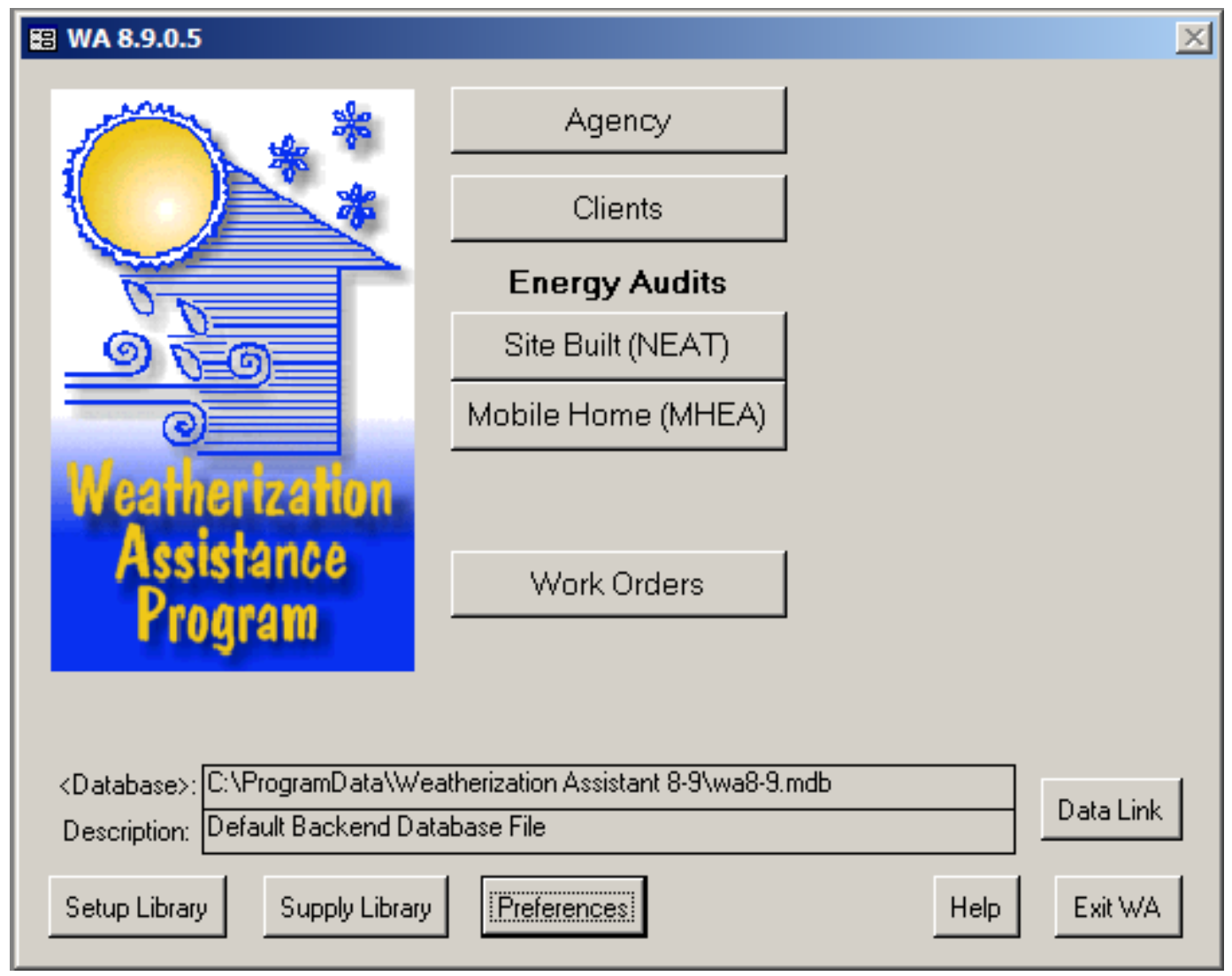

Figure 3.1. The Weatherization Assistant's Main Menu.

\subsection{Program Setup}

\section{Initializing Your Agency Information}

Select the “Agency” button from the Weatherization Assistant's Main Menu (see Figure 3.1) to display the Agency Information form (see Figure 3.2). Change the Agency Name from "Your Agency" to the actual name of your agency (the full name or perhaps an acronym) and the State from "US" to your state using the dropdown list. If the Weatherization Assistant is installed on multiple computers within your agency, you should make the Agency Names unique, perhaps by entering a dash (“-”) followed by the Installation ID that you used in Section 3.1, Initial Startup of the Weatherization Assistant, after the name of your agency. Although not absolutely necessary, you should select the Agency Type from the options in the drop-down list that best matches your agency. Make sure the checkbox in front of "Default agency to associate with new Client, Work Order..." is checked. Delete 


\section{Chapter 3: Setting Up the Weatherization Assistant}

the "Agency comment" from the Comment field. Section 6.1, Agency Information, should be reviewed for more information on these steps.

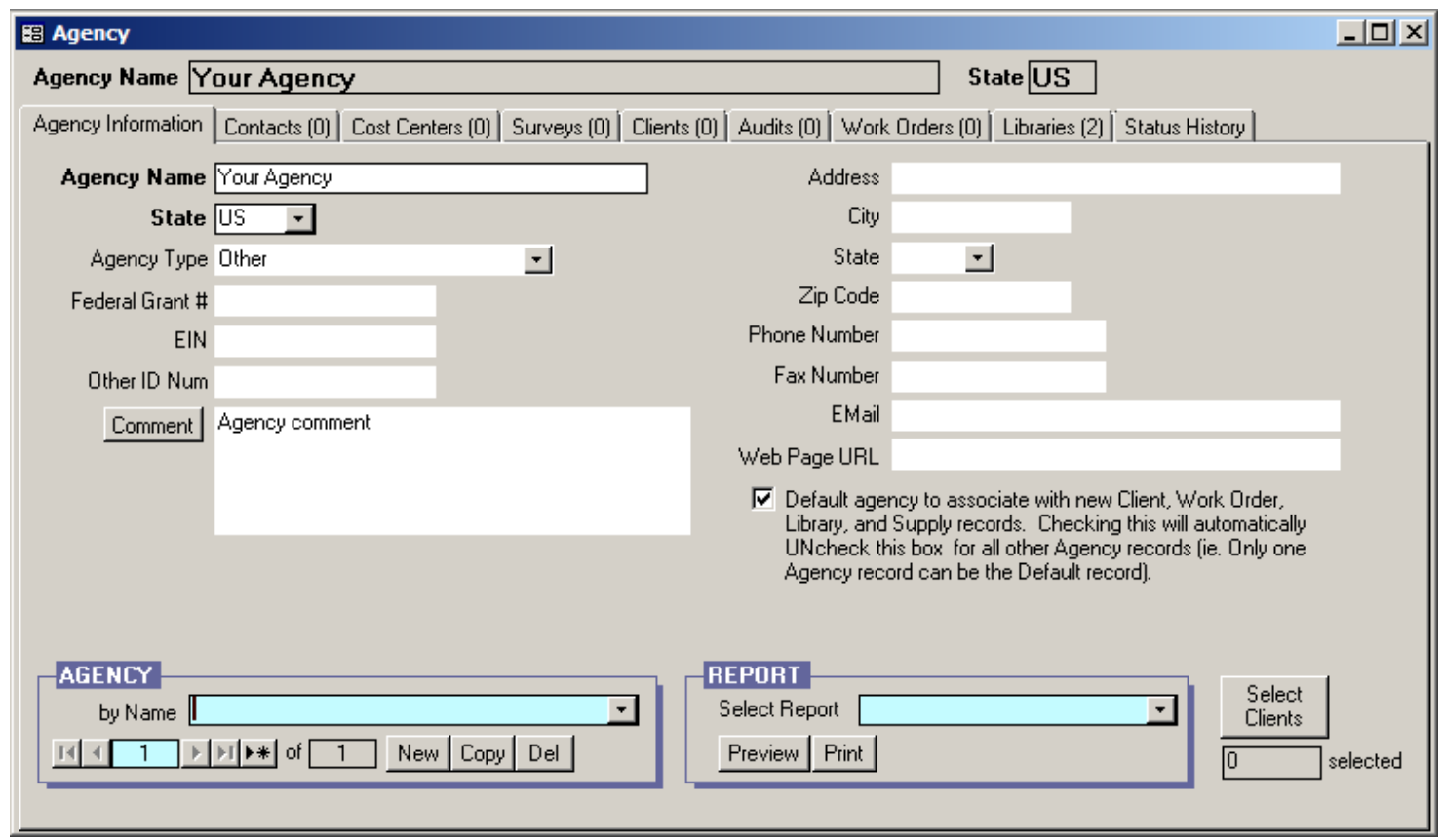

Figure 3.2. The Agency Information form under the Main Menu’s “Agency” button.

Although optional, you may wish to enter one or more "Contacts" for your agency. Select the Contacts tab under the Main Menu's “Agency” button to display the Contacts form (see Figure 3.3). Enter your name in the Contact Name field. Following this entry, make sure that the Name Detail field correctly identifies your First, MI (if you entered it), and Last names. Enter an abbreviated name, possibly your initials, first, or last name, in the User Name field and make sure the Active checkbox is checked. Enter your agency name in the Company field. If any of the checkboxes at the left center of the form apply to you, select them. An agency contact designated as an "Auditor" can be assigned responsibility for audits (see “Auditor” in Section 8.2, Audit Information).

If you want to use the Weatherization Assistant's User Logon feature to restrict who can open the software, select the "Change LogOn Group and Password" button. Select the "Admin" User Group and enter and confirm a password. Leave the "Confirm Old Password" field blank. Select the "Apply New Password" button (see Feature 4 in Section 16.4, Features for additional details). 


\section{Chapter 3: Setting Up the Weatherization Assistant}

You may enter additional agency contacts now or at a later time by selecting the "New" button in the Agency Contact record navigation block in the lower left corner of the form. Enter the new contact's Contact Name and User Name, and make sure the Active checkbox is checked. Enter the Company Name for the contact and any additional information related to the contact. For instance, if the contact was associated with a material supplier, the Company field would be the name of the company that supplies the material and the Supplier checkbox would be checked.

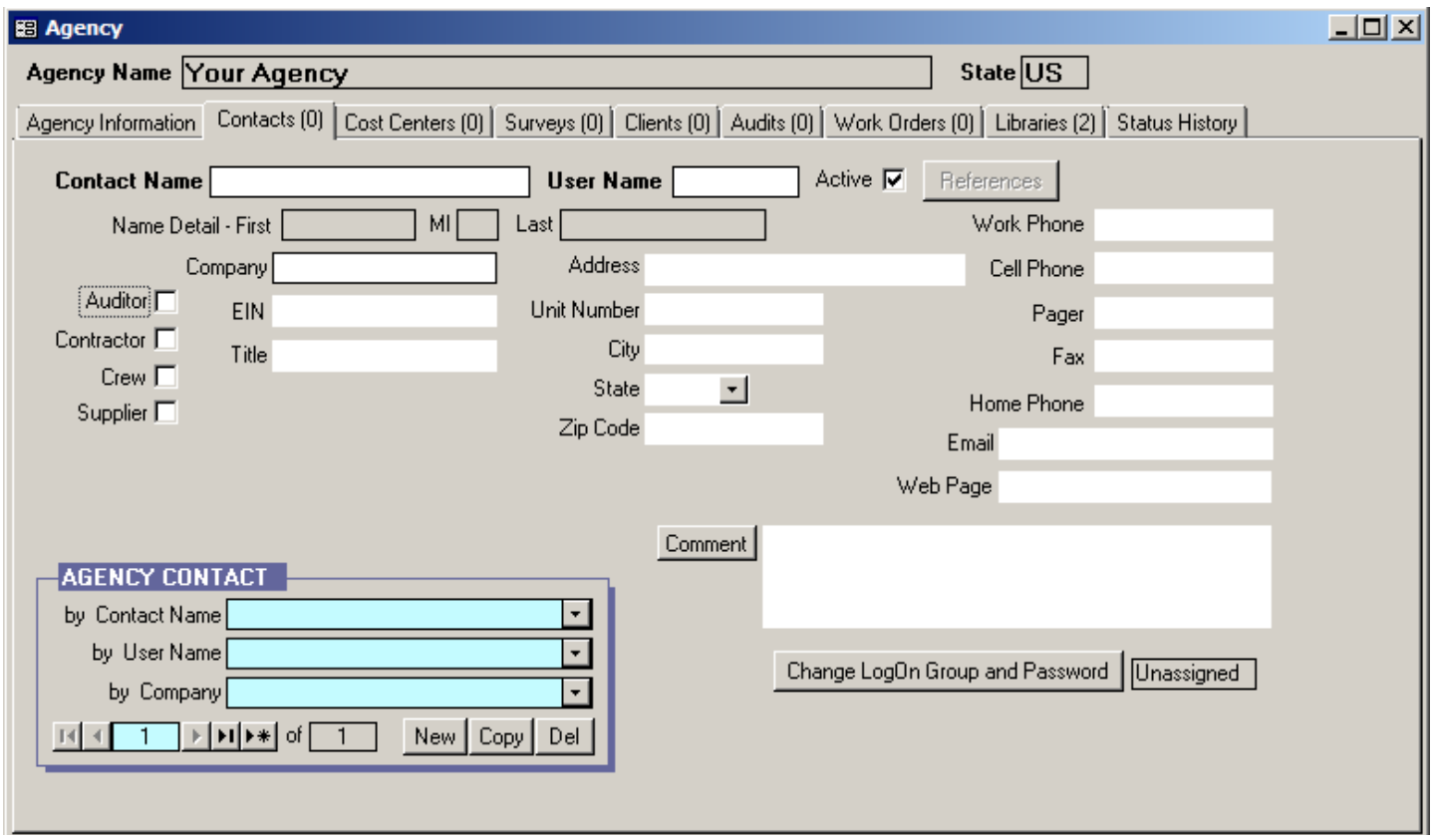

Figure 3.3. The Contacts form under the Main Menu’s “Agency” button.

Section 6.2, Contacts (Agency), should be consulted for more information on these steps. When finished, click on the X-box in the upper right corner of the "Agency" window to return to the Weatherization Assistant's Main Menu.

\section{Naming Your Supply Library}

Select the "Supply Library" button from the Weatherization Assistant's Main Menu (see Figure 3.1) to display the Supply Library's General Information form (see Figure 3.4). Rename the Supply Name from "Your Supply Library” to something more recognizable to you. It is recommended that you simply replace the "Your" with the acronym of your agency. If you have more than one installation of the software within your agency, it is also recommended that you 


\section{Chapter 3: Setting Up the Weatherization Assistant}

use the Installation ID in the name, perhaps by adding a dash ("--”) followed by the Installation ID. The name of your agency and your state, as entered previously, will be correctly listed in the Agency and State fields, respectively. Refer to Section 15.1, General Information (for the Supply Library) for more information on these steps. When finished, click on the X-box in the upper right corner of the "Supply" window to return to the Weatherization Assistant's Main Menu.

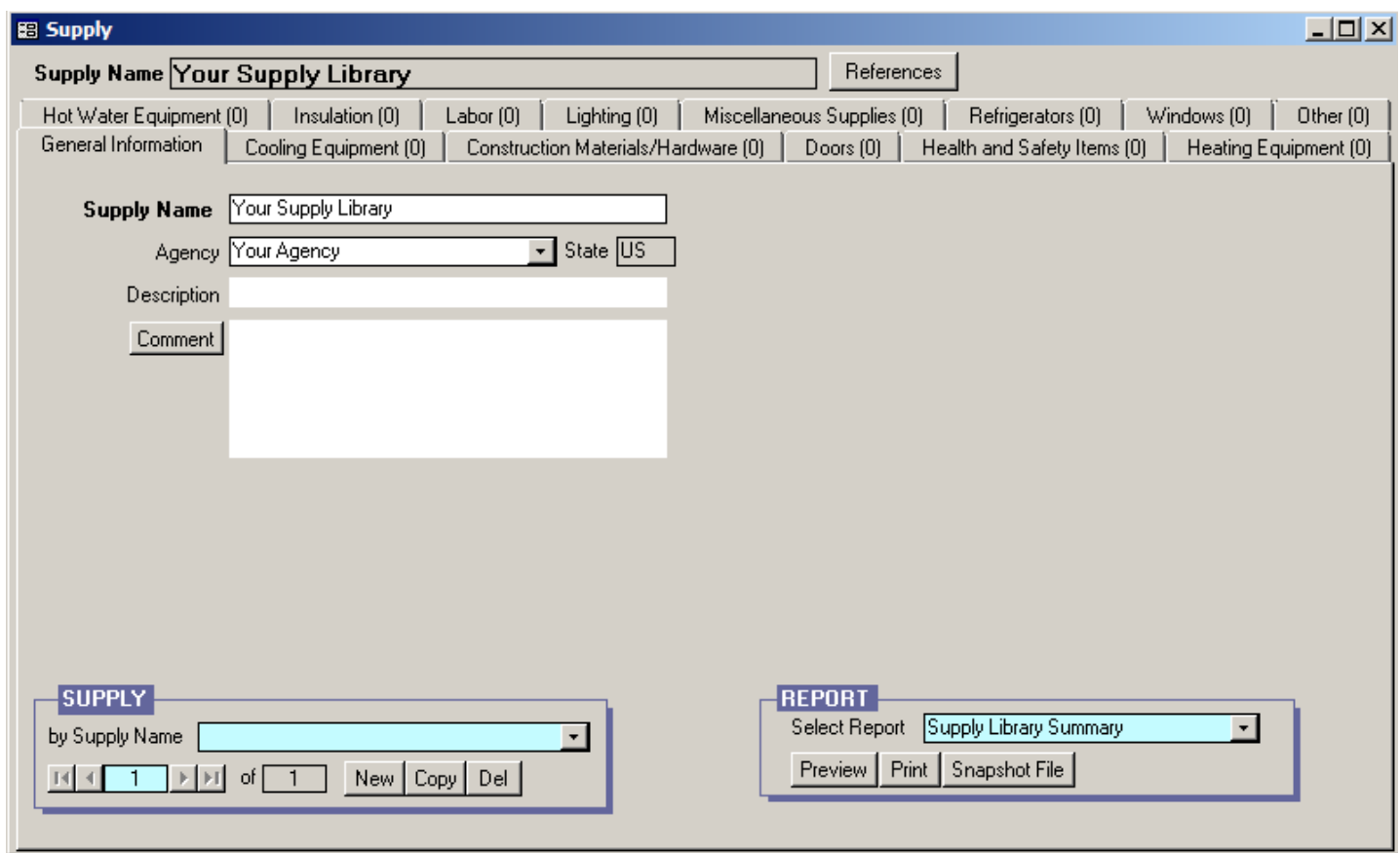

Figure 3.4. The General Information form under the Main Menu’s "Supply Library" button.

\section{Naming Your Setup Library}

Select the "Setup Library" button from the Weatherization Assistant's Main Menu (see Figure 3.1) to display the Setup Library Information form (see Figure 3.5). Rename the Library Name from "Your Setup Library" to something more recognizable to you. It is recommended that you simply replace the "Your" with the acronym of your agency. If you have more than one installation of the software within your agency, it is also recommended that you use the Installation ID in the name, perhaps by adding a dash (“-”) followed by the Installation ID. The name of your agency, state, and supply library as entered previously will be correctly listed 


\section{Chapter 3: Setting Up the Weatherization Assistant}

in the Agency, State, and Supply Library fields, respectively. Refer to Section 14.1, Setup Library Information, for more information on these steps.

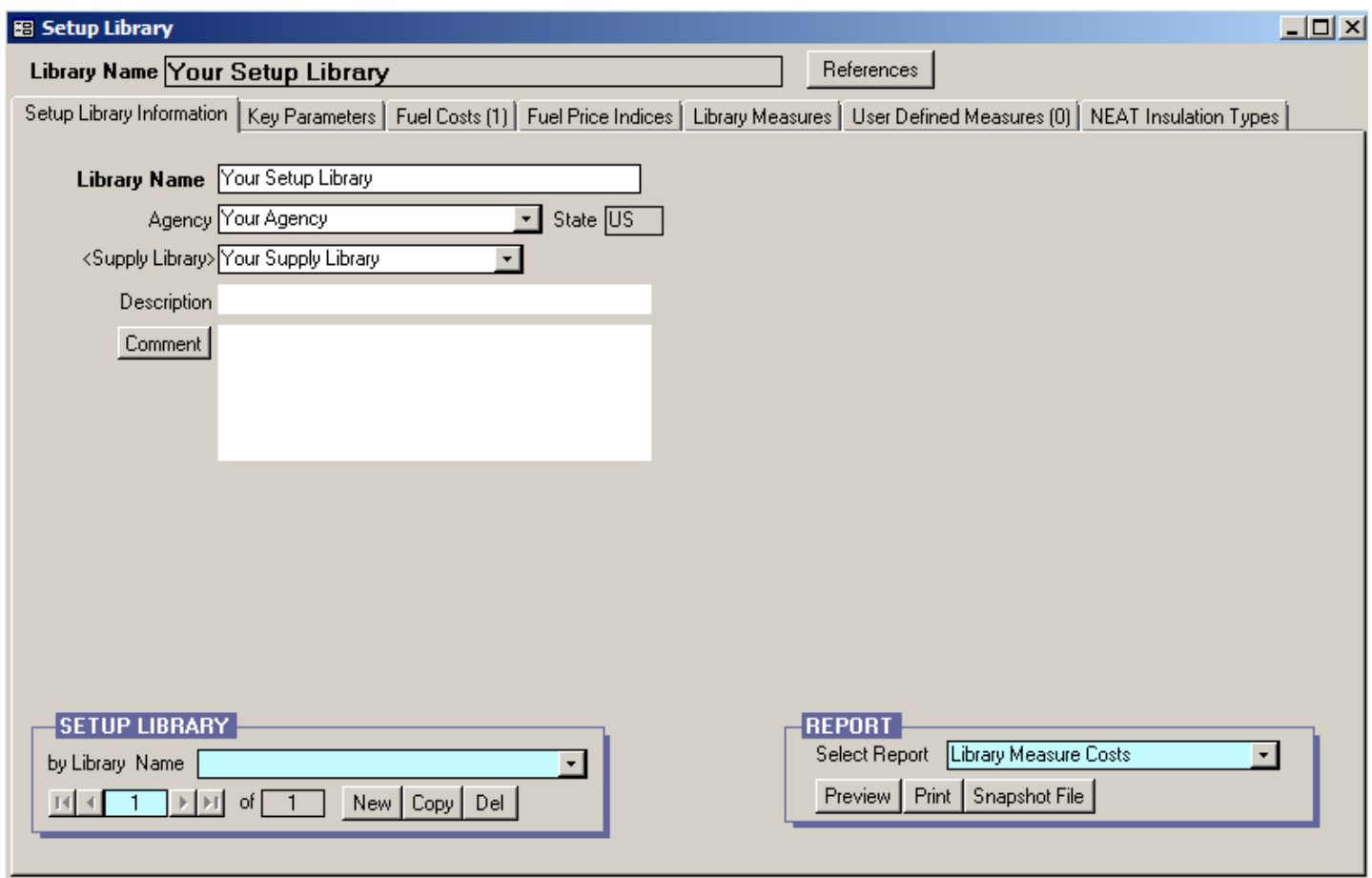

Figure 3.5. The Setup Library Information form under the Main Menu’s “Setup Library” button.

When finished, click on the X-box in the upper right corner of the "Setup Library" window to return to the Weatherization Assistant's Main Menu.

\section{Populating the Setup and Supply Libraries}

Before entering a client and running an audit, data specific to your agency must be entered into the Setup Library, such as your actual fuel costs, your actual costs for installing measures, and identifying which measures your agency can perform. Information needs to be entered into the Supply Library only if you replace refrigerators or water heaters, or if you will be using detailed material information in the optional work order feature.

If this is your first use of the Weatherization Assistant, it may take up to a week to compile the required information and then a day to enter it into the Weatherization Assistant. If you used a previous version of the Weatherization Assistant (e.g., Version 8.6.0.4), it may take less than a day for you to manually reenter the data 


\section{Chapter 3: Setting Up the Weatherization Assistant}

from the previous version into Version 8.9, although now might be a good time to take an extra day to update the information. If you were using a previous version of the Weatherization Assistant, you can easily print out all the measure costs you had entered in the Setup Library. Select the "Setup Library" button from the Weatherization Assistant's Main Menu (see Figure 3.1) in the previous version to display the Setup Library Information form (see Figure 3.5). Choose the Library Measure Costs report using the drop-down list in the Report block in the lower right corner of the Setup Library Information form, and then select the "Print" button in the Report block. Before using this previous measure cost data, it might be a good time to take an extra day to update the information.

To enter the information into Version 8.9, select the "Setup Library" button from the Weatherization Assistant's Main Menu in this version (see Figure 3.1) to display the Setup Library Information form (see Figure 3.5). Use the tabs shown in the "Setup Library" window to enter data specific to your agency. The Fuel Costs tab (to enter current fuel costs) and the Library Measures tab (to select the measures your agency installs and enter the costs for these measures in the units specified) are of greatest importance. Under the Fuel Costs tab, rename the Fuel Cost Table Name from "Default Costs" to something more recognizable to you, perhaps indicating when or from where the costs were obtained. You may also want to look at the Key Parameters tab to see if any of these items need to be altered. You will need to refer to Chapter 14, Setup Library, for details concerning the information stored in the setup library and how to change it. Exit the Setup Library by clicking on the X-box in the upper right corner of the "Setup Library" window to return to the Weatherization Assistant’s Main Menu.

From the Weatherization Assistant's Main Menu (see Figure 3.1), select the "Supply Library" button to display the General Information form (see Figure 3.4). If you are planning to replace refrigerators or install new water heaters as part of your weatherization program, you may want to enter the replacement models you will use by selecting the Refrigerators or Hot Water Equipment tabs, respectively (see "Additional Entries on the Hot Water Equipment and Refrigerator Forms" in Section 15.2, Materials/Labor Forms, for more details). Exit the Supply Library by clicking on the X-box in the upper right corner of the "Supply" window to return to the Weatherization Assistant's Main Menu. 


\section{Chapter 3: Setting Up the Weatherization Assistant}

\subsection{Importing Information from a Previous Version}

If you used a previous version (Version 8.2 or later) of the Weatherization Assistant, you can set up a new installation of Version 8.9 by importing your agency information, Setup and Supply Library information, as well as client and audit data from the previous version. (Importing from Version 7.4 is not possible.) This approach may reduce the time needed to reenter agency information and Setup and Supply Library data. However, an upgrade from Version 8.2 or later to Version 8.9 signifies changes in the database structure (e.g., added fields), default parameters, etc. Therefore, importing the Setup and Supply Libraries, clients, and/or audits may require some additional data entry or modification before they can be used in Version 8.9. Results (e.g., recommendations) obtained from different versions may also differ. See the end of this section for further details.

To begin, start your previous version (e.g., Version 8.6) of the Weatherization Assistant to display the Weatherization Assistant's Main Menu (similar to Figure 3.1), and then select the "Data Link" button to display the Data Link form (see Figure 3.6). Write down the path name of the database you are currently connected to and have been using, as shown in the Path field under "Currently Linked BACKEND DATABASE.” The path name will end with a

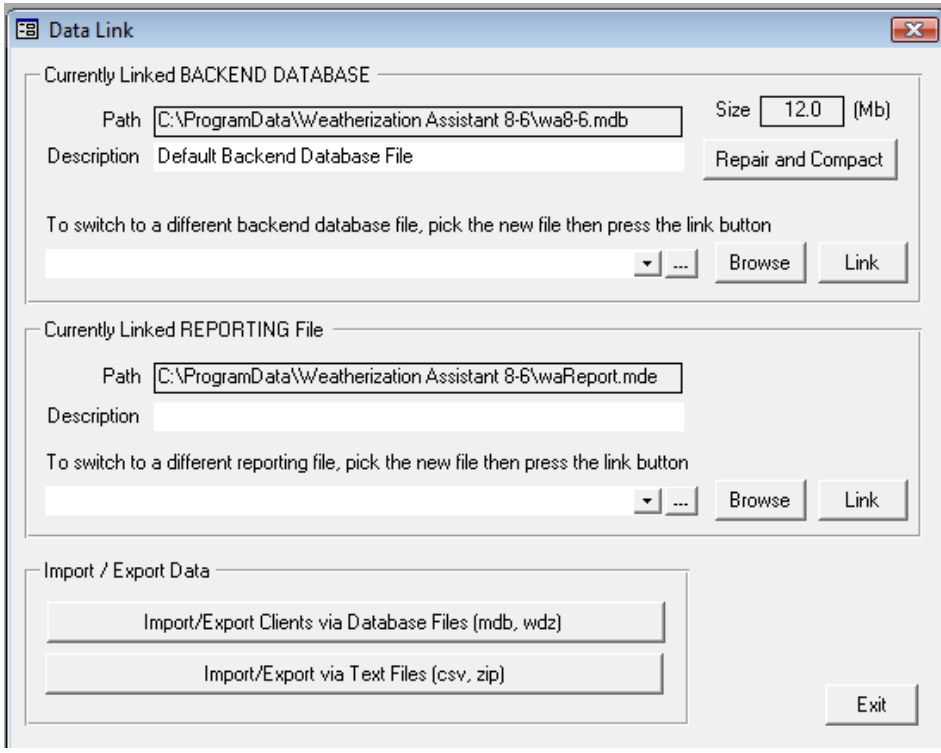

Figure 3.6. The Data Link form (Version 8.6). ".mdb." You can click into the Path field and use the keyboard navigation buttons to see the entire path name if needed. Alternatively, you can highlight the entire path name and use traditional Windows procedures (e.g., press the [Ctrl-C] key combination) to copy the name into your computer's memory for pasting later. Exit this previous version of the Weatherization Assistant when you are done.

Start Version 8.9 of the Weatherization Assistant using the icon displayed on your desktop. If this is the first time you have started Version 8.9 following installation, 


\section{Chapter 3: Setting Up the Weatherization Assistant}

follow the steps presented in Section 2.8, Starting the Weatherization Assistant, to correctly set up the Installation ID.

Select the "Data Link" button on the Weatherization Assistant's Main Menu (see Figure 3.1) to display the Data Link form in Version 8.9 (see Figure 3.7).

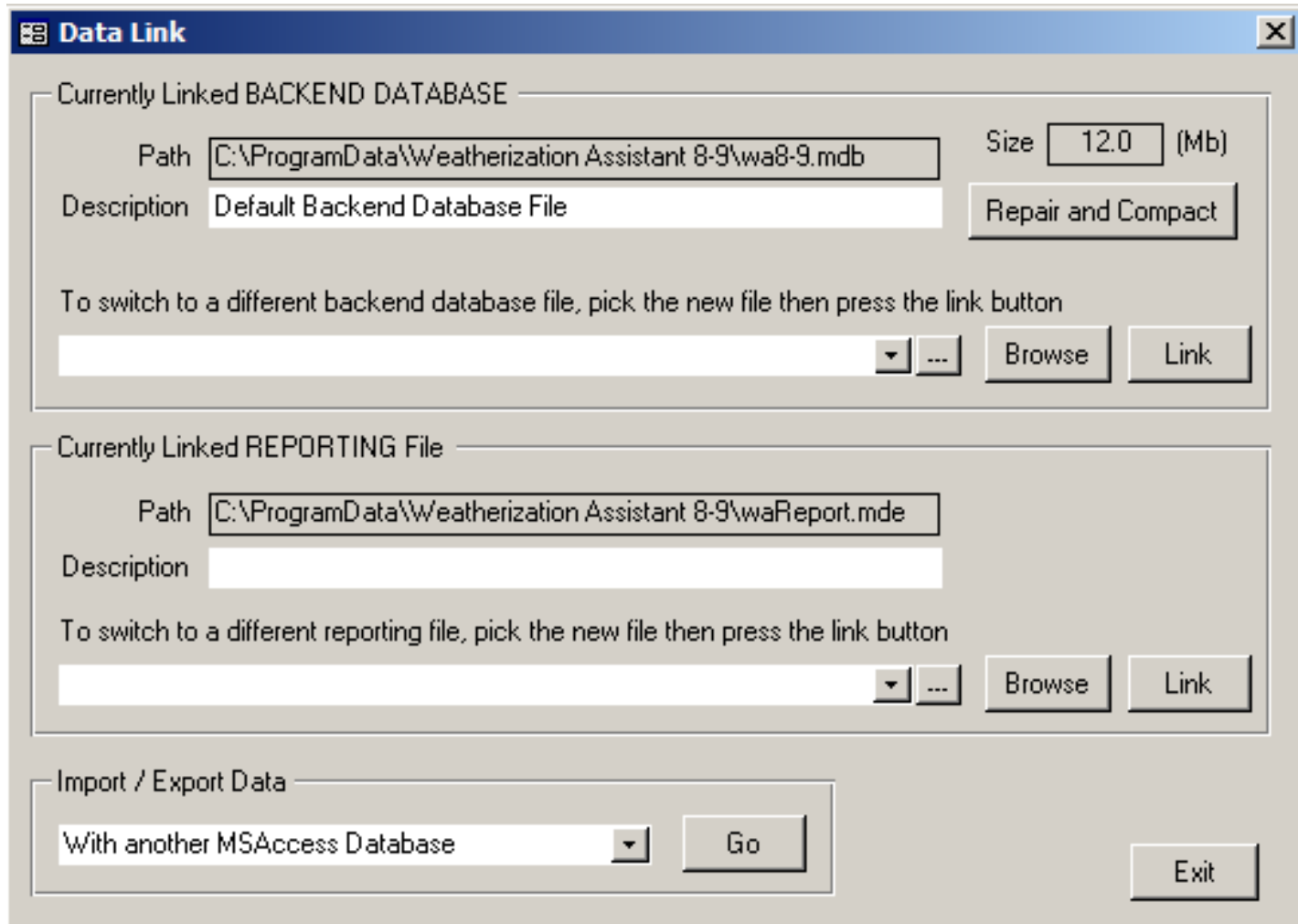

Figure 3.7. The Data Link form (Version 8.9).

Select "With another MSAccess Database" from the "Import/Export Data" dropdown list at the bottom of the form then click on the "Go" button to display a blank Transfer CLIENTS Between Databases form (see Figure 3.8).

If you copied the path name of your existing database into your computer's memory, you can click in the textbox beneath "External Database Path" then paste the path name into the textbox by pressing the [Ctrl-V] key combination. Pressing the Enter key on your keyboard will then display the clients in this database in the display window under "External Database Path" (see Figure 3.9). 


\section{Chapter 3: Setting Up the Weatherization Assistant}

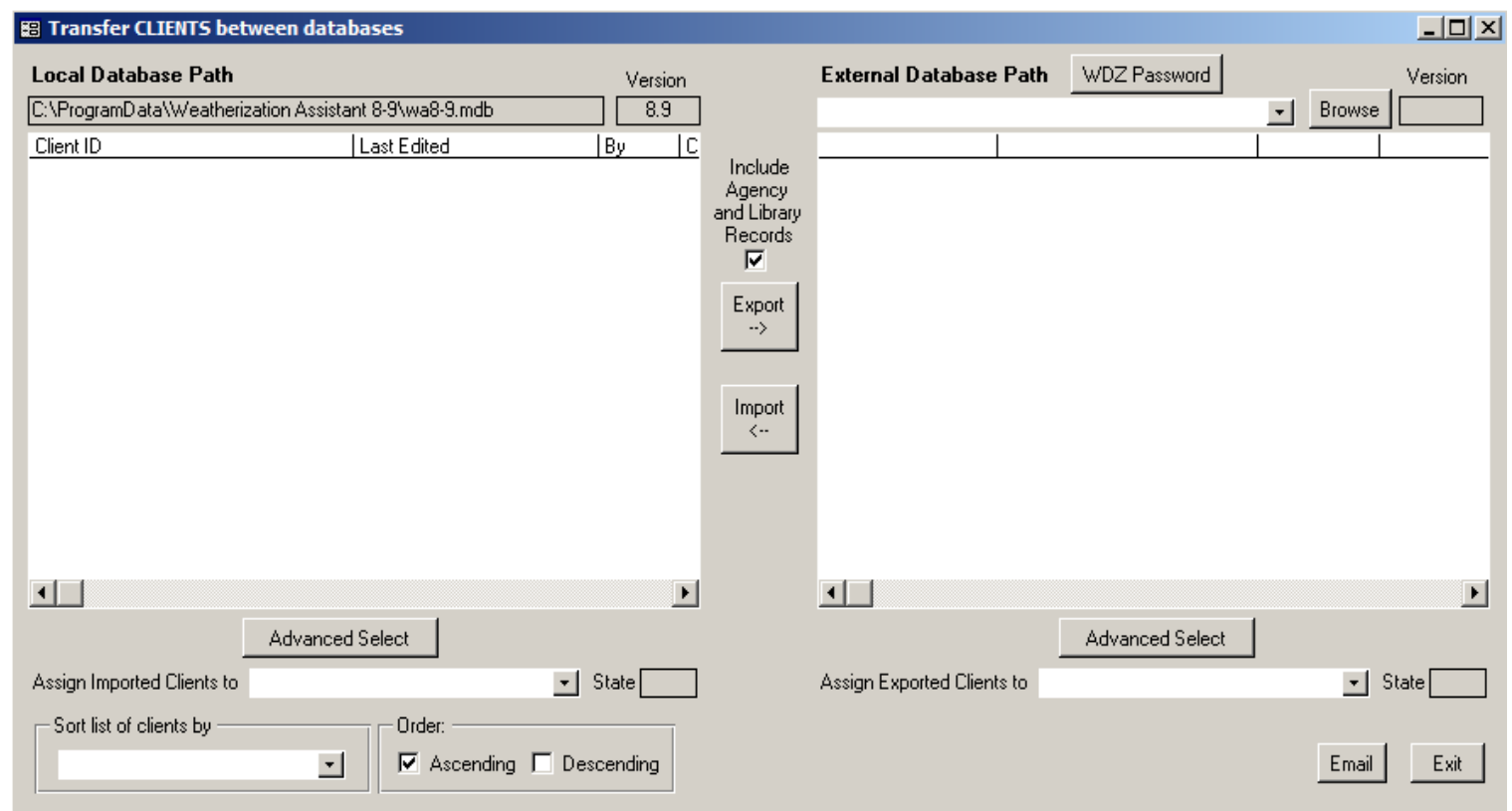

Figure 3.8. A blank Transfer CLIENTS Between Databases form.

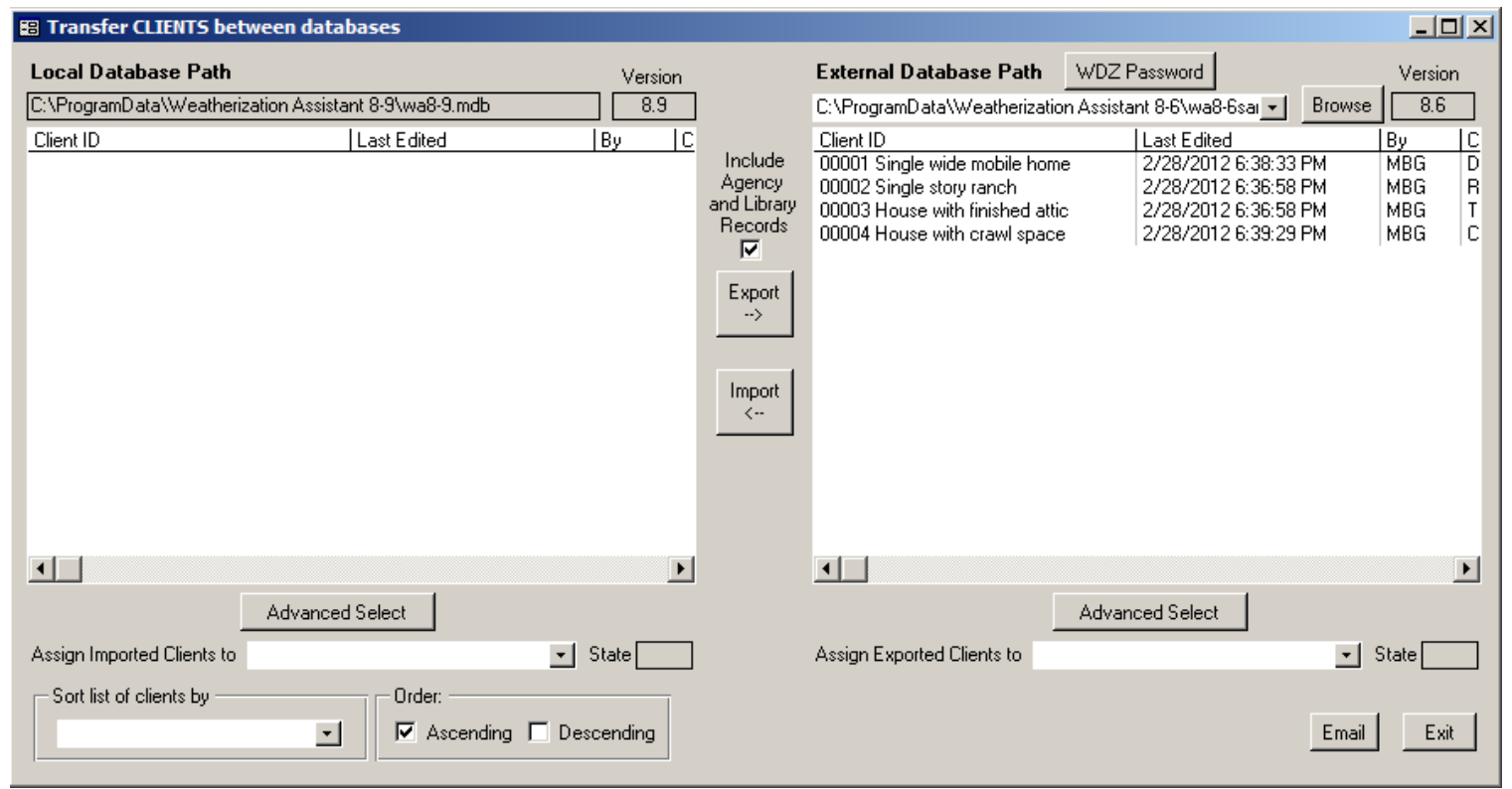

Figure 3.9. An example of the Transfer CLIENTS Between Databases form with client entries. 


\section{Chapter 3: Setting Up the Weatherization Assistant}

Otherwise, select the "Browse" button to display the Pick External Database for Import/Export form (see Figure 3.10). The default location first displayed on this form will either be the last location you visited using the form or the location where you installed Version 8.9. Use typical Windows navigation techniques to find the database that you were using in the previous version (refer to the path name you wrote down previously). Once you

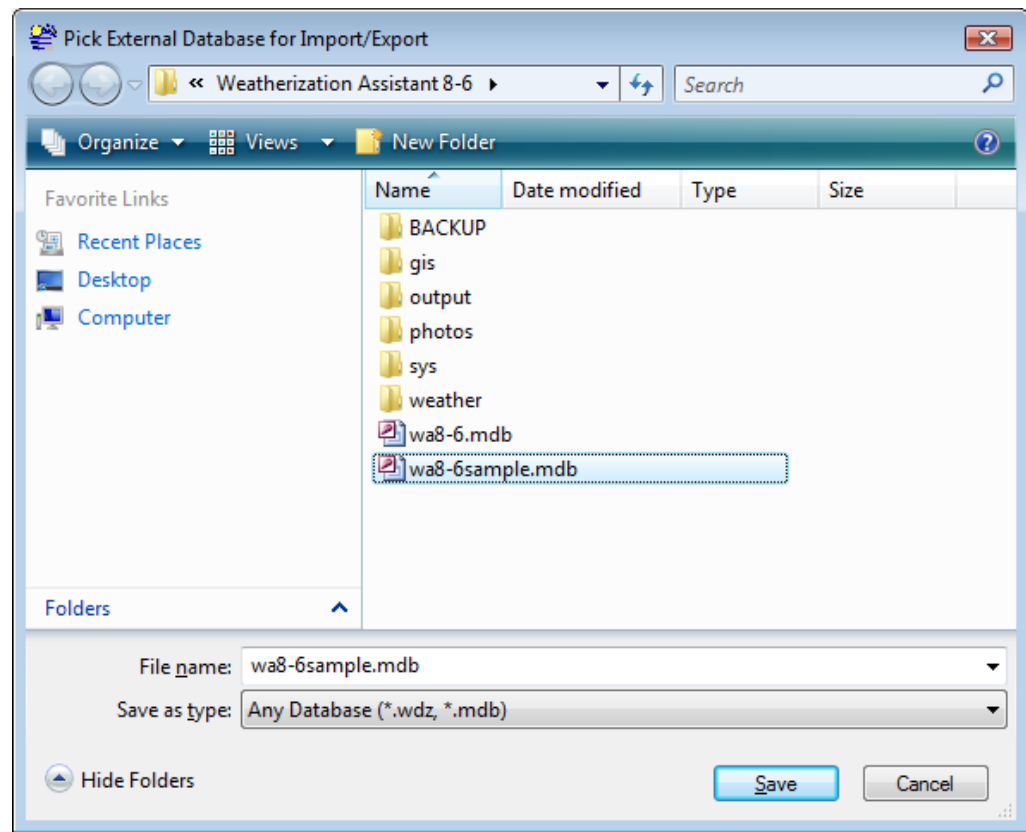

Figure 3.10. The Pick External Database for Import/Export form.

have found the database, click on the name of the database so that it is highlighted and its name appears in the "File name" field, and then select the "Save" button. You will be brought back to the "Transfer CLIENTS between databases" form (see Figure 3.9), where the path name for the database you were using in the previous version is displayed to the left of the "Browse" button and the clients entered in this database are shown in the display window under "External Database Path."

Information transferred between databases is grouped by clients. You specify what clients you want transferred and all the data associated with these clients is transferred. This includes all of the information entered under the "Client," "Energy Audits" (NEAT or MHEA), and "Work Orders" Main Menu buttons as well as the reports generated when running the audits or forming the work orders for these clients. If you so indicate, the transfer will also include the agency information and the Setup and Supply Libraries associated with these clients. See Chapters 6 through 15 for descriptions of the information contained in each of these categories.

If you want to transfer one or more clients from a previous version to Version 8.9 to create a contiguous Version 8.9 database, highlight the clients to be transferred by clicking on their Client IDs on the right side of the "Transfer CLIENTS 


\section{Chapter 3: Setting Up the Weatherization Assistant}

between databases" form. More than one client can be highlighted by holding down the [Ctrl] or [Alt] keys. If you are only interested in transferring agency and library information, highlight just one of your clients who accesses these libraries and is assigned to the agency of interest so that this information will be included in the transfer. You can delete this client from Version 8.9 once the transfer has occurred, while retaining the library and agency information. If you have multiple libraries you wish to transfer to Version 8.9, select multiple clients, each accessing a different library you wish to transfer.

Make sure that the "Include Agency and Library Records" checkbox is checked. Then, select the "Import <--" button to start the data transfer process. This may take several minutes depending on how many clients you highlighted. When the data transfer process is completed, the "Status" bar at the bottom right of the form will disappear and the client(s) that you transferred will be listed on the left side of the "Transfer CLIENTS between databases" form under "Local Database Path."

Select the "Exit" button to close the "Transfer CLIENTS between databases" form and return you to the Data Link form, and select the "Exit" button again to close the Data Link form and return you to the Weatherization Assistant's Main Menu.

Select the “Agency” button on the Weatherization Assistant's Main Menu (see Figure 3.1) to display the Agency Information form (see Figure 3.2). If the name shown in the Agency Name field is not the name of the agency that you used in the previous version, use the drop-down list in the Agency record navigation block in the lower left corner of the Agency Information form to call up the agency with the name that you were using. Make sure the checkbox in front of "Default agency to associate with new Client, Work Order, ...” is checked. You should examine the Agency Information forms for any other agencies that may exist in your Version 8.9 database (e.g., "Your Agency") to ensure that this checkbox is not selected. Verify that the number in parentheses on the Clients tab is the number of clients that you wanted to transfer from the previous version. When finished, click on the $\mathrm{X}$-box in the upper right corner of the "Agency" window to return to the Weatherization Assistant’s Main Menu.

If you transferred one or more clients only to transfer their associated library and/or agency information from the previous version to Version 8.9, you may wish to delete these clients from the Version 8.9 database so that they do not exist in more than one database. Deleting these clients will not delete the libraries imported 


\section{Chapter 3: Setting Up the Weatherization Assistant}

with them. Select the "Clients" button on the Weatherization Assistant's Main Menu (see Figure 3.1) to display the Client Information form (see Figure 3.11). If the name shown in the Client ID field near the top of the form is not one of the clients to be deleted, use one of the drop-down lists in the Client record navigation block in the lower left corner of the Client Information form to call up a client to be deleted. Once a correct client is shown in the Client ID field, select the "Del" button in the Client record navigation block. A warning box will appear that says "This will cause CASCADING DELETES to occur in all tables associated with this Client record.” Click “OK .” Repeat this process for any additional clients you used simply to transfer agency and/or library information. When finished, click on the X-box in the upper right corner of the "Client" window to return to the Weatherization Assistant’s Main Menu.

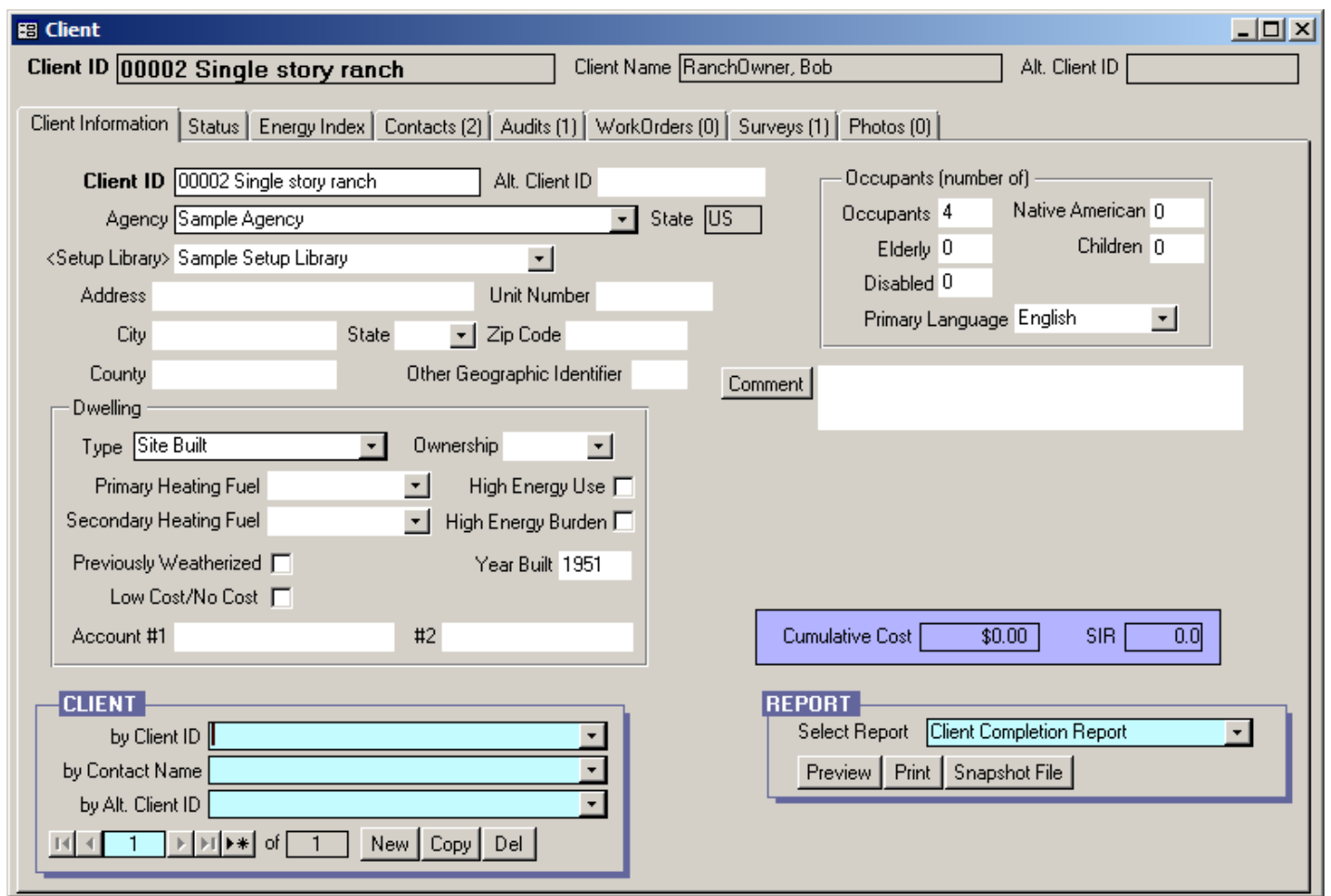

Figure 3.11. The Client Information form under the Main Menu's “Clients” button.

The methods outlined in this section have described how to import your previous version's Setup Library into Version 8.9. However, there are database structure differences between your former version's Setup Library and Version 8.9's library. For instance, Version 8.9 adds two new weatherization measures to NEAT, the Door Replacement measure and the White Roof Coating measure. You will need to decide whether these measures should be evaluated and, if so, what their costs 


\section{Chapter 3: Setting Up the Weatherization Assistant}

should be. Also, now might be a good time to review and update all of the information in the Setup Library, especially the fuel and measure costs.

If your import was from a version prior to 8.6, none of the user-defined NEAT insulation types from the previous version will be transferred since the formats are different. Therefore, you must enter these insulation types manually into Version 8.9 by selecting the "Setup Library" button from the Weatherization Assistant's Main Menu (see Figure 3.1) and then selecting the NEAT Insulation Types tab. See Section 14.7, NEAT Insulation Types, for more information. You must also enter the costs associated with these insulation types by selecting the Library Measures tab.

If you transferred one or more clients from a previous version to Version 8.9 to form a contiguous Version 8.9 database, you must check all the audit-related forms in Version 8.9 before rerunning these audits to make sure all the fields are filled in. Refer to the "Weatherization Assistant Update History" document that can be downloaded over the Internet from the same two web sites used to obtain the program files for the latest release of the Weatherization Assistant to help identify these fields:

- http://waptac.org/Energy-Audits/Weatherization-Assistant.aspx then select the "Update History" hyper link or

- http://eber.ed.ornl.gov/pub/weatherization/Update\%20History.pdf

In addition, rerunning an audit under Version 8.9 that was run under a previous version could result in changes in the Recommended Measures report (e.g., a different list of recommended measures; different energy savings, costs, and/or SIR for a recommended measure). Once the clients and audits have been transferred to Version 8.9, the Recommended Measures report generated for an audit under the previous version can still be viewed without rerunning the audit by selecting the Recommended Measures report in the Report block in the lower right corner of the NEAT or MHEA Audit Information forms. If you rerun the audit using Version 8.9, a new Recommended Measures report will be created and the previous report created under the previous version will be lost. Therefore, if you want to rerun the audit using Version 8.9 but keep the recommendations generated under the previous version, you should make a copy of the audit and then run the copied version using Version 8.9. 


\section{Chapter 4. Quick-Start Tutorial}

This chapter provides a quick overview of the basic steps needed to run an audit on a client home, which is the primary purpose of the Weatherization Assistant. These steps include entering a new client, initiating an energy audit for this client, and obtaining and understanding a recommendations report. Being familiar with these basic steps will allow you to start using the Weatherization Assistant. You will also be prepared to read other chapters of the user's manual to learn about the details of performing an audit and using other features of the program.

This chapter assumes that you are familiar with navigation and data entry techniques common to programs using a Windows user interface. If you are not, you should consult Chapter 5, Navigation and Data Entry, to learn how to navigate through the Weatherization Assistant's multiple forms and enter information into its data fields.

\subsection{Creating a New Client}

Before entering audit data for a client's home, you must first set up the client in the Weatherization Assistant and enter information related to the client. Select the "Clients" button from the Weatherization Assistant's Main Menu (see Figure 4.1) to display the Client Information form (see Figure 4.2).

If you have entered information previously for a client or have imported clients, the form will likely be filled in with information for one of these previously defined

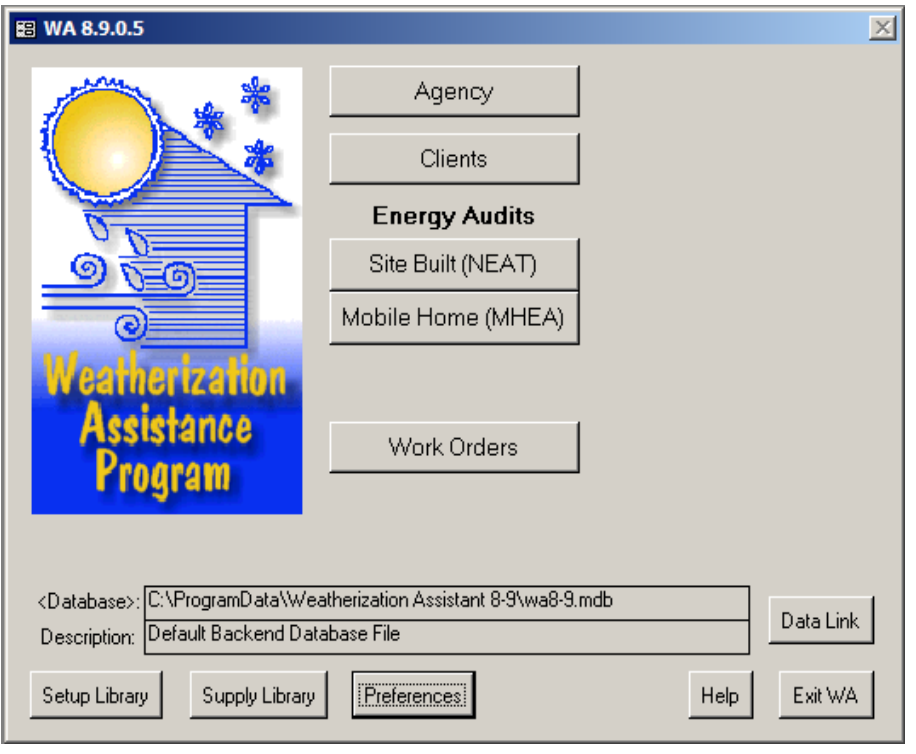

Figure 4.1. The Weatherization Assistant's Main Menu.

clients. Do not type over any information for an existing client. Click on the "New" button in the Client record navigation block in the lower left corner of the Client Information form to provide you with a blank Client Information form. If this is your first definition of a client for this installation of the Weatherization 


\section{Chapter 4: Quick-Start Tutorial}

Assistant, selecting the "Clients" button from the Main Menu will take you to a blank Client Information form with default Client ID of "Client (1).”

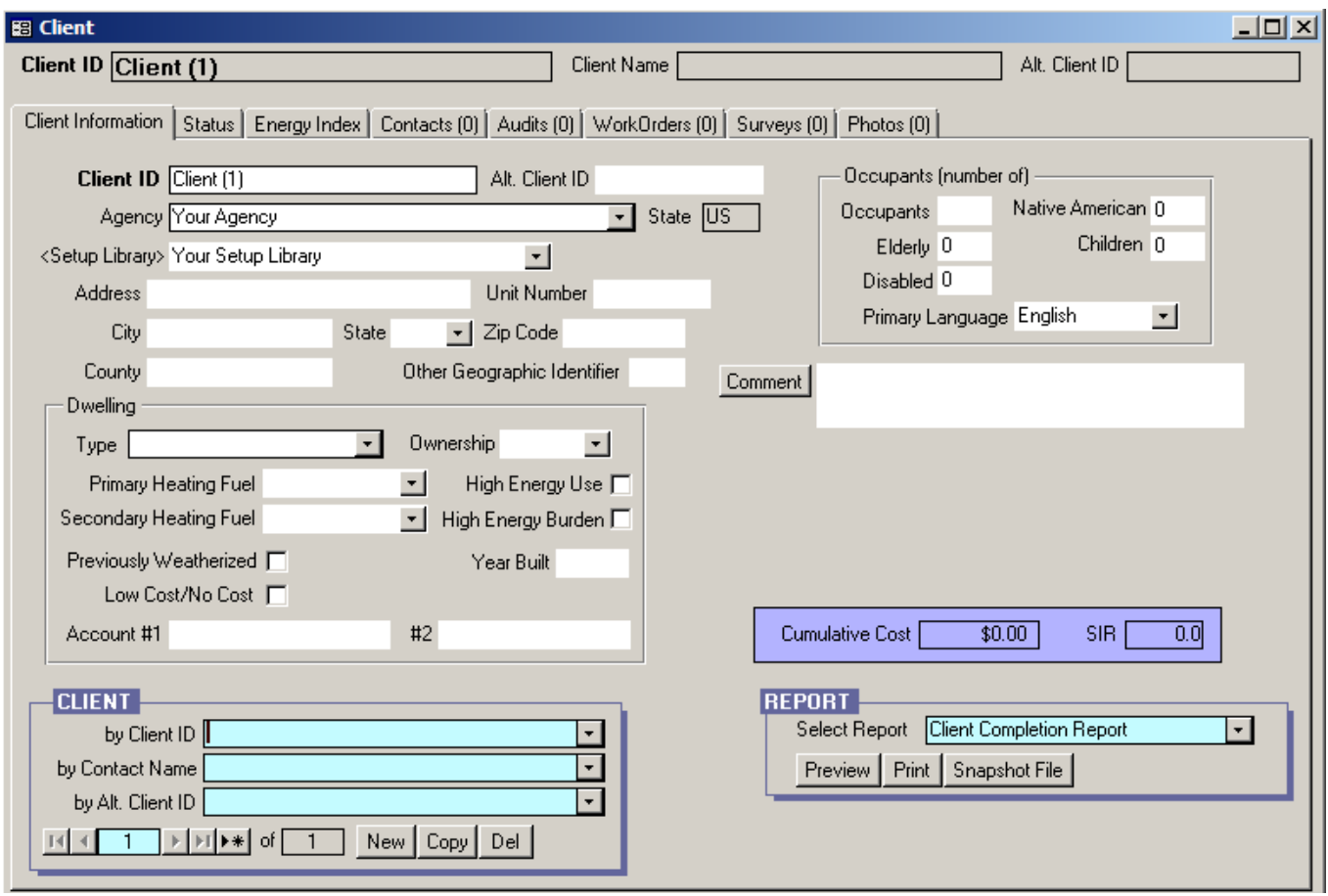

Figure 4.2. The Client Information form under the Main Menu's “Clients” button.

Change the default entry in the Client ID field that the Weatherization Assistant automatically created for this new client (i.e., "Client (xx)") to an ID your agency uses for the client, typically a job number. Make sure the name of your agency is listed in the Agency field. If not, select your agency using the drop-down list in the Agency field.

Although not required, you will want to assign a setup library to the client if you plan to use the work order feature (see Chapter 18, Work Orders). If you have only one setup library associated with your agency, then this setup library will be automatically selected in the Setup Library field. If you have more than one setup library associated with your agency, the Setup Library field will be blank, although you can select the correct setup library to use for this client by using the drop-down list. Select the correct Dwelling Type for the house using the drop-down list.

Although not absolutely necessary, you should enter the number of occupants in this client's house in the Number of Occupants field because NEAT and MHEA will use this number of occupants instead of a default number of 2 in its calculation 


\section{Chapter 4: Quick-Start Tutorial}

of internal loads. Enter any other information you desire for your new client in the other fields on this form. More detailed information is provided in Section 7.1, Client Information.

While still under the Main Menu's "Clients" button, select the Contacts tab to display the Contacts form (see Figure 4.3). Enter the client's name in the Full Name field. This should be the name of the person who applied for weatherization services. Make sure that the "Name Detail" fields correctly identify the "First," "MI" (if entered), and "Last" names. Check the Primary Applicant checkbox. Ensure that the Contact Type is "Applicant/Person of Record.” If not, use the dropdown list in the Contact Type field to select this entry. Enter any other information you desire for the client using the fields on this form. If you had entered address information for the client on the Client Information form, you can use the "Copy Client Addr" button to copy this address to the Contacts form.

Additional client contacts may be entered by choosing the "New" button in the Client Contact record navigation block. However, only one contact per client should be declared a Primary Applicant with Contact Type of "Applicant/Person of Record.” Choose any other appropriate Contact Type for subsequent contacts (e.g., "Other Contact for Applicant”).

For more information on how to use the Contacts form and enter additional contacts, see Section 7.4, Contacts (Client).

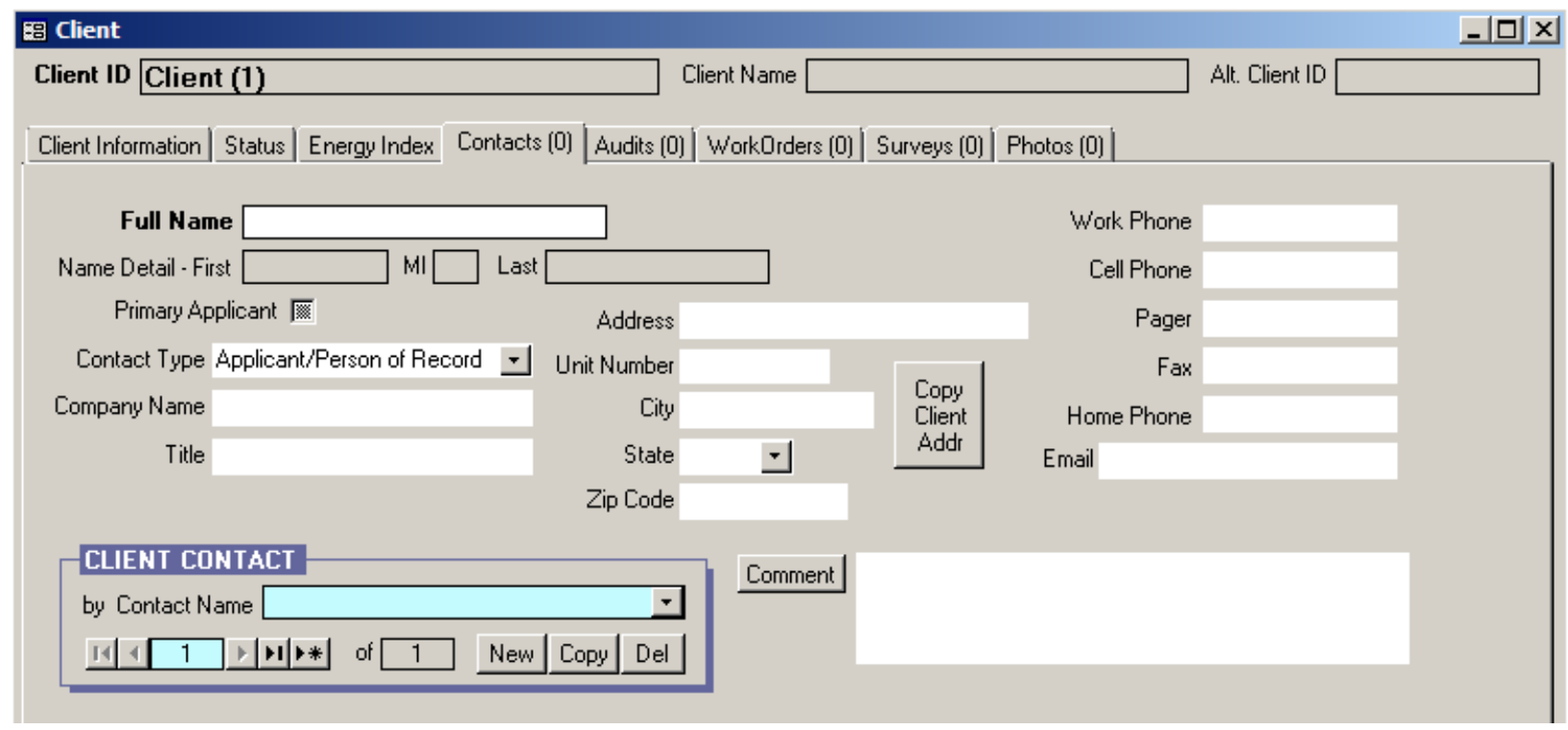

Figure 4.3. The Contacts form under the Main Menu's “Clients” button. 


\section{Chapter 4: Quick-Start Tutorial}

When finished, you can immediately start an audit for the client that you just entered by selecting the Audits tab. Alternatively, you can click on the X-box in the upper right corner of the "Client" window to return to the Weatherization Assistant's Main Menu and start an audit at a later time. Section 4.2, Starting a New Audit, discusses how to start an audit.

\subsection{Starting a New Audit}

Once you have created a client in the Weatherization Assistant, you can describe the client's home in either audit program (NEAT or MHEA), which will then allow you to run the audit to develop a set of recommended energy measures. You can start a new audit either by selecting the "Clients" button from the Weatherization Assistant's Main Menu (see Figure 4.1), or by selecting either the "Site Built (NEAT)" or "Mobile Home (MHEA)" buttons. Starting an audit from the "Client" window automatically enters the correct Client ID in the audit, which eliminates a common error, so this approach is recommended and described first.

If you just created a client as discussed in Section 4.1, Creating a New Client, and are still on either the Contacts form or Client Information form under the Main Menu's “Clients” button (see Figure 4.2 or Figure 4.3), you can start an audit for the client that you just entered by selecting the Audits tab to display the Audits form (see Figure 4.4). Otherwise, select the "Clients" button from the Weatherization Assistant's Main Menu (see Figure 4.1) to bring you to the Client Information form (see Figure 4.2), use the Client record navigation block in the lower left corner of the form to select the client that you want to create an audit for, and then select the Audits tab to display the Audits form (see Figure 4.4).

From the Audits form, select either the "Create New Site Built (NEAT) Audit" or "Create New Mobile Home (MHEA) Audit” button depending on which audit is applicable for the client's house. The buttons are on the bottom right on the form. This will bring you to the Audit Information form under the Main Menu's "NEAT" or "MHEA" button (see Figure 4.5 and Figure 4.6, respectively). 


\section{Chapter 4: Quick-Start Tutorial}

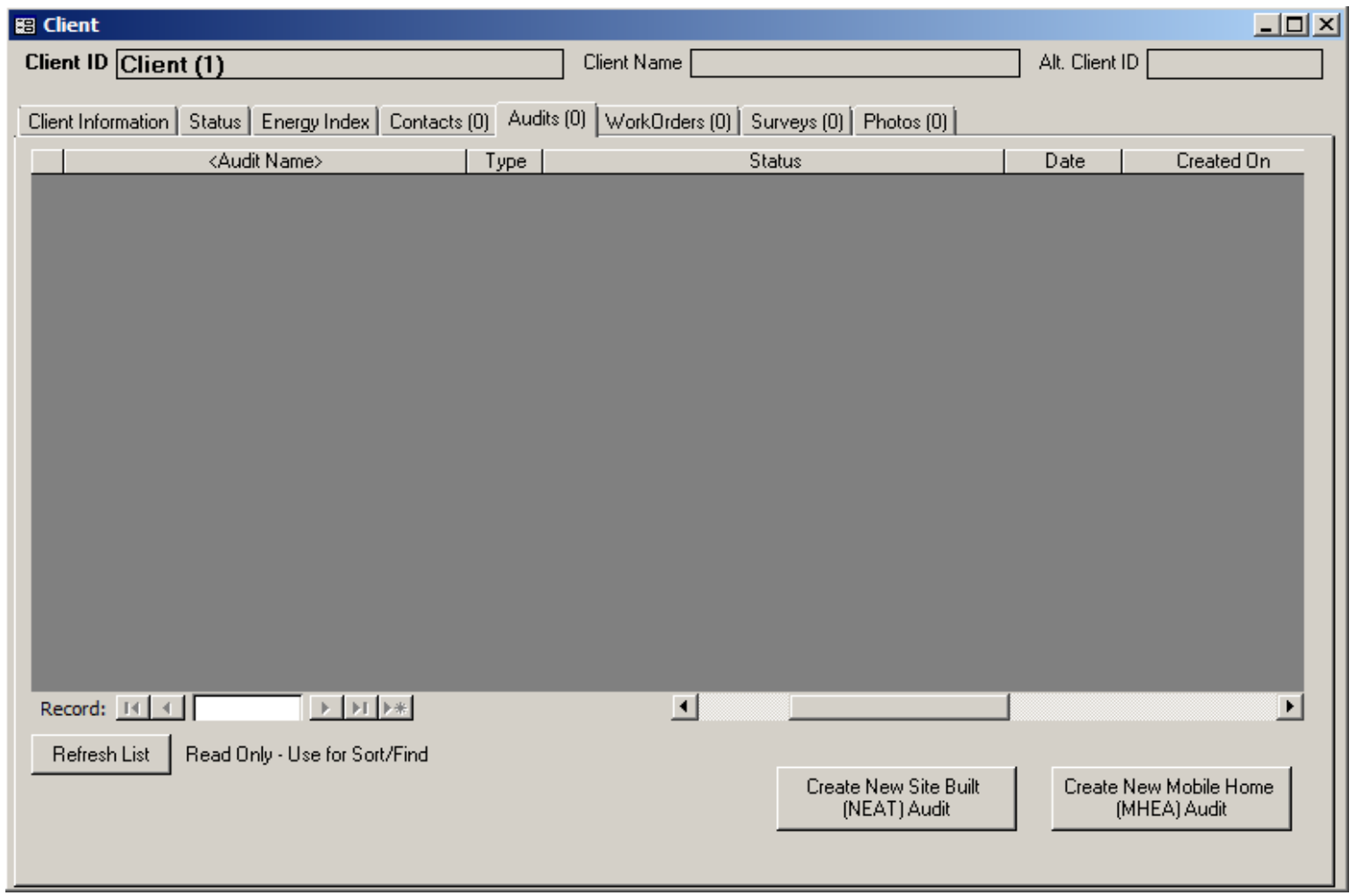

\section{Figure 4.4. The Audits form under the Main Menu's “Clients” button.}

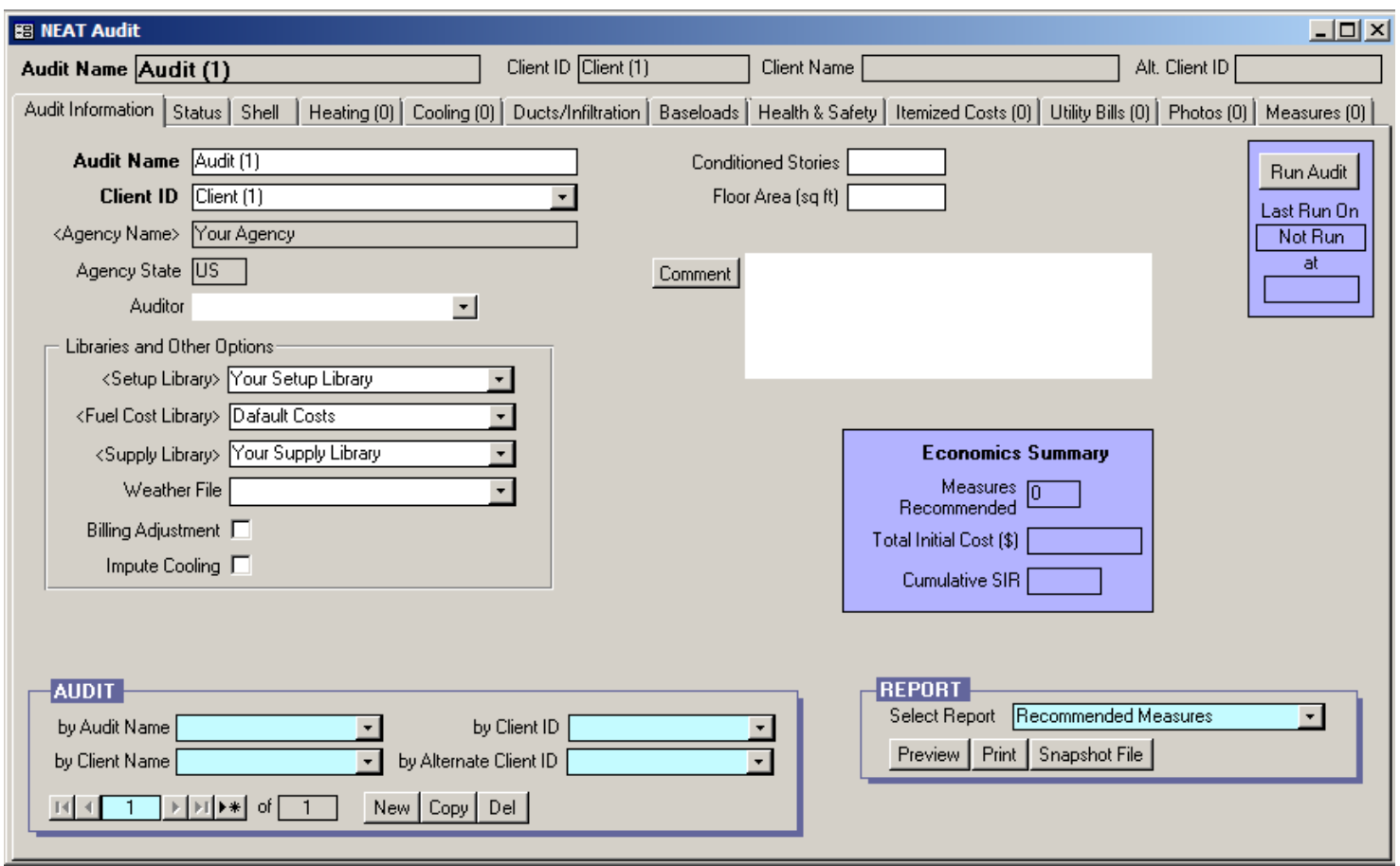

Figure 4.5. The Audit Information form under the Main Menu’s “NEAT” button. 


\section{Chapter 4: Quick-Start Tutorial}

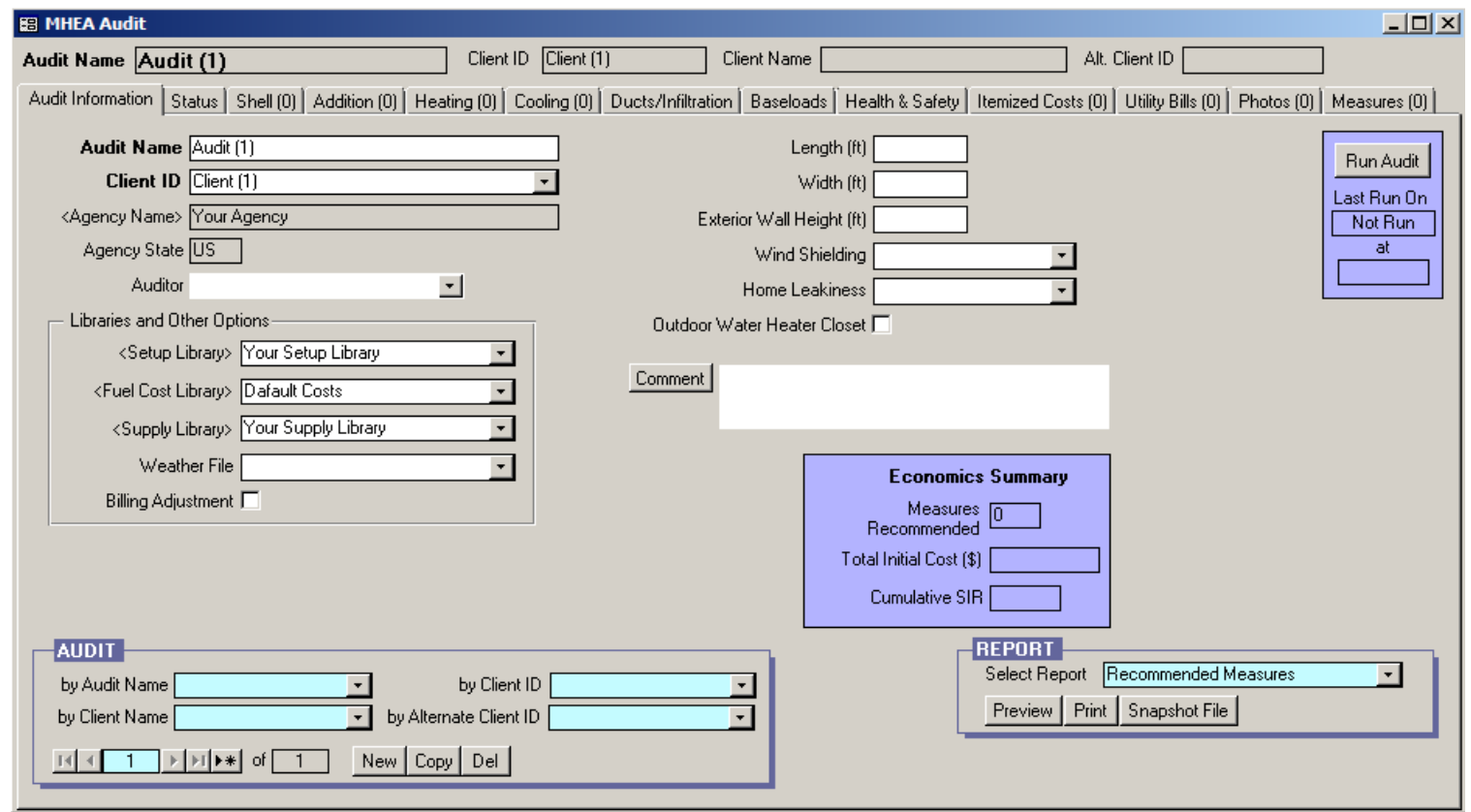

Figure 4.6. The Audit Information form under the Main Menu's “MHEA” button.

The Client ID, Agency Name, and Agency State fields will already be filled in using information you previously entered for the client. Change the Audit Name from the default name created for this new audit (e.g., "Audit (xx)") to a name conforming to the naming convention for the clients and audits you have developed. It is recommended that you use the Client ID in the Audit Name. For example, for a client named "John Smith” with a Client ID of “11_348," you might enter "11_348 Audit 1" as the Audit Name, signifying that this is the first (and probably only) audit you are creating for this client. Some may wish to include the client's last name in the Audit Name, such as "Smith Audit 1." However, if you have more than one client with last name "Smith", you may find locating the correct audit by the Audit Name difficult. Others have been known to use an Audit Name referencing the client's street address. This can become somewhat unwieldy.

Although not required, you may select the auditor to be assigned to this audit using the drop-down list in the Auditor field. If you selected yourself as an "Auditor" (see "Initializing Your Agency Information" in Section 3.2, Program Setup), you will be listed in the drop-down list, as will any other agency contacts declared as auditors.

Select the setup, fuel cost, and supply libraries to be assigned to this audit using the drop-down lists in the Setup Library, Fuel Cost Library, and Supply Library fields, 


\section{Chapter 4: Quick-Start Tutorial}

respectively. If you have not created more than one setup, fuel cost, or supply libraries for your agency, these entries will automatically be selected for you. The supply and setup libraries will be those that you named when you initially set up the Weatherization Assistant (see Section 3.2, Program Setup). The fuel cost library is a sub-library found in the setup library. You should have updated this fuel cost library and renamed it as part of the process of updating and populating the setup library (see Section 3.2, Program Setup). Select a city close to the client's home that has similar weather using the drop-down list in the Weather File field. Finally, enter audit data on the house in the remaining fields on the Audit Information form. Refer to Chapter 8, NEAT and MHEA Introduction, for more details.

At this point you may exit the "NEAT Audit" or "MHEA Audit" window and return later to enter the remaining house audit data, or you can enter all house data now under other tabs on the NEAT or MHEA audit forms (e.g., Shell, Heating, Cooling, Ducts/Infiltration, Baseloads, and Itemized Costs), as described in Chapters 9-11. In either case, when you are finished, click on the X-box in the upper right corner of the "NEAT Audit" or "MHEA Audit" window to return to the "Client" window, then click on the X-box in the upper right corner of the "Client” window to return to the Weatherization Assistant's Main Menu. One method of returning to the audit is somewhat the reverse of the above process used to exit the audit; that is, select the "Client" button from the Weatherization Assistant's Main Menu (see Figure 4.1) to bring you to the Client Information form (see Figure 4.2), use the Client record navigation block in the lower left corner of the form to select the client that you want to return to, select the Audits tab to display the Audits form (see Figure 4.4), and then double click on the name of the audit in the Audit Name column of the table that you want to return to.

The alternative method of starting a new audit for a client already entered under the Main Menu's "Client” button is to select either the "Site Built (NEAT)" or "Mobile Home (MHEA)" buttons from the Weatherization Assistant's Main Menu (see Figure 4.1), depending on which audit is applicable for the client's house. This will display the Audit Information form under the "NEAT Audit" or "MHEA Audit" window (see Figure 4.5 and Figure 4.6, respectively). This form will likely be filled in with information for a previously entered audit. Do not type over any information for an existing audit. 
Click on the "New" button in the Audit record navigation block in the lower left corner of the Audit Information form to obtain a new, essentially blank audit form. Select the client that this audit is being started for using the drop-down list in the Client ID field near the top of the form. The client information must have been previously entered as outlined in Section 4.1, Creating a New Client. Select "OK” if a warning box appears that says "This will assign the Audit to a new Client record.” The Agency Name and Agency State fields will be automatically filled in using the information you previously entered for the client. At this point, follow the same procedures outlined above for the other method of starting a new audit to fill in the information on the Audit Information form, starting with changing the Audit Name from the default name created for this new audit (e.g., "Audit (xx)") to a name conforming to the naming convention for the clients and audits you have developed.

As with the other approach of starting a new audit, you may exit the "NEAT Audit" or "MHEA Audit" window and return later to enter the remaining house audit data, or you can enter all house data now under the other tabs on the NEAT or MHEA audit forms, as described in Chapters 9-11. In either case, when you are finished, click on the X-box in the upper right corner of the "NEAT Audit" or "MHEA Audit" window to return to the Weatherization Assistant's Main Menu. To return to the audit, select either the "Site Built (NEAT)" or "Mobile Home (MHEA)" buttons from the Weatherization Assistant's Main Menu (see Figure 4.1) to bring you to the NEAT or MHEA Audit Information form (see Figure 4.5 and Figure 4.6, respectively), and then use the Audit record navigation block in the lower left corner of the form to select the audit that you want to return to.

\subsection{Running a New Audit}

When you are finished entering all the audit data for a house, you can run the audit by selecting the "Run Audit" button found in the upper right corner of each form under the "NEAT Audit" or "MHEA Audit" window. When you run an audit, a Recommended Measures report will automatically be displayed. The report lists all the cost-effective weatherization measures recommended for the house along with the estimated energy and cost savings, installation costs, and savings-to-investment ratios for the recommended measures. The report also provides other information such as the primary material quantities needed to perform each recommended measure, the total space-heating and space-cooling energy consumption estimated for the house, and the design heating and cooling loads for the house. The 


\section{Chapter 4: Quick-Start Tutorial}

Recommended Measures report is described in detail in Chapter 12, NEAT and MHEA Results.

The Recommended Measures report can be printed by clicking on the printer symbol in the menu bar located at the top of the report. Or, use the [Ctrl-P] key combination to call up your printer driver's form to further control the printing process. When finished reviewing the report, use the "Close" button in the menu bar located at the top of the report to exit the report and return to the audit. 


\section{Chapter 5. Navigation and Data Entry}

The Weatherization Assistant uses a graphic user interface (GUI) provided by Microsoft's Access XP (2003). The Weatherization Assistant uses all the standard Access forms, controls, and the concept of records contained in this software. Thus, if you are familiar with Access, you are probably already proficient with the mechanics of entering data into the Weatherization Assistant. Otherwise, this chapter will supply you with the basics needed for this task.

\subsection{Form and Record Navigation}

Data entry and display in the Weatherization Assistant uses forms. Each form contains information related to a specific topic, such as the Client Information form as found under the Main Menu's "Clients" button or the Heating form, as found under the Main Menu's "NEAT" and "MHEA" buttons. You navigate to the different forms found in the Weatherization Assistant using the tabs found under each of the Main Menu's buttons. In some cases, forms and/or sub-forms are accessed through buttons found on other forms.

The term "record" refers to the information contained on a specific form. The terms "record" and "form" are often used interchangeably, although you should keep the somewhat subtle difference in definitions in mind. To navigate to a record you are interested in on a given form, you can use the controls in the record navigation block located in the lower left corner of the form. The type of record you can find using the record navigation block is indicated in the upper left border of the block. For example, in Figure 5.1, audit records can be found using the Audit record navigation block that is located on the Audit Information form under the Main Menu's "NEAT" and "MHEA" buttons. There are three types of controls on record navigation blocks you can use:

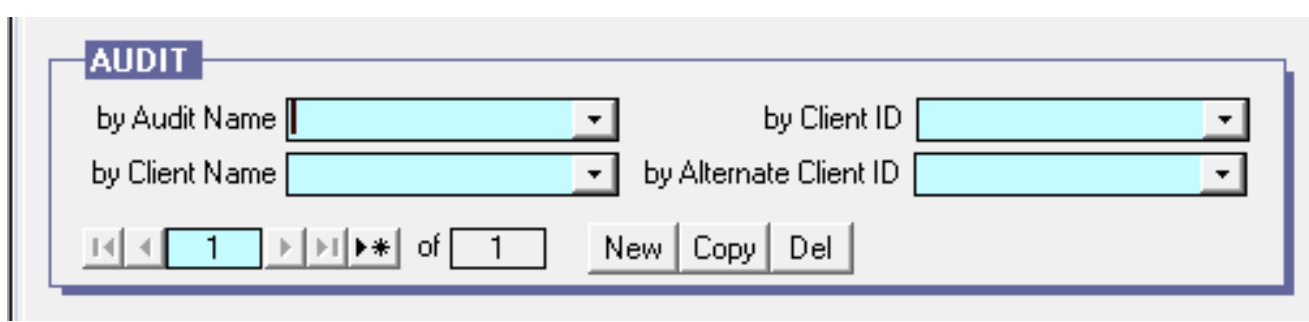

Figure 5.1. The Audit record navigation block found on the Audit Information form under the Main Menu's “NEAT Audit” and “MHEA Audit” buttons. 
- Drop-down Lists - The drop-down lists included in most of the record navigation blocks include listings of all available records arranged in alphanumeric order "by" various identifying fields. For example, in Figure 5.1, you can find a specific audit record by searching by the audit name, client name, client ID, or alternate client ID. Clicking your mouse on any item listed in one of the drop-down lists will position you to that record (i.e., will display that audit's records on the form).

- Navigation buttons - Records are stored in alphabetical order by one of the identifying fields (e.g., Audit Name or Client Name) and then numbered sequentially. You can use the navigation buttons at the bottom of the record navigation block to move to a particular record number by entering the appropriate number, using the first or last record buttons ( 1 . or $\mathbf{1 1}$, respectively), or using the next or previous record buttons ( $D$ or 1 , respectively). The total number of records currently stored is shown in the data field following the word "of."

- Other control buttons - Finally, remaining buttons in the record navigation block allow you to create, copy, or delete records. Use the "New" button to create a new blank record, the "Copy" button to copy the currently accessed record, and the "Del” button to delete the currently accessed record.

You can also locate and move to a desired client, audit, or work order record by accessing the Clients, Audits, or Work Orders forms found under the Main Menu's "Agency" and "Clients" buttons (see Figure 5.2). These display-only forms are meant just for navigation. They provide a relatively condensed view of all records of the type selected by displaying the available records in a spreadsheet format or data sheet view. Information in these forms cannot be altered, as indicated by the information being shaded. To navigate to a desired client, audit, or work order record, double click on the desired entry under the Client ID, Audit Name, or Work Order Name columns, or any column with a header that is in angle brackets $(<>)$. See Section 5.8, Data Sheet and Form Views of Records, for more information on how to use these navigation data sheet views.

The bookmarks feature is enabled when the Weatherization Assistant is first installed (see Feature 9 in Section 16.4, Features). If you select the "Agency," "Client," "Audit," "Work Order," "Setup Library,” or "Supply Library” buttons on the Main Menu with this feature enabled, the Weatherization Assistant will 


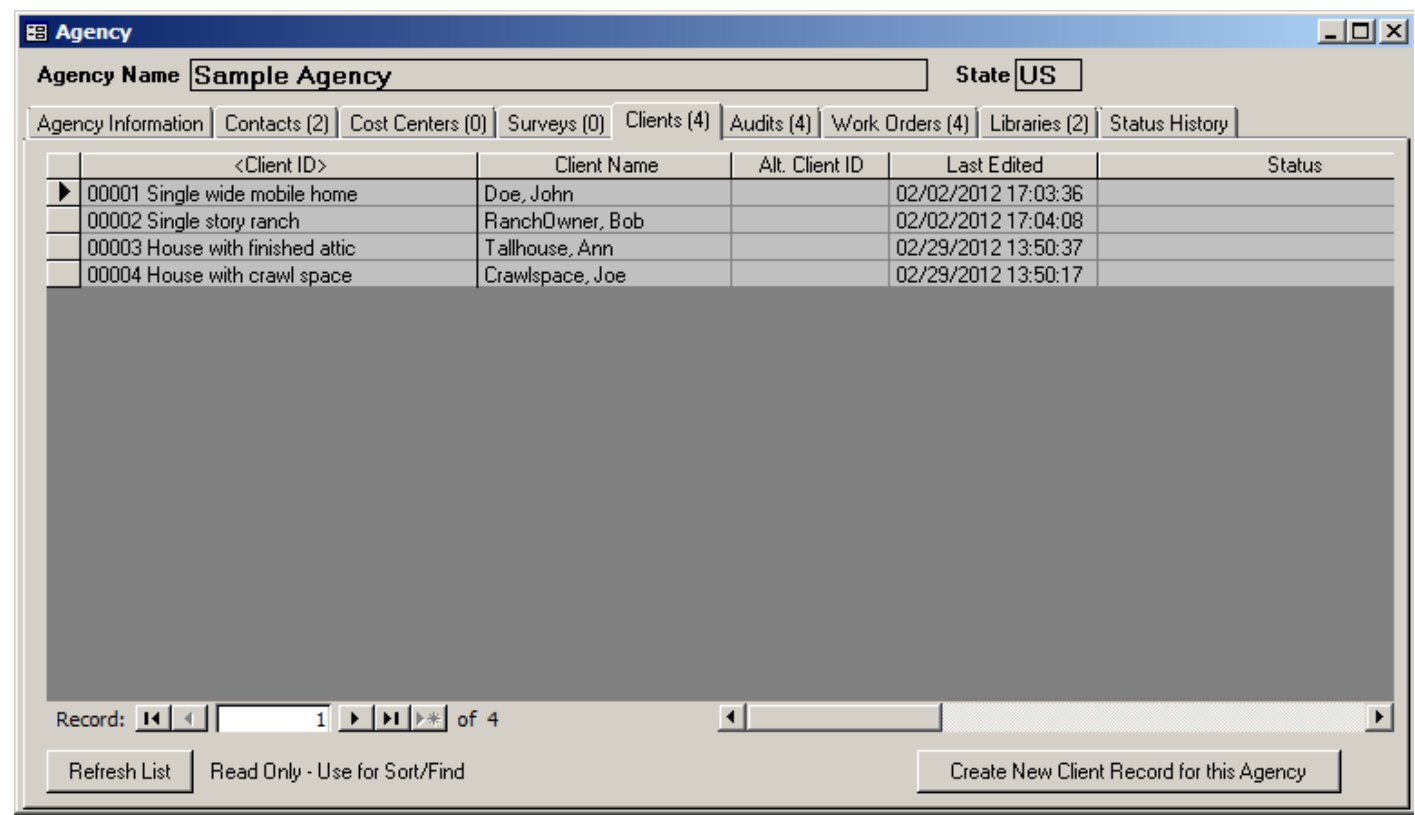

Figure 5.2. The Clients form under the Main Menu’s “Agency” button.

automatically return you to the same record you were last working on when you exited that record type to return to the Main Menu.

Data for a record are stored in your computer's temporary memory rather than to the Weatherization Assistant's database file that resides on a hard drive when you start filling in data on a blank form or modify data on an existing form. When you exit that form or navigate to a new record, the record you were working on gets saved to the Weatherization Assistant's database file, which typically resides on your hard drive or the hard drive of a network computer. There are a number of completeness and relational integrity checks performed by the Weatherization Assistant when the record is being saved. This is why you may get warning and error messages when you try to leave a form or navigate to a new record (see Section 5.11, Entry Errors).

\subsection{Entering Data into the Weatherization Assistant}

Forms contain three types of controls (or fields) that accept input from the user: textboxes, drop-down lists, and checkboxes.

Textboxes (see Figure 5.3) allow you to type in entries from the keyboard and are

\author{
Program Year 2005 \\ Description The above field must be numeric.
}

Figure 5.3. Two textboxes, one requiring a numeric entry. 


\section{Chapter 5: Navigation and Data Entry}

not restricted to any pre-selected set of responses. However, they often must satisfy certain conditions to be acceptable. For example, a field expecting a number cannot contain an alphabetic character.

Drop-down lists (see Figure 5.4) look like textboxes except that they have a square with a downward pointing arrow to the right of the field. Entries into these controls are restricted to one of several predefined responses.

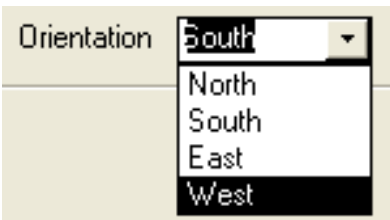

Figure 5.4. A drop-down list with its item list visible.

The drop-down list entries can be selected in one of three ways:

- Left click on the box with the arrow, and then click on the desired response from the item list that appears.

- Once you have navigated to the control, press the [Alt- $\uparrow$ ] key combination to display the item list, use the $[\uparrow]$ and $[\downarrow]$ keys to highlight the desired response.

- Whenever the entire field is selected (i.e., the entry is in reverse video), you may begin to type in one of the acceptable responses. As soon as the characters you type uniquely match one of the acceptable responses, the remainder of the field will be automatically filled in.

In all of the cases above, navigating from a drop-down list to a new control (e.g., pressing either the [Enter] or [Tab] keys) completes the selection. You will not be allowed to exit a drop-down list unless an acceptable response has been provided, or you press the [Esc] key to back out of your changes (see Section 5.4, The Escape Key).

Some drop-down boxes do not require the entry to be one of the predefined responses. The exceptions are the fields for the Existing Refrigerator and Water Heater Manufacturer and Model under the Audit Base Loads tab as well as the fields designating the location of zonal pressure and pressure pan readings under the Ducts and Infiltration tab of the audits. These fields are a combination of a drop-down list and a textbox, which lets you select an entry from the drop-down list as well as allows you to type in entries from the keyboard. However, you will obtain the data from the database of refrigerators and water heaters only if your 


\section{Chapter 5: Navigation and Data Entry}

entry exactly matches one that is in the database. See "Manufacturer and Model" in Section 11.4, Base Loads - Water Heating, and Section 11.5, Base Loads Refrigerators.

A green shaded drop-down list indicates that the selection is being made from a library of entries, normally from your Supply Library. In such circumstances, the information provided in the item list includes not only what will appear in the drop-down list, but most often additional data associated with each item.

When the characters in a textbox or drop-down list are highlighted (displayed in reverse video), the control is said to be in overwrite mode. Any characters that are highlighted in a control will be replaced by keystrokes you enter. Any number of characters can be highlighted by left clicking the mouse while dragging it across those you wish highlighted.

To switch to insert mode, position the mouse pointer to a location within the textbox or drop-down list and left click. This will allow you to insert characters between existing ones. While in insert mode within a textbox or drop-down list, the $[\leftarrow],[\rightarrow]$, [Home], [End], [Backspace], and [Delete] keys retain their traditional functions of positioning the cursor within the field. Single character highlighting (replacing only individual characters) can be obtained by pressing the [Insert] key on your keyboard.

The checkbox (see Figure 5.5) is a small white box in which a check mark may or may not be visible. If a check is visible, it implies a response of "Yes" to the question or statement associated with the box. You may change the response in a checkbox either by left clicking the mouse on the box or pressing the [Space]

Replacement Door Required $\nabla$ Include in SIR $\Gamma$

Figure 5.5. Two checkboxes, the top one being selected and with a "Yes" response.

key whenever the box has been selected (indicated by a dotted rectangle around the control name).

\subsection{Navigating Between Controls}

You can use either the mouse or keyboard to navigate among the controls on forms. You will typically use a mixture of both methods. The mouse is most intuitive for navigating between forms, menus, and tabs, but you may find the keyboard [Tab] key or other keys to be quite handy for navigating on forms. This 
may be especially true during lots of data entry when constantly moving between the mouse and keyboard can be tedious. Experiment and see what methods are most comfortable for you.

You can point and click the left mouse button on the textbox or drop-down list you want to edit or the checkbox you want to select. Accessing a textbox or drop-down list in this manner will automatically put you into the insert mode within the control, with a vertical line indicating where the next keyboard character will be inserted.

You can use the [Tab] key and the [Shift-Tab] key combination to cycle through the controls in forward or reverse order, respectively. These keys will place you in overwrite mode within a textbox or drop-down list, highlighting its entire contents. In most cases, the [Enter] key will act in the same manner as the [Tab] key. One exception is when positioned in a comment field. Here, the [Enter] key is reserved for moving the cursor to a new line within the comment. The $[\uparrow]$ and $[\downarrow]$ keys will work the same as the [Shift-Tab] key combination and [Tab] key, respectively, unless you are in the insert mode within a field, during which the keys will be inoperative.

\subsection{The Escape Key}

The [Esc] key is a useful way to back out of your changes. It is similar to an "undo" command. If you have made changes to a textbox or drop-down list but have not yet left the control, pressing the [Esc] key restores the value that was in the control prior to your change. Pressing the [Esc] key twice consecutively (or only once if you are not in a control) will back out of all changes you have made to all the controls on a form since you entered it. This can be particularly useful if, for some reason, entries you have made on a form produce an error in the input that will not allow you to exit the form. Sometimes identifying the error is difficult and you are, therefore, prevented from continuing data entry. Pressing the [Esc] key twice will place the form back to its original state, which was acceptable input. You will lose the data entered on the form but will be able to continue data entry.

Once you leave a form and navigate to another form, the changes in the former form are saved and cannot be reversed. Thus, if you have doubts whether many changes you will make to a record are desired, you might consider making a copy of the record prior to initiating the changes. 


\subsection{Required Versus Optional Textboxes and Drop-down Lists}

Required textboxes and drop-down lists have a single solid black border around the entry (see Figure 5.6). If you try to exit a form with a required textbox or drop-down list blank, you will be reminded to complete all required textboxes and drop-down lists, and the cursor will be positioned to the first required textbox or drop-down list that is missing an entry. If you decide NOT to save the record, you can use the [Esc] key to cancel your changes.

Textboxes and drop-down lists without the solid line border are not necessary for the Weatherization Assistant to execute or to allow saving the information on the form. However, the data may be necessary for clarity, record keeping requirements, or simply identification purposes.

In the remainder of this user's manual, the description of required entries will end with "Required" to clearly indicate that they are mandatory and must have an appropriate value entered in them before you can proceed. Other, non-required entries will be identified by descriptions that end with "Optional" to indicate that they may be left blank.

\subsection{Field Defaults}

Many of the numeric fields in the Weatherization Assistant, especially those under the Main Menu's "NEAT" and "MHEA" buttons, have a default value associated with them. This default value is displayed in the lower left corner of the screen in the status bar when you enter a field. A short description of the field is provided in the status bar followed by a set of square brackets (see Figure 5.7). If a default value has been assigned to this field, it will be displayed in this set of brackets as “Default Xx (TAB on blank field to accept)." If you leave a required numeric field using the [Enter] or [Tab] keys without having entered a value, the default value will be automatically entered. You can edit the default values that have been assigned to the numeric fields by selecting the Range Check and Default Values tab under the Main Menu's "Preferences” button (see Section 16.2, Range Check and Default Values). Some numeric fields have default values that are computed 


\section{Chapter 5: Navigation and Data Entry}

from other values you have already entered. An example is the Steady State Efficiency field on NEAT's Heating form. Computed defaults override values entered under Preferences, though either may be replaced by your own entry.

Number of conditioned stories [Min 1 , Max 4 ,Default 1 (TAB on blank field to accept) ]

Figure 5.7. A status bar showing the reasonable range and default value for the Number of Conditioned Stories field.

\subsection{Field Range Checking}

Numeric fields have range checks applied as soon as you exit the field. There are two ranges that are checked: the acceptable range and the reasonable range.

The acceptable range determines if a number entered is acceptable to the analysis engine. For example, it makes sure that all window dimensions are positive numbers. All numeric

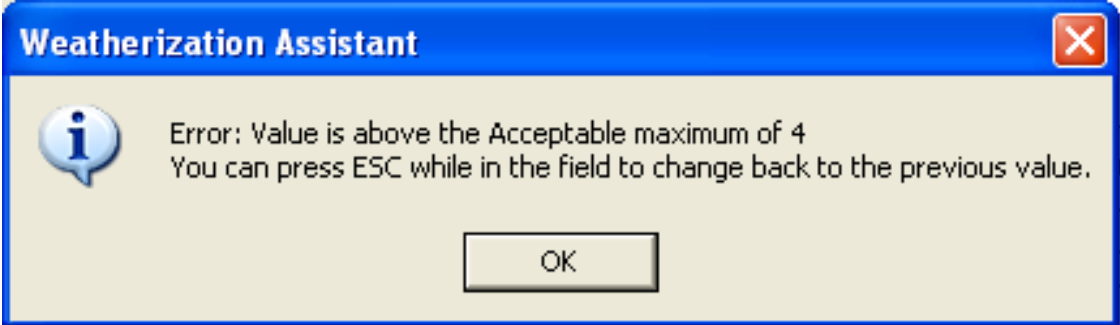

Figure 5.8. Error indicating that a numeric entry is outside the acceptable range.

\section{entries must fall within}

the acceptable range set for each field. If you enter a number outside the acceptable range for a field, you will get an error message (see Figure 5.8). You will then have to select the "OK" button to go back to the form to change the data entered in that field, or to use the [Esc] key to back out of the latest entry and change the entry back to its previous acceptable value (perhaps a blank field). You will not be allowed to continue entering data in any other field until you change the entry to an acceptable value. The acceptable ranges cannot be changed by the user.

The reasonable range checks to see if the number entered falls within a range of values that might be reasonable for the field in question. For example, for window dimensions, the reasonable range may be between 4 and 90 inches. The minimum and maximum values are displayed in the status bar in the lower left corner of the screen when you enter the field (see Figure 5.7). If you enter a value outside the reasonable range, you will get a warning message stating that the value is above or below the reasonable maximum or minimum value, respectively (See Figure 5.9). You can override the message to enter the value desired by selecting the "OK" 


\section{Chapter 5: Navigation and Data Entry}

button. Just be sure to check the accuracy of the value you entered if you choose to override the warning message. Otherwise, you will need to select the "Cancel" button to return to the field to correct the number entered. You can edit the reasonable minimum and maximum values that have been assigned to the individual numeric fields by selecting the Range Check and Default Values tab under the Main Menu's “Preferences” button (see Section 16.2, Range Check and Default Values).

\section{Weatherization Assistant}

Warning: Value is below the Reasonable minimum of 700

Press OK to override and continue.

Press CANCEL to return to the field to correct the value.

Press ESC while in the field to revert to the previous value.

You can also adjust the reasonable range using

Preferences'Range Check and Default Values from the main menu.

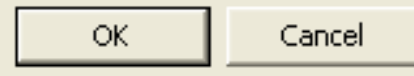

Figure 5.9. Warning indicating that a numeric entry is outside the reasonable range.

\subsection{Data Sheet and Form Views of Records}

The Weatherization Assistant provides you with the ability to display different views of your records on many of the forms, so you can select the view that best suits your needs. Multiple views are available more often for the forms found under the Main Menu's "NEAT" and "MHEA" buttons, although they are also available for some of the forms found under the Main Menu's "Agency," "Client," "Work Order," "Setup Library," and "Supply Library" buttons. There are three basic views supported, two of which allow you to edit the data displayed or enter new data in a new record:

- Form View - The form view is the most common view, allowing you to see and edit all of the information for a particular record, or enter new data into a blank record. The controls on the form are usually arranged or grouped in ways that will contribute to your understanding of the information requested. In those instances when a form has sub-forms, it is normally only in the form view that all of the sub-form information can also be viewed.

- Navigation Data Sheet View - This view displays a listing of all records of a particular type in a spreadsheet format (see Figure 5.2). The columns in the spreadsheet represent the information entered for a given control. Data displayed in the navigation data sheet view cannot be altered. This view is used primarily to provide a summary of records and to allow you to navigate to the 
record you choose. If the number of records is large, you may have to scroll vertically to see all the records. This view is distinguished from the editable data sheet view described below by noticing that the information presented in the navigation data sheet view are all shaded. See Section 5.1, Form and Record Navigation, for additional relevant discussion.

- Editable Data Sheet View - Like the navigation data sheet view, the editable data sheet view displays a listing of all records of a particular type in a spreadsheet format, with the columns in the spreadsheet representing the information entered for a given control. However, data displayed in the editable data sheet view can be edited, and data for new records can be entered. If the number of records is large, you may have to scroll vertically to see all the records. Typically, the number of controls or columns is too wide to fit on one screen, so you must also scroll horizontally to view all of the available controls. In some instances, all of the controls found on a form will not be displayed in the editable data sheet view.

The forms accessed by the Clients, Audits, or Work Orders tabs under the Main Menu's "Agency" and "Clients" buttons are set up to be displayed in the navigation data sheet view. These forms cannot be displayed in any of the other two views.

The form view is the default view for all other forms in the Weatherization Assistant. The editable data sheet view is also available for most of these other forms (and some sub-forms). These include the Contacts and Cost Centers tabs under the Main Menu's “Agency” button, the Contacts tab under the Main Menu's "Clients" button, the Measures tab under the Main Menu's "Work Orders" button, and many of the tabs under the Main Menu's "NEAT" and "MHEA" buttons. Before you use the editable data sheet view exclusively, you should view records in both the form view and the editable data sheet view until you become familiar with the contents of each record type.

It is often helpful to temporarily switch from form view to editable data sheet view to see all of your records for a given type. To switch from form view to editable data sheet view, right click anywhere on the form but not inside a control. A rectangular "Subform Datasheet" pop-up window will appear. Left click on the rectangular window and the view will change to the data sheet view. To change back to form view, position your cursor on the record you are interested in seeing 
in form view, then right click your mouse. The same "Subform Datasheet" window will appear. Left click on the rectangular window and you will be taken to the form view for that particular record.

The editable data sheet view also allows you to easily change all the entries in a given control. For example, if you wanted to change the Frame Type of all windows in a NEAT audit, place the Windows form under NEAT's Shell tab into the data sheet view, locate the Frame Type column, and then simply type in the first letter of the desired frame type in the drop-down list as you use the down arrow key to travel down the column. This allows the automatic fill feature of the drop-down list to complete the entry. Instead of entering the first letter of the desired frame type, you could change the entry in the first record (or make sure it is correct) and then use the [Ctrl-'] (control-apostrophe) key combination in subsequent records to copy the contents of the control from the previous record (which, in the data sheet view, is the field immediately above) into the current record.

The data sheet view is also handy for sorting the list of records shown. Click your mouse on the title header of the column of data that you want to sort the records by to display the entire column in reverse video. Then select either the "A-Z" or " $\mathrm{Z}$ A" buttons on the main application tool bar at the top of the application's window to sort the records in alphabetical or reverse alphabetical order, respectively. You can sort the records using multiple columns by selecting multiple adjacent columns in the data sheet. Just click and drag in the column header to select multiple columns. The sort order is determined by the position of the selected columns, from left to right. The ordering of columns can be changed by highlighting a column(s) then clicking and dragging the column to a new position. Several of the left most columns cannot be re-ordered because they are considered to be identifying information for the records. Column widths can be adjusted by dragging the column header borders. All column width and position settings affect only the current data sheet view. When you close the form, the original column widths and order will be restored.

A few specialized data sheet views will not allow sorting by multiple columns. In these data sheets, selecting an entire column of data will turn the "A-Z" and "Z-A" buttons grey, making them un-accessible. Instead, place your cursor in any single record of the type you wish to sort on. Then select either the "A-Z" or "Z-A" buttons. 
You may also "filter" the records when displayed in the editable data sheet view. Filtering forces the display of only those records that have a specified value for a given data item. For example, if one of the columns displayed the city in which the clients dwelt, you could choose to display only records for clients who lived in one specified city. To do so, click your mouse on any city entry of the particular city you are interested in, and then click on the filter button $\left(\nabla_{\ell}\right)$ at the top of the window. Your display will then include only those clients from the city selected. Be careful when using filtering. If you forget that your display of records is filtered, you may wonder what happened to some records you would otherwise expect to see in the display. To cancel filtering, select the remove filter button (可).

\subsection{Copying and Pasting Data}

Data records are normally copied while in the form view using the "Copy" button in the record navigation blocks (see Section 5.1, Form and Record Navigation). When this technique is used to create a copy of a record, you will be placed immediately into the form view for the new record. Most often, records have a data field that must be unique among all of the records of the specific type (e.g., the Client ID or the Audit Name). Whenever the "Copy" button is used to copy such a record, the copy process will provide you with a default entry for this data field that is unique (a significant advantage compared to the method of copying records while in the editable data sheet view describe below). In addition, your cursor will be positioned at this data field, with the field in reverse video, allowing you to immediately change the default entry to a value or name that makes more sense to you.

The process of copying a record while in the editable data sheet view is different. You must first select the record you wish to copy by clicking the record selection box at the very left of the record you wish to copy. This will display the entire row of data in reverse video. Copy the information in this record to a temporary location (referred to as the "clipboard" in most computer applications) by either entering the [Ctrl-C] key combination, selecting the "Copy" button (displayed as two small overlapping sheets of paper) from the toolbar at the top of the application window, or selecting the Edit/Copy menu items from the menu bar at the top of the application window. Select the blank record row by clinking the record selection box with the asterisk (“*”) in it. This will place the entire blank record row into reverse video. Finally, paste the copied record into the blank record by either entering the [Ctrl-V] key combination, selecting the "Paste" button 
(displayed as a clipboard with a small sheet of paper) from the toolbar at the top of the application, or selecting the Edit/Paste menu items from the menu bar at the top of the application window. This method of copying records does not provide you with default unique entries for those fields that must be unique. Therefore, immediately after pasting the copied record into the blank record row, you must provide a new unique entry yourself before proceeding.

Data presented in the data sheet view or any similar tabular format within the Weatherization Assistant can be copied and then pasted into an equivalent data range within the Weatherization Assistant. For example, you might want to copy the fuel prices from one setup library to another. To do this, go into the Fuel Costs tab of the setup library that you want to copy the fuel costs from. Click your mouse on the Unit Cost column header so that the entire column becomes displayed in reverse video. Use one of the techniques described above to copy this data to your computer's clipboard. Then, go to the Fuel Costs tab in the setup library that you want to paste the costs into. Select the Unit Cost column as before, and use one of the techniques described above to paste the copied data into this column. The existing fuel costs will be replaced with those that you copied. Care must be taken to always copy and paste the same amount and type of data.

Data presented in the data sheet view or any similar tabular format within the Weatherization Assistant can also be copied and then pasted into another Windows application. For example, the fuel costs copied above could also be pasted into Microsoft Excel. After copying the desired fuel costs, open the Excel application and position your cursor in the cell where you want the upper left corner of the data to be located. A single paste instruction will paste all of the copied data into the spreadsheet. You may need to adjust column widths to properly display the data, after which you can print the data from Excel.

The data entered in a single control or field can be copied and pasted, but make sure both controls or fields are the exact same type. To select the data to be copied, click and drag the mouse over the contents of the control or field, or use the [Tab] key to enter the field. Either way, the contents of the control or field will become highlighted and selected. Alternatively, if the control or field has only one word in it, you can simply double click on the control or field to highlight and select it. Then, use the copy and paste techniques described above to complete the process. 
You can also use the [Ctrl-'] (control-apostrophe) key combination when you are in a control or field to copy the contents of the same control or field from the previous record. This is particularly useful in the data sheet view when you want to make a copy of the field directly above the current field in the same data sheet column. See Section 5.8, Data Sheet and Form Views of Records, for more details.

\subsection{Deleting Data}

Once you have become familiar with all the techniques of selecting data described in the Section 5.9, Copying and Pasting Data, deleting data becomes relatively simple. To delete data in a single control, select the data you wish to delete and then either press the [Delete] key on your keyboard, select the "Cut" button (displayed as a pair of scissors) from the toolbar, or select the Edit/Cut menu items from the menu bar. Any deletion of data from a drop-down list must be replaced by another selection available from the drop-down list.

To delete an entire record while in form view, use the "Del" button in the record navigation block. This will delete the entire record currently accessed and of the type indicated by the navigation block. After hitting the "Del" button, the Weatherization Assistant will prompt you to make sure you really intend to delete the current record. When you delete a record, you also automatically delete all of the unique records that fall under it (called cascading deletes). For example, deleting a client record deletes all audits, work orders, and reports associated with that client, but it would not delete the setup and supply libraries associated with the client. A special case occurs in deleting setup or supply libraries. The Weatherization Assistant will not allow you to delete any library that is referenced by any agency, client, audit, or work order. You first have to reassign any of these references to another library before you can successfully delete it (see Section 5.14, The References Button).

To delete an entire record while in the editable data sheet view, click on the record selection box on the left of the record you wish to delete to display the entire row of data in reverse video. Then, use one of the three techniques described above to delete the data.

You can use the editable data sheet view to delete multiple records. Use the sorting feature of this view to make those records you wish to delete appear together. Select multiple records by moving the cursor to the record selector on the left side 
of the data sheet for the first record you wish to delete (the cursor will change to a right arrow), and then clicking and dragging the mouse across the record selectors until the records you want to delete are all highlighted. Press the [Delete] key, or use one of the other deletion methods described above. The Weatherization Assistant will ask you if you are sure you want to delete the number of records selected.

The Water Heating and Refrigerators forms found under the Main Menu's "NEAT" and "MHEA" buttons do not have a record navigation box in the form view and do not have an editable data sheet view available. If you have entered data on these forms and later wish to delete it, select the "Del" button at the lower left of the form. After confirming your intent to delete the record, the form will be blanked out. Choosing the "New" button, also in the lower left corner of the form, will not delete the entries on the form, but simply place your cursor in the Manufacturer field, preparatory to your selecting a different record from the database.

\subsection{Entry Errors}

The Weatherization Assistant may detect a data input error once the entry has been made or after you have selected the "Run Audit" button to run an audit. The error messages provided can be fairly descriptive, allowing you to clearly identify the problem. For drop-down lists, the most common error message is "The text you entered isn't an item in the list," indicating that you did not select one of the predesignated items from the drop-down list. Another possible data entry error message is "The value you entered isn't valid for this field." This would most likely occur because you accidentally input an alphabetic character rather than a numeric entry.

Occasionally, you may not completely understand the entire error message. However, the message may refer to a "table" that is causing the problem. The name of the table will lie in single quotes and will be the name that appears after "tbl." For example, an error message that refers to "tblAgency" is indicating a problem with a data entry in the Agency table. The table names generally coincide with the names found on the various tabs used within the Weatherization Assistant. Therefore, if you are fairly familiar with the Weatherization Assistant, you may be able to identify the form within the Weatherization Assistant that is creating the problem. For example, "tblAgency" refers to a problem with the Agency table that 


\section{Chapter 5: Navigation and Data Entry}

is associated with the Agency Information form found under the Main Menu's "Agency" button. In such instances, locate the form, examine the entries, and try to determine which entry does not conform to the requirements given in this manual.

\subsection{The Report Block}

A Report block (see Figure 5.10) appears in the lower right corner on the Information tabs under most of the Main Menu buttons: Agency, Clients, NEAT and MHEA Energy Audits, Work Orders, Setup and Supply Libraries.

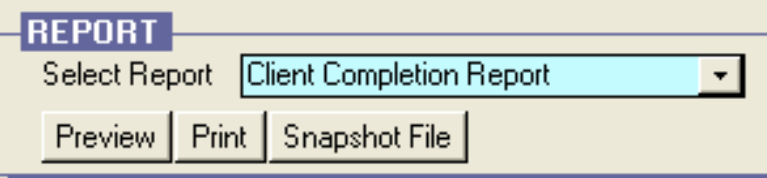

Figure 5.10. The Report block from the Client Information form.

The "Select Report" drop-down list in each Report block provides you with a list of all the reports available to you for the program segment you are currently accessing. Examples of all the standard reports provided with the Weatherization Assistant are shown in Appendix C, Reports. If you are familiar with Microsoft Access, you may be able to develop your own customized reports and add them to the report list following the procedures outlined in Appendix D, Development of Customized Reports. Choose the report you want to access using the standard mechanics for drop-down lists (see Section 5.2, Entering Data into the Weatherization Assistant).

The "Preview" button in the Report block displays the selected report as it would appear if printed. A standard Access toolbar will appear at the top of this display (see Figure 5.11). Three buttons on the toolbar allow you to display varying numbers of pages of the report. Each button looks like a single dark colored rectangle with light colored pages in it. A single page indicates viewing one page at a time, two pages side by side allows you to view two pages at one time, and the button with four small pages allows you to choose from an additional six predefined layouts. Depending on the resolution you have chosen for your graphics, many of these displays will not have large enough text to read. Clicking the mouse while positioning your cursor at a location on the display will enlarge the view about that 
point. You may also use the Zoom drop-down box, seen with "Fit" selected in Figure 5.11, to select a magnification.

Also on the toolbar is a print button, normally at the extreme left of the toolbar. Select this button to print the report using your default printer. You may also use the [Ctrl-P] key combination to display the Windows Print screen and print the report from there. This form allows you to select other printers you may have installed, choose which pages to print, and allows you to use other print options as well. If you have the PDF software, one of the printers available will be the PDF generator. Selecting this will save the report as a PDF file that can be distributed via most electronic media.

The right-most button on the toolbar is the "Output to Notepad" button. Select this button to export the report to a pure ASCII text file and display it in Notepad (a Windows application). From there, the file may be saved to any location on your computer with any file name you wish. It may also be edited as any other text file, attached to e-mails, etc.

Close the report display using the "Close" button on the toolbar. The standard Windows closure X-box in the upper right of the display may also be used for this purpose, but care must be taken to select the closure X-box associated with the report display, since other windows may also be open at the same time with their own closure X-boxes.

The "Print" button on the Report block acts the same as the "Preview" button except it automatically presents you with the Windows Print screen in addition to displaying the report.

The "Snapshot File" button on the Report block also displays the report, but only after creating a snapshot file and indicating the location where the snapshot file was placed on your computer (e.g., "Snapshot output file created: C:IProgram DatalWeatherization Assistant 8-9loutput\NAuOut-01.snp”). The filename is a default name that you may change using standard Windows procedures. A "Snapshot" file is a file formatted specifically for the "Snapshot" computer application. If you do not have this software on your computer, this option may not be of much use to you. 


\subsection{Getting Help}

You can get help anywhere within the Weatherization Assistant by pressing the [F1] key. The help material will usually be specific to the current control (textbox, drop-down list, or checkbox) that you are on. If help material for that specific control is not available, the Weatherization Assistant will display help material for a more general topic associated with the specific form that you are on.

The right pane of the help window will display the title of the help material in bold letters at the top with the help material underneath. The left pane of the window will contain one of three displays, depending on the tab chosen for the pane. The Contents tab displays a listing of all help topics available in the Weatherization Assistant. You can choose topics by browsing through the expandable Contents tree. Just double click on a topic in the tree to reveal any sub-topics associated with the main topic. The Index tab allows you to find topics by keywords, arranged in alphabetical order. If more than one help topic matches the word chosen, a subwindow will appear allowing you to choose which of the topics you want to view. You can also use the Search feature to search for a particular topic. Type a word in the field supplied and the Weatherization Assistant will search for all occurrences of that word in the help material.

Hypertext items (i.e., highlighted help text that you see in a different color) are found in some of the help material that provide you with more information on that topic or word. Move the mouse cursor to the highlighted word(s) and press the left mouse key to see this additional information.

The tabs to select these alternate help features may be hidden by selecting the "Hide" button at the top of the window. If you have viewed multiple topics during a single help session, you can use the "Back" button to go back to previous topics. The "Print" button allows you to print the material currently displayed. The “Options” button provides some additional options that may be of use.

Exit the help form and return to the main program by clicking on the X-box in the upper right corner. A blank "Weatherization Assistant 8-9 Help" window may persist. Use that window's X-Box to completely exit the help application. 


\subsection{The References Button}

"References" buttons exist on several forms in the Weatherization Assistant: the Contacts and Cost Centers tabs under the Main Menu's "Agency" button; beneath the banner line in the "Setup Library" window and under the Fuel Costs tab, both under the Main Menu's "Setup Library" button; and beneath the banner line in the "Supply” window under the Main Menu's "Supply Library" button. When selected from any of these locations, you are presented with a navigation data sheet window, each row listing a separate reference to the record accessed on the parent form (see Figure 5.12). The location of each reference is identified by the record type and the name you assigned the record. Some entries list multiple record types with the names of the records in successive columns in the table for the entry to further help you locate the reference.

The entries may be sorted or filtered (see Section 5.8, Data Sheet and Form View of Records). Entries can be hidden from view by using the same process described in Section 5.10, Deleting Data. From this form, however, no records are actually deleted. They will reappear the next time the window is opened. You can navigate directly to any record displayed by placing your cursor in the cell containing the name of the record you wish to access and double clicking your mouse.

The "Clear the References Listed Above" button at the lower left corner of the window will clear the reference being accessed from all of the records in the table. Be careful in using this feature. For example, if you clear the references to a setup library, any audits that had referenced the setup library will no longer run, though the audits and their recommendations will remain. However, you will no longer know what setup library was used in making the recommendations. Once this operation is performed, there is no way to undo it without going into each individual record and making the assignment manually. You may selectively clear references by hiding records you do not wish to be involved in the operation from the table before selecting the "Clear the References Listed Above" button.

You may wish to change the references in the records listed in the table to another reference of similar type. The drop-down list at the middle of the bottom of the window contains possible alternate references available for this reassignment. For example, you can change the auditor assigned to a group of audits. Thus, in viewing the references to a specific agency contact, the drop-down list will contain all agency contacts. Make your selection from the drop-down list and click on the 


\section{Chapter 5: Navigation and Data Entry}

"Change the References Listed Above to this new value" button. A selective reassignment may be accomplished by first hiding or filtering out from the table the records you do not wish to be reassigned.

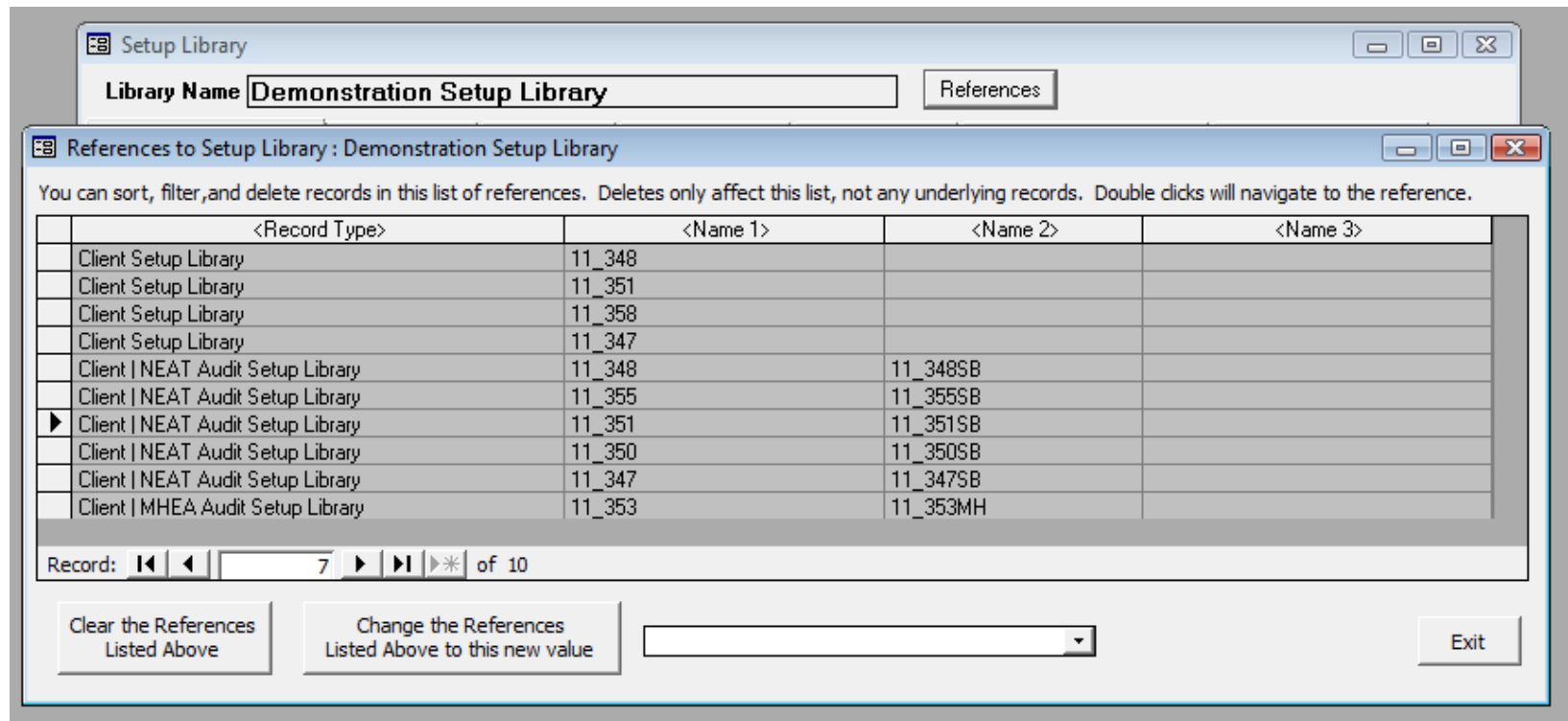

Figure 5.12. The "References to Setup Library:” window. 


\section{Chapter 6. Agency}

The Agency is the highest level record type within the Weatherization Assistant. Thus, all other records (Clients, Audits, Work Orders, etc.) must be associated with an Agency level record. Information related to your agency is organized under the Main Menu’s “Agency” button (see Figure 3.1).

The minimum agency information necessary to operate the Weatherization Assistant is already supplied by the program at installation; namely, an Agency Name and State. As discussed in Section 3.2, Program Setup, you should edit this information to tailor it to your agency unless you are setting up a new installation of the Weatherization Assistant by importing agency information from previous versions (see the introductory discussion associated with Chapter 3, Setting Up the Weatherization Assistant). You should also provide additional information about your agency as needed before substantial use of the Weatherization Assistant.

The remainder of this chapter is divided into sections describing the data and display items entered on each of the forms seen under the Main Menu's "Agency" button: Agency Information, Contacts, Cost Centers, Surveys, Clients, Audits, Work Orders, Libraries, and Status History. All but the first and last of the tabs associated with these forms have a number in parentheses following the tab name. These numbers indicate the number of records entered for each particular form for the agency currently selected. For example, in Figure 6.1, there are 12 client records and 7 audit records entered for the Demonstration Agency.

\subsection{Agency Information}

The Agency Information form (see Figure 6.1) allows you to enter general information about your agency. It also allows you to view and navigate to other agencies in your database, and to access agency level reports. Below are brief descriptions of the individual data items and controls found on the Agency Information form:

- Agency Name - Enter the name of the agency being described. The entry is one of only two required data items on the Agency Information form. The entry may be up to 80 characters in length. The name must be unique. Required. 


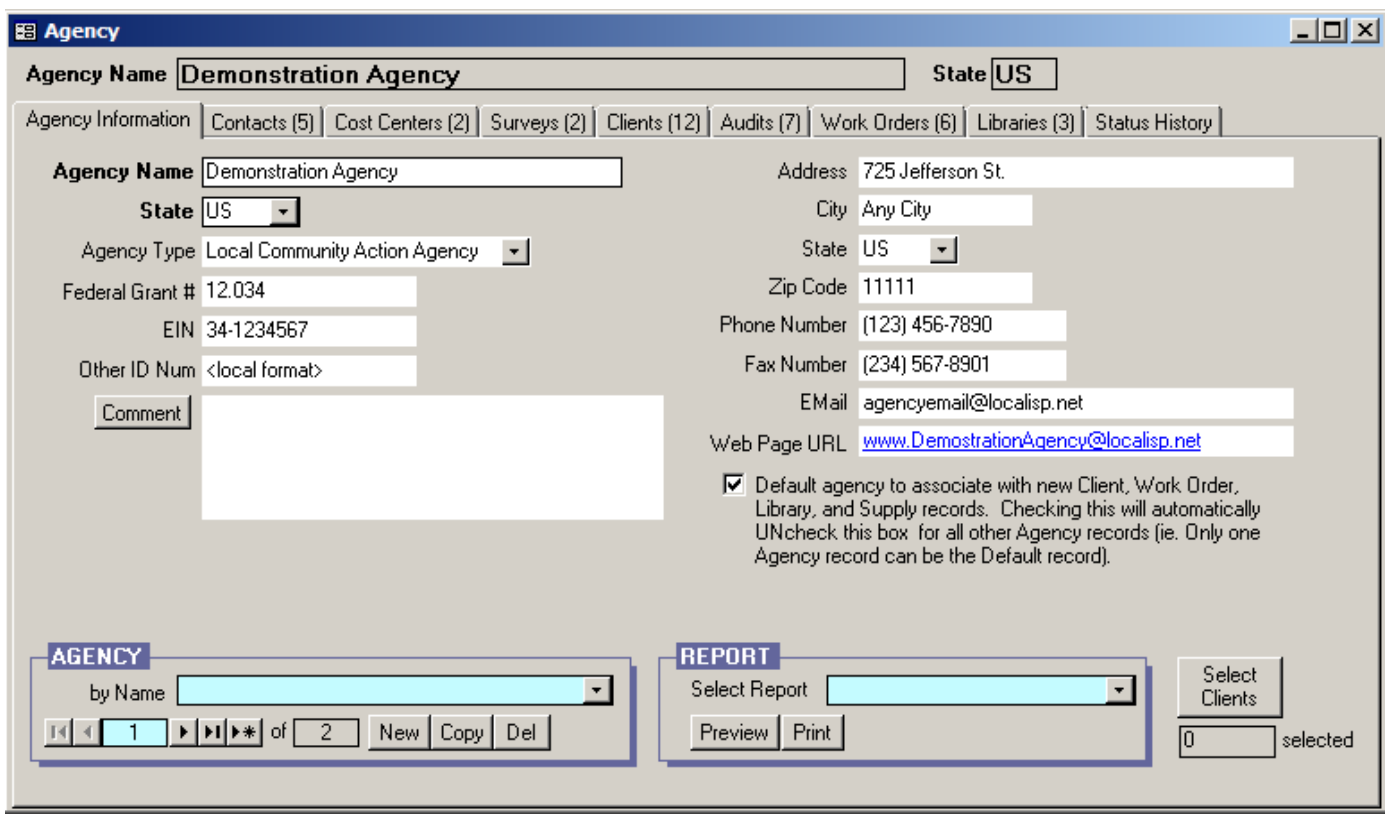

Figure 6.1. The Agency Information form under the Main Menu’s “Agency” button.

- State - Select the state in which the agency is located from the entries in the drop-down list. Required.

- Agency Type - Select the entry that best describes what type of agency this is. The choices are Local Community Action Agency, Tribal Organization, Unit of Local Government, or Other. Optional.

- Federal Grant \# - Enter the Federal Grant Number under which the agency performs Weatherization Assistance Program work, as seen on the DOE Program Application Forms. Optional.

- EIN - Enter the Federal Employer Identification Number assigned by the U.S. Internal Revenue Service to this agency, as entered on the Financial Status Reports of the DOE Program Application Form. Optional.

- Other ID Num - Enter any additional identification descriptor (numbers and/or letters) for this agency which may be of use to you or your state. The entry must be 50 characters or less. Optional.

- Comment - You may enter comments directly in the Comment field on the form, or you may enter them in the Comment Editor by selecting the "Comment" button to the left of the field. The Comment Editor is similar to 
Window's Notepad and may be used to enter extended comments. The total comment may have up to 65,000 characters. Text from other sources may be copied and pasted into the Comment Editor's window. The Agency Comment is not displayed anywhere else in the Weatherization Assistant or in any of its predefined reports. However, you may wish to include the comment in a userdesigned report (see Appendix D, Development of Customized Reports). Optional.

- Address, City, State, and Zip Code - Enter the complete mailing address for the agency. Optional.

- Phone and Fax Numbers - Enter a primary phone and fax number that others should use to contact the agency. Numbers for individuals within the agency may be separately recorded under the Contacts tab as, discussed in Section 6.2, Contacts (Agency). Optional.

- E-Mail - Enter an e-mail address that others should use to contact the agency. The Weatherization Assistant has the ability to e-mail client records from within the program. Setting up this feature will require you to enter this or another agency e-mail address (see Section 18.4, E-Mailing from Within Weatherization Assistant). Optional.

- Web Page URL - If the agency has a web site, enter its URL in this field. Optional.

- Default Agency - If selected, this checkbox indicates that the agency being described on the remainder of the form will be automatically associated with any new Client, Work Order, Setup Library, and Supply Library records created. Only one agency can be selected as the "Default Agency." Thus, selecting this checkbox for this agency will automatically uncheck it for any other agency.

- Agency Record Navigation Block - The Agency record navigation block is used to find and navigate to agency records for other agencies that have been entered, copy or delete the currently accessed agency, or create a new agency. These would not be a common activities because normally an agency has only one agency record. See Section 5.1, Form and Record Navigation, for information on using the Weatherization Assistant's record navigation blocks. 
- Report Block - The Report block provides you with access to all reports available under the Main Menu's “Agency” button. See Appendix C, Reports, for examples of these reports. The following reports are currently available: Quarterly Program Report (the unit production portion of the DOE Quarterly Program Report), Scheduled Audits (for NEAT and MHEA), Open Work Orders, and Economic Summary by Client, Client Surveys (Blank), and Work Order Totals by Category. See Section 5.12, The Report Block, for information on the mechanics of using the Weatherization Assistant's Report blocks. Appendix D, Development of Customized Reports, describes a method to create reports tailored to your needs and to list the reports in the Report block.

- Select Clients - If reports selected in the Report block require the selection of specific clients to be included in the report, the forms accessed from the "Select Clients” button are used to make this selection. Currently, none of the predefined reports at the Agency level require such a selection, although a customized report that you develop might. Transfer of client data between computers using text files requires this same client selection process. See Section 17.5, Selecting Client Records, for information on how to use the Select Clients feature.

If the Weatherization Assistant's Geographic Information System (GIS) feature has been turned on, you can select a group of clients using the forms accessed from the "Select Clients" button and display their locations on a map. Refer to Section 17.5, Selecting Client Records, as well as Section 18.2, Geographic Information System (GIS), for additional information on this mapping option.

\subsection{Contacts (Agency)}

The Contacts form under the Main Menu’s “Agency” button (see Figure 6.2) allows you to enter information on any person associated with the currently selected agency, including auditors, contractors, crew members, and suppliers. You can enter as many contacts as needed. If you have enabled the User Logon or Check-in/Check-out features (see Features 4 and 10 in Section 16.4, Features), you will have to enter users of the Weatherization Assistant. If you want to track work orders by contractor or add suppliers to your supply libraries, then you will have to enter these contacts as well. The Contacts form may be viewed and edited in the data sheet view, which provides a summary of all contacts already entered for the agency or allows quick changes to multiple entries. Below are descriptions of the 
individual data items and controls found on the Contacts form under the Main Menu’s “Agency” button:

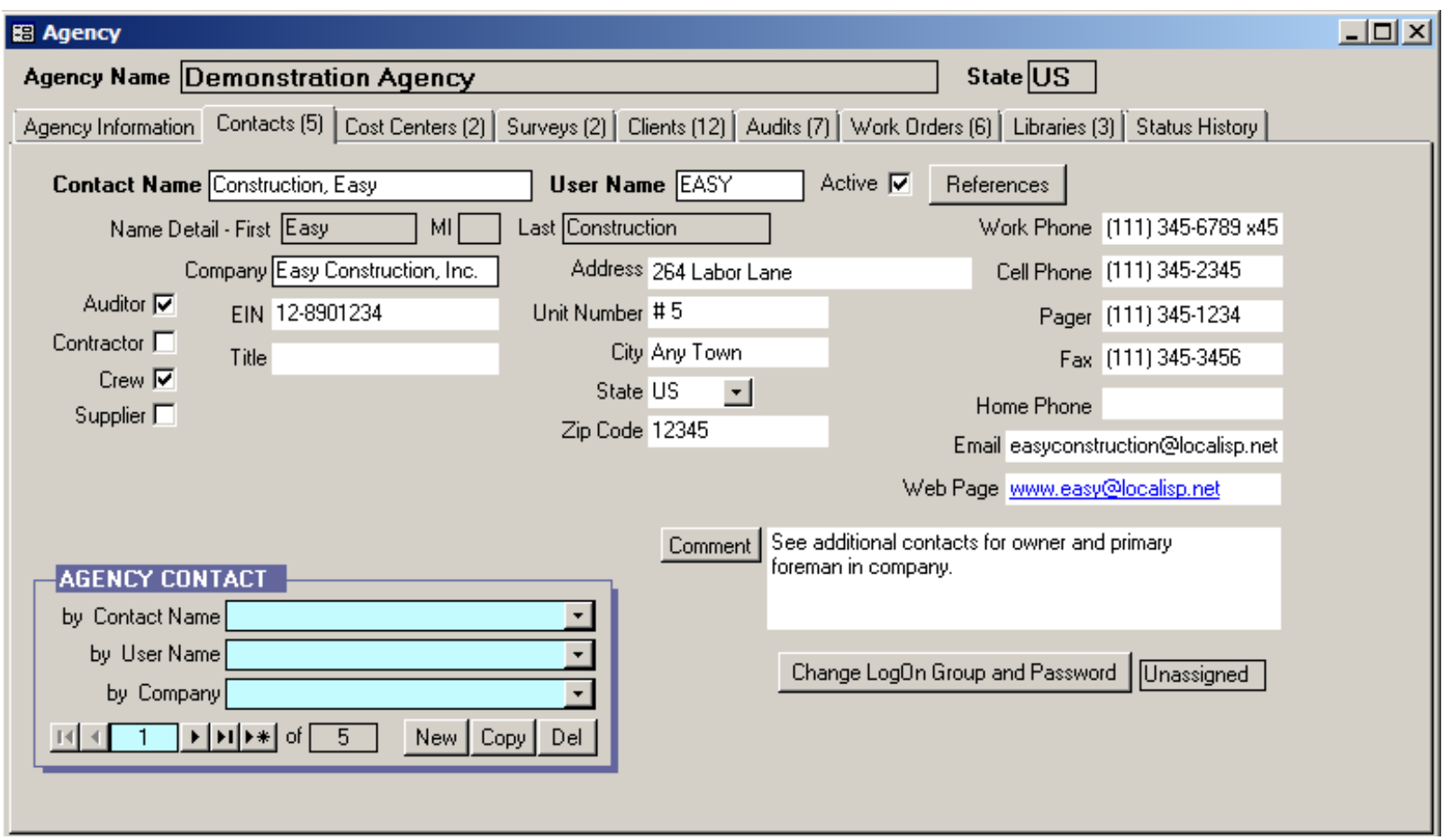

Figure 6.2. The Contacts form under the Main Menu's “Agency” button.

- Contact Name - Enter the name of the contact. You may enter the name with the given name first and the surname last, or with the surname first followed by a comma and then any given names. After you have entered the name and moved to another control, the Weatherization Assistant will automatically fill in the uneditable Name Detail fields (i.e., First, MI, and Last) to show you how the program sees the name that you have entered. If you are basically trying to enter the name of a company (e.g., a supplier of materials), you should enter the name of your primary contact within that company and then provide entries for the User Name and Company fields. Suppliers and contractors listed in dropdown lists in other parts of the Weatherization Assistant are identified by these User Name and Company fields rather than the Contact Name for a person within the company. The name must be unique. Required.

- User Name - Enter a short User Name (initials perhaps) that can be used to identify the contact. In some lists and reports, space does not permit displaying the full Contact Name, so the list or report will use the User Name to identify the contact instead. Be sure to use short User Names to make forms and reports 
more readable. If the User Logon feature is enabled (see Feature 4 in Section 16.4, Features), a valid User Name and password will be required to use the Weatherization Assistant. The name must be unique. Required.

- Active - This checkbox indicates if the contact is currently active with the weatherization program. Only contacts who are active will appear in appropriate drop-down lists throughout the program. See also the description for the Auditor, Contractor, Crew, and Supplier checkboxes provided below.

- References - Selecting this button displays the "References to Agency Contact" window, which lists all references to the contact currently being accessed. See Section 5.14, The References Button, for more information on the use of this feature.

- Company - Enter the company for whom the contact person works. Dropdown lists of contractors and suppliers used elsewhere in the Weatherization Assistant will identify the contact by the Company entry, as well as the User Name and/or Contact Name. Required.

- Auditor, Contractor, Crew, and Supplier - These checkboxes indicate whether the contact person holds any of the positions indicated. Contacts who are Auditors will be included in the drop-down list for the Auditor field on the Audit Information form under the Main Menu's "NEAT" and "MHEA" buttons (see Section 8.2, Audit Information). Contractors and Crews may be assigned to Work Orders (see Sections 13.1, Work Order Information, 14.5, Library Measures, and 14.6, User Defined Measures) and Suppliers may be listed as sources of inventoried items in the Supply Library (see Section 15.2, Materials/Labor Forms). These listings will occur only if a particular contact is declared as "Active" as described above.

- EIN - Enter the Federal Employer Identification Number of the company with whom the contact is associated, as assigned by the U.S. Internal Revenue Service. Optional.

- Title - Enter any title that may help describe the contact, such as "manager," "appliance manager," "crew chief," or "monitor.” This title will not appear in lists of contacts used throughout the Weatherization Assistant. Optional. 
- Address, Unit Number, City, State, and Zip Code - Enter the complete mailing address for the contact. Optional.

- Work, Cell, Pager, Fax, and Home Phone - Enter any of the indicated phone numbers for the contact. Optional.

- E-Mail - Enter an e-mail address for the contact. The Weatherization Assistant has the ability to e-mail client records from within the program. Entering an email address on the Contacts form will automatically enter this contact and their e-mail address in the Address Book of this feature (see Section 18.4, E-Mailing from Within Weatherization Assistant). Optional.

- Web Page - If available, enter the URL for this contact's web site. This could be particularly useful, for example, if the contact is a supplier whose web site contains a catalogue of available items. The web site may be accessed directly from the Contacts form by clicking your mouse on the address. Optional.

- Comment - You may enter comments directly in the Comment field on the form, or you may enter them in the Comment Editor by selecting the "Comment" button to the left of the field. The Comment Editor is similar to Window's Notepad and may be used to enter extended comments. The total comment may have up to 65,000 characters. Text from other sources may be copied and pasted into the Comment Editor's window. Comments entered on the Contacts form are not displayed anywhere else in the Weatherization Assistant or in any of its predefined reports. However, you may wish to include the comment in a user-designed report. Optional.

- Change LogOn Group and Password - If the User Logon feature is enabled (see Feature 4 in Section 16.4, Features), only contacts who have been entered under the Contacts form on the Main Menu's “Agency” button and assigned LogOn passwords will be able to open the Weatherization Assistant. In addition, only contacts who have been entered under the Contacts form and assigned a password can be identified as making a change to one of the Weatherization Assistant's status categories (see Section 18.3, Status Tracking).

Select the "Change LogOn Group and Password" button to obtain the User Group and Password sub-form to input the required password information (see Figure 6.3). Assign the contact to a User Group, either User or Admin. Contacts 
who are Users will be able to create new records and edit existing ones, but will not be allowed to delete client, audit, or work order records. In addition, a User cannot change the settings on the Features tab found under the Main Menu's "Preferences” button. Contacts who are assigned to the Admin group have full capabilities, including deletion of all records. If the contact does not currently have a password, you only need to enter a new password in the New Password and Confirm New Password fields. Otherwise, enter the contact's existing password in the Confirm Old Password field before entering a new password in the other two fields. Select the "Apply New Password" button when you are finished to complete the process. If the User Logon feature is not turned on, there are no restrictions enforced in the Weatherization Assistant and every user is considered to be in the Admin User Group.

- Agency Contact Record Navigation Block - The Agency Contact record navigation block is used to find and navigate to existing agency contacts, copy or delete the currently accessed contact, or create a new contact. See Section 5.1, Form and Record Navigation, for information on using the Weatherization Assistant's record navigation blocks.

\subsection{Cost Centers}

The Weatherization Assistant gives you the option of assigning the cost for each individual weatherization measure that is recommended and/or installed to a different cost center or funding source (see Sections 11.11, Measures (Audit); 13.3, Measures (Work Order); and 14.5, Library Measures, for more details). If you use this option, the Weatherization Assistant will track expenditures by cost center and provide you with summaries. If you enter the funding received or allocated to each cost center, the Weatherization Assistant will also provide you with summaries of the balance of funds remaining for each cost center. The Cost Centers form under the Main Menu's “Agency” button (see Figure 6.4) is used to set up these cost centers, enter the funding received for each cost center, and view summaries of expenditures and balances. Below are descriptions of the individual data items and controls found on the Cost Centers form under the Main Menu's “Agency” button: 


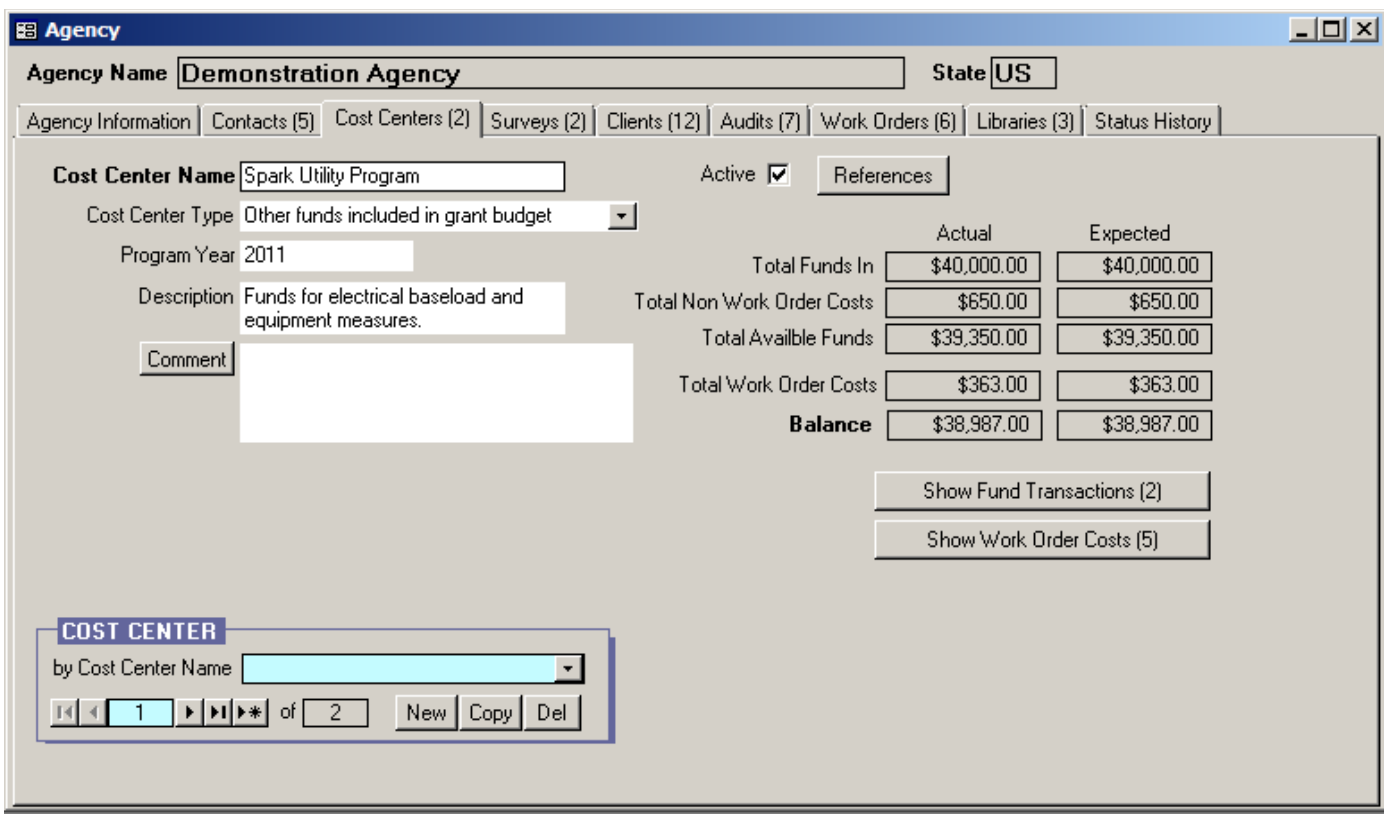

Figure 6.4. Cost Centers form under the Main Menu’s “Agency” button.

- Cost Center Name - Enter the name of the cost center that you are setting up. Usually this name identifies the source providing the funds for this cost center (e.g., the DOE Weatherization Program, LIHEAP, PVE, utility program, etc.). The name must be unique. Required.

- Active - This checkbox indicates whether the cost center is currently available to fund work. If the cost center is active, then it will appear in the drop-down lists found elsewhere in the Weatherization Assistant which assign recommended or installed measures to cost centers. If the cost center is not active, then it will not appear in these drop-down lists.

- References - Selecting this button displays the "References to Agency Cost Center" window, which lists all references to the cost center currently being accessed. The entries will include all instances where a measure whose cost has been assigned to the cost center has been recommended by an audit, any work order that includes the measure, as well as all measures whose costs have been pre-assigned to the cost center in a setup library (see Section 14.5, Library Measures). See Section 5.14, The References Button, for more information on the use of the References feature. 
- Cost Center Type - Indicate the type of funding source for this cost center. The options are DOE, Other funds included in grant budget, or Other funds not included in grant budget. Optional.

- Program Year - Enter the program year for which the funding and/or expenditures in this cost center are being tracked. If you want to track the same cost center by individual program years, you will need to set up a separate cost center for each year. For example, if you want to track DOE Weatherization Assistance Program funds over the 2008 and 2009 program years, you might create a cost center called "DOE 2008" and another called "DOE 2009." The entry does not allow a range of years to be entered. If such is your need, you might enter the beginning year in this field, then the expected span in the Comment field. Optional.

- Description - Enter any additional description you may need to identify this cost center. Optional.

- Comment - You may enter comments directly in the Comment field on the form, or you may enter them in the Comment Editor by selecting the "Comment" button to the left of the field. The Comment Editor is similar to Window's Notepad and may be used to enter extended comments. The total comment may have up to 65,000 characters. Text from other sources may be copied and pasted into the Comment Editor's window. Comments entered on the Cost Centers form are not displayed anywhere else in the Weatherization Assistant or in any of its predefined reports. However, you may wish to include the comment in a user-designed report. Optional.

- Cost Center Financial Summary Table - These fields are displayed on the Cost Center form in a table format. They summarize the income and expenditures for the cost center being referenced. The numbers in the "Actual" column are those debits or credits that have been declared as "Cleared" under the "Show Fund Transactions" button (see below) or that are listed as "Actual" expenditures on a work order (see "The Materials/Labor Details Sub-Form" in Section 13.3, Measures (Work Order), for details). The numbers in the "Expected" column include those in the "Actual" column as well as debits or credits that have been entered into the Transactions form but have not yet been "Cleared" and "Estimated" costs from active work orders that have not yet been declared "Actual.” Uneditable. 
The first three rows in the summary table show totals based on entries made under the "Show Fund Transactions" button (see below). The "Total Funds In" row lists the total funding received by or allocated to the cost center. The "Total Non Work Order Costs" row lists the total costs charged to the cost center that are not related to or accounted for in any work order. For example, these costs may be for overhead, administration, or training. The Total Available Funds row is simply the difference between the Total Funds In row and the total Non Work Order Costs row.

The Total Work Order Costs row lists the total costs from all work orders that have not been canceled. The individual entries that make up these totals may be viewed under the "Show Work Order Costs" button (see below). The Balance row displays the balance for the cost center (i.e., the difference between the Total Available Funds row and the Total Work Order Costs row).

- Show Fund Transactions - The individual entries that comprise the Total Funds In and the Total Non Work Order Costs in the cost center financial summary table are entered, edited, and displayed in the sub-form accessed by selecting the "Show Fund Transactions" button (see Figure 6.5). Enter the date of the transaction in the first column and a description of the transaction in the second column. Credits (allocations or income to the cost center) are entered in the third column while non-work order related debits (costs) are entered in the fourth column. Anticipated credits and debits may be entered. They will only affect totals under the "Expected" column on the financial summary table until the Cleared checkbox has been selected. Enter any comment associated with the transaction. Double clicking on the Comment field will bring up the Comment Editor permitting entry of extended length comments, if necessary. This subform cannot be viewed in the standard form view.

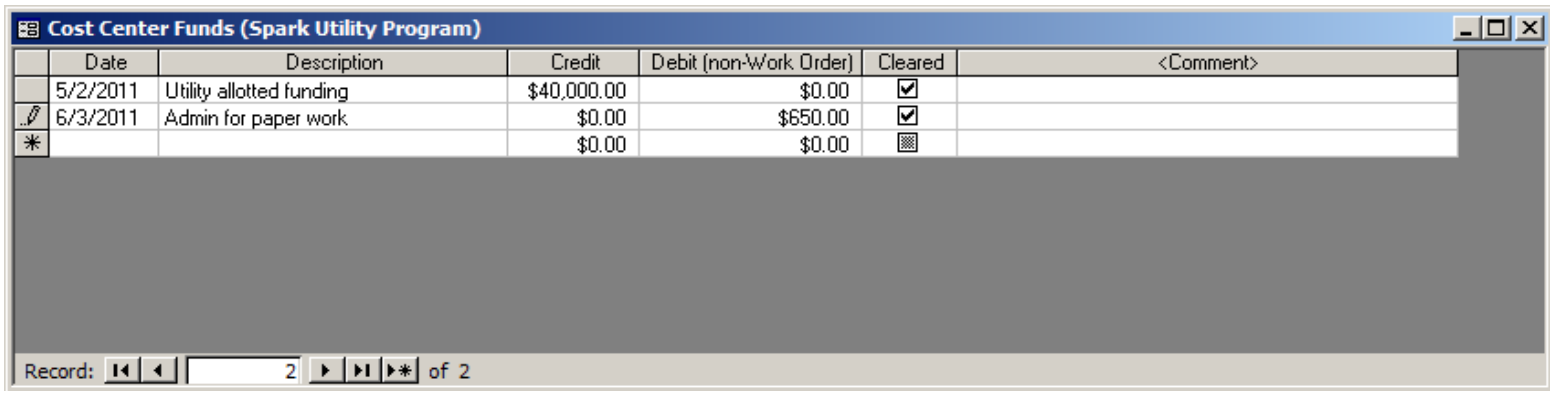

Figure 6.5. The Show Fund Transactions (or Cost Center Funds) sub-form. 
- Show Work Order Costs - An itemized listing of work order costs that comprise the Total Work Order Costs in the financial summary table are displayed in the sub-form accessed by selecting the "Show Work Order Costs" button (see Figure 6.6). This sub-form is "READ ONLY." No entries can be edited.

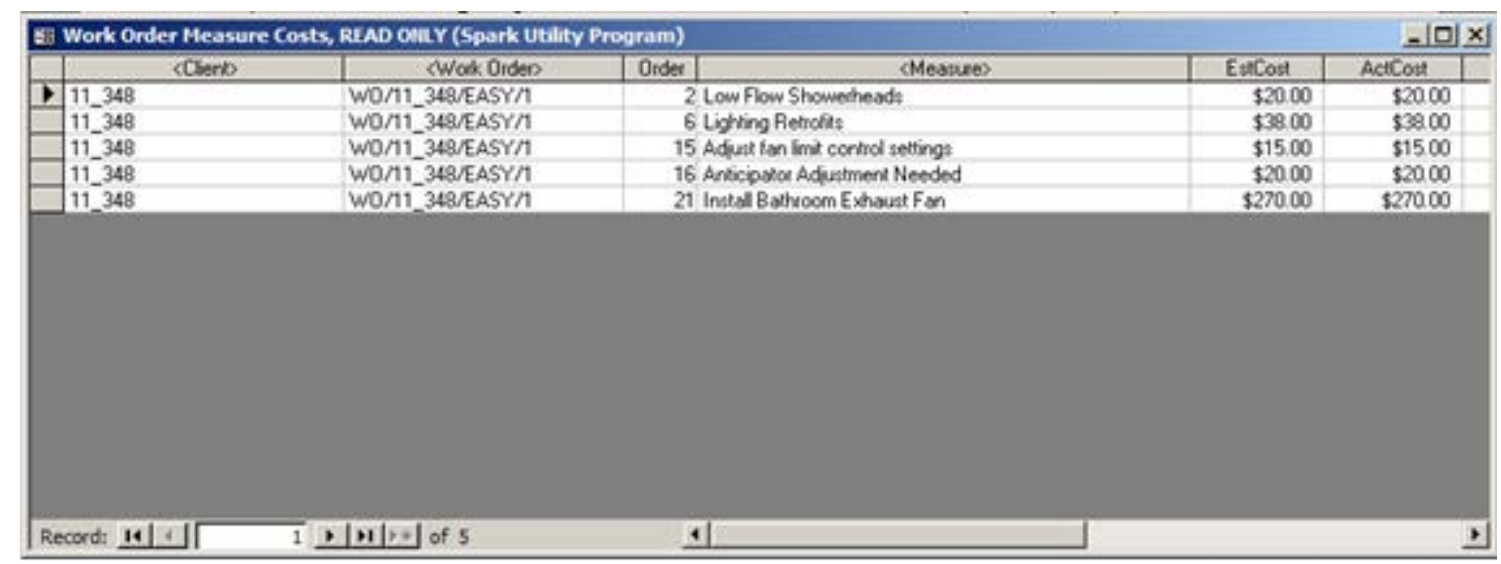

Figure 6.6. The Work Order Costs table under the Agency/Cost Centers tab.

Shown in their respective columns of entries are the Client ID of the client for whom the work is being performed, the specific work order under which the cost was incurred, the measure within the work order whose cost is being listed preceded by the order number of the measure within the work order, the estimated cost for the measure, and the actual cost for the measure if one has been entered. The "ActCost" entries on this sub-form are included in both the "Actual" and "Expected" entries of the financial summary table, whereas the "EstCost" costs are included in only the "Expected" totals of the financial summary table.

Modifications to the entries displayed in the sub-form can only be made in the respective work orders that generated the costs. The headers for the Client, Work Order, and Measure columns are in angle brackets $(<>)$. This implies that the actual client, work order, or measure may be accessed directly by double clicking on the record's entry in the form. If this technique of editing any of these records is used, it is highly recommended that you exit the record after making the modifications and return to the Work Order Measure Costs subform before navigating to other forms. Having too many inter-related forms open at the same time in the Weatherization Assistant can confuse the software and result in abnormal behavior and warnings. 
- Cost Center Record Navigation Block - The Cost Center record navigation block is used to find and navigate to existing cost centers, copy or delete the currently accessed cost center, or create a new cost center. See Section 5.1, Form and Record Navigation, for information on using the Weatherization Assistant's record navigation blocks.

\subsection{Surveys (Agency)}

The Surveys form (see Figure 6.7) under the Main Menu's “Agency” button is used to define custom surveys for your agency to collect additional information on your clients or their households. The surveys themselves are filled out for individual clients under the Main Menu's “Clients” button as described in Section

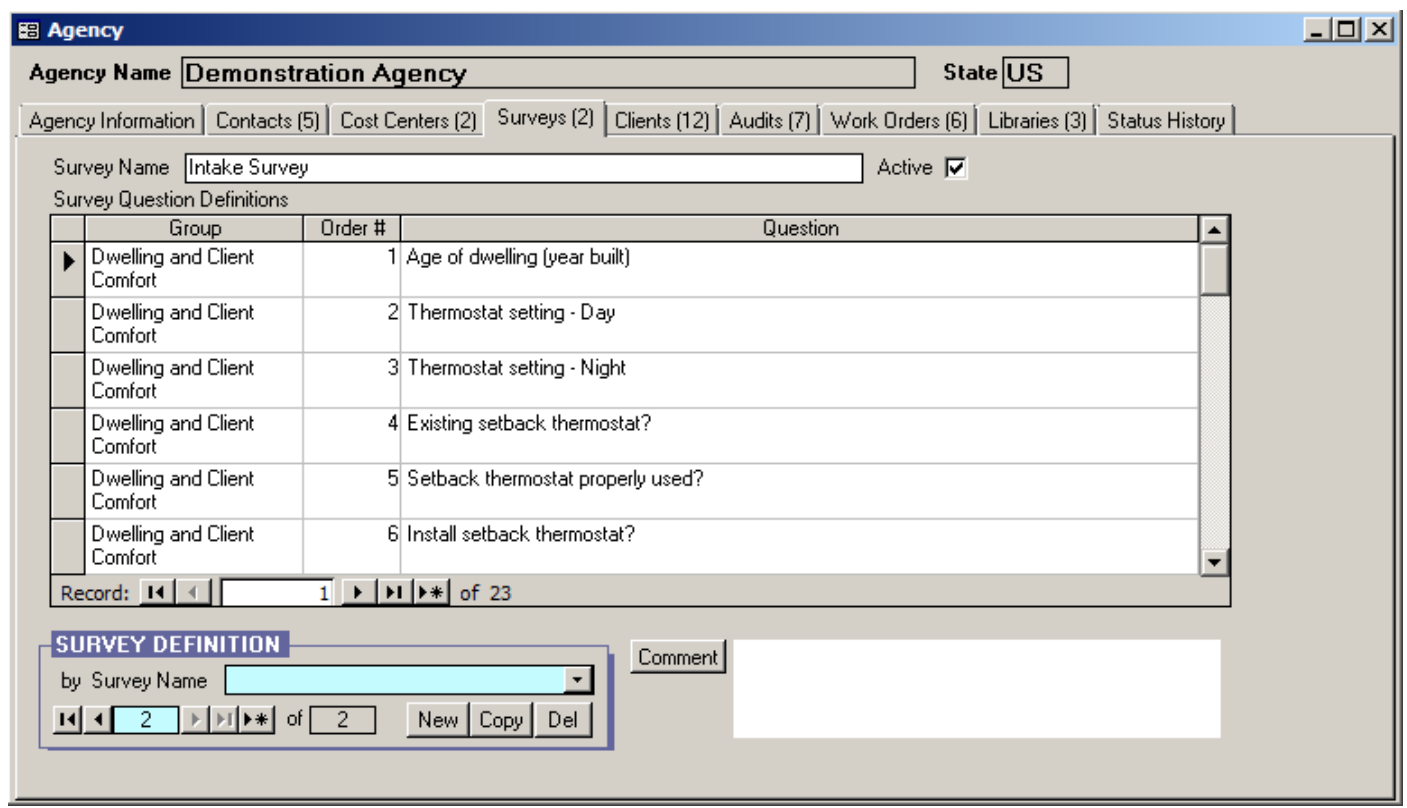

Figure 6.7. The Surveys form under the Main Menu's “Agency” button.

7.7, Surveys (Client). You can create any number of surveys. Surveys do not need to be limited to questions asked of the client. They could also be used to document other information related to the clients collected by the agency, such as income or other eligibility criteria. Below are descriptions of the individual data items and controls found on the Surveys form under the Main Menu's “Agency” button:

- Survey Name - Enter the name for the survey. The Survey Name will be used to identify the survey to be completed later under the Main Menu's “Clients" button. The name must be unique. Required. 
- Active - Use this checkbox to indicate whether the survey is considered "Active.” Only active surveys will be listed for selection in the drop-down lists under the Main Menu’s “Clients” button.

- Comment - You may enter comments that apply to the entire survey directly in the Comment field on the form, or you may enter them in the Comment Editor by selecting the "Comment" button to the left of the field. The Comment Editor is similar to Window's Notepad and may be used to enter extended comments. The total comment may have up to 65,000 characters. Text from other sources may be copied and pasted into the Comment Editor's window. Comments entered on the Cost Centers form are not displayed anywhere else in the Weatherization Assistant or in any of its predefined reports. However, you may wish to include the comment in a user-designed report (see Appendix D, Development of Customized Reports). Optional.

- Survey Definition Record Navigation Block - The Survey Definition record navigation block is used to find and navigate to existing surveys, copy or delete the currently accessed survey, or create a new survey. See Section 5.1, Form and Record Navigation, for information on using the Weatherization Assistant's record navigation blocks.

The Survey Question Definitions sub-form is used to define the questions to be included in the survey. A new question may be initiated by positioning the cursor at the first blank record or by selecting the new record button (*). Be careful to distinguish between the new record button associated with the Survey Question Definitions sub-form and the new record button in the Survey Definition record navigation block. The latter would initiate a whole new survey, not a new question within the existing survey. The entries in the sub-form are copy/paste friendly from both the Weatherization Assistant program and other text or word processing software. The multiple columns of the sub-form may be copied to a spreadsheet, such as Microsoft Excel, for printing. You can create a blank survey by not entering any questions. The survey can then be associated with any client when you want to create a totally unique set of questions specific to that client. Below are descriptions of the individual data items and controls found on the Survey Question Definitions sub-form:

- Group - You can assign each question to a group if desired. This may be desirable for organizational or presentation purposes. You could also use the 
Group designation to sort the questions on the sub-form using standard data sheet view sorting techniques. The entry is limited to 50 characters. Optional.

- Order \# - Establish the order the questions will appear on the Client Surveys report by assigning order numbers (see Section 7.1, Client Information). Optional.

- Question - Enter the actual question to be asked of the client or completed by agency staff. Each question may be 255 characters or less. Optional.

\subsection{Clients}

The Clients form under the Main Menu's “Agency” button (see Figure 6.8) shows all client records associated with the current agency in a navigation data sheet view. The form is used primarily to navigate to individual client records, although it can also be used to create new blank client records. The shading of the records displayed on this form is the visual indicator that this form is used for navigation

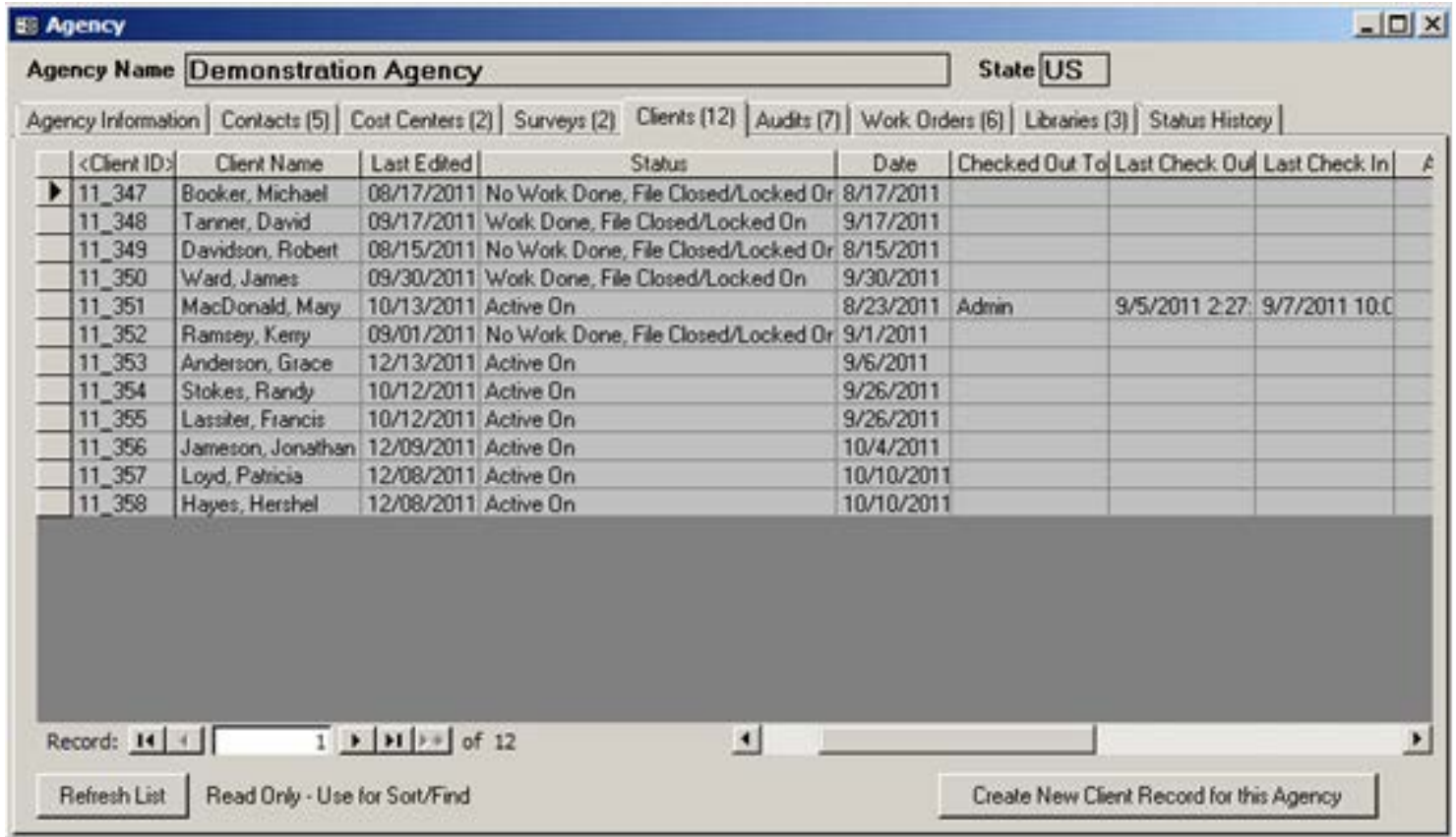

Figure 6.8. The Clients form under the Main Menu's “Agency” button. Columns not displayed in this figure include the Numerical ClientID, AgencyName, AgencyState, and Alt. Client ID. 
rather than data entry. You can double click with your mouse on the entries under the column headings in angle brackets $(<>)$ to navigate to the associated record. Like any data sheet, the order that the client records are displayed can be changed by highlighting a column and pressing one of the sort buttons in the tool bar. Some of the columns in the form may be collapsed (not shown) by default. You can double click the mouse near the borders of some of the columns in the header to have them expanded. You can also collapse columns that you do not want to see by dragging their widths to zero. You may need to use the vertical and horizontal scroll bars to display all the records and columns, respectively. Below are descriptions of the individual data items and controls found on the Clients form under the Main Menu's “Agency” button:

- Client ID, Agency Name (not shown), Agency State (not shown), Client Name, and Alt. Client ID (not shown) - These entries are those given on the Client Information form under the Main Menu's "Clients" button for each of the clients listed (see Section 7.1, Client Information).

- Numerical ClientID (not shown) - When a new client is created, the Weatherization Assistant automatically assigns a unique number to this client for internal software purposes. This field displays what this number is for each client. See Feature 6 in Section 16.4, Features, for more information.

- Last Edited - This field shows the date (and time, if the column is expanded) of the last change made to this client record while on the forms under the Main Menu's “Clients” button.

- Status - This field gives the latest client status for each client as set on the Status form under the Main Menu's “Clients” button for the client. See Section 7.2, Status (Clients), for more information.

- Date - This field provides the date that the latest status was set.

- Checked Out To, Last Checked Out, and Last Check In - If the Checkin/Check-out client records feature is turned on (see Feature 10 in Section 16.4, Features), the Weatherization Assistant tracks who checked a client out and when the client was checked out and checked back in. These fields provide this information for each client if applicable. 
- Refresh List - The "Refresh List" button can be used to update the information displayed on the Clients form to reflect any changes since the last time this form was opened.

- Create New Client Record for this Agency - You may use this button to create a new client. Selecting the button will take you to the Client Information form under the Main Menu's “Clients” button (see Section 7.1, Client Information), where descriptive data for the new client can be entered. When you exit the Client Information form, you will be returned to the Client form under the Main Menu's "Agency” button. A row with the new client should already be added; if not, use the "Refresh List" button on the form.

\subsection{Audits (Agency)}

The Audits form under the Main Menu’s “Agency” button (see Figure 6.9) shows all audit records associated with the current agency in a navigation data sheet view. The form is used primarily to navigate to individual audit records. The shading of the records displayed on this form is the visual indicator that this form is used for navigation rather than data entry. You can double click with your mouse on the

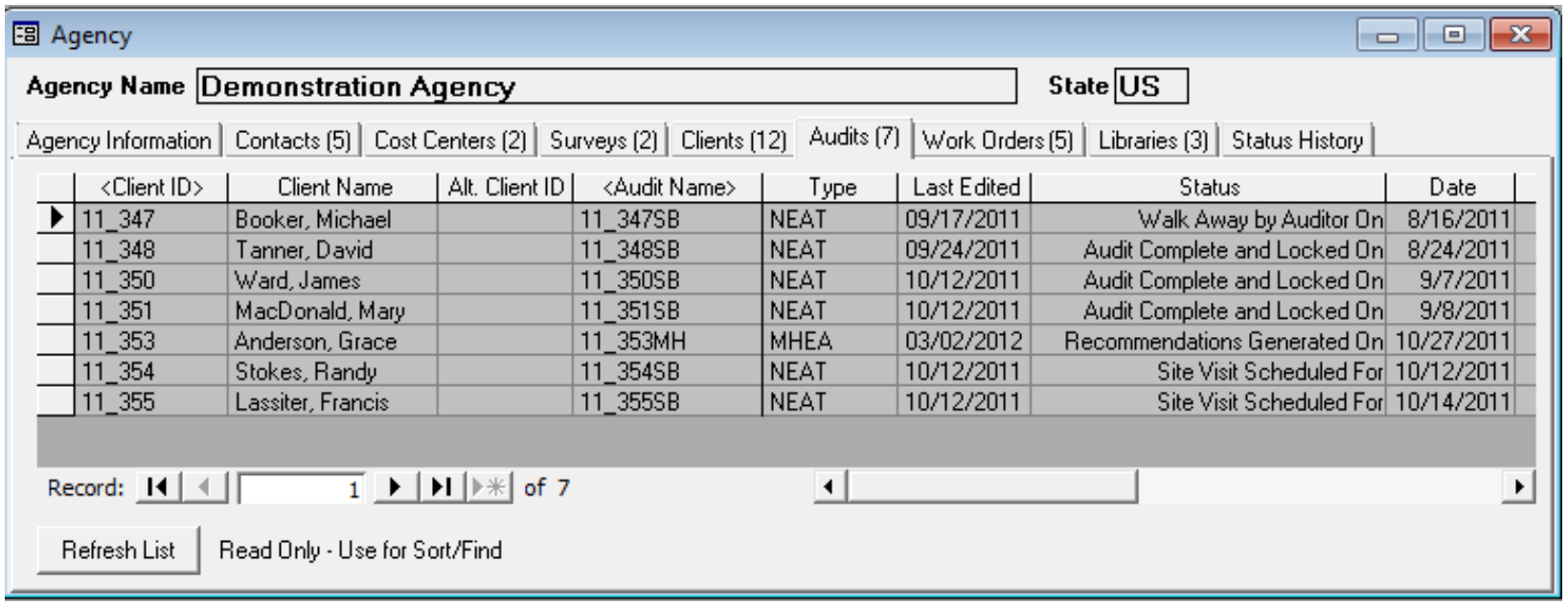

Figure 6.9. The Audits form under the Main Menu's “Agency” button.

Columns not displayed in this figure include the numerical ClientID, numerical JobID, agency name, and agency state.

entries under the column headings in angle brackets $(<>)$ to navigate to the associated record. Like any data sheet, the order that the audit records are displayed can be changed by highlighting a column and pressing one of the sort 
buttons in the tool bar. Some of the columns in the form may be collapsed (not shown) by default. You can double click the mouse near the borders of some of the columns in the header to have them expanded. You can collapse columns that you do not want to see by dragging their widths to zero. You may need to use the vertical and horizontal scroll bars to display all the records and columns, respectively.

Normally, there is only one audit record for each client. However, the Weatherization Assistant does allow you to create and execute more than one audit per client. This might be useful if you desire to see the effect of some modification in the building description on the recommendations of the audit. You might also want to update or correct an existing audit without losing the original description. If you have created and run more than one audit per client, you should ensure that only one set of active work orders exists for the client. Otherwise, confusion and double accounting of costs are likely to occur. Below are descriptions of the individual data items and controls found on the Audits form under the Main Menu’s “Agency” button:

- Client ID, Client Name, Alt. Client ID, Audit Name, Type, Agency Name (not shown), and Agency State (not shown) - These entries are those given on the Audit Information form under the Main Menu's "NEAT" and "MHEA" buttons for each of the audits listed (see Section 8.2, Audit Information).

- Last Edited - This field shows the date (and time, if the column is expanded) of the last change made to this audit record while in the forms under the Main Menu's "NEAT" or "MHEA" buttons.

- Status - This field gives the latest audit status for each audit as set on the Status form under the Main Menu's "NEAT" and "MHEA" buttons for the audit. See Section 11.1, Status (Audit), for more information.

- Date - This field provides the date that the latest status was set.

- Numerical ClientID and JobID (not shown) - When a new client and a new audit are created, the Weatherization Assistant automatically assigns a unique number to each of these for internal software purposes. These fields display what these numbers are for each audit. See Feature 6 in Section 16.4, Features, for more information. 
- Refresh List - The "Refresh List" button can be used to update the information displayed on the Audits form to reflect any changes since the last time this form was opened.

\subsection{Work Orders (Agency)}

The Work Orders form under the Main Menu's “Agency” button (see Figure 6.10) shows all work order records associated with the current agency in a navigation data sheet view. The form is used primarily to navigate to individual work order

\begin{tabular}{|c|c|c|c|c|c|c|c|c|c|}
\hline \multicolumn{8}{|l|}{ 凅 Agency } & \multicolumn{2}{|c|}{ 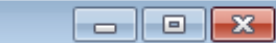 } \\
\hline \multicolumn{6}{|c|}{ Agency Name Demonstration Agency } & \multicolumn{2}{|c|}{ State US } & & \\
\hline Agency Information & on $\mid$ Contacts (5) $\mid \operatorname{Cos}$ & Centers (2) & Surveys (2) & Clients (12) Audits & (7) Work 0 & Orders (5) | Libra & aries (3) Stat & us History & \\
\hline$\langle$ Client |D $\rangle \mid$ & 〈Work Drder〉 & | Contractor| & Last Edited | & Status & Date & | Inspect Status| & Date & Pay Status & Date \\
\hline $11 \_348$ & WO/11_348/EASY/1 & EASY & $03 / 01 / 2012$ & Work Completed On & $9 / 6 / 2011$ & Passed On & $9 / 13 / 2011$ & Invoice Paid 0n & $9 / 15 / 201$ \\
\hline D11_348 & W0/11_348/JT/1 & JT & $08 / 16 / 2011$ & Work Completed On & $9 / 7 / 2011$ & Passed 0n & $9 / 13 / 2011$ & Invoice Paid On & $9 / 16 / 201$ \\
\hline $11 \_350$ & W0/11_350/JT/1 & JT & 10/13/2011 & Work Completed Dn & $9 / 22 / 2011$ & Passed 0n & $9 / 27 / 2011$ & Invoice Paid On & $9 / 29 / 201$ \\
\hline $11 \_351$ & W0/11_351/JT/1 & JT & 10/13/2011 & Work Completed On & $10 / 4 / 2011$ & Passed Dn & $10 / 10 / 2011$ & & \\
\hline 11_353 & W0/11_353/JT/1 & JT & 12/13/2011 & Work Started Dn & $10 / 6 / 2011$ & & & & \\
\hline Record: I| & 2 & 川米。 & of 5 & 1 & & & & & $\bullet$ \\
\hline Refresh List & Read Only - Use f & $t /$ Find & & & & & & & \\
\hline
\end{tabular}

Figure 6.10. The Work Orders form under the Main Menu's “Agency” button. Columns not displayed in this figure include the numerical ClientID, numerical WorkOrderID, agency name, and agency state.

records. The shading of the records displayed on this form is the visual indicator that this form is used for navigation rather than data entry. You can double click with your mouse on the entries under the column headings in angle brackets $(<>)$ to navigate to the associated record. Like any data sheet, the order that the work order records are displayed can be changed by highlighting a column and pressing one of the sort buttons in the tool bar. Some of the columns in the form may be collapsed (not shown) by default. You can double click the mouse near the borders of some of the columns in the header to have them expanded. You can collapse columns that you do not want to see by dragging their widths to zero. You may need to use the vertical and horizontal scroll bars to display all the records and columns, respectively.

More than one work order per client may be displayed on this form if more than one contractor is assigned work for a client. The work orders may have been 
generated automatically from the Measures form under the Main Menu's "NEAT" and "MHEA" buttons (see Section 11.11, Measures (Audit)) or manually under the Main Menu's “Work Orders” button (see Section 13.1, Work Order Information). Below are descriptions of the individual data items and controls found on the Work Orders form under the Main Menu’s “Agency” button:

- Client ID, Work Order, Agency Name (not shown), and Agency State (not shown) - These entries are those given on the Work Order Information form under the Main Menu's “Work Orders” button for each of the work orders listed (see Section 13.1, Work Order Information).

- Numerical ClientID (not shown) and WorkOrderID (not shown) - When a new client and a new work order are created, the Weatherization Assistant automatically assigns a unique number to each of these for internal software purposes. These fields display what these numbers are for each work order. See Section 16.4, Features, for more information.

- Contractor - This field shows the contractor assigned to this work order. This entry may be blank if a contractor has not been assigned. Contractors are defined on the Contacts tab under the Main Menu's “Agency” button as described in Section 6.2, Contacts (Agency), and assigned to each measure either by default in the Setup Library (see Section 14.5, Library Measures), when a work order is initiated from the Measure form under the Main Menu's "NEAT" and "MHEA" buttons (see Section 11.11, Measures (Audit)), or under the Main Menu's “Work Orders” button itself (see Section 13.1, Work Order Information).

- Last Edited - This field shows the date (and time, if the column is expanded) of the last change made to this work order record while on the forms under the Main Menu's "Work Orders" button, or the date the work order was generated from the Measures form of the Main Menu's "NEAT" and "MHEA" buttons if no modifications have been made.

- Status, Inspect Status, and Pay Status - There are three status categories for a work order: a general status, an inspection status, and a payment status. Each status category has its own column in the Work Orders form. These fields give the latest status of each category for each work order. These statuses are changed on the Status form under the Main Menu's “Work Orders” button for 
each individual work order. See Section 13.2, Status (Work Order), for more information.

- Dates - These fields provide the date that the latest status was set for each of the three work order status categories.

- Refresh List - The "Refresh List" button can be used to update the information displayed on the Work Orders form to reflect any changes since the last time this form was opened.

\subsection{Libraries}

The Libraries form under the Main Menu's “Agency” button (see Figure 6.11) shows all the setup libraries and supply libraries associated with the current agency in a navigation data sheet view. The form is used primarily for reference and to navigate to individual libraries. The shading of the records displayed on this form is the visual indicator that this form is used for navigation rather than data entry. You can double click with your mouse on a specific library of interest to directly access that library. Like any data sheet, the order that the libraries are shown can be changed by highlighting a column and pressing one of the sort buttons in the tool bar. Below are descriptions of the individual data items and controls found on the Libraries form under the Main Menu’s “Agency” button:

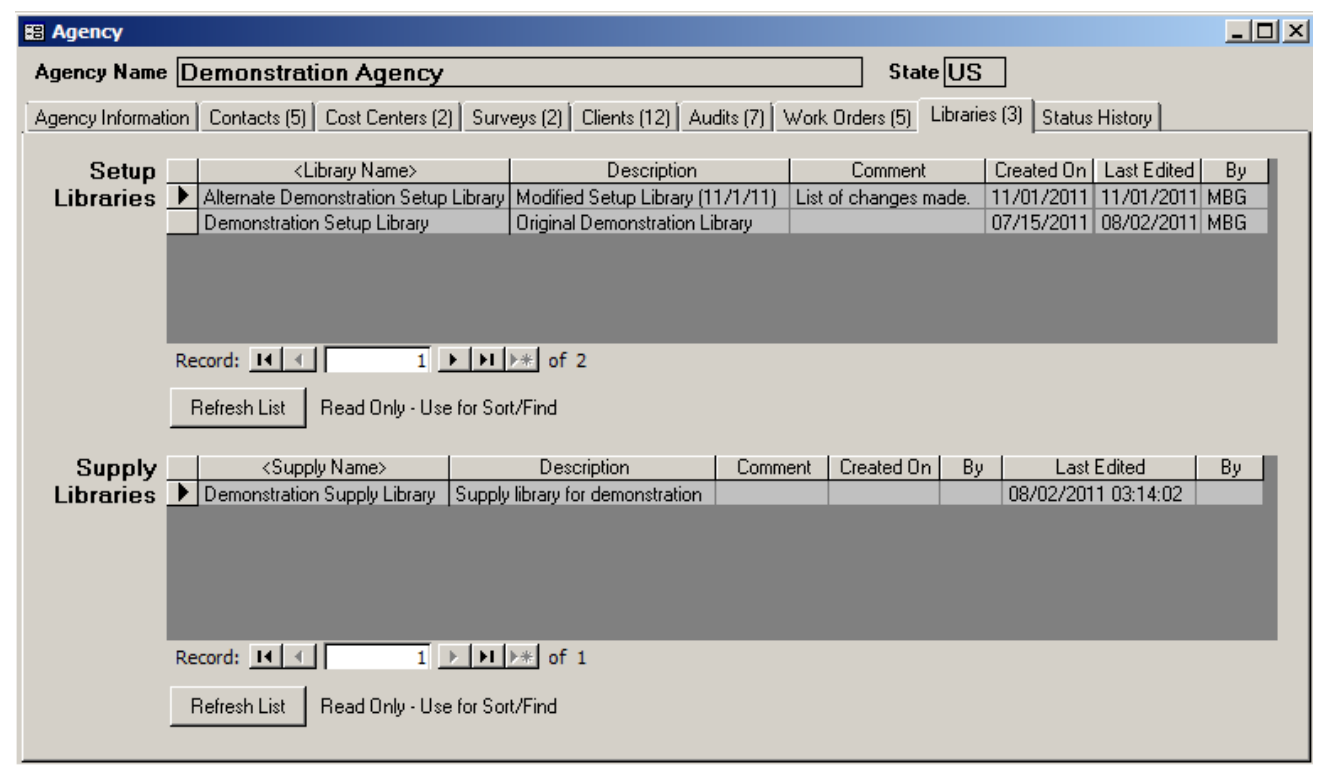

Figure 6.11. The Libraries form under the Main Menu’s “Agency” button. 
- Library Name, Description, and Comment - These entries are those given to each library under the Main Menu's "Setup Library" or "Supply Library" buttons (see Sections 14.1, Setup Library Information, and 15.1,General Information Form, respectively).

- Created On, By (not shown) - These entries give the date (and time if the column is expanded) and who was logged on when the library was created. The second entry requires use of the User Logon feature (see Feature 4 in Section 16.4, Features, for more information).

- Last Edited, By - These entries give the date the library was last modified and who was logged on when the modification was made. This second entry requires use of the User Logon feature (see Feature 4 in Section 16.4, Features, for more information).

- Refresh List - The "Refresh List" button can be used to update the information displayed on the Libraries form to reflect any changes since the last time this form was opened.

\subsection{Status History}

The Status History form under the Main Menu's “Agency” button lists the current status settings and the entire history of status changes for all status categories (types) for all clients belonging to the current agency (see Figure 6.12). You may need to use the vertical scroll bar to see all the status settings. The status categories used in the Weatherization Assistant are shown in Table 6.1.
Table 6.1. Status categories tracked under the Status History form

\begin{tabular}{|c|c|}
\hline Client Level & $\begin{array}{c}\text { Client (General) } \\
\text { Client Application }\end{array}$ \\
\hline Audit Level & $\begin{array}{c}\text { NEAT Audit } \\
\text { MHEA Audit }\end{array}$ \\
\hline $\begin{array}{c}\text { Work Order } \\
\text { Level }\end{array}$ & $\begin{array}{c}\text { Work Order (General) } \\
\text { Work Order Inspection } \\
\text { Work Order Payment }\end{array}$ \\
\hline
\end{tabular}

The Status History form is used to review the history of status changes rather than to edit individual records in the history. Changes in status are made under the Status forms of the respective "Client," "NEAT" or "MHEA," and "Work Orders" Main Menu buttons. See Sections 7.2, Status (Clients); 11.1, Status (Audit); and 13.2, Status (Work Order), for more details. Section 18.3, Status Tracking, provides a full discussion of the status tracking feature in the Weatherization 
Assistant, including a display of all of the status categories and the possible settings for each. You can double click your mouse on the entries under the column headings in angle brackets $(<>)$ to navigate to the associated record.

\begin{tabular}{|c|c|c|c|c|c|c|c|c|}
\hline 四 Agency & & & & & & & $-1 \square$ & ] $\underline{x}$ \\
\hline Agency Name & Demonstration A & gency & & State US & & & & \\
\hline Agency Information & $n|\operatorname{Contacts~(5)|}| \operatorname{Cost} C$ & ienters (2) Surveys (2) & Clients (12) & Audits (7) | Work Orders (5) | Libraries (3) & Status History & & & \\
\hline$\langle$ Client |D $\rangle$ & 〈Record Name〉 & StatusType & Date & Status & Changed On & By & . & - \\
\hline 11_347 & $11 \_347 \mathrm{SB}$ & NEAT Audit & $8 / 16 / 2011$ & Site Visit Scheduled For & $8 / 5 / 2011$ & admin & & \\
\hline $11 \_347$ & $11 \_347$ & Client Application & $8 / 5 / 2011$ & Approved On & $8 / 5 / 2011$ & admin & & \\
\hline $11 \_347$ & $11 \_347 \mathrm{SB}$ & NEAT Audit & $8 / 16 / 2011$ & Site Visit Completed On & $8 / 16 / 2011$ & admin & & \\
\hline $11 \_347$ & $11 \_347$ & Client & $8 / 17 / 2011$ & No Work Done, File Closed/Lockec & $8 / 17 / 2011$ & admin & & \\
\hline $11 \_347$ & 11_347SB & NEAT Audit & $8 / 16 / 2011$ & Walk Away by Auditor On & $8 / 16 / 2011$ & admin & & \\
\hline $11 \_347$ & $11 \_347$ & Client & $8 / 2 / 2011$ & Active $0 \mathrm{n}$ & $8 / 2 / 2011$ & admin & & \\
\hline $11 \_347$ & $11 \_347$ & Client Application & $8 / 2 / 2011$ & Received On & $8 / 2 / 2011$ & admin & & \\
\hline $11 \_348$ & $11 \_348$ & Client & $9 / 17 / 2011$ & Work Done, File Closed/Locked Or & $9 / 17 / 2011$ & admin & & \\
\hline $11 \_348$ & 11 11_88SB & NEAT Audit & $8 / 24 / 2011$ & Audit Complete and Locked On & $8 / 24 / 2011$ & admin & & \\
\hline $11 \_348$ & $11 \_348$ & Client Application & $8 / 2 / 2011$ & Received On & $8 / 2 / 2011$ & admin & & \\
\hline $11 \_348$ & W0/11_348/EASY/1 & Work Order Payment & $9 / 8 / 2011$ & Invoice Received On & 9/8/2011 & Admin & & \\
\hline $11 \_348$ & $11 \_3485 B$ & NEAT Audit & $8 / 22 / 2011$ & Site Visit Completed On & $8 / 22 / 2011$ & admin & & \\
\hline $11 \_348$ & Wo/11_348/JT/1 & Work Order Inspection & $9 / 13 / 2011$ & Passed On & $9 / 13 / 2011$ & Admin & & \\
\hline $11 \_348$ & WO/11_348/EASY/1 & Work Drder & $9 / 6 / 2011$ & Work Completed On & $9 / 6 / 2011$ & Admin & & \\
\hline $11 \_348$ & Wo/11_348/JT/1 & Work Order & $9 / 5 / 2011$ & Work Started On & $9 / 5 / 2011$ & Admin & & \\
\hline $11 \_348$ & WO/11_348/EASY/1 & Work Order Payment & $9 / 15 / 2011$ & Invoice Paid On & $9 / 15 / 2011$ & Admin & & \\
\hline $11 \_348$ & $11 \_348 S B$ & NEAT Audit & $8 / 19 / 2011$ & Site Visit Scheduled For & $8 / 9 / 2011$ & admin & & \\
\hline $11 \_348$ & W0/11_348/JT/1 & Work Drder & $8 / 24 / 2011$ & Work Order Created from Audit On & $8 / 24 / 2011$ & Admin & & \\
\hline $11 \_348$ & 11348 & Client Application & $8 / 9 / 2011$ & Approved On & $8 / 9 / 2011$ & admin & & -1 \\
\hline 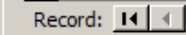 & $1+\|$ & D* of 85 & 1 & & & & $D$ & \\
\hline
\end{tabular}

Figure 6.12. The Status History form under the Main Menu’s “Agency” button.

The Weatherization Assistant retains a record of all status settings and changes. If you make a mistake in setting a status, correcting it will still leave the old setting in the history. Even though you cannot edit status records from the Status History form, you can delete entire status records using the form (see the discussion of deleting records in a data sheet view in Section 5.10, Deleting Data). Thus, the form is a good location to detect mistaken entries and delete them.

Combining the use of the filter and sort features in the Status History form can produce a useful display (see the discussion of filtering and sorting in Section 5.8, Data Sheet and Form Views of Records). If you filter the entries by a specific client and then sort the entries by date, you will display a chronological listing of all status entries associated with a specific client. A similar display can also be produced by selecting the "Overall Client Status History" button on the Status form under the Main Menu's "Clients" button and sorting the records by date as discussed in Section 7.2, Status (Clients).

Below are descriptions of the individual data items and controls found on the Status History form. 


\section{Chapter 6: Agency}

- Client ID - This entry is the Client ID given on the Client Information form under the Main Menu’s “Clients” button (see Section 7.1, Client Information).

- Record Name - This field identifies the Client ID, Audit Name, or Work Order associated with the status setting.

- Status Type - This field identifies the status category associated with this status setting. These categories are listed in Table 6.1.

- Date - This field displays the date that the status setting became effective.

- Status - This field displays the status setting. The possible settings for each status category are listed in Section 18.3, Status Tracking.

- Changed On - This field displays the date that the status setting was changed.

- By - This field displays the User Name of the person who changed the status setting. The names are restricted to those that have been entered on the Contacts form under the Main Menu's “Agency” button and have a password assigned as discussed in Section 6.2, Contacts (Agency). A User Name of “Admin” implies that a specific person was not identified as making the change.

- Comment (not shown) - Any comment that has been appended to this status setting will be shown in this field.

- Refresh List - The "Refresh List" button can be used to update the information displayed on the Status form to reflect any status changes that may have been made since the last time this form was opened. 


\section{Chapter 7. Clients}

Because audits and work orders are assigned to clients, setting up and entering information on clients is an essential task in the Weatherization Assistant. Information related to clients is organized under the Main Menu's "Clients" button of the Weatherization Assistant (see Figure 3.1).

The minimal information required for a specific client is a Client ID, the name of the agency handling the client, and the type of dwelling. However, you may store additional client information in case you are using the Weatherization Assistant as the main repository of client data.

The first task in using the Weatherization Assistant for a new client is to enter identifying information about the client under the Main Menu's "Clients" button. The most common and direct way to start a new client record is to select the "Clients" button on the Weatherization Assistant's Main Menu and then select the "New" button from the Client record navigation block on the Client Information form (see Figure 7.1). You can also start a new client by selecting the "Agency" button from the Weatherization Assistant's Main Menu, selecting the Clients tab, and then clicking on the "Create New Client Record for this Agency" button (see Section 6.5, Clients).

You may access the record for any existing client by selecting the "Clients" button from the Weatherization Assistant's Main Menu and then using one of the Client record navigation block drop-down lists to locate and select the desired client. Although this is the more direct method, you can also access an existing client by selecting the "Agency" button from the Weatherization Assistant's Main Menu, selecting the Clients tab, and then double clicking your mouse on the Client ID corresponding to the client of interest (see Section 6.5, Clients).

The remainder of this chapter is divided into sections describing the data and display items entered on each of the forms seen under the Main Menu's "Client" button: Client Information, Status, Energy Index, Contacts, Audits, Work Orders, Surveys, and Photos (if the photo browser tab method for attaching photos to records has been activated under the Main Menu's "Preferences" button as described under Feature 1 in Section 16.4, Features). All but the first three tabs associated with these forms have a number in parentheses following the tab name. These numbers indicate the number of records entered for each particular form for 


\section{Chapter 7: Clients}

the client currently selected. For example, in Figure 7.1, there are two contact records and one audit record entered for client 05_348.

\subsection{Client Information}

The Client Information form (see Figure 7.1) under the Main Menu's “Clients” button allows you to enter general information on a specific client and the client's dwelling. It also allows you to view and navigate to other clients in your database, and to access client level reports.

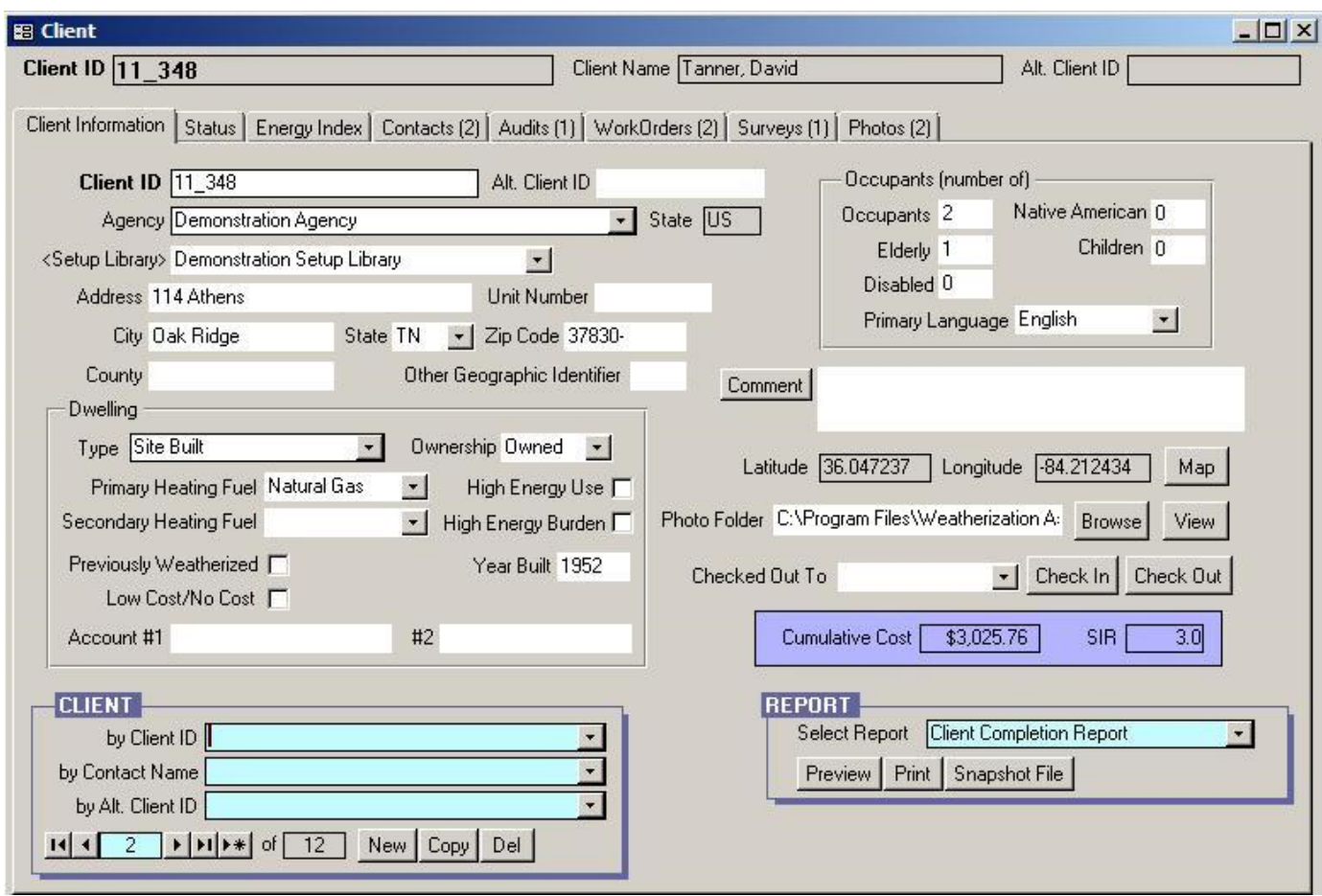

Figure 7.1. The Client Information form under the Main Menu's "Clients" button. Both options for linking photos to the client are shown (Photos tab and Photo Folder field with accompanying "Browse" and "View" buttons) even though only one can be selected and displayed at one time.

All of the data on the Client Information form are optional except the Client ID, Agency, and Dwelling Type. However, optional data may still be used elsewhere in the Weatherization Assistant or in reports that you generate. For example, if the number of occupants is entered, NEAT and MHEA will use this value rather than a default value to determine the amount of internal loads. As another example, the majority of information in the Dwelling and Occupants blocks of data are used to generate the Unit Production portion of the DOE Quarterly Report, accessed from 
the Report block under the Main Menu's “Agency” button (see Section 6.1, Agency Information).

Below are descriptions of the individual data items and controls found on the Client Information form. The data items and controls included in the Dwelling and Occupant blocks are described at the end, as are the data items and controls related to mapping, photographs, and checking out clients.

- Client ID - The Client ID is designed to be the key parameter identifying this client to you and to the computer. The Client ID is one of several fields you will use to locate and identify a specific client. Therefore, the Client ID must be unique. Typically, the Client ID should be the job number that agencies assign to their clients. Although the Client ID could be the name of the client, the names of the client and other contacts associated with the client should be entered under the Contacts form as discussed in Section 7.4, Contacts (Client). The entry may be up to 50 characters in length, although report footers can only report the first 20 characters. Required.

- Alt. Client ID - The Alternate Client ID field allows you to assign a second identifying parameter to each client if your agency or state has multiple means of identifying a client. The Alternate Client ID can be used to locate and identify a specific client. The entry may be up to 50 characters in length, although report footers can only report the first 20 characters. Optional.

- Agency - This field is used to select the agency associated with this client. A new client will automatically be assigned to the agency selected as the default agency under the Main Menu's "Agency" button (see Section 6.1, Agency Information), although any agency defined under the Main Menu's "Agency" button can be selected using the drop-down list. If the agency a client is assigned to is changed after audits and/or work orders have been created for this client, then information in the audits and/or work orders (e.g., Setup, Fuel Cost, and Supply Libraries) will have to be re-entered to retain consistency with the new agency assignment. Required.

- State - The State field that is to the right of the Agency field and shaded will be automatically filled in once the agency the client is assigned to is selected. This is the state associated with the selected agency as entered under the Main Menu’s “Agency” button (see Section 6.1, Agency Information). 
- Setup Library - The setup library to be associated with this client can be selected using the drop-down list. The setup libraries that will be listed in the drop-down list are those that have been created for the agency selected in the Agency field. The setup library needs to be selected using the Setup Library field only if work orders will be created without running a NEAT or MHEA audit so that user-defined measures entered in the setup library can be accessed from under the Main Menu's "Work Orders" button. The setup library that will be used in running NEAT or MHEA will be selected on the NEAT or MHEA Audit Information form (see Section 8.2, Audit Information). However, if you select a setup library on the Client Information form for this client, then the selected setup library will automatically be selected on the Audit Information form when an audit for this client is created (although you can change this selection on the Audit Information form if desired). Optional.

- Address, Unit Number, City, State, and Zip Code - Enter the address of the client's dwelling to be weatherized. If you are using the Weatherization Assistant's Geographic Information System (GIS) feature, the GIS will use this address to plot the location of the dwelling as explained in Section 18.2, Geographic Information System (GIS). The address may or may not be the same as any address you supply for contacts associated with the client as discussed in Section 7.4, Contacts (Client). Optional.

- County and Other Geographic Identifier - These fields can be used to identify the county the client's dwelling resides in or to locate the dwelling by some other geographic identifier (e.g., congressional district). The entries may be 20 characters or less. Optional.

- Comment - You may enter comments directly in the Comment field on the form, or you may enter them in the Comment Editor by selecting the "Comment" button to the left of the field. The Comment Editor is similar to Window's Notepad and may be used to enter extended comments. The total comment may have up to 65,000 characters. Text from other sources may be copied and pasted into the Comment Editor's window. Any comment that is entered is displayed in a variety of reports, including the Client Information Report and the NEAT/MHEA Data Collection Form (Client Specific) available from under the Main Menu's "Clients" button (see the Report Block below) and the Input Report available from under the Main Menu's "NEAT" and "MHEA" buttons (see Section 8.2, Audit Information). You may also wish to include the 
comment in a user-designed report (see Appendix D, Development of Customized Reports). Optional.

- Cumulative Cost and SIR - These two fields are filled in only if a work order has been created for the client and actual costs (rather than estimated costs) for weatherization measures have been entered into the work order. These fields show the actual cost of all measures performed on the client's dwelling and the savings-to-investment ratio (SIR) for these measures calculated using the actual costs and savings. See the discussion under "The Materials/Labor Details SubForm” in Section 13.3, Measures (Work Order), for more details. Uneditable.

- Client Record Navigation Block - The Client record navigation block is used to find and navigate to existing client records in your database, copy or delete the currently accessed client, or to create a new client. See Section 5.1, Form and Record Navigation, for information on using the Weatherization Assistant's record navigation blocks.

The default installation of the Weatherization Assistant turns on the "Use only the latest bookmarked Agency in the find record drop-down boxes" feature in Preferences (see Feature 9 in Section 16.4, Features). With this feature turned on, the drop-down lists in the Client record navigation block will display only the clients associated with the agency last visited using the Main Menu's "Agency" button. Normally, this will be your agency, so the drop-down lists will display only your agency’s clients. If you have more than one agency defined in your database and you want the drop-down lists to display clients from all defined agencies, turn this feature off.

- Report Block - The Report block provides you with access to all reports available under the Main Menu's “Clients” button. The following reports are currently available: Client Completion Report, Client Information Report, Client Information Form, NEAT/MHEA Data Collection Form (blank or client specific), and Client Surveys. See Appendix C, Reports, for examples of these reports. The reports display information already gathered and entered into the Weatherization Assistant while the forms are blank forms to be used to gather information. The NEAT and MHEA Data Collection Forms may be printed with general client information pre-entered or completely blank. See Section 5.12, The Report Block, for information on the mechanics of using the Weatherization Assistant's Report blocks. Appendix D, Development of 


\section{Chapter 7: Clients}

Customized Reports, describes a method to create reports tailored to your needs and to list the reports in the Report block.

\section{Dwelling Data Block}

The data items included in the Dwelling data block are intended to be used primarily to complete the Unit Production portion of the DOE Quarterly Program Report (see "Report Block" in Section 6.1, Agency Information). Definitions of the items should be consistent with this report, DOE guidance, and/or 10 CFR Part 440. All of the data entries are optional except for the Dwelling Type. Below are brief descriptions of the individual data items found in the Dwelling data block on the Client Information form:

- Type - Use the drop-down list to select the type of dwelling this client lives in. The choices are Site Built, Mobile Home, Duplex, Triplex, Fourplex, Multifamily (5 or more units per building), Shelter, and Other. The selection is used only in populating the Quarterly Program Report and does not restrict your assigning either the NEAT or MHEA audit to the client, though the two should be consistent. Required.

- Ownership - Indicate whether the client owns or rents the dwelling. If you select "Other," you may want to specify details in the Comment field. Optional.

- Primary Heating Fuel - Choose the fuel that is used to provide the most space heating in the dwelling using the drop-down list. The choices are None, Natural Gas, Oil, Electricity, Propane, Wood, Coal, Kerosene, and Other. If you select "Other," you may want to specify details in the Comment field. Optional.

- Secondary Heating Fuel - If more than one fuel is used to provide space heating to the dwelling, choose the next most used fuel using the drop-down list. The choices are the same as for the primary heating fuel. Optional.

- High Energy Use - Select this checkbox if the client/dwelling is considered to be a high energy user, defined as "a low-income household whose residential energy expenditures exceed the median level of residential expenditures for all low-income households in the state.” Optional. 


\section{Chapter 7: Clients}

- High Energy Burden - Select this checkbox if the client's energy costs are a high burden for them. A client with a high energy burden is defined as "a lowincome household whose residential energy expenditures divided by the annual income of that household (energy burden) exceeds the median level of energy burden for all low-income households in the state.” Optional.

- Previously Weatherized - Select this checkbox if the dwelling was previously weatherized as defined for the DOE Quarterly Program Report. Optional.

- Low Cost/No Cost - Select this checkbox if the work performed on the dwelling may be considered to be low cost or no cost as defined for the DOE Quarterly Program Report (typically a dwelling in which a maximum of \$50 materials were expended). Optional.

- Year Built - Enter an estimate of the year the dwelling was built. Optional.

- Account \#1 and \#2 - These fields are provided so that utility account numbers for the client can be entered. If both fields will be used, you should establish a consistent approach within your agency or state as to which fields are used for which accounts. For example, electric utility account numbers may always be entered in the Account \#1 field, and natural gas, propane, fuel oil, etc. account numbers are always entered in the Account \#2 field. A comment entry may also help clarify the type of account number entered in each field. Optional.

\section{Occupants Data Block}

The data items included in the Occupants data block are intended to be used primarily to complete the Unit Production portion of the DOE Quarterly Program Report. Definitions of the items should be consistent with this report, DOE guidance, and/or 10 CFR Part 440. All of the data entries are optional. Below are brief descriptions of the individual data items found in the Occupants data block on the Client Information form:

- Occupants - Enter the total number of occupants living in the dwelling. Although optional, this entry is used in NEAT and MHEA to determine the internal loads of the dwelling, estimate water usage, and modify the minimum leakage rate for the dwelling. A default number of occupants is used in NEAT and MHEA if no entry is made. Optional. 


\section{Chapter 7: Clients}

- Elderly - Enter the number of elderly people who live in the dwelling, defined as those 60 years of age or older. Optional.

- Disabled - Enter the number of disabled people who live in the dwelling, as defined in 10 CFR Part 40. Optional.

- Native American - Enter the number of occupants living in the dwelling who are members of a Native American Tribe. Optional.

- Children - Enter the number of children who live in the dwelling, defined as being 19 years of age or younger, or as defined by your state. Optional.

- Primary Language - Select the primary language spoken by the occupants of the dwelling using the drop-down list. The choices are English, Spanish, Other European Language, Asian Language, and Other. If you select "Other," you may want to specify details in the Comment field. Optional.

\section{Graphic Information System Data Items}

If you are using the Graphic Information System (GIS), two uneditable fields (Latitude and Longitude) and a "Map" button will be present on the Client Information form just below the Comment field. If the address entered on the Client Information form for the client is recognized by the Weatherization Assistant, the Latitude and Longitude fields will display the indicated data and the "Map" button will take you to a map showing this location. See Section 18.2, Geographic Information System (GIS), for more details.

\section{Photograph Data Items}

If you have chosen to use the third party photo browser method for attaching photos to records under the Main Menu's "Preferences" button (see Feature 2 in Section 16.4, Features), a Photo Folder field, "Browse" button, and "View" button will be present on the Client Information form below the Comment field. Use the "Browse" button to select a folder on your computer in which you have stored digital photos for this specific client. Once selected, the location of this folder will be displayed in the Photo Folder field. Select the "View" button to obtain a photo browser in which you may view and edit these photos. The Weatherization Assistant provides the option of attaching photos not only to clients, but also to 


\section{Chapter 7: Clients}

audits and work orders as well. Therefore, you should develop a consistent policy regarding where and how you store reference to your photos. See Section 18.1, Digital Photos, for more information on viewing and editing photos.

\section{Client Check Out Data Items}

If you have chosen to use the Check-in/Check-out feature of the Weatherization Assistant (see Feature 10 in Section 16.4, Features, and Section 17.3, Data Transfer - Import/Export With Another MSAccess Database), a Checked Out To field, “Check In” button, and "Check Out” button will be present on the Client Information form below the Comment field. If the client you are currently referencing has been checked out, the User Name of the user who checked out the record will appear in the "Checked Out To" drop-down list's field.

You may initialize the checking out of a client or change the user assigned to the check-out by using either the "Check Out" button or the "Checked Out To" dropdown list. You may choose any user to be recorded as the user having checked out the client using the drop-down list. If you use the "Check Out" button, you may only check the client out to the current user who is logged on, presumably yourself. Once a client has been checked out, you may check the client back in by selecting the "Check In" button, regardless of who had previously checked the client out. If there are multiple users who may check records in and out, you will need to coordinate this practice.

\subsection{Status (Clients)}

The Status form under the Main Menu's "Clients" button (see Figure 7.2) allows you to view the current status settings associated with the client being accessed for all of the status categories used in the Weatherization Assistant. The status categories used in the Weatherization Assistant and that appear in the left column of the Status form are shown in Table 7.1. The current status will be shown for only those categories for which a status has been set or for which a setting is possible. You may need to use the vertical scroll bar to see all the status settings, especially if multiple work orders exist
Table 7.1. The status categories used in the Weatherization Assistant

\begin{tabular}{|c|c|}
\hline Client Level & $\begin{array}{c}\text { Client (General) } \\
\text { Application }\end{array}$ \\
\hline Audit Level & Audit (NEAT or MHEA) \\
\hline $\begin{array}{c}\text { Work Order } \\
\text { Level }\end{array}$ & $\begin{array}{c}\text { Work Order (General) } \\
\text { Work Order Inspection } \\
\text { Work Order Payment }\end{array}$ \\
\hline
\end{tabular}




\section{Chapter 7: Clients}

because three status categories (general, inspection, and payment) are tracked separately for each work order. Below are brief descriptions of the individual data items and controls found on the Status form. The entries on the main Status form cannot be edited. You must use the "Edit" button on the form to change the current status for the client.

\begin{tabular}{|c|c|c|c|c|c|c|c|}
\hline Eg Client & & & & & & & $-1 \mathrm{C}$ \\
\hline Client ID $11 \_348$ & & Clier & ent Name Tanne & e, David & & Alt. Client ID [ & \\
\hline Client Information Status $\mid \mathrm{Er}_{\mathrm{r}}$ & ner & gy Index |Contacts (2) $\mid$ Audits (1) | W & WorkOrders [2] ] & Surveys (1)] & | Photos (2) & & \\
\hline Con & np & Current Status & Date & Changed & By & Comment & Edit History \\
\hline Client & $x$ & Work Done, File Closed/Locked On & 9/17/2011 & 9/17/2011 & admin & & Edit $\mathrm{H}$ \\
\hline Application & $x$ & Approved On & 8/9/2011 & $8 / 9 / 2011$ & admin & & Edit $\mathrm{H}$ \\
\hline Audit (11_348SB ) & $x$ & Audit Complete and Locked On & $8 / 24 / 2011$ & $8 / 24 / 2011$ & admin & & Edit $\mathrm{H}$ \\
\hline WO/11_348/EASY/1 & $x$ & Work Completed On & $9 / 6 / 2011$ & $9 / 6 / 2011$ & Admin & & Edit $\mathrm{H}$ \\
\hline $\begin{array}{l}\text { W0/11_348/EASY/1 } \\
\text { Inspection }\end{array}$ & $x$ & Passed On & 9/13/2011 & 9/13/2011 & Admin & & Edit $\mathrm{H}$ \\
\hline $\begin{array}{l}\text { WO/11_348/EASY/1 } \\
\text { Payment }\end{array}$ & $x$ & Invoice Paid On & $9 / 15 / 2011$ & 9/15/2011 & Admin & & Edit $\mathrm{H}$ \\
\hline W0/11_348/JT/1 & $x$ & Work Completed On & $9 / 7 / 2011$ & 9/7/2011 & Admin & & Edit $\mathrm{H}$ \\
\hline W0/11_348/JT/1 Inspection & $x$ & Passed On & 9/13/2011 & 9/13/2011 & Admin & & Edit $\mathrm{H}$ \\
\hline W0/11_348/JT/1 Payment & $x$ & Invoice Paid On & 9/16/2011 & 9/16/2011 & Admin & & Edit $\mathrm{H}$ \\
\hline & & & & & & & $\boldsymbol{I}$ \\
\hline Refresh List & & & & & & Overall Clie & ent Status History \\
\hline
\end{tabular}

Figure 7.2. The Status form under the Main Menu’s “Clients” button.

- <Status Category and Record Name> - The left most column of the form displays the status category (see Table 7.1). If the status category is an audit level or work order level category, the name of the audit or work order is also displayed.

- Current Status - This field displays the current status setting for the given status category. The possible settings for each category are listed in Section 18.3, Status Tracking.

- Completed - An "X" will be displayed in this field when the current status setting signifies completion or closure of the activity associated with the status category. Settings that represent completion are indicated by an asterisk $\left(^{*}\right)$ in Section 18.3, Status Tracking. 


\section{Chapter 7: Clients}

- Date - This field displays the date that the current status became effective.

- Changed - This field displays the date that the current status was last changed.

- By - This field displays the User Name of the person who changed the status. The names are restricted to those that have been entered on the Contacts form under the Main Menu's "Agency" button and have a password assigned as discussed in Section 6.2, Contacts (Agency). A User Name of "Admin" implies that a specific person was not identified as making the change.

- Comment - Any comment that has been appended to this current status setting will be shown in this field.

- Edit - Status settings are changed by selecting the "Edit" button in the row of the status category you wish to change. Use of the Status Editor form that appears is explained in Section 18.3, Status Tracking.

- History - Select the "H" button to see a history of all settings for a specific status category for the current client. The Status History form that appears is the same in appearance as that discussed in Section 6.9, Status History.

- Refresh List - The "Refresh" button can be used to update the information displayed on the Status form to reflect any status changes that may have been made since the last time this form was opened.

- Overall Client Status History - Select the "Overall Client Status History" button to display a listing of all status entries for all status categories associated with the current client. The Status History form that appears is the same in appearance as that discussed in Section 6.9, Status History. You can use of the sort feature (see Section 5.8, Data Sheet and Form Views of Records) while viewing this form to arrange the status settings in a desired order (e.g., chronologically).

\subsection{Energy Index}

The Energy Index form (see Figure 7.3) is used to compute an overall energy index for the client's dwelling in units of Btu per heating degree day per square foot. Note that the energy index may be significantly affected by the life styles of the 


\section{Chapter 7: Clients}

occupants, regardless of the dwelling's construction characteristics, because it is based on actual energy consumption. The energy index is typically used to determine how energy efficient the client's dwelling currently is and the potential to save energy in the client's dwelling from weatherization. The index can be used to compare or rank dwellings according to the potential energy savings that could be achieved from weatherization. The energy index calculated by the Weatherization Assistant is of most use in heating climates because the index is based on heating degree days. The energy index calculated on this form is independent of the detailed energy calculations and energy consumption estimates made under NEAT or MHEA. Use of the Energy Index form is totally optional.

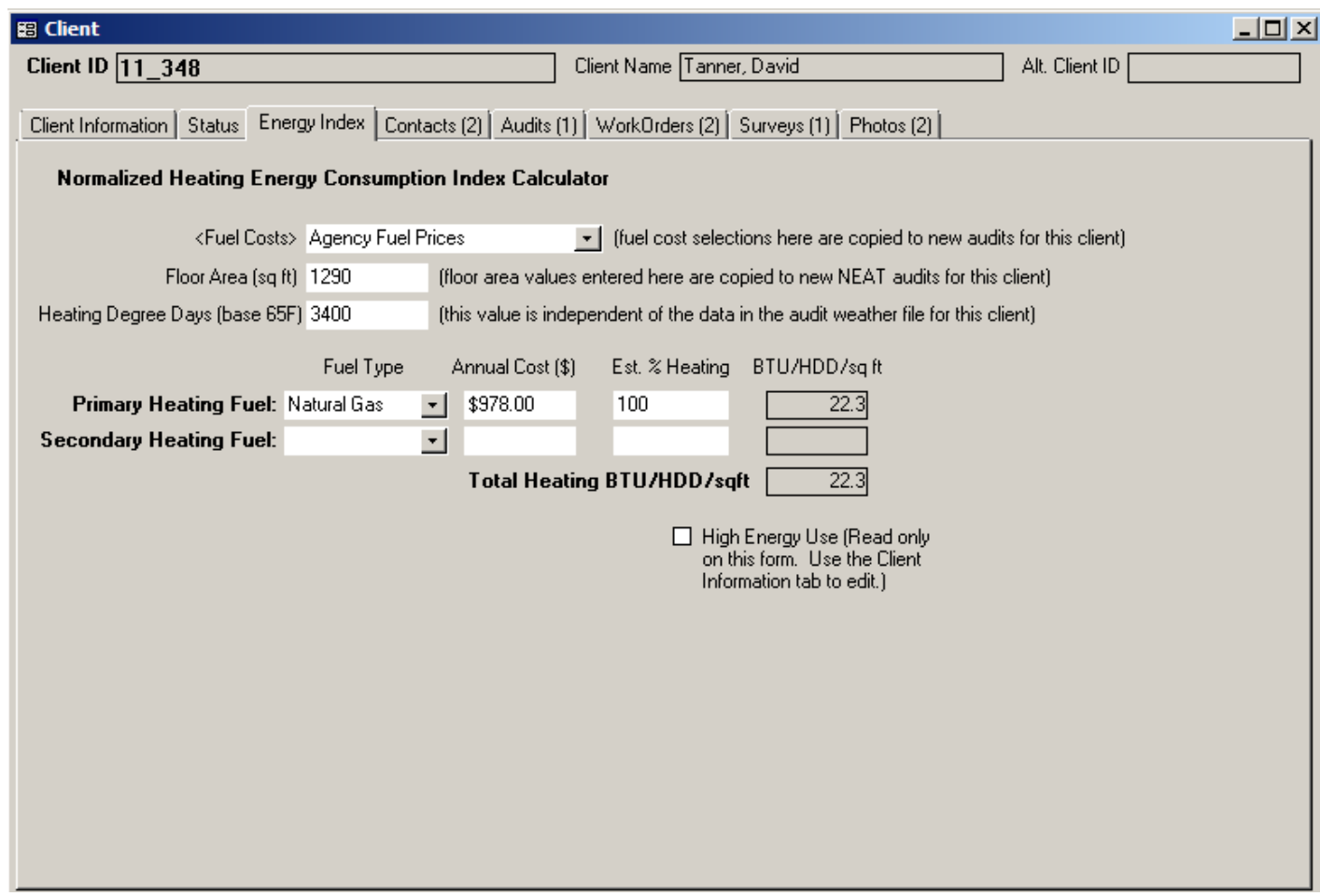

Figure 7.3. The Energy Index form under the Main Menu's “Clients” button.

Below are descriptions of the individual data items and controls found on the Energy Index form. All entries except those for the secondary fuel type are required for the Weatherization Assistant to calculate an energy index. You may, however, enter partial data and then return at a later time to complete the data entry.

- Fuel Costs - In order to translate the Annual Cost of energy entered in dollars (see below) into units of energy consumption, the Weatherization Assistant 
needs to know the fuel prices paid by the client. Use this drop-down list to select the Fuel Cost table that contains the fuel prices appropriate for this client. The fuel costs tables that will appear in the drop-down list are those that have been entered in any setup library associated with the agency that the client has been assigned to (see Section 14.3, Fuel Costs). If you have only one Setup Library with a single fuel cost table, this will be a trivial choice.

If you select a fuel cost table on the Energy Index form, then the selected fuel cost table will automatically be selected on the NEAT or MHEA Audit Information form (see Section 8.2, Audit Information) when an audit for this client is created (although you can change this selection on the Audit Information form if desired). If you do change the table on the Audit Information form, remember that the fuel costs used in the audit calculations may be different from those used to calculate the energy index.

- Floor Area - Enter the conditioned floor area of the client's dwelling in units of square feet. This is the floor area of the living space, not the foot print area. For example, a two-story home with a floor area of 1200 sq. ft. per story would have a total floor area of 2400 sq. $\mathrm{ft}$. If you enter a floor area on the Energy Index form, then this floor area will automatically be entered on the NEAT Audit Information form (see Section 8.2, Audit Information) when an audit for this client is created (although you can change this entry on the Audit Information form if desired).

- Heating Degree Days - Enter the number of annual Heating Degree Days (HDDs) at base $65^{\circ} \mathrm{F}$ for the location of the dwelling. The annual HDDs entered should correspond to the same time period as the annual energy costs entered below in order for the energy index to be most accurate. In addition, values entered for different clients should come from a consistent source. However, if the HDDs for this time period are not available, then average values over long time periods may be used. Such values may be available through the National Oceanic and Atmospheric Administration (NOAA) or The American Society of Heating, Refrigeration, and Air Conditioning Engineers (ASHRAE).

The next three fields exist for both a primary and a secondary heating fuel. Enter information into the fields for the secondary heating fuel only if more than one fuel is used to supply space heating to the dwelling (e.g., if an electric space heater is 


\section{Chapter 7: Clients}

used to heat a bedroom while the remainder of the house is heated by a gas furnace).

- Fuel Type - Select the fuel type that is being described as providing the space heat. The choices are None, Natural Gas, Oil, Electricity, Propane, Wood, Coal, Kerosene, and Other.

- Annual Cost - Enter the annual fuel bill for the fuel specified. Typically, this is the sum of 12 recent and consecutive monthly fuel bills.

- Est \% Heating - Enter an estimate of the portion of the Annual Cost for this fuel that was spent for space heating, as opposed to water heating, cooking, etc. A default value will be entered automatically based on the Fuel Type selected, but you should modify this value if you do not think it is representative of the actual conditions in the dwelling.

- BTU/HDD/sqft - Once all of the information described above has been entered, the Weatherization Assistant computes and displays an energy index for the primary and secondary fuels as well as a combined energy index for both fuels. This latter value is the energy index for the home (i.e., a measure of the home's energy efficiency).

- High Energy Use - This read-only checkbox is automatically filled out depending on your entry on the Client Information tab for this same data item (see Section 7.1, Client Information). Although DOE's definition of this parameter is not directly linked to the energy index for a home, consistent use of the energy index and designation of homes as high energy users should reveal some correlation between these two data items.

\subsection{Contacts (Client)}

The Contacts form under the Main Menu's “Clients” button (see Figure 7.4) allows you to enter information on people associated with the currently selected client. You can enter as many contacts as needed. The person requesting weatherization assistance should be one contact that is entered and will often be the only contact entered. Other contacts that may be entered for some clients include responsible relatives of elderly individuals who should be consulted, owners of rental units, or perhaps a utility representative from whom billing data will be obtained. 


\section{Chapter 7: Clients}

Multifamily buildings may have a superintendent or maintenance person assigned to the building who could be a valuable contact. The Contacts form may be viewed and edited in the data sheet view, which provides a summary of all contacts already entered for the client or allows quick changes to multiple entries. Client contacts are reported on the Client Completion Report, the Client Information Report, and the Client Survey Report, all of which are accessible from the Report block of the Client Information form (see Section 7.1, Client Information). Below are descriptions of the individual data items and controls found on the Contacts form under the Main Menu's “Clients” button:

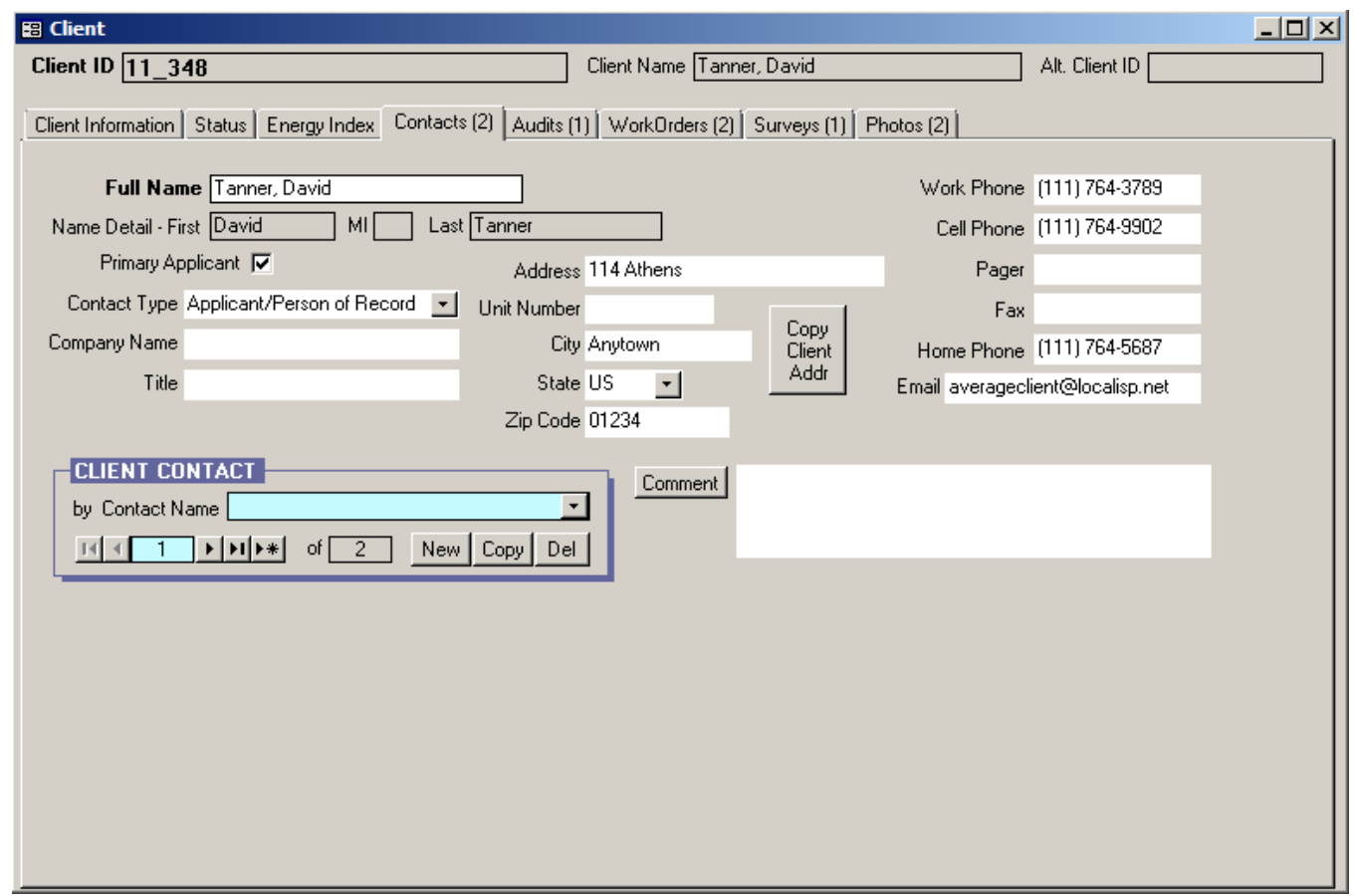

Figure 7.4. The Contacts form under the Main Menu's “Clients” button.

- Full Name - Enter the name of the contact. You may enter the name with the given name first and the surname last, or with the surname first followed by a comma and then any given names. After you have entered the name and moved to another control, the Weatherization Assistant will automatically fill in the uneditable Name Detail fields (i.e., First, MI, and Last) to show you how the program sees the name that you have entered. If you are trying to enter the name of a company (e.g., a supplier of materials), you should enter the name of your primary contact within that company and then provide entries for the User Name and Company fields. The name must be unique. Required. 
- Primary Applicant - Use this checkbox to indicate if the contact being entered is the name of the person who applied for weatherization. If checked, this person's name will also appear in the uneditable Client Name field, which is shown above the tabs on the "Client" window when this particular client is accessed. Reports will also display this individual as the client. Only one contact can be declared as the Primary Applicant for each client. Designating a contact as the Primary Applicant will automatically uncheck the checkbox for any other contact who had previously been declared as the Primary Applicant for this client. Changing the contact designated as the Primary Applicant is not recommended when other windows displaying this information are open.

- Contact Type - Select from the drop-down list the Contact Type that best applies to the contact. The choices are Applicant/Person of Record, NonApplicant/Person of Record, Other Contact for Applicant, Landlord/Owner, Superintendent, Maintenance Staff, and Other. The entry will default to "Applicant/Person of Record" when a new contact record is initiated. Normally, if the Primary Applicant checkbox is checked for this contact, then the Contact Type is "Applicant/Person of Record.” The "Non-Applicant/Person of Record" is intended for use in multifamily buildings to designate an occupant of a unit who can have weatherization work performed on their unit only because the required fraction of other units in the building qualify. Optional.

- Company Name - If applicable, enter the company associated with the contact. Remember that the contacts being entered here are for a specific client, not for your agency in general. Thus, companies you use as suppliers or contractors would likely be more appropriately entered on the Contacts form under the Main Menu's “Agency” button as discussed in Section 6.2, Contacts (Agency), unless this company supplied a specific need for this client alone. Optional.

- Title - If applicable, enter a title associated with the client. This entry could alternately be used to enter a position or relation of the contact to a primary applicant. Optional.

- Address, Unit Number, City, State, and Zip Code - Enter the complete mailing address of the contact. If the address is the same as the address entered for the dwelling on the Client Information form (see Section 7.1, Client Information), you can use the "Copy Client Addr" button to copy this address from the Client Information form to the Contacts form. Optional. 


\section{Chapter 7: Clients}

- Copy Client Addr - If the address entered for the dwelling on the Client Information form (see Section 7.1, Client Information) is the same as the address for the client, you can use the "Copy Client Addr" button to copy the address information from the Client Information form to the Contacts form.

- Work, Cell, Pager, Fax, and Home Phone - Enter any of the indicated phone numbers for the contact. Optional.

- E-Mail - Enter an e-mail address for the contact. Only e-mail addresses of contacts listed under the Main Menu’s “Agency” button will be entered automatically in the Address Book of the e-mail feature of the Weatherization Assistant. See Section 18.4, E-Mailing from Within Weatherization Assistant, for more information. Optional.

- Comment - You may enter comments directly in the Comment field on the form, or you may enter them in the Comment Editor by selecting the "Comment" button to the left of the field. The Comment Editor is similar to Window's Notepad and may be used to enter extended comments. The total comment may have up to 65,000 characters. Text from other sources may be copied and pasted into the Comment Editor's window. Comments entered on the Contacts form are not displayed anywhere else in the Weatherization Assistant or in any of its predefined reports. However, you may wish to include the comment in a user-designed report (see Appendix D, Development of Customized Reports). Optional.

- Client Contact Record Navigation Block - The Client Contact record navigation block is used to find and navigate to existing client contacts, copy or delete the currently accessed contact, or create a new contact. Only contacts associated with the current client will appear in the drop-down list. See Section 5.1, Form and Record Navigation, for information on using the Weatherization Assistant's record navigation blocks.

\subsection{Audits (Client)}

The Audits form (see Figure 7.5) under the Main Menu's “Clients” button shows all the audit records associated with the current client in a navigation data sheet view. This form is similar to the Audits form found under the Main Menu's "Agency” button as discussed in Section 6.6, Audits (Agency). The form is used 


\section{Chapter 7: Clients}

primarily to navigate to individual audit records. The shading of the records displayed on this form is the visual indicator that this form is used for navigation rather than data entry. You can double click with your mouse on the entries under the column headings in angle brackets $(<>)$ to navigate to the associated record. Like any data sheet, the order that the audit records are displayed can be changed by highlighting a column and pressing one of the sort buttons in the tool bar. Some of the columns in the form may be collapsed (not shown) by default. You can double click the mouse near the borders of some of the columns in the header to have them expanded. You can collapse columns that you do not want to see by dragging their widths to zero. You may need to use the horizontal scroll bar to display all the columns.

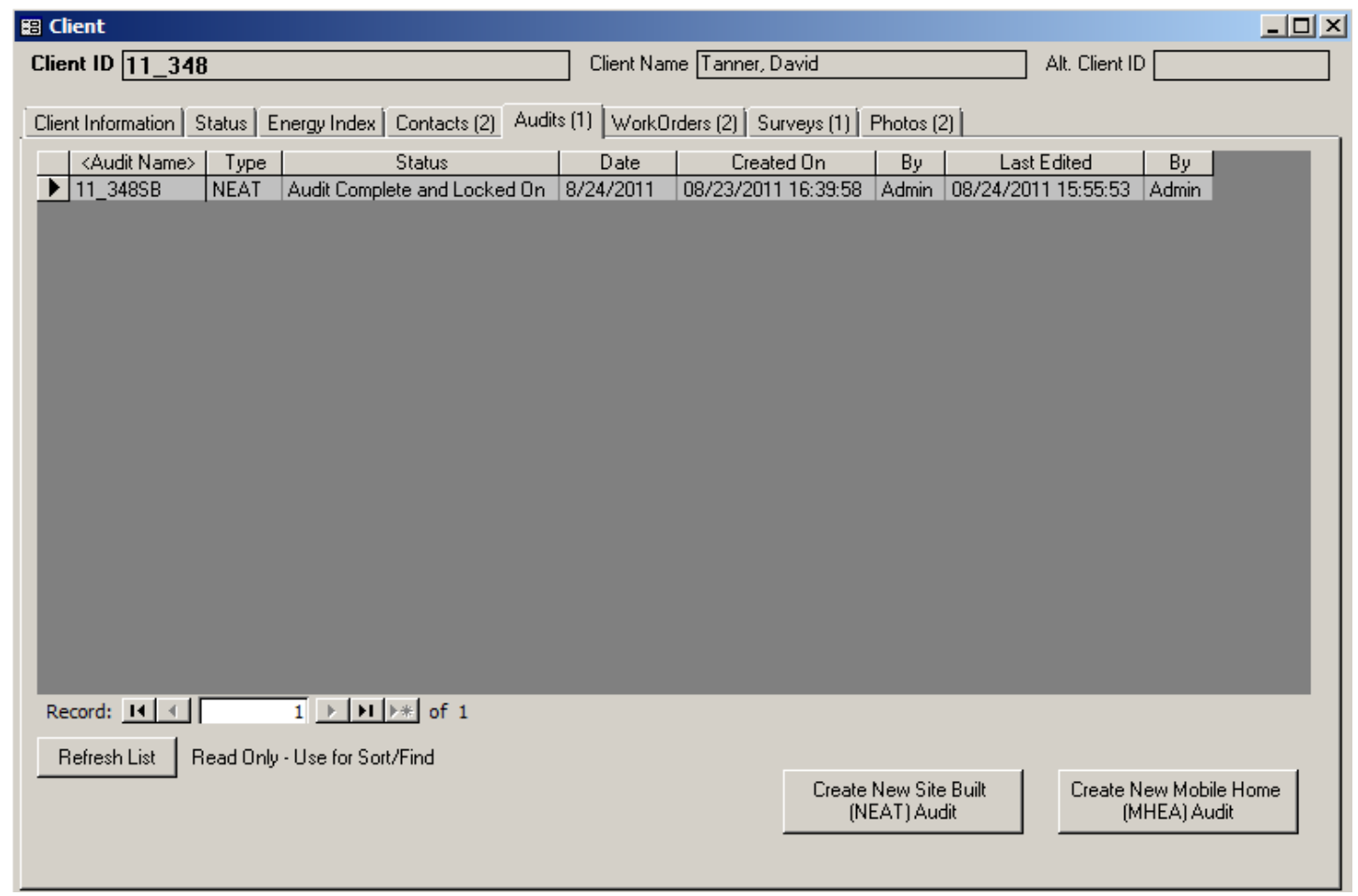

Figure 7.5. The Audits form under the Main Menu's “Clients” button.

Columns not displayed in this figure include the ClientID and numerical JobID.

Normally, there is only one audit record for each client. However, the Weatherization Assistant does allow you to create and execute more than one audit per client. This might be useful if you desire to see the effect of some modification in the building description on the recommendations of the audit. You might also want to update or correct an existing audit without losing the original description. If you have run more than one audit per client, you should ensure that only one set 
of active work orders exists for the client. Otherwise, confusion and double accounting of costs are likely to occur. Below are brief descriptions of the individual data items and controls found on the Audits form under the Main Menu's “Clients” button:

- Audit Name and Type - These entries are those given on the Audit Information form under the Main Menu's "NEAT" and "MHEA" buttons for each of the audits listed (see Section 8.2, Audit Information).

- Numerical JobID (not shown) - When a new audit is created, the Weatherization Assistant automatically assigns a unique number to the audit for internal software purposes. This field displays what this number is for each audit. See Feature 6 in Section 16.4, Features, for more information.

- ClientID (not shown) - The Client ID associated with each audit is also available on this form but not normally displayed since the same information is available immediately above the form (see Figure 7.5).

- Status - This field gives the latest audit status for each audit as set on the Status form under the Main Menu's “NEAT” or “MHEA” buttons. See Section 11.1, Status (Audit), and Section 18.3, Status Tracking, for more information.

- Date - This field provides the date that the latest status was set.

- Created On - This field gives the date (and time if the entry is expanded) that the audit was originally created, either by using the "Create New...Audit" buttons on this form, copying an existing audit, or selecting the "New" button on the Audit Information form.

- By - If the User Logon feature was enabled (see Feature 4 in Section 16.4, Features) when an audit was created or last edited, these columns will give the user that was logged on at that time.

- Last Edited - This field shows the date (and time, if the column is expanded) of the last change made to this audit record on forms under the Main Menu's "NEAT" and "MHEA" buttons. 
- Refresh List - The "Refresh List" button can be used to update the information displayed on the Audits form to reflect any changes since the last time this form was opened.

- Create New Site Built (NEAT) Audit and Create New Mobile Home (MHEA) Audit - You can select these buttons if you wish to initiate a new NEAT or MHEA audit for the current client. The Weatherization Assistant will create a new audit record, display the appropriate Audit Information form, and provide an initial default Audit Name. The Client ID, Agency Name, and Agency State fields on the form for the new audit will also be automatically entered based on the information entered for the current client and the agency assigned to that client. If no other entries are made on this new Audit Information form, you may immediately exit the form without adding any further information and the audit will be recorded for future reference but with blank status. If you enter any other information on the newly created audit form, the Weatherization Assistant will begin examining for required data entries and not allow you to exit the form until the required data entries have been made (or you have erased the other information by entering two consecutive [Esc] keys). See Sections 4.2, Starting a New Audit, and 8.2, Audit Information, for details.

\subsection{Work Orders (Client)}

The Work Orders form (see Figure 7.6) under the Main Menu's “Clients” button shows all work order records associated with the current client in a navigation data sheet view. This form is similar to the Work Orders form found under the Main Menu's “Agency” button as discussed in Section 6.7, Work Orders (Agency). The form is used primarily to navigate to individual work order records. The shading of the records displayed on this form is the visual indicator that this form is used for navigation rather than data entry. You can double click with your mouse on the entries under the column headings in angle brackets $(<>)$ to navigate to the associated record. Like any data sheet, the order that the work order records are displayed can be changed by highlighting a column and pressing one of the sort buttons in the tool bar. Some of the columns in the form may be collapsed (not shown) by default. You can double click the mouse near the borders of some of the columns in the header to have them expanded. You can collapse columns that you do not want to see by dragging their widths to zero. You may need to use the horizontal scroll bar to display all the columns. 


\section{Chapter 7: Clients}

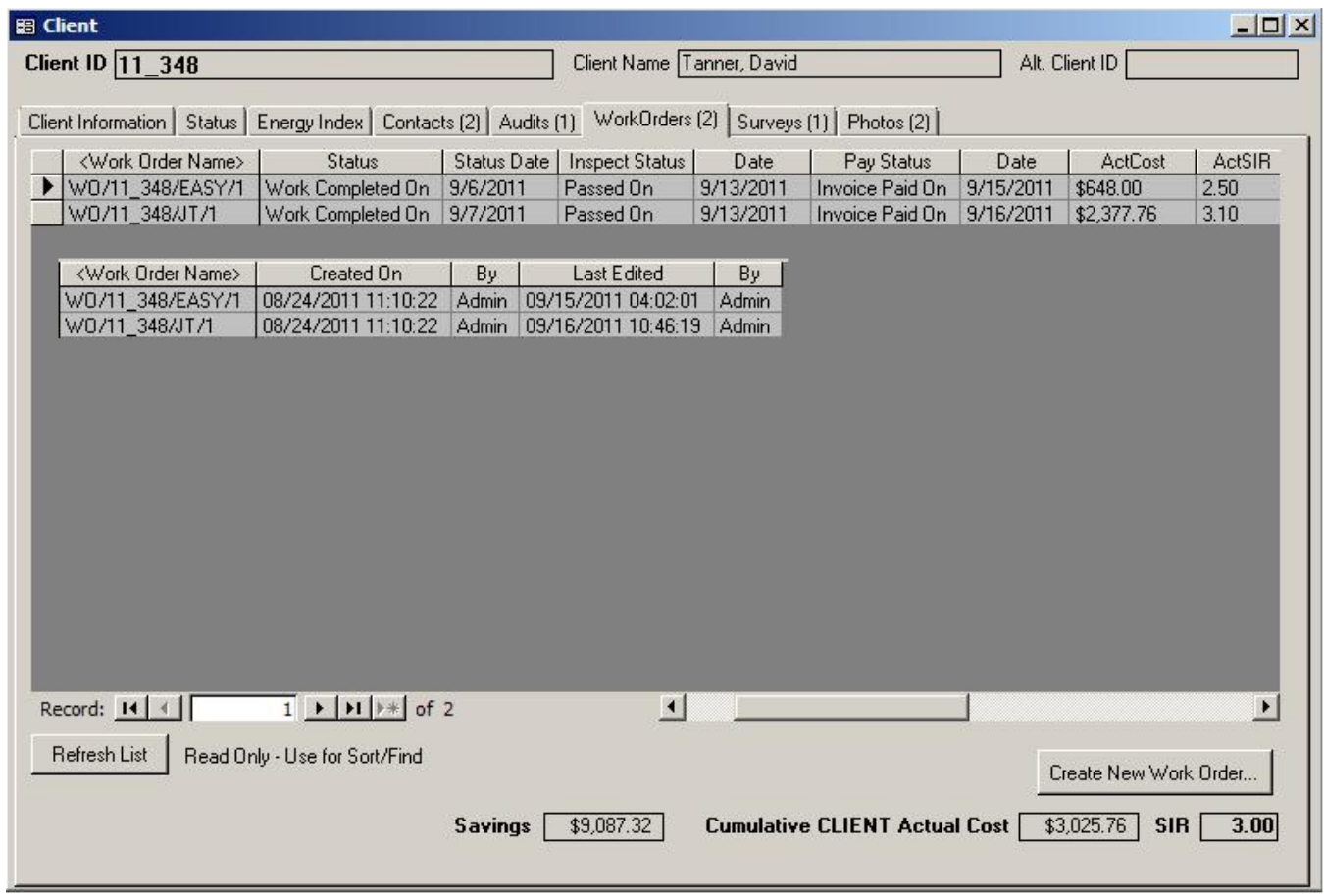

Figure 7.6. The Work Orders form under the Main Menu’s “Clients” button. Columns not displayed in this figure include the ClientID and numerical JobID. Note: The form has been displayed wrapped since scrolling would normally be necessary to see all columns.

More than one work order for a client will be displayed on this form if you have divided the work on a client's home into more than one work order, normally because more than one contractor is assigned the work. The work orders may have been generated automatically from the Measures form under the Main Menu's "NEAT" and "MHEA" buttons (see Section 11.11, Measures (Audit)) or manually from the Main Menu's “Work Orders” button (see Section 13.1, Work Order Information). Below are brief descriptions of the individual data items and controls found on the Work Orders form under the Main Menu's "Clients” button:

- Work Order Name - This entry is the name associated with the work order as prescribed on the Work Order Information form of the Main Menu's "Work Orders" button when it was first generated (see Section 13.1, Work Order Information).

- Numerical WorkOrderID (not shown) - When a new work order is created, the Weatherization Assistant automatically assigns a unique number to the work 
order for internal software purposes. This field displays what this number is for each work order. See Feature 6 in Section 16.4, Features, for more information.

- ClientID (not shown) - The Client ID associated with each work order is also available on this form but not normally displayed since the same information is available immediately above the form (see Figure 7.6).

- Status, Inspect Status, and Pay Status - There are three status categories for a work order: a general status, an inspection status, and a payment status. Each status category has its own column in the Work Orders form. These fields give the latest status of each category for each work order. These statuses are changed on the Status form under the Main Menu's “Work Orders” button for each individual work order. See Section 13.2, Status (Work Order), and Section 18.3, Status Tracking, for more information.

- Status Dates - These fields provide the date that the latest status was set for each of the three work order status categories.

- ActCost - If any actual costs have been entered for the measures listed on a work order, then the total of these costs will be displayed in this column. See “The Materials/Labor Details Sub-Form” in Section 13.3, Measures (Work Order), for more information. This entry may not be of much significance until the work order status has been declared "Completed" as discussed in Section 13.2, Status (Work Order).

- ActSave (not shown) - This field displays the total actual life cycle savings (over the life of the measures) for all measures listed on a work order that have energy savings associated with them. The actual savings reflects the "Actual/Estimated Adjustment Factors" imposed on the savings for each measure as discussed in Section 13.3, Measures (Work Order). This field is not shown (collapsed) by default because it may not be of interest on the work order level. To view the column, double click your mouse while positioned just to the right of the line between the ActCost and ActSIR column headers.

- ActSIR - If energy savings are associated with any of the measures listed on a work order and actual costs for these measures have been entered, then the ActSIR field will display the corresponding actual savings-to-investment ratio (SIR) for the work order as a whole. The value is the ratio of the ActSave to the 


\section{Chapter 7: Clients}

ActCost entries above. This entry may not be of much significance until the work order status has been declared "Completed" and all actual costs have been entered as discussed in Section 13.2, Status (Work Order).

- Created On - This field gives the date (and time if the entry is expanded) that the work order was originally created, either from having run a NEAT or MHEA audit, copying an existing work order, or selecting the "New" button on the Work Order Information form.

- By - If the User Logon feature was enabled (see Feature 4 in Section 16.4, Features) when a work order was created or last edited, these columns will give the user that was logged on at that time.

- Last Edited - This field shows the date (and time, if the column is expanded) of the last change made to this work order record on the forms under the Main Menu's "Work Orders" button or, if no modifications have been made, the date the work order was generated from the Measures form of the Main Menu's "NEAT" or "MHEA" buttons.

- Refresh List - The "Refresh List" button can be used to update the information displayed on the Work Orders form to reflect any changes since the last time this form was opened.

- Create New Work Order - Selecting this button will take you directly to the Work Order Information form under the Main Menu's "Work Orders" button (see Section 13.1, Work Order Information) and initiate a new work order for the current client. The Weatherization Assistant will provide an initial default Work Order name. The Client ID, Agency Name, and Agency State fields on the new work order will also be automatically entered based on the information entered for the current client and the agency assigned to that client. If a NEAT or MHEA audit has been entered for the client, the name of the audit will also be automatically entered. However, this entry may be altered if more than one audit has been performed for the client, or the entry may be deleted if the measures to be assigned to this work order come from some source other than a NEAT or MHEA audit, such as in the case of a priority list.

- Savings - This field displays the sum of the ActSave column (i.e., the cumulative actual life cycle savings for all measures for all work orders for the 


\section{Chapter 7: Clients}

client). If accurately tracked, this entry would eventually equal an estimate of the total life time dollar savings of all work performed for a client. Uneditable.

- Cumulative CLIENT Actual Cost - This field displays the sum of the ActCost column (i.e., the cumulative actual cost for all work orders for the client). If accurately tracked, this entry would eventually equal the total actual costs of all work performed for a client. Uneditable.

- Cumulative CLIENT Actual SIR - This field displays the actual SIR for all work performed for the client from all work orders displayed on the Work Orders form based on the entries in the ActSIR column. This entry may not be of much significance until the work order statuses for all work orders associated with the client have been declared "Completed" and all actual costs have been entered, as discussed in Section 13.2, Status (Work Order). Uneditable.

\subsection{Surveys (Client)}

The Surveys form (see Figure 7.7) under the Main Menu's “Clients” button is used to fill out the surveys you have previously developed under the Main Menu's “Agency” button (see Section 6.4, Surveys (Agency)) allowing you to collect

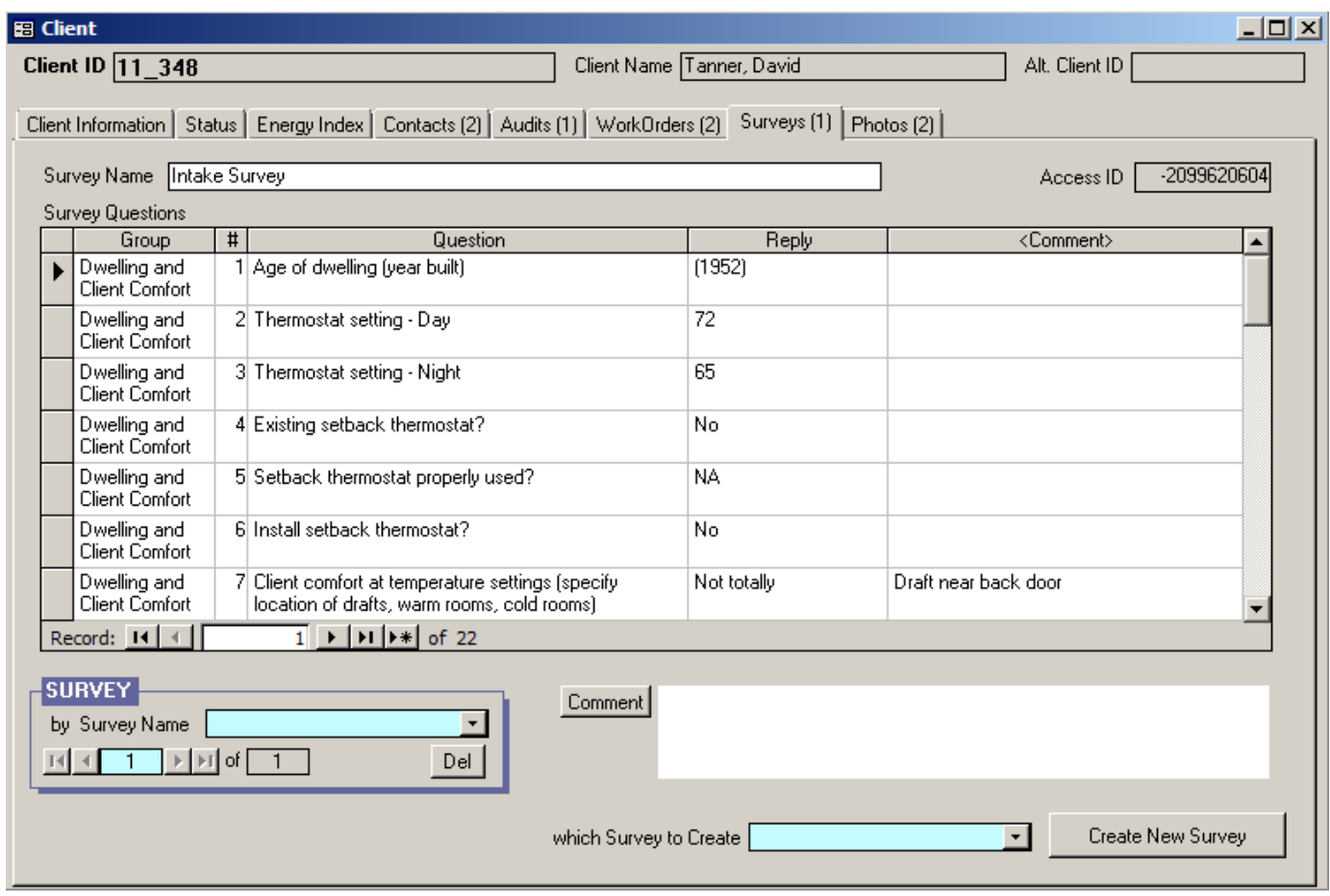

Figure 7.7. The Surveys form under the Main Menu’s “Clients” button. 


\section{Chapter 7: Clients}

additional information on your current client or his/her household. The predefined surveys you select for this client can be altered on this form without changing the originals. You may fill out multiple surveys for the same client. If you have predefined a blank survey, it can be selected for any client, then modified to create a unique survey specific to that client.

Below are descriptions of the individual data items and controls found on the Surveys form under the Main Menu's “Clients” button. Entries in the Survey Questions sub-form will be discussed afterwards.

- Survey Name - Once you have selected a survey from your predefined surveys (see the following control description), this field will contain the survey name given at the time of the survey's definition. You are free to change the name here without affecting the name of the original survey. The name should be unique amongst the survey names for a given client. Required.

- Which Survey to Create, Create New Survey - To select a predefined survey for the current client, use the "which Survey to Create" drop-down list to select the survey and then select the "Create New Survey" button to actually create and display the survey for your current client. The name of the survey will appear in the Survey Name field at the top of the form. Be careful. The Weatherization Assistant allows you to create more than one copy of the same survey for the same client, which can possibly create confusion.

- Survey Record Navigation Block - The Survey record navigation block is used to find and navigate to existing surveys that you have created and filled in for the current client, and to delete unwanted copies of surveys using the "Del" button. See Section 5.1, Form and Record Navigation, for information on using the Weatherization Assistant's record navigation blocks.

- Comment - You may enter comments pertinent to the entire survey directly in the Comment field on the form, or you may enter them in the Comment Editor by selecting the "Comment" button to the left of the field. The Comment Editor is similar to Window's Notepad and may be used to enter extended comments. The total comment may have up to 65,000 characters. Text from other sources may be copied and pasted into the Comment Editor's window. Any comment that is entered is displayed on the Client Surveys report accessed from the Client Information form (see Section 7.1, Client Information). Optional. 


\section{Chapter 7: Clients}

The Survey Questions sub-form is used to answer the questions listed in a predefined survey or alter a survey by adding, deleting, or modifying questions contained in it. Altering a survey on this form will not affect the surveys defined under the Main Menu's “Agency” button. You could develop a predefined survey under the Main Menu's “Agency” button that has no questions in it, recall this blank survey for the current client, and then develop a survey specific for the current client by adding whatever questions you like. A new question may be initiated by positioning the cursor at the first blank record or by selecting the new

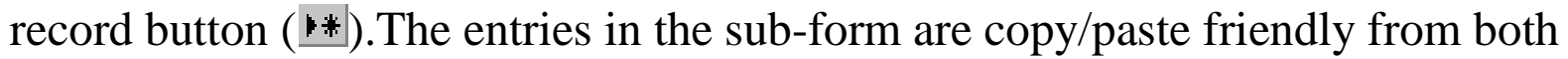
the Weatherization Assistant program and other text or word processing software. The multiple columns of the sub-form may be copied to a spreadsheet, such as Microsoft Excel, for printing. All surveys created for the current client can be printed from the Client Information form under the Main Menu's "Clients" button using the Report block (see Section 7.1, Client Information). Below are brief descriptions of the individual data items and controls found on the Survey Questions sub-form:

- Group - If not entered on the predefined survey, you can assign each question to a group if desired on this form. Any existing Group entry may be modified. Assigning groups may be desirable for organizational or presentation purposes. You could also use the Group designation to sort the questions on the sub-form using standard data sheet view sorting techniques. The entry is limited to 50 characters. Optional.

- \# - If not entered on the predefined survey, you can establish the order the questions will appear on the Client Surveys report by assigning order numbers on this form (see "Report Block" in Section 7.1, Client Information). Any existing assignments may be modified. Optional.

- Question - If not entered on the predefined survey, you can enter the actual question to be asked of the client or completed by agency staff on this form. Any existing question may be modified to be specific to the current client. Each question may be 255 characters or less. Optional.

- Reply - Individual question replies are entered as text entries in the Reply column and are limited to 50 characters or less. Number replies are seen as text and cannot be used in arithmetic operations. Optional. 


\section{Chapter 7: Clients}

- Comment - You can enter any comments associated with each question or answer directly in the Comment field on the form, or you may enter them in the Comment Editor by double clicking on the Comment field. Text from other sources may be copied and pasted into the Comment Editor's window. The comments appear on the Client Surveys report printed from the Client Information form under the Main Menu's “Clients” button using the Report block (see Section 7.1, Client Information). Optional.

\subsection{Photos (Client)}

If you have selected the "Use photo browser tab..." checkbox from the Features tab under the Main Menu's "Preferences" button (see Feature 1 in Section 16.4, Features), the Photos tab will appear under the Main Menu's "Clients" button. This feature allows you to access the Photos form (see Figure 7.8). From this form, you may select, view, and edit digital photos for the current client. See Section 18.1, Digital Photos, for more information on this feature. The ability to reference digital photos also exists under the Main Menu's "NEAT," "MHEA," and "Work Orders" buttons. Therefore, you should develop a consistent policy regarding where you reference your photos.

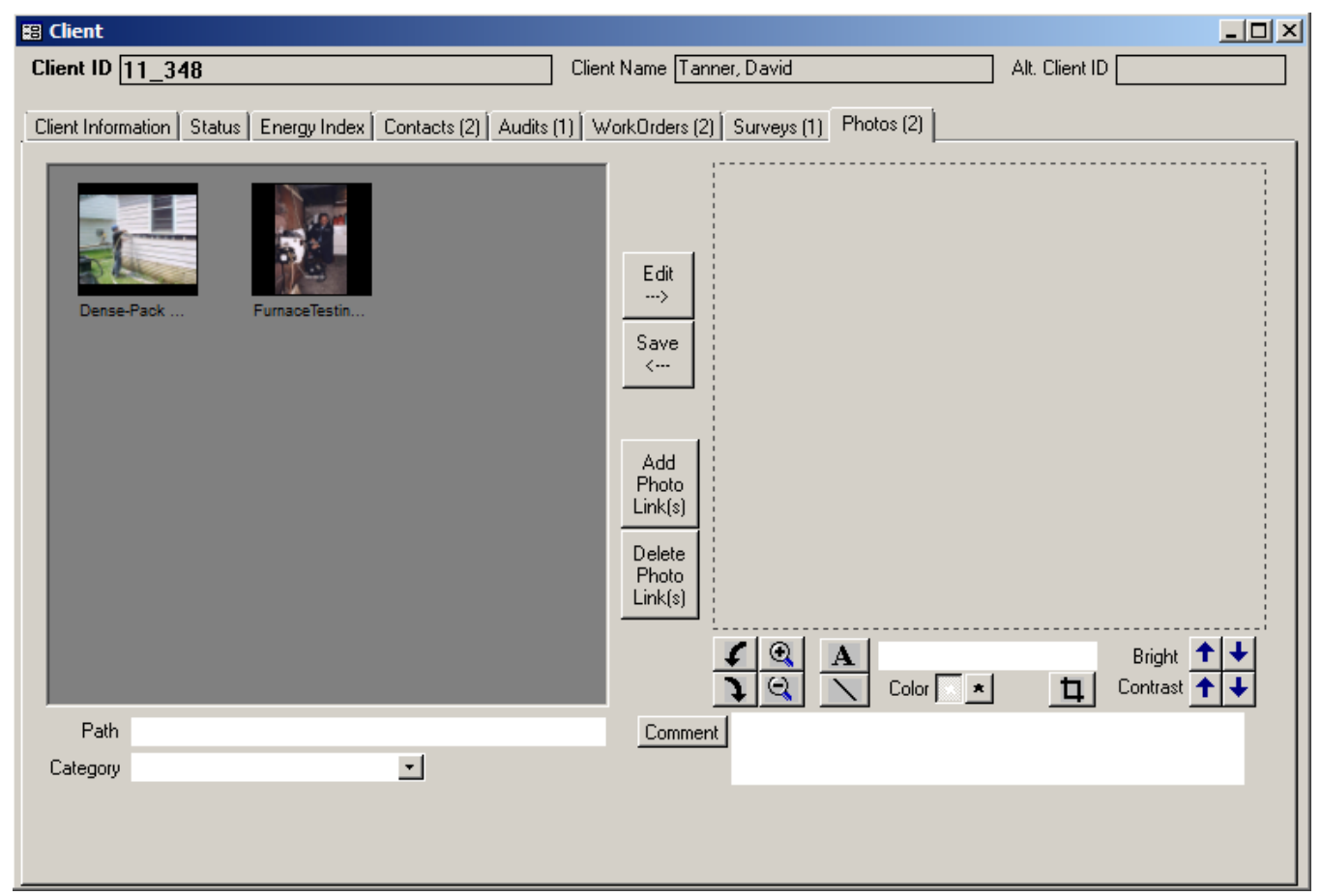

Figure 7.8. The Photos form under the Main Menu’s “Clients” button. 


\section{Chapter 8. NEAT and MHEA Introduction}

The primary purpose of the Weatherization Assistant is to make energy efficiency measure recommendations applicable to specific homes. This purpose is fulfilled by gathering audit information on each dwelling and entering this information into the program. This descriptive information is organized under either the Weatherization Assistant's Main Menu's "Site Built (NEAT)” button for site-built, single-family homes or the Main Menu's "Mobile Home (MHEA)" button for mobile homes (see Figure 3.1). This manual refers to information, forms, tabs, etc., accessed under these two buttons as being in the "NEAT Audit window" or "MHEA Audit window” or, simply, being "NEAT's” or "MHEA's."

The discussion of the data entry and display items found under these two Main Menu buttons is presented in five separate chapters. This chapter discusses the information entered on the Audit Information form found in both the "NEAT Audit" and "MHEA Audit" windows. The Audit Information forms for NEAT and MHEA are discussed together because most of the information entered on these forms is the same. Chapter 9, NEAT Building Description, discusses the data entered under NEAT's Shell, Heating, and Cooling forms to describe a site-built house, while Chapter 10, MHEA Building Description, discusses the data entered under MHEA's Shell, Addition, Heating, and Cooling forms to describe a mobile home. The NEAT forms are discussed separately from the MHEA forms because the descriptive data for a site-built home differs from the data for a mobile home. Chapter 11, NEAT and MHEA Common Elements, discusses forms that are common to both NEAT and MHEA. These include the Status, Ducts/Infiltration, Base Loads (Water Heating, Refrigerators, and Lighting Systems), Health \& Safety, Itemized Costs, Utility Bills, Photos, and Measures forms, as well as some optional sub-forms found on the Heating and Water Heating forms. Finally, the Recommended Measures report, typically displayed immediately after running a NEAT or MHEA audit, is discussed in Chapter 12, NEAT and MHEA Results.

\subsection{Starting a New Audit and Accessing Previous Audits}

Once a client has been entered into the Weatherization Assistant, audit information on the client's dwelling can be entered into the program and the audit can be run to obtain a list of recommended weatherization measures. The recommended way to start a new audit record for a client is to select the "Clients" button on the Weatherization Assistant's Main Menu (see Figure 3.1), select the client for which 


\section{Chapter 8: NEAT and MHEA Introduction}

the audit is to be performed, select the Audit tab, and then click on either the "Create New Site Built (NEAT) Audit” or "Create New Mobile Home (MHEA) Audit" button, whichever is appropriate for this specific dwelling. Alternatively, you may initiate a new audit for a previously entered client by entering either the "NEAT Audit" or "MHEA Audit" window from the Main Menu, then selecting the "New" button from the Audit record navigation block on the Audit Information form. You will then have to choose the correct client from the form's "Client ID" drop-down list. Either method will leave you on the Audit Information form for NEAT or MHEA with the audit correctly assigned to the client of your choice. These approaches are discussed in more detail in Sections 4.2, Starting a New Audit; 7.5, Audits (Client); and 8.2, Audit Information.

You may access any existing audit by selecting the appropriate "NEAT Audit" or "MHEA Audit" button from the Main Menu and then using one of the drop-down lists in the Audit record navigation block to locate and select the desired audit. Although this is the more direct method, you can also access an existing audit by selecting the "Clients" button from the Weatherization Assistant's Main Menu, navigating to the client of interest using the Client record navigation block, selecting the Audits tab, and then double clicking your mouse on the Audit Name corresponding to the audit of interest as discussed in Section 7.5, Audits (Client). Similarly, you can access an existing audit from the Main Menu's “Agency” button as discussed in Section 6.6, Audits (Agency).

\subsection{Audit Information}

The Audit Information forms under the Main Menu's "NEAT Audit" and "MHEA Audit" buttons (see Figure 8.1 and Figure 8.2, respectively) are used to enter overall audit information about the house and to identify the libraries the audit will reference for non-house specific data. They also allow you to view and navigate to other audits in your database, and to access audit level reports. Below are descriptions of the individual data items and controls found on the Audit Information forms. Most apply to both NEAT and MHEA. Those fields that are unique to NEAT or MHEA are described separately.

- Audit Name - The Audit Name is designed to be the key parameter identifying this audit to you and to the computer. Therefore, the Audit Name must be unique. Some convention should be adopted to supply each audit with a unique 


\section{Chapter 8: NEAT and MHEA Introduction}

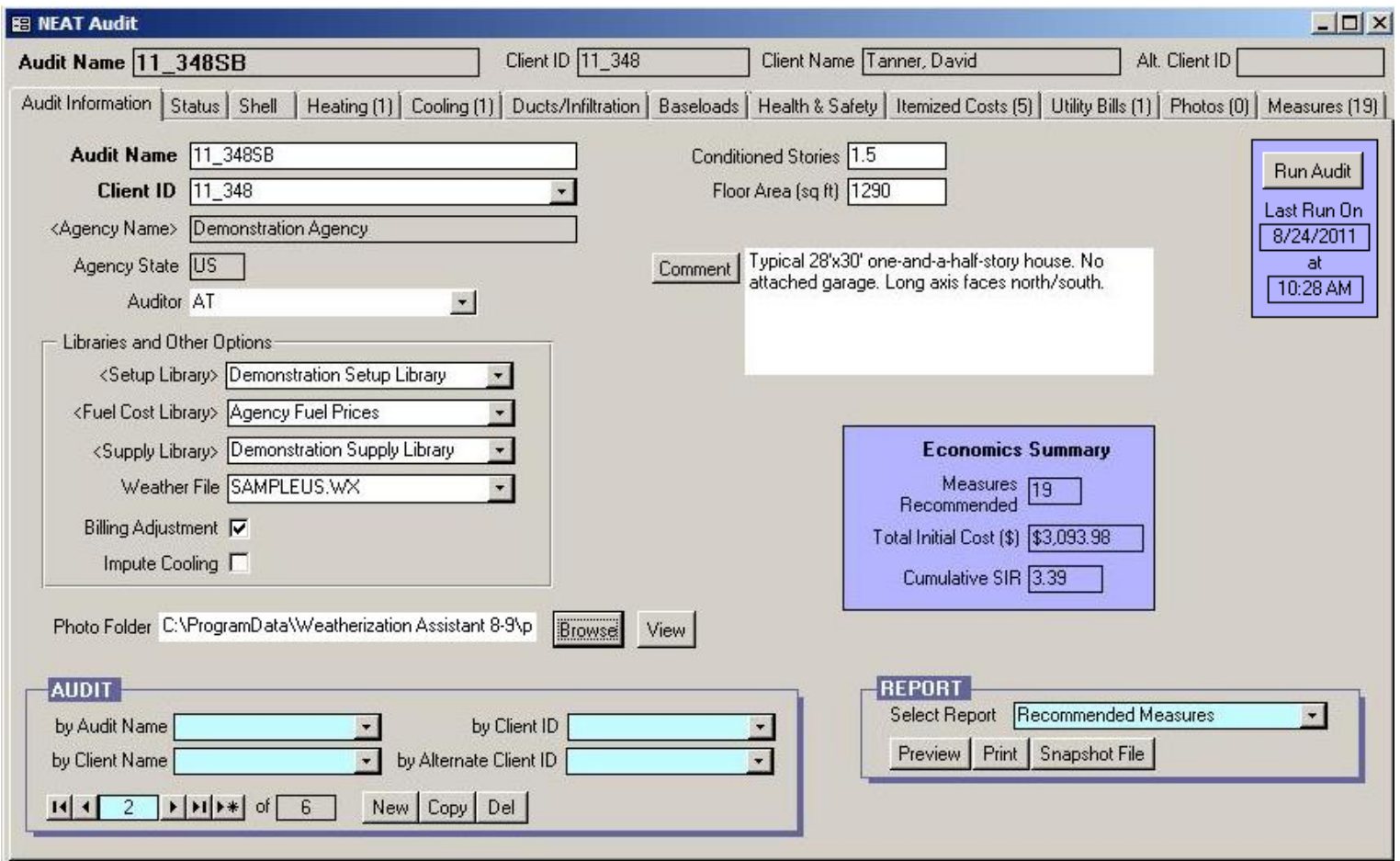

Figure 8.1. The Audit Information form under the Main Menu’s “NEAT” button".

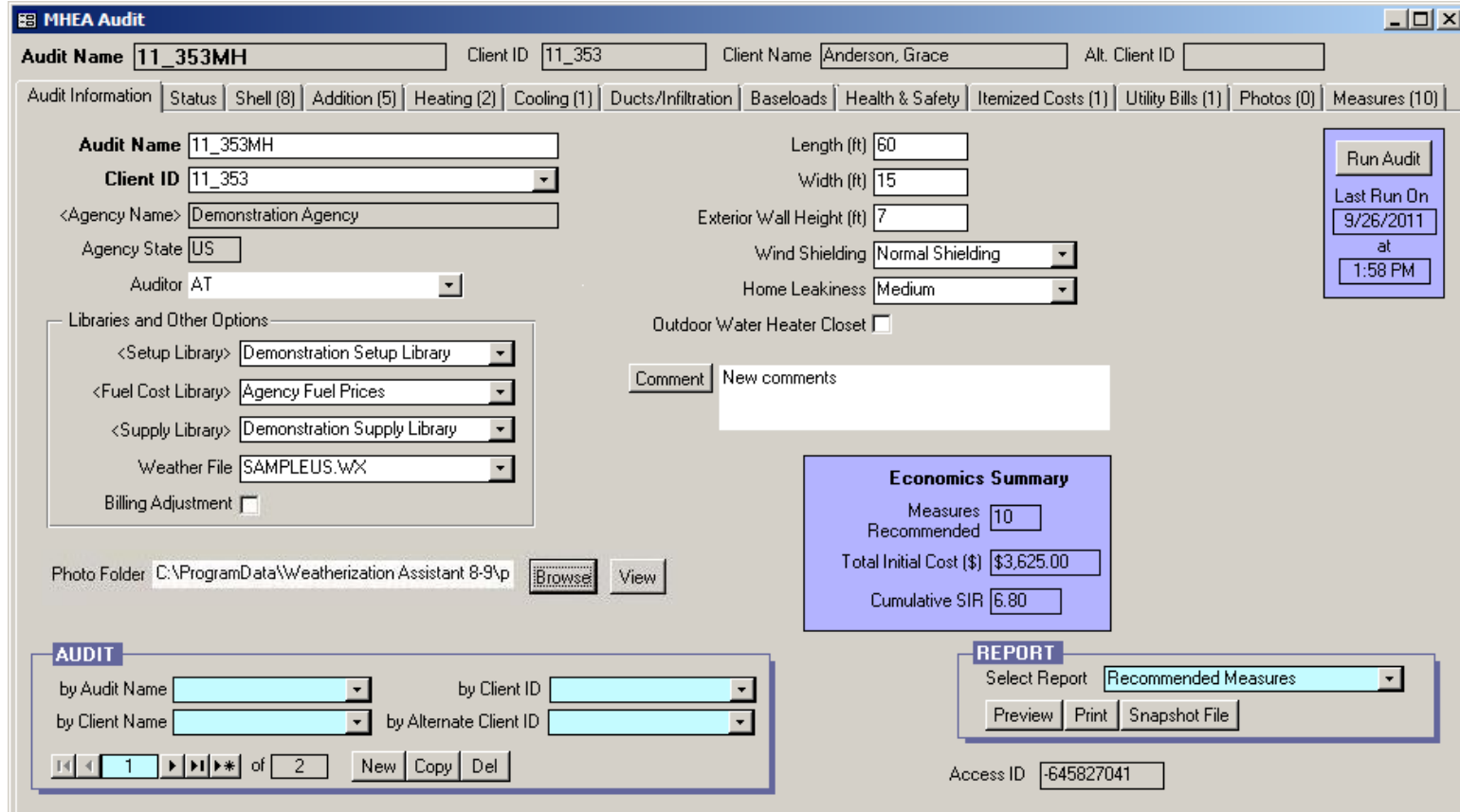

Figure 8.2. The Audit Information form under the Main Menu's “MHEA” button".

\footnotetext{
${ }^{2}$ Both options for linking photos to the audit are shown (the Photos tab and the Photo Folder field with accompanying "Browse" and "View" buttons) even though only one can be selected and displayed at one time.
} 


\section{Chapter 8: NEAT and MHEA Introduction}

Audit Name. When an audit is initially created, NEAT and MHEA will automatically give the new audit a unique name in the form of "Audit (\#)." You should replace this default name with a name of your choosing. The name of the audit might contain or somehow reference the Client ID because the Client ID must be unique for each client. Use of the occupant's name rather than the Client ID is not recommended because it does not ensure uniqueness, especially if only last names are used. Most clients will usually have only one audit associated with it; however, the possibility of creating and running multiple audits for a client should be considered. Required.

- Client ID - This field will display the Client ID of the client for whom the audit is being performed. If the audit was initiated using the "Create New Site Built (NEAT) Audit" or "Create New Mobile Home (MHEA) Audit” buttons on the Audits form of the Main Menu's "Clients" button as discussed in Section 7.5, Audits (Client), then this field will automatically be filled in with that client's Client ID. If the audit was initiated using the "New" button in the Audit record navigation block, then the Client ID will remain the same as the Client ID on the Audit Information form prior to requesting the new audit. In this case, you will have to use the drop-down list associated with the Client ID field to choose the correct client from those entered in the database. When you do attempt to change the client, you will get a warning because changing the client associated with an existing audit is usually not a normal operation except for when a new audit is being created. NEAT and MHEA simply do not know that this is a new audit. Required.

- Agency Name and Agency State - These fields display the name of the agency that this client is assigned to and the state of this agency. The fields will be automatically filled in based on the information entered on the Client Information form (see Section 7.1, Client Information) for the client identified in the Client ID field. Changing the Client ID will immediately update the Agency Name and Agency State, if needed. Uneditable.

- Auditor - This field displays the User Name of the auditor who is assigned to or responsible for this audit. Use the drop-down list associated with this field to select the auditor. The list will contain only those contacts declared as auditors on the Contacts form under the Main Menu's “Agency” button corresponding to the agency responsible for the client as discussed in Section 6.2, Contacts (Agency). Optional. 
- Comment - You may enter comments pertinent to the audit directly in the Comment field on the form, or you may enter them in the Comment Editor by selecting the "Comment" button to the left of the field. The Comment Editor is similar to Window's Notepad and may be used to enter extended comments. The total comment may have up to 65,000 characters. Text from other sources may be copied and pasted into the Comment Editor's window. Any comment that is entered is displayed on the Recommended Measures report and the Input Report available from the Report block as described below. You may also wish to include the comment in a user-designed report (see Appendix D, Development of Customized Reports). Optional.

- Photo Folder, Browse, and View - If you have chosen to use the third party photo browser method for attaching photos to records under the Main Menu's "Preferences" button (see Feature 2 in Section 16.4, Features), a "Photo Folder" field, "Browse" button, and "View" button will be present on the Audit Information form below the Libraries and Other Options data block. Use the "Browse" button to select a folder on your computer in which you have stored digital photos for this specific client. Once selected, the location of this folder will be displayed in the Photo Folder field. Select the "View" button to obtain a photo browser in which you may view and edit these photos. The Weatherization Assistant provides the option of attaching photos not only to audits, but also to clients and work orders as well. Therefore, you should develop a consistent policy regarding where and how you store reference to your photos. See Section 18.1, Digital Photos, for more information on viewing and editing photos.

\section{Libraries and Other Options Data Block}

The first four entries in the Libraries and Other Options data block tell NEAT and MHEA what libraries to reference for non-house specific data when running the audit.

- Setup Library - Select the setup library to be associated with this audit using the drop-down list. If you selected a setup library on the Client Information form for this client (see Section 7.1, Client Information), then the selected setup library will automatically be entered in the Setup Library field, although you can change this selection using the drop-down list if desired. The Setup Library contains measure costs, candidate measure information, fuel costs, and other 


\section{Chapter 8: NEAT and MHEA Introduction}

key parameters that will be used by NEAT and MHEA when the audit is run. The setup libraries that will be listed in the drop-down list are those that have been created for the agency identified in the Agency Name field. If there is only one setup library associated with the agency chosen for the audit, the setup library will automatically be entered into the field. See Chapter 14, Setup Library, for more information on how to create setup libraries and the need to update the Setup Library to reflect your local costs and weatherization approaches. Required.

- Fuel Cost Library - Select the fuel cost library to be associated with this audit using the drop-down list. The fuel cost library contains the fuel costs to be used by the audit in calculating the dollar value of energy savings. If you have more than one utility supplying a particular fuel in your agency's service territory, you may need to have more than one fuel cost library. Fuel cost libraries are a subset of data contained in a setup library. Therefore, the fuel cost libraries that will be listed in the drop-down list are only those that have been created in the setup library identified in the Setup Library field. If you change the setup library to be used by the audit, entry in the Fuel Cost Library field will be automatically deleted, indicating the need to choose another entry from the new setup library. If the assigned setup library has only one fuel cost library, that library will automatically be assigned. See Section 14.3, Fuel Costs, for more details. Required.

- Supply Library - Select the supply library to be associated with this audit using the drop-down list. Although the supply library is used primarily with work orders, the audit uses entries in the supply library for replacement refrigerators and water heaters. See "Additional Entries on the Hot Water Equipment and Refrigerator Forms” in Section 15.2, Materials/Labor Forms, for more details on how to populate the supply library with the replacement refrigerators and hot water heaters that you use. Unless an unusual situation arises, an agency will have only a single supply library. If this is the case, this supply library will be entered automatically. Required.

- Weather File - The audit must be told what climatic data to use in estimating the energy consumptions for the dwelling. Select one of the approximately 220 weather cities provided in NEAT and MHEA using the drop-down list. The cities are listed in alphabetical order, first by state, then by city. You may wish 
to consider not only those cities in your state, but also cities near you in states that border your own. Required.

- Billing Adjustment - NEAT and MHEA will adjust their energy savings estimates and develop a second set of recommended weatherization measures based on the actual pre-weatherization energy consumption of the house if the Billing Adjustment checkbox is selected and pre-retrofit utility billing data have been entered on the Utility Bills form (see Section 11.9, Utility Bills). Both unadjusted and adjusted results will be reported in the Recommended Measures report. See Chapter 12, NEAT and MHEA Results, for more details.

\section{NEAT Specific Entries}

- Conditioned Stories - Enter the number of conditioned stories. The value is used to determine an approximate distance between the lowest and highest points at which air infiltration can enter and exit the house. Therefore, include a finished attic if the space is heated or cooled, and a basement if it is heated or cooled and a significant portion of its wall area is above-grade (e.g., a walk-out basement). You may enter a decimal (e.g., 1.5) for a finished attic, split level, or half story upstairs, although this degree of accuracy is normally not necessary. Required.

- Floor Area - Enter the approximate floor area of the house that is heated or cooled in units of square feet. This is the floor area of the living space, not the foot print area. For example, a two-story home with a floor area of $1200 \mathrm{sq}$. ft. per story would have a total floor area of $2400 \mathrm{sq}$. ft. If you entered a floor area on the Energy Index form under the Main Menu's “Clients” button (see Section 7.3, Energy Index), then this floor area will automatically be entered in the Floor Area field, although you can change this entry if desired. Required.

- Impute Cooling - This checkbox, which appears in the Libraries and Other Options data block, is intended to accommodate audits in extremely southern climates where neither heating nor cooling equipment is present in the house. The feature is designed to allow installation of measures that could add to the occupants' comfort even though energy cannot be saved. If the checkbox is selected, NEAT will "impute" a cooling energy use assuming that an air conditioner with an SEER as specified in the Key Parameters/Equipment form 


\section{Chapter 8: NEAT and MHEA Introduction}

under the Main Menu’s “Setup Library” button (see Section 14.2, Key

Parameters) is present in the house.

\section{MHEA Specific Entries}

- Length - Enter the length of the home in units of feet (see Figure 8.3). The home length is the length of the living space. Do not include the length of the towing tongue that extends out from one end of the manufactured home. Required.

- Width - Enter the width of the home in units of feet (see Figure 8.3). Required.

- Exterior Wall Height - Enter the height of the exterior wall of the home in units of feet as measured from the floor to the ceiling inside the home (see Figure 8.3). Make this measurement in any portion of the house that does not have a raised or cathedral ceiling. Required.

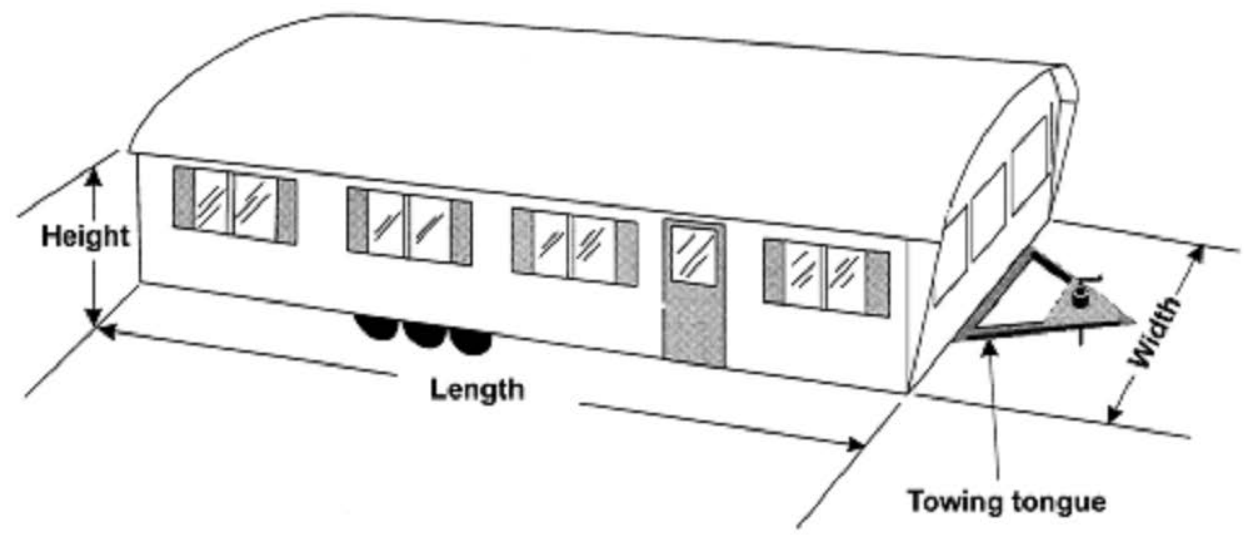

Manufactured Home Dimensions

Figure 8.3. Dimensions associated with a manufactured home.

- Wind Shielding - Select the option that best describes how well the manufactured home is shielded from the wind. The options are Well Shielded, Normal Shielding, or Exposed. A correction factor is included in the infiltration calculation for the relative amount of external wind shielding. A well-shielded home may be surrounded by thick vegetation, in a small valley that seldom experiences windy conditions, or closely surrounded by other homes and buildings. A normally shielded home may be one that is found in a typical 
manufactured home housing park where there are surrounding homes and some surrounding vegetation. An exposed home has no surrounding vegetation or buildings to protect it from the wind. Required.

- Home Leakiness - Select the option that best describes the air leakage rate of the home prior to your weatherization work. The options are Tight, Medium, and Loose. Leakage rates in units of cfm at $50 \mathrm{~Pa}$ associated with these selections are set in the Key Parameters/Heat Transfer form under the Main Menu's “Setup Library” button (see Section 14.2, Key Parameters). These values will be used as the existing air leakage rate for the home before weatherization if actual values are not entered on the Ducts/Infiltration form (see Section 11.2, Ducts/Infiltration - Air and Duct Leakages). Required.

- Outdoor Water Heater Closet - Select this checkbox to indicate that the water heater is located in an unconditioned closet with an exterior access. If an outdoor closet exists, MHEA will assume a "30 × 30" inch water heater closet in its calculations of wall, floor, and ceiling areas.

\section{Other Controls}

- Audit Record Navigation Block - The Audit record navigation block is used to find and navigate to existing audits in your database, copy or delete the currently accessed audit, or create a new audit. See Section 5.1, Form and Record Navigation, for information on using the Weatherization Assistant's record navigation blocks.

The default installation of the Weatherization Assistant turns on the "Use only the latest bookmarked Agency in the find record drop-down boxes" feature in Preferences (see Feature 9 in Section 16.4, Features). With this feature turned on, the drop-down lists in the Audit record navigation block will display only the audits associated with the agency last visited using the Main Menu's "Agency" button. Normally, this will be your agency, so the drop-down lists will display only your agency's audits. If you have more than one agency defined in your database and you want the drop-down lists to display audits from all defined agencies, turn this feature off.

- Report Block - The Report block provides you with access to all reports available under the Main Menu's "NEAT" and "MHEA" buttons. The 


\section{Chapter 8: NEAT and MHEA Introduction}

following reports are currently available: Recommended Measures, Input Report, Heating System Summary, Pressure Diagnostics Report/Form, and Health \& Safety Summary. See Appendix C, Reports, for examples of these reports. The Recommended Measures report provides information on the weatherization measures recommended by NEAT or MHEA for a house and is available only after an audit has been run. The Input Report provides a listing of all the building description data entered in the audit as well as a summary of the data entered on the client. The Heating System Summary report provides a listing of just the heating system information entered in the audit while the Pressure Diagnostics Report/Form lists just the air leakage and other pressure diagnostic measurements that were entered in the audit. Finally, the Health \& Safety Summary report displays issues associated with health and safety and any instances that were identified on any of the audit forms. See Section 5.12, The Report Block, for information on the mechanics of using the Weatherization Assistant's Report blocks. Appendix D, Development of Customized Reports, describes a method to create reports tailored to your needs and to list the reports in the Report block.

- Economics Summary Block - Once an audit has been run, the following three fields in the Economics Summary block of data will summarize the audit recommendations: Measures Recommended, Total Initial Cost, and Cumulative SIR. The Measures Recommended field identifies the number of measures recommended. The Total Initial Cost field provides the total cost for the recommended measures as estimated by NEAT or MHEA (as opposed to actual costs, which may be entered in the Weatherization Assistant as part of a work order). The Cumulative SIR field provides the savings-to-investment ratio (SIR) estimated by NEAT or MHEA for all the measures recommended for the house. If the audit has not yet been run, the Measures Recommended field will be "0" and the other two fields will be blank. Uneditable.

- Run Audit Block - After entering all the audit data for a house using the other forms under the Main Menu's "NEAT" or "MHEA" buttons, you can select the "Run Audit" button to have NEAT or MHEA perform its calculations and recommend weatherization measures for the house. The Run Audit data block contains two uneditable fields that show when the audit was last run (i.e., the "Last Run On" date and time). If the audit has not yet been run, the date field will display "Not Run.” The Run Audit data block is provided on all the forms under the "NEAT Audit" and "MHEA Audit" windows. Thus, an audit can be 


\section{Chapter 8: NEAT and MHEA Introduction}

run from any of these forms once you have decided that the audit input is complete.

An audit may be run any number of times. However, rerunning an audit will cause the recommendations from the previous run to be overwritten. If you wish to rerun an audit but save the recommendations from the previous run, use the "Copy" button in the Audit record navigation block to copy the audit. Give the copied audit a new Audit Name (possibly a variant of the original name), make any modifications to the audit desired, and then select the "Run Audit" button to produce a new set of recommendations. 


\section{Chapter 9. NEAT Building Description}

This chapter discusses the information entered on the building description forms under the Main Menu's "NEAT" button that is needed to describe site-built, singlefamily houses and that is unique as compared to mobile homes. Prior to entering information on the forms described in this chapter, you must have already entered information about the client whose house is being audited (see Section 7.1, Client Information), chosen to run a NEAT audit for this client, and entered required information on the Audit Information form for this audit (see Section 8.2, Audit Information).

The Shell tab contains information related to Walls, Windows, Doors, Unfinished Attics, Finished Attics, and Foundations of the home. Although unrealistic, only one wall description need be entered under the Shell tab in order to satisfy the minimum requirements of the program. This allows describing single units of a small multifamily building where no heat is lost to the outside through some of the envelop components.

The Heating and Cooling forms allow descriptions of the home's HVAC equipment. NEAT requires at least one heating system, but cooling systems are optional. The entries on these forms are also discussed in this chapter.

Chapter 11, NEAT and MHEA Common Elements, discusses the remaining forms under the Main Menu's "NEAT" button, those common to both NEAT and MHEA. These include the Status, Ducts/Infiltration, Base Loads (Water Heating, Refrigerators, and Lighting Systems), Health \& Safety (Whole House, Equipment, and Building Shell), Itemized Costs, Utility Bills, Photos, and Measures forms, as well as some optional sub-forms found on the Heating and Water Heating forms. Of these forms, only the Ducts/Infiltration form is required.

On many of the tabs and sub-tabs under the Main Menu's "NEAT” button, numbers in parentheses are displayed. These numbers indicate the number of descriptions, or records, that have been entered for that particular component type. For example, a tab titled "Walls (4)" indicates that four wall descriptions have thus far been entered for the house currently being viewed.

Finally, the Recommended Measures report that is typically displayed immediately after running a NEAT audit is discussed in Chapter 12, NEAT and MHEA Results. 


\section{Chapter 9: NEAT Building Description}

\subsection{Shell - Walls}

Before you can run NEAT to produce weatherization recommendations, you must enter data for at least one exterior wall (as well as one heating system) using the Walls form (see Figure 9.1) under the Main Menu's “NEAT” button. However, you will normally describe at least one exterior wall for each of the four cardinal directions. Walls with different orientations differ in their heat transfer characteristics because of the different amounts of sunlight that fall on them. This is particularly important for the windows that may lie on the walls. Walls that differ in their construction or insulation level should also be described separately because the cost of insulating the wall and the energy savings from insulating the wall will differ. In order to describe all of the exterior walls of a house most efficiently, you may combine multi-storied walls that have the same orientation and construction. A maximum of 18 wall descriptions may be entered.

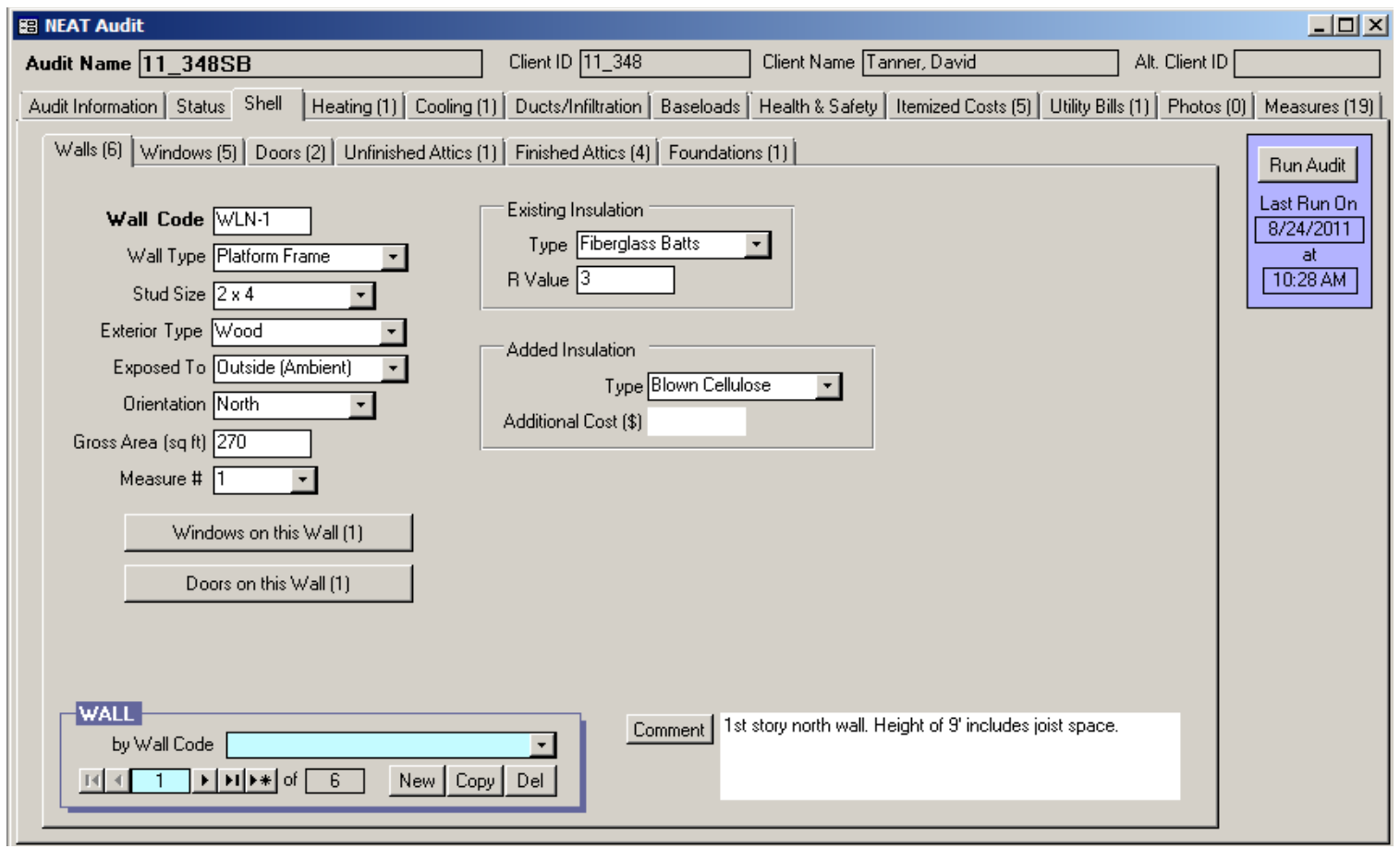

Figure 9.1. The Walls form under the Main Menu’s “NEAT” button.

Below are descriptions of the individual data items and controls found on the Walls form. All items on the Walls form are required except the Additional Cost and Comment fields. 
- Wall Code - The Wall Code identifies wall sections to you and the Weatherization Assistant. The wall codes must be unique for all wall descriptions within a given audit. NEAT automatically provides a default entry for all new walls that are created in the form of "WL\#" where the "\#” is an integer insuring uniqueness of the wall code. You may choose to enter your own wall code to include characters that indicate the orientation, exposure, or some other characteristics of the wall section. The wall code may have up to 20 characters, although space restrictions in many reports suggest a lesser number of characters should be used (perhaps 3 to 5) to preserve readability. Entries are not case-sensitive. The wall code allows NEAT to subtract the correct window and door areas entered in the Windows and Doors forms (see Sections 9.2, Shell - Windows, and 9.3, Shell - Doors, respectively) from the corresponding wall area. NEAT will also use the wall codes in output reports and work orders to identifying which walls should receive recommended insulation. The wall codes may also be helpful when labeling your drawing of the house's floor plan. Required.

- Wall Type - Enter the type of load-bearing structure for this wall. The choices are Balloon Frame, Platform Frame, Masonry or Stone, Concrete Block, Adobe, and Other. NEAT does not differentiate between a balloon frame and platform frame structure. This distinction is for the benefit of you and the insulation subcontractor. The thermal characteristics of the Other wall type can be defined in the Setup Library (see Section 14.2, Key Parameters). The Wall Type selected may affect the measures considered. See "Added Insulation Type" below. Required.

- Stud Size - If the Wall Type selected is Balloon Frame or Platform Frame, the Stud Size field will appear. Enter the stud size used in framing this wall section. The choices are $2 \times 2,2 \times 3,2 \times 4,2 \times 6$, and $2 \times 8$. The material cost for insulating frame walls with loose fill insulation will be adjusted to reflect the available space in the wall cavity as determined by the stud size and any existing insulation. Conditionally Required.

- Exterior Type - Select the type of siding installed on the exterior surface of the wall. The choices are Wood, Metal or Vinyl, Stucco, Brick or Stone, None, and Other. The thermal characteristics of the Other exterior siding type can be defined in the Setup Library (see Section 14.2, Key Parameters). Required. 
- Exposed To - Enter what the wall is exposed to on the outside of the wall. The choices are Outside (Ambient) if the wall is exposed to the outside air, Buffered Space if the wall is exposed to an unconditioned but enclosed space such as a garage or porch, and Attic Space if the wall is exposed to an unconditioned attic. A wall adjacent to an unconditioned attic may be described on the Walls form or as a Kneewall on the Finished Attics form (see Section 9.5, Shell Finished Attics). Required.

- Orientation - Enter the closest cardinal compass direction that the wall component faces. The choices are North, South, East, and West. Required.

- Gross Area - Enter the gross area of the wall in square feet. This is the total area, including the windows and doors in that wall section (NEAT will automatically subtract out the area of any windows and doors assigned to the wall section in its calculations). Values to the nearest ten square feet are normally sufficient, although a decimal point can be used. Inaccuracy in this entry will mostly affect the estimated quantity of wall insulation needed should wall insulation be recommended. Required.

- Measure \# - The Measure Number field allows individual wall segments to be combined into groups. For each group, an SIR to insulate all the wall segments in that group will be calculated, and one recommendation for insulating all the wall segments in that group will be given. Enter the same measure number to all walls you wish to be grouped together. Measure numbers must be used in consecutive order, starting with 1 . For example, a measure number of 3 cannot be entered until measure numbers 1 and 2 have been used. Walls having an Added Insulation Type of None or an Added Insulation Type selected that is not appropriate for the wall will be automatically dropped from a group (see Added Insulation Type below). Required.

You will likely want to obtain a single SIR for insulating all the walls in a house if all the walls have the same construction. In this case, enter a measure number of 1 for all the wall segments. Reasons for giving some walls a different measure number might be because they have different existing insulation levels, different wall or exterior types, or different exposures, all of which can impact installation costs and potential savings. However, walls with these differences might still be grouped together if you wanted to determine if insulating all the walls in a house as one retrofit measure was cost-effective. 
- Existing Insulation Type - Select the type of existing insulation installed in the wall segment. The choices are None, Blown Cellulose, Blown Fiberglass, Rockwool, Fiberglass Batts, Polystyrene Board, and Other. If the Wall Type is Balloon or Platform Frame, NEAT assumes that the Polystyrene Board and Other insulation types are installed over the wall studs rather than between the studs as is assumed for the remaining insulation types. If both cavity insulation and exterior sheathing insulation are present, enter the type that has the greatest $\mathrm{R}$-value (you should also note this in the Comment field described below). If loose fill insulation exists in the wall cavity, NEAT will not consider adding any additional loose fill insulation. Required.

- Existing Insulation R Value - If the Existing Insulation Type is an entry other than None, then the Existing Insulation R Value field will appear. Enter the Rvalue of the existing insulation found in this wall segment. The default R-values displayed in the status bar located in the lower left corner of the Weatherization Assistant window assume a $31 / 2$ inch cavity and a standard R-value/inch for the insulation type indicated by the user. If both cavity insulation and exterior sheathing insulation are present, their total R-value should be entered in this field. Required.

- Added Insulation Type - Select the type of insulation you would install in this wall segment if NEAT recommends wall insulation. The choices are None, Blown Cellulose, and any wall insulation types that you have entered on the NEAT Insulation Types form under the Setup Library (see Section 14.7, NEAT Insulation Types). Select None if conditions prohibit insulating this particular wall segment (you should note what the conditions are in the Comment field described below). NEAT will not recommend wall insulation if the Added Insulation Type is a loose fill insulation and (1) the Existing Insulation Type is also a loose fill insulation; (2) the Wall Type is Masonry or Stone, Concrete Block, or Adobe; (3) and the air space in the cavity is less than 1.5 inches. NEAT considers a wall insulation material to be loose fill if the "Units" for the material on the NEAT Insulation Types form have been chosen as "R/in." Required.

- Additional Cost - Enter any additional cost associated with insulating this specific wall segment in units of dollars. This would be a cost that is not normally associated with the wall insulation measure and, therefore, is not accounted for in the measure costs entered on the Library Measures form under 
the Setup Library (see Section 14.5, Library Measures). The cost is the total additional cost to insulate this specific wall segment; it is not a cost per square foot of wall area. The amount entered will be added to the normal cost computed from the wall area and the cost per square foot specified on the Library Measures form in the Setup Library. A blank entry indicates no additional cost. If a cost is entered, you should briefly explain the reason for this additional cost in the Comment field described below. Optional.

- Comment - You may enter comments pertinent to the wall segment directly in the Comment field on the form, or you may enter them in the Comment Editor by selecting the "Comment" button to the left of the field. The Comment Editor is similar to Window's Notepad and may be used to enter extended comments. The total comment may have up to 65,000 characters. Text from other sources may be copied and pasted into the Comment Editor's window. Any comment that is entered is displayed on the Recommended Measures report and the Input Report available from the Audit Information form (see Section 8.2, Audit Information). In addition, any comment that is entered can be imported into any specific work order (see Section 13.1, Work Order Information). Thus, you may wish to enter comments related to any observation that might affect the installation of needed measures or repair work. Optional.

- Windows on this Wall - Selecting this button will take you to a data sheet view of the Windows form (see Section 9.2, Shell - Windows) that displays only those windows installed in the wall you are currently accessing. You may use this form to enter the windows installed in this wall segment and to make modifications to any window that you have already entered. The form is the same as that described for the main Windows form except that a form view is not available and you cannot run NEAT from this view. Users may want to enter windows using this button rather than the main Windows form because they find it easier to enter the windows installed on a wall at the same time the wall is described rather than after all wall segments have been entered.

However, special care must be taken to keep the Window Codes entered unique since the codes already entered but for windows on other walls will not be visible from this form.

- Doors on this Wall - Selecting this button will take you to a data sheet view of the Doors form (see Section 9.3, Shell - Doors) that displays only those doors installed in the wall you are currently accessing. You may use this form to enter 


\section{Chapter 9: NEAT Building Description}

the doors installed in this wall segment and to make modifications to any door that you have already entered. The form is the same as that described for the main Doors form except that a form view is not available and you cannot run NEAT from this view. Users may want to enter doors using this button rather than the main Doors form because they find it easier to enter the doors installed on a wall at the same time the wall is described rather than after all wall segments have been entered. However, special care must be taken to keep the Door Codes entered unique since the codes already entered but for doors on other walls will not be visible from this form.

- Wall Record Navigation Block - The Wall record navigation block is used to find and navigate to existing wall descriptions for the audit being edited, copy or delete the currently accessed wall description, or create a new wall description for the audit. See Section 5.1, Form and Record Navigation, for information on using the Weatherization Assistant's record navigation blocks.

\subsection{Shell - Windows}

The Windows form (see Figure 9.2) under the Main Menu's "NEAT” button is used to enter information on all the windows installed in the house.

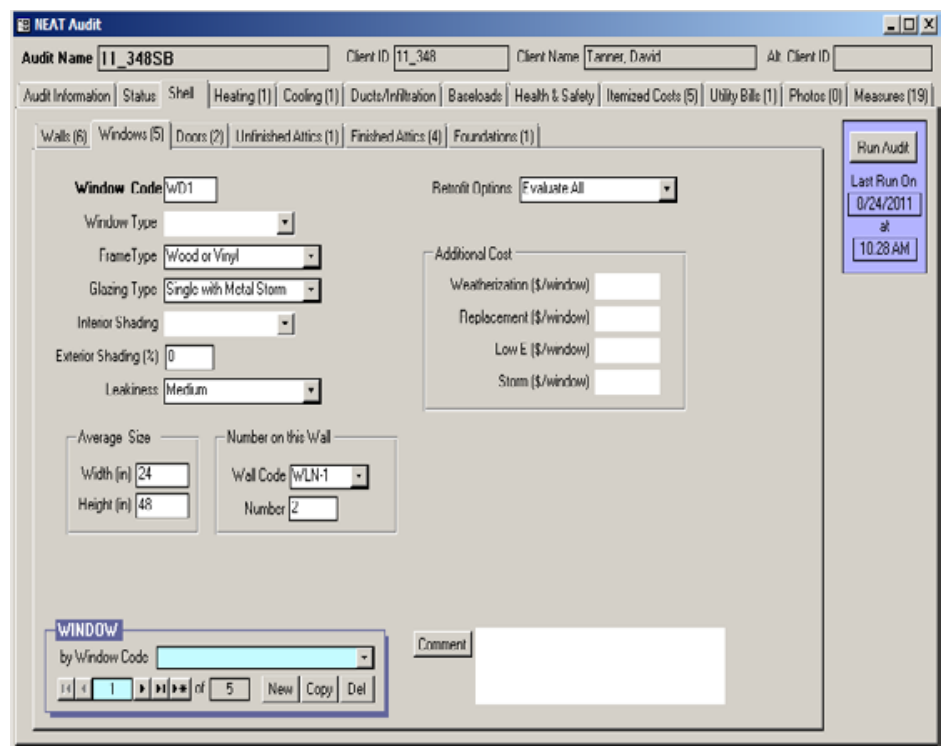

Figure 9.2. The Windows form under the Main Menu's “NEAT” button.

A total of 24 window descriptions (i.e., Window Codes) may be entered. Multiple windows installed on the same wall segment and with the same characteristics may 


\section{Chapter 9: NEAT Building Description}

be entered as a single window description by indicating the number of windows having this description. If the 24 window records are not enough to describe all the windows, you will have to enter windows with characteristics that are close to others under one common window description. Avoid combining the areas of two or more windows into one so that just one window needs to be entered. Window treatments are generally priced in NEAT either per window unit or window area. Combining two or more window areas into one large area can lead to errors in estimating the cost of the window treatments depending on the pricing scheme used.

Below are descriptions of the individual data items and controls found on the Windows form.

- Window Code - The Window Code identifies window descriptions to you and the Weatherization Assistant. If more than one window has the same description, you may enter the description once and indicate the number of windows with this description using the Number field described below. The window codes must be unique for all window descriptions within a given audit. NEAT automatically provides a default entry for all new windows that are created in the form of "WD\#" where the "\#” is an integer insuring uniqueness of the window code. You may choose to enter your own window code to include characters that indicate the orientation or some other characteristics of the window. The window code may have up to 20 characters, although space restrictions in many reports suggest a lesser number of characters should be used (perhaps 3 to 5) to preserve readability. Entries are not case-sensitive. NEAT will use the window codes in output reports and work orders to identify which windows should receive weatherization measures. The window codes may also be helpful when labeling your drawing of the house's floor plan. Required.

- Window Type - Select what basic type of window this is. The choices are Jalousie, Awning, Slider, Fixed, Door Window, Sliding Glass Door, and Skylight. These choices are shown in Figure 9.3 and described below. NEAT does not use the window type in any of its calculations. This entry is provided for your benefit, so that you can clearly identify and recall the type of window being described. In addition, you can better determine the correct entry for the Leakiness field described below if you know the window type. Optional. 


\section{Chapter 9: NEAT Building Description}

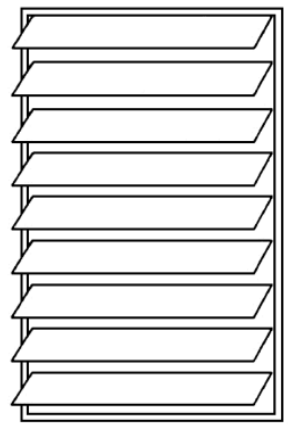

Jalousie

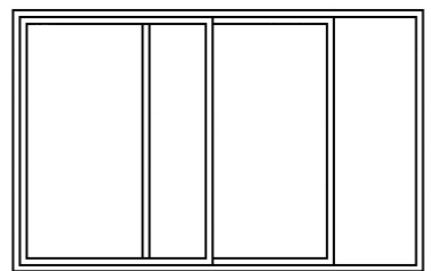

Horizontal Slider

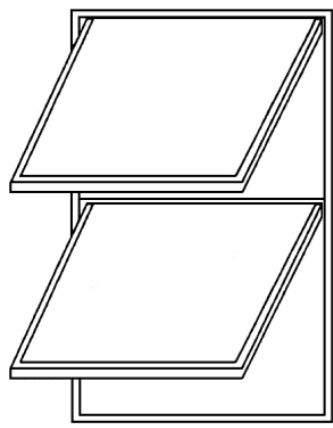

Awning

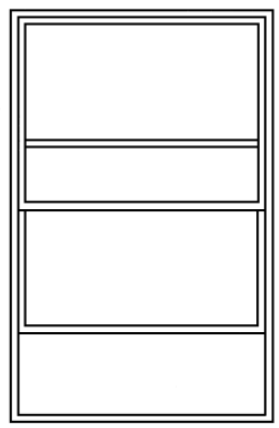

Vertical Slider

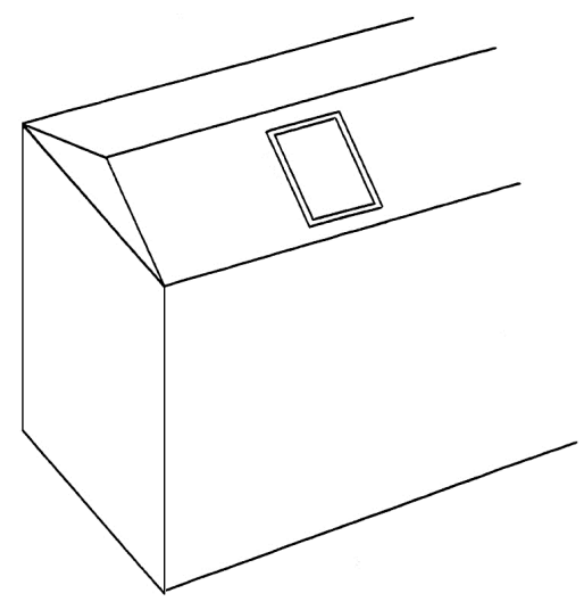

Skylight

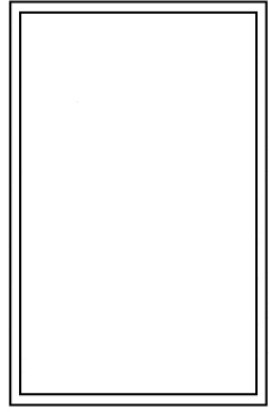

Fixed

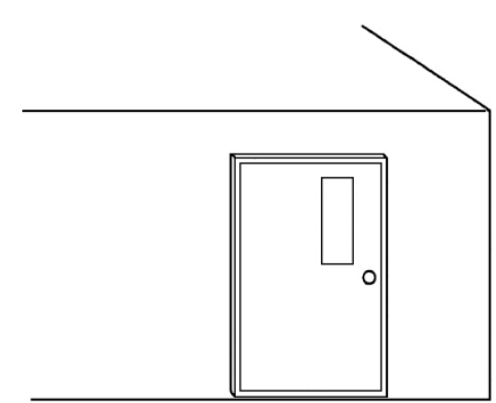

Door

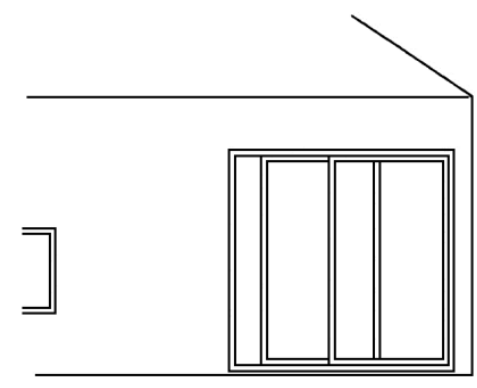

Sliding Glass Door

Figure 9.3. Basic types of windows. 
Jalousie windows are constructed of multiple horizontal panes of glass (about 2-6 inches wide) that open at the same angle when a crank near the bottom of the window is turned. Jalousie windows are always single pane. When closed, large gaps between the glass panes often exist, which results in high infiltration rates through the window.

Awning windows are constructed of 1-3 glass sashes that are hinged at the top, bottom, or side of the sashes. When the window is opened (often by turning a crank), the sashes angle out from the home's exterior. The sashes either angle down, up, or to the side depending on where the sashes are hinged to the window frame. An awning window with a single sash of glass is often called a casement window if it is hinged on the side or a hopper window if it is hinged on the bottom.

Slider windows usually have two sashes of glass. One or both sashes can slide past the other either horizontally or vertically when the window is opened.

Fixed windows are sealed in the window frame and cannot be opened.

Door windows are usually sealed in the window frame of a door and cannot be opened, although sometimes they are made to be opened.

Sliding Glass Doors are large windows that extend from the floor to almost the ceiling. You may use this selection to describe picture windows even though they are fixed and cannot be opened.

Skylights are glass or plastic windows in the ceiling.

- Frame Type - Select the window frame and sash construction materials. The choices are Wood or Vinyl, Metal, and Improved Metal (i.e., metal frame with a thermal break). Required.

- Glazing Type - Select the glazing type depending on the number of panes and type of glass in the primary window and the characteristics of any storm window that may cover the primary window. The choices are Single Pane, Single with Wood Storm, Single with Metal Storm, Single with Bad Storm, Double Pane, and Double Pane Low-e. NEAT treats a Single with Bad Storm window as a single pane window without a storm. The Single with Bad Storm 
glazing type is provided for your use in conveying additional information. Required.

- Interior Shading - Select the interior window coverings that are present which, when closed, prevent sunlight from entering the home and also slightly increase the insulating value of the window. The options are Drapes, Blinds or Shades, Drapes with Blinds or Shades, and None. NEAT does not use this information in any of its calculations at this time. Optional.

- Exterior Shading - Enter the approximate percentage of window area that is shaded by trees, overhangs, or other exterior barriers when sunlight could otherwise strike the window. Do not enter the \% sign with the number. As guidance, consider that a window directly under a roof eave may have $20 \%$ of its area shaded and windows completely covered by a large porch may be shaded $100 \%$ of the time. For houses in heating climates and/or when spaceheating energy consumption is the primary concern, determine the percentage of shading that occurs during the winter. For example, deciduous trees lose their leaves in the winter, so their shading during that season may be minimal. NEAT already accounts for the effect of a window's orientation on the sunlight striking it, unless an unusual house configuration causes additional shading, e.g., a window opening into a small interior courtyard being shaded by other walls surrounding the courtyard. Required.

- Leakiness - Provide an estimate of how leaky the window is. The choices are Very Tight, Tight, Medium, Loose, and Very Loose. Typical selections by window type are listed below. Degrade the leakiness one level if 2 to 9 sq. in. of glass is missing in the window and two levels if 9 to 25 sq. in. of glass is missing. Specify the window to be Very Loose if more than 25 sq. in. of glass is missing in the window. Upgrade the leakiness one level if a storm window in average or better condition is installed. Additional guidance is provided in Appendix E, Window Leakiness. Required.

Jalousie window - The leakiness of a jalousie window is typically Loose.

Awning window - The leakiness of an awning window that has a single sash of glass and a latching/locking mechanism that seals the window by forcing the sash against the frame and any installed weather stripping is typically Tight. Such a window is often called a casement window if it is hinged on the side or a 


\section{Chapter 9: NEAT Building Description}

hopper window if it is hinged on the bottom. The leakiness of an awning window with 2 or 3 sashes of glass or without a latching/locking mechanism is typically Medium.

Slider window and sliding glass door - The leakiness of a vertical slider window is typically Medium if it is older and Tight if it is newer. The leakiness of a horizontal slider window or sliding glass door is typically Medium.

Fixed window, door window, and skylight - The leakiness of a fixed window, door window, or skylight is typically Very Tight.

- Width and Height - Enter the width and height of the window (including the window frame) in units of inches. Accuracy to the nearest inch is usually satisfactory. NEAT will use these dimensions together with the number of windows being described by this Window Code (see Number below) to estimate the total window area, and it will subtract this total area from the appropriate wall area based on the Wall Code (see below). Required.

- Wall Code - Enter the Wall Code of the exterior wall on which the window is installed. The acceptable Wall Codes are determined by your input on the Walls form (see Section 9.1, Shell - Walls) and will be displayed in the drop-down list associated with the field. Thus, you must enter a wall description before attempting to describe any windows that may lie on that wall. Specifying the Wall Code for each window allows NEAT to subtract the window area from the appropriate gross wall area. Required.

- Number - Enter the number of windows that can be described by the entries on this form. If the number of windows is greater than one, then this indicates that more than one window lies on the indicated wall because one of the data entry items making up the window description is the Wall Code. Be aware that the number in parentheses on the Windows tab (see Figure 9.2) is the number of window descriptions, not necessarily the total number of windows. Required.

- Retrofit Options - Select one of the approaches for retrofitting the window. The options are Evaluate All, Weatherize, Replace, Replace with LowE, Add Storm, and Evaluate None. Evaluate All allows NEAT to determine if it is costeffective to weatherize, replace, or install a storm window on the window. If more than one of these retrofits is cost-effective, NEAT will select the most 
cost-effective measure. If none of the measures are cost-effective, no measure will be recommended. Choosing Weatherize (weatherizing the window using caulk, weather stripping, incidental repairs, etc.), Replace or Replace with LowE (replacing the window, often because it is damaged beyond repair), or Add Storm (adding an exterior storm window to a window that currently does not have a storm window) indicates your decision to perform the selected retrofit measure regardless of its cost-effectiveness and to not perform the other three measures. These selections tell NEAT to make the selected retrofit a mandatory measure. NEAT will recommend the measure regardless of its costeffectiveness and will not evaluate the other three options. However, NEAT will estimate the energy savings and SIR for the measure. If the SIR for the measure is less than your accepted minimum value, you will need to reconsider your selection or consider the measure a repair. Evaluate None prevents NEAT from evaluating the cost-effectiveness of weatherizing, replacing, or installing a storm window on the window, which effectively turns off all retrofits for this window. Reasons for selecting any option other than Evaluate All for this window description would be an appropriate entry in the Comment field described below. The thermal characteristics of replacement windows and storm windows are specified in the Key Parameters/Windows form under the Main Menu's “Setup Library” button (see Section 14.2, Key Parameters) and may be altered if the user sees a need to do so. Required.

- Include in SIR - If the Retrofit Option selected is Weatherize, Replace, Replace with LowE, or Add Storm, an Include in SIR checkbox will appear on the form. This checkbox allows you to indicate that you wish to have the selected measure included in the calculation of the whole house (package) SIR for this audit. Measures not included in the whole house SIR are normally related to health and safety. Current Weatherization Program policy dictates that window replacement cannot be considered a health and safety measure. Check the guidance for your program in deciding when you can mandate a window treatment and if it can be excluded from the SIR calculation. An alternate reason for excluding a measure from the whole house SIR is if it is funded outside of the Weatherization Assistance Program. Whether a measure is or is not included in the whole house SIR is indicated by its location in the list of measures produced for the Recommended Measures Report (see Chapter 12, NEAT and MHEA Results). 


\section{Chapter 9: NEAT Building Description}

- Additional Cost - If the Retrofit Option selected is any option other than Evaluate None, then Additional Cost fields will appear separately for Weatherization, Replacement, Low E, and Storm windows. Enter any additional cost associated with performing these retrofits on the window being described that is in addition to the cost that NEAT will automatically calculate using the cost information you entered in the Library Measures form of the Setup Library (see Section 14.5, Library Measures). For example, these might include the cost for removing any security bars before replacement or storm windows can be installed. The cost to be entered is a cost per window, not a total cost for all the windows indicated in the Number field. The reason for this extra cost would be an appropriate entry in the Comment field described below. Optional.

- Comment - You may enter comments pertinent to the window directly in the Comment field on the form, or you may enter them in the Comment Editor by selecting the "Comment" button to the left of the field. The Comment Editor is similar to Window's Notepad and may be used to enter extended comments. The total comment may have up to 65,000 characters. Text from other sources may be copied and pasted into the Comment Editor's window. Any comment that is entered is displayed on the Recommended Measures report and the Input Report available from the Audit Information form (see Section 8.2, Audit Information). In addition, any comment that is entered can be imported into any specific work order (see Section 13.1, Work Order Information). Thus, you may wish to enter comments related to any observation that might affect the installation of needed measures or repair work. Optional.

- Window Record Navigation Block - The Window record navigation block is used to find and navigate to existing window descriptions for the audit being edited, copy or delete the currently accessed window description, or create a new window description for the audit. See Section 5.1, Form and Record Navigation, for information on using the Weatherization Assistant's record navigation blocks.

\subsection{Shell - Doors}

The Doors form (see Figure 9.4) under the Main Menu's "NEAT” button is used to enter information on all the doors installed in the house. A total of 10 door descriptions (i.e., Door Codes) may be entered. Multiple doors installed on the same wall segment and with the same characteristics may be entered as a single 


\section{Chapter 9: NEAT Building Description}

door description by indicating the number of doors having this description. If the 10 door records are not enough to describe all the doors, you will have to enter doors with characteristics that are close to others under one common door description.

Installation of a storm door or replacement of the primary door would seldom be cost-effective based solely on heat conduction savings. NEAT will attempt to account for air leakage reduction accomplished by replacing a primary exterior door, which can allow the measure to be cost-effective. Care must be taken not to over-estimate the leakiness of the existing door (see "Leakiness" below).

Alternatively, replacing or weatherizing a door may be considered as part of the air-leakage reduction work performed on the house and the associated cost included as part of the Infiltration Reduction Cost (see Section 11.2, Ducts/ Infiltration - Air and Duct Leakages). In such cases, the Door Replacement measure can be turned off (see Section 14.5, Library Measures) or the leakage of the existing door declared as Tight, reflecting the replacement or weatherized door.

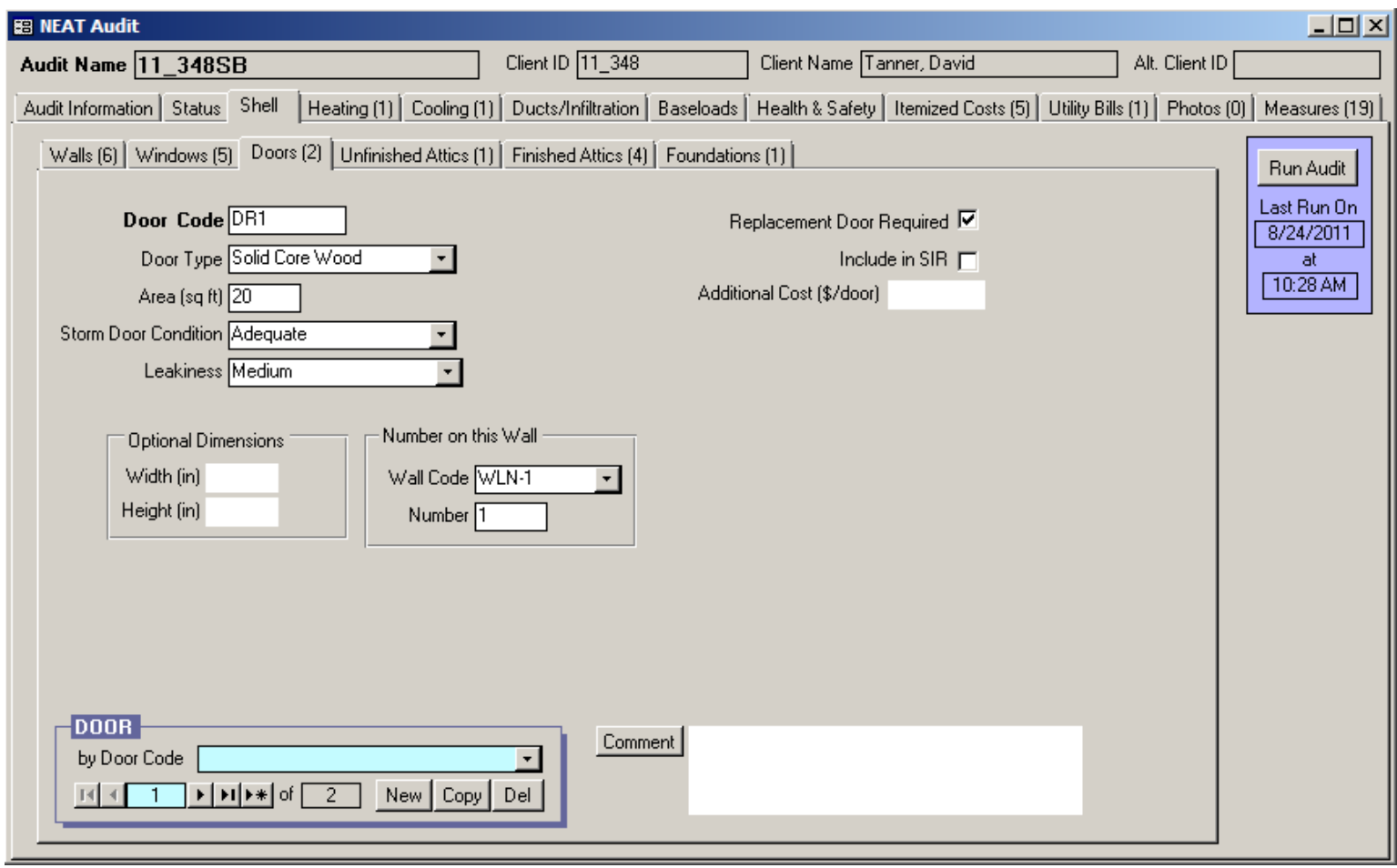

Figure 9.4. The Doors form under the Main Menu's “NEAT” button. 


\section{Chapter 9: NEAT Building Description}

Below are descriptions of the individual data items and controls found on the Doors form.

- Door Code - The Door Code identifies door descriptions to you and the Weatherization Assistant. If more than one door has the same description, you may enter the description once and indicate the number of doors with this description using the Number field described below. The door codes must be unique for all door descriptions within a given audit. NEAT automatically provides a default entry for all new doors that are created in the form of "DR\#" where the "\#” is an integer insuring uniqueness of the door code. You may choose to enter your own door code to include characters that indicate the orientation or some other characteristics of the door. The door code may have up to 20 characters, although space restrictions in many reports suggest a lesser number of characters should be used (perhaps 3 to 5) to preserve readability. Entries are not case-sensitive. NEAT will use the door codes in output reports. The door codes may also be helpful when labeling your drawing of the house's floor plan. Required.

- Door Type - Select what type of door this is. The choices are Hollow Core Wood, Solid Core Wood, Insulated Steel, Single Pane Sliding Glass, and Double Pane Sliding Glass. For a door with glazing, you will usually enter the door on the Doors form and the glazing on the Windows Form (see Section 9.2, Shell - Windows), although in some cases you may want to enter it just as a door or a window depending on which has the greatest fraction of area. Required.

- Area - Enter the area of the door in units of square feet (accuracy to the nearest square foot is usually satisfactory). NEAT will compute the total door area for the door type being described using this door area and the number of doors meeting this description (see Number below) and subtract this total area from the appropriate wall area based on the Wall Code (see below). Required.

- Storm Door Condition - Select the condition of any storm door installed on this door. The choices are Adequate, Deteriorated, and None. NEAT treats Deteriorated the same as None. The distinction allows you to convey added information. Required. 
- Leakiness - Provide an estimate of how leaky the door is. The choices are Tight, Medium, and Loose. Typical characteristics of these three categories of leakiness are listed below. Required.

Tight doors will have the door and frame squared, no warping, functioning weather stripping in good condition around the door, a good seal at the threshold, no holes or structural damage, and latches that keep the door securely shut. If windows exist in the door, they will be fixed and well sealed.

Doors with medium leakiness will have some characteristics of loose doors, but retain substantial integrity. However, they would likely benefit from air sealing efforts.

Loose doors will exhibit many, if not most, of the following problems: door and/or frame out of square, warping, weather stripping missing or severely damaged, no seal at the threshold, holes or significant structural damage, and latches that do not keep the door securely shut.

- Width and Height - If a replacement door or storm door installation is needed, you can use these two fields to enter the width and height of the door opening in units of inches. The dimensions are not used in any NEAT calculations. They are for your reference only. Therefore, the fields may be left blank. Alternatively, you could enter the dimensions in the Comment field. Optional.

- Wall Code - Enter the Wall Code for the exterior wall on which the door is installed. The acceptable Wall Codes are determined by your input on the Walls form (see Section 9.1, Shell - Walls) and will be displayed in the drop-down list associated with the field. Thus, you must enter a wall description before attempting to describe any doors that may lie on that wall. Specifying the Wall Code for each door allows NEAT to subtract the door area from the appropriate gross wall area. Required.

- Number - Enter the number of doors that can be described by the entries on this form. If the number of doors is greater than one, then this indicates that more than one door lies on the indicated wall because one of the data entry items making up the door description is the Wall Code. Be aware that the number in parentheses on the Doors tab (see Figure 9.4) is the number of door descriptions, not necessarily the total number of doors. Required. 
- Replacement Door Required - Selecting this checkbox indicates your decision to replace the door(s) described regardless of the cost-effectiveness, that is, making the measure mandatory. If the SIR of the measure is less than your accepted minimum value, you will have to reconsider requiring the door be replaced or consider its replacement a repair, if your program permits, or as part of your infiltration reduction efforts. If unchecked, NEAT will recommend this measure only if it is cost-effective.

- Include in SIR - If the Replacement Door Required checkbox is selected, an Include in SIR checkbox will appear on the form. This checkbox allows you to indicate whether you wish to have the door replacement included in the calculation of the whole house (package) SIR for this audit. Measures not included in the whole house SIR are normally related to health and safety. Check the guidance for your program in deciding when you can mandate a door replacement and if it can be excluded from the SIR calculation. An alternate reason for excluding a measure from the whole house SIR is if the measure is being funded outside of the Weatherization Assistance Program. Whether a measure is or is not included in the whole house SIR is indicated by its location in the list of measures produced for the Recommended Measures Report (see Chapter 12, NEAT and MHEA Results).

- Additional Cost - Enter any additional cost associated with replacing the door(s) being described that is in addition to the cost that NEAT will automatically calculate using the cost information you entered in the Library Measures form of the Setup Library (see Section 14.5, Library Measures). For example, an unforeseen cost to repair or modify the door frame. The cost to be entered is a cost per door, not a total cost for all the doors indicated in the Number field. The reason for this extra cost would be an appropriate entry in the Comment field described below. Optional.

- Comment - You may enter comments pertinent to the door directly in the Comment field on the form, or you may enter them in the Comment Editor by selecting the "Comment" button to the left of the field. The Comment Editor is similar to Window's Notepad and may be used to enter extended comments. The total comment may have up to 65,000 characters. Text from other sources may be copied and pasted into the Comment Editor's window. Any comment that is entered is displayed on the Recommended Measures report and the Input Report available from the Audit Information form (see Section 8.2, Audit 


\section{Chapter 9: NEAT Building Description}

Information). In addition, any comment that is entered can be imported into any specific work order (see Section 13.1, Work Order Information). Thus, you may wish to enter comments related to any observation that might affect the installation of needed measures or repair work. Optional.

- Door Record Navigation Block - The Door record navigation block is used to find and navigate to existing door descriptions for the audit being edited, copy or delete the currently accessed door description, or create a new door description for the audit. See Section 5.1, Form and Record Navigation, for information on using the Weatherization Assistant's record navigation blocks.

\subsection{Shell - Unfinished Attics}

The Unfinished Attics form (see Figure 9.5) under the Main Menu's "NEAT" button is used to enter information on unfinished attics in the house (i.e., attic areas that are not conditioned).

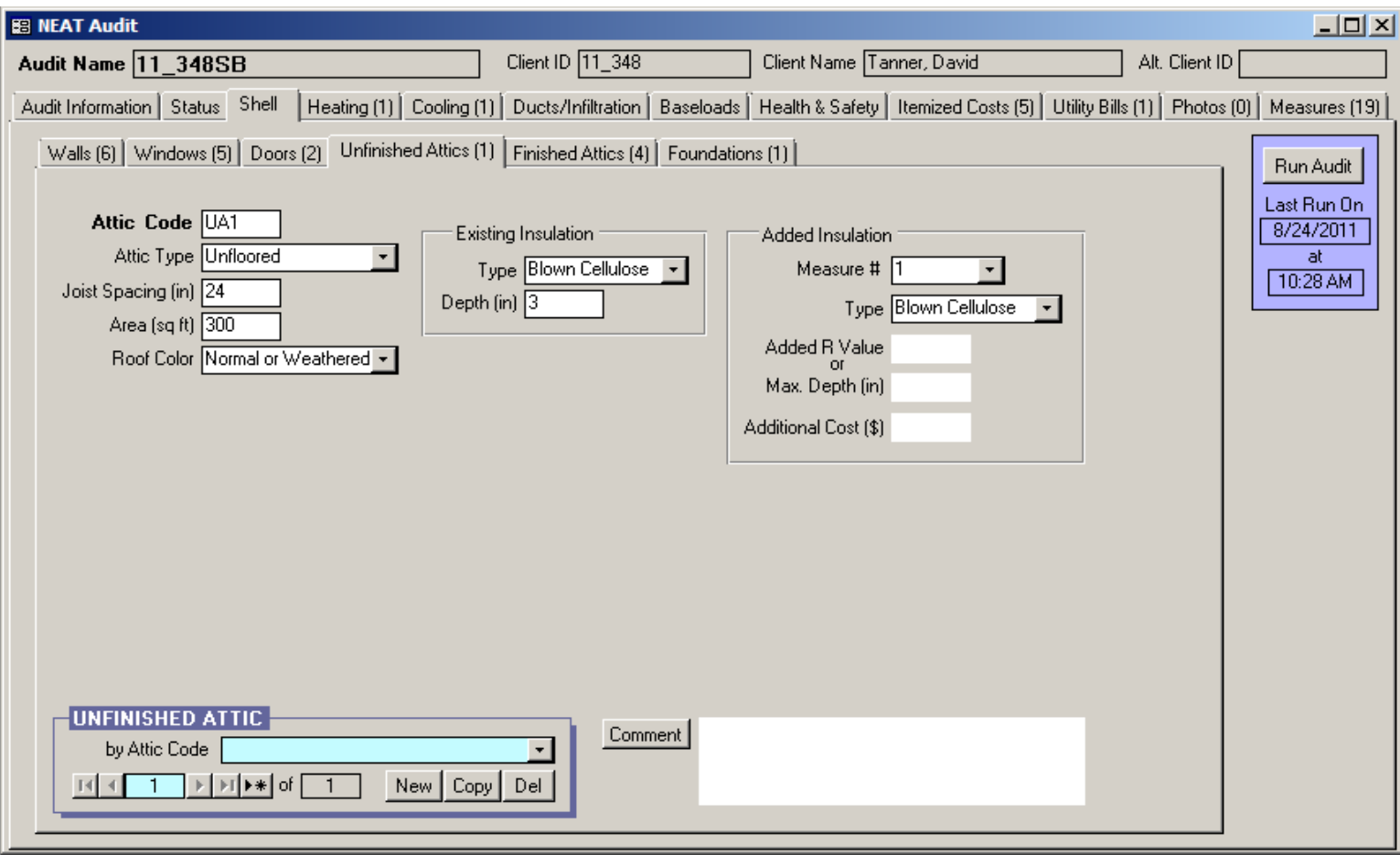

Figure 9.5. The Unfinished Attics form under the Main Menu's “NEAT” button.

A total of seven unfinished attic areas can be described in NEAT. Many houses will require only one unfinished attic description, but homes with separate 


\section{Chapter 9: NEAT Building Description}

unfinished attic areas may require two or more unfinished attic descriptions, especially if the attic areas differ in their characteristics. This occurs especially in homes that have had additions built onto them, where the attics in the additions differ in characteristics from the attic in the original home. Use the Finished Attics form (see Section 9.5, Shell - Finished Attics) to describe attic areas that are included in the conditioned area of the home.

Below are descriptions of the individual data items and controls found on the Unfinished Attics form.

- Attic Code - The Attic Code identifies attic components to you and the Weatherization Assistant. The attic codes must be unique for all attic descriptions within a given audit. This includes the attic codes entered on the Unfinished Attics form as well as those entered on the Finished Attics form. NEAT automatically provides a default entry for all new attic areas that are created in the form of "A\#" where the "\#” is an integer insuring uniqueness of the attic code. You may choose to enter your own attic code to include characters that indicate location or some other characteristics of the attic. The attic code may have up to 20 characters, although space restrictions in many reports suggest a lesser number of characters should be used (perhaps 3 to 5) to preserve readability. Entries are not case-sensitive. NEAT will use the attic codes in output reports and work orders to identifying which attic areas should receive weatherization measures. The attic codes may also be helpful when labeling your drawing of the house's floor plan. Required.

- Attic Type - Select what type of attic this is. The choices are Unfloored, Floored, and Cathedral or Flat. NEAT does not treat Unfloored and Floored attics differently. However, if the attic has a floor (e.g., plywood installed over the attic joists so that items may be stored in the attic), you will likely need to enter the restriction on the total depth of insulation that can be installed in the Max. Depth field (see below). You may also need to enter an additional cost for insulating this attic area because of this obstruction in the Additional Cost field (see below). A Cathedral or Flat attic is any attic area where the roof and interior ceiling surfaces are parallel. Required.

- Joist Spacing - Enter the spacing between the attic joists in units of inches. NEAT does not use this number in any of its calculations. This number is for 
your reference only. For example, it may be important to an insulation contractor installing batt insulation in the attic. Required.

- Area - For a Floored or Unfloored attic area, enter the area of the attic floor that borders a conditioned space in units of square feet. For a Cathedral or Flat attic area, enter the actual area of the sloped ceiling in units of square feet. Required.

- Roof Color - Enter the color of the roof. The two choices are "White, Reflective or Shaded" and "Normal or Weathered". A White or Reflective roof appears white or shiny metallic, is clean, and has little or no discoloration due to weathering. Roofs completely shaded by trees in the summer should be categorized as white or reflective roof regardless of their finish. The majority of roofs will be classified as Normal or Weathered. Roof surfaces designated as White or Reflective will not be considered when evaluating the White Roof Coating measure. Required.

- Existing Insulation Type - Select the type of existing insulation installed in the attic area. The choices are None, Blown Cellulose, Blown Fiberglass, Rockwool, Fiberglass Batts, and Other. NEAT assumes that the R-value per inch of insulation for the Other insulation type is $3.09 \mathrm{~h}-\mathrm{ft}^{2}-\mathrm{F} / \mathrm{Btu}-\mathrm{in}$. If more than one type of attic insulation is present, enter the type that has the greatest Rvalue (you should also note this in the Comment field described below). Required.

- Existing Insulation Depth - If the Existing Insulation Type is an entry other than None, then the Existing Insulation Depth field will appear. Enter the average depth of the existing insulation found in this attic segment, in units of inches. You can ignore compression or different depths of insulation around eaves. If more than one type of attic insulation is present, their total depth should be entered in this field. Required.

- Measure \# - If the Added Insulation Type field is an entry other than None, then the Measure \# field will appear. The Measure Number field allows individual attic segments to be combined into groups. For each group, an SIR to insulate all the attic segments in that group will be calculated, and one recommendation for insulating the attic segments in that group will be given. Enter the same measure number to all attics you wish to be grouped together. 
Measure numbers must be used in consecutive order, starting with 1. For example, a measure number of 3 cannot be entered until measure numbers 1 and 2 have been used. Required.

You will likely want to obtain a single SIR and recommendation for insulating all the attics in a house if all the attics have the same construction. In this case, enter a measure number of 1 for all the attic segments. Reasons for giving some attics a different measure number might be because they have different existing insulation levels or depth restrictions, which impacts potential savings and installation costs. In this case, each attic will receive the insulation recommendation that is most cost-effective in light of its existing condition.

- Added Insulation Type - Select the type of insulation you would install in this attic segment if NEAT recommends attic insulation. The choices are None, Blown Cellulose, Blown Fiberglass, and any attic insulation name(s) that you have entered on the NEAT Insulation Types form under the Setup Library (see Section 14.7, NEAT Insulation Types). Select None if conditions prohibit insulating this particular attic segment (you should note what the conditions are in the Comment field described below). Unless entries are present in either the Added R Value or Max. Depth fields described below, NEAT will evaluate the cost-effectiveness of installing standard levels of insulation (R-11, R-19, R-30, $\mathrm{R}-38$, and R-49) for the type chosen over the existing insulation and recommend the most cost-effective level, if any. Required.

- Added R Value - If the Added Insulation Type field is an entry other than None, then the Added R Value field will appear. If you want NEAT to evaluate only a specific R-value of added attic insulation, enter the R-value in this field. If the field is left blank, NEAT will determine the most cost-effective level of insulation for you from its standard levels (R-11, R-19, R-30, R-38, and R-49). If you enter an R-value in this field, all the standard levels of insulation will be ignored. In addition, the installation of the specified level of insulation will be considered mandatory and will be listed on the Recommended Measures report whether or not it is cost-effective. If it is not cost-effective, it would be wise to run NEAT again with a different R-value or with this field left blank to allow NEAT to choose a cost-effective level of insulation to add, if one exists. This entry might be used if you want to test adding a specific amount of insulation to that presently installed in the attic so that the total R-value of insulation in the 
attic (existing plus added) would be equal to a value specified by your state for new construction. Optional.

- Max. Depth - If the Added Insulation Type field is an entry other than None, then the Max. Depth field will appear. If there is a restriction to the total depth of insulation that can exist in an attic, enter the total depth (depth of any existing insulation plus the space for any additional insulation) in this field in units of inches. You should leave this field blank if no restriction exists or the restriction is greater than 15 in. (NEAT assumes unlimited insulation may be added if the restriction is greater than $15 \mathrm{in}$.). It is expected that the insulation depth might be restricted at the eaves, so this restriction does not need to be entered. For floored attics, this depth will usually be the height of the attic floor joists (usually 3.5 or 5.5 in.). If you enter a maximum depth restriction for a particular attic segment, NEAT will evaluate adding not only the standard levels of insulation (R-11, R-19, R-30, R-38, and R-49) that still fit into the available space, but also the level of insulation that exactly fills the available space. NEAT determines the cost for installing an amount of insulation that exactly fills the available space by interpolating between costs for installing the standard levels of insulation as entered in the Setup Library (see Section 14.5, Library Measures). Optional.

- Additional Cost - If the Added Insulation Type field is an entry other than None, then the Additional Cost field will appear. Enter any additional cost associated with insulating this specific attic segment in units of dollars. This would be a cost that is not normally associated with insulating an attic and, therefore, is not accounted for in the measure costs entered on the Library Measures form under the Setup Library (see Section 14.5, Library Measures). For instance, enter the additional costs associated with cutting an opening into the attic or for insulating beneath an attic floor. The cost is the total additional cost to insulate this specific attic segment; it is not a cost per square foot of attic area. The amount entered will be added to the normal cost computed from the attic area and the cost per square foot specified on the Library Measures form in the Setup Library. A blank entry indicates no additional cost. If a cost is entered, you should briefly explain the reason for this additional cost in the Comment field described below. Optional.

- Comment - You may enter comments pertinent to the attic segment directly in the Comment field on the form, or you may enter them in the Comment Editor 


\section{Chapter 9: NEAT Building Description}

by selecting the "Comment" button to the left of the field. The Comment Editor is similar to Window's Notepad and may be used to enter extended comments. The total comment may have up to 65,000 characters. Text from other sources may be copied and pasted into the Comment Editor's window. Any comment that is entered is displayed on the Recommended Measures report and the Input Report available from the Audit Information form (see Section 8.2, Audit Information). In addition, any comment that is entered can be imported into any specific work order (see Section 13.1, Work Order Information). Thus, you may wish to enter comments related to any observation that might affect the installation of needed measures or repair work. Optional.

- Unfinished Attic Record Navigation Block - The Unfinished Attic record navigation block is used to find and navigate to existing unfinished attic descriptions for the audit being edited, copy or delete the currently accessed unfinished attic description, or create a new unfinished attic description for the audit. See Section 5.1, Form and Record Navigation, for information on using the Weatherization Assistant's record navigation blocks.

\subsection{Shell - Finished Attics}

The Finished Attics form (see Figure 9.6) under the Main Menu's "NEAT” button is used to enter information on finished attics in the house (i.e., attic areas that are included in the conditioned area of the home). Four types of finished attic components can be described in NEAT: outer ceiling joist, collar beam, knee wall, and roof rafter (see Figure 9.7 and the Attic Area Type field described below).

You may enter into NEAT as many as 16 finished attic components in any combination of the four types. Use the Unfinished Attics form (see Section 9.4, Shell - Unfinished Attics) to describe attic areas that are not conditioned.

Most of the entries on the Finished Attics form are the same as those for the Unfinished Attics form. Therefore, refer to Section 9.4, Shell - Unfinished Attics, for descriptions of most of the individual data items and controls. Fields that are new or need additional explanation are discussed below. 


\section{Chapter 9: NEAT Building Description}

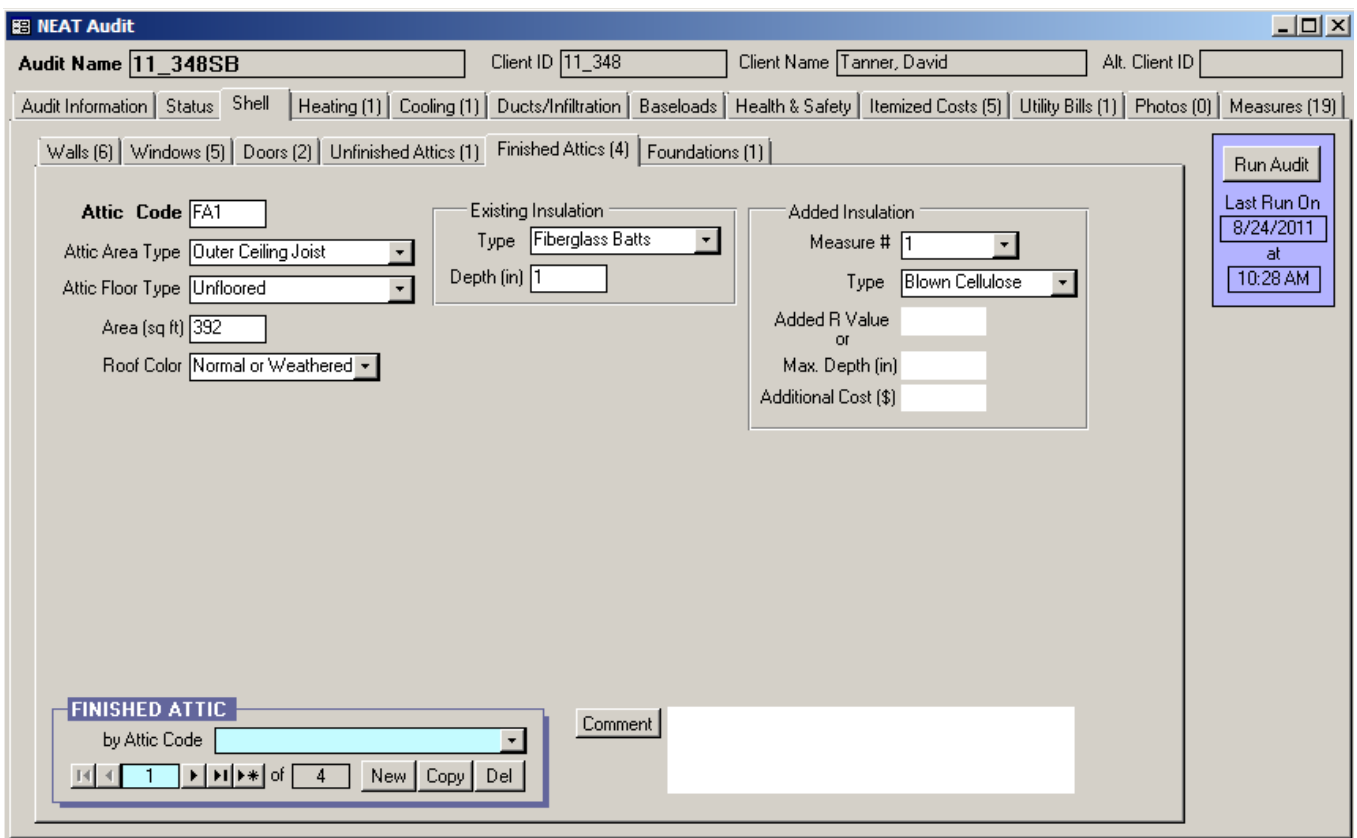

Figure 9.6. The Finished Attics form under the Main Menu’s “NEAT” button.

- Attic Area Type - Select what type of finished attic area this is. The choices as shown in Figure 9.7 are Outer Ceiling Joist, Collar Beam, Kneewall, and Roof Rafter. Required.

- Attic Floor Type - If the Attic Area Type is Outer Ceiling Joist or Collar Beam, the Attic Floor Type field will appear. This field is analogous to the Attic Type field on the Unfinished Attics form, although the only options are Unfloored and Floored. The

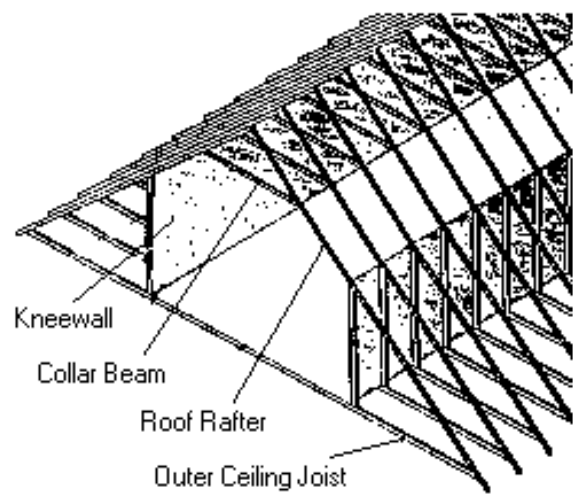

Figure 9.7. Components of a finished attic. Cathedral or Flat option is not provided because NEAT assumes that only finished attic areas of type Roof Rafter will have this construction. The Attic Floor Type field is not shown when the Attic Area Type is Kneewall or Roof Rafter because these component types do not have floors. Required.

- Measure \# - The Measure \# field will not appear if the Attic Area Type is Kneewall because NEAT always evaluates the insulation of a knee wall 


\section{Chapter 9: NEAT Building Description}

separately. Be careful in grouping finished attic sections because an insulation depth restriction entered for one section will be applied to all sections grouped together. Required.

- Added Insulation Type - The options for Added Insulation Type for knee walls differ from those for other finished or unfinished attic types. They are None, Fiberglass Batts, and any knee wall insulation type(s) that you have entered on the NEAT Insulation Types form under the Setup Library (see Section 14.7, NEAT Insulation Types).

- Added R Value and Max. Depth - The Added R Value and Max. Depth fields will not appear if the Attic Area Type is Kneewall because these fields do not pertain to knee walls. The R-value of insulation added to knee walls is the value specified on the NEAT Insulation Types form under the Setup Library (see Section 14.7, NEAT Insulation Types) for insulations of type "Knee Wall."

- Finished Attic Record Navigation Block - The Finished Attic record navigation block is used to find and navigate to existing finished attic descriptions for the audit being edited, copy or delete the currently accessed finished attic description, or create a new finished attic description for the audit. See Section 5.1, Form and Record Navigation, for information on using the Weatherization Assistant's record navigation blocks.

\subsection{Shell - Foundations}

The Foundations form (see Figure 9.8) under the Main Menu's “NEAT” button is used to describe the foundations of the dwelling, including basements, crawl spaces, slab-on-grade foundations, and floors exposed to the outside air. Foundations can be insulated by insulating the floor, sill box, and/or wall of the foundation depending on what type of foundation it is, the intended use of any foundation space, and what areas of the foundation are currently insulated. For basements and crawlspaces, NEAT will never recommend both floor insulation and sill and/or wall insulation for the same foundation

Below are descriptions of the individual data items and controls found on the Foundations form. 


\section{Chapter 9: NEAT Building Description}

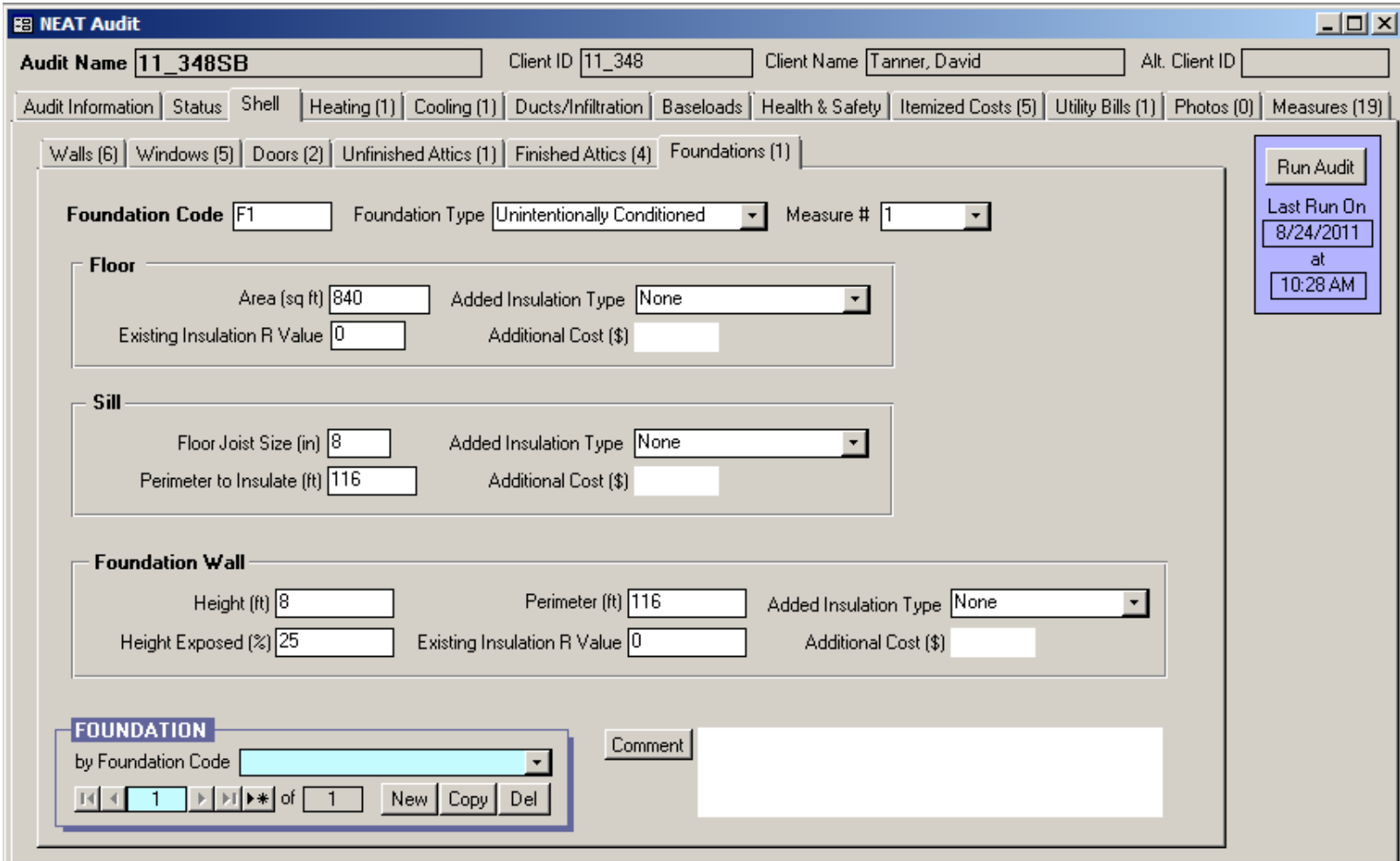

Figure 9.8. The Foundations form under the Main Menu’s “NEAT” button.

- Foundation Code - The Foundation Code identifies foundation spaces to you and the Weatherization Assistant. The foundation codes must be unique for all foundation space descriptions within a given audit. NEAT automatically provides a default entry for all new foundations that are created in the form of "F\#” where the “\#” is an integer insuring uniqueness of the foundation code. You may choose to enter your own foundation code to include characters that indicate the type of foundation or some other characteristics of the foundation space. The foundation code may have up to 20 characters, although space restrictions in many reports suggest a lesser number of characters should be used (perhaps 3 to 5) to preserve readability. Entries are not case-sensitive. NEAT will use the foundation codes in output reports and work orders to identifying which foundation spaces should receive recommended insulation. The foundation codes may also be helpful when labeling your drawing of the house's floor plan, particularly if there are more than one foundation spaces. Required. 
- Foundation Type - Select what type of foundation space this is. The choices are Conditioned, Non Conditioned, Vented Non Conditioned, Unintentionally Conditioned, Uninsulated Slab, Insulated Slab, and Exposed Floor. The first four choices are used to describe basements, crawlspaces, and any other type of enclosed foundation space. Conditioned means that the space is purposefully heated (or more rarely cooled) by a heating or cooling system to maintain a temperature at or near the rest of the dwelling. For example, a basement heated by supply registers or radiators in the space would be conditioned, as would a basement heated continuously by a space heater. Unintentionally Heated means that there is a heat source in the space that adds heat unintentionally to the space so that the temperature of the space is maintained above the outside temperature or ground temperature. For example, a basement that is heated because a furnace or water heater is located in the basement or because uninsulated ductwork runs through the basement would be unintentionally heated. Non Conditioned means that there are no sources of heat in the space other than conduction through walls, floors, and perhaps insulated ductwork. Vented Non Conditioned means that there are no sources of heat in the space other than conduction through walls, floors and perhaps uninsulated ductwork, and the space is vented directly to the outdoors. For example, a crawlspace with foundation vents would be Vented Non Conditioned. An Exposed Floor is used to describe a floor that is exposed directly to the outside air. For example, a second-story floor overhang would be an Exposed Floor, as would the floor of a house built on stilts or using pier and beam construction such that ambient air can freely flow beneath the house. Required.

NEAT assumes that all Conditioned, Unintentionally Conditioned, Non Conditioned, and Vented Non Conditioned foundations (i.e., basements and crawl spaces) are enclosed by walls with thermal characteristics of concrete block walls. Walls built with other cementitious materials, including solid concrete walls, likely have similar thermal characteristics to block walls.

All of the data fields shown in Figure 9.8 are not needed if the Foundation Type is Uninsulated Slab, Insulated Slab, or Exposed Floor. If the Foundation Type is changed while editing an existing foundation from a type that does not require certain fields to one that requires these same fields, a warning will appear if you attempt to leave the Foundations form before entering the required data. You will not be permitted to leave the form until the missing entries are entered. 
- Measure \# - The Measure Number field allows individual foundation spaces to be combined into groups. For each group, an SIR to insulate all the foundation spaces in that group will be calculated, and one recommendation for insulating all the foundation spaces in that group will be given. Enter the same measure number to all foundation spaces you wish to be grouped together. Measure numbers must be used in consecutive order, starting with 1 . For example, a measure number of 3 cannot be entered until measure numbers 1 and 2 have been used. Required.

You should usually give each foundation space a different measure number unless they are basically identical and you select the same insulation strategies (i.e., the same Added Insulation Types). The cost-effectiveness of insulating a foundation will vary depending on the type of foundation, existing insulation levels and locations, and the retrofit approach.

- Comment - You may enter comments pertinent to the foundation space directly in the Comment field on the form, or you may enter them in the Comment Editor by selecting the "Comment" button to the left of the field. The Comment Editor is similar to Window's Notepad and may be used to enter extended comments. The total comment may have up to 65,000 characters. Text from other sources may be copied and pasted into the Comment Editor's window. Any comment that is entered is displayed on the Recommended Measures report and the Input Report available from the Audit Information form (see Section 8.2, Audit Information). In addition, any comment that is entered can be imported into any specific work order (see Section 13.1, Work Order Information). Thus, you may wish to enter comments related to any observation that might affect the installation of needed measures or repair work. Optional.

- Foundation Record Navigation Block - The Foundation record navigation block is used to find and navigate to existing foundation descriptions for the audit being edited, copy or delete the currently accessed foundation description, or create a new foundation description for the audit. See Section 5.1, Form and Record Navigation, for information on using the Weatherization Assistant's record navigation blocks. 


\section{Floor Data Block}

The data items included in the Floor data block are intended to describe the floor of the house that separates the conditioned space inside the house from the foundation space, the outside, or the ground. For enclosed foundations, some people think of this floor as being the ceiling of the foundation space. All of the data items described below will be shown if the Foundation Type is Conditioned, Non Conditioned, Vented Non Conditioned, Unintentionally Conditioned, or Exposed Floor. If the Foundation Type is Uninsulated Slab or Insulated Slab, only the Area field will appear. For these foundation types, the Existing Insulation R Value field is not needed because NEAT knows that there is no insulation present if the Foundation Type is Uninsulated Slab and assumes a standard R-value for the insulation if the Foundation Type is Insulated Slab. The other two fields are not needed because NEAT does not currently evaluate an insulation retrofit for slabs.

- Area - For enclosed foundation spaces, enter the area of the floor directly above the foundation space you are describing in units of square feet. For slabon-grade foundations, enter the area of the slab floor in the living space. For exposed floors, enter the area of the floor that is directly exposed to the outside. Required.

- Existing Insulation R Value - Enter the R-value of the existing insulation installed in the floor being described. Required.

- Added Insulation Type - Select the type of insulation you would install under this floor if NEAT recommends floor insulation. The choices are None, Fiberglass Batts, and any floor insulation type(s) that you have entered on the NEAT Insulation Types form under the Setup Library (see Section 14.7, NEAT Insulation Types). Select None if conditions prohibit insulating this particular floor (you should note what the conditions are in the Comment field described above). For any entry other than None, NEAT will evaluate the costeffectiveness of installing standard levels of insulation (R-11, R-19, R-30, and R-38) for the insulation type chosen. However, the depth of any existing insulation plus the added insulation must fit within the joist depth, as specified by the Floor Joist Size, entered under the Sill data block. NEAT will recommend the most cost-effective level, if any. Required. 
- Additional Cost - Enter any additional cost associated with insulating the floor of the foundation being described in units of dollars. This would be a cost that is not normally associated with insulating the floor and, therefore, is not accounted for in the measure costs entered on the Library Measures form under the Setup Library (see Section 14.5, Library Measures). For instance, enter the additional labor and material costs associated with freeze protecting water pipes. The cost is the total additional cost to insulate the floor; it is not a cost per square foot of floor area. The amount entered will be added to the normal cost computed from the floor area and the cost per square foot specified on the Library Measures form in the Setup Library. A blank entry indicates no additional cost. If a cost is entered, you should briefly explain the reason for this additional cost in the Comment field described above. Optional.

\section{Sill Data Block}

The data items included in the Sill data block are intended to describe the sill portion of an enclosing foundation wall or the sill associated with an exposed floor. All of the data items described below will be shown if the Foundation Type is Conditioned, Non Conditioned, Vented Non Conditioned, Unintentionally Conditioned, or Exposed Floor. If the Foundation Type is Uninsulated Slab or Insulated Slab, none of the fields in the Sill data block will appear because these foundation types do not have a sill.

- Floor Joist Size - Enter the size of the floor joist (i.e., the band or rim joist) in units of inches. The levels of insulation that will be evaluated for addition to a floor are restricted to those that allow the total of existing and added insulation to fit within this joist depth. Required.

- Perimeter to Insulate - Enter the length of the sill perimeter (i.e., the band or rim joist) that is exposed to the outdoor air and is uninsulated in units of feet. This entry is used with the Floor Joist Size to determine the sill area that could be insulated. Required.

- Added Insulation Type - Select the type of insulation you would install in this sill if NEAT recommends sill (i.e., band or rim joist) insulation. The choices are None, Fiberglass Batts, and any sill insulation type(s) that you have entered on the NEAT Insulation Types form under the Setup Library (see Section 14.7, NEAT Insulation Types). Select None if conditions prohibit insulating this sill 


\section{Chapter 9: NEAT Building Description}

(you should note what the conditions are in the Comment field described above). Regardless of your entry, NEAT will not evaluate sill insulation if the Foundation Type is Vented Non Conditioned, Uninsulated Slab, Insulated Slab, or Exposed Floor. For any entry other than None, NEAT will evaluate the costeffectiveness of installing the R-value specified on the NEAT Insulation Types form under the Setup Library for the insulation type chosen. Required.

- Additional Cost - Enter any additional cost associated with insulating the sill of the foundation being described in units of dollars. This would be a cost that is not normally associated with insulating a sill and, therefore, is not accounted for in the measure costs entered on the Library Measures form under the Setup Library (see Section 14.5, Library Measures). The cost is the total additional cost to insulate the sill; it is not a cost per square foot of sill area. The amount entered will be added to the normal cost computed from the sill area and the cost per square foot specified on the Library Measures form in the Setup Library. A blank entry indicates no additional cost. If a cost is entered, you should briefly explain the reason for this additional cost in the Comment field described above. Optional.

\section{Foundation Wall Data Block}

The data items included in the Foundation Wall data block are intended primarily to describe the walls that enclose the foundation. All of the data items described below will be shown if the Foundation Type is Conditioned, Non Conditioned, Vented Non Conditioned, or Unintentionally Conditioned. If the Foundation Type is Uninsulated Slab, Insulated Slab, or Exposed Floor, only the Perimeter field will appear because these foundation types are not enclosed by walls.

- Height - Enter the height of the wall enclosing the foundation space (i.e., the basement or crawlspace wall) in units of feet. Enter an average height if the height of the wall is not uniform. If the wall height entered is less than two feet, NEAT will not evaluate the installation of floor, sill, or foundation wall insulation for the space because of access concerns. Required.

- Height Exposed - Enter the percentage of the Height field that is exposed to the outside air (i.e., is above grade). Do not enter the \% sign with the number. Enter an average percentage if the exposure is not uniform. Required. 
- Perimeter - Enter the length of the floor perimeter that borders the outdoors or ground in units of feet. Do not include the perimeter that borders another foundation space. The value is used with the Height and Height Exposed fields to determine the foundation wall area that borders the outside and ground. The default is the perimeter of a square with area given in the Area field. The value must be greater than zero. Required.

- Existing Insulation R-Value - Enter the R-value of the existing insulation currently installed on the foundation walls. NEAT assumes this coverage is uniform. If only part of the wall is insulated, enter an area weighted average Rvalue, or enter just the uninsulated length of the wall in the Perimeter field and enter zero in this field. Required.

- Added Insulation Type - Select the type of insulation you would install on the foundation wall if NEAT recommends foundation wall insulation. The choices are None, Rigid Foam Board, and any floor insulation type(s) that you have entered on the NEAT Insulation Types form under the Setup Library (see Section 14.7, NEAT Insulation Types). Select None if conditions prohibit insulating this particular floor (you should note what the conditions are in the Comment field described above). For any entry other than None, NEAT will evaluate the cost-effectiveness of installing the R-value specified on the NEAT Insulation Types form under the Setup Library for the insulation type chosen. Required.

- Additional Cost - Enter any additional cost associated with insulating the walls of the foundation being described in units of dollars. This would be a cost that is not normally associated with the Foundation Wall Insulation measure and, therefore, is not accounted for in the measure costs entered on the Library Measures form under the Setup Library (see Section 14.5, Library Measures). The cost is the total additional cost to insulate the foundation wall; it is not a cost per square foot of foundation wall area. The amount entered will be added to the normal cost computed from the foundation wall area and the cost per square foot specified on the Library Measures form in the Setup Library. A blank entry indicates no additional cost. If a cost is entered, you should briefly explain the reason for this additional cost in the Comment field described above. Optional. 


\section{Chapter 9: NEAT Building Description}

\subsection{Heating - General Description Data}

The Heating form (see Figure 9.9) under the Main Menu's "NEAT" button is used to describe the heating systems installed in the dwelling. NEAT allows the definition of a primary heating system and as many secondary systems as are required to describe all of the heat sources in a home. The primary system is the system that supplies the majority of the heat to the home and on which NEAT will consider installing energy efficiency measures. You should enter information and describe the primary system first before creating additional records to describe secondary systems.

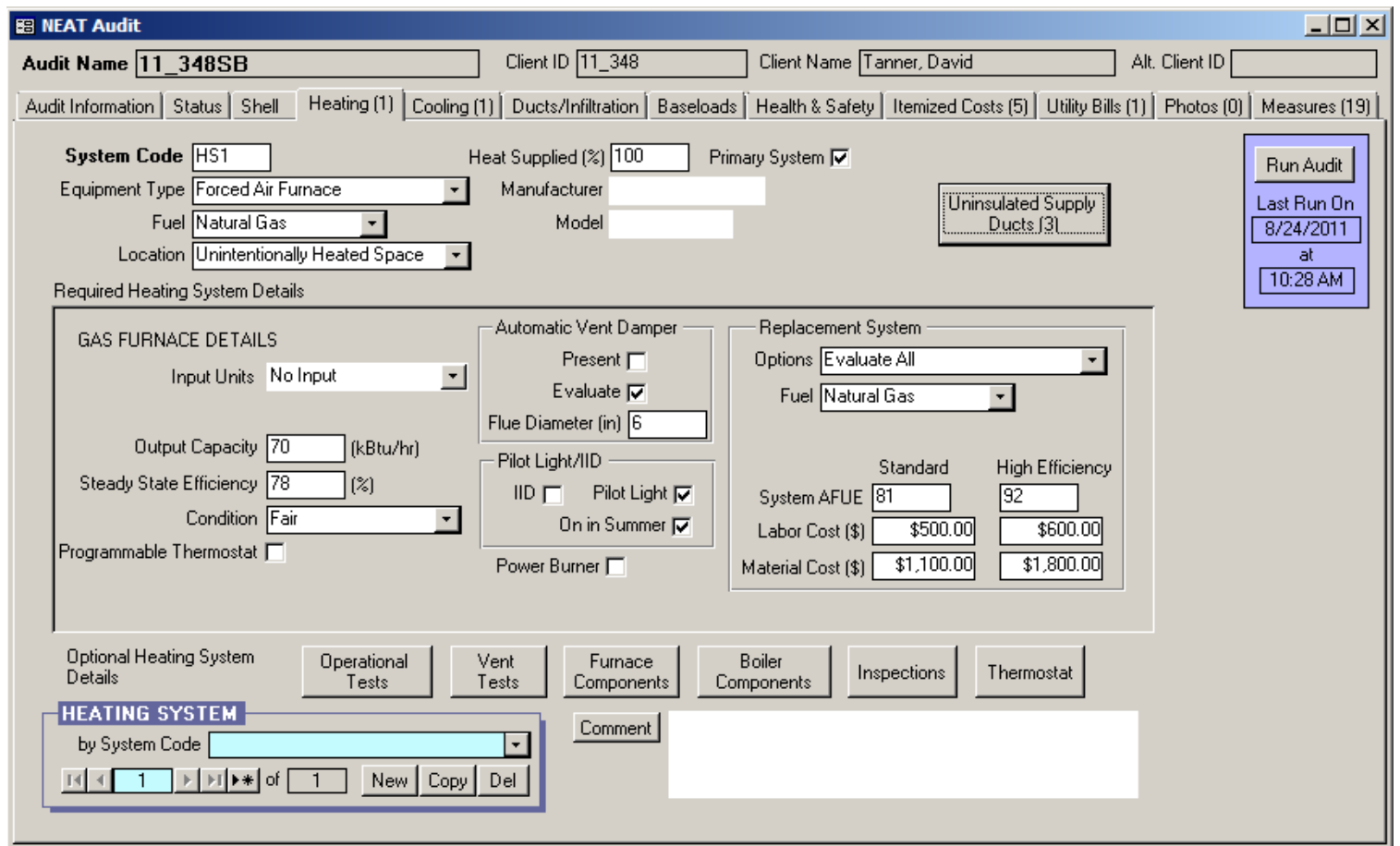

Figure 9.9. The Heating form under the Main Menu’s “NEAT” button.

You may encounter homes where even the primary heating system is inoperative. Do not attempt to describe these systems to NEAT by entering a value of 0 for either the Steady-State Efficiency or Heat Supplied (see the corresponding field descriptions in the discussions that follow). Rather, describe the existing heating equipment as supplying the amount of heat (Heat Supplied) as it was intended to supply and an efficiency that is typical, but at the lowest end of the possible range, for equipment of its type. Then, follow your state's recommendations regarding repair, tune-up, or replacement of the equipment, which may involve issues of cost-justifying a replacement or replacement as a health and safety measure. 
Alternatively, if you know that the inoperative system is going to be replaced, you could describe the replacement as the existing system on the heating forms.

The Heating form is divided into four sections: a general description of the heating system at the top of the form, a Required Heating System Details sub-form in the middle of the form, buttons for entry of Optional Heating System Details below the sub-form, and a Heating System record navigation block and Comment field at the very bottom of the form. Always enter information on the general description of the system first because the data fields in the sub-form change depending on the selections made in the Equipment Type and Fuel fields. The Optional Heating System Details buttons can be used to document an extensive number of heating system measurements and observations made while auditing a dwelling, though their entry does not affect the recommendations of the audit.

Descriptions of the individual data items and controls found in the general description portion of the Heating form are provided below, along with descriptions of the Heating System record navigation block and Comment field. The Required Heating System Details sub-form is discussed in Section 9.8, Required Heating System Details Sub-Form, and the optional information that can be entered using the Optional Heating System Details buttons is described in Section 11.12, Optional Heating System Details.

- System Code - The System Code identifies heating systems to you and the Weatherization Assistant. The system codes must be unique for all heating system descriptions within a given audit. NEAT automatically provides a default entry for all new heating systems that are created in the form of "HS\#" where the "\#” is an integer insuring uniqueness of the system code. You may choose to enter your own system code to include characters that indicate some characteristics of the heating system. The system code may have up to 20 characters, although space restrictions in many reports suggest a lesser number of characters should be used (perhaps 3 to 5) to preserve readability. Entries are not case-sensitive. NEAT will use the system codes in output reports and work orders to identifying which heating systems should receive recommended energy efficiency measures. The system codes may also be helpful when labeling your drawing of the house's floor plan, particularly if there are more than one heating system. Required. 
- Equipment Type - Select what type of heating system this is. The choices are Gravity Furnace, Forced Air Furnace, Steam Boiler, Hot Water Boiler, Fixed Electric Resistance, Portable Electric Resistance, Heat Pump, Vented Space Heater, Unvented Space Heater, and Other. Required.

- Fuel - Select the type of fuel used by the heating system. The choices are Natural Gas, Oil, Electricity, Propane, Wood, Coal, Kerosene, and Other. You can set the heat content and price for the Other fuel type on the Fuel Costs tab of the Setup Library (see Section 14.3, Fuel Costs). Required.

- Location - Select the location of the heating system. The choices are Heated Space, Unconditioned Space, and Unintentionally Heated Space. A Heated Space is purposefully heated by a heating system to maintain a desired temperature, usually utilizing a thermostat to control the space temperature. For example, a basement heated by supply registers or radiators in the space would be heated, as would a basement heated continuously by a space heater. An Unintentionally Heated Space is partially heated by a heat source in the space that adds heat unintentionally to the space. For example, a basement that is heated because a furnace, boiler, or water heater are located in the basement or because uninsulated ductwork runs through the basement would be unintentionally heated. An Unconditioned Space is not heated because there are no sources of heat in the space other than conduction through walls, floors, and perhaps insulated ductwork. The choice will affect the savings attributed to vent dampers. Required.

- Heat Supplied - Enter an estimate of the percent of the floor area that is heated by the heating system being described. Do not enter the \% sign with the number. The sum of the percentages for all heating system described must be 100 or less. Required.

- Primary System - Use this checkbox to identify that this is the primary heating system installed in the dwelling. The primary system supplies the majority of heat for the dwelling and is the system to which heating retrofits will apply. If this checkbox is left unchecked, NEAT will assume that the heating system is a secondary system unless it is the only heating system described. Checking the checkbox for any heating system will automatically uncheck the checkbox for any other system you may have previously indicated as being the primary system. It is best if the first heating system you describe is the Primary system. 
- Manufacturer and Model - Enter the name of the manufacturer and the model number of the existing heating system. This information may be needed for reference to establish an appropriate replacement. Optional.

- Eliminate with Primary System Replacement - This checkbox will appear if the Primary System checkbox is not checked (i.e., if this is a secondary heating system). Use this checkbox to identify that this secondary heating system is to be permanently turned off or removed from the dwelling if the primary system is replaced. NEAT will assign the percent of the floor area that was heated by this secondary heating system to the replacement primary system (i.e., NEAT will assume that any heat formerly supplied by this secondary system will be supplied by the new primary system following its installation).

- Comment - You may enter comments pertinent to the heating system directly in the Comment field on the form, or you may enter them in the Comment Editor by selecting the "Comment" button to the left of the field. The Comment Editor is similar to Window's Notepad and may be used to enter extended comments. The total comment may have up to 65,000 characters. Text from other sources may be copied and pasted into the Comment Editor's window. Any comment that is entered is displayed on the Recommended Measures report and the Input Report available from the Audit Information form (see Section 8.2, Audit Information). In addition, any comment that is entered can be imported into any specific work order (see Section 13.1, Work Order Information). Thus, you may wish to enter comments related to any observation that might affect the installation of needed measures or repair work. Heating system comments may include observations of equipment conditions, reasons for selecting specific retrofit options, or references to health and safety items. Optional.

- Heating System Record Navigation Block - The Heating System record navigation block is used to find and navigate to existing heating system descriptions for the audit being edited, copy or delete the currently accessed heating system description, or create a new heating system description for the audit. See Section 5.1, Form and Record Navigation, for information on using the Weatherization Assistant's record navigation blocks. 


\section{Chapter 9: NEAT Building Description}

\section{Uninsulated Supply Ducts}

The "Uninsulated Supply Ducts" button will appear only when the Equipment Type is Gravity Furnace, Forced Air Furnace, Heat Pump or Other, and the Primary System checkbox is checked. Also, if the Duct Insulation measure has been turned off in the Library Measures form of the Main

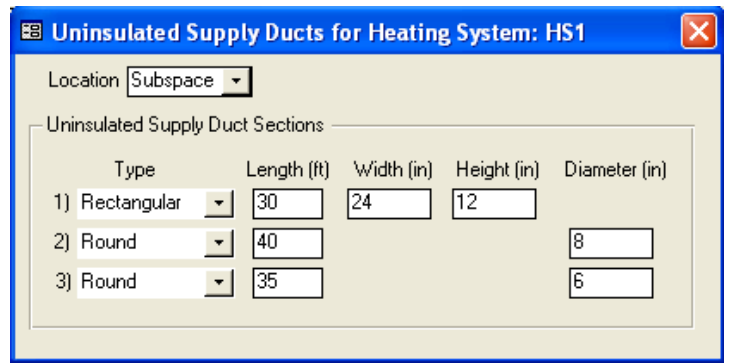

Figure 9.10. The Uninsulated Supply Ducts for Heating System sub-form. Menu's “Setup Library” button (see 14.5, Library Measures), this button will not appear. NEAT assumes that all other equipment types and all secondary systems do not have a forced air distribution system. Selecting the "Uninsulated Supply Ducts" button causes the Uninsulated Supply Ducts sub-form to appear (see Figure 9.10). This form is used to enter information on uninsulated supply ducts that are located outside the conditioned space of the dwelling. Insulated ducts, ducts located within the conditioned space of the dwelling, and return ducts should not be entered. NEAT will use this information to evaluate the cost-effectiveness of supply duct insulation. Duct leakage characteristics can be entered on the Ducts/Infiltration form described in Section 11.2, Ducts/Infiltration - Air and Duct Leakages.

Duct systems in homes are often comprised of ducts of different types and of varying sizes. At this time, up to three uninsulated supply duct sections located outside the conditioned space can be described. If the three duct sections are not enough to describe all the different ducts, you will have to enter duct sections with characteristics that are close to others under one common duct section description. For example, you may have to describe ducts with 6 in. and 8 in. diameters together, entering 7 in. as their average diameter. Although the quantity of insulation and the total cost for insulating the ducts might be somewhat affected by having to describe ducts with different characteristics together, the costeffectiveness of insulating the ducts will most likely not be significantly affected.

Below are descriptions of the individual data items and controls found on the Uninsulated Supply Ducts sub-form.

- Location - Select the location of the uninsulated ducts to be described. The choices are Attic or Subspace. For an Attic location, NEAT assumes that an attic environment surrounds the ducts. For a Subspace location, NEAT assumes 
that the ducts are located in the largest unintentionally heated foundation space that you have described on the Foundations forms (see Section 9.6, Shell Foundations). If there is no unintentionally heated foundation space, then NEAT will assume that the ducts are located in an unconditioned space. If uninsulated ducts exist in both the Attic and Subspaces, choose the location that has the greatest uninsulated duct surface area. Required.

- Type - Select what type of duct this is. The choices are Round or Rectangular. Required.

- Length - Enter the length of the uninsulated supply duct in units of feet. Required.

- Width, Height, and Diameter - The Width and Height fields will appear if the Type is Rectangular, and the Diameter field will appear if the Type is Round. Enter these dimensions for the uninsulated duct in units of inches. NEAT will use these dimensions together with the Length to determine the surface area of the uninsulated duct in computing the savings and insulation quantity for the Duct Insulation measure. If duct sections with different characteristics need to be combined together because only three duct sections can be described on the Uninsulated Supply Ducts form, then usually ducts with different widths and heights or with different diameters will need to be described together. In this case, enter average values. Required.

\subsection{Required Heating System Details Sub-Form}

The Required Heating System Details sub-form found on the Heating form (see Section 9.7, Heating - General Description Data) is used to describe details for the heating system being described. The data fields in the sub-form change depending on the selections made in the Equipment Type and Fuel fields on the general heating system portion of the Heating form. There are five possible subforms (see Table 9.1). Each sub-form will be discussed separately. However, the three sub-forms for fuel types other than Electricity are similar. Therefore, a complete description of the fields on the Gas Furnace Details sub-form will be given first, then only the fields that differ from these discussed for the other two sub-forms corresponding to the fuel types other than Electricity. 


\section{Chapter 9: NEAT Building Description}

Table 9.1. Selection of Equipment Type and Fuel to display different Required Heating System Details sub-form

\begin{tabular}{|c|c|c|c|}
\hline \multirow[b]{2}{*}{ EQUIPMENT TYPE } & \multicolumn{3}{|c|}{ FUEL } \\
\hline & $\begin{array}{l}\text { Natural Gas, } \\
\text { Propane, Wood, } \\
\text { Coal, or Other }\end{array}$ & Oil, Kerosene & Electricity \\
\hline $\begin{array}{l}\text { Gravity Furnace, } \\
\text { Forced Air Furnace, } \\
\text { Steam Boiler or Hot } \\
\text { Water Boiler }\end{array}$ & $\begin{array}{c}\text { Gas Furnace Details } \\
\text { Sub-Form }\end{array}$ & $\begin{array}{c}\text { Oil, Kerosene } \\
\text { Furnace/Boiler } \\
\text { Details Sub-Form }\end{array}$ & \\
\hline $\begin{array}{l}\text { Vented Space Heater, } \\
\text { Unvented Space Heater, } \\
\text { or Other }\end{array}$ & \multicolumn{2}{|c|}{ Space Heater Details Sub-Form } & $\begin{array}{l}\text { Electric Resistance } \\
\text { Heating System } \\
\text { Details Sub-Form }\end{array}$ \\
\hline $\begin{array}{l}\text { Fixed Electric } \\
\text { Resistance or Portable } \\
\text { Electric Resistance }\end{array}$ & \multicolumn{2}{|c|}{ - N/A - } & \\
\hline Heat Pump & \multicolumn{2}{|c|}{ - N/A - } & $\begin{array}{l}\text { Heat Pump Details } \\
\text { Sub-Form }\end{array}$ \\
\hline
\end{tabular}

\section{Gas Furnace Details Sub-Form}

If the selected equipment type is Gravity Furnace, Forced Air Furnace, Steam Boiler or Hot Water Boiler, and the selected fuel is Natural Gas, Propane, Wood, Coal or Other, the Gas Furnace Details sub-form is presented (see Figure 9.11). Below are descriptions of the individual data items and controls found on the Gas Furnace Details sub-form.

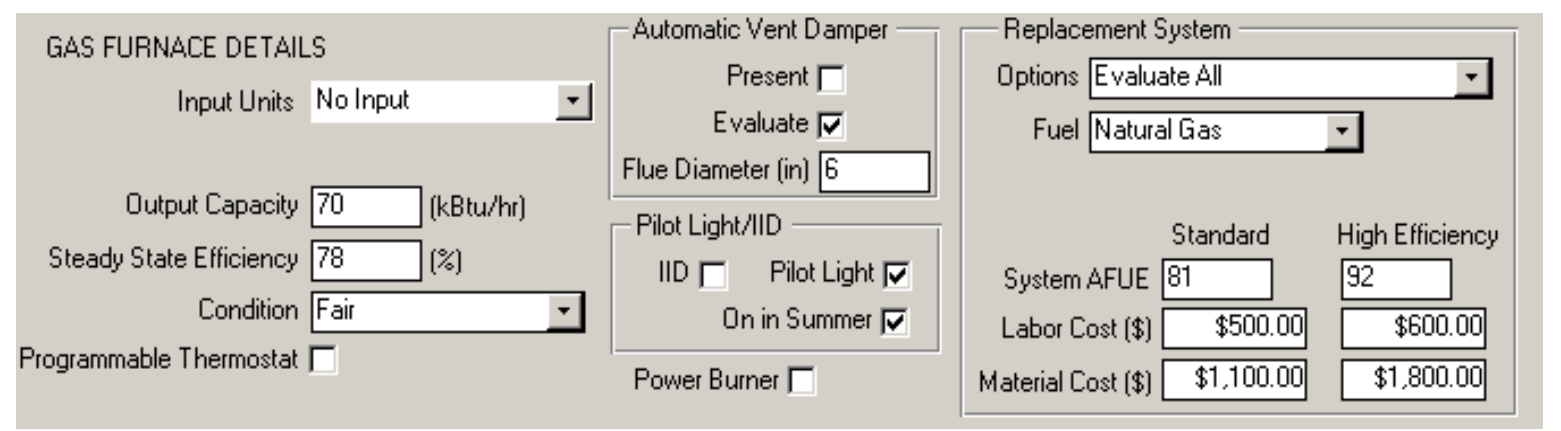

Figure 9.11. The Gas Furnace Details sub-form under the Main Menu’s “NEAT” button. 
- Input Units and Input Rating - The input rating of the heating system is used only with the output capacity to calculate a default steady-state efficiency for the heating system. If the steady-state efficiency of the heating system will be measured by performing a flue-gas analysis or if the steady-state efficiency will be obtained by some other means, then there is no need to enter the input rating. In this case, choose "No Input" in the Input Units field, in which case the Input Rating field will be hidden. If you want to enter the input rating, first select the energy units for the input rating in the Input Units field. The choices for the Input Units field are No Input, kBtu per Hour, Gallons per Hour, Lbs per Hour, and Cubic Centimeters per Minute. Then enter the actual value of the input rating in the Input Rating field. The input rating can be measured or taken directly from the nameplate of the heating system. Optional.

- Output Capacity - Enter the output capacity of the heating system in units of $\mathrm{kBtu} / \mathrm{h}$. The output capacity is usually obtained directly from the nameplate of the heating system or from an inspection of nozzle sizes, etc. If this information is not available, the output capacity can be approximated or, in rare cases, measured. Required.

- Steady-State Efficiency - Enter the steady-state efficiency of the heating system in units of percent (do not enter the \% sign with the number). This parameter should be as accurate as possible because it affects not only the savings calculated for heating system weatherization measures but also the savings of most envelope measures. There are online and published sources of heating equipment efficiencies by manufacturer and model. However, these efficiencies are for new units installed and tuned properly or may have been determined under different conditions. Thus, the values may differ significantly from those for the unit as it is actually operating. Therefore, if possible, the steady-state efficiency should be measured by performing a flue-gas analysis. If no other information on the steady-state efficiency is available, NEAT will compute a default value for you if you have entered values for the Input Units, Input Rating, and Output Capacity fields, described above (see Section 5.6, Field Defaults). Beware of replacing a heating system based on anything but a measured efficiency. Required.

- Condition - Describe the current condition of the heating system by selecting either Good, Fair, or Poor (but working). The condition is used to estimate the 
efficiency increase resulting from a tune-up. The poorer the condition, the greater will be the estimated efficiency increase. Required.

- Programmable Thermostat - Use this checkbox to indicate whether the existing thermostat is a programmable model that allows automatic setback of the heating set-point. The number of degrees assumed for the setback is entered in the Setup Library (see Section 14.2, Key Parameters). The presence of an existing programmable thermostat will prevent the Setback (Smart) Thermostat retrofit measure from being evaluated and recommended for this house. This checkbox should also be checked to indicate that the occupants would not benefit from a programmable thermostat because they already manually set their thermostat back in winter, do not want a programmable thermostat, and/or would not be able to use a programmable thermostat. In these cases, the reason for checking the checkbox should be noted in the Comment field described below.

- Automatic Vent Damper - Present - Use this checkbox to indicate that the heating system flue is equipped with an automatic vent damper. The presence of an existing automatic vent damper will prevent an automatic vent damper retrofit measure from being evaluated and recommended for this heating system.

- Automatic Vent Damper - Evaluate - This checkbox will appear only if the Automatic Vent Damper - Present checkbox is not checked. Use this checkbox to indicate that the installation of an automatic vent damper should be evaluated and recommended if it is found to be cost-effective. In some areas, local codes prohibit their use. If not selected, NEAT will not evaluate installing an automatic vent damper.

- Automatic Vent Damper - Flue Diameter - This field will appear only if the Automatic Vent Damper - Evaluate checkbox is checked. If installation of an automatic vent damper is recommended, this entry records the diameter in inches required for installation. This information might also be recorded in the Comment field to assist the contractor in obtaining the correct size and allowing it to be imported into a work order (see Comment in Section 13.1, Work Order Information). Conditionally Required. 
- IID and Pilot Light - Use these checkboxes to indicate whether the heating system uses an intermittent ignition device (IID) or pilot light to ignite the main burner of the heating system (an IID consists of an electric igniter that eliminates the need for a standing pilot light). Only one of the two checkboxes can be checked at the same time. If you checked a box but want to change your selection, uncheck the current selection before selecting the other checkbox. The presence of an existing IID will prevent an IID from being evaluated and recommended for this heating system.

- On in Summer - This checkbox is visible only if the Pilot Light checkbox has been selected. Use this checkbox to indicate that the pilot light on the heating system is left on (i.e., remains lit) throughout the summer, which increases the energy savings calculated for the IID retrofit measure.

- Power Burner - Use this checkbox to indicate that the heating system is equipped with a power burner. Power burners use blowers to better mix the fuel and combustion air, and are normally found on oil or coal furnaces that have been converted to burn natural gas.

The Replacement System data block will appear on the right side of the Required Heating System Details sub-form if the heating system being described is the primary heating system. The Replacement System data block will not appear for existing systems where the Fuel has been selected as Wood, Coal, or Other, since the replacement system options described below are not considered for these types of systems. Individual data items and controls presented in this data block allow you to enter information associated with tune-up and replacement retrofit measures for this primary system. Below are descriptions of the individual data items and controls found in the Replacement System data block.

- Options - Select one of six choices regarding tune-up or replacement of the primary heating unit, as listed below. Required.

1. Evaluate All. NEAT will evaluate both heating system replacement (standard and high efficiency) and system tune-up and recommend the most cost-effective option, if any. Replacement system efficiencies and costs for both standard and high efficiency replacements will be requested in subsequent fields. 
2. Tune-up Performed - Evaluate Replacements Only. A tune-up of the system has already been performed. The System Efficiency given on the form is the efficiency of the tuned up system. NEAT will not evaluate the system Tuneup measure. Replacement system efficiencies and costs for both standard and high efficiency replacements will be requested in subsequent fields.

3. Tune-up Mandatory. Inspection of the heating system has indicated that a system tune-up is all that is necessary. NEAT will evaluate and recommend the system Tune-up measure, regardless of its cost-effectiveness. The tuned up system will then be used in evaluating other measures not considered mandatory. No system replacement will be considered.

4. Standard Efficiency Replacement Mandatory. NEAT will consider replacement of the existing heating system with a standard efficiency system as mandatory. This may be the result of an unsafe yet unrepairable existing system. NEAT will evaluate and recommend the replacement regardless of its cost-effectiveness. The replacement system efficiency and cost for the standard unit will be requested in subsequent fields. The efficiency of the replacement system will be used in evaluating other measures not considered mandatory. No system tune-up or high efficiency replacement will be considered.

5. High Efficiency Replacement Mandatory. NEAT will consider replacement of the existing heating system with a high efficiency system as mandatory. This may be the result of an unsafe yet unrepairable existing system. NEAT will evaluate and recommend the replacement regardless of its costeffectiveness. The replacement system efficiency and cost for the high efficiency unit will be requested in subsequent fields. The efficiency of the replacement system will be used in evaluating other measures not considered mandatory. No system tune-up or standard efficiency replacement will be considered.

6. Don't Replace - Evaluate Tune-up Only. No system replacement (either standard or high efficiency) will be considered. The tune-up measure, however, will still be considered and recommended if cost-effective.

7. Evaluate None. No system replacement or tune-up will be considered. 
The Replacement System Options setting can help you direct NEAT in its evaluation process related to the heating systems in two ways not necessarily obvious from the field descriptions. NEAT was originally designed to compare the cost-effectiveness of replacing the existing system with either a standard efficiency heating system or a high efficiency system. However, if you are not interested in this comparison and only want to evaluate the cost-effectiveness of either the standard efficiency or high efficiency systems, choose "Evaluate All” or "Tuneup Performed - Evaluate Replacements Only" for the Options, then enter a large total cost (see Labor and Material Cost field descriptions below) for the replacement system you do not want to be recommended. Since the system with the large cost will never be cost-effective, you have essentially eliminated the comparison of the two different replacements.

The selection of "Tuneup Performed - Evaluate Replacements Only" for the Replacement System Options was intended to indicate that a heating system had been tuned up during an initial visit to a home and that the measure should not be evaluated further by the audit. However, if inspection of the heating system indicates that a tune-up most likely can't be performed successfully, yet replacement of the system still needs to be cost justified, this same setting can also be used to direct NEAT to not evaluate the Tune-up measure under these circumstances.

In both of the instances described above, the inputs being used to direct NEAT are not being used in the original manner they were intended. Thus, these techniques should only be implemented with proper authorization and should be documented appropriately (e.g., using the Comments).

- Fuel - If the System Retrofit Option selected is Evaluate All, Tune-up Performed - Evaluate Replacements Only, Standard Efficiency Replacement Mandatory, or High Efficiency Replacement Mandatory, a "Fuel” drop-down list will appear on the form. Select the type of fuel used by the replacement heating system. The choices are Natural Gas, Oil, Electricity, Propane, Wood, Coal, Kerosene, and Other. You can set the heat content and price for the Other fuel type on the Fuel Costs tab of the Setup Library (see Section 14.3, Fuel Costs). Conditionally Required.

- Include in SIR - If the System Retrofit Option selected is Tuneup Mandatory, Standard Efficiency Replacement Mandatory, or High Efficiency Replacement 
Mandatory, an Include in SIR checkbox will appear on the form. This checkbox allows you to indicate that you wish to have the selected measure included in the calculation of the whole house (package) SIR for this audit. Measures not included in the whole house SIR are normally related to health and safety. Check the guidance for your program in deciding when you can mandate an HVAC measure and if it can be excluded from the SIR calculation. Even if the measure can be considered related to health and safety, if it is shown to be costeffective, you may choose to still include it in the whole house SIR by selecting this checkbox. By doing so, it will not be seen as a health and safety measure, thus preserving funds dedicated for other such measures. An alternate reason for excluding a measure from the whole house SIR is if it is funded outside of the Weatherization Assistance Program. Whether a measure is or is not included in the whole house SIR is indicated by its location in the list of measures produced for the Recommended Measures Report (see Chapter 12, NEAT and MHEA Results).

- System AFUE - Enter the AFUE (Annual Fuel Utilization Efficiency) for the replacement unit. The AFUE will most often be given on the unit label or in the unit specifications. Entries will be required for either or both the standard and high efficiency units, depending on the choice made for System Retrofit Options. Conditionally Required.

- Labor and Material Costs - Enter the Labor and Material costs associated with replacing the primary heating system. Entries will be displayed for either or both the standard and high efficiency units, depending on the choice made for System Retrofit Options. NEAT adds the labor and equipment costs in computing the SIR and reporting costs. Thus, the total cost (labor plus equipment) may be entered in either field, with $\$ 0.00$ entered for the other of the two fields, with no adverse consequences. Do not enter $\$ 0.00$ in both Labor and Material Cost fields for either replacement unit. Conditionally Required.

\section{Oil/Kerosene Furnace/Boiler Details Sub-Form}

If the selected equipment type is Gravity Furnace, Forced Air Furnace, Steam Boiler or Hot Water Boiler, and the selected fuel is Oil or Kerosene, the Oil/Kerosene Furnace/Boiler Details sub-form is presented (see Figure 9.12). 


\section{Chapter 9: NEAT Building Description}

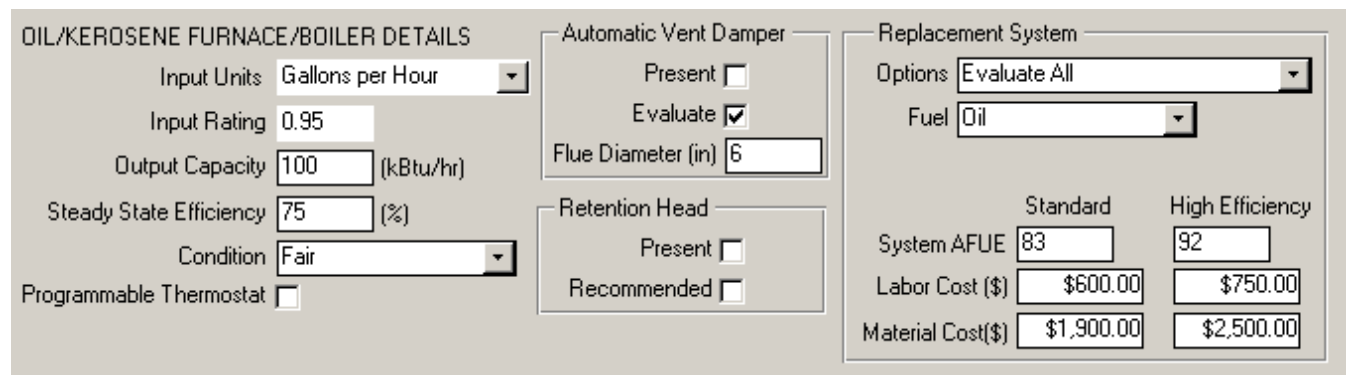

Figure 9.12. The Oil/Kerosene Furnace/Boiler Details sub-form under the Main Menu's “NEAT” button.

This sub-form is nearly identical with the Gas Furnace Details sub-form except that the Pilot Light/IID and Power Burner entries (see Figure 9.11) are replaced by the Retention Head entries (see Figure 9.12 above and descriptions below). See the discussion under "Gas Furnace Details Sub-Form” above for descriptions of the other entry fields.

- Retention Head Present - Indicate whether the primary heating system is equipped with a Retention Head Oil Burner. This device provides higher combustion efficiency by mixing the oil and air more vigorously.

- Retention Head Recommended - Indicate whether the installation of a retention head burner would be recommended if found cost-effective. If selected, NEAT will recommend installation only if found cost-effective. If not selected, the measure will not be considered. This checkbox will be visible only if you have indicated that a retention head burner does not already exist.

The Replacement System data block of the Oil/Kerosene Furnace/Boiler Details sub-form is identical to that described previously for the Gas Furnace Details subform and the response of NEAT to its entries is the same.

\section{Electric Resistance Heating System Details Sub-Form}

If the selected equipment type is other than Heat Pump and the selected fuel is Electricity, the Electric Resistance Heating System Details sub-form is presented (see Figure 9.13). Electrical equipment is assumed 100\% efficient, although distribution losses may be described by the data associated with the Uninsulated Supply Ducts fields (see Section 9.7, Heating - General Description Data) and the Ducts and Infiltration form (see Section 11.2 ). Electric furnaces do require entry of an Output Capacity. 


\section{Chapter 9: NEAT Building Description}

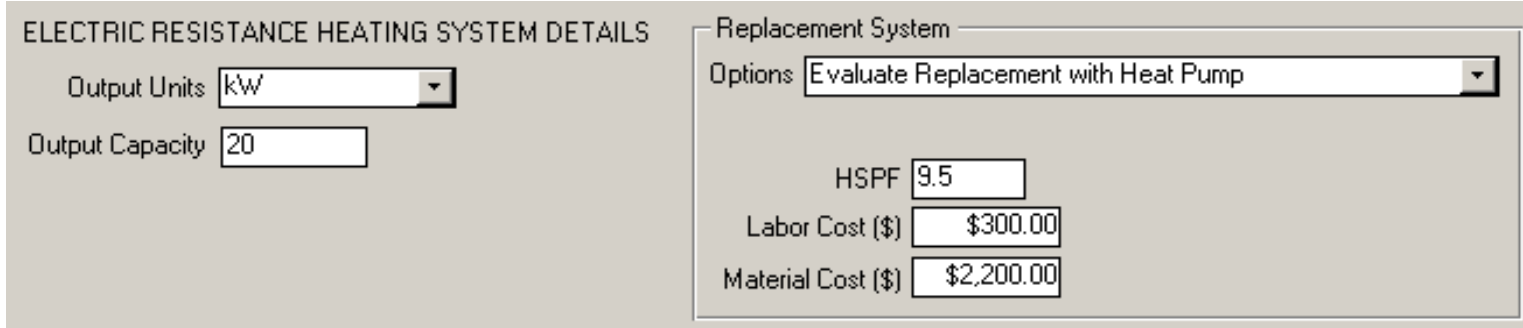

Figure 9.13. The Electric Resistance Heating System Details sub-form under the Main Menu's “NEAT” button.

- Output Units and Output Capacity - Select the energy units for the output capacity in the Output Units field. The choices are kW and kBtu per hour. Enter the output capacity of the heating system in the units selected in the Output Units field. The output capacity is usually obtained directly from the nameplate of the heating system. If this information is not available, the output capacity can be approximated or, in rare cases, measured. Required.

The Replacement System data block will appear on the right side of the Required Heating System Details sub-form if the heating system being described is the primary heating system. Individual data items and controls presented in this data block allow you to enter information associated with the replacement of this primary system. The entries do not allow evaluation of replacing electric heating equipment with a fossil-fueled system.

Any time NEAT recommends installation of a heat pump, any existing cooling equipment that has been described (see Section 9.9, Cooling) will be assumed replaced by the heat pump, with characteristics on the cooling side as specified in the Setup Library (see “Equipment” in Section 14.2, Key Parameters). If no existing cooling equipment has been specified, NEAT will include only heating savings in its estimates of total savings and SIR for the heat pump installation as well as for other measures being evaluated.

Below are descriptions of the individual data items and controls found in the Replacement System data block on the Electric Resistance Heating System Details sub-form.

- Options - Select one of four choices regarding replacement of the primary heating unit. Required. 
1. Evaluate Replacement with Heat Pump. NEAT will evaluate replacing the existing heating system, and any existing cooling equipment, with a heat pump and recommend the replacement only if it is cost-effective. See assumptions associated with installation of a heat pump in the introductory paragraph to the Replacement System data block, above. Replacement system efficiency and cost will be requested in subsequent fields.

2. Replacement with Heat Pump Mandatory. NEAT will consider replacing the existing heating system with a heat pump as mandatory. However, if existing cooling equipment has been described (see Section 9.9, Cooling), the replacement of the first cooling unit must also be declared mandatory in order for the heat pump replacement to be treated as mandatory. See additional assumptions associated with installation of a heat pump in the introductory paragraph to the Replacement System data block, above. Mandatory replacement of a heating system may be the result of an unsafe yet unrepairable existing system. NEAT will recommend the replacement regardless of its cost-effectiveness. The replacement system efficiency and cost will be requested in subsequent fields. The efficiency of the replacement system will be used in evaluating other measures not considered mandatory.

3. Replacement with Electric Resistance System Mandatory. NEAT will consider replacing the existing heating system with an electric resistance system as mandatory. Mandatory replacement of a heating system may be the result of an unsafe yet unrepairable existing system. NEAT will evaluate and recommend the replacement regardless of its cost-effectiveness. The replacement system cost for the electric resistance unit will be requested in subsequent fields. The efficiency of the replacement system will be used in evaluating other measures not considered mandatory.

4. Don't Replace - Evaluate None. No system retrofit option will be considered.

- Include in SIR - If the System Retrofit Option selected is Replacement with Heat Pump Mandatory or Replacement with Electric Resistance System Mandatory, an Include in SIR checkbox will appear on the form. This checkbox allows you to indicate that you wish to have the selected measure included in the calculation of the whole house (package) SIR for this audit. Measures not 


\section{Chapter 9: NEAT Building Description}

included in the whole house SIR are normally related to health and safety. Check the guidance for your program in deciding when you can mandate an HVAC measure and if it can be excluded from the SIR calculation. Even if the measure can be considered related to health and safety, if it is shown to be costeffective, you may choose to still include it in the whole house SIR by selecting this checkbox. By doing so, it will not be seen as a health and safety measure, thus preserving funds dedicated for other such measures. An alternate reason for excluding a measure from the whole house SIR is if it is funded outside of the Weatherization Assistance Program. Whether a measure is or is not included in the whole house SIR is indicated by its location in the list of measures produced for the Recommended Measures Report (see Chapter 12, NEAT and MHEA Results).

- HSPF - If the System Retrofit Option selected is Evaluate Replacement with Heat Pump or Replacement with Heat Pump Mandatory, an HSPF field will appear on the form. Enter the HSPF (Heating System Performance Factor) for the replacement unit. The HSPF will most often be given on the unit label or in the unit specifications. Conditionally Required.

- Labor and Material Costs - Enter the Labor and Material costs associated with replacing the primary heating system. NEAT adds the labor and equipment costs in computing the SIR and reporting costs. Thus, the total cost (labor plus equipment) may be entered in either field, with $\$ 0.00$ entered for the other of the two fields, with no adverse consequences. Do not enter $\$ 0.00$ in both Labor and Material Cost fields. Required.

\section{Heat Pump Details Sub-Form}

If the selected Equipment Type is Heat Pump, the Heat Pump Details sub-form is presented (see Figure 9.14). Below are descriptions of the individual data items and controls found on the Heat Pump Details sub-form.
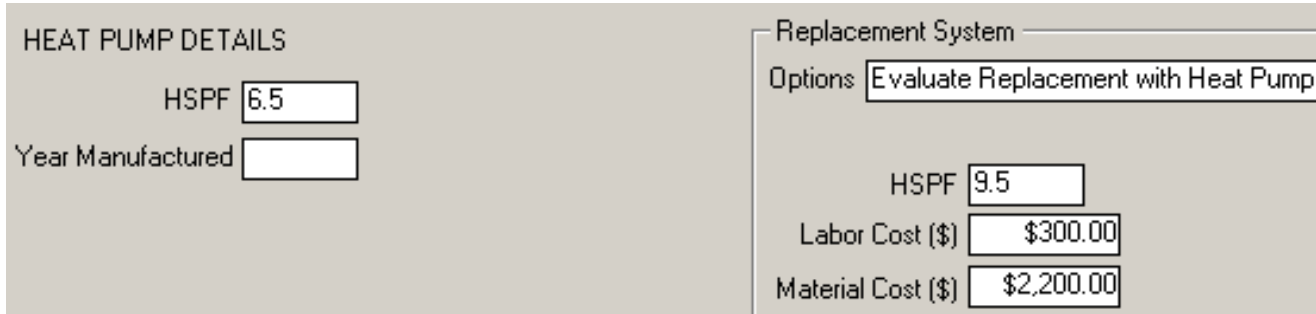
Figure 9.14. The Heat Pump Details sub-form under the Main Menu’s “NEAT” button.

- $\mathbf{H S P F}$ - Provide an estimate of the heating seasonal performance factor (HSPF) of the existing system. Newer systems are required to display the HSPF rating on the unit. There are online and published sources of heat pump efficiencies by manufacturer and model. If no estimate is available, you may leave the field blank and proceed to the Year Manufactured field (see entry below). All of these sources represent the system efficiency for a new unit operating correctly. Conditionally required.

- Year Manufactured - Enter the approximate year the unit was manufactured or purchased new. Use the full year (e.g., “1986,” not “"96”). This entry is hidden if a value has been provided in the HSPF field above. Otherwise the year is used to estimate the HSPF. Conditionally required.

The Replacement System data block will appear on the right side of the Required Heating System Details sub-form if the heating system being described is the primary heating system. Individual data items and controls presented in this data block allow you to enter information associated with the replacement of this primary system. The entries do not allow evaluation of replacing electric heating equipment with a fossil-fueled system.

Any time NEAT recommends installation of a heat pump, any existing cooling equipment that has been described (see Section 9.9, Cooling) will be assumed replaced by the heat pump, with characteristics on the cooling side as specified in the Setup Library (see "Equipment” in Section 14.2, Key Parameters). If no existing cooling equipment has been specified, NEAT will include only heating savings in its estimates of total savings and SIR for the replacement as well as for other measures being evaluated.

Below are descriptions of the individual data items and controls found on the Heat Pump Details sub-form.

- Options - Select one of three choices regarding replacement of the primary heating unit. Required.

1. Evaluate Replacement with Heat Pump. NEAT will evaluate replacing the existing heating system, and any existing cooling equipment, with a heat 
pump and recommend the replacement only if it is cost-effective. See assumptions associated with installation of a heat pump in the introductory paragraph to the Replacement System data block, above. Replacement system efficiency and cost will be requested in subsequent fields.

2. Replacement with Heat Pump Mandatory. NEAT will consider replacing the existing heating system with a heat pump as mandatory. However, if existing cooling equipment has been described (see Section 9.9, Cooling), the replacement of the first cooling unit must also be declared mandatory in order for the heat pump replacement to be treated as mandatory. See additional assumptions associated with installation of a heat pump in the introductory paragraph to the Replacement System data block, above. Mandatory replacement of a heating system may be the result of an unsafe yet unrepairable existing system. NEAT will recommend the replacement regardless of its cost-effectiveness. The replacement system efficiency and cost will be requested in subsequent fields. The efficiency of the replacement system will be used in evaluating other measures not considered mandatory.

3. Don’t Replace - Evaluate None. No system replacement will be considered.

- Include in SIR - If the System Retrofit Option selected is Replacement with Heat Pump Mandatory, an Include in SIR checkbox will appear on the form. This checkbox allows you to indicate that you wish to have the selected measure included in the calculation of the whole house (package) SIR for this audit. Measures not included in the whole house SIR are normally related to health and safety. Check the guidance for your program in deciding when you can mandate an HVAC measure and if it can be excluded from the SIR calculation. Even if the measure can be considered related to health and safety, if it is shown to be cost-effective, you may choose to still include it in the whole house SIR by selecting this checkbox. By doing so, it will not be seen as a health and safety measure, thus preserving funds dedicated for other such measures. An alternate reason for excluding a measure from the whole house SIR is if it is funded outside of the Weatherization Assistance Program. Whether a measure is or is not included in the whole house SIR is indicated by its location in the list of measures produced for the Recommended Measures Report (see Chapter 12, NEAT and MHEA Results). 


\section{Chapter 9: NEAT Building Description}

- (Replacement System) HSPF - If the System Retrofit Option selected is Evaluate Replacement with Heat Pump or Replacement with Heat Pump Mandatory, an HSPF field will appear on the form. Enter the HSPF (Heating System Performance Factor) for the replacement unit. The HSPF will most often be given on the unit label or in the unit specifications. Required.

- Labor and Material Costs - Enter the Labor and Material costs associated with replacing the primary heating system. NEAT adds the labor and equipment costs in computing the SIR and reporting costs. Thus, the total cost (labor plus equipment) may be entered in either field, with $\$ 0.00$ entered for the other of the two fields, with no adverse consequences. Do not enter $\$ 0.00$ in both Labor and Material Cost fields for the replacement unit. Required.

\section{Space Heater Details Sub-Form}

If the selected equipment type is Unvented Space Heater, Vented Space Heater or Other, and the selected fuel is any type other than Electricity, the Space Heater Details sub-form is presented (see Figure 9.15).

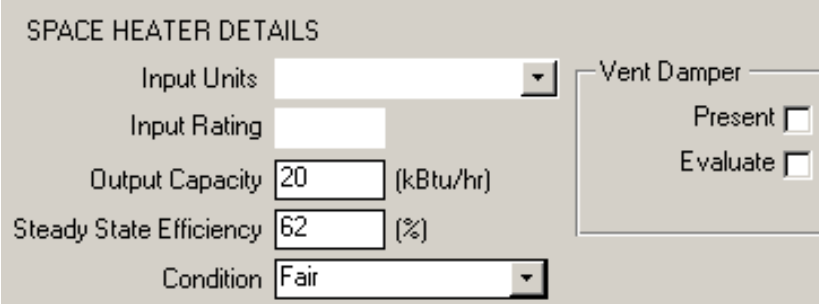

Figure 9.15. The Space Heater Details sub-form under the Main Menu's “NEAT” button.

This sub-form is identical with the Gas Furnace Details sub-form except that the entries related to the pilot light, IID, and power burner have been eliminated as well as those related to high efficiency replacements. Discussion of the entry fields given under the Gas Furnace Details sub-form section should be referred to for the space heater equipment types along with the additional information below.

If the selected equipment type is Unvented Space Heater, it would be assumed that the Steady State Efficiency entered would be essentially 100\% (the "\%" sign is not entered), the only exception possibly being a wall-mounted unit where heat may be lost through the wall. For a unit with 100\% efficiency, the Input Rating is the same 


\section{Chapter 9: NEAT Building Description}

as the Output Capacity (if expressed in the same units) and only the latter value needs to be entered. Also, for an unvented space heater, the Vent Damper entries are not applicable and would be left unchecked.

The Replacement System data block will appear on the right side of the Space Heater Details sub-form if the heating system being described is the primary heating system and the selected fuel is Natural Gas, Propane or Oil. Individual data items and controls presented in this data block are the same as described under the Gas Furnace Details sub-form except no entries related to the high efficiency replacement unit will appear. Only a standard efficiency replacement is assumed available.

In Version 8.9 of the Weatherization Assistant, the Tune-up measure has been eliminated for space heaters. However, the possible selections under the "Replacement Systems Options" drop-down list do not reflect this change. Any selection directing NEAT to evaluate the Tune-up measure for a space heater will be ignored.

\subsection{Cooling}

The Cooling form (see Figure 9.16) under the Main Menu's “NEAT” button is used to describe the cooling systems installed in the dwelling. NEAT allows the definition of up to five cooling systems in a home. Below are the descriptions of the individual data items and controls found on the Cooling form. 


\section{Chapter 9: NEAT Building Description}

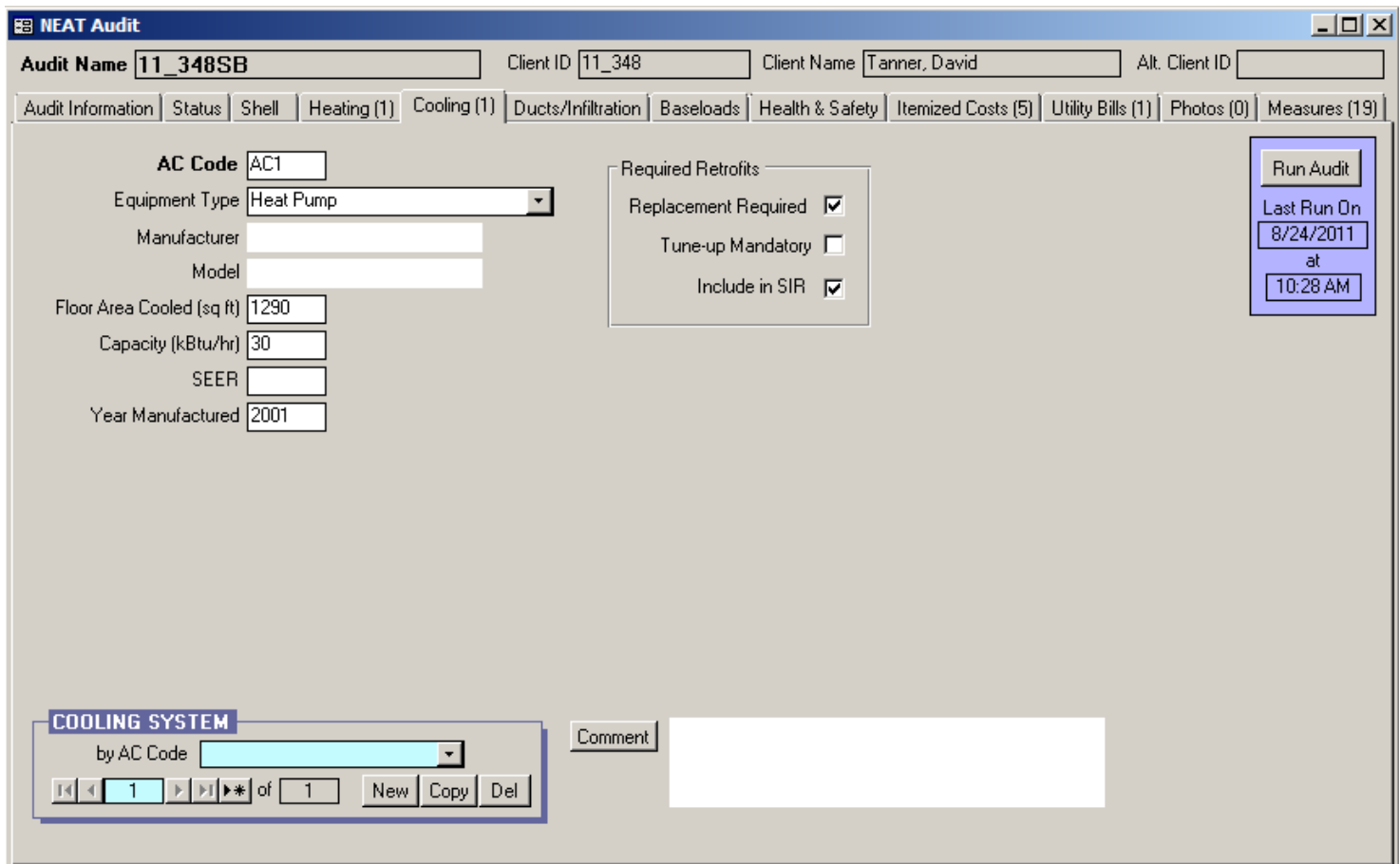

Figure 9.16. The Cooling form under the Main Menu's “NEAT” button.

- AC Code - The AC Code identifies cooling systems installed in the house to you and the Weatherization Assistant. The AC code must be unique for all cooling system descriptions within a given audit. NEAT automatically provides a default entry for all new cooling systems that are created in the form of "AC\#” where the "\#” is an integer insuring uniqueness of the AC code. You may choose to enter your own AC code to include characters that indicate the location, type, or some other characteristics of the cooling system. The AC code may have up to 20 characters, although space restrictions in many reports suggest a lesser number of characters should be used (perhaps 3 to 5) to preserve readability. Entries are not case-sensitive. NEAT will use the AC codes in output reports and work orders to identify which cooling system should receive recommended retrofit measures. The AC codes may also be helpful when labeling your drawing of the house's floor plan in case there are more than one unit (e.g., multiple window air conditioners). Required.

- Equipment Type - Select what type of cooling system this is. The choices are Central Air Conditioner, Window or Room Air Conditioner, Heat Pump, and Evaporative Cooler. If Evaporative Cooler has been selected, the subsequent fields requesting system performance characteristics (i.e., Capacity, SEER, and Year Manufactured) will be automatically hidden. Required. 


\section{Chapter 9: NEAT Building Description}

- Manufacturer and Model - Enter the manufacturer and model of the existing air-conditioning unit if needed for reference, possibly in establishing an appropriate replacement. See also description of Capacity below. Optional.

- Floor Area Cooled - Enter an estimate of the floor area that is cooled by the cooling system being described in square feet. The sum of all areas cooled by primary and secondary equipment should be less than or equal to the floor area of the house entered on the Audit Information form under the Main Menu's "NEAT" button. NEAT uses this information to calculate the cooling energy consumption as well as the savings from cooling system retrofit measures. Required.

- Capacity - Enter the output capacity of the unit in $\mathrm{kBtu} / \mathrm{h}$. The value is often printed on the nameplate of the air conditioner. For central systems and heat pumps, the value may be given in tons of cooling, where 1 ton equals 12 $\mathrm{kBtu} / \mathrm{h}$. Sometimes the model number will contain a value divisible by 6 , since cooling equipment is normally sold with capacities that are integral multiples of $1 / 2$ ton (e.g., a model xxx30xxx is likely a $30 \mathrm{kBtu} / \mathrm{h}$ or $2-1 / 2$ ton system). Otherwise enter an estimate. Required.

- SEER - Enter the efficiency of the cooling system in units of SEER (Seasonal Energy Efficiency Ratio). Usually, SEER is used in rating central air conditioners and heat pumps. Window air conditioners are rated in EER (Energy Efficiency Ratio). SEER is annual average efficiency of a cooling unit, while EER is a measure of efficiency averaged over several hours of constant operating condition. Both have units of Btu/watt-hour. An exact conversion between EER and SEER is not possible since they are measured at different operating conditions. An approximate conversion may be obtained using the following formulae:

SEER $=(1.2 \times$ EER $)-0.7$ (if the unit's fan runs only when conditioning is required).

SEER $=(0.9 \times$ EER $)+0.1$ (if the fan runs continuously while the unit is in use). 
If an SEER cannot be estimated, leave the field blank and press [Enter] while accessing the field. NEAT will display the Year Purchased field, allowing an alternate entry. Conditionally required.

- Year Manufactured - Enter the approximate year the unit was purchased new. Use the full year (e.g., “1986,” not “"96”). The purchase year is used to estimate the SEER, if it is not provided. This entry is hidden if a value has been provided in the SEER field. Conditionally required.

NEAT will evaluate replacing a central system with another central system and a window unit with another window unit. Costs associated with these replacements are specified in the Library Measures form of the Setup Library (see Section 14.5, Library Measures), with the replacement efficiencies as indicated in the Key Parameters/Equipment form under the Main Menu's “Setup Library” button (see Section 14.2, Key Parameters).

If your description of the heating equipment (see Sections 9.7, Heating - General Description Data, and 9.8, Required Heating System Details Sub-Form) allows for installation of a heat pump (i.e., the existing primary heating is supplied by a heat pump or electric resistance), NEAT will evaluate replacing the heating system and all cooling equipment by the single heat pump and recommend this if costeffective and more so than replacing each individual air conditioner.

The Required Retrofits data block in the middle of the Cooling form allows you to specify that system replacement or tune-up should be considered mandatory. Only one of the two selections can be chosen for each cooling equipment description. Selection of one when the other is checked will automatically eliminate the initial selection.

- Replacement Required - Select this checkbox if you have determined that the existing air conditioner being described must be replaced. NEAT will recommend the replacement regardless of its cost-effectiveness. In order for your selection of "Replacement with Heat Pump Mandatory" (see "Options" under the Electric Resistance Heating System or Heat Pump Details sub-forms in Section 9.8, Required Heating System Details Sub-Form) to be effective, this checkbox must be selected for the first cooling equipment description. 
- Tune-up Mandatory - Select this checkbox if inspection of the existing air conditioner indicates that a system tune-up is all that is necessary. NEAT will evaluate and recommend the system tune-up, regardless of its costeffectiveness. The tuned up system will then be used in evaluating other measures not considered mandatory. No system replacement will be considered.

- Include in SIR - If either the Replacement Required or Tune-up Mandatory checkboxes have been selected, an Include in SIR checkbox will appear on the form. This checkbox allows you to indicate that you wish to have the selected measure included in the calculation of the whole house (package) SIR for this audit. Measures not included in the whole house SIR are normally related to health and safety. Check the guidance for your program in deciding when you can mandate an HVAC measure and if it can be excluded from the SIR calculation. Even if the measure can be considered related to health and safety, if it is shown to be cost-effective, you may choose to still include it in the whole house SIR by selecting this checkbox. By doing so, it will not be seen as a health and safety measure, thus preserving funds dedicated for other such measures. An alternate reason for excluding a measure from the whole house SIR is if it is funded outside of the Weatherization Assistance Program. Whether a measure is or is not included in the whole house SIR is indicated by its location in the list of measures produced for the Recommended Measures Report (see Chapter 12, NEAT and MHEA Results).

- Comment - You may enter comments pertinent to the cooling system directly in the Comment field on the form, or you may enter them in the Comment Editor by selecting the "Comment" button to the left of the field. The Comment Editor is similar to Window's Notepad and may be used to enter extended comments. The total comment may have up to 65,000 characters. Text from other sources may be copied and pasted into the Comment Editor's window. Any comment that is entered is displayed on the Recommended Measures report and the Input Report available from the Audit Information form (see Section 8.2, Audit Information). In addition, any comment that is entered can be imported into any specific work order (see Section 13.1, Work Order Information). Thus, you may wish to enter comments related to any observation that might affect the installation of needed measures or repair work. Cooling system comments may include observations of equipment conditions, reasons for selecting specific retrofit options, or the need for specific maintenance (e.g., low refrigerant charge or dirty coils). Optional. 
- Cooling System Record Navigation Block - The Cooling System record navigation block is used to find and navigate to existing cooling system descriptions, copy or delete the currently accessed cooling system description, or create a new cooling system description for the audit. See Section 5.1, Form and Record Navigation for information on using the Weatherization Assistant's record navigation blocks. 


\section{Chapter 10. MHEA Building Description}

This chapter discusses the information entered on the building description forms under the Main Menu's "MHEA" button that is needed to describe manufactured homes and that is unique to this building type, as opposed to site-built, singlefamily houses. Prior to entering information on the forms described in this chapter, you must have already created the client whose house is being audited (see Section 7.1, Client Information), chosen to run a MHEA audit for this client, and entered required information on the Audit Information form for this audit (see Section 8.2, Audit Information).

The Shell tab contains information related to the Walls, Windows, Doors, Ceiling, and the Floor of the manufactured home. At least one form under each of these categories must be entered.

The Addition tab allows the description of an addition to the mobile home and has the same catagories of components as the Shell tab-that is, Walls, Windows, Doors, Ceiling, and Floor. All entries under this tab may be left blank, but if entry is begun on any one of the Walls, Ceiling, or Floor forms, all three of these forms must be completed. The Windows and Doors forms under the Addition tab are optional.

The Heating (Primary, Secondary, and Replacement), and Cooling (Primary, Secondary, and Replacement) forms allow descriptions of the home's HVAC equipment. The Primary Heating form must be filled out for each home, but the remaining forms under these two tabs are optional. The entries on the above forms are discussed in this chapter.

Chapter 11, NEAT and MHEA Common Elements, discusses the remaining forms under the Main Menu's "MHEA" button, those common to both NEAT and MHEA. These include the Status, Ducts/Infiltration, Base Loads (Water Heating, Refrigerators, and Lighting Systems), Health \& Safety (Whole House, Equipment, and Building Shell), Itemized Costs, Utility Bills, Photos, and Measures forms, as well as some optional sub-forms found on the Heating and Water Heating forms. Of these forms, only the Ducts/Infiltration form is required.

On many of the tabs and sub-tabs under the Main Menu's "MHEA” button, numbers in parentheses are displayed. These numbers indicate the number of 


\section{Chapter 10: MHEA Building Description}

descriptions, or records, that have been entered for that particular component type. Some of the component types in MHEA can have only a single record described, for example, Walls. Thus the number indicated for walls will never be greater than 1. See the individual descriptions below for additional information regarding the number of records possible for each component type.

Finally, the Recommended Measures report that is typically displayed immediately after running a MHEA audit is discussed in Chapter 12, NEAT and MHEA Results.

\subsection{Shell - Walls}

Unlike NEAT, MHEA uses only one form to describe the characteristics of the walls (see Figure 10.1). MHEA assumes that the house is rectangular in shape with wood-frame construction, and the walls facing different orientations have identical construction characteristics.

Below are brief descriptions of the individual data items and controls found on the Walls form. All items on the Walls form are required except the Uninsulatable Wall Area, Additional Cost, and Comment fields.

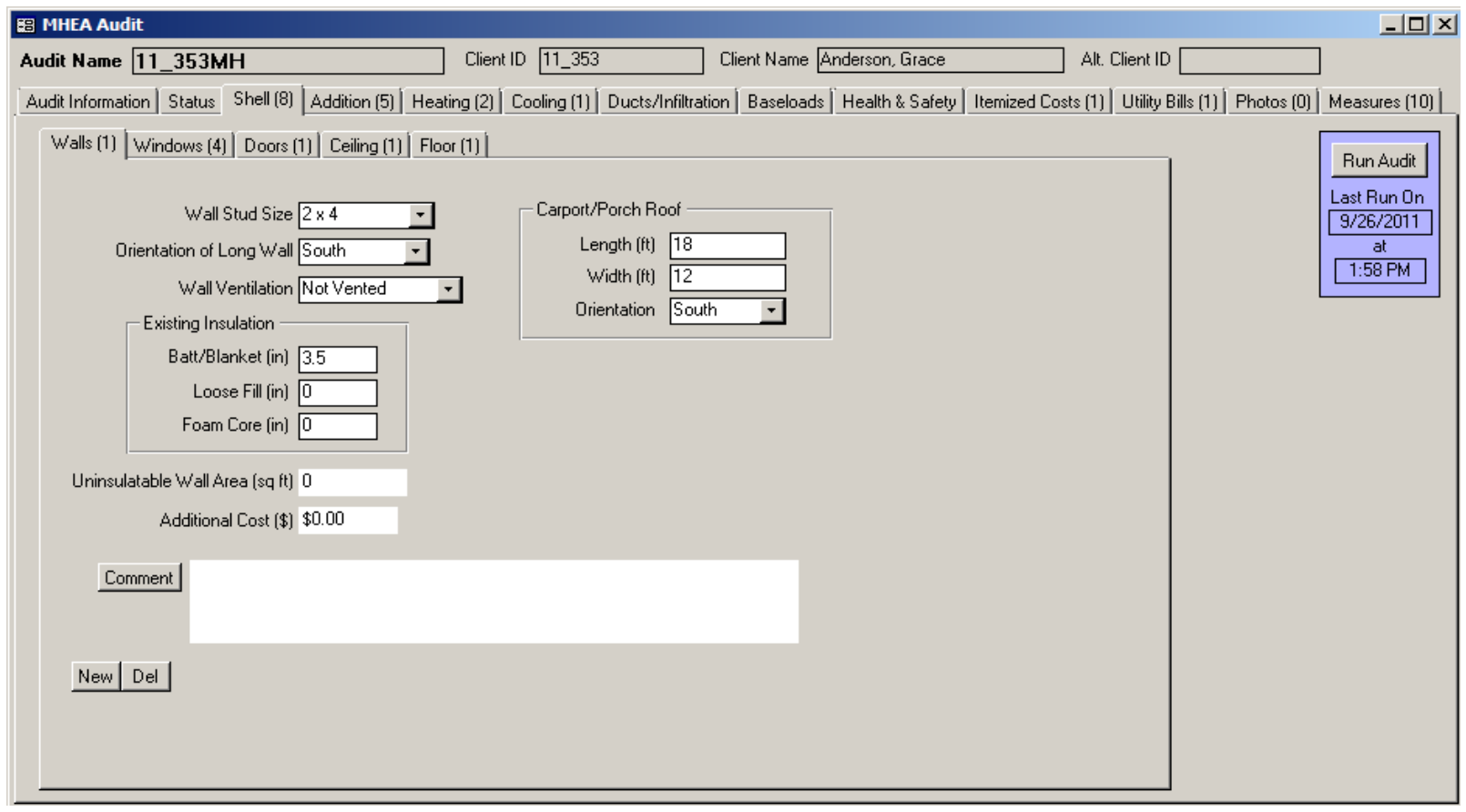

Figure 10.1. The Shell - Walls form under the Main Menu's “MHEA” button. 
- Wall Stud Size - Select the stud size used in framing the walls. The choices are $2 \times 2,2 \times 3,2 \times 4$, and $2 \times 6$. MHEA uses this information to calculate the wall Rvalue and the volume of wall cavity available for added insulation. Required.

- Orientation of Long Wall - Select the closest cardinal compass direction that one of the long walls of the home faces. The choices are North, South, East, and West. For this entry, MHEA considers North the same as South and East the same as West, but you might adopt a convention such as entering the orientation of the front wall as the long wall. MHEA uses this information to calculate solar loads on the home. Required.

- Wall Ventilation - Indicate whether the walls are vented or not vented. The walls of the mobile home may be intentionally ventilated to remove accumulated moisture by having corrugated metal siding open at the bottom to provide space for air to flow between the exterior and interior wall materials. Small holes in the siding at the bottom and top of the exterior wall surface may be visible, allowing the air to circulate. MHEA degrades the wall R-value for ventilated walls. Required.

- Existing Insulation Type and Thickness - MHEA assumes that exterior walls of the mobile home may have Batt/Blanket, Loose Fill, or Foam Core insulation. Enter the thickness of existing insulation type in inches. Enter 0 for insulation types that do not exist. MHEA uses this information to calculate the wall R-value and the volume of wall cavity available for additional insulation. You may view, and, if necessary, adjust the Rs/Inch MHEA assigns to these existing insulation types from the Key Parameters/Insulation form in MHEA's Setup Library (see Section 14.2, Key Parameters). A default value of 3.5 inches exists. Required.

- Carport/Porch Roof Length, Width and Orientation - A carport/ porch roof is a shelter with no walls that extends out from one wall of the mobile home (see Figure 10.2). Often, this shelter is used to park a car or shade an outdoor sitting area. Enter the length and width of this carport/porch roof in feet. The length is the dimension parallel to the mobile home. Enter 0 for the length and width if no carport/porch roof exists. 


\section{Chapter 10: MHEA Building Description}

If the length or width of the carport/porch roof is greater than 0 , the Orientation field appears. Enter the orientation of the wall to which the carport/porch is attached. MHEA allows description of only one carport or porch. For more than one carport or porch attached to the home, describe the one that provides the most shading to the walls. Thus, if you have to choose, you should

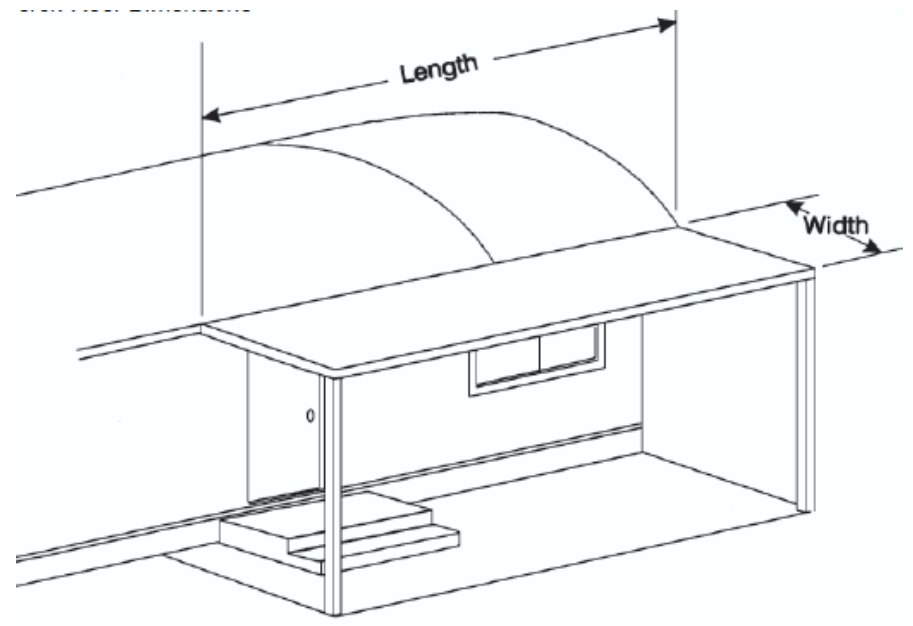

Figure 10.2. Carport/Porch Roof Dimensions. first consider omitting the description of porch attached to the north wall of the home. The carport/porch description can also be used to describe extensive shading of a wall due to trees or shrubs. Enter pseudo dimensions sufficient to represent the desired degree of shading and orientation of the shaded wall. Required.

- Uninsulatable Wall Area - Enter the gross area of the wall in square feet that is not accessible for insulating. MHEA already accounts for wall studs and framing around windows and doors. An example of uninsulatable wall is a wall segment between an attached unconditioned space and the home. Note: Wall area associated with a conditioned addition to the home that is described under the Addition tab is automatically excluded. Optional.

- Additional Cost - Enter any additional cost associated with insulating the walls in units of dollars. This would be a cost that is not normally associated with insulating the walls and, therefore, is not accounted for in the measure costs entered on the Library Measures form under the Setup Library (see Section 14.5, Library Measures). The cost is the total additional cost to insulate the walls; it is not a cost per square foot of wall area. The amount entered will be added to the normal cost computed from the wall area and the cost per square foot specified on the Library Measures form in the Setup Library. A blank entry indicates no additional cost. If a cost is entered, you should briefly explain the reason for this additional cost in the Comment field described below. Optional. 
- Comment - You may enter comments pertinent to the walls directly in the Comment field on the form, or you may enter them in the Comment Editor by selecting the "Comment" button to the left of the field. The Comment Editor is similar to Window's Notepad and may be used to enter extended comments. The total comment may have up to 65,000 characters. Text from other sources may be copied and pasted into the Comment Editor's window. Any comment that is entered is displayed on the Recommended Measures report and the Input Report available from the Audit Information form (see Section 8.2, Audit Information). In addition, any comment that is entered can be imported into any specific work order (see Section 13.1, Work Order Information). Thus, you may wish to enter comments related to any observation that might affect the installation of needed measures or repair work. Optional.

- New/Del - Since there can be only one Wall record in MHEA, the "New" button will not initiate a new blank Walls form. Instead, it takes you back to the first field on the existing form, the Wall Stud Size field, while not changing any existing entries. The "Del" button will erase all entries on the Walls form. You will receive a warning of the deletion only if the form had been previously filled in prior to your entering the form.

\subsection{Shell - Windows}

The Windows form (see Figure 10.3) under MHEA's Shell tab is used to enter information on all the windows installed in the house. A total of 24 window descriptions (i.e., Window Codes) may be entered. Multiple windows with the same characteristics facing any orientation may be entered as a single window description by indicating the number of windows facing different orientations having this description. However, similar windows considered for different retrofit measures should be described separately. If the 24 window records are not enough to describe all the windows, you will have to enter windows with characteristics that are close to others under one common window description. Avoid combining the areas of two or more windows into one so that just one window needs to be entered. Window treatments in MHEA are priced either per window unit, by window area, or per united inch. Combining two or more window areas into one large area can lead to errors in estimating the cost of the window treatments regardless of pricing scheme used. The thermal characteristics of existing and replacement windows, with or without storms, are specified in the Key 


\section{Chapter 10: MHEA Building Description}

Parameters/Windows form under the Main Menu's “Setup Library” button (see Section 14.2, Key Parameters).

Below are brief descriptions of the individual data items and controls found on the Windows form. All items on the Windows form are required except the Frame Type, Additional Cost and Comment fields.

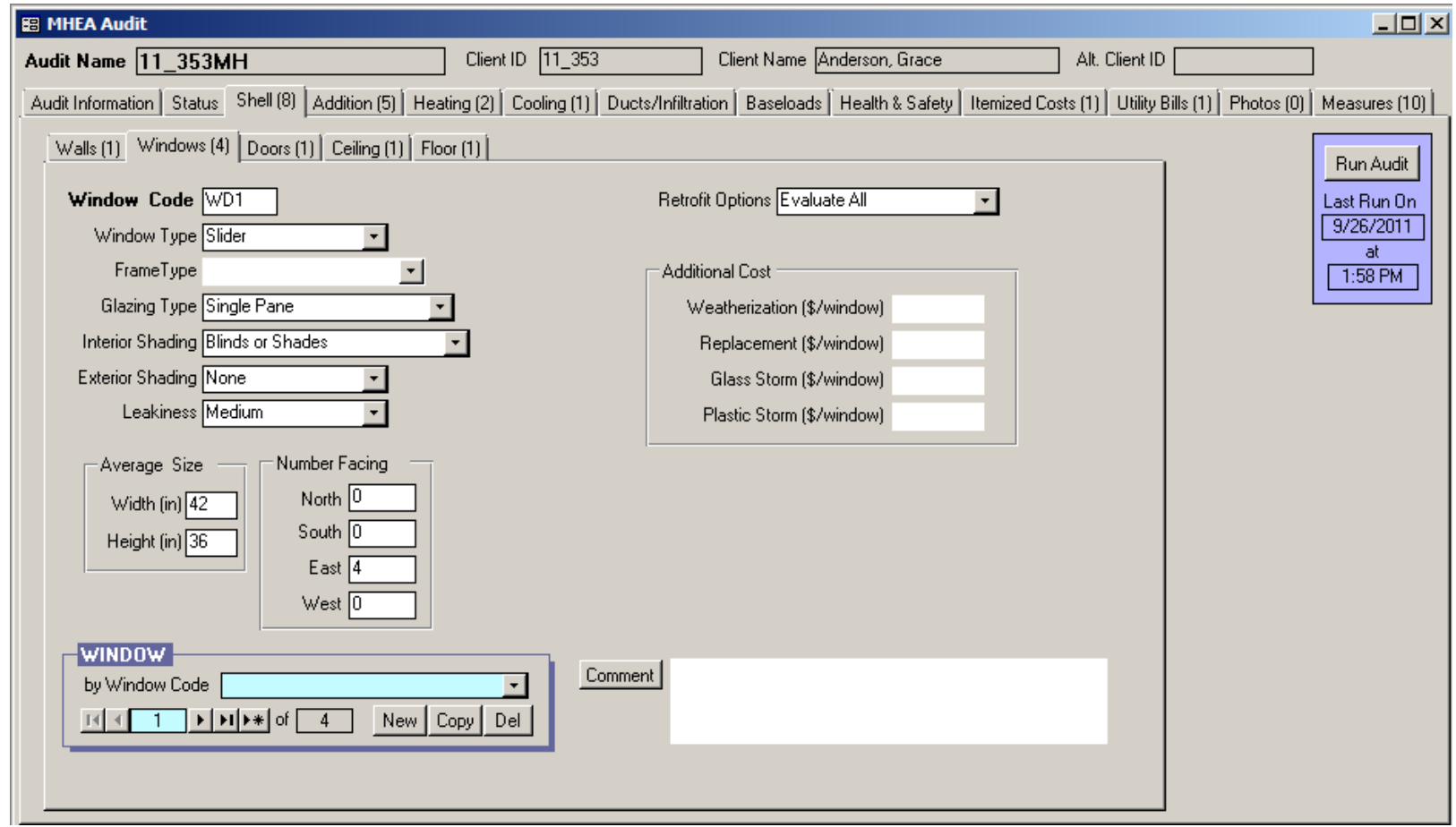

Figure 10.3. The Shell - Windows form under the Main Menu's “MHEA” button.

- Window Code - The Window Code identifies window descriptions to you and the Weatherization Assistant. If more than one window has the same description, you may enter the description once and indicate the number of windows with this description using the Number Facing field described below. The window codes must be unique for all window descriptions within a given audit. MHEA automatically provides a default entry for all new windows that are created in the form of "WD\#" where the "\#” is an integer insuring uniqueness of the window code. You may choose to enter your own window code to include characters that indicate some characteristics of the window. The window code may have up to 20 characters, although space restrictions in many reports suggest a lesser number of characters should be used (perhaps 3 to 5) to preserve readability. Entries are not case-sensitive. MHEA will use the window codes in output reports and work orders to identify which windows should 
receive weatherization measures. The window codes may also be helpful when labeling your drawing of the house's floor plan. Required.

- Window Type - Select what basic type of window this is. The choices are Jalousie, Awning, Slider, Fixed, Door Window, Sliding Glass Door, and Skylight. These choices are shown in Figure 10.4 and described below. MHEA does not use the window type in any of its calculations, except to exclude those of type "Sliding Glass Door" and "Door Window" from being considered for replacement. Otherwise, this entry is provided for your benefit, so that you can clearly identify and recall the type of window being described. In addition, you can better determine the correct entry for the Leakiness field described below if you know the window type. Required.

Jalousie windows are constructed of multiple horizontal panes of glass (about 2-6 inches wide) that open at the same angle when a crank near the bottom of the window is turned. Jalousie windows are always single pane. When closed, large gaps between the glass panes often exist, which results in high infiltration rates through the window.

Awning windows are constructed of 1-3 glass sashes that are hinged at the top, bottom, or side of the sashes. When the window is opened (often by turning a crank), the sashes angle out from the home exterior. The sashes either angle down, up, or to the side depending on where the sashes are hinged to the window frame. An awning window with a single sash of glass is often called a casement window if it is hinged on the side or a hopper window if it is hinged on the bottom.

Slider windows usually have two sashes of glass. One or both sashes can slide past the other either horizontally or vertically when the window is opened.

Fixed windows are sealed in the window frame and cannot be opened.

Door windows are usually sealed in the window frame of a door and cannot be opened, although sometimes they are made to be opened.

Sliding Glass Doors are large windows that extend from the floor to almost the ceiling. You may use this selection to describe picture windows even though they are fixed and cannot be opened. 


\section{Chapter 10: MHEA Building Description}

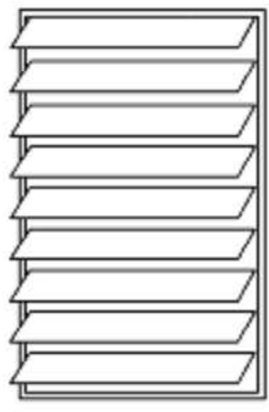

Jalousie

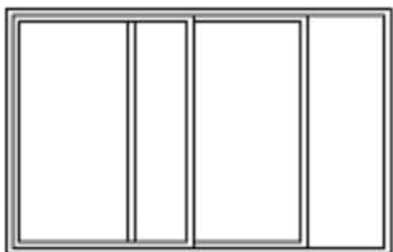

Horizontal Slider

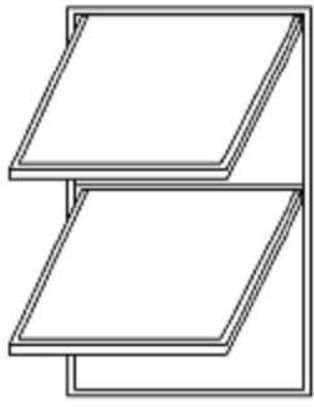

Awning

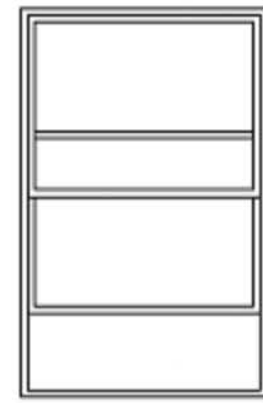

Vertical Slider

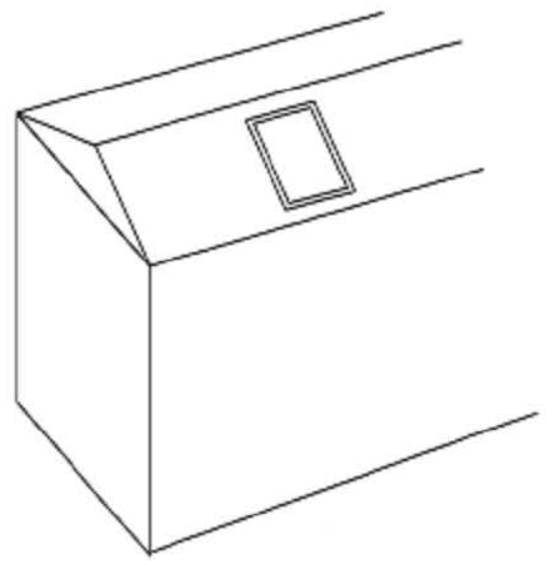

Skylight

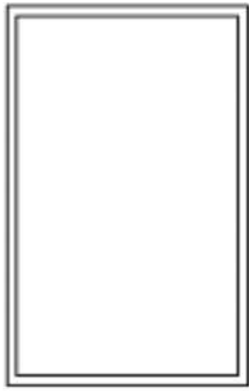

Fixed

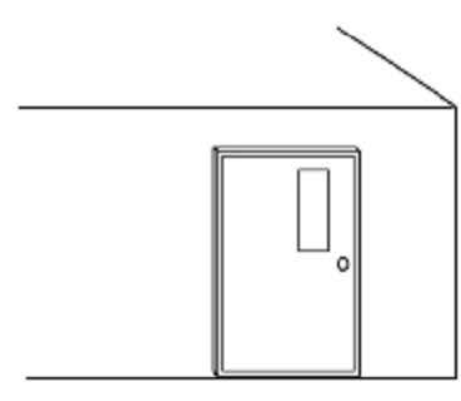

Door

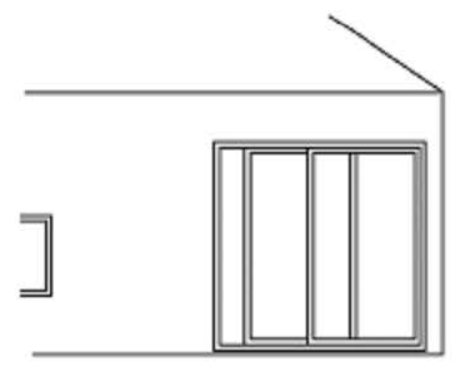

Sliding Glass Door

Typica/Manufactured Home Window Types

Figure 10.4. Basic types of windows. 
Skylights are glass or plastic windows in the ceiling.

- Frame Type - Select the window frame and sash construction materials. The choices are Wood or Vinyl, Metal, and Improved Metal (i.e., metal frame with a thermal break). MHEA does not use this information at this time and assumes all window frames in the mobile home to be improved metal. This assumption is reflected in the U-values assigned to windows in the Setup Library and can be altered by the user if desired (see Section 14.2, Key Parameters). Optional.

- Glazing Type - Select the glazing type depending on the number of panes of glass in the primary window and the characteristics of any storm window that may cover the primary window. The choices are Single Pane, Single with Glass Storm, Single with Plastic Storm, Double Pane, Double with Glass Storm, and Double with Plastic Storm. Required.

- Interior Shading - Select the interior window coverings that are present which, when closed, prevent sunlight from entering the home and also slightly increase the insulating value of the window. The options are Drapes, Blinds or Shades, Drapes with Blinds or Shades, and None. Drapes are heavy curtains that hang in loose folds over the window. Decorative drapes that don't block much sun should be ignored. Blinds or shades are lightweight window coverings that hang flat over the window and include Venetian blinds, minishades, and roller shades. Select drapes with shades if the occupant has both drapes and blinds/shades on the window. Required.

- Exterior Shading - Select the type of exterior window shading present outside the window and which reduces the sunlight striking the window. The choices are Awning, Carport or Porch, Low-e Film, Sunscreen, and None. An awning is a device that is mounted above a window and extends out from the exterior of the home. It is usually constructed of aluminum, fiberglass, or fabric. A carport/porch roof is a shelter that has no walls and extends out from one wall of the manufactured home. Often, the home occupant will park a car under this shelter or use it to shade an outdoor sitting area. A low-e film is a reflective film applied to the exterior surface of glass window panes by the window manufacturer. A sun screen is a mesh screen that is installed or hung on the exterior side of a window. You may need to estimate the effect of other objects that consistently shade a window using one of the entries provided. For example, an object that reduces the amount of sunlight striking a window by 


\section{Chapter 10: MHEA Building Description}

$50 \%$ might be described as an Awning. If the window is shaded most of the time, you could use the Carport or Porch entry. Required.

- Leakiness - Provide an estimate of how leaky the window is. The choices are Very Tight, Tight, Medium, Loose, and Very Loose. Typical selections by window type are listed below. Degrade the leakiness one level if 2 to 9 sq. in. of glass is missing in the window and two levels if 9 to 25 sq. in. of glass is missing. Specify the window to be Very Loose if more than 25 sq. in. of glass is missing in the window. Upgrade the leakiness one level if a storm window in average or better condition is installed. Additional guidance is provided in Appendix E, Window Leakiness. Required.

Jalousie window - The leakiness of a jalousie window is typically Loose.

Awning window - The leakiness of an awning window that has a single sash of glass and a latching/locking mechanism that seals the window by forcing the sash against the frame and any installed weather stripping is typically Tight. Such a window is often called a casement window if it is hinged on the side or a hopper window if it is hinged on the bottom. The leakiness of an awning window with 2 or 3 sashes of glass or without a latching/locking mechanism is typically Medium.

Slider window and sliding glass door - The leakiness of a vertical slider window is typically Medium if it is older and Tight if it is newer. The leakiness of a horizontal slider window or sliding glass door is typically Medium.

Fixed window, door window, and skylight - The leakiness of a fixed window, door window, or skylight is typically Very Tight.

- Width and Height - Enter the width and height of the window (including the window frame) in units of inches (accuracy to the nearest inch is usually satisfactory). MHEA will use these dimensions together with the number of windows being described by this Window Code (see Number Facing below) to estimate the total window area facing different orientations, and it will subtract this total area from the appropriate wall area based on the wall orientation. Required. 
- Number Facing North, South, East, and West - Enter the number of windows with this window description facing closest cardinal directions. Required.

- Retrofit Options - Select one of the approaches for retrofitting the window. The options are Evaluate All, Weatherize, Replace, Add Glass Storm, Add Plastic Storm, and Evaluate None. Evaluate All allows MHEA to determine if it is cost-effective to weatherize, replace, or install a glass or plastic storm window on the windows. If more than one of these retrofits is cost-effective, MHEA will select the best measure. If none of the measures are cost-effective, then no measure will be recommended. Choosing Weatherize (weatherizing the window using caulk, weather stripping, incidental repairs, etc.), Replace (replacing the window, often because it is damaged beyond repair), or Add Glass Storm or Plastic Storm (adding a glass or a plastic storm window to a window that currently does not have a storm window) indicates your decision to perform the selected retrofit measure regardless of its cost-effectiveness and to not perform the other two measures. These selections tell MHEA to make the selected retrofit a mandatory measure. MHEA will recommend the measure regardless of its cost-effectiveness and will not evaluate the other two options. However, MHEA will estimate the energy savings and SIR for the measure. If the SIR for the measure is less than your accepted minimum value, you will need to reconsider your selection or consider the measure a repair. Evaluate None prevents MHEA from evaluating the cost-effectiveness of weatherizing, replacing, or installing a glass or a plastic storm window on the window, which effectively turns off all retrofits for this window. Reasons for selecting any option other than Evaluate All for this window description would be an appropriate entry in the Comment field described below. Required.

- Include in SIR - If the Retrofit Option selected is Weatherize, Replace, Add Glass Storm, or Add Plastic Storm, an Include in SIR checkbox will appear on the form. This checkbox allows you to indicate that you wish to have the selected measure included in the calculation of the whole house (package) SIR for this audit. Measures not included in the whole house SIR are normally related to health and safety. Current Weatherization Program policy dictates that window replacement cannot be considered a health and safety measure. Check the guidance for your program in deciding when you can mandate a window treatment and if it can be excluded from the SIR calculation. An alternate reason for excluding a measure from the whole house SIR is if it is 
funded outside of the Weatherization Assistance Program. Whether a measure is or is not included in the whole house SIR is indicated by its location in the list of measures produced for the Recommended Measures Report (see Chapter 12, NEAT and MHEA Results).

- Additional Cost - If the Retrofit Option selected is any option other than Evaluate None, then Additional Cost fields will appear separately for Weatherization, Replacement, Glass Storm and Plastic Storm windows. Enter any additional cost associated with performing these retrofits on the window being described that is in addition to the cost that MHEA will automatically calculate using the cost information you entered in the Library Measures form of the Setup Library (see Section 14.5, Library Measures). For example, these might include the cost for removing any security bars before replacement or storm windows can be installed. The cost to be entered is a cost per window, not a total cost for all the windows indicated in the Number field. The reason for this extra cost would be an appropriate entry in the Comment field described below. Optional.

- Comment - You may enter comments pertinent to the window directly in the Comment field on the form, or you may enter them in the Comment Editor by selecting the "Comment" button to the left of the field. The Comment Editor is similar to Window's Notepad and may be used to enter extended comments. The total comment may have up to 65,000 characters. Text from other sources may be copied and pasted into the Comment Editor's window. Any comment that is entered is displayed on the Recommended Measures report and the Input Report available from the Audit Information form (see Section 8.2, Audit Information). In addition, any comment that is entered can be imported into any specific work order (see Section 13.1, Work Order Information). Thus, you may wish to enter comments related to any observation that might affect the installation of needed measures or repair work. Optional.

- Window Record Navigation Block - The Window record navigation block is used to find and navigate to existing window descriptions for the audit being edited, copy or delete the currently accessed window description, or create a new window description for the audit. See Section 5.1, Form and Record Navigation for information on using the Weatherization Assistant's record navigation blocks. 


\section{Chapter 10: MHEA Building Description}

\subsection{Shell - Doors}

The Doors form (see Figure 10.5) under the Main Menu's "MHEA" button is used to enter information on all the doors installed in the house. A total of 10 door descriptions (i.e., Door Codes) may be entered. Multiple doors with the same characteristics facing any orientation may be entered as a single door description by indicating the number of doors facing different orientations having this description. However, similar doors considered for different retrofit measures should be described separately. If the 10 door records are not enough to describe all the doors, you will have to enter doors with characteristics that are close to others under one common door description.

Below are brief descriptions of the individual data items and controls found on the Doors form. All items on the Doors form are required except the Additional Cost and Comment fields.

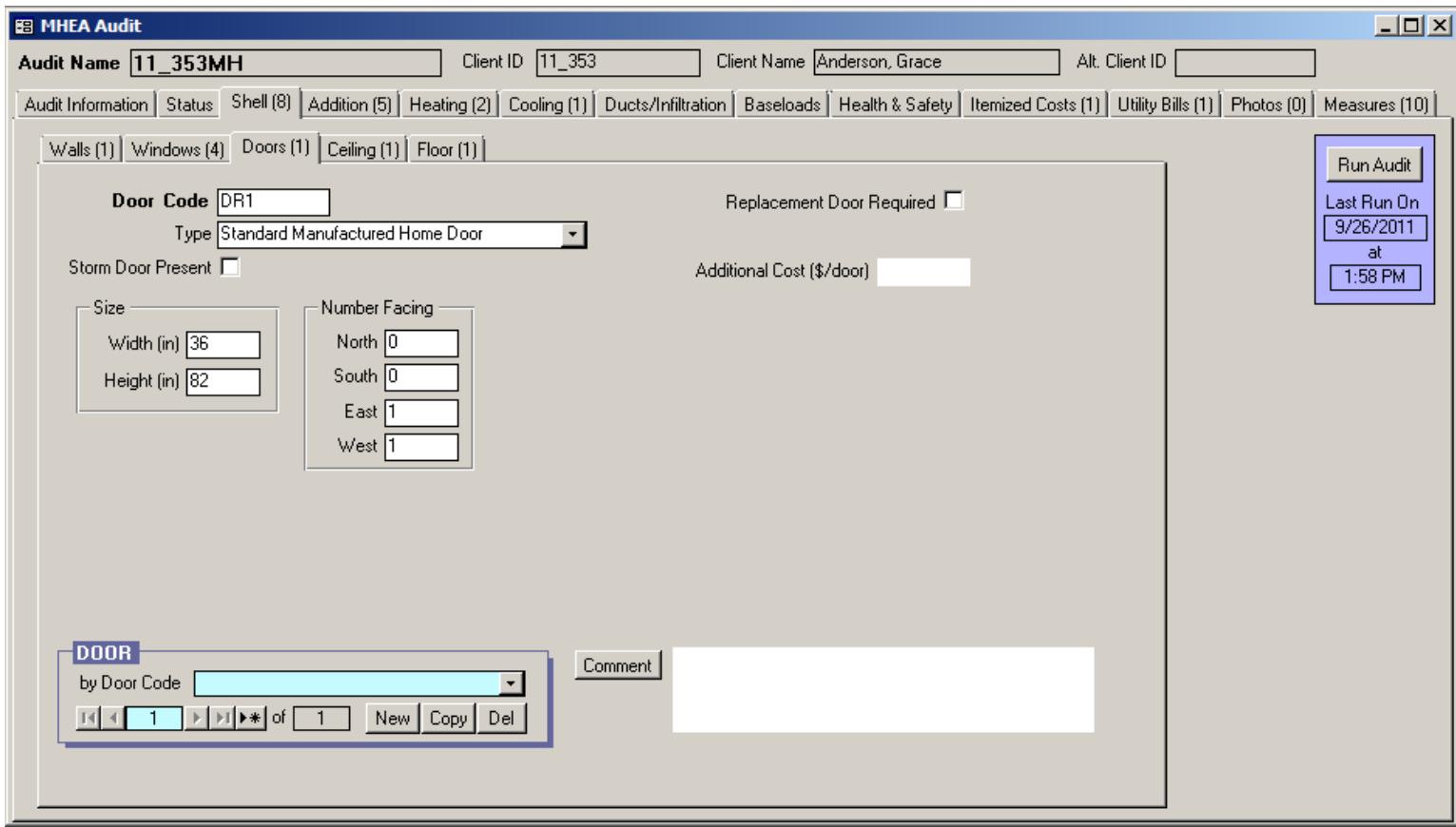

Figure 10.5. The Shell - Doors form under the Main Menu's “NEAT” button.

- Door Code - The Door Code identifies door descriptions to you and the Weatherization Assistant. If more than one door has the same description, you may enter the description once and indicate the number of doors with this description using the Number Facing field described below. The door codes 


\section{Chapter 10: MHEA Building Description}

must be unique for all door descriptions within a given audit. MHEA automatically provides a default entry for all new doors that are created in the form of "DR\#” where the "\#” is an integer insuring uniqueness of the door code. You may choose to enter your own door code to include characters that indicate the orientation or some other characteristics of the door. The door code may have up to 20 characters, although space restrictions in many reports suggest a lesser number of characters should be used (perhaps 3 to 5) to preserve readability. Entries are not case-sensitive. MHEA will use the door codes in output reports. The door codes may also be helpful when labeling your drawing of the house's floor plan. Required.

- Door Type - Select what type of door this is. The choices are Hollow Core Wood, Solid Core Wood, Standard Manufactured Home Door, and Insulated Steel. The U-value assumed for each of these types is specified in the Key Parameters/Doors form under the Main Menu's "Setup Library” button (see Section 14.2, Key Parameters). Required.

- Storm Door Present - Select this checkbox to indicate if any storm door is installed on this door. If this field is unchecked, MHEA will evaluate installing a storm door as one of the retrofit measure, if this measure is selected on the Library Measures form of the Main Menu's “Setup Library” button (see Section 14.5, Library Measures).

- Width and Height - Enter the width and height of the door opening in units of inches. Required.

- Number Facing North, South, East, and West - Enter the number of exterior doors with this door description facing the closest cardinal directions. Required.

- Replacement Door Required - Select this checkbox to indicate that door replacement is required (often because it is in poor condition and beyond repair). MHEA will make this a mandatory measure and recommend it regardless of its cost-effectiveness. If the SIR of the measure is less than your accepted minimum value, you will have to reconsider requiring the door be replaced or consider its replacement a repair. If unchecked, MHEA will recommend this measure only if it is cost-effective. The U-value assumed for any replaced door is specified in the Key Parameters/Doors form under the Main Menu’s “Setup Library” button (see Section 14.2, Key Parameters). 
- Include in SIR - If the Replacement Door Required checkbox is selected, an Include in SIR checkbox will appear on the form. This checkbox allows you to indicate whether you wish to have the door replacement included in the calculation of the whole house (package) SIR for this audit. Measures not included in the whole house SIR are normally related to health and safety. Check the guidance for your program in deciding when you can mandate a door replacement and if it can be excluded from the SIR calculation. An alternate reason for excluding a measure from the whole house SIR is if the measure is being funded outside of the Weatherization Assistance Program. Whether a measure is or is not included in the whole house SIR is indicated by its location in the list of measures produced for the Recommended Measures Report (see Chapter 12, NEAT and MHEA Results).

- Additional Cost - Enter any additional cost associated with door replacement that is in addition to the cost that MHEA will automatically calculate using the cost information you entered in the Library Measures form of the Setup Library (see Section 14.5, Library Measures). The cost to be entered is a cost per door, not a total cost for all the doors indicated in the Number field. The reason for this extra cost would be an appropriate entry in the Comment field described below. Optional.

- Comment - You may enter comments pertinent to the door directly in the Comment field on the form, or you may enter them in the Comment Editor by selecting the "Comment" button to the left of the field. The Comment Editor is similar to Window's Notepad and may be used to enter extended comments. The total comment may have up to 65,000 characters. Text from other sources may be copied and pasted into the Comment Editor's window. Any comment that is entered is displayed on the Recommended Measures report and the Input Report available from the Audit Information form (see Section 8.2, Audit Information). In addition, any comment that is entered can be imported into any specific work order (see Section 13.1, Work Order Information). Thus, you may wish to enter comments related to any observation that might affect the installation of needed measures or repair work. Optional.

- Door Record Navigation Block - The Door record navigation block is used to find and navigate to existing door descriptions for the audit being edited, copy or delete the currently accessed door description, or create a new door 


\section{Chapter 10: MHEA Building Description}

description for the audit. See Section 5.1, Form and Record Navigation, for information on using the Weatherization Assistant's record navigation blocks.

\subsection{Shell - Ceiling}

The Ceiling form (see Figure 10.6) under the Main Menu's "MHEA" button is used to enter information on roof/ceiling in the house. Unlike NEAT, only one roof/ceiling segment can be described in MHEA.

Below are brief descriptions of the individual data items and controls found on the Ceiling form. All items on the Ceiling form are required except the Existing Insulation, Additional Cost and Comment fields.

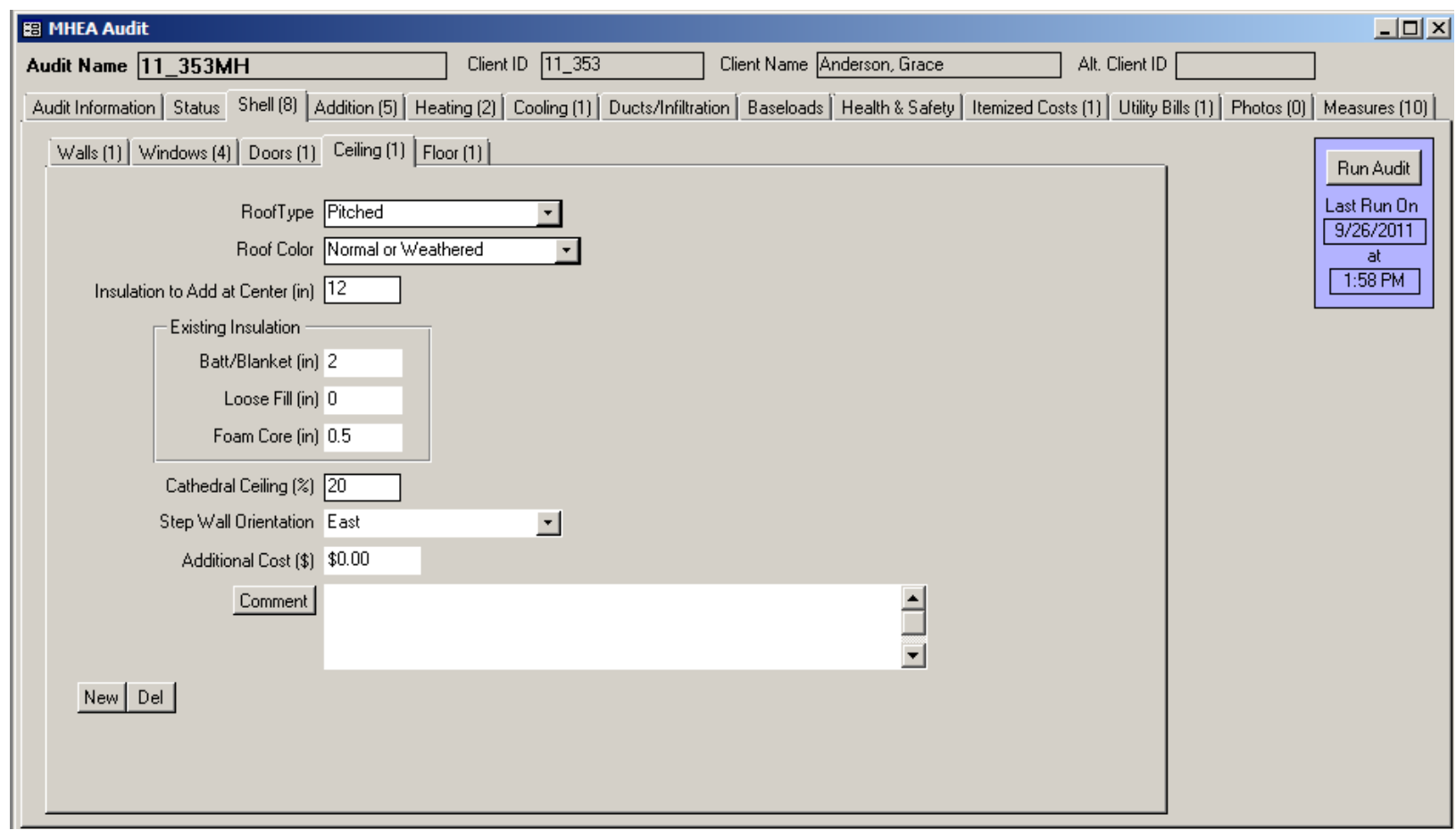

Figure 10.6. The Shell - Ceiling form under the Main Menu's “NEAT” button.

- Roof Type - Select what type of roof this is. The choices are Flat, Bowstring, and Pitched. These are the typical roof configurations found in mobile homes (see Figure 10.7). The characteristics of these roof types and how MHEA treats them are described below. 


\section{Chapter 10: MHEA Building Description}

- Flat roofs have an interior surface (ceiling) and an exterior surface (roof) attached directly to either side of the wood framing, similar to wall construction.

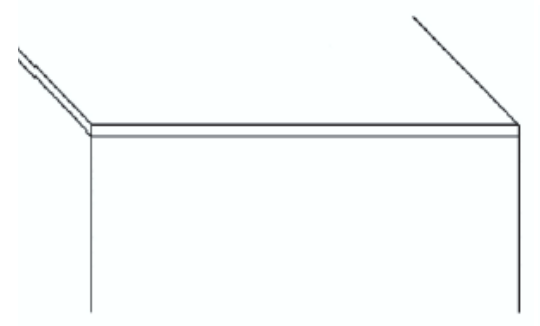

Flat Roof

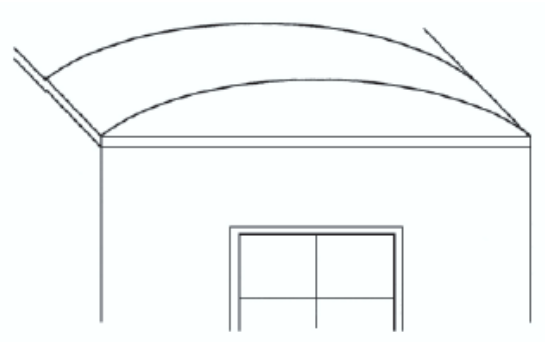

Bowstring Roof

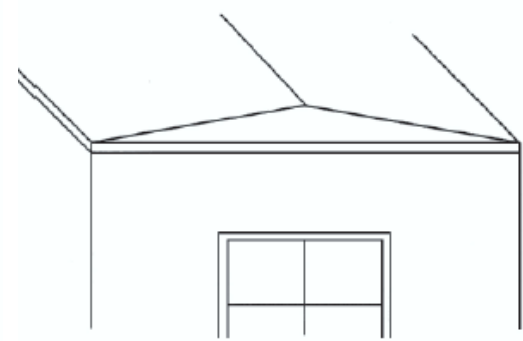

Pitched Roof

Figure 10.7. Typical Manufactured Home Roof Configuration.

- Bowstring roofs have a lightweight exterior roofing material (usually aluminum) that is stretched over the roof frame structure. The roof has a slight curve with the highest point in the middle of the home width. MHEA uses the home width and the height of the roof you specify to calculate the volume of a bowstring roof section.

- Pitched roofs are sloped to a higher peak point. When viewing the home width from the outdoors, the roof line creates a triangle. Pitched roofs are also often shingled, unlike flat and bowstring roofs. MHEA uses the width of the home and the height of added insulation you provide to calculate the volume of added insulation for the roof insulation measures.

NOTE: In some cases, there will be a second roof constructed over the original manufactured home roof. Enter construction information describing the original manufactured home roof since it is under this roof that insulation may be added. Enter the roof color of the second exterior roof. Required.

- Roof Color - Enter the color of the roof. The two choices are "White, Reflective or Shaded" and "Normal or Weathered". A White or Reflective roof appears white or shiny metallic, is clean, and has little or no discoloration due to weathering. Roofs completely shaded by trees in the summer should be categorized as white or reflective regardless of their finish. The majority of roofs will be classified as Normal or Weathered. Roof surfaces designated as White or Reflective will not be considered when evaluating the White Coat Roof measure. Required. 
- Joist Size - If the roof type selected is Flat, a Joist Size field will appear on the form. The choices are $2 \times 4,2 \times 6$, and $2 \times 8$. MHEA uses this information to calculate the volume of roof cavity available for adding insulation. Conditionally required.

- Height of Roof at Center - If the roof type selected is Bowstring, a Height of Roof at Center field will appear on the form. Enter the maximum height in inches of the roof above the ceiling, disregarding any existing insulation. MHEA uses this information to determine the available space for additional insulation. This field is not provided if a flat or pitched roof is selected. Conditionally required.

- Insulation to Add at Center - If the roof type selected is Pitched, an Insulation to Add at Center field will appear on the form. Enter the thickness of added insulation in inches you want MHEA to evaluate. The pitch of the roof may be such that filling the roof cavity would not be cost-effective in your climate, whereas a lesser level would. Local standard practice or experience with the predictions of MHEA will help establish the most appropriate levels to use. MHEA will not consider adding more than 12 inches of insulation. Enter 0 if you do not want MHEA to evaluate any roof insulation measures. Conditionally required.

- Existing Insulation Type and Thickness - MHEA assumes that the roof of the mobile home may have Batt/Blanket, Loose Fill, or Foam Core insulation. Enter the thickness of existing insulation by type in inches measured at the center of the roof/ceiling section. Enter 0 or leave the field blank for insulation types that do not exist. MHEA uses this information to calculate the roof/ceiling $\mathrm{R}$-value and also to calculate the volume in the roof/ceiling cavity for flat and bowstring roofs that is available for additional insulation. You can ignore compression or different depths of insulation across the roof/ceiling. You may view, and, if necessary, adjust the Rs/Inch MHEA assigns to these existing insulation types from the Key Parameters/Insulation form in MHEA's Setup Library (see Section 14.2, Key Parameters). Optional.

- Cathedral Ceiling - A Cathedral ceiling is a sloped or vaulted ceiling where the ceiling and roof planes are parallel. Enter the percent of floor area that is under a cathedral ceiling. Enter 0 if no segment of the mobile home floor has a cathedral ceiling. Required. 


\section{Chapter 10: MHEA Building Description}

\section{- Step Wall}

Orientation - If the roof type selected is flat or pitched and the percent of cathedral ceiling is greater than 0 , the Step Wall

Orientation field will appear. Some

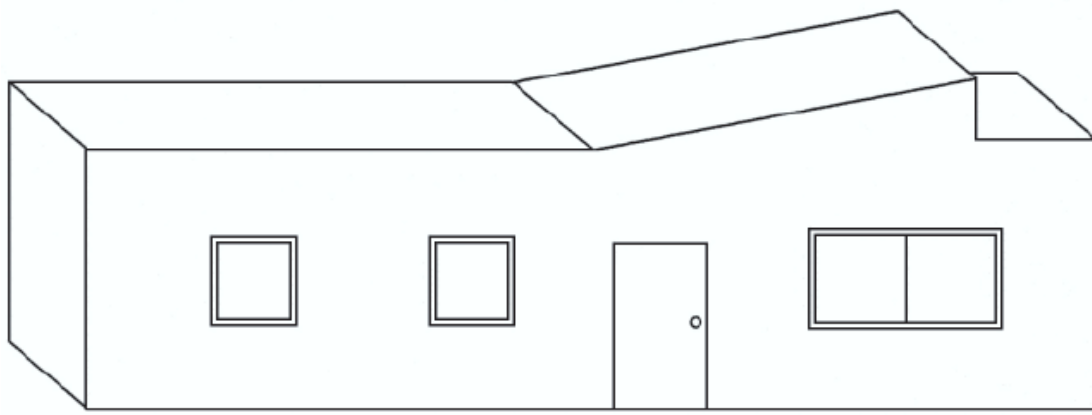

Figure 10.8. Mobile Home Cathedral Ceiling with Step Wall. manufactured home ceilings slope up to the top of a short wall. This short wall then steps vertically down to the average home height (see Figure 10.8). Select the closest cardinal direction that the step wall faces. The choices are North, South, East, West, and No Step Wall. Enter No Step Wall if a step wall does not exist. Optional.

- Additional Cost - Enter any additional cost associated with insulating this roof/ceiling cavity in dollars. This would be a cost that is not normally associated with insulating the roof and, therefore, is not accounted for in the measure costs entered on the Library Measures form under the Setup Library (see Section 14.5, Library Measures). For instance, enter the additional costs associated with cutting an opening into the roof to access the cavity. The cost is the total additional cost to insulate the entire cavity; it is not a cost per square foot of attic area. The amount entered will be added to the normal cost computed from the attic characteristics and costs specified on the Library Measures form in the Setup Library. A blank entry indicates no additional cost. If a cost is entered, you should briefly explain the reason for this additional cost in the Comment field described below. Optional.

- Comment - You may enter comments pertinent to the roof/ceiling section directly in the Comment field on the form, or you may enter them in the Comment Editor by selecting the "Comment" button to the left of the field. The Comment Editor is similar to Window's Notepad and may be used to enter extended comments. The total comment may have up to 65,000 characters. Text from other sources may be copied and pasted into the Comment Editor's window. Any comment that is entered is displayed on the Recommended Measures report and the Input Report available from the Audit Information form (see Section 8.2, Audit Information). In addition, any comment that is 


\section{Chapter 10: MHEA Building Description}

entered can be imported into any specific work order (see Section 13.1, Work Order Information). Thus, you may wish to enter comments related to any observation that might affect the installation of needed measures or repair work. Optional.

- New/Del - Since there can be only one Ceiling record in MHEA, the "New" button will not initiate a new blank Ceiling form. Instead, it takes you back to the first field on the existing form, the Roof Type field, while not changing any existing entries. The "Del” button will erase all entries on the Ceiling form. You will receive a warning of the deletion only if the form had been previously filled in prior to your entering the form.

\subsection{Shell - Floor}

The Floor form (see Figure 10.9) under the Shell tab of the Main Menu's "MHEA" button is used to describe the floor of a mobile home.

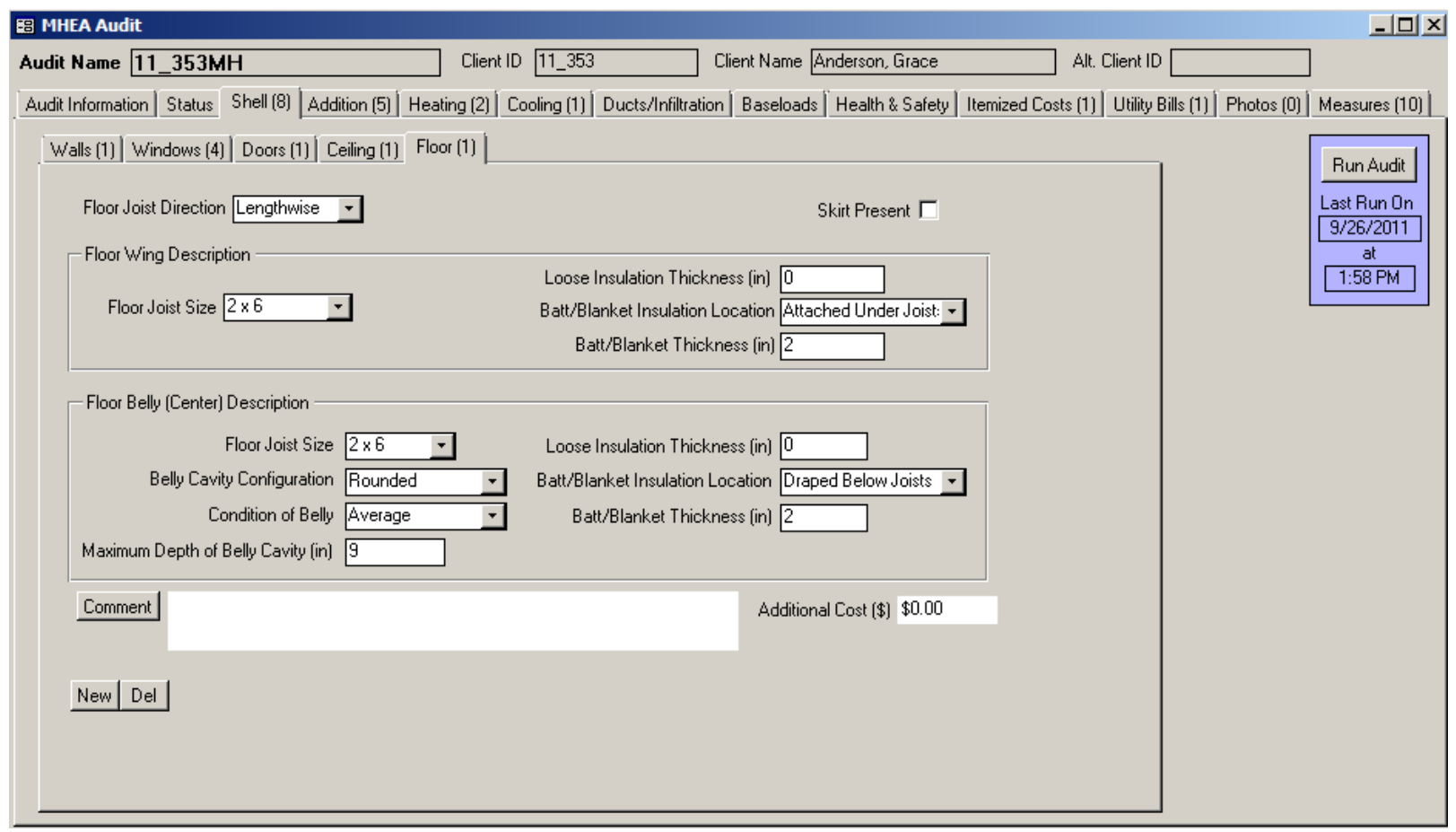

Figure 10.9. The Shell - Floor form under the Main Menu's “NEAT” button. 
The Floor form is divided into four sections: a general description, floor wing description, floor belly description, and the Comment and Additional Cost fields. The floor of a mobile home is constructed of floor joists spanning across the length or width of the mobile home. The floor is supported on a pair of I-beams spanning across the length of the mobile home. The floor wing is the part of the floor outside the supports, and the floor belly is the part between the supports. The floor wing and belly sections are protected from outside elements including water, wind, and rodents using a wrap attached to the underside of floor joists, and may contain insulation.

Figure 10.10 illustrates a section of the floor of a typical mobile home. The mobile home may also have skirting installed around the perimeter of the floor.

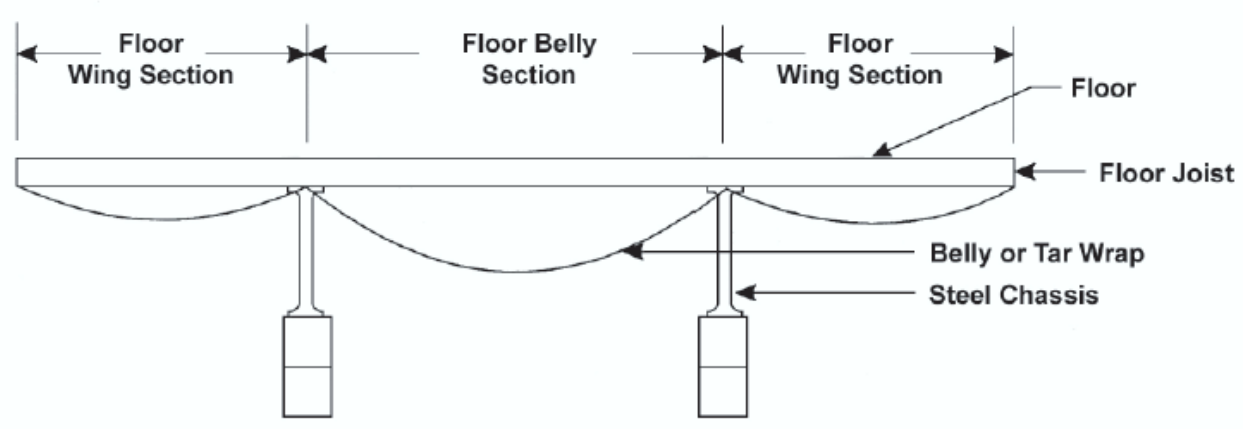

Figure 10.10. Section of a Typical Mobile Home Floor.

Below are descriptions of the individual data items and controls found on the Floor form. All items are required except the Comment and Additional Cost fields.

- Floor Joist Direction - The floor joist is the wood framing that supports the interior floor. Select the direction the floor joists are installed. The choices are Lengthwise and Widthwise. Lengthwise floor joists are installed parallel to the long dimension (length) of the home. Widthwise floor joists are installed parallel to the short dimension (width) of the home (see Figure 10.11). The floor joist direction is needed to calculate the volume of the belly section and the R-value of the floor. It also indicates if the central air supply duct is located 


\section{Chapter 10: MHEA Building Description}

between the joists (for lengthwise floor joists) or below the joists (for widthwise floor joists). Required.

- Skirt Present - Select this checkbox to indicate that a skirt exists around the exterior of the home. The skirt must be solid and intact, not simply a decorative lattice. Research has shown that skirting only protects the mobile home belly from exposure to the wind. If skirting exists, MHEA adjusts the R-value of the floor to account for the absence of wind.

- Floor Joist Size [Wing and Belly] -Select the dimensions of the floor joists. The choices are $2 \times 4,2 \times 6$, and $2 \times 8$. Sometimes, the floor joist size is different in the wing section than in the belly section. MHEA needs this information to calculate the floor R-value and the volume of the floor/belly section. Required.

- Loose Insulation Thickness [Wing and Belly]

- Enter the amount of existing loose-fill insulation in inches for both wing and belly

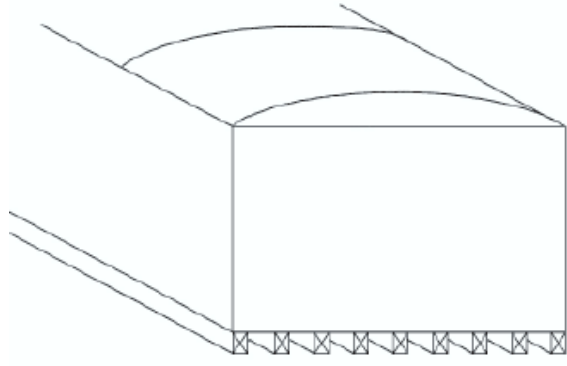

Lengthwise floor joists

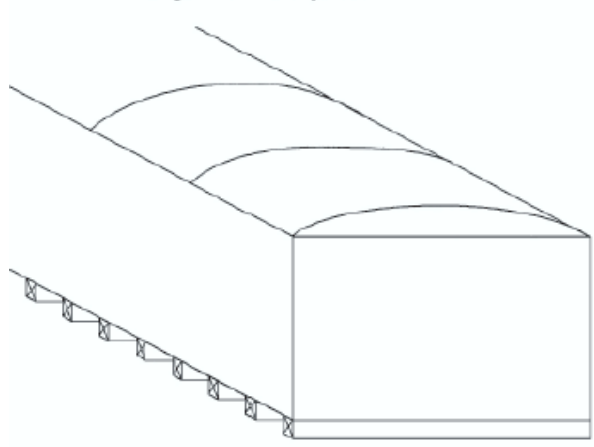

Widthwise floor joists

Figure 10.11. Lengthwise and Widthwise Floor Joists. sections. Occasionally, the insulation existing in the wing section is different than that in the belly section. Enter 0 if insulation does not exist. MHEA uses this information to calculate the R-value and the volume of the wing and belly sections available for additional insulation. Required. 


\section{Chapter 10: MHEA Building Description}

- Batt/Blanket Insulation Location

[Wing and Belly] - Indicate where the batt/blanket insulation is located in the wing and belly sections. The choices are Attached to Flooring, Between Joists, Attached Under Joists, Draped Below Joists (only belly section), and None. Figure 10.12 shows the four configurations. Attached to Flooring refers to the case when the batt/blanket insulation is attached to the underside of the flooring. Between Joists refers to the case when the insulation lies between but at the bottom of the joists, leaving the potential for an air space between the top of the insulation and the flooring. Attached to Joist refers to the case when the insulation is attached to the underside of the floor joists. Insulation in the belly section that is draped below the underside of the floor joists (attached
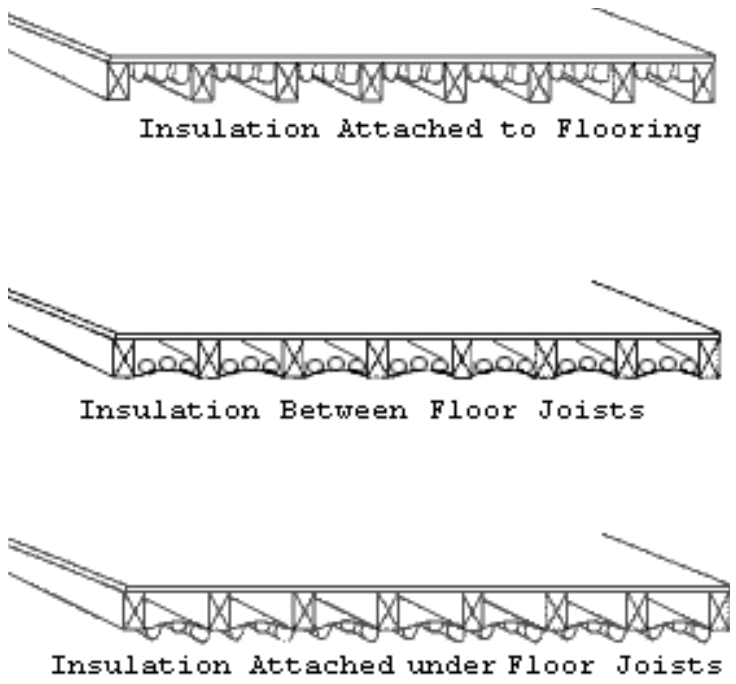
only around the perimeter of the belly section) is described as Draped Below Floor Joist. Enter None if batt/blanket insulation does not exist in the wing and belly sections.

- Batt/Blanket Thickness [Wing and Belly] - Enter the amount of existing batt/blanket insulation in inches for both wing and belly sections. Occasionally, the insulation existing in the wing section is different than that in the belly section. Enter 0 if insulation does not exist. MHEA uses this information to calculate the R-value and the volume of the wing and belly sections available for additional insulation. Required. 


\section{Chapter 10: MHEA Building Description}

- Belly Cavity Configuration [Belly] The belly cavity is the deep section that runs across the length of the home and usually houses the main supply air duct. Select the configuration of the belly cavity. The choices are Square, Rounded, and Flat. Figure 10.13 shows the three configurations. A square cavity configuration has vertical sides and a horizontal base. Usually, the main iron support beams act as the vertical sides. For a rounded belly cavity configuration, the belly wrap is draped in the middle portion of the home. Usually, the wrap is draped between the main iron support beams of the home. A flat belly cavity configuration is one in which there is no

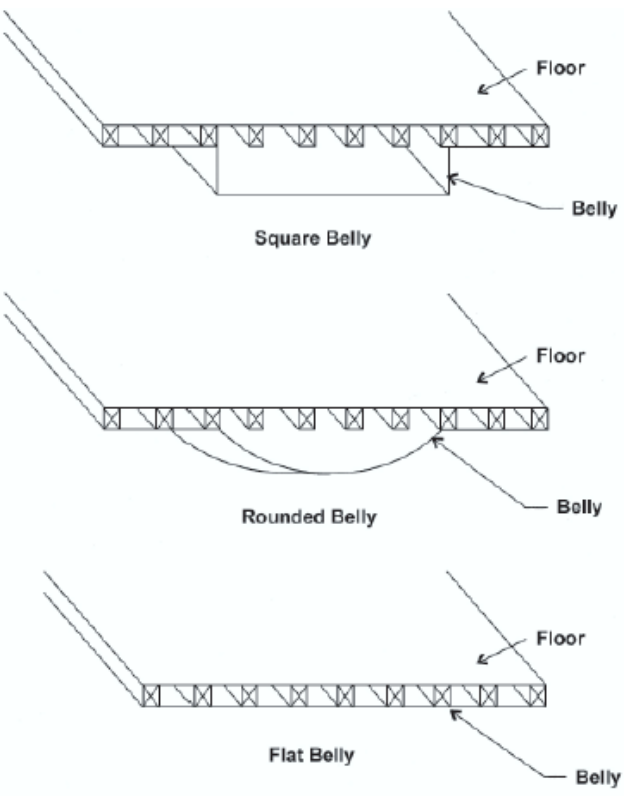

Figure 10.13. Typical Mobile Home Belly Configurations. center belly section lower than the belly wing section. This type of belly is often found on homes where the main air supply duct is located between lengthwise floor joists or where the main supply air duct is located in the roof section. Required

- Condition of Belly -Enter the condition of the existing belly wrap. The choices are Good, Average, and Poor. A belly wrap in good condition does not have any tears, holes, or other openings and is tightly attached around its entire perimeter. An average belly wrap condition has some small tears, holes, or other openings in the belly wrap or may not be well attached at its perimeter. A belly wrap with large tears, holes, or other openings or which is not well attached at its perimeter is in poor condition. MHEA uses this information to adjust the Rvalue of the floor and the effectiveness of sealing any ducts lying within the belly. If the belly is in other than Good condition and you want MHEA to evaluate floor insulation, you should include the cost associated with repairing the belly as an Additional Cost on this form or as a repair item on the Itemized Costs form (see Sections 11.8, Itemized Costs, and 14.6, User Defined Measures). Required.

- Maximum Depth of Belly Cavity [Belly] - Enter the maximum depth of the belly in inches as measured from the underside of the flooring to the lowest part 
of the belly, disregarding any existing insulation. MHEA uses this information to calculate the volume of the belly section available for additional insulation. Required.

- Additional Cost - Enter any additional cost associated with insulating the floor in units of dollars. This would be a cost that is not normally associated with insulating the floor and, therefore, is not accounted for in the measure costs entered on the Library Measures form under the Setup Library (see Section 14.5, Library Measures). For instance, enter the additional labor and material costs associated with repairing the belly wrap. The cost is the total additional cost to insulate the floor; it is not a cost per square foot of floor area. The amount entered will be added to the normal cost computed from the floor characteristics and costs specified on the Library Measures form in the Setup Library. A blank entry indicates no additional cost. If a cost is entered, you should briefly explain the reason for this additional cost in the Comment field described below. Optional.

- Comment - You may enter comments pertinent to floor directly in the Comment field on the form, or you may enter them in the Comment Editor by selecting the "Comment" button to the left of the field. The Comment Editor is similar to Window's Notepad and may be used to enter extended comments. The total comment may have up to 65,000 characters. Text from other sources may be copied and pasted into the Comment Editor's window. Any comment that is entered is displayed on the Recommended Measures report and the Input Report available from the Audit Information form (see Section 8.2, Audit Information). In addition, any comment that is entered can be imported into any specific work order (see Section 13.1, Work Order Information). Thus, you may wish to enter comments related to any observation that might affect the installation of needed measures or repair work. Optional.

- New/Del - Since there can be only one Floor record in MHEA, the "New" button will not initiate a new blank Floor form. Instead, it takes you back to the first field on the existing form, the Floor Joist Direction field, while not changing any existing entries. The "Del" button will erase all entries on the Floor form. You will receive a warning of the deletion only if the form had been previously filled in prior to your entering the form. 


\section{Chapter 10: MHEA Building Description}

\subsection{Addition}

An addition to a mobile home is defined as any add-on structure that is attached to the mobile home through a shared wall and is conditioned by the mobile home heating and cooling systems (see Figure 10.14). If the addition is separated from the mobile home (e.g., by closing the door to an entrance vestibule), it should not be included in the energy audit. MHEA assumes that the addition is

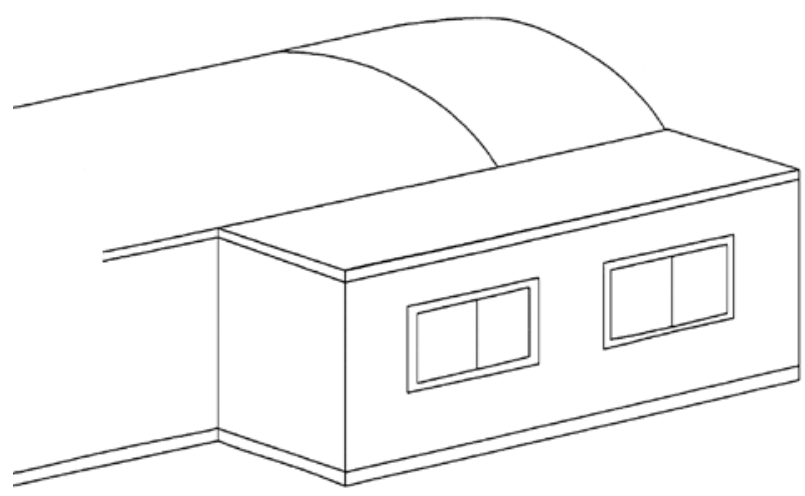

Figure 10.14. Addition of a Mobile Home. rectangular in shape and has woodframe construction.

The characteristics of the envelope of an addition are described on the Walls, Windows, Doors, Ceiling, and Floor forms under the Addition tab of the Main Menu's "MHEA" button. You may access these forms in any order. However, it is recommended that you complete the forms in a logical order so that you do not overlook any components of the addition. If there is no addition, you do not need to access the addition forms. However, if entry is begun on any one of the Walls, Ceiling, or Floor forms, all three of these forms must be completed.

The Windows and Doors forms and many items on other forms are analogous to the forms and items on the forms under the Shell tab for the mobile home proper.

\section{Addition - Walls}

The Walls form (see Figure 10.15) under the Addition tab of the Main Menu's "MHEA" button is used to describe the exterior walls of the addition. MHEA assumes that the three exposed walls of the addition have identical construction characteristics, and therefore, uses only one form to describe them. 


\section{Chapter 10: MHEA Building Description}

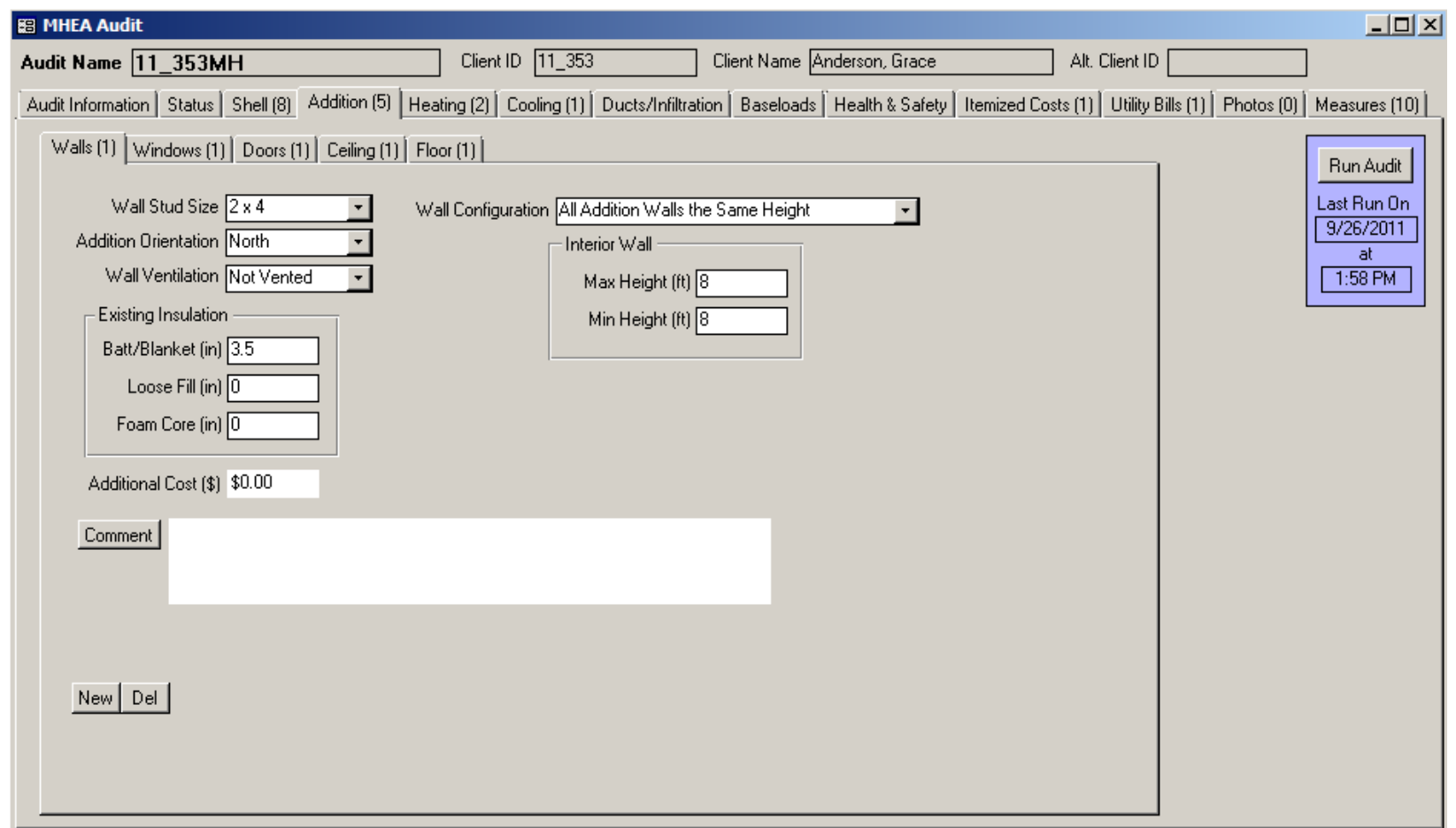

Figure 10.15. The Addition - Walls form under the Main Menu's “MHEA” button.

Below are brief descriptions of the individual data items and controls found on the Walls form. All items on the Walls form are required except the Additional Cost and Comment fields.

- Wall Stud Size - Select the stud size used in framing the walls. The choices are $2 \times 2,2 \times 3,2 \times 4$, and $2 \times 6$. MHEA uses this information to calculate the wall Rvalue and the volume of wall cavity available for added insulation. Required.

- Addition Orientation - Select the closest cardinal compass direction that the addition of the mobile home faces. It is the orientation of the wall of the mobile home to which the addition is attached. The choices are North, South, East, and West. MHEA uses this information to calculate solar loads on the addition. Required.

- Wall Ventilation - Indicate whether the walls are vented or not vented. The walls of the mobile home addition may be intentionally ventilated to remove accumulated moisture by having corrugated metal siding open at the bottom to provide space for air to flow between the exterior and interior wall materials. Small holes in the siding at the bottom and top of the exterior wall surface may 


\section{Chapter 10: MHEA Building Description}

be visible, allowing the air to circulate. MHEA degrades the wall R-value for ventilated walls. Required.

- Existing Insulation Type and Thickness - MHEA assumes that exterior walls of the mobile home addition may have Batt/Blanket, Loose Fill, or Foam Core insulation. Enter the thickness of existing insulation type in inches. Enter 0 for insulation types that do not exist. MHEA uses this information to calculate the wall R-value and the volume of wall cavity available for additional insulation. You may view, and, if necessary, adjust the Rs/Inch MHEA assigns to these existing insulation types from the Key Parameters/Insulation form in MHEA's Setup Library (see Section 14.2, Key Parameters). A default value of 3.5 inches exists. Required.

- Wall Configuration - Select the wall configuration of the mobile home addition. The choices are Maximum Wall Height at Interior Wall, Maximum Wall Height at Center of Addition, and All Addition Walls the Same Height. Select Maximum Wall Height at Interior Wall if the roof of the addition slopes down from the shared wall of the mobile home and the addition to the opposite exterior wall of the addition. Select Maximum Wall Height at Center of Addition if the roof peaks along the center of the addition and slopes down towards the two exterior walls that are perpendicular to the mobile home. Finally, select All Addition Walls the Same Height if the addition has a flat roof. Required.

- Interior Wall Max. and Min. Height - Enter the height in feet of the addition walls as seen from the addition's conditioned space. If the walls are of varying height, enter the maximum and minimum wall heights. If the walls are all the same height, enter the same value in both the maximum and minimum height fields. Required.

- Additional Cost - Enter any additional cost associated with insulating the walls of the addition, in units of dollars. This would be a cost that is not normally associated with insulating the walls of an addition to a mobile home and, therefore, is not accounted for in the measure costs entered on the Library Measures form under the Setup Library (see Section 14.5, Library Measures). The cost is the total additional cost to insulate the walls; it is not a cost per square foot of wall area. The amount entered will be added to the normal cost computed from the wall area and the cost per square foot specified on the 
Library Measures form in the Setup Library. A blank entry indicates no additional cost. If a cost is entered, you should briefly explain the reason for this additional cost in the Comment field described below. Optional.

- Comment - You may enter comments pertinent to walls directly in the Comment field on the form, or you may enter them in the Comment Editor by selecting the "Comment" button to the left of the field. The Comment Editor is similar to Window's Notepad and may be used to enter extended comments. The total comment may have up to 65,000 characters. Text from other sources may be copied and pasted into the Comment Editor's window. Any comment that is entered is displayed on the Recommended Measures report and the Input Report available from the Audit Information form (see Section 8.2, Audit Information). In addition, any comment that is entered can be imported into any specific work order (see Section 13.1, Work Order Information). Thus, you may wish to enter comments related to any observation that might affect the installation of needed measures or repair work. Optional.

- New/Del - Since there can be only one Wall record in MHEA, the "New" button will not initiate a new blank Walls form. Instead, it takes you back to the first field on the existing form, the Wall Stud Size field, while not changing any existing entries. The "Del" button will erase all entries on the Walls form. You will receive a warning of the deletion only if the form had been previously filled in prior to your entering the form.

\section{Addition - Windows}

The Windows form (see Figure 10.16) under the Addition tab of the Main Menu's "MHEA" button is used to enter information on all the windows installed in the mobile home addition. This form is analogous to the Windows form under the Shell tab of the Main Menu's "MHEA" button. See Section 10.2, Shell - Windows for descriptions of the individual data items and controls found on the Windows form. 


\section{Chapter 10: MHEA Building Description}

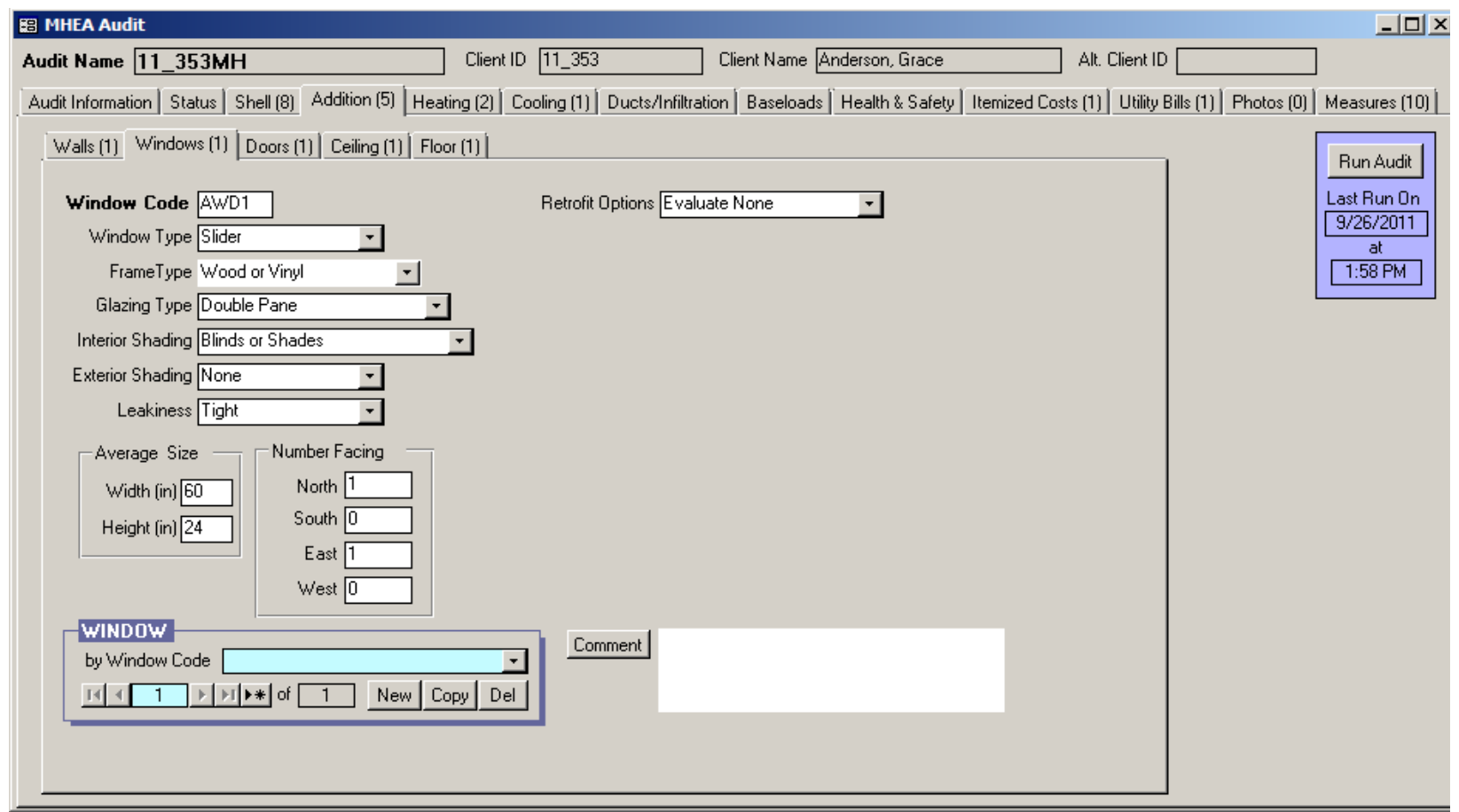

Figure 10.16. The Addition - Windows form under the Main Menu’s “MHEA” button.

\section{Addition - Doors}

The Doors form (see Figure 10.17) under the Addition tab of the Main Menu's "MHEA" button is used to enter information on all the doors installed in the mobile home addition. This form is analogous to the Doors form under the Shell tab of the Main Menu's "MHEA" button. See Section 10.3, Shell - Doors, for descriptions of the individual data items and controls found on the Doors form. The Replacement Door Required and the Include in SIR checkboxes, which appear on the Doors form under the Shell tab, do not appear on the Doors form under the Addition tab.

NOTE: Do not describe the doors installed on the shared wall between the mobile home and the addition. 


\section{Chapter 10: MHEA Building Description}

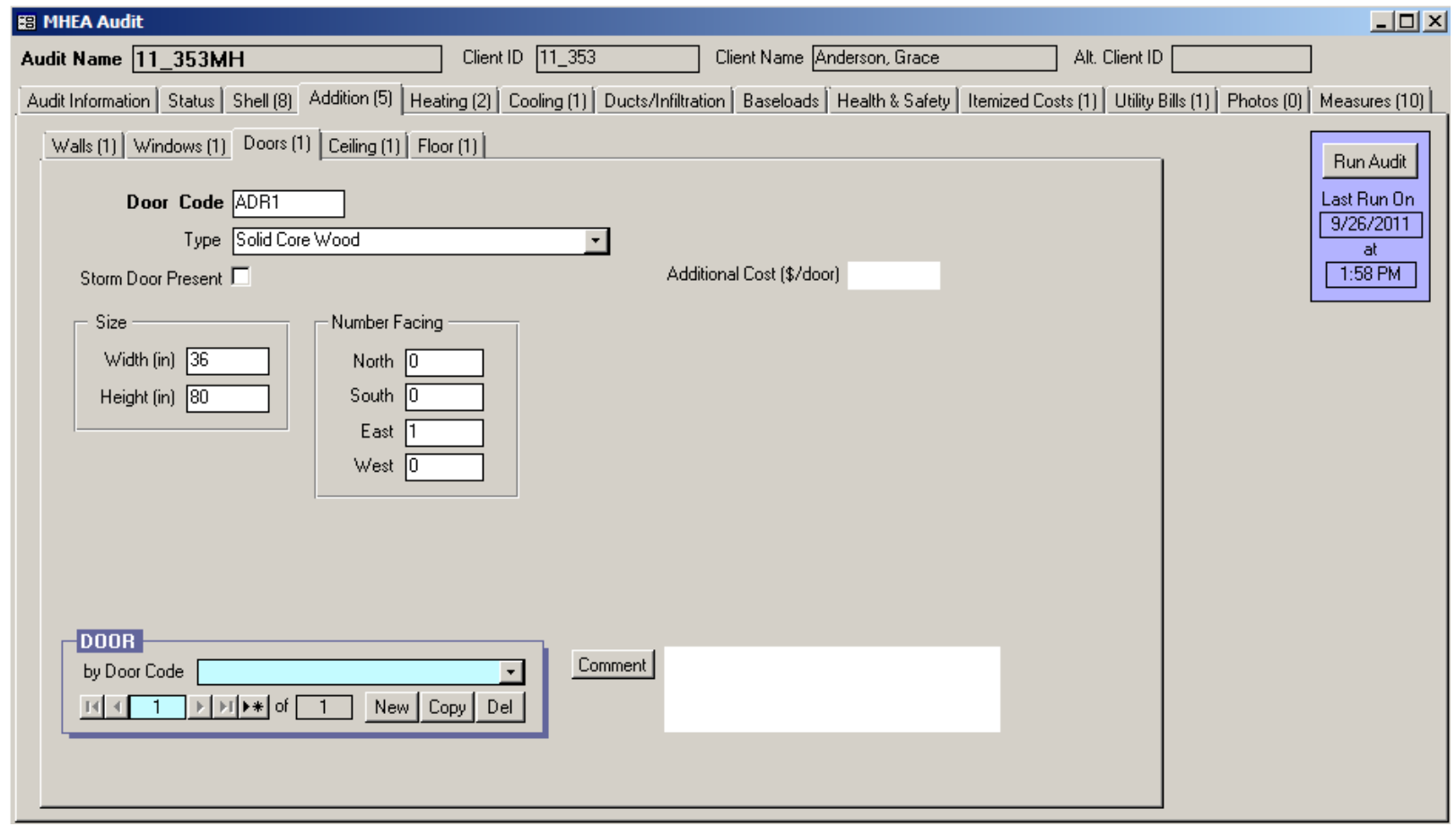

Figure 10.17. The Addition - Doors form under the Main Menu's “MHEA” button.

\section{Addition - Ceiling}

The Ceiling form (see Figure 10.18) under the Addition tab of the Main Menu's "MHEA" button is used to enter information on the ceiling of the mobile home addition.

- Joist Size - Enter the joist size of the roof of the mobile home addition. The choices are $2 \times 4,2 \times 6$, and $2 \times 8$. MHEA uses this information to calculate the volume of roof cavity available for adding roof insulation. Required.

- Roof Color - Enter the color of the roof of the mobile home addition. The two choices are "White, Reflective or Shaded" and "Normal or Weathered". A White or Reflective roof appears white or shiny metallic, is clean, and has little or no discoloration due to weathering. Roofs completely shaded by trees in the summer should be categorized as white or reflective regardless of their finish. The majority of roofs will be classified as Normal or Weathered. Roof surfaces designated as White or Reflective will not be considered when evaluating the White Coat Roof measure. Required. 


\section{Chapter 10: MHEA Building Description}

- Existing Insulation Type and Thickness - MHEA assumes that the roof of the mobile home addition may have Batt/Blanket, Loose Fill, or Foam Core insulation. Enter the thickness of existing insulation by type in inches measured at the center of the roof/ceiling section. Enter 0 or leave the field blank for other insulation types that do not exist. MHEA uses this information to calculate the roof/ceiling R-value and also to calculate the volume in the roof/ceiling cavity that is available for additional insulation. You can ignore compression or different depths of insulation across the roof/ceiling. You may view, and, if necessary, adjust the Rs/Inch MHEA assigns to these existing insulation types from the Key Parameters/Insulation form in MHEA's Setup Library (see Section 14.2, Key Parameters). Optional.

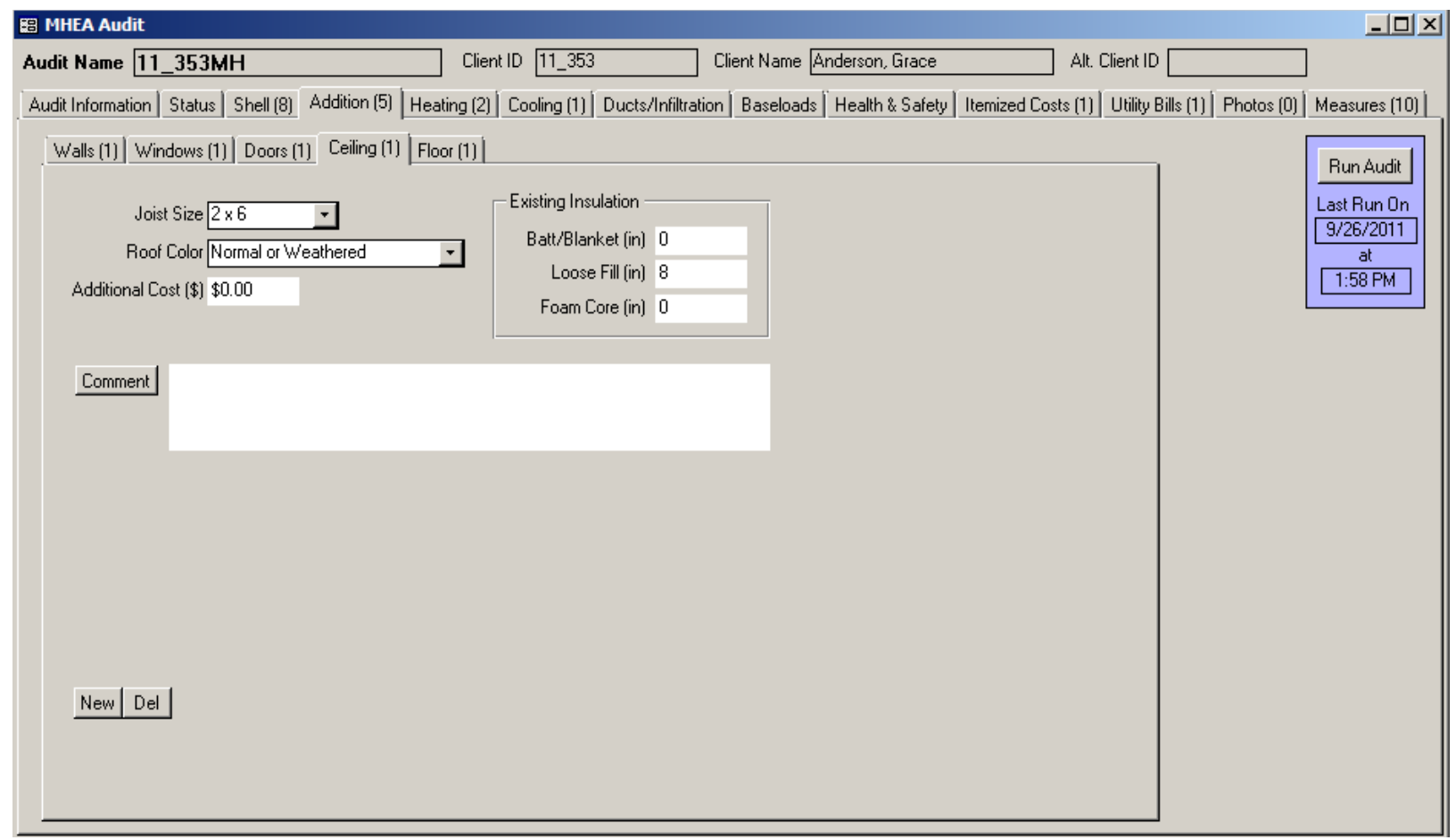

Figure 10.18. The Addition - Ceiling form under the Main Menu's “MHEA” button.

- Additional Cost - Enter any additional cost associated with insulating this roof/ceiling cavity in dollars. This would be a cost that is not normally associated with insulating the roof/ceiling of an addition to a mobile home and, therefore, is not accounted for in the measure costs entered on the Library Measures form under the Setup Library (see Section 14.5, Library Measures). For instance, enter the additional costs associated with cutting an opening into the roof to access the cavity. The cost is the total additional cost to insulate the 
entire cavity; it is not a cost per square foot of attic area. The amount entered will be added to the normal cost computed from the attic characteristics and costs specified on the Library Measures form in the Setup Library. A blank entry indicates no additional cost. If a cost is entered, you should briefly explain the reason for this additional cost in the Comment field described below. Optional.

- Comment - You may enter comments pertinent to the roof/ceiling section directly in the Comment field on the form, or you may enter them in the Comment Editor by selecting the "Comment" button to the left of the field. The Comment Editor is similar to Window's Notepad and may be used to enter extended comments. The total comment may have up to 65,000 characters. Text from other sources may be copied and pasted into the Comment Editor's window. Any comment that is entered is displayed on the Recommended Measures report and the Input Report available from the Audit Information form (see Section 8.2, Audit Information). In addition, any comment that is entered can be imported into any specific work order (see Section 13.1, Work Order Information). Thus, you may wish to enter comments related to any observation that might affect the installation of needed measures or repair work. Optional.

- New/Del - Since there can be only one Ceiling record in MHEA, the "New" button will not initiate a new blank Ceiling form. Instead, it takes you back to the first field on the existing form, the Joist Size field, while not changing any existing entries. The "Del" button will erase all entries on the Ceiling form. You will receive a warning of the deletion only if the form had been previously filled in prior to your entering the form.

\section{Addition - Floor}

The Floor form (see Figure 10.19) under the Addition tab of Main Menu's "MHEA" button is used to describe the floor of the mobile home addition, including crawl space, slab-on-grade foundations, and floors exposed to the outside air. The floor can be retrofitted by adding insulation depending on the floor type and space available for adding insulation. Below are descriptions of the individual data items and controls found on the Floor form. All items are required except the Comment field. 


\section{Chapter 10: MHEA Building Description}

- Floor Type - Select the type of floor for the mobile home addition. The choices are Crawl Space, Slab-on-Grade, and Exposed Floor. The choice affects the heat conduction seen through the floor. All of the data fields shown in Figure 10.19 are not needed if the Floor Type is Slab-on-Grade. Required.

- Floor Joist Size -The Floor Joist Size field appears when the floor type is other than Slab-on-Grade. Select the dimensions of floor joists in inches. The choices are $2 \times 4,2 \times 6$, and $2 \times 8$. MHEA uses this information to calculate the floor Rvalue and the volume of the addition floor section. Required.

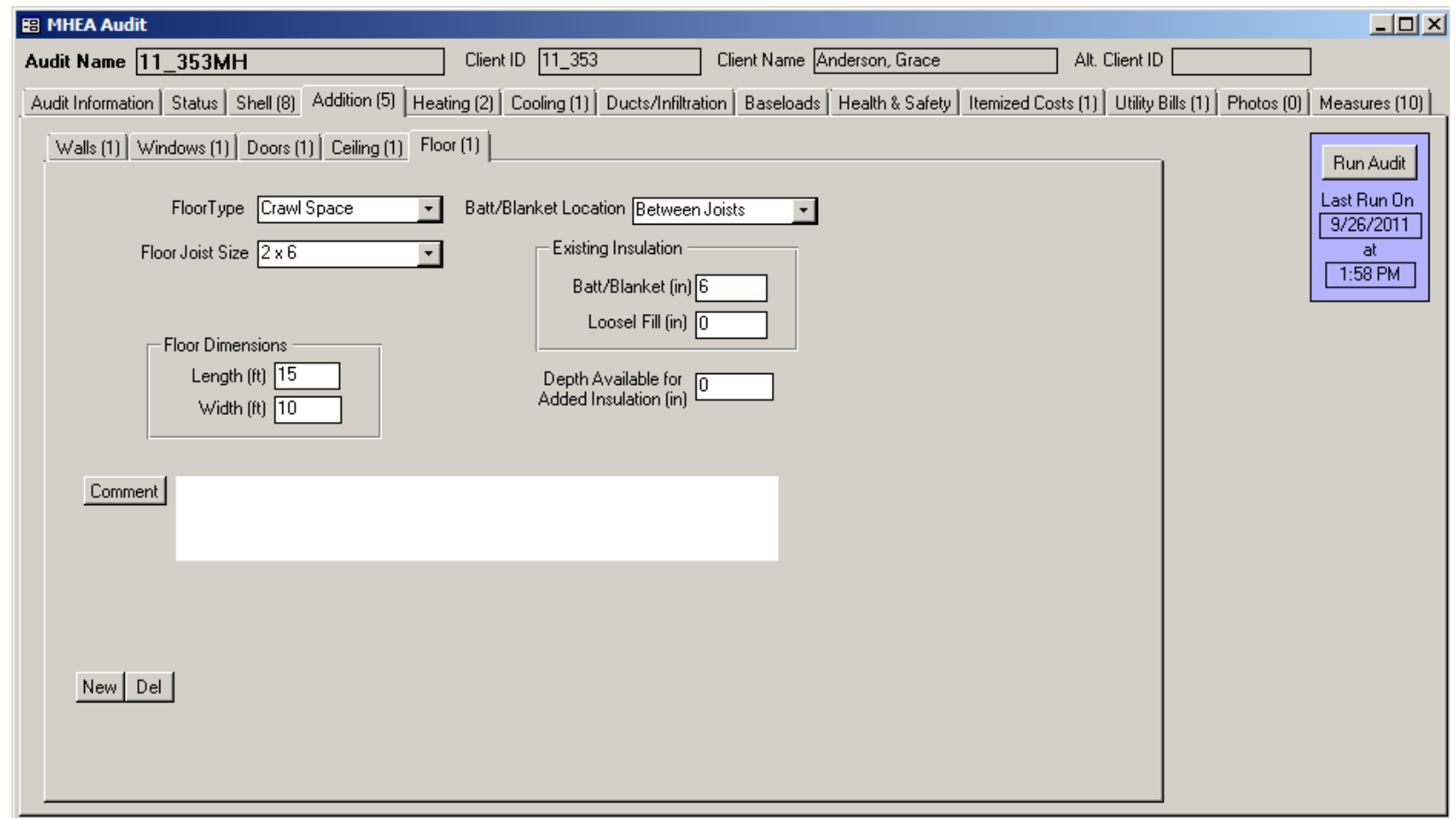

Figure 10.19. The Addition - Floor form under the Main Menu's “MHEA” button.

- Floor Length and Width - Enter the dimensions of the addition floor in feet. The floor length is the dimension parallel to the wall shared with the mobile home and the floor width is the dimension perpendicular to the wall (see Figure 10.20). MHEA recognizes an addition to the mobile home only when floor dimensions are entered. Required. 


\section{Chapter 10: MHEA Building Description}

- Batt/Blanket Location - The Batt/Blanket Location field appears when the floor type is other than Slab-on-Grade. Indicate where the batt/blanket insulation is located in the addition floor. The choices are Attached to Flooring, Between Joists, Attached Under Joists, and None. Figure 10.21shows the three configurations. Attached to Flooring refers to the case when the batt/blanket insulation is attached to the underside of the flooring.

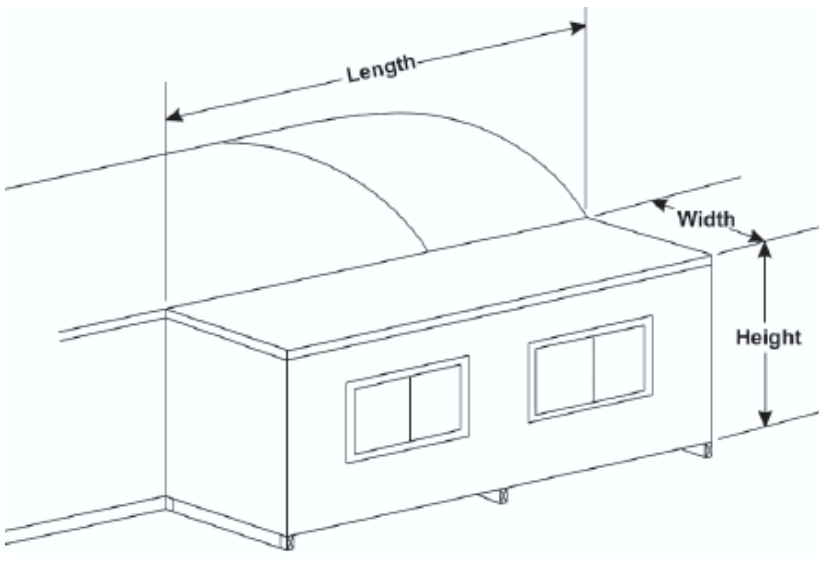

Figure 10.20. Dimensions of Mobile Home Addition.

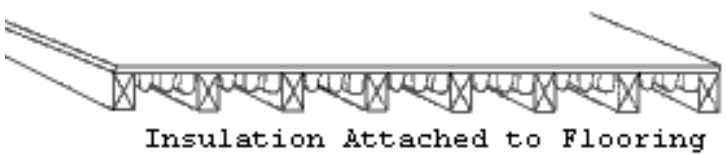
at the bottom of the joists, le between the top of the insulation and the flooring. Attached to Joist refers to the case when the insulation is attached to the underside of the floor joists. Enter None if batt/blanket insulation does not exist in the addition floor. Required.

- Existing Insulation Type and Thickness - The Existing Insulation fields appear when the floor type is other than Slab-on-

Grade. MHEA assumes that the mobile home addition floor, if not a slab-ongrade, may have Batt/Blanket or Loose Fill insulation. Enter the thickness of existing insulation by type in inches. Enter 0 for any insulation type that does not exist. MHEA uses this information to calculate the addition floor R-value and the volume of addition floor cavity available for additional insulation. You may view, and, if necessary, adjust the Rs/Inch MHEA assigns to these existing insulation types from the Key Parameters/Insulation form in MHEA's Setup Library (see Section 14.2, Key Parameters). Required. 


\section{Chapter 10: MHEA Building Description}

- Depth Available for Added Insulation - The Depth Available for Added Insulation fields appear when the floor type is other than Slab-on-Grade. Enter the depth of space available for additional insulation in the addition floor section in inches. MHEA uses this information to evaluate insulation measures for the addition floor. Required.

- Comment - You may enter comments pertinent to the addition floor directly in the Comment field on the form, or you may enter them in the Comment Editor by selecting the "Comment" button to the left of the field. The Comment Editor is similar to Window's Notepad and may be used to enter extended comments. The total comment may have up to 65,000 characters. Text from other sources may be copied and pasted into the Comment Editor's window. Any comment that is entered is displayed on the Recommended Measures report and the Input Report available from the Audit Information form (see Section 8.2, Audit Information). In addition, any comment that is entered can be imported into any specific work order (see Section 13.1, Work Order Information). Thus, you may wish to enter comments related to any observation that might affect the installation of needed measures or repair work. Optional.

- New/Del - Since there can be only one addition Floor record in MHEA, the "New" button will not initiate a new blank Floor form. Instead, it takes you back to the first field on the existing form, the Floor Type field, while not changing any existing entries. The "Del" button will erase all entries on the Floor form. You will receive a warning of the deletion only if the form had been previously filled in prior to your entering the form. 


\section{Chapter 10: MHEA Building Description}

\subsection{Heating - Primary, Secondary, and Replacement}

The Primary and Secondary forms (see Figure 10.22 and 10.23) under the Heating tab of the Main Menu's "MHEA" button are used to describe the heating systems installed in the mobile home. MHEA allows the definition of a primary and a secondary heating system in a home. The primary system is the system that supplies the majority of the heat to the home and on which MHEA will consider installing energy efficiency measures. You must describe an existing primary heating system before MHEA will execute. The Replacement form (see Figure 10.24) is used to describe the equipment you want MHEA to evaluate for the primary heating system replacement as a possible retrofit measure. MHEA will properly evaluate the effects of fuel switching as part of the Replace Heating System measure. You should enter information and describe the primary system first before describing the secondary system and/or the replacement system. If the primary system supplies all of the heat for the home, leave the Secondary form blank. If no replacement is to be considered, leave the Replacement form blank.

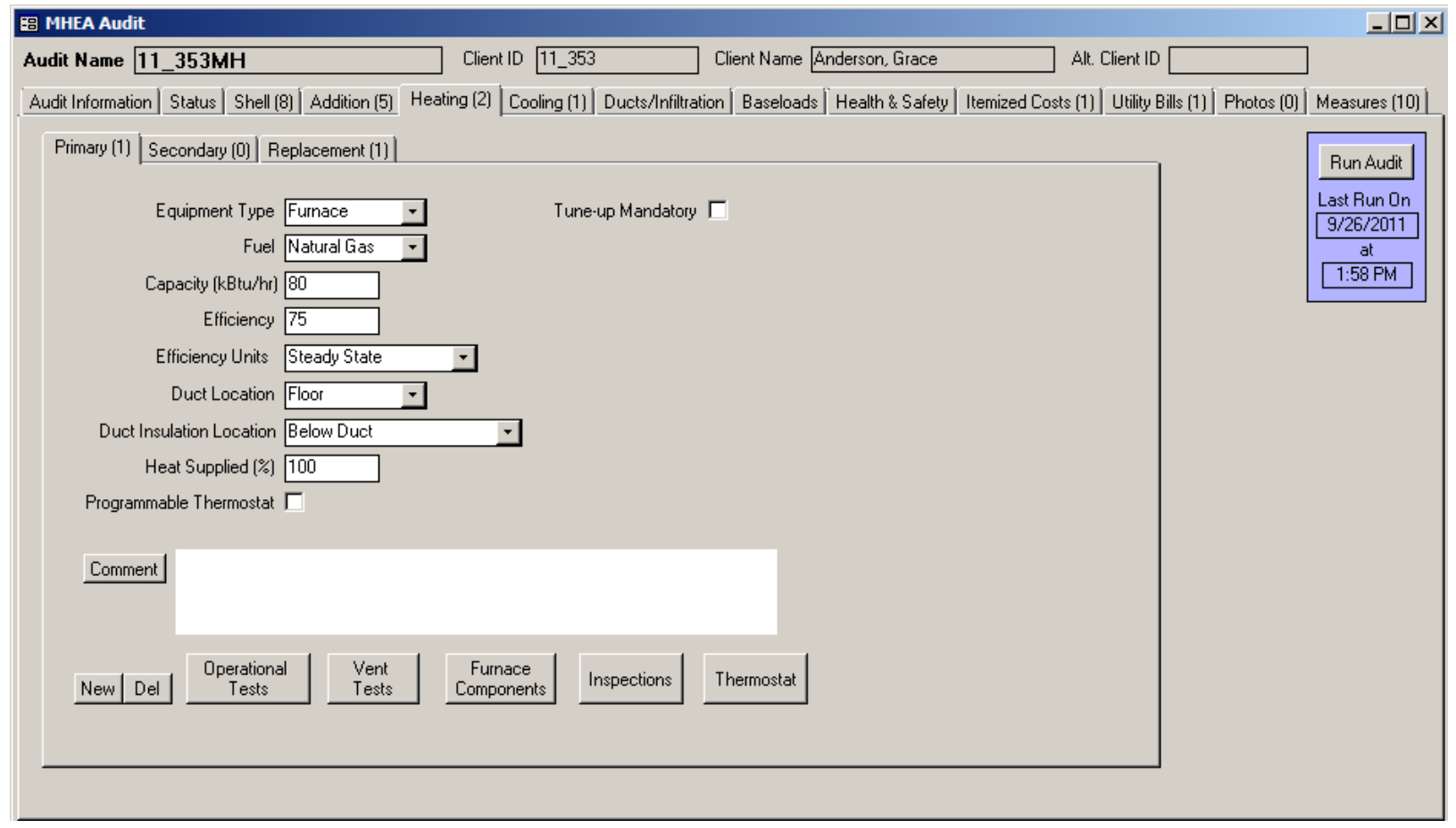

Figure 10.22. The Primary Heating System form under the Main Menu's “MHEA” button. 


\section{Chapter 10: MHEA Building Description}

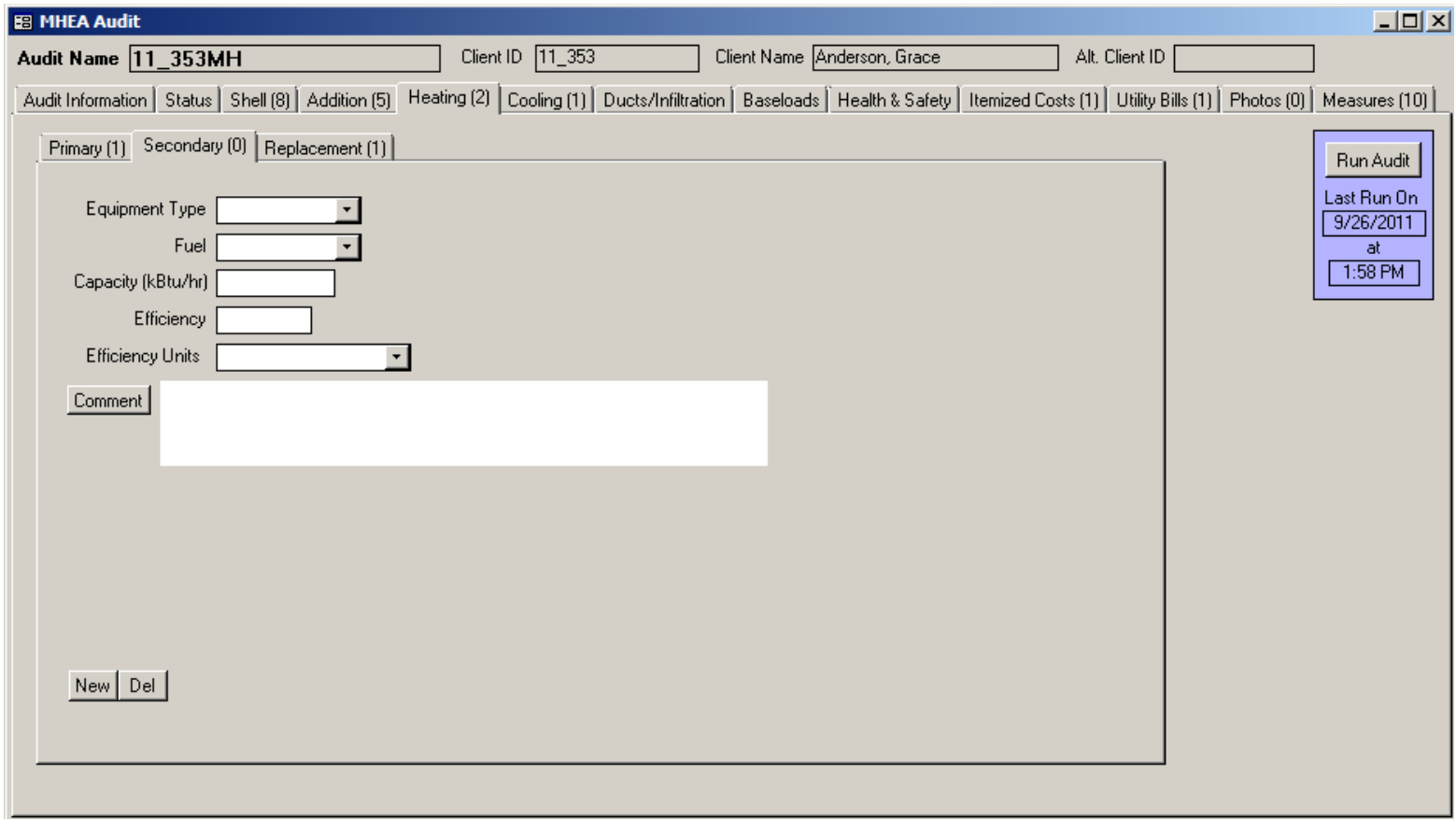

Figure 10.23. The Secondary Heating System form under the Main Menu's “MHEA” button.

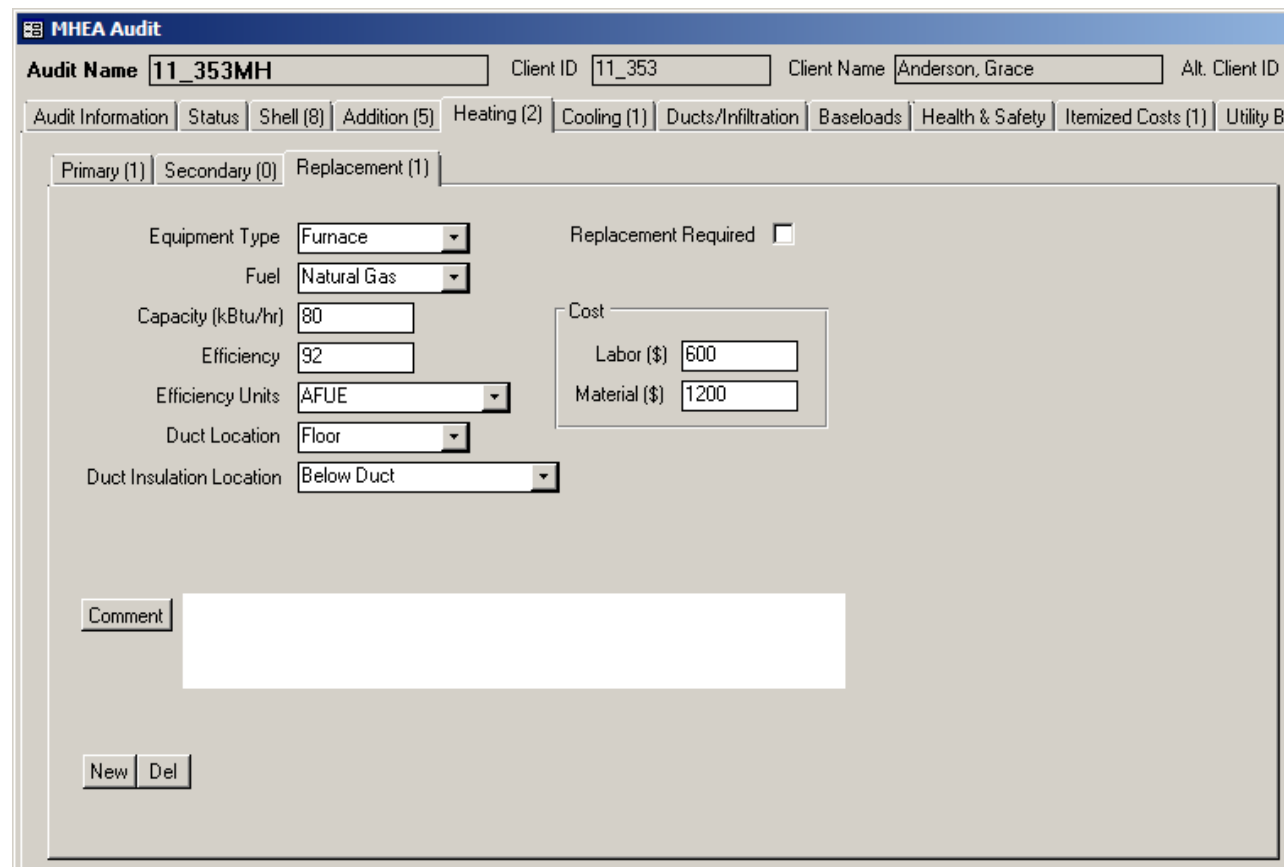

$-[\mathrm{x} x$

Audit Name 11_353MH

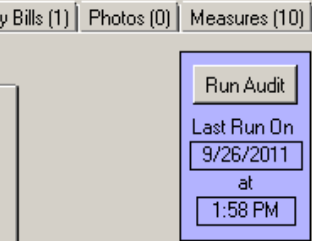

Figure 10.24. The Replacement Heating System form under the Main Menu's "MHEA" button. 
You may encounter homes where even the primary heating system is inoperative. Do not attempt to describe these systems to MHEA by entering a value of 0 for either the Efficiency or Heat Supplied (see the corresponding field descriptions in the discussions that follow). Rather, describe the existing heating equipment as supplying the amount of heat (Heat Supplied) as it was intended to supply and an efficiency that is typical, but at the lowest end of the possible range, for equipment of its type. Then, follow your state's recommendations regarding repair, tune-up, or replacement of the equipment, which may involve issues of cost-justifying a replacement or replacement as a health and safety measure. Alternatively, if you know that the inoperative system is going to be replaced, you could describe the replacement as the existing system on the heating forms.

Below are the descriptions of the individual data items and controls found in the Primary, Secondary and Replacement forms. All items on these forms are required except the Comment field. Items that appear on more than one form are analogous. Items that are not common on all three forms are indicated by specifying the name of the applicable form(s) in brackets.

- Equipment Type - Select what type of heating system this is. The choices are Furnace, Heat Pump, Space Heater, and None.

Heating and cooling sides of a heat pump are treated as separate components requiring descriptions under both the Heating and Cooling tabs. For correct modeling of replacing the existing primary heating and cooling systems with a heat pump, the replacement for both the heating and cooling sides of the existing units must be specified as heat pumps and the total cost of the replacement split between the Heating Replacement and Cooling Replacement descriptions. To have the replacement seen as cost-effective, this split in cost should be adjusted in order to show an SIR for replacement of both the heating and cooling components as cost-effective. See Appendix B.16, Replace Heating System. Required.

- Fuel - Select the type of fuel used by the heating system. The choices are Natural Gas, Oil, Electricity, Propane, Wood, Coal, Kerosene, and Other. You can set the heat content and price for all fuel types, including the Other fuel type, on the Fuel Costs tab of the Setup Library (see Section 14.3, Fuel Costs). Required. 
- Capacity - Enter the rated input capacity in kBtu/h for natural gas, electric, propane, oil, and kerosene furnaces and space heaters. Enter the rated output capacity in $\mathrm{kBtu} / \mathrm{h}$ for heat pumps. The capacity can often be obtained directly from the nameplate of the heating system or from an inspection of nozzle sizes, etc. If this information is not available, the output capacity can be approximated or, in rare cases, measured. The capacity for coal and wood systems is not required. NOTE: If MHEA determines that the capacity of the equipment is insufficient to meet the home's load, the annual energy consumption predicted by the program for the house will be reduced, also reducing the savings that might be predicted for individual measures. If this has occurred for any individual execution of MHEA, it will be noted under the Special Notes on the Recommended Measures Report. See Special Notes in Section 12.2, Recommended Measures Report. Conditionally required.

- Efficiency and Efficiency Units - Enter the efficiency of the heating system in the Efficiency Units selected. The choices for Efficiency Units are Steady State, AFUE, HSPF, and COP. Steady State and AFUE (Annual Fuel Utilization Efficiency) entries apply to furnaces and space heaters and are expressed as percentages typically ranging from $50 \%$ to $100 \%$. Steady state efficiency is an instantaneous measure of efficiency while AFUE is an annual average efficiency, which is often displayed on the unit label or in the unit specifications. An electric furnace or unvented space heater is $100 \%$ efficient as long as it is located in the living space of the manufactured home. COP (Coefficient of Performance) and HSPF (Heating Seasonal performance factor) are used in rating heat pump equipment. COP is an instantaneous measure of efficiency while HSPF is annual average efficiency. Newer systems are required to display the HSPF rating on the unit. Older systems may list a COP value. COP entries are pure numbers with typical values between 1.5 and 4.0. HSPF values are also pure numbers with typical values between 5.0 and 10.0.

This parameter should be as accurate as possible because it affects not only the savings calculated for heating system weatherization measures but also the savings of most envelope measures. There are online and published sources of heating equipment efficiencies by manufacturer and model. However, these efficiencies are for new units installed and tuned properly or may have been determined under different conditions. For fossil-fueled heating systems, if possible the steady-state efficiency should be measured by performing a fluegas analysis. Required. 
- Duct Location [Primary and Replacement] - Select the location of the main supply air duct for the heating system. The choices are Floor, Ceiling, and None. Usually, mobile homes have a single duct that runs along the center of the home, or two ducts, each running along the length of the home and connected by a cross-over duct. Indicate if the main supply duct is located under the floor or above the ceiling. In some cases, no main supply air duct exists. Enter None, if the supply air duct has been disconnected from the central heating system and there are no plans to reconnect it. Required.

- Duct Insulation Location [Primary and Replacement] - Select the location of insulation, if any, associated with the main supply air duct. The choices are Above Duct, Blow Duct, Around Duct or Ductboard, and No Insulation. Insulation may be located only on the top of the duct, only on the bottom of the duct, or wrapped completely around the duct. Ductboard is a rigid insulation used to either insulate ducts or to create a plenum that is used as a duct. Select the appropriate choice if duct is insulated or None if the duct is uninsulated. Required.

- Heat Supplied [Primary] - Enter the percent of the floor area that is heated by the primary heating system. Do not enter the \% sign with the number. Required.

- Programmable Thermostat [Primary] - Use this checkbox to indicate whether the existing thermostat is a programmable model that allows automatic setback of the heating set-point. The number of degrees assumed for the setback is entered in the Setup Library (see Section 14.2, Key Parameters). The presence of an existing programmable thermostat will prevent a Setback Thermostat retrofit measure from being evaluated and recommended for this house. This checkbox should also be checked to indicate that the occupants would not benefit from a programmable thermostat because they already manually set their thermostat back in winter, do not want a programmable thermostat, and/or would not be able to use a programmable thermostat. In these cases, the reason for checking the checkbox should be noted in the Comment field described below.

- Tune-up Mandatory [Primary] - Use this checkbox to indicate that a system tune-up is all that is necessary based on the inspection of the heating system. MHEA will evaluate and recommend the system tune-up, regardless of its cost- 
effectiveness. The tuned up system will then be used in evaluating other measures not considered mandatory. No system replacement will be considered.

- Replacement Required [Replacement] - Use this checkbox to indicate that a system replacement is necessary based on the inspection of existing heating system. This may be the result of an unsafe yet unrepairable existing system. Make sure that the Tune-up Mandatory checkbox on the Primary Heating form is unchecked. MHEA will evaluate and recommend system replacement, regardless of its cost-effectiveness. The replacement system will then be used in evaluating other measures not considered mandatory. No primary heating system tune-up will be considered.

- Include in SIR [Primary and Replacement] - If the Tune-up Mandatory [Primary] checkbox or the Replacement Required [Replacement] checkbox is selected, the Include in SIR checkbox will appear on the form. This checkbox allows you to indicate that you wish to have the selected measure included in the calculation of the whole house (package) SIR for this audit. Measures not included in the whole house SIR are normally related to health and safety. Check the guidance for your program in deciding when you can mandate an HVAC measure and if it can be excluded from the SIR calculation. Even if the measure can be considered related to health and safety, if it is shown to be costeffective, you may choose to still include it in the whole house SIR by selecting this checkbox. By doing so, it will not be seen as a health and safety measure, thus preserving funds dedicated for other such measures. An alternate reason for excluding a measure from the whole house SIR is if it is funded outside of the Weatherization Assistance Program. Whether a measure is or is not included in the whole house SIR is indicated by its location in the list of measures produced for the Recommended Measures Report (see Chapter 12, NEAT and MHEA Results).

- Labor and Material Costs [Replacement] - Enter the Labor and Material cost associated with replacing the primary heating system. MHEA adds the labor and equipment costs in computing the SIR and reporting costs. Thus, the total cost (labor plus equipment) may be entered in either field, with $\$ 0.00$ entered for the other of the two fields, with no adverse consequences. Do not enter $\$ 0.00$ in both Labor and Material Cost fields for either replacement unit. Required. 
- Comment - You may enter comments pertinent to the heating system directly in the Comment field on the form, or you may enter them in the Comment Editor by selecting the "Comment" button to the left of the field. The Comment Editor is similar to Window's Notepad and may be used to enter extended comments. The total comment may have up to 65,000 characters. Text from other sources may be copied and pasted into the Comment Editor's window. Any comment that is entered is displayed on the Recommended Measures report and the Input Report available from the Audit Information form (see Section 8.2, Audit Information). In addition, any comment that is entered can be imported into any specific work order (see Section 13.1, Work Order Information). Thus, you may wish to enter comments related to any observation that might affect the installation of needed measures or repair work. Heating system comments may include observations of equipment conditions, reasons for selecting specific retrofit options, or references to health and safety items. Optional.

- Optional Heating System Detail Buttons [Primary] - Buttons at the bottom of the Primary Heating form can be used to document an extensive number of heating system measurements and observations made while auditing a dwelling. These buttons are described in Section 11.12, Optional Heating System Details.

- New/Del -The "New" button will take you back to the first field on the existing form, the Equipment Type field, while not changing any existing entries. The "Del" button will erase all entries on the Primary Heating form. You will receive a warning of the deletion only if the form had been previously filled in prior to your entering the form.

\subsection{Cooling - Primary, Secondary, and Replacement}

The Primary and Secondary forms (see Figure 10.25 and 10.26) under the Cooling tab of the Main Menu's "MHEA" button are used to describe the cooling systems installed in the mobile home. MHEA allows the definition of a primary and a secondary cooling system in a home. The primary system is the system that supplies the majority of the cooling to the home and on which MHEA will consider installing energy efficiency measures. The Replacement form (see Figure 10.27) is used to describe the equipment you want MHEA to evaluate for the primary cooling system replacement as a possible retrofit measure. You should 


\section{Chapter 10: MHEA Building Description}

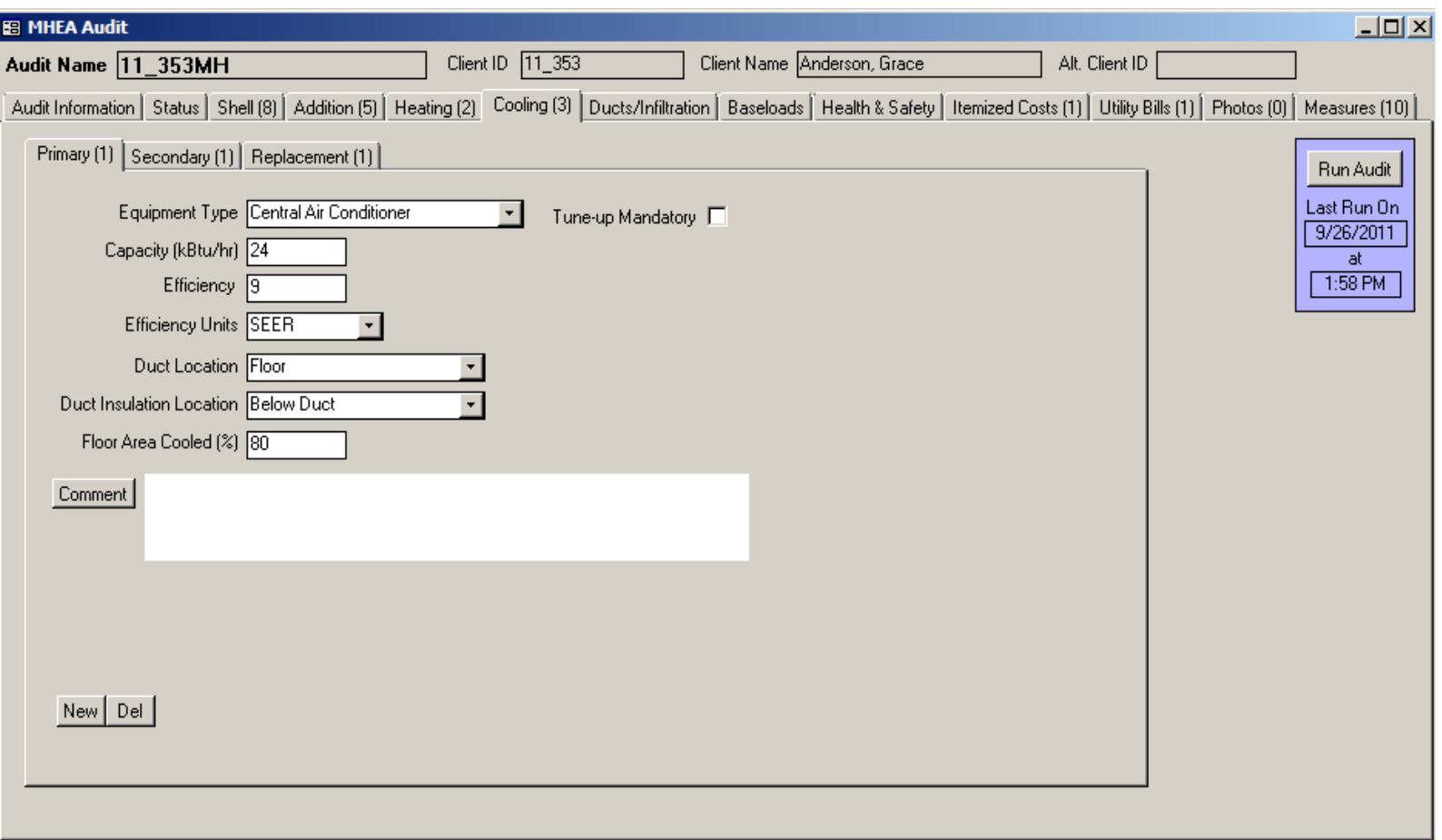

Figure 10.25. The Primary Cooling System form under the Main Menu's “MHEA” button.

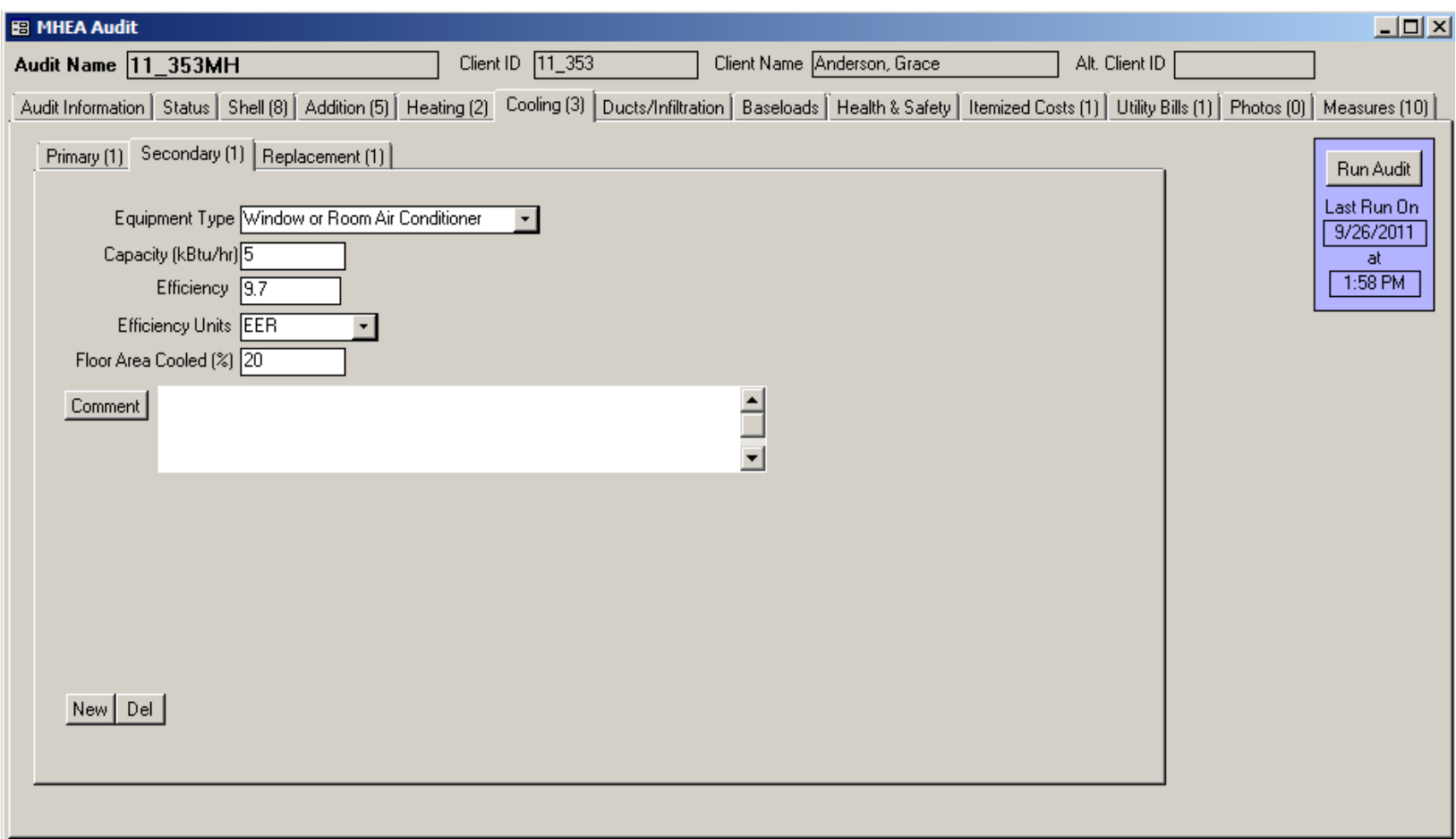

Figure 10.26. The Secondary Cooling System form under the Main Menu's “MHEA” button. 


\section{Chapter 10: MHEA Building Description}

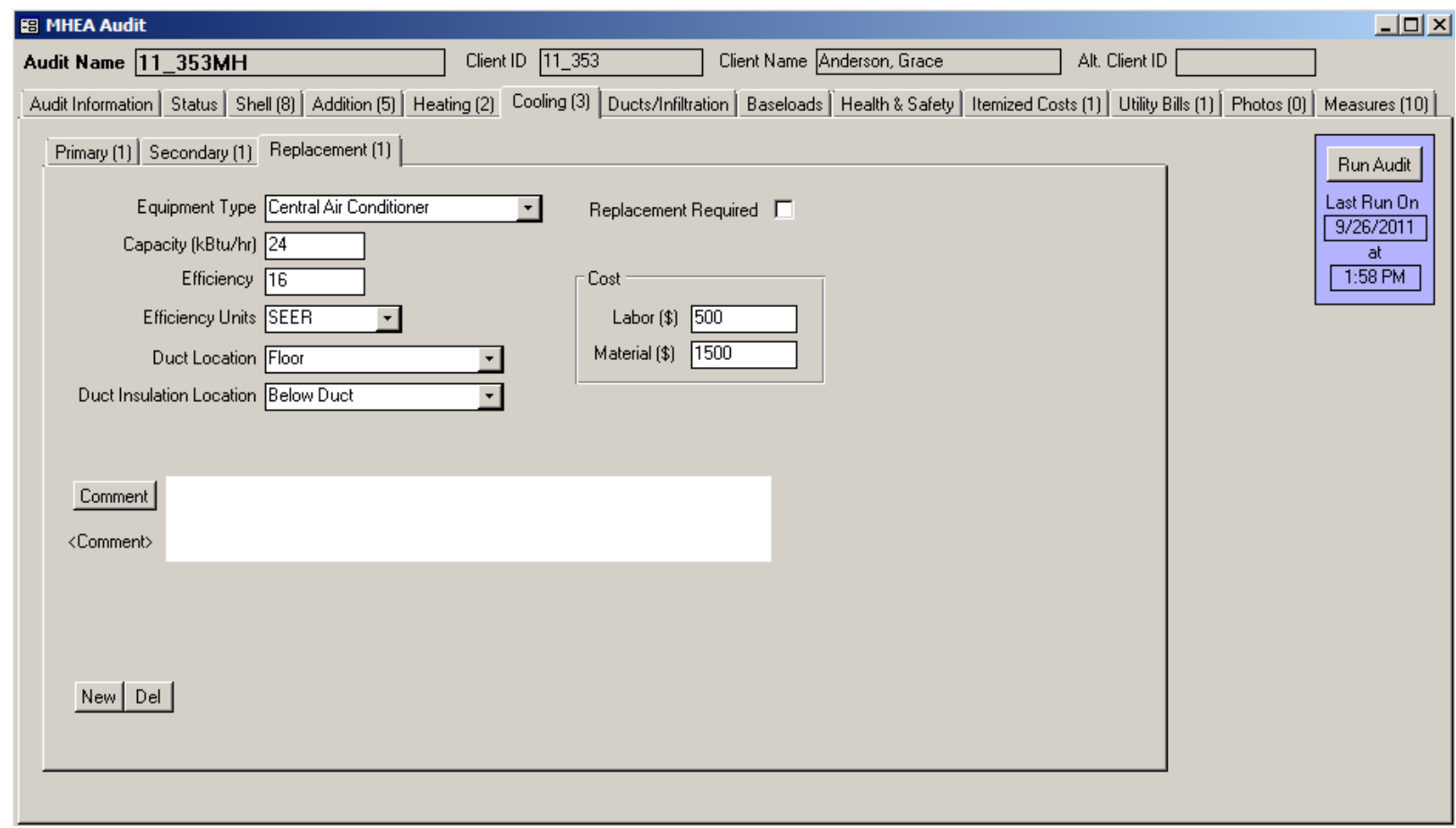

Figure 10.27. The Replacement Cooling System form under the Main Menu’s “MHEA” button.

enter information and describe the primary system first before describing the secondary system and/or the replacement system.

Below are descriptions of the individual data items and controls found in the Primary, Secondary and Replacement forms. All items on these forms are required except the Comment field. Items that appear on more than one form are analogous. Items that are not common on all three forms are indicated by specifying the name of the applicable form(s) in brackets.

- Equipment Type - Select what type of cooling system this is. The choices are Central Air Conditioner, Window or Room Air Conditioner, Heat Pump, Evaporative Cooler, or None. If Evaporative Cooler or None has been selected, fields not needed will be automatically hidden.

Heating and cooling sides of a heat pump are treated as separate components requiring descriptions under both the Heating and Cooling tabs. For correct modeling of replacing the existing primary heating and cooling systems with a heat pump, the replacement for both the heating and cooling sides of the existing units must be specified as heat pumps and the total cost of the 
replacement split between the Heating Replacement and Cooling Replacement descriptions. To have the replacement seen as cost-effective, this split in cost should be adjusted in order to show an SIR for replacement of both the heating and cooling components as cost-effective. See B.20, Replace DX Cooling . Required.

- Capacity - Enter the cooling capacity of the unit in $\mathrm{kBtu} / \mathrm{h}$. The value is often printed on the nameplate of the air conditioner. For central systems and heat pumps, the value may be given in tons of cooling, where 1 ton equals 12 $\mathrm{kBtu} / \mathrm{h}$. Sometimes the model number will contain a value divisible by 6 , since cooling equipment is normally sold with capacities that are integral multiples of $1 / 2$ ton (e.g., a model $x x x 30 x x x$ is likely a $30 \mathrm{kBtu} / \mathrm{h}$ or $2-1 / 2$ ton system). If no capacity is available, enter an estimate. NOTE: If MHEA determines that the capacity of the equipment is insufficient to meet the home's load, the annual energy consumption predicted by the program for the house will be reduced, also reducing the savings that might be predicted for individual measures. If this has occurred for any individual execution of MHEA, it will be noted under the Special Notes on the Recommended Measures Report. See Special Notes in Section 12.2, Recommended Measures Report. Required.

- Efficiency and Efficiency Units - Enter the efficiency of the cooling system in the Efficiency Units selected. The choices for Efficiency Units are EER, SEER, and COP. EER (Energy Efficiency Ratio) is used in rating window air conditioners. SEER (Seasonal Energy Efficiency Ratio) and COP (Coefficient of Performance) are used in rating central air conditioners and heat pumps. COP is an instantaneous measure of efficiency. EER is a measure of efficiency averaged over several hours of constant operating condition, while SEER is annual average efficiency. COP is unitless, while EER and SEER have units of Btu/watt-hour. Newer systems are required to display the EER or SEER rating on the unit. Older systems may list a COP value. COP has typical values between 1.5 and 4.0. EER and SEER have typical values between 5.0 and 20.0. Enter the SEER, if it is given on the nameplate. Required.

- Duct Location [Primary and Replacement] - If Central Air Conditioner or Heat Pump has been selected for Equipment Type, the Duct Location field will appear. Select the location of the main supply air duct for the cooling system. The choices are Floor, Ceiling, and None. Usually, mobile homes have a single duct that runs along the center of the home, or two ducts, each running along 
the length of the home and connected by a cross-over duct. Indicate if the main supply duct is located under the floor or above the ceiling. In some cases, no main supply air duct exists. Enter None, if the supply air duct has been disconnected from the central heating system and there are no plans to reconnect it. Conditionally required.

- Duct Insulation Location [Primary and Replacement] - If Central Air Conditioner or Heat Pump has been selected for Equipment Type, the Duct Insulation Location field will appear. Enter the location of insulation, if any, associated with the main supply air duct. The choices are Above Duct, Below Duct, Around Duct or Ductboard, and No Insulation. Insulation may be located only on the top of the duct, only on the bottom of the duct, or wrapped completely around the duct. Ductboard is a rigid insulation used to either insulate ducts or to create a plenum that is used as a duct. Select appropriate choice if duct is insulated or None if duct is uninsulated. The entry will be available only if the Duct Location is other than None. Conditonally Required.

- Floor Area Cooled [Primary and Secondary] - Enter an estimate of the percent of the floor area that is cooled by the cooling system being described. Do not enter the \% sign with the number. The sum of all areas cooled by primary and secondary equipment should be less than or equal to $100 \%$. Required.

- Tune-up Mandatory [Primary] - Use this checkbox to indicate that a system tune-up is all that is necessary based on the inspection of the cooling system. MHEA will evaluate and recommend the system tune-up, regardless of its costeffectiveness. The tuned up system will then be used in evaluating other measures not considered mandatory. No system replacement will be considered.

- Replacement Required [Replacement] - Use this checkbox to indicate that a system replacement is necessary based on the inspection of existing cooling system. Make sure that the Tune-up Mandatory checkbox on the Primary Cooling form is unchecked. MHEA will evaluate and recommend system replacement, regardless of its cost-effectiveness. The replacement system will then be used in evaluating other measures not considered mandatory. No primary cooling system tune-up will be considered. 
- Include in SIR [Primary and Replacement] - If Tune-up Mandatory [Primary] or Replacement Required [Replacement] is selected, an Include in SIR checkbox will appear on the form. This checkbox allows you to indicate that you wish to have the selected measure included in the calculation of the whole house (package) SIR for this audit. Measures not included in the whole house SIR are normally related to health and safety. Check the guidance for your program in deciding when you can mandate an HVAC measure and if it can be excluded from the SIR calculation. Even if the measure can be considered related to health and safety, if it is shown to be cost-effective, you may choose to still include it in the whole house SIR by selecting this checkbox. By doing so, it will not be seen as a health and safety measure, thus preserving funds dedicated for other such measures. An alternate reason for excluding a measure from the whole house SIR is if it is funded outside of the Weatherization Assistance Program. Whether a measure is or is not included in the whole house SIR is indicated by its location in the list of measures produced for the Recommended Measures Report (see Chapter 12, NEAT and MHEA Results).

- Labor and Material Costs [Replacement] - Enter the Labor and Material cost associated with replacing the primary cooling system. MHEA adds the labor and equipment costs in computing the SIR and reporting costs. Thus, the total cost (labor plus equipment) may be entered in either field, with $\$ 0.00$ entered for the other of the two fields, with no adverse consequences. Do not enter $\$ 0.00$ in both Labor and Material Cost fields for either replacement unit. Required.

- Comment - You may enter comments pertinent to the cooling system directly in the Comment field on the form, or you may enter them in the Comment Editor by selecting the "Comment" button to the left of the field. The Comment Editor is similar to Window's Notepad and may be used to enter extended comments. The total comment may have up to 65,000 characters. Text from other sources may be copied and pasted into the Comment Editor's window. Any comment that is entered is displayed on the Recommended Measures report and the Input Report available from the Audit Information form (see Section 8.2, Audit Information). In addition, any comment that is entered can be imported into any specific work order (see Section 13.1, Work Order Information). Thus, you may wish to enter comments related to any observation that might affect the installation of needed measures or repair work. Cooling 


\section{Chapter 10: MHEA Building Description}

system comments may include observations of equipment conditions, reasons for selecting specific retrofit options, or references to health and safety items. Optional.

- New/Del -The "New" button will take you back to the first field on the existing form, the Equipment Type field, while not changing any existing entries. The "Del" button will erase all entries on the Primary Heating form. You will receive a warning of the deletion only if the form had been previously filled in prior to your entering the form. 


\section{Chapter 11. NEAT and MHEA Common Elements}

Chapter 9, NEAT Building Description, and Chapter 10, MHEA Building Description, of this manual addressed entry of building description data specific to NEAT and MHEA, respectively. The Weatherization Assistant contains additional building and audit description data that is common to both site-built and mobile homes. This chapter describes the information entered on the forms found under Main Menu's "NEAT Audit" and "MHEA Audit" buttons which is common to both building types. This includes the Status, Ducts/Infiltration, Base Loads (Water Heating, Refrigerators, and Lighting Systems), Health \& Safety, Itemized Costs, Utility Bills, Photos, and Measures forms, as well as the optional sub-forms found on the Heating and Water Heating forms.

The only form of these that must be visited is the Ducts/Infiltration form. Entries under the Baseloads tab may generate recommendations for base load measures modeled by NEAT and MHEA. The Health \& Safety and Itemized Costs forms can be used to create recommendations to remediate health and safety issues, enter repair activities and administrative costs, or define energy saving measures not evaluated by NEAT or MHEA. The Utility Bills tab can store data from utility bills, which can be used to adjust the audits' recommendations based on actual consumption. The Status and Photos tabs are associated with optional features that do not affect the recommendations of the audits. The Measures tab is the starting point from which the optional work order feature of the program can be employed (see Chapter 13, Work Orders).

\subsection{Status (Audit)}

The Status form under the "NEAT Audit" and "MHEA Audit" windows (see Figure 11.1) allows you to view the status associated with the current audit. The setting and tracking of statuses is an optional feature in the Weatherization Assistant. See Section 18.3, Status Tracking, for more detailed information on this feature.

Below are descriptions of the individual data items and controls found on the Status form. The entries on the main Status form cannot be edited. You must use the "Edit" button on the form to change the current status for the audit. 


\section{Chapter 11: NEAT and MHEA Common Elements}

- Audit (<Record Name $>$ ) - The left most column of the form displays the status category (i.e., Audit) followed by the name of the audit for which the status is being reported.

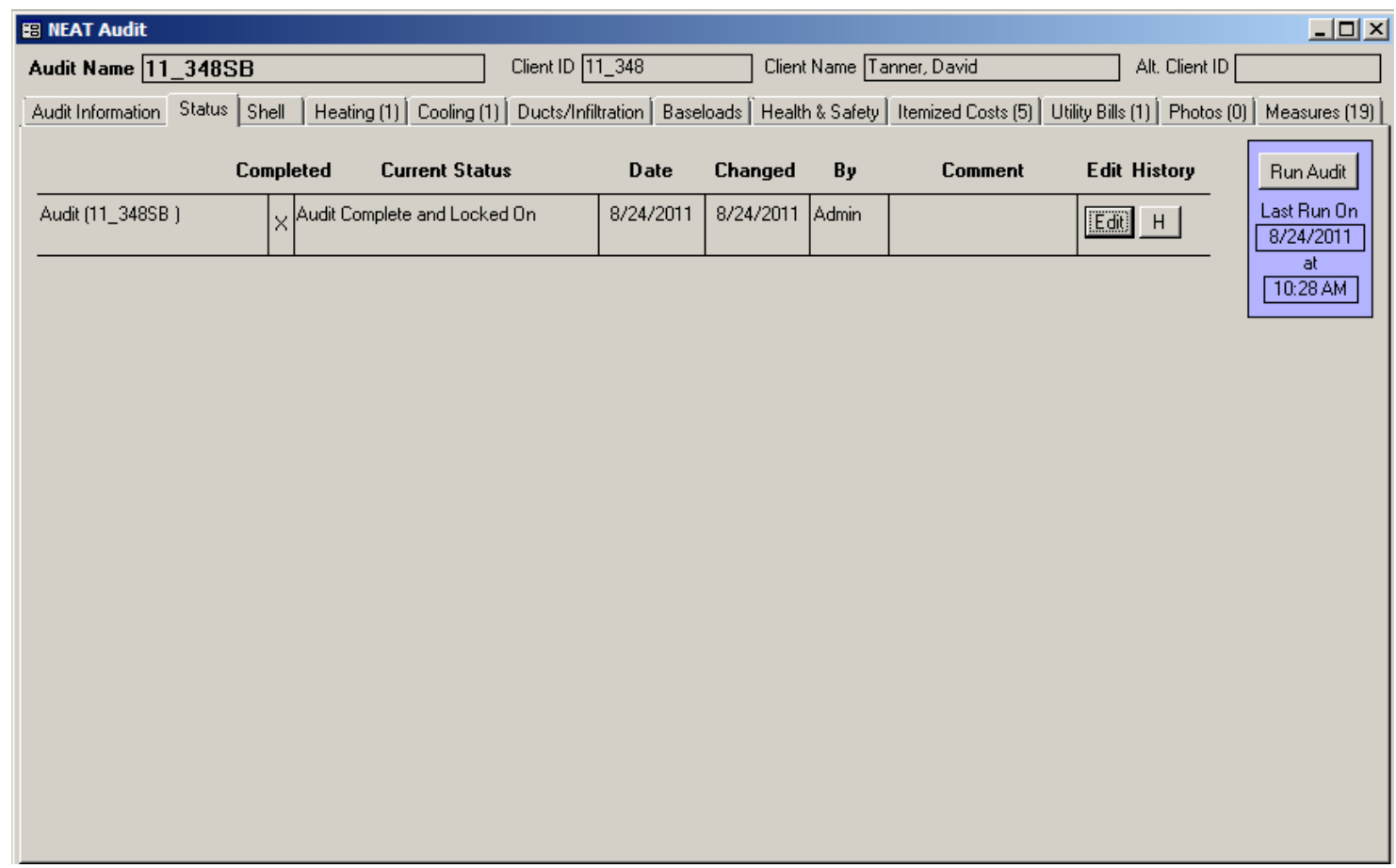

Figure 11.1. The Status form under the Main Menu's “NEAT” button.

- Current Status - This field displays the current status setting for the audit. The possible settings for audits are listed in Section 18.3, Status Tracking.

- Completed - An "X" will be displayed in this field when the current status setting signifies completion or closure of the activity associated with the status category. Settings that represent completion are indicated by an asterisk $\left(^{*}\right.$ ) (see Table 18.1, Status Categories and Settings).

- Date - This field displays the date that the current status became effective.

- Changed - This field displays the date that the current status was last changed.

- By - This field displays the User Name of the person who changed the status. The names are restricted to those that have been entered on the Contacts form under the Main Menu's “Agency” button and have a password assigned as 


\section{Chapter 11: NEAT and MHEA Common Elements}

discussed in Section 6.2, Contacts (Agency). A User Name of “Admin” implies that a specific person was not identified as making the change.

- Comment - Any comment that has been appended to this current status setting will be shown in this field.

- Edit - Status settings for the current audit are changed by selecting the "Edit" button. Use of the Status Editor form that appears is explained in Section 18.3, Status Tracking.

- History - Select the "H" button to see a history of all settings for the current audit. The Status History form that appears is the same in appearance as that discussed in Section 6.9, Status History.

\subsection{Ducts/Infiltration - Air and Duct Leakages}

Both NEAT and MHEA provide means to evaluate the effectiveness of infiltration reduction work and duct sealing. However, the programs do not direct this work. They assume that available procedures of locating and repairing air leaks are being used. Air leakage reductions from specific weatherization activities cannot be predicted accurately with calculations. What information you provide regarding infiltration and ducts will depend on what measurements you are accustomed to taking during an audit.

Blower doors depressurize (or pressurize) a house to some pressure differential (measured in Pascal, Pa) with respect to the outside. They can then measure the cubic feet per minute (CFM) of air passing through leaks in the house at that pressure differential. Since the pressure differential is greater than under normal circumstances, the air leaks are exaggerated above what they would be normally, allow easier detection. A duct blower works in a similar manner as the blower door, except that it pressurizes only the ducts in the house with respect to the remainder of the house and outdoors. Similarly, it will provide the CFM leaking from the ducts at this pressure differential.

The appearance of the Air and Duct Leakages form changes depending on whether you wish the Weatherization Assistant to evaluate duct sealing and, if so, which method is to be used. However, some entries and features appear regardless of your choice. Below are descriptions of these individual data items and controls. Others, 


\section{Chapter 11: NEAT and MHEA Common Elements}

which are unique to the choice of duct sealing method, will be described separately with the discussion of the specific method. See Figure 11.2 for the positioning of these items on the form.

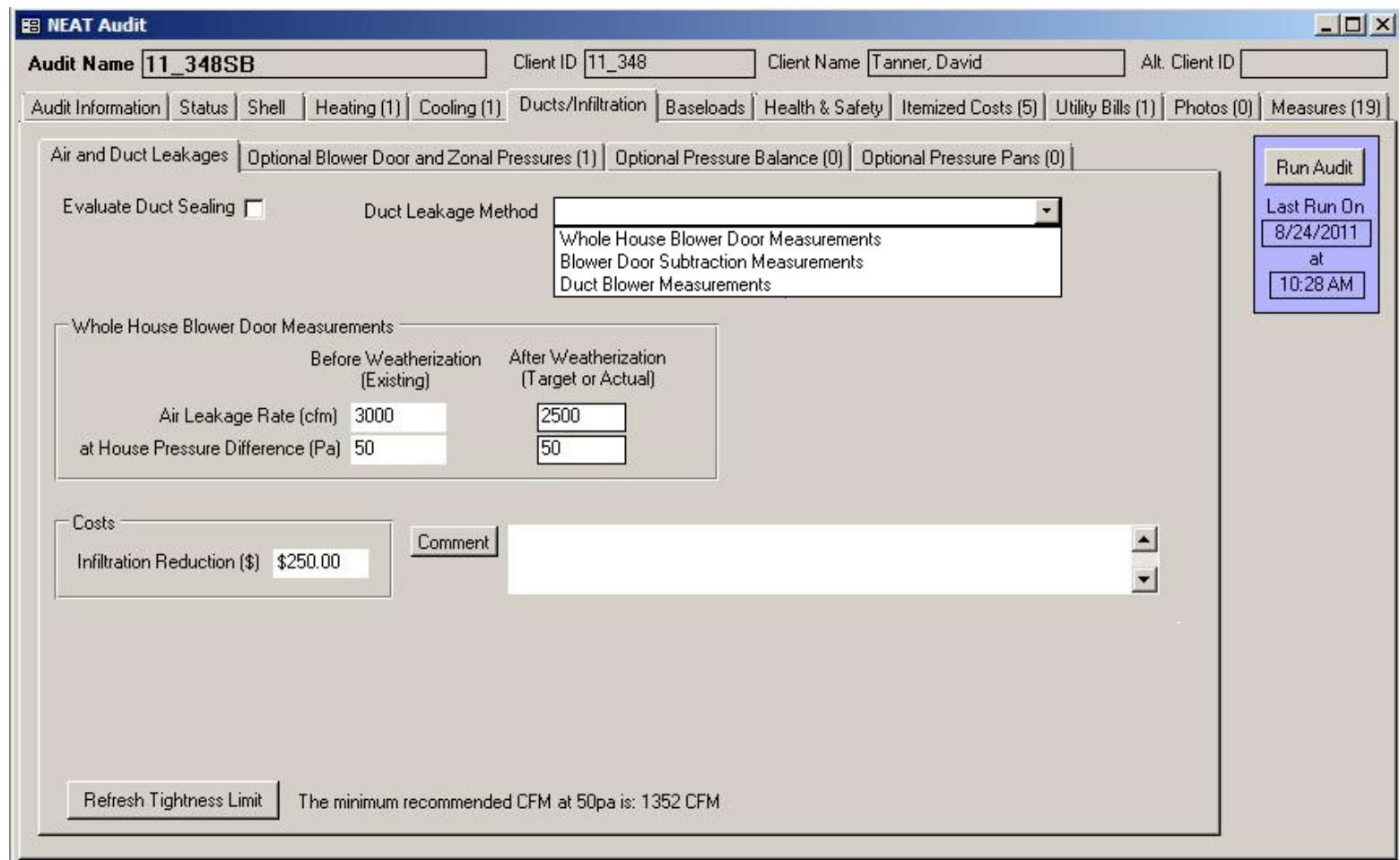

Figure 11.2. The Air and Duct Leakages form under the Main Menu’s “NEAT” button.

- Evaluate Duct Sealing - Select this checkbox if you wish to evaluate duct sealing and duct leakage measurements are available. If this checkbox is unchecked, only the air sealing of the building envelope is evaluated using two sets of air leakage measurements - before and after weatherization. If this checkbox is selected, you will be required to provide additional air leakage or duct leakage data before and after duct sealing.

- Duct Leakage Method - If the Evaluate Duct Sealing checkbox is selected, the Duct Leakage Method field will be presented. Select a desired method for duct leakage measurements. The choices are Whole House Blower Door Measurements, Blower Door Subtraction Measurements, Duct Blower Measurements, and Pressure Pan Measurements. The Pressure Pan Measurements option is available only in MHEA. NEAT and MHEA assume that the duct sealing efforts are performed prior to any whole house air 


\section{Chapter 11: NEAT and MHEA Common Elements}

infiltration reduction work having been performed. The entries required for each of these methods are described below in sections specific to the method chosen.

- Cost of Infiltration Reduction - If the Evaluate Duct Sealing checkbox is left unchecked, you may enter the cost in dollars associated with reducing the infiltration from the pre-weatherization value to an actual or target postweatherization level. NEAT and MHEA use this information to calculate the SIR for the infiltration reduction measures. If the cost of infiltration reduction is not provided, the SIR for infiltration reduction will not be computed or reported on the Recommendation Measure Report. If the Evaluate Duct Sealing checkbox is checked and a Duct Leakage Method is selected, you must enter the costs associated with reducing the infiltration from the post-duct sealing value (before other weatherization measures are implemented) to the target post-weatherization level in dollars. Conditionally Required.

- Cost of Duct Sealing - If the Evaluate Duct Sealing checkbox is checked and a Duct Leakage Method is selected, a field for entering the cost of duct sealing will be displayed. You must enter the costs associated with duct sealing in dollars to reduce duct leakage from pre-duct sealing value to post-duct sealing value (before other weatherization measures are implemented). NEAT and MHEA use this information in computing the SIR for the Seal Ducts measure. Required.

- Comment - You may enter comments pertinent to the air and duct leakages directly in the Comment field on the form, or you may enter them in the Comment Editor by selecting the "Comment" button to the left of the field. The Comment Editor is similar to Window's Notepad and may be used to enter extended comments. The total comment may have up to 65,000 characters. Text from other sources may be copied and pasted into the Comment Editor's window. Any comment that is entered is displayed on the Recommended Measures report and the Input Report available from the Audit Information form (see Section 8.2, Audit Information). In addition, any comment that is entered can be imported into any specific work order (see Section 13.1, Work Order Information). Thus, you may wish to enter comments related to any observation that might affect the installation of needed measures or repair work. If leakage sites are located during the initial blower door setup, you could 


\section{Chapter 11: NEAT and MHEA Common Elements}

include comments to identify to the crew or contractor where these sites are. Optional.

- Refresh Tightness Limit - NEAT and MHEA use building data entered on the Audit Information form under the "NEAT Audit" and "MHEA Audit" windows to calculate the building tightness limit (BTL) and report that on the Air and Duct Leakages form as "The minimum recommended CFM at $50 \mathrm{~Pa}$ is: <BTL> CFM." You may use this value as the minimum limit for target post weatherization air leakage rate to ensure that the building is not over-tight after weatherization. If you alter the building data on the Audit Information form, you may select the "Refresh Tightness Limit" button on this form to update the building tightness limit reported on this form. If you have an alternate method of computing a building tightness limit or if ASHRAE 62.2 is being used, this limit may be considered irrelevant.

The Air and Duct Leakages form has four different sub-forms-one if you do not wish to evaluate duct sealing and three that evaluate duct sealing using three different methods. MHEA has an additional sub-form for a fourth duct sealing method available only for mobile homes. Each sub-form and its associated inputs will be discussed separately below.

\section{Whole House Blower Door Measurements for Air Leakage Sub-Form}

If the duct leakage measurements are unavailable, the Evaluate Duct Sealing checkbox should be left unchecked. The sub-form, then presented, includes the Whole House Blower Door Measurements data block consisting of two sets of data fields for entering air leakage data before and after weatherization (see Figure 11.3).

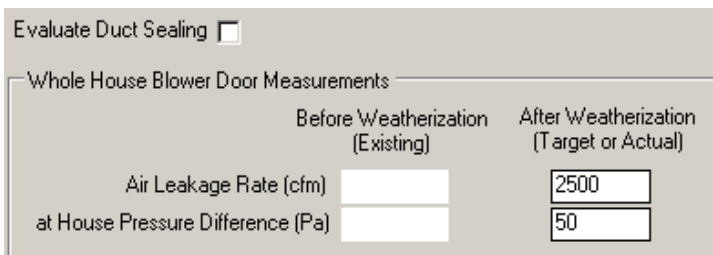

Figure 11.3. The Whole House Blower Door Measurements for Air Leakage subform.

- Before Weatherization (Existing) Air Leakage Rate and House Pressure Difference - If pre-weatherization blower door measurements are available, enter the measured air leakage rate in units of CFM (cubic feet per minute) and the corresponding house pressure difference in units of Pa (Pascal). The air leakage rate is normally measured at a pressure differential of $50 \mathrm{~Pa}$, if such can 


\section{Chapter 11: NEAT and MHEA Common Elements}

be reached. Leave the fields blank if blower door measurements are unavailable. NEAT and MHEA will use this information only to estimate energy savings from air sealing. The entries will not impact the energy saving estimates for other measures. The default for House Pressure Difference is 50 Pa. Optional.

- After Weatherization (Target or Actual) Air Leakage Rate and House Pressure Difference - Enter the post-weatherization air leakage rate in units of CFM (cubic feet per minute) and the corresponding house pressure difference in units of $\mathrm{Pa}$ (Pascal). If you are entering data before performing any work on a house, and your audit procedure has established a "target" post-weatherization air leakage rate, enter the value here. If the target air leakage information is in air changes per hour (ACH) at natural conditions, an approximate conversion may be accomplished by multiplying the $\mathrm{ACH}$ by one-third of the volume of the house to obtain air leakage rate in CFM at $50 \mathrm{~Pa}$ (CFM50). If no target value is available and no pre-retrofit air leakage data has been entered, established default values may be entered (e.g., 2500 CFM at 50 PA). If no air sealing work is deemed necessary and no other weatherization measure is likely to reduce air leakage, enter the pre-weatherization blower door measurements in the post-weatherization data fields. If a post-weatherization blower door test is eventually conducted, you may re-run the audit using these blower door measurements to verify the previous results or maintain a record of actual air leakage reduction and energy savings accomplished. The values entered for post-weatherization leakage rate can affect the savings of other measures, particularly HVAC measures. The default for House Pressure Difference is 50 Pa. Required.

Entry of target or default values for the post-weatherization leakage data is required to allow the Weatherization Assistant to make logical recommendations for the other measures it considers. Estimates of these savings will be more accurate if they are based on the air leakage characteristics of the house after infiltration reduction is accomplished, rather than before.

If only the required post-weatherization leakage data is provided, NEAT and MHEA will not produce any measure listing for infiltration reduction on the Recommended Measures report (see Section 12.2, Recommended Measures Report). If both pre- and post-weatherization leakage data is entered, the programs will produce an entry in this report indicating the savings for infiltration reduction. 


\section{Chapter 11: NEAT and MHEA Common Elements}

If, in addition, a cost for the infiltration reduction has been entered on the Whole House Blower Door Measurements sub-form, the Recommended Measures report will display an estimated SIR for the measure. If you routinely provide entries for all of the air leakage data and the SIRs for infiltration reduction shown in the Recommended Measures report are consistently less than the minimum acceptable value (normally 1.0), then you should review your procedure for determining the targets used, the costs being assumed, or your infiltration reduction procedure.

\section{Common Entries on the Duct Leakage Sub-Forms - Duct Operating Pressures}

All of the duct leakage measurement methods require entries for duct operating pressures. These entries will be described below and not repeated for each individual method. See Figure 11.4 to view the Duct Operating Pressures data block.

- Before Duct Sealing Supply and Return Duct Operating Pressures - Enter the pre-weatherization (i.e., pre-duct sealing) supply and return duct operating pressures with respect to the space surrounding the ducts, in units of Pascal, measured during normal conditions with the air handler fan on. These measurements can be taken by inserting pressure probes through small holes drilled in the supply plenum (near the air handler) and the return plenum. The holes should be repaired afterwards. Required.

- After Duct Sealing Supply and Return Duct Operating Pressures - Enter the post-duct sealing supply and return duct operating pressures with respect to space surrounding the ducts, in units of Pascal, measured during normal conditions with the air handler fan on. These measurements can be taken by inserting pressure probes through small holes drilled in the supply plenum (near the air handler) and the return plenum. The holes should be repaired afterwards. Required.

\section{Whole House Blower Door Measurements for Duct Leakage Sub-Forms}

Under this duct leakage measurement method, whole house blower door measurements are taken before and after duct sealing to estimate the duct leakage reduction. For the method to provide this estimate, duct sealing must be accomplished first, followed by the remainder of the weatherization efforts, 


\section{Chapter 11: NEAT and MHEA Common Elements}

including infiltration reduction. The entries required are from readings you would normally take to estimate the whole house infiltration. These readings are simply taken before any duct leakage or infiltration reduction work has been performed, then after duct sealing is accomplished - but before any other weatherization efforts, then a third time after all of the weatherization work has been performed, including infiltration reduction. For all readings, the registers and grills of the duct system should remain open, thus making the ducts as much a part of the conditioned space as possible.

This sub-form (see Figure 11.4) includes a Whole House Blower Door Measurements data block consisting of the three sets of data fields requiring air leakage data and the Duct Operating Pressure data block, discussed under "Common Entries on the Duct Leakage Sub-Forms - Duct Operating Pressures," above. All data items on this sub-form are required.

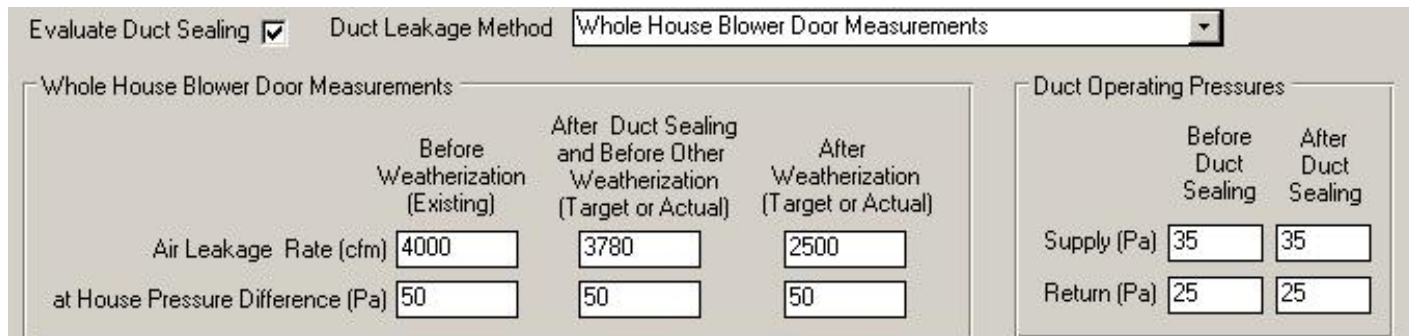

Figure 11.4. The Whole House Blower Door Measurements for Duct Leakage sub-form.

- Before Weatherization (Existing) Air Leakage Rate and House pressure Difference - Enter the pre-weatherization blower door measurements including the measured air leakage rate in units of CFM (cubic feet per minute) and the corresponding house pressure difference in units of $\mathrm{Pa}$ (Pascal). The air leakage rate is normally measured at a pressure differential of $50 \mathrm{~Pa}$, if such can be reached. NEAT and MHEA use this information with the measurements conducted after duct sealing and before other weatherization to estimate energy savings from duct sealing. The default for House Pressure Difference is $50 \mathrm{~Pa}$. Required.

- After Duct Sealing and Before Other Weatherization (Target or Actual) Air Leakage Rate and House pressure Difference - Enter the post-duct sealing air leakage rate in units of CFM (cubic feet per minute) and the corresponding house pressure difference in units of $\mathrm{Pa}$ (Pascal). If you are 


\section{Chapter 11: NEAT and MHEA Common Elements}

entering data before duct sealing efforts have actually been performed, enter "target" post-duct sealing air leakage data your audit procedure has established for the house being audited. NEAT and MHEA use this information with the measurements conducted before weatherization to estimate energy savings from duct sealing. The default for House Pressure Difference is 50 Pa. Required.

- After Weatherization (Target or Actual) Air Leakage Rate and House Pressure Difference - Enter the post-weatherization air leakage rate in units of CFM (cubic feet per minute) and the corresponding house pressure difference in units of $\mathrm{Pa}$ (Pascal). If you are entering data before weatherizing the house, enter an established "target" post-weatherization air leakage rate. If the target air leakage information is in air changes per hour (ACH) at natural conditions, an approximate conversion may be accomplished by multiplying the ACH by one-third of the volume of the house to obtain air leakage rate in CFM at $50 \mathrm{~Pa}$ (CFM50). If no target value is available, established default values may be entered (e.g., 2500 CFM at 50 PA). Make sure the target or default values indicate the same or less air leakage than the post-duct sealing values. If no air sealing work is deemed necessary and no other weatherization measure is likely to reduce air leakage, enter the post-duct sealing blower door measurements in the post-weatherization data fields. If a post-weatherization blower door test is eventually conducted, you may re-run the audit using these blower door measurements to verify the previous results or maintain a record of actual air leakage reduction and energy savings accomplished. NEAT and MHEA use this information with the measurements conducted after duct sealing and before other weatherization efforts to estimate energy savings from air sealing. The values entered for post-weatherization leakage rate can affect the savings of other measures, particularly HVAC measures. The default for House Pressure Difference is $50 \mathrm{~Pa}$. Required.

\section{Blower Door Subtraction Measurements for Duct Leakage Sub-Form}

Under this duct leakage measurement method, two sets of whole house blower door measurements are taken, both before and after duct sealing. The first set of measurements is taken with the registers and grills of the duct system open, making the ducts as much a part of the conditioned space as possible. The second set of measurements is taken with all supply registers and return grills covered with cardboard, plastic, tape, etc., to have the duct system sealed off from the rest of the house. These measurements allow the program to subtract the house leakage from 


\section{Chapter 11: NEAT and MHEA Common Elements}

the house-plus-duct leakage, thus isolating the duct leakage. One additional reading is recorded while the duct system is sealed off - the duct-to-house pressure difference, allowing a determination of the duct leakage to the house. This method of determining duct leakage is not useful if this duct-to-house pressure difference is less than $20 \mathrm{~Pa}$, indicating large duct leakage to the house. This is commonly true in homes that use building cavities for a significant part of the ductwork.

This sub-form includes a Whole House Blower Door Measurements data block and a Duct Operating Pressure data block, discussed under "Common Entries on the Duct Leakage Sub-Forms - Duct Operating Pressures,” above (see Figure 11.5).

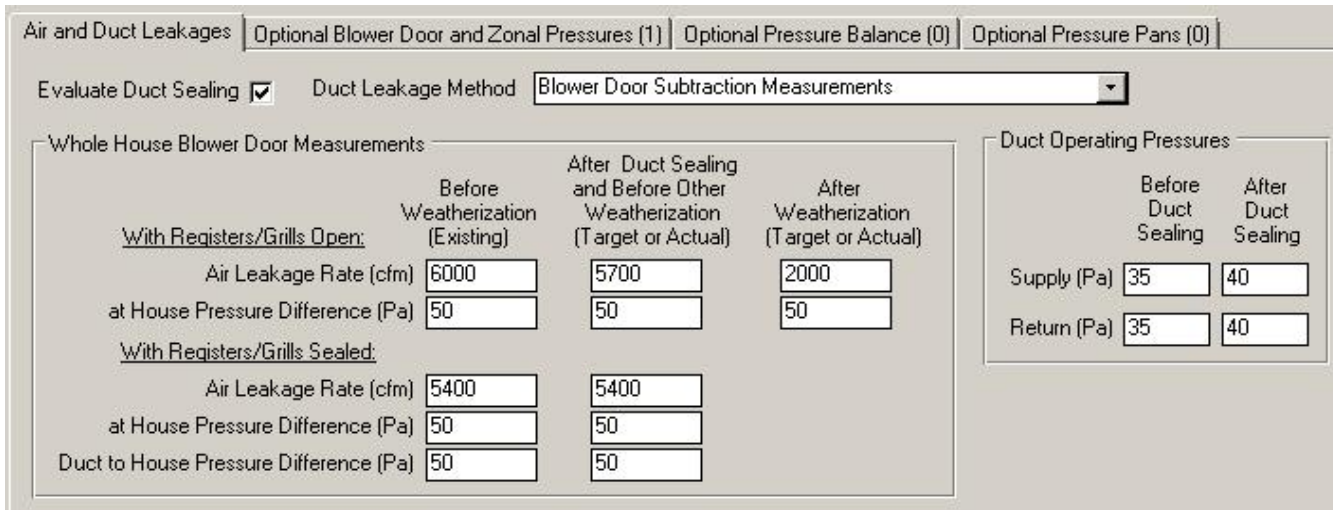

Figure 11.5. The Blower Door Subtraction Measurements for Duct Leakage sub-form.

- Before Weatherization (Existing) Air Leakage Rate and House Pressure Difference, With Registers/Grills Open - Enter the pre-weatherization air leakage rate in units of CFM (cubic feet per minute) and the corresponding house pressure difference in units of $\mathrm{Pa}$ (Pascal) with the registers and grills open. The air leakage rate is normally measured at a pressure differential of 50 $\mathrm{Pa}$, if such can be reached. The default for House Pressure Difference is $50 \mathrm{~Pa}$. Required.

- Before Weatherization (Existing) Air Leakage Rate, House Pressure Difference, and Duct-to-House Pressure Difference, With Registers/Grills Sealed - Enter the pre-weatherization air leakage rate in units of CFM (cubic feet per minute) and the corresponding house pressure difference with respect to the outside and the duct-to-house pressure difference in units of $\mathrm{Pa}$ (Pascal), all with the registers and grills sealed off. The air leakage rate is normally measured at a pressure differential of $50 \mathrm{~Pa}$, if such can be reached. The ductto-house pressure difference can be measured by inserting a pressure probe 
through a small hole drilled in either the supply plenum (near the air handler) or the return plenum, or by punching a small hole through the temporary covering over a supply register or return grill. The default for House Pressure Difference is $50 \mathrm{~Pa}$. Required.

- After Duct Sealing and Before Other Weatherization (Target or Actual) Air Leakage Rate and House Pressure Difference, With Registers/Grills Open - Enter the post-duct sealing air leakage rate in units of CFM (cubic feet per minute) and the corresponding house pressure difference in units of $\mathrm{Pa}$ (Pascal), with the registers and grills open. The air leakage rate is normally measured at a pressure differential of $50 \mathrm{~Pa}$, if such can be reached. The input form indicates possible use of "Target" values for these inputs. If your audit procedure has established such targets, they may be entered. However, due to the unique nature of duct leakage in homes, availability of such values is less likely. The default for House Pressure Difference is $50 \mathrm{~Pa}$. Required.

- After Duct Sealing and Before Other Weatherization (Target or Actual) Air Leakage Rate, House Pressure Difference, and Duct to House Pressure Difference, With Registers/Grills Sealed - Enter the post-duct sealing air leakage rate in units of CFM (cubic feet per minute) and the corresponding house pressure difference and duct-to-house pressure difference in units of $\mathrm{Pa}$ (Pascal), all with the registers and grills sealed off. The duct-to-house pressure difference can be measured by inserting a pressure probe through a small hole drilled in either the supply plenum (near the air handler) or the return plenum, or punching a small hole through the temporary covering over a supply register or return grill. The input form indicates possible use of "Target" values for these inputs. If your audit procedure has established such targets, they may be entered. However, due to the unique nature of duct leakage in homes, availability of such values is less likely. The default for House Pressure Difference is $50 \mathrm{~Pa}$. Required.

- After Weatherization (Target or Actual) Air Leakage Rate and House Pressure Difference, With Registers/Grills Open - Enter the postweatherization air leakage rate in units of CFM (cubic feet per minute) and the corresponding house pressure difference in units of $\mathrm{Pa}$ (Pascal). If you are entering data before weatherizing the house, enter an established "target" postweatherization air leakage rate. If the target air leakage information is in air changes per hour (ACH) at natural conditions, an approximate conversion may 


\section{Chapter 11: NEAT and MHEA Common Elements}

be accomplished by multiplying the $\mathrm{ACH}$ by one-third of the volume of the house to obtain air leakage rate in CFM at $50 \mathrm{~Pa}$ (CFM50). If no target value is available, established default values may be entered (e.g., 2500 CFM at 50 PA). Make sure the target or default values indicate the same or less air leakage than the post-duct sealing values. If no air sealing work is deemed necessary and no other weatherization measure is likely to reduce air leakage, enter the post-duct sealing blower door measurements in the post-weatherization data fields. If a post-weatherization blower door test is eventually conducted, you may re-run the audit using these blower door measurements to verify the previous results or maintain a record of actual air leakage reduction and energy savings accomplished. NEAT and MHEA use this information with the measurements conducted after duct sealing and before other weatherization efforts to estimate energy savings from air sealing. The values entered for post-weatherization leakage rate can affect the savings of other measures, particularly HVAC measures. The default for House Pressure Difference is $50 \mathrm{~Pa}$. Required.

\section{Duct Blower Measurements for Duct Leakage Sub-Form}

Under this duct leakage measurement method, a duct blower (see Figure 11.6) and a blower door are used. A duct blower pressurizes the duct system, which is sealed off (by taping the registers and grills) from the remainder of the house. The measured CFM through the duct blower is then equal to the total air leakage from the ducts to both the outside and interior of the house. To obtain leakage to only the outside, the blower door is used to pressurize the entire house to the same pressure, with respect to the outside, as the ducts. With no pressure differential between the house and the ducts, any leakage recorded by the duct blower must then be to the outside. The leakage will be at whatever duct/outside pressure differential is established by the duct blower. This technique of determining duct leakage is more accurate than the other means, but also the most time-consuming.

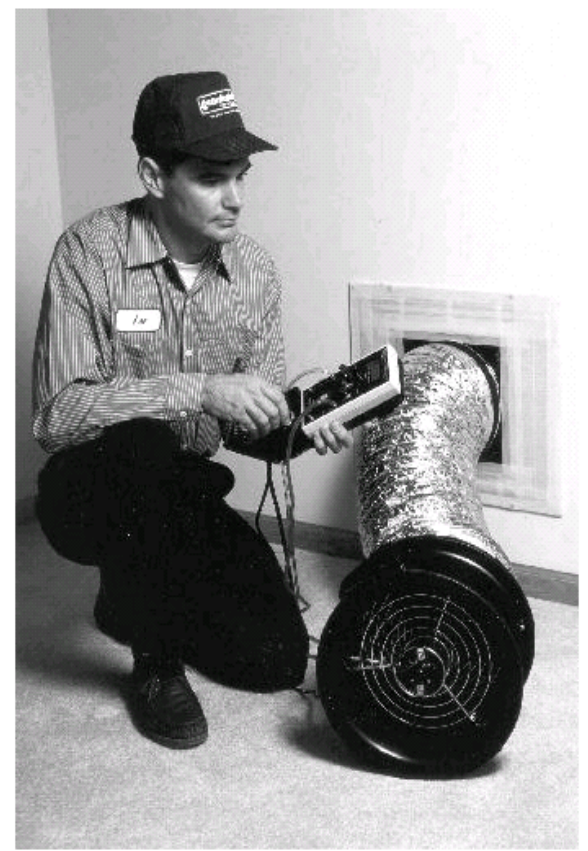

Figure 11.6. The Duct Blower (Photo courtesy of The Energy Conservatory). 


\section{Chapter 11: NEAT and MHEA Common Elements}

This sub-form includes a Whole House Blower Door Measurements data block, a Duct Operating Pressure data block, and a Duct Blower Measurements data block (see Figure 11.7). The Whole House Blower Door Measurements data block is the same as used for evaluation of air leakage without consideration of duct leakage, discussed earlier in this chapter. Entries for the Duct Operating Pressure data block are the same as for all other duct leakage measurement methods and will not be described here. They are discussed under "Common Entries on the Duct Leakage Sub-Forms - Duct Operating Pressures," earlier in this chapter.

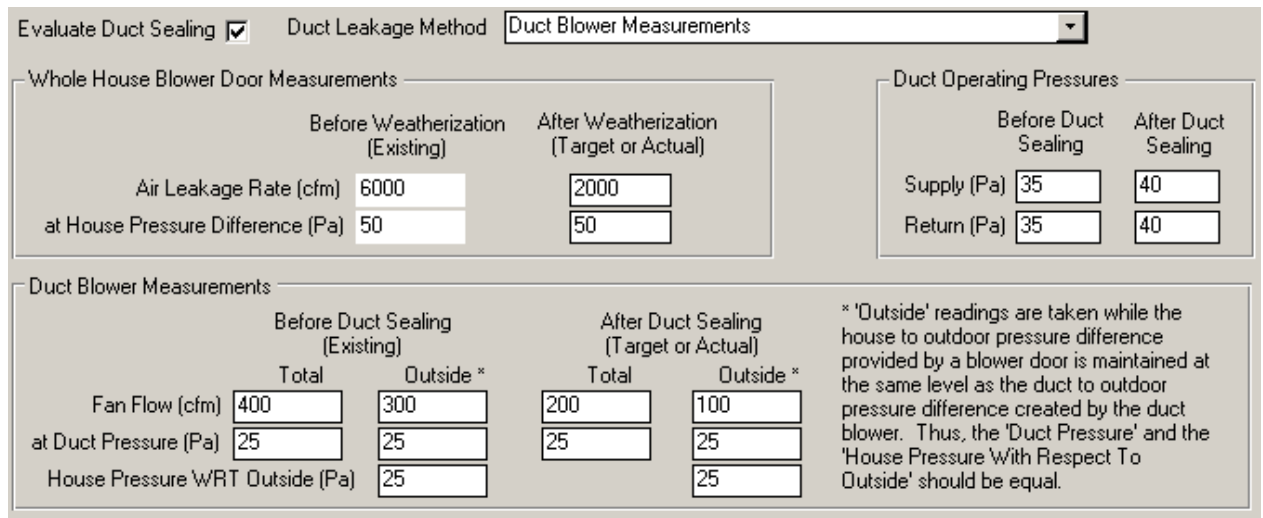

Figure 11.7. The Duct Blower Measurements for Duct Leakage sub-form.

- Before Weatherization (Existing) Air Leakage Rate and House Pressure Difference - If pre-weatherization blower door measurements are available, enter the measured air leakage rate in units of CFM (cubic feet per minute) and the corresponding house pressure difference in units of Pa (Pascal). The air leakage rate is normally measured at a pressure differential of $50 \mathrm{~Pa}$, if such can be reached. Leave the fields blank if blower door measurements are unavailable. NEAT and MHEA will use this information only to estimate energy savings from air sealing. The entries do not impact the energy saving estimates for other measures. The default for House Pressure Difference is 50 Pa. Optional.

\section{- After Weatherization (Target or Actual) Air Leakage Rate and House}

Pressure Difference - Enter the post-weatherization air leakage rate in units of CFM (cubic feet per minute) and the corresponding house pressure difference in units of $\mathrm{Pa}$ (Pascal). If you are entering data before performing any work on a house, and your audit procedure has established a "target" post-weatherization air leakage rate, enter the value here. If the target air leakage information is in air changes per hour (ACH) at natural conditions, an approximate conversion 


\section{Chapter 11: NEAT and MHEA Common Elements}

may be accomplished by multiplying the ACH by one-third of the volume of the house to obtain air leakage rate in CFM at $50 \mathrm{~Pa}$ (CFM50). If no target value is available and no pre-retrofit air leakage data has been entered, established default values may be entered (e.g., 2500 CFM at 50 PA). If no air sealing work is deemed necessary and no other weatherization measure is likely to reduce air leakage, enter the pre-weatherization blower door measurements in the post-weatherization data fields. If a post-weatherization blower door test is eventually conducted, you may re-run the audit using these blower door measurements to verify the previous results or maintain a record of actual air leakage reduction and energy savings accomplished. The values entered for post-weatherization leakage rate can affect the savings of other measures, particularly HVAC measures. The default for House Pressure Difference is 50 Pa. Required.

- Before Duct Sealing Total Fan Flow and Duct Pressure - These measurements are taken before duct sealing with the registers and grills temporarily sealed off (taped) from the rest of the house and the duct blower pressurizing the ducts with respect to the house and outside. Enter the duct blower fan flow rate in CFM and the corresponding ducts-to-house pressure difference in Pascal. You might want to open a window during the measurement to ensure that there is no pressure differential between the house and the outside. Required.

- Before Duct Sealing Outside Fan Flow, Duct Pressure, and House Pressure WRT Outside - These measurements are taken before duct sealing with the registers and grills temporarily sealed off (taped) from the rest of the house, the duct blower pressurizing the ducts, and the blower door pressurizing the house such that the house-to-outdoor pressure difference is maintained at the same level as the ducts-to-outdoor pressure difference. Enter the duct blower fan flow rate in CFM and the ducts-to-outdoor and house-to-outdoor pressure differences in Pascal. Note, for accurate determination of the duct leakage, the house-tooutdoor pressure difference should be the same as, or as close as possible to, the ducts-to-outdoor pressure difference. Required.

- After Duct Sealing Total Fan Flow and Duct Pressure - These measurements are taken after duct sealing with the registers and grills temporarily sealed off (taped) from the rest of the house, and the duct blower pressurizing the ducts with respect to the house and outside. Enter the duct 


\section{Chapter 11: NEAT and MHEA Common Elements}

blower fan flow rate in CFM and the corresponding ducts-to-house pressure difference in Pascal. You might want to open a window during the measurement to ensure that there is no pressure differential between the house and the outside. Required.

- After Duct Sealing Outside Fan Flow, Duct Pressure, and House Pressure WRT Outside - These measurements are taken after duct sealing with the registers and grills temporarily sealed off (taped) from the rest of the house, the duct blower pressurizing the ducts, and the blower door pressurizing the house such that the house-to-outdoor pressure difference is maintained at the same level as the ducts-to-outdoor pressure difference. Enter the duct blower fan flow rate in CFM, and the ducts-to-outdoor and house-to-outdoor pressure differences in Pascal. Note, for accurate determination of the duct leakage, the house-to-outdoor pressure difference should be the same as, or as close as possible to, the ducts-to-outdoor pressure difference. Required.

\section{Pressure Pan Measurements for Duct Leakage Sub-Form (For MHEA Only)}

A correspondence has been found to exist between the sum of pressure pan measurements from all of the supply registers in a manufactured home and the duct leakage to the outside. The pressure pan measurements, that is, the duct-to-house pressure difference at each supply register, are taken with the blower door depressurizing the house to 50 Pascal and a pressure pan attached to a manometer. No pressure difference indicates that the supply duct leading to the register is at the same pressure as the house and that little or no leaks to the outside exist in that branch of the ducts. The sum of these measurements from all supply registers corresponds to the total leakage of the ducts to the outside. This method of estimating duct leakage is normally appropriate only for manufactured homes.

This sub-form includes a Whole House Blower Door Measurements data block, a Duct Operating Pressure data block, and a Pressure Pan Measurements data block (see Figure 11.8). The Whole House Blower Door Measurements data block is the same as used for evaluation of air leakage without consideration of duct leakage, discussed earlier in this chapter. Entries for the Duct Operating Pressure data block are the same as for all other duct leakage measurement methods and will not be described here. They are discussed under "Common Entries on the Duct Leakage Sub-Forms - Duct Operating Pressures," earlier in this chapter. 


\section{Chapter 11: NEAT and MHEA Common Elements}

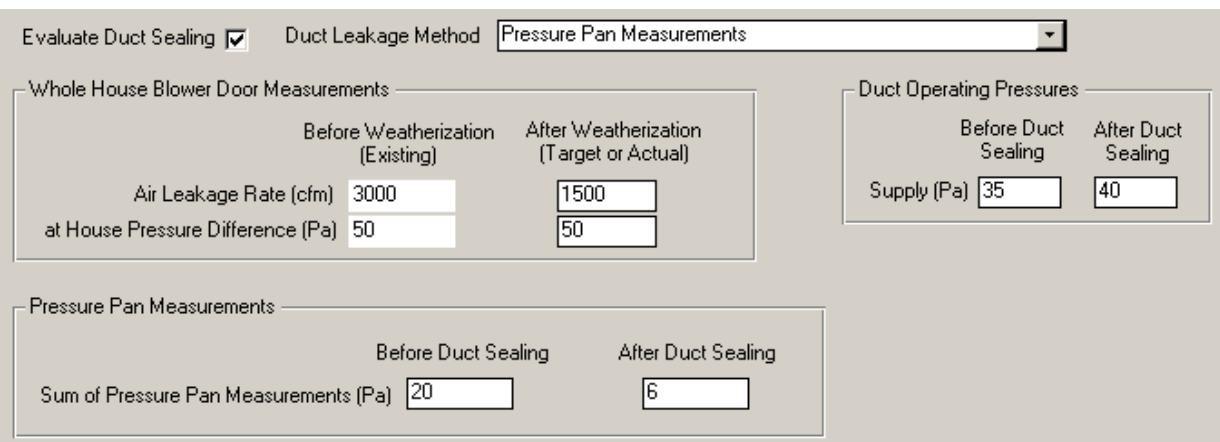

Figure 11.8. The Pressure Pan Measurements for Duct Leakage sub-form (MHEA only).

- Before Weatherization (Existing), Air Leakage Rate and House Pressure Difference - If pre-weatherization blower door measurements are available, enter the measured air leakage rate in units of CFM (cubic feet per minute) and the corresponding house pressure difference in units of $\mathrm{Pa}$ (Pascal). The air leakage rate is normally measured at a pressure differential of $50 \mathrm{~Pa}$, if such can be reached. Leave the fields blank if blower door measurements are unavailable. NEAT and MHEA will use this information only to estimate energy savings from air sealing. The entries do not impact the energy saving estimates for other measures. The default for House Pressure Difference is 50 Pa. Optional.

\section{- After Weatherization (Target or Actual), Air Leakage Rate and House} Pressure Difference - Enter the post-weatherization air leakage rate in units of CFM (cubic feet per minute) and the corresponding house pressure difference in units of $\mathrm{Pa}$ (Pascal). If you are entering data before performing any work on a house, and your audit procedure has established a "target" post-weatherization air leakage rate, enter the value here. If the target air leakage information is in air changes per hour (ACH) at natural conditions, an approximate conversion may be accomplished by multiplying the ACH by one-third of the volume of the house to obtain air leakage rate in CFM at $50 \mathrm{~Pa}$ (CFM50). If no target value is available and no pre-retrofit air leakage data has been entered, established default values may be entered (e.g., 2500 CFM at 50 PA). If no air sealing work is deemed necessary and no other weatherization measure is likely to reduce air leakage, enter the pre-weatherization blower door measurements in the post-weatherization data fields. If a post-weatherization blower door test is eventually conducted, you may re-run the audit using these blower door measurements to verify the previous results or maintain a record of actual air leakage reduction and energy savings accomplished. The values entered for 


\section{Chapter 11: NEAT and MHEA Common Elements}

post-weatherization leakage rate can affect the savings of other measures, particularly HVAC measures. The default for House Pressure Difference is 50 Pa. Required.

- Before Duct Sealing Sum of Pressure Pan Measurements - Enter the preduct sealing sum of the pressure differentials between the supply duct registers and the house with the house depressurized to $50 \mathrm{~Pa}$ with respect to the outside. Required.

- After Duct Sealing Sum of Pressure Pan Measurements - Enter the postduct sealing sum of the pressure differentials between the supply duct registers and the house with the house depressurized to $50 \mathrm{~Pa}$ with respect to the outside. Required.

\subsection{Duct/Infiltration - Optional Forms}

The Weatherization Assistant has an optional capability of documenting additional air and duct leakage measurements that may be taken during the audit for diagnostic purposes. These measurements are entered on three optional forms under the Ducts/Infiltration tab in the "NEAT Audit" and "MHEA Audit" windows: the Optional Blower Door and Zonal Pressures form, the Optional Pressure Balance form, and the Optional Pressure Pans form. Below are the descriptions of the procedures for obtaining the measurements and recording them on these forms.

\section{Optional Blower Door and Zonal Pressures}

Multiple blower door tests may be conducted during an audit, perhaps to assess the effects of weatherization measures (e.g., high density blown insulation installation) on the air and duct leakage rate, during different stages of weatherization, or during an inspection. The Optional Blower Door and Zonal Pressures form (see Figure 11.9) allows recording any additional blower door measurements as well as zonal pressure and/or pressure pan readings taken during the blower door tests. Multiple records of data may be entered accommodating any number of sets of readings. 


\section{Chapter 11: NEAT and MHEA Common Elements}

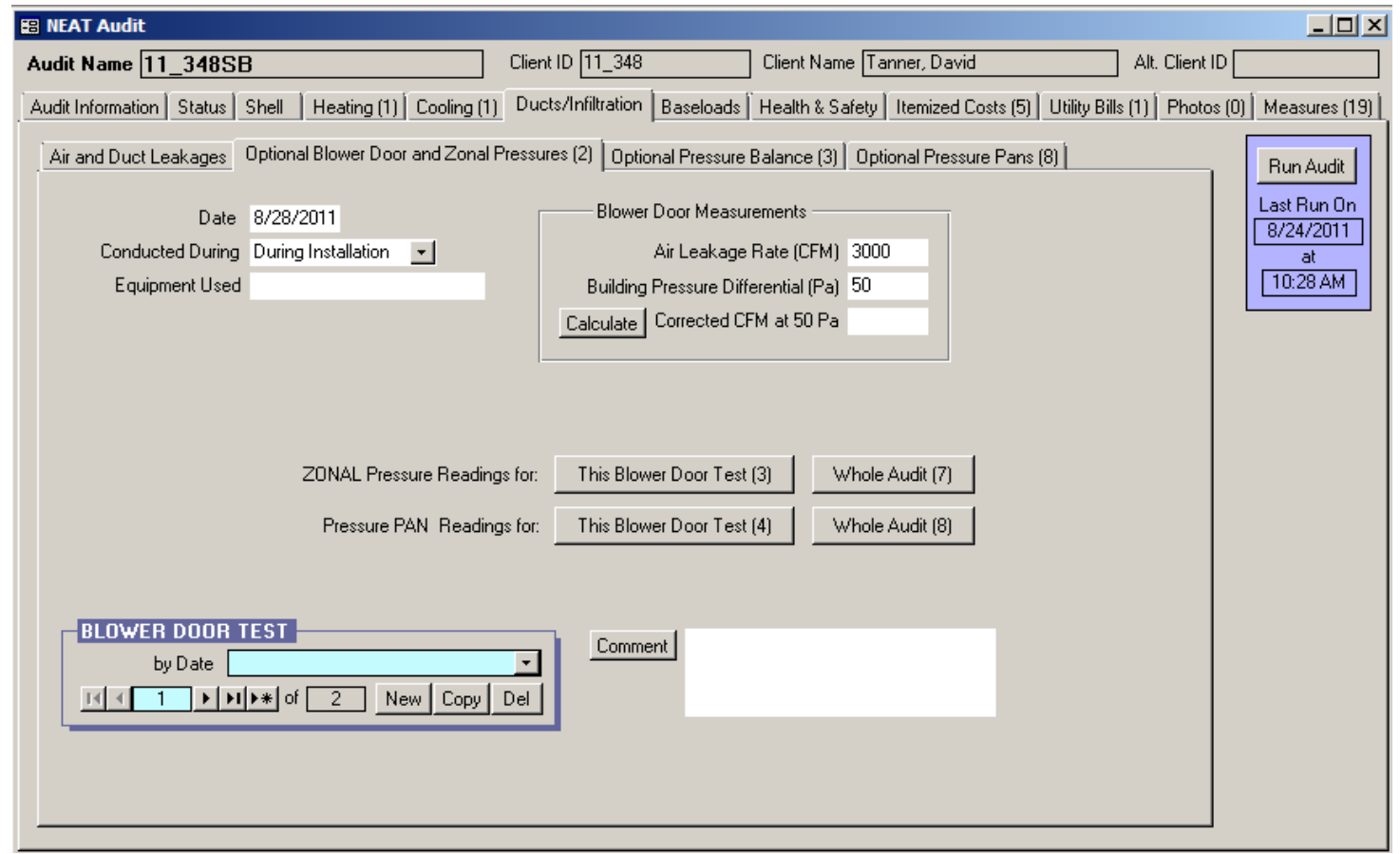

Figure 11.9. The Optional Blower Door and Zonal Pressures form under the Main Menu's “NEAT” button.

Enter when the blower door test was conducted in the Date field. The default date will be the current date. Entering the Date and any other field on the form will preserve the record, allowing all other fields to be optional. Select the occasion when the test was conducted using the Conducted During drop-down list. The choices are Audit, Pre-Installation, During Installation, Post-Installation, Inspection, and Other. Enter the name of the equipment used for taking the measurements in the Equipment Used field. Enter the blower door measurements including the Air Leakage Rate in CFM and the corresponding Building Pressure Differential with respect to outside in Pascal. If the pressure differential is other than 50 Pascal (which occurs in dwellings with high air leakage where 50 Pa house pressure cannot be reached), use the "Calculate" button to have the audit provide the equivalent air leakage rate at $50 \mathrm{~Pa}$ in the Corrected CFM at $50 \mathrm{~Pa}$ field. Use the Blower Door Test record navigation block to access additional blower door tests or create new ones. Enter any comments pertaining to the current blower door test in the Comment field.

If Audit has been selected in the Conducted During drop-down list, you will be asked on exiting the form if you wish to overwrite any existing Pre-retrofit blower door measurements entered on the Air and Duct Leakages form - the values used 


\section{Chapter 11: NEAT and MHEA Common Elements}

by the audits in computing energy savings from infiltration reduction efforts - with the leakage rates just entered. Choose "OK" to copy the data to the Air and Duct Leakages form or "Cancel” to reject the offer. Data on the Optional Blower Door and Zonal Pressures sub-form will be unaffected, regardless of your choice.

Zonal pressures are pressure differences between the living zone of the house where the blower door is installed and other zones of the house, such as attics, basements, attached garages, etc. Zonal pressure readings taken during blower door tests may help identify the possible location of leakage sites, locate where the functioning air barrier is, or identify potential health and safety problems associated with a combustion appliance.

Zonal pressure readings can be recorded for a specific blower door setup using the “This Blower Door Test" button displayed on the form next to the ZONAL Pressure Readings for: text. Selecting this button will display the Zonal Pressures $<$ Date $>-<$ Conducted During $>$ sub-form (see Figure 11.10). If you are revisiting this form, your entries from the Date and Conducted During fields on the parent form will be displayed following the "Zonal Pressures" header. On this form you may enter the name of the zone using the Location drop-down list or by typing a new location if it is not listed. Enter the corresponding pressure differential in Pascal in the Pressure field. Select the Ducts Present checkbox if any ducts are present in the zone. You may enter any comments pertaining to each entry in the Comment field.

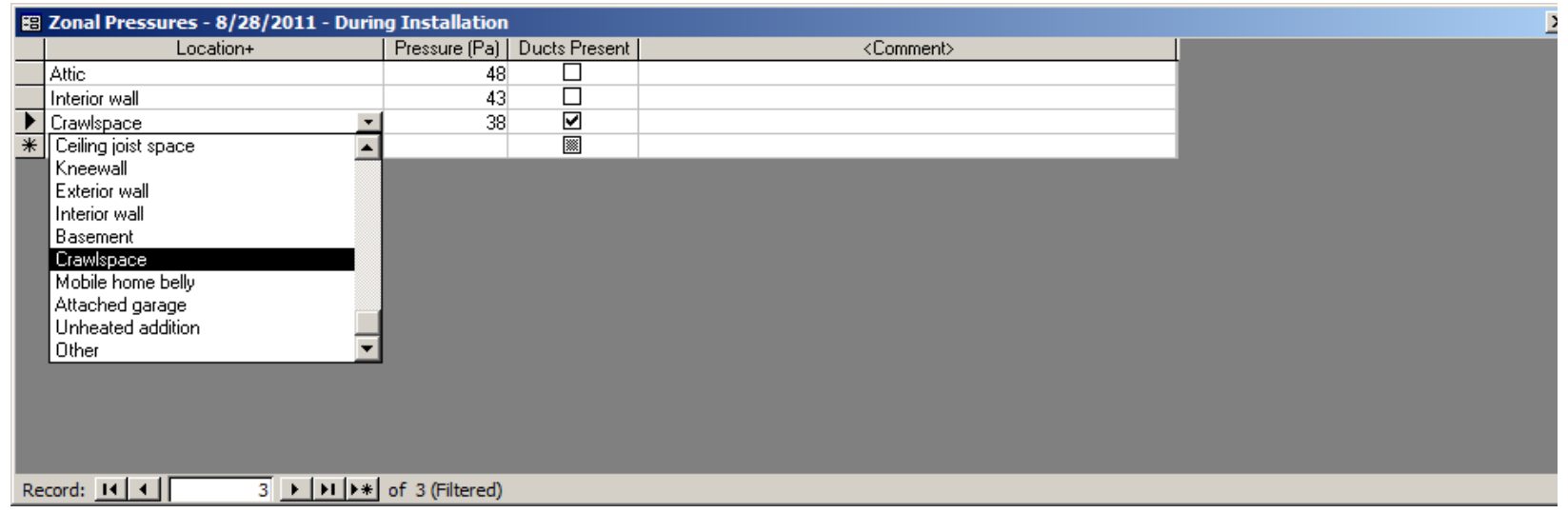

Figure 11.10. The Zonal Pressures sub-form.

Zonal pressure readings recorded for all zones from all blower door setups can be viewed and altered using the "Whole Audit" button displayed next to the "This Blower Door Test” button. Selecting this button will take you to the Zonal 


\section{Chapter 11: NEAT and MHEA Common Elements}

Pressures $-<$ Audit $>$ sub-form, which is similar to Zonal Pressures sub-form accessed using the "This Blower Door Test” button, except for an additional Blower Door Test column that lists the Date and Conducted During information you have entered for each blower door setup. The $<$ Audit $>$ indicated in the header is the Audit Name designated on the Audit Information Form.

The "This Blower Door Test" button and the "Whole Audit" button associated with the Pressure PAN Readings for: text on the Optional Blower Door and Zonal Pressure tab (see Figure 11.9) behave in the same manner as the analogous buttons associated with the zonal pressure readings, described above. They allow you to view and alter either pressure pan readings for the specific blower door setup currently being viewed on the form (see Figure 11.11) or pressure pan readings recorded for all blower door setups. If revisiting the sub-form for "This Blower Door Test," the readings from the current blower door setup, your entries from the Date and Conducted During fields on the parent form will be displayed following the "Pressure Pans" header. If viewing readings for the "Whole Audit," the subform will have an additional Blower Door Test column listing the Date and Conducted During information you have entered for each blower door setup. For a discussion on the use of pressure pans and the information entered on the Pressure Pans sub-form, see the section, “Optional Pressure Pans,” which follows.

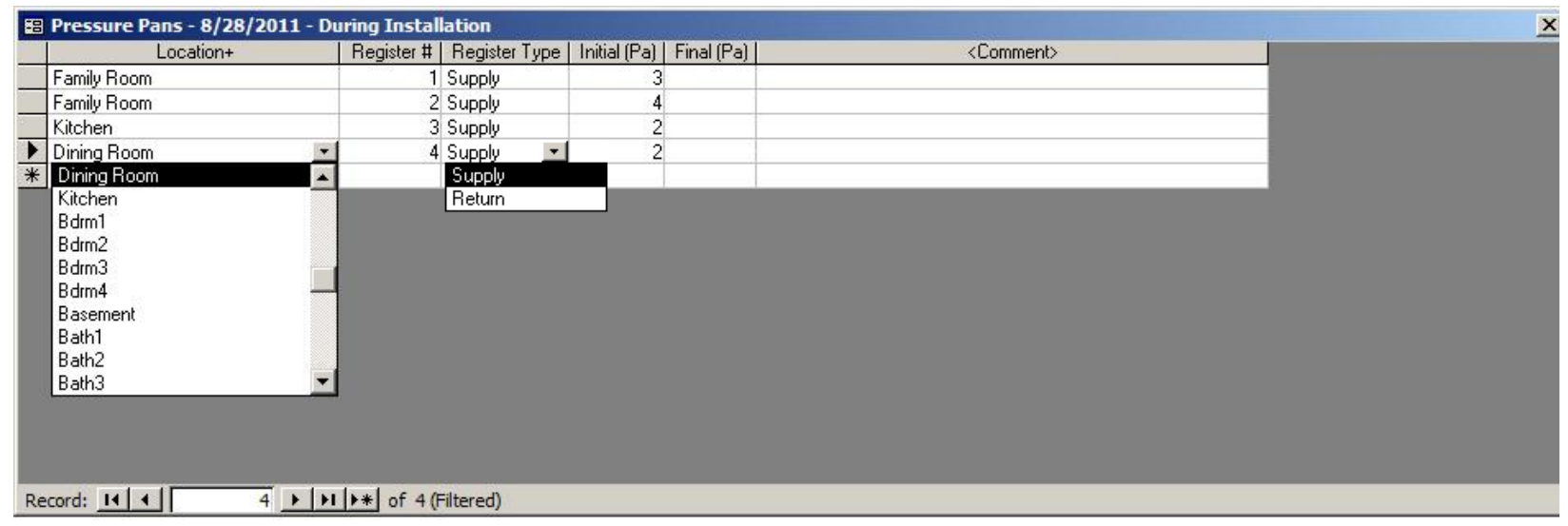

Figure 11.11. The Pressure Pans sub-form. 


\section{Chapter 11: NEAT and MHEA Common Elements}

\section{Optional Pressure Balance}

Pressure Balance measurements help identify factors within the building envelope that may increase infiltration. When the air handler of a central forced air furnace or air conditioner is on, it supplies conditioned air to rooms throughout a house. The supplied air is intended to be returned to the furnace or air conditioner through the return registers. If supply air is blocked from reaching a return, pressure differences can be created between the space and the location of the return register(s). This can hinder the supply air from being delivered to the space as well as create a negative pressure in the remainder of the house, increasing infiltration. The Optional Pressure Balance form (see Figure 11.12) allows you to record pressure differences between various rooms of the house and the space where the return registers exist. The measurements are taken with the air handler operating. The measurements do not involve the use of a blower door.

Enter the name of the space using the Location drop-down list or by typing a new location if it is not listed. Enter the pressure differential in Pascal measured between the room and the location where the return registers exist before, after, or both before and after any pressure balancing efforts (such as undercutting a door) in the Initial and Final pressure fields. You may enter comments pertaining to each entry in the Comment field.

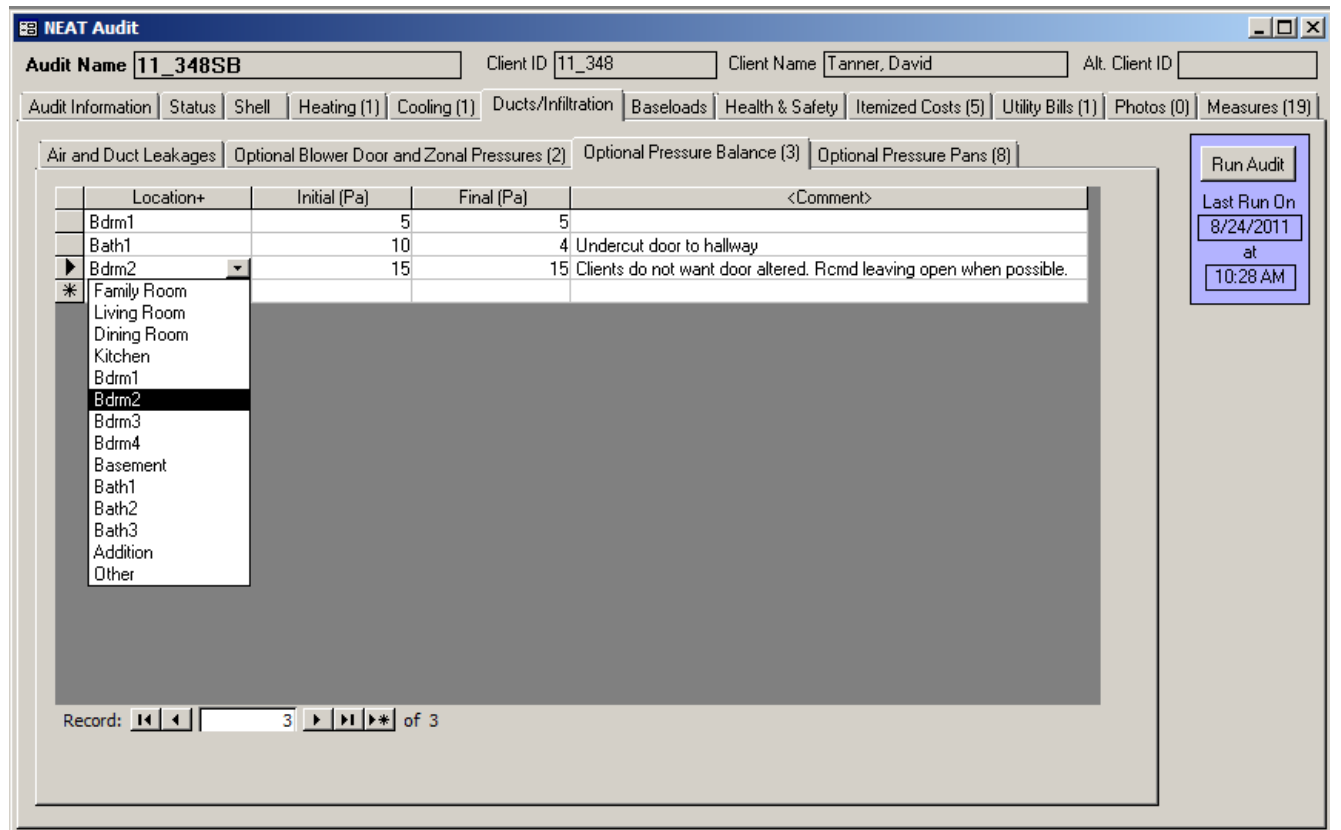

Figure 11.12. The Optional Pressure Balance form under the Main Menu's “NEAT" button. 


\section{Optional Pressure Pans}

Pressure pan readings are pressure differences between the house and the supply or return registers measured during a blower door test by attaching a digital manometer to a pressure pan that fits snugly over the register. Pressure pan readings may help identify the possible location of duct leakage. No pressure difference indicates that the duct leading to the register is at the same pressure as the house and that little or no leaks to the outside exist in that branch of the ducts. Pressure pan readings can be recorded on the Optional Pressure Pans form (see Figure 11.13) under the Ducts/Infiltration tab in the "NEAT Audit" and MHEA Audit” windows.

Enter an optional Register \# to uniquely identify the register, possibly referenced in a drawing. Enter the location of the register using the Location drop-down list or by typing a new location if it is not listed. Identify the register as Supply or Return using the Register Type drop-down list. Enter the pressure differential in Pascal between the register and the home before, after, or both before and after any duct sealing efforts in the Initial Pressure and Final Pressure fields. NEAT and MHEA audits do not use this information in any calculation. This form is to assist you in identifying duct leakage and potential locations for duct sealing. You may enter comments pertaining to each entry in the Comment field.

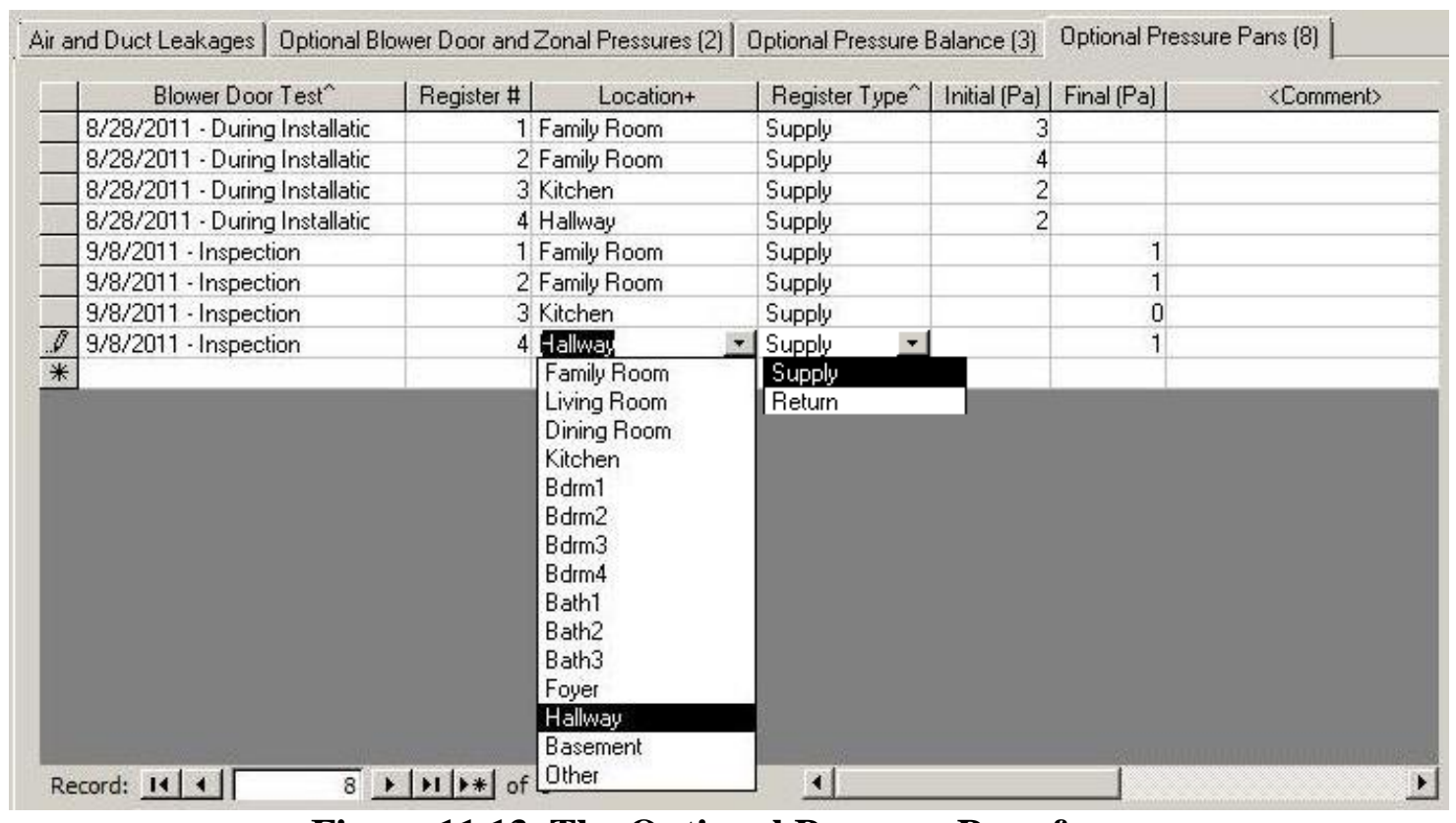

Figure 11.13. The Optional Pressure Pans form. 


\section{Chapter 11: NEAT and MHEA Common Elements}

\subsection{Base Loads - Water Heating}

The Water Heating form (see Figure 11.14) under the Baseloads tab in the "NEAT Audit" and "MHEA Audit" windows is used to enter information necessary to evaluate four water heating measures: tank insulation, pipe insulation, low-flow showerheads, and water heater replacement. Only those data entry fields necessary to evaluate the water heating measures you have turned on in the Library Measures form (see Section 14.5, Library Measures) of the Setup Library associated with the audit will appear on the form. If none of the four water heating measures are to be evaluated for a specific audit, the Water Heating form need not be accessed.

If water heater replacement is to be evaluated, the existing water heater or its equivalent must be found in the database provided by the program. Both NEAT and MHEA will evaluate replacing an existing unit with a unit using a different fuel. However, if this is to be considered, the replacement costs should include whatever cost is involved in making the fuel for the replacement unit available (e.g., piping the natural gas to the location of the replacement unit).

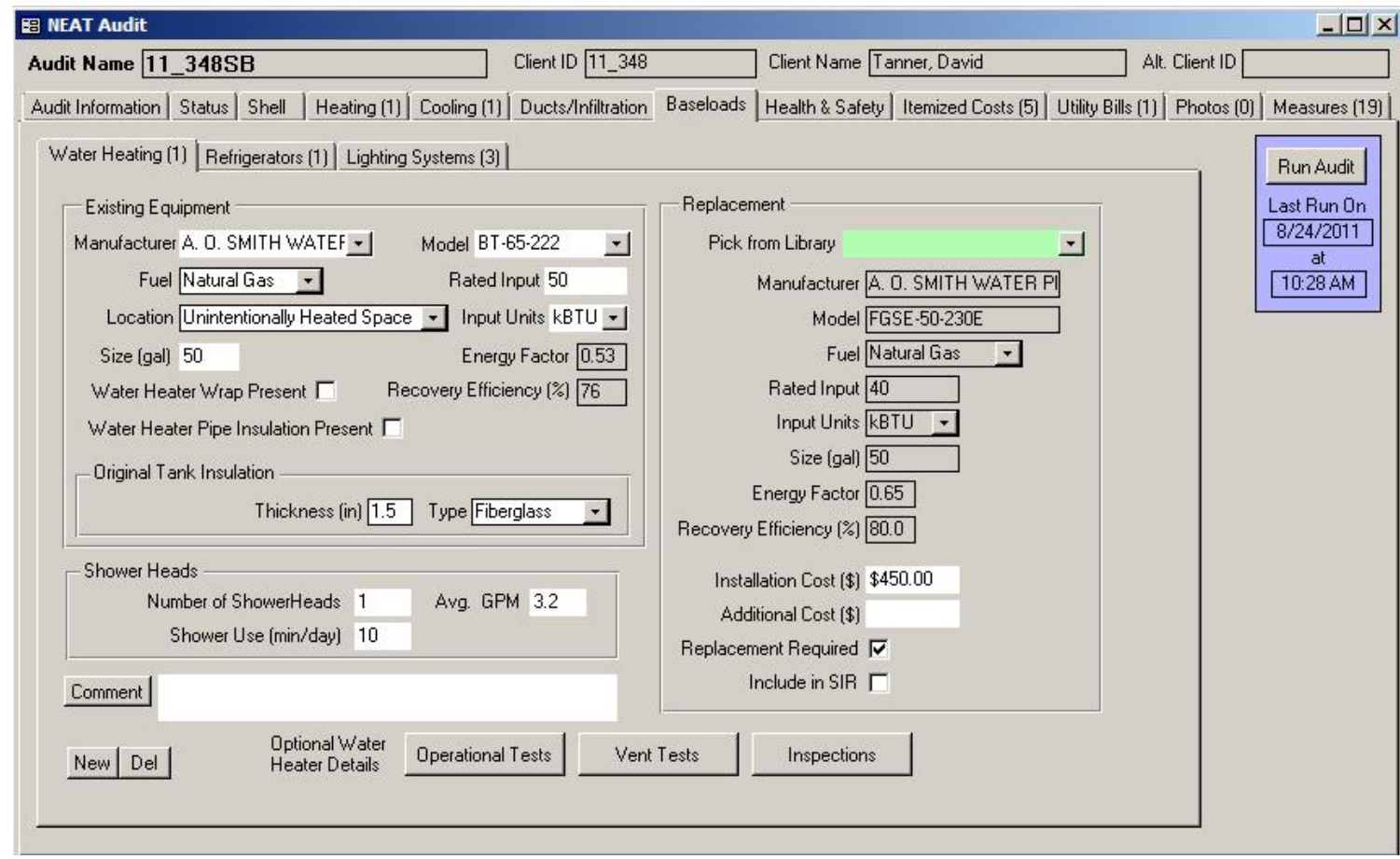

Figure 11.14. The Water Heating form under the Main Menu's “NEAT” button.

The Water Heating form is divided into four sections: the "Existing Equipment" description, the "Shower Heads" section, both on the left of the form, the 


\section{Chapter 11: NEAT and MHEA Common Elements}

"Replacement" equipment description on the right of the form, and the Comment field, water heater record creation buttons and buttons for the entry of Optional Water Heater Details at the bottom of the form. Always enter information on the general description of the existing water heater first. The Optional Water Heater Details buttons can be used to document an extensive number of water heater measurements and observations made while auditing a dwelling.

Below are descriptions of the individual data items and controls found on the Water Heating form. The optional information that can be entered using the Optional Water Heater Details buttons is described in Section 11.13, Optional Water Heater Details.

\section{Existing Equipment Data Block}

- Manufacturer and Model - Enter the existing water heater's manufacturer and model number. You may select the manufacturer and model number from the database using the drop-down lists or type them in if they are not listed. However, if you are evaluating water heater replacement, you must select the manufacturer and model of the unit or its equivalent from the database in order for the audit to provide the efficiency data necessary to evaluate the replacement. Selecting a manufacturer from the "Manufacturer" drop-down list first will restrict the listings in the "Model" drop-down list to those models of that manufacturer and make the selection of model easier. Otherwise, the Model list will include all models from all manufacturers. Conditionally required.

- Fuel Type - Select the type of fuel used by the water heater. The choices are Natural Gas, Electricity, and Propane. If the manufacturer and model have been selected from the database, this field will be entered automatically. Required.

- Location - Select the location of the existing water heater from the drop-down list. The choices are Heated Space, Unconditioned Space, and Unintentionally Heated Space. A Heated Space is purposely heated to the living space temperature, possibly under thermostat control or using a space heater. A basement may be considered a heated space if maintained at the living space temperature. An Unintentionally Heated Space is partially heated by a heat source in the space that adds heat unintentionally. For example, a basement without heating registers, but having a furnace, boiler, water heater, or uninsulated ductwork would be unintentionally heated. An Unconditioned 


\section{Chapter 11: NEAT and MHEA Common Elements}

Space is not heated because there are no sources of heat in the space other than conduction through walls, floors, and perhaps insulated ductwork. The choice will affect the savings of the water heating measures. Required.

- Size - Enter the capacity of the water heater in gallons. If the manufacturer and model have been selected from the database, this field will be entered automatically. If you are evaluating water heater replacement and the capacity of the replacement unit differs substantially from the capacity of the existing unit, you will receive a warning. Required for replacement.

- Water Heater Wrap Present -Use this checkbox to indicate if water heater tank insulation already exists. If the Water Heater Tank Insulation measure has been turned off in the Library Measures form of the Main Menu's "Setup Library” button (see Section 14.5, Library Measures), this checkbox will not appear.

- Water Heater Pipe Insulation Present - Use this checkbox to indicate if pipe insulation exists on at least the first five feet of pipe exiting the water heater. If the Water Heater Pipe Insulation measure has been turned off in the Library Measures form of the Main Menu's “Setup Library” button (see Section 14.5, Library Measures), this checkbox will not appear.

- Rated Input and Input Units - Enter the rated input of the water heater in units selected. The choice for input units are $\mathrm{kBtu}$ and $\mathrm{kW}$. If the manufacturer and model have been selected from the database, this field will be entered automatically. Required for replacement.

- Energy Factor and Recovery Efficiency - These fields display the Energy Factor and Recovery Efficiency of the water heater if the manufacturer and model have been selected from the database. These fields are not displayed if the Water Heater Replacement measure has been turned off on the Library Measure form of the Main Menu's "Setup Library” button (see Section 14.5, Library Measures). Uneditable.

- Original Tank Insulation R-value, Thickness, and Type - If the Water Heater Wrap Present checkbox is left unchecked, the Original Tank Insulation fields for entering the R-value, thickness, and type of tank insulation are displayed. Enter either the R-value or both the type and thickness of tank 


\section{Chapter 11: NEAT and MHEA Common Elements}

insulation. If you enter the R-value, the Type and Thickness fields disappear. If you enter the thickness, the R-value field disappears. Select the insulation type using the drop-down menu. The choices are Fiberglass and Polyurethane. You may remove the access plate or examine the relief valve penetration to view the insulation and determine its characteristics. Conditionally required.

\section{Shower Heads Data Block}

If the Low-flow Showerheads measure has been turned off in the Library Measures form of the Main Menu's “Setup Library” button (see Section 14.5, Library Measures), this data block will not appear. The entries are optional unless the Showerheads measure is to be evaluated.

- Number of Shower Heads - Enter the number of showerheads used in the house for which replacement with low-flow models needs to be evaluated.

- Shower Use - Enter the total time the showerheads are in use in minutes per day for which replacement with low-flow models needs to be evaluated. Combine the times for all showerheads being considered for replacement.

- Average GPM - Enter the average flow rate in gallons per minute of all showerheads being considered for replacement. You can determine this easily using a container of known volume and a stop watch.

\section{Replacement Data Block}

Most of the data items in the Replacement data block are uneditable. They are automatically filled in after you select a replacement water heater from the "Pick from Library" drop-down list. The fields are analogous to those described in the Existing Equipment data block except they apply to the replacement unit. See the discussions of these fields in the material above. The three additional fields are discussed below. If the Water Heater Replacement measure has been turned off in the Library Measures form of the Main Menu's "Setup Library” button (see Section 14.5, Library Measures), this data block will not appear.

- Pick from Library - Select the replacement water heater using the drop-down list. The listed items are those you have entered in the Supply Library (see "Additional Entries on the Hot Water Equipment and Refrigerator Forms" in 


\section{Chapter 11: NEAT and MHEA Common Elements}

Section 15.2, Materials/Labor Forms). If you do not have any replacement water heaters in the Supply Library being accessed by this audit, you will receive a notice giving you brief instructions on how to add these records.

- Installation Cost - If you have entered unit costs for your replacement water heaters in the Supply Library, these costs will be copied into this field when a unit is selected for use. You may alter this cost here if the unit cost changed. Also, if the unit cost entered in the Supply Library does not include the labor cost associated with installation, you would add it to this value, unless you choose to enter this cost in the Additional Cost field. Required for replacement.

- Additional Cost - Enter any additional cost associated with installation of the water heater not included in the value entered above. This may include labor costs, delivery changes, or the cost of disposing of the old unit. Optional.

- Replacement Required - Use this checkbox to indicate that a water heater replacement is necessary based on the inspection of existing unit. This may be the result of an unsafe yet un-repairable existing system. NEAT and MHEA will evaluate and recommend system replacement, regardless of its costeffectiveness.

- Include in SIR - If the Replacement Required checkbox is selected, the Include in SIR checkbox will appear on the form. This checkbox allows you to indicate that you wish to have the Water Heater Replacement measure included in the calculation of the whole house (package) SIR for this audit. Measures not included in the whole house SIR are normally related to health and safety. Check the guidance for your program in deciding when you can mandate a water heater be replaced and if the replacement can be excluded from the SIR calculation. Even if the replacement is related to health and safety, if the measure is shown to be cost-effective, you may choose to still include it in the whole house SIR by selecting this checkbox. By doing so, it will not be seen as a health and safety measure, thus preserving funds dedicated for such measures. Another reason you may not want a measure included in the whole-house SIR calculation is if it is being funded outside of the Weatherization Assistance Program. Whether a measure is or is not included in the whole house SIR is indication by its location in the list of measures produced for the Recommended Measures Report (see Chapter 12, NEAT and MHEA Results). 


\section{Chapter 11: NEAT and MHEA Common Elements}

- Comment - You may enter comments pertinent to the water heating system or associated retrofit measures directly in the Comment field on the form, or you may enter them in the Comment Editor by selecting the "Comment" button to the left of the field. The Comment Editor is similar to Window's Notepad and may be used to enter extended comments. The total comment may have up to 65,000 characters. Text from other sources may be copied and pasted into the Comment Editor's window. Any comment that is entered is displayed on the Recommended Measures report and the Input Report available from the Audit Information form (see Section 8.2, Audit Information). In addition, any comment that is entered can be imported into any specific work order (see Section 13.1, Work Order Information). Thus, you may wish to enter comments related to any observation thaat might affect the installation of needed measures or repair work. Optional.

\subsection{Base Loads - Refrigerators}

The Refrigerator form (see Figure 11.15) under the Baseloads tab in the "NEAT Audit" and "MHEA Audit" windows is used to enter information necessary to evaluate the Refrigerator Replacement measure. If this measure is not to be evaluated for a specific audit, the Refrigerator form need not be accessed.

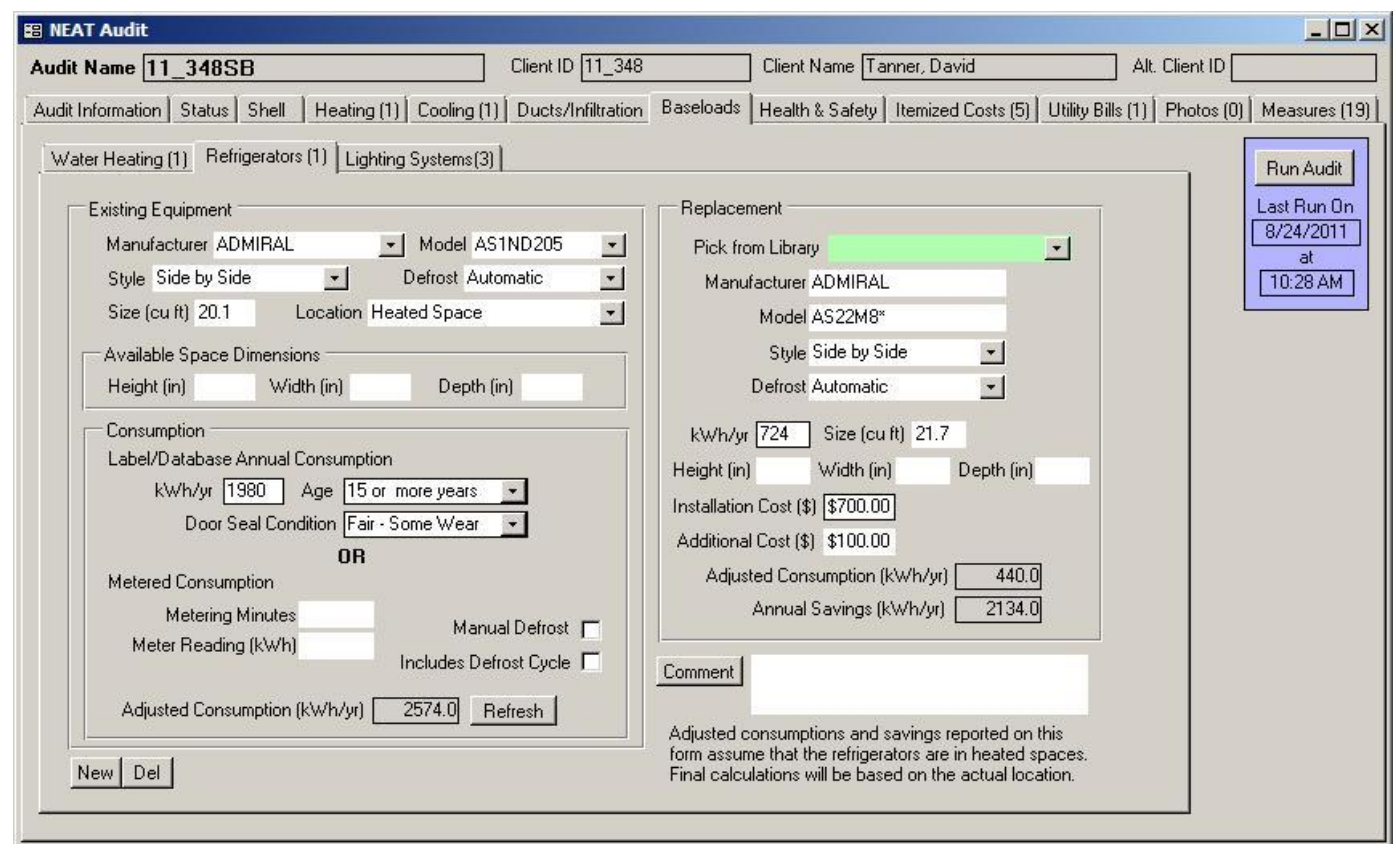

Figure 11.15. The Refrigerator form under the Main Menu's “NEAT” button. 


\section{Chapter 11: NEAT and MHEA Common Elements}

The Refrigerator form is divided into four data blocks: the Existing Equipment description and energy Consumption information for the existing refrigerator on the left of the form, the description of the Replacement unit on the right, and the comment field and record creation/deletion buttons at the bottom of the form. Below are descriptions of the individual data items and controls found on the refrigerator form.

\section{Existing Equipment Data Block}

- Manufacturer and Model - Enter the existing refrigerator's manufacturer and model number. You may select the manufacturer and model from the database using the drop-down lists or type them in if they are not listed. Selecting a manufacturer from the "Manufacturer" drop-down list first will restrict the listings in the "Model" drop-down list to those models of that manufacturer and make the selection of model easier. Otherwise, the Model list will include all models from all manufacturers. If you select the manufacturer and model of the unit from the database, the general characteristics of the unit and the energy consumption data will be entered automatically. Optional.

- Style - Select the style of the existing unit from the drop-down list. The choices are Top Freezer, Bottom Freezer, Side by Side, Single Door, Single Door w/Freezer, and Other. If the manufacturer and model are selected from the database, this information will be entered automatically. Optional.

- Defrost - Select the defrost characteristics of the existing unit from the dropdown list. The choices are Automatic, Manual, Partial Automatic, and Other. If the manufacturer and model are selected from the database, this information will be entered automatically. Optional.

- Size - Enter the size of the existing unit in cubit feet. If the size of the existing and replacement units are not within $20 \%$, the programs will produce a warning indicating a possible size problem related to the replacement. Optional.

- Location - Select the location of the existing refrigerator from the drop-down list. The choices are Heated Space, Unconditioned Space, and Unintentionally Heated Space. A Heated Space is purposely heated to the living space temperature, possibly under thermostat control or using a space heater. A basement may be considered a heated space if maintained at the living space 


\section{Chapter 11: NEAT and MHEA Common Elements}

temperature. An Unintentionally Heated Space is partially heated by a heat source in the space that adds heat unintentionally. For example, a basement without heating registers, but having a furnace, boiler, water heater, or uninsulated ductwork would be unintentionally heated. An Unconditioned Space is not heated because there are no sources of heat in the space other than conduction through walls, floors, and perhaps insulated ductwork. The choice will affect the savings of the water heating measures. Required.

- Height, Width, Depth [Available Space Dimensions] - Enter the dimensions of the available space in inches, where the existing refrigerator is installed. If entered, NEAT and MHEA use this information along with the dimensions of the replacement unit to determine if the replacement unit could fit in the available space and produce a warning if the replacement unit is oversized. Optional.

\section{Consumption Data Block}

The Consumption data block accommodates entries required to estimate the existing unit's current annual consumption. This data can be obtained either by selecting a unit from the database, reading the energy guide label of the unit, or metering the energy consumption of the refrigerator.

If the consumption is to be estimated from an energy guide label or from values in the database, the following three entries will be used.

- $\mathbf{k W h} / \mathbf{Y r}$ - Enter the annual energy consumption listed on the energy guide label for the existing unit, if one exists. If the manufacturer and model are selected from the database, this data will be entered automatically. However, you may change the value obtained from the database on this form. The entry is not required if you are using metered consumption data. Conditionally required.

- Age - Select the age of the existing unit from the drop-down list. The choices are Less than 5 years, 5 to 9 years, 10 to 14 years, and 15 or more years. If the manufacturer and model are selected from the database, this information will be entered automatically. The age in the database is based on the first year the model was sold. If you know that the existing model is newer than that indicated from the database, change the entry. The entry is not required if you are using metered consumption data. Conditionally required. 


\section{Chapter 11: NEAT and MHEA Common Elements}

- Door Seal Condition - Select the entry that best describes the current condition of the existing refrigerator's door seals. The choices are Good, Fair - Some Wear, and Poor - Gaps Visible. The entry is not required if you are using metered consumption data. Conditionally required.

If you use metering to determine the consumption of the existing refrigerator, enter data into the "Metered Consumption" portion of the Consumption data block.

- Metering Minutes - If you are using metered consumption data, enter the time period in minutes for which you metered the existing refrigerator. Metering should be performed for at least 180 minutes (3 hours). You should attempt to prevent refrigerator door openings during the metering period. This entry is not required if the $\mathrm{kWh} / \mathrm{yr}$ field has been used to provide the consumption data. Conditionally required.

- Meter Reading - Enter the metered consumption in $\mathrm{kWh}$ for the period specified by the Metering Minutes. This entry is not required if the kWh/yr field has been used to provide the consumption data. Conditionally required.

- Manual Defrost - Use this checkbox to indicate that the existing unit has manual defrost. This entry is used for metered consumption only.

- Includes Defrost Cycle - Use this checkbox to indicate if the metered consumption entered above included a defrost cycle. This can be determined if you observe a peak in either the metered consumption or the monitored refrigerator interior temperature which is several times higher than the surrounding peaks. Estimation of annual refrigerator consumption from metered data is less accurate if the metering period includes a defrost cycle.

- Adjusted Consumption - This field displays the adjusted annual energy consumption in $\mathrm{kWh} / \mathrm{yr}$, based on the location, age, door seal condition, and the presence of defrost cycle during metering. Uneditable.

- Refresh - Select this button to display or update the adjusted consumption after you have entered or changed the data for the existing refrigerator. 


\section{Chapter 11: NEAT and MHEA Common Elements}

\section{Replacement Data Block}

The Replacement data block is displayed on the right side of the Refrigerator form. Individual data items and controls presented in this data block allow you to enter information on the replacement refrigerator. For most of the data items in this data block, you may either type the information on this form or recall the data entered on the Refrigerator form of the Main Menu's "Supply Library” button (see "Additional Entries on the Hot Water Equipment and Refrigerator Forms" in Section 15.2, Materials/Labor Forms). If the Refrigerator Replacement measure has been turned off in the Library Measures form of the Main Menu's "Setup Library” button (see Section 14.5, Library Measures), this data block will not appear.

- Pick from Library - Use this drop-down box to select a replacement unit from your library of refrigerators. If this field is used, the entry must match a unit listed in your library. If you do not have any replacement refrigerators in the Supply Library being accessed by this audit, you will receive a notice giving you brief instructions on how to add these records. Optional.

- Manufacturer and Model - Enter the manufacturer and model number of the replacement refrigerator. If the unit is selected from the library, this information will be entered automatically. Optional.

- Style - Select the style of the replacement unit from the drop-down list. The choices are Top Freezer, Bottom Freezer, Side by Side, Single Door, Single Door w/Freezer, and Other. If the unit is selected from the library, this information will be entered automatically. Optional.

- Defrost - Select the defrost characteristics of the replacement unit from the drop-down list. The choices are Automatic, Manual, Partial Automatic, and Other. If the unit is selected from the library, this information will be entered automatically. Optional.

- $\mathbf{k W h} / \mathbf{Y r}$ - Enter the annual energy consumption in $\mathrm{kWh} / \mathrm{Yr}$ for the replacement refrigerator. You may find this information from the energy guide label of the replacement unit. If the unit is selected from the library and the energy details were already provided in the library, this information will be entered automatically. Required. 


\section{Chapter 11: NEAT and MHEA Common Elements}

- Size - Enter the size of the replacement unit in cubit feet. If the size of the existing and replacement units are not within $20 \%$, the programs will produce a warning indicating a possible size problem related to the replacement. Optional.

- Height, Width, Depth, Size [Replacement Unit] - Enter the dimensions of the replacement unit. If entered, NEAT and MHEA use this information along with the dimensions of the available space, if entered in the Existing Equipment data block, to determine if the replacement unit could fit in the available space. NEAT and MHEA will produce a warning if the replacement unit is oversized. If the unit is selected from the library and the dimensions were already provided in the library, this information will be entered automatically. Optional.

- Installation Cost - Enter the installation cost of the replacement refrigerator including the labor cost. If the unit was selected from the library and you have entered unit costs for your replacement refrigerator in the Supply Library, these costs will be copied into this field when a unit is selected for use. You may alter this cost here if the unit cost changed. Also, if the unit cost entered in the Supply Library does not include the labor cost associated with installation, you would add it to this value, unless you choose to enter this cost in the Additional Cost field. Required for replacement.

- Additional Cost - Enter any additional cost associated with replacing the refrigerator not included in the value entered above. This may include labor costs, delivery changes, or the cost of disposing of the old unit. Optional.

- Adjusted Consumption - This field displays an adjusted annual energy consumption for the replacement unit in $\mathrm{kWh} / \mathrm{yr}$, based on the number of occupants specified on the Client Information form of the Client main Menu item (see Section 7.1, Client Information). The adjusted consumption will be higher than the value entered/displayed in the $\mathrm{kWh} / \mathrm{yr}$ field above, if the number of occupants is larger increasing the frequency of opening the refrigerator door. Uneditable.

- Annual Savings - This field displays the annual energy savings in $\mathrm{kWh} / \mathrm{yr}$, which is the difference between the adjusted consumption of the existing and replacement refrigerators. NEAT and MHEA use this value to determine the SIR of the Refrigerator Replacement measure if the refrigerator is located in a Heated Space. If the refrigerator is outside the heated space, additional 


\section{Chapter 11: NEAT and MHEA Common Elements}

temperature dependency adjustments will be made when computing the SIR which cannot be performed until the audit is run. Uneditable.

- Comments - You may enter comments pertinent to the refrigerator replacement directly in the Comment field on the form, or you may enter them in the Comment Editor by selecting the "Comment" button to the left of the field. The Comment Editor is similar to Window's Notepad and may be used to enter extended comments. The total comment may have up to 65,000 characters. Text from other sources may be copied and pasted into the Comment Editor's window. Any comment that is entered is displayed on the Recommended Measures report and the Input Report available from the Audit Information form (see Section 8.2, Audit Information). In addition, any comment that is entered can be imported into any specific work order (see Section 13.1, Work Order Information). Thus, you may wish to enter comments related to any observation that might affect the installation of needed measures or repair work. Optional.

\subsection{Base Loads - Lighting Systems}

The Lighting Systems form (see Figure 11.16) under the Baseloads tab in the "NEAT Audit" and "MHEA Audit" windows is used to describe the existing lighting systems for which you want to evaluate the replacement measure. If this measure is not to be evaluated for a specific audit, the Lighting Systems form need not be accessed. If the Lighting Retrofits measure has been turned off in the Library Measures form of the Main Menu's “Setup Library” button (see Section 14.5, Library Measures), this form will not be accessible.

Below are descriptions of the individual data items and controls found on the Lighting Systems form.

- Light Code - The Light Code identifies the lighting systems to you and the Weatherization Assistant. The light codes within a given audit must be unique for lighting systems that are dissimilar in terms of lamp type, size, or use, or which are retrofitted differently. NEAT automatically provides a default entry for all new lighting systems that are created in the form of "LT\#” where the "\#” is an integer insuring uniqueness of the light code. You may choose to enter your own light code to include characters that indicate the room, location, or any other lighting system characteristics. The light code may have up to 20 


\section{Chapter 11: NEAT and MHEA Common Elements}

characters, although space restrictions in many reports suggest a lesser number of characters should be used (perhaps 3 to 5) to preserve readability. Entries are not case-sensitive. NEAT and MHEA will also use the light codes in output reports and work orders to identifying which lamps should be replaced. The light codes may also be helpful when labeling your drawing of the house's floor plan. Required.

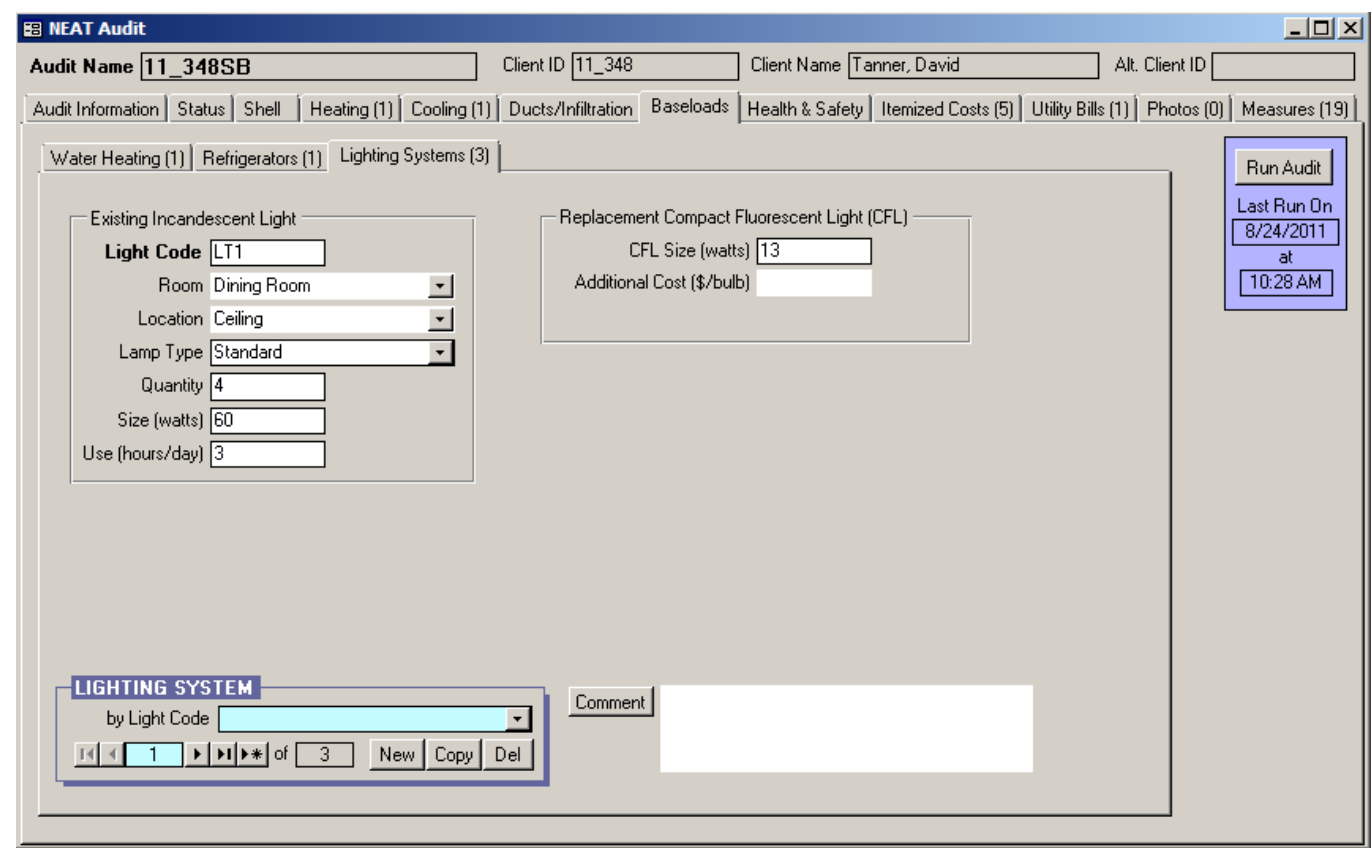

Figure 11.16. The Lighting Systems form under the Main Menu's “NEAT” button.

- Room - Select the name of the room where the lamps being described are located. The choices are Kitchen, Family Room, Living Room, Recreation Room, Dining Room, Bedroom, Bathroom, Utility Room, and Other. This information may be needed for reference to locate the lamps considered for replacement. Optional.

- Location - Select the location of the lighting in the room from the drop-down list. The choices are Ceiling, Floor, Table, Wall, and Other. This information may be needed for reference to locate the lamps considered for replacement. Optional.

- Lamp Type - Select the type of existing incandescent lamp from the dropdown list. The choices are Standard, Flood, and Other. NEAT and MHEA use this information along with the Size to provide the default wattage and cost for 


\section{Chapter 11: NEAT and MHEA Common Elements}

the replacement CFL. This information is reported in the Materials section of the Recommended Measures report (see Section 12.2, Recommended Measures Report) to indicate which replacement CFL type would be required. Required.

- Quantity - Enter the number of lamps represented by this record which are candidates for CFL replacement. Required.

- Size - Enter the size of the existing incandescent lamp in watts. NEAT and MHEA use this information along with the lamp type to provide a default wattage for the replacement CFL and to calculate energy savings from the replacement measure. Required.

- Use - Enter the average time period, in hours per day, the lamp is normally on. The value must be 24 or less. This value has a significant effect on the savings from lamp replacement. You should target the replacement measure to lamps with substantial on-times. Required.

- CFL Size [Replacement CFL] - Enter the size of the replacement CFL in watts. NEAT and MHEA use the standard replacement wattage closest to your entry. The default for this field is the wattage of the CFL with approximately the same light output as the existing incandescent lamp characterized by the Size. Required.

- Additional Cost [Replacement CFL] - Enter any added cost, in dollars per lamp, not normally associated with installation of a CFL and not included in the standard material costs. Replacing a fixture's harp to allow the CFL to fit, or other size modification, are examples. Optional.

- Lighting System Record Navigation Block - The Lighting System record navigation block is used to find and navigate to existing lighting system descriptions for the audit being edited, copy or delete the currently accessed lighting system description, or create a new lighting system description for the audit. See Section 5.1, Form and Record Navigation, for information on using the Weatherization Assistant's record navigation blocks.

- Comments - You may enter comments pertinent to the lighting system directly in the Comment field on the form, or you may enter them in the Comment Editor by selecting the "Comment" button to the left of the field. The Comment 


\section{Chapter 11: NEAT and MHEA Common Elements}

Editor is similar to Window's Notepad and may be used to enter extended comments. The total comment may have up to 65,000 characters. Text from other sources may be copied and pasted into the Comment Editor's window. Any comment that is entered is displayed on the Recommended Measures report and the Input Report available from the Audit Information form (see Section 8.2, Audit Information). In addition, any comment that is entered can be imported into any specific work order (see Section 13.1, Work Order Information). Thus, you may wish to enter comments related to any observation that might affect the installation of measures. Optional.

\subsection{Health and Safety}

The forms under the Health \& Safety tab in the "NEAT Audit" and "MHEA Audit" windows, which include the Whole House, Equipment, and Building Shell forms (see Figure 11.17 to 11.20), can be used to enter information about the health and safety related observations in the house. Items on these forms, and on the optional sub-forms under the Heating and Water Heating forms (see Sections 11.12, Optional Heating System Details, and 11.13, Optional Water Heater Details), help identify potential health and safety hazards. Each form also provides the opportunity to record extended comments. Below are descriptions of these forms as well as the handling of health and safety hazards in the Weatherization Assistant.

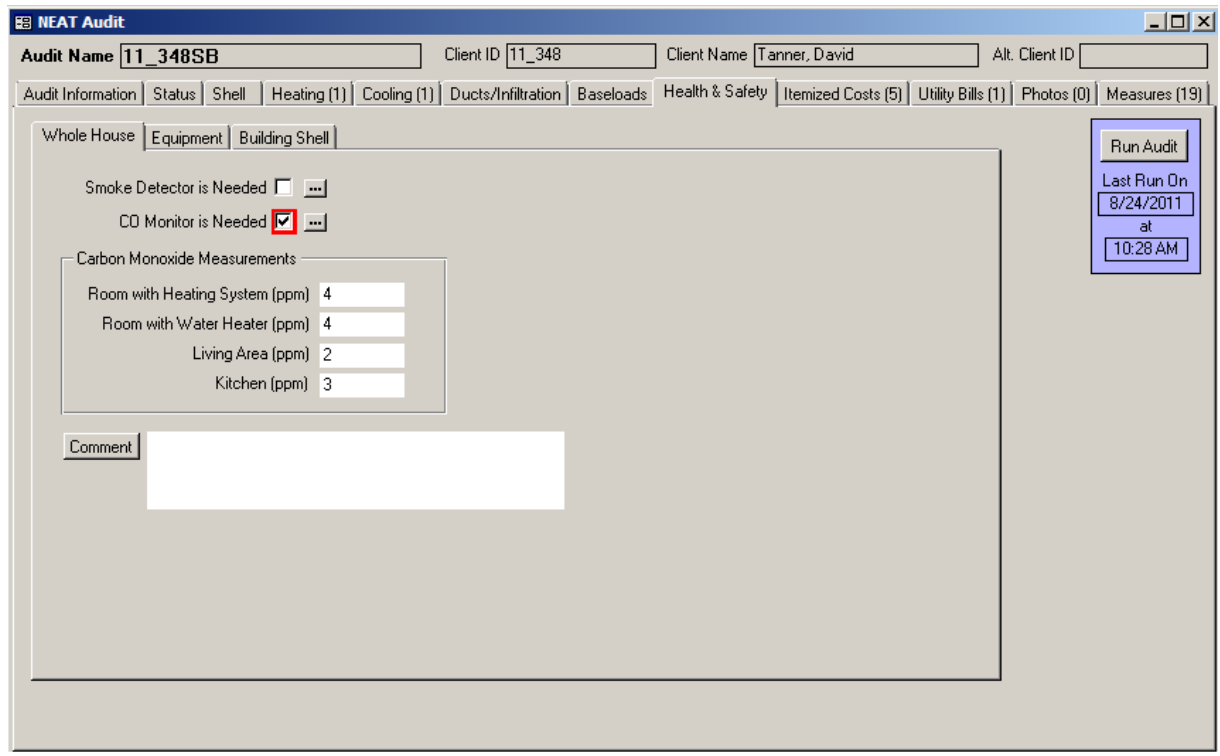

Figure 11.17. The Health \& Safety - Whole House form under the Main Menu's “NEAT” button. 


\section{Chapter 11: NEAT and MHEA Common Elements}

\section{Whole House}

The Whole House form (see Figure 11.17) can be used to identify the need for smoke detectors and carbon monoxide monitors and to record the carbon monoxide concentrations in various rooms of the house. The carbon monoxide concentration should be recorded for rooms having combustion-based space heating or water heating, a kitchen having a gas/propane cook stove, and the living area.

\section{Equipment}

The Equipment form (see Figure 11.18) can be used to identify health and safety issues related to equipment in the house. Worst case condition draft measurements may be recorded for any combustion-based heating systems described under the Heating tabs of NEAT and MHEA and any water heaters (see Figure 11.19). Improper venting is a concern for wood stoves, fireplaces, clothes dryers, and exhaust fans. Wood stoves and fireplaces may also have inadequate combustion air and bathroom or kitchen exhaust fans may be missing or not operational.

Individual burner and oven carbon monoxide measurements can be recorded for a gas cook stove and a gas leak associated with the gas stove may also be identified. The existence of an air-to-air heat exchanger may be indicated.

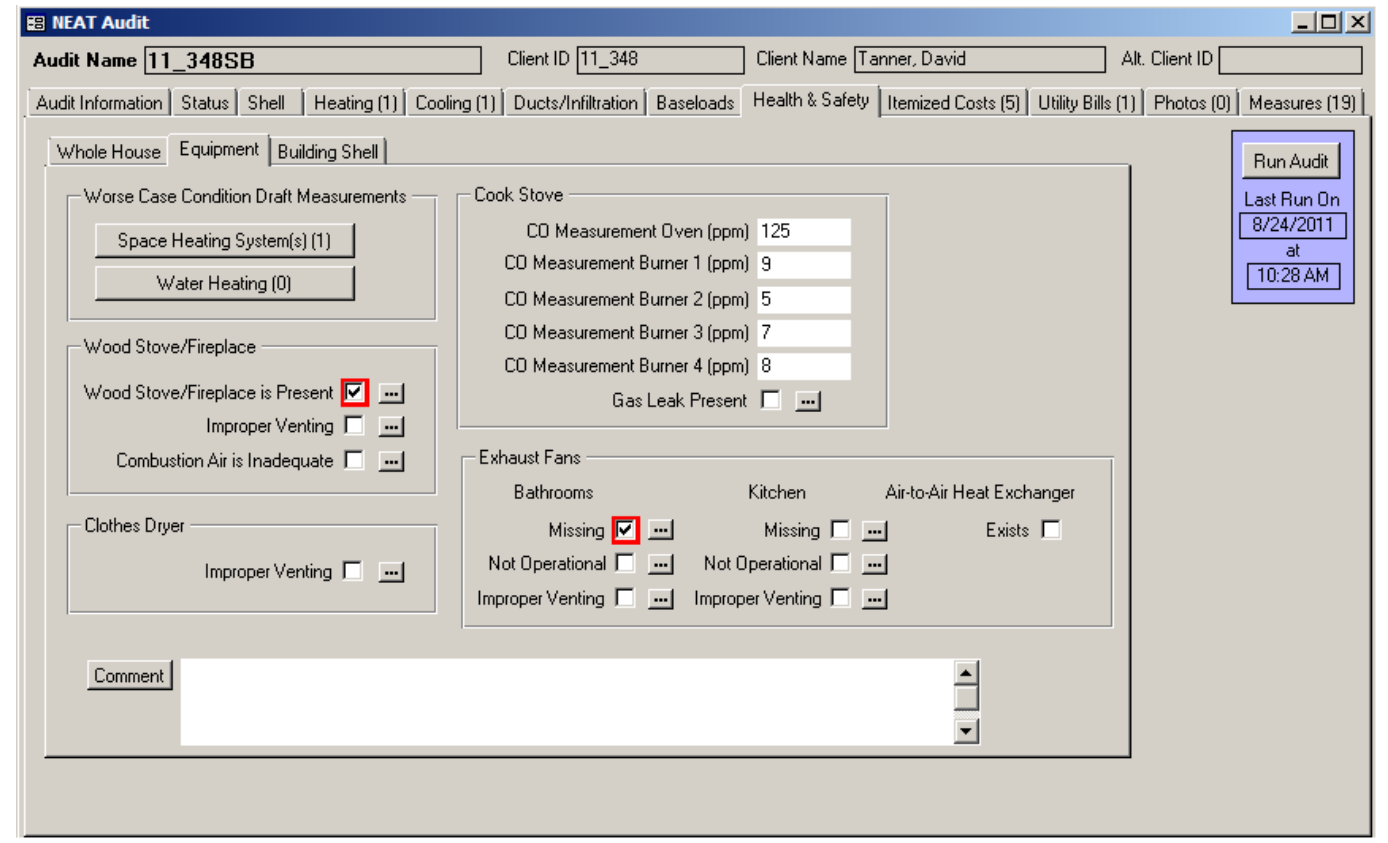

Figure 11.18. The Health \& Safety - Equipment form under the Main Menu's “NEAT" button. 


\section{Chapter 11: NEAT and MHEA Common Elements}

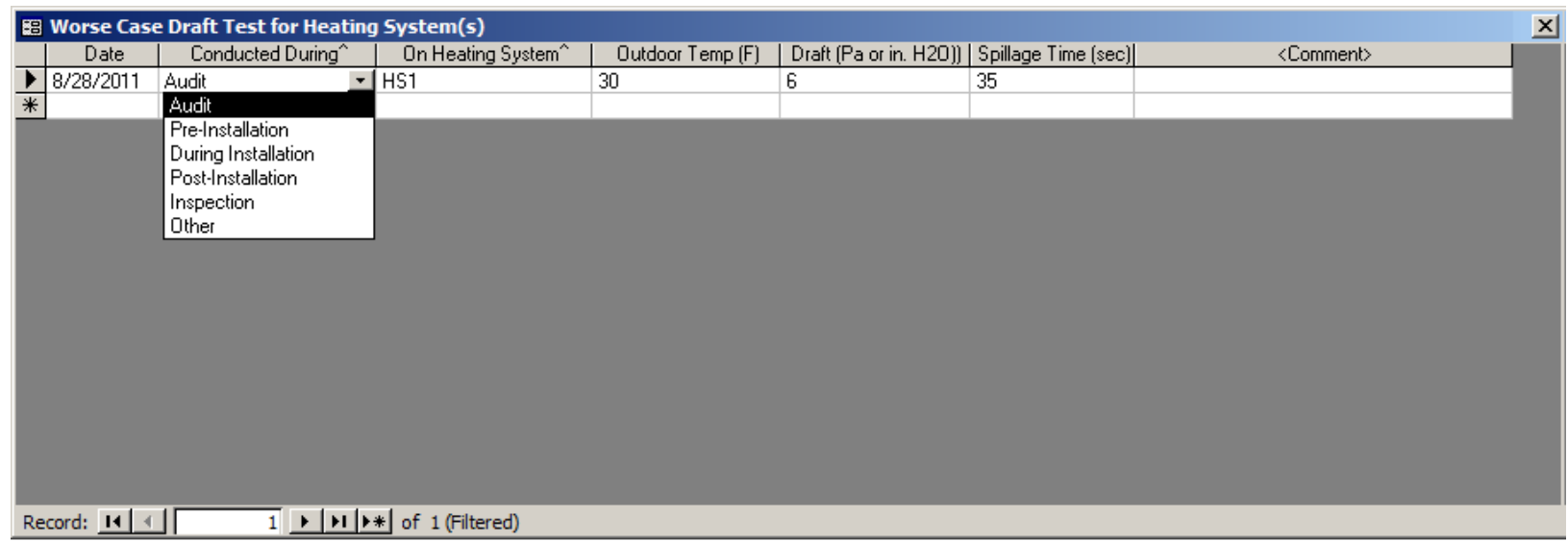

Figure 11.19. The Worst Case Draft Test for Heating System(s) sub-form.

\section{Building Shell}

The Building Shell form (see Figure 11.20) can be used to identify health and safety issues in the attics, walls, and basement/crawlspaces of the house. The health and safety issues that can be identified include wiring problems, water leaks,

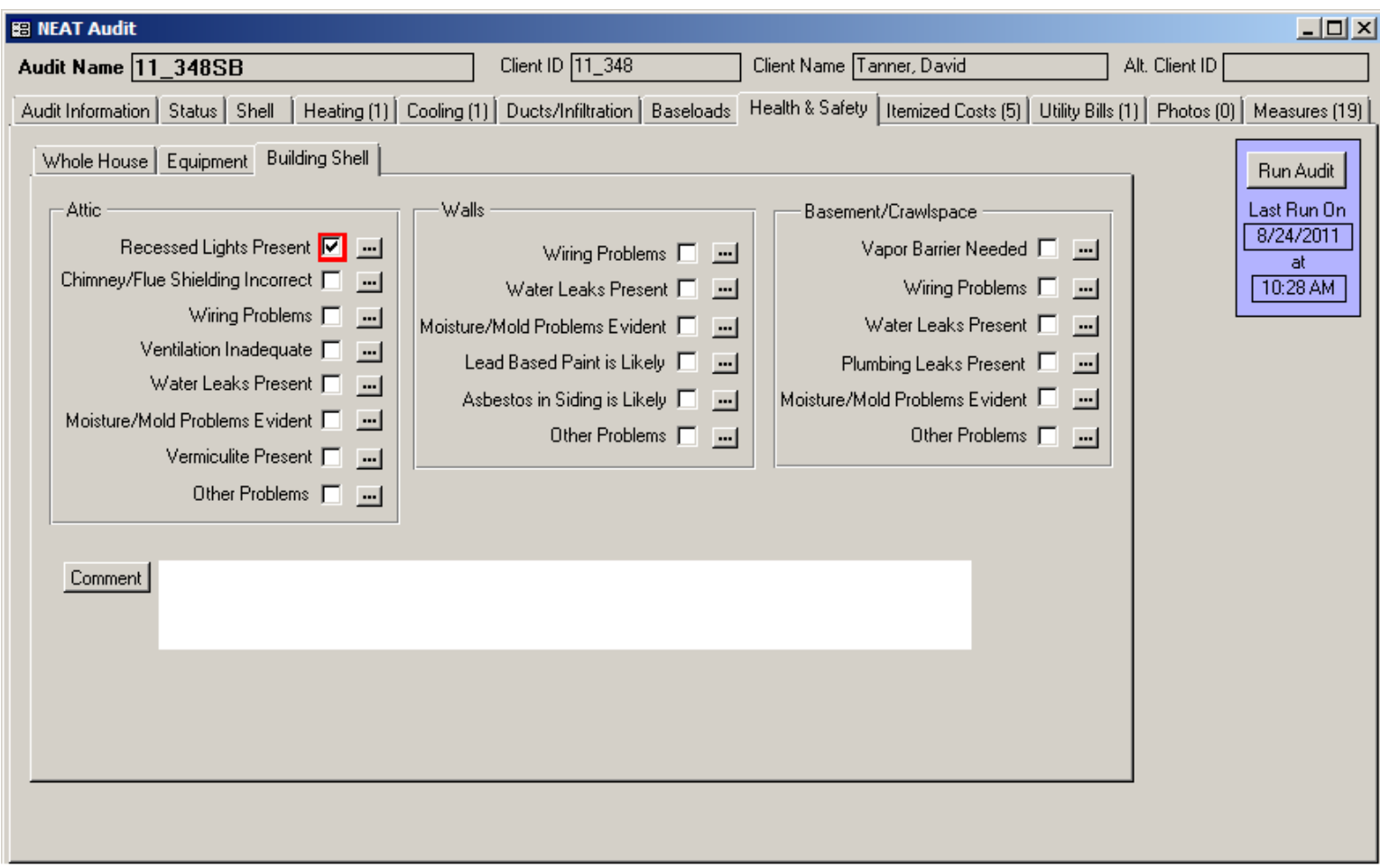

Figure 11.20. The Health \& Safety - Building Shell form under the Main Menu's "NEAT" button. 


\section{Chapter 11: NEAT and MHEA Common Elements}

plumbing leaks, moisture/mold problems, the presence of lead-based paint in walls, asbestos in vermiculite attic insulation or wall siding, and recessed lighting, as well as problems associated with the ventilation and the chimney/flue, and the need of a vapor barrier in the basement. All areas can also be described as having Other Problems, which could be explained in the comments.

\section{Handling of Health and Safety Hazards}

Most of the checkboxes on the forms under the Health \& Safety tab and on the optional details sub-forms under the Heating and Water Heating forms, when checked, show a red border around them (see Figure 11.21) indicating potential hazards. All such hazards are reported under both the Heating System and the Health \& Safety Summary Reports for the audit. For each potential health and safety hazard, remedial measures are predefined under the User Defined Measures form of the Setup Library (see Section 14.6, User Defined Measures).

\author{
Air-to-Air Heat Exchanger \\ Exists $\sqrt{\nabla}$ \\ Not Operational [ $[$... \\ Figure 11.21. Two \\ checkboxes, one \\ indicating a hazard.
}

If you have selected Feature 11, "Automatically generate itemized cost records for health and safety problems," on the Features form of the Main Menu's "Preferences" button (see Section 16.4, Features), when checking/unchecking a checkbox indicating a potential hazard, you will be asked if you wish to ADD/ERASE an associated health and safety measure to/from the Itemized Costs form under the "NEAT Audit" or "MHEA Audit" windows (see Section 11.8, Itemized Costs). In the same request to ADD a measure, you will be notified if the cost of the measure has not been initialized, indicated by a default cost of $\$ 999$. You may use the button with three periods, $\overrightarrow{-}$, to the right of the checkbox on the form, to go to the User Defined Measures form under the Main Menu's "Setup Library" button (see Section 14.6, User Defined Measures), which addresses the specific health and safety item and allows you to initialize the cost. Thus, this feature allows you to automatically generate health and safety measures to supplement your recommended energy measures list.

\subsection{Itemized Costs}

The primary use of the Itemized Costs form under the "NEAT Audit" and "MHEA Audit" windows is to describe costs you encounter during weatherization, such as travel, administrative, or repair costs, or the costs of health and safety measures. 


\section{Chapter 11: NEAT and MHEA Common Elements}

The form was later expanded to include an ability to describe energy saving measures defined by the user and not addressed under the Library Measures tab of the Main Menu's “Setup Library” button (see Section 14.5, Library Measures). However, in such cases, since the measure is being defined by you, the user, you must determine and enter the associated annual energy savings. Once fully defined on the Itemized Costs form, a user-defined measure that saves energy is treated by NEAT and MHEA the same as the library measures.

Itemized cost records may be created in three ways. First, you may define measures by supplying entries to each individual data field on a blank Itemized Costs form in the specific audit you are currently referencing. Second, NEAT and MHEA provide you with a mechanism to predefine measures that you commonly encounter by defining them in the Setup Library, then copying them into any audit to which they apply. NEAT and MHEA also allow you to copy from a library of predefined standard health and safety measures that is a part of all setup libraries (see Section 14.6, User Defined Measures). Third, if you have selected Feature 11, "Automatically generate itemized cost records for health and safety problems" on the Features form of the Main Menu's "Preferences" button (see Section 16.4, Features), any health and safety issues you have identified on the Health \& Safety forms or under the Heating and Water Heating forms will create itemized cost records to remediate the hazards (see "Handling of Health and Safety Hazards" in Section 11.7, Health and Safety).

\section{Entering a User-Defined Measure for an Individual Audit}

Below are descriptions of the individual data items and controls found on the Itemized Costs form as you would enter them individually for a specific audit. See Figure 11.22 for an example of a simple cost measure and Figure 11.23 for an example of a user-defined measure having energy savings. See the following section, "Copying a Predefined Measure from the Setup Library," for the alternative means of providing these entries. See Section 12.2, Recommended Measures Report, for references to this report in the field descriptions below.

- Measure Name - Enter a brief description of the measure. This description will be displayed in the "Recommended Measure" column of the Recommended Measures report. 


\section{Chapter 11: NEAT and MHEA Common Elements}

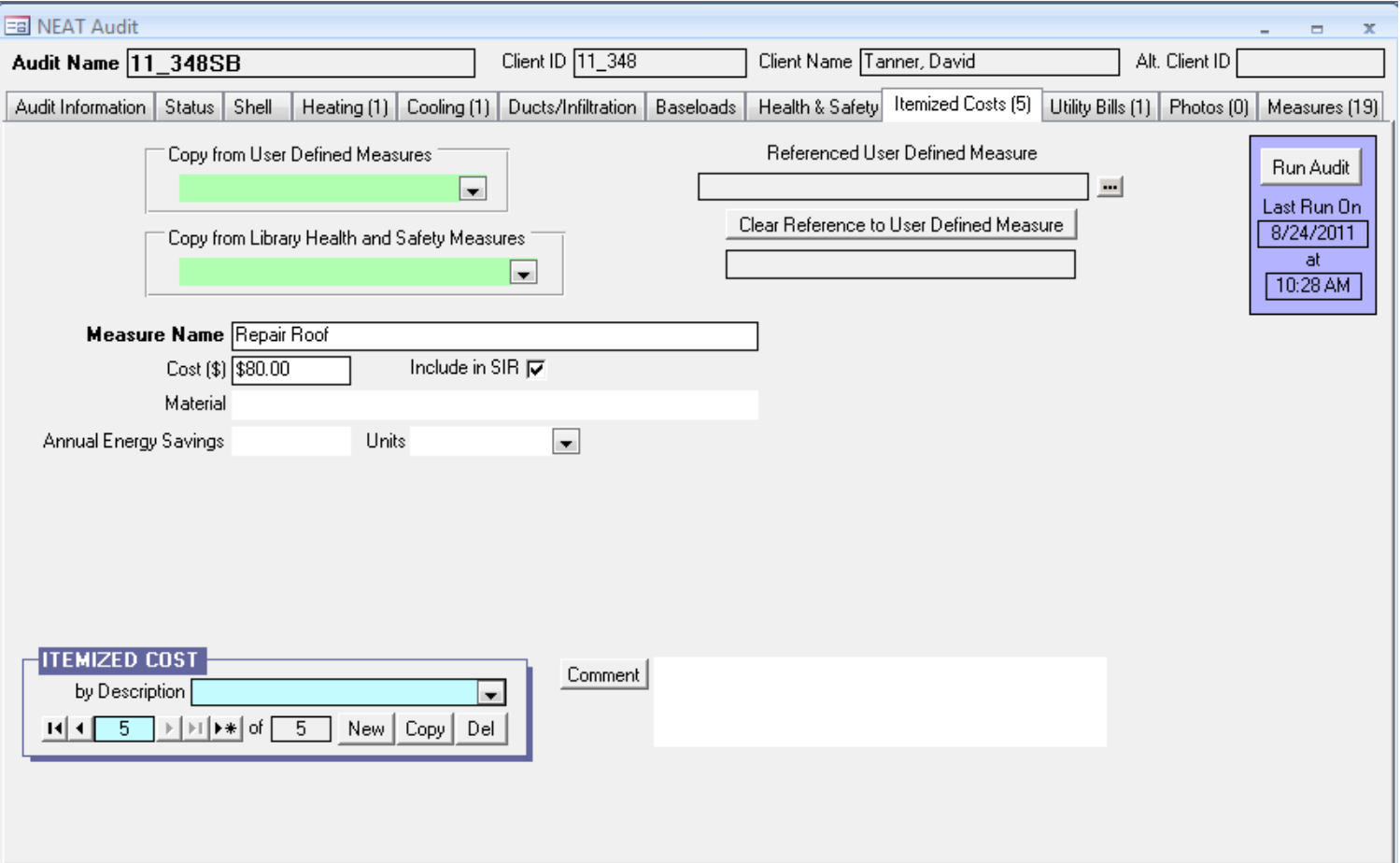

Figure 11.22. The Itemized Costs form under the Main Menu's “NEAT” button. A simple cost.

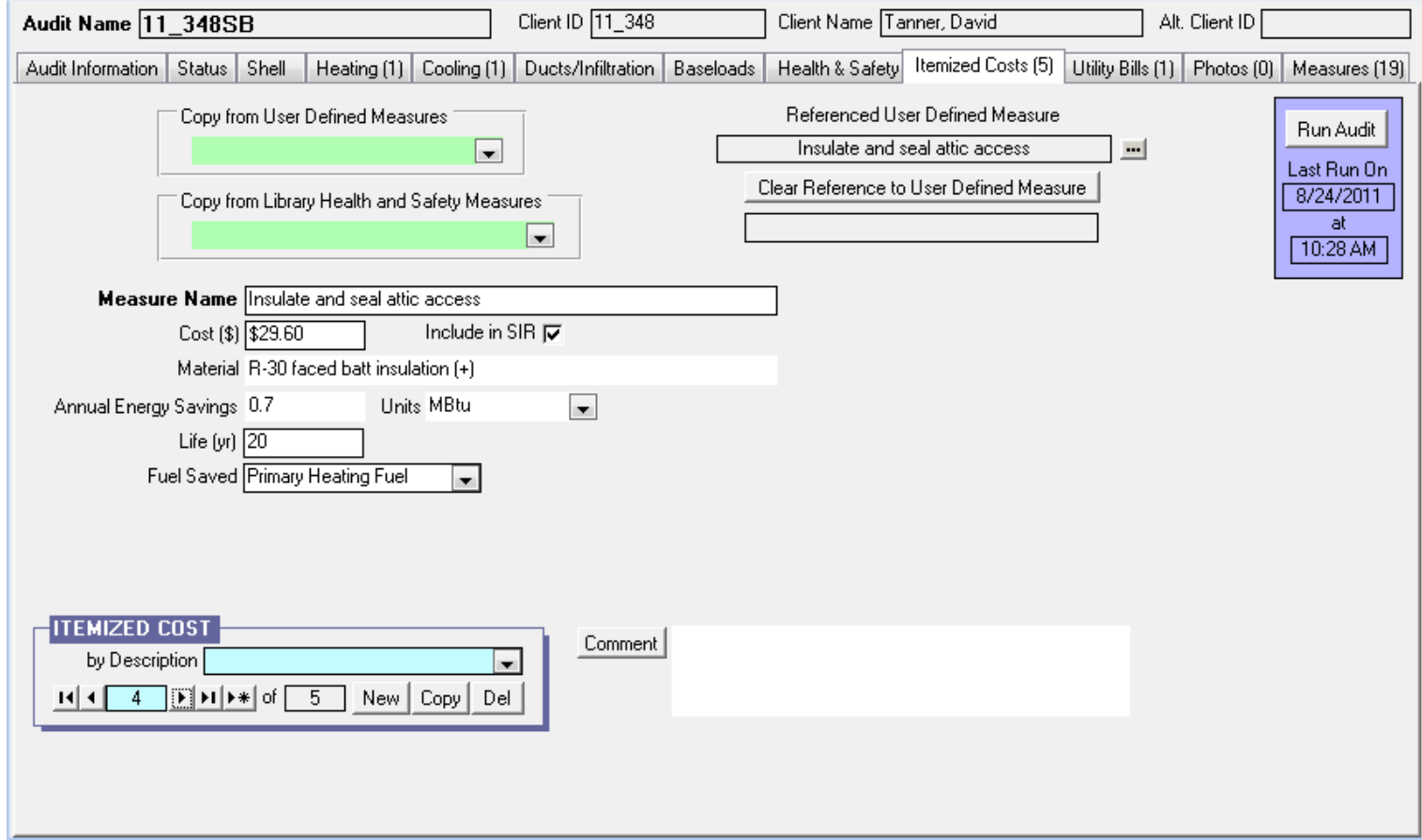

Figure 11.23. The Itemized Costs form under the Main Menu's “NEAT” button. A user-defined measure with energy savings. 


\section{Chapter 11: NEAT and MHEA Common Elements}

- Cost - Enter the total cost of the measure in dollars. The cost will be displayed in the "Measure Cost" column under the Energy Saving Measure Economics section of the Recommended Measures report. Required.

- Include in SIR - This checkbox allows you to indicate that you wish to have the measure included in the calculation of the whole house (package) SIR for this audit. Measures not included in the whole house SIR are normally related to health and safety. An alternate reason for excluding a measure from the whole house SIR is if it is funded outside of the Weatherization Assistance Program. Whether a measure is or is not included in the whole house SIR is indicated by its location in the list of measures produced for the Recommended Measures Report (see Chapter 12, NEAT and MHEA Results). You must select this checkbox in order to have the form present you with the fields necessary to define a measure having energy savings.

- Material - If you enter a material associated with the measure, this material will be displayed under the Materials section of the Recommended Measures Report. Optional.

- Annual Energy Savings and Units - If the Include in SIR checkbox is checked, the Annual Energy Savings and Units fields appear. If you are describing an energy saving measure, enter an estimate of the annual energy savings in the units selected in the adjacent drop-down list. The choices for units are $\mathrm{kWh}$, MBtu or Therms. You may need to use standard conversion factors to arrive at savings in these units. This information is used in calculating the measure's SIR. Required for an energy saving measure.

- Life - If annual energy savings is entered, the Life field will appear. Enter the lifetime of the measure/materials associated with the measure in years. Industry standards have been established for many materials. Required for an energy saving measure.

- Fuel Saved - If annual energy savings is entered, the Fuel Saved field will appear. Select the type of fuel saved by the user-defined measure. The choices are Primary Heating Fuel, Water Heating Fuel, Natural Gas, Oil, Electricity, Propane, Wood, Coal, Kerosene, and Other. Required for an energy saving measure. 
- Comment - You may enter comments pertinent to the measure directly in the Comment field on the form, or you may enter them in the Comment Editor by selecting the "Comment" button to the left of the field. The Comment Editor is similar to Window's Notepad and may be used to enter extended comments. The total comment may have up to 65,000 characters. Text from other sources may be copied and pasted into the Comment Editor's window. Any comment that is entered is displayed on the Recommended Measures report and the Input Report available from the Audit Information form (see Section 8.2, Audit Information). In addition, any comment that is entered can be imported into any specific work order (see Section 13.1, Work Order Information). Thus, you may wish to enter comments related to any observation that might affect the installation of needed measures or repair work. Optional.

- Itemized Cost Record Navigation Block - The Itemized Cost record navigation block is used to find and navigate to existing measure descriptions, copy or delete the currently accessed description, or create a new measure description for the audit. See Section 5.1, Form and Record Navigation, for information on using the Weatherization Assistant's record navigation blocks.

\section{Copying a Predefined Measure from the Setup Library}

If you have predefined measures in your Setup Library (see Section 14.6,User Defined Measures), the Itemized Costs form allows you to simply copy any of these measures into an audit whenever they apply. All of the fields on the Itemized Costs form will be entered automatically with the data provided by the measure definition in the Setup Library. If any data existed in the form's fields prior to the selection, it will be overwritten. Most often, no further data entry will be necessary.

There are major differences between a measure defined by supplying individual entries on the Itemized Costs form and one copied from the Setup Library. The former can have only one material associated with it, the one entered in the Material field on the form. However, a measure defined in the Setup Library can have multiple material/labor components, each with its own individual cost associated with it (see Section 14.6,User Defined Measures). In this latter case, only the component designated by the user in the Setup Library (presumably the major component) will be displayed in the Material field, followed by a plus sign in parentheses, $(+)$, (see Figure 11.23) indicating that there is more to the material description of the measure than this single entry. The Cost field, however, will 


\section{Chapter 11: NEAT and MHEA Common Elements}

display the total cost of the measure, comprised of the sum of the individual component costs defined in the Setup Library. If after copying a measure from the Setup Library into an audit, the Cost field displays “\$999”, it is an indication that you have not initialized the cost of the measure in the Setup Library.

You may review the complete description of a user-defined measure in the Setup Library by clicking on the small button with three periods in it, $\overrightarrow{-\cdot}$, located just to the right of the uneditable display of the measure name on the Itemized Costs form. This button takes you to the actual Setup Library entry. Be aware that any changes to the measure made at this point will change the measure description for all future references. If you do access the measure in the Setup Library, use the X-box in the upper right corner of the "Setup Library" window to close the Setup Library and return to the Itemized Costs form.

Although the display of a measure copied from the Setup Library will show only one component on the Itemized Costs form of the audit, the complete description with all of its individual components will be available in any work order created from the audit. Any modifications to the measure can be made at that time. See Section 13.3, Measures (Work Order). If you have chosen to copy a predefined measure from the Setup Library into an audit and you do not want this detailed material costing to be forwarded to the work orders, click on the "Clear Reference to User Defined Measure” button. You are then free to alter any of the entries displayed on the audit's Itemized Cost form for this item. The changes will not affect the measure definition in the Setup Library. Only the single Material and Cost visible on the Itemized Cost form will be associated with the measure and be forwarded to the work orders. WARNING: Modifying the information on the Itemized Costs form for a user-defined measure generated by copying an item from your Setup Library without first clearing the reference to the predefined measure will cause inconsistencies in data reported in the NEAT or MHEA Recommended Measures report and that reported in the work orders.

The following summarizes the information regarding copying a predefined measure from the Setup Library as it relates to the specific data items and controls on the Itemized Costs form.

- Copy from User Defined Measure - If you wish to include a measure you have previously described on the User Defined Measures form under the Main Menu's "Setup Library" button into an audit, select it from the "Copy from 


\section{Chapter 11: NEAT and MHEA Common Elements}

User Defined Measure” drop-down list. The drop-down list includes all measures you have described on the User Defined Measures form in the Setup Library accessed by the audit.

- Copy from Library Health and Safety Measures - If you wish to include a measure from the library of health and safety measures into an audit, select it from the "Copy from Library Health and Safety Measure" drop-down list. The drop-down list includes fifty health and safety measures that come as part of the program. Although all setup libraries contain these same health and safety measures, the costs for the measures will be taken from the specific Setup Library accessed by the audit.

- Referenced User Defined Measure - If you have selected a measure from the Setup Library using either option above, this field will display the measure name as given in the library. If the measure was selected from the "Copy from Library Health and Safety Measures" drop-down list, the text "Health \& Safety Item \#" appears in red in the uneditable field below this field, where \# is a unique number pre-assigned to each measure of the library of health and safety measures. See Figure 11.24. Uneditable.

- Clear Reference to User Defined Measure - If a user-defined measure has been copied from the Setup Library, this button can be used to remove the reference of the measure in the audit from the user-defined measure defined in the Setup Library. The detailed material/labor detail associated with the measure in the Setup Library will no longer be available to any work order that might be created from the audit. Only the information on the Itemized Costs form remains as defining the measure. However, that information can be altered without causing a discrepancy between data seen in the Recommended Measures report and work orders generated from the audit.

The Itemized Costs form can be viewed and edited in the data sheet view (see Figure 11.25). The data sheet view provides a summary of all measures entered on this form and allows quick changes to multiple entries. However, care must be taken if any changes are made from this view, as it may not be obvious which measures have been copied from the Setup Library. 


\section{Chapter 11: NEAT and MHEA Common Elements}

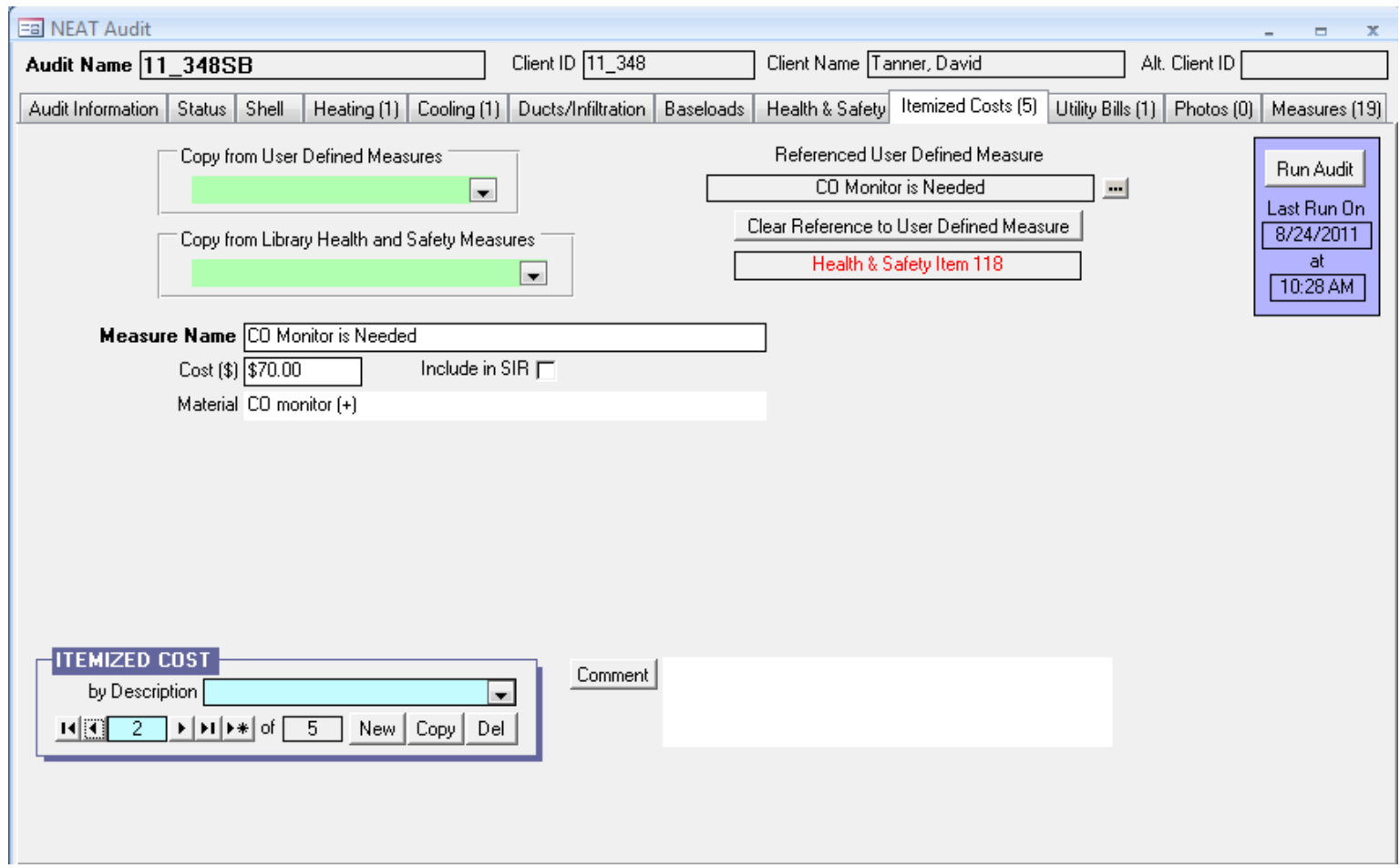

Figure 11.24. The Itemized Costs form under the Main Menu's “NEAT” button. A measure copied from Library Health and Safety Measures.

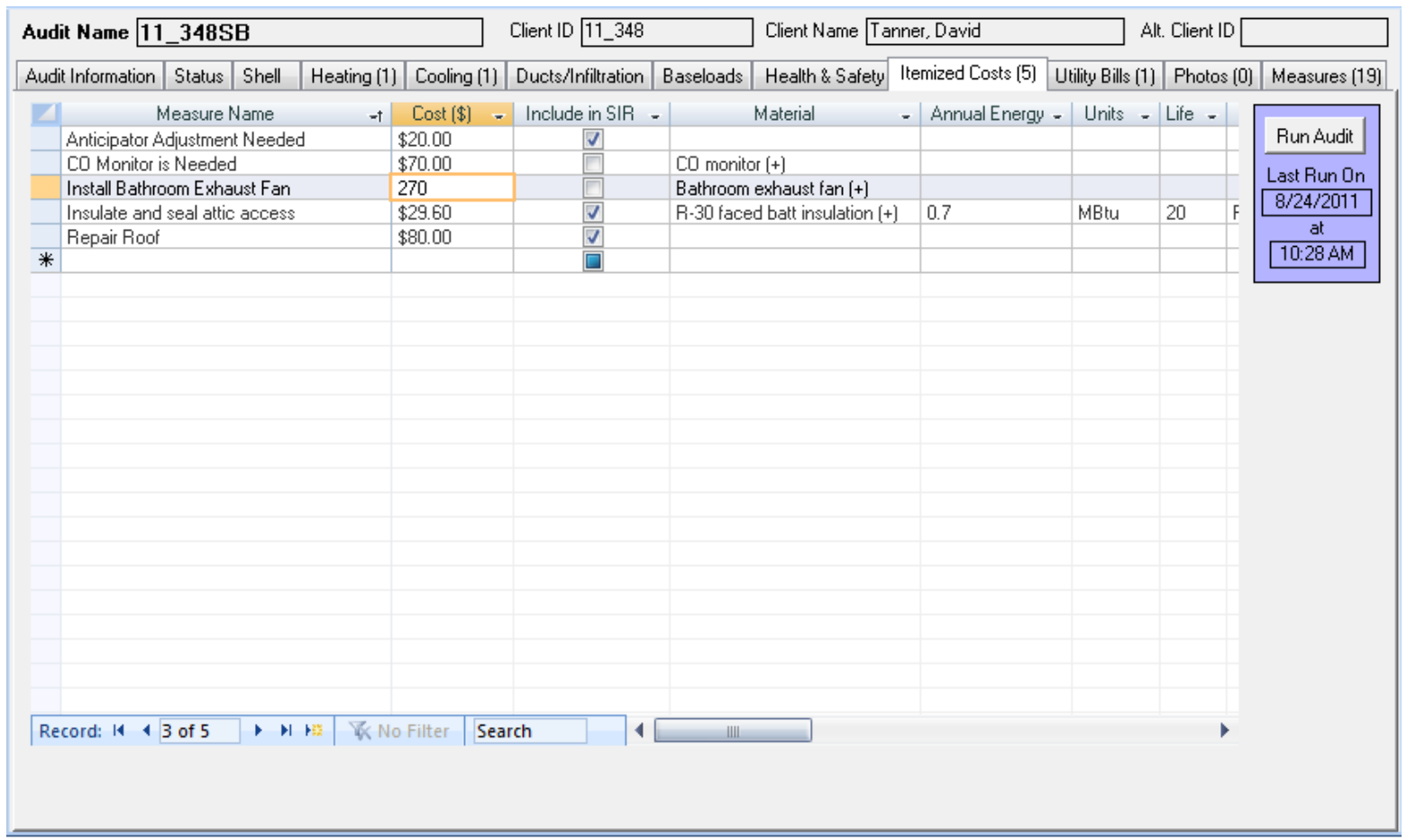

Figure 11.25. Data sheet view of the Itemized Costs form.

Columns not displayed include "Fuel Saved," "Health \& Safety Item \#," and "Comment." 


\subsection{Utility Bills}

The Weatherization Assistant has an optional feature of storing utility billing data for the homes you weatherize and using that data to adjust energy savings estimates from the measures. The Utility Bills form under the "NEAT Audit" and "MHEA Audit" windows (see Figure 11.26) can be used to enter four sets of energy use data: pre-retrofit heating, pre-retrofit cooling, post-retrofit heating, and post-retrofit cooling energy use. NEAT and MHEA will compare their consumption predictions with the pre-retrofit billing data (see "Heating and Cooling Energy Consumption Comparisons" Section 12.2, Recommended Measures Report). If the Billing Adjustment checkbox has been selected in the Audit Information form (see "Billing Adjustment" in Section 8.2, Audit Information), NEAT and MHEA will adjust their energy savings estimates and develop a second set of recommended weatherization measures based on the actual pre-weatherization energy consumption of the house. Post-retrofit billing data may also be entered and stored with the other data for a house but is not used or displayed by the programs. Computer software exists which can use both pre- and post-weatherization billing data to derive actual energy savings estimates, possibly useful in program evaluations.

The billing data feature works best with metered fuels, such as natural gas and electric. Bulk fuels, such as propane or fuel oil, which are delivered in bulk and stored in tanks at the house, are less appropriate. Unless deliveries are relatively frequent and approximately the same amount of fuel remains in the tank at the time of each delivery, use of billing data for these fuels can lead to substantial inaccuracy.

Below are descriptions of the individual data items and controls found on the Utility Bills form under the "NEAT Audit" and "MHEA Audit" windows.

- Type - Select the predominant energy end use, either Heating or Cooling, for which the billing data is entered. NOTE: Billing data may include both heating and cooling energy use when the same fuel is used for both end uses, such as in a house with electric heating and cooling or a heat pump. In these cases, you are advised to not use this feature unless you can successfully separate heating and cooling end uses prior to entering the data. The billing data may also include non-heating or non-cooling end uses, such as for cooking or water heating. In 


\section{Chapter 11: NEAT and MHEA Common Elements}

these cases, you will have to enter an estimate of this base load in a subsequent field. Required.

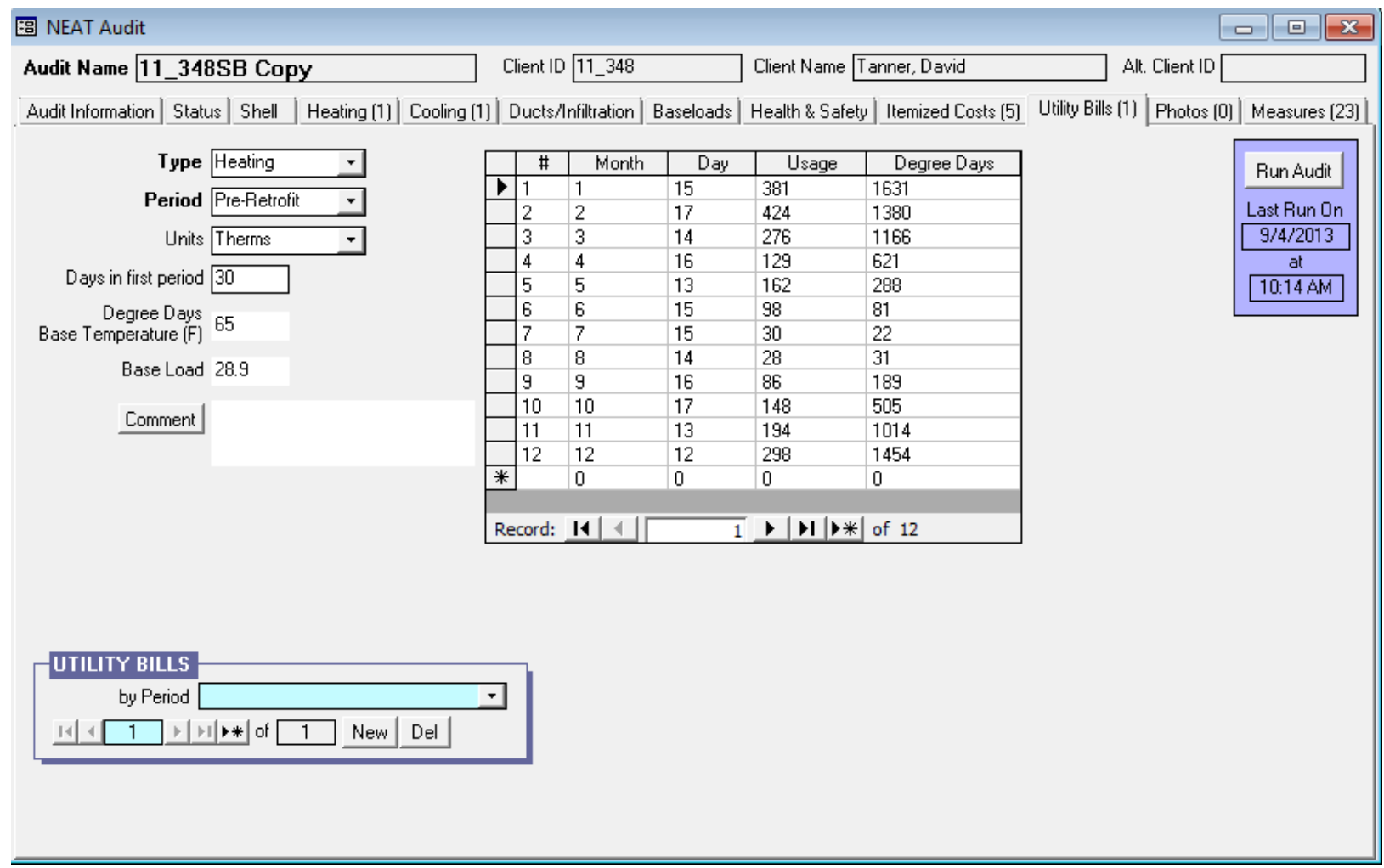

Figure 11.26. The Utility Bills form under the Main Menu's “NEAT” button.

- Period - Select whether the data being entered pertains to a Pre-Retrofit or Post-Retrofit period. Only pre-retrofit data will be used for measure savings adjustments. Required.

- Units - Select the units of energy use data, either Therms or kWh, depending on the fuel source for the data being entered. Normally cooling data will be in kilowatt-hours of electricity and heating data in either therms or kilowatt-hours.

The utility may document natural gas consumption in therms, mcf (thousand cubic feet), or ccf (hundred cubic feet). Mcf and ccf can be converted to therms by multiplying by a value obtained from the local gas utility. Because heat content of natural gas varies according to composition, ask the utility for the number of therms contained in a mcf or ccf delivered to homes in the area. This value (in therms per ccf) should be entered in the Setup Library (see Section 14.3, Fuel Costs). Most natural gas contains approximately ten therms per mcf 


\section{Chapter 11: NEAT and MHEA Common Elements}

and one therm per ccf of natural gas. Other fuels may require additional conversion, such as gallons of propane or oil. However, note the warning at the beginning of this section regarding recording bulk fuel consumption. Required.

- Days in First Period - Enter the number of days included in the first billing period. Subsequent periods are assumed to be contiguous, thus allowing determination of their lengths. Required.

- Degree Days Base Temperature - The Utility Bills Data Entry screens provide an option for entering heating degree days (HDDs) and cooling degree days (CDDs) for natural gas and electricity billing data, respectively. The degree-day information will not affect any adjustments to the measure savings. It simply allows you to compare degree-days used by NEAT and MHEA with those from the actual billing periods. Heating and cooling degree days are available from local utilities, state energy offices or the U.S. Department of Commerce National Weather Service, Asheville, NC.

If degree-day information is to be entered, base temperatures for these HDD or $\mathrm{CDD}$ values are required. Assume a base temperature of $65^{\circ} \mathrm{F}$ for HDDs and 78 ${ }^{\circ} \mathrm{F}$ for CDDs, unless different base temperatures are listed on the utility bills. Optional.

- Base Load - Enter an estimate of the average base load consumption per month in the units chosen in "Units" above. The period consumptions entered likely result from not only heating or cooling, but also from use of appliances, such as stoves, water heaters, or refrigerators. The Weatherization Assistant needs to separate this "base load" consumption from the heating and cooling consumption. This entry is an estimate of this base load consumption for an average 30-day month.

To estimate the base load for the heating fuel in homes with fossil fueled heating systems, select a billing period when the heating equipment did not operate, most likely during the summer. This period should be one of normal household occupancy - no long term visitors or household vacations. If the billing period chosen is significantly more or less than 30 days, you should adjust the consumption by the ratio of 30 over the number of days in the period. This consumption in the units selected in the Units field is an estimate of the heating fuel base load. 


\section{Chapter 11: NEAT and MHEA Common Elements}

To estimate the base load for a fuel with Type "Cooling," select a billing period with normal household occupancy having no or minimal electric heating or airconditioning consumption, most likely during fall or spring. Make any adjustment for the period length, as described above. This adjusted kilowatthours consumed is the base load.

During the audit runs, NEAT and MHEA will adjust the base load entries to correspond to each of the billing periods, regardless of their duration. You may wish to leave this field blank until after entering the actual billing data for the periods. If possible, the programs will compute default values for the base loads that reflect the period data entered. Optional.

- Comments - You may enter comments pertinent to each set of billing data directly in the Comment field on the form, or you may enter them in the Comment Editor by selecting the "Comment" button to the left of the field. The Comment Editor is similar to Window's Notepad and may be used to enter extended comments. The total comment may have up to 65,000 characters. Text from other sources may be copied and pasted into the Comment Editor's window. Any comment that is entered is displayed on the Recommended Measures report and the Input Report available from the Audit Information form (see Section 8.2, Audit Information). Optional.

\section{Billing Periods}

If you wish to enter billing data, ask the client for up to 12 months of utility bills. If the client has discarded these bills, he or she may request consumption records from the local utility. Or, the client may sign a release that grants you authority to directly obtain the data. The billing periods entered must be consecutive. They may extend from one year into the next, but must span one year or less. Thus, no two periods can include any of the same days of the year in two years. The number of periods entered must be 12 or less. Examples of entries are twelve consecutive monthly readings, one annual consumption, or any number of consecutive periods, 12 or less, spanning less than a year. The following data are required for each billing period within a set. They appear on the right side of the input screen in a tabular format.

The data need not be entered row by row, but, instead, could be entered column by column. For example, if the meter is read on the $15^{\text {th }}$ of every month, you may 


\section{Chapter 11: NEAT and MHEA Common Elements}

wish to travel down the month column entering $1,2,3,4 \ldots$, then moving to the Day column entering 15 in each entry, using the down arrow key to travel from on field in a column to the next.

- \# - Enter consecutive integers for the entry numbers, beginning with 1. Required.

- Month - Enter the number of the month in which the meter reading corresponding to the billing period was taken (i.e., 1 for January, 2 for February, etc.). Required.

- Day - Enter the day of month on which the meter reading corresponding to the billing period was taken. Required.

- Usage - Enter the energy consumption during the billing period. The units are those selected in the Units field to the left. Required.

- Degree Days - Enter the number of heating or cooling degree-days (depending on the "Type" selected) corresponding to the period. Optional.

\subsection{Photos (Audit)}

If you have selected the "Use photo browser tab..." checkbox from the Features tab under the Main Menu's "Preferences" button (see Feataure 1 in Section 16.4, Features), the Photos tab will appear under the "NEAT Audit" and "MHEA Audit" windows. This feature allows you to access the Photos form (see Figure 11.27). From this form, you may select, view, and edit digital photos for the current audit. See Section 18.1, Digital Photos, for more information on this feature. The ability to reference digital photos also exists under the Client and Main Menu's "Work Orders” buttons. Therefore, you should develop a consistent policy regarding where you reference your photos. 


\section{Chapter 11: NEAT and MHEA Common Elements}

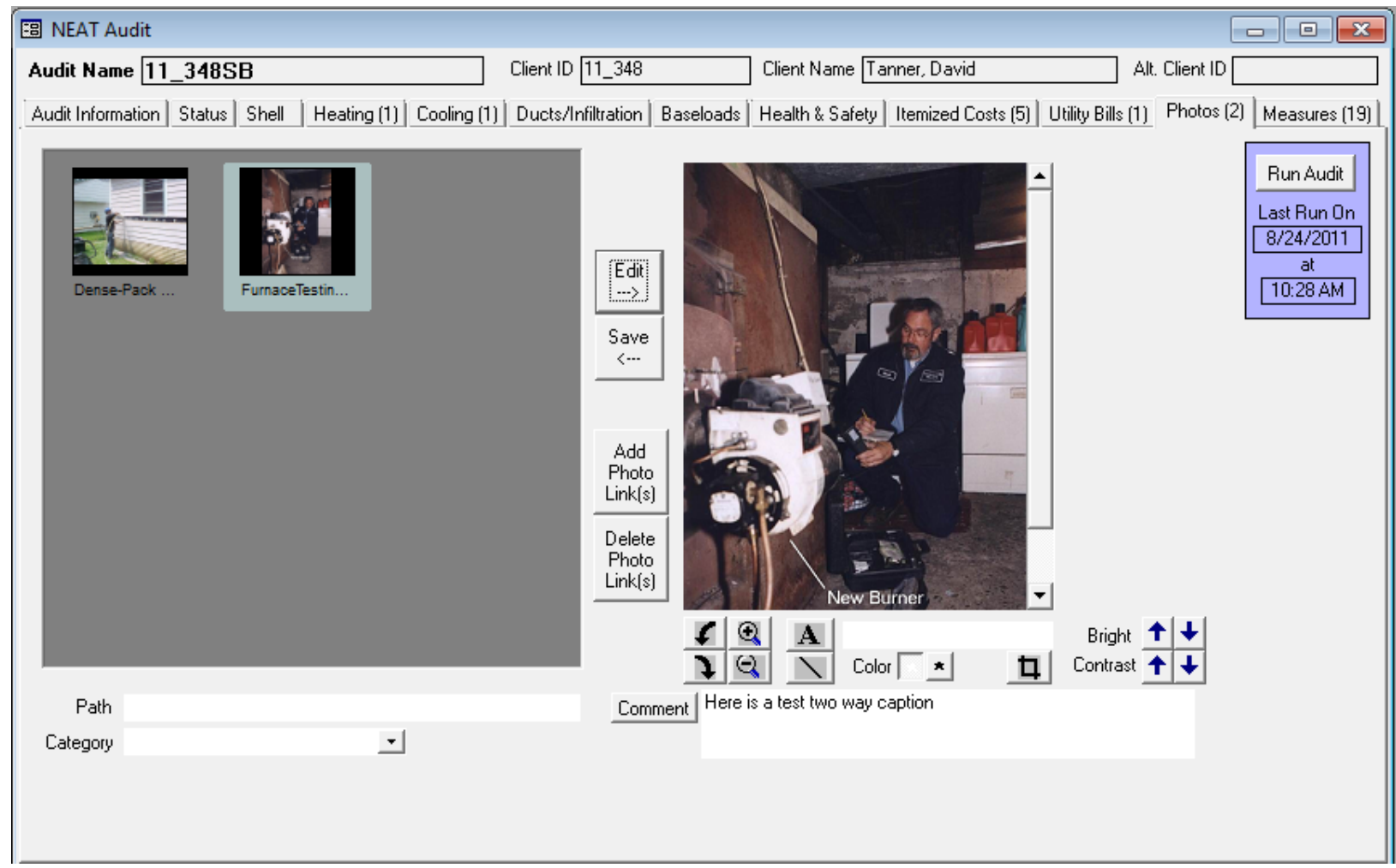

Figure 11.27. The Photos form under the Main Menu’s “NEAT” button.

\subsection{Measures (Audit)}

The Weatherization Assistant has an optional feature of creating work orders based on the results from running NEAT or MHEA. An alternate use of the work order feature is to modify the recommendations generated by NEAT and MHEA to reflect actual work performed with associated actual costs or to add as much detail to the measure descriptions as desired (see Chapter 13, Work Orders). The Measures form (see Figure 11.28) under the "NEAT Audit" and "MHEA Audit" windows is the starting point for implementing these capabilities.

Following execution of either NEAT or MHEA, the Measures form will list all measure recommendations, similar to the listing in the Recommended Measures report (see Section 12.2, Recommended Measures Report) except in a different format. Any corresponding data items will have the same values in both.

Below are the descriptions of the individual data items and controls found on the Measures form. The uneditable fields reflect the data and assumptions used by the audits in generating the recommendations. 


\section{Chapter 11: NEAT and MHEA Common Elements}

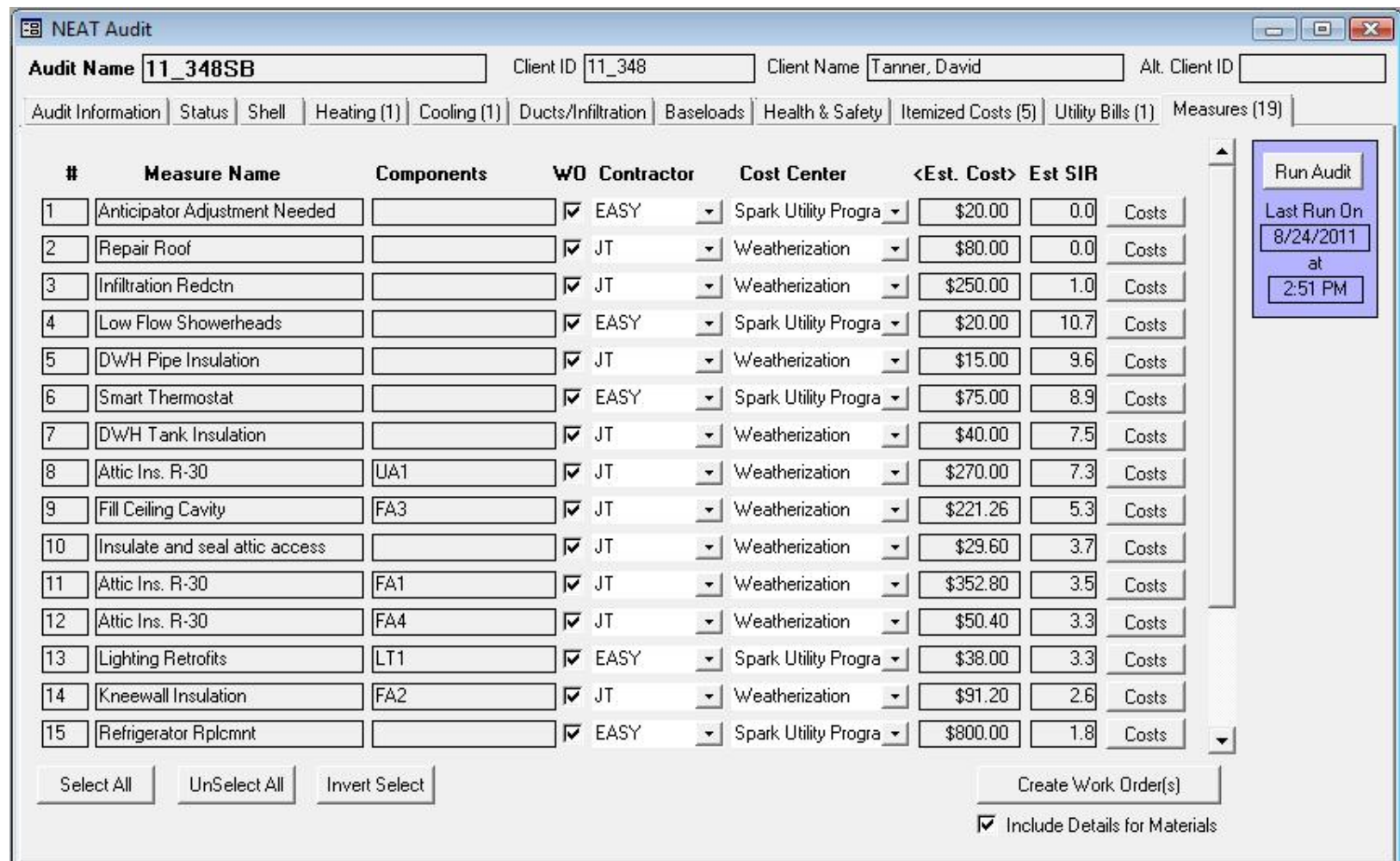

Figure 11.28. The Measures form under the Main Menu’s “NEAT” button.

- \# - This field displays the order in which measures appear on the Recommended Measures report. Uneditable.

- Measure Name - This field displays the name of the measure as given under the Library Measures tab of the Main Menu's “Setup Library” button (see Section 14.5, Library Measures) or on the Itemized Costs form for the audit (see Section 11.8, Itemized Costs). Uneditable.

- Components - This field displays the component names associated with the measure. These are primarily the Codes you have assigned to records during entry of the building description in either NEAT or MHEA. For example a Wall Code, "WL1.” Uneditable.

- WO? - The header name is short for "Include in Work Order?" Select this checkbox for each measure you want transferred to a work order. Measures not selected will not be sent to a work order. You may wish not to send a specific measure to a work order if a work order already exists which includes the measure or if you do not want to consider certain recommended measures. 
- Contractor -This field allows you to assign each measure to a contractor. The selection will determine how many work orders are created from the measure list. All measures assigned the same contractor will be included in the same work order. The choices displayed in the drop-down list will be all agency contacts defined as Contractor or Crew (see Section 6.2, Contacts (Agency)). The entry is optional. All measures with an unassigned Contractor that are selected for transfer to a work order will be assigned to the same work order with the contractor undesignated. If you have assigned a Default Contractor to a particular measure in the Setup Library (see "Default Contractor/Crew" under "The General Task Description” in Section 14.6, User Defined Measures) and that measure is recommended, the default contractor will automatically be entered for you. However, you may still change the selection if you desire. Optional.

- Cost Center - If you are tracking costs that are incurred by different funding sources or programs, assign the cost of implementing this particular measure to the appropriate Cost Center. The choices available in the drop-down list will be those you have defined under the Cost Center tab on the Main Menu's "Agency" button for your agency (see 6.3, Cost Centers). If the entry is left unassigned, work orders can still be developed, but total costs over multiple audits will not be tracked. The costs assigned to the various cost centers are not the estimated costs displayed on this Measures tab. They are the actual costs declared in the work orders themselves (see "Quantity (Actual)" and "Unit Cost (Actual)” under “The Materials/Labor Details Sub-Form” in Section 13.3, Measures (Work Order)). If you have assigned a Default Cost Center to a particular measure in the Setup Library (see "Default Cost Center" under "The General Task Description” in Section 14.6, User Defined Measures) and that measure is recommended, the default cost center will automatically be entered for you. However, you may still change the selection if you desire. Optional.

- <Est. Cost > - This field displays the estimated cost used by the audits to make their initial recommendations, which may not be the same as actual costs following implementation of the measures, as referred to above. The angle brackets surrounding the header name are to remind you that the values displayed in the column could be the sum of individual component costs and that the breakdown of the total may be viewed by using the "Costs" buttons to the right of the entries. Uneditable. 


\section{Chapter 11: NEAT and MHEA Common Elements}

- Est. SIR - This field displays the estimated SIR used by the audits to make their initial recommendations, which may not be the same as actual SIRs seen following implementation of the measures. Uneditable.

- Costs (button) - Selecting this button will display the Unit Costs for Measure sub-form, which shows a breakdown of the costs for the standard audit measures. The entries of the sub-form are uneditable. For measures copied to the audit from the Setup Library (see Section 11.8, Itemized Costs), this subform will include as many costing components as were defined in the Setup Library (see "The Materials/Labor Details Sub-Form” in Section 14.6, User Defined Measures).

- Select All, UnSelect All, Invert Select (buttons) - The "Select All," "UnSelect All," and "Invert Select” buttons allow you to accept all of the recommended measures, reject (unselect) all, or invert the current selection under WO?.

- Include Details for Materials - Select this checkbox if you wish to transfer the material details, including the estimated costs, associated with the selected measures to the work orders. This will ensure that the estimated costs in the work orders match those used by the audit, as seen in the detail displayed by selecting the "Costs" buttons on the form. You may choose to leave the checkbox unchecked and create work orders with no predefined cost data associated with the measures. This may be chosen in order to enter costing of the measures in the work orders, perhaps expected to have greater detail than available from the estimated costs used by the audits. However, the cost data used by the audits will still be available to you in the work orders and you may still transfer these costs to the work orders, measure by measure, if desired.

- Create Work Order(s) - Select this button when you are ready to create the work orders based on the information provided on the Measures form. If you have previously created work orders from this specific audit, the Create Work Order sub-form (see Figure 11.29) will be displayed asking your preference among three options: 


\section{Chapter 11: NEAT and MHEA Common Elements}

1. SAVE the previously existing work orders and create new ones. NOTE, this could lead to duplicate measures in multiple work orders.

2. REPLACE the previously generated work orders with new ones, or

3. CANCEL the request to

Create Work Order

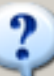

SAVE the previously generated work orders and create new ones

c REPLACE the previously generated work orders with new ones

C CANCEL creation of work order

OK

Figure 11.29. The Create Work Order sub-form.

create work orders.

Any work orders not created from this audit will be unaffected by the request to create work orders from the Measures form of this audit. Thus, if you used the Work Orders form under the Main Menu's “Clients” button to create work orders for a client, then ran an audit for the same client and asked to create work orders based on the audit recommendations, the two sets of work orders will exist for the same client. This is intended to address situations where agencies may implement some measures outside an audit (such as from a priority list), but use the audit for recommendations on other measures.

The program will next display a window (see Figure 11.30) indicating how many work orders were created as a result of your selections on the Measures form. This should correspond to the number of unique

Weatherization Assistant $\mathrm{X}$ contractors, including undesignated, you indicated on the form. Selecting the "OK” button will take you to the Main Menu's "Work Orders” button for the first work order. Having created work orders from the audit recommendations, you are prepared to make any modifications to them before printing out the actual work orders. See Chapter 13, Work Orders, for additional information. 


\section{Chapter 11: NEAT and MHEA Common Elements}

\subsection{Optional Heating System Details}

The Optional Heating System Details buttons on the Heating form under the Main Menu's “NEAT" button and Heating [Primary] form under the Main Menu's "MHEA" button (see Figure 11.31) can be used to document an extensive number of heating system measurements and observations made while auditing a dwelling. The measurements and observations are entered on the sub-forms accessed from these buttons. Some of these data items help to identify potential health and safety hazards. You must complete the description of the current heating system before accessing these optional sub-forms. Not all buttons will be applicable to the heating system you describe on the Heating form. No attempt is made in this manual to describe correct procedures for obtaining the measurements or performing the inspections. You should refer to literature on standard HVAC practices or consult with a qualified HVAC contractor. Other than the indication of potential health and safety issues, the information entered on the sub-forms is only for documentation, and not used anywhere else in the Weatherization Assistant.

Below are brief descriptions of the Optional Heating System Details buttons found on the Heating forms. Each form allows entry of an extended comment if necessary. Each form must be closed before any other form can be accessed. Close the forms using the customary Windows $\mathrm{X}$ box in the upper right corner.

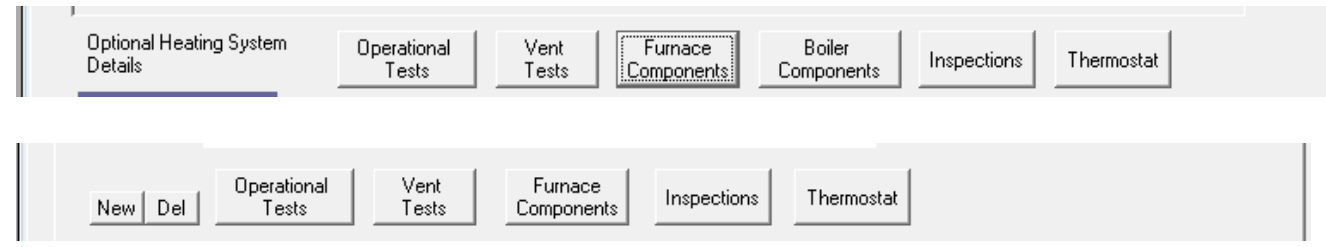

Figure 11.31. The Optional Heating System Details buttons on the Heating form of the Main Menu's "NEAT" button (above) and Heating [Primary] form of the Main Menu's “MHEA” button (below).

\section{Operational Tests}

Selecting the "Operational Tests" button displays the Operational Tests for Heating System sub-form (see Figure 11.32), which allows recording information about the operation of the heating system. Three groups of measurements can be recorded: flue gas analysis, carbon monoxide measurements, and the heat rise across the heat exchanger. For each item in these groups, pre-retrofit (i.e., during the "Audit”) and post-retrofit (i.e., during the "Inspection”) measurements can be recorded. On 


\section{Chapter 11: NEAT and MHEA Common Elements}

exiting the form, either the pre-retrofit or post-retrofit Steady State Efficiency entry can replace the steady state efficiency previously entered on the Heating form if you indicate so by choosing "Yes" on the screen that appears when you exit either of the two fields. Optional.

\section{Vent Tests}

Selecting the "Vent Tests" button displays the Venting Tests for Heating System sub-form (see Figure 11.33), which allows recording information about the characteristics of the flue and damper as well as pre- and post-retrofit draft measurements on the flue associated with the heating system. Any potential safety hazard due to venting related problems may also be noted. See the description under Section 11.7, Health and Safety, on how the Weatherization Assistance handles health and safety hazards. Optional.

\section{Furnace Components}

Selecting the "Furnace Components" button displays the Other Components for Heating System sub-form (see Figure 11.34), which allows recording information about the characteristics for various furnace components including the fan limit controls, burner and pilot, blower and belts, accessories (humidifier, air cleaner, and air conditioner coil), and air filter. In general, the type, size/dimensions, operation settings, and physical condition can be recorded here. The potential safety hazard due to malfunctioning fan limit control may also be noted. See the description under Section 11.7, Health and Safety, on how the Weatherization Assistance handles health and safety hazards. Optional.

\section{Boiler Components}

The "Boiler Components" button is available only on the Heating form under the Main Menu's "NEAT" button. Selecting this button displays the Other Boiler Components for Heating System sub-form (see Figure 11.35), which allows recording information about the components of the boiler, boiler type, its overall condition, and the condition of the expansion tank and drain valve. Additional information can be entered describing the associated controls and convectors. The presence of asbestos associated with the boiler constitutes a potential hazard and may also be noted. See the description under Section 11.7, Health and Safety, on how the Weatherization Assistance handles health and safety hazards. Optional. 


\section{Chapter 11: NEAT and MHEA Common Elements}

\section{Inspections}

Selecting the "Inspections" button displays the Inspections for Heating System sub-form (see Figure 11.36), which allows recording information about additional items not addressed otherwise. The checkboxes, if selected, indicate potential safety hazards associated with the heating system. See the description under Section 11.7, Health and Safety, on how the Weatherization Assistance handles health and safety hazards. Optional.

\section{Thermostat}

Selecting the "Thermostat" button displays the Thermostat Details for Heating System sub-form (see Figure 11.37), which allows recording information about the characteristics associated with the thermostat used by the heating system including its type, set-points, and anticipator settings. Optional.

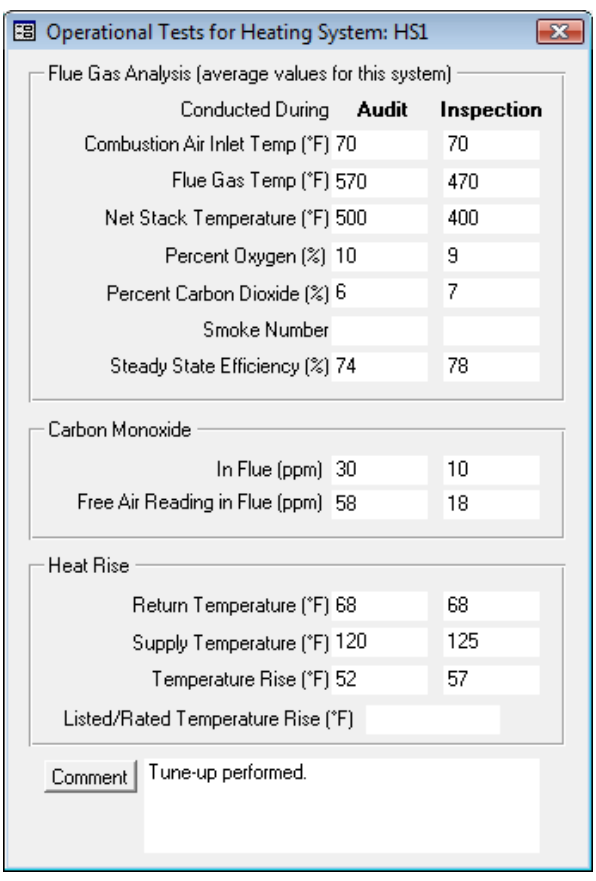

Figure 11.32. The Operational Tests for Heating System sub-form.

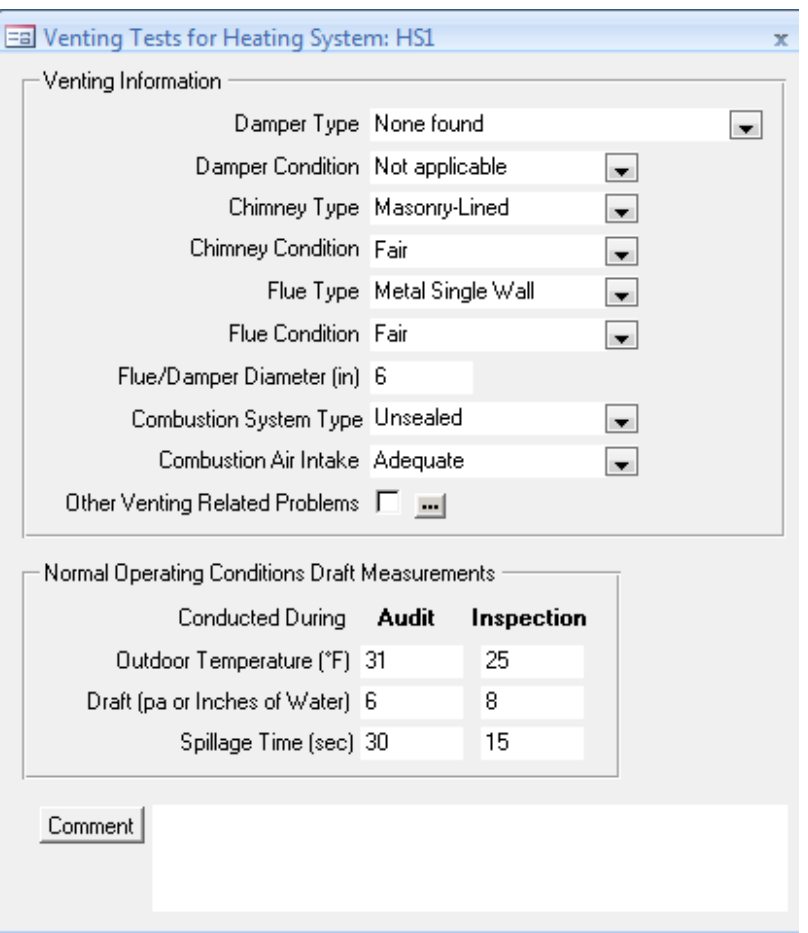

Figure 11.33. The Vent Tests for Heating System sub-form. 


\section{Chapter 11: NEAT and MHEA Common Elements}

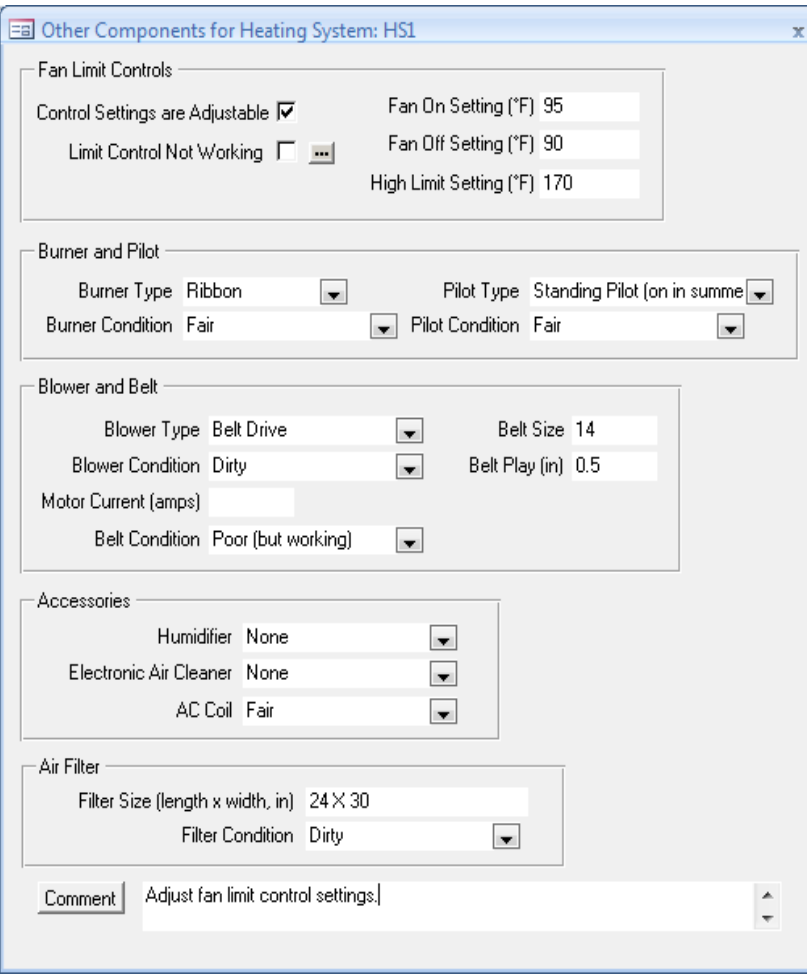

Figure 11.34. The Other Components for Heating System sub-form.

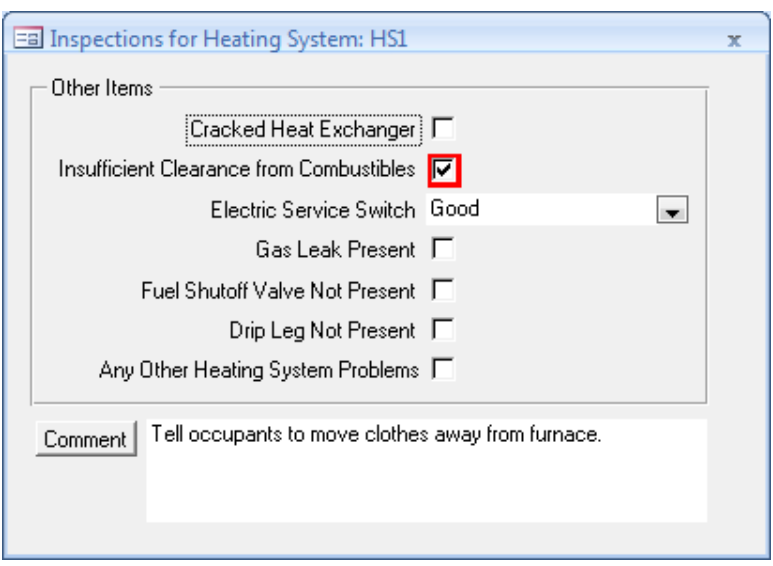

Figure 11.36. The Inspections for Heating System sub-form.

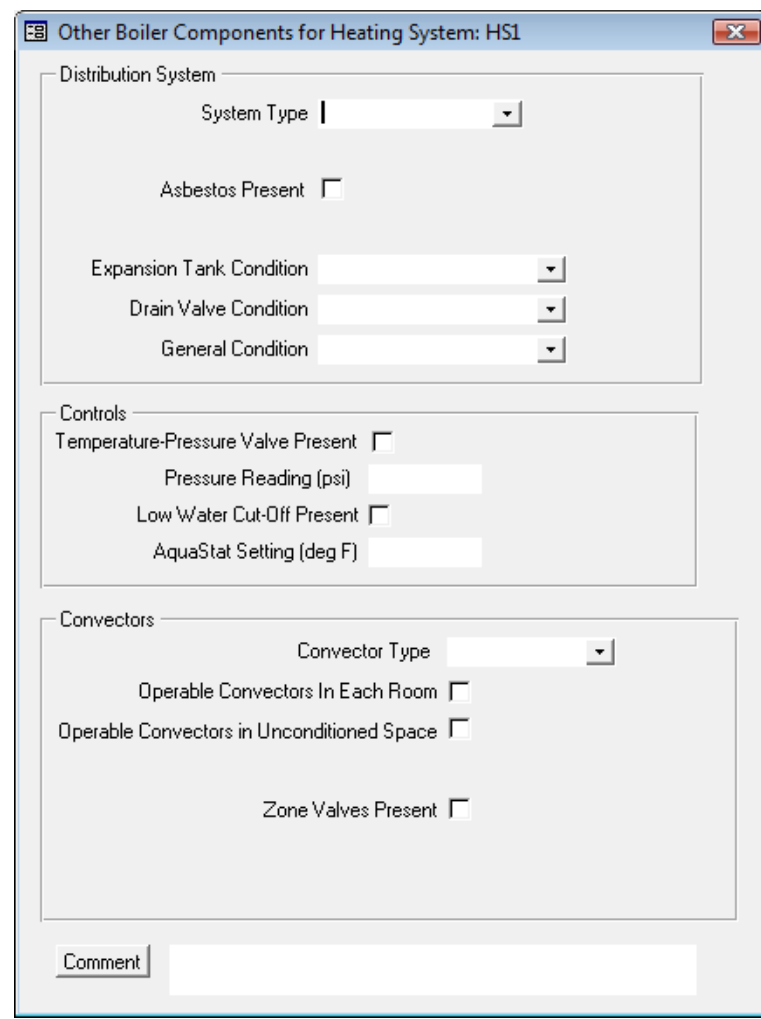

Figure 11.35. The Other Boiler Components for Heating System sub-form (NEAT only).

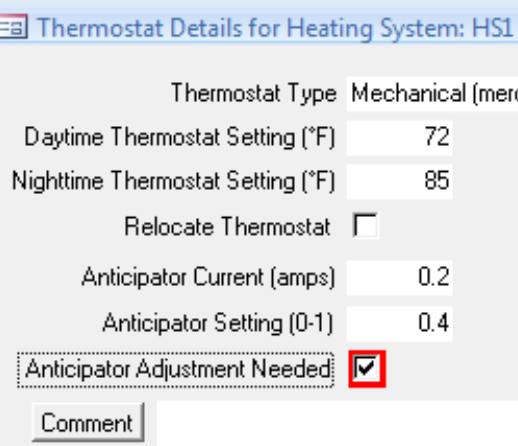

Figure 11.37. The Thermostat Details for Heating System sub-form. 


\subsection{Optional Water Heater Details}

The Optional Water Heater

Details buttons on the Water

Heating form under the Main

Menu's "NEAT Audit” or

"MHEA Audit" buttons (see

Figure 11.38) can be used to document an extensive number of water heater measurements and observations made while auditing a dwelling. The measurements and observations are entered on the sub-forms accessed from these buttons. Some of these data items help to identify potential health and safety hazards. You must complete the description of the water heater before accessing these optional sub-forms. Not all buttons will be applicable to the water heater you describe on the Water Heating form. No attempt is made in this manual to describe correct procedures for obtaining the measurements or performing the inspections. You should refer to literature on standard practices or consult with a qualified contractor. The information entered on the sub-forms is only for documentation and not used anywhere else in the Weatherization Assistant.

Below are brief descriptions of the Optional Water Heater Details buttons found on the Water Heating form. Each form allows entry of an extended comment if necessary. Each sub-form must be closed before any other sub-form can be accessed. Close the forms using the customary Windows $\mathrm{X}$ box in the upper right corner.

\section{Operational Tests}

Selecting the "Operational Tests" button displays the Operational Tests for Water Heater sub-form (see Figure 11.39), which allows recording information about the operation of the water heater. Two groups of measurements can be recorded: flue gas analysis and carbon monoxide measurements. For each item in these groups, pre-retrofit (i.e., during the "Audit”) and post-retrofit (i.e., during the "Inspection") measurements can be recorded. Optional.

\section{Vent Tests}

Selecting the "Vent Tests" button displays the Venting Tests for Water Heater subform (see Figure 11.40), which allows recording information about the 


\section{Chapter 11: NEAT and MHEA Common Elements}

characteristics of the flue and damper as well as pre- and post-retrofit draft measurements on the flue associated with the water heater. Any potential safety hazard due to venting related problems may also be noted. See the description under Section 11.7, Health and Safety, on how the Weatherization Assistance handles health and safety hazards. Optional.

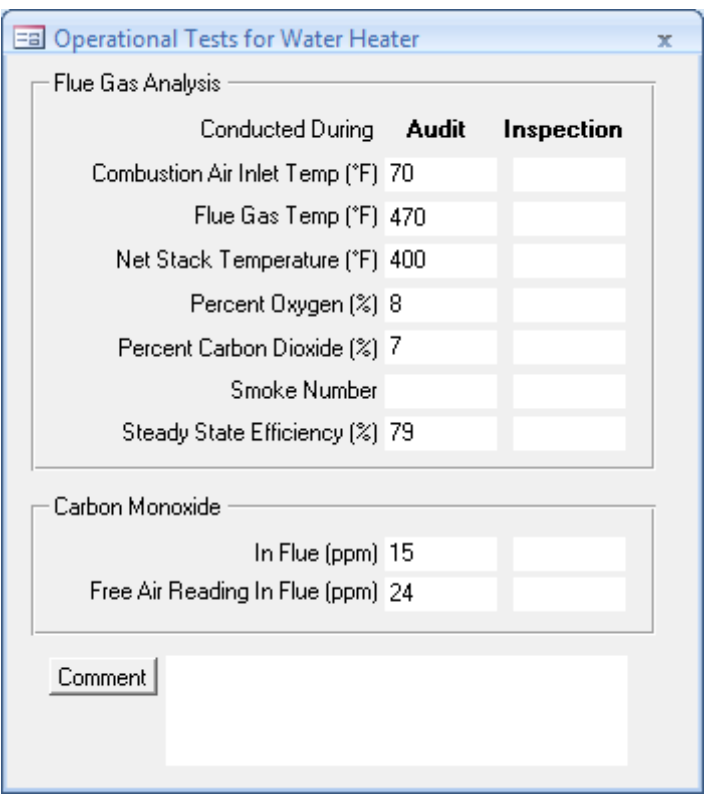

Figure 11.39. The Operational Tests for Water Heater sub-form.

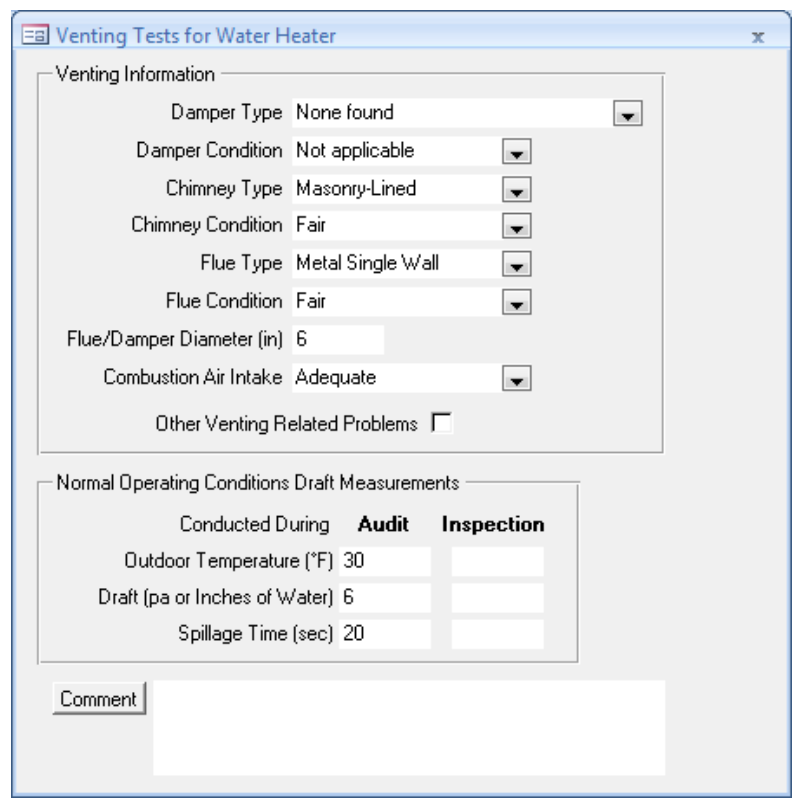

Figure 11.40. The Vent Tests for Water Heater sub-form.

\section{Inspections}

Selecting the "Inspections" button displays the Inspections for Water Heater sub-form (see Figure 11.41), which allows recording information about additional items not addressed otherwise. The checkboxes, if selected, indicate potential safety hazards associated with the heating system. See the description under Section 11.7, Health and Safety, on how the Weatherization Assistance handles health and safety hazards. Optional.

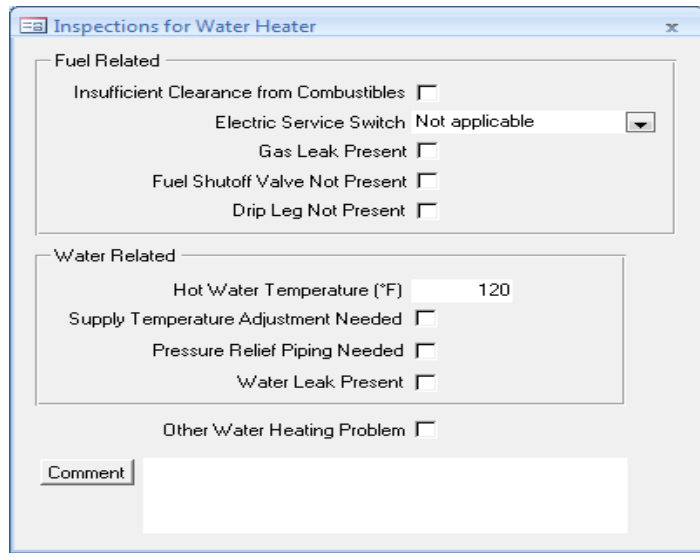

Figure 11.41. The Inspections for Water Heater sub-form. 


\section{Chapter 12. NEAT and MHEA Results}

This chapter describes how to have NEAT and MHEA perform the computations necessary to produce the measure recommendations. The chapter also describes the Recommended Measures report, the primary location for displaying data related to these recommendations and the specific audit from which they were derived. Additional reports are available from the Audit Information tab's Report block. For brief discussions on these other reports, see "Report Block" in Section 8.2, Audit Information. See Appendix C, Reports, for examples of all the reports available in the Weatherization Assistant. For the mechanics involved in viewing and printing reports, see Section 5.12, The Report Block.

\subsection{Running an Audit and Viewing the Results}

After entering all the audit data for a house on the forms under the Main Menu's "NEAT Audit" or "MHEA Audit" buttons, select the "Run Audit" button found on the upper right corner of each form to run the audit. The Weatherization Assistant will examine your input to see that all necessary information is available. Although the programs perform checks on each form's content every time you exit a form, they do not know until you request execution whether all the forms with required information have been visited. If a required form has not been entered, a warning box will appear, indicating the form that contains the required data and its need of being visited. Running a NEAT audit requires that at least the information on the Audit Information, Shell-Walls, Heating, and Ducts/Infiltration forms be provided. Running a MHEA audit requires that the information on the Audit Information, all Shell components, Heating - Primary, and Ducts/Infiltration forms be provided.

Provided all necessary data is available, selecting the "Run Audit" button will change your mouse pointer to its "Busy" form while the calculations are being performed. You will also see the word "Calculating" in the status bar at the bottom of the window. Wait for the operations to be completed before attempting further interaction with the Weatherization Assistant.

Depending on your settings in Preferences (see "Run Control" under Section 16.1, General), either the Measures form under the Main Menu's "NEAT Audit" or "MHEA Audit" buttons or the Recommended Measures report will be displayed in a separate window. The "Last Run On field" below the "Run Audit" button will also be updated with the current time stamp. If you are not presented with the 


\section{Chapter 12: NEAT and MHEA Results}

Recommended Measures report after execution, you may view it using the Report block under the Audit Information form. See Section 5.12, The Report Block, for information about viewing the report.

\subsection{Recommended Measures Report}

The information available on the Recommended Measures report is divided into sections. By default, all sections are displayed. You may choose which sections are displayed using the Report Sections form under the Main Menu's "Preferences" button (see Section 16.3, Report Sections). All sections of the Recommended Measures report will be discussed individually below with descriptions of the data each displays.

\section{The Introductory Section}

Regardless of your settings under Preferences, the report will always include an introductory section identifying at least your Agency, the Client ID, Audit Name, the Weather File used, and the Setup Library Name associated with the audit - all information provided on the Audit Information tab (see Section 8.2, Audit Information). If you made entries in the Comment or Auditor fields on this form, they will also be displayed here. Figure 12.1 is an example introductory section of the Recommended Measures report for a MHEA audit.

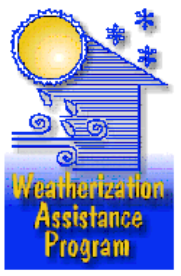

\section{MHEA Recommended Measures}

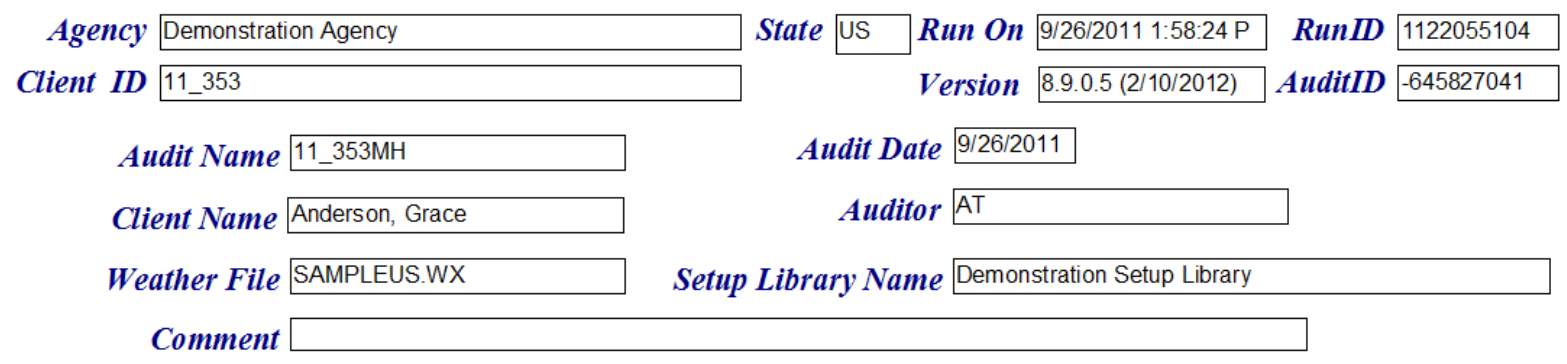

Figure 12.1. The Introductory section of the Recommended Measures report. 


\section{Chapter 12: NEAT and MHEA Results}

If you provided a client name under the Contacts tab of the Main Menu's "Clients" button (see Section 7.4, Contacts (Client)), it will be displayed in this introductory section. Other fields in the section give the date of the audit and the version of the Weatherization Assistant that created the recommendations. The RunID and AuditID fields uniquely identify where the recommendations on the report came from. All audits (i.e., complete building descriptions) have unique Audit IDs. If an audit is copied, it will be assigned a different Audit ID from its parent audit. However, a given audit may be executed any number of times using the "Run Audit" button. Each time an audit is executed, even though it retains the same Audit ID number, it will be given a different Run ID number.

\section{Annual Energy and Cost Savings}

The Annual Energy and Cost Savings section of the Recommended Measures report lists the individual measure recommendations generated by NEAT or MHEA that reflect the input you provided and displays the savings data associated with each (see Figure 12.2). Each recommendation is identified by its name, as given on the Library Measures form under the Main Menu's "Setup Library" button (see Section 14.5, Library Measures). If the audit producing the report included a user-defined measure with energy savings that is seen as cost-effective, it will appear in the list with the name you gave the measure on the Itemized Costs form (see Section 11.8, Itemized Costs). MHEA may append to a measure name additional information associated with the measure. For example, the Lighting retrofit will display what room the auditor indicated the lights were in and how many lights met that particular description (e.g., “[Dining][4]”). Activities having no savings, such as health and safety measures or repair costs, will not appear in this section of the report.

\section{Annual Energy and Cost Savings}

\begin{tabular}{|c|c|c|c|c|c|c|c|c|c|}
\hline \multirow[b]{2}{*}{$\#$} & \multirow{2}{*}{$\begin{array}{l}\text { Recommended } \\
\text { Measure }\end{array}$} & \multirow[b]{2}{*}{ Components } & \multicolumn{2}{|c|}{ Heating } & \multicolumn{2}{|c|}{ Cooling } & \multicolumn{2}{|c|}{ BaseLoad } & \multirow{2}{*}{$\begin{array}{r}\text { Total } \\
(M M B t u)\end{array}$} \\
\hline & & & (MMBtu) & (\$) & $(\mathrm{kWh})$ & (\$) & $(k W h)$ & (\$) & \\
\hline 1 & General Air Sealing & & 9.3 & 155 & 1 & 0 & 0 & 0 & $\overline{9.3}$ \\
\hline 2 & Setback [heating] & & 3.2 & 53 & 0 & 0 & 0 & 0 & 3.2 \\
\hline 3 & Roof Cellulose Loose & & 15.3 & 255 & 388 & 27 & 0 & 0 & 16.6 \\
\hline 4 & DWH Pipe Insulation & & 0.0 & 0 & 0 & 0 & 223 & 8 & 0.8 \\
\hline 5 & DWH Tank Insulation & & 0.0 & 0 & 0 & 0 & 531 & 18 & 1.8 \\
\hline 6 & Belly Fiberglass Loose & & 4.6 & 76 & -2 & 0 & 0 & 0 & 4.6 \\
\hline 7 & Refrigerator Replacement & & 0.0 & 0 & 0 & 0 & 922 & 65 & 3.1 \\
\hline
\end{tabular}

Figure 12.2. The Annual Energy and Cost Savings section of the Recommended Measures report. 


\section{Chapter 12: NEAT and MHEA Results}

The order of measures in the list will be as follows:

1. Those measures assumed to be cost-effective outside of NEAT and MHEA, that is, infiltration reduction and duct sealing. These measures will appear on the report only if sufficient information has been given in the audit to determine their energy savings (see Section 11.2, Ducts/Infiltration - Air and Duct Leakages).

2. Measures declared mandatory (such as the replacement of an unsafe furnace) that are to be included in determining the single SIR for all work performed on the home (the "package" SIR). These could include measures you may have indicated as having already been performed (such as a heating system tune-up).

3. Measures not declared mandatory whose recommendation depends on their cost-effectiveness, after all interactions with other measures have been considered. These measures will be listed in order of their cost-effectiveness (as determined by their Measure SIR, displayed in the succeeding report section). Only measures with SIR equal to or greater than the cut-off value selected in Key Parameters of the Setup Library (see Section 14.2, Key Parameters), normally 1.0, will be displayed.

4. Measures declared mandatory but which are not to be included in determining the package SIR, normally either health and safety measures, if they have energy savings, or measures not funded by the Weatherization Assistance Program.

Following the measure name, but in a separate column, the component codes of those components to which the measure is to be applied will appear. For example, one recommendation might be to install "Wall Insulation" on walls "WL1," "WL2," "WL3," and "WL4." See the building description inputs discussed in Chapters 9 and 10 for information on component codes.

For each measure recommended, the annual energy and dollar savings associated with the measure are given. These savings parameters will be divided into four categories, Heating, Cooling, Base Load, and Total for each measure. The heating energy savings is always given in MMBtu (million Btu), while the energy savings for cooling and base load measures (hot water measures, refrigerator replacement, and lighting retrofits) will be in $\mathrm{kWh}$. The savings from any user-defined measures 


\section{Chapter 12: NEAT and MHEA Results}

with energy savings will appear in the Base Load column with energy savings given in $\mathrm{kWh}$, regardless of the fuel they may save. A total energy savings in MMBtu is given in the last column of this report section. This entry may not be of use, particularly if it is a total of contributions in differing units (i.e., MMBtu and $\mathrm{kWh})$.

\section{Energy Saving Measure Economics}

The Energy Saving Measure Economics section of the Recommended Measures report gives individual and cumulative economic parameters for the recommended measures (see Figure 12.3). All of the measures displayed in the Annual Energy and Cost Savings section of the report will appear in this section. However, in addition, this report section will also list activities having no savings, such as health and safety measures and repair costs. The listing will contain the same measures as seen on the Measures tab of the audit from which the report was created (see Section 11.11, Measures (Audit)).

\section{Energy Saving Measure Economics}

\begin{tabular}{|c|c|c|c|c|c|c|c|c|}
\hline \# & $\begin{array}{l}\text { Recommended } \\
\text { Measure }\end{array}$ & Components & $\begin{array}{r}\text { Measure } \\
\text { Savings } \\
(\$ / y r)\end{array}$ & $\begin{array}{r}\text { Measure } \\
\text { Cost } \\
\text { (\$) }\end{array}$ & $\begin{array}{r}\text { Measure } \\
\text { SIR }\end{array}$ & $\begin{array}{r}\text { Cost } \\
\text { (\$) }\end{array}$ & $\begin{array}{c}\text { Cumul } \\
\text { Savings } \\
\text { (\$/yr) }\end{array}$ & ative \\
\hline 1 & Fix roof leak & & 0 & 180 & 0.0 & 180 & 0 & 0.0 \\
\hline 2 & General Air Sealing & & 155 & 250 & 6.0 & 430 & 155 & 3.5 \\
\hline 3 & Setback [heating] & & 53 & 75 & 9.9 & 505 & 208 & 4.4 \\
\hline 4 & Roof Cellulose Loose & & 282 & 701 & 7.0 & 1206 & 490 & 6.0 \\
\hline 5 & DWH Pipe Insulation & & 8 & 15 & 5.5 & 1221 & 498 & 5.9 \\
\hline 6 & DWH Tank Insulation & & 18 & 40 & 4.9 & 1261 & 516 & 5.9 \\
\hline 7 & Belly Fiberglass Loose & & 76 & 555 & 2.4 & 1816 & 592 & 4.9 \\
\hline 8 & Refrigerator Replacement & & 65 & 620 & 1.2 & 2436 & 657 & 3.9 \\
\hline 9 & Fix Wiring Problems (Attic) & & 0 & 120 & 0.0 & 2556 & 657 & 0.0 \\
\hline
\end{tabular}

Figure 12.3. The Energy Saving Measure Economics section of the Recommended Measures report.

For each measure, the following data is listed in this report section:

- Index (\#) - A consecutive line number, possibly used when referencing the measures of the report. The indices could differ from those in the previous section due to the addition of activities having no energy savings. However, they will be the same as those in the audit's Measures tab. 


\section{Chapter 12: NEAT and MHEA Results}

- Recommended Measure - The Measure name, as described in the previous section. The measure names of any itemized costs or health and safety entries will be the same as seen on the Itemized Costs tab of the audit that produced the report. See Section 11.8, Itemized Costs.

- Components - The Component codes, as described in the previous report section.

- Measure Savings - The total dollar savings per year. The value will be the sum of the three dollar savings amounts for each measure, as displayed in the previous section.

- Measure Cost - The dollar cost to install the measure. For library measures, this cost will be computed from cost data contained in the setup library together with the applicable building description data you entered during the audit, including any Additional Costs you specified. For itemized costs or health and safety measures, the measure costs will be taken from the measure descriptions on the Itemized Costs tab of the audit.

- Measure SIR - The Savings-to-Investment Ratio (SIR) for each individual recommended energy conservation measure (computed over the measure's lifetime).

Values in columns under the "Cumulative" designation are such that a value in a given row represents the parameter value that accounts for the measure on that row and all measures in the rows above it.

- Cumulative Cost (\$) - The cumulative cost of the recommended measures. If all measures listed in the Recommended Measures report are installed in a home, the cumulative cost displayed on the last row of the listing will be an estimate of the total cost of weatherizing the home.

- Cumulative Savings(\$/yr) (MHEA Only) - The cumulative dollar savings of the recommended measures. If all measures listed in the Recommended Measures report are installed in a home, the cumulative savings displayed on the last row of the listing will be an estimate of the total annual dollar savings for the home owner. 
- Cumulative SIR - The cumulative SIR of the recommended measures. If all measures listed in the Recommended Measures report are installed in a home, the last non-zero cumulative SIR displayed will be the SIR for all of the work performed on the home - referred to in some documents as the "package" SIR. The " 0.0 " values often seen at the bottom of this column indicate that the measures in such rows are considered health and safety measures that, according to Weatherization Program policy, do not have to be included in the package SIR.

The order of measures in the listing will be the same as in the previous report section but with the addition of those measures having no energy savings (e.g., itemized costs and health and safety measures). These added measures are displayed with " 0 " savings and " 0.0 " SIR values. They fall into two categories: those that are required to affect the "package" SIR (the SIR for all work performed on the home) and those that are not required to affect this parameter.

Within the Weatherization Assistance Program, health and safety measures are permitted to be excluded from affecting the package SIR. Measures not funded by the Program might also be included in this category. All other costs, including repair costs and any administrative costs, should be accounted for in determining the package SIR. The Energy Saving Measure Economics section treats these two categories of measures differently in order to report a package SIR that reflects these policies. Which of the two categories a measure falls into is determined by your selection in the Include in SIR checkbox associated with the measure on the Itemized Costs form where the measure was defined (see Section 11.8, Itemized Costs). If you indicated that a measure should not be included in the package SIR, the measure will be listed at the end of the recommended measure list such that its cost does not affect the entry representing the package SIR (see the description of the Cumulative SIR parameter, above). Its cost will still be added to the cumulative cost so that the last Cumulative Cost entry represents all work performed on a home. If, on the other hand, you have requested that a cost be included in the SIR, the measure will be listed at the top of the measure list and its effect felt in both the cumulative cost and cumulative SIR values further down the listing.

\section{Materials}

This report section lists the major materials and their quantities necessary to install the recommended measures reported in the Energy Saving Measure Economics 


\section{Chapter 12: NEAT and MHEA Results}

report section. Some material descriptions include a material type as well as a name. For example, the material "Wall Insulation" may have type, "Blown Cellulose." In NEAT, if a heating system replacement has been recommended, the corresponding line in the Materials section will give estimates of the pre-retrofit and range of post-retrofit sizes for the equipment. Minor materials, such as fasteners and other hardware, are not listed.

\section{Pre/Post Retrofit Energy and Loads (NEAT)}

NEAT provides estimates of annual loads and energy consumption for both heating and cooling, before and after installing the recommended measures. The loads represent the energy addition or extraction the heating and cooling equipment must deliver over an entire year to keep the conditioned spaces at the specified set-point temperature. The energy is the consumption of the equipment in meeting these loads. The loads and energy differ essentially by a factor of the seasonal efficiency of the equipment. Both the loads and consumption for heating and cooling have units of MMBtu (million Btu) per year.

Also provided are estimates of the peak rate of heating and cooling energy that the equipment must deliver. The "Heat loss/gain" values are these peak rates prior to application of duct loss factors and the "Output required" the peaks with the duct loss factors applied. The units for the Heat loss/gain values are $\mathrm{kBtu} / \mathrm{h}$ for both the heating and cooling entries. For the Output required values, the units are $\mathrm{kBtu} / \mathrm{h}$ for heating and ton for cooling. One ton of cooling is equivalent to $12 \mathrm{kBtu} / \mathrm{h}$. The Output required values are more often referred to as the "size" of the equipment (see "Approximate Component Contributions to Peak HEATING and COOLING Loads (Equipment Sizing)” below).

\section{Pre/Post Retrofit Energy Consumption (MHEA)}

MHEA's Recommended Measures report displays a Pre/Post Retrofit Energy Consumption section, which provides estimates of annual energy consumption for heating, cooling, and base loads before and after installing the recommended measures. The units for the Heating entries are MMBtu (million Btu) and kWh for the Cooling and Base Load entries. The values given for the Base Loads reflect only the appliances described on the Baseloads tab of the audit description (see Sections 11.4 through 11.6). 


\section{Billing Data Adjusted Results}

Pre-retrofit utility billing data you enter into either NEAT or MHEA (see Section 11.9, Utility Bills) allows these programs to show you a comparison of their energy consumption predictions and this billing data (see "Heating and Cooling Energy Consumption Comparisons" below). Then, if you desire, the programs will adjust their measure recommendations and savings to reflect the data.

Results in three of the above sections (Annual Energy and Cost Savings, Energy Saving Measure Economics, and the Materials) are altered whenever you enter this billing data and request that the results be adjusted accordingly - by using the Include Billing Adjustment checkbox on the General Information form (see Section 8.2, Audit Information). If so requested, NEAT and MHEA will then generate these same three sections except with adjusted values. You may choose to have the report include both the unadjusted and the adjusted sections, or, to avoid confusion, only the adjusted if you have made the decision to weatherize according to billing adjusted recommendations (see Section 16.3, Report Sections).

Note, however, the Weatherization Assistant was designed to make its recommendations based on average weather and occupancy characteristics rather than the specific life styles of the occupants. If adjustment is requested, the recommendations will then be specific to the life styles of the occupants during the period in which the billing data were gathered.

\section{Heating and Cooling Energy Consumption Comparisons}

The Heating and Cooling Energy Comparison reports allow you to see a comparison of the annual consumptions predicted by the Weatherization Assistant and the billed consumptions you enter on the Utility Bills form in either NEAT or MHEA. These report sections are available only if the corresponding heating and/or cooling billing data have been previously entered.

The predicted values are based on the building description you have entered and assumptions used in estimating measure savings. They correspond to the same time intervals for which the billing data were taken, allowing a one-to-one comparison. They will be displayed side-by-side with actual values taken from the billing data. If you have entered the value on the Ducts/Infiltration form, NEAT will use the pre-retrofit air leakage data in computing the predicted consumptions. Otherwise, 


\section{Chapter 12: NEAT and MHEA Results}

NEAT will use the post-retrofit air leakage data. In MHEA, if no pre-retrofit air leakage data is available, the program will use the rate corresponding to your selection for Home Leakiness on the Audit Information form.

Also displayed side-by side will be the heating or cooling degree-days assumed by the programs in their computations as well as those you entered with the billing data (if available). Both will be based on the base temperature you indicated during entry of the billing data. Comparison of predicted and actual consumptions and degree-day totals should assist you in determining whether to use the adjusted recommendations based on the billing data entered. Percent differences of the totals of these two quantities are displayed to further aid in the decision. Note, however, NEAT and MHEA were designed to make their recommendations based on average weather and occupancy characteristics rather than the specific life styles of the occupants. Thus, the programs should not be expected to reproduce the billing data with great accuracy.

\section{Approximate Component Contributions to Peak HEATING and COOLING Loads (Equipment Sizing)}

The size of a home's heating equipment should correspond to the peak heat loss of the house, normally expressed in thousand Btu per hour $(\mathrm{kBtu} / \mathrm{h})$. Peak heat gain, including solar, can also be expressed in $\mathrm{kBtu} / \mathrm{h}$ or in tons (each ton being equal to $12 \mathrm{kBtu} / \mathrm{h}$ ). NEAT estimates these sizes both before and after weatherization using the building description information you provide and formulas taken with permission from tables published by the Air Conditioning Contractors of America (ACCA) in their Seventh Edition (1986) of Manual J, Load Calculation for Residential Winter and Summer Air Conditioning. MHEA's peak heating load estimates are not based on published values. The program uses the same building component characteristics used in estimating the annual heating consumption and recommended measure savings. MHEA does not provide peak cooling load estimates.

You should verify NEAT and MHEA's sizing estimates with the results of an actual equipment sizing procedure for the types of building components common in your area before regularly using them to guide the purchase of new replacement equipment. The sizing estimates calculated by NEAT may differ from those of other techniques designed specifically to size equipment in several ways: 


\section{Chapter 12: NEAT and MHEA Results}

1. the range of building components available in NEAT may not be as extensive as the range in other equipment sizing techniques;

2. NEAT performs the estimate on the building as a whole, not by zones or even room by room;

3. generic duct-loss factors are used in NEAT, whereas other techniques may use unique duct loss factors for each zone; and

4. the methods of computing specific component contributions may have changed since NEAT's component contributions were developed.

MHEA's assumptions of building component characteristics may also vary from those used in techniques specifically designed to size HVAC equipment.

The Approximate Component Contributions to Peak Heating/Cooling Load sections of the Recommended Measures report will list each building component's contribution to the total design heat loss or gain, in units of Btu/h. Also listed for each component is a size parameter (e.g., area, volume, or a number). You can compare these individual component contributions with those from other sizing procedures for an indication of variations in the procedures.

NEAT and MHEA convert the total building heat loss/gain to a required heating/cooling system output by multiplying the former by a duct-loss factor. The programs do not have sufficient information to accurately predict this factor. Standard sizing techniques recommend computing the building heat loss/gain zone-by-zone, then assigning a specific duct-loss factor for the duct supplying air to each zone.

NEAT assumes no duct loss if the heating system is either a space heater or a portable electric resistance heater. Otherwise, it uses a factor of 1.15, assuming that either exposed duct has been insulated by the weatherization of the house or the duct is not in an unconditioned space. Pre-retrofit duct-loss factors of 1.20 are assigned to systems having more than ten feet of uninsulated duct in an unconditioned space. MHEA's duct-loss factors range from 1.0 to 1.20 depending on the winter design temperature and whether or not the ducts are insulated. 


\section{Chapter 12: NEAT and MHEA Results}

These duct-loss factors may be inappropriate for hot water or steam boilers, which may have lower or higher heat loss through their pipes than forced-air systems have through ducts. You may be able to assign a more accurate duct-loss (or "pipe loss") factor based upon your examination of a home after weatherization.

For example, a hydronic heating system with insulated pipes or a forced-air system with insulated ducts may merit a lower "duct-loss" factor. A steam system with uninsulated pipes may merit a higher "duct-loss" factor. You can estimate the heating system output required by multiplying this factor by the building heat loss reported on the Sizing report.

If a heating system replacement is recommended, NEAT's material list (see the section "Materials" above) will give both a pre- and a post-retrofit equipment size. The pre-retrofit size reported is taken from your input of Capacity on the Heating Systems form, not NEAT's sizing estimate. You may wish to compare your entered value with the estimate reported here in the Component Contributions section. The minimum post-retrofit equipment size reported in the Materials List is NEAT's estimate as described in this section.

\section{Special Notes}

This report section displays notes generated internally by the audit as it executes. They should be reviewed to see if any may alter your retrofit decisions. It is in this report section that MHEA will indicate its determination that the HVAC capacities you have entered for the equipment are insufficient to heat or cool a home. See the Capacity field descriptions for MHEA's Heating and Cooling equipment in Sections 10.7 and 10.8 . The note will have the form, "Pre/Post-weatherization heating/cooling load may not be met in all months," then give you the number of the months (e.g., "12, 1," indicating December and January). This situation can affect the savings and recommendations of measures.

\section{Comments}

The Comments section contains all comments you may have entered during the building description input. Each is associated with both a component type and the specific component code you chose for the component, if applicable. 


\section{Retrofit Measures Not Considered}

This report section lists all of the library measures that have chosen to not be considered in the audit having produced the report, as taken from the Library Measures tab of the Setup Library accessed by the audit (see the Active field description in Section 14.5, Library Measures). 


\section{Chapter 13. Work Orders}

The Weatherization Assistant's optional Work Order feature allows you to prepare detailed listings of tasks to be performed as part of the weatherization of a house. A work order can include as much material and costing detail as you wish to specify. You may create as many work orders for a client as is necessary. Tasks assigned to work orders can be developed from the NEAT or MHEA audits or a listing of measures you have previously defined in the Setup Library (as perhaps from a priority list), or be described individually on the work order itself. The status of individual work orders can be tracked. Work orders also allow you to track inventory and costs by cost center (funding source). An alternate use of the work order feature is to document, to any detail desired, exactly what was done to a client's home.

Before you can create a work order, you must first have a client defined under the Main Menu's "Clients” button on whose dwelling the work is to be performed (see Chapter 7, Clients). You may initiate a new work order in any of three ways:

1. If you have already run an audit for a client, select the "Create Work Order(s)" button on the Measures form in either the "NEAT Audit" or "MHEA Audit" windows (see Section 11.11, Measures (Audit)) - This will automatically assign the work order(s) to the Client for whom the audit was run and populate them with the measure recommendations from the audit. If you have assigned contractors to the measures on the Measures form, separate work orders will be created for each contractor with the contractor information included in each.

2. Select the "Create New Work Order..." button on the Work Orders form under the Main Menu's “Clients” button (see Section 7.6, Work Orders (Client)) This will automatically assign a new work order to the current client, but have no measures associated with it.

3. Use the new record button (-.) or "Copy" button on the Work Order Information form under the Main Menu's "Work Orders" button - This will initiate a new work order and assign it, by default, to the same client assigned to the work order displayed when the new record button was selected. This default assignment can be easily changed using the "Client ID" drop-down list on the form. 


\section{Chapter 13: Work Orders}

The remainder of this chapter is divided into sections describing the data and display items entered on each of the forms seen under the Main Menu's "Work Orders” button: Work Order Information, Status, Measures, and Photos (if the photo browser tab method for attaching photos to records has been activated in the Main Menu's “Preferences” button as described in Section 16.4, Features). See Appendix C, Reports, for an example of an actual work order generated from information entered under Main Menu’s “Work Orders” button.

\subsection{Work Order Information}

The Work Order Information form under the Main Menu's “Work Orders” button (see Figure 13.1) is used to enter general information about the work order, to identify the client and contractor, and to specify a supply library from which inventoried items can be selected. It also allows you to view and navigate to other work orders in your database and access work order reports for viewing and printing, including the actual work orders.

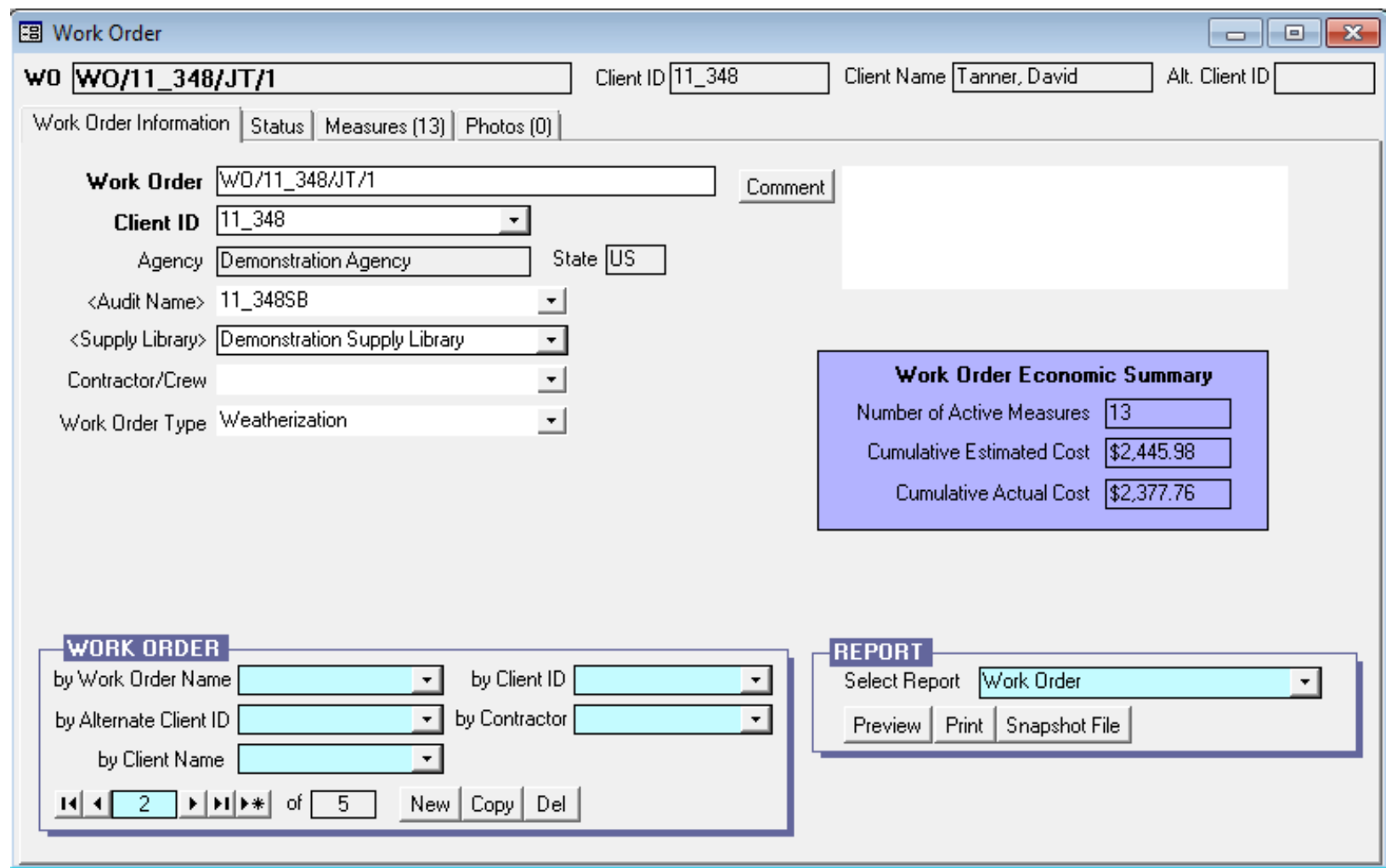

Figure 13.1. The Work Order Information form under the Main Menu’s “Work Orders” button. 
Below are brief descriptions of the individual data items and controls found on the Work Order Information form:

- Work Order - This field contains a unique name to identify the current work order. You may develop your own naming convention or use the default names. If the work order is initiated from under the Main Menu's "Clients," "NEAT Audit," or "MHEA Audit" buttons, the default work order name will have the form "WO/<Client ID $>/<$ Contractor ID $>/ \#$." The $<$ Client ID $>$ is the Client ID entered on the Client Information form of the Main Menu's “Clients” button. The $<$ Contractor ID $>$ is the User Name, if entered and designated as a Contractor or Crew, on the Contacts form of the Main Menu's "Agency" button. The "\#” is an integer assigned to ensure uniqueness of the name, since there may be multiple work orders for the same client and assigned to the same contractor. If the work order is initiated from this, the Work Order Information form, the default work order name will be simply "Work Order (\#)," where the \# is an integer insuring uniqueness of the name. In such cases, it is advised that you replace this name with a more descriptive name. The Work Order name is printed on the work order. Required.

- Client ID - This field identifies the Client for whom the work is being performed. The entry will likely be supplied for you as the program anticipates the assignment. If incorrect, use the drop-down list to select the correct Client. Client and Client Contact information are printed on the work order. Required.

- Agency/State - These fields will automatically be set to correspond to the agency performing the work for the client identified in the Client ID field above. Changing the Client ID will immediately update the Agency Name and State, if need be. Agency Information will be printed on the work order. Uneditable.

- Audit Name - If the work order was initiated by selecting the "Create Work Order(s)" button on the Measures form in either the "NEAT Audit" or "MHEA Audit” windows (see Section 11.11, Measures (Audit)), this field will display the name of the audit from which the work order was generated. Otherwise the field will be blank. If an audit name is displayed, the audit may be accessed simply by double clicking on the name. The drop-down list will show any audits assigned to the client identified in the Client ID field above. Note that it would be an unusual occurrence to change the audit to which a work order was 
associated or even to have more than one audit associated with a given client. If supplied, the Audit Name will be printed on the work order. Optional.

- Supply Library - This field identifies the Supply Library (see Chapter 15, Supply Library) containing the inventory of materials from which any materials used by the work order may be taken. In addition, if you have specified costs for materials in the Supply Library, reference to these materials in the work order will automatically use these costs. Only supply libraries associated with the agency specified in the Agency field on the form will be visible in the dropdown list, though most agencies would likely have only one supply library. If the work order was created from an audit or copied from an existing work order, this field will be set initially to the same supply library assigned to the record from which the work order was created. Required.

- Contractor/Crew - The Contractor/Crew field identifies the contractor assigned to perform the work described by the work order. Only those Contacts defined as Contractors or Crew for the Agency indicated in the Agency field on the Work Order Information form will be displayed in the drop-down list. If the work order was initiated from the Measures form in either the "NEAT Audit" or "MHEA Audit" windows, the entry will comply with your settings on that form. If the Contractor/Crew is specified, the Contractor Company, Address, and Contractor Contact Name as well as phone numbers and e-mail address will be printed on the Work Order. Optional.

- Work Order Type - Choose the type that best describes this work order. The choices are Weatherization, Re-Weatherization, Emergency Repair or Replacement, Response to Client Request, and Other. The Work Order Type is printed on the work order, if available. Optional.

- Comment - You may enter comments pertinent to the entire work order directly in the Comment field on the form, or you may enter them in the Comment Editor by selecting the "Comment" button to the left of the field. The Comment Editor is similar to Window's Notepad and may be used to enter extended comments. Text from other sources may be copied and pasted into the Comment Editor's window. Unique to the comment fields on any form within the "Work Order" window is the ability to import comments from the audit you have specified in the Audit Name field above by selecting the "Use an Audit Comment” drop-down list displayed at the bottom of the Comment Editor. This 


\section{Chapter 13: Work Orders}

drop-down list includes references to all comments entered on all forms of the audit. You can import multiple comments by selecting items one-by-one. The total comment may have up to 65,000 characters. Any comment that is entered in this comment field will be displayed in the header section of the work order. Optional.

- Work Order Economic Summary Block - This block displays three fields:

1. The Number of Active Measures assigned to the work order. A work order description under the "Work Order" window may contain more measures than have been declared "Active." Measures are declared "Active” under the Measures tab on the form corresponding to each individual measure (see “Active” in Section 13.3, Measures (Work Order)). Only active measures are printed in the work order. Note that a single measure may be composed of many components (see "The Materials/Labor Details Sub-Form” in Section 13.3). When a measure is indicated as not being active, all of its components are excluded from the work order when it is printed. Uneditable.

2. The Cumulative Estimated Cost of all active measures assigned to the work order. This value is the sum of the "Estimated" costs for all active measures, as displayed on the individual measure forms. See "Estimated/Actual Cost and SIR" in Section 13.3. Uneditable.

3. The Cumulative Actual Cost of all active measures assigned to the work order. This value is the sum of the "Actual" costs for all active measures, as displayed on the individual measure forms. See "Estimated/Actual Cost and SIR" in Section 13.3. A measure for which an active cost has been declared will still have an estimated cost as well. Both costs will contribute to the appropriate cumulative cost displayed in this block. Uneditable.

- Work Order Record Navigation Block - The Work Order record navigation block is used to find and navigate to existing work orders, copy or delete the currently accessed work order, or create a new work order. See Section 5.1, Form and Record Navigation, for information on using the Weatherization Assistant's record navigation blocks. If you use the "Copy" button to copy the current work order, the program will ask you if you wish to copy all of the measures as well. A "Yes" response will copy the measures and all associated 
data (e.g., costs and quantities). A "No" response will copy only the information on the Work Order Information form. Whether you use the "New" or "Copy" button, the resulting work order will be assigned a default Work Order name that you can modify.

The default installation of the Weatherization Assistant turns on the "Use only the latest bookmarked Agency in the find record drop-down boxes" feature in Preferences (see Feature 9 in Section 16.4, Features). With this feature turned on, the drop-down lists in the Work Order record navigation block will display only the work orders associated with the agency last visited using the Main Menu's “Agency” button. Normally, this will be your agency, so the drop-down lists will display only your agency's work orders. If you have more than one agency defined in your database and you want the drop-down lists to display work orders from all defined agencies, turn this feature off.

- Report Block - The Report block provides you with access to all reports available under the Main Menu's “Work Orders” button. See Appendix C, Reports, for examples of these reports. The following reports are currently available:

1. "Work Order" - The standard work order containing estimated costs for the measures.

2. "Work Order (Bid Form)" - The work order with costs unspecified, to solicit bids from the contractor on the costs for the measures).

3. "Work Order (Bid Form with Only Client ID)" - The same as the Bid Form above except using only the Client ID to identify the client in order to protect the privacy of the client.

See Section 5.12, The Report Block, for information on the mechanics of using the Weatherization Assistant's Report blocks. Appendix D, Development of Customized Reports, describes a method to create reports tailored to your needs and to list the reports in the Report block. 


\section{Photographic Data Items}

If you have chosen to use the third party photo browser method for attaching photos to records under the Main Menu's "Preferences” button (see Section 16.4, Features), a Photo Folder field, "Browse" button, and "View" button will be present on the Work Order Information form below the Comment field. Use the "Browse" button to select a folder on your computer in which you have stored digital photos for this specific work order. Once selected, the location of this folder will be displayed in the Photo Folder field. Select the "View" button to obtain a photo browser in which you may view and edit these photos. The Weatherization Assistant provides the option of attaching photos not only to work orders, but also to audits and clients as well. Therefore, you should develop a consistent policy regarding where and how you store reference to your photos. See Section 18.1, Digital Photos, for more information on viewing and editing photos.

\subsection{Status (Work Order)}

The setting and tracking of statuses is an optional feature of the Weatherization Assistant. The Status form under the Main Menu's “Work Orders" button (see Figure 13.2) allows you to view the current status settings associated with the current work order for three work order status categories: General, Inspection, and Payment. The current status will be shown for only those categories for which a status has been set or for which a setting is possible. Below are brief descriptions of the individual data items and controls found on the Status form.

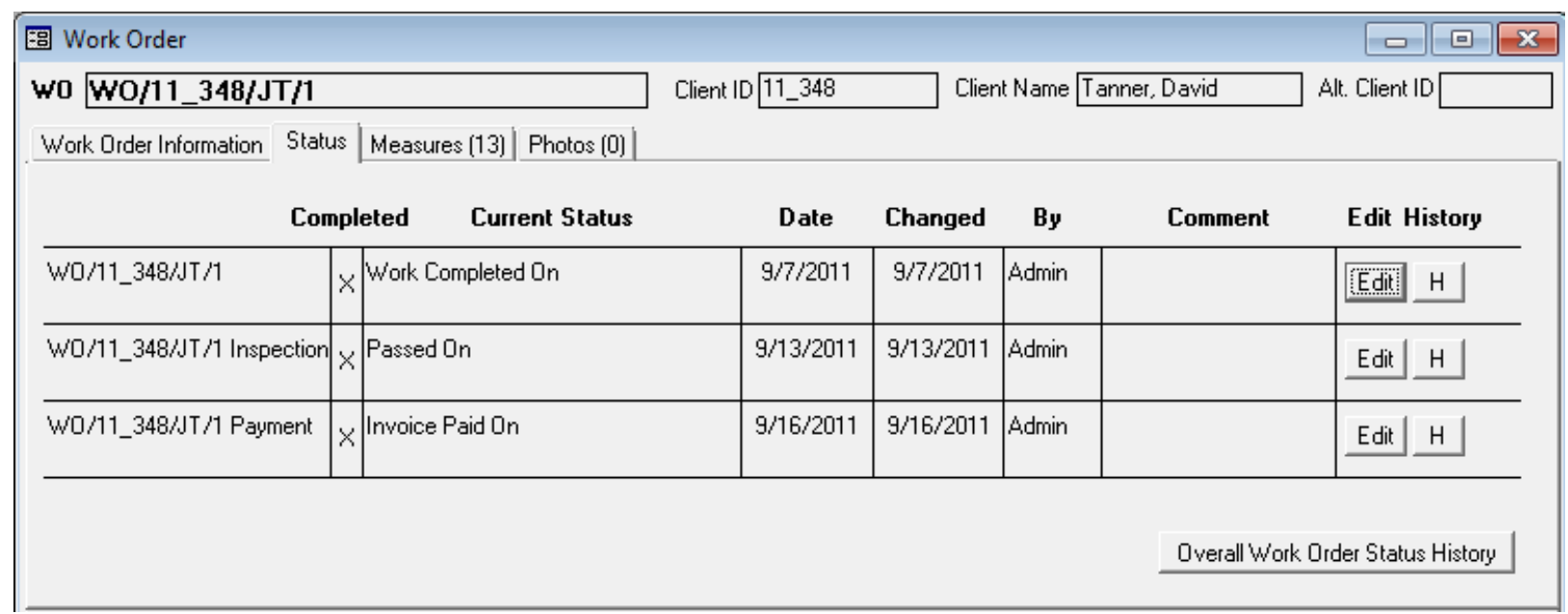

Figure 13.2. The Status form under the Work Order main Menu Item. 
- WO/ $/<$ Record Name $>/<$ Work Order Status Category $>-$ The left most column of the form displays the work order status category and the name of the work order for which the status is being reported.

- Current Status - This field displays the current status setting for the given work order status category. The possible settings for each work order category are listed in Section 18.3, Status Tracking.

- Completed - An "X" will be displayed in this field when the current status setting signifies completion or closure of the activity associated with the work order status category. Settings that represent completion are indicated by an asterisk (*) in Section 18.3, Status Tracking.

- Date - This field displays the date that the current status became effective.

- Changed - This field displays the date that the current status was last changed.

- By - This field displays the User Name of the person who changed the status. The names are restricted to those that have been entered on the Contacts form under the Main Menu's “Agency” button and have a password assigned, as discussed in Section 6.2, Contacts (Agency). A User Name of "Admin” implies that a specific person was not identified as making the change.

- Comment - Any comment that has been appended to this current status setting will be shown in this field.

- Edit - Status settings are changed by selecting the "Edit" button in the row of the work order category you wish to change. Use of the Status Editor form that appears is explained in Section 18.3, Status Tracking. The status of a work order may also be changed on the Status tab of the Main Menu's "Client" button (see Section 7.2 Status (Clients)).

- History - Select the " $H$ " button to see a history of all settings for a specific work order status category for the current work order. The Status History form that appears is the same in appearance as that discussed in Section 6.9, Status History. 


\section{Chapter 13: Work Orders}

- Overall Work Order Status History - Select the "Overall Work Order Status History" button to display a listing of all status entries for all work order categories associated with the current work order. The Status History form that appears is the same in appearance as that discussed in Section 6.9, Status History. You can use of the sort feature (see Section 5.8, Data Sheet and Form Views of Records) while viewing this form to arrange the status settings in a desired order (e.g., chronologically).

\subsection{Measures (Work Order)}

The Measures form under the Main Menu's “Work Orders” button (see Figure 13.3) is used to develop detailed task descriptions for weatherization measures to be installed in a home. If a work order is initiated by executing a NEAT or MHEA audit, this form will automatically have records corresponding to the recommended measures that you have selected on the Measures form in either the "NEAT Audit" or "MHEA Audit" windows (see Section 11.11, Measures (Audit)). If a work order is initiated from the Work Orders form of the Main Menu's "Clients" button or the Work Order Information form of the Main Menu's "Work Orders” button, the Measures form will have no measure records.

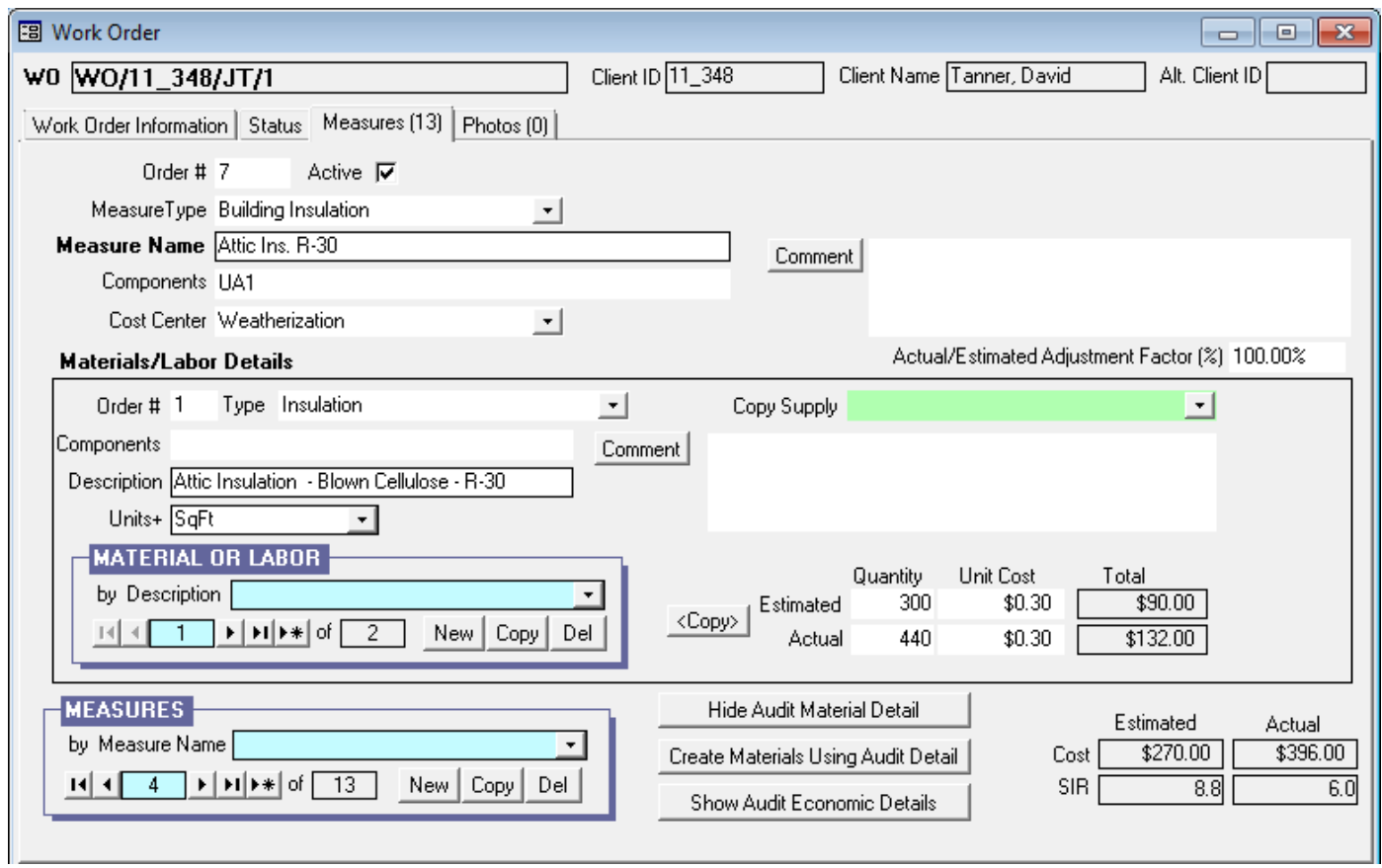

Figure 13.3. The Measures form under the Main Menu’s “Work Orders” button (form view). 


\section{Chapter 13: Work Orders}

The Measures form is divided into three sections: a general description of measures at the top of the form, a Materials/Labor Details sub-form in the middle of the form, and the Measures record navigation block, three buttons for accessing details, and a measure summary block at the bottom of the form. Always enter information on the general description of the measure first before describing materials and labor components on the Materials/Labor Details sub-form. The Materials/Labor Details sub-form may contain multiple records associated with the current measure. This sub-form will be described under its own topic, as will the sub-form accessed by selecting the "Show Audit Economic Details" button. Below are descriptions of the individual data items and controls found in the Measures form.

- Order \# - Enter an integer to prescribe the order in which you want the measures to be listed in the Measures record navigation block (see "Measures Record Navigation Block" below) and in the work order (see "Report Block" in Section 13.1, Work Order Information). All measures not assigned unique order numbers will be listed first in random order in the work order. Optional.

- Active - Use this checkbox to include the measure in the work order. If unchecked, it is recommended you include a comment indicating the reason for not including the measure in the work order.

- Measure Type - Select the type for the measure using the drop-down list. The choices are Base Loads, Building Insulation, Client Education, Doors and Windows, General Heat Waste and Air Infiltration, General Repairs, Health and Safety, HVAC Systems, and Other. A measure generated by a NEAT or MHEA audit, which corresponds to a library measure, will automatically be assigned the measure type as prescribed on the Library Measures form of the Main Menu's “Setup Library” button (see "Measure Type” in Section 14.5, Library Measures). The measure type does not appear on any report. However, it can be used to sort the measure list when the Measures form is viewed in the data sheet view. Optional.

- Measure Name - Enter the name of the measure to identify it in the work order. Any measure generated by a NEAT or MHEA audit (i.e., corresponding to a library measure, an itemized cost, or a health and safety measure) will automatically be assigned the measure name listed on the Measures form of the 


\section{Chapter 13: Work Orders}

Main Menu's "NEAT Audit” or "MHEA Audit” buttons (see "Measure Name” in Section 11.11, Measures (Audit)). Required.

- Components - List the identifying name or code of all the building components to which the measure will be applied. A library measure generated by a NEAT or MHEA audit will automatically be assigned the component codes entered on the respective forms under the Main Menu's "NEAT Audit" or "MHEA Audit" buttons. (See the building description inputs discussed in Chapters 9 and 10 for information on component codes.) The component codes can also be entered here under the Work Orders Measures form without reference to an audit. They may indicate some characteristic of the components or correspond to codes used on a sketch of the house, which could be scanned and attached electronically to the work order (see Section 13.4, Photos (Work Order)). For these codes to be of use to the contractor or crew performing the weatherization, their correlation to the floor plan of the house must somehow be conveyed. The list of component codes may have up to 255 characters, although the space restrictions in work orders suggest a lesser limit in the number of characters (perhaps 15 to 30) to preserve readability. Optional.

- Cost Center - If you want to track the costs incurred by different funding sources or programs, select the cost center (funding source) from the drop-down list to which the actual cost of the measure will be charged. The drop-down list will display the cost centers defined under the Cost Centers form of the Main Menu's “Agency” button (see Section 6.3, Cost Centers) for the agency identified on the Work Order Information form. A measure generated by a NEAT or MHEA audit will automatically be assigned a cost center if you have assigned a default cost center for the measure on the Library Measures or User Defined Measures forms of the Main Menu's “Setup Library” button (see "Default Cost Center" in Sections 14.5, Library Measures, and 14.6, User Defined Measures) or a cost center for the measure on the Measures form of the Main Menu's "NEAT Audit" or "MHEA Audit" buttons (see "Cost Center" in Section 11.11, Measures (Audit)). However, you may alter the cost center on this form using the drop-down list. Optional.

- Copy from Library Measures -If you want to include a measure from the library measures without running a NEAT or MHEA audit, select the "New" button on the Measures record navigation block (see "Measures Record Navigation Block” below). The “Copy from Library Measures” drop-down list 
will appear on the top right of the Measures form. The drop-down list will include the 45 library measures for NEAT or the 49 library measures for MHEA listed on the Library Measures form of the Main Menu's "Setup Library” button (see Section 14.5, Library Measures). You can select a desired measure using the drop-down list.

NOTE: If you include a measure from the library measures that has multiple material descriptions (such as Building Insulation (except Fill Ceiling Cavity), Replace AC, and Lighting Retrofits in NEAT, and Replace DX Cooling Equipment and Lighting Retrofits in MHEA), you will receive a message (see Figure 13.4) indicating that more than three material detail records (i.e., material, labor, and other) were added.

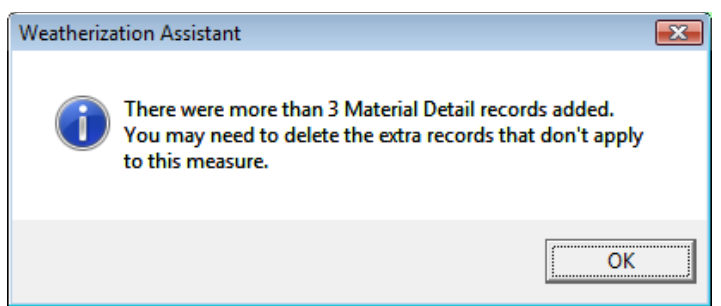

Figure 13.4. Message when copying a measure from Library Measures to a work order.

Delete the extra records in the sub-form that you do not intend to use for this measure.

- Copy from User Defined Measures - If you want to include a measure from the user-defined measures without running a NEAT or MHEA audit, select the "New" button on the Measures record navigation block (see "Measures Record Navigation Block" below). The "Copy from User Defined Measure” drop-down list will appear on the top right of the Measures form. The drop-down list will include those library health and safety measures and the user-defined measures you have described on the User Defined Measures form of the setup library assigned to the client on the Client Information form and for which you have also selected the Active checkbox and the Available for Use in NEAT or MHEA checkboxes on that form (see Section 7.1, Client Information, and Section 14.6, User Defined Measures). Select the desired measure using the drop-down list.

- Comment - You may enter comments pertinent to the measure directly in the Comment field on the form, or you may enter them in the Comment Editor by selecting the "Comment" button to the left of the field. The Comment Editor is similar to Window's Notepad and may be used to enter extended comments. Text from other sources may be copied and pasted into the Comment Editor's window. Unique to the comment fields on any form within the "Work Order" 


\section{Chapter 13: Work Orders}

window is the ability to import comments from the audit you have specified in the Audit Name field above by selecting the "Use an Audit Comment" dropdown list displayed at the bottom of the Comment Editor. This drop-down list includes references to all comments entered on all forms of the audit. You can import multiple comments by selecting items one-by-one. The total comment may have up to 65,000 characters. Any comment that is entered in this comment field will be displayed in the work order immediately under the measure name. Optional.

- Actual/Estimated Adjustment Factor (\%) - Enter an adjustment factor, in percent, if you discover that the actual energy savings from the measure would likely be different from the estimated energy savings calculated by NEAT or MHEA. For example, if you discover that only $80 \%$ of the wall area of your initial input can actually be insulated, you would expect that the actual energy savings from the wall insulation measure might be approximately $80 \%$ of the initial energy saving estimate calculated by NEAT or MHEA. In this case, you would enter 80 in the Actual/Estimated Adjustment Factor field. Such an adjustment would also likely be desired in your conversion of estimated costs to actual costs on the Materials/Labor Details sub-form for the measure (see Quantity / Unit Cost (Actual) entries under "The Materials/Labor Details SubForm” below).

- Measures Record Navigation Block - The Measures record navigation block is used to find and navigate to existing measure descriptions, copy or delete the currently accessed measure description, or create a new measure description for the work order being created. See Section 5.1, Form and Record Navigation, for information on using the Weatherization Assistant's record navigation blocks. If you choose the "New" button, you will be presented with a blank Measures form displaying the "Copy from Library Measures" and "Copy from User Defined Measures” drop-down boxes (see descriptions of these fields above).

- Estimated/Actual Cost and SIR - This block of data, located in the lower right corner of the Measures form, summarizes the total Estimated and Actual Costs and SIRs for the measure. Estimated Costs are those initially coming from whatever source generated the measure, such as the execution of either NEAT or MHEA, although even these may be altered under the Materials/Labor Details sub-form (see below). Actual costs are assigned 
whenever you declare them under the Materials/Labor Details sub-form. Care must be taken in interpreting these "Actual” parameters. If you have only declared part of the costing components as "Actual” under the Materials/Labor Details sub-form (see below), this cost may not reflect the total cost of implementing the measure. The SIR will display " 0.0 ” for measures without energy savings associated with them.

- Show/Hide Audit Material Detail - If a measure was generated by a NEAT or MHEA audit, selection of this button will display the costing components used by the audit (see Figure 13.5), as specified in the Setup Library (see "Costs" in Section 14.5, Library Measures, or "The Materials/Labor Details Sub-Form” in Section 14.6, User Defined Measures).

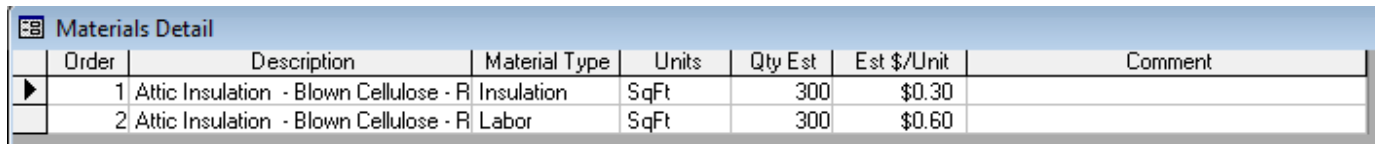

Figure 13.5. The "Materials Detail" sub-form displayed by selecting the "Show Audit Material Detail” button on the Work Order Measures form.

These audit cost components are designed to be approximate costs used by the audits in estimating the cost-effectiveness of the measures for determining the recommendations. However, they may not be as detailed or as precise as you may wish for the purposes of generating work orders for the measures. Nevertheless, you may still wish to refer back to them. If, in initially forming the work orders from audit recommendations, you chose to "Include Details for Materials” (see Section 11.11, Measures (Audit)), these costing components will have already been transferred to the work order. This would make what you view by selecting the "Show Audit Material Detail" button the same as what is displayed in the Materials/Labor Details sub-form, particularly if the latter is viewed in data sheet view. However, if you did not choose to transfer these components or you have since altered the detail in the sub-form, this button still allows you to refer back to what the audits used for costing components in making their recommendations. If the measure was generated by some other means than the audits, no entries will be seen when selecting this button.

It is recommended that prior to selecting the "Show Audit Material Detail" button you maximize the window the Weatherization Assistant program uses. 
Otherwise, the sub-window used to display this added data may fall outside the program window, making it hidden.

Once the "Show Audit Material Detail" button has been selected, the name of the button will change to "Hide Audit Material Detail.” It is recommended that when you no longer require viewing the audit material detail and prior to navigating to another measure, you close the sub-window by selecting the "Hide Audit Material Detail" button instead of using the traditional Windows X box in the upper right corner of the sub-window. This allows the naming of the button to remain synchronized with the display.

- Create Materials Using Audit Detail - This button is used to transfer the audit material detail for the measure being accessed (viewed using the "Show Audit Material Detail" button) to the work order. This would not be necessary if, when generating the work order from the Measures form under the Main Menu's "NEAT Audit” or "MHEA Audit" buttons, you chose "Include Details for Materials" (see Section 11.11, Measures (Audit). The detail would have already been transferred. However, if this was not your choice and you now wish to transfer the information, this button will accomplish the action. Even if you initially chose to transfer the audit data but have since altered it using the Materials/Labor Detail sub-form, you may still go back to the original audit data using this button. However, you will be warned that this transfer will overwrite the existing data for this measure. If the measure was not generated from running either the NEAT or MHEA audits, selecting this button will indicate "No material detail records to add."

- Show Audit Economic Details - Selecting this button will display the Energy Savings for Measure: <Measure Name> sub-form (see Figure 13.6) that allows you to view the energy and economic details about the measure as calculated by NEAT or MHEA. The values for the annual energy and dollar savings and the savings-to-investment ratio (SIR) will be the same as those seen in the Recommended Measures report (see Section 12.2, Recommended Measures Report). If the measure was not produced by running an audit, most of the entries will be zero. The values displayed on this sub-form will be described in the following section, “The Energy Savings for Measure Sub-Form.” 


\section{Chapter 13: Work Orders}

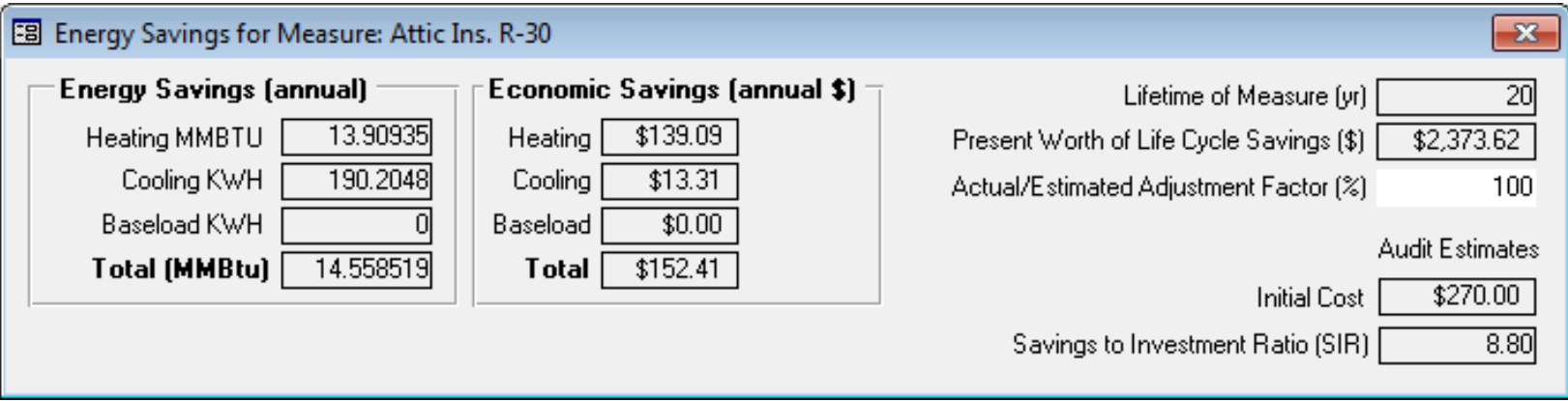

Figure 13.6. The Energy Savings for Measure: <Measure Name $>$ sub-form.

\section{The Energy Savings for Measure Sub-Form}

- Energy Savings - This set of fields shows the annual heating energy savings in units of MMBtu, annual cooling energy savings in units of $\mathrm{kWh}$, and annual base load energy savings in units of kWh estimated by NEAT or MHEA for the measure. The total savings displayed in MMBtu converts the cooling and base load savings from kWh to MMBtu, then adds their sum to the heating savings, already in MMBtu. Uneditable.

- Economics Savings - This set of fields shows the heating, cooling, and base load energy cost savings in units of dollars estimated by NEAT or MHEA for the measure in the first year. Uneditable.

- Lifetime of Measure - This field displays the life of measure, in years, used by NEAT or MHEA. These values are taken from the Library Measures form under the Main Menu’s “Setup Library” button. Uneditable.

- Present Worth of Life Cycle Savings - This field displays the energy savings of the measure in the first year multiplied by the lifetime of the measure, adjusted to account for the fact that future dollar savings are not worth as much as current dollars (i.e., “discounted”). The value also attempts to adjust for forecasted changes in fuel costs. Uneditable.

- Actual/Estimated Adjustment Factor (\%) - This is the only editable field on the Energy Savings for Measure sub-form. It is identical to the entry of the same name that appears on the Measures form (see above). Changing the value here will automatically change the value displayed on the Measures form. Since this parameter only affects the "Actual” savings and SIR, changing it on this 
sub-form will not affect any other entries on the form. It is included here to allow you to make some conclusion about actual energy savings while viewing the savings estimated by the audits. Windows of the program can be arranged such that both this window and the parent Measures form are both visible, in which case you will be able to see the effect of the "Actual/ Estimated Adjustment Factor (\%)" on the Actual SIR viewed on this latter form. Optional.

- Initial Cost - This field displays the cost of the measure, in dollars, used by NEAT or MHEA. Uneditable.

- Savings to Investment Ratio (SIR) - This field displays the SIR of the measure computed by NEAT or MHEA, which is the ratio of the present worth of life cycle savings and the cost of implementing the measure, both displayed on this sub-form immediately above this field. Uneditable.

\section{The Materials/Labor Details Sub-Form}

The Materials/Labor Details sub-form (see Figure 13.7) is used to describe individual materials and labor components for a measure. You may enter as much information as you feel necessary to sufficiently describe implementation of each measure. There must be at least one material/labor record described for a measure in order to have the measure printed on the work order.

A measure generated by a NEAT or MHEA audit will have this information entered automatically, if you have so chosen (see "Include Details for Materials" in Section 11.11, Measures (Audit)). The library measures have three cost components: material, labor, and other, as specified in the Setup Library (see "Costs" in Section 14.5, Library Measures). The user-defined measures and library of health and safety measures may have as many cost components as you feel necessary (see "The Materials/ Labor Details Sub-Form” in Section 14.6, User Defined Measures). This detail is the same data viewed by selecting the "Show Audit Material Detail" button described above, as well as under the "Costs" button for the measure on the Measures form of the audit that generated the work order.

The Materials/Labor Details sub-form is also where you indicate use of materials from your Supply Library (see Chapter 15, Supply Library) which then get subtracted from your inventory once the material costs have been declared "Actual." 


\section{Chapter 13: Work Orders}

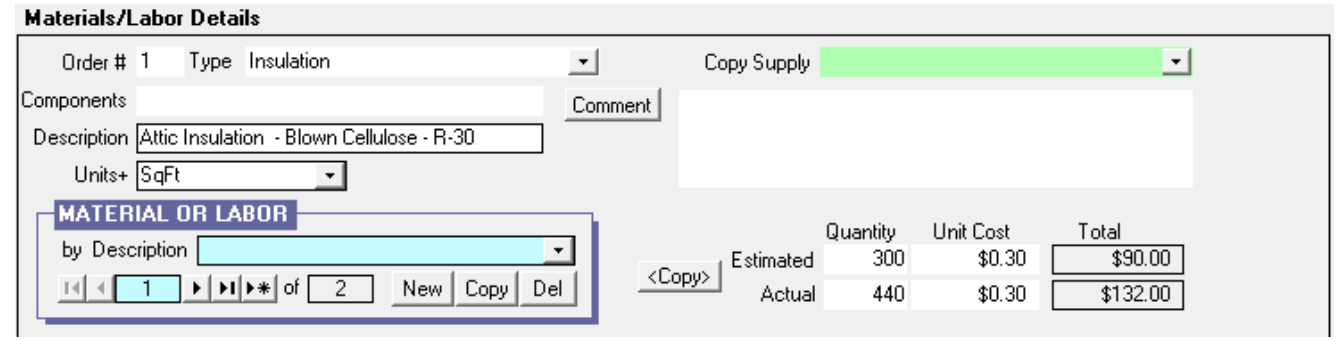

Figure 13.7. The Materials/Labor Details sub-form (form view).

The Materials/Labor Details sub-form can also be viewed in data sheet view (see Figure 13.8 below and Section 5.8, Data Sheet and Form Views of Records). Although both views allow full data entry capabilities, the form view is likely easier to understand since the controls have their standard appearance and do not need to be cast in a spreadsheet format. Therefore, this view will be assumed in the following descriptions. However, the alternate data sheet view still offers the added ability to quickly see all components of the measure detail at once, not possible in the form view.

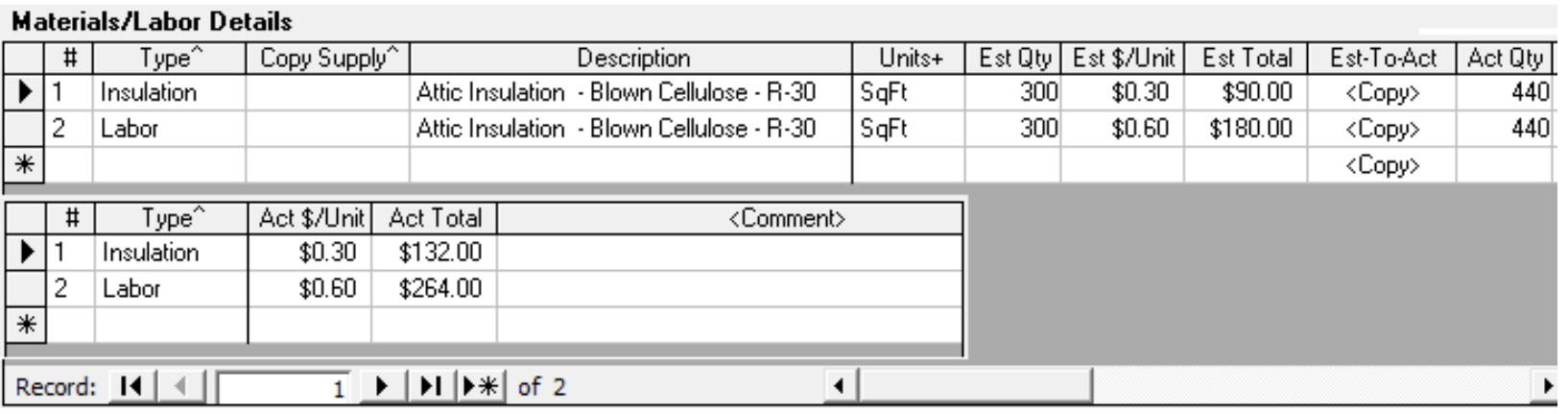

Figure 13.8. The Materials/Labor Details sub-form (data sheet view).

Note: The form has been displayed wrapped since scrolling would normally be necessary to see all columns.

Below are descriptions of the individual data items and controls found on the Materials/Labor Details form.

- Order \# - An integer entry that prescribes the order in which the costing components of the measure will be listed on the work order. Any components not given an order number will be listed first on the work order, but all those without a number will have random order amongst themselves. Optional. 


\section{Chapter 13: Work Orders}

- Type - If you are using the Supply Library (see Chapter 15, Supply Library) to store information about materials you use in your weatherization activities and possibly taking advantage of the Weatherization Assistant's inventory feature, select the category under which the material is listed in the library. With the exception of "Unspecified," these selections correspond to the material categories in the Supply Library. The choices are as follows.

Cooling Equipment
Construction Materials/Hardware
Doors
Health and Safety Items
Heating Equipment
Hot Water Equipment
Insulation

Labor
Lighting
Miscellaneous Supplies
Refrigerators
Windows
Other
Unspecified

The entry is used to narrow your search for the material when using the "Copy Supply" drop-down list at the upper right corner of the sub-form. If you do not plan to locate this material detail component in your Supply Library, this entry may be left blank or designated as "Unspecified." If the measure to which this detail belongs originated from a NEAT or MHEA audit recommendation, this entry will automatically be assigned. Optional (unless locating the material in your Supply Library).

- Components - This field is normally visible only in the form view of the Materials/Labor Details sub-form. It must not be confused with the Components field in the general description portion of the Measures form. Figure 13.3 displays both of these fields. The Components field on the Materials/Labor Details sub-form is never pre-populated and it is not displayed on the work order. You may use it for a note regarding the material, but it will be visible only within the Weatherization Assistant.

- Description - Enter a brief description of the component detail of the measure. If the "Copy Supply" drop-down list was used to select the material, this field will be automatically entered. In such cases, you may change the description, but it may complicate your ability to identify the item as one tracked in your inventory. The entry will also be automatically filled in if the material entry stems from an audit recommendation. However, this does not link the entry to an item in your Supply Library. You must still use the "Copy Supply" drop- 
down list if you wish to associate the material with one in that library. The entry may be 80 characters or less. Required.

- Units - Enter the units to be associated with the Estimated and Actual Quantity fields on the sub-form. You may enter a unit not in the drop-down list (e.g., "gallon”). WARNING: If the detail entry was copied from the Supply Library using the "Copy Supply" drop-down list, do not change the Units entry that is automatically entered. The inventory kept in the Supply Library will expect the Quantity and Unit Cost entries on the sub-form to be in these original units. Required.

- Copy Supply - This entry is used in conjunction with the Type entry on the sub-form to locate a specific material from your Supply Library. Selecting the material using this drop-down list will automatically fill in most of the remaining fields on the sub-form, provided you have specified the analogous entries in the Supply Library. Locating a material in the Supply Library is essential if you are using the inventory feature of the program for the specific material being described. Optional.

- Comment - You may enter comments pertinent to the current material/labor component directly in the Comment field on the form, or you may enter them in the Comment Editor by selecting the "Comment" button to the left of the field. If in data sheet view, double clicking on the field will display the Comment Editor. The Comment Editor is similar to Window's Notepad and may be used to enter extended comments. Text from other sources may be copied and pasted into the Comment Editor's window. Unique to the comment fields on any form within the "Work Order" window is the ability to import comments from the audit you have specified in the Audit Name field on the Work Order Information form by selecting the "Use an Audit Comment" drop-down list displayed at the bottom of the Comment Editor. This drop-down list includes references to all comments entered on all forms of the audit. You can import multiple comments by selecting items one-by-one. The total comment may have up to 65,000 characters. However, the field on the work order that will display this comment is only about 20 characters in width. The Weatherization Assistant will wrap the comment on the report, but excessively long comments become awkward to display. Any comment that is entered in this comment field will be displayed in the work order immediately under the material description. Optional. 
If the measure being viewed is a library measure or a user-defined measure, and you had entered comments for any component detail while defining the measure in the Setup Library, those comments will be automatically copied to this form provided the request was made to carry along the component detail when generating the work order (see "Include Details for Materials" in Section 11.11, Measures (Audit), or “Create Materials Using Audit Detail” in Section 13.3, Measures (Work Order)).

- Quantity (Estimated) - This is an estimate of the quantity of the material needed to install this component of the measure, in the units specified in the Units field. If this specific Materials/Labor Details entry originated from an audit recommendation, this field will initially be the quantity specified in the recommendation. However, you may alter this value if a new, more accurate estimate becomes available. You may always review the value from the audit recommendation by selecting the "Show Audit Material Detail" button. Although the entry is optional with regard to form completeness checks, if left blank, no value will appear in the Work Order report. You may enter the Estimated Quantity without entering the associated Unit Cost. However, unless both the Estimated Quantity and Estimated Unit Cost are entered, no Estimated Total will be computed or displayed. Optional.

- Unit Cost (Estimated) - This is an estimate of the cost per unit (as given in the Units field) of the material used in installing this component of the measure. If this specific Materials/Labor Details entry originated from an audit recommendation, this field will initially be the unit cost used to calculate cost and SIR of the recommendation. However, you may alter this value if a new, more accurate unit cost estimate becomes available. You may always review the value from the audit recommendation by selecting the "Show Audit Material Detail” button. Although the entry is optional with regard to form completeness checks, if left blank, no value will appear in the Work Order report. You may enter an Estimated Unit Cost without an associated Estimated Quantity. However, unless both the Estimated Quantity and Estimated Unit Cost are entered, no Estimated Total will be computed or displayed. Optional.

- Total (Estimated) - This field is the product of the Estimated Quantity and the Estimated Unit Cost entries described above. If either of these values is changed, this total will automatically change (you may need to access the field for this update to occur). This total estimated cost will be reported in the work 
order as an indication to the contractor of your estimate of the cost for this particular component of the measure's installation. The sum of the Total Estimated costs from all components of a measure is displayed in the Estimated Cost field in the lower right corner of the Measures form (see "Estimated/ Actual Cost and SIR” earlier in this section). Uneditable.

- Quantity (Actual) - This field allows you to record an actual quantity of material used in installing this component of the measure, in the units specified in the Units field. This value may not be available until after the work has actually been performed. A blank field on the Work Order report for this quantity allows the contractor to record it such that it can be reported back to the agency. However, if entered prior to the Work Order report being printed, the value will appear on the report in this field instead. If the Actual Quantity and Actual Unit Cost are found to be the same as the estimated values, clicking on the "Copy" button will automatically copy the estimated values to the Actual fields. If the material has been selected from your Supply Library, it is only after the quantity of the material has been declared "Actual" that the quantity will be subtracted from your inventory tracked in that Supply Library (see "Used (button)" in Section 15.2, Materials/Labor Forms, and the Copy Supply field described above). You may enter the Actual Quantity without entering the associated Unit Cost. However, unless both the Actual Quantity and Actual Unit Cost are entered, no Actual Total will be computed or displayed. Optional.

- Unit Cost (Actual) - This field allows you to record an Actual Unit Cost of the material used in installing this component of the measure, in the units specified in the Units field. This value may not be available until after the work has actually been performed. A blank field on the Work Order report for this quantity allows the contractor to record it such that it can be reported back to the agency. However, if entered prior to the Work Order report being printed, the value will appear on the report in this field instead. If the Actual Quantity and Actual Unit Cost are found to be the same as the estimated values, clicking on the "Copy" button will automatically copy the estimated values to the actual fields. You may enter the Actual Unit Cost without entering the associated Quantity. This may be useful if a Unit Cost for the material has been set by a contract, but the Actual Quantity is left for the contractor to determine at the time the work is performed. However, unless both the Actual Quantity and Actual Unit Cost are entered, no Actual Total will be computed. Optional. 


\section{Chapter 13: Work Orders}

- Total (Actual) - This field is the product of the Actual Quantity and the Actual Unit Cost entries described above. If either of these values is changed, this total will automatically change (you may need to access the field for this update to occur). The entries necessary for the program to compute this value may not be available until after the work has been performed. However, if available at the time the Work Order report is printed, the value will appear on the report. The sum of the Total Actual costs from all components of a measure is displayed in the Actual Cost field in the lower right corner of the Measures form (see "Estimated/ Actual Cost and SIR" earlier in this section). Uneditable.

You may also view all tasks within the work order in data sheet view (see Section 5.8, Data Sheet and Form Views of Records, and Figure 13.9, below). Although you can make changes while in this view, it is not recommended as standard practice since the form has more information associated with it than can be displayed from this view (in particular the information within the Materials/ Labor Details sub-form).

\begin{tabular}{|c|c|c|c|c|c|c|c|c|c|c|c|}
\hline 圆 Wo & ork & Orde & & & & & & & & 0 & 回 $x$ \\
\hline wo 1 & $W C$ & $0 / 1$ & $1 \_348 / J T / 1$ & & Client I & $11 \_348$ & Client $\mathrm{N} a$ & ame Tanner, David & & Client ID & \\
\hline Work & Ord & der Ir & fformation Status Measures & & & & & & & & \\
\hline & & $\#$ & Measure Name & Active & Type ${ }^{\wedge}$ & Components & EstSIR & \begin{tabular}{|l|l} 
ActSIR & EstCost
\end{tabular} & ActCost & & \\
\hline & + & 2 & Repair Roof & $\nabla$ & & & 0.0 & $0.0 \$ 80.00$ & $\$ 135.00$ & & \\
\hline & + & 3 & Infiltration Redctn & $\nabla$ & & & 1.0 & $1.0 \$ 250.00$ & $\$ 250.00$ & & \\
\hline & + & 5 & DWH Pipe Insulation & $\nabla$ & Baseloads & & 9.6 & $9.6 \$ 15.00$ & $\$ 15.00$ & & \\
\hline & + & 7 & DWH Tank Insulation & $\nabla$ & Baseloads & & 7.5 & $7.5 \$ 40.00$ & $\$ 40.00$ & & \\
\hline & + & 8 & Attic Ins. R-30 & $\square$ & Building Insulati & UA.1 & 7.3 & $5.0 \$ 270.00$ & $\$ 396.00$ & & \\
\hline$D$ & +2 & 9 & Fill Ceiling Cavity & $\square$ & Building Insulati & FA3 & 5.3 & $5.3 \$ 221.26$ & $\$ 221.26$ & & \\
\hline & + & 10 & Insulate and seal attic access & $\nabla$ & & & 3.7 & $3.7 \$ 29.60$ & $\$ 29.60$ & & \\
\hline & + & 11 & Attic Ins. R-30 & $\nabla$ & Building Insulati & FA1 & 3.5 & $3.5 \$ 352.80$ & $\$ 352.80$ & & \\
\hline & + & 12 & Attic Ins. R-30 & $\nabla$ & Building Insulati & FA4 & 3.3 & $3.3 \$ 50.40$ & $\$ 50.40$ & & \\
\hline & + & 14 & Kneewall Insulation & $\square$ & Building Insulati & FA2 & 2.6 & $2.6 \quad \$ 91.20$ & $\$ 91.20$ & & \\
\hline & + & 16 & Wall Insulation & $\nabla$ & Building Insulati & WLE-1,WLN-1,W & 1.2 & $1.2 \$ 854.46$ & $\$ 717.10$ & & \\
\hline & + & 17 & Window Replacement & $\nabla$ & Doors and Wine & WD4 & 0.5 & $1.9 \$ 346.00$ & $\$ 96.00$ & & \\
\hline & + & 18 & CO Monitor is Needed & $\square$ & Health and Saft & & 0.0 & $0.0 \$ 70.00$ & $\$ 70.00$ & & \\
\hline 米 & & & & $\nabla$ & & & & & & & \\
\hline $\operatorname{Rec}$ & cord & d: 1 & $6 \mapsto \mid 1$ & 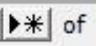 & & 1 & & & & & - \\
\hline
\end{tabular}

Figure 13.9. The Measures form under the Main Menu’s “Work Orders” button (data sheet view).

Note: “Cost Center" and “Comment” fields not displayed. 


\section{Chapter 13: Work Orders}

\subsection{Photos (Work Order)}

If you have selected the "Use photo browser tab..." checkbox from the Features tab under the Main Menu's "Preferences" button (see Feature 1 in Section 16.4, Features), the Photos tab will appear under the Main Menu's "Work Orders" button. This feature allows you to access the Photos form (see Figure 13.10). From this form, you may select, view, and edit digital photos for the current work order. See Section 18.1, Digital Photos, for more information on this feature. The ability to reference digital photos also exists under the Main Menu's "Clients," "NEAT," and "MHEA" buttons. Therefore, you should develop a consistent policy regarding where you reference your photos.

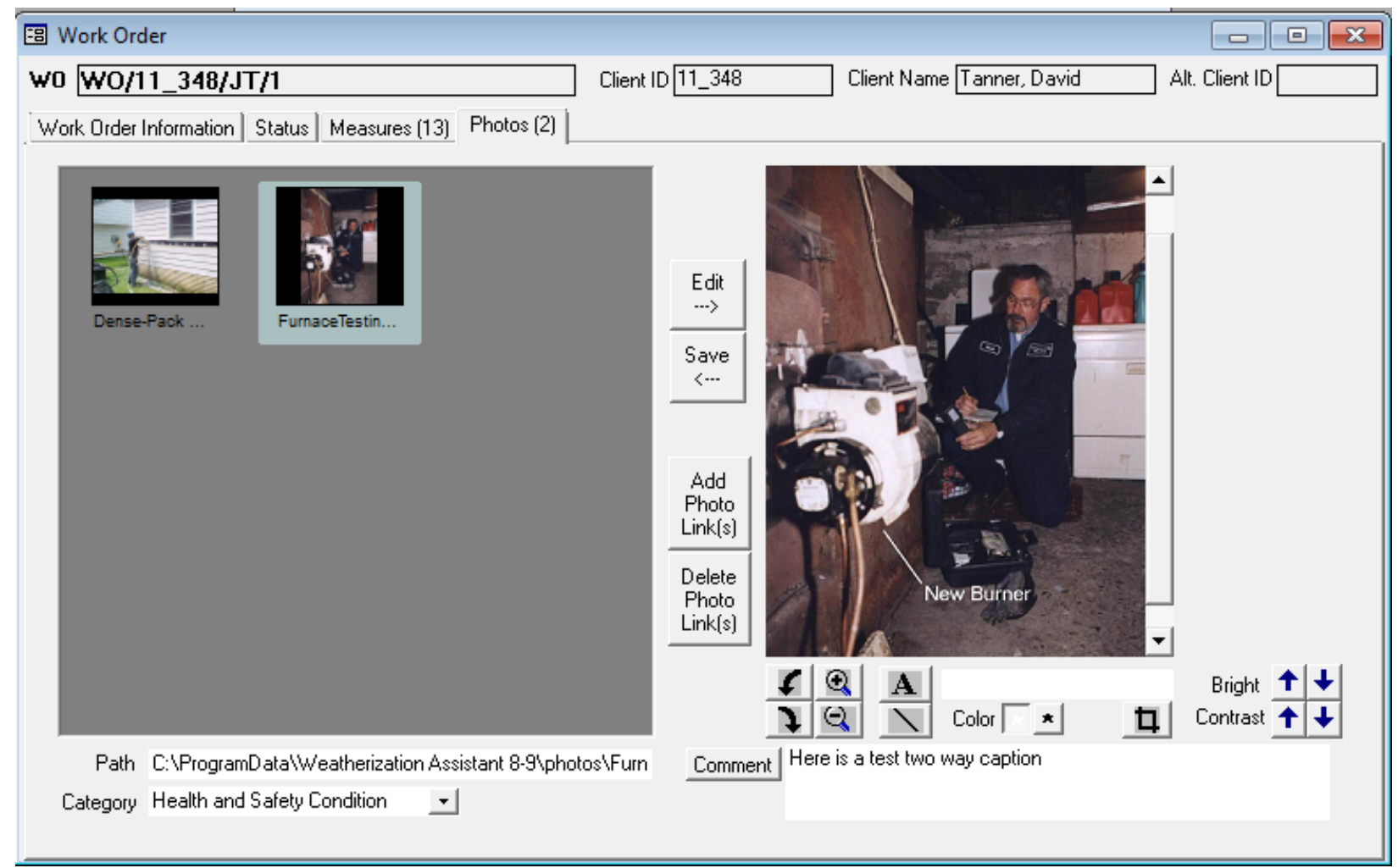

Figure 13.10. The Photos form under the Main Menu's “Work Orders” button. 


\section{Chapter 14. Setup Library}

NEAT and MHEA require more than a home's description in order to select energy efficiency measures for a dwelling. Using the Setup Library, you customize the programs to your area by supplying fuel costs, material costs, and additional key parameters. These values are not expected to change house-by-house. The Setup Library is also where you have the opportunity to tell the programs which measures to consider. This is most often decided with input from the state. You may also define those tasks regularly performed as part of your weatherization work that are not otherwise addressed by the programs. If you know energy savings associated with any of these tasks, you may define user-defined measures, which NEAT and MHEA will treat exactly as they do the predefined library measures. The Setup Library is also where you may designate the characteristics of insulation types not already available in NEAT.

As many setup library records as needed may be defined, each with its individual set of material costs, settings, etc. For example, you may have more than one contractor who has bid on work you perform. Thus, you would need a separate Setup Library to contain the costs associated with each contractor. In addition, each Setup Library itself can have multiple sets of fuel costs (see Section 14.3, Fuel Costs) to accommodate the possibility of different utility service areas. See "Setup Library Record Navigation Block" below for a discussion on how to create additional setup libraries. Only one Setup Library can be associated with each audit.

The remainder of this chapter is divided into sections describing the data entered on each of the tabs seen under the Main Menu's “Setup Library” button: Setup Library Information, Key Parameters, Fuel Costs, Fuel Price Indices, Library Measures, User Defined Measures, and NEAT Insulation Types. Data under the Setup Library Information, Fuel Costs, and Fuel Price Indices tabs are used by both NEAT and MHEA. Under the Key Parameters, Library Measures, and User Defined Measures tabs, you designate which of the programs' data you wish to view using the "View" drop-down list in the lower left corner of the form. For the latter of these tabs, you also have the choices to look at "All User-Defined Measures" or only the "Health \& Safety" measures defined in the Weatherization Assistant (see Section 14.6, User Defined Measures). 


\subsection{Setup Library Information}

The Setup Library Information form (see Figure 14.1) under the Main Menu's "Setup Library" button identifies the Setup Library being displayed and the Agency to which the Setup Library is assigned. Individual entry items on the form are discussed below:

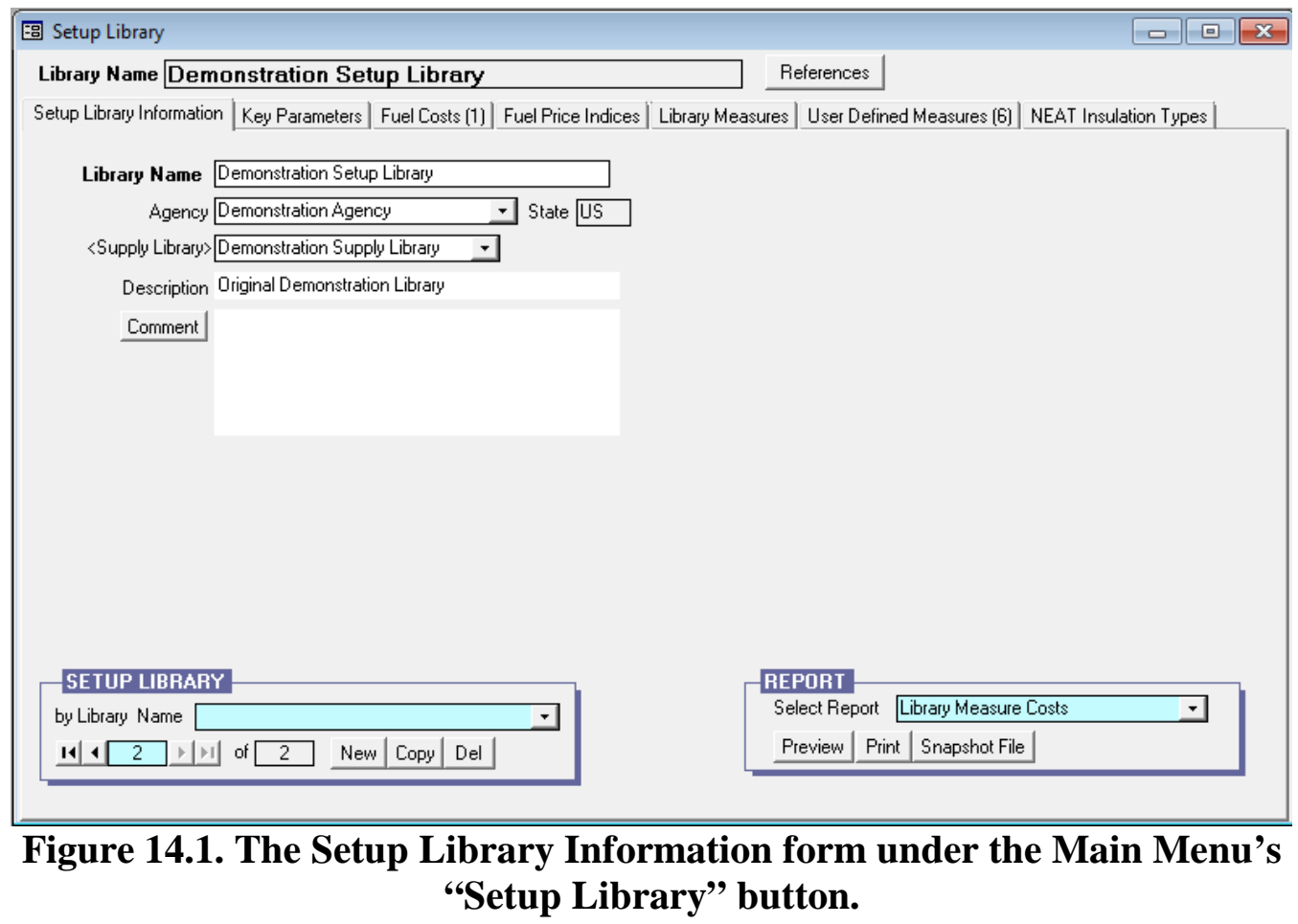

- References - Selecting this button, located just below the banner line of the "Setup Library" window, displays the "References to Setup Library" window, which lists all references to the Setup Library currently being accessed. See Section 5.14, The References Button, for more information on the use of this feature.

- Library Name - Enter in this field a name by which the specific Setup Library can be identified. If you have more than one Setup Library in your agency, the Library Name may contain some reference to how this library differs from any other. However, a complete description of the library should be saved for the Description or the Comment fields. A default name will be supplied when a new Setup Library is created. The name will use your installation's ID (see Section 3.1, Initial Start-up of the Weatherization Assistant), if available. 
Otherwise the default name will contain the time the library was created. Entries may be 50 characters or less. Required.

- Agency - This field identifies the agency to which the Setup Library is associated. Since the only way to create a new Setup Library is to copy an existing one (see "Setup Library Record Navigation Block" below) or import one with a client (see both topics on "Importing Clients" in Sections 17.3, Data Transfer - Import/Export With Another MSAccess Database, and 17.4, Data Transfer - Import/Export Indirectly with Text Files), this entry will always be at least initially filled in for you. The field is more for identification purposes, possibly on a state level, identifying the agency within the state using the set of parameters in the library. WARNING: The program allows you to change the Agency assignment of a Setup Library. However, doing so when existing audits use the library will hide the link between the audits and the library. Therefore, re-assigning the agency assignment of a Setup Library is not recommended unless the library has just been created, insuring no other references to it exist. Required.

- State - This field displays the state associated with the Agency entered in the previous field. Uneditable.

- Supply Library - This entry indicates what Supply Library is associated with the Setup Library. The Supply Library is where your inventory of materials and supplies exists. The Setup Library uses this designation to permit you to include materials from your inventory in defining user-defined measures. If these materials are so chosen, actual implementation of the user-defined measure will automatically update your inventory within the specified Supply Library. (See Section 14.6, User Defined Measures). The Supply Library entry will most often be automatically entered for you whenever a new Setup Library is created or when associated with an imported client (see both topics on "Importing Clients” in Sections 17.3, Data Transfer - Import/Export With Another MSAccess Database, and 17.4, Data Transfer - Import/Export Indirectly with Text Files). WARNING: Changing the Supply Library assigned to a Setup Library can cause confusion, unless the library has just been created. Even though the material assignments used in existing work orders will remain unchanged, the Supply Library and Setup Library designations in Audits and Work Orders may become inconsistent and make it difficult to distinguish where inventoried materials are being drawn from. Required. 
- Description - You may use this field to briefly describe the intended use of the Setup Library, particularly if you use more than one Setup Library in your agency. If you have multiple contracts for work with different costs, this field would be ideal to record the contractor's name, the geographic area in which they work, etc. The entry may be 255 characters or less. Optional.

- Comment - You may enter comments pertinent to the Setup Library directly in the Comment field on the form, or you may enter them in the Comment Editor by selecting the "Comment" button to the left of the field. The Comment Editor is similar to Window's Notepad and may be used to enter extended comments. The total comment may have up to 65,000 characters. Text from other sources may be copied and pasted into the Comment Editor's window. Any comment that is entered is displayed on the Library Measure Costs and User Defined Measure Costs reports (see "Report Block" below). Optional.

- Setup Library Record Navigation Block - The Setup Library record navigation block is used to find and navigate to setup libraries, delete existing libraries, or create a new library by copying an existing one. See Section 5.1, Form and Record Navigation, for information on using the Weatherization Assistant's record navigation blocks.

By default, the drop-down list will display only the setup libraries associated with your agency, since it is likely that your agency has been "bookmarked" by being the last agency visited using the Main Menu’s “Agency” button. If you have more than one agency defined in your database (as might be the case for a state's installation) and you want the drop-down list to display setup libraries from all defined agencies, uncheck the "Use only the last bookmarked Agency in the find record drop down boxes," Feature 9 from the Preferences/Features tab (see the indicated topic in Section 16.4, Features).

For the Setup Library record navigation block, the "New" button does not create a blank setup library. Instead it creates a setup library which is a copy of the library that came with the program, originally named "Your Setup Library," prior to you having made any changes to it (see "Naming Your Setup Library" in Section 3.2, Program Setup). If you wish to create a setup library having modifications to one you are already using, access it using the record navigation block, use the "Copy" button, and make the changes you wish in the resulting 
copy. Do not use the "New" button for this, since the new setup library will not have any of your current data in it.

- Report Block - The Report block provides you with access to all reports available under the Main Menu's “Setup Library” button. The following reports are currently available: Library Measure Costs and User Defined Measure Costs. See Appendix C, Reports, for examples of these reports. The Library Measure Costs report will display the costs for all of the library measures (see "Costs" in Section 14.5, Library Measures). For each measure, all three cost components (material, labor, and other) will be displayed. For insulation measures having multiple insulation types for each application, these three components will be given for each material. The entries are divided by Measure Type and Measure Name. The User Defined Measure Costs report displays the costs for all of the measures you have defined under the User Defined Measures tab of the setup library as well as all of the library health and safety measures contained in the Weatherization Assistant (see Section 14.6, User Defined Measures). User-defined measures may have as many materials or components to their costing scheme as you wish. All of these components will be listed in the report for these measures. See Section 5.12, The Report Block, for the mechanics of using the Weatherization Assistant's Report blocks. Appendix D, Development of Customized Reports, describes a method to create reports tailored to your needs and to list the reports in the Report block.

\subsection{Key Parameters}

The forms under the Key Parameters tab in the "Setup Library" window provide you with the flexibility of modifying some of NEAT's and MHEA's inputs and assumptions. If you have information about the parameters included on these forms that is more appropriate for your agency than the existing values, you may enter them by replacing the existing values. Caution should be exercised in altering parameters. The Weatherization Assistant attempts to recommend measures for houses with typical occupancy characteristics. No effort should be made to adjust parameters to coincide with the lifestyles of individual occupants unless possibly for health and safety considerations of a specific occupant. (If this is done, make sure to alter a copy of your standard setup library so that other audits won't be affected by the change.) 


\section{Chapter 14: Setup Library}

Key parameters for both NEAT and MHEA are accessible under the same Key Parameters tab. Choose which set of parameters you view by using the "View" drop-down list in the lower left corner of the Key Parameters form. The name of the program (either "NEAT" or "MHEA") will appear just above the "View" dropdown list indicating which set is currently being displayed.

The key parameters for both programs are subdivided into categories, such as "Economics" or "Insulation," each under a separate sub-form. These parameters will be discussed below in order of the program, then category.

\section{Site Built (NEAT) Key Parameters}

\section{Economics}

- Real discount rate - The rate at which dollars saved in the future are discounted to a worth in current dollars. DOE supplies this rate each year and has specified that it must be changed no less than every five years. This parameter is updated with each release of the Weatherization Assistant.

- Minimum acceptable SIR - The savings to investment ratio (SIR) for an individual measure below which the measure will not be recommended. A minimum SIR of 1.0 has been specified by DOE.

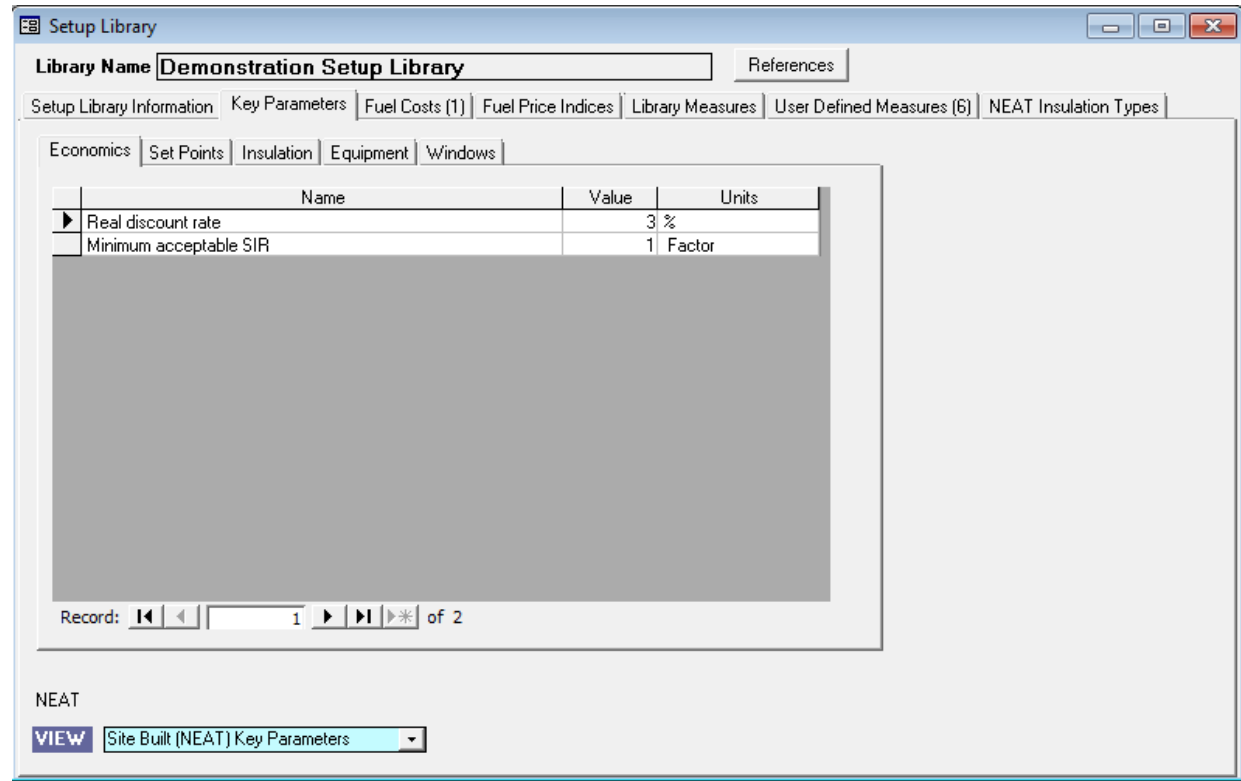

Figure 14.2. The NEAT Key Parameters - Economics form under the Main Menu's “Setup Library” button. 


\section{Set Points}

- Heating and Cooling setpoints for daytime and nighttime (F) - The assumed thermostat set-points in the house being audited. NEAT has been designed to make recommendations based on typical occupancy. The set-point values should not be altered to reflect any unusual lifestyles of the occupants.

- Night setback (F) - The number of degrees Fahrenheit below the normal heating nighttime set-point that an automatic set-back thermostat decreases the heating set-point during the night.

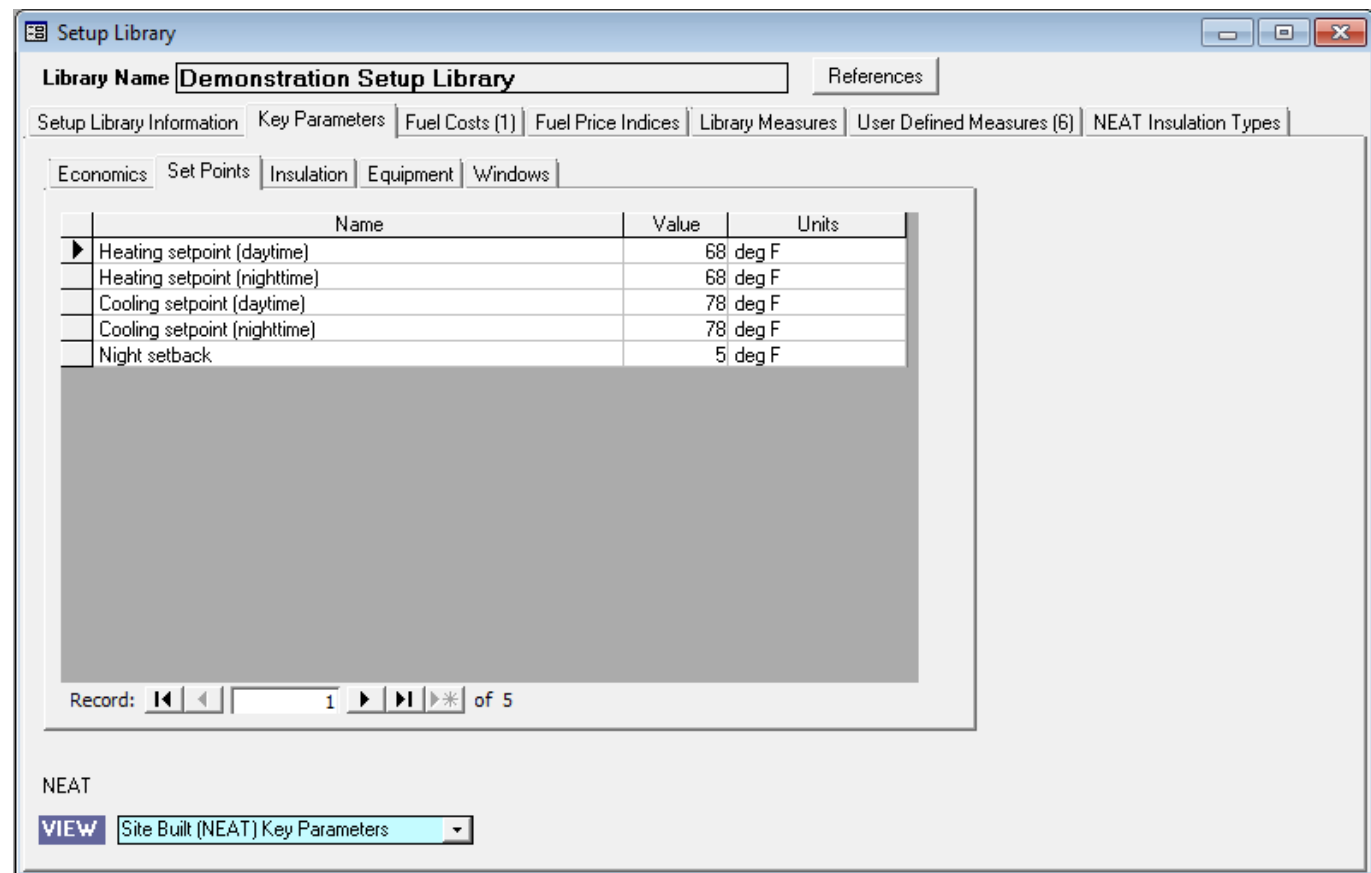

Figure 14.3. The NEAT Key Parameters - Set Points form under the Main Menu's “Setup Library” button.

\section{Insulation}

- Average annual outside film coefficient (Btu/h-ft $\left.{ }^{2}-\mathbf{F}\right)$ - The winter/summer average film coefficient assumed for exterior surfaces.

- Uninsulated R-Value for "Other" Wall Type (h-ft $\left.\mathbf{t}^{2}-\mathbf{F} / \mathbf{B t u}\right)$ - The R-value associated with an uninsulated wall given Wall Type “Other” on NEAT's Walls form. 


\section{Chapter 14: Setup Library}

- R-Value for "Other" exterior siding type (h-ft $\left.{ }^{2}-\mathbf{F} / \mathbf{B t u}\right)$ - The R-value associated with the Exterior Type (siding) “Other” on NEAT's Walls form.

- R-Value per inch for "Other" existing ceiling insulation type (h-ft'-F/Btuin) - This value is not used in the program. The parameter entry was left in the key parameters by mistake. The value used is $3.09 \mathrm{~h}-\mathrm{ft}^{2}-\mathrm{F} / \mathrm{Btu}-\mathrm{in}$.

- Added duct insulation R-value (h-ft ${ }^{2}$-F/Btu) - The R-value assumed added to the supply ducts by the Duct Insulation measure.

- Water heater wrap added $\mathbf{R}$ value (h-ft $\left.{ }^{2}-\mathbf{F} / \mathbf{B t u}\right)$ - The insulation $\mathrm{R}$-value used to wrap water heaters in the Water Heater Tank Insulation measure.

- Base value of free heat from internals (Btu/h) - The heat from lights and appliances that is released into a home. NEAT adds to this base amount the heat generated by as many as two adults (276 Btu/h per adult) and one child (224 $\mathrm{Btu} / \mathrm{h}$ ) for every occupant above two in number.

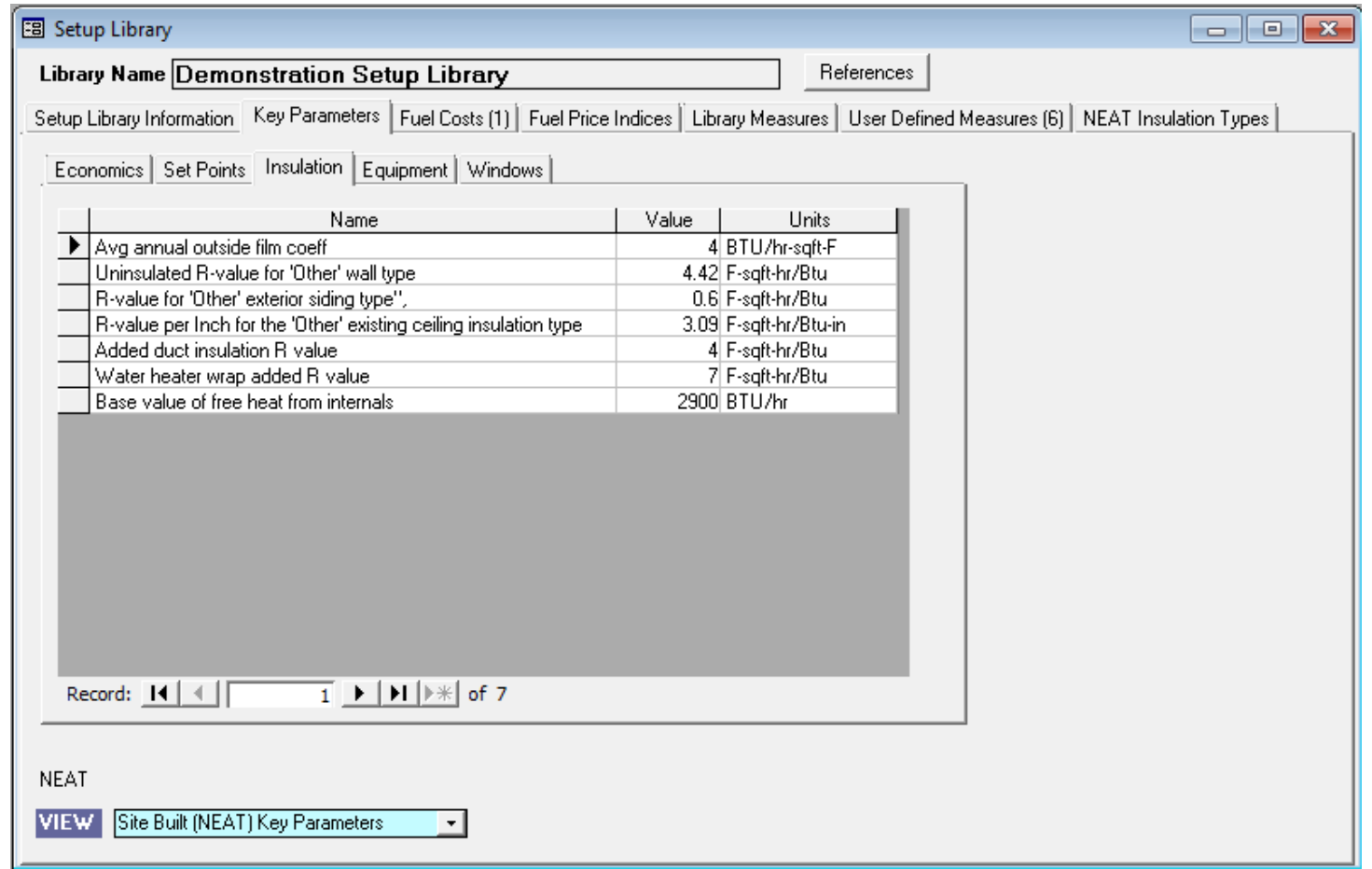

Figure 14.4. The NEAT Key Parameters - Insulation form under the Main Menu's “Setup Library” button. 


\section{Equipment}

- Window A/C replacement SEER - The SEER (seasonal energy efficiency ratio) of the replacement window air conditioner.

- Central A/C replacement SEER - The SEER of the replacement central air conditioner.

- Heat pump replacement SEER (cooling) - The SEER of the replacement heat pump when operating in cooling mode.

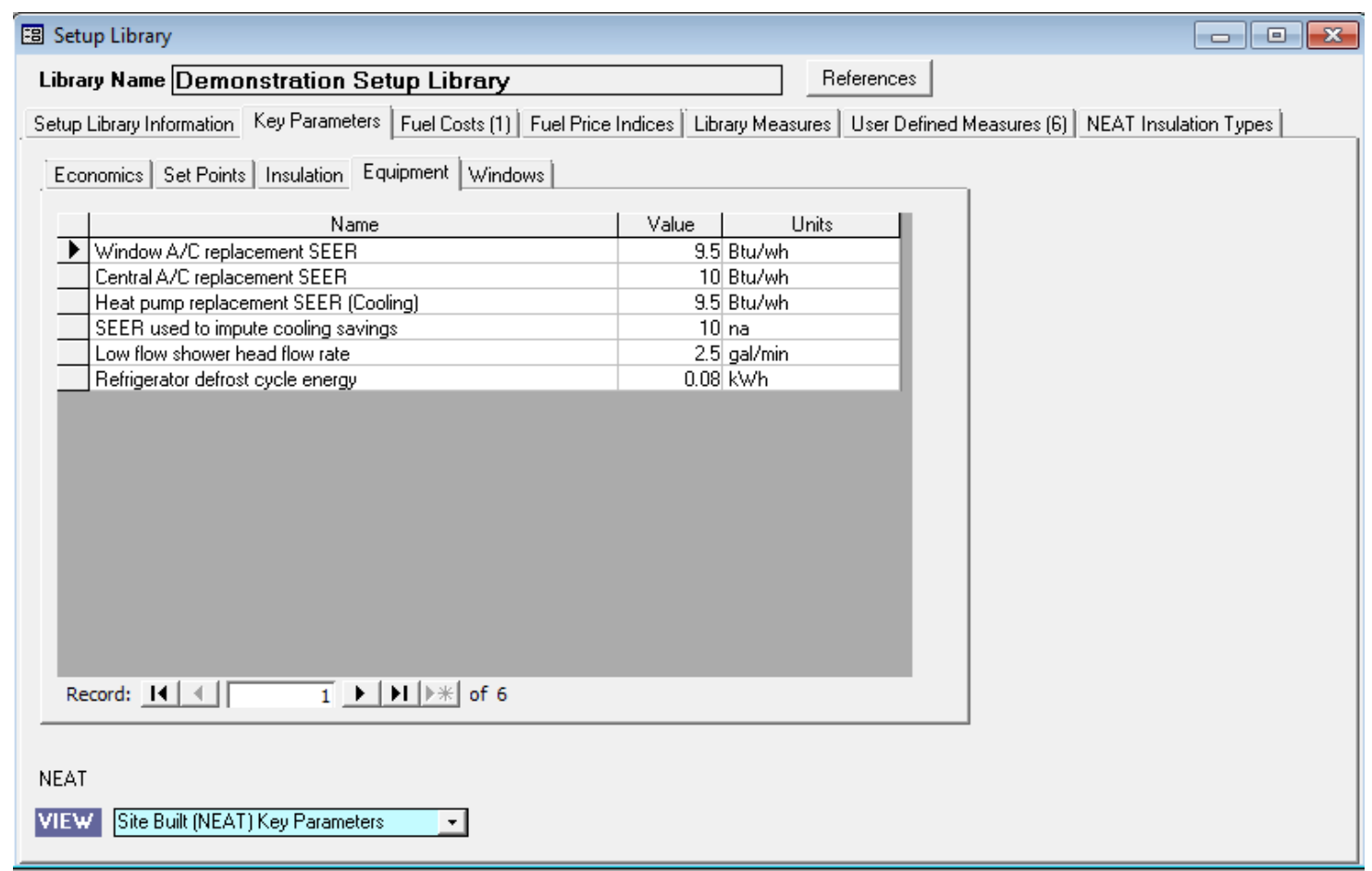

Figure 14.5. The NEAT Key Parameters - Equipment form under the Main Menu's “Setup Library” button.

- SEER used to impute cooling savings - The SEER of an air-conditioning unit that would most likely be installed in the home sometime in the future. This data item controls NEAT's optional feature of computing cooling savings for measures in a home without air-conditioning and little heating needs, as might be found in the extreme South. No cooling energy is actually saved in such a home. However, most measures that would save cooling energy in a home with air-conditioning can make living conditions more comfortable during the summer in homes without air-conditioning. Thus, you may "impute" a savings 
in these homes in order to install measures to increase the comfort. The homes may install air-conditioning in the future, thus eventually allowing this imputed savings to occur. The feature is activated from NEAT's Audit Information form (see “Impute Cooling” in Section 8.2, Audit Information).

- Low flow shower head flow rate (gal/min) - The flow rate in gallons per minute of replacement shower heads used in your program.

- Refrigerator defrost cycle energy (kWh) - The electrical energy in kWh assumed consumed by the existing refrigerator's defrost cycle. The value is used to adjust the metered consumption of the refrigerator if the user indicates that the metering period included a defrost cycle.

\section{Windows}

- Replacement Window U-value (Btu/h-ft $\left.{ }^{2}-\mathbf{F}\right)$ - The U-value assumed for the replacement window. Unless changed by the user, the value represents a typical double pane, wood or vinyl frame window.

- Replacement Window Solar Heat Gain Coefficient - The solar heat gain coefficient (SHGC) assumed for the replacement window. Unless changed by the user, the value represents a typical double pane, wood or vinyl frame window.

- Replacement Low-e Window U-value (Btu/h-ft $\left.{ }^{2}-\mathbf{F}\right)$ - The U-value assumed for the replacement low-e window. Unless changed by the user, the value represents a typical double pane, low-e, wood or vinyl frame window.

- Replacement Low-e Window Solar Heat Gain Coefficient - The solar heat gain coefficient (SHGC) assumed for the replacement low-e window. Unless changed by the user, the value represents a typical double pane, low-e, wood or vinyl frame window.

- Retrofit Storm Window Emittance - The emittance assumed for the Storm Windows retrofit measure. Unless changed by the user, the value represents a typical single pane wood frame glass storm window. If your agency installs low-e storm windows, this value should be changed to reflect the low-e storm's manufacturer's specifications. 
- Retrofit Storm Window Solar Heat Gain Coefficient - The solar heat gain coefficient (SHGC) assumed for the Storm Windows retrofit measure. Unless changed by the user, the value represents a typical single pane wood frame glass storm window. If your agency installs low-e storm windows, this value should be changed to reflect the low-e storm's manufacturer's specifications.

- Retrofit Window Film Surface Emittance - The surface emittance assumed for the Window Film retrofit measure. There is a wide range of reported window film characteristics. If your agency installs window films, this value should be changed to reflect the window film's manufacturer's specifications.

- Retrofit Window Film Solar Heat Gain Coefficient (including frame) - The solar heat gain coefficient (SHGC) assumed for the retrofit window film measure. There is a wide range of reported window film characteristics. If your agency installs window films, this value should be changed to reflect the window film's manufacturer's specifications.

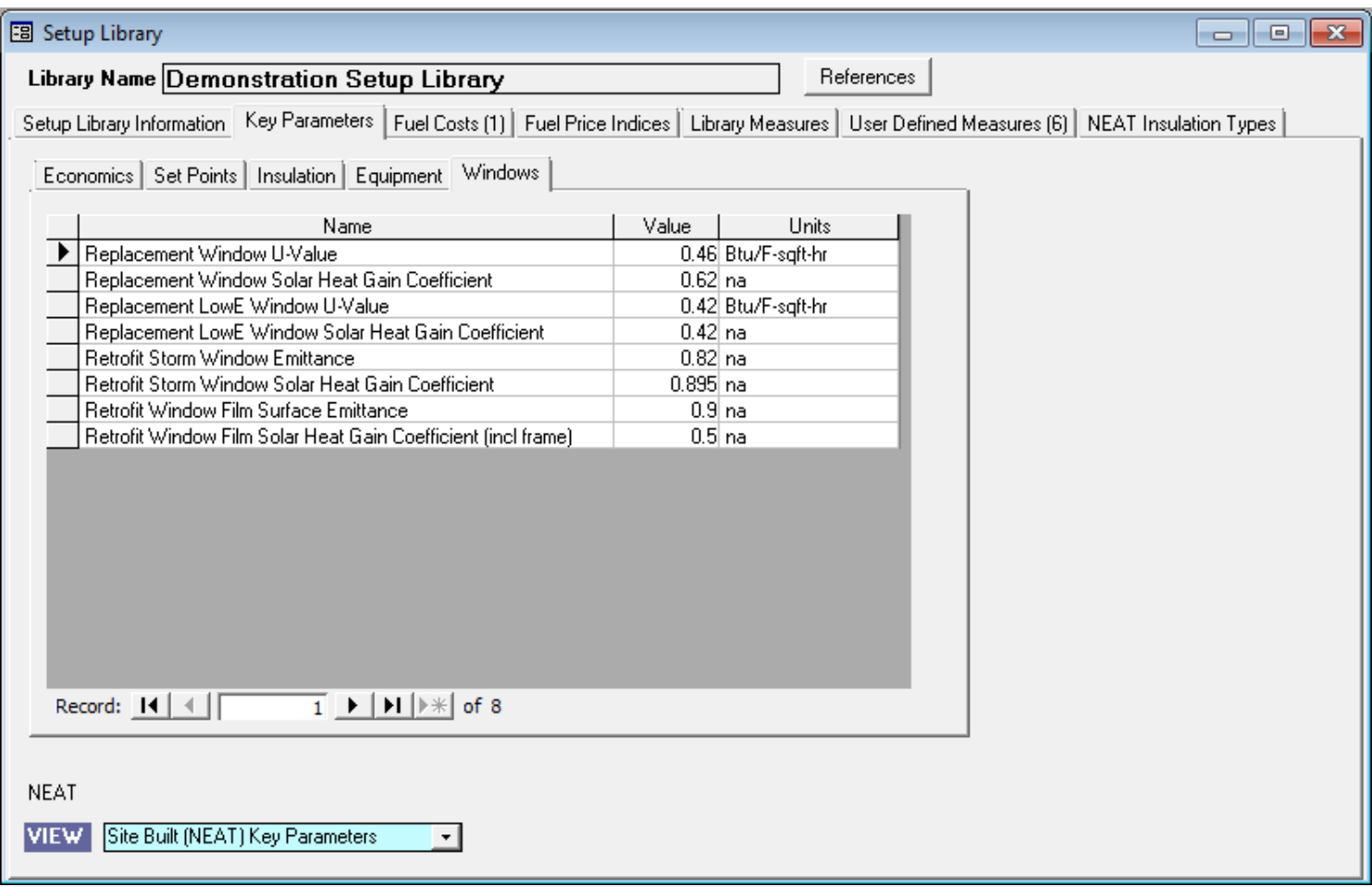

Figure 14.6. The NEAT Key Parameters - Windows form under the Main Menu’s "Setup Library" button. 


\section{Mobile Home (MHEA) Key Parameters}

\section{Economics}

- MHEA Real Discount Rate - The rate at which dollars saved in the future are discounted to a worth in current dollars. DOE supplies this rate each year and has specified that it must be changed no less than every five years. This parameter is updated with each release of the Weatherization Assistant.

- MHEA Minimum Acceptable SIR - The savings to investment ratio (SIR) for an individual measure below which the measure will not be recommended. A minimum SIR of 1.0 has been specified by DOE.

- Spending limit for package of measures (\$) - The average per dwelling expenditure of financial assistance allowed under the Weatherization Assistance Program as prescribed in the annual program guidance. If this limit is exceeded for an specific dwelling, a warning will be printed under the "Special Notes" section of the Recommended Measures Report (see Section 12.2, Recommended Measures Report).

\section{Set Points}

- MHEA Heating and Cooling Setpoints for daytime and nighttime (F) - The assumed thermostat set-points in the house being audited. MHEA has been designed to make recommendations based on typical occupancy. The set-point values should not be altered to reflect any unusual lifestyles of the occupants.

- Thermostat setback amount (F) - The number of degrees Fahrenheit below the normal heating nighttime set-point that an automatic set-back thermostat decreases the heating set-point during the night.

- Length of night thermostat setback (Hours) - The number of hours each day that a set-back thermostat, if one exists, affects the thermostat set-point. 


\section{Chapter 14: Setup Library}

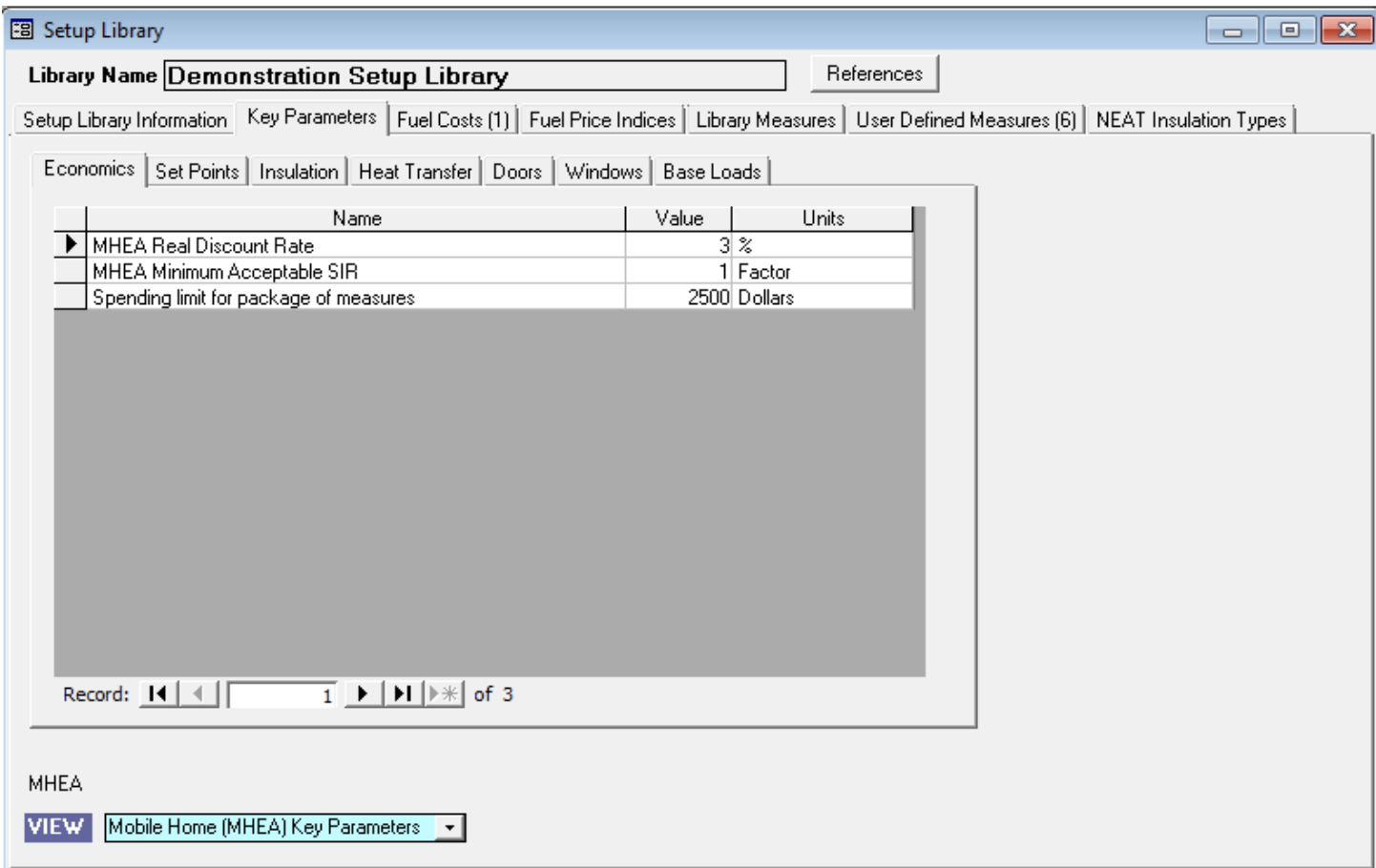

Figure 14.7. The MHEA Key Parameters - Economics form under the Main Menu's “Setup Library” button.

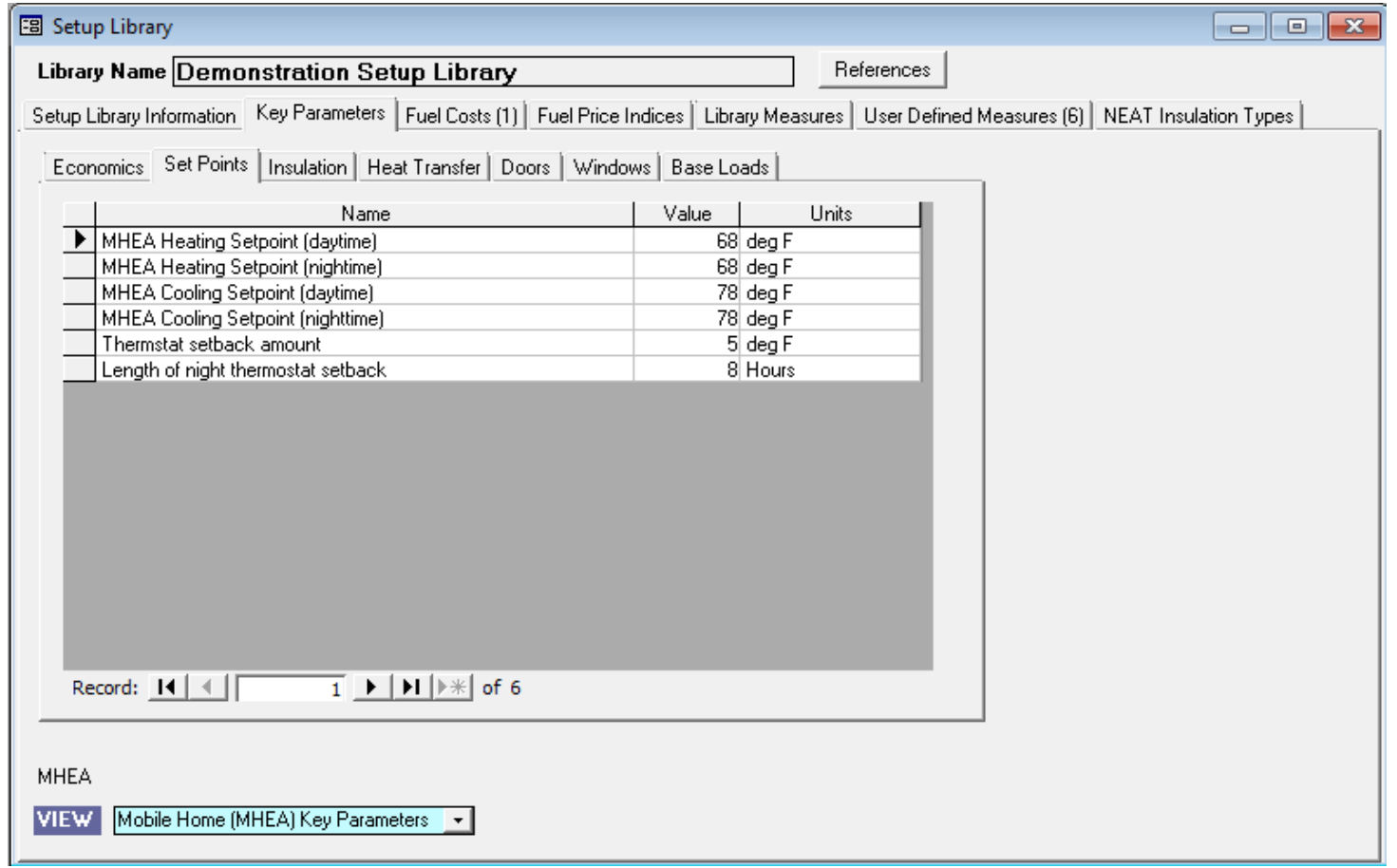

Figure 14.8. The MHEA Key Parameters - Set Points form under the Main Menu's “Setup Library” button. 


\section{Insulation}

- Bag size for loose cellulose and fiberglass insulation (lb) - The weight of the bags of cellulose and fiberglass insulation purchased to perform the insulation retrofits. These values are used with the Installed Density entries above to determine the number of bags of insulation necessary to perform the associated insulation retrofits.

- Density of added loose cellulose and fiberglass insulation $\left(\mathbf{l b} / \mathbf{f t}^{3}\right)$ - The anticipated density of cellulose and fiberglass insulation to be installed. These values are used with the Bag Size entries that follow to determine the number of bags of insulation necessary to perform the associated insulation retrofits.

- Interior wall R-value: Summer, Winter (h-ft $\left.{ }^{2}-\mathbf{F} / \mathbf{B t u}\right)$ - The R-value of the interior wall components, excluding insulation, for the summer and winter seasons. The wall components may simply include the interior wall material and the surface resistance of the air. Refer to literature related to approximating $\mathrm{R}$-values of building components for further detail.

- Interior ceiling R-value: Summer, Winter (h-ft' $\left.{ }^{2}-\mathbf{F} / \mathbf{B t u}\right)$ - The R-value of the interior ceiling components, excluding insulation, for the summer and winter seasons. The ceiling components may simply include the ceiling material and the surface resistance of the air. Refer to literature related to approximating Rvalues of building components for further detail.

- Interior floor R-value: Summer, Winter (h-ft $\left.{ }^{2}-\mathbf{F} / \mathbf{B t u}\right)$ - The R-value of the interior floor components, excluding insulation, for the summer and winter seasons. The floor components may simply include the flooring material, floor covering, and the surface resistance of the air. Refer to literature related to approximating R-values of building components for further detail.

- Outside wall R-value: Summer, Winter (h-ft $\left.{ }^{2}-\mathbf{F} / \mathbf{B t u}\right)$ - The R-value of the outside wall components for the summer and winter seasons. The wall components may simply include the exterior wall material and the surface resistance of the air. Refer to literature related to approximating R-values of building components for further detail. 


\section{Chapter 14: Setup Library}

- Existing Batt/Blanket, Loose, Rigid, Foamcore insulation R-value per inch (h-ft ${ }^{2}$-F/Btu-in) - The R-value per inch for the insulation to be used in MHEA energy calculations. This value is used to evaluate the insulating effect of existing insulation described on the input forms as well as the insulating effect of new insulation added during weatherization. Note that rigid insulation is typically found in the roof section and that foamcore insulation is typically found in the wall sections of the manufactured home.

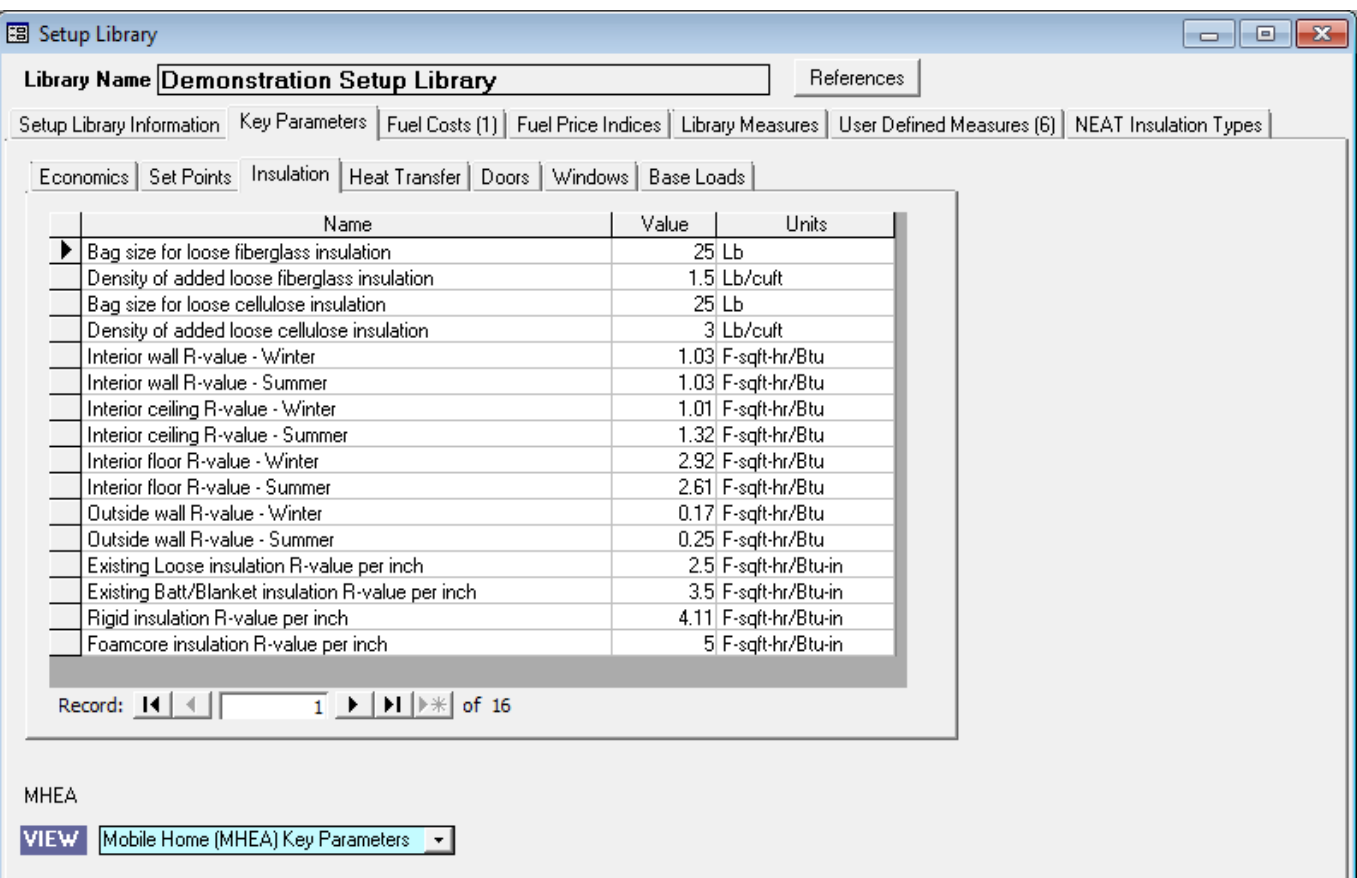

Figure 14.9. The MHEA Key Parameters - Insulation form under the Main Menu's “Setup Library” button.

\section{Heat Transfer}

- Home leakiness - Tight, Medium, and Loose (cfm) - The pre-retrofit blower door readings in CFM at 50 Pa pressure differential which are associated with the selection of Home Leakiness on the Audit Information form of the MHEA audit (see "Home Leakiness" in Section 8.2, Audit Information). The values are used if none are entered for "Before Weatherization (Existing) Air Leakage Rate and House Pressure" on the Air and Duct Leakages form of the audit (see Section 11.2, Ducts and Infiltration - Air and Duct Leakages). 


\section{Chapter 14: Setup Library}

- Free heat from interior sources: Day, Night (Btu/h) - The heat released into a home from activities taking place within the home, including cooking, hot water use, appliance use, and lighting. MHEA assumes daytime hours of 8:00 AM through 8:00 PM with the remaining hours considered to be during the nighttime.

- Duct-sealing and Duct insulation distribution loss reduction (\%) - These parameters are no longer used in the program. Duct leakage measurements are taken from the forms used to evaluate duct sealing efforts (see Section 11.2, Ducts and Infiltration - Air and Duct Leakages).

- Evaporative cooler actual saturating efficiency (\%) - The saturating efficiency of the evaporative cooler. Refer to manufacturer's literature or literature describing evaporative cooler operation for actual saturating efficiency values.

- Saturating efficiency for evaporative tune-up (\%) - The saturating efficiency after a tune-up has been performed on an evaporative cooler.

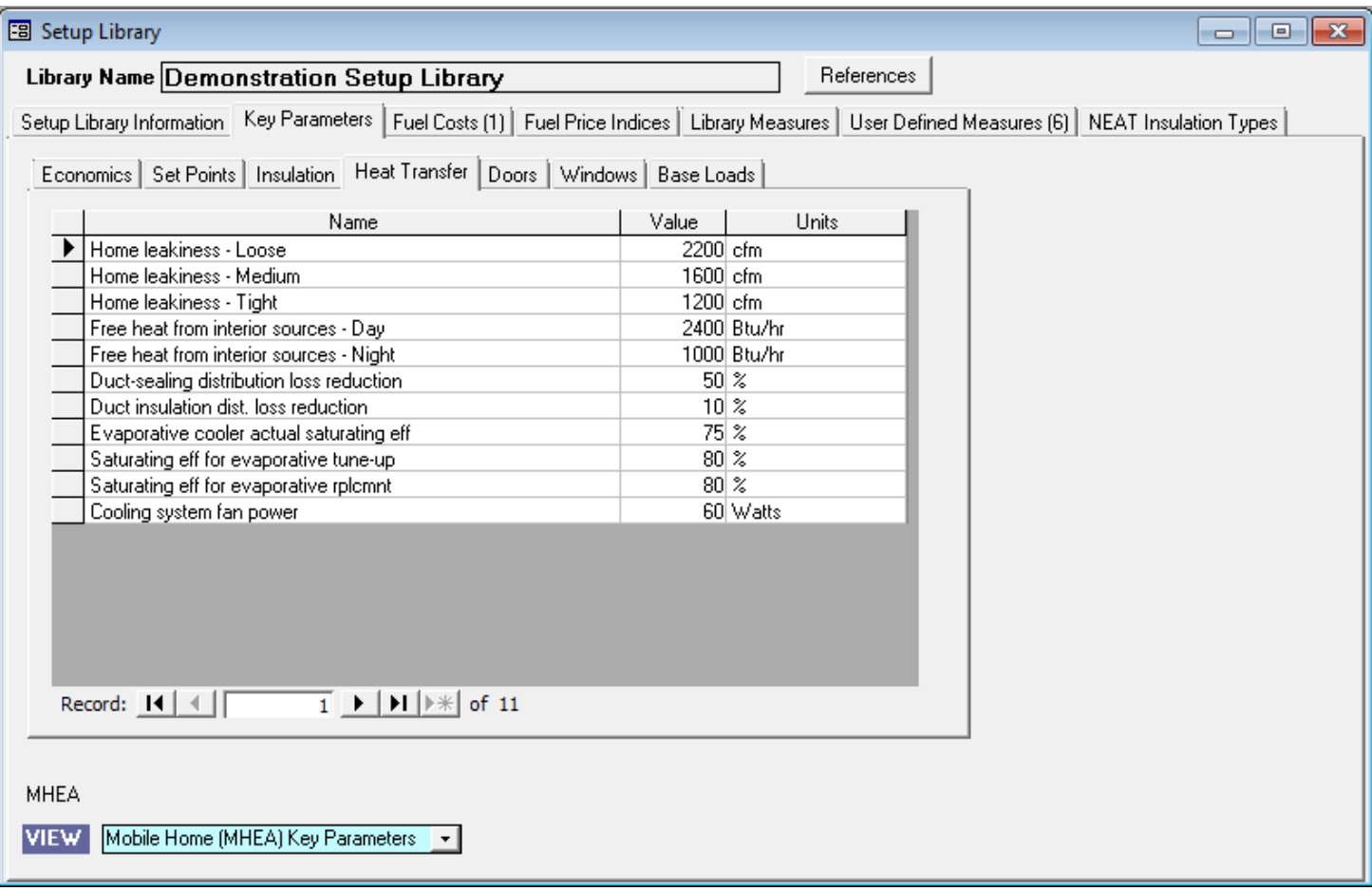

Figure 14.10. The MHEA Key Parameters - Heat Transfer form under the Main Menu's “Setup Library” button. 
- Saturating efficiency for evaporative replacement (\%) - The saturating efficiency of a replacement evaporative cooler. The manufacturer's literature should help indicate the new evaporative cooler saturating efficiency.

- Cooling system fan power (Watts) - The fan power of the cooling system fan. This value is used in the equipment cooling capacity and equipment energy consumption calculations.

\section{Doors}

- Door U-value: wood with solid core, wood with hollow core, standard manufactured home door (Btu/h-ft' $\left.{ }^{2}-\mathbf{F}\right)$ - The U-value for each type of door. These values are used to evaluate the insulating effect of the existing doors described on the input screens. Note that MHEA assumes a standard manufactured home door has a solid core and a vinyl or fiberglass skin.

- U-value of replacement door (Btu/h-ft $\left.{ }^{2}-\mathbf{F}\right)$ - The U-value for a replacement manufactured home door. This value is used to evaluate the insulating effect of the replacement doors.

\section{Windows}

- Window U-value: Winter, Summer; single pane, double pane (Btu/h-ft $\left.{ }^{2}-\mathrm{F}\right)$ - Enter the U-value of single and double pane windows with no storm windows for the summer and winter seasons. Refer to manufacturer's literature or literature related to approximating window U-values for further detail.

- Window U-value with glass storm: Winter, Summer; single pane, double pane (Btu/h-ft' $\left.{ }^{2}-\mathbf{F}\right)$ - The U-value of single and double pane windows with a glass storm for the summer and winter seasons. Refer to manufacturer's literature or literature related to approximating window $U$-values for further detail.

- Window U-value with plastic storm: Winter, Summer; single pane, double pane (Btu/h-ft $\left.{ }^{2}-\mathbf{F}\right)$ - The U-value of single and double pane windows with a plastic storm for the summer and winter seasons. Refer to manufacturer's literature or literature related to approximating window U-values for further detail. 
- Skylight U-value: Winter, Summer; single pane, double pane (Btu/h- $\left.\mathrm{ft}^{2}-\mathrm{F}\right)$

- The U-value of single and double pane skylights with no storms for the summer and winter seasons. Refer to manufacturer's literature or literature related to approximating skylight $\mathrm{U}$-values for further detail.

- Skylight U-value with glass storm: Winter, Summer; single pane, double pane (Btu/h-ft $\left.{ }^{2}-\mathbf{F}\right)$ - The U-value of single and double pane skylights with a glass storm for the summer and winter seasons. Refer to manufacturer's literature or literature related to approximating skylight U-values for further detail.

- Skylight U-value with plastic storm: Winter, Summer; single pane, double pane (Btu/h-ft $\left.{ }^{2}-\mathbf{F}\right)$ - The U-value of the skylights with a plastic storm for the summer and winter seasons. Refer to manufacturer's literature or literature related to approximating skylight $\mathrm{U}$-values for further detail.

- Window shading R-value: drapes, blinds or shades, drapes and shades (h$\mathbf{f t}^{2}$-F/Btu) - The R-value of the interior window covering. Refer to manufacturer's literature or literature related to approximating R-values of interior window shading devices for further detail.

- Sun screen solar transmittance reduction: Winter, Summer (\%) - The percent reduction of solar transmittance in the winter and summer due to an exterior window sun screen. The solar transmittance is the fraction of solar energy that travels through the window into the living space of the manufactured home. Refer to manufacturer's literature or literature related to sun screen solar transmittance for further detail.

- Ratio of awning depth to window height - The ratio of the exterior window awning depth to the height of the window (depth/height). The depth of the awning is the distance the awning extends from the exterior of the manufactured home. This value is used to calculate the shading effect of the awning on the window. 


\section{Chapter 14: Setup Library}

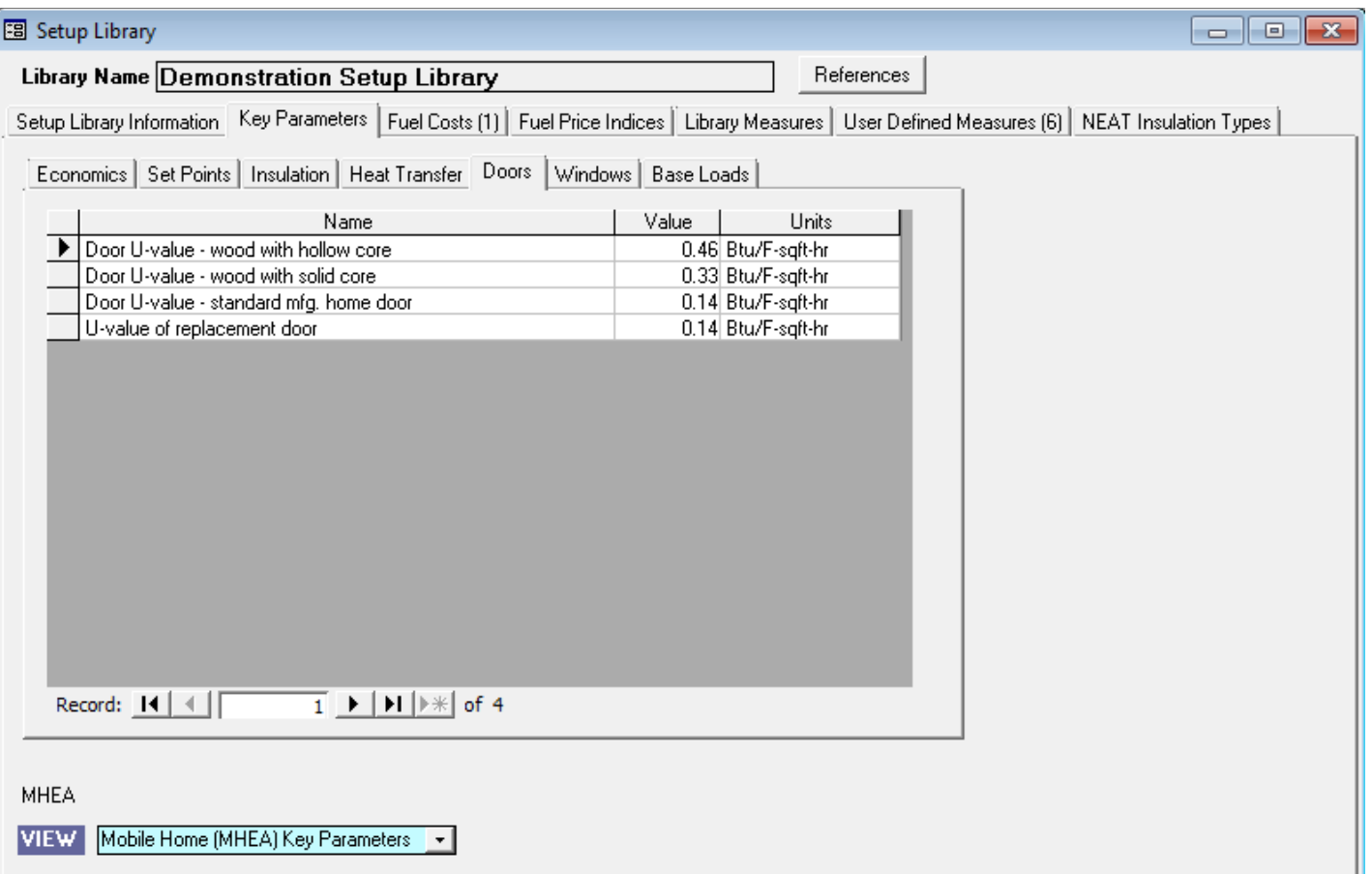

Figure 14.11. The MHEA Key Parameters - Doors form under the Main Menu's “Setup Library” button.

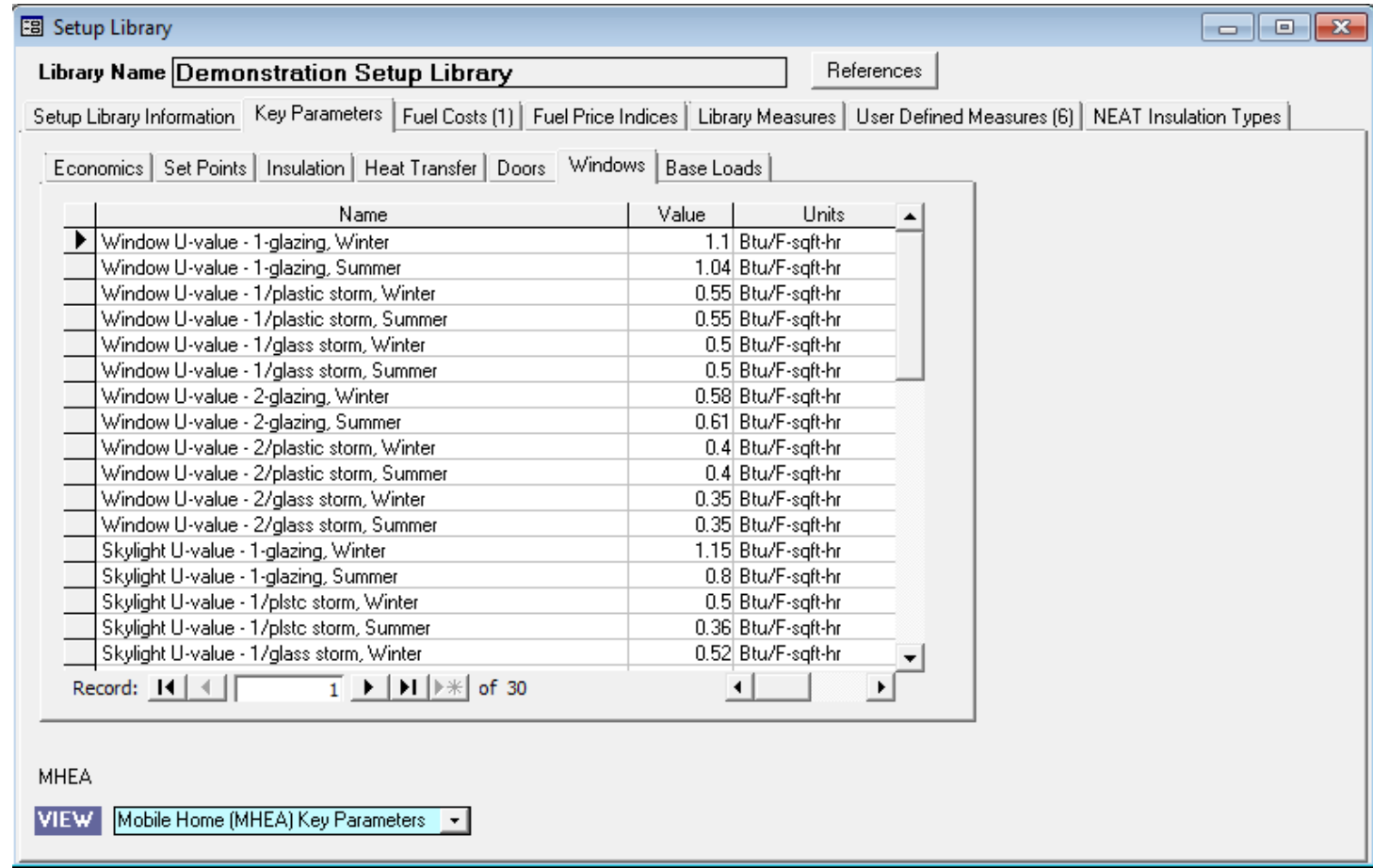

Figure 14.12. The MHEA Key Parameters - Windows form under the Main Menu's “Setup Library” button. 


\section{Chapter 14: Setup Library}

\section{Base Loads}

- MHEA Low-flow shower head flow rate (gal/min) - The flow rate in gallons per minute of replacement shower heads used in your program.

- MHEA Water heater wrap added R-value (h-ft $\left.{ }^{2}-\mathbf{F} / \mathbf{B t u}\right)$ - The R-value of insulation used to wrap water heaters in the Water Heater Tank Insulation measure.

- MHEA Refrigerator defrost cycle energy (kWh) - The electrical energy in $\mathrm{kWh}$ assumed consumed by the existing refrigerator's defrost cycle. The value is used to adjust the metered consumption of the refrigerator if the user indicates that the metering period included a defrost cycle.

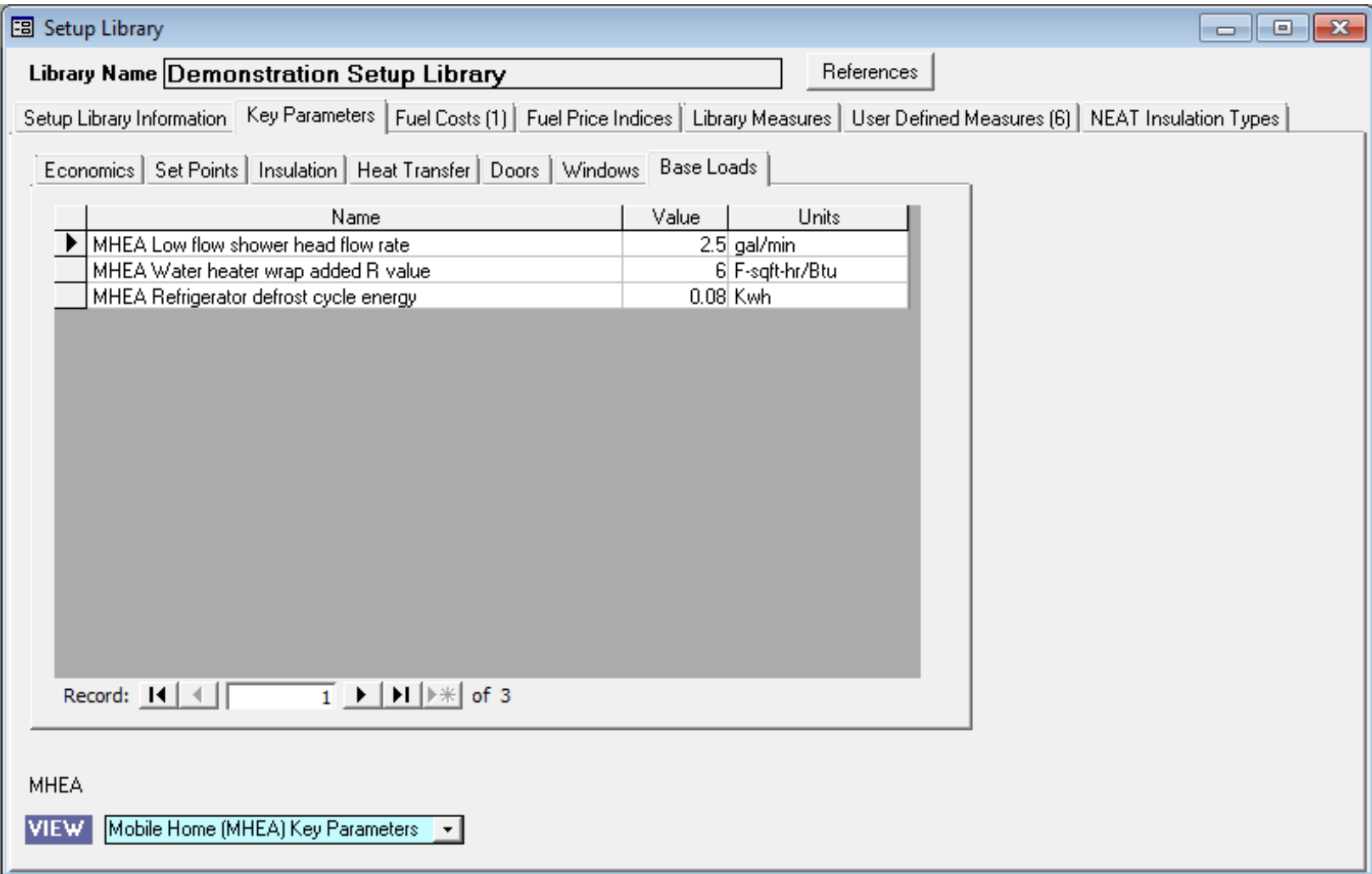

Figure 14.13. The MHEA Key Parameters - Base Loads form under the Main Menu's "Setup Library" button. 


\section{Chapter 14: Setup Library}

\subsection{Fuel Costs}

The Fuel Costs form (see Figure 14.14) under the Main Menu's "Setup Library" button is used to record the average fuel prices in your agency's area. The prices will be used in any NEAT or MHEA audit that specifies use of the Setup Library and the Fuel Cost Library within a Setup Library (see "Libraries and Other Options Data Block" under Section 8.2, Audit Information). Since fuel costs vary widely throughout the United States, the values in the "Default Costs" Fuel Cost Library, installed with the program, will likely not reflect costs in your location and should be changed (see "Populating the Setup and Supply Libraries" in Section 3.2, Program Setup, and the "Unit Cost" field description below). Check the costs of fuels in your area at least once a year and update the costs, if necessary. Fuel costs should be typical—avoid high or low short-term values.

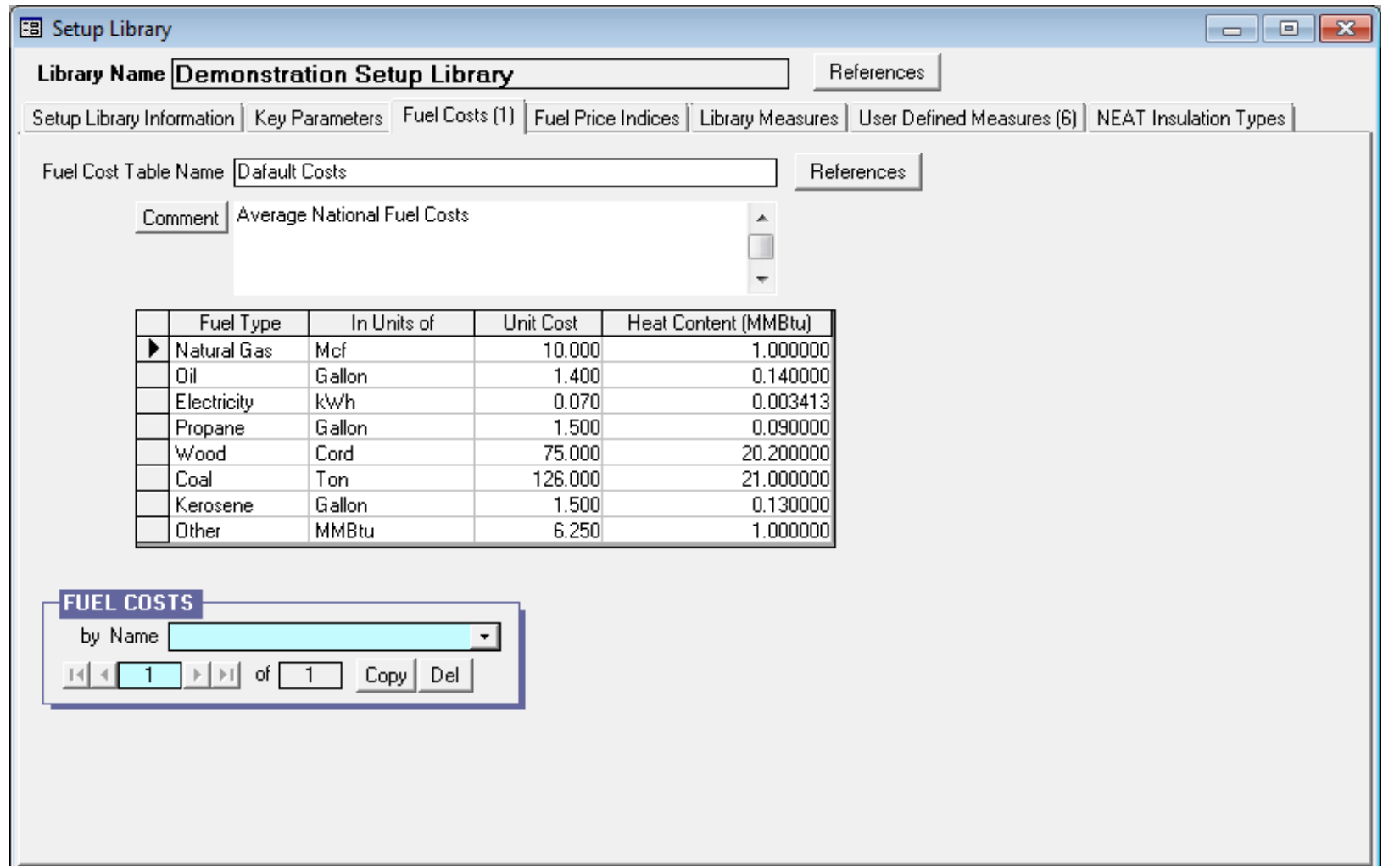

Figure 14.14. The Fuel Costs form under the Main Menu's “Setup Library” button.

You may have multiple fuel cost libraries in any given setup library, but each must contain prices for all fuels. Thus, if your area is served by two different natural gas companies and two different electric companies, in order to account for all 
possibilities of service from these four companies, you would need four different fuel cost libraries. As the number of service companies increases, the number of fuel cost libraries needed to cover all possibilities can become unmanageable. Under these circumstances, it is recommended that you attempt to average prices for a given fuel from providers whose prices do not differ significantly. Some states have decided to use state-wide average fuel costs in order to ensure equity among all of its clients.

Below are descriptions of the individual data items and controls found under the Fuel Costs form and the associated Fuel Costs Table.

- Fuel Cost Table Name - Use the Fuel Cost Table Name to identify the applicability of this particular set of fuel prices, particularly if you have multiple sets. The Name may be 80 characters or less.

- References - Selecting this button will display the "References to Fuel Cost Table" window, which lists all the references to the current fuel cost table. You may clear the references to the current fuel cost table or change the references to any other fuel cost table you have already created under the currently accessed setup library. See Section 5.14, The References Button, for more information on the use of this feature.

- Fuel Cost Record Navigation Block - The Fuel Cost record navigation block is used to find and navigate to existing fuel cost tables under the currently accessed setup library, copy or delete the current fuel cost table, or create a new fuel cost table. See Section 5.1, Form and Record Navigation, for information on using the Weatherization Assistant's record navigation blocks.

- Comment - You may enter comments pertinent to the current fuel cost table directly in the Comment field on the form, or you may enter them in the Comment Editor by selecting the "Comment" button to the left of the field. The Comment Editor is similar to Window's Notepad and may be used to enter extended comments. The total comment may have up to 65,000 characters. Text from other sources may be copied and pasted into the Comment Editor's window. If you have multiple fuel cost tables, use this Comment field to describe how this fuel cost table differs from the others. Optional. 


\section{The Fuel Cost Table}

- Fuel Type - This column lists all fuel types considered in the Weatherization Assistant. These include: Natural Gas, Oil, Electricity, Propane, Wood, Coal, Kerosene, and Other. You may use "Other" by providing the unit cost and heat content of a fuel not listed. Uneditable.

- In Units of - This field shows the unit in which the fuel cost is specified for different fuel types. These include: Mcf (1,000 cubic feet) for natural gas, gallons for \#2 oil, kWh for electricity, gallons for propane, cords for wood, tons for coal, and gallons for kerosene, and MMBtu (million British thermal units) for other. Uneditable.

- Unit Cost - The fuel price in dollars per unit of fuel given in the previous column. If you obtain information regarding fuel prices from a fuel bill or communication with a utility company, you may not be able to immediately obtain the appropriate values for use in the Weatherization Assistant. First, note carefully the units that are given for the prices from any source. If they do not match the units required by the Weatherization Assistant, as given in the "In Units of" field, you will have to convert the values. The most common need for conversion is seen in the prices given for natural gas. The Weatherization Assistant requires the natural gas price to be in \$/Mcf (dollars per thousand cubic feet of gas). Use the following conversions if the natural gas price is given in different units:

Multiply \$/MBtu by 1.025 to obtain the cost in units of \$/Mcf. Multiply $\$$ /therm by 10.25 to obtain the cost in units of $\$ /$ Mcf. Multiply $\$ /$ ccf by 10 to obtain the cost in units of $\$ /$ Mcf.

Particularly if you take a fuel price from a utility bill, you may have to account for fixed charges. The prices required by the Weatherization Assistant must exclude any fixed charges, such as a $\$ 2.00$ per month fee for bill processing. However, if the fee is charged per unit of consumption, such as a delivery cost for propane in dollars per gallon, then this cost should be included in the price used in the Weatherization Assistant.

You may experience situations where the price per unit depends on the total amount consumed each month, sometimes referred to as "tiered" rates. The fuel 
price to be used in the Weatherization Assistant should be the price per unit from the highest tier normally encountered by your clients. Or, you may find seasonal rates where the price per unit is different during the winter versus the summer. If the fuel for which you encounter this situation is used predominately during one of the seasons, use the price for that season. If you feel that the fuel is used almost equally during both seasons, take an average of the prices.

- Heat Content -The number of MMBtu (million British thermal units) derived from burning one unit of the fuel. If you have information providing more accurate heat contents than listed for the fuels you commonly encounter, enter these values here. Some natural gas companies post the heat content of their gas on their bills or would certainly be able to give you an average if contacted. They do vary, but normally not more than a few percent.

\subsection{Fuel Price Indices}

The Fuel Price Indices form (see Figure 14.15) under the Main Menu's "Setup Library" button specifies the fuel price indices, which are used to adjust the dollar savings of measures to account for anticipated changes in fuel prices over their life. Those distributed with the program and viewed under this tab are national average indices, published annually by the Department of Commerce, for the year closest to program's release (2011 for Version 8.9.0.5). Weatherization Program rules require these indices to be updated at least every five years. Thus, unless your release of the program is more than five years old, you do not need to make any changes in these entries.

- Fuel Type - This column lists all fuel types considered in the Weatherization Assistant. These include: Natural Gas, Oil, Electricity, Propane, Wood, Coal, Kerosene, and Other. The "Other" fuel type corresponds to the "Other" fuel type found under the Fuel Costs form and can be used for a fuel not otherwise listed. Uneditable.

- Year - This column displays the year beyond the current year for which the Price Index corresponds. Uneditable. 


\section{Chapter 14: Setup Library}

圆 Setup Library

ㅁ $\quad x$

Library Name Demonstration Setup Library

References

Setup Library Information | Key Parameters $\mid$ Fuel Costs (1) Fuel Price Indices |Library Measures | User Defined Measures (6) | NEAT Insulation Types |

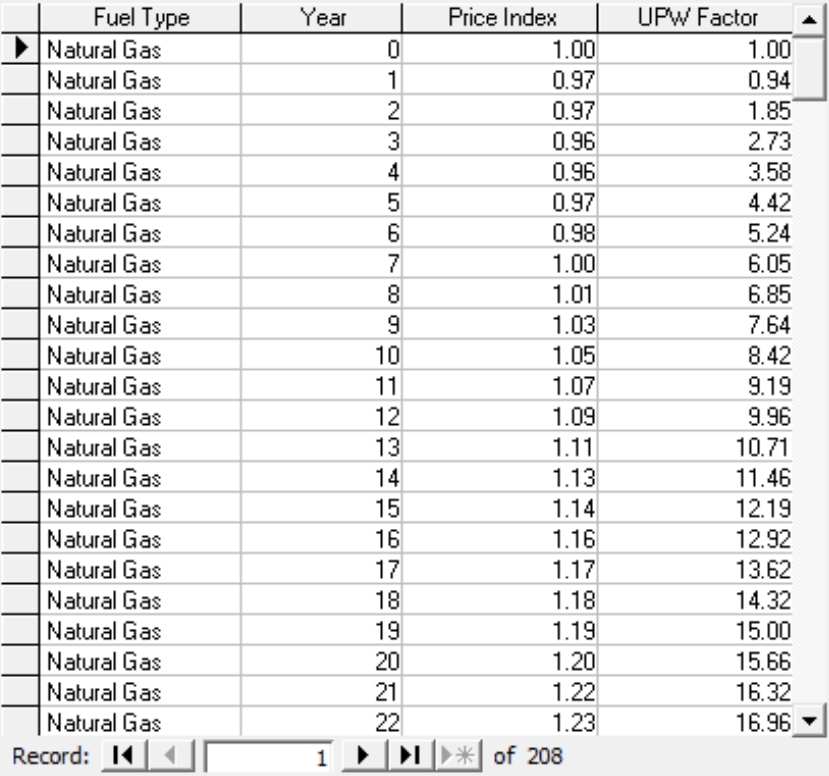

Figure 14.15. The Fuel Price Indices form under the Main Menu’s “Setup Library” button.

- Price Index - This column displays the projected Fuel Price Indices, as taken from the Department of Commerce publication. Those distributed with the Weatherization Assistant are national average indices, even though the Department of Commerce also publishes separate indices for the four U.S. census regions.

- UPW Factor - The Uniform Present Worth (UPW) factor is computed from the Fuel Price Indices, described above, and the "Real discount rate," entered on the Key Parameters/Economics form. The values give the factor by which the first year's dollar savings of a measure is to be multiplied to obtain its "discounted" lifetime dollar savings. The discounted lifetime dollar savings of a measure is the value which, when divided by the cost of installing the measure, gives the savings-to-investment ratio (SIR) reported by NEAT and MHEA. Uneditable. 


\section{Chapter 14: Setup Library}

\subsection{Library Measures}

The Library Measures form (see Figure 14.16) is used to enter information about the measures you want NEAT and MHEA to consider while evaluating an audit. The "Library" measures are those which have been programmed into NEAT and MHEA, as opposed to any user-defined measures you may have generated (see Section 14.6, User Defined Measures). Both NEAT and MHEA measures are accessed under the same Library Measures tab in the "Setup Library" window. Use the "View" drop-down list at the lower left of the form to choose which program's measures you wish to display.

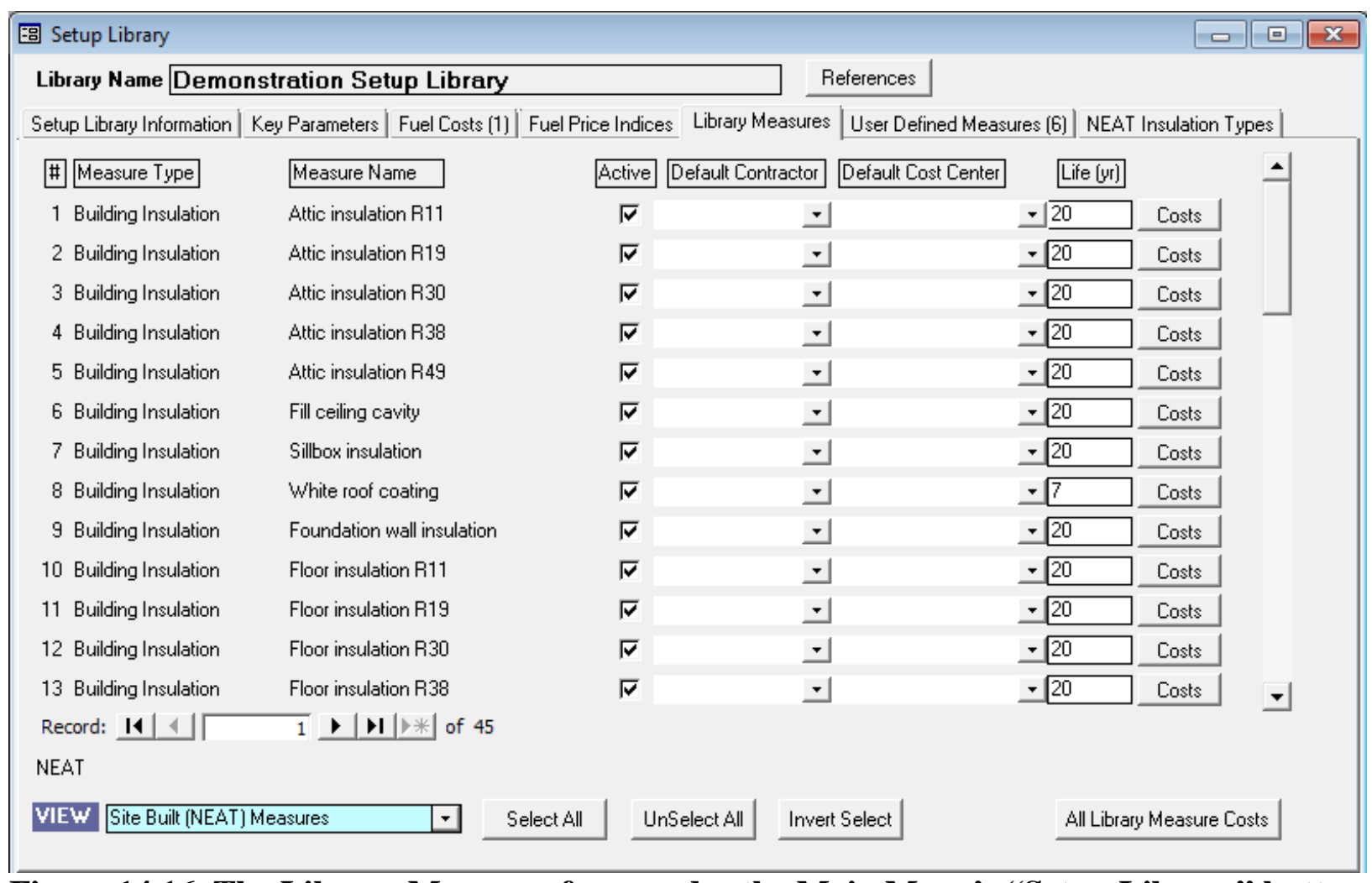

Figure 14.16. The Library Measures form under the Main Menu’s “Setup Library” button.

Below are descriptions of the individual data items and controls found on the Library Measures form.

- Measure Type - This field displays the type of measure assigned to the NEAT and MHEA library measures. Examples are "Building Insulation" and "HVAC Systems.” The measure type does not appear on any report. However, if a work 
order is initiated from a NEAT or MHEA audit, the measure types for the transferred library measures are displayed on the Measures form under the Main Menu's "Work Orders" button (see "Measure Type" in Section 13.3, Measures (Work Order)), where they can be used to sort the measure list when the Measures form is viewed in the data sheet view. Uneditable.

- Measure Name - This is the name the library measure will be identified by in all locations throughout the program, including audits, work orders, and all reports that might be printed. Note that the measure names of similar measures may differ in NEAT versus MHEA. Uneditable.

- Active - Selection of this checkbox indicates your desire that the program consider this measure when forming its recommendations. Even if selected here, however, a measure will not be recommended unless it is cost-effective or is a measure that has been declared mandatory and is so designated within a specific audit. The "Select All," "UnSelect All," and "Invert Select” buttons at the bottom of the form allow you to make all of the measures active, deactivate (unselect) all measures, or invert the selection currently seen from the checkboxes.

- Default Contractor - The Weatherization Assistant gives you the optional feature of designating the contractor who will install each measure. Measures recommended for the same dwelling but assigned different contractors will be automatically added to different work orders, if the work order feature has been implemented (see Chapter 13, Work Orders). The "Default Contractor" dropdown list allows you to assign a default contractor for each measure. This assignment can be changed either when the measure is recommended (see "Contractor" in Section 11.11, Measures (Audit)) or when assigned to a specific work order (see "Contractor/Crew" in Section 13.1, Work Order Information). Thus an entry here does not commit you to retain the assignment, but simply may reduce your effort making such assignments later.

The choices available from the drop-down list will include only those agency contacts declared as "Contractors" or "Crew" under the Contacts tab of the Main Menu's “Agency” button for the agency associated with this Setup Library (see Section 6.2, Contacts (Agency)). Optional. 
- Default Cost Center - The Weatherization Assistant gives you the option to attribute costs incurred in installing measures to different Cost Centers or funding sources. With this, and additional information, the program can provide you with a record of income and expenditures by cost center (see Section 6.3, Cost Centers). The "Default Cost Center" drop-down list allows you to assign a default cost center for each measure. This assignment can be changed either when the measure is recommended (see "Cost Center" in Section 11.11, Measures (Audit)) or when assigned to a specific work order (see "Cost Center" in 13.3, Measures (Work Order)). Thus an entry here does not commit you to retain the assignment, but may reduce your effort making such assignments later.

The choices available from the drop-down list will include only those entries made under the Cost Center tab of the Main Menu's "Agency” button for the agency associated with this Setup Library (see Section 6.3, Cost Centers). Cost Center assignments need to be made only if you are tracking funds within the Weatherization Assistant. Optional.

- Life - NEAT and MHEA allow you to change the "Life" of each measure. The lifetimes in the programs as shipped reflect industry standards and will likely be sufficient for your use. However, if you have documented cause to alter them, you may do so on this form. For some measures, the life may depend on the specific material being used in your program. Note that lifetimes for "Lighting retrofits" are in thousands of hours burn time for the replacement compact fluorescent, not years. Required.

- Costs - Each measure listed on the Library Measures form has a "Costs" button on the right side of the form. Selecting this button for a measure will take you to the Unit Costs of Measure sub-form (see Figure 14.17), where the cost components for implementing the measure can be entered. The exceptions are the measures for replacing heating systems, cooling systems (only in MHEA), water heaters, and refrigerators. These measures require their costs to be entered on the audit forms. NEAT and MHEA use these costs for evaluating the costeffectiveness of measures. These costs can be transferred to work orders. However, they may not represent the actual cost or detail cost breakup you may wish to have for the work orders. Therefore, they may be adjusted in the work orders (see "Estimated/Actual Costs and SIR" in Section 13.3, Measures (Work Order)). 
On this form, the cost components for a measure are restricted to three entries: Material (or Insulation), Labor, and Other. Most of the insulation measures, cooling system, and lighting retrofit measures have multiple materials that may be used in installing the measure. In such instances, each material will have its own set of Material, Labor, and Other cost components.

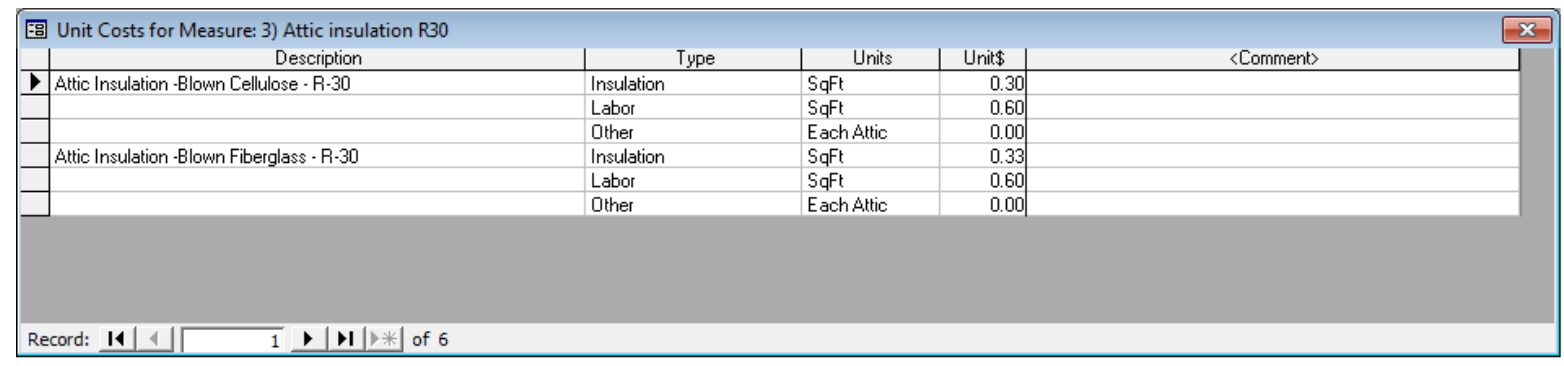

Figure 14.17. The Unit Costs of Measure sub-form for the Attic Insulation R-30 measure.

Only the Unit\$ and the Comment fields are editable. Enter into the Unit\$ column the dollar cost per "Unit" for installing the material. Separate entries may be made for the dollar cost per unit to purchase the material and the dollar cost per unit for labor to install the material. Both NEAT and MHEA simply add these two quantities together (they always have the same "Units" associated with them) in determining the cost of installing the measure.

The third component to the cost for each material, "Other," will likely have different units than the material and labor components. Entry in this column may be handled differently in NEAT versus MHEA and may not be appropriate for many measures in NEAT. For example, an "Other" cost for an attic insulation measure in NEAT has units of "Each Attic," implying that this dollar cost will be added to the cost of insulating each individual attic segment, not to the total cost for insulating all of the attics in an entire dwelling. Only in the case of a home having a single attic segment qualifying for insulation would the entry have the likely intended purpose of a setup cost for equipment necessary to install the insulation. MHEA does not have the option to describe multiple attic segments for the mobile home, so doesn't suffer from this problem. The "Other" cost column in NEAT is more appropriately used for measures such as window treatments where you have an option to cost the measure per window ("Each") or per square foot of window area. Paying close attention to the "Units" of the "Other" cost component will assist you in deciding when it is appropriate to use this entry for any specific measure. 
The entries in the Unit\$ column are required, though they are permitted to be zero, as long as no material has zero cost per unit for all three components. The total cost for a measure will be the sum of the costs from the three components, using the quantities in the units indicated from the specific audit being evaluated.

The Comment field is a standard comment field capable of storing 65,000 characters or less, even though only a few of these would be visible in this data sheet view. However, by double clicking your mouse on a particular Comment field, the Comment Editor window will open allowing better entry and viewing of longer comments. The comments entered here for each component of a measure cost will be copied to the component costs displayed for each measure seen using the "Costs" button on the Measures form under the Main Menu's "NEAT Audit" or "MHEA Audit" buttons if the measure gets recommended (see Section 11.11, Measures (Audit)). Also, the comment will be available on any work order that includes the measure using the material (see "Show/Hide Audit Material Detail” and "Create Materials Using Audit Detail” in Section 13.3, Measures (Work Order)).

- Record Navigation Bars - Both the Unit Costs of Measure sub-form and the Library Measures form have abbreviated record navigation bars near the lower left of the forms, allowing you to move to a desired entry. However, both of these forms will have a vertical scroll bar at the right of the entries (if necessary) allowing you to access any of its entries without having to use these navigation bars. The Unit Costs of Measure sub-form for most measures is of sufficient size such that all components associated with the measure will fit into the Costs sub-form window, eliminating the need for the scroll bar. In most cases, it will be easier to use your mouse or arrow keys on your keyboard to select a desired entry.

- All Library Measure Costs - Selecting the “All Library Measure Costs” button at the lower right of the Library Measures form presents you with a form view of all measures' costing components in a single window. In the standard setup of Version 8.9, there are a total of 332 costing components. However, this number will increase if you define additional NEAT insulation types (see Section 14.7, NEAT Insulation Types). Vertical scrolling will be necessary to see all of these components. This format may not be the most favorable for editing the costs. However, one key advantage of the form is that it allows you 
to quickly copy the cost table or any portion of the table to another location for printing or electronic mailing purposes (see Section 5.9, Copying and Pasting Data).

\subsection{User Defined Measures}

The User Defined Measures form under the Main Menu's "Setup Library” button (see Figure 14.18) can be used to predefine weatherization activities that are not addressed within the library measures but commonly encountered during an audit. Defining these measures on this form allows you to copy them to any audit as an itemized cost (see Section 11.8, Itemized Costs) or to a work order (see "Copy from User-Defined Measures” in Section 13.3, Measures (Work Order)).

Also accessible from this form is a library of 50 predefined health and safety measures (see Figure 14.19). These measures are not actually "user-defined" because they come with the program. Their names cannot be altered by the user and they cannot be deleted since these are measures associated with specific health and safety hazards which may be observed during an audit (see "Handling of Health and Safety Issues” under Section 11.7, Health and Safety).

Activities that you define (those other than the library health and safety measures) may or may not have energy savings associated with them. Those without energy savings are referred to as "itemized costs." They may be repair costs, administrative costs, etc. Those for which you have assigned energy savings will be considered exactly as any of the library measures defined in the program.

They will be ranked by SIR and recommended only if their SIR meets the established criteria. Additional input is required for this type of user-defined measure, as described below.

The User Defined Measures form is divided into three sections: the general task description on the top of the form, a Materials/Labor Details sub-form in the middle, and the Measures record navigation block, the Measure Comment field, the "View" drop-down list and the "All User Measures Costs" button at the very bottom of the form. The Materials/Labor Details sub-form is multi-component, allowing any number of material or labor components to be associated with each user-defined measure. 


\section{Chapter 14: Setup Library}

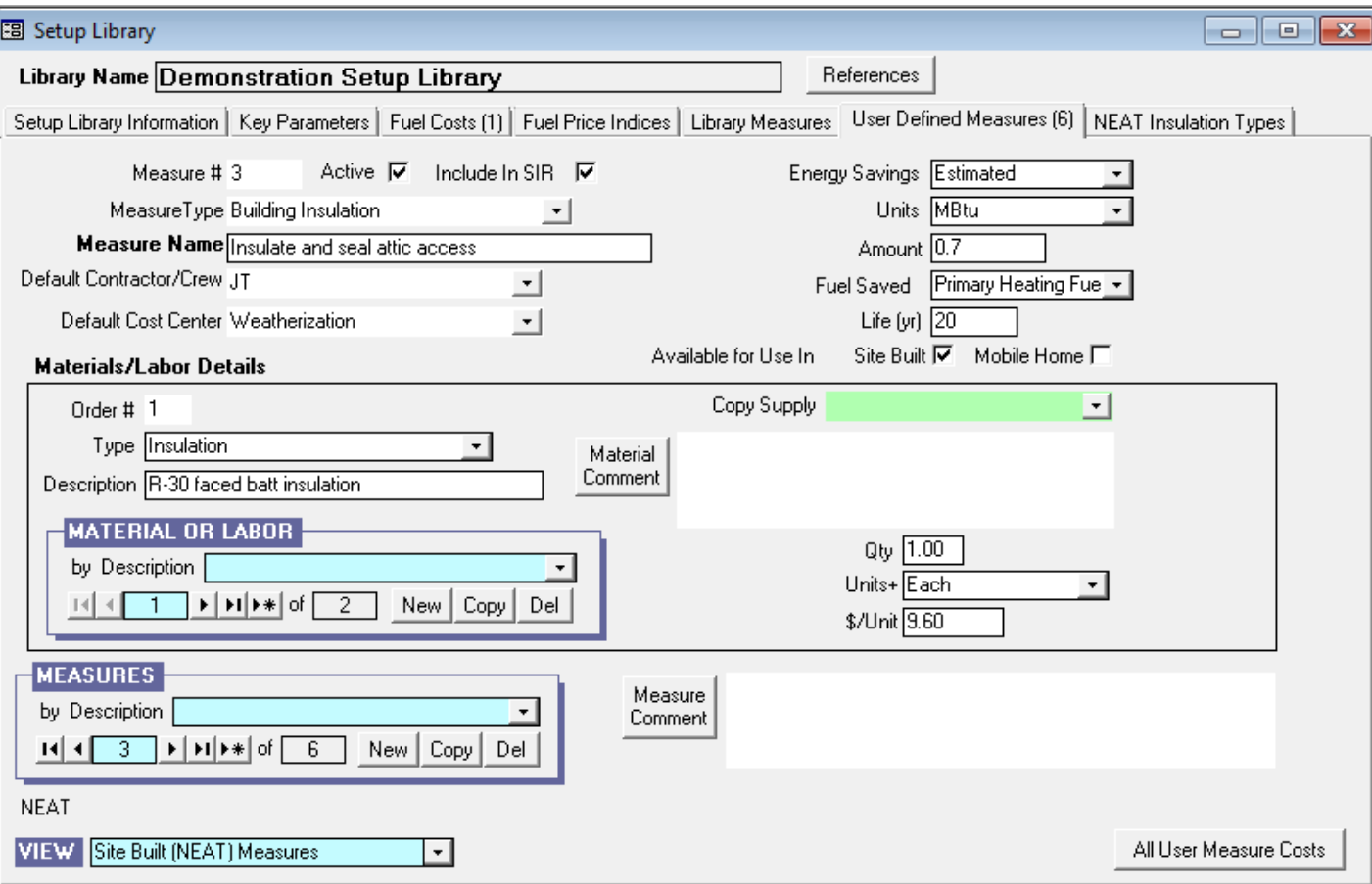

Figure 14.18. The User Defined Measures form under the Main Menu's “Setup Library” button (with energy savings, Materials/Labor Details sub-form in form view).

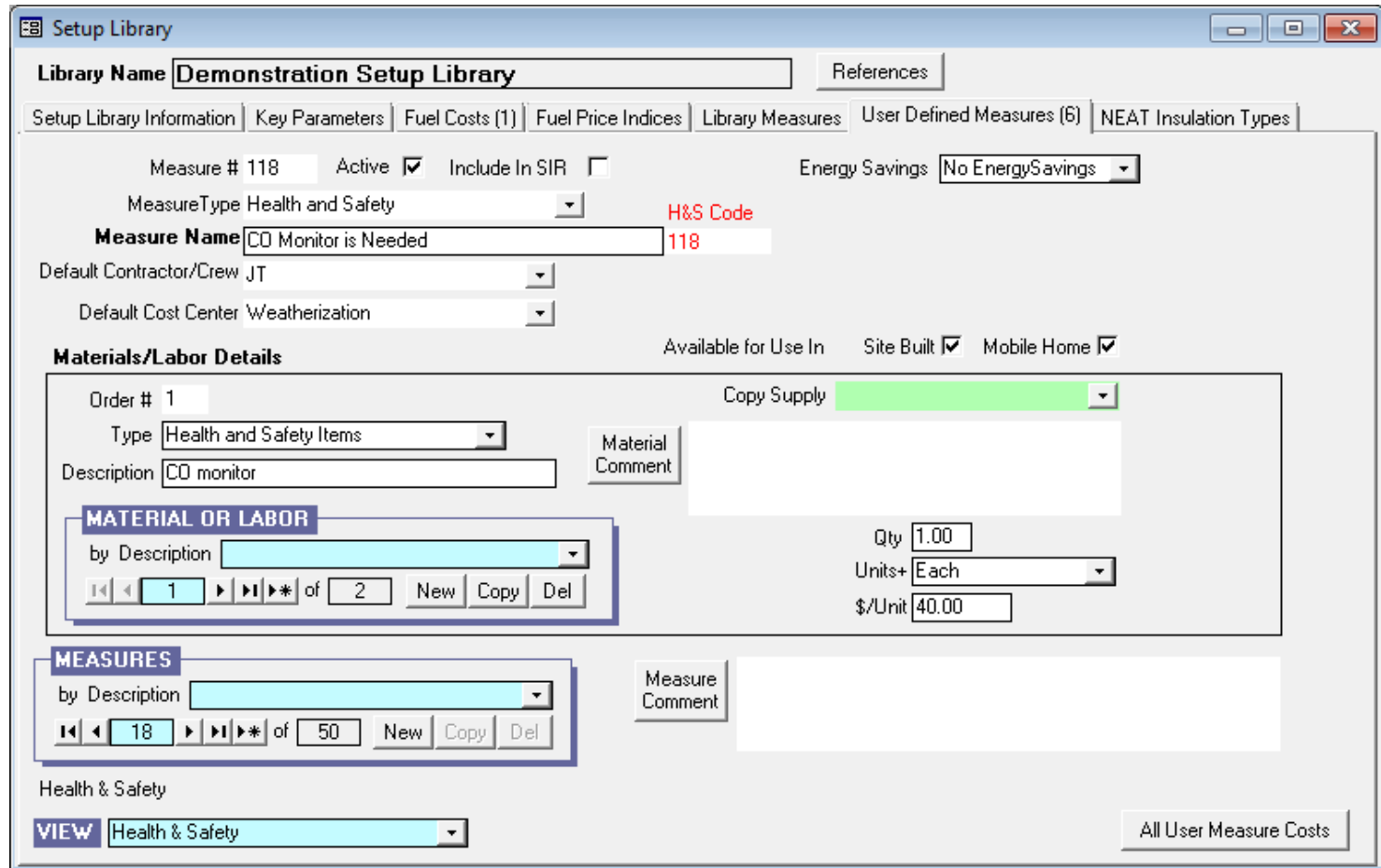

Figure 14.19. The User Defined Measures form under the Main Menu's "Setup Library" button (Health and Safety measure, Materials/Labor sub-form in data sheet view). 
The general task description area can be viewed in form view or data sheet view (Section 5.8, Data Sheet and Form Views of Records). The data sheet view (see Figure 14.20) allows you to see more of the records at one time, possibly making it easier to locate a particular record. However, the detail involved in each make it normally more feasible to make changes while in the form view. The measures displayed will be those of type selected from the "View" drop-down list.

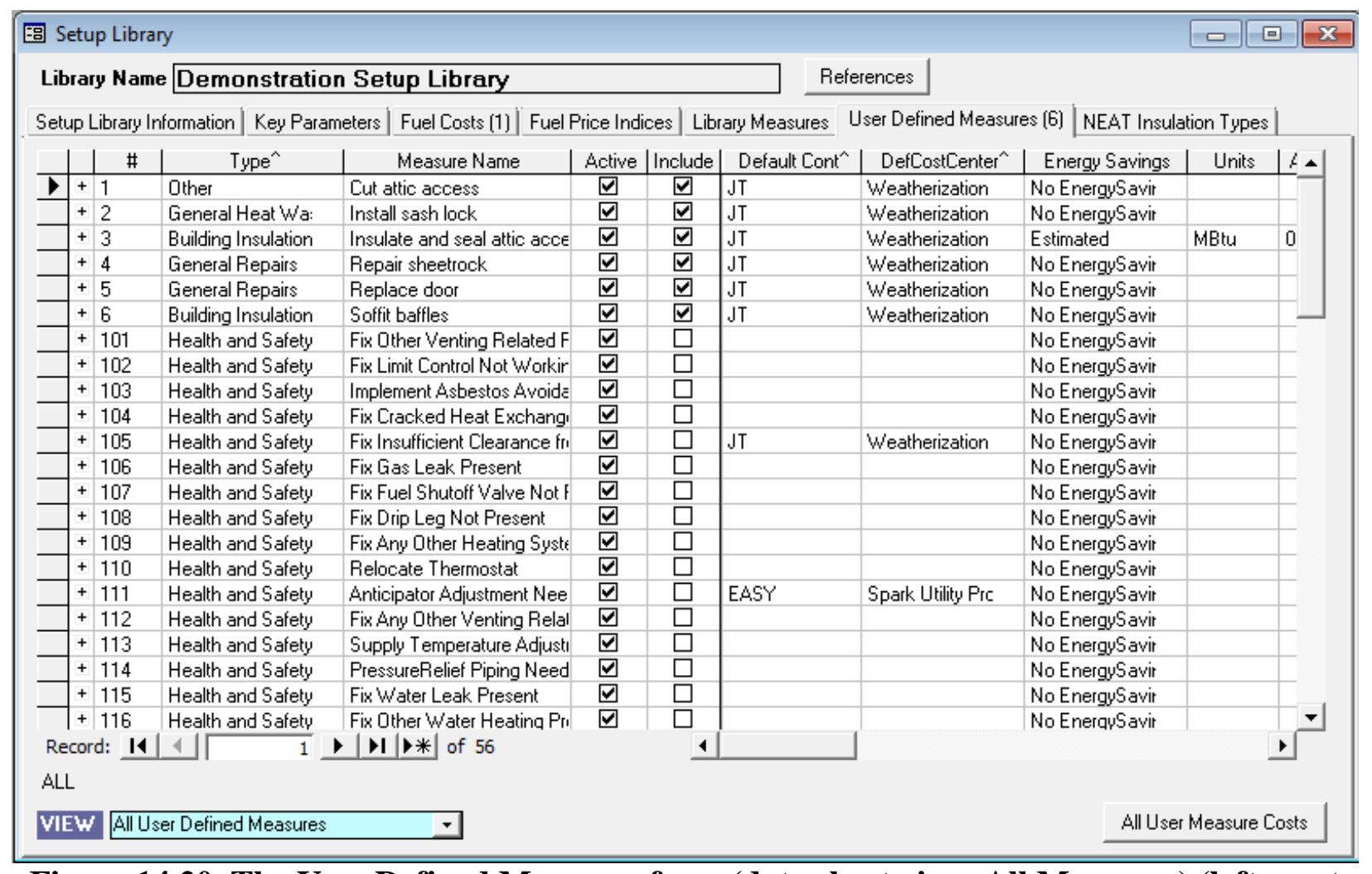

Figure 14.20. The User Defined Measures form (data sheet view, All Measures) (left-most columns).

\section{The General Description}

Below are descriptions of the individual data items and controls found in the general task description portion of the User Defined Measures form. Discussion of the Materials/Labor Details sub-form and the controls at the bottom of the form will follow. The item descriptions below are phrased as if you were entering a new user-defined measure. For any measure previously defined, the program will display those entries made at the time the measure was defined or previously modified. 
- Measure \# - If you want to prescribe the order in which the user-defined measures will be displayed in the "Measures" drop-down list on the form or when the form is displayed in data sheet view, assign a number to the measure by entering a unique integer in this field. The library health and safety measures have pre-assigned measure numbers from 101 through 150. You may change, reuse, or delete pre-assigned or new measure numbers in this field in any desired manner. However, this is not recommended for the health and safety measures. All measures with no number assigned will be listed first but in a random order amongst themselves. If a measure number is changed, you must exit the currently accessed form in order to see the measures in the altered order. When in data sheet view, the ordering of the measures may be temporarily changed using the Sort feature (see Section 5.8, Data Sheet and Form Views of Records), but this ordering will be lost once a setup library has been exited. Optional.

- Active - If selected, this checkbox makes the current measure accessible to either NEAT or MHEA audits (see "Copying a Predefined Measure from the Setup Library” in Section 11.8, Itemized Costs) and work orders (see "Copy from User Defined Measures” in Section 13.3, Measures (Work Order)). If the current measure is a library health and safety measure with this checkbox selected, the measure can be automatically added to the Itemized Costs form of a NEAT or MHEA audit if the potential health and safety hazard is identified on the Health \& Safety forms or under the Heating and Water Heating forms of the audits (see "Handling of Health and Safety Issues" under Section 11.7, Health and Safety). If this checkbox is unchecked, it would be wise to include a comment indicating the reason for excluding the measure.

- Include in SIR - Use this checkbox to indicate if you wish the cost of this measure to be included in the cumulative SIR on the Recommended Measures report (see Section 12.2, Recommended Measures Report). If checked, the measure will appear at the top of the recommended measure list and will be included in the cumulative cost and SIR for the job. If unchecked, the measure is placed at the end of the measure list after a final cumulative SIR has been displayed. In this case, the cost is added to the cumulative cost but not used to compute the cumulative SIR. You must check this checkbox for all user-defined measures having energy savings associated with them. 
- Measure Type - Select the measure type category from the drop-down list. The choices are Base Loads, Building Insulation, Client Education, Doors and Windows, General Heat Waste and Air Infiltration, General Repairs, Health and Safety, HVAC Systems, and Other. This information does not appear on any report. However, if entered, it can be used to sort measures by measure type on this form or on the Measures form of the Main Menu's "Work Orders" button (see Section 13.3, Measures (Work Order)). Optional.

- Measure Name - If you want to define a measure other than a library health and safety measure, enter a unique name for the measure in this field. A measure is identified by the measure name elsewhere in the program. The library health and safety measures already have the measure name assigned. Their names cannot be changed. If you attempt to, you will receive a message that the program can't save the record. If you are accessing one of the library health and safety measures, an uneditable field displaying the pre-assigned measure number and the text "H\&S Code" in red will appear to the right of the Measure Name field. Required.

- Default Contractor/Crew - If you wish to pre-assign a measure to a specific contractor or crew, enter this indication here. The choices in the drop-down list for this field will be those entries on the Contacts tab under the Main Menu's "Agency" button which have been designated as either "Contractor" or "Crew" (see Section 6.2, Contacts (Agency)). This information will be carried with the measure anytime it is copied to an audit, although it may then be changed. Anytime a measure is copied to a work order, it will automatically be assigned the Contractor or Crew affiliated with that work order. Optional.

- Default Cost Center - If you are tracking costs that are incurred by different funding sources or programs, the Cost Center is that source of funding to which the actual cost of implementing the measure will be charged. Cost centers are defined under the Cost Centers tab of the Main Menu's “Agency” button for your agency (see Section 6.3, Cost Centers). The cost center entered here will be carried with the measure anytime the measure is copied to an audit or work order, although it may be changed in either of these locations. Optional.

- Energy Savings - Indicate in this drop-down list whether you have an estimate of annual energy savings to associate with the user-defined measure being described. Otherwise, the record is viewed as an itemized cost without energy 
savings. Your selection here determines whether additional fields will be displayed below this one in which information necessary to define an energy efficiency measure is to be entered. NOTE: In order to have energy information associated with a user-defined measure transferred to a work order, the measure must have been implemented into either the NEAT or MHEA audit that was then used to create the work order.

- Units - If Estimated is selected in the "Energy Savings" drop-down list, the "Units" drop-down list appears. Select the units of the annual energy savings to be assigned to the measure. The choices are $\mathrm{kWh}$, MBtu (million Btu), and Therms. Required.

- Amount - If Estimated is selected in the "Energy Savings" drop-down list, the Amount field appears. Enter the numeric value of the amount of annual energy to be saved in the units indicated in the "Units" drop-down list. Required.

- Fuel Saved - If Estimated is selected in the "Energy Savings" drop-down list, the "Fuel Saved" drop-down list appears. Select the type of fuel to be saved. The choices are Primary Heating Fuel, Water Heating Fuel, Natural Gas, Oil, Electricity, Propane, Wood, Coal, Kerosene, and Other. If Primary Heating Fuel or Water Heating Fuel is selected, the type of fuel saved will be set the same as the fuel specified for the primary heating system or water heater, respectively, in the audit into which the measure is copied. Required.

- Life - If Estimated is selected for the "Energy Savings" drop-down list, the Life field appears. Enter the expected life of the measure being defined in years. Required.

- Available for Use In Site Built and Mobile Home - Select either or both of these checkboxes to indicate the applicability of the measure to the building type(s), Site Built and/or Mobile Home. Your selection will determine which user-defined measures will appear in the "Copy from User Defined Measures" drop-down lists on the Itemized Costs forms under the "NEAT Audit" or "MHEA Audit” windows (see Section 11.8, Itemized Costs).

- Measure Comment - You may enter comments pertinent to the current measure directly in the Comment field on the form, or you may enter them in the Comment Editor by selecting the "Measure Comment" button to the left of 
the field. The Comment Editor is similar to Window's Notepad and may be used to enter extended comments. The total comment may have up to 65,000 characters. Text from other sources may be copied and pasted into the Comment Editor's window. The comment that is entered will be imported with the measure to any audit in which you choose to use the measure. The comment will then be displayed on that audit's Recommended Measures report and the Input Report, available from the Audit Information form (see Section 8.2, Audit Information). If a work order is created from the audit, the comment will be carried with the measure to the work order. Or, the measure may be imported directly to a work order, carrying the comment with. Either way a measure with a comment becomes part of a work order, it will then be displayed in the Work Order report which can be printed from the Work Order Information form (see Section 13.1, Work Order Information). Optional.

- Measures Record Navigation Block - The Measures record navigation block is used to find and navigate to existing measure descriptions for the setup library being edited, copy or delete the currently accessed measure description, or create a new measure description for the setup library. See Section 5.1, Form and Record Navigation, for information on using the Weatherization Assistant's record navigation blocks. The Measures record navigation block on the User Defined Measures form differs somewhat from other navigation blocks. Although the "by Description" drop-down list will contain all user-defined and health and safety measures in the setup library being accessed, the arrow buttons and numeric values displayed in the block pertain only the category of measures selected in the "View" drop-down list described below.

- View - Use this drop-down list to indicate which group of measures you wish to be displayed when viewing the User Defined Measures form in the data sheet view. The choices are Site Built (NEAT) Measures, Mobile Home (MHEA) Measures, Health \& Safety, and All User Defined Measures. Selecting Site Built (NEAT) Measures or Mobile Home (MHEA) Measures will restrict the display to those user-defined measures that you have defined and selected using the Available for Use In checkboxes. Selecting Health \& Safety will restrict the display to the fifty library health and safety measures. Selecting All User Defined Measures will display all measures that you have defined and the library health and safety measures, irrespective of them being selected or not selected for NEAT and MHEA. 
- All User Measure Costs - Selecting this button will display the Cost Detail for All User Defined Measures sub-form in data sheet view (see Figure 14.21). This form lists all costs associated with all of the user-defined measures having cost components assigned, as well as the library health and safety measures. The columns displayed include: NEAT, MHEA, \#, Measure Name, Material Description, Type, Units, <first material/labor component indicator>, Unit \$ and Comment. The first four columns correspond to the fields of the general description area of the User Defined Measures form. The remaining six fields correspond to the fields of the Materials/Labor Details sub-form (see below). Since each measure may have multiple material/labor components, the first material/labor components associated with a measure is indicated by a right pointing arrow (-->) in the column to the left of the Unit\$ column. On this subform, NEAT, MHEA, \#, Unit \$ and Comment columns are editable.

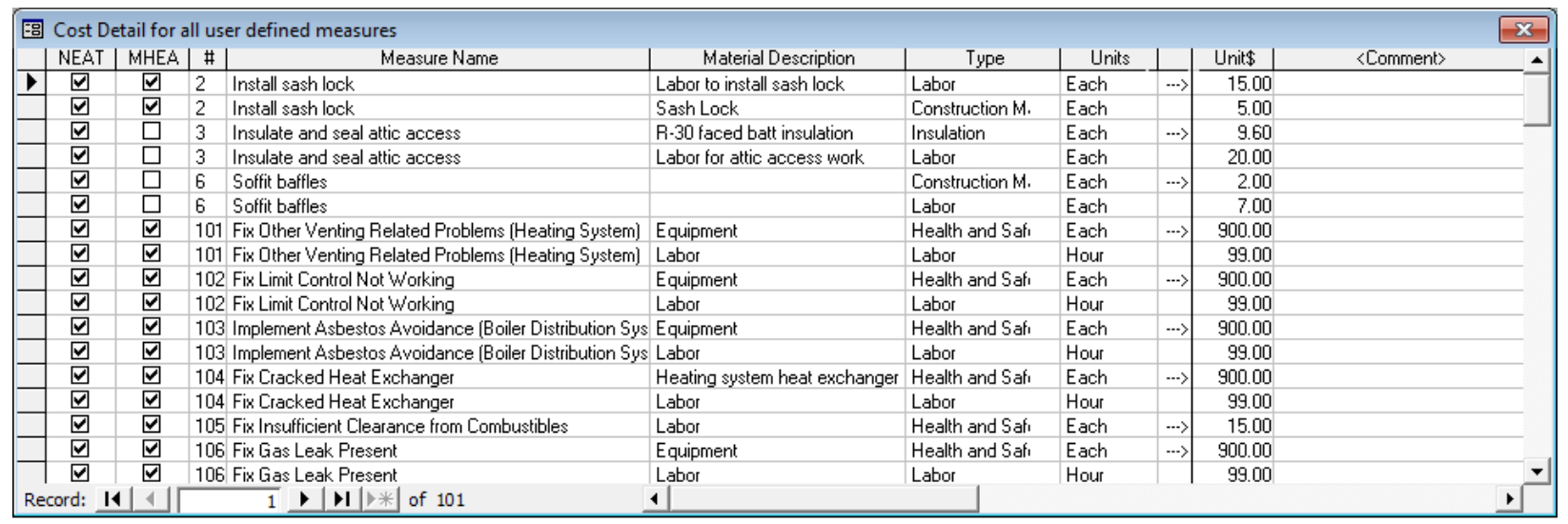

Figure 14.21. The All User Measures Cost sub-form.

\section{The Materials/Labor Details Sub-Form}

The Materials/Labor Details sub-form is used to enter information about all material/labor components needed to install a measure. The sub-form may be viewed in either form view (see Figure 14.22) or data sheet view (see Figure 14.23) (see Section 5.8, Data Sheet and Form Views of Records). Both views have the same capabilities for entering data, except that the form view displays only one material/labor component at a time while the data sheet view displays all material/labor components defined under a measure. Thus, the form view uses a record navigation block to allow access to all material/labor components. A measure may have as many cost components in its details as necessary but should normally have at least one. A user-defined measure with no detail may be 


\section{Chapter 14: Setup Library}

copied into and audit, but it would have to have a cost assigned at that time and have its reference to the user-defined measure in the setup library cleared (see "Clear Reference to User Defined Measure” in Section 11.8, Itemized Costs). If copied to a work order, a cost would also have to be designated at that time. Health and safety measures should have at least one cost component or an error message will be produced if the program attempts to automatically use the measure as a result of a hazard noted in an audit (see "Handling of Health and Safety Issues" in Section 11.7, Health and Safety).

When a user-defined measure is copied from a setup library into a work order (see “Copy from User Defined Measures” in Section 13.3, Measures (Work Orders)), all cost components given in the Materials/Labor Details sub-form are carried along with the measure and will appear in the work order's Material/Labor Details sub-form. However, when a user-defined measure with multiple cost components is copied to an audit, only one of those components can be displayed on the audit's Itemized Costs form (see "Copying a Predefined Measure from the Setup Library" in Section 11.8, Itemized Costs, and the Order \# field below). However, the measure will retain reference to the entire detail unless "Clear Reference to User Defined Measure" is chosen in the audit, in which case only the component visible on the audit's Itemized cost form is retained. However, the cost assigned will still be the total cost of all the former cost components.

Below are descriptions of individual data items and controls found on the Materials/Labor Details sub-form of the setup library's User Defined Measures form.

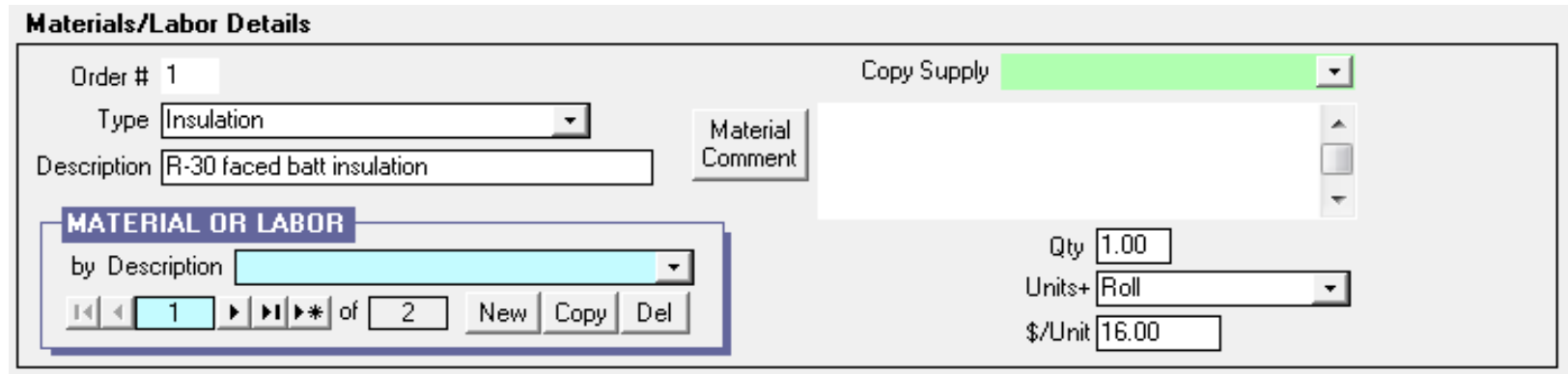

Figure 14.22. The Materials/Labor Details sub-form (form view). 


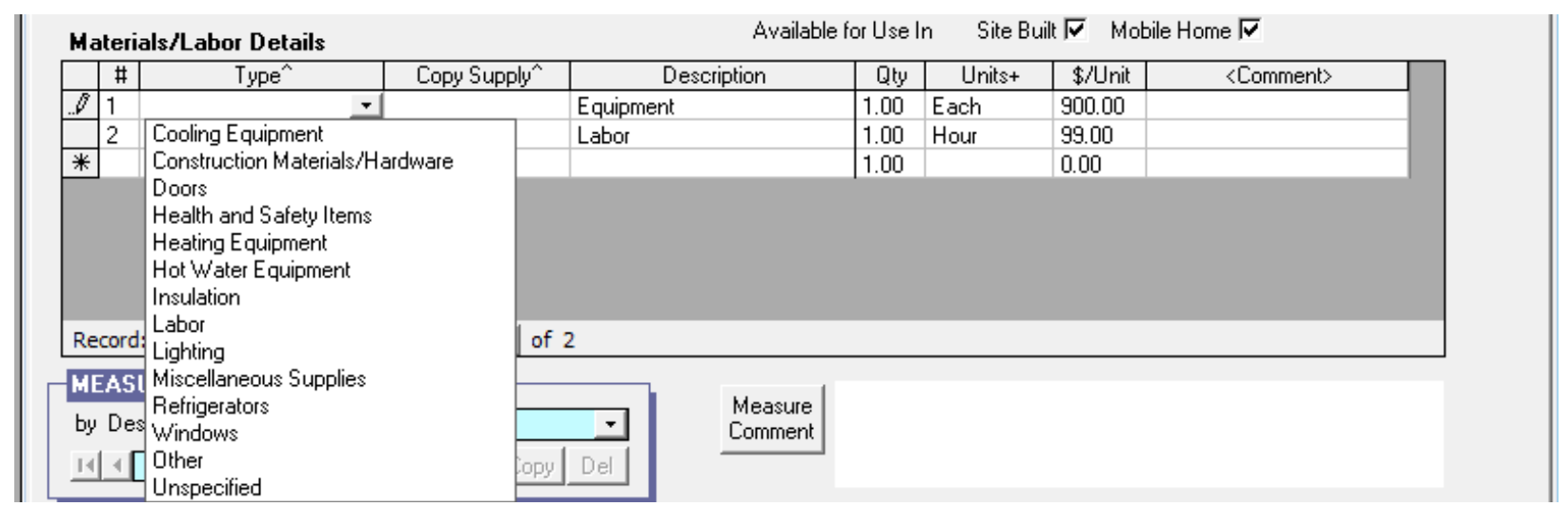

Figure 14.23. The Materials/Labor Details sub-form (data sheet view).

- Order \# - Enter an integer that prescribes the order in which the costing components of the measure will be listed on a work order when the measure is implemented (see “Order \#” in Section 13.3, Measures (Work Order)). Any component not assigned an order number will be listed first in the work order, but all those without a number will have random order amongst themselves.

If the measure being described here in the Setup Library is copied to an audit (see Section 11.8, Itemized Costs) and it has more than one Material/Labor Detail component, only one of these components can be displayed in the audit's Material field on the Itemized Costs form, even though all will get carried along with the measure. The audit will choose the detail component with Order \# of "1" then add " $(+)$ " after the detail name in the audit indicating additional detail is associated with the measure. The exception to this convention is if the userdefined measure has a cost component with Order \# of " 1 ” whose Description (see below) is "Equipment." This is seen as a nondescript detail whose inclusion on the audit form would not be of benefit. For such a measure, the Material field on the audit's Itemized Cost form is left blank. Optional.

- Type - If you are using the Supply Library to store information about materials you use in your weatherization activities and track inventory, select the category using the drop-down list under which the material/labor is listed in that library. The choices are the thirteen material/labor categories in the Supply Library (see Chapter 15, Supply Library) and Unspecified (see Figure 14.23). The entry is used to narrow your search for the material when using the "Copy Supply" drop-down list on the sub-form. If you do not plan to locate this material/labor component in your Supply Library, this entry may be left blank or designated as "Unspecified.” Conditionally Required. 
- Copy Supply - This entry is used in conjunction with the Type field, described above, to locate a specific material/labor component in the Supply Library (see Chapter 15, Supply Library). Selecting the material using this drop-down list will automatically fill in most of the remaining fields on the sub-form, provided you have specified the analogous entries in the Supply Library. Copying a material/labor component from the Supply Library is essential if you want to track its inventory. The library health and safety measures have pre-entered materials, but none that reference a Supply Library. If you wish to have these measures reference Supply Library materials, make that association here. Optional.

- Description - Enter a brief description of the material/labor component. If the material/labor component has been copied from the Supply Library, this field will be entered automatically. In such cases, you may change the description, but it may complicate your ability to identify the component as one tracked in your inventory. The library health and safety measures have predefined material descriptions, though you may change them here. The entry may be 80 characters or less. Required.

- Qty (Quantity) - Enter the quantity of the material/labor component needed to install the measure, in the units selected in the "Units" drop-down list, described below. This data is copied to a work order or carried through an audit to a work order if the measure becomes part of a work order. However, you may alter the quantity in the work order if a new, more accurate estimate becomes available. When you create a new user-defined measure from the Measures record navigation block a default value of "1.00” will automatically be entered. Alter this value as needed. Required.

- Units - Select the units in which the Quantity (above) and cost, \$/Unit (below), of the material/labor component are specified, using the drop-down list. The choices are Each, Hour, Bag, Roll, SqFt, Linear Foot, Inch, Pound, Case, Box, and United Inch. You may also enter a unit of your own choosing that is not in the drop-down list. The program will retain this new unit as part of the component description where ever the user-defined measure is used. If the material/labor component has been copied from the Supply Library, this field will be entered automatically. If this is the case, do not change the Units entry provided. The inventory kept in the Supply Library will expect the Quantity and \$/Unit entries for the component to be in these original units. Required. 
- $\$$ /Unit - Enter the cost of the material/labor component, in dollars per unit selected in the Units field, described above, needed to install the measure. If the material/labor component is copied from the Supply Library, this information will be entered automatically. A library health and safety measure will initially have material and labor costs of $\$ 900.00$ and $\$ 99.00$, respectively, indicating that the user has not provided costs for the measure (see Figure 14.23). Required.

- Material Comment - You may enter comments pertinent to the current material/labor component directly in the Comment field on the form, or you may enter them in the Comment Editor by selecting the "Comment" button to the left of the field. If in data sheet view, double clicking on the field will display the Comment Editor. The Comment Editor is similar to Window's Notepad and may be used to enter extended comments. Text from other sources may be copied and pasted into the Comment Editor's window. The total comment may have up to 65,000 characters. However, the field on the work order which will display this comment is only about 20 characters in width. The Weatherization Assistant will wrap the comment on the report, but excessively long comments become awkward to display. A comment that is entered in this comment field will be displayed in any work order that includes the userdefined measure containing the material/labor component. Optional.

- Material or Labor Record Navigation Block - If viewed in form view, the Material or Labor record navigation block is used to find and navigate to existing material/labor component descriptions for a measure, copy or delete the currently accessed material/labor component descriptions, or create a new material/labor component description for a measure. See Section 5.1, Form and Record Navigation, for information on using the Weatherization Assistant's record navigation blocks.

\subsection{NEAT Insulation Types}

The Added Insulation Type fields on the Walls, Unfinished Attics, Finished Attics and Foundations forms in the NEAT building description allow specification of the type of insulation to be considered when insulating these components (see the "Added Insulation Type" topics for these shell components in Chapter 9, NEAT Building Description. The NEAT Insulation Types form in the Setup Library 


\section{Chapter 14: Setup Library}

allows you to define additional insulation types for each of these components to meet local needs (see Figure 14.24).

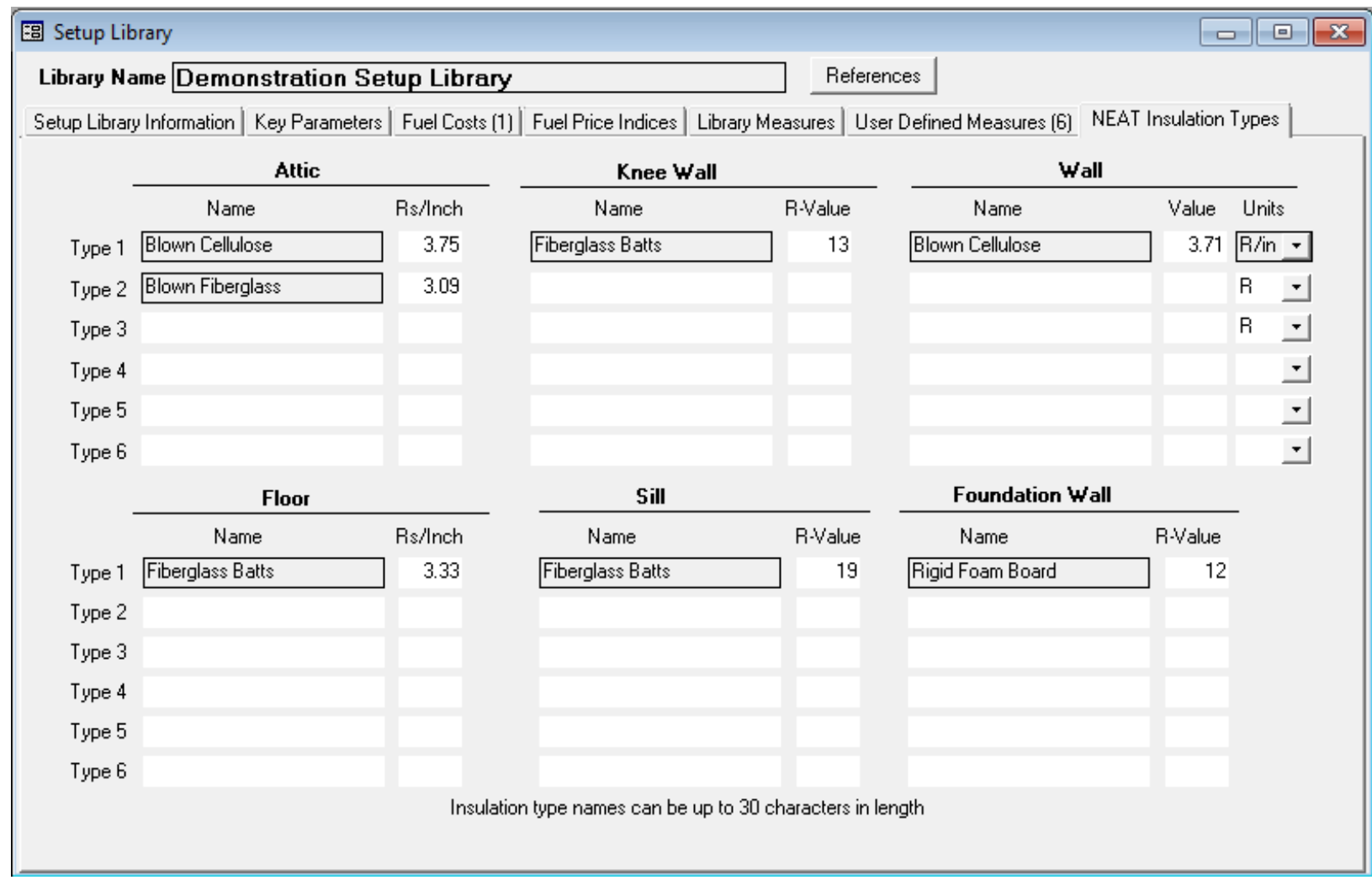

Figure 14.24. NEAT Insulation Types.

When the Weatherization Assistant is initially installed, the added insulation types available for each of the shell components are displayed in greyed, outlined fields on the NEAT Insulation Types form. The names cannot be changed. Below these insulation type names are blank, editable fields into which you may enter the names of additional insulation types of your choosing. Once entered, these names will appear in the Library Measures Costs sections of the Setup Library (see "Costs" in Section 14.5, Library Measures), in the building description forms of audits accessing the Setup Library, and in any reports that recommend a measure using these materials.

To the right of these material names are numeric fields giving the R-values per inch or total R-Value of the material you are defining. The R-values have dimensions $\mathrm{h}-\mathrm{ft}^{2}-\mathrm{F} / \mathrm{Btu}$. Typical values corresponding to various insulation types may be found in building manufacturers' data sheets or in ASHRAE's (American Society of Heating, Refrigerating, and Air-Conditioning Engineers) Handbook of 


\section{Chapter 14: Setup Library}

Fundamentals. Entries in these fields permit your defined insulation types to have the physical characteristics needed by the NEAT audit that you desire. Required for named insulation types.

As indicated above, any insulation type added on the NEAT Insulation Types form will automatically add the type to the appropriate Library Measures Costs form. By default, the Unit\$ field for the insulation type on this form will be assigned a cost of "9999.00" with the Comment of "Not considered unless cost is specified." Thus, in order for the program to seriously consider use of an insulation type you add, a reasonable cost must be entered on Library Measures Costs form corresponding to the envelope component using the insulation. For example, if you add "Spray Foam” as a Sill insulation type, you must access the "Costs" button for NEAT's measure 7, "Sillbox insulation," under the Library Measures form in the Setup Library and enter a reasonable cost for installing sill insulation using this insulation type. 


\section{Chapter 15. Supply Library}

The Supply Library provides you with an optional ability to record material properties and labor rates related to your weatherization work. If you use the inventory feature of the Weatherization Assistant, the Supply Library becomes the mechanism for tracking your inventory. It provides you with a means of recording material purchases, tracking use of the materials through the work orders, and tallying up your total remaining items. Energy-related information that can be stored in the Supply Library includes thermal properties, equipment efficiencies, physical dimensions, lifetimes, etc. The Supply Library is accessed from the Main Menu’s “Supply Library” button. (see Figure 3.1).

Items in the Supply Library are accessed from audits, work orders, and setup libraries. The NEAT and MHEA audits use the Supply Library as a repository for replacement refrigerators and water heaters (see Sections 11.4, Base Loads - Water Heat, and 11.5, Base Loads - Refrigerators), the data possibly coming from a visit to a home building supply store. Items from the Supply Library may be referenced directly from the setup library forms when specifying the materials/labor components needed for installing user-defined measures (see "Copy Supply" under "The Materials/Labor Details Sub-Form” in Section 14.6, User Defined Measures). Items from the Supply Library may also be referenced directly from the work order forms when specifying the materials/labor components needed in generating detailed work orders (see "Copy Supply" under "The Materials/Labor Details SubForm” in Section 13.3, Measures (Work Order)).

NOTE: Referencing materials from the Supply Library is optional for running the audits and/or creating the work orders except when considering the Water Heater Replacement measure under the NEAT or MHEA audits. See "Additional Entries on the Hot Water Equipment and Refrigerator Forms” in Section 15.2, Materials/Labor Forms, for the exception related to water heater replacement.

You will likely need only one supply library, though any number of supply libraries may be defined for an agency. More than one supply library might be used if materials are stored in multiple locations and you wish to track the inventory at these locations separately. However, you would need to be continually aware of this situation and know when a given library should be referenced. Considering the Supply Library as an inventory of items may help you determine if more than one library is necessary and how many should be used. All supply libraries associated 


\section{Chapter 15: Supply Library}

with the current agency are listed under the Libraries form under the Main Menu's “Agency” button (see Section 6.8, Libraries).

This chapter describes all the data items and controls found under the Main Menu's “Supply Library” button. The Supply Library consists of 14 forms that include General Information, Cooling Equipment, Construction Materials/Hardware, Doors, Health and Safety Items, Heating Equipment, Hot Water Equipment, Insulation, Labor, Lighting, Miscellaneous Supplies, Refrigerators, Windows, and Other. All forms except the General Information form represent materials/labor categories, which contain similar data items and controls. This chapter will discuss the General Information form first. Then, in a separate section, the data items and controls on the materials/labor forms are described, noting any exceptions to the commonality of the forms. Finally, the entries on the Energy Details sub-forms are discussed individually, since they differ for the different material categories.

\subsection{General Information Form}

The entry items and controls on the General Information tab (see Figure 15.1) of the Main Menu’s “Supply Library” button are described below.

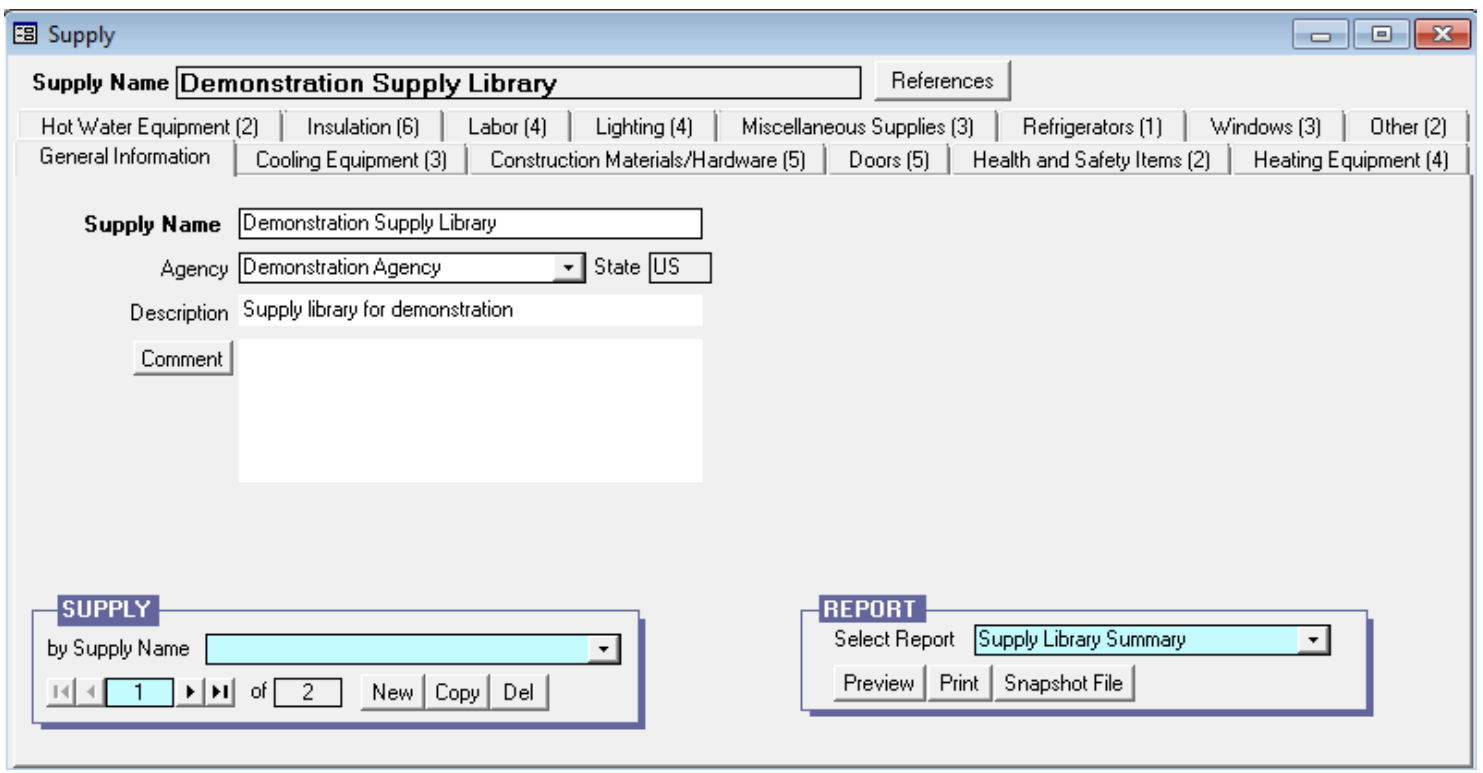

Figure 15.1. The General Information form under the Main Menu’s “Supply Library” button.

- References - Selecting this button, located just below the banner line of the "Supply Library" window, displays the "References to Supply Library" 
window, which lists all references to the Supply Library currently being accessed. See Section 5.14, The References Button, for more information on the use of this feature.

- Supply Name - Enter a name that will identify this particular Supply Library throughout the program. Most often, an agency will have only one supply library. If more than one is created, the name may contain an abbreviated indication as to why one differs from another. Save any lengthier explanations for the Description and Comment fields. A default name will be supplied when a new supply library is created. The name will use your installation's ID (see Section 3.1, Initial Start-up of the Weatherization Assistant), if available. Otherwise the default name will contain the time the library was created. The entry may be 50 characters or less. Required.

- Agency - This field identifies the agency to which the Supply Library is associated. Since the only way to create a new supply library is to copy an existing one or import one with a client (see "Importing Clients" in Sections 17.3, Data Transfer - Import/Export With Another MSAccess Database, and 17.4, Data Transfer - Import/Export Indirectly with Text Files), this entry will always be at least initially filled in for you. The field is more for identification purposes, possibly on a state level, identifying the agency within the state using the materials in the library. Required.

WARNING: The program allows you to change the agency assignment of a supply library. However, doing so when existing setup libraries, audits, or work orders use the Supply Library will likely cause confusion. Therefore, reassigning the agency assignment of a supply library is not recommended unless the library has just been created, insuring no other references to it exist.

- State - This field displays the state associated with the agency entered in the previous field. Uneditable.

- Description - You may use this field to briefly describe the intended use of the Supply Library, particularly if you use more than one supply library in your agency. The entry may be 255 characters or less. Optional.

- Comment - You may enter comments directly in the Comment field on the form, or you may enter them in the Comment Editor by selecting the 
"Comment" button to the left of the field. The Comment Editor is similar to Window's Notepad and may be used to enter extended comments. The total comment may have up to 65,000 characters. Text from other sources may be copied and pasted into the Comment Editor's window. Comments entered on this General Information form are not displayed anywhere else in the Weatherization Assistant or in any of its predefined reports. However, you may wish to enter a comment that provides helpful information about the Setup Library. The comment could then be included in a user-designed report (see Appendix D, Development of Customized Reports). Optional.

- Supply Record Navigation Block - The Supply record navigation block is used to find and navigate to supply libraries, or copy or delete existing libraries. See Section 5.1, Form and Record Navigation, for information on using the Weatherization Assistant's record navigation blocks. The drop-down lists will display only the supply libraries associated with the agency currently selected ("bookmarked") on the Agency Information tab under the Main Menu's “Agency” button. Normally the agency selected on this tab is your agency, so the drop-down lists will display only your agency's supply libraries. If you have more than one agency defined in your database and you want the drop-down lists to display supply libraries from all defined agencies, uncheck the "Use only the last bookmarked Agency in the find record drop down boxes," Feature 9 from the Preferences/Features tab (see the indicated topic in Section 16.4, Features).

- Report Block - The Report block provides you with access to the Supply Library Summary report. See Appendix C, Reports, for an example of this report. The report lists the Category, Description, and Manufacturer of each item in the Supply Library for which you have indicated at least one purchase (see “Inventory Data Block” in Section 15.2, Materials/Labor Forms). For each of these items, the report also lists the number purchased, the number designated as actually having been used (see "Quantity (Actual)" under "The Materials/Labor Details Sub-Form” in Section 13.3, Measures (Work Order)), and the number yet available to be used. See Section 5.12, The Report Block, for the mechanics of using the Weatherization Assistant's Report blocks. 


\section{Chapter 15: Supply Library}

\subsection{Materials/Labor Forms}

The following entry items and controls are common to all materials/labor forms in the Supply Library. Exceptions are noted in square brackets following the field or control name. All these forms may be viewed and edited in either form or data sheet view (see Section 5.8, Data Sheet and Form Views of Records). See Figures 15.2 and 15.3 for examples. The items displayed in these figures are only examples and most likely will not represent materials you would actually need in your supply library. The information on the Energy Details sub-form is not available in data sheet view for some of the category's forms. Nevertheless, the data sheet view offers a convenient way of reviewing the materials in your library, especially if it grows large. Records in the view may also be copied and pasted either between Weatherization Assistant databases or from the program to a spreadsheet application. Additional entries that are not common are given in "Additional Entries on the Hot Water Equipment and Refrigerator Forms" in Section 15.2, Materials/Labor Forms.

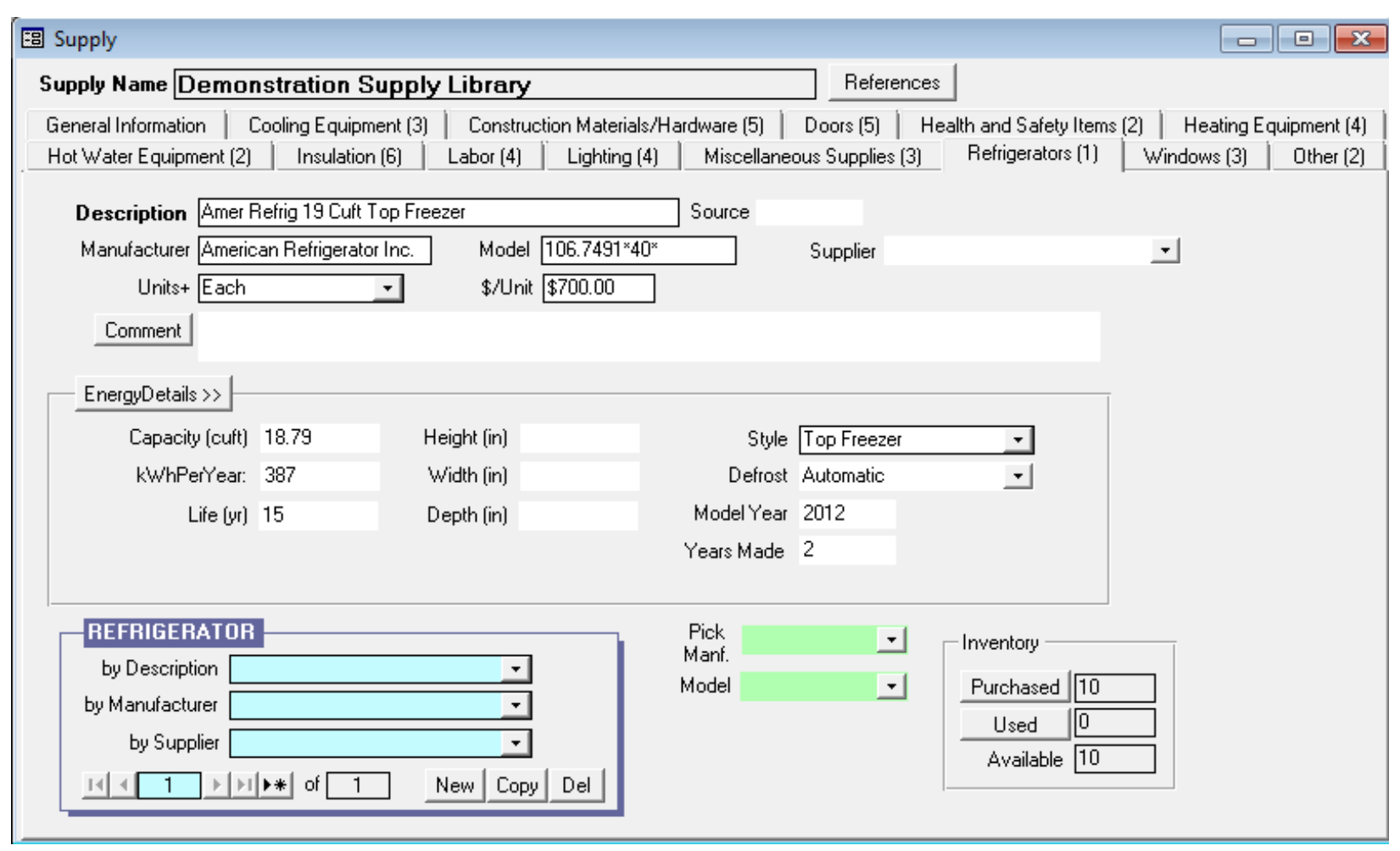

Figure 15.2. A typical form under Main Menu’s “Supply Library” button (form view). 


\section{Chapter 15: Supply Library}

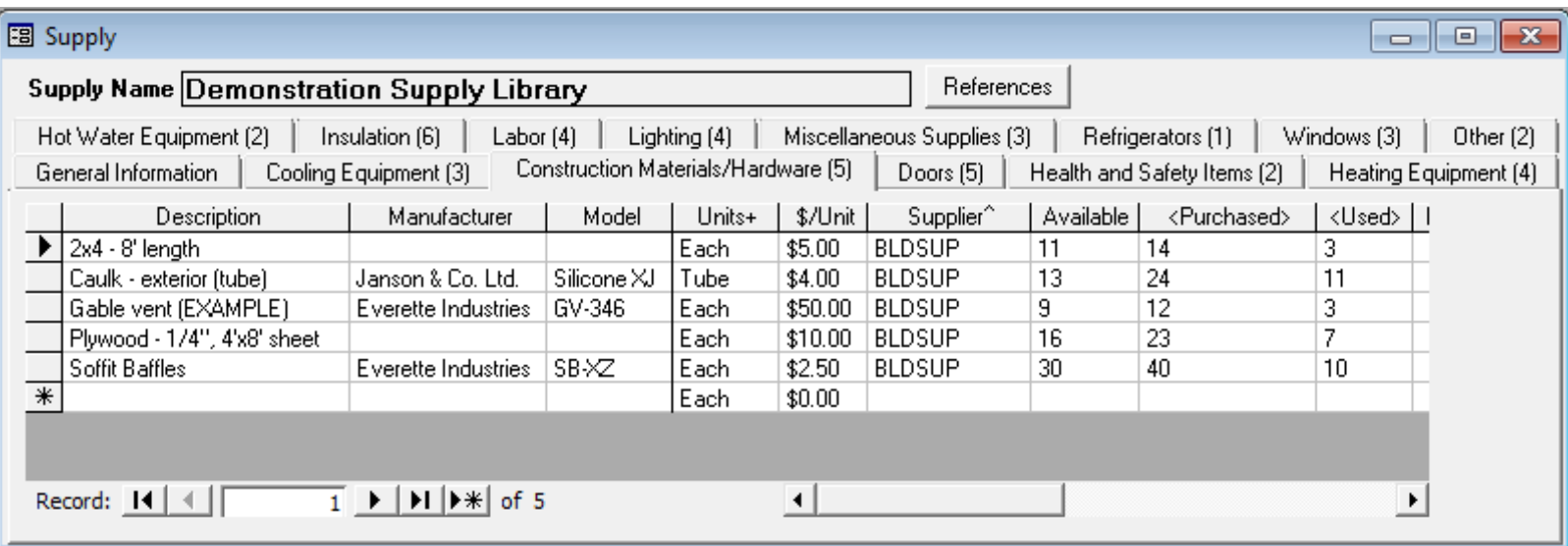

Figure 15.3. The Construction Materials/Hardware Materials form (data sheet view). ${ }^{3}$

\section{General Description Data}

- Description - Enter a description of the item or labor category. Entries may be 80 characters or less. Required.

- Manufacturer [except Labor] - Enter the name of the manufacturer of the item. Your inventory may contain multiple entries for the same item but manufactured by different companies, though their descriptions must still be unique. Entry may be 50 characters or less. Optional.

- Model [except Labor] - Enter the model of the item being described. Your inventory may have multiple entries of a specific item type but of varying models, though their descriptions must still be unique. Thus, you may alternatively decide to include model information in the Description. Entries may be 50 characters or less. Optional.

- Supplier [except Labor] - Choose the Supplier of the material being described. Choices in the drop-down list will be those agency contacts declared as "Supplier" on the Contacts tab under the Main Menu's “Agency" button. See Section 6.2, Contacts (Agency). Optional.

- Units - Enter the units by which the item is costed. The choices provided are Each, Hour, Bag, Roll, SqFt, Linear Foot, Inch, Pound, Case, Box, and United Inch. You may also enter a unit of your own choosing that is not in the dropdown list. The program will retain this new unit as part of the item description

\footnotetext{
${ }^{3}$ Above is an example for one category of material. The Comment field is not displayed
} 
where ever the material is used. The units for analogous materials used in the audits, as prescribed in the Setup Library, and those in the Supply Library do not need to be the same. However, if you are using the inventory feature for the material, you would need to manually convert the quantity required when translating the audit recommendation to a task in a work order. If this is undesirable, care should be taken to set the Units here to be the same as those used in the audits. Required.

- $\mathbf{\$} /$ Unit - Enter the cost of the item in the Units entered in the previous field. Both the Units and the \$/Unit will be copied to any use of the item in the Work Orders. Required.

- Comment - Supply material comments may be entered directly in the comment field on the form. Or, select the "Comment" button to the left of the field, which will take you to the Comment Editor. This text editor is similar to Window's Notepad and may be used to edit extended comments. The total comment may have up to 65,000 characters. Text from other sources may be copied and pasted into the Comment Editor's window. Supply material Comments are carried with the material whenever the material is used in a user-defined measure or work order. You may also wish to include the comment in a user-designed report (see Appendix D, Development of Customized Reports) Optional.

- Supply Item Record Navigation Block - The supply item record navigation block, located at the bottom left of each material category tab, is used to find, access, copy, delete, or initiate new materials under the category being referenced. See Section 5.1, Form and Record Navigation, for information on using the Weatherization Assistant's record navigation blocks. The listings of items in a given category may be ordered by their Description, Manufacturer, or Supplier in the drop-down lists of the navigation block.

\section{Inventory Data Block [Except Labor]}

The following data items and buttons fall under the Inventory data block positioned in the lower right corner of all material category forms except Labor (see Figure 15.4). 
- Purchased (data item) - This value displays the accumulated number of the item you have indicated as having been purchased. Uneditable.

- Purchased (button) - This button takes you to a data sheet displaying all your entries documenting purchases or removal of the item currently being accessed.

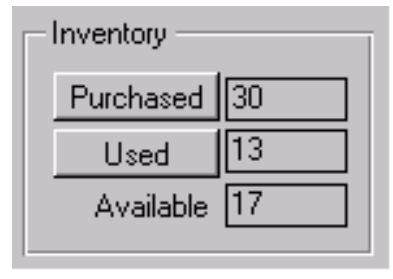

Figure 15.4. Inventory control block.

圆 Inventory Summary for: 13 Watt Bulb
\begin{tabular}{|c|c|c|c|c|c|}
\hline & Date & Added & Removed & Cleared & \\
\hline & $1 / 8 / 2011$ & 35.0 & & $\square$ & \\
\hline & $2 / 10 / 2011$ & & 5.0 & $\square$ & \\
\hline * & $2 / 10 / 2011$ & & & (Comment> & \\
\hline
\end{tabular}

Figure 15.5. Inventory Purchase/Removal Summary.

Enter the date of purchase or removal, the number of items (in the units displayed on the parent form) purchased or removed, and any comment that you may wish to associate with the entry. Only entries "Cleared" will actually affect the inventory. Uncleared entries may be anticipatory, rather than actual. The current date is the default, though this may be changed. Items "Removed" are in addition to those that will automatically be removed by use in work orders (see "Used (button)" below). Entire records may be deleted from the table by selecting the entire row of data and pressing your [Delete] key (see Section 5.10, Deleting Data).

- Used (data item) - This value displays the total number of the items that have been used in work orders. See "Used (button)" below. Uneditable.

- Used (button) - The "Used” button takes you to a data sheet displaying all instances in which the specific item being referenced has been used in work orders.

\begin{tabular}{|c|c|c|c|c|c|c|c|c|c|}
\hline \multicolumn{9}{|c|}{ 凅 Inventory Used for: 13 Watt Bulb } & \multirow{2}{*}{$\begin{array}{l}\square \text { 回 } x \\
\text { Comment }\end{array}$} \\
\hline & $\mid\langle$ Client> & Other ID & 〈Work Drder〉 & Status & Changed & Order & $\langle$ Measure〉 & Qty Actual & \\
\hline$D$ & 11_353 & & W0/11_353/JT/1 & Work Started On & $10 / 6 / 2011$ & & Lighting Retrofits & 1 & \\
\hline & $11 \_351$ & & W0/11_351/JT/1 & Work Completed On & $10 / 4 / 2011$ & & Lighting Retrofits & 5 & \\
\hline & $11 \_350$ & & W0/11_350/JT/1 & Work Completed On & $9 / 22 / 2011$ & & Lighting Retrofits & 3 & \\
\hline & $11 \_348$ & & W0/11_348/EASY/1 & Work Completed On & $9 / 6 / 2011$ & & Lighting Retrofits & 4 & \\
\hline
\end{tabular}

Figure 15.6. Inventory Used Summary. 
Only those materials declared as being "Actual" in their use will be included in the table (see "Quantity (Actual)” under "The Materials/Labor Details SubForm" of Section 13.3, Measures (Work Order)). The table displays the Client ID, the Alternate Client ID, the Work Order in which the item was used, the status of the work order and when this status was last changed, the material number within the measure on the work order (if entered), the specific measure in the work order in which the item is used, the quantity used by the specific work order entry, and any comment you entered on the work order related to this item's use. See “The Materials/Labor Details Sub-Form” referenced above for more information on most of these data items. Note that the headers for "Client," Work Order," and "Measure" in the table are within angle brackets (< $>$ ), indicating that double clicking your mouse in any entry in these columns will take you to the specific record indicated by the entry. This provides a quick method of reminding yourself of any use of an item. The items within the table may appear to be editable, but attempts to change them will fail. Changes must be made in the actual record from which the data originated.

- Available - This field displays the difference between the number of items "Purchased" and those "Used", as given in the fields immediately above. Uneditable.

\section{Additional Entries on the Hot Water Equipment and Refrigerator Forms}

The supply library's listings for Hot Water Equipment and Refrigerators have an additional purpose in the Weatherization Assistant beyond listing materials for use in the work orders. They are the only categories of materials in the library which have data that may be copied to an audit and used in the audit calculations in determining the cost-effectiveness of measures (i.e., the water heater and refrigerator replacement measures). Although information about a replacement refrigerator may be entered directly into NEAT's and MHEA's audit input forms (see Section 11.5, Base Loads - Refrigerators), data on replacement water heaters must be entered in the program's Supply Library in order for the audits to consider replacing a water heater (see Section 11.4, Base Loads - Water Heating).

In addition to the fields described in Section 15.2, Materials/Labor Forms, the Hot Water Equipment and Refrigerator forms have two drop-down lists. These dropdown lists access the Weatherization Assistant's database of almost 8,000 water 
heaters and 34,000 refrigerators. The green shading of the drop-down list is an indication that you are accessing an entire database.

- Pick Manuf. and Model - Select the manufacturer and model from the drop-down lists. Choosing a Manufacturer first will restrict the listings in the "Model" drop-down list to those models of that manufacturer. Otherwise, the Model list will include all models from all manufacturers. If you don't

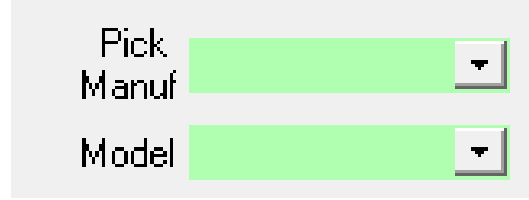

Figure 15.7. The additional entries on the Hot Water Equipment and Refrigerator forms. find a model by first choosing the manufacturer, you might attempt to leave the Manufacturer field blank and search for the model without specifying the manufacturer. Some manufactures may be listed in more than one way in the "Pick Manufacturer" drop-down list.

Selecting a model from the databases automatically fills in all required data on both the main form and the Energy Details form (see "Hot Water Equipment" and "Refrigerator" in Section 15.3, Energy Details) except for the Description on the main form, which you must provide. Choosing from the database is optional. However, if not chosen from the database and you plan to use the listing in the NEAT or MHEA appliance replacement measures, all required data on both the main form and Energy Details form must then be manually supplied.

\subsection{Energy Details Sub-Form}

The Energy Details sub-form allows you to record energy-related data for each entry under certain material categories of the Supply Library: Cooling Equipment, Doors, Heating Equipment, Hot Water Equipment, Insulation, Lighting, Refrigerator, and Windows. The Energy Details sub-form can be accessed by selecting the "Energy Details" button on the forms corresponding to these material categories of the Main Menu's "Supply Library" button. The fields under the Energy Details sub-form depend on the specific category of material being accessed. On the Hot Water Equipment and Refrigerators forms, the fields under Energy Details are required to provide sufficient information for use in the NEAT and MHEA audits. For other material categories, the fields under Energy Details sub-form are optional and the data provided is for documentation purposes only, 


\section{Chapter 15: Supply Library}

having no bearing on the material properties assumed by the NEAT and MHEA audits.

Below are brief descriptions of the individual data items and controls found on the Energy Details sub-forms for each material category under the Main Menu's "Supply Library" button. Energy Details are available only from the form view.

\section{Cooling Equipment}

- Equipment Type - Select what type of cooling equipment is being described. The choices are the same as in the audits: Central Air Conditioner, Window or Room Air Conditioner, Heat Pump, and Evaporative Cooler. Optional.

- Efficiency Units, Efficiency - If you want to enter the efficiency of the unit, first select the units for efficiency input. The choices are EER, SEER and COP. EER ratings are commonly found on window or room air-conditioning units while SEER ratings are more common on central systems. COP ratings are rarely found. Optional.

- Capacity - Enter the output capacity of the unit in $\mathrm{kBtu} / \mathrm{h}$. The value is often printed on the nameplate of the air conditioner. Central systems have their capacity rated in "tons." One ton is approximately $12 \mathrm{kBtu} / \mathrm{h}$. Optional.

- Life (yr) - Enter the life of the unit in years. If you are in doubt regarding what lifetime to use for a material and a similar material is used in one of NEAT's or MHEA's standard library measures (see "Life" in Section 14.5, Library Measures), you may use these values. Optional.

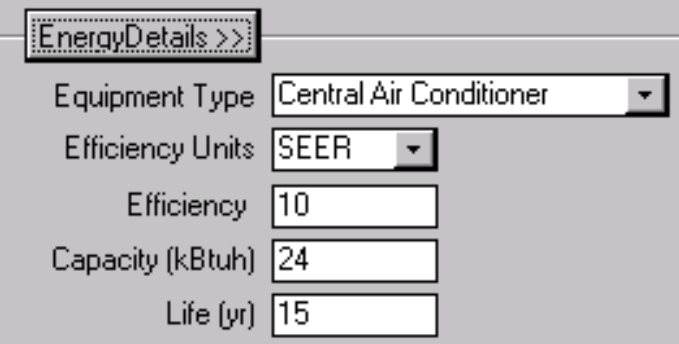

Figure 15.8. The Energy Details sub-form for Replacement Cooling Equipment. 


\section{Doors}

- Rated U Value - Enter the Rated U Value of the door, likely included on a label attached to the door when new. Optional.

- Life - Enter the life of the door in years. Most doors could be assumed to have a life of 20 years. Optional.

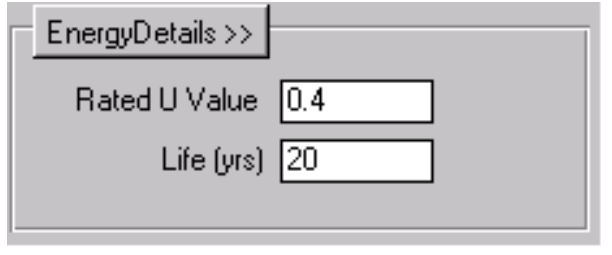

Figure 15.9. The Energy Details sub-form for Replacement Doors.

\section{Heating Equipment}

- Equipment Type -The choices for Equipment Type are Furnace, Heat Pump, and Space Heater. If you need to be more specific regarding the type (e.g., vented versus un-vented space heater), you may do so using either the Description or Comment fields for the item. Optional.

- Fuel Type - Select the type of fuel used by the heating system. The choices are Natural Gas, Oil, Electricity, Propane, Wood, Coal, Kerosene, and Other. The "Other" fuel type should correspond to what you have defined this fuel to be in your agency’s Setup Library (see Section 14.3, Fuel Costs). Optional.

- Efficiency Units, Efficiency - If you want to enter the efficiency of the system, first select the units for efficiency input. The choices are Steady State, AFUE, HSPF, and COP. Steady State and AFUE ratings apply to fossil fueled or electric resistance systems while HSPF (and less commonly COP) are used to rate the efficiencies of heat pumps. Optional.

- Capacity - Enter the output capacity of the heating system in units of kBtu/h. The output capacity is usually obtained directly from the nameplate of the heating system or from an inspection of nozzle sizes, etc. If this information is not available, the output capacity can be approximated or, in rare cases, measured. Optional.

- Life (yr) - Enter the life of the unit in years. If you are in doubt regarding what lifetime to use for a material and a similar material is used in one of NEAT's or MHEA's standard library measures (see "Life" in Section 14.5, Library Measures), you may use these values. Optional. 


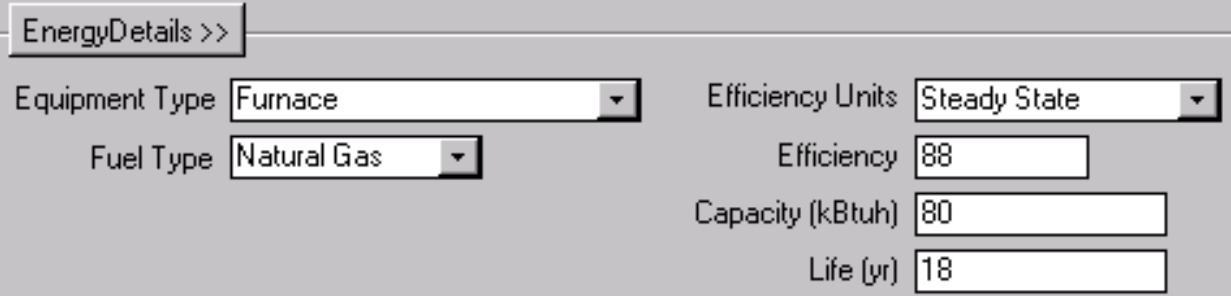

Figure 15.10. The Energy Details sub-form for Replacement Heating Equipment.

\section{Hot Water Equipment}

If you are considering the Water Heater Replacement measure in the NEAT or MHEA audit, you are required to enter information about your replacement units in the Energy Details sub-form. If you have chosen a unit from the database of water heaters, the information on the Energy Details sub-form for the unit will automatically be entered (see "Additional Entries on the Hot Water Equipment and Refrigerator Forms" in Section 15.2, Materials/Labor Forms). Otherwise, enter the indicated information.

- Fuel Type - Select the type of fuel used by the equipment. The choices are Natural Gas, Electricity and Propane. Required.

- Capacity - Enter the capacity of the water heater in gallon. Required.

- Input Units, Input - First select the unit for the Input rating. The choices are $\mathrm{kBtu}$ or $\mathrm{kW}$. Then, enter the Input rating of the water heater. Required.

- Energy Factor, Recovery Efficiency - The Energy Factor and Recovery Efficiency are ratings of a water heater's efficiency and are required for estimating the savings of replacing the existing water heater. They are available in the database of water heaters or from your local distributor. Required.

- Life - Enter the life of water heater in years. The standard life used for water heaters is 15 years. Required. 


\section{Chapter 15: Supply Library}

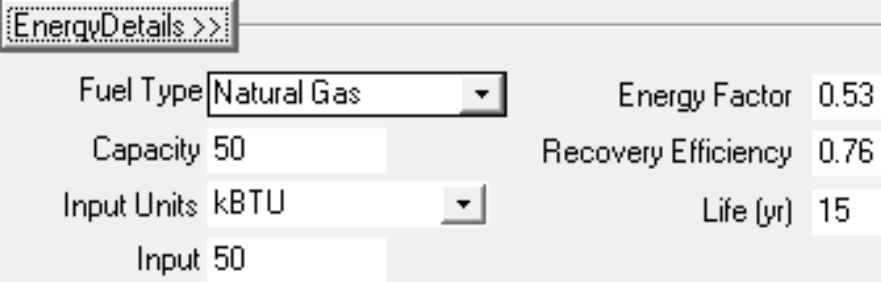

Fuel Type Natural Gas

Capacity 50

Input Units kBTU

Input 50

Energy Factor 0.53

Recovery Efficiency 0.76

Life [ur] 15

Figure 15.11. The Energy Details sub-form for Replacement Hot Water Equipment.

\section{Insulation}

- Insulation Type - Select the insulation type from the available choices: Batts or rolls (unfaced), Batts or rolls (faced), Loose (blown or pumped), and Rigid. Specify the material (e.g., fiberglass) in the Description field. Optional.

- R-value, R Value Units - Enter the R-value of the insulation either as "R-value (overall)" or "R-value per inch of thickness." Select the corresponding R-value units from these two choices. Optional.

- Installed Density - If the Insulation Type is "Loose," you will be asked for the Installed Density" in "lb/cuft." Optional.

- Bag Size - If the Insulation Type is "Loose," you will be asked for the bag size in pounds (lb). Optional.

- Suitable for - Use the checkboxes to indicate what applications the insulation will be used for. Select all applicable choices: Attics, Walls, Sill Boxes, and Foundations. Optional.

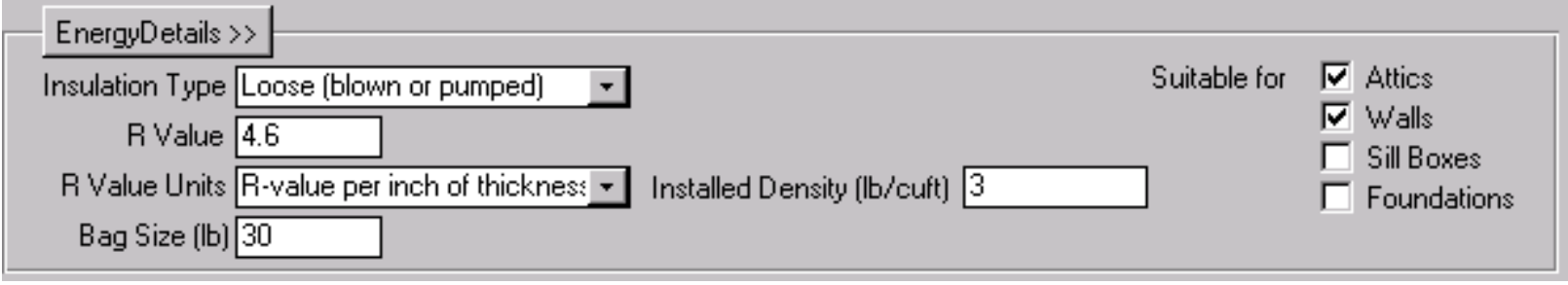

Figure 15.12. The Energy Details sub-form for Insulation. 


\section{Chapter 15: Supply Library}

\section{Lighting}

Enter information that describes each replacement lamp in your inventory.

- Lamp Type-Enter the replacement lamp type. The choices are Compact Spiral (a-lamp replacement), Spiral, Globe (decorative), Chandelier (decorative), Circline screw in, and Other. Optional.

- Life - Enter the estimated life of the lamp in hours. Optional.

- Lumens - Enter the light output in lumens. Optional.

- Watts - Enter the wattage of the lamp. Optional.

- Color Temp. - Enter the color temperature of the lamp in Kelvin (K). Optional.

- Three-way, Dimmable - Use the checkboxes to indicate whether the bulb is three way and/or dimmable. Optional.

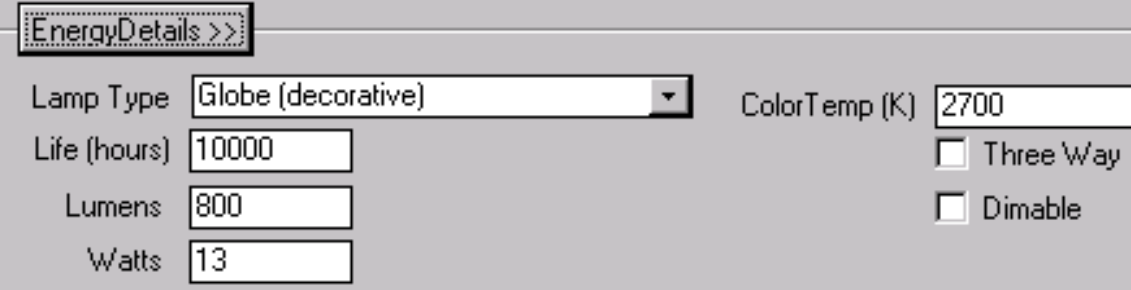

Figure 15.13. The Energy Details sub-form for Replacement Lamps.

\section{Refrigerators}

If you are considering the Refrigerator Replacement measure in the NEAT or MHEA audit, you may enter information about your replacement units in the Energy Details sub-form. If you have chosen a unit from the database of refrigerators, the information on the Energy Details sub-form for the unit will be entered automatically (see "Additional Entries on the Hot Water Equipment and Refrigerator Forms” in Section 15.2, Materials/Labor Forms). Otherwise, enter the indicated information. 


\section{Chapter 15: Supply Library}

EnerguDetails $>>$

Capacity (cuft) 18.79

kWhPerYear: 387

Life [ur] 15
Height (in)

Width (in)

Depth (in)
Style Top Freezer

Defrost Automatic

Model Year 2012

Years Made 2

Figure 15.14. The Energy Details sub-form for Replacement Refrigerators.

- Capacity - Enter capacity of the replacement unit in cuft. NEAT and MHEA compare this capacity (if specified here) with the capacity of the existing unit, if specified on the Refrigerator form under the audits, and produce a warning if the entries are not within $20 \%$, indicating a possible size problem related to the replacement (see "Capacity” in Section 11.5, Base Loads - Refrigerators). Optional.

- $\quad \mathbf{k W h}$ per Year - Enter an estimate of the annual energy consumed by the unit in $\mathrm{kWh} /$ Year, possibly from its energy guide label. Optional.

- Life - Enter the expected life of the unit. The standard life used for water heaters is 15 years. Optional.

- Height, Width, Depth - Enter the dimensions of the refrigerator in inches. NEAT or MHEA compare these dimensions (if specified here) with the available space dimensions of the existing unit, if specified on the Refrigerator form under the audits, and produce a warning if the entries indicate a possible size problem related to the replacement. (see "Height, Width, Depth" in Section 11.5, Base Loads - Refrigerators). Optional.

- Style - Select the style of the unit. The choices are Top Freezer, Bottom Freezer, Side by Side, Single Door, Single Door with Freezer, and Other. Optional.

- Defrost - Select the defrost type for the replacement unit. The choices are Automatic, Manual, Partial Automatic, and Other. Optional.

- Model Year - Enter the year when the specific model was first sold. If unknown, the entry may be estimated or left blank. Optional. 


\section{Chapter 15: Supply Library}

- Years Made - Enter the number of years the model was available. If unknown, the entry may be estimated or left blank. Optional.

\section{Windows}

Enter data that describes the replacement windows you have in your inventory. Most of the information requested can be found on the new window's label. Many manufacturers have adopted the National Fenestration Rating Council's (NFRC) labeling, which includes the information, though the leakage coefficient is optional for manufacturers to report.

- Rated U Value - Enter the U-Value of the window (Btu/hr- $\left.\mathrm{ft}^{2}-\mathrm{F}\right)$, a measure of the heat conduction property of the window.

- Rated SHGC - The SHGC (solar heat gain coefficient) measures how well the window blocks heat from the sun. Values are between 0.0 and 0.87 .

- Rated Leakage Coefficient - Enter the rated infiltration in $\mathrm{cfm} / \mathrm{ft}^{2}$, which is NFRC's air leakage parameter in cubic feet per minute of air per square foot of window area at $75 \mathrm{~Pa}$.

- Life - Enter the life of the window. A standard glass window may be normally assigned a life of 20 years.

If you have standard window sizes in your inventory, make separate entries in the Supply Library for each standard size, indicating the size in the Description field of the main form for the Windows.

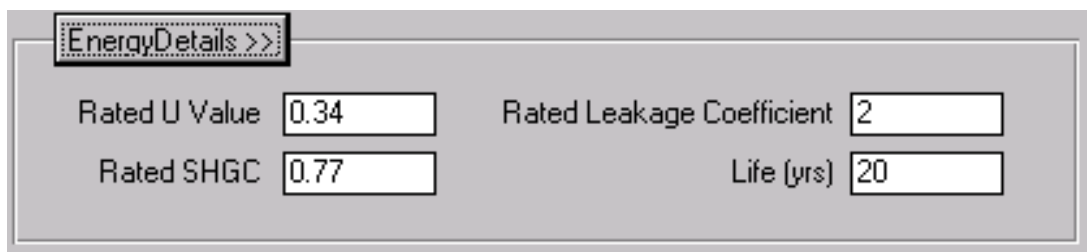

Figure 15.15. The Energy Details sub-form for Replacement Windows. 


\section{Chapter 16. Preferences}

Forms under the Main Menu's "Preferences" button (see Figure 3.1) allow you to choose various options available in the Weatherization Assistant which modify the appearance and operation of the interface to the program. These options do not affect the audits or influence the recommendations from the audits.

If you are using the Weatherization Assistant on a network, it will be important for you to understand the difference between the locations where your data are stored and where the settings for these preferences reside. Normal use of a network for the program is to have each terminal linked to a database on the network server, where all of the data you and others enter into the program are stored (see the introductory remarks in Chapter 17, Data Link). Thus, all users linked to the server's database file share the same information. However, the settings for the Preferences are stored locally at each terminal, regardless of where you store your other data. Thus, you may see a difference in behavior of the program from one terminal to the next, despite being linked to the same database. In Access terminology, your data are stored in the "backend" (or "mdb") file, while the settings for the Preferences are stored in the "frontend" (or "mde") file.

This chapter describes all the data entry and display items on the four forms found under the Main Menu's “Preferences” button: General, Range Check and Default values, Report Sections, and Features. Each form is discussed individually in the sections below.

\subsection{General}

The General form (see Figure 16.1) under the Main Menu’s "Preferences” button allows you to make settings for some in-depth run analyses, which may assist developers investigate any apparent unusual behavior in the program, as well as allow the user to designate images and text to appear at the top of reports that the program generates. Below are brief descriptions of the settings and controls found on the General form.

- Installation ID - This field allows you to specify (or change) the Installation ID, which was previously requested on the Installation ID form displayed when the Weatherization Assistant was started the first time following its installation. The Installation ID can be used to identify one installation of the 


\section{Chapter 16: Preferences}

Weatherization Assistant from another, possibly in the same office or agency. See Section 2.8, Starting the Weatherization Assistant, for information on the use of Installation ID in the Weatherization Assistant. Optional.

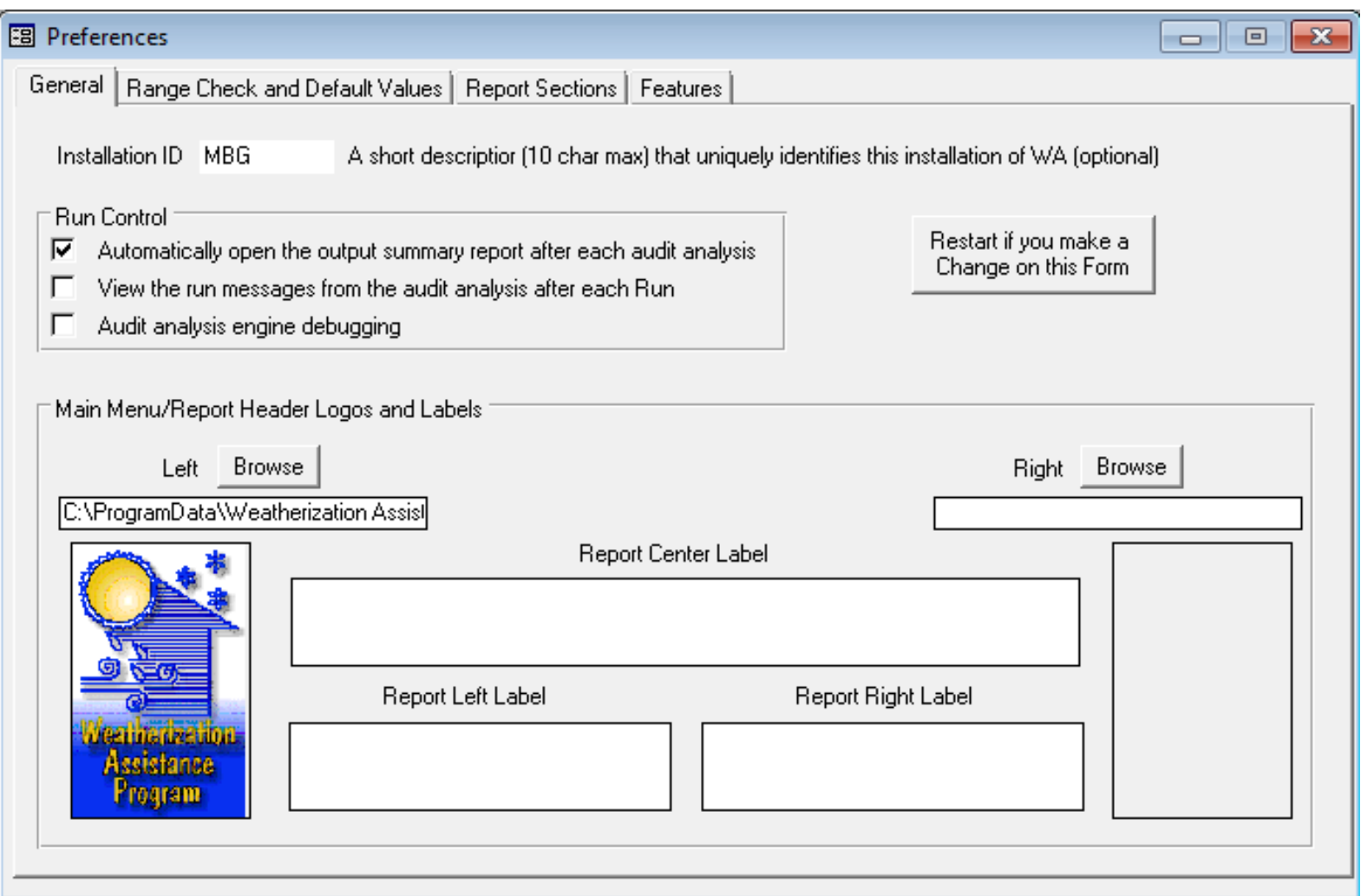

Figure 16.1. The General form under the Main Menu’s “Preferences” button.

- Restart - The "Restart" button should be selected whenever changes are made on the form to ensure that the options selected take effect during the current session of program use. Following the button's selection, you will be taken to the Main Menu.

\section{Run Control Block}

The Run Control block provides three checkboxes controlling reports that may be automatically displayed upon executing an audit. If none of the checkboxes are selected, no obvious change, other than the final disappearance of the <busy $>$ cursor, will be seen on your computer screen following execution. 
- Automatically open the output summary report after each audit analysis This checkbox controls whether the Recommended Measures report is displayed after execution (see "Report Block" in Section 8.2, Audit Information). The report displays information related to the audit recommendations for the house being analyzed. See Appendix C, Reports, for a sample of this report. The default setting for this checkbox is checked.

- View the run messages from the audit analysis after each Run - If this checkbox is selected, the program will display run messages produced during the execution of an audit. This display requires that your computer has access to Microsoft WordPad, which is normally part of your Windows installation. Use the normal Windows close button to exit the message display. The program will also write these additional diagnostic messages to a file named "last_neat_run.txt" or "last_mhea_run.txt," depending on which audit was last executed, and save the file in the folder created during installation (e.g., “C: Program Files \Weatherization Assistant 8-9" or “C: $\backslash$ ProgramData\ Weatherization Assistant 8-9”). Note: Unless you rename these message files, they will be overwritten each time you execute an audit of the same type, NEAT or MHEA. These diagnostics data would likely be useful to program developers in determining causes for unusual behavior of the program. The files may be viewed with any text editor. The default setting for this checkbox is unchecked, suitable for normal execution of the program.

- Audit analysis engine debugging - If selected, this checkbox will add to the above diagnostic messages output detailing the calculations performed by the audits. The display requires access to Microsoft WordPad. The output can be lengthy and is not designed to be of significant use to normal users. However, it may assist support personnel in identifying problems that might be occurring. Use the normal Windows close button to exit the display. The information is saved to your computer, may be viewed at any time using a text editor, and is overwritten each time an audit of the same type is executed. The default setting for this checkbox is unchecked, suitable for normal execution of the program.

\section{Main Menu/Report Header Logos and Labels Block}

Fields in this block allow you to customize report headers and add your own logo to the Main Menu display. Below are discussions of the data items and controls found in the Main Menu/Report Header Logos and Labels block. 
- Browse - Use the Left and Right "Browse" buttons to locate and choose logos to be displayed on the upper left and right, respectively, of your reports and the Weatherization Assistant's Main Menu. Selection of the buttons takes you to normal Windows browse displays where you can select any file in most graphic formats (e.g., jpg, bmp, etc.) to be used as logos. The graphics you use may have to be correctly sized using other graphics applications. The field under each browse button displays the path and file name of the graphic file you have chosen. The boxes below the path name fields display the actual graphics that have been selected. For a Weatherization Program agency, it is customary to retain the Program logo on the left.

- Report Label - Enter the text you wish to have displayed in report headers into the Label fields provided. Provision is made for Left, Right and Center text. Note, however, that no attempt is made to truncate or create multiple lines of header from this text. Thus, if text is entered for all three positions and the text is too lengthy, it has the possibility of overlapping. Figure 16.2 shows an example of a report header with left and right logos and text entered for all three positions. Notice that the text almost overlaps. If you have multiple installations of the Weatherization Assistant in your agency, you may wish to coordinate these settings.

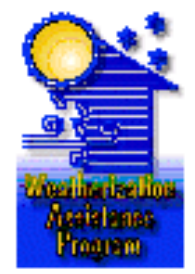

\section{NEAT Recommended Measures}

Sample Left TextSample Center TextSample Right text

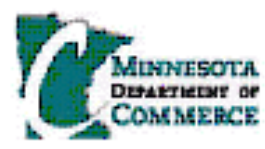

Figure 16.2. Report displaying optional logo and header text.

\subsection{Range Check and Default Values}

To assist you in properly entering data, the Weatherization Assistant provides you with default values and range checks for many of the numeric input parameters (See Sections 5.6, Field Defaults, and 5.7, Field Range Checking). Though values for these defaults and ranges are set during installation of the program, you may alter these values on the Range Check and Default Values form (see Figure 16.3) under the Main Menu's "Preferences” button. 
On this form, each parameter is listed by the Main Menu window ("Form") and form ("Location") on which it lies. Designated columns allow you to adjust the "Min," "Max," and "Default" values to meet your needs. No other fields on this form are editable and no records can be added or deleted. If a field on this form is left blank, no default will be available or no range checking will occur for that particular parameter. Also, any default computed by either of the audits overrides the default entered on this form. The Range Check and Default Values form must be exited before any changes made during a visit to the form will take effect.

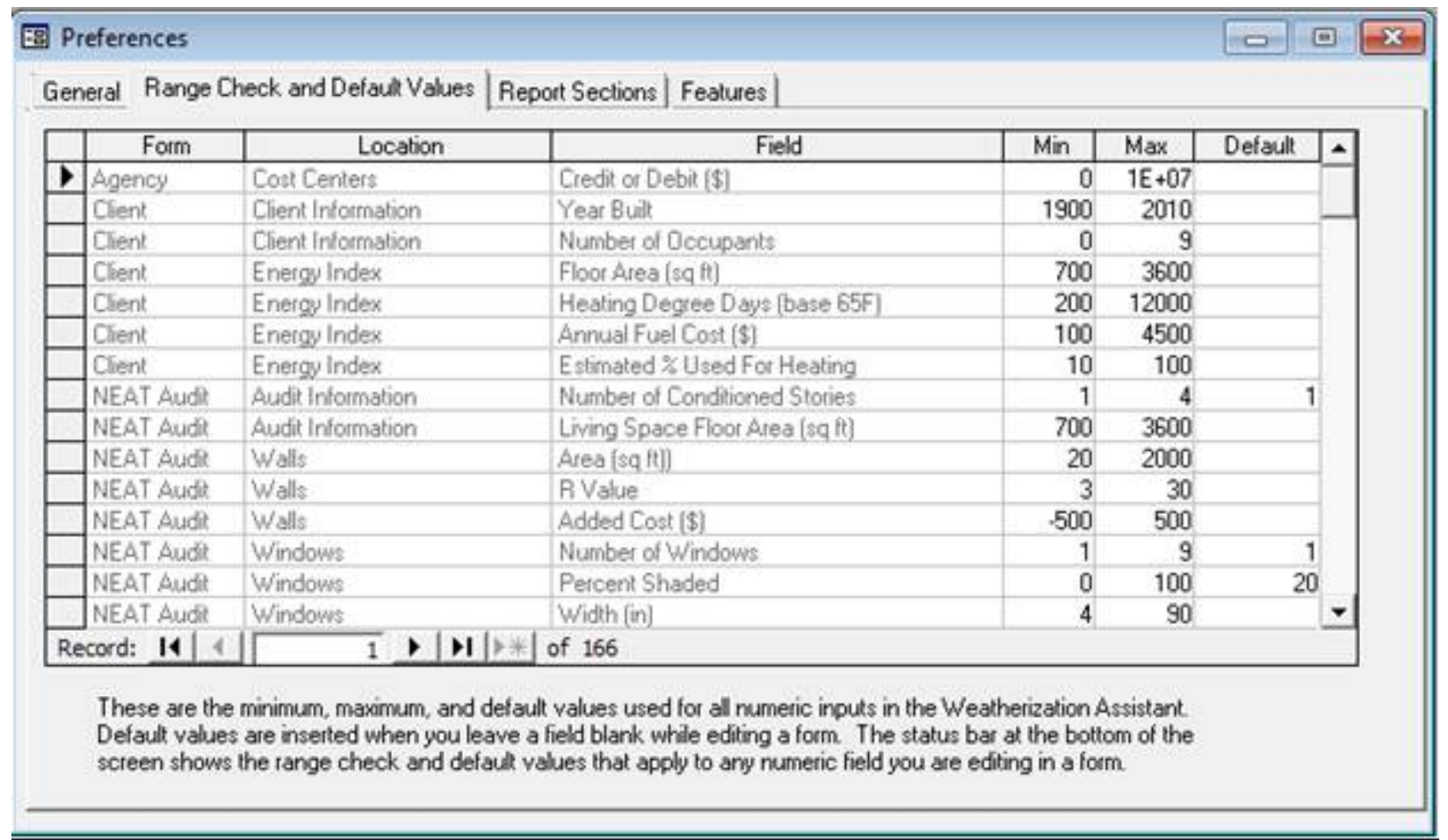

Figure 16.3. Range Check and Default Values form under the Main Menu's “Preferences" button.

\subsection{Report Sections}

Two of the reports available from the NEAT and MHEA audits are the Recommended Measures and the Input Report (see "Report Block" in Section 8.2, Audit Information). Both of these reports have multiple sections (see Appendix C, Reports). The Report Sections form (see Figure 16.4) under the Main Menu's "Preferences" button allows you to select which sections of these reports are displayed and/or printed. Below are brief descriptions of the settings and controls found on the Report Sections form. 


\section{Chapter 16: Preferences}

- Which Report - This drop-down list displays the names of the reports for which the settings can be edited. The list includes: NEAT Recommended Measures, NEAT Input Report, MHEA Recommended Measures, and MHEA Input Report.

- Sections to Hide or Make Visible Block - This block displays the Section Names of the report selected in the "Which Report" drop-down list, and allows you to select the sections you wish to be displayed on the report. Select the checkboxes under Visible to indicate your preference. The default setting for all sections is Checked.

- Select All, UnSelect All, Invert Select (buttons) - The "Select All," "UnSelect All," and "Invert Select" buttons allow you to select all listed sections, unselect all, or invert the selection currently set from the checkboxes.

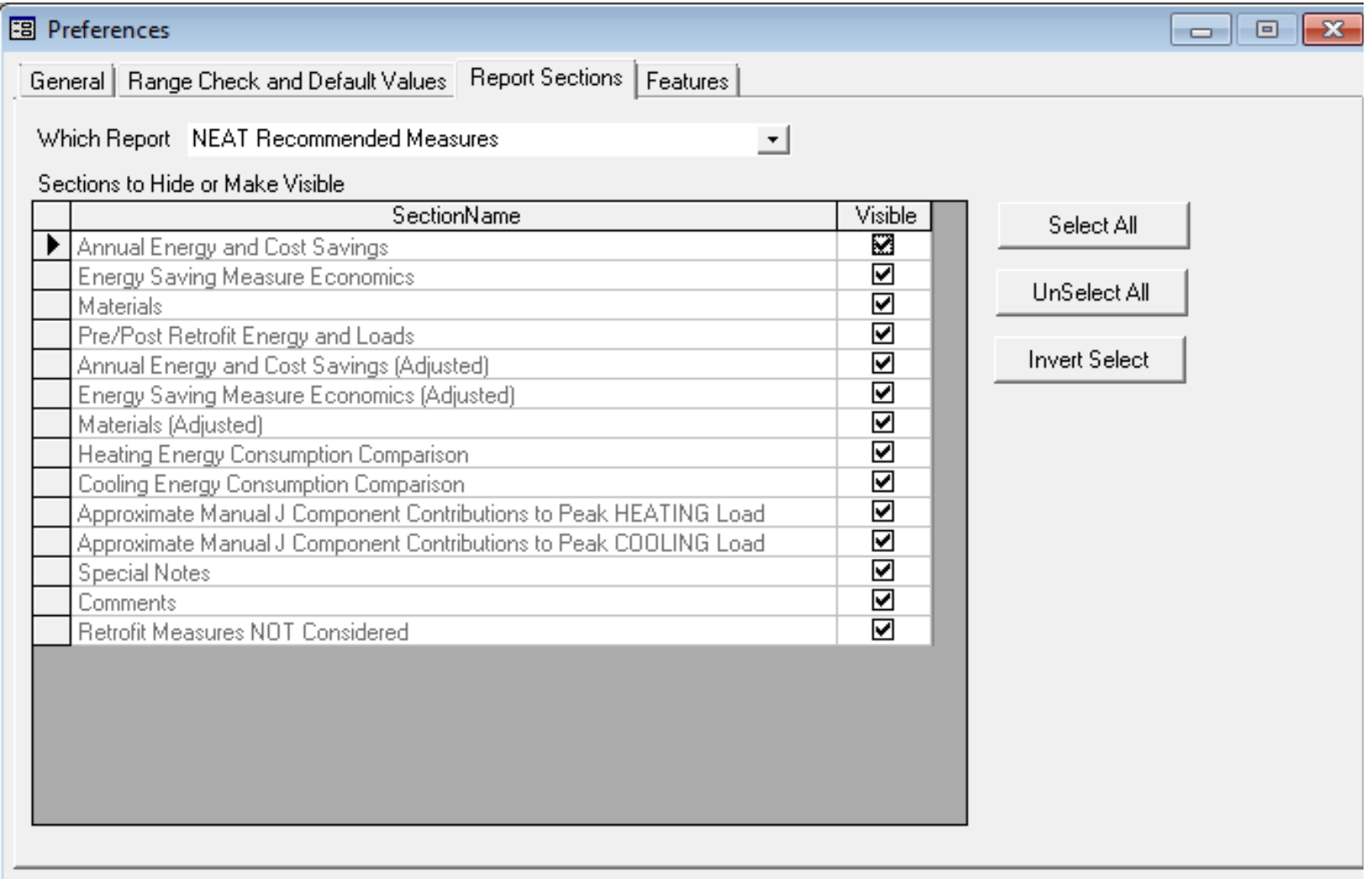

Figure 16.4. The Report Sections form under the Main Menu's “Preferences” button. 


\subsection{Features}

The Features form (see Figure 16.5) under the Main Menu's "Preferences" button is used for setting preferences regarding many features of the Weatherization Assistant. You can activate these features by selecting the corresponding checkboxes. The feature selections are independent of one another, except for the two related to Digital Photo Options. Below are brief descriptions of the settings and controls found on the Features form.

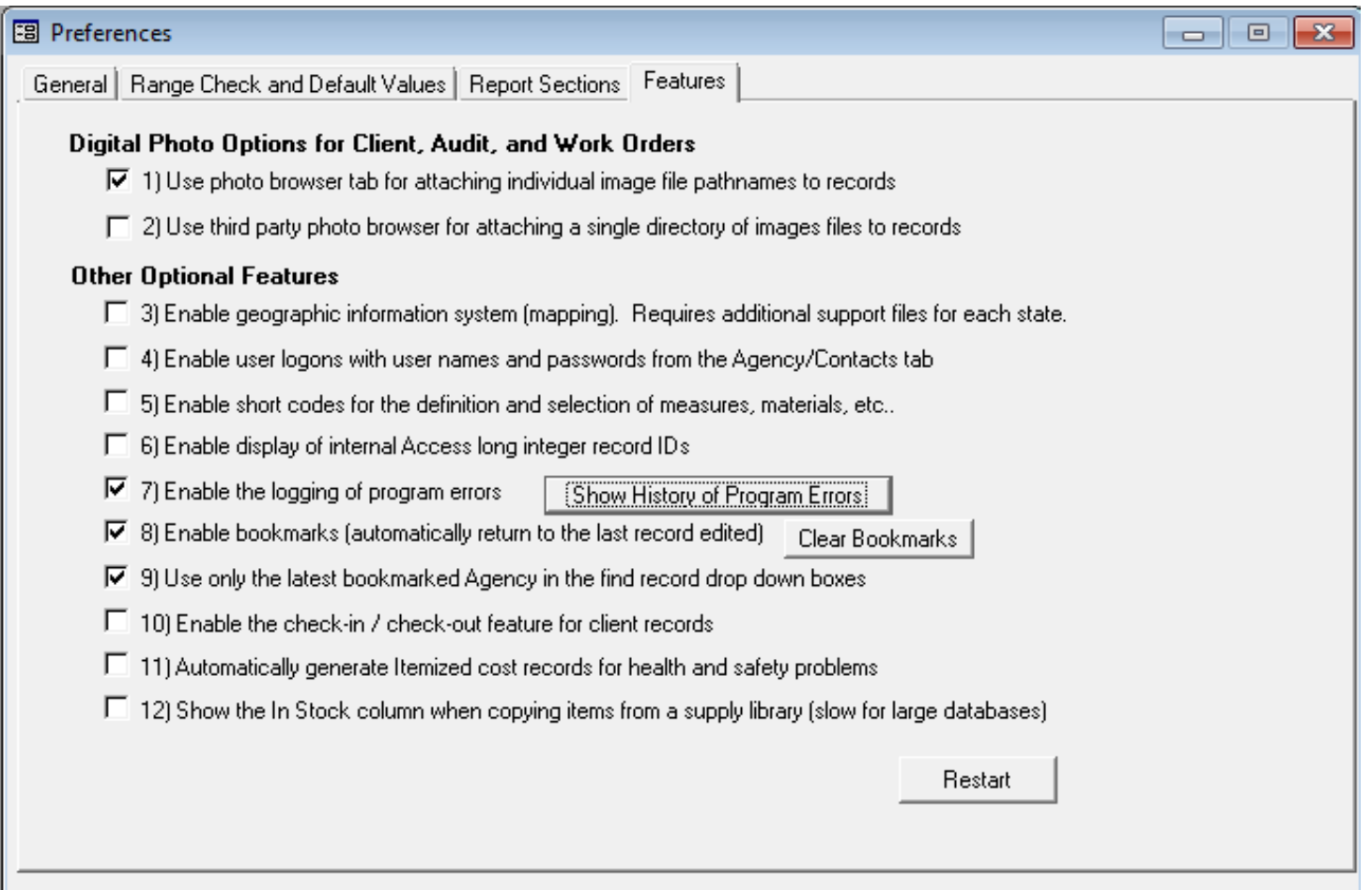

Figure 16.5. The Features form under the Main Menu’s “Preferences” button.

- Restart - The "Restart" button should be selected whenever changes are made on the form to ensure that the options selected take effect during the current session of program use. Following the button's selection, you will be taken to the Main Menu.

- (1) Use photo browser tab for attaching individual image file pathnames to records - The Weatherization Assistant allows storage of digital images associated with your weatherization work. These photos can be stored, viewed, and edited on your computer using one of two methods. The method selected by this first checkbox allows you to store the location on your computer of each 
individual photo image file. Thus, photos accessible from any location in the Weatherization Assistant need not be in any particular location. Choice of this method will add a tab to any Main Menu window to which photos can be attached. This is the photo feature initially selected. See Section 18.1, Digital Photos, for more information on the use of the photo browsers.

- (2) Use third party photo browser for attaching a single directory of image files to records - This second method of storing, viewing, and editing photos requires you to assign a location on your computer where all photo files associated with a given record for each Main Menu window of the program (e.g., Audits, Work Orders, etc.) will be stored. Thus, all photos associated with a given work order must be stored together and all those for the audit of a client in another (or the same if so chosen). If this method is selected, the Weatherization Assistant will display a Photo Folder field on the Information forms of the appropriate Main Menu windows asking for this location. Use the "Browse" button to find and select the location, then the "View" button to view the photos stored in that location. See Section 18.1, Digital Photos, for more information on the photo browsers.

Photo Folder C:MrogramDataWWeatherization Assistant 8-9 Browse View

Figure 16.6. Photo Folder field for third party photo browser.

- (3) Enable geographic information system (mapping) - This checkbox turns on the Geographic Information System (GIS), which allows you to map the locations of clients' dwellings (see Section 18.2, Geographic Information System (GIS)). Using this feature requires downloading additional files and an installation. If the feature is selected without having performed this installation, you will receive a message upon restarting the program that the installation has not been performed and that the feature is subsequently turned off until installation is complete. If the required installation for the feature has been performed, selecting this feature will place a "Map" button on the Client Information form (see "Geographic Information System Data Items" in Section 7.1, Client Information) and under the "Select Clients" button of the Agency Information form (see "Select Clients" in Section 6.1, Agency Information).

- (4) Enable user logons with user names and passwords from the Agency/ Contacts tab - This feature allows regulation of the users for a specific 
installation of the program. If activated, users must logon with a User Name and Password to enter the program. See "Change LogOn Group and Password" in Section 6.2, Contacts (Agency), for additional details on this feature. If this feature is selected, the user will receive a "User Logon Notice" window with the warning: "Turning on the logon feature will require you to select a user name and enter a password using records entered on the Agency/Contact tab the next time the program starts. Be sure you have a record entered on that tab with your user name and an assigned password before turning on this feature.” The form will then allow you to either continue or cancel the selection of the feature. If the feature is activated without the requisite user name and password available, you will be locked out of using the Weatherization Assistant.

- (5) Enable short codes for the definition and selection of measures, materials, etc. - This feature was never fully implemented into the Weatherization Assistant. The selection here will make no difference in the operation of the program.

- (6) Enable display of internal Access long integer record IDs - Whenever a record (such as a client, audit, or work order) is created, Access assigns the record a random integer ID number unique to the record. This ID number will not change, even if you change the other information related to the record or export the record to another computer. Under normal operation of the program, this Access ID number is of little use. Thus, the default mode is to not have them displayed. However, they can be used to uniquely identify a record, regardless of its location. Program analysts who may use other Microsoft products to debug or otherwise analyze the execution of the program with relation to a particular record may require the ID. Whenever a record is copied, the new record is given a different, unique, integer record ID.

- (7) Enable the logging of program errors - Although many possible conditions that would cause the Weatherization Assistant to not function properly have been anticipated and addressed by provided warnings or messages to the user, some likely remain unaddressed. Under such circumstances the user will receive an Access or System error message, which the user is not expected to understand. If this feature is turned on, these "unhandled" errors will be logged giving more detailed information regarding the condition of the program at the time the error occurred (see Figure 16.7). This information, together with the user's description of what operation was being 


\section{Chapter 16: Preferences}

performed when the error occurred and possibly the database being used at the time of the error, is normally sufficient to allow developers of the program to determine the cause of the problem.

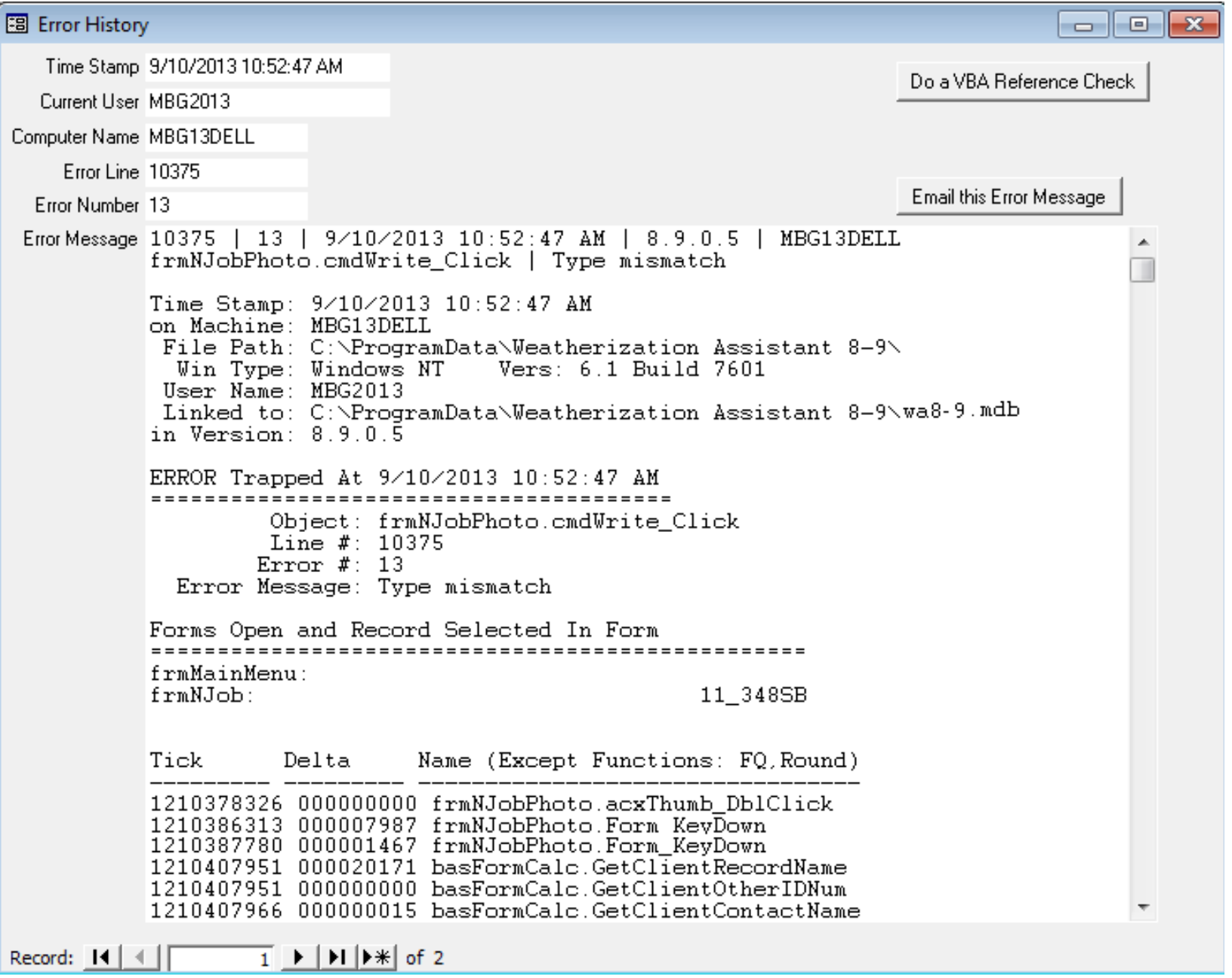

Figure 16.7. Program Error History form.

- Show History of Program Errors - Selecting this button to the right of this feature's description will display an Error History form showing the latest error (if any) that has occurred with all of the information the software can provide related to it. Note that the "Error Message" window will likely have a vertical scroll bar associated with it. The abbreviated navigation block at the bottom left of the form can be used to access additional error entries, if they exist. The "Do a VBA Reference Check" button on this form initiates an installation test, which might be of use to program developers in diagnosing irregular operation of a specific installation. You may view the error records in data sheet view (see 
Section 5.8, Data Sheet and Form Views of Records), from which you may delete any records no longer desired (see Section 5.10, Deleting Data). It is likely good policy to keep the error messages recorded to a minimum so that if an error that causes considerable disruption in your operations occurs, it can be easily recognized in the error history. If an error that you need assistance to resolve occurs and this feature is not activated, turn it on and then try to reproduce the error. If you have set up the e-mail feature of the Weatherization Assistant (see Section 18.4, E-Mailing from Within Weatherization Assistant), you may click on the "Email this Error Message" button to quickly e-mail the error message to program support personnel.

- (8) Enable bookmarks (automatically return to the last record edited) This feature keeps track of the last record you visited under each of the Main Menu buttons (e.g., “Agency,” Client,” "Energy Audits,” etc.), and displays that same record the next time that button is selected. Otherwise, the program will present the first record in its list of records of that type. This order may not be obvious and more likely will require you to access the desired record via the record navigation block. This same order will be initiated for all record types if the "Clear Bookmarks" button is selected. The default setting for this feature is checked.

- (9) Use only the latest bookmarked Agency in the find record drop-down boxes -This feature will restrict the records displayed in the record navigation blocks (see Section 5.1, Form and Record Navigation) on the Information forms under the "Clients," "Energy Audits," and "Work Orders” Main Menu buttons to those belonging to the agency last visited via the Main Menu's "Agency" button. If this feature has not been selected, the record navigation drop-down lists will display records associated with all agencies in the database you are currently linked to. For most agencies using the program, this feature will have no effect, since their agency will be the only one in the database. However, for a state director with clients from all of the state's agencies imported into a common database, lists of clients, audits, work orders, etc. would likely be long, making locating a specific record difficult. With this feature activated, the director may view only records from a specific agency at any given time. The feature is initially activated with the agency present at installation bookmarked. 
- (10) Enable the check-in/check-out feature for client records - This feature is used by the Client Export routines (see both topics on "Exporting Clients" in Sections 17.3, Data Transfer - Import/Export With Another MSAccess Database, and 17.4, Data Transfer - Import/Export Indirectly with Text Files) of the Weatherization Assistant. It is used in conjunction with the User Logon feature (Feature 4, described above). If activated, the program tracks which clients have been exported and who was logged on when each was exported. The feature may be of use if you routinely pass client information between computers. A client record that is "checked out" will show what user checked the record out on the Client Information form for that specific client (see "Checked Out To" in Section 7.1, Client Information) as well as on the forms associated with the Import/Export feature, referenced above.

When this feature is activated, you will receive a notice from the program that the User Logon feature will also be activated. See the warning stated relating to this feature. If you are not prepared to have the User Logons feature activated, be sure to deactivate it before proceeding.

- (11) Automatically generate Itemized cost records for health and safety problems - This feature automatically adds a library health and safety measure to the Itemized Costs forms of an audit corresponding to each health and safety problem identified on the Health \& Safety forms, the Optional Details for Heating Systems sub-forms, and the Optional Details for Water Heater subforms of the audit. After running the audit, these measures are listed on the Recommended Measures report. See "Handling of Health and Safety Issues" in Section 11.7, Health and Safety, for more information on this feature.

- (12) Show the In Stock column when copying items from a supply library (slow for large databases) - If you are using the inventory feature of the Weatherization Assistant, you will be using the "Copy Supply" drop-down list to locate items in the Supply Library and indicate their use in work orders (see "Copy Supply” under "The Materials/Labor Details Sub-Form” in Section 13.3, Measures (Work Orders)). One of the columns in this drop-down list is the "In Stock" column. To obtain this number, the Weatherization Assistant searches through its database for all the instances that the material has been used. This search can require considerable time for large databases. This feature allows you to turn off this computation, thus speeding up the presentation of this dropdown list, but at the expense of eliminating the "In Stock" column. 


\section{Chapter 17. Data Link}

The facilities under the Main Menu's “Data Link” button allow you to group client information (such as for different program years), quickly link to various database files, and share information on individual clients or groups of clients with others.

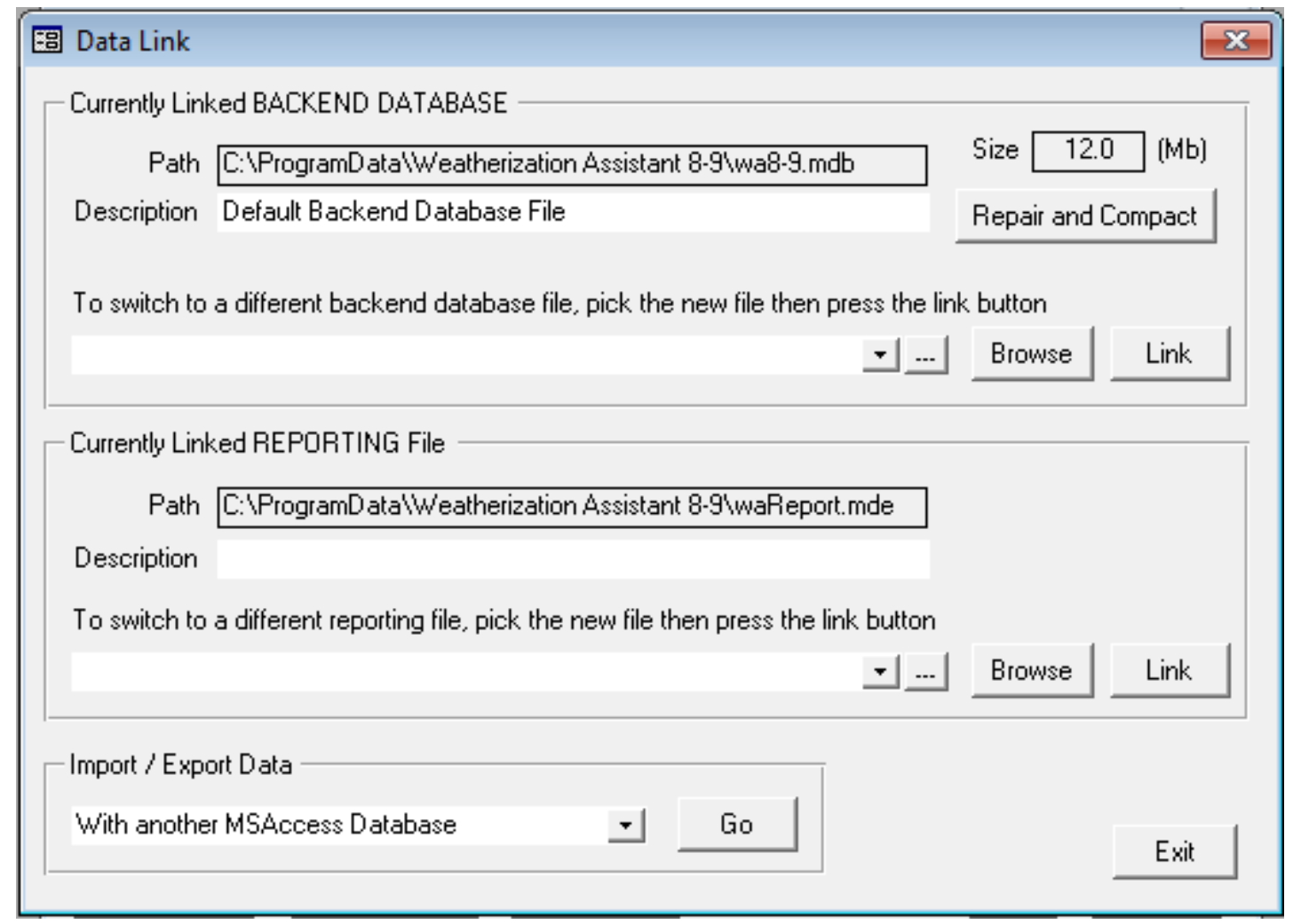

Figure 17.1. The Data Link form.

All user-supplied data and audit results in the Weatherization Assistant are stored in a file on the computer. The file is a Microsoft Access database file, termed the "backend" file in Access terminology. Files on your computer are designated by a name and an extension with the two separated by a period (e.g., "wa8-9.mdb"). This is, in fact, the default name of the file in which data in Version 8.9 of the Weatherization Assistant will be stored, the "mdb" standing for "Microsoft database.” You may have as many database files as you feel necessary with whatever names you choose, as long as their extensions are "mdb." You may wish to have a separate database file for each program year. A state may have a different database file for each agency within their state. The Data Link feature of the Weatherization Assistant allows you to quickly "link" to various database files. 
When linked to a specific database file, the program views only the client information contained in the file, thus allowing you to more easily locate records.

Understanding the concept of the database file allows you to perform some relatively simple operations outside of the Weatherization Assistant using standard Windows capabilities. Although means within the program may accomplish the same objectives, those with knowledge of Windows may prefer to use its features instead. For example, database files may be copied using Windows, though the files' names must remain unique. If, immediately after installing the Weatherization Assistant, the original "mdb" file (e.g., "wa8-9.mdb") is copied, this original file may be saved as an empty database file for future use. If you plan to keep separate database files for each program year, you might name the copied database file after the year whose data it will contain (e.g., "wa8-9 PY2011.mdb”), then link to this database file (see Section 17.1, Currently Linked Backend Data File) for use during that year. When you are ready to begin the 2012 program year, make another copy of the original, empty database file and name it after the new program year (e.g., "wa8-9 PY2012.mdb”), link to it, and begin using it. Thus, you are provided with separate files, each with all the data applicable to a specific program year. You may think of other reasons to have multiple database files with names you choose to indicate specific characteristics.

In addition to allowing you to link to various database files, the facilities on the Data Link form also provide means to share information with others. Although this could be accomplished by simply sending an entire database file, an "mdb" file, this may not be the best method under some situations. The added features on the Data Link form provide you with two independent methods of sending others all or portions of the contents of database files. These features might be used to move client data from a laptop used in the field to the central agency computer in the office or to upload data periodically to the state agency. You may select specific clients to include in your data transmission, you may send them in a "compressed" and "encrypted" format, or you may send them in a format more appropriate for use by other software applications. Files created by these alternate approaches will have different "extensions" than the "mdb" files, but they still contain similar information.

Report formats that are available in the Weatherization Assistant are stored in another file, waReport.mde. The program must also link to this file before you may utilize these reports. With the ability in Version 8 to have multiple reporting 
modules (see Appendix D, Development of Customized Reports), you may need to select between various reporting modules. The Data Link facility allows you to perform this operation.

The following sections discuss in more detail the facilities available on the Main Menu's "Data Link" button of the Weatherization Assistant. The discussion is subdivided into topics whose related data input fall within separate blocks on the Data Link form.

\subsection{Currently Linked Backend Data File}

As discussed above, you may have multiple database files in which your client information is stored, perhaps dividing the data into different program years or as coming from different sources. This facility under the "Currently Linked Backend Database” block allows you to quickly access any of these databases. The fields and controls under this block are discussed separately.

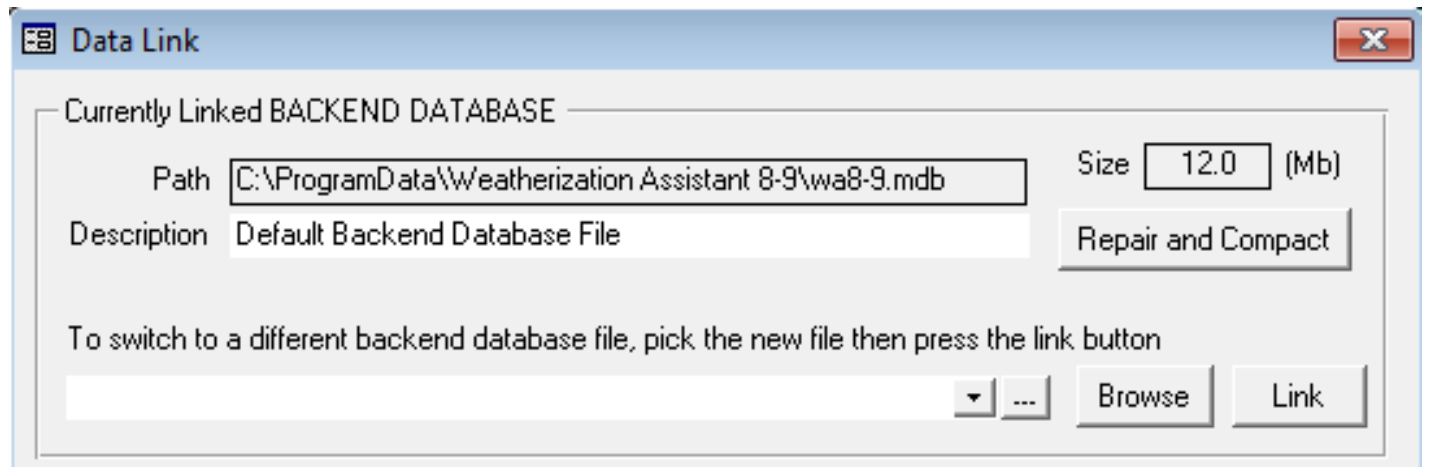

Figure 17.2. The “Currently Linked Backend Database” block.

- Path - This field displays the path (location in your computer) and name of the database file you are currently linked to. Thus, any data viewed anywhere within the Weatherization Assistant will come from this file. If the path designation is long, you may need to use the [Home] or [End] keys to be able to view the entire entry. Uneditable.

- Size - This field reports the size of the database file displayed in the Path field, in Megabytes (Mb). This may be important to note if you intend to send your entire database file via means that may have restrictions on the size of files that 
can be transmitted or stored. Also, the time required to send files by e-mail will likely depend on the size. Uneditable.

- Description - This field provides you with a means of identifying the characteristics or contents of the database file designated in the Path field. If the file was sent to you, it may reveal identifying information to you regarding the file. Optional.

- Repair and Compact - With extended use, a database file may become disjoint or even damaged. The "Repair and Compact" button examines the database file identified in the Path field and attempts to repair any damaged segments, then compact the file into its most efficient form. If selected, the operation will notify you that it "requires exclusive use of the backend database." Thus, "If the currently linked backend [database] is shared on a network, be sure everyone has exited the program...”, or are not linked to the database. You are then given the opportunity to continue or cancel the operation. It is a good idea to execute the Repair and Compact operation periodically to ensure your database remains free of errors. If you should have an abnormal termination of the program, such as due to a power failure, it would be particularly advisable to execute it. Note, under such circumstances, some of the most recent data may be lost, but the remaining data would be intact and in proper format for future use.

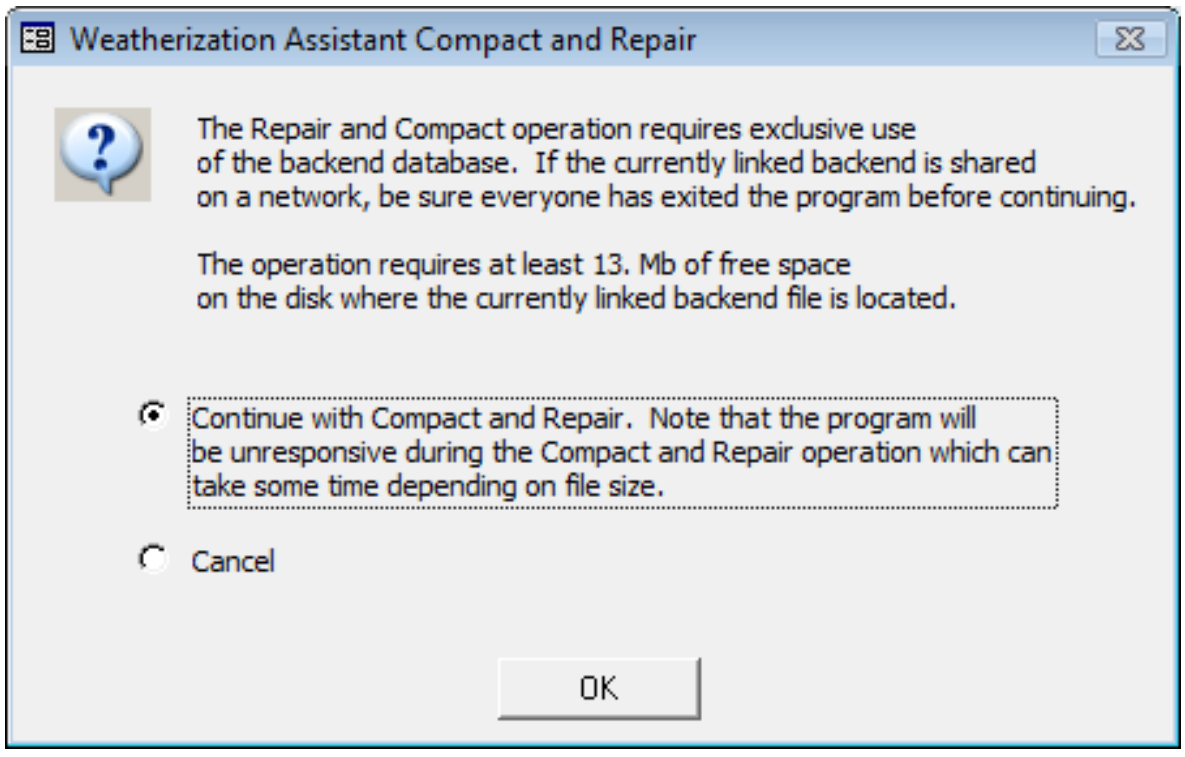

Figure 17.3. The Compact and Repair window. 


\section{Chapter 17: Data Link}

- To switch to a different backend database file, pick the new file then press the link button [drop-down list and "Browse" and "Link" buttons] - A history of database files you have accessed using this drop-down list is kept by the program. These files are displayed in this drop-down list. Selecting the database file from the list will automatically place the database path and file name into the drop-down list's field. If the file no longer exists, you will be notified in a message. Otherwise, you may then immediately select the "Link" button to link to the specific database file.

Selecting the box containing three periods just to the right of this drop-down list, $--\cdot$, produces a datasheet view of all the entries that appear in the dropdown list. The form allows you to delete any entries no longer of use or no longer in existence (see Section 5.10, Deleting Data).

You may also use the associated "Browse" button to locate files you want to link to. Selecting the "Browse" button will take you to the "Pick Database to Link" window, a standard Windows Operating System browse window (see Figure 17.4).

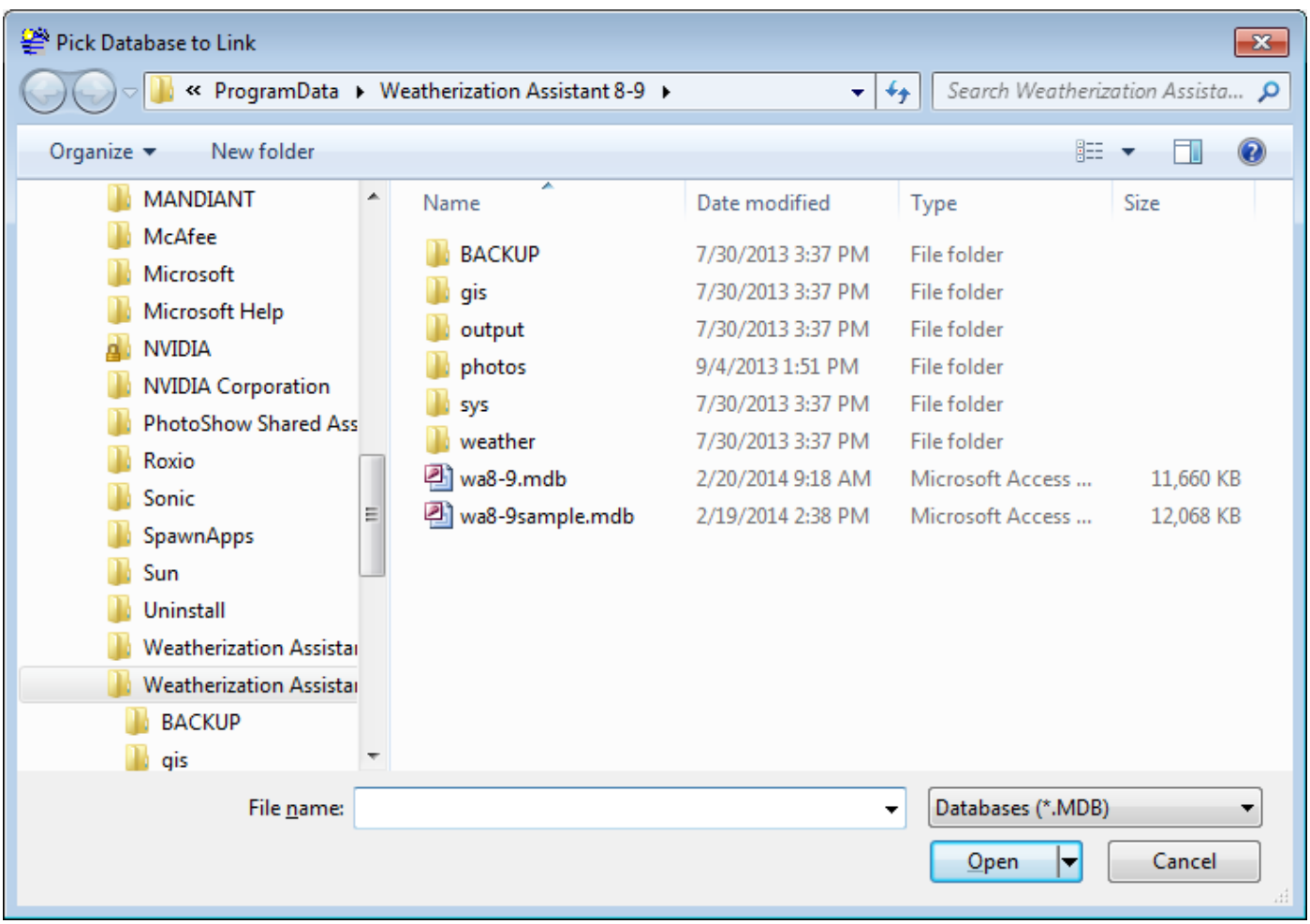

Figure 17.4. The Pick Database to Link window. 
The default location when entering this window will be the location from which you last chose a database using this window. If you have not yet used the "Browse" button, this location will be the folder where you installed the Weatherization Assistant, typically C: ProgramDatalWeatherization Assistant 8-9. However, you may navigate to anywhere on your computer or any network to which you are attached. Locate the database file you wish to link to. Select the file by either double clicking on it or single clicking on it and choosing the "Open" button in the lower right corner of the window. Doing either will take you back to the Data Link form with the database path and file name entered into the drop-down list's field in the block. Then, select the "Link" button to link to the database file.

Whenever the "Link" button is selected, you will be shown a progress bar in a dialog box that monitors the progress of the linking operation. When completed, the message "Data Link was successful" should appear. Select the "OK" button in this window. A similar linking process will then be performed for the Reporting module associated with the database, which will indicate that "Reporting Data Link was successful” when completed. Again, select “OK.”

Following a successful linking operation, one of two courses will be taken by the program. If you do not use the User Logon feature (see Feature 4, "User logons with user names and passwords from the Agency/Contacts tab" in Section 16.4, Features), the program will take you to the Main Menu with the newly linked database file information now visible in the $<$ Database $>$ and Description fields (see Figure 2.9). If you do use the User Logon feature, you will instead be taken to the Weatherization Assistant's Log On form (see Section 3.1, Initial Start-up of the Weatherization Assistant) where you will have to logon as a user of this specific database. In either case, you are now ready to access all the information in the database just linked to.

\subsection{Currently Linked Reporting File}

This data block acts the same as the "Currently Linked Backend Database" block discussed above except that it links only to a Reporting file. Under most circumstances, linking to a report file will be accomplished for you, either on installation of the program or as you change database files (see Section 17.1, Currently Linked Backend Data File). However, Version 8 of the Weatherization Assistant allows for multiple reporting modules (see Appendix D, Development of 


\section{Chapter 17: Data Link}

Customized Reports). This data block allows you to choose which reporting module will be used in the current session of running the program.

\subsection{Data Transfer - Import/Export with Another MSAccess Database}

The Weatherization Assistant provides you with two methods of sharing data with others. The method is selected using the drop-down list in the "Import/Export Data" block at the bottom of the Data Link form (see Figure 17.1). The transfer "With another MS Access Database" method is most applicable to sending portions of your database or even single client records. The method creates files either in the same format as your entire database (i.e., "mdb" files), or compressed and encrypted "wdz" files, where the designations relate to the "extensions" given to the file names of the files created. Whereas you may directly link to "mdb" files, the "wdz" files must be "imported" before their information can be accessed. However, the latter files are smaller and their information is "encrypted" such that only those with the Weatherization Assistant software can view their contents.

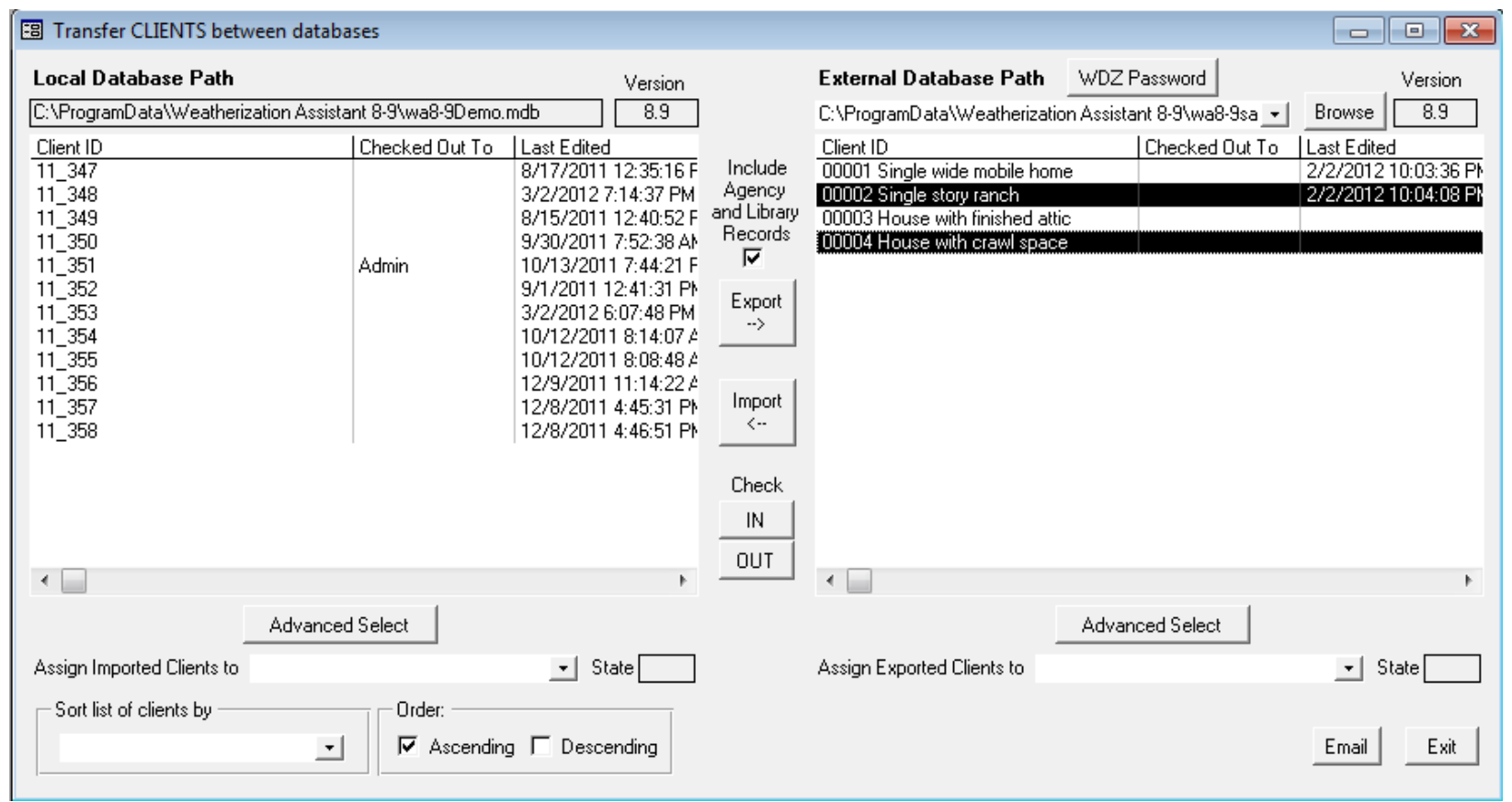

Figure 17.5. Import/Export via MSAccess Database (Transfer CLIENTS between databases) - Check-in/Check-out feature activated (two clients selected for import).

Choosing this method from the drop-down list, then selecting the "Go" button to the right, will present you with the "Transfer CLIENTS between databases" form, 
displayed in Figure 17.5, though initially there will be no entries in the panel on the right. The figure has been produced with the Check-in/Check-out feature activated. This adds the "Checked Out To" columns in each of the form's two panels and the "Check IN/OUT" buttons, which would not be present without this feature activated (see Feature 10, "Enable the check-in/check-out feature for client records” in Section 16.4, Features).

In the left panel of this form you will see a list of clients in the database you are currently accessing (linked to). The database path and file name are displayed in the "Local Database Path" box at the top of this half of the form as well as a reminder of what version of the program created these entries. Additional columns of data may be seen by using the horizontal scroll bar at the bottom of each panel. The data columns available are

Client ID

Last Edited / By

Contact Name
Alt. Client ID

City / Zip

Agency Name / State
Work Status / Date

Last Imported

Last Exported

\section{Importing Clients (from another MS Access database)}

The following discussions that describe specific data items or controls found on the “Transfer CLIENTS between databases" form, as they pertain to importing clients from MS Access database files, are bulleted. Those applying to the process in general lie outside any bullets.

- External Database Path [drop-down list and "Browse" button] - To import clients to your database from another MS Access database, you must first locate the database file containing the clients you wish to import. If you have imported or exported clients previously, the database files you used will be displayed in the "External Database Path" drop-down list at the top of the right half of the "Transfer CLIENTS between databases" form. You may select one of these previously accessed files to use again on this occasion. Otherwise, select the "Browse" button to the right of this drop-down list. This will take you to the "Pick External Database for Import/Export” window, a standard Windows Operating System browse window, an example of which is shown in Figure 17.6. 
Chapter 17: Data Link

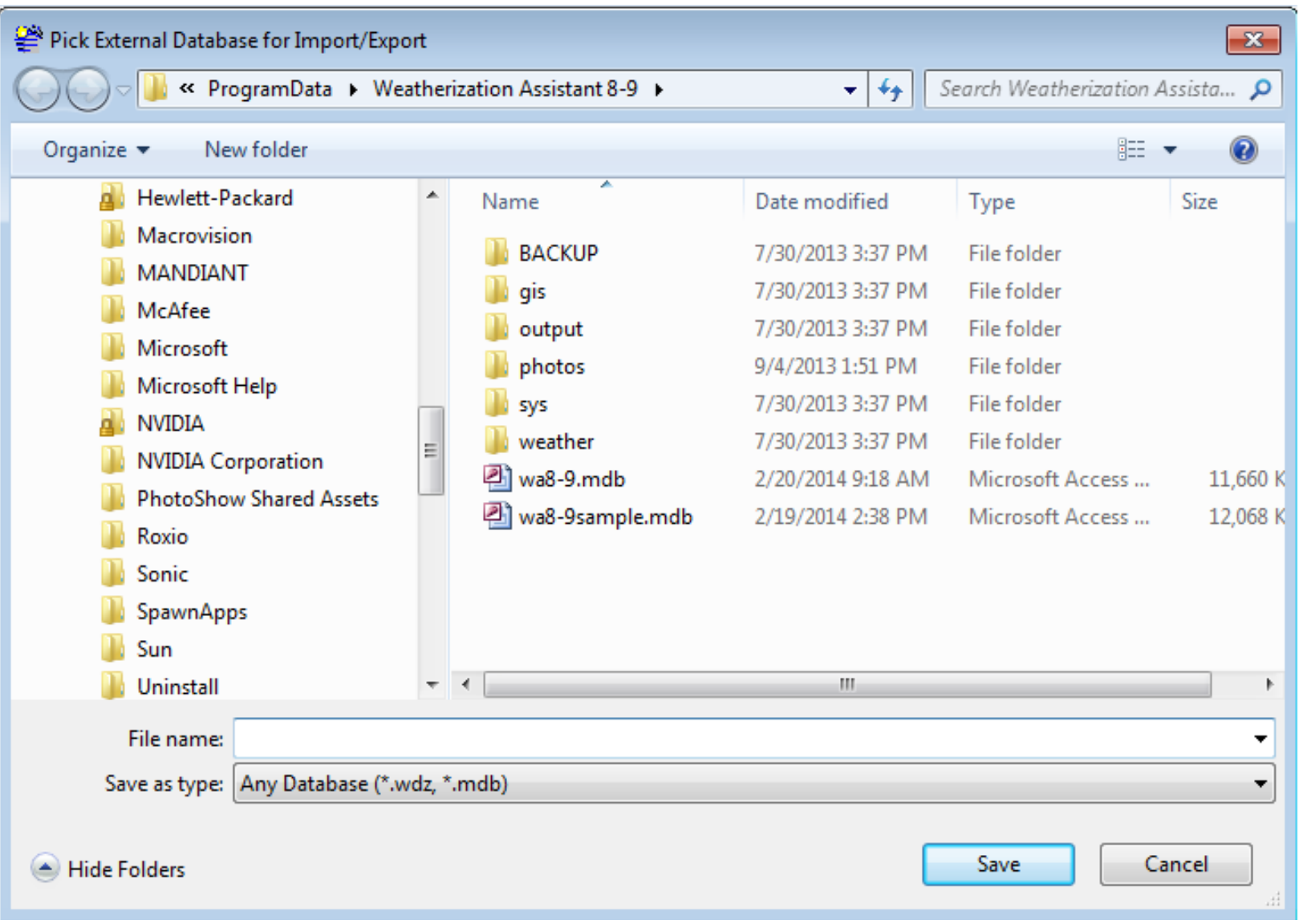

Figure 17.6. The Pick External Database for Import/Export window.

Use standard Windows techniques to locate the "mdb" or "wdz" file that contains the desired clients to be imported. The default location when entering this window will be the location from which you last chose a database. If you have not yet used the "Browse" button, this location will be the folder where you installed the Weatherization Assistant, typically C: ProgramData। Weatherization Assistant 8-9. The drop-down list near the top of the form will present you with a list of "Previous Locations" accessed from the form. Other locations accessible to your computer can be located using the left panel, possibly requiring use of its associated vertical scroll bar. Once a location is selected, the contents of the folder will appear in the right panel. Clicking your mouse on the desired file from this listing will transfer the file name to the "File name" field near the bottom of the window. Select the "Save" button to accept this file as the one containing the desired clients, or "Cancel" to abort the search without having selected a file. Either will return you to the "Transfer CLIENTS between databases” form. 
The right half of the "Transfer CLIENTS between databases" form will now contain a listing of clients in the selected database from which you are going to import (see Figure 17.5 for an example). Note the display under the "Version" title indicates the version of the database whose clients are being displayed. Only clients from databases produced by versions of the Weatherization Assistant having version numbers beginning with " 8 " can be imported. The data in prior versions (Versions 7 and below) have data structures significantly different, making their conversion impractical.

Select the clients to be imported by clicking on rows containing their information in the listing on the right of the screen, and using the other standard Windows item selection procedures. For example, you can select multiple clients lying adjacent to each other by clicking on the first or last, then holding down the [Shift] key while using the [Up Arrow] or [Down Arrow] keys to select others. Holding down the [Ctrl] key while clicking on clients will allow you to select multiple clients that are not adjacent to each other. To assist in the selection process, you may sort the listings by any of the client identifying criteria, using the "Sort list of clients by" drop-down list at the lower left of the form. The sort may be performed in "Ascending" or "Descending" order, depending on the selection made in the checkboxes under "Order" to the right of the drop-down list. The sorting will sort clients on both halves of the form. Selected clients' entries will appear in reverse video. In the example used here, Figure 17.5, two clients have been selected.

- Advanced Select - An alternate method of selecting the clients to import uses the "Advanced Select" button beneath the panel on the right of the form. Selecting this button takes you to the "Client Selector" window. This window is the primary means of selecting clients in the second method of transferring data. See Section 17.5, Selecting Client Records, for more information on this feature.

- Include Agency and Library Records - Before actually performing the import, you must decide whether you want the library and agency information associated with the clients to be imported as well. If you do, make sure the checkbox under "Include Agency and Library Records" between the two panels is checked. If selected, the import will copy the setup and supply libraries used by the clients and their audits to your computer. If it is your intent to be able and run the audits associated with the clients being imported and replicate the results originally obtained, this will be necessary. However, if you import many 


\section{Chapter 17: Data Link}

clients from the same source, there is a greater possibility of conflicting records and/or overwriting libraries in the database receiving the import with this option chosen.

- Assign Imported Clients to - If the "Include Agency and Library Records" checkbox is not selected, no libraries will be transferred with the import. Also, you will have to tell the Weatherization Assistant what agency already in your database you wish to assign the records to during the import by using the "Assign Imported Clients to" drop-down list near the bottom left of the form. Displayed in this list will be all the agencies existing in the database you are currently linked to. In many cases, your agency may be the only one listed.

- WDZ Password - One final consideration that may need to be addressed prior to actually performing the import procedure regards the possible use of password protection on "wdz" files. If you are importing clients from a "wdz" file that originated from an export that used a password, that password would have to have been conveyed to you in order to allow you to import the clients. To accomplish this, choose the "WDZ Password" button near the top of the right half of the "Transfer CLIENTS between databases" window (see Figure 17.5). The "WDZ/ZIP Password" window will appear. In the field, "ZIP Password for Import and Export" in the window, type the password given you, paying attention to punctuation and capitalization. Close the window and proceed with the importing process. The Weatherization Assistant will leave this password set until you change it. So, unless the password is expected to be used in repeated importing and exporting sessions, make sure to return to this form and delete the password.

- Import - Once you have completed the selection of clients and any other necessary settings, click on the left pointing arrow, "Import," which lies between the two halves of the form. The clients will be copied over into the database file you are currently linked to and the Client IDs and associated information will appear on the left half of the form. A status bar in the middle near the bottom of the form will monitor the progress of the operation, indicating each record as it is transferred.

During the importing process, there is the potential that a record being imported is seen as already existing in the database you are currently linked to, that is, the database into which the records are being imported. If this occurs, the program will 
display a window titled "WARNING: Overwrite with older data?" An example of this window is given in Figure 17.7.

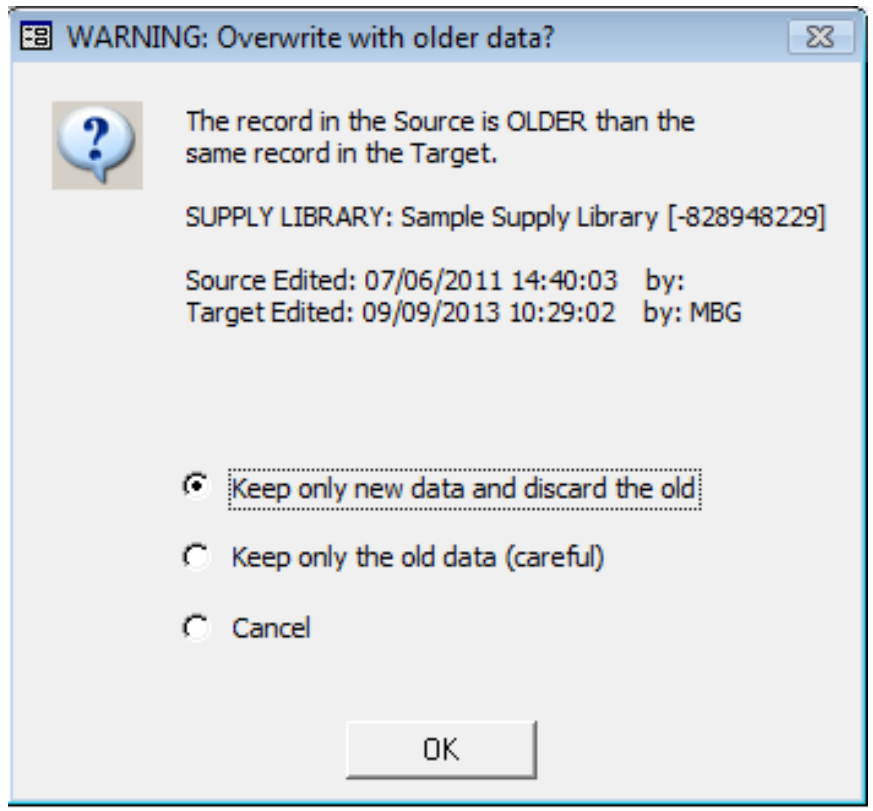

Figure 17.7. The WARNING: Overwrite with older data? window.

In this example, the Weatherization Assistant sees a supply library being imported as the same as one already existing in the database. For all records except agency records, two records are considered to be the same if they have the same Access long integer ID number. You can choose to have the program display these numbers on the applicable forms (see Feature 6 in Section 16.4, Features). In the warning, this ID number is displayed in square brackets following the identification of the record type. The example warning is indicating that the supply library being imported, "in the Source," is older than the supply library considered identical in the "Target," or in the database you are currently linked to and into which you are importing the record. This is confirmed by displaying the dates and times the source and target records were last edited.

The warning window gives you three options to resolve the situation. The first, "Keep only new data and discard the old," prevents the imported record from replacing the corresponding record in your database. This is a relatively safe choice in that you do not change any parameters in your database that may have been or will be used in your weatherization work. However, it does not ensure that you will be able to replicate the results associated with the imported client or even examine the parameters contained in the offending record associated with the client. The 
second choice, "Keep only the old data (careful)," will result in overwriting the record in your database. This would normally be seen as unwanted, since any audits, work orders, etc., that had references to the record would now likely see different data. Thus, the "careful" appended to the choice description. The last choice, "Cancel," cancels the import of the client that resulted in the conflict, but proceeds with attempting to import any additional clients you may have designated as part of this particular importing session.

\section{Exporting Clients (via database files)}

Exporting is the reverse process to importing, described above. You can export any set of clients in the database you are currently linked to and place them in either another existing database or have a new database created just for the clients you wish to export. However, if exporting to an existing database, the database must have been formed using the same version of the Weatherization Assistant. No backward compatibility has been provided. To export clients to a database file ("wdz" or "mdb”), enter the "Transfer CLIENTS between databases" form by choosing "With another MSAccess Database" in the "Import/Export Data" block on the Data Link form and selecting the "Go" button (see Figures 17.1 and 17.5).

- External Database Path -Your first task will be to designate to the Weatherization Assistant the name and location of the file you wish to have the clients' data transferred to. If you have exported clients previously, the files to which your exports were sent will be displayed in the "External Database Path" drop-down list at the top of the right half of the "Transfer CLIENTS between databases" form. You may select one of these previously accessed files to use again. Any clients you export to the file on this occasion will be added to the clients previously sent to the file.

- Browse - If the file you wish to use for exporting is not in the "External Database Path" drop-down list, or you wish to create a new database file to export clients to, select the "Browse" button to the right of the "External Database Path" drop-down list. This will take you to the "Pick External Database for Import/Export” window, a standard Windows Operating System browse window (see Figure 17.6 for an example). If you plan to export clients to a database file that already exists but did not find in the "External Database Path" drop-down list, follow the directions in the paragraph below Figure 17.6 
to locate and select the file. When finished, you will be taken back to the “Transfer CLIENTS between databases” form.

If you are creating a new database file, use the portion of these same directions below Figure 17.6 to designate the location on your computer where you wish to have the new file placed. One option might be your "Desktop." Enter the name you wish assigned to this new database in the "File name" field at the bottom of the window. Then choose the type of database file you wish to create, either a standard "mdb" database file or a zipped "wdz" file, using the "Save as type:" drop-down list at the bottom of the form. Either “.wdz" or ".mdb” will be appended to the file name you entered in the File name field, depending on your choice. The "wdz" files are compressed to about one tenth the size of the standard file. They are also automatically encrypted to allow safe transport of confidential client information over public media. Next, click on the "Save" button to indicate your selection is complete. If the database file did not exist previously, you will receive a notice stating this and that the program is "Creating a database file so you can copy jobs to that database." You will then be taken back to the "Transfer CLIENTS between databases" form.

Choose the clients you wish to export from those currently in your database, listed on the left of the "Transfer CLIENTS between databases" form. Multiple client selections may be accomplished using standard Windows selection techniques (i.e., the [Shift] and [Up/Down Arrow] or [Ctrl] [Mouse Click] key combinations), as described above in the discussion on importing clients. When your client selection is complete, click on the right pointing arrow to initiate the export procedure. The status bar at the lower left of the form will monitor the progress of the operation. This process may be repeated to export additional clients, until all the desired clients have been exported to the specified database. The exported client information will now appear on the right panel of the form.

- Email - If you have setup the e-mail feature of the Weatherization Assistant (see Section 18.4, E-Mailing from Within Weatherization Assistant), you may click on the "Email" button to initiate sending the database file as an attachment to an e-mail. The file name may be automatically entered into the Attach Files field on the form. If not, you may copy and paste the database path and file name from the External Database Path field into the Attach Files field using standard techniques (see Section 5.9, Copying and Pasting Data). You will need to enter the e-mail address of the recipient and any Subject and Text you 
wish to include in the mailing. Then click on the "Send Email" button to send your e-mail. If this e-mailing feature has not been set up within the Weatherization Assistant, simply use your standard e-mail application, attaching the database file just created. Be aware that if you chose to export the clients to an "mdb" file, the file size may prohibit attachment to an e-mail.

- Check IN/OUT - If you have activated the Check-in/Check-out feature of the Weatherization Assistant (see Feature 10 in Section 16.4, Features), you can use the "Check IN" and "Check OUT" buttons that appear on the form to change the status of any client record appearing on either the left (Local Database) or right (External Database) halves of the "Transfer CLIENTS between databases" form. Simply select the desired client as you would for importing or exporting, then click on the appropriate button. If you choose the "Check OUT" button, the client record will be "Checked Out To" the current logged-on user. Checking in a client deletes the entry in this field, although the date in the Last Check Out field, wherever it is displayed, will remain. Any status change made on this form will also appear on the Client Information form (see Section 7.1, Client Information) as well as any time the Client Selector form is viewed (see Section 17.5, Selecting Client Records, and the "Select Client” button description in Section 6.1, Agency Information).

\subsection{Data Transfer - Import/Export Indirectly with Text Files}

The Weatherization Assistant provides you with a second method of sharing data. The transfer "Indirectly with text files" method is most applicable to sending large quantities of data. It also gives the user greater control over individual data items within the client file. The method uses data formatted in "csv" (comma separated value) files which, if you choose, can be compressed into a single "zip" file for any given exporting session. These files are text files that could be read with any text editor, though the arrangement of data would be difficult to use directly. As text files in this specific format, other computer applications could be made to accept the data and integrate the information with other data.

Access this mode of data transfer by choosing "Indirectly with text files" in the "Import/Export Data” drop-down list under the Main Menu's "Data Link” button, then selecting the "Go" button to the right. You will be presented with a "Transfer between databases with intermediate CSV/ZIP files” form, designed specifically 


\section{Chapter 17: Data Link}

for transferring data using text files (see Figure 17.8). The form has three data blocks: "Import," "Export," and "Progress Messages.” Each will be addressed separately below. For brevity, this form will hereafter be referred to simply as the “Transfer between databases...” form.

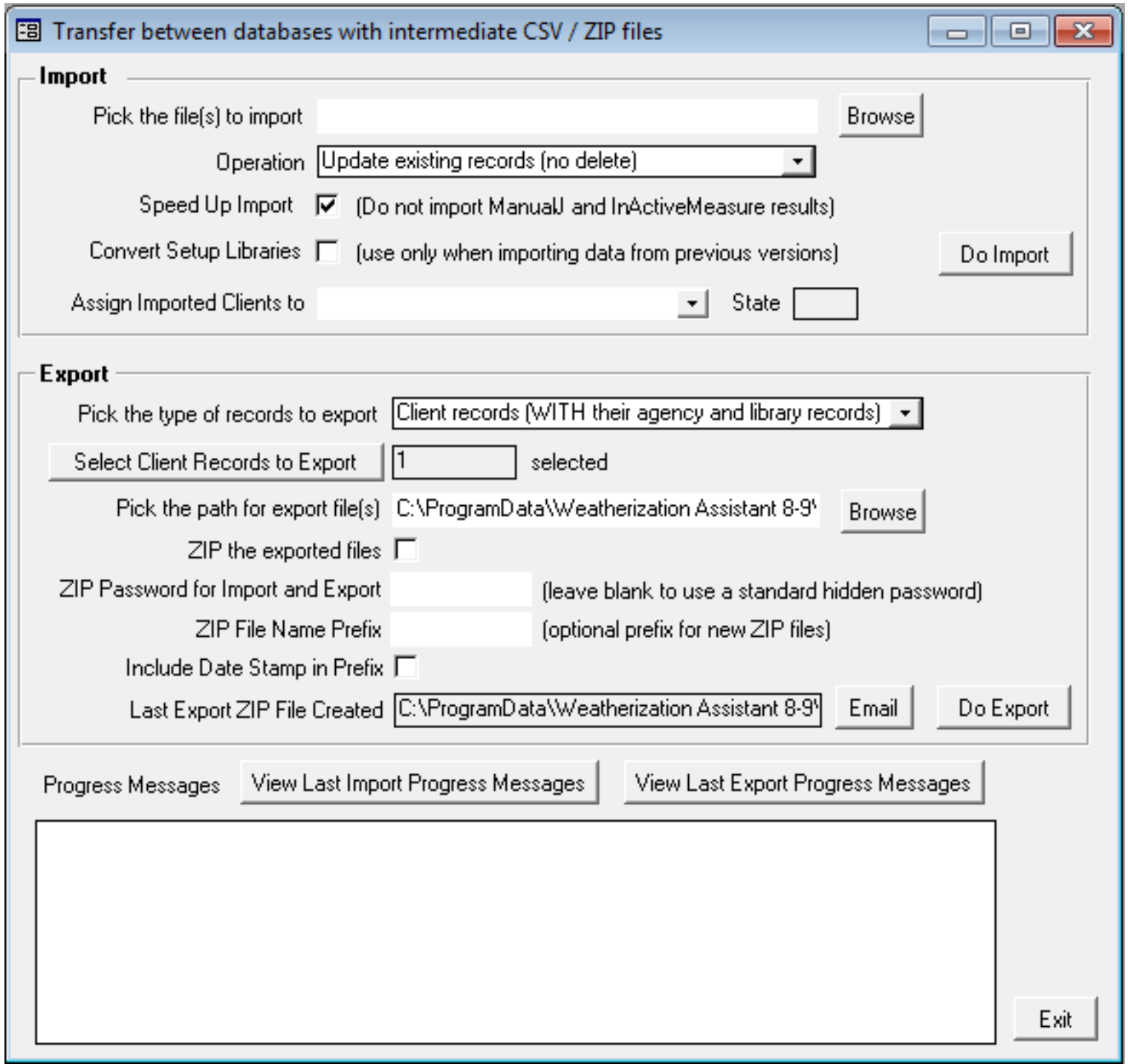

Figure 17.8. The Transfer between databases form (via Text Files).

\section{Importing Clients (via text files)}

The "Import" block on the "Transfer between databases..." form is shown in Figure 17.8, above. The block on the form is used specifically to import data in the "csv" format, whether from individual "csv" files or combined and compressed 


\section{Chapter 17: Data Link}

into "zip" files. Discussions of specific data items or controls found on the "Transfer between databases..." form, as they pertain to importing clients from text files, are bulleted. Those applying to the process in general lie outside any bullets.

- Pick the file(s) to import - In order to import information, you must first "Pick the files(s) to import.” Although you could type in the path and name of a file into this field directly, most often you will use the "Browse" button to the right of this field in making the file selection. The button presents you with the "Pick Import File(s)" window, a standard Windows Operating System browse window. Figure 17.9. displays an example of the window you will see.

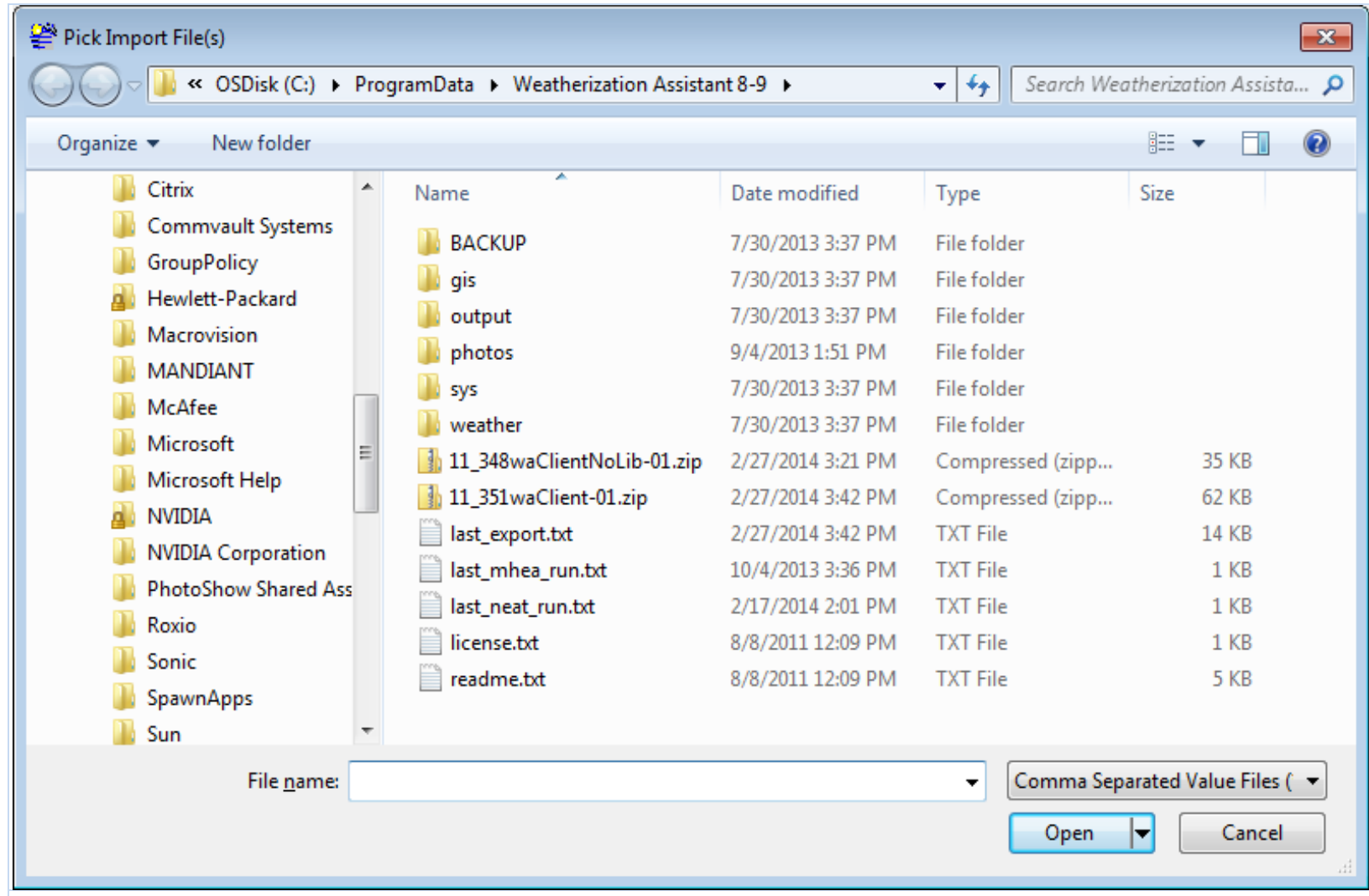

Figure 17.9. Browse window for selecting files to import via text files.

Use standard Windows techniques to locate the folder where the file or files you wish to import are stored. Most of the time you will be looking for files with extension "zip." The two "zip" files seen in the figure above are just such files, having been created using the Weatherization Assistant's client export via text files feature (see "Exporting Clients (via text files)" in the following topic). 
Follow the directions in the paragraph below Figure 17.6 to locate and select the "zip" file from which you wish to import, using the "Open" button to conclude your choice. Only one file of type "zip" can be imported at a time.

Though possibly used less often, the program can import individual or groups of records of information using separate "csv" files. All of the data related to any given client is composed of many individual records of data, each related to a different area of information (e.g., address, contacts, wall descriptions, heating systems, etc.). If, on exporting information (see the following topic), the user has chosen to export record-by-record instead of by entire client, individual "Csv" files will be created for each record type (see Figure 17.10) instead of a single "zip" file containing all records for a given client. Thus, on import, you could choose to import only data corresponding to specific record types. Adding to the flexibility, each "csv" file may contain information of the same type but for multiple clients. Thus, for example, you could choose to import only all of the billing data records for a group of clients in a single import. Thus, the potential exists for a utility to be able to provide client billing data in the "csv" format, which could then be directly imported to the Weatherization Assistant. The Browse window may be used to select single or multiple "csv" files for importing (using the standard Windows [Shift][Arrow] and [Control][Mouse Click] techniques). Doing so will transfer multiple file names into the "File name" field.

\section{4-qryX1+tblNHeating.csv}

"JobID", "SystemCode", "TypeID", "HeatingEquipTypeID", "FuelTypeID", "HeatingEquipLocationID" ," 1704471506, "HS1", 0, 3, 1, 1, 100.00,0, 1, ,80.00, , , 2, 1, 0, , 72.00,0,0,0, 1, 0, 0, 0, 6, 0, 81.00, 92.00 -858011473, "HS1", 0, 3, 1, 1, $100.00,0,1,1,80.00, \ldots, 2,1,0,, 72.00,0,0,0,1,0,0,0,6,0,81.00,92.00$ 1359998494 , "HS1", $0,3,1,1,100.00,0,1,1,80.00,1,1,2,1,0,72.00,0,0,0,1,0,0,0,6,0,81.00,92.00$ 874207113, "HS1", 0,3,1,1,100.00,0, 1, ,80.00, , , 2, 1, 0, ,72.00,0,0,0,1,0,0,0,6,0,81.00,92.00, 1147117665, "HS1", $0,3,1,1,100.00,0,1,, 80.00,1,1,2,1,0,72.00,0,0,0,1,0,0,0,6,0,81.00,92.00$ -675163965, "HS1", 0, 3, 1, 1, $100.00,0,1,1,80.00,1,1,2,1,0,72.00,0,0,0,1,0,0,0,6,0,81.00,92.00$ -546134610 , "HS1", 0, 3, 1, 1, 100.00,0, 1, , 80.00, , , , 2, 1, 0, ,72.00,0, 0, 0, 1, 0, 0, 0, 6, 0, 81.00, 92.00 1140526127, "HS2", 0, 6, 3, 2, 33.00,0, , 2, , 30.00, "Trane", "TWH030B14", 1992, , 0, 0, 0, , , 0, 0, 0, 0, 0, 0, 0 1140526127, "HS1", 0, 6, 3, 2, 67.00,0, , 2, 64.00, "Trane", "TWH064P150C1", 1992, , 0, 1, 0, , 0, 0, 0, 0, 0, -1028560058, "HS2", 0, 6, 3, 2, 33.00, 0, , 2, , 30.00, "Trane", "TWH030B14", 1992, , 0, 0, 0, , 0, 0, 0, 0, 0, 0, -1028560058 , "HS1", 0, 6, 3, 2, 67.00,0, ,2, ,64.00, "Trane", "TWH064P150C1", 1992, , 0, 1, 0, , , 0, 0, 0, 0, 0 1337885691, "HS2", 0, 6, 3, 2, 33.00,0, ,2, , 30.00, "Trane", "TWH030B14", $1992,, 0,0,0,1,0,0,0,0,0,0,0$ 1337885691, "HS1", 0, 6, 3, 2, 67.00,0, ,2, 64.00, "Trane", "TWH064P150C1", 1992, ,0, 1, 0, , 0, 0, 0, 0, 0,

Figure 17.10. Example “CSV” file for heating system records.

Clicking on the "Open" button completes the file selection and returns you to the "Transfer between databases..." form with the file's path and name automatically transferred to the "Pick the file(s) to import" field. If more than 


\section{Chapter 17: Data Link}

one file has been chosen in the file selection process ("csv" files only), the field will indicate "Multiple Files Selected."

- Operation - You must next choose what the importing process should do if client information for the any specific client you are importing already exists in the database you are currently linked to. The choice is made using the "Operation” drop-down list. The choices are, "Replace any existing records (delete+write)," "Update existing records (no delete)," and "Just show list of clients (test only-no write).” In making your choice, you need to think of individual records of client information and the data items or fields within the records. For example, a wall record within the NEAT audit for a client contains fields describing the wall's existing insulation type, its orientation, etc. If a given record of specific information already exists for the same client for which the importing process is being performed, selecting "Replace..." will replace the entire record with the same record from the imported file. If "Update..." is selected, existing records are updated field-by-field, preserving any fields in the existing record that don't have information for matching fields in the record being imported.

Choose "Just show list of clients (test only, no write)" in the "Operation" dropdown list if you wish to have the program perform a "dry run" of the importing process without actually writing any information to the database you are currently linked to. This will show you, in the Progress Messages window at the bottom of the form, the list of records that would be imported, without actually performing the import (i.e., altering your existing database information). At the end of the messages produced in any import process, including an import using the "test only" option, a list of Clients for whom records are included in the import will be shown. This could be useful to review prior to actually performing the import, which will alter your database.

- Speed Up Import - Importing information related to the peak heating and cooling loads (see "Approximate Component Contributions to Peak HEATING and COOLING loads (Equipment Sizing)" in Section 12.2, Recommended Measures Report) has been found to be time consuming when importing data for many clients. If this information is not required and your import is already large, selecting the Speed Up Import checkbox will shorten the time required to accomplish the import. 
- Convert Setup Libraries - The Convert Setup Libraries checkbox is designed for importing records of Setup Libraries from different versions. The feature will convert any Version 8 setup library to one compatible with Version 8.9.0.5 (e.g., Version 8.3.3.11 or 8.6.0.4). No means of data conversion from versions prior to Version 8 are yet available.

- Assign Imported Clients to - If you are importing a client that was exported without agency information (see "Exporting Clients (via text files)" in the next section), use the "Assign Imported Clients to" drop-down list to assign an agency to the client. The drop-down list will display those agencies in the database you are currently linked to. If you attempt to import such a client without providing this assignment, the Progress Message associated with the import will indicate "Import Failed" and no information will be added to your database.

If you import a client with agency information, this field may be left blank and the agency information associated with the imported client will be added to your database, if it doesn't already exist. If you designate a different agency in this field than is assigned to the client(s) to be imported, you will receive a warning window allowing you to "Assign Clients to: <the agency designated>” or "Keep original agency assignments.” Make your selection, then click on "OK" to exit the window

- Do Import - When all of the specifications for the import have been established, select the "Do Import" button to actually initiate the import process.

- Progress Messages - In the white space below the "Progress Messages" header, record-by-record progress of the importing process will be displayed. This may be too detailed for most users to be concerned with. However, at the end of the process, the message will indicate the success or failure of the import as well as the clients for which information was imported. If a failure should occur, the messages will indicate exactly what record caused the failure. This would allow an analyst to examine a specific location in the file being imported to determine the potential cause of the failure. With the increased information collected with Version 8, importing data can be relatively slow. It may be wise to copy the files to be used in the import to a local drive on your computer, if necessary, before initiating the process. Be patient and wait for the <busy> 


\section{Chapter 17: Data Link}

cursor to disappear and the Progress Message to report the outcome of the process.

- View Last Import/Export Progress Messages - The Weatherization Assistant saves the text of the Progress Messages from the last import and export sessions in files on your computer. These files may be displayed by selecting the "View Last Import Progress Messages” and "View Last Export Progress Messages” buttons, respectively.

- Exit - Use the "Exit" button to return to the Main Menu's Data Link form whenever you have completed your import session.

\section{Exporting Clients (via text files)}

The "Export" block on the "Transfer between databases..." form is shown in Figure 17.11, below. It is used to export information to text files in the "csv" format, either as individual "csv" files or combined into "zip" files.

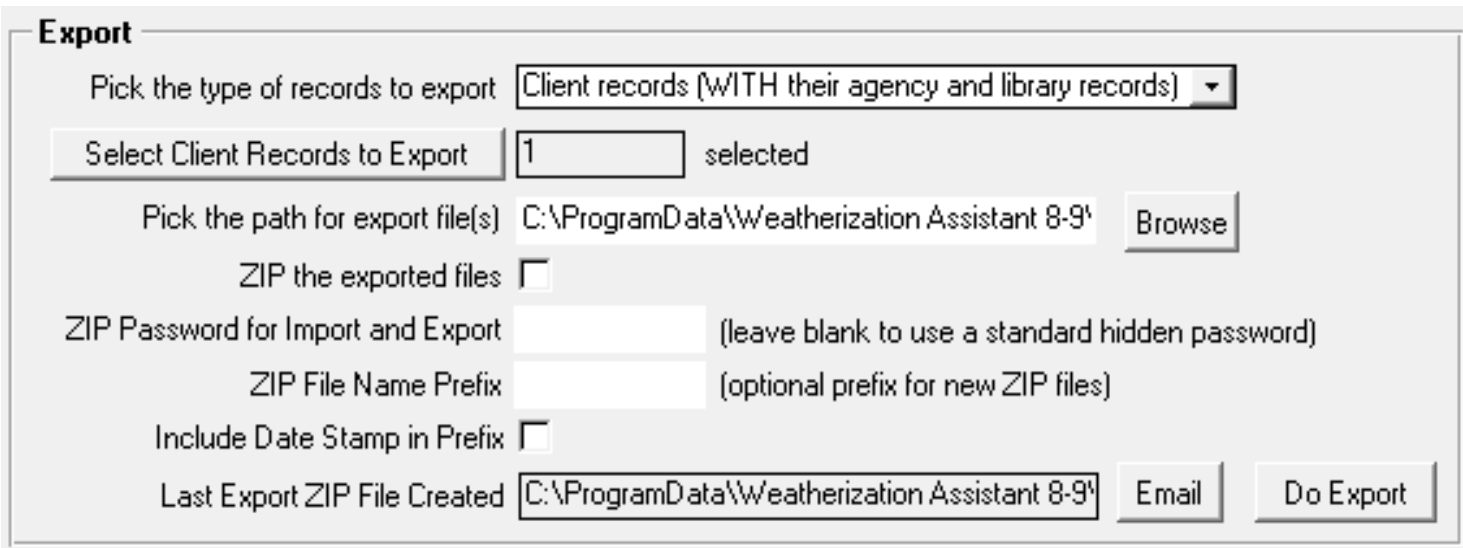

Figure 17.11. Exporting Clients (via text files).

- Pick the type records to export - To export information, you must first pick the type of export to be performed. The program allows you to export three types of information, "Client records (WITH/WITHOUT agency and library records)," "Error History (all runtime errors)," and "Database Dump (all tables, all clients)." The two "Client records" options allow you to choose whether to include data related to the exported clients' agencies and library records. The "Error History" export creates a single "csv" formatted file of the run-time error messages. These are the same error messages reported under the Main Menu's 
"Preferences" button (see Feature 7, "Enable the logging of program errors" in Section 16.4, Features). The "Database Dump" option does the same as the "Client records (WITH their agency and library records)" option, except that it performs the process for all client records in the database you are currently linked to. This may be used by local agencies who report regularly to the state.

- Select Client Records to Export - If the type of export was chosen as one of the "Client records" options, you must tell the exporting process which clients in your current database you wish to have exported. Do this by clicking on the "Select Client Records to Export" button. Doing so will present you with a Client Selector form. This form has applicability elsewhere in the program as well, specifically if any user-generated reports require selection of clients (see "Select Clients" in Section 6.1, Agency Information). For an explanation in the use of the Client Selector form, see Section 17.5, Selecting Client Records.

- Pick the path for export file(s) - You must next tell the exporting procedure where to place the files it will create. You may enter a path directly into the "Pick the path for export file(s)" field. If you have performed an export previously, the field will use the location used in that export as a default. Otherwise, use the "Browse" button to the right of this field to designate the path. The button takes you to a standard Windows directory tree from which to select the path. Use the tree to locate the desired folder, highlighting the folder when found. Then click on "OK." The path will automatically be transferred to the Select Client Records to Export field in the Export block of the "Transfer between databases...” form. If, in the next step, you do not choose to create a single "zip" file from your export, you may wish to select an empty folder, previously created outside the Weatherization Assistant, at this step to prevent the numerous "csv" files created from becoming confused with other files, possibly from another export procedure. If you have chosen to perform a Database Dump, it would be wise to export to a local drive on your computer to speed the process.

- Zip the exported files - Indicate with the "ZIP the exported files" checkbox whether you wish to create a single "zip" file containing all of the individual "csv" files as a result of the export. If the checkbox is not selected, individual "csv" files will be created, each containing data for a different record type, over multiple clients if you chose more than one client under the Client Selector form (see Figure 17.10). Note that "zip" files are customarily about a tenth the 
size of their corresponding unzipped files, making even the single "csv" file created by exporting the Error History a considerably smaller "zip” file.

- ZIP Password for Import and Export - The "ZIP Password for Import and Export” field gives users an optional added control over the security of information in the files created. All "zip" files created with the export process in the Weatherization Assistant have their information "encrypted," preventing non-users of the program from viewing their contents. However, the encryption is standard with the program, allowing any user to import the information and view it. If you wish to further restrict the accessibility of the information in the files, enter a password in this field when exporting the data. That password will then be required by any person importing the data in order to successfully accomplish the import. WARNING: If you assign a password and it is forgotten, the information in the exported files will no longer be retrievable by any means.

- ZIP File Name Prefix / Include Date Stamp in Prefix - If a single "zip" file will be created by the export, the next two entry items simply give you flexibility in specifying the name of the file that will be created. The standard default name for a Client Export "zip" file is "waClient-\#\#.zip” or "waClientNoLib-\#\#.zip" depending on whether you have or have not chosen to include the agency and library records with the export. For a Database Dump "zip" file, the default name is "waDump-\#\#.zip"; and for an Error History "zip" file, "waErrors-\#\#.zip." In all three cases, the “\#\#” represents a two digit integer insuring uniqueness of the file. The integer will be selected automatically based on other existing files in the location to which the file is to be exported. To add greater specificity you may enter characters in the "ZIP File Name Prefix" field. If entered, these characters will be added as a prefix to the default name. Similarly, if the "Include Data Stamp in Prefix" checkbox is selected, a date will be used as part of a prefix in the file name. The date format will be "yyyymm-dd-dow,” for example, “Prefix2005-09-30-Fri-waClient-01.zip.”

- Do Export - When all of the specifications for the export have been established, select the "Do Export" button to initiate the export process.

- Progress Messages - In the white space below the "Progress Messages" header, record-by-record progress of the exporting process will be displayed. This may be too detailed for most users to be concerned with. However, at the 
end of the process, the message will indicate the success or failure of the export as well as confirm the exported "zip" file name (if a "zip" file was created) and that the process is "done." If a failure should occur, the messages will indicate exactly what record caused the failure. This would allow an analyst to more likely determine the potential cause of the failure. With the increased information collected with Version 8, exporting can be relatively slow. It would be wise to export the file(s) to a local drive on your computer. Be patient and wait for the <busy> cursor to disappear and the Progress Message to report the outcome of the process.

- Email - If you have setup the e-mail feature of the Weatherization Assistant (see Section 18.4, E-Mailing from Within Weatherization Assistant), you may click on the "Email" button to initiate sending the file(s) created by the export as an attachment to an e-mail. If a single "zip" file was created by the export, the file name will be automatically entered into the Attach Files field on the form. You will need to enter the e-mail address of the recipient and any Subject and Text you wish to include in the mailing. Then click on the "Send Email" button to send your e-mail.

- View Last Import/Export Progress Messages - The Weatherization Assistant saves the text of the Progress Messages from the last import and export sessions in files on your computer. These files may be displayed by selecting the "View Last Import Progress Messages" and "View Last Export Progress Messages" buttons, respectively.

- Exit - Use the "Exit” button to return to the main Main Menu's Data Link form whenever you have completed your export session.

\subsection{Selecting Client Records}

The Client Selector form (see Figure 17.12) is used anytime there is a need to select specific clients from your entire list of clients in a database. This occurs when specifying which clients' data are to be exported to a text file or included in a user-defined report (see "Select Clients” in Section 6.1, Agency Information). 


\section{Chapter 17: Data Link}

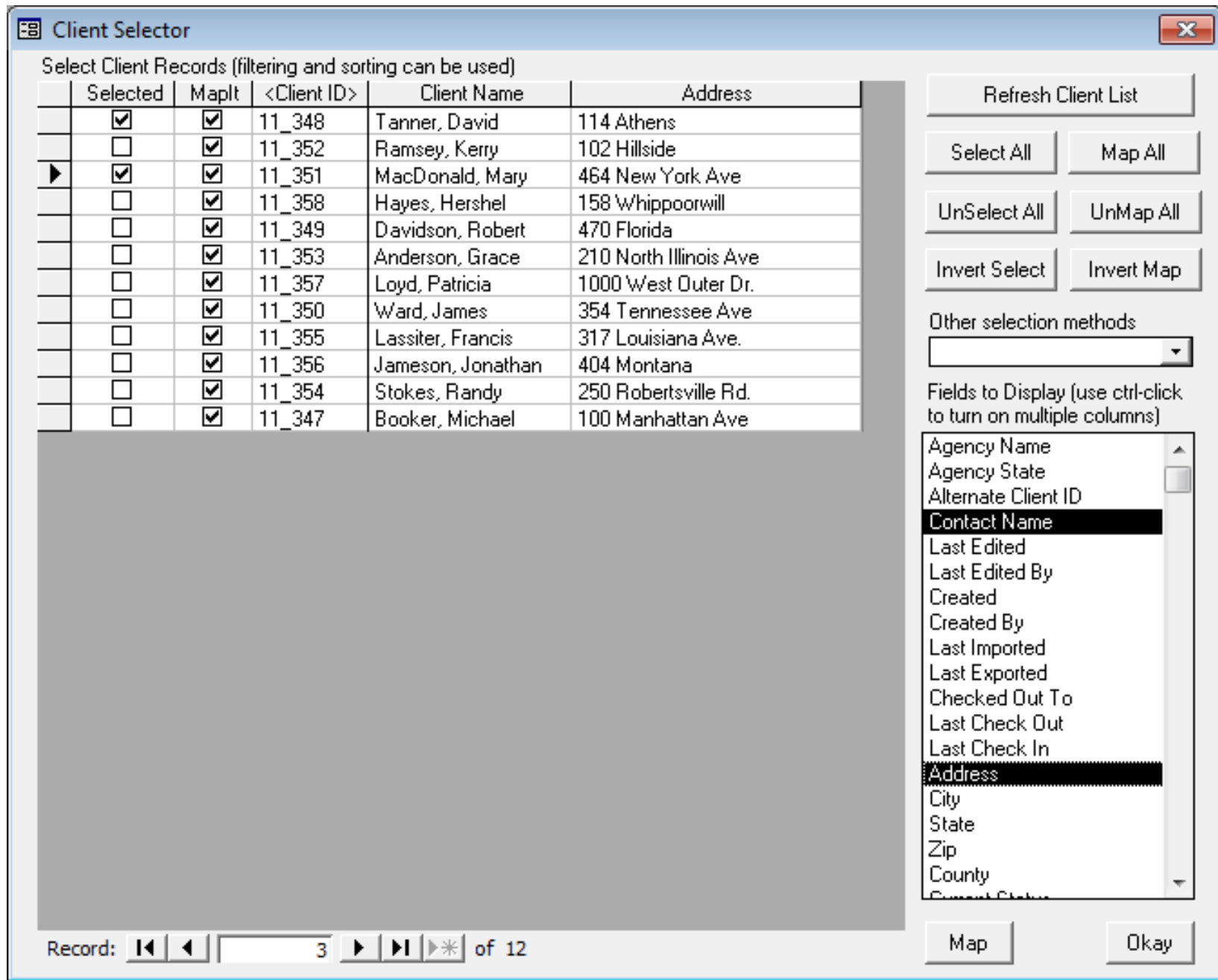

Figure 17.12. The Client Selector form (GIS feature on).

The form displays a list of the Client IDs for all clients in your database on the left side of the form along with a column of checkboxes denoted as "Selected." To select clients, click on the checkboxes next to the clients you wish to have included in the selection. To assist in identifying the clients, the form will also display additional information for each client, as chosen by the user in the "Fields to Display" window. Use the standard Windows selection technique of the [Ctrl][Mouse-Click] combination to select which client information fields you wish displayed. If you select a significant number of fields, you may need to use a horizontal scroll bar, which will appear at the bottom right of the listing, to view some of the fields. 
The form allows sorting and filtering of the entries. Once you have chosen the client information fields to be displayed, you may right click your mouse in any client's cell in the column of information you wish to sort by. You will receive the Sort/Filter menu window seen in Figure 17.13. Select either "Sort Ascending" or "Sort Descending". The records displayed will be sorted in alpha-numeric order according to the data item chosen.

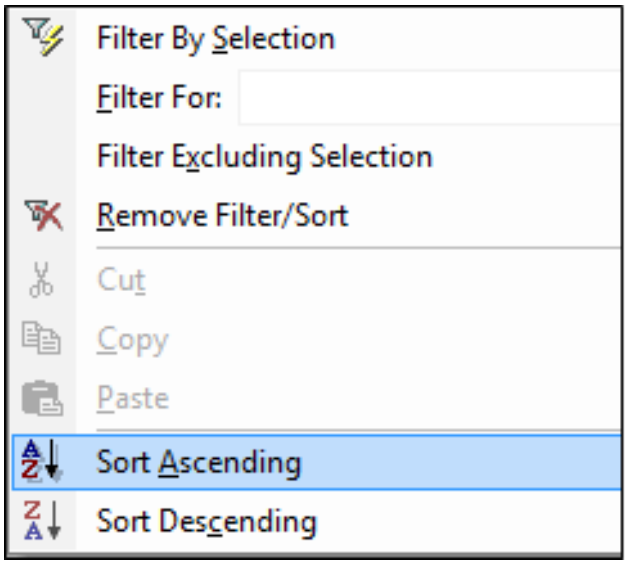

Figure 17.13. The Sort/Filter menu window.

You may also "filter" the records to be displayed. For example, if one of the fields you chose to have displayed included the City and clients in your database lived in various cities, position your cursor in a cell under the City column containing the name of the city on which you wish to filter the entries. Right click your mouse to obtain the Sort/Filter menu window. Select "Filter by Selection.” Your display will then include only those clients from the city chosen. You could also select your filtering criteria by entering the text by which you wish to filter into the Filter For field in the Sort/Filter menu window. However, the entry must match a data item exactly or you will filter out all of the entries. The entry in this field might best be obtained by copying an existing data item and pasting it into this field.

Selecting "Filter Excluding Selection" will eliminate all entries matching the chosen data item, the opposite of filtering by the selection. Be careful when using filtering. If you forget that your display of records is filtered, you may wonder what happened to some records you would otherwise expect to see in the display. To cancel filtering, select "Remove Filter/Sort" in the Sort/Filter menu window.

Descriptions of the functions for other controls on the form are given below.

- Select All / UnSelect All / Invert Select - Buttons on the Client Selector form allow alternate methods of selecting clients. The "Select All" button will select all of the clients displayed. You could then individually uncheck the checkboxes of those clients you do not want selected. The "UnSelect All" button will eliminate all previously selected clients, allowing you to begin the selection process again. The "Invert Select" button will cause all of the 


\section{Chapter 17: Data Link}

currently selected clients to become un-selected and all of the un-selected clients to be selected.

- Other selection methods - If you use the Check-in/Check-out feature of the Weatherization Assistant (see "Check-in/Check-out client records" Feature 10 in Section 16.4, Features) or have exported or imported specific client records, as described elsewhere in this chapter, the client selection methods under the "Other selection methods" drop-down list may also be useful. The selections from the list are

Checked out to Me

Edited since last Check out

Edited since last Check in
Edited since last Import

Edited since last Export

The choices on the left in the list above apply only if you are using the Checkin/Check-out feature. The first category will display only those client records currently checked out by you, the current user logged on. The next two choices will display all records that have been altered since they were last checked out or in, regardless of who performed the check-out or check-in. This can be useful, not only in selecting client records, but also in identifying records that you personally have checked out or those that have been altered since having been checked out, possibly an undesirable situation. The choices on the right in the table look for all clients that have been exported or imported.

- Mapit / Map All / UnMap All / Invert Map - If you have implemented the Geographic Information System (GIS) feature (see Section 18.2, Geographic Information System (GIS), and Feature 3 in Section 16.4, Features) of the Weatherization Assistant, an additional column will appear in the list of clients and several buttons will be added to the form. The "Mapit" column of checkboxes allows you to select which clients you wish to have mapped. This is a separate selection process from that using the "Selected" checkboxes. The three buttons, "Map All," "UnMap All,” and "Invert Map” have the analogous effect on the mapping selection as do the three corresponding buttons described above for the actual client selection process. Having made the selection of clients to map, clicking on the "Map" button will display a map with the locations of the clients' homes indicated with stick pins. This may be useful, for example, in deciding what homes are in the same vicinity for establishing a visit schedule. However, there are also capabilities within the mapping routine to 


\section{Chapter 17: Data Link}

"select" homes on the map for the actual client selection process (see the GIS reference given above). Having made the selection using the map will automatically check the "Selected" checkboxes on the Client Selector form corresponding to the homes selected on the map.

- Refresh Client List - Use the "Refresh Client List" button if the Client Selector form has remained open while other program activities have been performed which might change the listing of clients included on the form.

- Map - If you have enabled the Geographic Information System (GIS) feature (see Section 18.2, Geographic Information System (GIS), and Feature 3 in Section 16.4, Features), clicking on of the "Map" button, which will appear on the form, will use your selection of clients under the "Mapit" column to display a map with the indicated clients' residences located with stick pins. If you have not checked any clients under the "Mapit" column or if none that you have selected have addresses that can be located by the GIS feature, you will be given an error window stating, "ERROR! There are no Clients that can be displayed on the map.”

- Okay - Clicking on the "Okay" button will return you to whatever feature you were using when you accessed the Client Selector form with the clients indicated under the "Selected" column as having been selected. 


\section{Chapter 18. Auxiliary Features}

The Weatherization Assistant has several additional features whose use is optional and which do not affect the recommendations in any way. However, they may assist you in the administration of your program. They have been referred to earlier in the manual, but never fully described. These features include the ability to associate digital photos to specific Weatherization Assistant records, map the locations of your clients, track the status of your work with your clients, and use a directory internal to the program to send e-mails.

\subsection{Digital Photos}

Weatherization Assistant allows you to organize digital photographs related to your weatherization work. Photos may be saved at several levels, as associated with the Client, an Audit, or a specific Work Order. Read Sections 7.8, Photos (Client); 11.10, Photos (Audit); and 13.4, Photos (Work Order), for additional information.

There are two methods of photo storage and editing supported in the Weatherization Assistant: the pathname method and the third party photo browser. Use the Features tab under Preferences (see Items 1 and 2 in Section 16.4, Features) to choose which method is displayed on your forms. Both methods assume that you know how to use a digital camera to take pictures and that you know how to move those pictures as JPG (jpeg) files to folders on your computer. The Weatherization Assistant also gives you tools for cropping, rotating, adjusting, and annotating each of your photographs. Your annotations are actually stored in the JPG file header thus preserving your notes if the JPG files are moved.

With networked installations you can store all the digital JPG files in shared folders with each client computer accessing the same images. However, note that in order to have each client computer access these photos, each must have assigned the same drive letter to the shared folder on the network server such that the pathnames stored refer to the correct location.

\section{Pathname Method of Photo Storage}

The first method uses a photo tab displayed on the Client, Audit, and Work Order forms to access the

Photos

Figure 18.1. Tab for pathname method of photo storage. 


\section{Chapter 18: Auxiliary Features}

photo feature (see Figure 18.1). When selected, you will be presented with the pathname photo browser form. The form is the same regardless of which of the three Main Menu windows access it. The form accessed for a work order is shown in Figure 18.2.

Photos are added to the collection by using the "Add Link" button in the center of the two panes on the form. The button will take you to a standard Windows browse window designed for selecting files. This window is used in the same manner as it

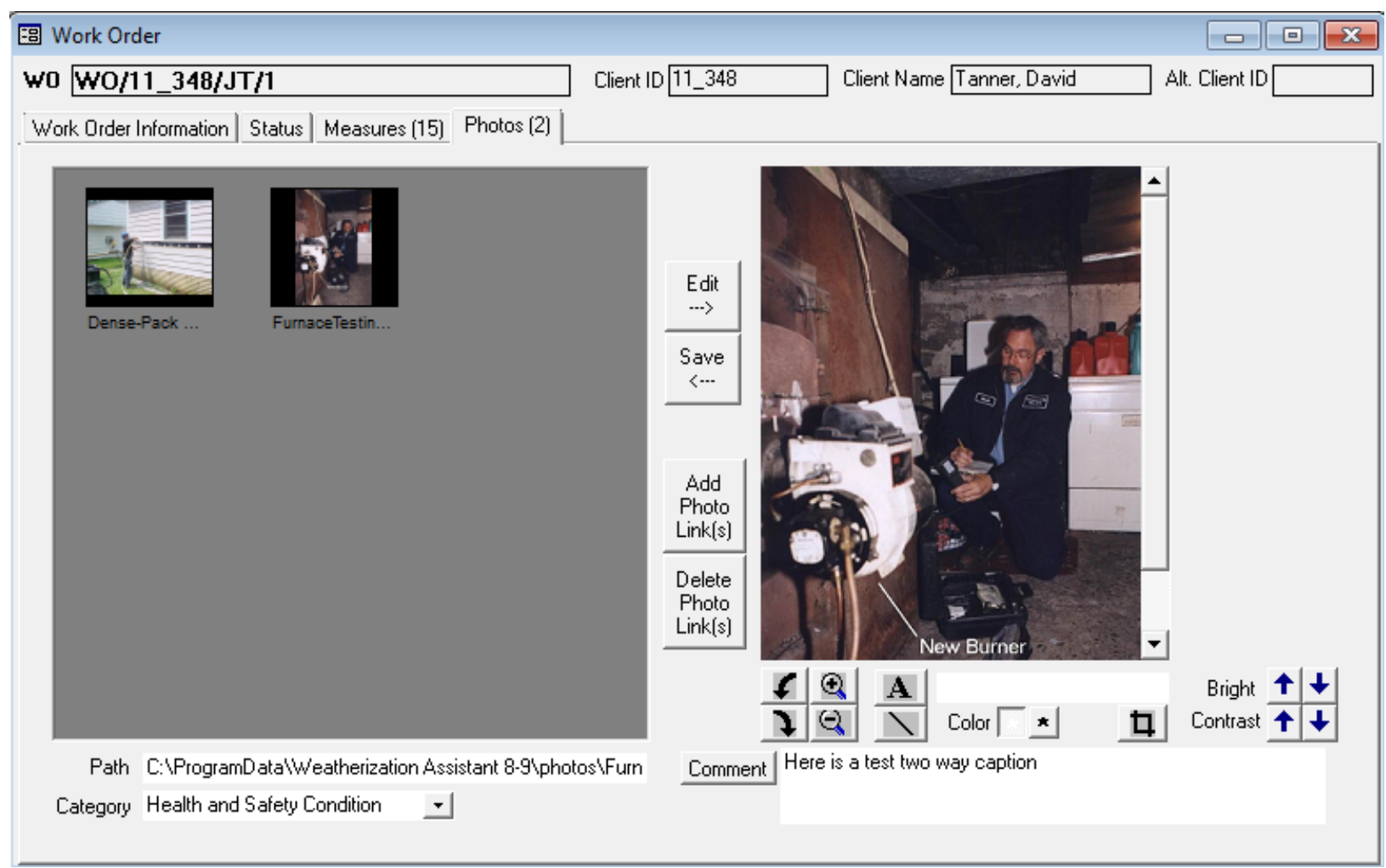

Figure 18.2. Pathname photo browser.

is to choose files to be imported (see "Importing Clients (via text files)" in Section 17.4, Data Transfer - Import/Export Indirectly with Text Files), except now you will be looking for the JPG files you downloaded from your digital camera. If you are familiar with Windows Explorer, you may also use the standard drag and drop procedure between this application and the Weatherization Assistant's photo browser to add photos to a collection. Simply drop the photo files into the left pane in Weatherization Assistant's photo browser window. The full pathname for each JPG file is stored in the database and associated with each photo. This way, the JPG files can be stored in whatever folders make sense for your installation. For 
example, you might want each auditor to have their own shared folder for storing their photographs or place all your digital photos in a single shared folder.

Each photo added to your collection will display a thumbnail in the browse pane (see Figure 18.2). Clicking on any of these thumbnails will select it and display the path to the photo file in the Path field below the browse pane. You may also assign a category to the photo using the "Category" drop-down list. Choices are Existing Conditions, Health and Safety Condition, Work Completed, Drawings and Documents, and Other. A photo is deleted from your collection by selecting the "Del Link" button. The actual JPG file is not deleted, only the application's link to the file, thus deleting the thumbnail and accessibility to the file.

Highlighting a photo's thumbnail in the browse pane and pressing the "Edit" button or double clicking on a thumbnail will open the photo in the editor pane on the right portion of the form. When the photo is opened in the editor, you can crop, rotate, adjust brightness and contrast, add text or lines, and add comments of any length using the controls below the image. Most of these processes are selfevident. However, to add text to your photo, enter the text in the field to the right of the " $A$ " button below the photo, select the color of the text (white or black) with the buttons just below the text, press the " $A$ " button, locate the cross-hair cursor at the point in the photo you want the upper left corner of the text to appear, and click your mouse. Pressing the "Save" button saves your edits to the file indicated in the Path field at the lower left of the window. If you want to preserve your original photo, change this path and/or file name before saving any of your changes. You may need to use the [End] key on your keyboard while in this field to see the file name at the end of the entry.

\section{Third Party Photo Browser}

The second method of photo storage and editing available in the Weatherization

Assistant uses a licensed

Photo Folder Browse

Figure 18.3. Controls on Information tab for third party photo browser.

program (IrfanView), referred to as the "third party photo browser" under the Main Menu's "Preferences" button (see Feature 2 in Section 16.4, Features). The method assumes that you will be storing all of the JPG photo files for each client, audit, and work order in specified folders (perhaps sub-folders under a photos folder on a 


\section{Chapter 18: Auxiliary Features}

shared network drive or in sub-folders under the Weatherization Assistant's "photos" folder). With this method, only the path name to the folder is associated with the record. The Photo Folder field on the Information tabs of the Client, NEAT and MHEA Audit, and Work Order windows contains the path name to the folder containing the JPG files (see Figure 18.3). Once you enter the path, either manually or using the "Browse" button, it will remain with the record unless you change it. Select the "View" button to start IrfanView. A window showing thumbnails of all the files in that folder will be displayed (see Figure 18.4). If chosen to be viewed, a directory tree will also appear displaying the location of the folder currently being accessed. Double clicking on any thumbnail will take you to the photo editor.

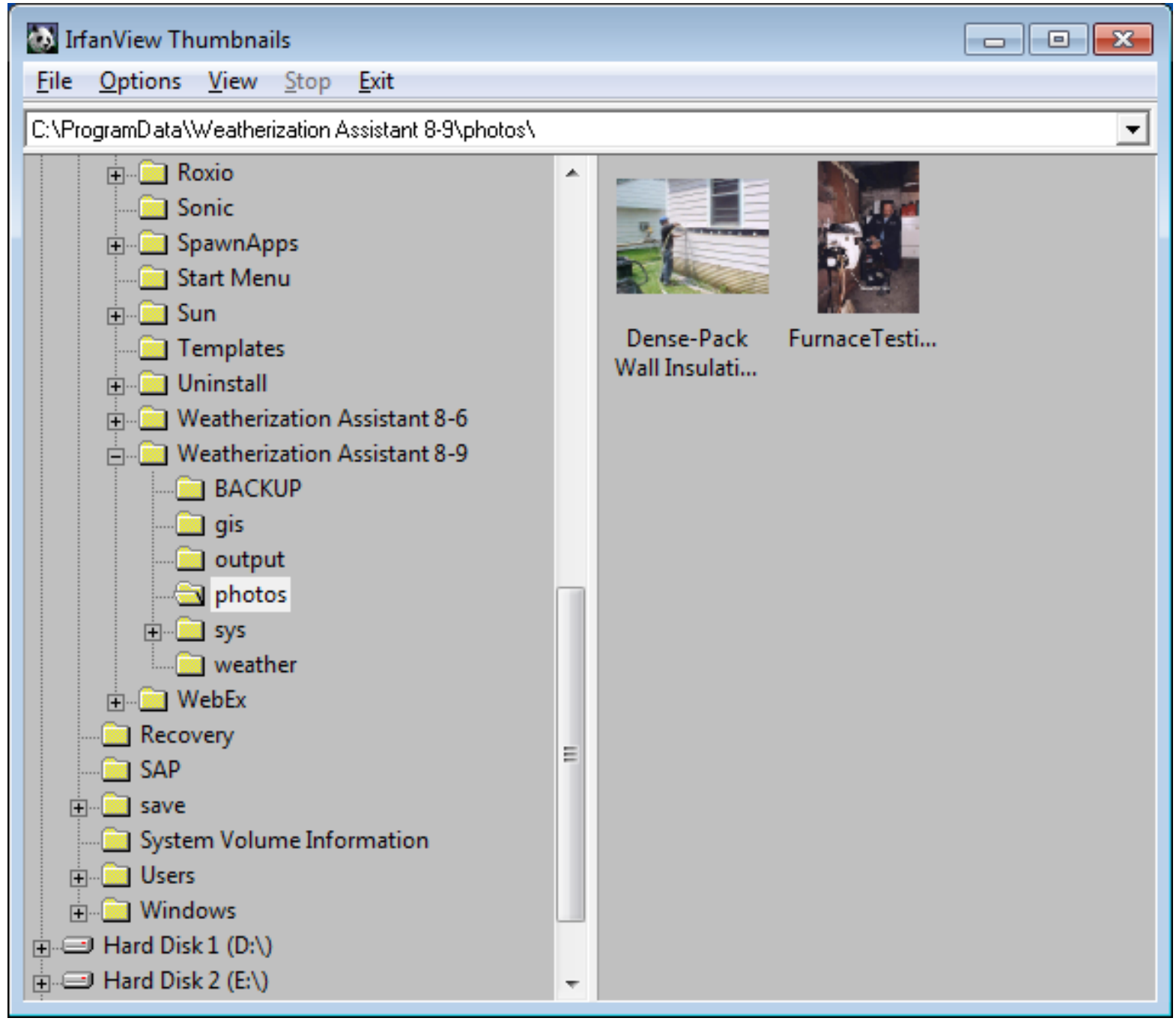

Figure 18.4. Third party photo browser navigation window. 


\section{Chapter 18: Auxiliary Features}

The editor for the third party photo browser contains numerous tools to crop, rotate, adjust, and annotate the JPG files (see Figure 18.5). It is a full featured photo editor whose capabilities are beyond the scope of this manual to totally describe. It is suggested that you experiment with the editor using a photo of no value or a copy of a photo, though you could simply choose to not save your changes. Use the "IPTC info" button under the Image/Information menu option in the editor to add detailed comments related to each photo. Save your changes to the photo using the File/Save or Save as menu item. Exit the editor using the [Esc] key or the standard Windows X-box in the upper right corner of the editor.

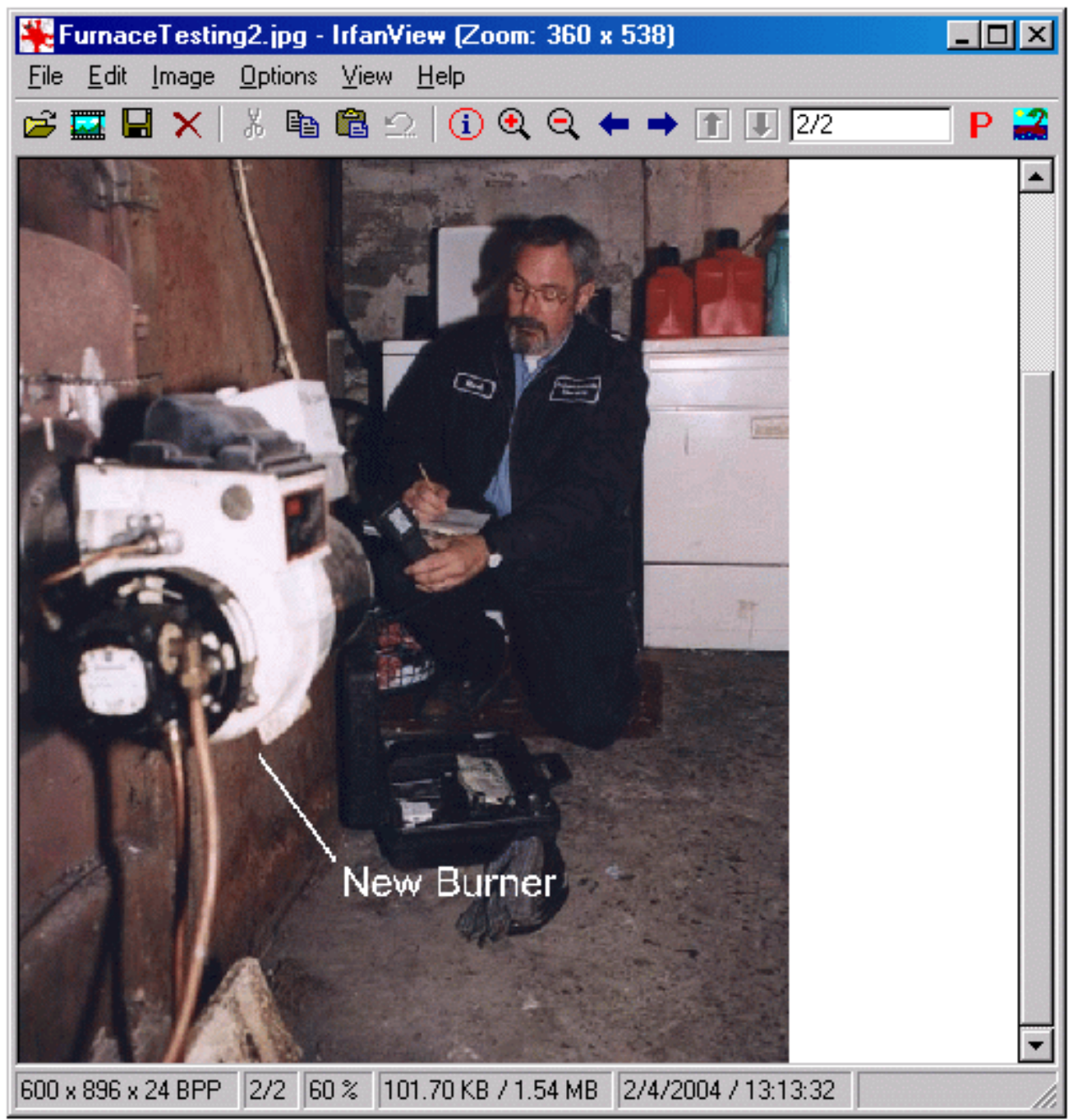

Figure 18.5. Editor of third party photo browser. 


\section{Chapter 18: Auxiliary Features}

\subsection{Geographic Information System (GIS)}

An optional feature of the Weatherization Assistant Version 8 is geographical encoding of each client location performed by the Graphic Information System (GIS). The feature allows you to map the location of a single client or a group of clients. It may also be used to select clients to include in a user-defined report or to select client data to export.

The Geographic Information System requires download of two additional files (from the same Weatherization Assistance Program sponsored site on which the main program is posted) and an installation procedure. The first file you will need to download, "base.zip," is common to all states. Choose the second based on your state of residence (e.g., "tn.zip” for Tennessee). You may download and install files for more than one state if needed. Both the "base.zip" and the state specific file are "zip" files requiring "unzipping." Most recent versions of Windows will include a routine to do this. Once downloaded to a location on your computer, right click on the zip file and choose "Extract All." You will be presented with a request for a location on your computer where the files are to be placed once unzipped. Select the "GIS" folder under the location where you installed the Weatherization Assistant, normally, "C:\Program Files\Weatherization Assistant 8-9\GIS.” Unzip both the base.zip and your state specific file into this location. Make sure that all individual files are in this same location, not in any sub-folders of this location. Sometimes the unzipping routine will place the state specific files in a separate folder named after the abbreviation for the state. If this occurs, use Windows Explorer, or some other file handling application, to move them to the GIS folder.

Make sure to turn the feature on in Preferences the next time you start the Weatherization Assistant program (see Feature 3, "Enable geographic information system (mapping)" in Section 16.4, Features). After successful installation of the feature, the "zip" files are no longer needed. All files associated with the GIS feature are used under a license agreement and cannot be duplicated or further distributed.

Once installed and activated, the GIS "Map” button will appear in two locations within the program: the Client Information form (see Section 7.1, Client Information) and the Client Selector form (see Section 17.5, Selecting Client Records), the latter accessible from the Agency Information tab and the Main Menu's "Data Link" button. Use of the feature, as accessed from these two 


\section{Chapter 18: Auxiliary Features}

locations, differs as do some of the controls on the resulting form. When accessed from the Client Information tab, the primary use is to locate a single client. Thus, only one location will be indicated on the map. When accessed from the Client Selector form, the purpose is to select a group of clients from all those in your database. Thus, many locations may be displayed on a single map. The two different applications will be discussed separately below.

\section{Client Location}

When the "Map" button on the Client Information form of the Main Menu's "Clients" button (see the discussion of the "Map" button in Section 7.1, Client Information) is selected for a client having an address understood by GIS, a map with the location indicated is displayed (see Figure 18.6).

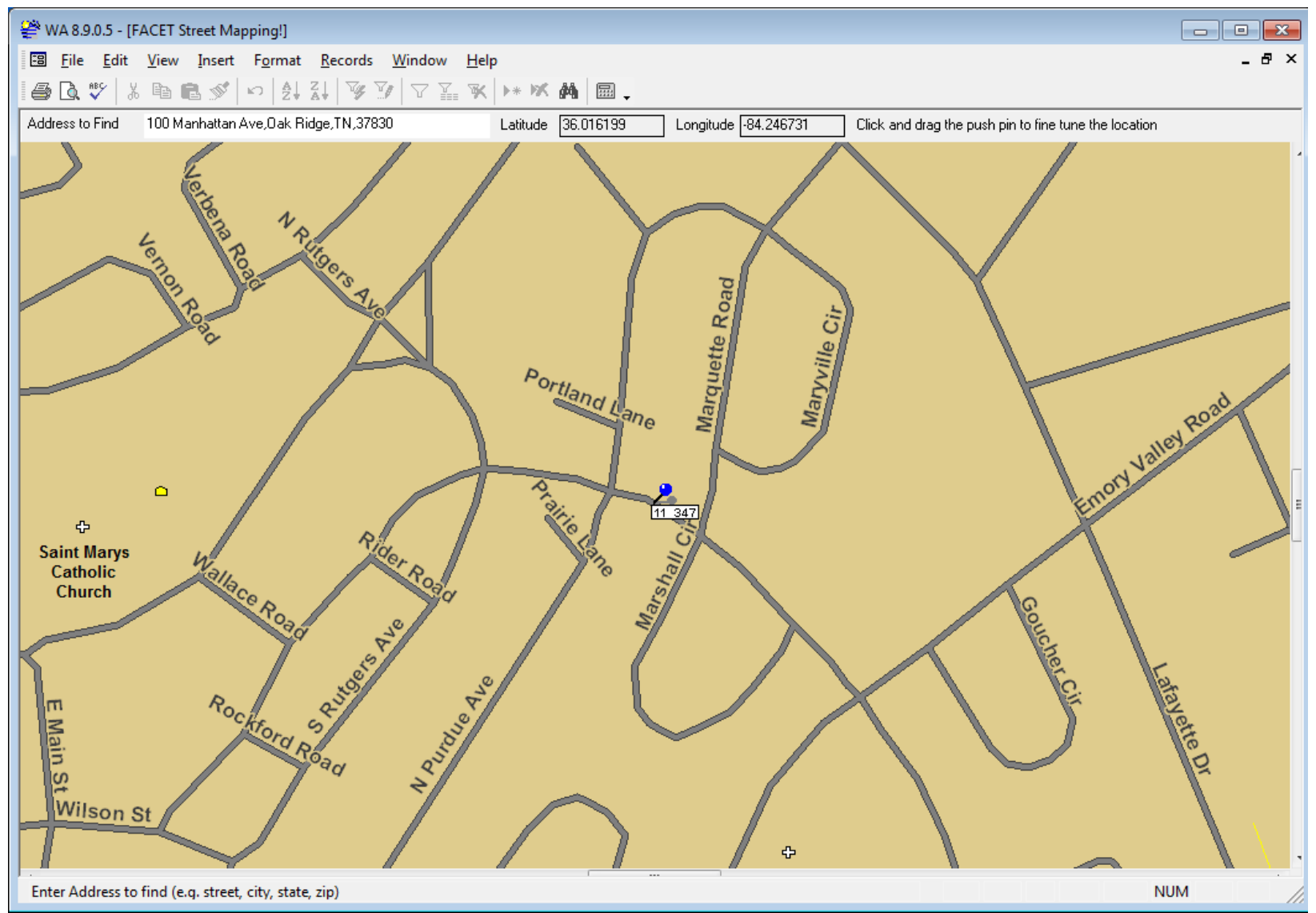

Figure 18.6. GIS Map for Single Client Location.

Note that the address of the location is automatically entered in the "Address to Find” field with the location's Latitude and Longitude also indicated. Attached to 


\section{Chapter 18: Auxiliary Features}

the pin denoting the location is the Client ID. A feature of the Client Location GIS map is that if you find the location to be different from the originally displayed location, you may click on the pin and drag it to its correct location. This will not change the address, but will update the coordinates of the location, which preserves your change for future references via the GIS mapping. On exiting the map, you will be asked to confirm your desire to preserve this change.

Most of the useful features associated with this use of GIS are accessed by right clicking your mouse on the map. Doing so will produce a menu from which you may zoom in or out, return to the previous scale ("Unzoom"), have the location displayed on a map of the entire state or country, enter a different address to locate, or print the map. An alternative method of zooming in is to outline a rectangular sub-area of the map by holding down your left mouse button while moving your mouse across the map. When you release the mouse button, the area outlined will then fill the window.

\section{Client Selection}

The second use for the GIS feature is to select clients based on their location using the Client Selector form, although the form may also be used to simply view the locations of a group of clients without actually selecting them (see Section 17.5, Selecting Client Records). The Client Selector form, containing a "Map" button, is accessible from the "Select Clients" button located on the Agency Information form (see "Select Clients" in Section 6.1, Agency Information) or from the "Select Client Records to Export" button on the "Transfer between databases with intermediate CSV/ZIP files” form under the Main Menu's "Data Link” button (see "Exporting Clients (via text files)" in Section 17.4, Data Transfer - Import/Export Indirectly with Text Files). The Client Selector form may also be involved in generating user-defined reports (see Appendix D, Development of Customized Reports).

The map displayed when initiated in this mode will likely have multiple locations denoted by pins (see Figure 18.7). Note that the pins are of two different colors, red and green. The map was initiated from a Client Selector form in which some clients may have already been selected. The map indicates this by having the selected clients' locations designated with the green pins. Un-selected clients have red pins. 


\section{Chapter 18: Auxiliary Features}

One of the uses of the GIS map initiated from the Client Selector form is to give you an alternative method of selecting clients. You may change a client from being un-selected to selected, or vice versa, by simply clicking on the pin. If all the locations within a specific area are to have their selection status changed, activate the Selects checkbox at the top middle of the window, place your mouse at a corner of a rectangle used to enclose the clients of interest, and drag your mouse to the opposite corner of the rectangle. All clients within the rectangle you have outlined will have their selection status reversed.

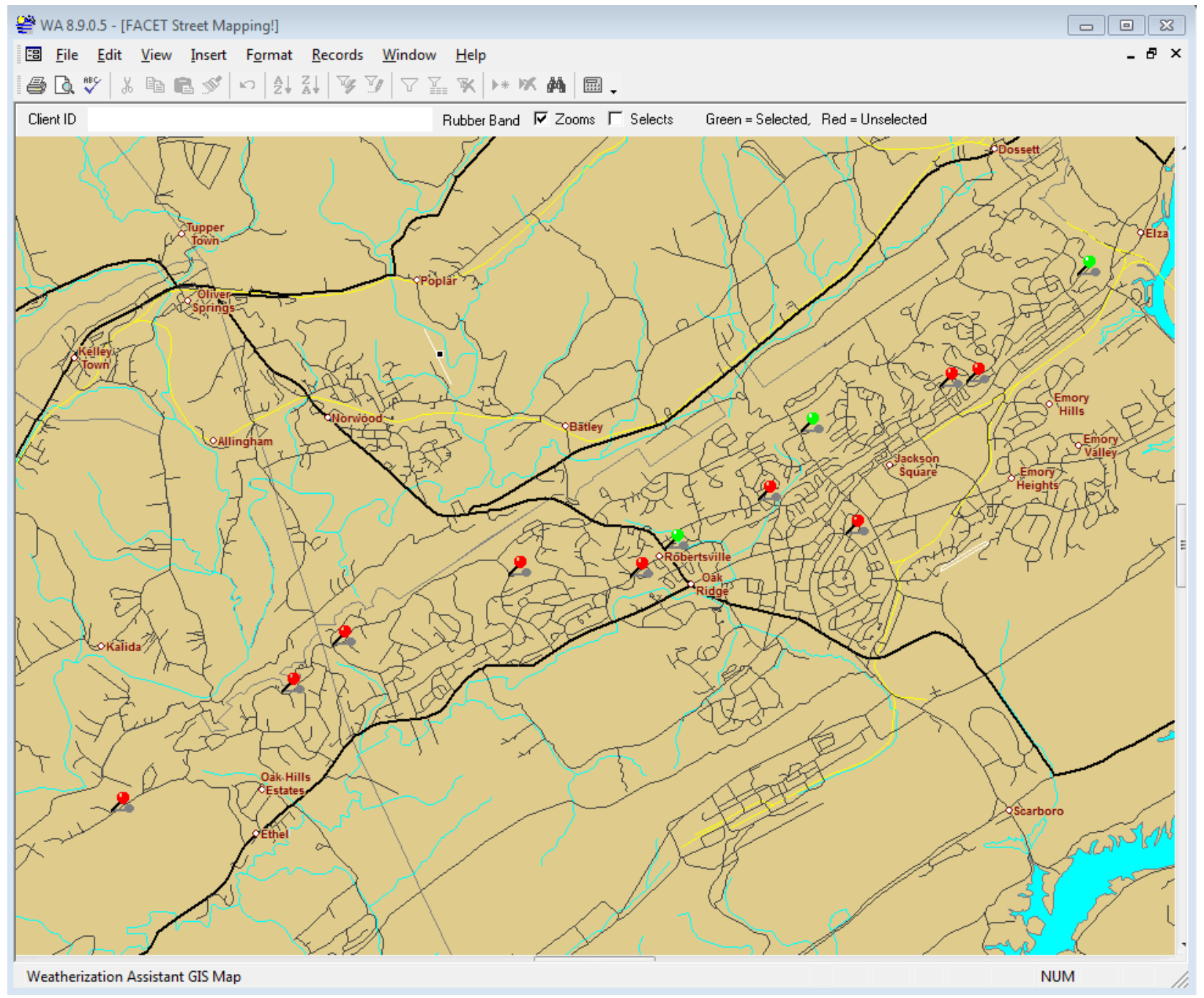

Figure 18.7. GIS Map for Client Selection.

Any changes made in the selection status of clients made from the map will be automatically transferred back to the client selector form from which the map was initiated. 


\section{Chapter 18: Auxiliary Features}

When you click your mouse on a specific client's pin, that client's ID will appear in the Client ID field. Double clicking on this field will then take you to the Client Information form (see Section 7.1, Client Information) for that client.

As with the map used to locate a single client, the client selector mode of the GIS map has additional features accessible by right clicking your mouse on the map. The menu then displayed allows you to zoom in or out, return to the previous scale ("Unzoom"), have the location displayed on a map of the entire state or country, enter a different address to locate, or print the map. An alternative method of zooming in is available. Activate the Zooms checkbox at the top middle of the window. Then, outline a rectangular sub-area of the map by holding down your left mouse button while moving your mouse across the map. When you release the mouse button, the area outlined will then fill the window.

\subsection{Status Tracking}

Status tracking is an optional feature that allows you to track the status of Clients, Audits, and Work Orders. The status categories for these three record types and their available settings are listed in Table 18.1. Settings of these statuses are normally performed from the Status tab in each of the three corresponding Main Menu windows. However, the Status tab under the Main Menu's "Clients" button allows you to view and edit the settings for all of the status categories associated with the current client. See Sections 7.2, Status (Clients); 11.1, Status (Audit); and 13.2, Status (Work Order), for discussions on the Status tab. See Section 6.9, Status History for information on reviewing the history of all status settings. Status settings are changed using the Status Editor sub-form (see Figure 18.8), which is accessed by selecting the "Edit" button in the row of the setting category you wish to change on the Status tab. All status settings for a given client are displayed in that client's Client Completion Report (see Appendix C, Reports).

Below are brief descriptions of the individual data items and controls found on the Status Editor sub-form.

- Status of - This field reminds you of the status category (type) you are about to change. Uneditable.

- For Client ID, Alt Client ID - These fields display the client identification parameters. Uneditable. 


\section{Chapter 18: Auxiliary Features}

Table 18.1. Status categories and settings

\begin{tabular}{|c|c|c|}
\hline Main Menu Window & Status Categories & Settings $^{4}$ \\
\hline \multirow[t]{2}{*}{ Client Level } & Client (General) & $\begin{array}{l}\text { Active On } \\
\text { Work Done, File Closed/Locked On* } \\
\text { No Work Done, File Closed/Locked On* } \\
\text { File Locked On } \\
\text { Delayed On } \\
\text { Other }\end{array}$ \\
\hline & Application & $\begin{array}{l}\text { Received On } \\
\text { Eligibility Verified On } \\
\text { Approved On* } \\
\text { Referred On* } \\
\text { Denied On* } \\
\text { Delayed On } \\
\text { Other }\end{array}$ \\
\hline Audit Level & $\begin{array}{l}\text { Audit (NEAT or } \\
\text { MHEA) }\end{array}$ & $\begin{array}{l}\text { Site Visit Scheduled For } \\
\text { Site Visit Completed On } \\
\text { Billing Data Collected On } \\
\text { Recommendations Generated On } \\
\text { Audit Complete and Locked On* } \\
\text { Walk Away by Auditor On* } \\
\text { Delayed On } \\
\text { Denied On } \\
\text { Other }\end{array}$ \\
\hline
\end{tabular}

\footnotetext{
${ }^{4}$ Statuses tagged with an asterisk $(*)$ are considered to represent completion.
} 


\section{Chapter 18: Auxiliary Features}

Table 18.1. Status categories and settings (cont.)

\begin{tabular}{|c|c|c|}
\hline Main Menu Window & Status Categories & Settings $^{4}$ \\
\hline \multirow[t]{3}{*}{ Work Order Level } & Work Order (General) & $\begin{array}{l}\text { Work Order Created from Audit On } \\
\text { Work Order Details Completed On } \\
\text { Work Order Approved On } \\
\text { Work Order Issued On } \\
\text { Work Scheduled to Start On } \\
\text { Work Started On } \\
\text { Work Completed On* } \\
\text { Client Signed Off On* } \\
\text { Work Refused by Client On* } \\
\text { Work Order Canceled On* } \\
\text { Work Order Modified On } \\
\text { Rework Completed On* } \\
\text { Delayed On } \\
\text { Other }\end{array}$ \\
\hline & Work Order Inspection & $\begin{array}{l}\text { Scheduled For } \\
\text { Passed On* } \\
\text { Failed On } \\
\text { Not Required* } \\
\text { Delayed On } \\
\text { Other }\end{array}$ \\
\hline & Work Order Payment & $\begin{array}{l}\text { Invoice Received On } \\
\text { Invoice Approved On } \\
\text { Invoice Paid On* } \\
\text { Not Required* } \\
\text { Delayed On } \\
\text { Other }\end{array}$ \\
\hline
\end{tabular}




\section{Chapter 18: Auxiliary Features}

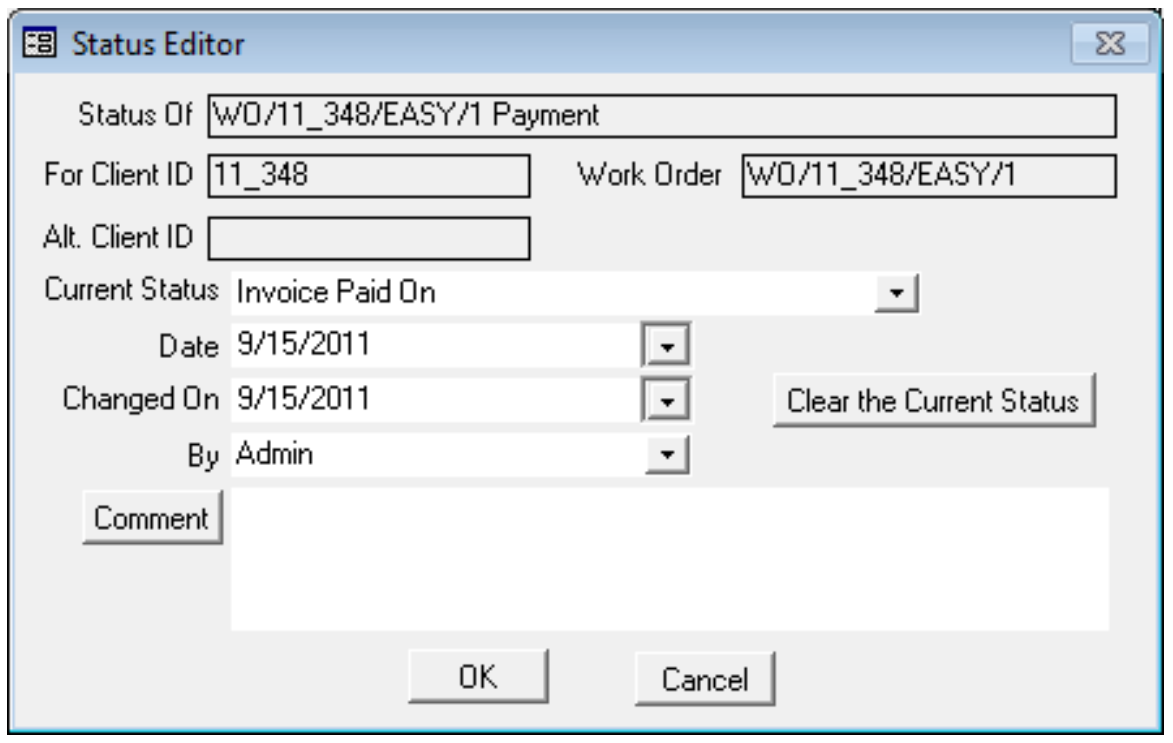

Figure 18.8. The Status Editor sub-form.

- Client ID/Audit Name/Work Order - This field displays the record name (either the Client ID, Audit Name or Work Order) for which you are about to change the status setting. Uneditable.

- Current Status - The Current Status field will indicate the existing status, if one exists. If this is the first time you have accessed this status category, the field will be blank. Use the "Current Status" drop-down list to change the status to the appropriate setting (i.e., one of those listed in the Table 18.1).

- Date - Following the designation of each current status are two dates, a reference to an agency contact or user, and a comment. Click on the "Date" drop-down list arrow to be presented with a calendar to select the date on which the status became effective. The current date will be circled in red on the calendar. Use the left and right arrow boxes at the top of the calendar to display other months, if necessary. Click on the date you wish to select, and it will automatically be entered in the Date field. If you save the status change without entering a date in the Date field, it will automatically be assigned the current date.

- Changed On - Perform the same steps as above to set the "Changed On" date. However, this may not be necessary since this date will be set to the current date if left blank, which is likely what you would have entered. 
- By - Use the "By" drop-down list to select who is making this change, likely yourself. The names that will appear are those you have entered in the Contacts tab under the Main Menu's “Agency” button. If left blank, this field will be automatically set to the agency contact who has logged on for this specific use of the program, if you are using the Logon Feature (see Feature 4, "Enable user logons with user name and passwords from the Agency/Contacts tab" in Section 16.4, Features). Otherwise, it will be set to "admin," indicating that if the Logon Feature is not being used, all users are assumed to have administrative privileges.

- Clear the Current Status - Use this button to clear the current status setting being viewed in the form. Selecting the button will send you back to the status form from which the "Edit" button had been selected with the row of the status category cleared, though the row with the status category designated will remain. It is anticipated that at some future time, the current status for the status category will be revisited and an entry made. Clearing a current status setting only effects the "Current Status" entry. It does not affect the "Status History" listing. The two sets of statuses are separate. Status history records must be deleted from a Status History form, often accessed using the " $\mathrm{H}$ " buttons just to the right of the "Edit" buttons used to access the Status Editor (see the references at the beginning of this section).

- Comment - Comments related to the Status may be entered directly in the comment field on the form. Or, select the "Comment" button to the left of the field, which will take you to the Comment Editor. This text editor is similar to Window's Notepad and may be used to edit extended comments. The total comment may have up to 65,000 characters. Text from other sources may be copied and pasted into the Comment Editor's window. The Comment is displayed only on the Client Status form and in the Client Completion Report (see Appendix C, Reports). Optional.

- OK, Cancel - When satisfied with your entries, select the "OK” button to save the changes or select the "Cancel" button to exit without saving your changes to the status category. You will be returned to the Status tab form. 


\section{Chapter 18: Auxiliary Features}

\subsection{E-Mailing from Within Weatherization Assistant}

The Weatherization Assistant allows you to send e-mails directly from within the program using an address book of e-mail addresses generated from the Contacts tab under the Main Menu's “Agency” button. Messages and/or attachments may be automatically linked to an e-mail when initiated using the "E-mail this Error Message" button located on the Error History form (see Feature 7, "Enable the logging of program errors" in Section 16.4, Features) or the "E-mail" button on either of the transfer between databases forms used to export client information (see discussions on "Exporting Clients" in Sections 17.3, Data Transfer Import/Export With Another MSAccess Database, and 17.4, Data Transfer Import/Export Indirectly with Text Files).

For example, Figure 18.9 shows the form displayed having selected the "Email this Error Message" button on the Error History form.

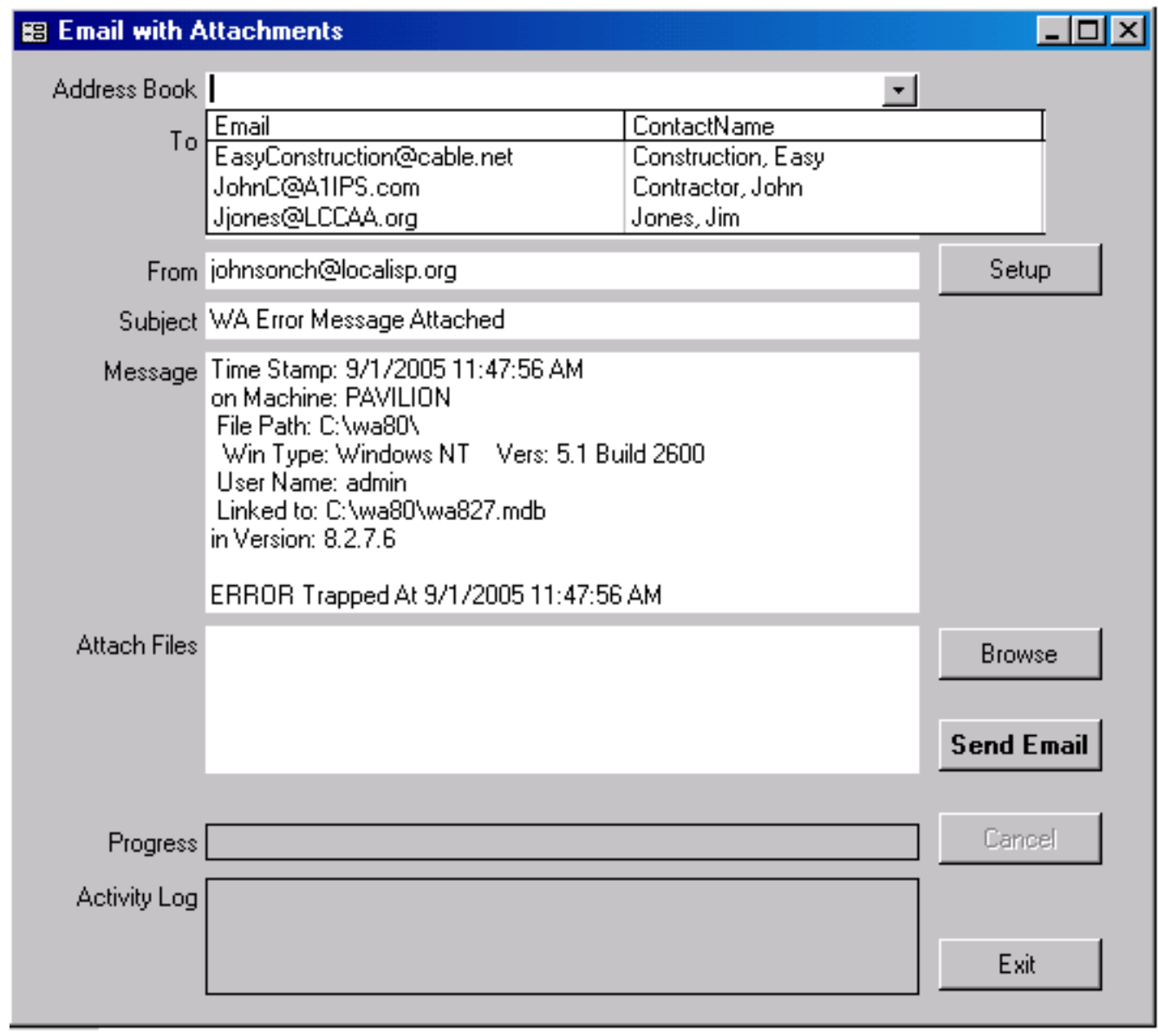

Figure 18.9. E-mail specification form for reporting error message. 
- Address Book and To - The form is shown with the "Address Book" dropdown list displaying a sample list of e-mail addresses that were entered under the Agency Contacts tab. Selecting one of the entries in this drop-down list will automatically transfer the address to the "To" field. Or, you may type an address directly into the field.

- From - The From field is automatically filled in as the "From Address" entered in the E-mail Account Setup form accessed by selecting the "Setup" button (see Figure 18.10 below). Or, you may type an address directly into the field.

- Subject and Message - In this example, the Subject and Message fields are automatically filled in, reflecting the entry in the Error History from which the e-mail was initiated. You may alter and add to the message to give any additional information.

- Attach Files - If the e-mail had been initiated from one of the Export forms having created a single file with the exported information, the file path would have been automatically entered in the "Attached Files" field. Otherwise, use the "Browse" button to locate and select files to attach.

- Send Email - Once completed with the e-mail specification, click the "Send Email” button to send the message and attached file(s).

- Progress - The Progress field will notify you of the progress in sending the email. If attachments are large, it may take some time to complete the process. The field will indicate to you how much (how many bytes of data) of the transmission has been sent and how large (total bytes) the total transmission is. Uneditable.

- Activity Log - The “Activity Log” will contain any messages from the e-mail server, more than likely the connection status and any problems that might be encountered. The messages could be used by a system administrator to debug any problem with e-mail transmissions that might occur. Uneditable.

- Setup - In order to use the e-mail feature, you must have an e-mail account on a server somewhere. The Setup for the e-mailing capability in the Weatherization Assistant need be performed only once, though it may be 


\section{Chapter 18: Auxiliary Features}

altered at any time. The form presented upon selecting the "Setup" button is shown in Figure 18.10.

Contact your system administrator for the information required to successfully initiate your e-mail account according to local procedures. The administrator may also need to address information on the two additional tabs: "Advanced" and "Fire Wall."

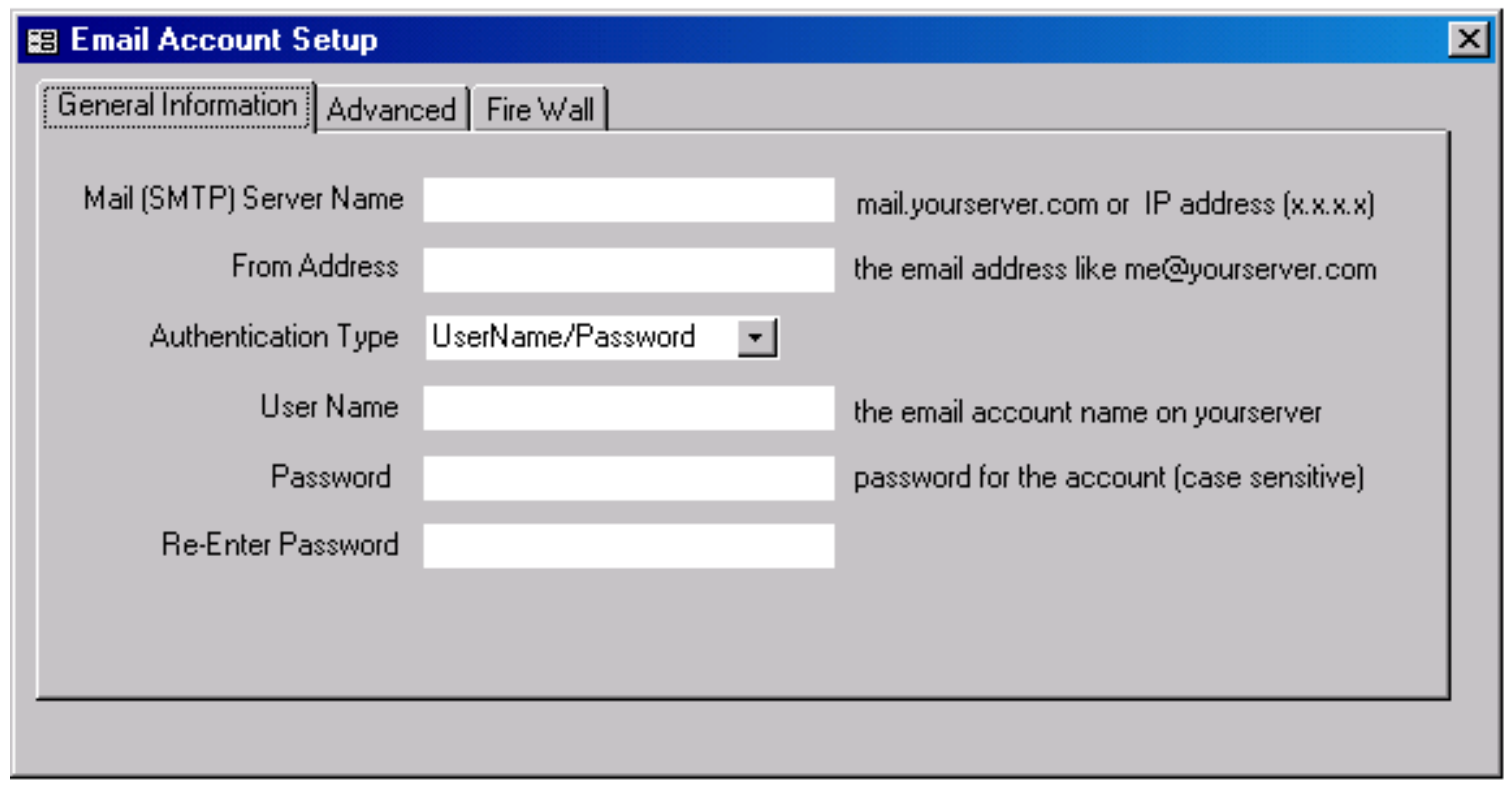

Figure 18.10. E-mail Setup form.

- Cancel/Exit - Use the "Cancel" button to cancel a transmission in progress. This may be necessary if an attached file is of un-expected size that creates problems for the server or the server goes down during the transmission. Use the "Exit" button to exit the form at any other time, whether the e-mail has been sent or not. 


\section{Appendix A. NEAT Weatherization Measures}

The list of weatherization measures considered by NEAT can be accessed in the Library Measures form under the Main Menu's “Setup Library” button (see Section 14.5, Library Measures). NEAT currently examines 30 weatherization measure types:

1. Attic insulation (R-11, 19, 30, 38, 49, Specified level, Fill cavity)

2. Sill box (band joist) insulation

3. White roof coating

4. Foundation wall insulation

5. Floor insulation (R-11, 19, 30, and 38)

6. Wall insulation and knee wall insulation

7. Duct insulation

8. Window sealing

9. Door replacement

10. Storm windows

11. Window replacement (standard and low-e)

12. Window shading (awnings, sun screens, and window films)

13. Vent damper (thermal and electric)

14. IID (Intermittent ignition device) and Electric vent damper/IID combined

15. Flame retention burner

16. Furnace tune-up

17. Replace heating system (furnace/boiler - standard/high efficiency)

18. Smart (set-back) thermostat

19. Tune-up air conditioner

20. Replace air conditioner (window and central)

21. Evaporative cooler

22. Install/Replace heat pump

23. Seal ducts

24. Lighting retrofits

25. Refrigerator replacement

26. Water heater tank insulation

27. Water heater pipe insulation

28. Low-flow showerheads

29. Water heater replacement

30. Infiltration reduction 


\section{Appendix A: NEAT Weatherization Measures}

The first seven weatherization measure types are designed to reduce heat loss or gain through walls, floors, ceilings, and ducts. Measure types 8 through 12 affect the way windows and doors behave in the house. They can alter the conduction and infiltration heat loss through windows and doors, and solar gain through windows. Window shading reduces solar gain - a major element of total heat gain. Shading the windows may affect the heating load, because shade blocks some solar heat from the house during the heating season. Measure types 13 through 18 improve the heating system efficiency by decreasing heating energy consumed by the house. They do not affect heat loss through the envelope, however. Measure types 19 through 22 improve cooling efficiency during the cooling season. Evaporative coolers and new air conditioners can reduce cooling costs markedly, if properly installed. Measure type 23, Seal Ducts, can increase the efficiency for both heating and cooling, depending on the HVAC equipment in the home. Measure types 24 through 29 reduce the energy consumption not directly related to the climate. Such measures are referred to as "base load" measures. They can have minor effects on the heating and cooling costs. Measure type 30, Infiltration Reduction, evaluates the success of your efforts to reduce the air infiltration in the home, reducing both heating and cooling costs.

For each of the envelope components of a home where insulation may be added (see Sections A.1, A.2, and A.4 through A.6 below), there are one or two insulation types standard to NEAT that may be chosen to be installed. However, NEAT allows the user to define additional insulation types for each of these envelope locations in the Setup Library (see Section 14.7, NEAT Insulation Types). For each of these types, the insulation characteristics (R-value per inch or total R-value) are also specified on this form.

\section{A.1 Attic Insulation}

For any attic type or attic component (except knee wall ${ }^{5}$ ), attic insulation can be evaluated in NEAT in three ways:

- Add Standard Insulation Levels - If the R-value of added insulation or the maximum depth of total insulation (see Section 9.4, Shell - Unfinished Attics) is not specified, NEAT will evaluate the cost-effectiveness of adding all standard

\footnotetext{
${ }^{5}$ For knee wall insulation, see Appendix A.6, Wall Insulation.
} 
levels of attic insulation (R-11, R-19, R-30, R-38 and R-49) as separate measures and recommend the most cost-effective insulation level.

- Add Specified Insulation Level - If the user has specified an R-value of added attic insulation, NEAT will evaluate its addition as a mandatory measure and display it in the recommended measures list, even if it is not cost-effective. If the specified level proves not to be cost-effective, it might be wise to run NEAT again, allowing the program to choose a cost-effective level, if one exists.

- Fill Ceiling Cavity (given depth restriction) - If the user has specified a depth restriction for attic insulation (depth of any existing insulation plus the space for any additional insulation), NEAT will evaluate adding all standard levels of insulation that fit into the available space as well as the level of insulation that exactly fills the available space, and recommend the most cost-effective insulation level.

For determining the level of insulation that exactly fills the available space, NEAT uses R-values per inch entered on the NEAT Insulation Types form under the Setup Library (see Section 14.7, NEAT Insulation Types).

For determining the installation cost of added insulation levels other than the standard insulation levels, NEAT interpolates using the costs of installing the standard levels entered on the Library Measures form under the Main Menu's “Setup Library” button (see Section 14.5, Library Measures).

\section{A.2 Sill Box (Band joist) Insulation}

For houses with an enclosed foundation space or an exposed floor, the sill portion of the foundation wall or floor may be uninsulated. Insulating the sill is often costeffective. No air leakage reduction through the sill is assumed to occur due to implementing this measure. NEAT will compute the change in the overall conductance of the foundation space that results from installing sill insulation. NEAT evaluates installing fiberglass batts or any other sill insulation materials, at their indicated R-values, as specified by the user in the NEAT Insulation Types form under the Setup Library. The insulation is added to the uninsulated perimeter of sill exposed to the outdoors, as indicated by the user. 


\section{Appendix A: NEAT Weatherization Measures}

Sill Box and Floor Insulation measures are considered mutually exclusive; that is, NEAT will not recommend both measures, because the program assumes that if floor insulation is installed, it extends to the sill, thus insulating the sill as well.

\section{A.3 White Roof Coating}

An elastomeric, low-emissivity roof-coating retrofit measure is applied only to homes with a Roof Color of "Normal or Weathered" (see Section 9.4, Shell Unfinished Attics) and an existing cooling system. The benefit comes from decreasing the solar load on the roof, which in turn decreases the home cooling load. This retrofit measure is commonly installed only in cooling climates.

\section{A.4 Foundation Wall Insulation}

For houses with an enclosed foundation space, the Foundation Wall Insulation measure assumes a uniform addition of insulation to both above- and below-grade foundation space walls. NEAT evaluates installing rigid foam board or any other foundation wall insulation materials, at their indicated R-values, as specified by the user in the NEAT Insulation Types form under the Setup Library. No insulation will be added to foundation walls of a subspace having average height less than two feet.

See Section A.4, Floor Insulation, below for information regarding selecting between the foundation wall insulation and floor insulation measures.

\section{A.5 Floor Insulation}

For houses with an enclosed foundation space or an exposed floor, adding insulation to the floor between the living space and the foundation space can be evaluated. NEAT will evaluate the cost-effectiveness of adding standard levels of insulation (R-11, R-19, R-30, and R-38) as separate measures and recommend the most cost-effective insulation level. The levels evaluated are restricted to those that fit within the joists, as determined by the entry for the Floor Joist Size on the Foundations form of the audit (see Section 9.6, Shell - Foundations) and the Rvalues per inch entered on the NEAT Insulation Types form under the Setup Library for the floor insulation material chosen to be installed. 
The Foundation Wall Insulation and Floor Insulation measures are mutually exclusive; that is, NEAT will not recommend both. You may choose to have NEAT evaluate either or both measures, depending on your choice of Added Insulation Type in the Floor and Foundation Wall data blocks on the Foundations form. A choice of "None" in either or both of these fields prevents NEAT from evaluating installing insulation in the location. If both measures are specified, NEAT will evaluate insulating the foundation walls and all standard levels of floor insulation as separate measures and recommend the most cost-effective measure, if the SIR is greater than the minimum allowable SIR.

\section{A.6 Wall Insulation and Knee Wall Insulation}

NEAT will evaluate installing insulation either into the cavity of a frame wall or board insulation over the entire surface of a wall, depending on the Wall Type and Added Insulation Type chosen on the Walls form of an audit (see Section 9.1, Shell - Walls). NEAT will not attempt to add loose fill insulation to a wall that is not frame, a frame wall already having loose fill insulation present, or where the existing insulation depth is seen as providing less than 1.5 inches of available space. In determining the available space, the program takes into account the stud size, the R-value of existing insulation, and the insulation's thermal characteristics (R-value per inch). NEAT considers a wall insulation material to be loose fill if the "Units" for the material on the NEAT Insulation Types form have been chosen as "R/in."

NEAT's Wall Insulation measure not only affects the conduction through a wall, but also reduces the free solar heat transmission through the wall. If a wall is located next to a sheltered but unheated area, NEAT reduces the effective temperature difference seen across the wall by one-third.

For houses with a finished attic, NEAT will evaluate the savings from insulating a knee wall (a wall next to an unconditioned attic space) using a measure dedicated to this purpose. NEAT evaluates installing fiberglass batts or any other insulation material, at the indicated R-value, as specified by the user in the NEAT Insulation Types form under the Setup Library. 


\section{A.7 Duct Insulation}

The Duct Insulation measure can be evaluated for a house where the primary heating system is a furnace or heat pump and uninsulated supply ducts are located in an unconditioned attic or foundation space. NEAT uses information you supply under the Uninsulated Supply Ducts sub-form under the Heating form of the audit (see Section 9.7, Heating - General Description Data).

NEAT evaluates this measure by adding a specified R-value (see "Site Built (NEAT) Key Parameters” in Section 14.2, Key Parameters) to existing R-1.5 supply ducts and calculating the heat lost from the supply duct during winter as well as heat gained by the duct during summer (if central air-conditioning is present). These heat exchanges are assumed to occur between the duct and the space where the duct is located, either a foundation space or the attic, depending on your input.

\section{A.8 Window Sealing}

NEAT estimates the savings that can be obtained from weatherizing an existing window to reduce its leakiness. Both heating and cooling (latent and sensible) energy is considered in this estimate. The amount of energy saved depends on your declaration of each window's pre-retrofit leakiness, as indicated on the Windows input form (see Section 9.2, Shell - Windows) as well as the overall house leakiness described on the Ducts/Infiltration form (see Section 11.2, Ducts/ Infiltration).

NEAT translates your leakiness designation into an estimate of air leakage through the window that results primarily from a pressure differential created by wind. It then uses empirically-based relations to reduce this air leakage through the window to reflect caulking, weather-stripping, and general repairs to the window. The energy saved from this reduction is computed in much the same way savings from whole house infiltration reduction is determined.

Note that if pre-retrofit whole house infiltration data has been entered, NEAT subtracts from it that leakage determined for the windows. Thus, if window treatments are performed, the savings from general air sealing will likely be reduced, so as to avoid accounting for the window sealing twice. 
The Window Sealing measure is one of four mutually exclusive window measures, along with Storm Windows and Window Replacement (both standard and low-e). Only the measure found most cost-effective from a set of mutually exclusive measures will be recommended. Thus, NEAT would never recommend both sealing and replacing the same window. The only exception to the costeffectiveness criteria is when a specific measure has been declared mandatory, as might be the case for the Window Replacement measure for a window whose frame is so rotted that weatherization or use of a storm window is not practical. Check your program's policy regarding mandating measures. How the measure is viewed depends on the selection you make for Retrofit Status on the Windows form during data input.

\section{A.9 Door Replacement}

This retrofit measure is evaluated only if the existing door is not a sliding glass door. The benefit assigned to this measure is to increase the R-value through the door and possibly reduce air leakage through the door. The infiltration savings depends on your indication of the existing door's initial leakiness (see "Leakiness" in Section 9.3, Shell - Doors). This savings is computed in a manner similar to that used for windows (see the discussion of the Window Sealing measure above for more information).

\section{A.10 Storm Windows}

NEAT assumes that installation of a storm window introduces an additional air space to the window assembly. The added R-value due to this air space and extra pane of glass is taken from published data for air space R-values with one face having the emittance the user provides in the Key Parameters of the Setup Library (see "Site Built (NEAT) Key Parameters" in Section 14.2, Key Parameters). This allows for the modeling of installing a low-e storm window. The addition of the storm window will also change the solar transmission through the window, depending on additional data provided in the key parameters. Unless altered by the user, the key parameters are set to model the storm window as a single pane of clear glass in a wood or vinyl frame.

Lastly, the storm window could produce an additional benefit of reducing air leakage. The change in air leakage through the window system with and without a storm window is estimated in NEAT from published and empirical data as well as 


\section{Appendix A: NEAT Weatherization Measures}

your indication of the existing window's initial leakiness (see the discussion of the Window Sealing measure above for more information).

This measure is mutually exclusive with other window retrofit measures. See the discussion of the Window Sealing measure above for the implications of mutual exclusiveness.

\section{A.11 Window Replacement (Standard and Low-E)}

NEAT assumes the installation of a quality window with thermal and transmittance properties specified in the Setup Library (see "Site Built (NEAT) Key Parameters" in Section 14.2, Key Parameters) and leakage characteristics that meet code standards. The energy savings estimated for the measure considers conduction, solar, and infiltration effects.

The Low-e Window measure replaces the existing window with a low-e window with properties also specified in the Key Parameters. Low-e windows save energy during the winter by reflecting heat rays originating in the house back indoors. During the summer, low-e windows reflect solar heat outdoors. Low-e windows also have a higher R-value than single- or standard double-pane windows.

Both window replacement measures may also produce infiltration savings, depending on your indication of the existing window's initial leakiness (see the discussion of the Window Sealing measure above for more information). Any such savings will be subtracted from the general air sealing savings so as to avoid accounting for this infiltration savings twice.

The window replacement measures are mutually exclusive with other window retrofit measures. See the discussion of the Window Sealing measure above for the implications of mutual exclusiveness.

Unless changed by the user, the standard Window Replacement measure assumes installation of a quality double pane window with a thermally broken metal frame. The Low-e Window measure assumes installation of a double-pane window with a metalized coating on one of the interior glass surfaces. 


\section{A.12 Window Shading}

NEAT evaluates window shading on all windows not facing north and shaded less than 50\% without the measure. Shading measures are most beneficial in cooling climates, since their effect during the heating season is detrimental, blocking sunlight that would otherwise help heat the home. Three types of window shading are evaluated: Awnings, Sun screens, and Window films. The Window Shading measures are mutually exclusive among themselves, as well as with the Storm Windows measure and the Low-E Windows measure.

- Awning - Awnings reduce direct solar radiation incident on a window. NEAT's affect is season-dependent since the sun is lower in the sky during the winter than in the summer. Awnings are an effective conservation measure because they stop solar heat before it contacts the house. However, awnings are expensive, they require more planning, and they restrict views through windows more than other conservation measures.

- Sun Screen (Fabric or Louvered) - NEAT assumes that fabric sun screens block about 66 percent of solar radiation and that aluminum louvered sun screens block about 89 percent of solar radiation. Fabric sun screens are less expensive and easier to assemble than louvered sun screens. Both affect the view through the window.

- Window Film - There is a large variety of window films available having a range of optical and thermal properties. The more reflective films are highly metalized and appear mirror-like from outdoors. All-season films, sometimes called low-e films, also reflect heat energy from inside the house during the heating season. In all but the hottest, sunniest climates, these low-e films are more cost-effective than films that merely reflect solar heat. NEAT assumes that the window films installed have the characteristics specified in the Key Parameters of the Setup Library (see "Site Built (NEAT) Key Parameters" in Section 14.2, Key Parameters). Unless changed by the user, they represent an average window film, though more effective products do exist.

NEAT assumes that these window treatments, if installed, will cover the entire glazed portion of the window. 


\section{A.13 Vent Damper (Thermal and Electric)}

A vent damper reduces heat loss from a heating system by closing the chimney when the burner is not operating. The vent damper prevents most of the residual heat from escaping the heat exchanger, allowing the pump or fan to deliver the leftover heat to the house instead of losing it up the chimney.

NEAT evaluates installing electric vent dampers on natural gas, propane, or oilfueled furnaces or boilers that currently have intermittent ignition devices (IID) or will have an IID installed during retrofit. Electric vent dampers use a solenoid or a small electric motor. A solenoid is a magnetically operated lever that opens the damper when the burner fires and closes it when the burner goes off. Vent dampers for use with oil systems or with gas systems with IIDs may have dampers that close almost completely. NEAT will not evaluate installing an electric vent damper on a heating system having a pilot light.

NEAT evaluates installing thermal vent dampers on natural gas or propane-fueled furnaces, boilers, or vented space heaters, with and without pilot lights. These dampers open a bimetallic damper when they sense heat. Thermal vent dampers close when the burner is off and the chimney cools. Thermal vent dampers may be dangerous in heating systems with marginal draft and may not be permitted by some local codes. Some locations may have regulations barring installation of any vent dampers. Should a damper fail in the closed position, serious health problems may be incurred by the occupants.

NEAT will not consider installing a vent damper of either type on a heating system that is in a conditioned space or has either a power or retention head burner. The savings attributed to the measure ranges from $4 \%$ to $9 \%$ of monthly heating energy consumption, depending on the system type, fuel, and location.

\section{A.14 IID (Intermittent Ignition Device) and Electric Vent Damper/IID Combined}

An intermittent ignition device (IID) saves energy by eliminating the need for a standing pilot light. The IID consists of a special gas valve, an electric igniter, and a pilot light that remains on only while the main burner is lit. NEAT assumes that an electric vent damper will be installed with an IID, or that an IID is present 
before an electric vent damper is recommended. NEAT will evaluate this measure only for furnaces or boilers fueled with natural gas or propane.

NEAT uses formulas to estimate savings for two possible conditions: 1 - Pilot light on during the summer; 2 - Pilot off during the summer. Indicate on the heating screen (see Section 9.8, Required Heating System Details Sub-Form) which of these conditions exists. Rather than computing savings based on a percentage of annual consumption, NEAT links savings to the location's heating degree days. For most climates, this results in annual savings from 4 to 6 percent of gas consumed by the heater.

\section{A.15 Flame Retention Burner}

Flame retention burners provide higher combustion efficiency by mixing the mist of oil and air more vigorously than conventional burners. Furnaces and boilers with flame retention burners have steady-state efficiencies of 80 percent or more. Replacing an existing oil burner with a flame retention burner is usually costeffective if the existing steady-state efficiency is less than 75 percent. NEAT evaluates installing a flame retention burner only on oil-fueled furnaces or boilers.

When a conventional oil burner is replaced with a flame retention burner, the burner nozzle is usually reduced a size to account for the oversizing of the original burner, the higher efficiency of the new burner, and improvements to the envelope of the house. NEAT does not account for any additional savings that may result from this down-rating of capacity.

The combustion chamber of the existing heating unit may be re-lined with a ceramic liner - installed like a plaster cast - when the burner is replaced. If installation of a flame retention burner in your program includes this procedure, the price of this measure should include its cost.

\section{A.16 Furnace Tune-Up}

Heating systems may function far below their potential efficiency or may be unsafe due to the following common problems:

- Dirt, soot, or other materials interfering with the burner flame;

- Dirt, soot, or corrosion on heat exchanger surfaces; 


\section{Appendix A: NEAT Weatherization Measures}

- Electric controls malfunctioning or out of adjustment;

- Air hotter than necessary to heat the house;

- Incomplete combustion;

- Faulty draft;

- Incorrect fuel input;

- Blockages or leaks in heating distribution system; or

- Faulty thermostatic setting, anticipator adjustment, or location.

Heating technicians performing tune-ups should have specific training on increasing the efficiency of heating systems. Technicians should have test equipment to measure efficiency, air temperature, carbon monoxide, draft, and all other parameters relevant to the seasonal efficiency of each heating system.

NEAT will evaluate the Furnace Tune-Up measure only for furnaces or boilers fueled by oil, natural gas, propane, or kerosene. NEAT assumes that if the measure is performed by a qualified technician who addresses the issues listed above, it will save from 0 to 14.5 percent of the fuel used for heating, depending on the Equipment Type and Fuel entered under NEAT's Heating tab and the Condition of the furnace and Steady State Efficiency, as indicated by the user's entries on the Required Heating System Details sub-form of the tab (see Sections 9.7, Heating General Description Data, and 9.8, Required Heating System Details Sub-Form). The measure is mutually exclusive with the Replace Heating System measure; that is, only one of the two will ever be recommended, if either.

\section{A.17 Replace Heating System (Furnace/Boiler - Standard/High Efficiency}

Replacing the heating system makes economic sense when the steady-state efficiency of the existing system is low and can't be significantly improved. Today's standard efficiency natural gas heating systems have fans that draw in the combustion air and propel the combustion products out of the heater. They also have intermittent ignition devices that eliminate the energy consumed by a standing pilot light. With these improvements, standard efficiency gas heaters achieve seasonal efficiencies of 80 percent or more.

Today's standard efficiency oil heaters use flame retention oil burners. They also eliminate the natural draft chimney and the barometric draft control by using an 


\section{Appendix A: NEAT Weatherization Measures}

induced draft fan and a smaller flue pipe. These improved oil heaters achieve seasonal efficiencies of around 85 percent.

The most efficient gas heaters cause water vapor formed in the combustion process to condense into liquid water. These condensing heaters reclaim the heat in the water vapor and achieve seasonal efficiencies of more than 90 percent.

NEAT provides for two separate furnace/boiler replacement measures: a standard efficiency heating unit (80+ percent) and a high-efficiency unit (90+ percent). The user may have NEAT weigh the two and recommend the most cost-effective, if either; force NEAT to evaluate only one of the two options; or, in the case of a health and safety issue with the existing unit, dictate either option as mandatory (see “Options” in Section 9.8, Required Heating System Details Sub-Form).

The cost-effectiveness of replacing a heating system heavily depends on the Steady State Efficiency of the existing system, entered on the Required Heating System Details sub-form. If a heating system is to be replaced solely based on the savings

of the measure, it is strongly recommended that this entry be obtained from an accepted efficiency measurement, as opposed to data from the name plate of the unit or a data base of heating system efficiencies. The measure is mutually exclusive with the Furnace Tune-Up measure; that is, only one of the two will ever be recommended, if either.

NEAT will also evaluate the cost-effectiveness of replacing any fossil-fueled (including propane and kerosene) furnace or boiler to a system consuming a different fossil fuel. It is left to the user to determine the appropriateness of such a replacement in his/her program.

\section{A.18 Smart (Set-Back) Thermostat}

Automatic set-back (smart) thermostats can be cost-effective for people who have regular schedules and who understand how to set the thermostats to follow those schedules. NEAT evaluates savings accomplished by setting back the set-point temperature during the heating season only. Therefore, the measure will not show cost-effectiveness in cooling climates. You may set the number of degrees Fahrenheit of the nightly setback in the Key Parameters of the Setup Library (see "Site Built (NEAT) Key Parameters" in Section 14.2, Key Parameters). Unless altered, NEAT will assume a five degree Fahrenheit setback. 


\section{A.19 Tune-Up Air Conditioner}

Significant efficiency increases for an air conditioner may be possible through proper maintenance and periodic tune-ups. The following steps are suggested in performing the tune-up of an existing air conditioner:

- Replace or clean existing air filters and grills,

- Check and clean condensate trough and drain,

- Clean evaporator (indoor) and condenser (outside) coils,

- Straighten bent or flattened coil fins if necessary,

- Ensure unobstructed air flow to the condenser coil,

- Check for proper refrigerant charge,

- Remove dust and dirt from fan blades,

- Examine and oil motor and fan bearings,

- Inspect and/or tighten electrical connections and contacts,

- Check for blockages or leaks in the supply and return ducts.

Properly trained HVAC technicians should perform the tune-up insuring the use of proper equipment, especially in checking the refrigerant charge and the electrical wiring, and straightening the coil fins.

NEAT will evaluate the Tune-Up Air-Conditioner measure for any central or window air-conditioner having an SEER below 10.0. NEAT assumes that a thorough air conditioner tune-up, addressing the issues listed above, will increase the system's SEER from 0 to 36 percent, depending on its existing condition.

\section{A.20 Replace Air Conditioner (Window and Central)}

Air conditioners move heat from indoors to outdoors with a cooling coil (the evaporator), a heating coil (the condenser), and a compressor, which moves a heat transfer fluid (the refrigerant) between the two coils. The federal government requires all air conditioners to carry a yellow energy label listing its annual cost of operation and a measure if its efficiency, EER for room air conditioners and SEER for central systems. A room air conditioner with an EER of 12 will use half as much electricity for cooling as one with an EER of 6.

NEAT considers replacing each existing unit individually with an equivalent unit with similar characteristics, but higher efficiency. The program will not evaluate 


\section{Appendix A: NEAT Weatherization Measures}

replacing multiple window units with one central unit. NEAT will not replace an air-conditioner having SEER of nine or above.

The costs of replacing individual air conditioners having each of three capacities for both room and central systems are entered under the "Costs" button for the Replace AC measure on the Library Measures form of the Setup Library (see Section 14.5, Library Measures). For room air conditioners, NEAT will interpolate to arrive at a cost of replacing the size of unit specified in the building description. For central systems, the program will use the capacity of the nearest $1 / 2$-ton increment to the specified size, using interpolation or extrapolation of the costs for the three standard sizes.

Since NEAT accepts only Seasonal Energy Efficiency Ratio (SEER) values, you may need to convert EER to SEER values (see Section 9.9, Cooling, for converting EER to SEER). Replacement SEERs may be altered in the Key Parameters of the Setup Library (see "Site Built (NEAT) Key Parameters" in Section 14.2, Key Parameters). Unless changed by the user, the SEERs for the replacement window and central air-conditioners are 11 and 13, respectively.

\section{A.21 Evaporative Cooler}

In the warm, dry climates of the western United States, evaporative coolers (also called swamp coolers) are a popular and energy efficient cooling device. The lower the summertime relative humidity, the more the evaporative cooler will drop the indoor temperature. Evaporative coolers use one-quarter to one-sixth of the energy of air conditioners and they cost about one-half as much to install. Unlike central air conditioners, evaporative coolers provide a steady stream of fresh, humid air to the house.

NEAT will evaluate the Evaporative Cooler measure only in climates where the relative humidity is less than $50 \%$ during $90 \%$ of the months when the average monthly temperate is above $78^{\circ} \mathrm{F}$. However, even in some climates where these conditions hold, evaporative coolers may not be available or seen as applicable. They have maintenance requirements that may be difficult for some clients to accomplish. If this measure is not to be considered for your program, the measure should be turned off (see Section 14.5, Library Measures) since its high costeffectiveness will usually place the measure high on the recommended measures 


\section{Appendix A: NEAT Weatherization Measures}

list, preventing other mutually exclusive cooling measures from being recommended and making other cooling measures less cost-effective.

\section{A.22 Install/Replace Heat Pump}

Heat pumps operate like air conditioners except that the heating and cooling coils can switch functions to supply heat as well as cooling. The Install/Replace Heat Pump measure will evaluate the cost-effectiveness of (1) installing a heat pump to replace any electric resistance heating system with or without existing cooling and (2) replacing an existing heat pump with one of higher efficiency.

If your existing primary heating system is electric resistance, regardless of the equipment type (see Sections 9.7, Heating - General Description Data, and 9.8, Required Heating System Details Sub-Form), you may request NEAT evaluate installation of a heat pump. NEAT will evaluate replacing the heating system and all cooling equipment with a single heat pump and recommend this if costeffective and more so than replacing each individual air conditioner and leaving the heating system unaltered. If no existing cooling equipment has been specified, NEAT will include only heating savings in its estimates of the total savings and SIR for the replacement, as well as for other measures being evaluated.

For NEAT to evaluate replacing an existing heat pump, the primary heating system and at least one of the cooling systems should have been described as heat pumps. Any existing cooling equipment, regardless of its type (see Section 9.9, Cooling), will be replaced by the new heat pump.

The heating efficiency (HSPF) of the replacement system is designated in the Replacement System data block on the Required Heating System Details sub-form. The cooling efficiency (SEER) of the replacement heat pump is specified in the Setup Library (see "Equipment” in Section 14.2, Key Parameters). The costs for the heat pump installation are determined from the user-supplied costs for three sizes of heat pumps supplied in the Material Cost screens of Setup (see Section 14.5, Library Measures).

\section{A.23 Seal Ducts}

NEAT's Seal Ducts measure estimates the heating savings from sealing ducts of a forced air furnace or heat pump. Cooling savings will also be determined if the 
cooling system is a central air conditioner. The measure has no entry on NEAT's Library Measures form of the Setup Library. It is activated by the user selecting the Evaluate Duct Sealing checkbox on the audit's Ducts/Infiltration form (see Section 11.2, Ducts/Infiltration - Air and Duct Leakages). Costs associated with the work are also entered on this form.

NEAT translates your pre- and post-retrofit input data from either of the three duct leakage measurement techniques (whole house blower door, blower door subtraction, or duct blower) into parameters accepted by the ASHRAE 152P "Method of Test for Determining the Design Seasonal Efficiencies of Residential Thermal Distribution Systems.” The program applies the resulting pre- and postretrofit efficiencies to the heating and cooling loads to estimate the savings of the duct sealing efforts.

\section{A.24 Lighting Retrofits}

Replacing existing incandescent interior lighting with compact fluorescent lights is a relatively straight forward way to reduce the electric consumption of a home. The development of the compact fluorescent bulb has made this retrofit an extremely simple one to accomplish because most compact fluorescent lights are fitted with screw-in bases identical to the incandescent bulbs, which allow a quick and easy one-for-one replacement.

NEAT computes the savings of using the lower wattage compact fluorescent bulb to replace an existing incandescent bulb. The watt reduction is determined from the user's input of existing and replacement bulb consumption, though default values for replacement wattage give the standard replacement for the existing bulb (see Section 11.6, Base Loads - Lighting Systems). NEAT also accounts for the longer life of the fluorescent bulb over that of the incandescent lamp.

You must enter the cost for various wattage compact fluorescent bulbs under the "Costs" button for the Lighting Retrofits measure on the Library Measures form of the Setup Library (see Section 14.5, Library Measures). NEAT will use these costs to interpolate or extrapolate to obtain a cost for a wattage not specified in this listing. Therefore, your costs should reflect a reasonable pattern with respect to wattages for the entire range of entries. 


\section{A.25 Refrigerator Replacement}

On an average, refrigerators use nine percent of a home's total energy consumption. They can consume as much as $2000 \mathrm{kWh} /$ year to as little as 400 $\mathrm{kWh} /$ year - a factor of five! Significant energy efficiency improvements have been made in refrigerator construction. Units manufactured prior to 1990 will most often use substantially more energy than today's units. NEAT evaluates the costeffectiveness of replacing an older unit with a more energy efficient model.

The savings associated with refrigerator replacement is computed from the difference in the annual consumptions of the existing and replacement units. Several methods of data collection can be used to determine this information (see Section 11.5, Base Loads - Refrigerators). Depending on the method used, NEAT makes adjustments for the location of the unit, its age, existence of defrost cycles, the condition of the door seals, and door openings (based on occupancy). The characteristics for the replacement refrigerator can either be entered on the Refrigerators form or copied from a listing of replacements in your Supply Library (see "Additional Entries on the Hot Water Equipment and Refrigerator Forms" in Section 15.2, Materials/Labor Forms).

\section{A.26 Water Heater Tank Insulation}

Older water heaters will likely have less insulation surrounding the hot water tank than is economically justified. NEAT evaluates installing a water heater wrap with R-value specified in the Setup Library (see "Insulation” under "Site Built (NEAT) Key Parameters" in Section 14.2, Key Parameters). Greater dollar savings will occur from this measure when applied to electric units in unconditioned spaces. Common practice is to also insulate the tops of electric units, but not gas or propane due to potential fire hazards associated with the flue or interference with the draft diverter. The measure uses information you provide on the Water Heating form (see Section 11.4, Base Loads - Water Heating).

Due to relatively low cost of the measure, it will often be found cost-effective.

\section{A.27 Water Heater Pipe Insulation}

NEAT evaluates the savings from insulating the first five feet of both the cold and hot water pipes entering and exiting a water heater. This not only reduces direct 


\section{Appendix A: NEAT Weatherization Measures}

conductive heat loss from the hot water pipe, but also loss from convection of the water in both the hot and cold pipes. The insulation is assumed to be R-1.85, $1 / 2$ in. elastomer foam rubber. The savings estimates are based on first principle heat transfer calculations.

Due to the measures relatively low cost, it will often be cost-effective.

\section{A.28 Low-Flow Showerheads}

Low-flow showerheads are inexpensive, easily installed, energy efficiency measures. NEAT uses your input of the approximate minutes of shower use per day and the gallons per minute discharged from existing showerheads to estimate an energy savings associated with installing low-flow heads. Replacement showerheads are assumed to discharge 2.5 gallons per minute, unless changed by the user in the Setup Library (see "Equipment” under "Site Built (NEAT) Key Parameters" in Section 14.2, Key Parameters). The temperature difference of water discharged from the replacement showerheads is assumed to be four degrees Fahrenheit greater than water from the existing showerheads. This is to compensate for the lower output from the replacement heads.

Due to their relative low cost, low-flow showerheads will often be recommended as long as they are not already installed and there is at least average shower use.

\section{A.29 Water Heater Replacement}

NEAT will evaluate the cost-effectiveness of replacing an existing water heater with a higher efficiency model. NEAT allows you to evaluate the effectiveness of fuel switching. However, you must be sure that all costs associated with the switch are included in the Water Heater Replacement measure cost and allowed in your program.

NEAT will also evaluate replacing the existing water heater with a heat pump water heater and, if the unit is located in a heated space, model the effect the heat pump water heater has on the heating and cooling consumption in the home. For a heat pump water heater, use a value of EF that represents its efficiency (e.g., 2.2). An RE of 0.98 is normally appropriate. 


\section{Appendix A: NEAT Weatherization Measures}

The derivation of the savings for this measure requires the water heater characteristics of energy factor, recovery efficiency, and input rating, available for many models from the GAMA database referenced by NEAT. Estimates of daily hot water consumption are based on the number of occupants you indicate on the Client Information form (see Section 7.1, Client Information).

\section{A.30 Infiltration Reduction}

NEAT's Infiltration Reduction measure uses pre- and post-retrofit ("Before Weatherization" and "After Weatherization") blower door readings entered on the Ducts/Infiltration form (see Section 11.2, Ducts/Infiltration - Air and Duct Leakages) to estimate the heating and cooling (sensible and latent) energy savings from your air sealing efforts. NEAT translates these leakage rates you enter at the pressure differential specified into infiltration rates under natural conditions, taking into account wind speeds and outdoor temperatures recorded in the weather file for the location of the home as well as the height of the home, as determined from the Conditioned Stories (see Section 8.2, Audit Information). If the recommended measures produced by running an audit (see Section 12.2, Recommended Measures Report) include window and door measures that would reduce the infiltration through these envelop components, NEAT subtracts this infiltration reduction from the infiltration reduction recorded for this measure so as to not count its effect twice.

The measure has no entry on NEAT's Library Measures form of the Setup Library (see Section 14.5, Library Measures). It is activated by the user's input on the Ducts/Infiltration form. If only a post-retrofit blower door reading is entered on the form, no Infiltration Reduction measure will appear on the Recommended Measures report. If only pre- and post-retrofit blower door readings are entered, but no cost, NEAT's recommendations will include an estimate of the energy saved by the measure, but no "Measure Economics" (e.g., savings-to-investment ratio [SIR]). If both blower door readings and a cost for the measure are entered on the form, the Infiltration Reduction measure displayed in the report will have a complete set of results, similar to all other measures recommended. 


\section{Appendix B. MHEA Weatherization Measures}

The list of weatherization measures considered by MHEA can be accessed in the Library Measures form under the Main Menu's “Setup Library” button (see Section 14.5, Library Measures). MHEA currently examines 25 weatherization measure types:

1. Seal ducts

2. General air sealing

3. Wall fiberglass batt insulation

4. Wall cellulose or fiberglass loose-fill insulation

5. Floor cellulose or fiberglass loose-fill insulation

6. Roof cellulose or fiberglass loose-fill insulation

7. Add skirting

8. White coat roof

9. Replace wooden/marked doors (standard and mandatory)

10. Storm doors

11. Window sealing

12. Replace single-paned windows

13. Plastic/glass storm windows

14. Add awnings or shade screens)

15. Setback thermostat

16. Replace heating system

17. Tune heating system

18. Evaporative cooling

19. Tune cooling system

20. Replace DX cooling equipment

21. Lighting retrofits

22. Refrigerator replacement

23. Water heater tank insulation

24. Water heater pipe insulation

25. Low-flow showerheads

26. Water heater replacement

The first eight weatherization measure types are designed to reduce heat loss or gain through walls, floors, ceilings, and ducts. Measure types 9 through 13 affect the way windows and doors behave in the house. They can alter the conduction heat loss through windows and doors, and the solar gain and infiltration through 


\section{Appendix B: MHEA Weatherization Measures}

windows. Window shading reduces solar gain - a major element of total heat gain. Shading the windows may affect the heating load, because shade blocks some solar heat from the house during the heating season. Measures types 14 through 16 improve heating system efficiency by decreasing heating energy consumed by the house. They do not affect heat loss through the envelope, however. Measure types 17 through 19 improve cooling efficiency during the cooling season. Evaporative coolers and new air conditioners can reduce cooling costs markedly, if properly installed. Measure types 20 through 25 reduce the energy consumption not directly related to the climate. Such measures are referred to as "base load" measures. They can have minor effects on the heating and cooling costs.

All of the envelope measure types (3-13) have separate entries in the Library Measures form of the Setup Library for their application to the manufactured home proper versus any addition to the home. They will also be listed separately in any reports involving the measures.

\section{B.1 Seal Ducts}

MHEA's Seal Ducts measure estimates the heating savings from sealing ducts of a forced air furnace or heat pump. In addition, the entry in the "Duct Location" dropdown list cannot be "None." Cooling savings will also be determined if the cooling system is a central air conditioner or heat pump. For the measure to be evaluated, it must be indicated as being active in the MHEA's Library Measures form of the Setup Library (see Section 14.5, Library Measures) as well as be activated for a specific audit by selecting the Evaluate Duct Sealing checkbox on the audit's Ducts/Infiltration form (see Section 11.2, Ducts/Infiltration - Air and Duct Leakages). Costs associated with the work are also entered on this form.

MHEA translates your pre- and post-retrofit input data from either of the four duct leakage measurement techniques (whole house blower door, blower door subtraction, duct blower, or pressure pan) into parameters accepted by the ASHRAE 152P "Method of Test for Determining the Design Seasonal Efficiencies of Residential Thermal Distribution Systems.” The program applies the resulting pre- and post-retrofit efficiencies to the heating and cooling loads to estimate the savings of the duct sealing efforts. 


\section{B.2 General Air Sealing}

MHEA's General Air Sealing measure uses pre- and post-retrofit ("Before Weatherization" and "After Weatherization") blower door readings entered on the Ducts/Infiltration form (see Section 11.2, Ducts/Infiltration - Air and Duct Leakages) to estimate the heating and cooling (sensible and latent) energy savings from your air sealing efforts. MHEA translates these leakage rates at the pressure differential specified into infiltration rates under natural conditions, taking into account wind speeds, modified by the choice of Wind Shielding from the MHEA Audit Information form, and the outdoor temperatures recorded in the weather file for the location of the home. If the recommended measures produced by running an audit (see Section 12.2, Recommended Measures Report) include window measures that would reduce the infiltration through these envelop components, NEAT subtracts this infiltration reduction from the infiltration reduction recorded for this measure so as to not count its effect twice.

For MHEA to evaluate the Infiltration Reduction measure, the measure must be activated in the Setup Library (see Section 14.5, Library Measures) and the postretrofit blower door reading and the Infiltration Reduction Cost entered on the Ducts/Infiltration form. If the user has not entered a pre-retrofit leakage rate on the form, MHEA will use the leakage rates assigned in the "Home Leakiness" dropdown list on the Audit Information form (see "MHEA Specific Entries" in Section 8.2, Audit Information, and "Mobil Home (MHEA) Key Parameters" in Section 14.2, Key Parameters).

\section{B.3 Wall Fiberglass Batt Insulation}

This measure adds 3-1/2 inches of fiberglass batt insulation to the walls. It will be evaluated if the available space in the walls is 1 inch or greater. If the specified Wall Stud Size and depths of Existing Insulation (see Section 10.1, Shell - Walls) indicate that compression of the batt will occur, the effect of this compression on the R-value of added insulation is taken into account. If the wall had been declared "Vented" on the Walls form of the audit, this measure will change this characteristic to "Not Vented," increasing the effectiveness of any existing batt insulation. A 15\% framing factor is assumed and any wall area indicated as "Uninsulatable” will also be excluded. 


\section{Appendix B: MHEA Weatherization Measures}

\section{B.4 Wall Cellulose or Fiberglass Loose-Fill Insulation}

These measures add either cellulose or fiberglass loose-fill insulation to walls. They will be evaluated if the available space in the walls is 1 inch or greater. The quantity of insulation required for the measures accounts for the compression of any existing batt insulation in the cavity, as determined by the density of the added loose-fill insulation, specified in the Key Parameters (see "Mobile Home (MHEA) Key Parameters" in Section 14.2, Key Parameters). If the wall had been declared "Vented" on the Walls form of the audit (see Section 10.1, Shell - Walls), this measure will change this characteristic to "Not Vented," increasing the effectiveness of any existing batt insulation. A 15\% framing factor is assumed and any wall area indicated as "Uninsulatable" will also be excluded.

\section{B.5 Floor Cellulose or Fiberglass Loose-Fill Insulation}

These measures fill the available space in the wing sections with cellulose or fiberglass loose-fill insulation and add up to eight inches of cellulose or fiberglass loose-fill insulation to the belly, space permitting. The quantity of insulation required for the measures accounts for the compression of any existing batt insulation in the floor, as determined by the density of the added loose-fill insulation, specified in the Key Parameters (see "Mobile Home (MHEA) Key Parameters" in Section 14.2, Key Parameters). The wing and center sections are each assumed to be half the total area of the entire belly. The measures will not be evaluated if the air space in the center section is less than two inches. A $10 \%$ framing factor is assumed and the volume of ducts is also excluded, if the user's input indicates their presence in the belly.

\section{B.6 Roof Cellulose or Fiberglass Loose-Fill Insulation}

These measures fill the available space in the roof with cellulose or fiberglass loose-fill insulation. The quantity of insulation required for the measures accounts for the compression of any existing batt insulation in flat or bowstring roof types, as determined by the density of the added loose-fill insulation, specified in the Key Parameters (see "Mobile Home (MHEA) Key Parameters" in Section 14.2, Key Parameters). The quantity of insulation required for the measures also depends on the roof type and dimensions of the home. A 10\% framing factor is assumed and the volume of ducts is also excluded, if the user's input indicates their presence in the ceiling. 


\section{Appendix B: MHEA Weatherization Measures}

\section{B.7 Add Skirting}

This measure installs exterior skirting around the home. It is evaluated only if you have not indicated an existing skirt on MHEA's Floor form and the belly insulation measures have been turned off (see Section 14.5, Library Measures). The skirt is not assumed to be insulated, but must be capable of blocking the wind. Its effect is to increase the film resistance between the air and the belly to represent still air.

\section{B.8 White Coat Roof}

An elastomeric, low-emissivity roof-coating retrofit measure is applied only to homes with either a bowstring or flat roof, whose Roof Color is "Normal or Weathered," and having an existing cooling system. The benefit is to decrease the solar load on the roof, which in turn decreases the home cooling load. This retrofit measure is commonly installed in cooling climates.

\section{B.9 Replace Wooden/Marked Doors (Optional and Mandatory)}

- Standard - This retrofit measure is evaluated for all door types other than "Steel Insulated." The benefit assigned to this measure is to increase the Rvalue through the door. The R-values corresponding to the existing door types and the replacement door are specified in the Setup Library (see "Mobile Home (MHEA) Key Parameters” in Section 14.2, Key Parameters).

- Mandatory - This measure forces the door replacement for the specified door description to be recommended, whether or not it proves to be cost-effective. If it is not cost-effective, the replacement would normally have to be viewed as a repair item. Alternative approaches are to include the door replacement as part of the infiltration reduction or to list it as a repair item on the Itemized Cost form (see Section 11.8, Itemized Costs).

\section{B.10 Storm Doors}

This retrofit measure is evaluated for doors not having an existing storm door. The effect of installing a storm door is to add $1 \mathrm{R}$-value (h- $\mathrm{ft}^{2}$-F/Btu) to the storm door/ home door combination. 


\section{Appendix B: MHEA Weatherization Measures}

\section{B.11 Window Sealing}

MHEA estimates the savings that can be obtained from weatherizing an existing window to reduce its leakiness. Both heating and cooling (latent and sensible) energy is considered in this estimate. The amount of energy saved depends on your declaration of each window's pre-retrofit leakiness, as indicated on the Windows input form (see Section 10.2, Shell - Windows) as well as the overall house leakiness described on the Ducts/Infiltration form (see Section 11.2, Ducts/ Infiltration).

MHEA translates your leakiness designation into an estimate of air leakage through the window that results primarily from a pressure differential created by wind. It then uses empirically-based relations to reduce this air leakage through the window to reflect caulking, weather-stripping, and general repairs to the window. The energy saved from this reduction is computed in much the same way savings from whole house infiltration reduction is determined.

If MHEA's General Air Sealing measure is being evaluated, the program will subtract from the savings of this measure any savings resulting from the infiltration reduction included as savings for the Window Sealing measure, so as to avoid accounting for the window sealing twice.

The Window Sealing measure is one of four mutually exclusive window measures, along with Plastic/Glass Storm Windows and Replace Single-Paned Windows. Only the measure found most cost-effective from a set of mutually exclusive measures will be recommended. Thus, MHEA would never recommend both sealing and replacing the same window. The only exception to the costeffectiveness criteria is when a specific measure has been declared mandatory, as might be the case when replacing a window whose frame is so rotted that weatherization or use of a storm window is not practical. Check your program's policy regarding mandating measures. How the measure is viewed depends on the selection you make for Retrofit Option on the Windows form during data input.

\section{B.12 Replace Singe-Paned Windows}

MHEA replaces the existing window with one having the U-value and solar heat gain coefficient (SHGC) specified in the Key Parameters of the Setup Library (see "Mobile Home (MHEA) Key Parameters" in Section 14.2, Key Parameters). The 


\section{Appendix B: MHEA Weatherization Measures}

measure is not applied to sliding glass doors, door windows, or skylights. An existing double pane window will be replaced only if the user indicates that this measure is mandatory.

The energy savings estimated when replacing a window considers conduction, solar, and infiltration effects. The infiltration savings will depend on your indication of the existing window's initial leakiness (see the discussion of the Window Sealing measure above for more information). Any such savings will be subtracted from the savings for the General Air Sealing measure so as to avoid accounting for this infiltration savings twice.

The Replace Single-Paned Window measure is mutually exclusive with other window retrofit measures. See the discussion of the Window Sealing measure above for the implications of mutual exclusiveness.

\section{B.13 Plastic/Glass Storm Windows}

This retrofit measure is evaluated for windows not already having a storm window. No storms will be evaluated for sliding glass doors. The U-value of the storm window/window combination is taken from the values specified in the Key Parameters of the Setup Library (see "Mobile Home (MHEA) Key Parameters" in Section 14.2, Key Parameters). MHEA assumes that the plastic or glass storm window is installed on the interior of the window assembly and is used only during the heating-dominated months. The plastic storm may either be purchased or can be fabricated using an acrylic window film/sheet cut to the size of the window and held in place with magnetic strips or Velcro ${ }^{\mathrm{TM}}$.

\section{B.14 Add Awnings or Shade Screens}

The two window shading measures (Add Awnings and Add Shade Screens) apply exterior awnings or fabric mesh window sun screens to all windows not facing north and to those not having any existing Exterior Shading, as indicated by the user on MHEA's Windows form. The two measures are mutually exclusive; that is, only one of the two will ever be recommended, if either. The measures increase the shade on the windows, which decreases the solar load. 


\section{Appendix B: MHEA Weatherization Measures}

\section{B.15 Setback Thermostat}

Automatic set-back thermostats can be cost-effective for people who have regular schedules and who understand how to set the thermostats to follow those schedules. MHEA evaluates savings accomplished by setting back the set-point temperature during the heating season only. Therefore, the measure will not show cost-effectiveness in cooling climates. You may set the number of degrees Fahrenheit of the nightly setback in the Key Parameters of the Setup Library (see “Mobile Home (MHEA) Key Parameters" in Section 14.2, Key Parameters). Unless altered, MHEA will assume a three degree Fahrenheit setback for eight hours.

\section{B.16 Replace Heating System}

The cost-effectiveness of replacing a heating system heavily depends on the Efficiency of the existing system, entered on the Primary sub-form under the Heating tab. If a heating system is to be replaced solely based on the savings of the measure, it is strongly recommended that this entry be obtained from an accepted efficiency measurement, as opposed to data from the name plate of the unit or a data base of heating system efficiencies.

In order for the measure to be evaluated, you must fill in the required data on both the Primary and Replacement sub-forms under MHEA's Heating tab. The replacement will not eliminate any secondary system you may have identified or change the percentage of heat supplied by the primary and secondary systems. MHEA will evaluate the cost-effectiveness of replacing a heating system that uses one fuel with one consuming a different fuel. However, it is left to the user to determine the appropriateness of such a replacement in his/her program. Depending on the characteristics of the two fuels, evaluation of the savings over the life of the replacement system can produce unexpected, yet valid results. The measure is mutually exclusive with the Tune Heating System measure; that is, only one of the two will ever be recommended, if either.

MHEA Versions 8 and earlier treat the heating and cooling sides of a heat pump as separate components requiring descriptions under both the Heating and Cooling tabs. For correct modeling of replacing the existing primary heating and cooling systems with a heat pump, the replacement for both the heating and cooling sides of the existing units must be specified as heat pumps and the total cost of the 
replacement split between the Heating Replacement and Cooling Replacement descriptions. To have the replacement seen as cost-effective, this split in cost should be adjusted in order to show an SIR for replacement of both the heating and cooling components as cost-effective.

\section{B.17 Tune Heating System}

Heating systems may function far below their potential efficiency or may be unsafe due to the following common problems:

- Dirt, soot, or other materials interfering with the burner flame;

- Dirt, soot, or corrosion on heat exchanger surfaces;

- Electric controls malfunctioning or out of adjustment;

- Air hotter than necessary to heat the house;

- Incomplete combustion;

- Faulty draft;

- Incorrect fuel input;

- Blockages or leaks in heating distribution system; or

- Faulty thermostatic setting, anticipator adjustment, or location.

Heating technicians performing tune-ups should have specific training on increasing the efficiency of heating systems. Technicians should have test equipment to measure efficiency, air temperature, carbon monoxide, draft, and all other parameters relevant to the seasonal efficiency of each heating system.

MHEA will evaluate the Tune Heating System measure only for furnaces that are not electric or fueled by wood or the "Other" fuel type. MHEA assumes that if the measure is performed by a qualified technician who addresses the issues listed above, it will save from 0 to 13 percent of the fuel used for heating, depending on the Equipment Type, Fuel, and Efficiency, as indicated by the user's entries on MHEA's Primary sub-form under the Heating tab. The measure is mutually exclusive with the Heating System Replacement measure; that is, only one of the two will ever be recommended, if either.

\section{B.18 Evaporative Cooling}

In the warm, dry climates of the western United States, evaporative coolers (also called swamp coolers) are a popular and energy efficient cooling device. The lower 


\section{Appendix B: MHEA Weatherization Measures}

the summertime relative humidity, the more the evaporative cooler will drop the indoor temperature. Evaporative coolers use one-quarter to one-sixth of the energy of air conditioners and they cost about one-half as much to install. Unlike central air conditioners, evaporative coolers provide a steady stream of fresh, humid air to the house.

MHEA will evaluate replacing any existing DX (direct expansion) cooling system with an evaporative cooler if the existing cooling equipment is a central system or a room air conditioner with a COP of less than 2.5. The measure will replace either or both the primary or secondary systems if they meet the replacement criteria.

\section{B.19 Tune Cooling System}

Significant efficiency increases for an air conditioner may be possible through proper maintenance and periodic tune-ups. The following steps are suggested in performing the tune-up of an existing air conditioner:

- Replace or clean existing air filters and grills,

- Check and clean condensate trough and drain,

- Clean evaporator (indoor) and condenser (outside) coils,

- Straighten bent or flattened coil fins if necessary,

- Ensure unobstructed air flow to the condenser coil,

- Check for proper refrigerant charge,

- Remove dust and dirt from fan blades,

- Examine and oil motor and fan bearings,

- Inspect and/or tighten electrical connections and contacts,

- Check for blockages or leaks in the supply and return ducts.

Properly trained HVAC technicians should perform the tune-up insuring the use of proper equipment, especially in checking the refrigerant charge and the electrical wiring, and straightening the coil fins.

MHEA will evaluate the Tune Cooling System measure for any Primary cooling system except an evaporative cooler. Tune-ups of Secondary systems are not considered. MHEA assumes that a thorough air conditioner tune-up, addressing the issues listed above, will increase the system's COP to 3.0. The measure will not be evaluated for systems with existing efficiency above this. 


\section{Appendix B: MHEA Weatherization Measures}

\section{B.20 Replace DX Cooling Equipment}

Air conditioners move heat from indoors to outdoors with a cooling coil (the evaporator), a heating coil (the condenser), and a compressor, which moves a heat transfer fluid (the refrigerant) between the two coils. This type of cooling is sometimes referred to as "DX cooling," where the "DX" stands for "direct expansion." It is the expansion of the refrigerant in the evaporator coil that actually produces the cooling. The federal government requires all air conditioners to carry a yellow energy label listing its annual cost of operation and a measure if its efficiency, EER for room air conditioners and SEER for central systems. A room air conditioner with an EER of 12 will use half as much electricity for cooling as one with an EER of 6. See Section "Efficiency and Efficiency Units" in Section 10.8, Cooling - Primary, Secondary, and Replacement, for further discussion of the various units of cooling efficiency.

MHEA considers replacing the Primary cooling unit with the unit described under the Replacement tab. If a Secondary cooling unit has been described, the replacement measure does not affect the percent of floor area cooled by the two systems. MHEA will not replace an air-conditioner whose adjusted (for local weather) COP is greater than or equal to 2.5. All equipment types may be designated as being replaced with any other equipment type except the evaporative cooler. If an existing evaporative cooler is mandated to be replaced by a DX unit, it will be recommended but with negative savings and SIR. It is left to the user to determine the appropriateness of the replacements in his/her program.

MHEA Versions 8 and earlier treat the heating and cooling sides of a heat pump as separate components requiring descriptions under both the Heating and Cooling tabs. For correct modeling of replacing the existing primary heating and cooling systems with a heat pump, the replacement for both the heating and cooling sides of the existing units must be specified as heat pumps and the total cost of the replacement split between the Heating Replacement and Cooling Replacement descriptions. To have the replacement seen as cost-effective, this split in cost should be adjusted in order to show an SIR for replacement of both the heating and cooling components as cost-effective. 


\section{Appendix B: MHEA Weatherization Measures}

\section{B.21 Lighting Retrofits}

Replacing existing incandescent interior lighting with compact fluorescent lights is a relatively straight forward way to reduce the electric consumption of a home. The development of the compact fluorescent bulb has made this retrofit an extremely simple one to accomplish because most compact fluorescent lights are fitted with screw-in bases identical to the incandescent bulbs, which allow a quick and easy one-for-one replacement.

MHEA computes the savings of using the lower wattage compact fluorescent bulb to replace an existing incandescent bulb. The watt reduction is determined from the user's input of existing and replacement bulb consumption, though default values for replacement wattage give the standard replacement for the existing bulb (see Section 11.6, Base Loads - Lighting Systems). MHEA also accounts for the longer life of the fluorescent bulb over that of the incandescent lamp.

You must enter the cost for various wattage compact fluorescent bulbs under the "Costs" button for the Lighting Retrofits measure on the Library Measures form of the Setup Library (see Section 14.5, Library Measures). MHEA will use these costs to interpolate or extrapolate to obtain a cost for a wattage not specified in this listing. Therefore, your costs should reflect a reasonable pattern with respect to wattages for the entire range of entries

\section{B.22 Refrigerator Replacement}

On an average, refrigerators use nine percent of a home's total energy consumption. They can consume as much as $2000 \mathrm{kWh} /$ year to as little as 400 $\mathrm{kWh} /$ year - a factor of five! Significant energy efficiency improvements have been made in refrigerator construction. Units manufactured prior to 1990 will most often use substantially more energy than today's units. MHEA evaluates the costeffectiveness of replacing an older unit with a more energy efficient model.

The savings associated with refrigerator replacement is computed from the difference in the annual consumptions of the existing and replacement units. Several methods of data collection can be used to determine this information (see Section 11.5, Base Loads - Refrigerators). Depending on the method used, MHEA makes adjustments for the location of the unit, its age, existence of defrost cycles, the condition of the door seals, and door openings (based on occupancy). The 
characteristics for the replacement refrigerator can either be entered on the Refrigerators form or copied from a listing of replacements in your Supply Library (see "Additional Entries on the Hot Water Equipment and Refrigerator Forms" in Section 15.2, Materials/Labor Forms).

\section{B.23 Water Heater Tank Insulation}

Older water heaters will likely have less insulation surrounding the hot water tank than is economically justified. MHEA evaluates installing a water heater wrap with R-value specified in the Setup Library (see "Base Loads" under "Mobile Home (MHEA) Key Parameters” in Section 14.2, Key Parameters). Greater dollar savings will occur from this measure when applied to electric units in unconditioned spaces. Common practice is to also insulate the tops of electric units, but not gas or propane due to potential fire hazards associated with the flue or interference with the draft diverter. The measure uses information you provide on the Water Heating form (see Section 11.4, Base Loads - Water Heating).

Due to relatively low cost of the measure, it will often be found cost-effective.

\section{B.24 Water Heater Pipe Insulation}

MHEA evaluates the savings from insulating the first five feet of both the cold and hot water pipes entering and exiting a water heater. This not only reduces direct conductive heat loss from the hot water pipe, but also loss from convection of the water in both the hot and cold pipes. The insulation is assumed to be R-1.85, $1 / 2 \mathrm{in}$. elastomer foam rubber. The savings estimates are based on first principle heat transfer calculations.

Due to the measures relatively low cost, it will often be cost-effective

\section{B.25 Low-Flow Showerheads}

Low-flow showerheads are inexpensive, easily installed, energy efficiency measures. MHEA uses your input of the approximate minutes of shower use per day and the gallons per minute discharged from existing showerheads to estimate an energy savings associated with installing low-flow heads. Replacement showerheads are assumed to discharge 2.5 gallons per minute, unless changed by the user in the Setup Library (see "Base Loads" under "Mobile Home (MHEA) 


\section{Appendix B: MHEA Weatherization Measures}

Key Parameters" in Section 14.2, Key Parameters). The temperature difference of water discharged from the replacement showerheads is assumed to be four degrees Fahrenheit greater than water from the existing showerheads. This is to compensate for the lower output from the replacement heads.

Due to their relative low cost, low-flow showerheads will often be recommended as long as they are not already installed and there is at least average shower use.

\section{B.26 Water Heater Replacement}

MHEA will evaluate the cost-effectiveness of replacing an existing water heater with a higher efficiency model. MHEA allows you to evaluate the effectiveness of fuel switching. However, you must be sure that all costs associated with the switch are included in the Water Heater Replacement measure cost and allowed in your program.

MHEA will also evaluate replacing the existing water heater with a heat pump water heater. However, MHEA does not model the effect the heat pump water heater has on the heating and cooling consumption in the home when it is located in a heated space. For a heat pump water heater, use a value of EF that represents its efficiency (e.g., 2.2). An RE of 0.98 is normally appropriate.

The derivation of the savings for this measure requires the water heater characteristics of energy factor, recovery efficiency, and input rating, available for many models from the GAMA database referenced by MHEA. Estimates of daily hot water consumption are based on the number of occupants you indicate on the Client Information form (see Section 7.1, Client Information). 


\section{Appendix C. Reports}

This appendix displays samples of reports available in the Weatherization Assistant, Version 8. They are accessed from the Report blocks at the lower right of the General Information tabs for each of the Main Menu windows: Agency, Client, Audit (NEAT and MHEA), and Work Orders. A listing of these reports is given below.

\section{Agency Reports}

Quarterly Program Report (Unit Production) ................................................... C-3

Scheduled Audits (NEAT or MHEA) ……................................................... C-4

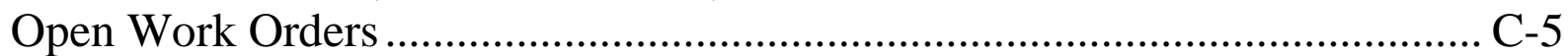

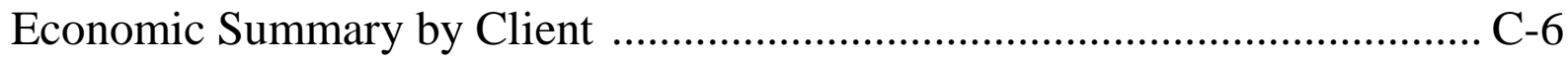

Client Surveys (Blank)......................................................................... C-10

\section{Client Reports}

Client Completion Report .............................................................................. C-11

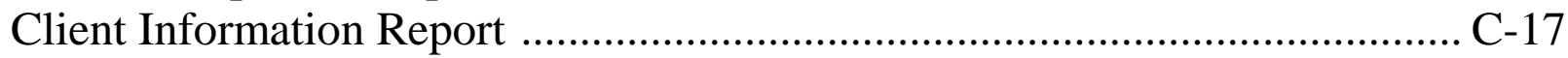

Client Information Form ..................................................................... C-18

NEAT Data Collection Form (Blank)............................................................... C-19

MHEA Data Collection Form (Blank) ....................................................... C-53

Client Surveys ….......................................................................................... C-92

\section{NEAT Audit Reports}

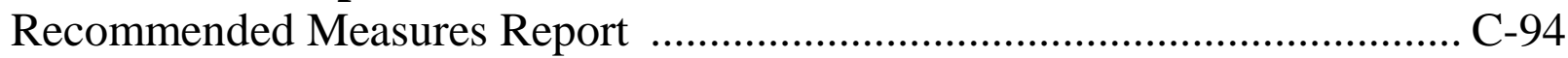

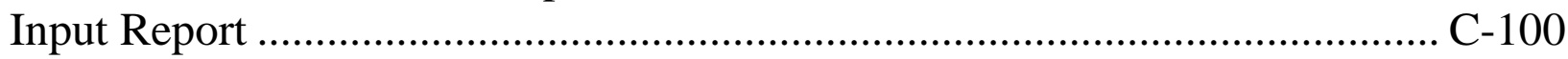

Heating System Summary …..................................................................... C-117

Pressure Diagnostics Report/Form ........................................................... C-120

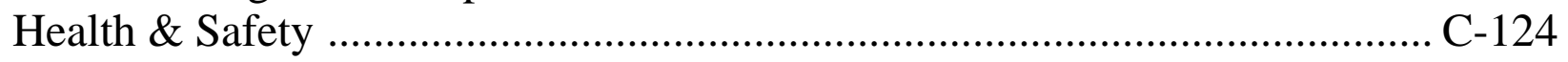

\section{MHEA Audit Reports}

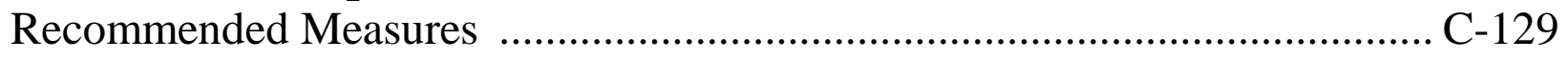

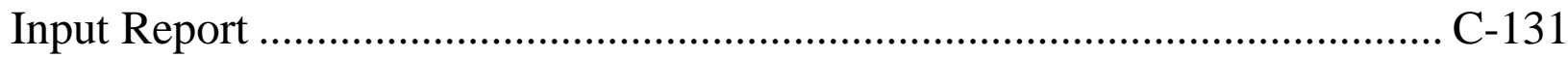

Heating System Summary …............................................................. C-148

Pressure Diagnostics Report/Form .............................................................. C-152

Health \& Safety ..................................................................................... C-156 


\section{Work Order Reports}

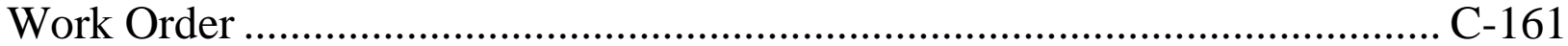

Work Order (Bid Form) …............................................................................... C-169 


\section{Quarterly Program Report}

Report Period: Begin 9/1/2011 to End 10/1/2011

Assistance

Assistance

Program

Agency Name: Demonstration Agency

1. Units By Dwelling Type

Owner-Occupied Single Family Site Built

Renter-Occupled Single Family Site Built

Multi-Family (5 or more units per bullding)

Owner-Occupied Mobile Home

Renter-Occupied Mobile Home

Shelter

Other

UNCATEGOAIZED

\begin{tabular}{|l|}
\hline 1 \\
\hline 0 \\
\hline 0 \\
\hline 0 \\
\hline 0 \\
\hline 0 \\
\hline 0 \\
\hline 1 \\
\hline
\end{tabular}

TOTAL DOE Units:

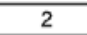

2: Units By Primary Heating Fuel Type

Natural Gas
Fuel Oil
Electricity
Propane/LPG
Kerosene
Wood
Other
UNCATEGORIZED

\begin{tabular}{|l|}
\hline 1 \\
\hline 0 \\
\hline 0 \\
\hline 0 \\
\hline 0 \\
\hline 0 \\
\hline 0 \\
\hline 1 \\
\hline
\end{tabular}

3: Units By Occupancy

Elderly-Occupied

Native American-Occupied

Children-Occupied

High Energy Use

High Energy Cost Burden

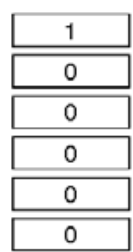

4. Other Unit Categories

ReWeatherized

Low Cost / No Cost

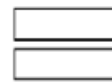

TOTAL Other Units:

5. Total People Assisted with Grant Funds

Elderly

Persons with Disabilities

Native American

Children

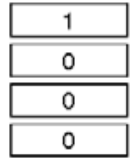

Agency Name: 


\section{Appendix C: Reports}

\section{Scheduled Audits (NEAT)}

Report Period: Begin 10/1/2011 to End 11/1/2012

Assistance

Program

Agency Name: Demonstration Agency

Office Phone: (123) 456-7890

Address: 725 Jefferson St.

Office Email: agencyemail@localisp.net

Any City, US 11111

\begin{tabular}{llll}
$\begin{array}{l}\text { Audit Name } \\
\begin{array}{l}\text { Auditor } \\
\text { Dwelling Type }\end{array}\end{array}$ & $\begin{array}{l}\text { Client Name } \\
\text { Client ID } \\
\text { Alt. Client ID }\end{array}$ & Client Address & $\begin{array}{l}\text { Audit Status } \\
\text { Audit Status Date } \\
\text { Comments }\end{array}$ \\
\hline $11 \_354$ SB & $\begin{array}{l}\text { Stokes, Randy } \\
11 \_354\end{array}$ & $\begin{array}{l}250 \text { Robertsville Rd. } \\
\text { Oak Ridge, TN } 37830\end{array}$ & $\begin{array}{l}\text { Site Visit Scheduled For } \\
10 / 12 / 2011\end{array}$ \\
Site Built & & & \\
\hline $11 \_355 S B$ & Lassiter, Francis & 317 Louisiana Ave. & Site Visit Scheduled For \\
Tor, Audrey & $11 \_355$ & Oak Ridge, TN 37830 & $10 / 14 / 2011$
\end{tabular}

Site Built 


\section{Open Work Orders}

Assistance

Program

Agency Name: Demonstration Agency

Office Phone: (123) 456-7890

Address: 725 Jefferson St.

Office Email: agencyemail@localisp.net

Any City, US 11111

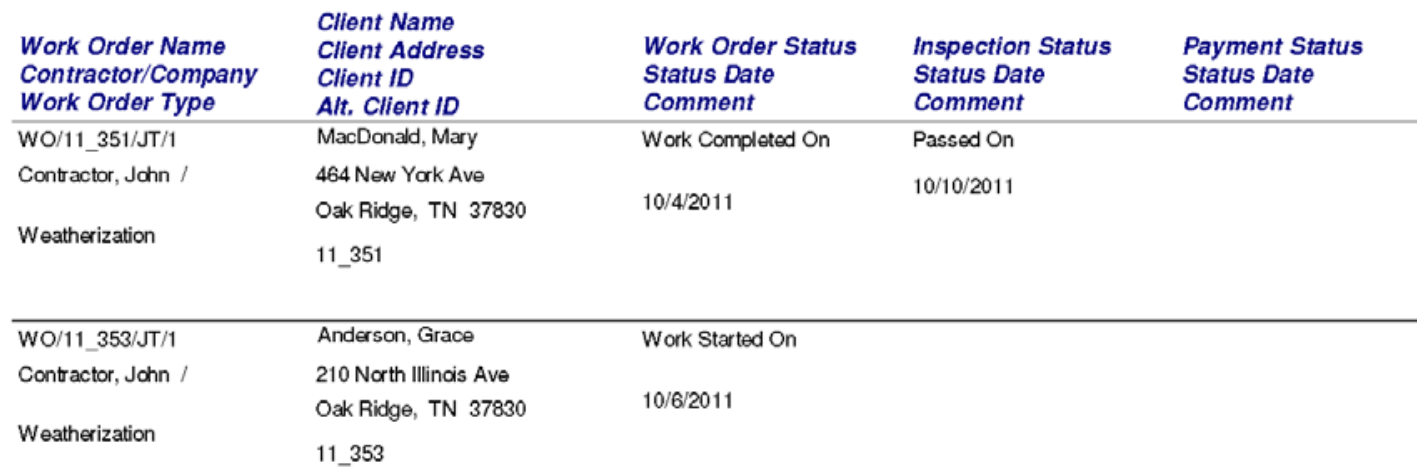

Agency Name: 


\section{Appendix C: Reports}

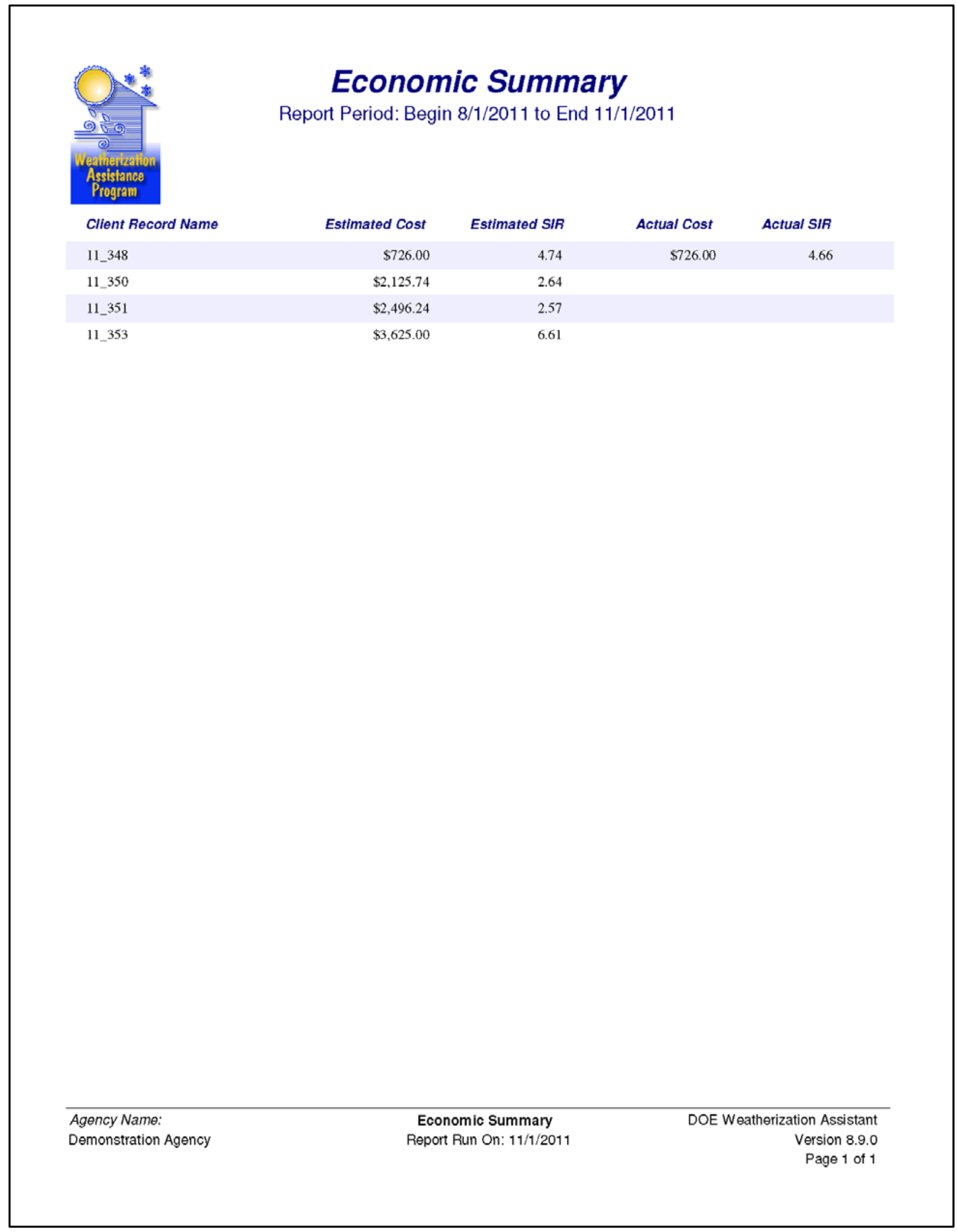




\section{Client Surveys (Blank)}

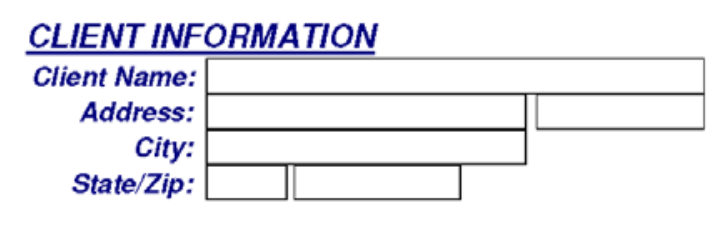

\section{Surver Name Client Satisfaction}

\section{Question}

1 Is the client generally satisfied with the work performed?

2 Was any instruction given to the client regarding operating new equipment, etc. If so, describe.

3 Does the client recognize any specific improvements in his living conditions? If so describe.

\section{Answer}
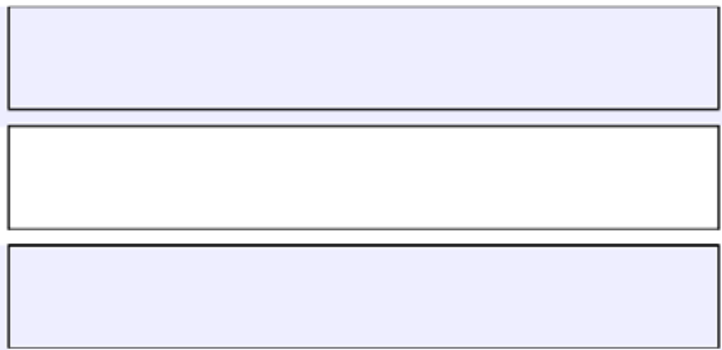

Agency Name: Demonstration Agency
Client Surveys (Blank) Report Run On: 11/1/2011
DOE Weatherization Assistant

Version 8.9 .0

Paae 1 of 4 


\section{Appendix C: Reports}

\section{Survey Name Intake Survey}

\section{Question}

1 Age of dwelling (year built)

2 Thermostat setting - Day

3 Ther mostat setting - Night

$4 \quad$ Existing setback thermostat?

5 Setback thermostat properly used?

6 Install setback thermostat?

7 Client comfort at temperature settings (specify location of drafts, warm rooms, cold rooms)

8 Suuply/returns in cold rooms? Specify

9 Basement used as living space? If yes, describe.

10 Basement temperature during winter?

11 Attic use (storage, other)

12 How will attic use affect attic insulating?

13 Rooms closed off during winter (locate and explain)?

\section{Answer}
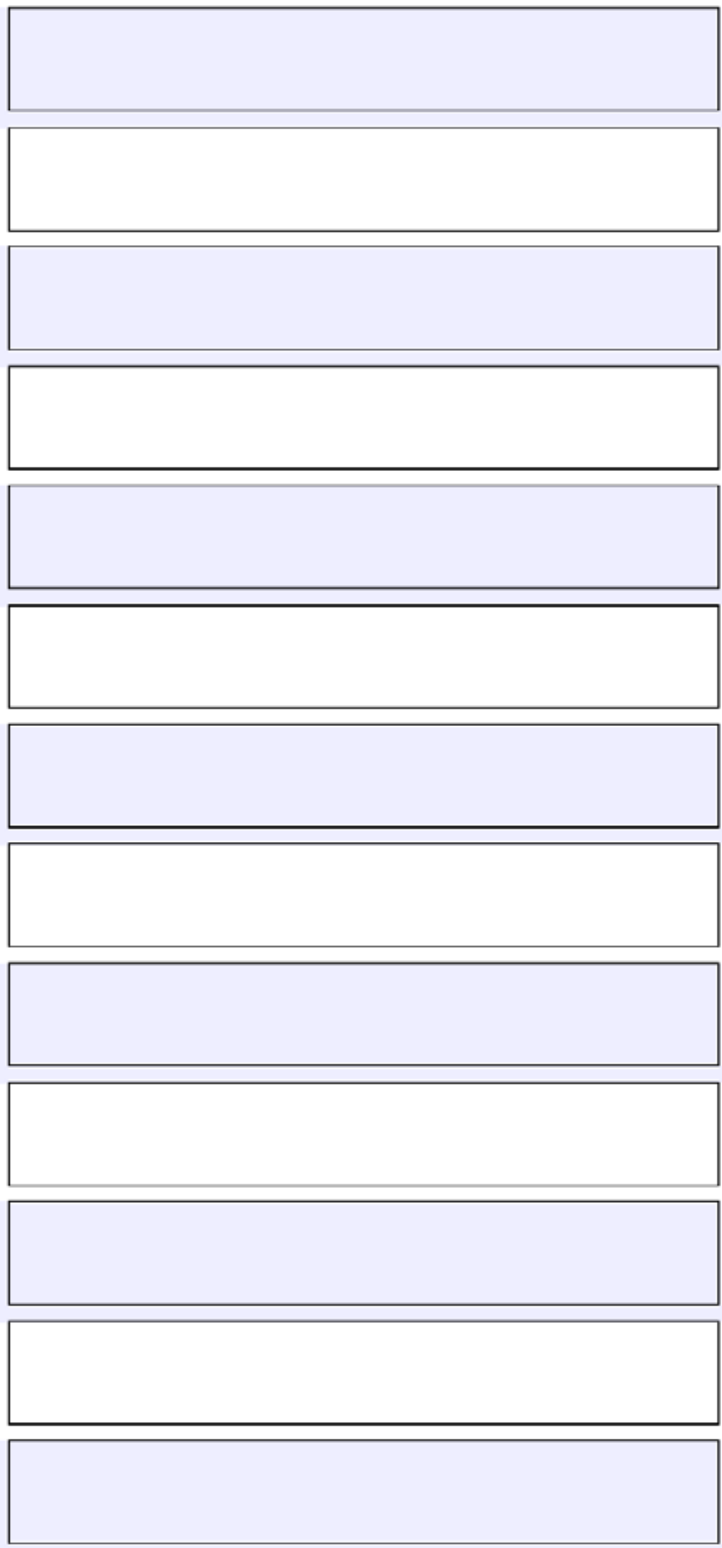

Client Surveys (Blank)

Report Run On: 11/1/2011
DOE Weatherization Assistant

Version 8.9.0

Paae 2 of 4 


\section{Survey Name Intake Survey}

Question

14 Age (years)

15 Describe repairs in last 3 years

16 Routine maintenance (Yes or No)?

17 Describe routine maintenance

18 Does the dwelling have icicles or ice dams (Yes or No)? Explain if Yes.

19 Does the dwelling have moisture problems, mold or mildew (Yes or No)? Explain if Yes.

20 Does the dwelling have freezing pipes (Yes or No)? Explain if Yes.

21 Does the client have recurrent headaches, itching or burning eyes while at home (Yes or No)? Explain if Yes.

22 Other (specify)

23 Do yoyu feel any drafts

\section{Answer}
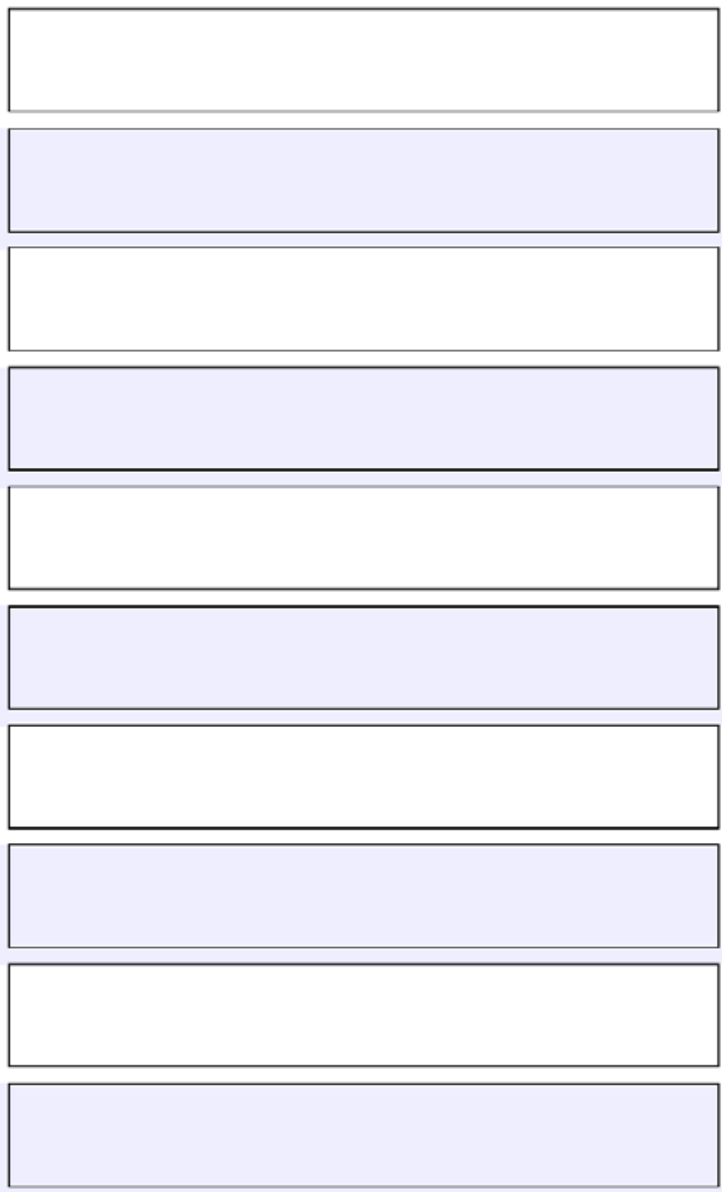

Agency Name: Demonstration Agency
Client Surveys (Blank)

Report Run On: 11/1/2011
DOE Weatherization Assistant

Version 8.9 .0

Paae 3 of 4 


\section{Appendix C: Reports}

\section{Work Order Totals by Category}

The development of the Work Order Totals by Category form was not completed at the time the Weatherization Assistant Version 8.9.0.5 was released. It does not contain identifiable information of use to the user. 


\section{Client Completion Report}

CLIENT INFORMATION

Client ID: 11_348

Client Name: Tanner, David

Alt. Client ID:

Address: 114 Athens

Anytown

US 01234

\section{AGENCY INFORMATION}

Agency Name: Demonstration Agency

Address: 725 Jefferson St.

Any City

US 11111

Office Phone: (123) 456-7890

Office Email: agencyemail@localisp.net

\begin{tabular}{lccclcc} 
Contact Name & Home Ph & Work Ph & Cell Ph & Contact Type & $\begin{array}{c}\text { Primary } \\
\text { Applican Comment }\end{array}$ \\
\hline Tanner, David & $(111) 764-5687$ & $(111) 764-3789$ & $(111) 764-9902$ & $\begin{array}{c}\text { Applicant/Person of } \\
\text { Record }\end{array}$ & $\checkmark$ & \\
Tanner, John & & $(254) 567-8908$ & & $\begin{array}{c}\text { Applicant/Person of } \\
\text { Record }\end{array}$ & $\square$ & Son of primary applicant
\end{tabular}

\section{DWELLING INFORMATION}

Dwelling Type: Site Built

Occupancy (own/rent): Owned

Primary heating fuel: Natural Gas

Secondary heating fuel:

$\square$ Previously Weatherized $\quad \begin{gathered}\text { Year Previously } \\ \text { Weatherized }\end{gathered}$

Year Built 1952

\section{ENERGY INDEX}

$\begin{array}{cc}\text { Floor Area (sq ft) } & 1290 \\ \text { Heating Degree Days } & 3400 \\ \text { (base 65 F) }\end{array}$

\begin{tabular}{ccccc} 
& \multicolumn{5}{c}{ Estim.\% } & & \\
Pnnual Cost & for heating & & \\
Primary Heating Fuel & $\$ 978.00$ & 100 & $\begin{array}{c}\text { Total Heating } \\
(B T U / H D D / s q ~ f t)\end{array}$ & 22.3 \\
Secondary Heating Fuel & & & &
\end{tabular}

\section{WORK HISTORY}

Client Application

$\begin{array}{lccc}\text { Status } & \text { Date } & \text { Auditor } & \text { Comment } \\ \text { Received On } & 8 / 2 / 2011 & \text { admin } & \\ \text { Approved On } & 8 / 9 / 2011 & \text { admin } & \\ \text { Active On } & 8 / 2 / 2011 & \text { admin } & \\ \text { Work Done, File Closed/Locked On } & 9 / 17 / 2011 & \text { admin } & \end{array}$

$\underline{\text { Audit }}$

\begin{tabular}{|c|c|c|c|}
\hline Audit Name: 11_348SB & & & Audit Job ID: -1909609271 \\
\hline $\begin{array}{l}\text { Status } \\
\text { Audit Complete and Locked On }\end{array}$ & $\begin{array}{l}\text { Date } \\
8 / 24 / 2011\end{array}$ & $\begin{array}{l}\text { Auditor } \\
\text { admin }\end{array}$ & Comment \\
\hline
\end{tabular}

$\begin{array}{llr}\text { Client Name: Tanner, David } & \text { Client Completion Report } & \text { DOE Weatherization Assistant } \\ \text { Client ID: } 11 \_348 & \text { Report Run On: 11/3/2011 } & \text { Version } 8.9 .0 \\ \text { Ait. Client ID: } & & \text { Page } 1 \text { of } 6\end{array}$




\section{Appendix C: Reports}

Audit Name: 11 348SB

Status

Recommendations Generated On

Site Visit Completed On

Site Visit Scheduled For

\section{Date}

8/24/2011

$8 / 22 / 2011$

$8 / 19 / 2011$
Audit Job ID: -1909609271

Auditot Comment

admin

admin

admin

\section{Work Orders}

\begin{tabular}{|c|c|c|c|}
\hline Work Order Name: WO/11_348/JT/1 & & & Work Order ID: 12491995 \\
\hline Status & Date & Auditor & Comment \\
\hline Passed On & $9 / 13 / 2011$ & Admin & \\
\hline Invoice Received On & $9 / 9 / 2011$ & Admin & \\
\hline Invoice Paid On & $9 / 16 / 2011$ & Admin & \\
\hline Work Started On & $9 / 5 / 2011$ & Admin & \\
\hline Work Completed On & $9 / 7 / 2011$ & Admin & \\
\hline Work Order Created from Audit On & $8 / 24 / 2011$ & Admin & \\
\hline Work Order Name: WO/11_348/EASY/1 & & & Work Order ID: 1554965122 \\
\hline Status & Date & Auditor & Comment \\
\hline Passed On & $9 / 13 / 2011$ & Admin & \\
\hline Invoice Received On & $9 / 8 / 2011$ & Admin & \\
\hline Invoice Paid On & $9 / 15 / 2011$ & Admin & \\
\hline Work Started On & $9 / 1 / 2011$ & Admin & \\
\hline Work Completed On & $9 / 6 / 2011$ & Admin & \\
\hline Work Order Created from Audit On & $8 / 24 / 2011$ & Admin & \\
\hline
\end{tabular}

\begin{tabular}{llr}
\hline Client Name: Tanner, David & Client Completion Report & DOE Weatherization Assistant \\
Client ID: $11 \_348$ & Report Run On: 11/3/2011 & Version 8.9 .0 \\
Alt. Client ID: & & Page 2 of 6
\end{tabular}




\section{COST SUMMARY BY WORK ORDER / MEASURE}

Work Order Name: WO/11_348/EASY/1

\begin{tabular}{|c|c|c|c|c|}
\hline \multirow[b]{2}{*}{ Measure Name / Components } & \multicolumn{3}{|c|}{ Actual Costs } & \multirow[b]{2}{*}{ Funding Source } \\
\hline & Materials & Labor & Total & \\
\hline Install Bathroom Exhaust Fan & $\$ 190.00$ & $\$ 80.00$ & $\$ 270.00$ & Spark Utility Program \\
\hline Anticipator Adjustment Needed & $\$ 20.00$ & $\$ 0.00$ & $\$ 20.00$ & Spark Utility Program \\
\hline Low Flow Showerheads & $\$ 5.00$ & $\$ 15.00$ & $\$ 20.00$ & Spark Utility Program \\
\hline Smart Thermostat & $\$ 50.00$ & $\$ 25.00$ & $\$ 75.00$ & Spark Utility Program \\
\hline $\begin{array}{l}\text { Lighting Retrofits } \\
\qquad \text { LT1 }\end{array}$ & $\$ 26.00$ & $\$ 12.00$ & $\$ 38.00$ & Spark Utility Program \\
\hline${ }_{H S 1}$ & $\$ 150.00$ & $\$ 75.00$ & $\$ 225.00$ & Spark Utility Program \\
\hline Work Order Sub Total: & $\$ 441.00$ & $\$ 207.00$ & $\$ 648.00$ & \\
\hline
\end{tabular}

Work Order Name: WO/11_348/JT/1

\begin{tabular}{|c|c|c|c|c|}
\hline \multirow[b]{2}{*}{ Measure Name / Components } & \multicolumn{3}{|c|}{ Actual Costs } & \multirow[b]{2}{*}{ Funding Source } \\
\hline & Materials & Labor & Total & \\
\hline Infiltration Redctn & $\$ 250.00$ & $\$ 0.00$ & $\$ 250.00$ & Weatherization \\
\hline Repair Roof & $\$ 135.00$ & $\$ 0.00$ & $\$ 135.00$ & Weatherization \\
\hline CO Monitor is Needed & $\$ 40.00$ & $\$ 30.00$ & $\$ 70.00$ & Weatherization \\
\hline $\begin{array}{l}\text { Attic Ins. R-30 } \\
\text { UA1 }\end{array}$ & $\$ 90.00$ & $\$ 180.00$ & $\$ 270.00$ & Weatherization \\
\hline DWH Pipe Insulation & $\$ 5.00$ & $\$ 10.00$ & $\$ 15.00$ & Weatherization \\
\hline DWH Tank Insulation & $\$ 15.00$ & $\$ 25.00$ & $\$ 40.00$ & Weatherization \\
\hline $\begin{array}{l}\text { Wall Insulation } \\
\text { WLN-1,WLN-2,WLS-2,WLW-1 }\end{array}$ & $\$ 130.00$ & $\$ 375.00$ & $\$ 505.00$ & Weatherization \\
\hline $\begin{array}{l}\text { Window Replacement } \\
\text { WD4 }\end{array}$ & $\$ 346.00$ & $\$ 0.00$ & $\$ 346.00$ & Weatherization \\
\hline $\begin{array}{l}\text { Fill Ceiling Cavity } \\
\text { FA3 }\end{array}$ & $\$ 73.77$ & $\$ 147.49$ & $\$ 221.26$ & Weatherization \\
\hline $\begin{array}{l}\text { Attic Ins. R-30 } \\
\text { FA4 }\end{array}$ & $\$ 16.80$ & $\$ 33.60$ & $\$ 50.40$ & Weatherization \\
\hline $\begin{array}{l}\text { Kneewall Insulation } \\
\text { FA2 }\end{array}$ & $\$ 31.20$ & $\$ 60.00$ & $\$ 91.20$ & Weatherization \\
\hline $\begin{array}{l}\text { Attic Ins. R-30 } \\
\text { FA1 }\end{array}$ & $\$ 117.60$ & $\$ 235.20$ & $\$ 352.80$ & Weatherization \\
\hline Insulate and seal attic access & $\$ 11.10$ & $\$ 20.00$ & $\$ 31.10$ & Weatherization \\
\hline Work Order Sub Total: & $\$ 1,261.47$ & $\$ 1,116.29$ & $\$ 2,377.76$ & \\
\hline All Work Orders Grand Total: & $\$ 1,702.47$ & $\$ 1,323.29$ & $\$ 3,025.76$ & \\
\hline
\end{tabular}

$\begin{array}{lrr}\text { Client Name: Tanner, David } & \text { Client Completion Report } & \text { DOE Weatherization Assistant } \\ \text { Client ID: } 11 \_348 & \text { Report Run On: 11/3/2011 } & \text { Version 8.9.0 } \\ \text { Alt. Client ID: } & & \text { Page } 3 \text { of } 6\end{array}$




\section{Appendix C: Reports}

\section{ECONOMICS SUMMARY BY MEASURE TYPE / MEASURE}

Measure Type:

\begin{tabular}{|c|c|c|c|c|c|c|c|c|c|}
\hline \multirow[b]{3}{*}{ Measure Name / Components } & \multicolumn{6}{|c|}{ Energy Savings } & \multirow{3}{*}{$\begin{array}{c}\text { Total } \\
\text { Annual } \\
\text { Savings } \\
\$\end{array}$} & \multirow{3}{*}{$\begin{array}{c}\text { Total } \\
\text { Cost } \\
\$\end{array}$} & \multirow{3}{*}{$\begin{array}{c}\text { Actua } \\
\text { SIR }\end{array}$} \\
\hline & \multicolumn{2}{|c|}{ Heating } & \multicolumn{2}{|c|}{ Cooling } & \multicolumn{2}{|c|}{ Baseload } & & & \\
\hline & MMBtu & $\$$ & $k W h$ & $\$$ & $k W h$ & $\$$ & & & \\
\hline Infiltration Redctn & 3.4 & $\$ 34$ & 28 & $\$ 2$ & 0 & $\$ 0$ & $\$ 36$ & $\$ 250.00$ & 1.2 \\
\hline Repair Roof & 0.0 & $\$ 0$ & 0 & $\$ 0$ & 0 & $\$ 0$ & $\$ 0$ & $\$ 135.00$ & 0.0 \\
\hline Measure Type Sub Total: & 3.4 & $\$ 34$ & 28 & $\$ 2$ & 0 & $\$ 0$ & $\$ 36$ & $\$ 385.00$ & \\
\hline
\end{tabular}

\begin{tabular}{|c|c|c|c|c|c|c|c|c|c|}
\hline \multicolumn{10}{|l|}{ Measure Type: Baseloads } \\
\hline \multirow[b]{3}{*}{ Measure Name / Components } & \multicolumn{6}{|c|}{ Energy Savings } & \multirow{3}{*}{$\begin{array}{c}\text { Total } \\
\text { Annual } \\
\text { Savings } \\
\$\end{array}$} & \multirow{3}{*}{$\begin{array}{c}\text { Total } \\
\text { Cost } \\
\$\end{array}$} & \multirow{3}{*}{$\begin{array}{c}\text { Actua } \\
\text { SIR }\end{array}$} \\
\hline & \multicolumn{2}{|c|}{ Heating } & \multicolumn{2}{|c|}{ Cooling } & \multicolumn{2}{|c|}{ Baseload } & & & \\
\hline & MMBtu & $\$$ & $k W h$ & $\$$ & $k W h$ & $\$$ & & & \\
\hline Low Flow Showerheads & 0.0 & $\$ 0$ & 0 & $\$ 0$ & 259 & $\$ 18$ & $\$ 18$ & $\$ 20.00$ & 10.7 \\
\hline DWH Pipe Insulation & 0.0 & \$0 & 0 & $\$ 0$ & 195 & $\$ 14$ & $\$ 14$ & $\$ 15.00$ & 9.5 \\
\hline DWH Tank Insulation & 0.0 & $\$ 0$ & 0 & $\$ 0$ & 405 & $\$ 28$ & $\$ 28$ & $\$ 40.00$ & 7.4 \\
\hline $\begin{array}{l}\text { Lighting Retrofits } \\
\text { LT1 }\end{array}$ & 0.0 & $\$ 0$ & 0 & $\$ 0$ & 823 & $\$ 58$ & $\$ 58$ & $\$ 38.00$ & 3.3 \\
\hline Measure Type Sub Total: & 0.0 & $\$ 0$ & 0 & $\$ 0$ & 1,683 & $\$ 118$ & $\$ 118$ & $\$ 113.00$ & \\
\hline
\end{tabular}

Measure Type: Building Insulation

\begin{tabular}{|c|c|c|c|c|c|c|c|c|c|}
\hline \multirow[b]{3}{*}{ Measure Name / Components } & \multicolumn{6}{|c|}{ Energy Savings } & \multirow{3}{*}{$\begin{array}{c}\text { Total } \\
\text { Annual } \\
\text { Savings } \\
\$\end{array}$} & \multirow{3}{*}{$\begin{array}{c}\text { Total } \\
\text { Cost } \\
\$\end{array}$} & \multirow{3}{*}{$\begin{array}{l}\text { Actua } \\
\text { SIR }\end{array}$} \\
\hline & \multicolumn{2}{|c|}{ Heating } & \multicolumn{2}{|c|}{ Cooling } & \multicolumn{2}{|c|}{ Baseload } & & & \\
\hline & MMBtu & $\$$ & $k W h$ & $\$$ & $k W h$ & $\$$ & & & \\
\hline $\begin{array}{l}\text { Wall Insulation } \\
\text { WLN-1,WLN-2,WLS-2,WLW-1 }\end{array}$ & 5.0 & $\$ 50$ & 37 & $\$ 3$ & 0 & $\$ 0$ & $\$ 52$ & $\$ 505.00$ & 1.6 \\
\hline $\begin{array}{l}\text { Attic Ins. R-30 } \\
\text { UA1 }\end{array}$ & 13.9 & $\$ 139$ & 190 & $\$ 13$ & 0 & $\$ 0$ & $\$ 152$ & $\$ 270.00$ & 8.8 \\
\hline Insulate and sea attic access & 0.0 & $\$ 0$ & 0 & $\$ 0$ & 0 & $\$ 0$ & $\$ 0$ & $\$ 31.10$ & 3.5 \\
\hline $\begin{array}{l}\text { Fill Ceiling Cavity } \\
\text { FA3 }\end{array}$ & 8.3 & $\$ 83$ & 122 & $\$ 9$ & 0 & $\$ 0$ & $\$ 91$ & $\$ 221.26$ & 6.4 \\
\hline $\begin{array}{l}\text { Attic Ins. R-30 } \\
\text { FA4 }\end{array}$ & 1.2 & $\$ 12$ & 15 & $\$ 1$ & 0 & $\$ 0$ & $\$ 13$ & $\$ 50.40$ & 4.0 \\
\hline $\begin{array}{l}\text { Kneewall Insulation } \\
\quad \text { FA2 }\end{array}$ & 1.8 & $\$ 18$ & 3 & $\$ 0$ & 0 & $\$ 0$ & $\$ 18$ & $\$ 91.20$ & 3.2 \\
\hline $\begin{array}{l}\text { Attic Ins. R-30 } \\
\text { FA1 }\end{array}$ & 8.6 & $\$ 86$ & 103 & $\$ 7$ & 0 & $\$ 0$ & $\$ 93$ & $\$ 352.80$ & 4.1 \\
\hline Measure Type Sub Total: & 38.7 & $\$ 387$ & 471 & $\$ 33$ & 0 & $\$ 0$ & $\$ 420$ & $\$ 1,521.76$ & \\
\hline
\end{tabular}

$\begin{array}{llr}\text { Client Name: Tanner, David } & \text { Client Completion Report } & \text { DOE Weatherization Assistant } \\ \text { Client ID: } 11 \_348 & \text { Report Run On: 11/3/2011 } & \text { Version } 8.9 .0 \\ \text { Alt. Client ID: } & & \text { Page } 4 \text { of } 6\end{array}$


Measure Type: Doors and Windows

\begin{tabular}{|c|c|c|c|c|c|c|c|c|c|}
\hline \multirow[b]{3}{*}{ Measure Name / Components } & \multicolumn{6}{|c|}{ Energy Savings } & \multirow{3}{*}{$\begin{array}{c}\text { Total } \\
\text { Annual } \\
\text { Savings } \\
\$\end{array}$} & \multirow{3}{*}{$\begin{array}{c}\text { Total } \\
\text { Cost } \\
\$\end{array}$} & \multirow{3}{*}{$\begin{array}{c}\text { Actua } \\
\text { SIR }\end{array}$} \\
\hline & \multicolumn{2}{|c|}{ Heating } & \multicolumn{2}{|c|}{ Cooling } & \multicolumn{2}{|c|}{ Baseload } & & & \\
\hline & MMBtu & $\$$ & $k W h$ & $\$$ & $k W h$ & $\$$ & & & \\
\hline $\begin{array}{l}\text { Window Replacement } \\
\text { WD4 }\end{array}$ & 1.4 & $\$ 14$ & -8 & $(\$ 1)$ & 0 & $\$ 0$ & $\$ 14$ & $\$ 346.00$ & 0.6 \\
\hline Measure Type Sub Total: & 1.4 & $\$ 14$ & -8 & $(\$ 1)$ & 0 & $\$ 0$ & $\$ 14$ & $\$ 346.00$ & \\
\hline
\end{tabular}

Measure Type: Health and Safety

\begin{tabular}{|c|c|c|c|c|c|c|c|c|c|}
\hline \multirow[b]{3}{*}{ Measure Name / Components } & \multicolumn{6}{|c|}{ Energy Savings } & \multirow{3}{*}{$\begin{array}{c}\text { Total } \\
\text { Annual } \\
\text { Savings } \\
\$\end{array}$} & \multirow{3}{*}{$\begin{array}{c}\text { Total } \\
\text { Cost } \\
\$\end{array}$} & \multirow{3}{*}{$\begin{array}{c}\text { Actual } \\
\text { SIR }\end{array}$} \\
\hline & \multicolumn{2}{|c|}{ Heating } & \multicolumn{2}{|c|}{ Cooling } & \multicolumn{2}{|c|}{ Baseload } & & & \\
\hline & MMBtu & $\$$ & $k W h$ & $\$$ & $k W h$ & $\$$ & & & \\
\hline CO Monitor is Needed & 0.0 & $\$ 0$ & 0 & $\$ 0$ & 0 & $\$ 0$ & $\$ 0$ & $\$ 70.00$ & 0.0 \\
\hline Anticipator Adjustment Needed & 0.0 & $\$ 0$ & 0 & $\$ 0$ & 0 & $\$ 0$ & $\$ 0$ & $\$ 20.00$ & 0.0 \\
\hline Install Bathroom Exhaust Fan & 0.0 & $\$ 0$ & 0 & $\$ 0$ & 0 & $\$ 0$ & \$o & $\$ 270.00$ & 0.0 \\
\hline Measure Type Sub Total: & 0.0 & $\$ 0$ & 0 & $\$ 0$ & 0 & $\$ 0$ & $\$ 0$ & $\$ 360.00$ & \\
\hline
\end{tabular}

Measure Type: HVAC Systems

\begin{tabular}{|c|c|c|c|c|c|c|c|c|c|}
\hline \multirow[b]{3}{*}{ Measure Name / Components } & \multicolumn{6}{|c|}{ Energy Savings } & \multirow{3}{*}{$\begin{array}{c}\text { Total } \\
\text { Annual } \\
\text { Savings } \\
\$\end{array}$} & \multirow{3}{*}{$\begin{array}{c}\text { Total } \\
\text { Cost } \\
\$\end{array}$} & \multirow{3}{*}{$\begin{array}{c}\text { Actual } \\
\text { SIR }\end{array}$} \\
\hline & \multicolumn{2}{|c|}{ Heating } & \multicolumn{2}{|c|}{ Cooling } & \multicolumn{2}{|c|}{ Baseload } & & & \\
\hline & MMBtu & $\$$ & $k W h$ & $\$$ & $k W h$ & $\$$ & & & \\
\hline Smart Thermostat & 6.6 & $\$ 66$ & 0 & $\$ 0$ & 0 & $\$ 0$ & $\$ 66$ & $\$ 75.00$ & 10.7 \\
\hline IID & 5.9 & $\$ 59$ & 0 & $\$ 0$ & 0 & $\$ 0$ & $\$ 59$ & $\$ 225.00$ & 2.2 \\
\hline \multicolumn{10}{|l|}{ HS1 } \\
\hline Measure Type Sub Total: & 12.4 & $\$ 124$ & 0 & $\$ 0$ & 0 & $\$ 0$ & $\$ 124$ & $\$ 300.00$ & \\
\hline All Measure Types Grand Total: & 56.0 & $\$ 560$ & 490 & $\$ 34$ & 1,683 & $\$ 118$ & $\$ 712$ & $\$ 3,025.76$ & \\
\hline
\end{tabular}

$\begin{array}{lrr}\text { Client Name: Tanner, David } & \text { Client Completion Report } & \text { DOE Weatherization Assistant } \\ \text { Client ID: } 11 \_348 & \text { Report Run On: 11/3/2011 } & \text { Version } 8.9 .0 \\ \text { Alt. Client ID: } & & \text { Page } 5 \text { of } 6\end{array}$




\section{COST SUMMARY BY FUNDING SOURCE}

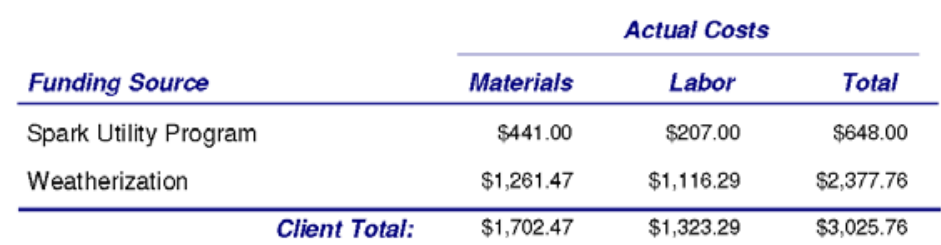

Client Name: Tanner, David

Client ID: 11_348

Alt. Client ID:
DOE Weatherization Assistant

Version 8.9.0

Page 6 of 6 


\section{Client Information Report}

\section{CLIENT INFORMATION

\begin{tabular}{|c|c|c|}
\hline Client ID & 11_348 & Alt. Client ID \\
\hline Client Name & Tanner, David & \\
\hline Address & $11 \overline{4}$ Athens & \\
\hline $\begin{array}{r}\text { Unit No. } \\
\text { City }\end{array}$ & $= \pm---j$ State & Zip $[012 \overline{1234}$ \\
\hline County & I Other & Geo. Ident. \\
\hline
\end{tabular}

\begin{tabular}{|c|c|c|c|}
\hline \multicolumn{2}{|l|}{ Dwelling } & \multirow[b]{2}{*}{ Ownership } & \multirow[b]{2}{*}{ Owned } \\
\hline Dwelling Type & Site Built & & \\
\hline Primary Heat. Fuel & Natural Gas & \multirow{2}{*}{\multicolumn{2}{|c|}{$\begin{array}{l}\square \text { High Energy Use } \\
\square \text { High Energy Burden }\end{array}$}} \\
\hline Secondary Heat. Fuel & & & \\
\hline \multicolumn{2}{|c|}{$\square$ Previously Weatherized } & Year Built & 1952 \\
\hline Year & & & \\
\hline
\end{tabular}

\section{Energy Index}

Floor Area (sq ft) 1290

Heating Degree Days 3400

(base 65 F)

Primary Heating Fuel

Secondary Heating Fuel
Total Heating (BTU/HDD/sq ft)

22.3

Estim. \%

Annual Cost for heating

100

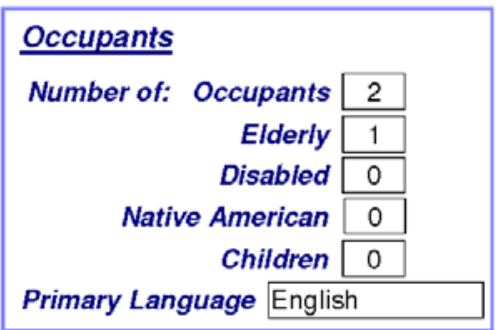

Primary Language English

\section{Comment}

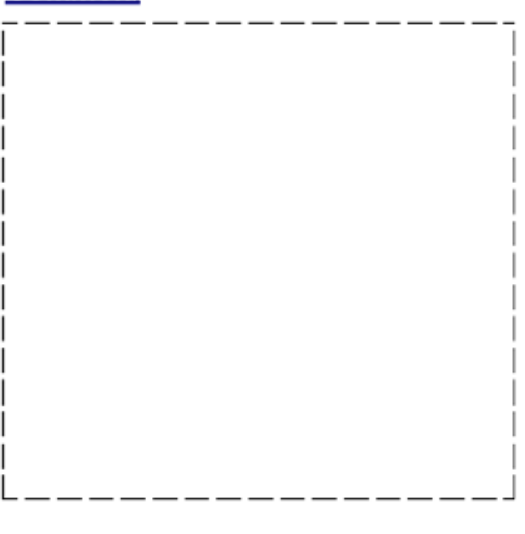

\section{CLIENT CONTACT INFORMATION}

$\begin{array}{lllll}\text { Tanner, David } & \text { (111) } 764-5687 & \text { (111) } 764-3789 & \text { (111) } 764-9902 & \begin{array}{c}\text { Applicant/Person of } \\ \text { Record }\end{array} \\ \text { Tanner, John } & \text { (254) } 567-8908 & \begin{array}{c}\text { Applicant/Person of } \\ \text { Record }\end{array} & \square\end{array}$

Client Name: Tanner, David

Client ID: 11_348

Alt. Client ID:
Client Information Report

Report Run On: 11/2/2011
DOE Weatherization Assistant

Version 8.9 .0

Page 1 of 1 


\section{Appendix C: Reports}

\section{Client Information Form}

\section{CLIENT INFORMATION}

\begin{tabular}{|r|r|} 
Client ID & Alt. Client ID \\
Client Name & \\
Address & \multicolumn{1}{c|}{ Unit No. } \\
City & \multicolumn{1}{|c|}{ State $\square$ Zip } \\
County & Other Geo. Ident.
\end{tabular}
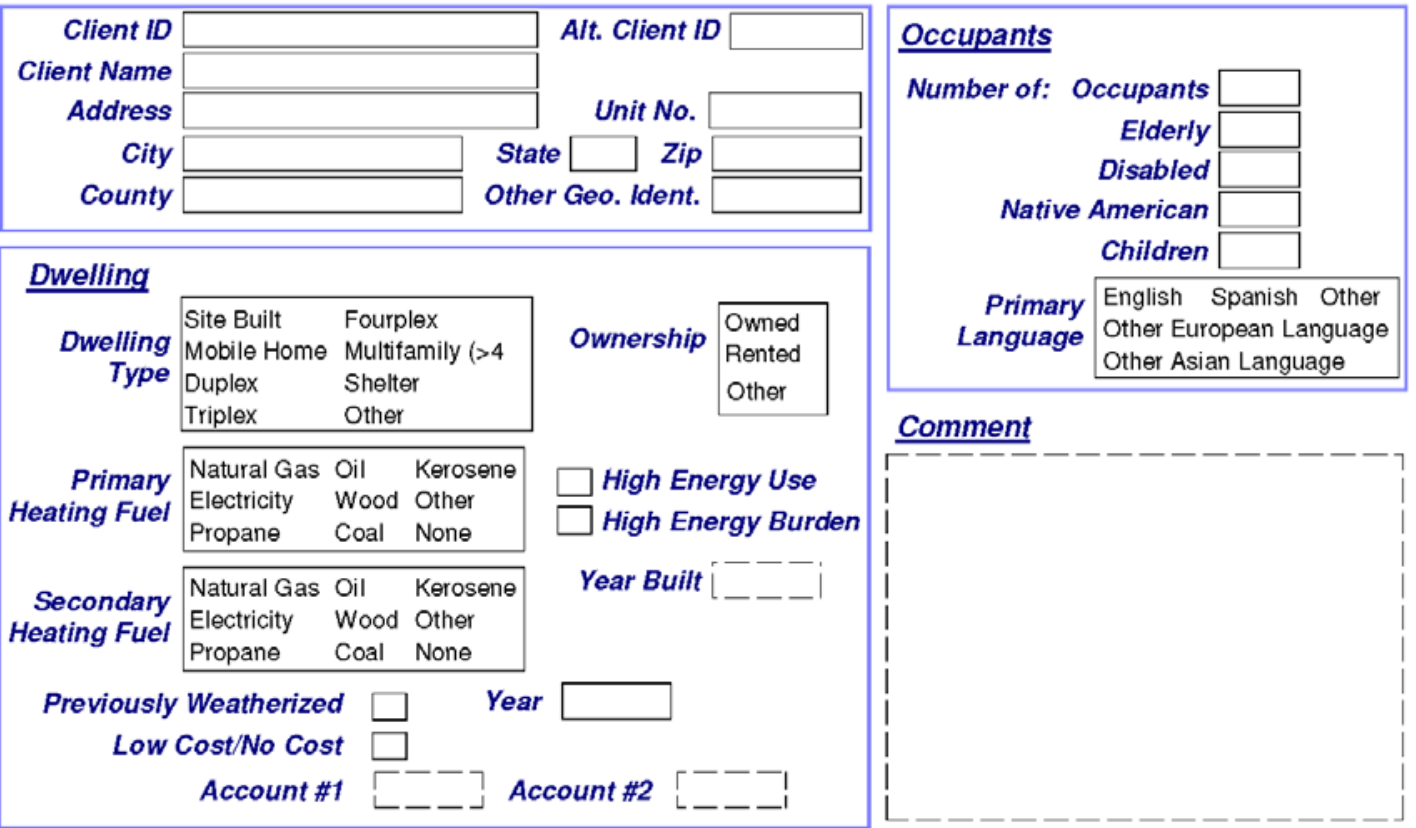

\section{Comment}
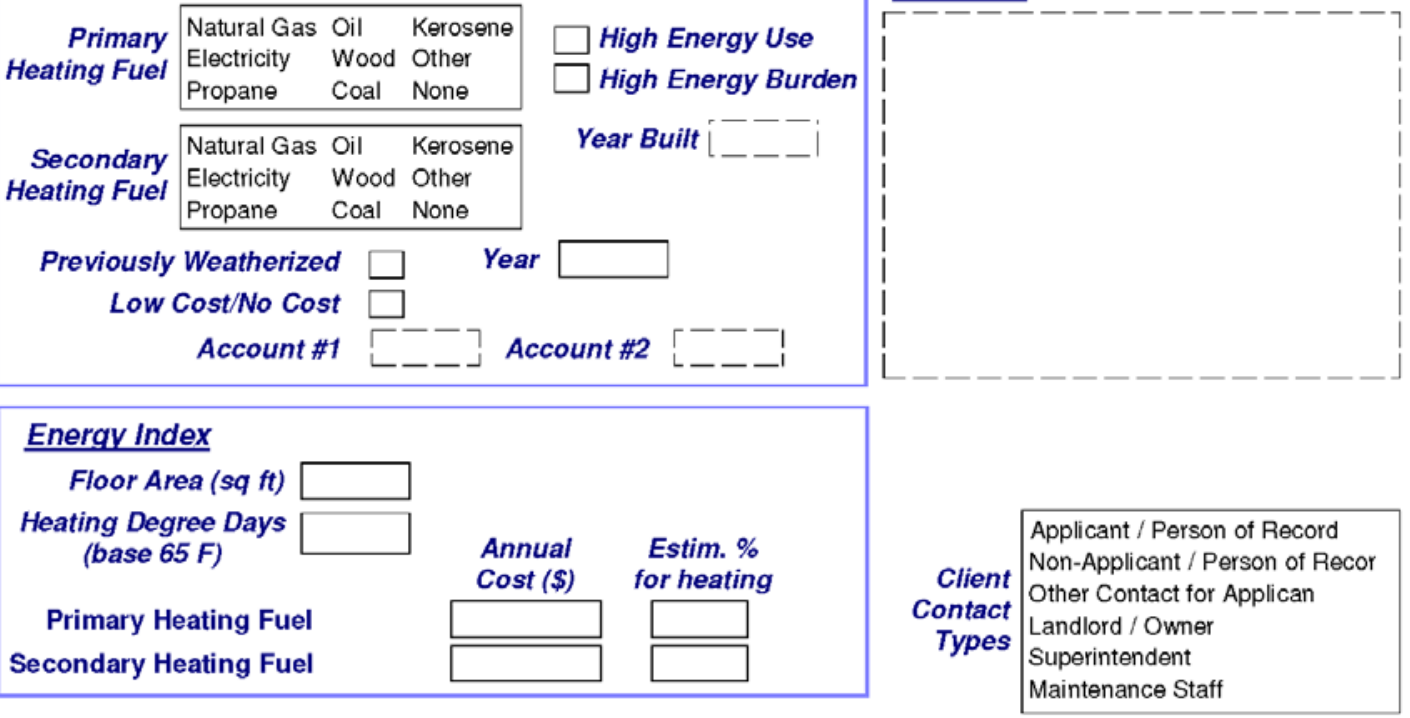

\section{CLIENT CONTACT INFORMATION}

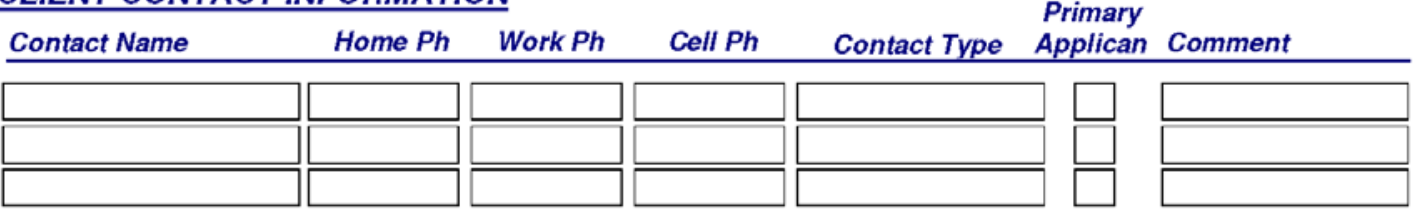




\section{NEAT Data Collection Form}

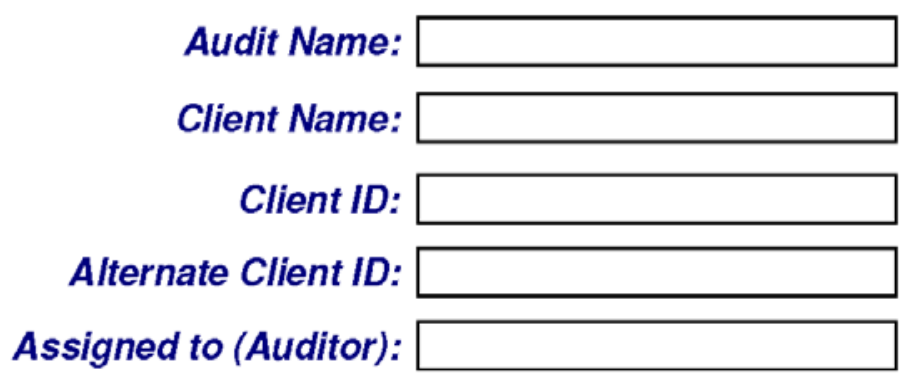

Number of Conditioned Stories:

Floor Area (sq. ft.):

Comment:

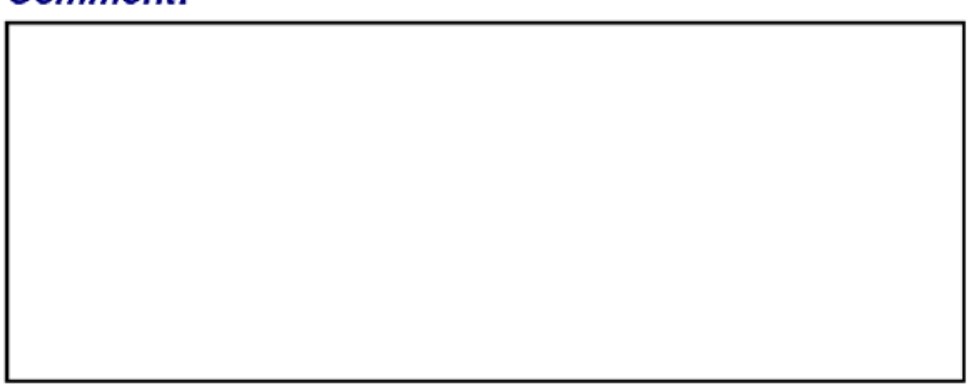




\section{Appendix C: Reports}

\section{Site Diagram}

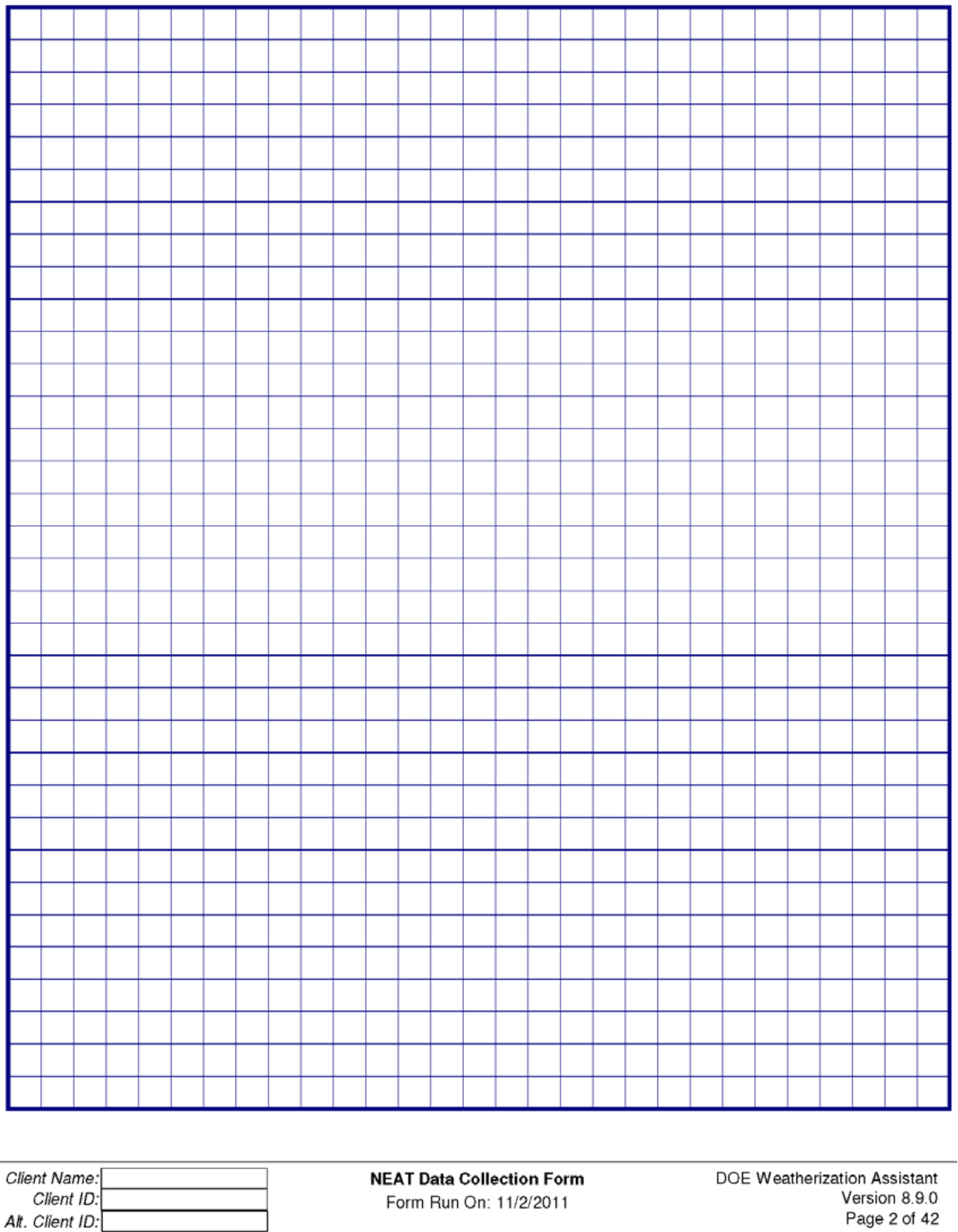


Walls

\begin{tabular}{|c|c|c|c|c|c|}
\hline \multirow{3}{*}{$\begin{array}{l}\text { Wall Code } \\
\text { Wall Type }\end{array}$} & & \multirow{2}{*}{$\begin{array}{r}\text { Existing } \\
\text { Insul Type }\end{array}$} & \multirow{2}{*}{\begin{tabular}{|l|} 
None \\
Blown cellulose \\
Blown fiberglass \\
\end{tabular}} & \multirow{2}{*}{\multicolumn{2}{|c|}{$\begin{array}{l}\text { Rockwool Other } \\
\text { Fiberglass batts } \\
\text { Polystyrene board }\end{array}$}} \\
\hline & $\begin{array}{ll}\text { Balloon frame } & \text { Concrete bloc } \\
\text { Platform frame } & \text { Adobe }\end{array}$ & & & & \\
\hline & Masonry or Stone Other & Existing & & & \\
\hline Stud Size & $\begin{array}{lllll}2 \times 2 & 2 \times 3 & 2 \times 4 & 2 \times 6 & 2 \times 8\end{array}$ & & & & \\
\hline Exterior Type & $\begin{array}{|lll|}\text { Wood } & \text { Metal or Vinyl } & \text { Other } \\
\text { Stucco } & \text { Brick or Stone } & \text { None } \\
\end{array}$ & $\begin{array}{r}\text { Added } \\
\text { Insul Type }\end{array}$ & $\begin{array}{l}\text { None } \\
\text { Blown cellulose }\end{array}$ & $\begin{array}{l}\text { User type } 1 \\
\text { User type } 2 \\
\end{array}$ & \\
\hline Exposure & \begin{tabular}{|lll} 
Outside & Buffered & Attic \\
\end{tabular} & $\begin{array}{r}\text { Additional } \\
\text { Cost }(\$)\end{array}$ & $\left\{\begin{array}{l}----1 \\
----1\end{array}\right.$ & & \\
\hline Orientation & North South East West & Comment & & & \\
\hline $\operatorname{Area}(s q f t)$ & & 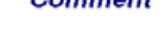 & & & \\
\hline Measure No. & & & & & \\
\hline
\end{tabular}

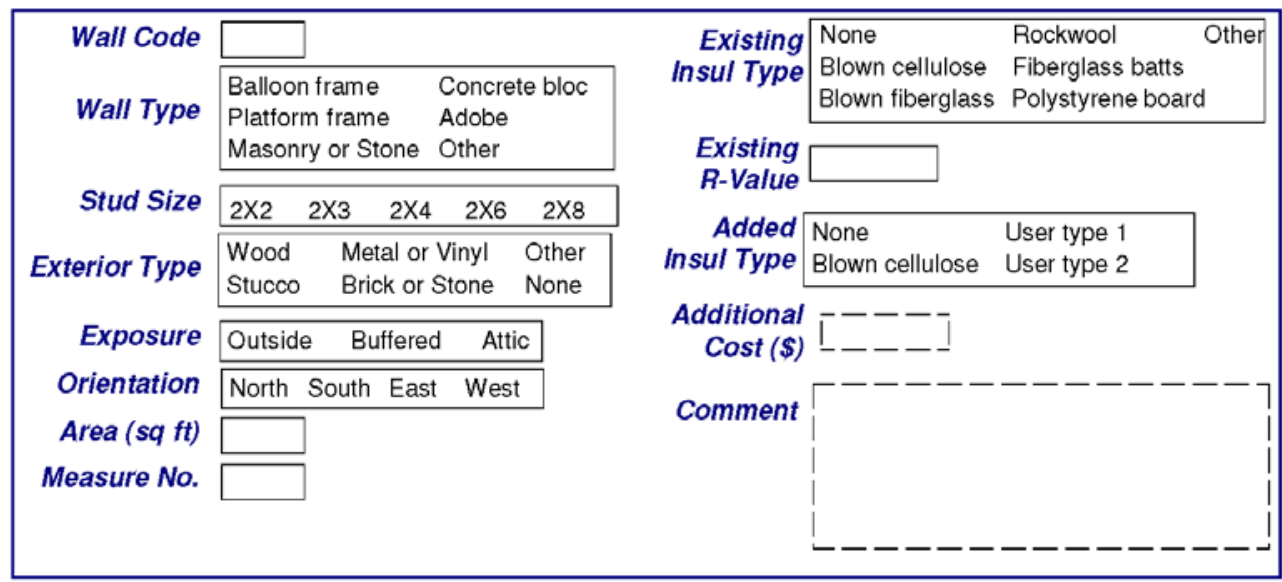

Client Name:
Client ID:
Alt. Client ID:

NEAT Data Collection Form

Form Run On: 11/2/2011
DOE Weatherization Assistant

Version 8.9.0

Page 3 of 42 


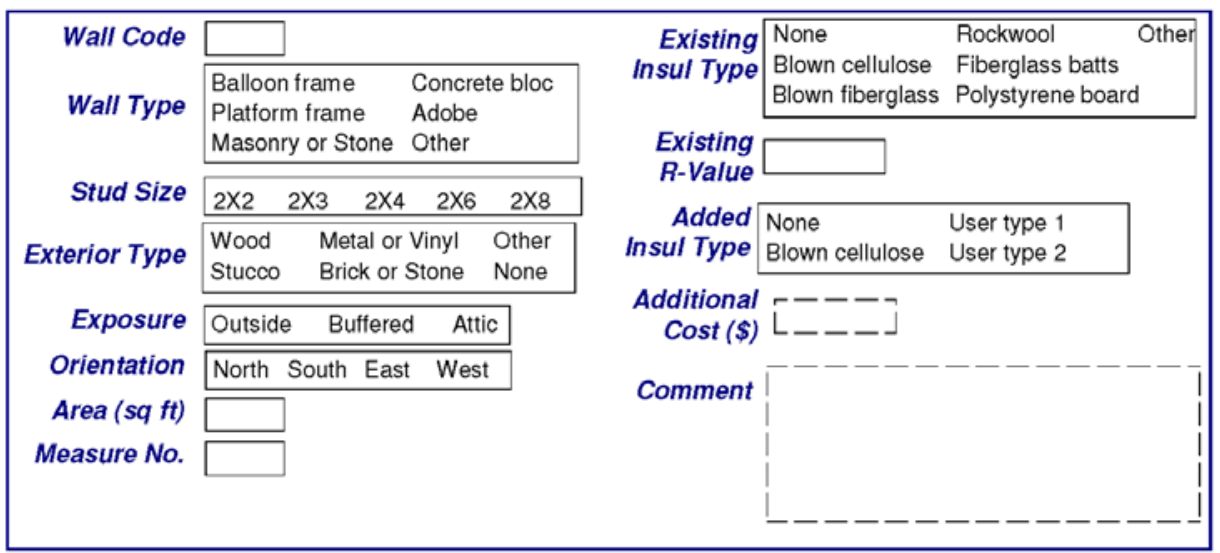

Notes :

\begin{tabular}{|c|c|c|}
\hline Client Name: & NEAT Data Collection Form & DOE Weatherization Assistant \\
\hline $\begin{array}{r}\text { Client ID: } \\
\text { Alt Client ID. }\end{array}$ & Form Run On: 11/2/2011 & $\begin{array}{l}\text { Version } 8.9 .0 \\
\text { Page } 4 \text { of } 42\end{array}$ \\
\hline
\end{tabular}

Two additional sets of Walls input forms exist in the NEAT Data Collection Forms. 


\section{Windows}

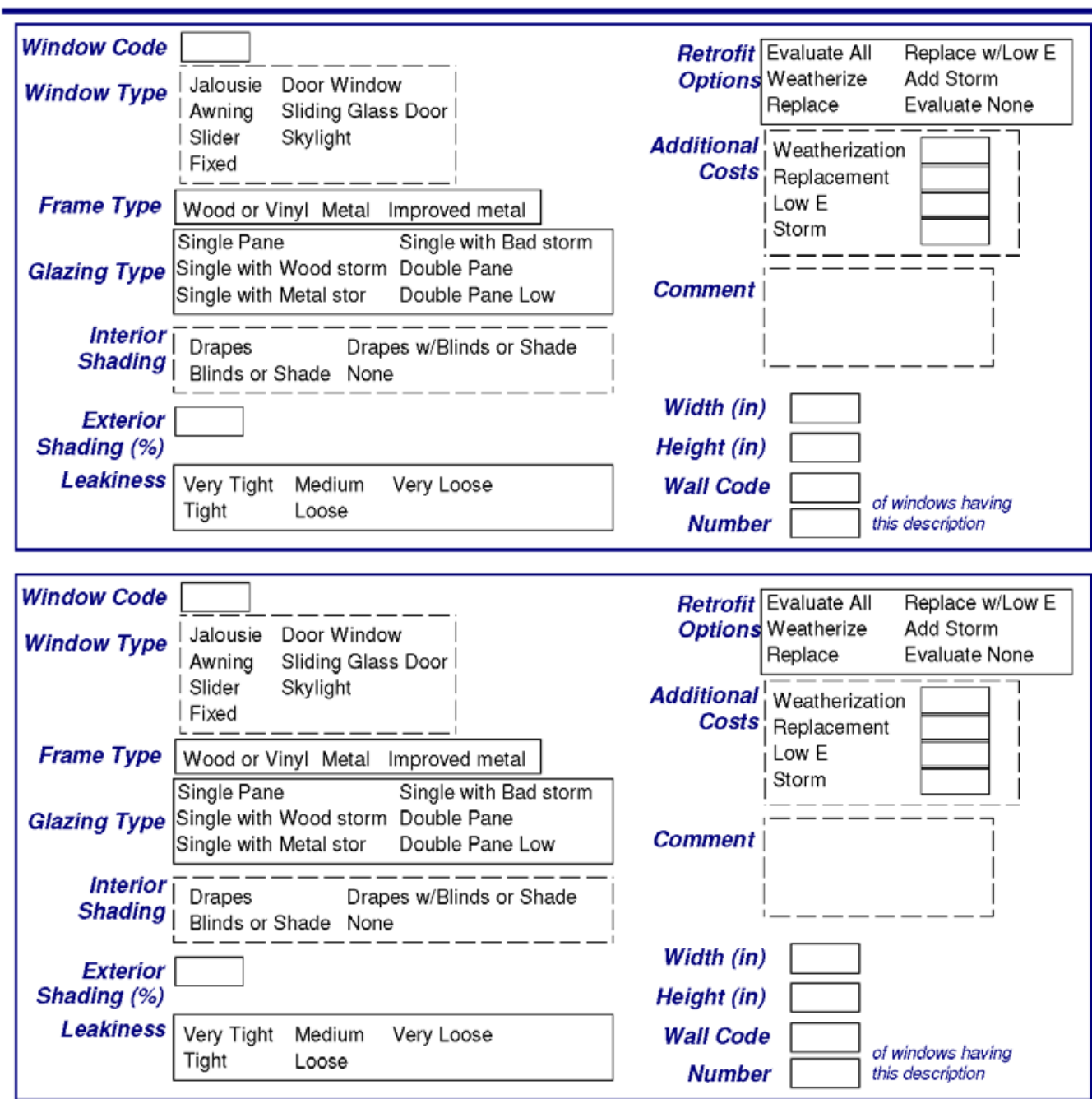

Client Name:
Client ID:
Alt. Client ID:

NEAT Data Collection Form

Form Run On: 11/2/2011
DOE Weatherization Assistant

Version 8.9.0

Page 9 of 42 


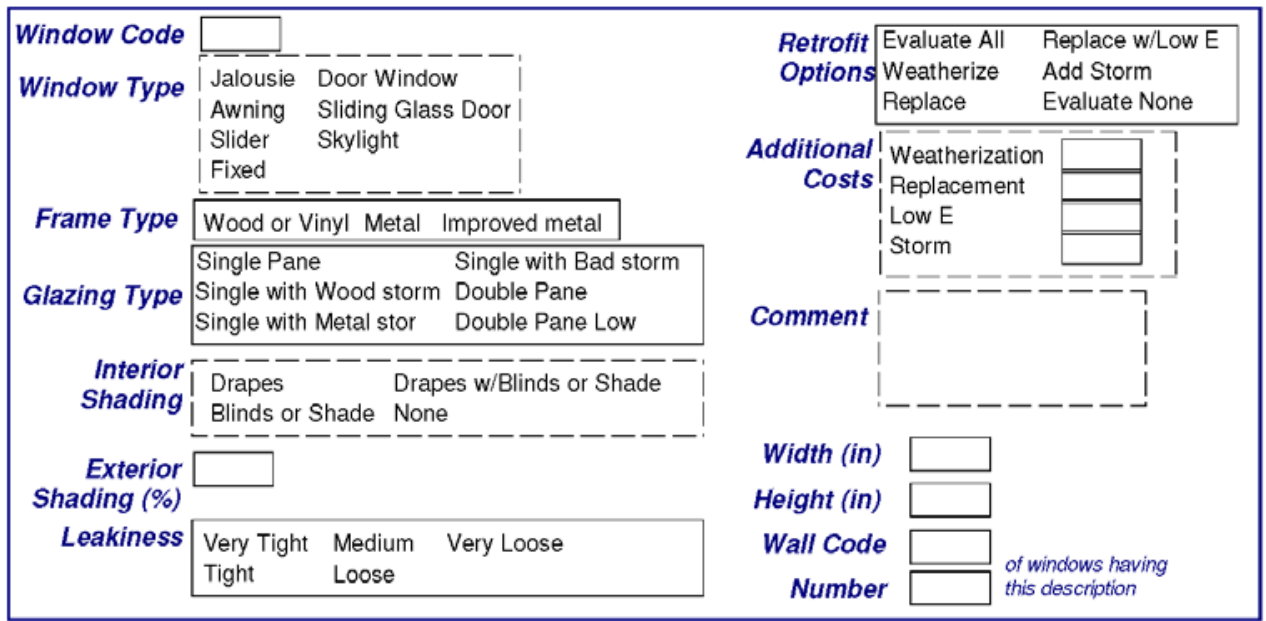

Notes :

\begin{tabular}{|c|c|c|}
\hline Client Name: & \multirow{2}{*}{$\begin{array}{l}\text { NEAT Data Collection Form } \\
\text { Form Run On: 11/2/2011 }\end{array}$} & \multirow{2}{*}{$\begin{array}{r}\text { DOE Weatherization Assistant } \\
\text { Version } 8.9 .0 \\
\text { Page } 10 \text { of } 42\end{array}$} \\
\hline Client ID: & & \\
\hline
\end{tabular}

Two additional sets of Windows input forms exist in the NEAT Data Collection Forms. 


\section{Doors}

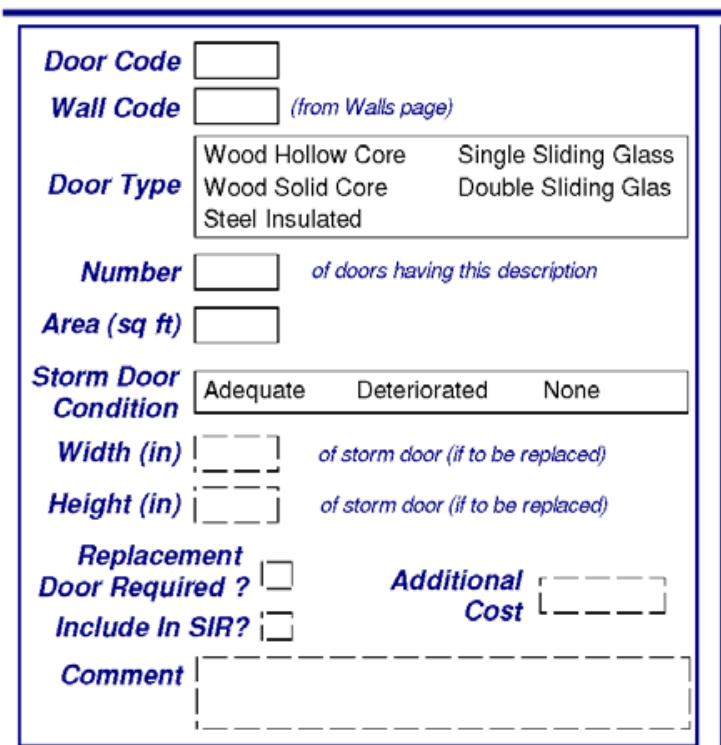

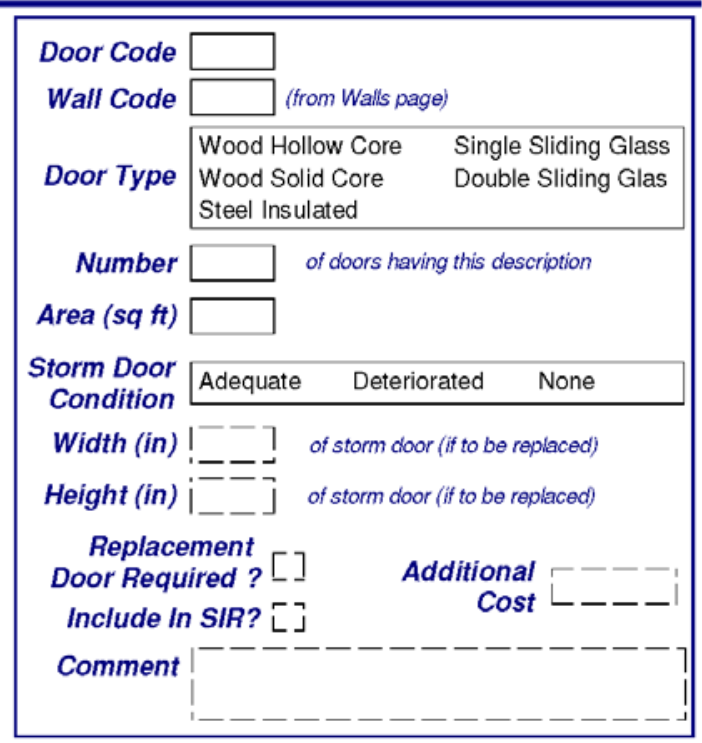

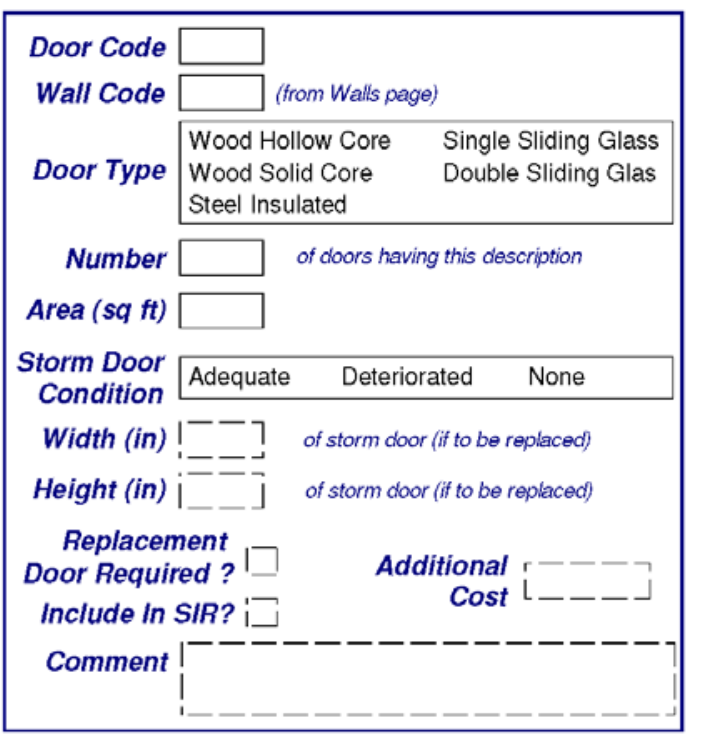

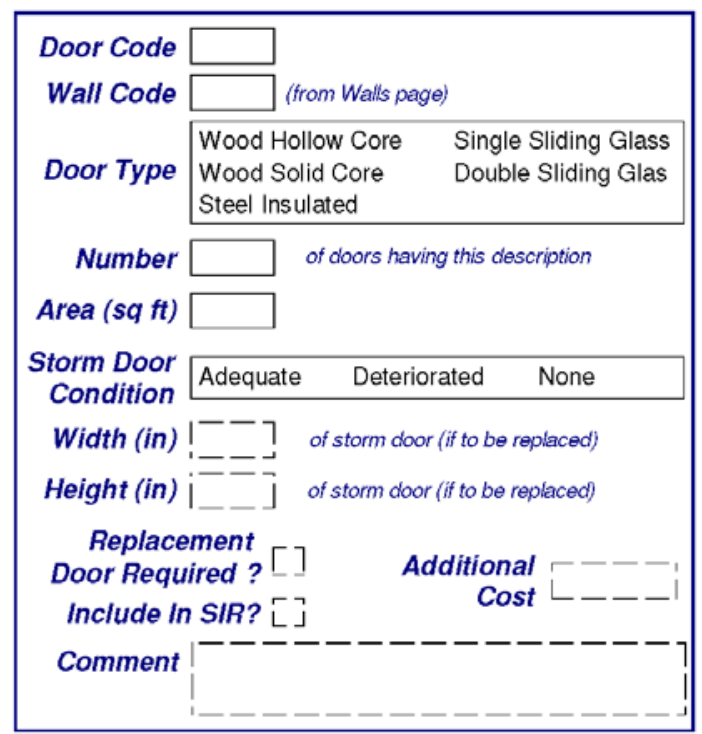

\section{Notes:}

\begin{tabular}{l}
\hline Client Name: \\
Client ID: \\
Att. Client ID:
\end{tabular}

NEAT Data Collection Form
Form Run On: 11/2/2011
DOE Weatherization Assistant

Version 8.9 .0

Page 15 of 42 


\section{Appendix C: Reports}

\section{Unfinished Attics}
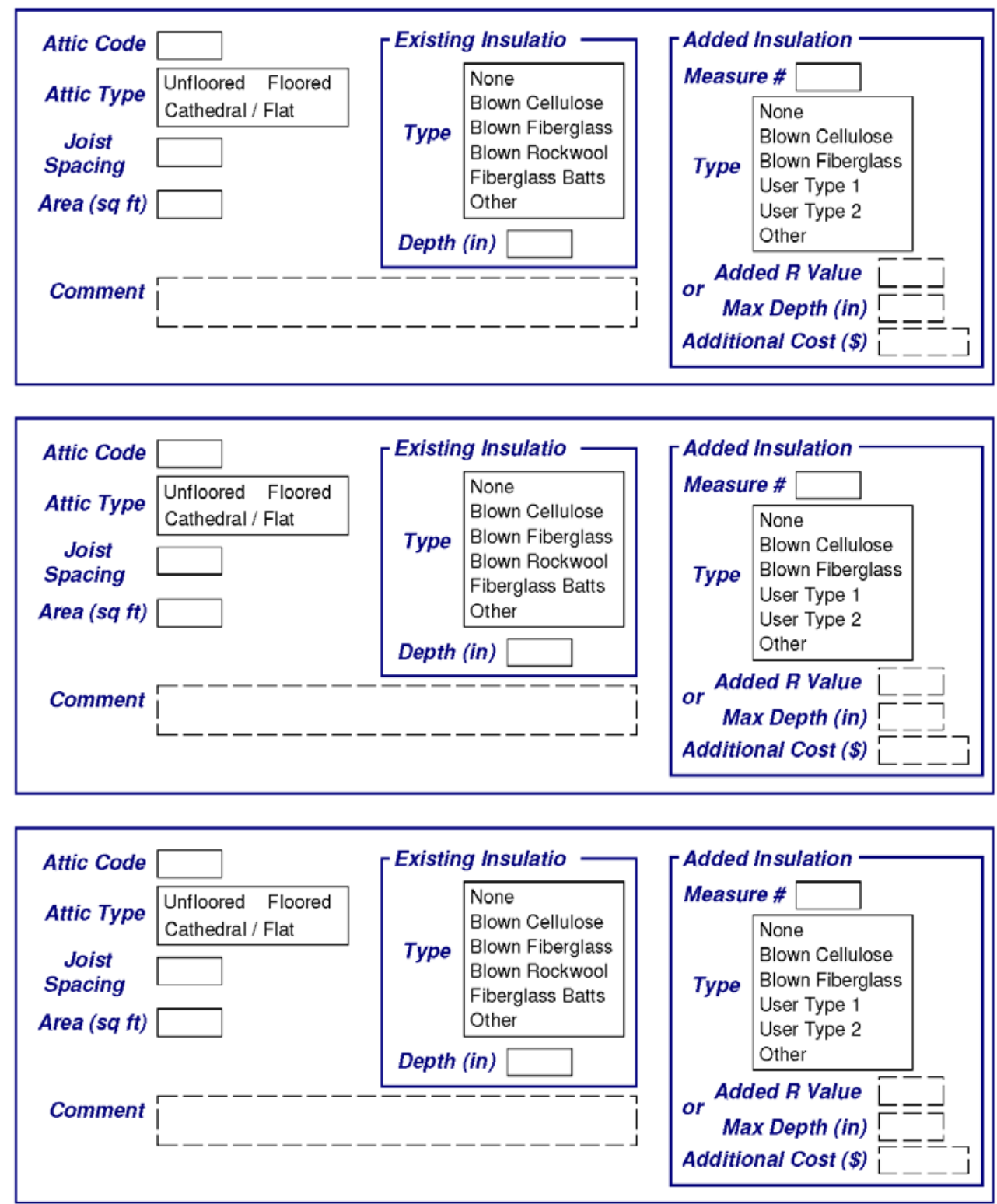

\section{Notes :}

\begin{tabular}{|c|c|c|}
\hline Client Name: & NEAT Data Collection Form & DOE Weatherization Assistant \\
\hline Client ID: & Form Run On: 11/2/2011 & Version 8.9 .0 \\
\hline Alt. Client ID: & & Page 16 of 42 \\
\hline
\end{tabular}


Finished Attics

\begin{tabular}{|c|c|c|c|c|c|}
\hline Attic Code & & \multicolumn{2}{|c|}{ [Existing Insulation } & Added Insulation & Type \\
\hline $\begin{array}{c}\text { Attic } \\
\text { Area Type }\end{array}$ & \begin{tabular}{|l} 
Outer Ceiling Jois \\
Collar Beam \\
Kneewall \\
Roof Rafter \\
\end{tabular} & Type & \begin{tabular}{|l} 
None \\
Blown Cellulose \\
Blown Fiberglass \\
Rockwool
\end{tabular} & $\begin{array}{l}\text { Measure \# } \square \\
\text { Added } R \text { Value } L_{-}--i\end{array}$ & $\begin{array}{l}\text { None } \\
\text { Blown Cellulose } \\
\text { Blown Fiberglass } \\
\text { Bockwn }\end{array}$ \\
\hline $\begin{array}{l}\text { Attic } \\
\text { Floor }\end{array}$ & \begin{tabular}{|l} 
Unfloored \\
Floored
\end{tabular} & & $\begin{array}{l}\text { Fiberglass Batts } \\
\text { Other }\end{array}$ & $\begin{array}{c}\text { Max } \\
\text { Depth (in) }\end{array}$ & $\begin{array}{l}\text { Fiberglass Batts } \\
\text { Other }\end{array}$ \\
\hline Area (sq ft) & & Depth & (in) & Additional Cost (\$) & --7 \\
\hline
\end{tabular}

\begin{tabular}{|c|c|c|c|c|c|}
\hline Attic Code & & \multicolumn{2}{|c|}{ [Existing Insulation - } & \multicolumn{2}{|c|}{ - Added Insulation Type } \\
\hline $\begin{array}{c}\text { Attic } \\
\text { Area Type }\end{array}$ & \begin{tabular}{|l} 
Outer Ceiling Jois \\
Collar Beam \\
Kneewall \\
Roof Rafter \\
\end{tabular} & Type & \begin{tabular}{|l|} 
None \\
Blown Cellulose \\
Blown Fiberglass \\
Rockwool
\end{tabular} & Measure \# $\square$ & $\begin{array}{l}\text { None } \\
\text { Blown Cellulose } \\
\text { Blown Fiberglass } \\
\text { Rockwool }\end{array}$ \\
\hline $\begin{array}{l}\text { Attic } \\
\text { Floor }\end{array}$ & \begin{tabular}{|l|} 
Unfloored \\
Floored
\end{tabular} & & $\begin{array}{l}\text { Fiberglass Batts } \\
\text { Other }\end{array}$ & Depth (in) ${ }_{---}$ & $\begin{array}{l}\text { Fiberglass Batts } \\
\text { Other }\end{array}$ \\
\hline $\operatorname{Area}(s q f t)$ & & Depth & (in) & Additional Cost (\$) & $-1-7$ \\
\hline
\end{tabular}

\begin{tabular}{|c|c|c|c|c|c|}
\hline Attic Code & & \multicolumn{2}{|c|}{ [ Existing Insulation - } & Added Insulation & Type \\
\hline $\begin{array}{c}\text { Attic } \\
\text { Area Type }\end{array}$ & \begin{tabular}{|l} 
Outer Ceiling Jois \\
Collar Beam \\
Kneewall \\
Roof Rafter \\
\end{tabular} & Type & \begin{tabular}{|l} 
None \\
Blown Cellulose \\
Blown Fiberglass \\
Rockwool
\end{tabular} & Measure \# & \begin{tabular}{|l} 
None \\
Blown Cellulose \\
Blown Fiberglass \\
Rockwool
\end{tabular} \\
\hline $\begin{array}{l}\text { Attic } \\
\text { Floor }\end{array}$ & \begin{tabular}{|l} 
Unfloored \\
Floored
\end{tabular} & & $\begin{array}{l}\text { Fiberglass Batts } \\
\text { Other }\end{array}$ & $\begin{array}{c}\text { Max } \\
\text { Depth (in) }\end{array}$ & $\begin{array}{l}\text { Fiberglass Batts } \\
\text { Other }\end{array}$ \\
\hline $\operatorname{Area}(s q f t)$ & & Depth & (in) & Additional Cost (\$) & -7 \\
\hline
\end{tabular}

\begin{tabular}{|c|c|c|c|c|c|}
\hline Attic Code & & \multirow{3}{*}{\multicolumn{2}{|c|}{\begin{tabular}{l} 
Existing Insulation \\
$\qquad$\begin{tabular}{|l} 
Type \\
Bone \\
Blown Cellulose \\
Blown Fiberglass \\
Rockwool \\
Fiberglass Batts \\
Other
\end{tabular} \\
\cline { 2 - 3 }
\end{tabular}}} & \multirow{3}{*}{$\begin{array}{l}\text { Added Insulation } \\
\text { Measure \# } \\
\text { Added R Value } \\
\text { or Max } \\
\text { Depth (in) }\end{array}$} & \multirow{3}{*}{$\begin{array}{l}\text { Type } \\
\begin{array}{|l}\text { None } \\
\text { Blown Cellulose } \\
\text { Blown Fiberglass } \\
\text { Rockwool } \\
\text { Fiberglass Batts } \\
\text { Other }\end{array} \\
\end{array}$} \\
\hline $\begin{array}{c}\text { Attic } \\
\text { Area Type }\end{array}$ & $\begin{array}{l}\text { Outer Ceiling Jois } \\
\text { Collar Beam } \\
\text { Kneewall } \\
\text { Roof Rafter }\end{array}$ & & & & \\
\hline $\begin{array}{l}\text { Attic } \\
\text { Floor }\end{array}$ & $\begin{array}{l}\text { Unfloored } \\
\text { Floored }\end{array}$ & & & & \\
\hline $\operatorname{Area}(s q f t)$ & & Depth & (in) & Additional Cost (\$) & -7 \\
\hline
\end{tabular}

Client Name:
Client ID:
Att. Client ID:

NEAT Data Collection Form
Form Run On: 11/2/2011
DOE Weatherization Assistant

Version 8.9 .0

Page 17 of 42 


\section{Finished Attics}

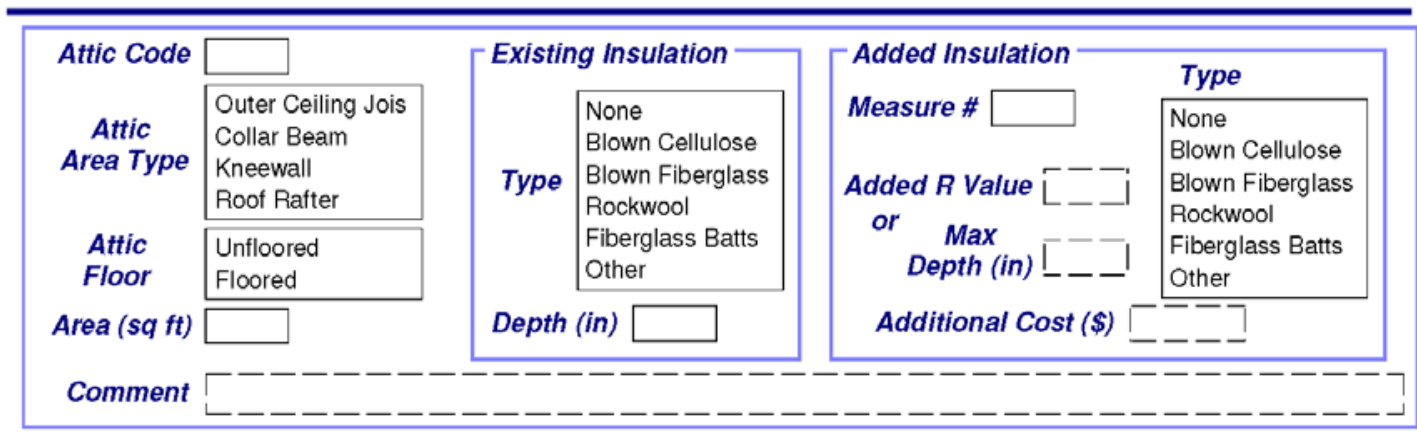

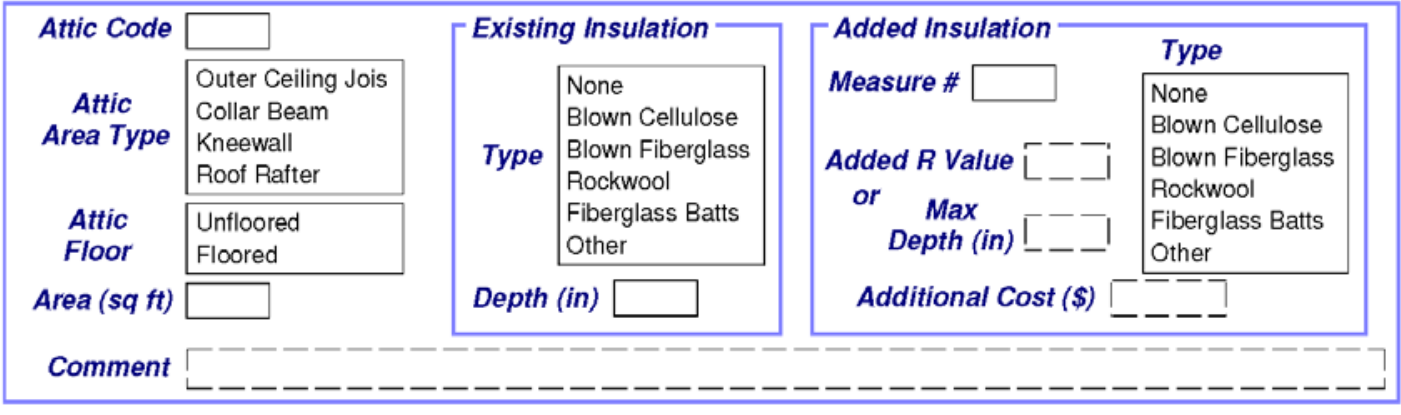

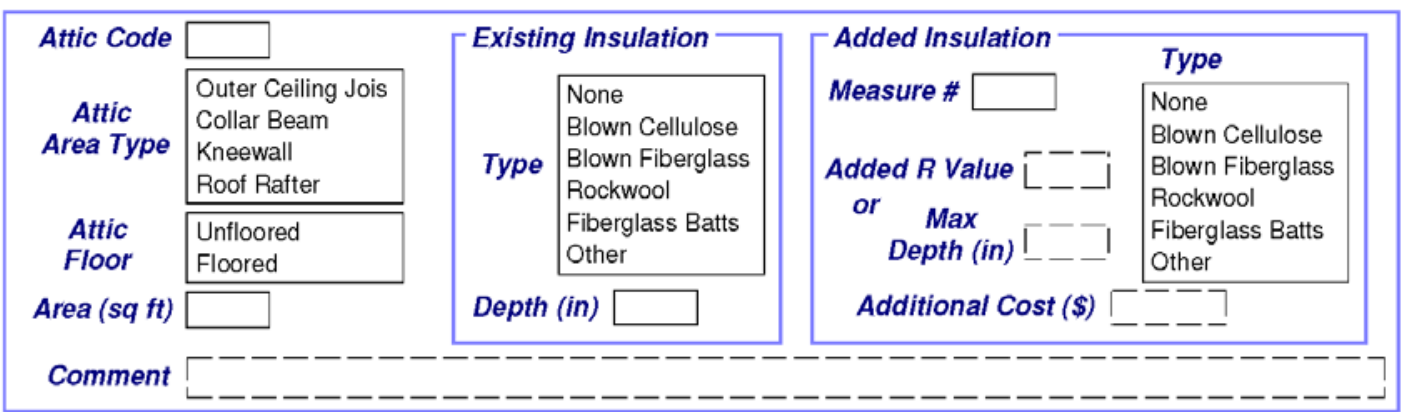

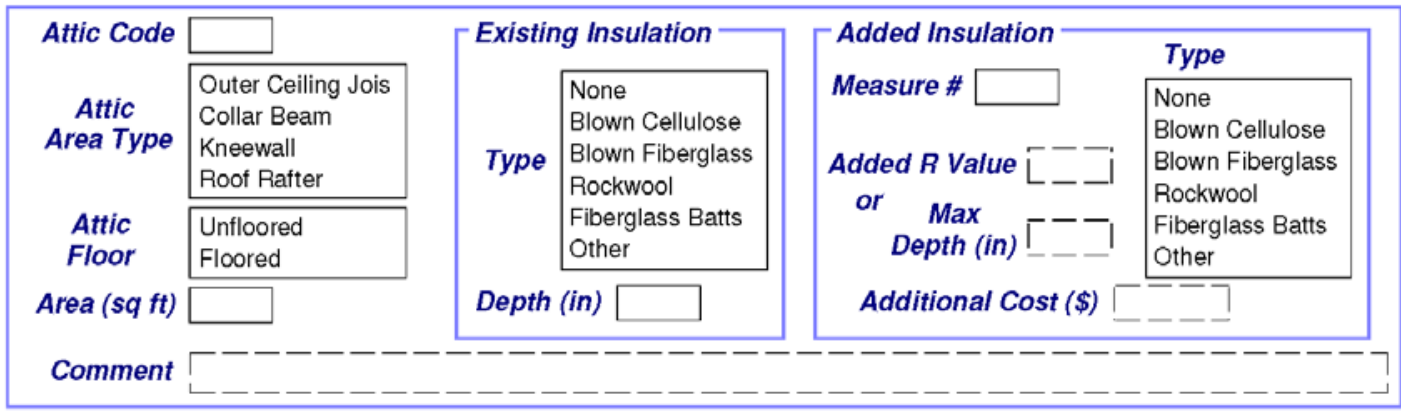

Client Name:
Client ID:
Att. Client ID:

NEAT Data Collection Form

Form Run On: 11/2/2011
DOE Weatherization Assistant

Version 8.9.0

Page 18 of 42 


\section{Foundations}

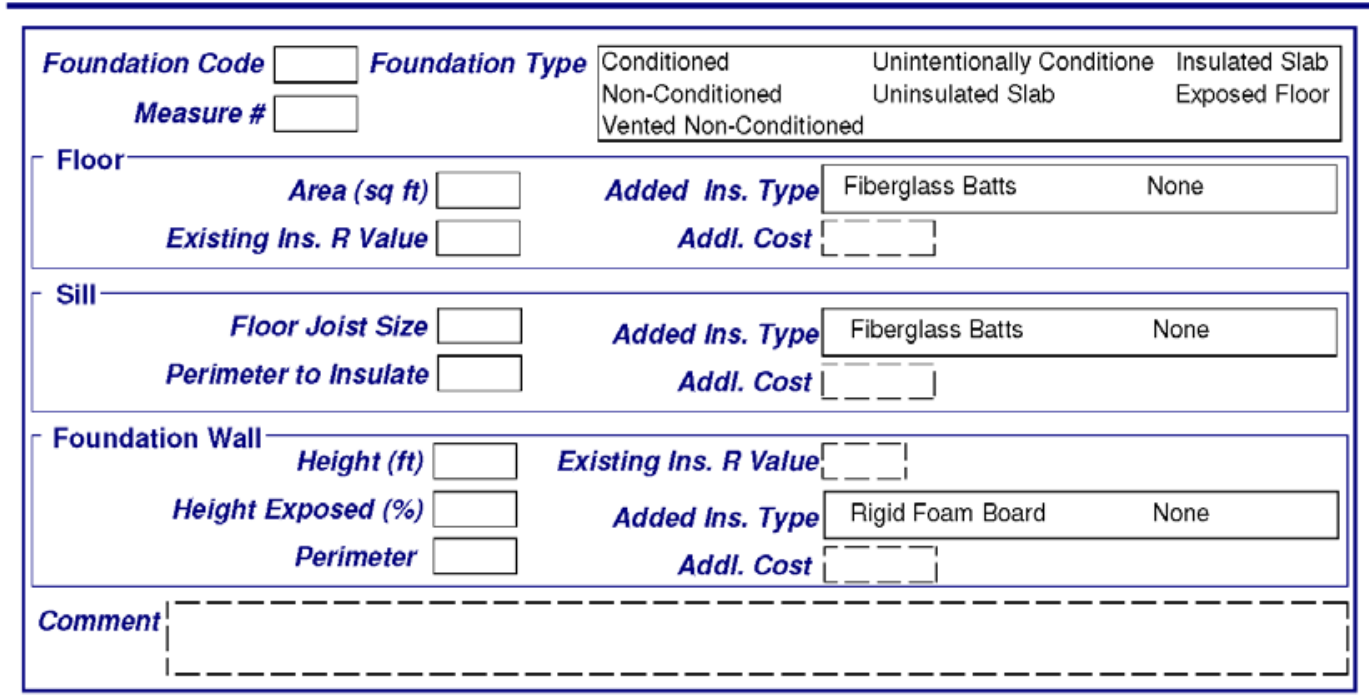

\begin{tabular}{|c|c|c|}
\hline $\begin{array}{r}\text { Foundation Code } \\
\text { Measure \# }\end{array}$ & \begin{tabular}{|l|l|} 
Foundation Type & $\begin{array}{l}\text { Conditioned } \\
\text { Non-Conditioned } \\
\text { Vented Non-Conditione }\end{array}$ \\
\end{tabular} & $\begin{array}{ll}\begin{array}{l}\text { Unintentionally Conditione } \\
\text { Uninsulated Slab }\end{array} & \text { Insulated Slab } \\
\text { d } & \text { Exposed Floor } \\
\end{array}$ \\
\hline [ Floor- & $(s q f t)[$ & $\begin{array}{ll}\text { Added Ins. Type Fiberglass Batts } \\
\end{array}$ \\
\hline \multicolumn{2}{|c|}{ Existing Ins. $R$ Value } & Addl. Cost $\begin{array}{l}\mathrm{L}_{-}-\square \\
\end{array}$ \\
\hline \multicolumn{3}{|l|}{ Sill- } \\
\hline \multicolumn{2}{|c|}{ Floor Joist Size [ } & Added Ins. Type Fiberglass Batts \\
\hline \multicolumn{2}{|c|}{ Perimeter to Insulate } & Addl. Cost \\
\hline \multicolumn{3}{|l|}{ Foundation Wall- } \\
\hline \multicolumn{2}{|c|}{ Height Exposed (\%) } & Added Ins. Type Rigid Foam Board \\
\hline \multicolumn{2}{|c|}{ Perimeter } & Addl. Cost $\mathrm{L}_{--}$ \\
\hline \multicolumn{3}{|c|}{ 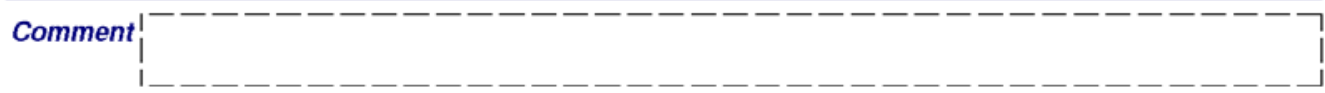 } \\
\hline
\end{tabular}

Client Name:
Client ID:
Alt. Client ID:

NEAT Data Collection Form
Form Run On: $11 / 2 / 2011$
DOE Weatherization Assistant

Version 8.9.0

Page 19 of 42 


\section{Appendix C: Reports}

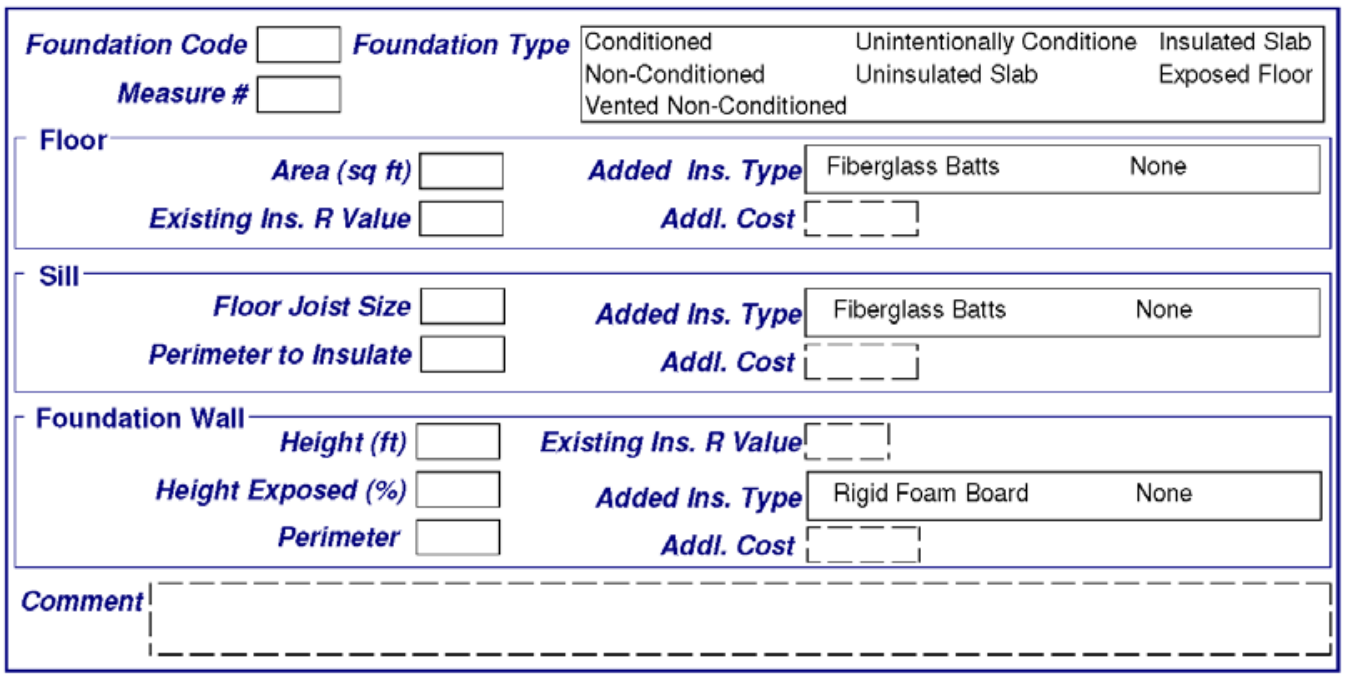

Notes:

Client Name:
Client ID:
Att. Client ID:

NEAT Data Collection Form

Form Run On: 11/2/2011
DOE Weatherization Assistant

Version 8.9.0

Page 20 of 42 


\section{Heating Systems}

\begin{tabular}{|c|c|c|c|c|c|c|}
\hline \multirow[t]{3}{*}{ System Code } & & & & & Primary Sys & tem $\square$ \\
\hline & \multirow{5}{*}{\multicolumn{2}{|c|}{$\begin{array}{l}\text { Gravity Furnace } \\
\text { Forced Air Furnace } \\
\text { Steam Boiler } \\
\text { Hot Water Boiler } \\
\text { Fixed Electric Resistance } \\
\text { Portable Electric Resistance } \\
\text { Heat Pump } \\
\text { Vented Space Heater } \\
\text { Unvented Space Heater } \\
\text { Other }\end{array}$}} & \multicolumn{2}{|c|}{ 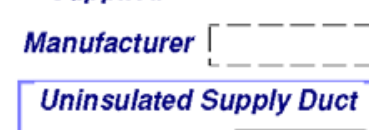 } & & \\
\hline & & & \multirow{5}{*}{\multicolumn{2}{|c|}{\begin{tabular}{|r|} 
Uninsulated Supply Duct \\
Type \\
Round \\
Rectangula \\
Length (ft) \\
Width (in) \\
Height (in)
\end{tabular}}} & \multirow{4}{*}{\multicolumn{2}{|c|}{$\begin{aligned} & \text { Unin sulated Supply Duct } \\
& \text { Type } \begin{array}{l}\text { Round } \\
\text { Rectangula }\end{array} \\
& \text { Length (ft) } \\
& \text { Width (in) } \\
& \text { Height (in) }\end{aligned}$}} \\
\hline \multirow[t]{5}{*}{ Equipment Type } & & & & & & \\
\hline & & & & & & \\
\hline & & & & & & \\
\hline & \multirow{3}{*}{$\begin{array}{l}\text { Natural Ga } \\
\text { Electricity } \\
\text { Wood } \\
\text { Kerosene } \\
\end{array}$} & & & & \multirow{3}{*}{$\begin{array}{l}\text { Diameter (in) } \\
\quad \text { Location }\end{array}$} & \\
\hline & & Propane & Diameter (in) & & & \\
\hline Fuel & & $\begin{array}{l}\text { Coal } \\
\text { Other }\end{array}$ & Location & $\begin{array}{l}\text { Attic } \\
\text { Subspace }\end{array}$ & & \begin{tabular}{|l|} 
Attic \\
Subspace
\end{tabular} \\
\hline Location & \multicolumn{2}{|c|}{$\begin{array}{l}\text { Heated Space } \\
\text { Unconditioned Space } \\
\text { Unintentionally Heated Space }\end{array}$} & Comment & & & \\
\hline
\end{tabular}

HEATING SYSTEM DETAILS * Equipment Type and Fuel Determine which fields are required

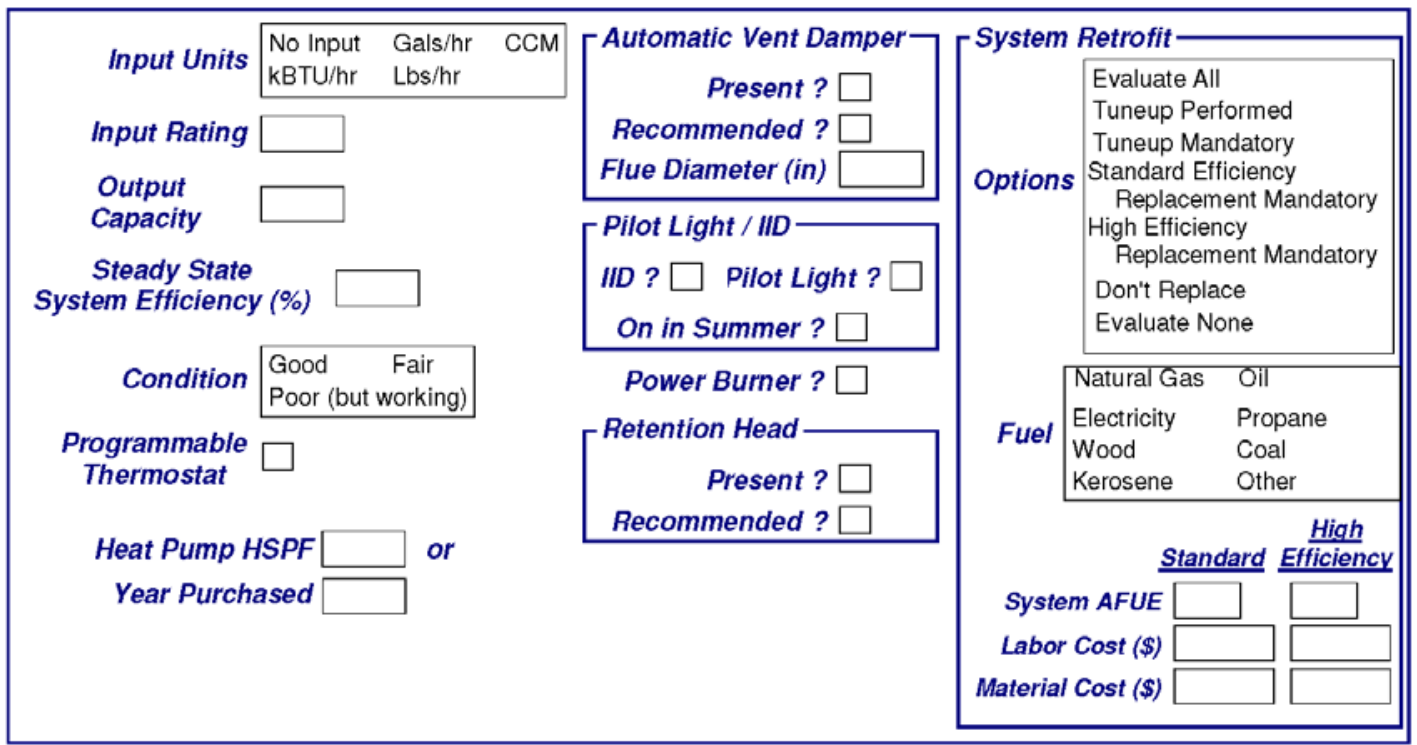

\section{Notes :}

Client Name:
Client ID:
Att. Client ID:

NEAT Data Collection Form
Form Run On: $11 / 2 / 2011$
DOE Weatherization Assistant

Version 8.9 .0

Page 21 of 42 
Heating Systems (Continued)

OPTIONAL HEATING SYSTEM DETAILS

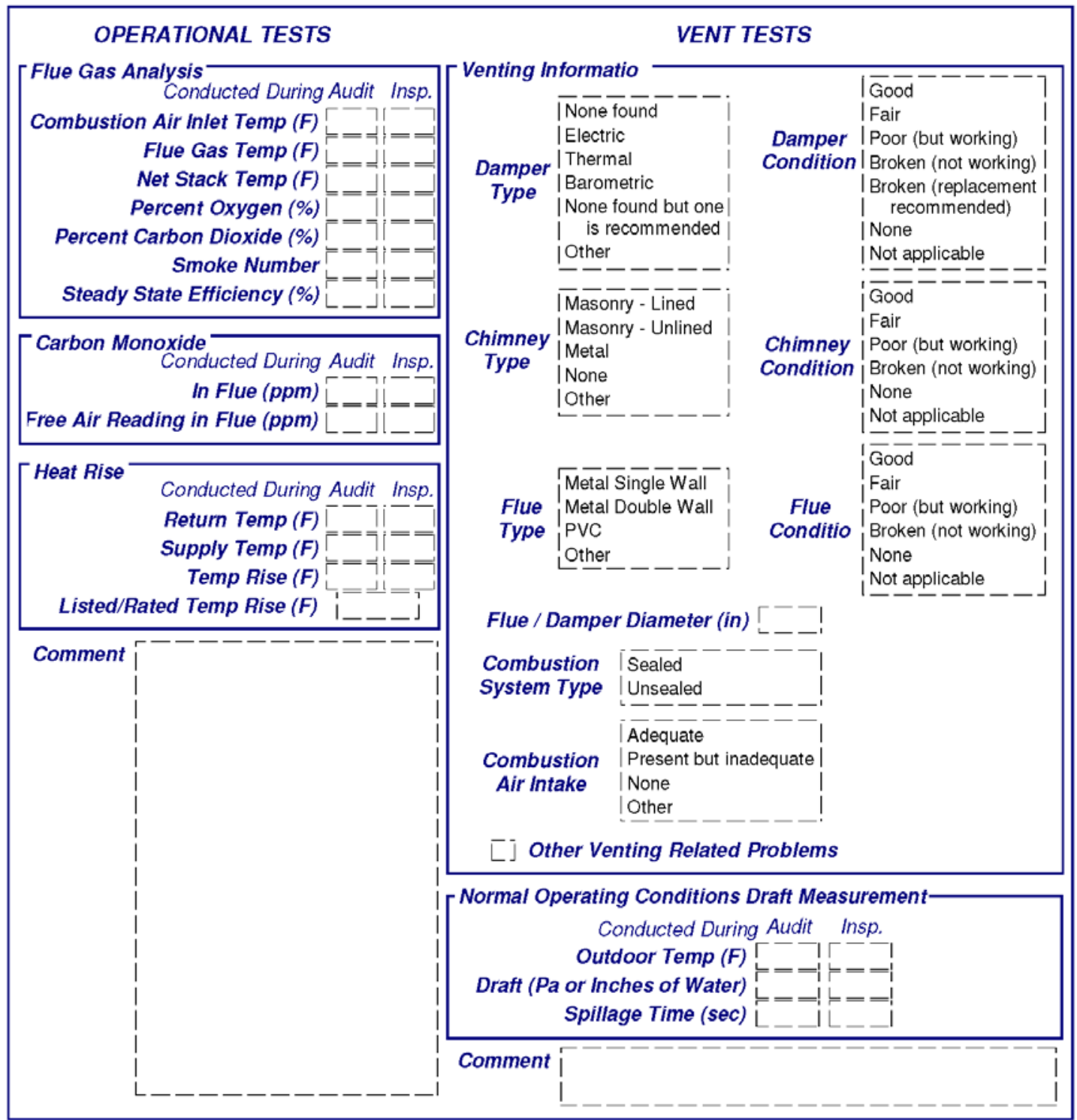

Notes:

Client Name:
Client ID:
Alt. Client ID:

NEAT Data Collection Form

Form Run On: 11/2/2011
DOE Weatherization Assistant

Version 8.9 .0

Page 22 of 42 


\section{Heating Systems (Continued)}

\section{OPTIONAL HEATING SYSTEM DETAILS (Continued)}

\begin{tabular}{|c|c|}
\hline$\left[\begin{array}{l}\text { Fan Limit Controls } \\
\sqsubset\urcorner \text { Control Settings are Adjustable } \\
\lceil\rceil \text { Limit Controls Not Working }\end{array}\right.$ & $\begin{array}{l}\text { Setting }(F) \longleftarrow=\dashv \\
\text { f Setting }(F) \longleftarrow=\rfloor \\
\text { Setting }(F) !\end{array}$ \\
\hline 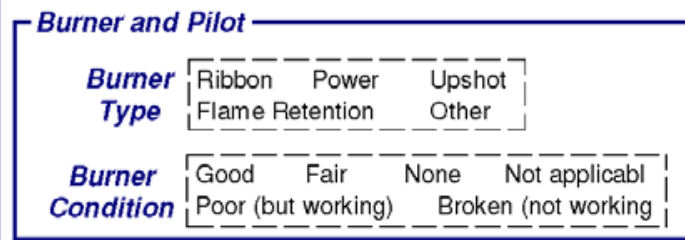 & 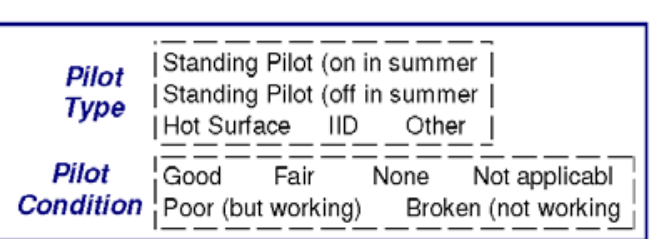 \\
\hline 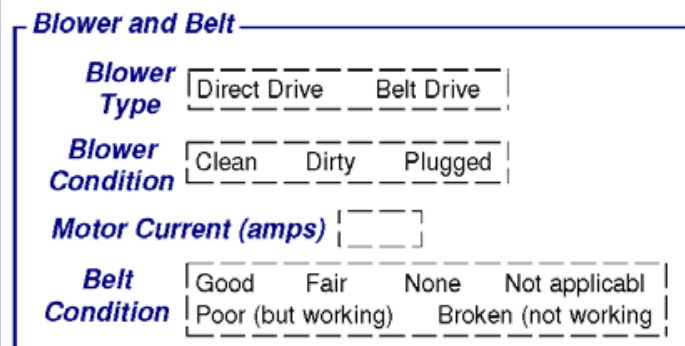 & 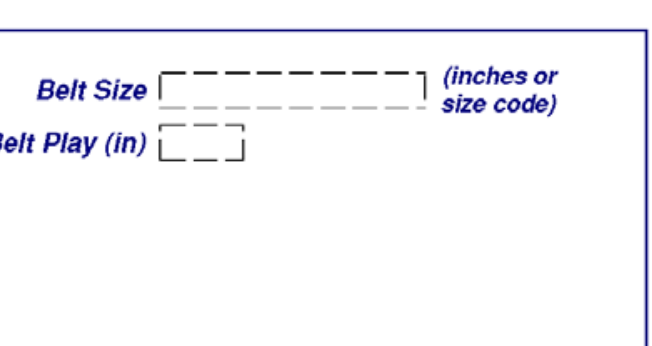 \\
\hline 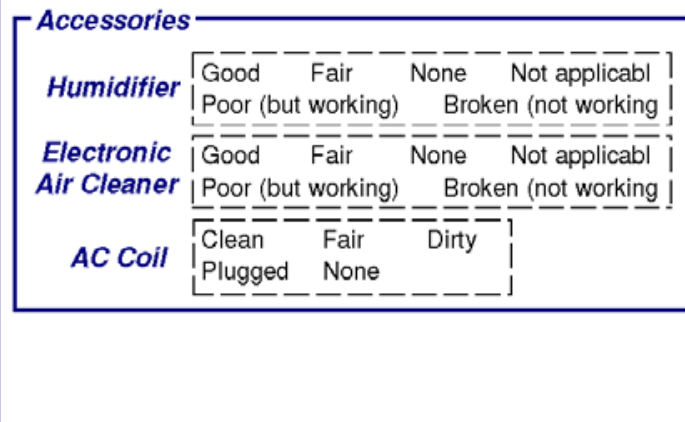 & 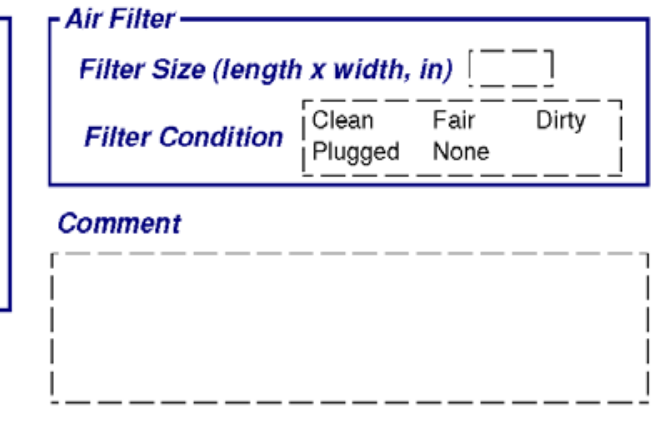 \\
\hline
\end{tabular}

\section{Notes :}

Client Name:
Client ID:
Alt. Client ID:

NEAT Data Collection Form

Form Run On: 11/2/2011
DOE Weatherization Assistant

Version 8.9 .0

Page 23 of 42 


\section{Appendix C: Reports}

\section{Heating Systems (Continued)}

OPTIONAL HEATING SYSTEM DETAILS (Continued)

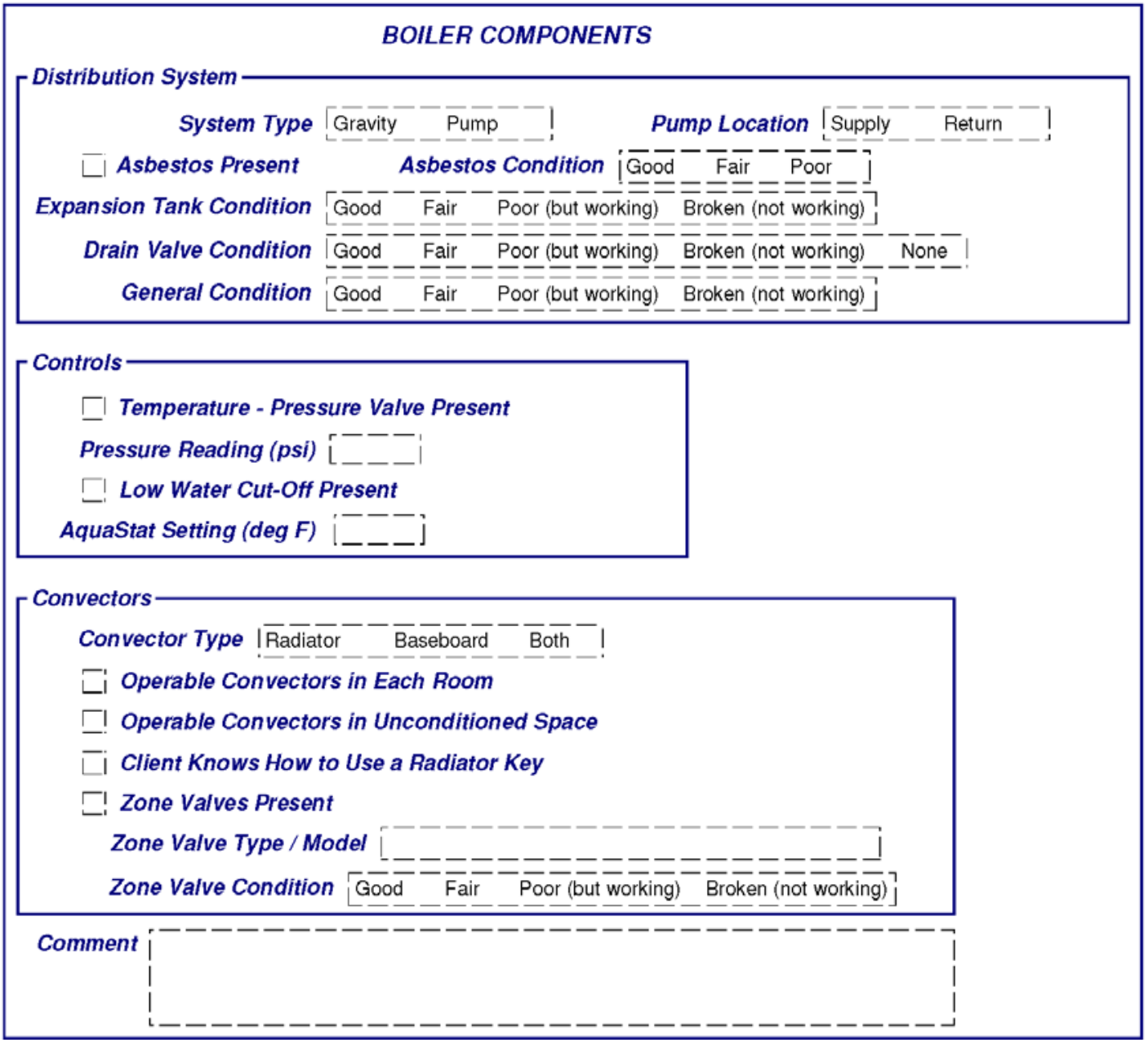

Notes:

Client Name:
Client ID:
Att. Client ID:

NEAT Data Collection Form

Form Run On: 11/2/2011
DOE Weatherization Assistant

Version 8.9 .0

Page 24 of 42 


\section{Heating Systems (Continued)}

\section{OPTIONAL HEATING SYSTEM DETAILS (Continued)}

\begin{tabular}{|c|c|}
\hline 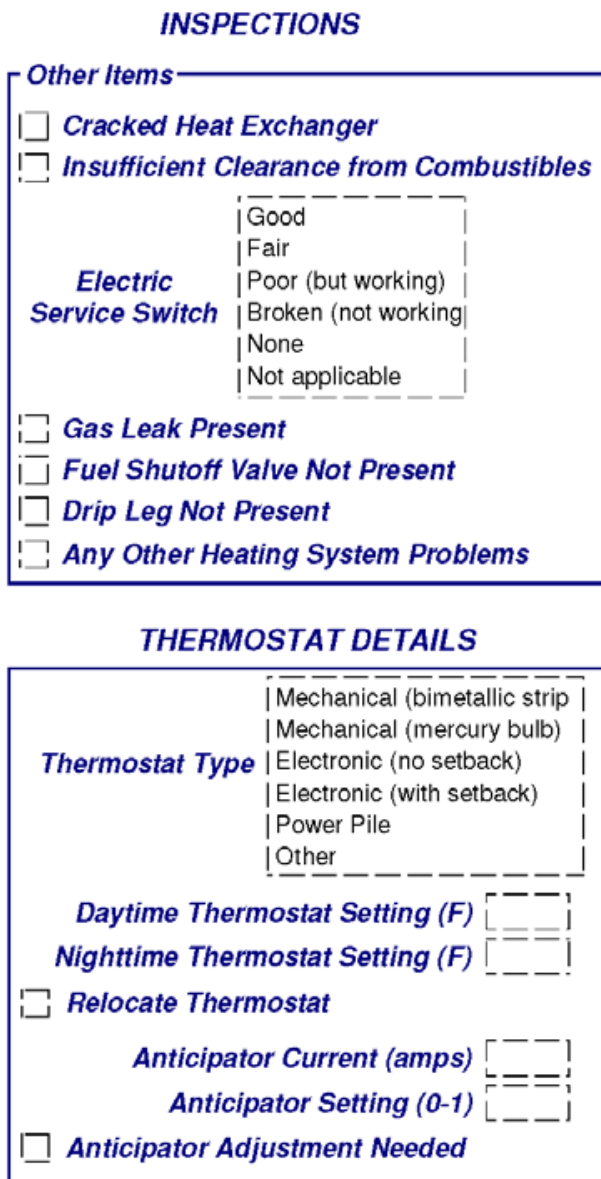 & $\left\{\begin{array}{l}\text { Comment } \\
\mid \begin{array}{r}\mid \\
---------------1\end{array}\end{array}\right.$ \\
\hline
\end{tabular}

Notes :

Client Name:
Client ID:
Att. Client ID:




\section{Appendix C: Reports}

Cooling

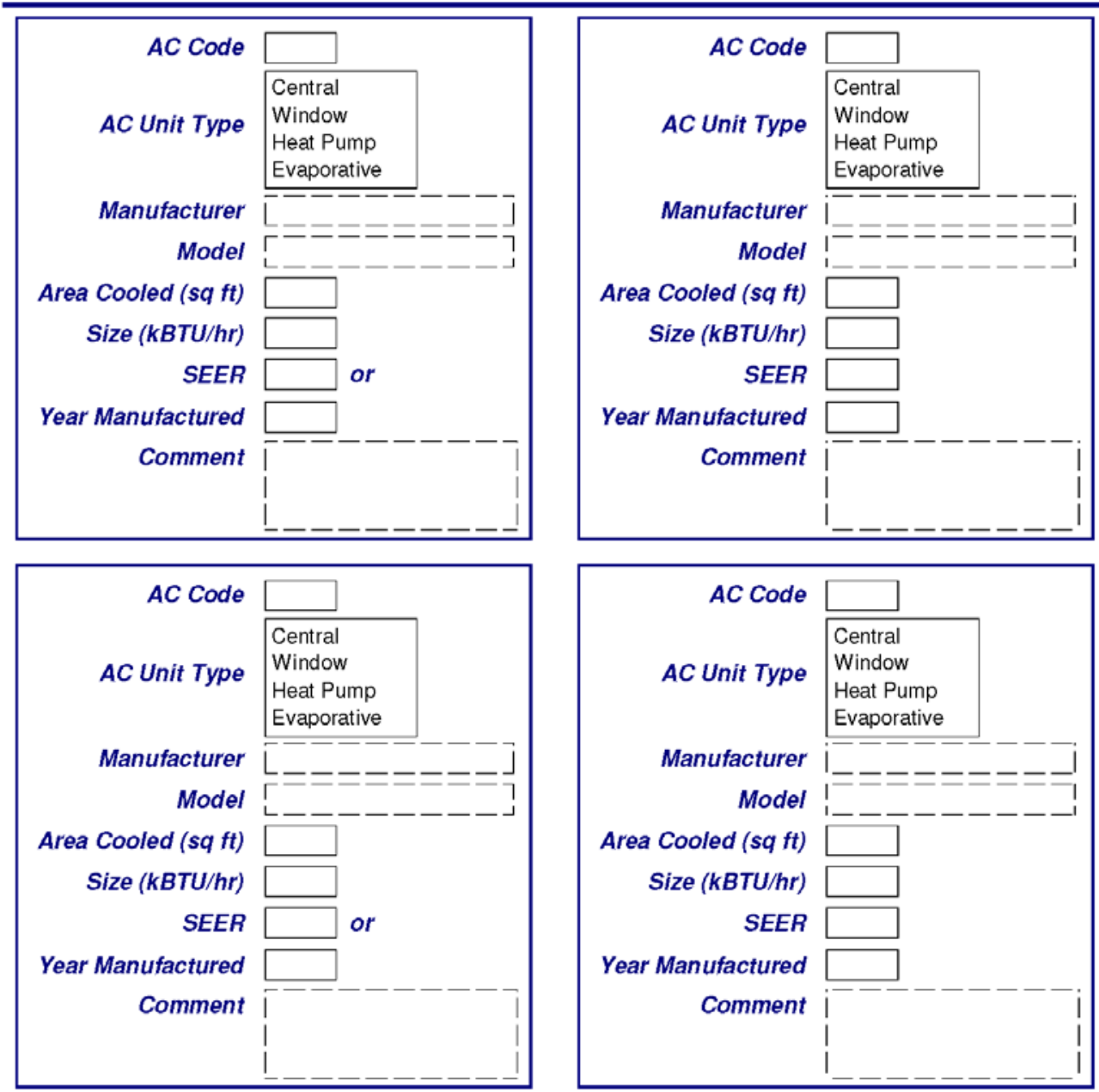

Notes :

Client Name:
Client ID:
Att. Client ID:

NEAT Data Collection Form

Form Run On: 11/2/2011
DOE Weatherization Assistant

Version 8.9.0

Page 26 of 42 
Ducts / Infiltration

\section{AIR and DUCT LEAKAGES}

Evaluate Duct Sealing?

Duct Leakage

Whole House Blower Door Measurement

Method

Blower Door Subtraction (sealed and unsealed registeres and grills)

Duct-Blower Measurements
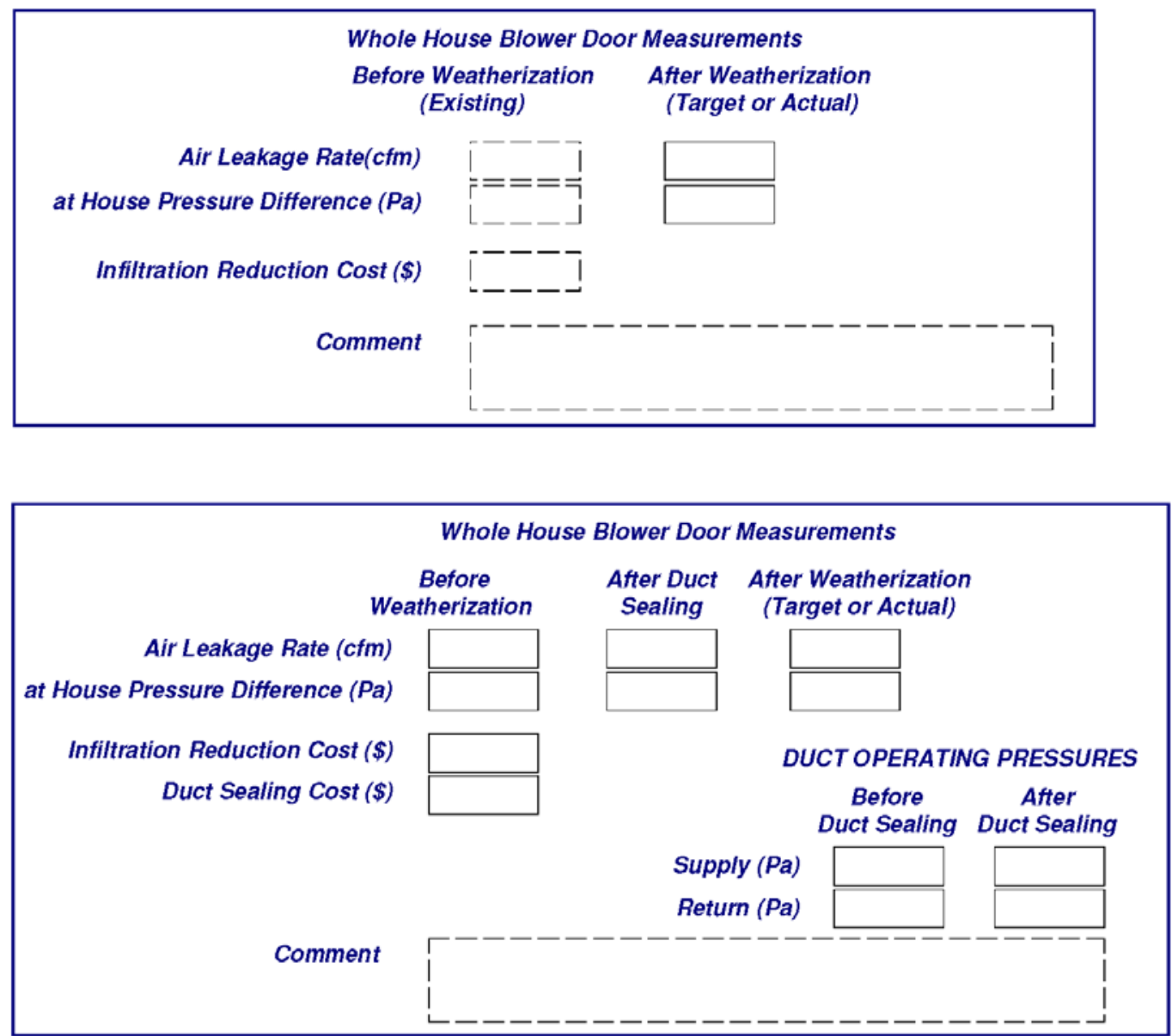

Client Name:
Client ID:
Att. Client ID:

NEAT Data Collection Form

Form Run On: 11/2/2011
DOE Weatherization Assistant

Version 8.9.0

Page 27 of 42 
Ducts / Infiltration (Continued)

\section{AlR and DUCT LEAKAGES (Continued)}

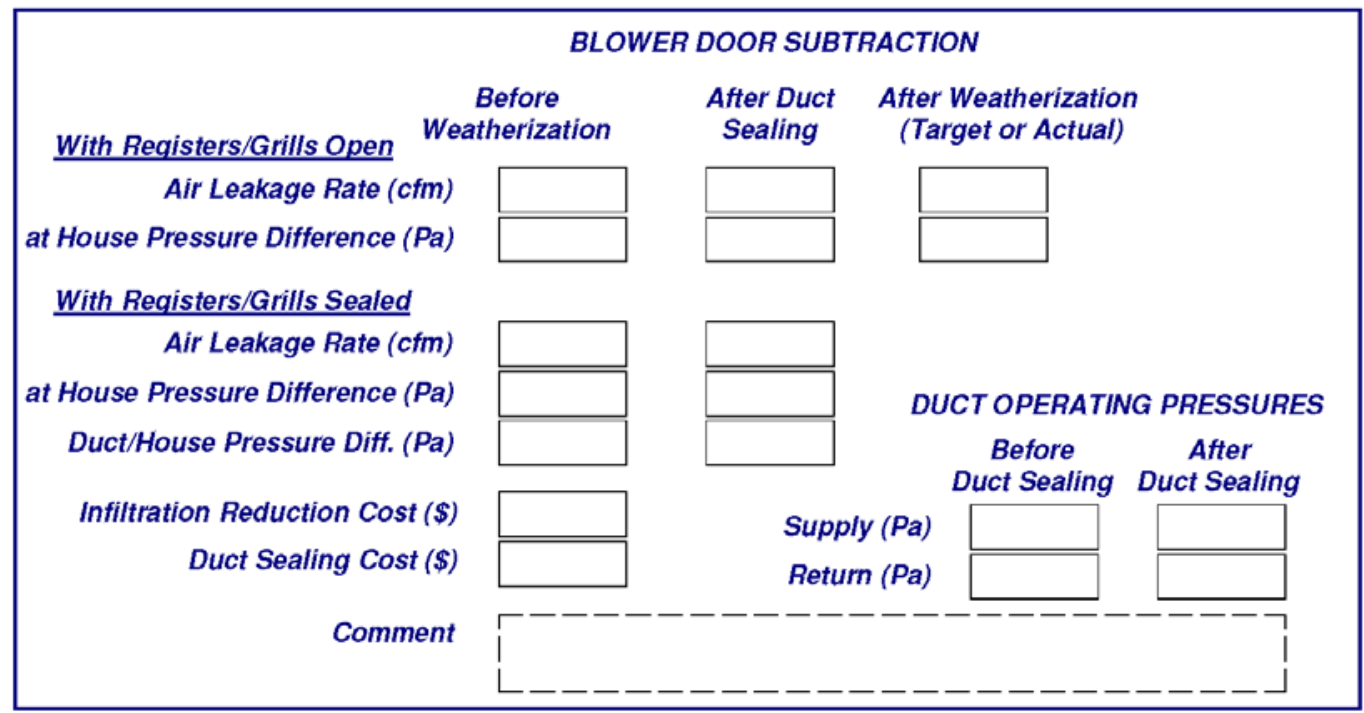

WHOLE HOUSE BLOWER DOOR MEASUREMENT

Before Weatherizatio
DUCT OPERATING PRESSURES Before Duct Sealing Duct Sealing

Air Leakage Rate (CFM)

at House Pressure Difference $(\mathrm{Pa})$
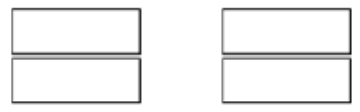

Supply (Pa)

Return (Pa)

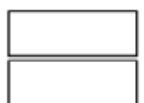

DUCT BLOWER MEASUREMENTS

Before Duct Sealing

After Duct Sealing

Total Outside *

Total Outside *

" 'Outside' readings are taken while the house / outdoor pressure differential provided by a blower door is maintained at the same

Fan Flow (CFM) at Duct Pressure (Pa) House Pressure $(\mathrm{Pa})$ wrt outside
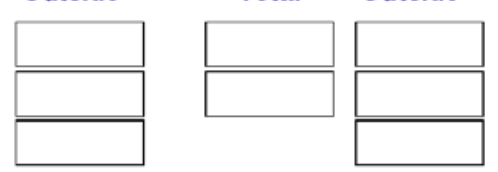
level as the duct / outdoor pressure differential created by the ductblower. Thus the 'Duct Pressure' and the 'House Pressure wrt outside' should be equal.

Infiltration Reduction Cost (\$) Duct Sealing Cost (\$)
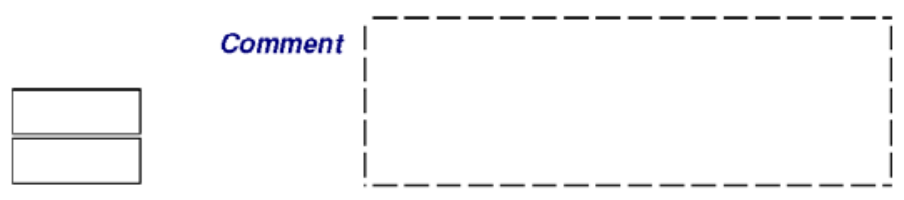

Client Name:
Client ID:
Att. Client ID:

NEAT Data Collection Form

Form Run On: 11/2/2011
DOE Weatherization Assistant

Version 8.9 .0

Page 28 of 42 
Notes : Att. Client ID:
NEAT Data Collection Form

Form Run On: 11/2/2011
DOE Weatherization Assistant

Version 8.9 .0

Page 29 of 42 


\section{Appendix C: Reports}

Ducts / Infiltration (Continued)

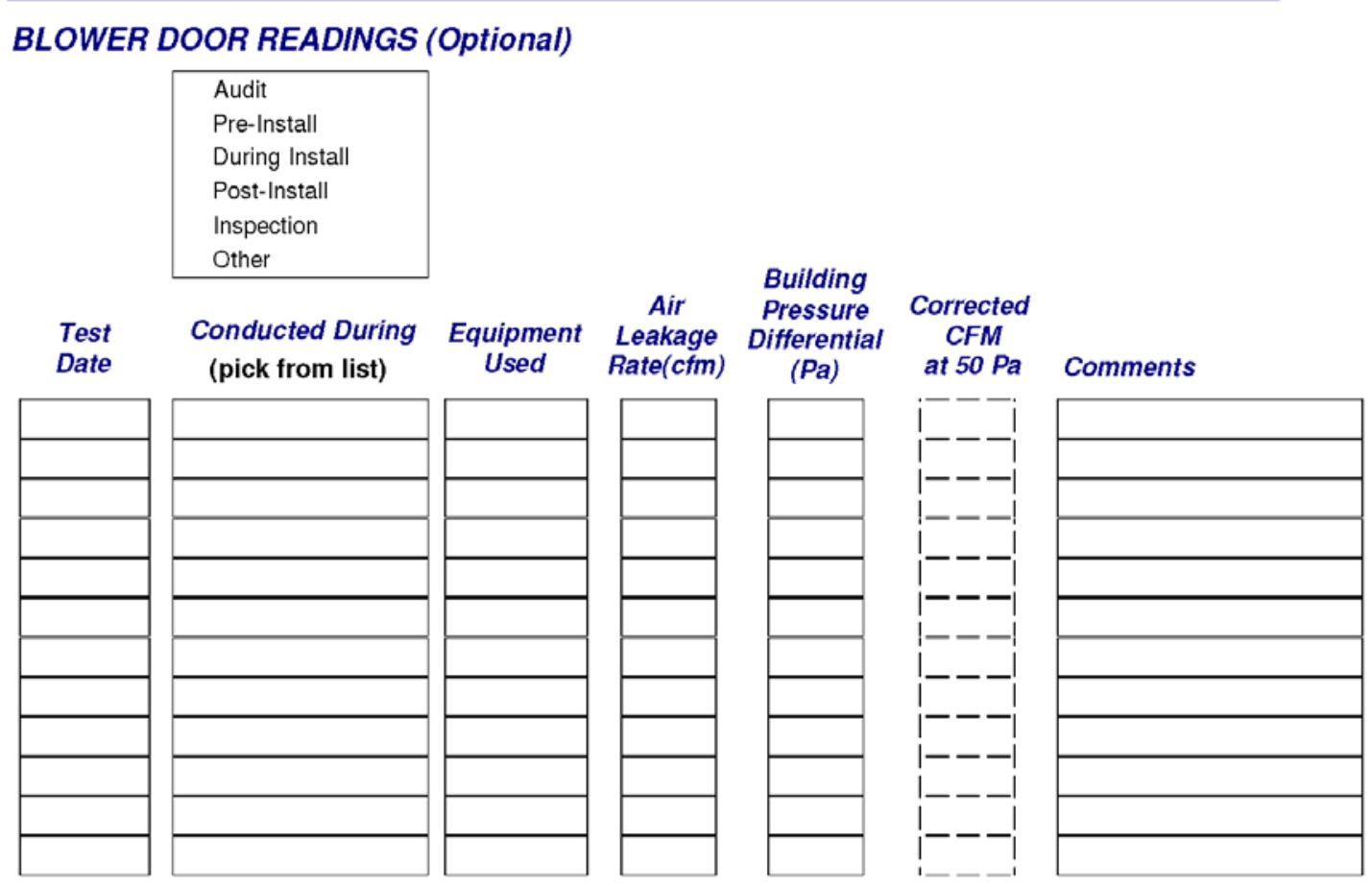

Notes:

Client Name:
Client ID:
Att. Client ID:


Ducts / Infiltration (Continued)

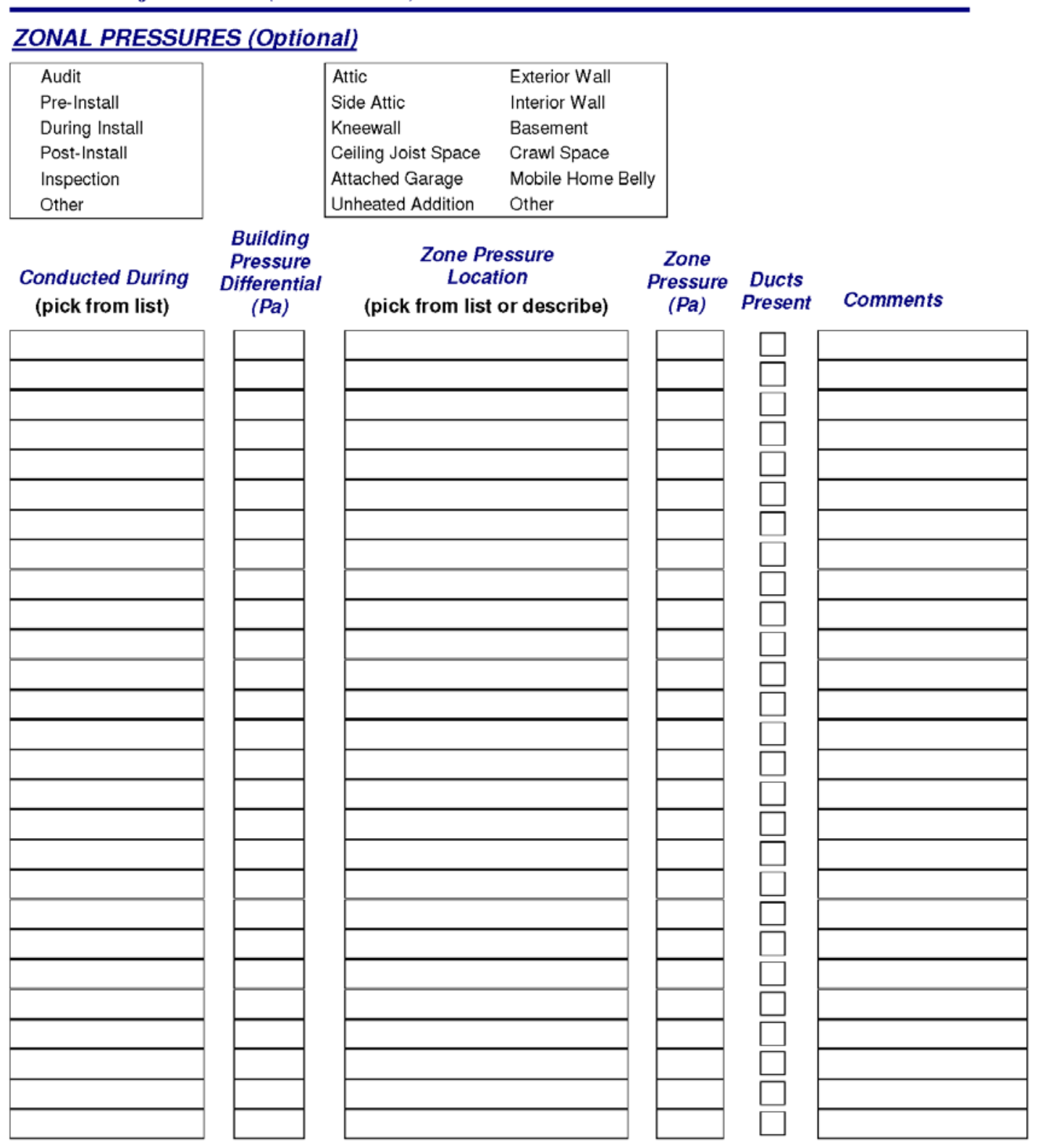

Notes :

Client Name:
Client ID:
Att. Client ID:

NEAT Data Collection Form

Form Run On: 11/2/2011
DOE Weatherization Assistant

Version 8.9.0

Page 31 of 42 


\section{Appendix C: Reports}

Ducts / Infiltration (Continued)

\section{PRESSURE BALANCE READINGS (Optional)}

\begin{tabular}{|ll|}
\hline Family Room & Bdrm1 \\
Living Room & Bdrm2 \\
Dining Room & Bdrm3 \\
Kitchen & Bdrm4 \\
Bath1 & Basement \\
Bath2 & Addition \\
Bath3 & Other \\
\hline
\end{tabular}

Location

Initial

Final

(pick one or describe) Pressure $(\mathrm{Pa}) \quad$ Pressure $(\mathrm{Pa})$

\section{Comments}
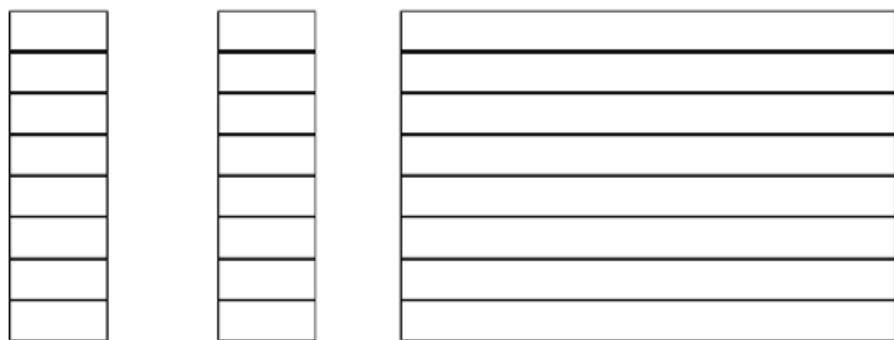

Notes :

Client Name:
Client ID:
Att. Client ID:


Ducts / Infiltration (Continued)

\section{PRESSURE PAN READINGS (Optional)}

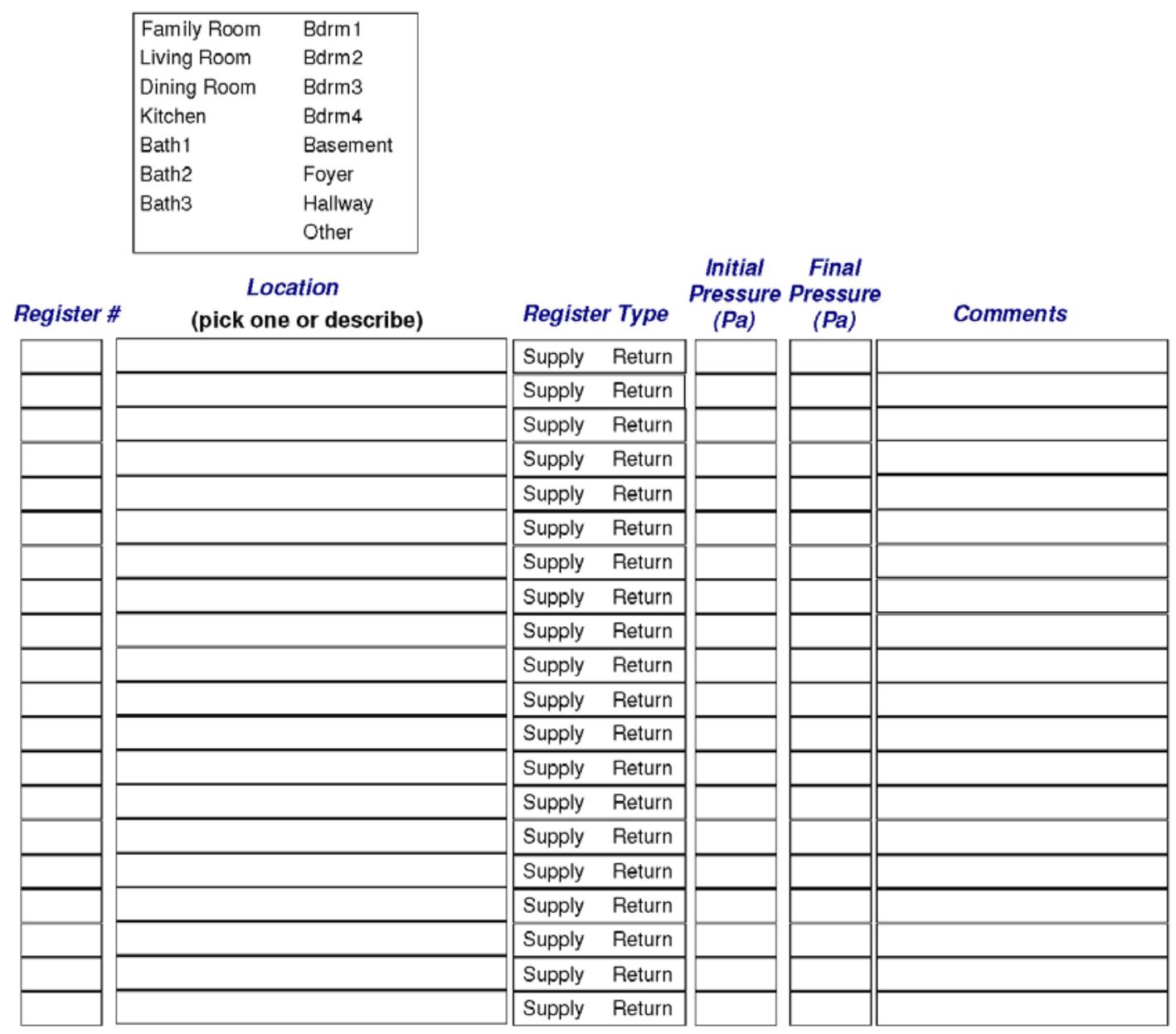

Notes :

Client Name:
Client ID:
Att. Client ID:




\section{Appendix C: Reports}

Base Load - Water Heater

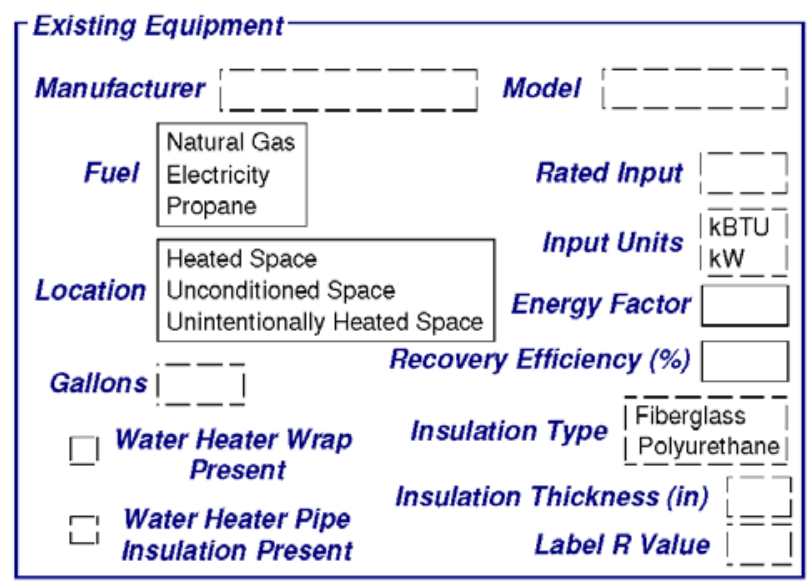

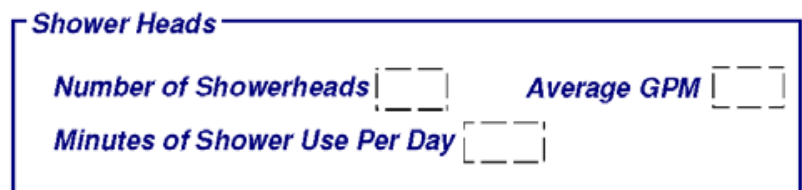

Comment

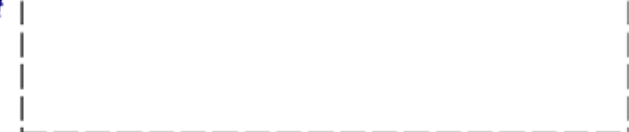

[Replacement Equipment

Manufacturer

Model

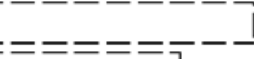

$\mathrm{L}===-\mathrm{J}$

| Natural Gas |

Fuel | Electricity

I Propane

Rated Input $\mathrm{i}==$

Input Units $\left\lceil_{\mathrm{kW}}^{\overline{\mathrm{kB}} \overline{\overline{\mathrm{T}}}\rceil}\right.$

Gallons $: \stackrel{:=1}{=}$

$\mathrm{L}= \pm \_-1$

Installation Cost $(\$){ }_{\mathrm{L}}^{\top}====$

Additional Cost (\$) i

Energy Factor

Recovery Efficiency (\%)

Notes:

Client Name:
Client ID:
Att. Client ID:

NEAT Data Collection Form

Form Run On: 11/2/2011
DOE Weatherization Assistant

Version 8.9 .0

Page 34 of 42 
Base Load - Water Heater (Continued)

OPTIONAL WATER HEATING SYSTEM DETAIL

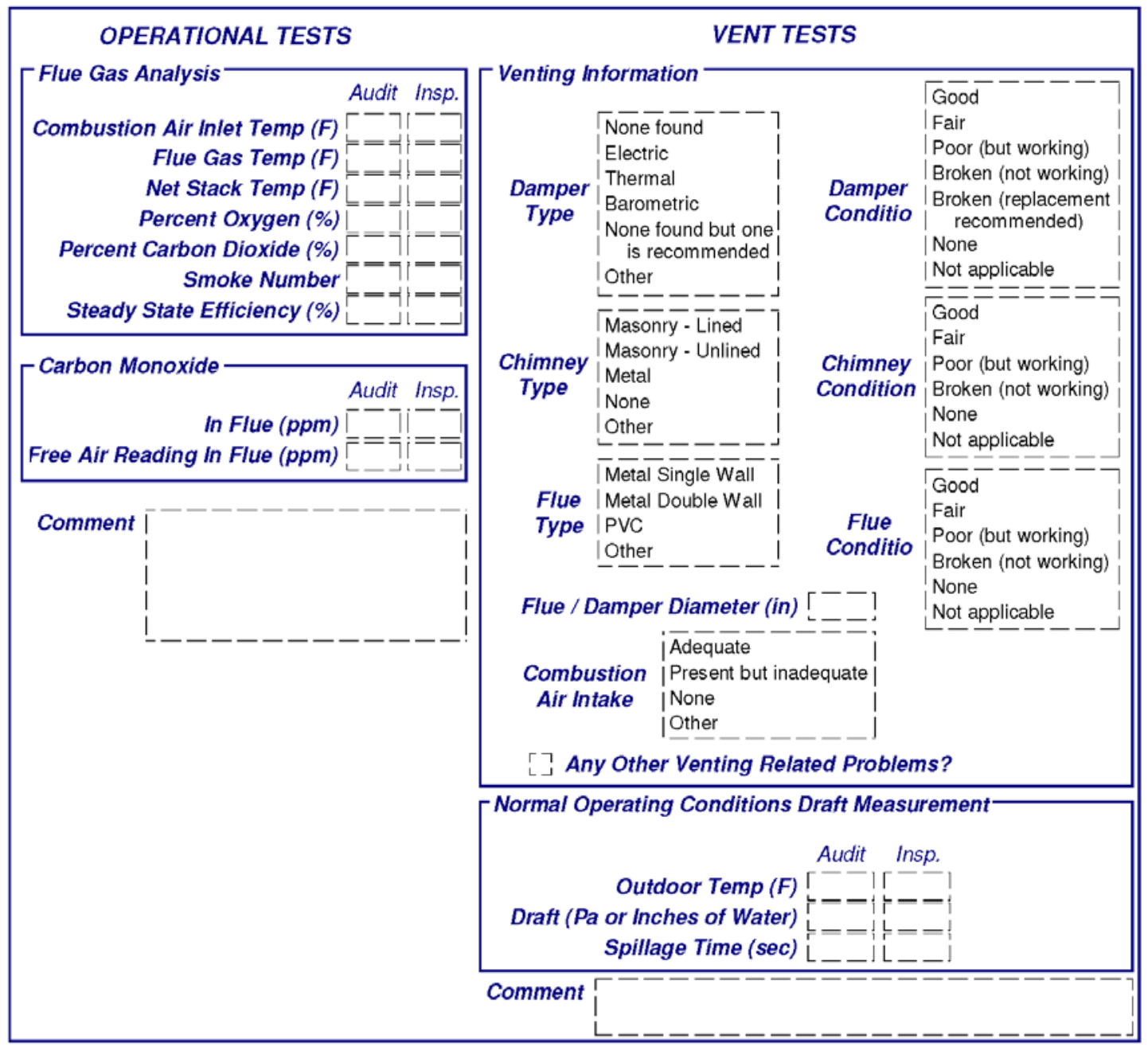

\section{Notes :}

Client Name:
Client ID:
Alt. Client ID:

NEAT Data Collection Form

Form Run On: 11/2/2011
DOE Weatherization Assistant

Version 8.9 .0

Page 35 of 42 


\section{Appendix C: Reports}

Base Load - Water Heater (Continued)

OPTIONAL WATER HEATING SYSTEM DETAILS (Continued)

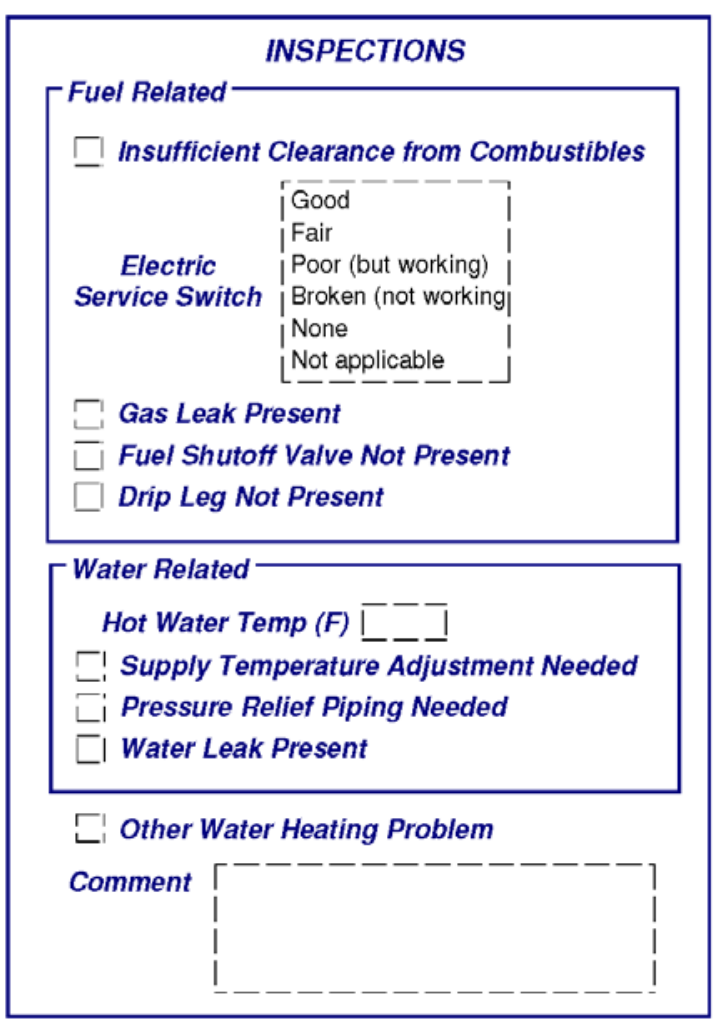

Notes :

Client Name:
Client ID:
Att. Client ID:


Base Load - Refrigerator

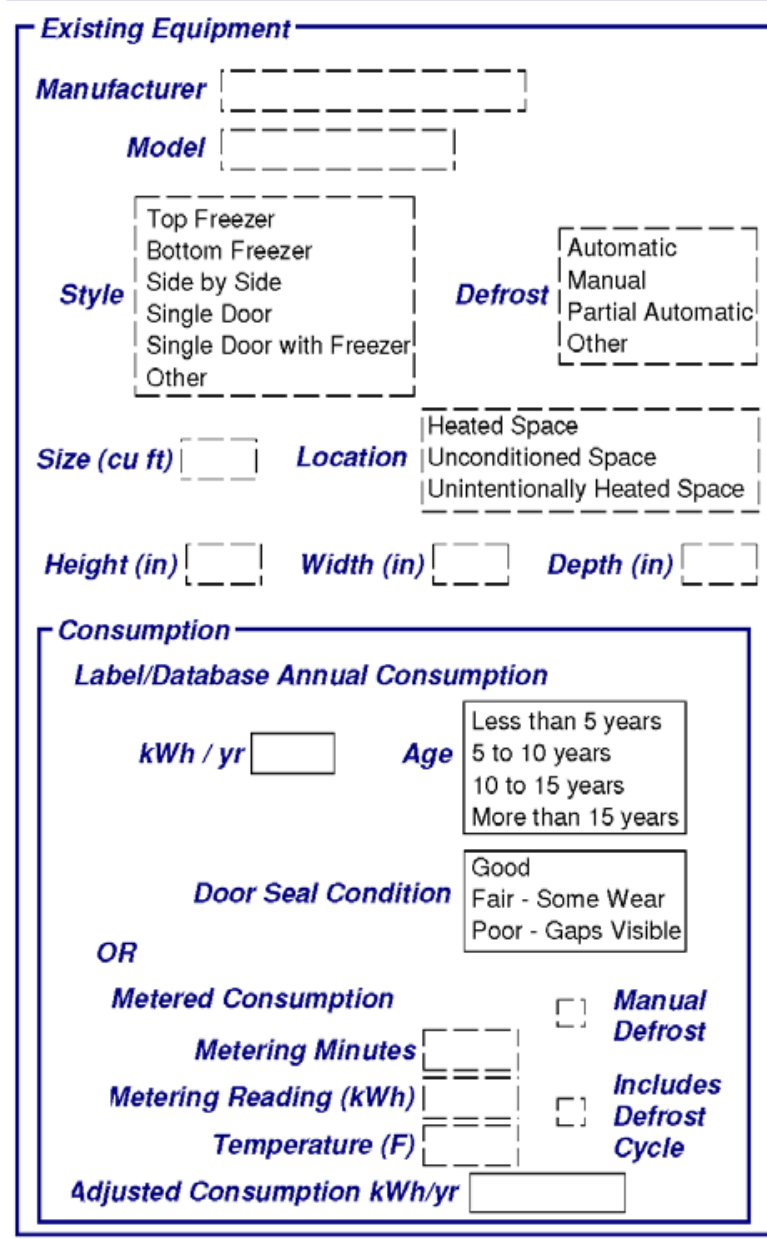
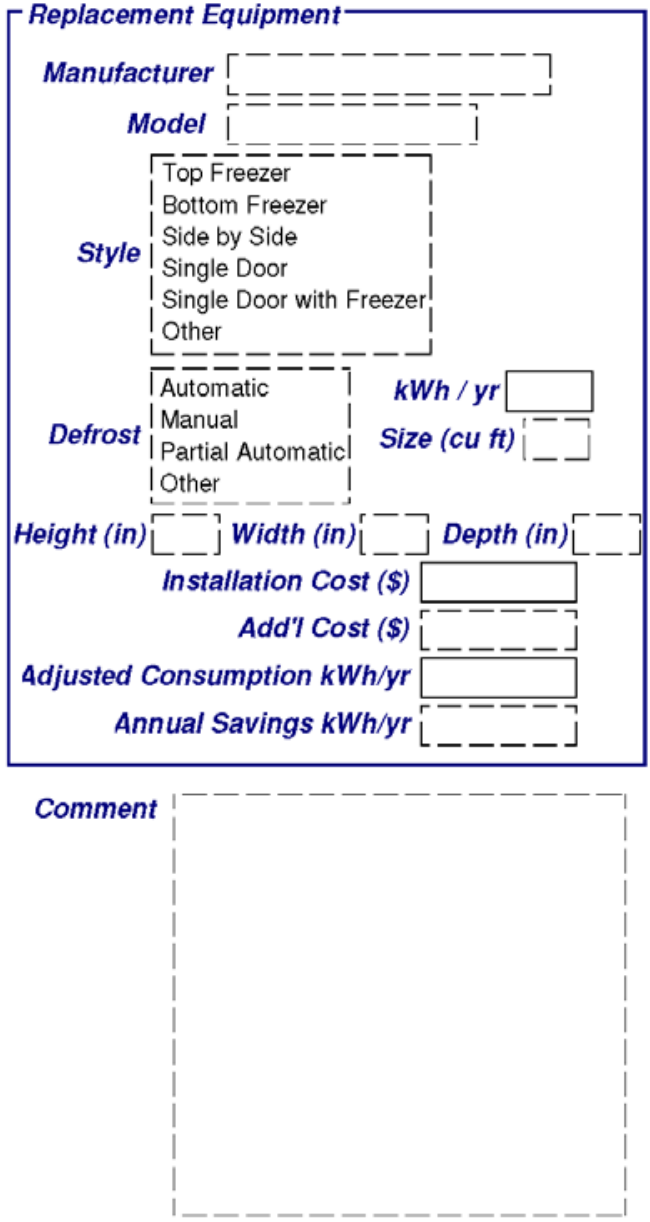

Notes:

Client Name:
Client ID:
Alt. Client ID:

NEAT Data Collection Form

Form Run On: 11/2/2011
DOE Weatherization Assistant

Version 8.9 .0

Page 37 of 42 


\section{Appendix C: Reports}

Baseload - Lighting Systems

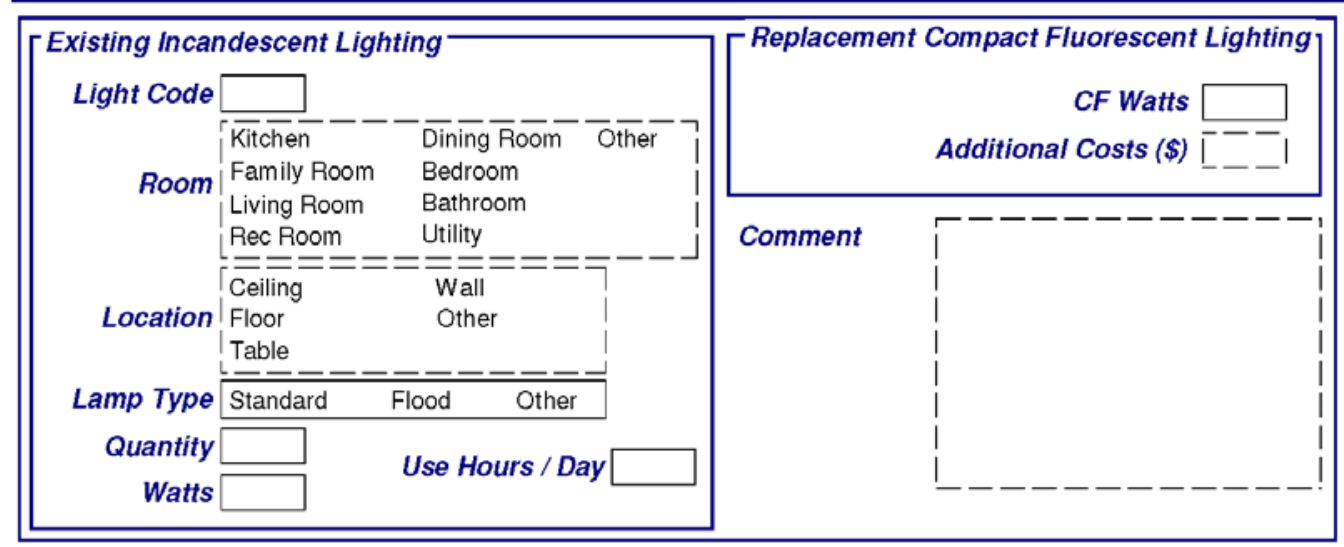
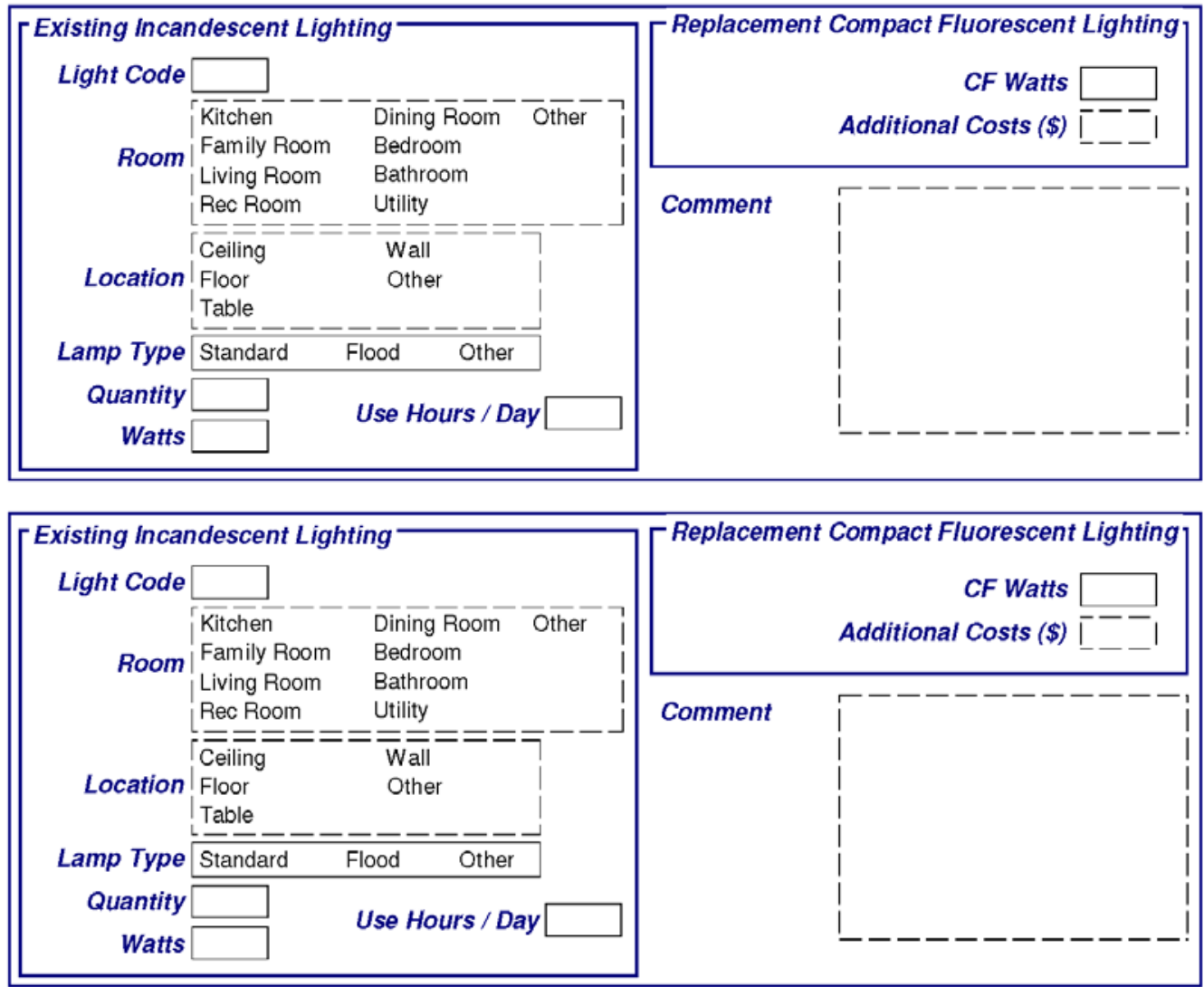

Client Name:
Client ID:
Att. Client ID:




\section{Health \& Safety}

\section{WHOLE HOUSE}

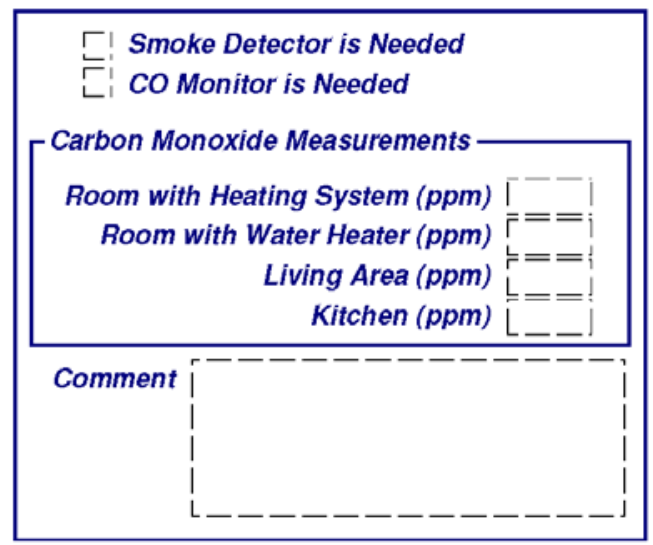

\section{BUILDING SHELL}

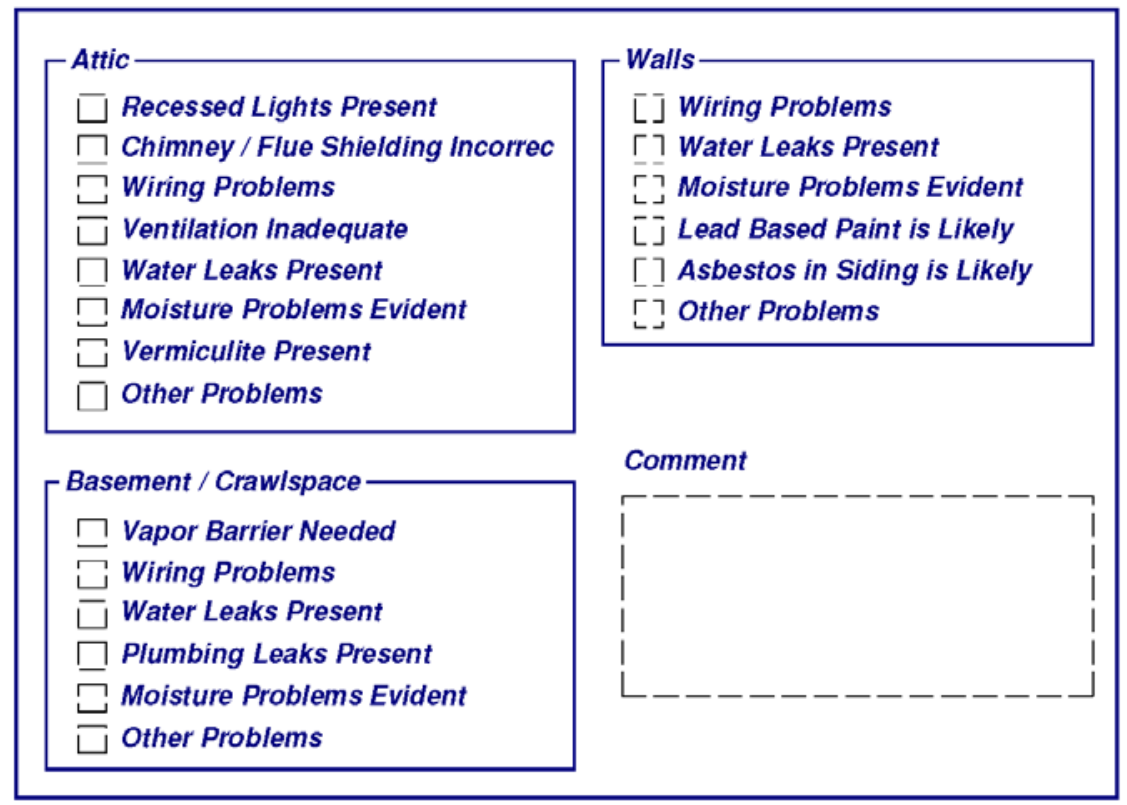

\section{Notes :}

Client Name:
Client ID:
Alt. Client ID:

NEAT Data Collection Form

Form Run On: 11/2/2011
DOE Weatherization Assistant

Version 8.9.0

Page 39 of 42 


\section{Appendix C: Reports}

\section{Health \& Safety (Continued)}

\section{EQUIPMENT}

Worst Case Condition Draft Measurements - Space Heating System(s)

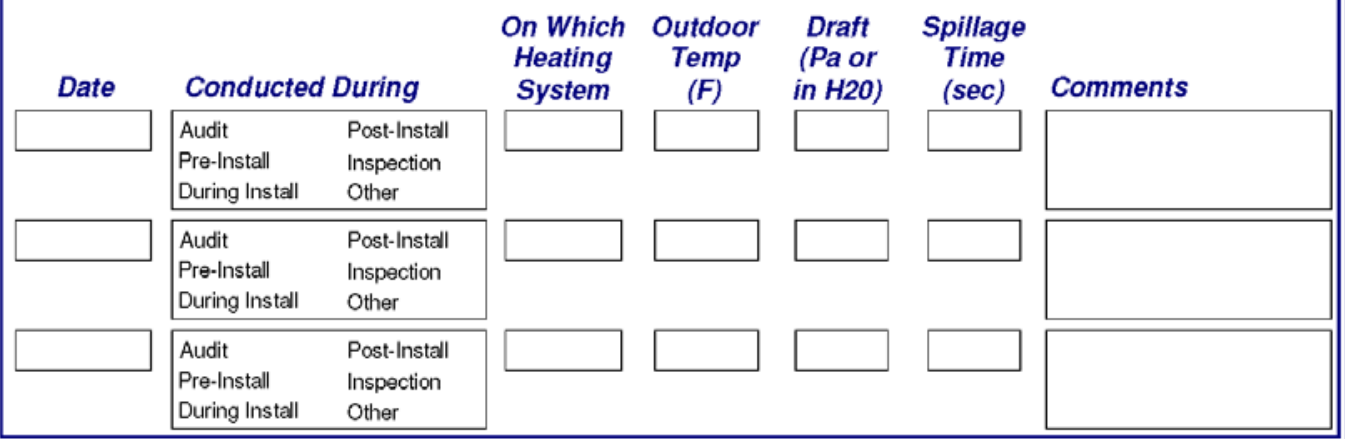

Worst Case Condition Draft Measurements - Water Heatin

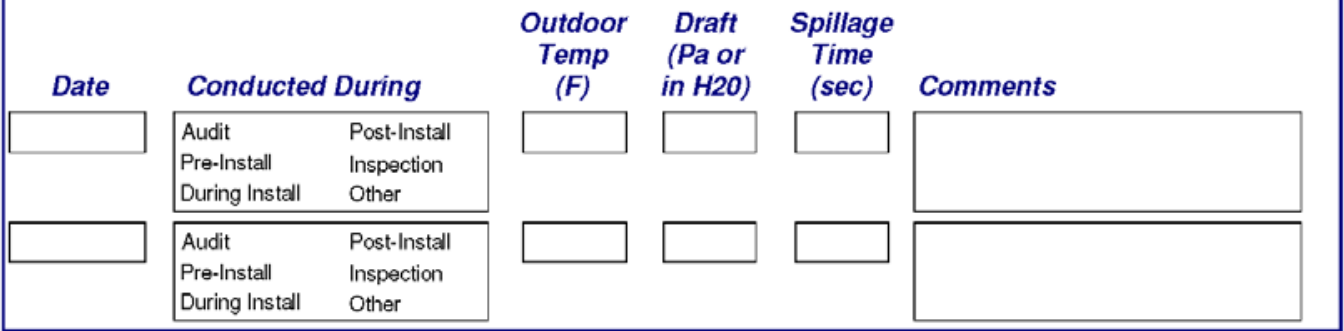

Wood Stove / Fireplace

\lceil\urcorner Wood Stove / Fireplace is Present

¿ᄃ] Improper Venting

[.] Combustion Air is Inadequate

Clothes Dryer

$\ulcorner$ Improper Venting

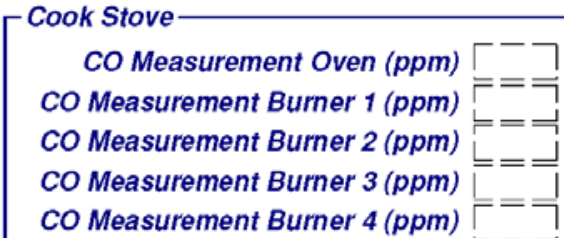

[j Gas Leak Present

\begin{tabular}{|c|c|c|}
\hline Bathrooms & Kitchen & Air-to-Air Heat Exchangen \\
\hline Бi Missing & Missing & Ej Exists \\
\hline $\begin{array}{l}\text { ¿ Not Operational } \\
\square \text { Improper Venting }\end{array}$ & $\begin{array}{l}\text { Not Operational } \\
\text { Improper Venting }\end{array}$ & [] Not Operational \\
\hline
\end{tabular}

Comment

Client Name:
Client ID:
Att. Client ID:

NEAT Data Collection Form

Form Run On: 11/2/2011
DOE Weatherization Assistant

Version 8.9 .0

Page 40 of 42 


\section{Itemized Costs}

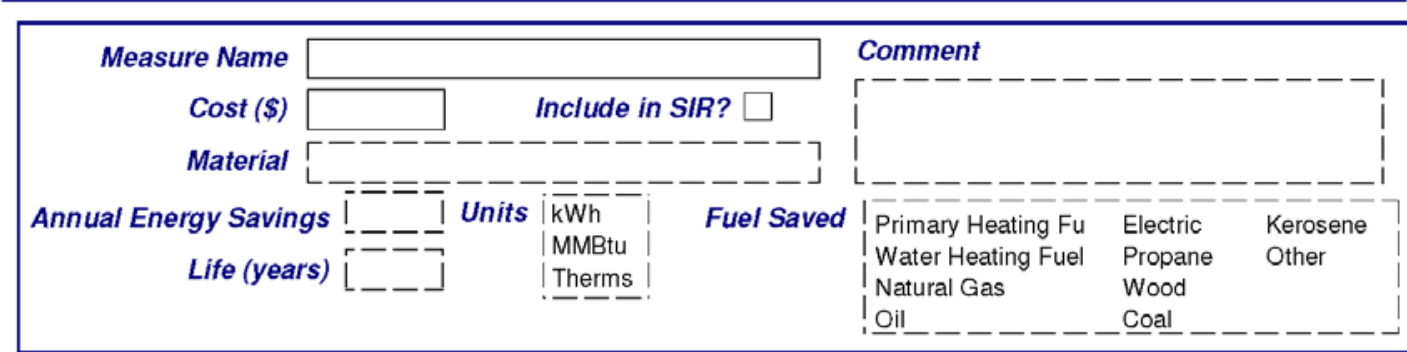

\begin{tabular}{|c|c|c|c|c|}
\hline \multirow{2}{*}{$\begin{array}{r}\text { Measure Name } \\
\text { Cost (\$) }\end{array}$} & & \multicolumn{3}{|l|}{ Comment } \\
\hline & Include in SIR? $\square$ & & & \\
\hline Material $\left[\begin{array}{l}{ }_{L}---- \\
-\end{array}\right.$ & $\begin{array}{l}---ー \jmath \\
----\jmath\end{array}$ & i & & \\
\hline $\begin{array}{c}\text { Annual Energy Savings } \mid+\_ \text {Units } \\
\text { Life (years) }\end{array}$ & \begin{tabular}{|l|} 
kWh \\
$\mid$ MMBtu \\
Therms
\end{tabular} & $\begin{array}{l}\text { Primary Heating Fu } \\
\text { Water Heating Fuel } \\
\text { Natural Gas } \\
\text { Oil_- - - - - }\end{array}$ & $\begin{array}{l}\text { Electric } \\
\text { Propane } \\
\text { Wood } \\
\text { Coal }\end{array}$ & $\begin{array}{l}\text { Kerosene } \\
\text { Other }\end{array}$ \\
\hline
\end{tabular}

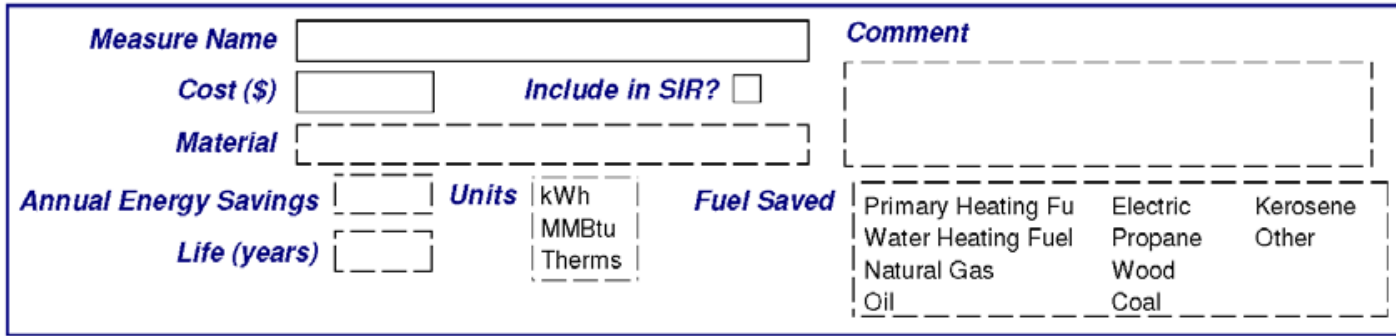

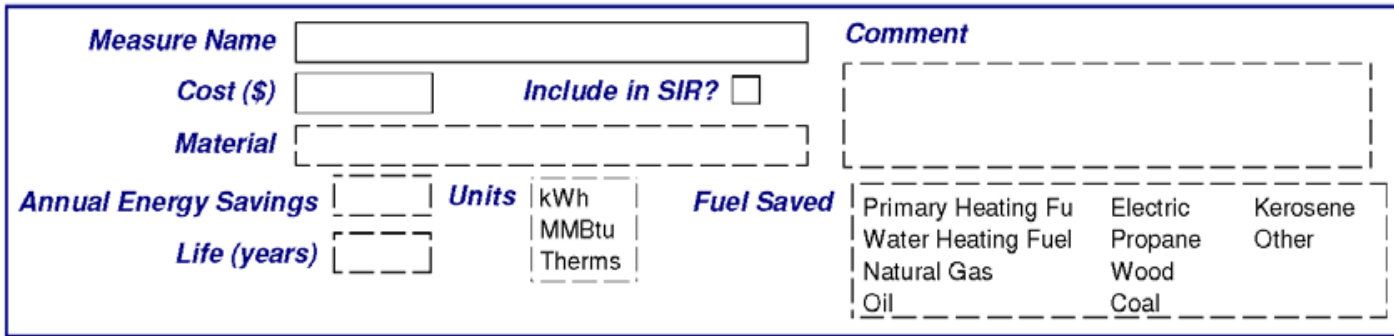

\section{Notes:}

Client Name:
Client ID:
Alt. Client ID:

NEAT Data Collection Form

Form Run On: 11/2/2011
DOE Weatherization Assistant

Version 8.9 .0

Page 41 of 42 


\section{Appendix C: Reports}

\section{Itemized Costs (Continued)}

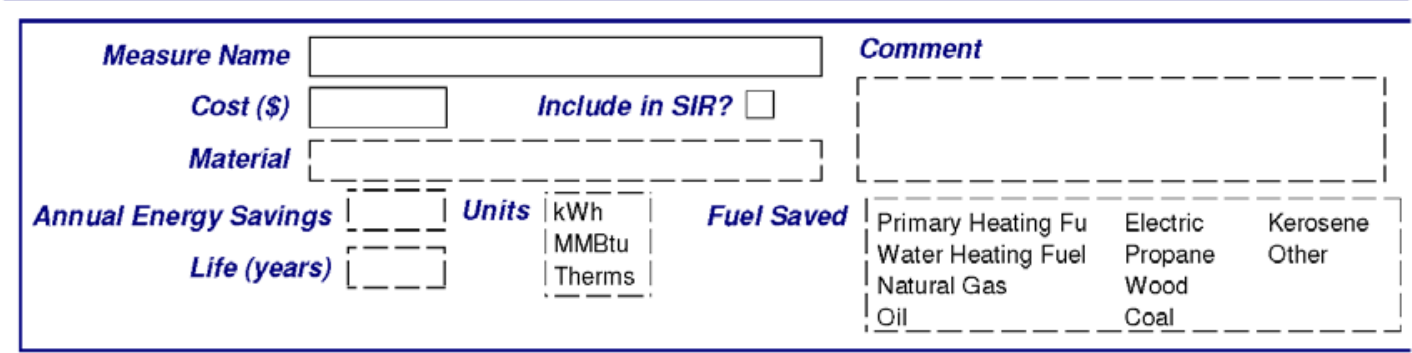

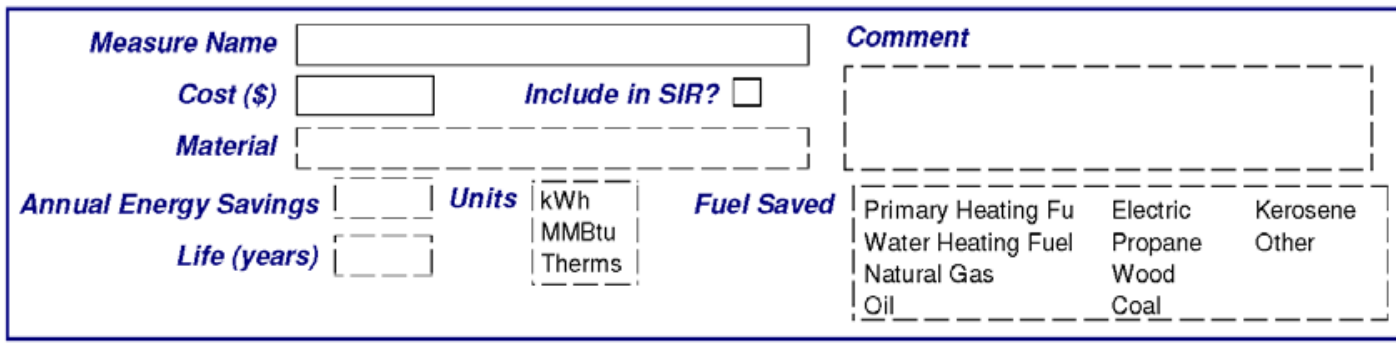

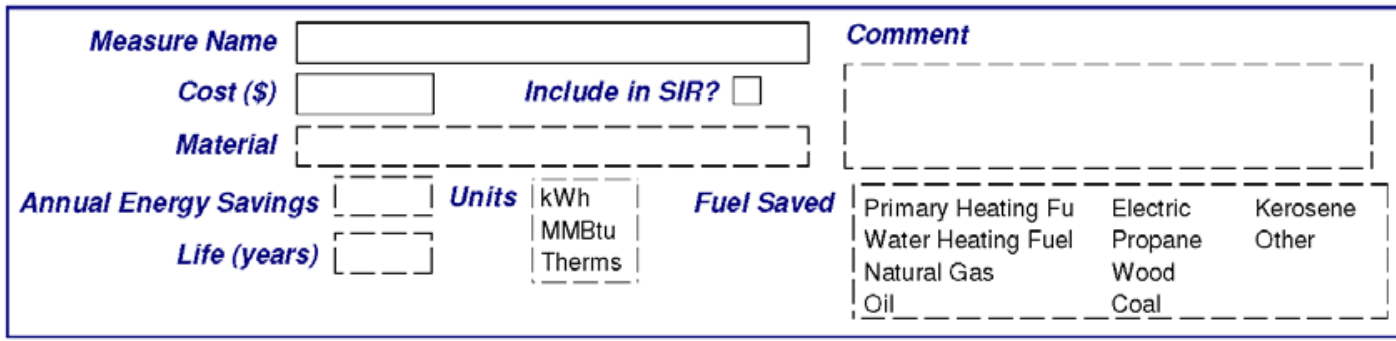

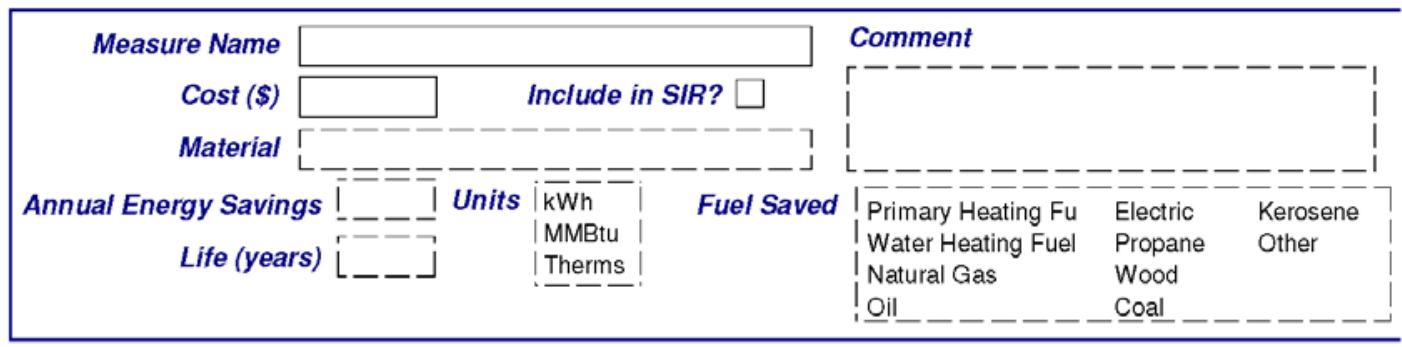

Notes :

Client Name:
Client ID:
Alt. Client ID:

NEAT Data Collection Form

Form Run On: 11/2/2011
DOE Weatherization Assistant

Version 8.9.0

Page 42 of 42 


\section{MHEA Data Collection Form}

Audit Name:

Client Name:

Client ID:

Alternate Client ID:

Assigned to (Auditor):

Length:

Width:

Height:

Wind Shielding: Well Shielded Normal Shielding Exposed

Home Leakiness: North Medium Loose

Outdoor Water Heater Closet:

Comment:

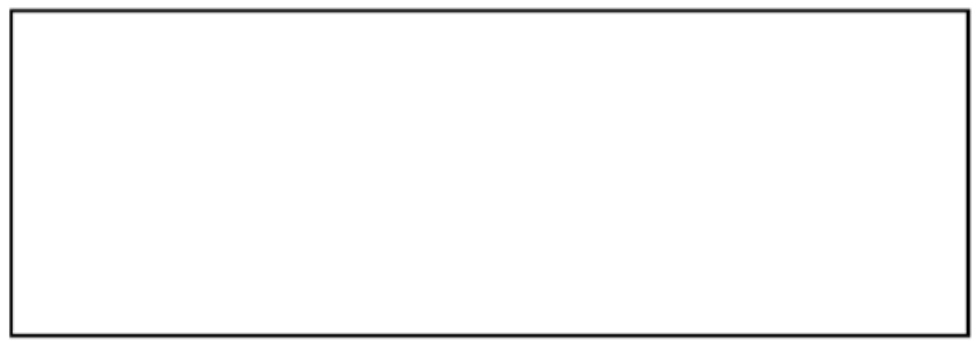




\section{Appendix C: Reports}

\section{Site Diagram}

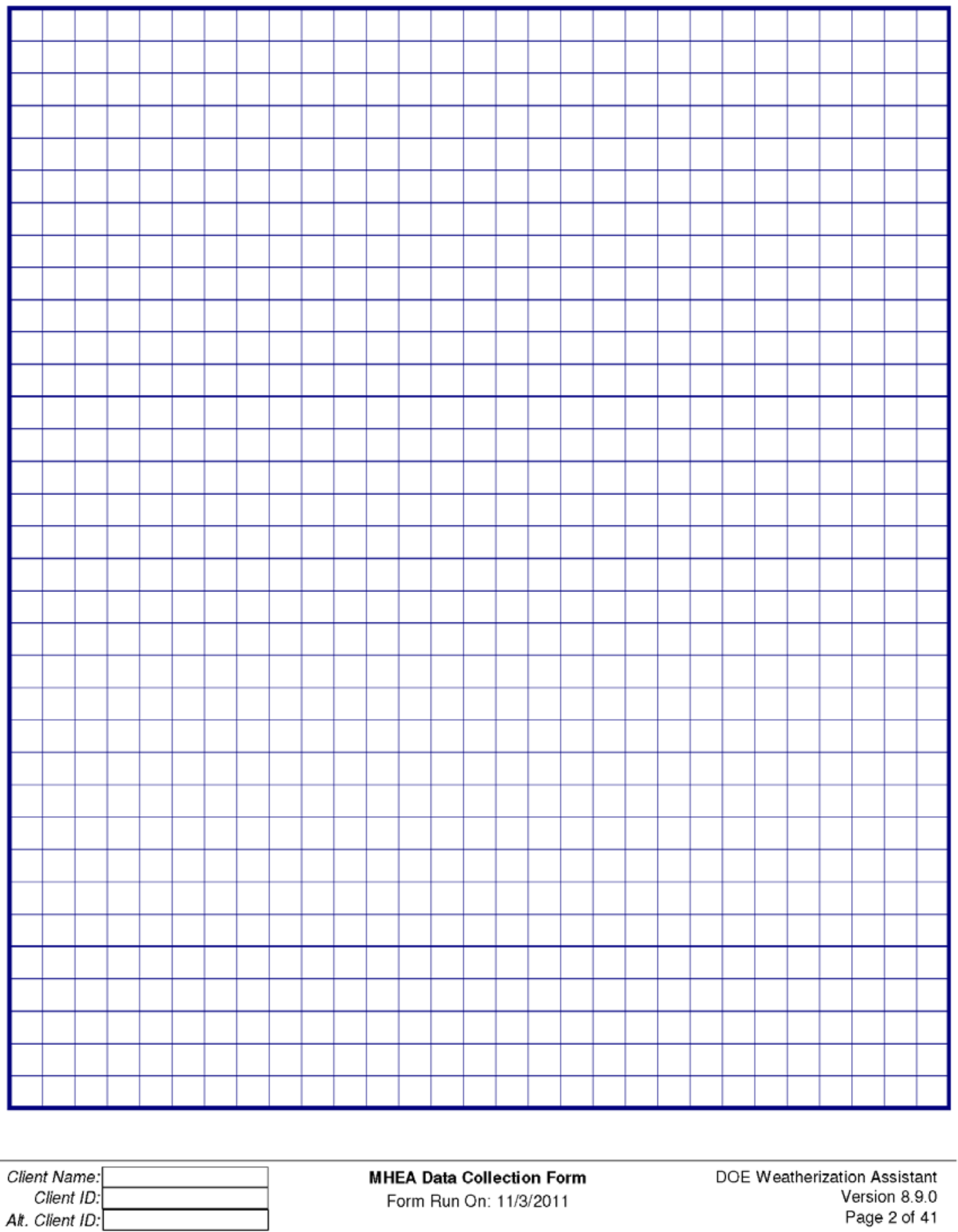


Walls

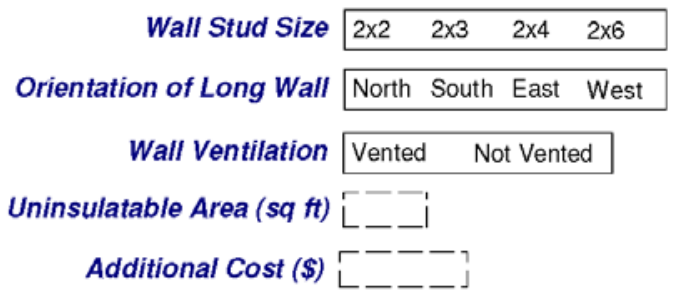

\begin{tabular}{|c|} 
Insulation Type Thickness \\
Batt/Blanket (in) \\
Loose Fill (in) $\square$ \\
Foam Core (in) $\square$
\end{tabular}$\quad\left[\begin{array}{c}\text { Carport/Porch Roof- } \\
\text { Length (ft) } \\
\text { Width (ft) } \\
\text { Orientation North South East West }\end{array}\right.$

Comment $\begin{array}{r}-------------------------- \\ \text { - }--------------------------\end{array}$

Client Name:
Client ID:
Att. Client ID:

MHEA Data Collection Form

Form Run On: 11/3/2011
DOE Weatherization Assistant

Version 8.9 .0

Page 3 of 41 


\section{Appendix C: Reports}

\section{Windows}

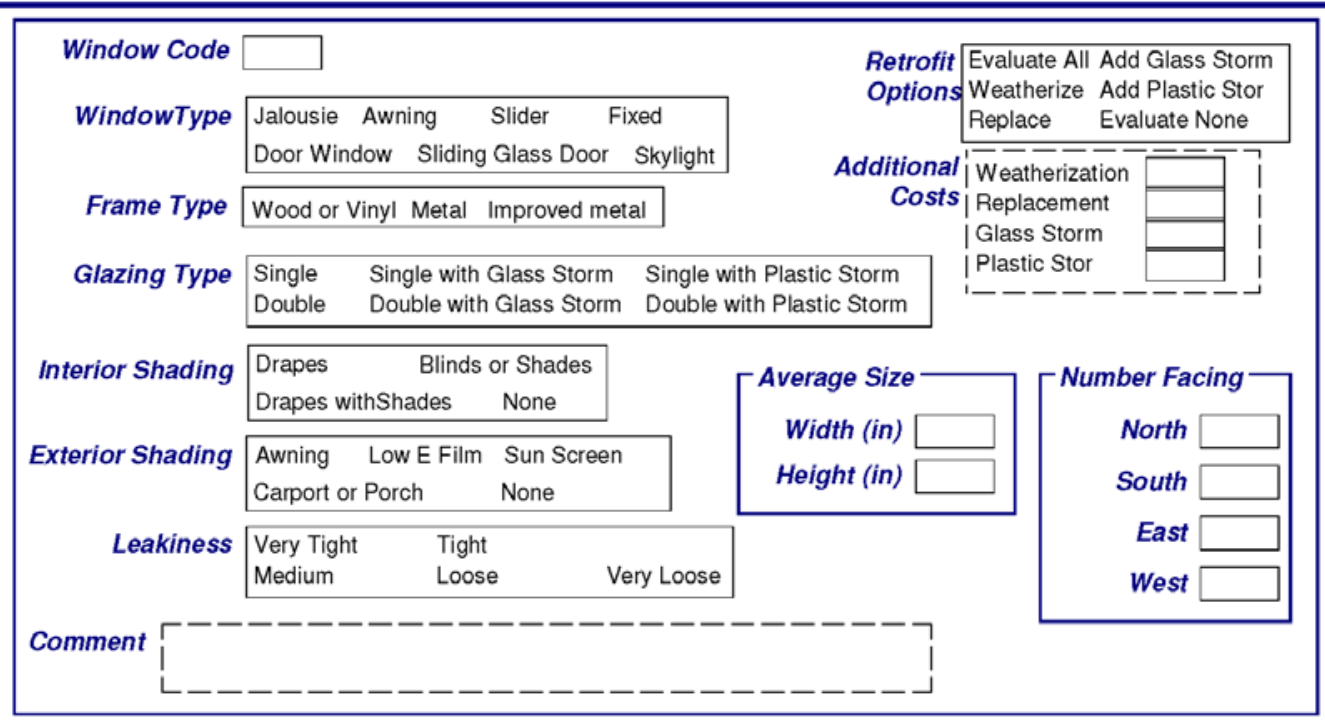

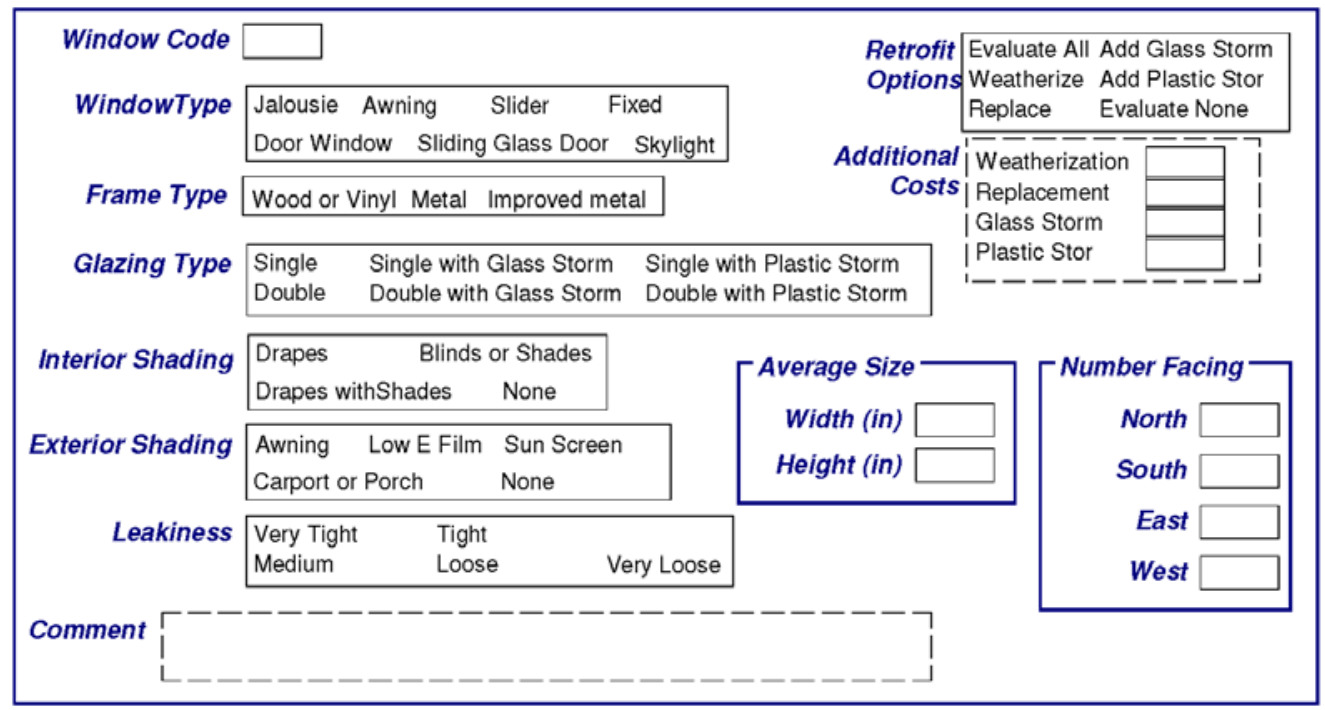

\section{Notes :}

\begin{tabular}{|c|c|c|}
\hline Client Name: & MHEA Data Collection Form & DOE Weatherization Assistant \\
\hline Client ID: & Form Run On: 11/3/2011 & $\begin{array}{l}\text { Version } 8.9 .0 \\
\text { Page } 4 \text { of } 41\end{array}$ \\
\hline
\end{tabular}

Two additional pages of Windows input forms exist in the MHEA Data Collection Forms. 


\section{Doors}

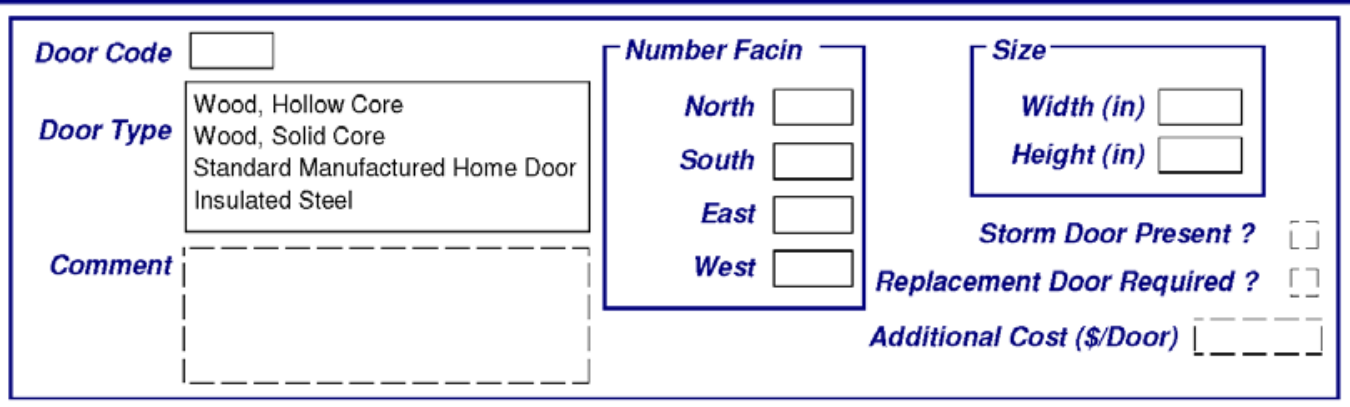

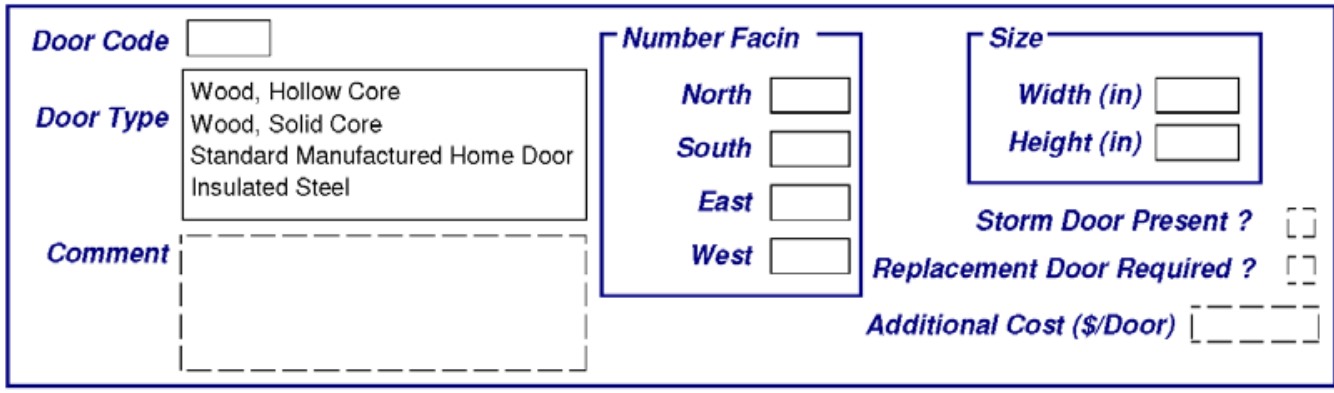

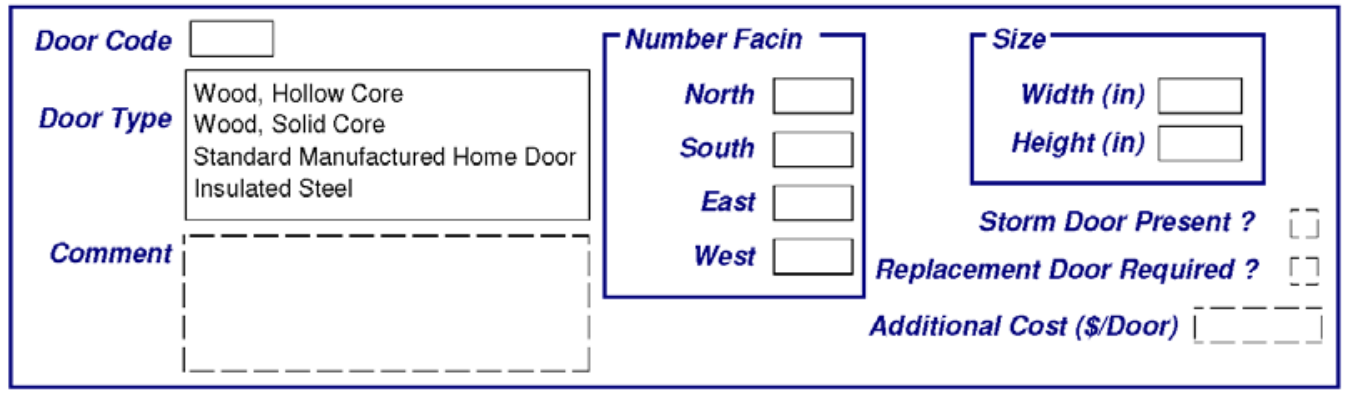

\section{Notes :}

Client Name:
Client ID:
Att. Client ID:

MHEA Data Collection Form

Form Run On: 11/3/2011
DOE Weatherization Assistant

Version 8.9.0

Page 7 of 41 


\section{Ceiling}

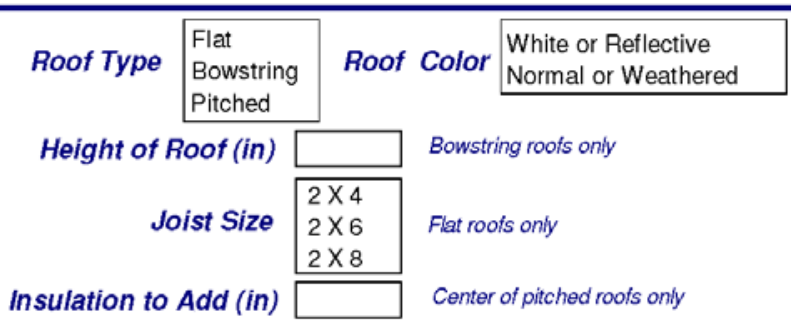

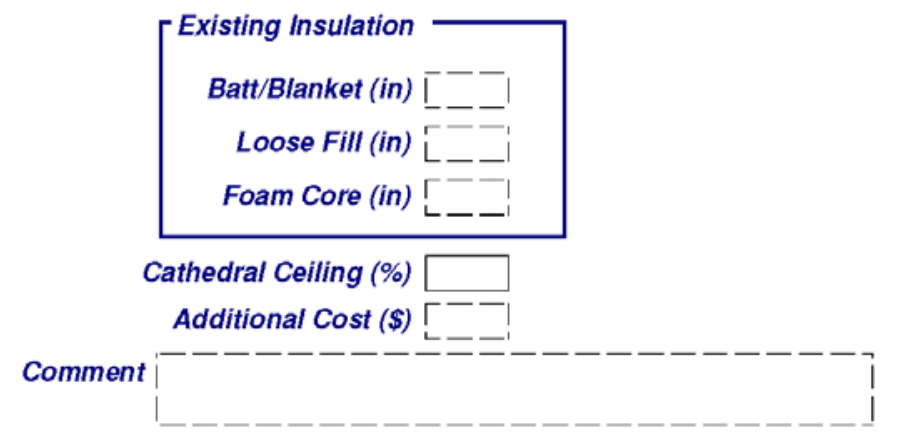

Client Name:
Client ID:
Att. Client ID:

MHEA Data Collection Form

Form Run On: 11/3/2011
DOE Weatherization Assistant

Version 8.9.0

Page 8 of 41 
Floor Joist Direction Lengthwise Widthwise

Is There a Skirt ?

\begin{tabular}{|c|c|c|c|}
\hline \multirow{3}{*}{$\begin{array}{r}\text { Floor Joist } \\
\text { Size }\end{array}$} & & \multirow{2}{*}{ Batt/Blanket Insulation Location } & \\
\hline & $\begin{array}{l}2 \times 6 \\
2 \times 8\end{array}$ & & $\begin{array}{l}\text { Attached to Flooring } \\
\text { Between Joists } \\
\text { Attached Under Joists } \\
\text { None }\end{array}$ \\
\hline & & Batt/Blanket Thickness (in) & \\
\hline
\end{tabular}

[Floor Belly (Center) Description

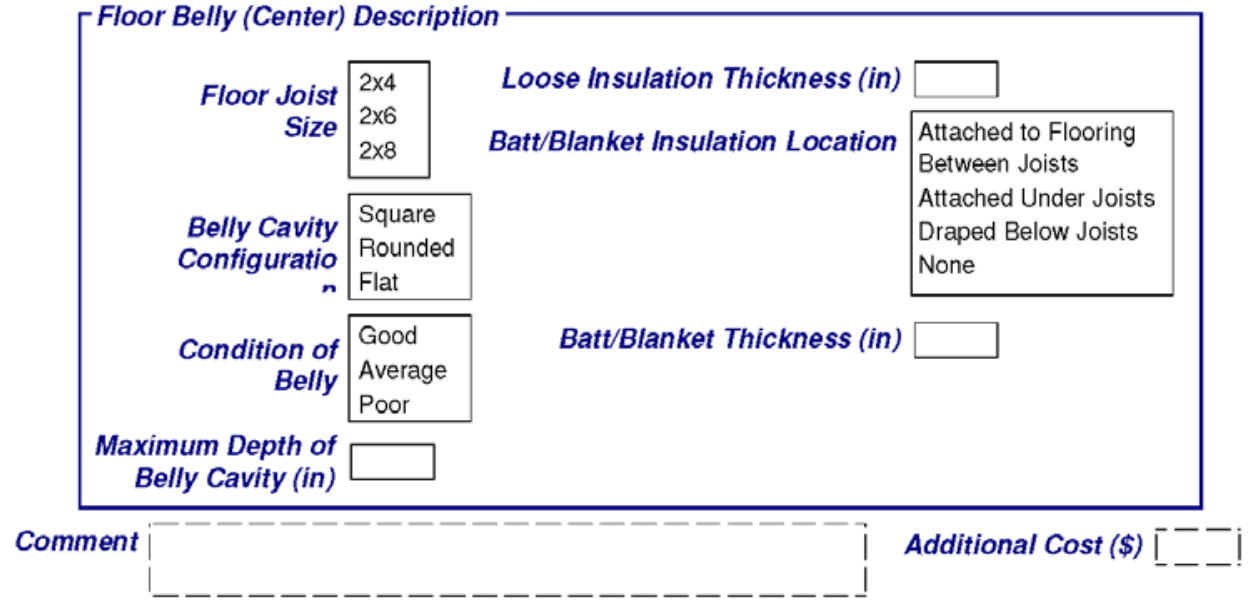

Client Name:
Client ID:
Alt. Client ID:

MHEA Data Collection Form

Form Run On: 11/3/2011
DOE Weatherization Assistant

Version 8.9 .0

Page 9 of 41 


\section{Appendix C: Reports}

\section{Walls (Addition)}

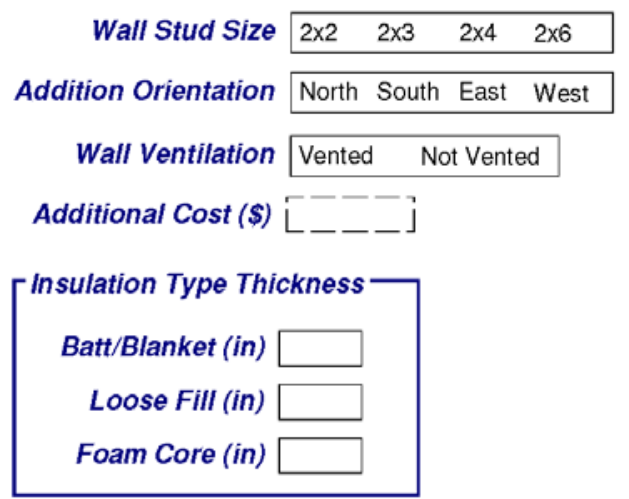

Wall Configuration Maximum Wall Height at Interior Wall

Maximum Wall Height in Center

of Addition

All Addition Wall the Same Height

Interior wall

Max Height (ft)

Min Height (ft)

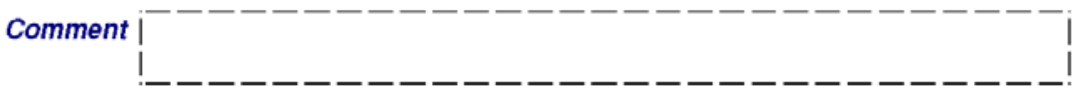

Client Name:
Client ID:
Att. Client ID:


Windows (Addition)

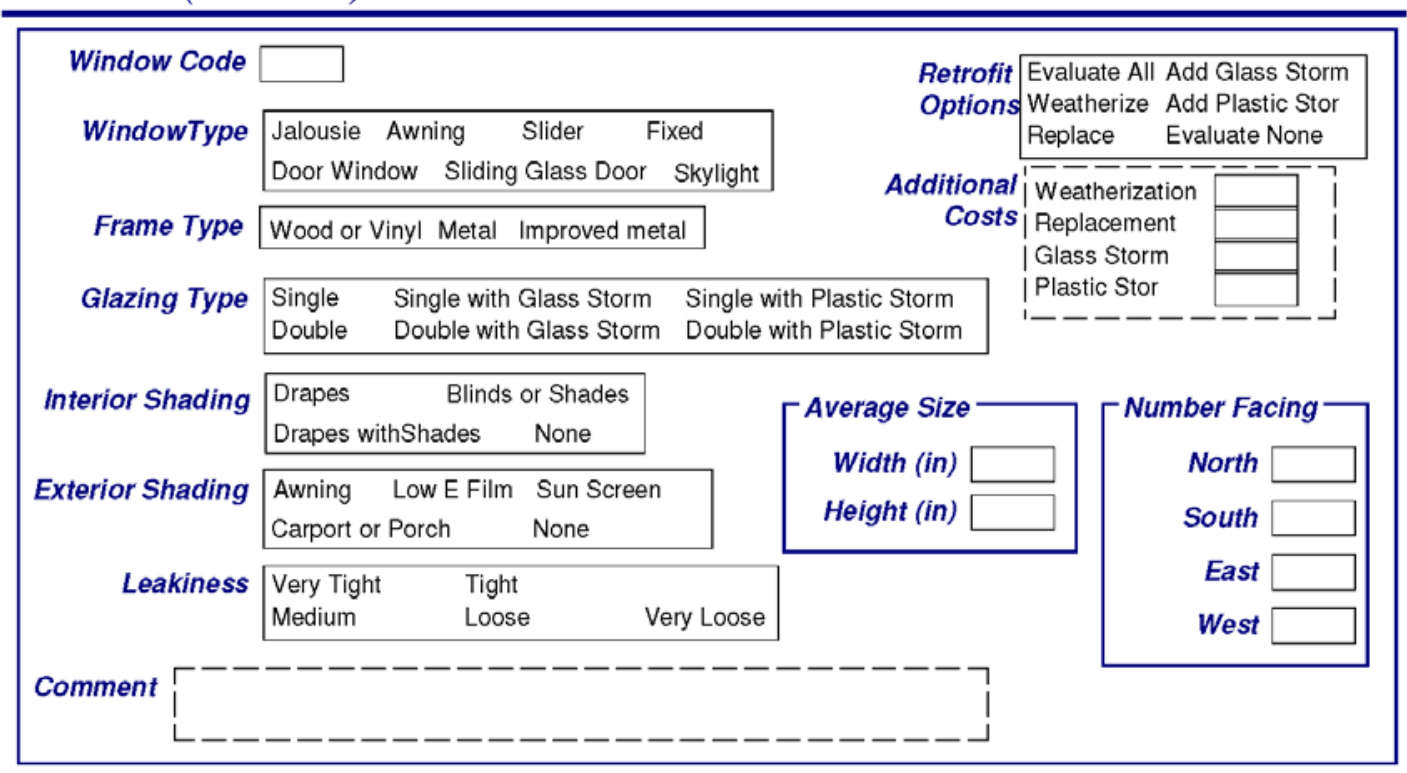

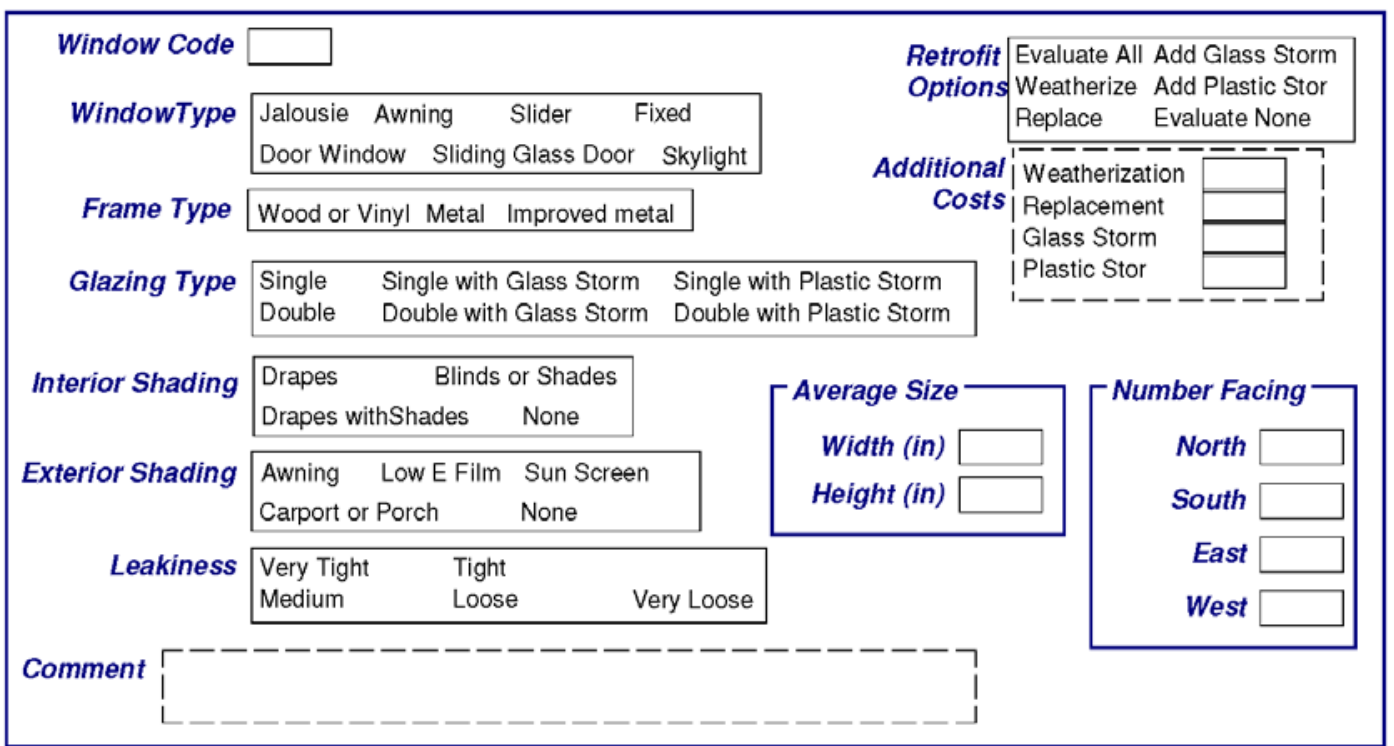

Notes:

Client Name:
Client ID:
Att. Client ID:

MHEA Data Collection Form

Form Run On: 11/3/2011
DOE Weatherization Assistant

Version 8.9.0

Page 11 of 41 


\section{Appendix C: Reports}

Windows (Addition)

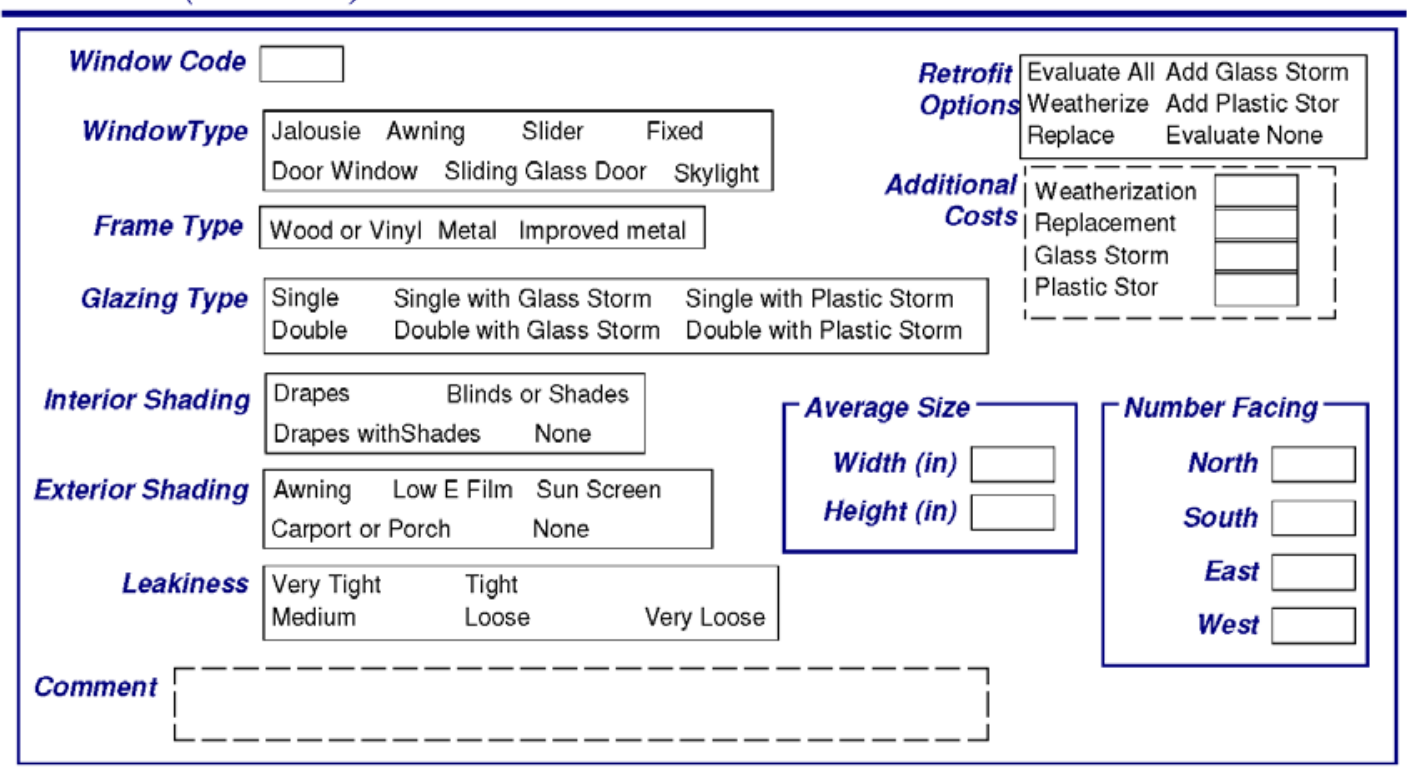

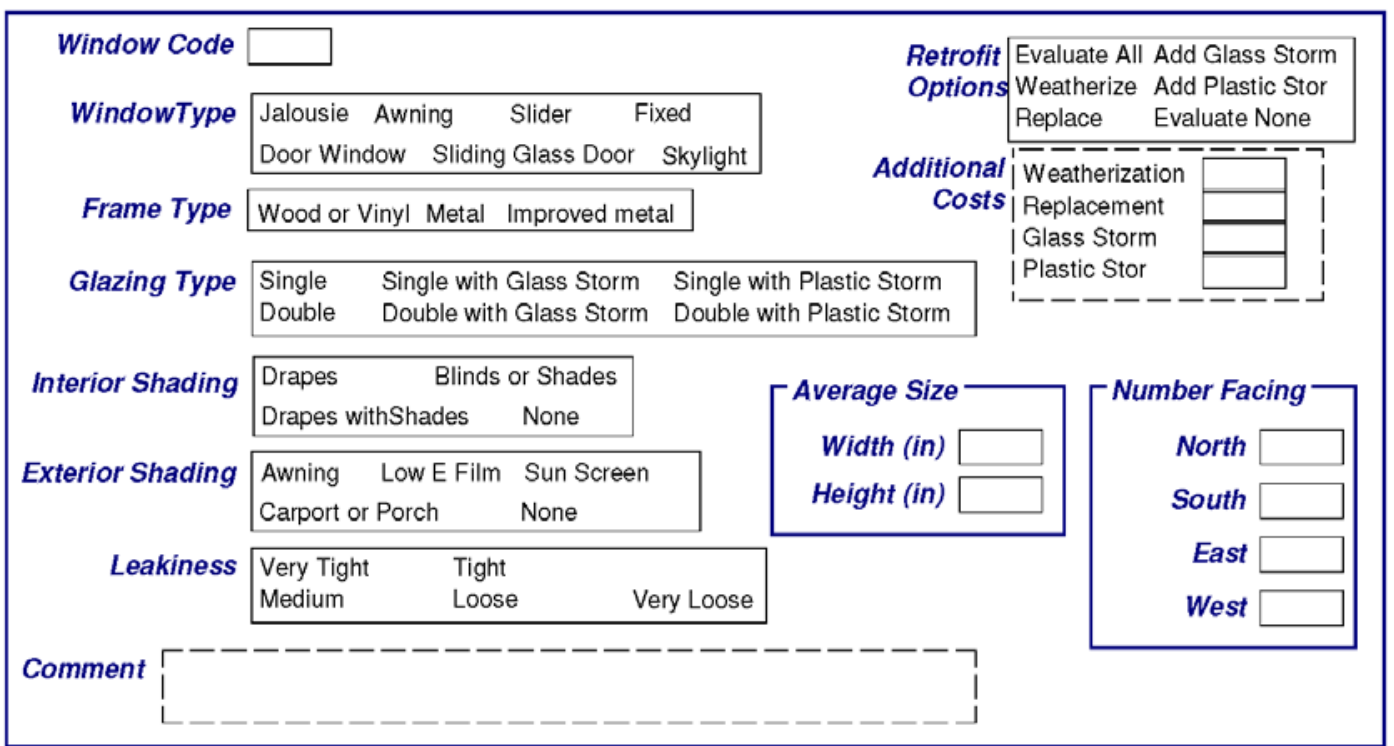

Notes:

\begin{tabular}{rrr}
\hline Client Name: & MHEA Data Collection Form & DOE Weatherization Assistant \\
Client ID: & Version 8.9 .0 \\
Alt. Client ID: & Form Run On: $11 / 3 / 2011$ & Page 12 of 41
\end{tabular}




\section{Doors (Addition)}

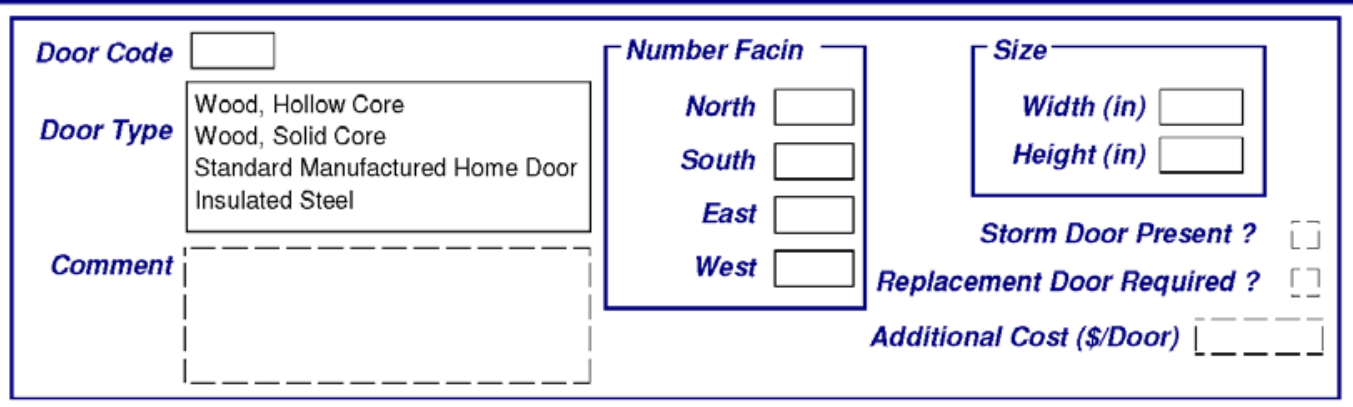

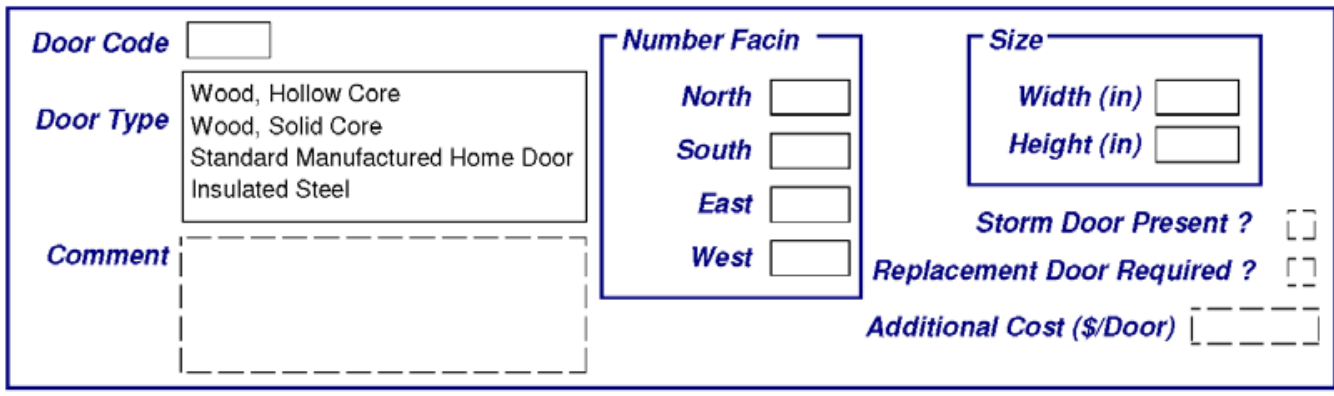

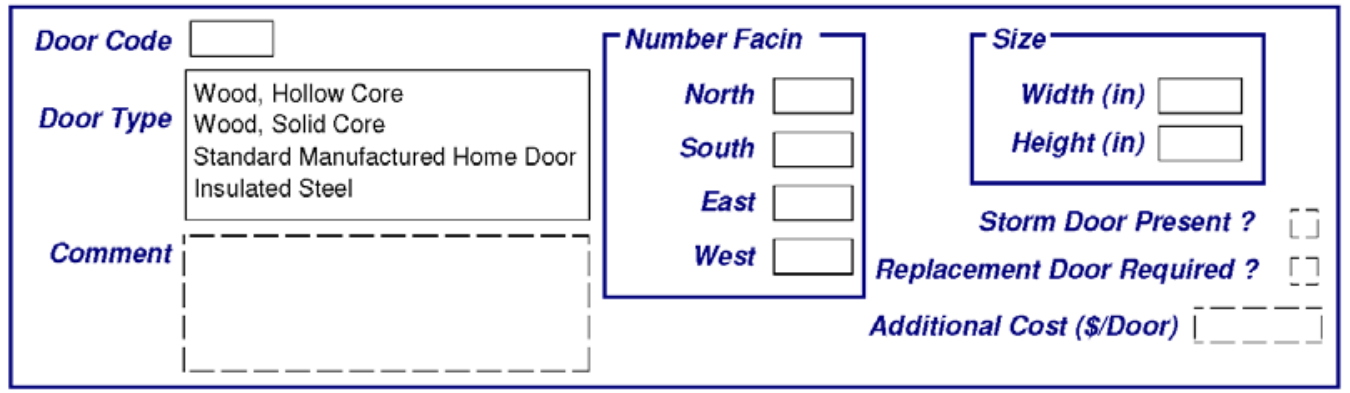

\section{Notes :}

Client Name:
Client ID:
Att. Client ID:

MHEA Data Collection Form

Form Run On: 11/3/2011
DOE Weatherization Assistant

Version 8.9.0

Page 13 of 41 


\section{Appendix C: Reports}

\section{Ceiling (Addition)}

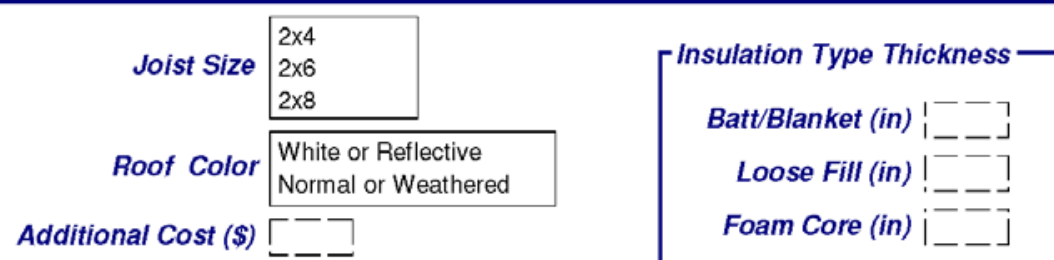

Comment 5 - - - - - - - - - - - - - - - - - - - - - - -

Client Name:

Client ID

Alt. Client 10
MHEA Data Collection Form

Form Run On: 11/3/2011
DOE Weatherization Assistant

Version 8.9.0

Page 14 of 41 
Floor (Addition)

\begin{tabular}{c|l|} 
Floor Type & $\begin{array}{l}\text { Crawl Space } \\
\text { Slab on Grade } \\
\text { Exposed Floor }\end{array}$ \\
\hline \multirow{4}{*}{ Joist Size } & $\begin{array}{l}2 \times 4 \\
2 \times 6 \\
2 \times 8\end{array}$ \\
\hline
\end{tabular}

\begin{tabular}{|l|l|}
\hline Batt/Blanket Location & Attached to Flooring \\
Between Joists \\
Attached Under Joists \\
None
\end{tabular}

$\left[\begin{array}{c}\text { Floor Dimensions } \\ \text { Length (ft) } \square \\ \text { Width (ft) } \square\end{array}\right.$

$\left[\begin{array}{r}\text { Insulation Type Thickness } \\ \text { Batt/Blanket (in) } \\ \text { Loose Fill (in) }\end{array}\right.$

Depth Available for Added Insulation (in)

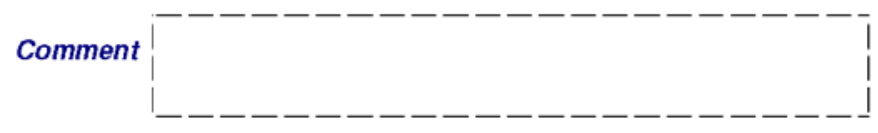

Client Name:
Client ID:
Att. Client ID:




\section{Appendix C: Reports}

\section{Heating System (Primary)}

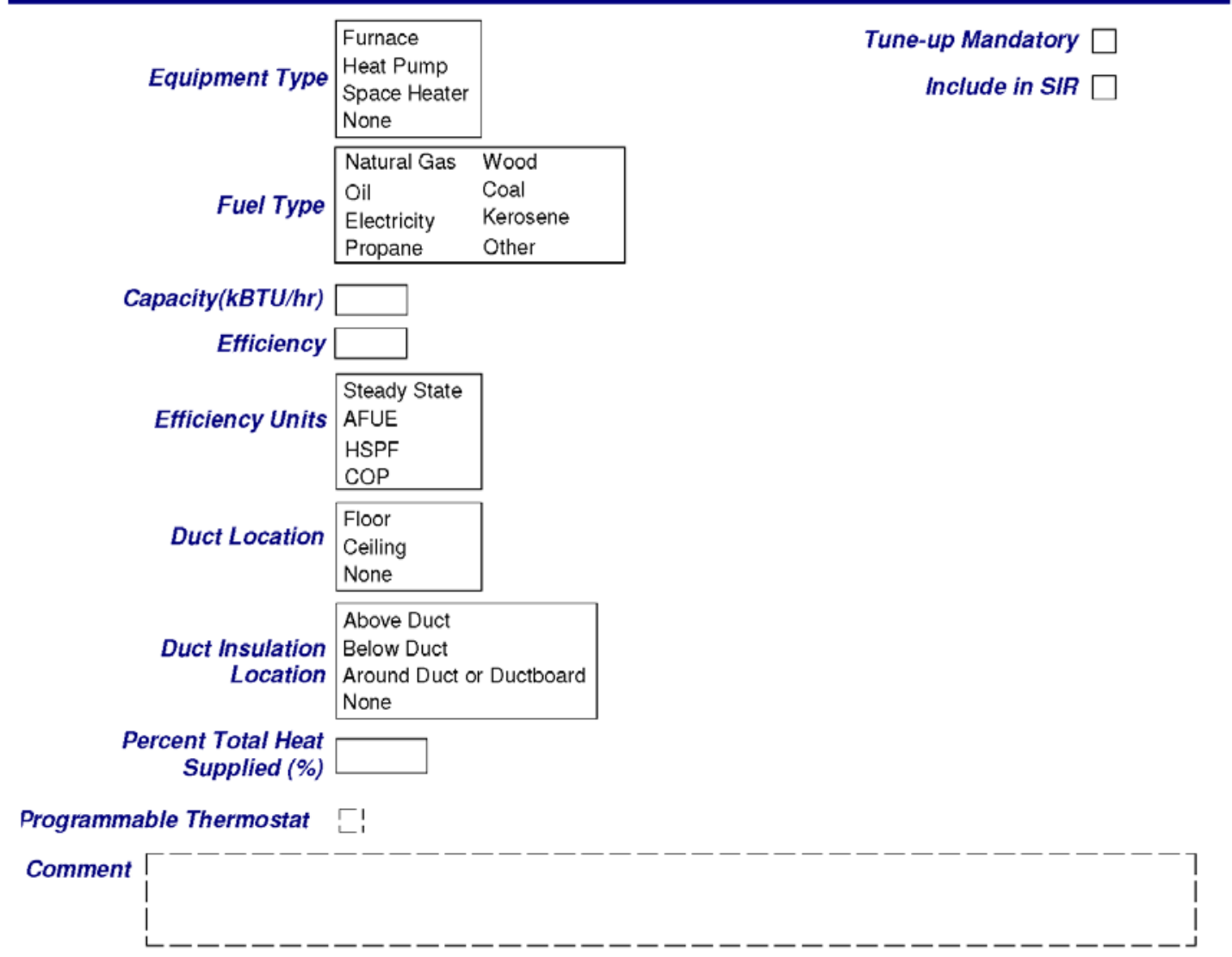

\section{Notes :}

Client Name:
Client ID:
Att. Client ID:




\section{Heating System (Secondary)}

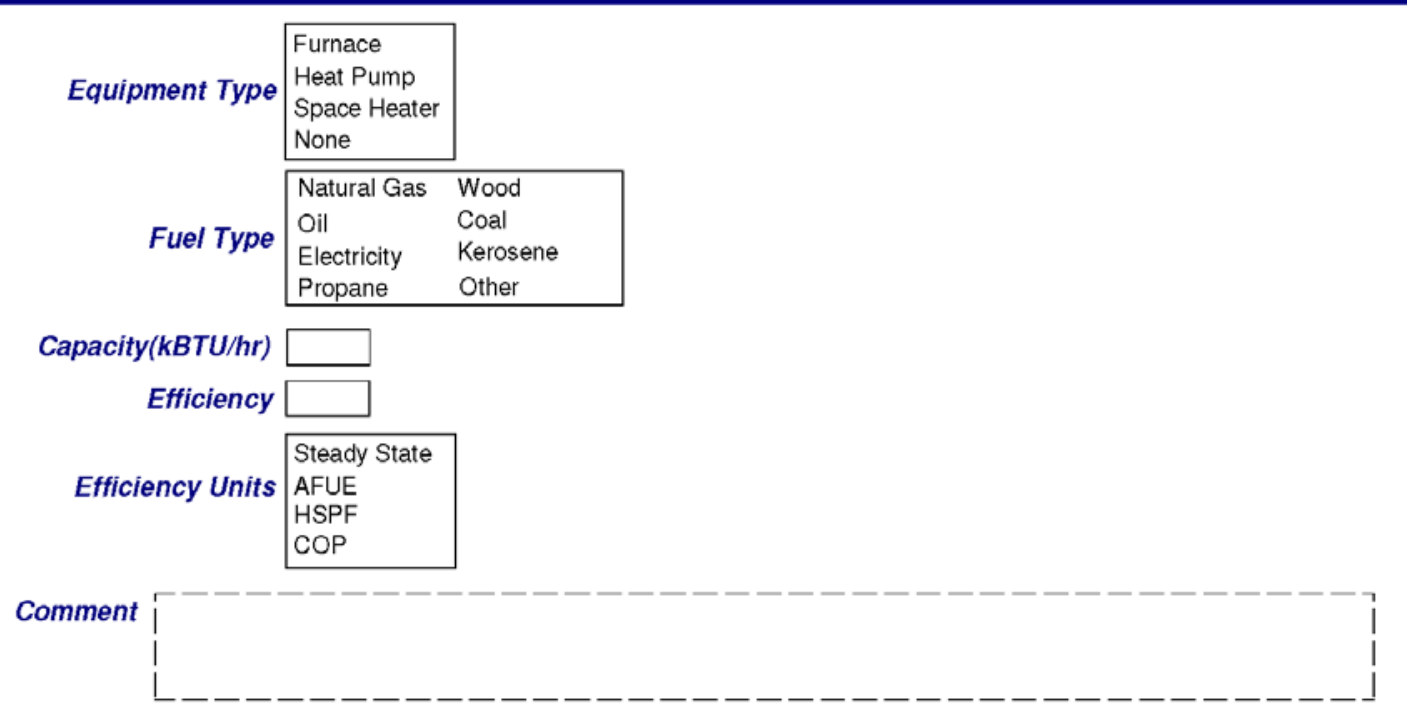

Notes:

Client Name:
Client ID:
Att. Client ID:




\section{Appendix C: Reports}

\section{Heating System (Replacement)}

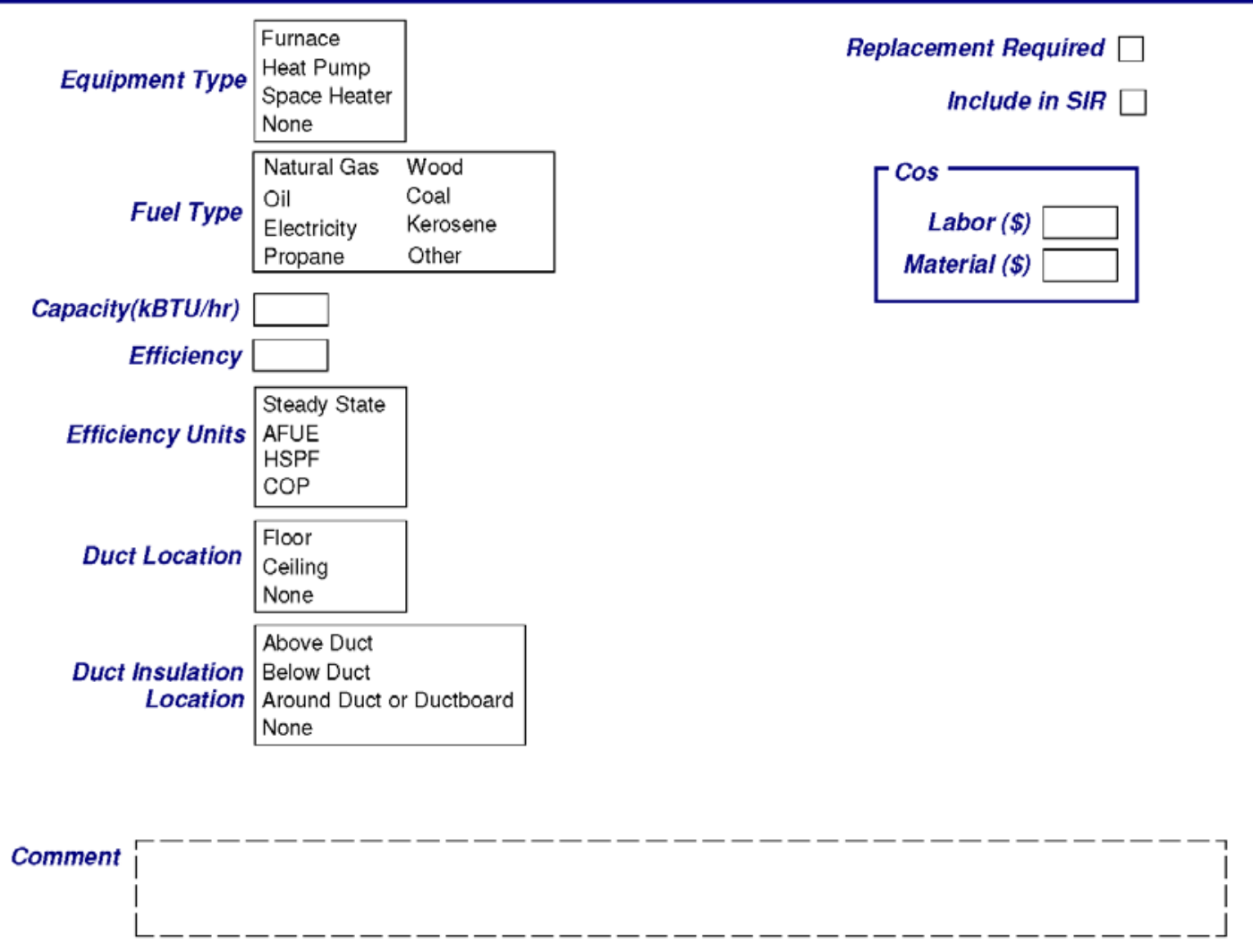

Notes:

Client Name:
Client ID:
Att. Client ID:




\section{Heating Systems (Continued)}

OPTIONAL HEATING SYSTEM DETAILS

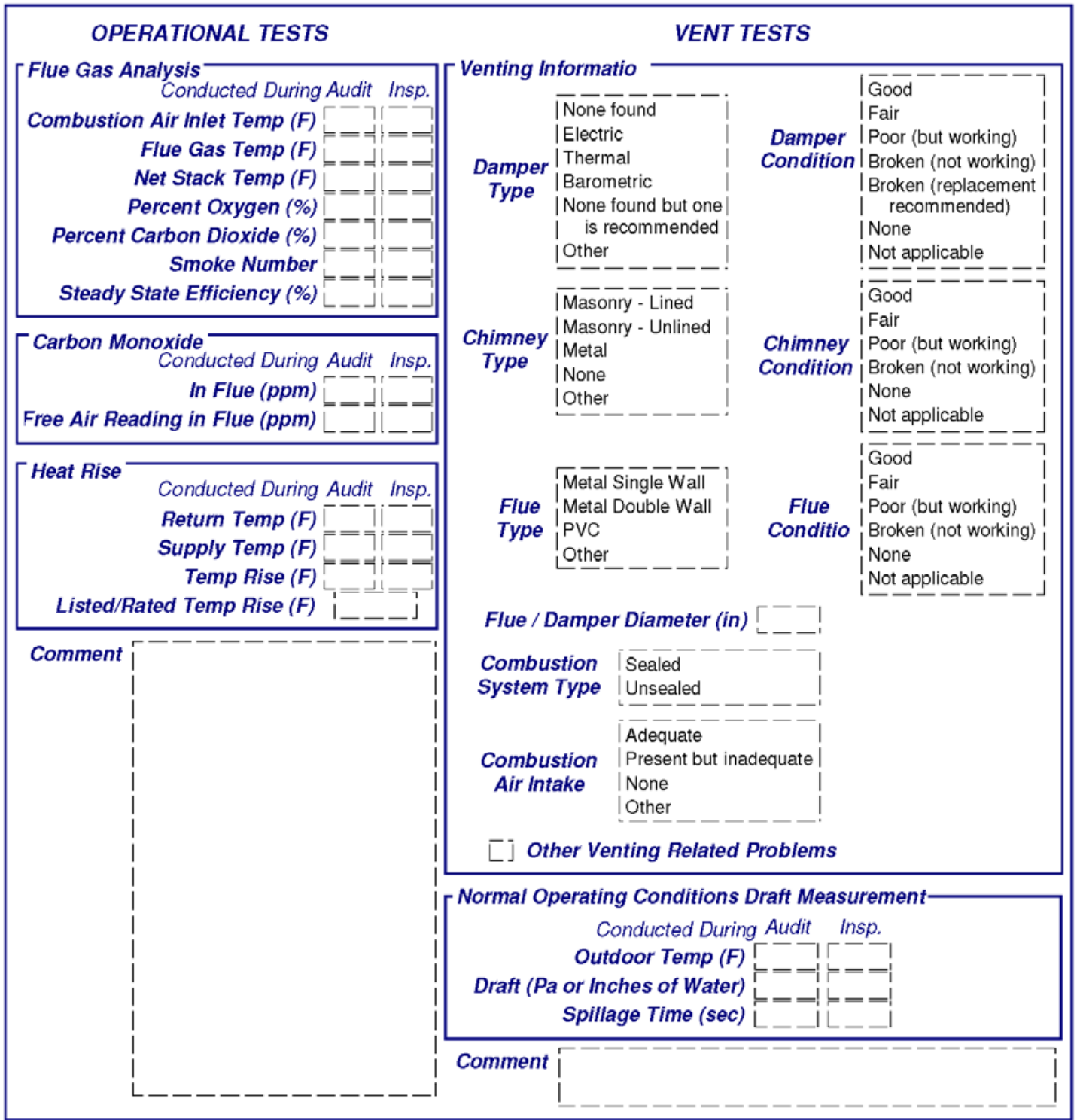

Notes:

Client Name:
Client ID:
Alt. Client ID:

MHEA Data Collection Form

Form Run On: 11/3/2011
DOE Weatherization Assistant

Version 8.9.0

Page 19 of 41 


\section{Appendix C: Reports}

\section{Heating Systems (Continued)}

OPTIONAL HEATING SYSTEM DETAILS (Continued)

\begin{tabular}{|c|c|}
\hline$\left[\begin{array}{l}\text { Fan Limit Controls } \\
\sqsubset\urcorner \text { Control Settings are Adjustable } \\
\lceil\rceil \text { Limit Controls Not Working }\end{array}\right.$ & $\begin{array}{l}\text { Setting }(F) \longleftarrow=\dashv \\
\text { f Setting }(F) \longleftarrow=\rfloor \\
\text { Setting }(F) !\end{array}$ \\
\hline 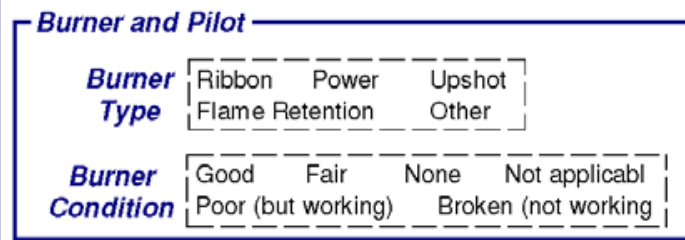 & 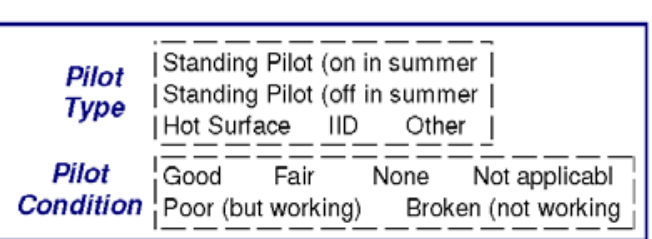 \\
\hline 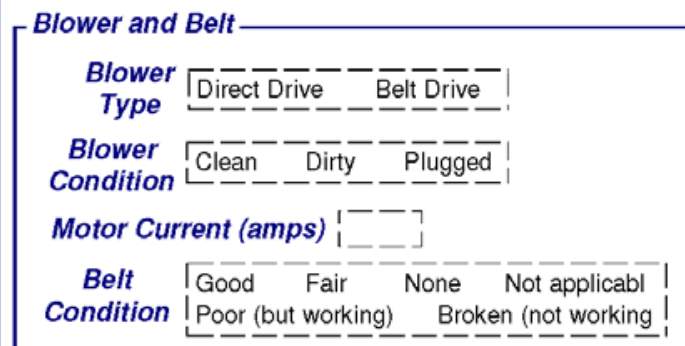 & 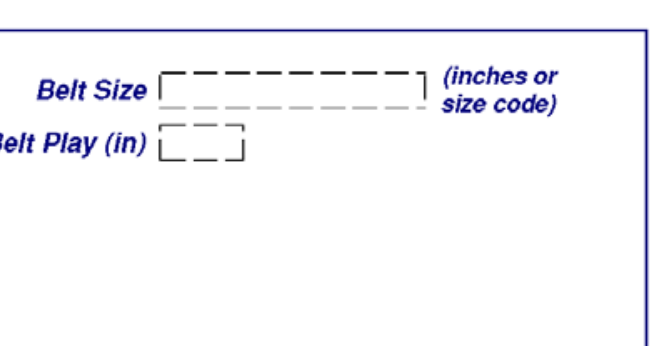 \\
\hline 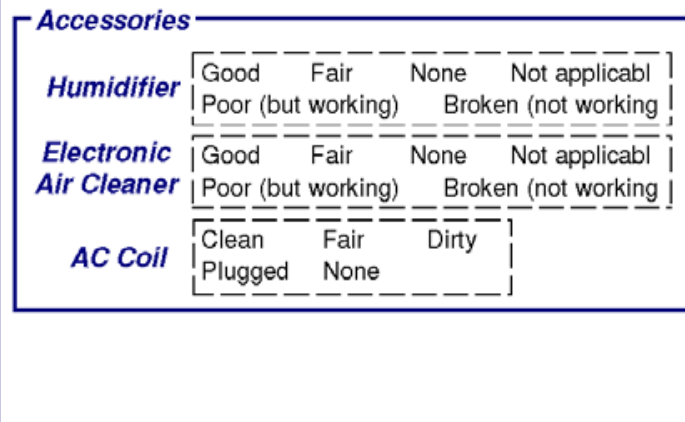 & 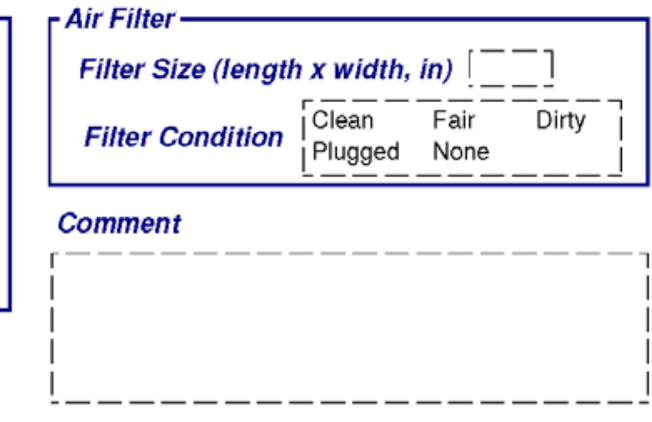 \\
\hline
\end{tabular}

\section{Notes :}

Client Name:
Client ID:
Alt. Client ID:

MHEA Data Collection Form

Form Run On: 11/3/2011
DOE Weatherization Assistant

Version 8.9.0

Page 20 of 41 


\section{Heating Systems (Continued)}

\section{OPTIONAL HEATING SYSTEM DETAILS (Continued)}

\begin{tabular}{|c|c|}
\hline 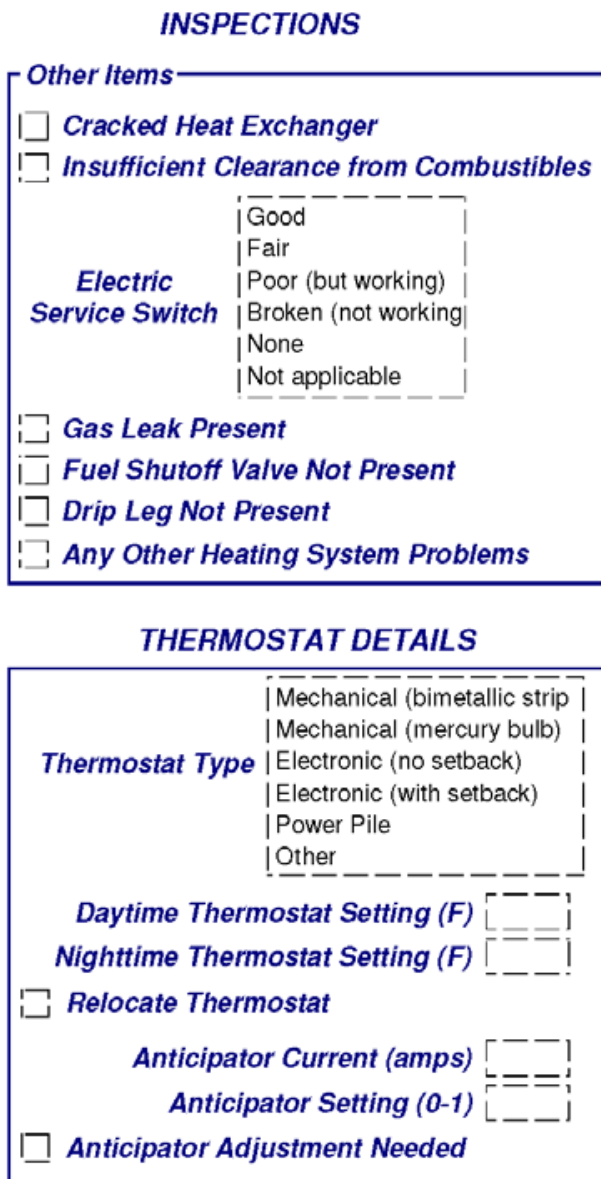 & $\left\{\begin{array}{l}\text { Comment } \\
\mid \begin{array}{r}\mid \\
---------------1\end{array}\end{array}\right.$ \\
\hline
\end{tabular}

Notes :

Client Name:
Client ID:
Att. Client ID:




\section{Appendix C: Reports}

\section{Cooling System (Primary)}

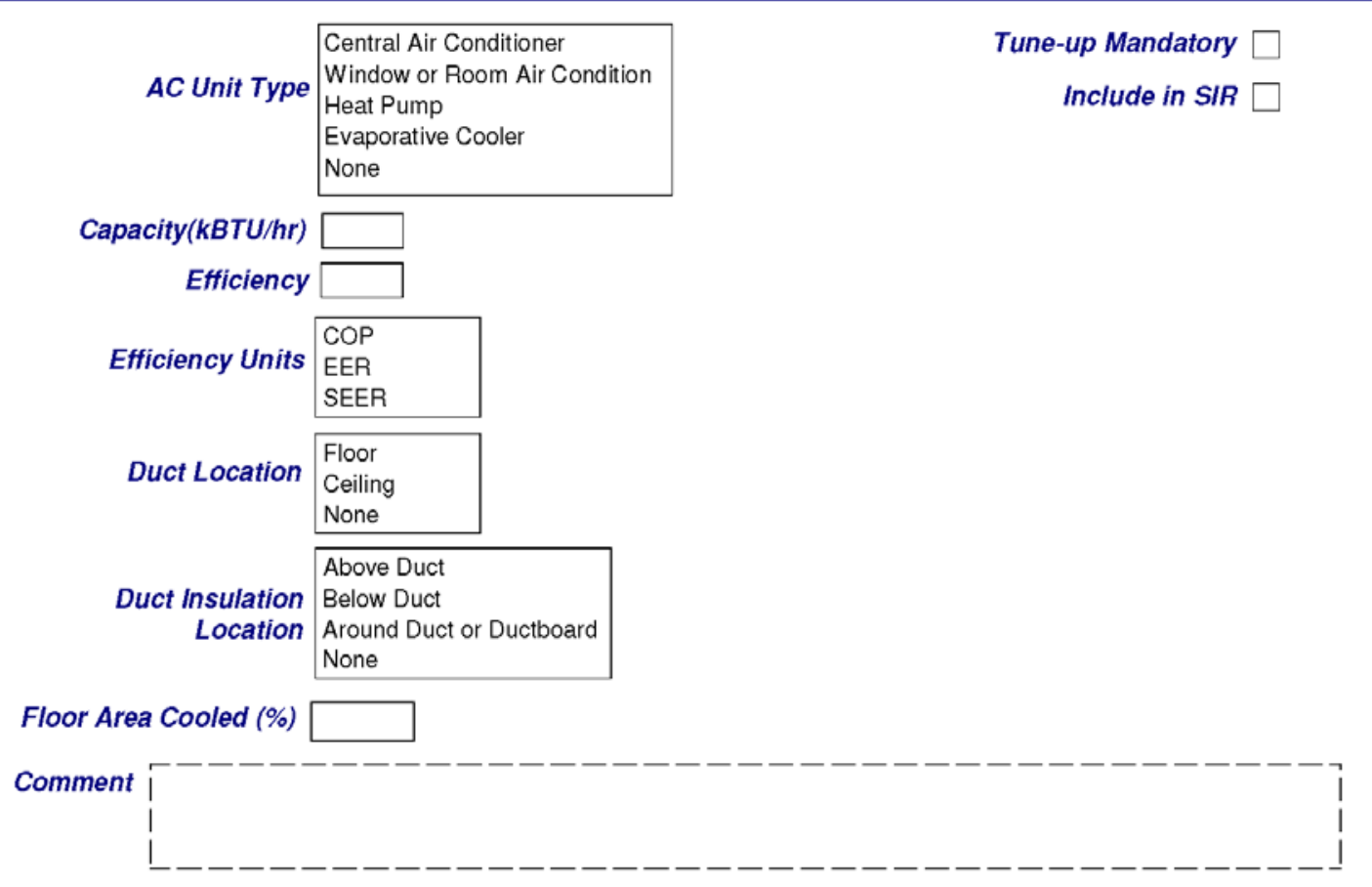

Notes:

Client Name:
Client ID:
Att. Client ID:




\section{Cooling System (Secondary)}

AC Unit Type \begin{tabular}{|l|} 
Central Air Conditioner \\
Window or Room Air Condition \\
Heat Pump \\
Evaporative Cooler \\
None
\end{tabular}

Capacity (kBTU/hr)

Efficiency

\begin{tabular}{l|l|} 
Efficiency Units & COP \\
EER \\
SEER
\end{tabular}

Floor Area Cooled (\%)

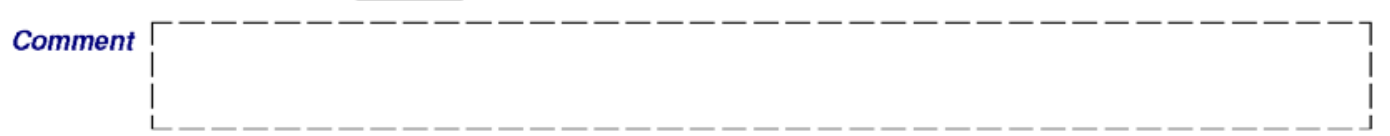

\section{Notes:}

Client Name:
Client ID:
Att. Client ID:




\section{Appendix C: Reports}

\section{Cooling System (Replacemen}

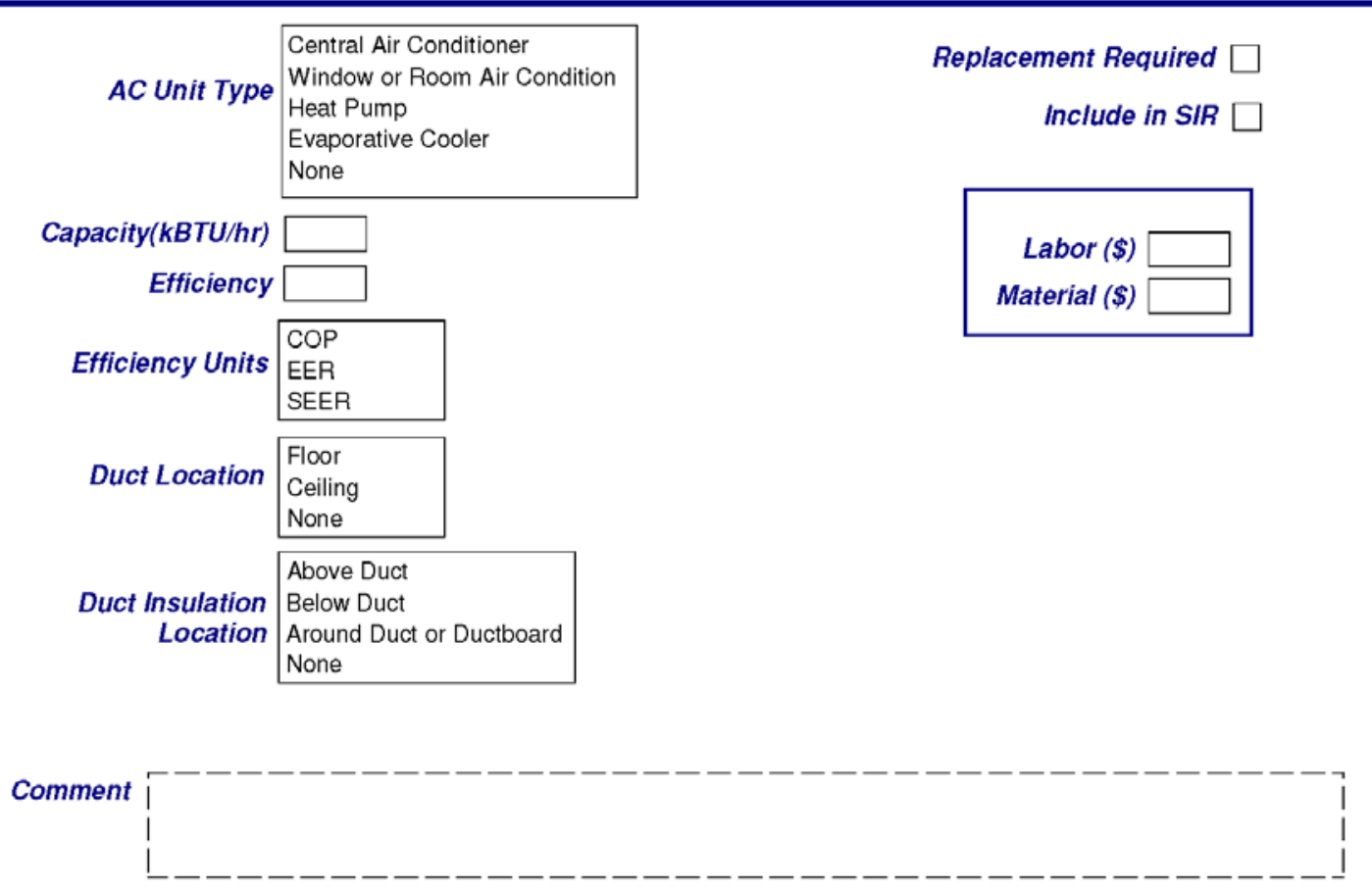

Notes :

Client Name:
Client ID:
Att. Client ID:




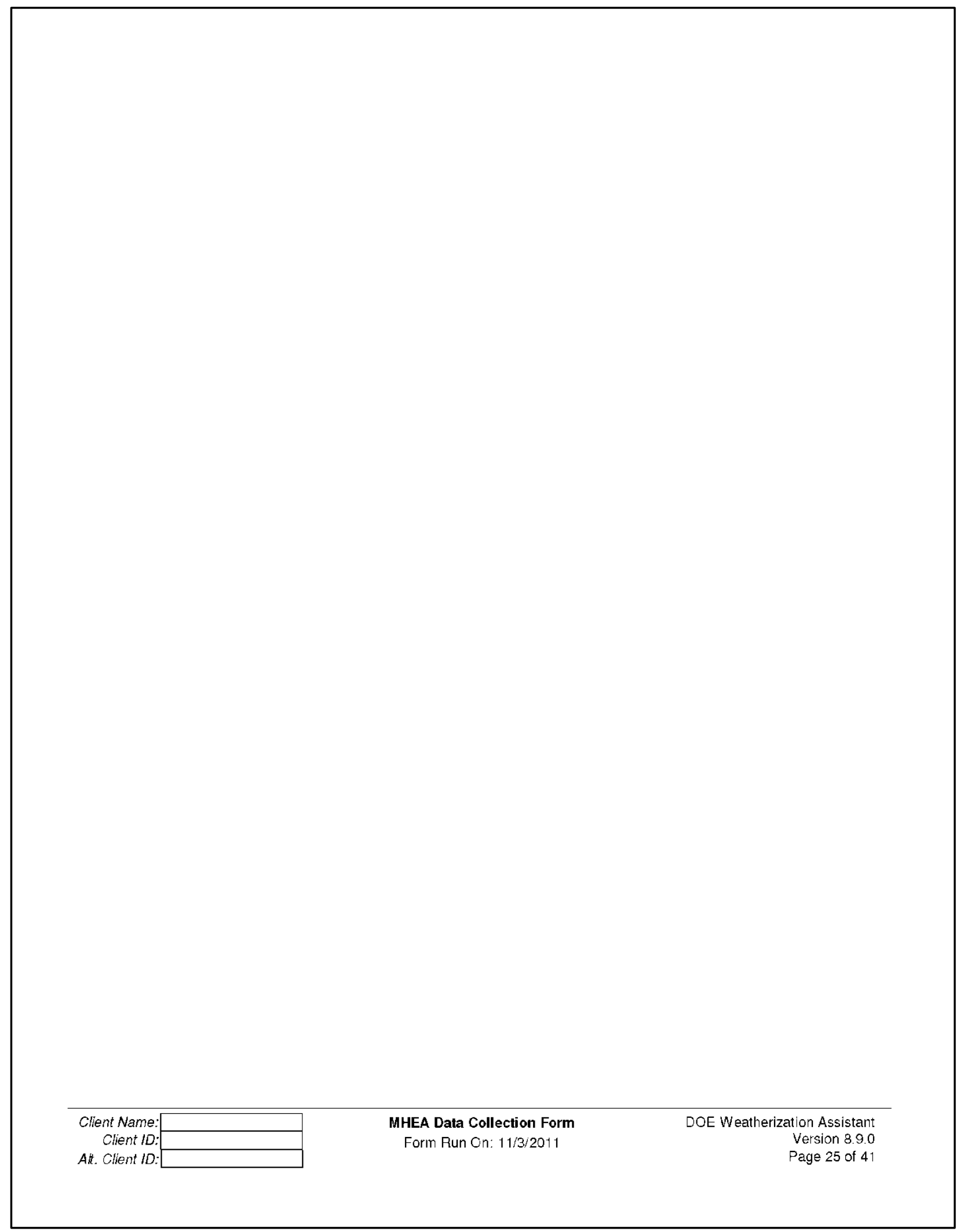




\section{Appendix C: Reports}

\section{Ducts / Infiltration}

\section{AlR and DUCT LEAKAGES}

Evaluate Duct Sealing?

Whole House Blower Door Measurement

Method Blower Door Subtraction (sealed and unsealed registers and grills)

Duct-Blower Measurements

Pressure Pan Measurements
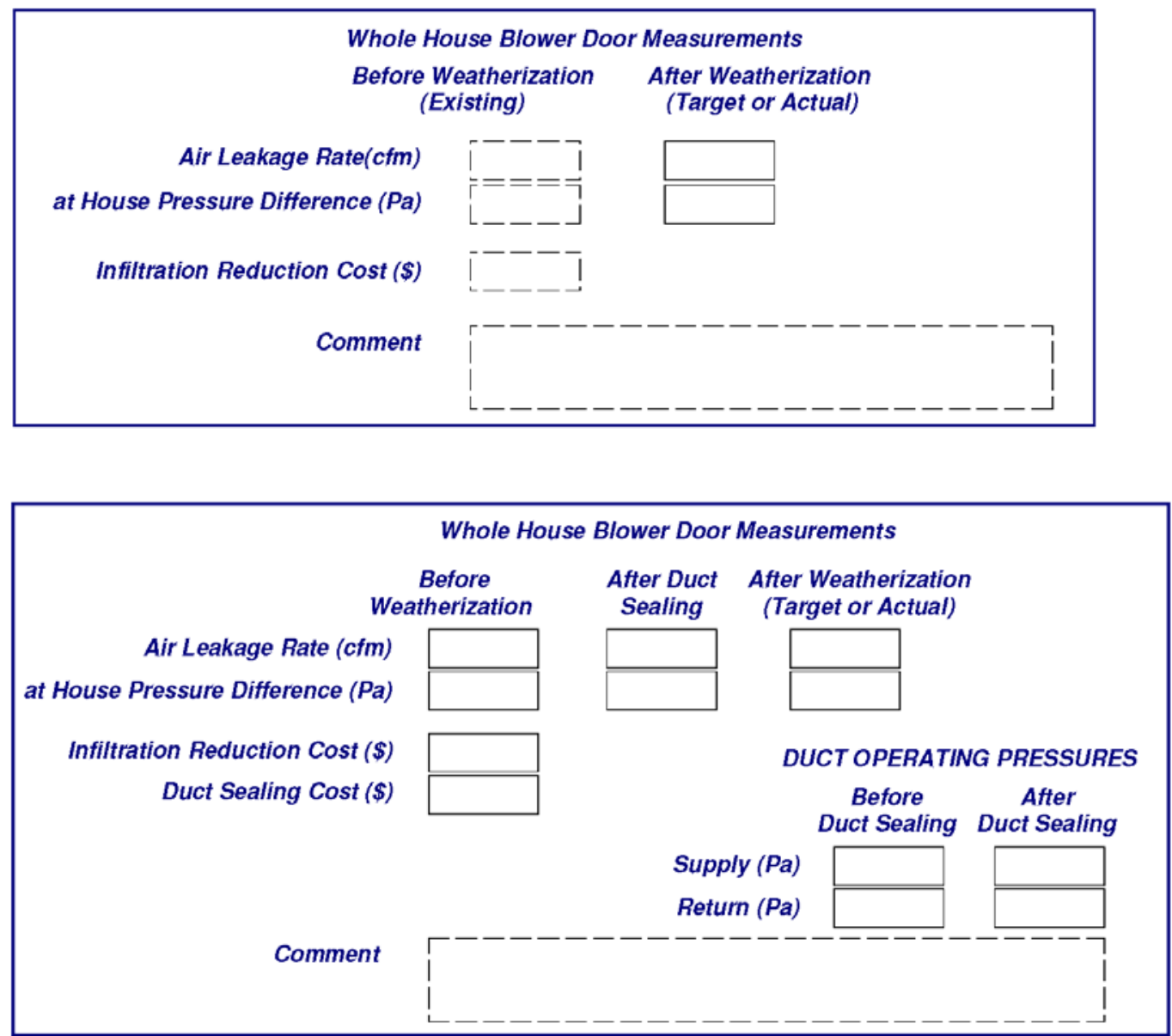

Client Name:
Client ID:
Att. Client ID:

MHEA Data Collection Form

Form Run On: 11/3/2011
DOE Weatherization Assistant

Version 8.9.0

Page 26 of 41 
Ducts / Infiltration (Continued)

\section{AIR and DUCT LEAKAGES (Continued)}

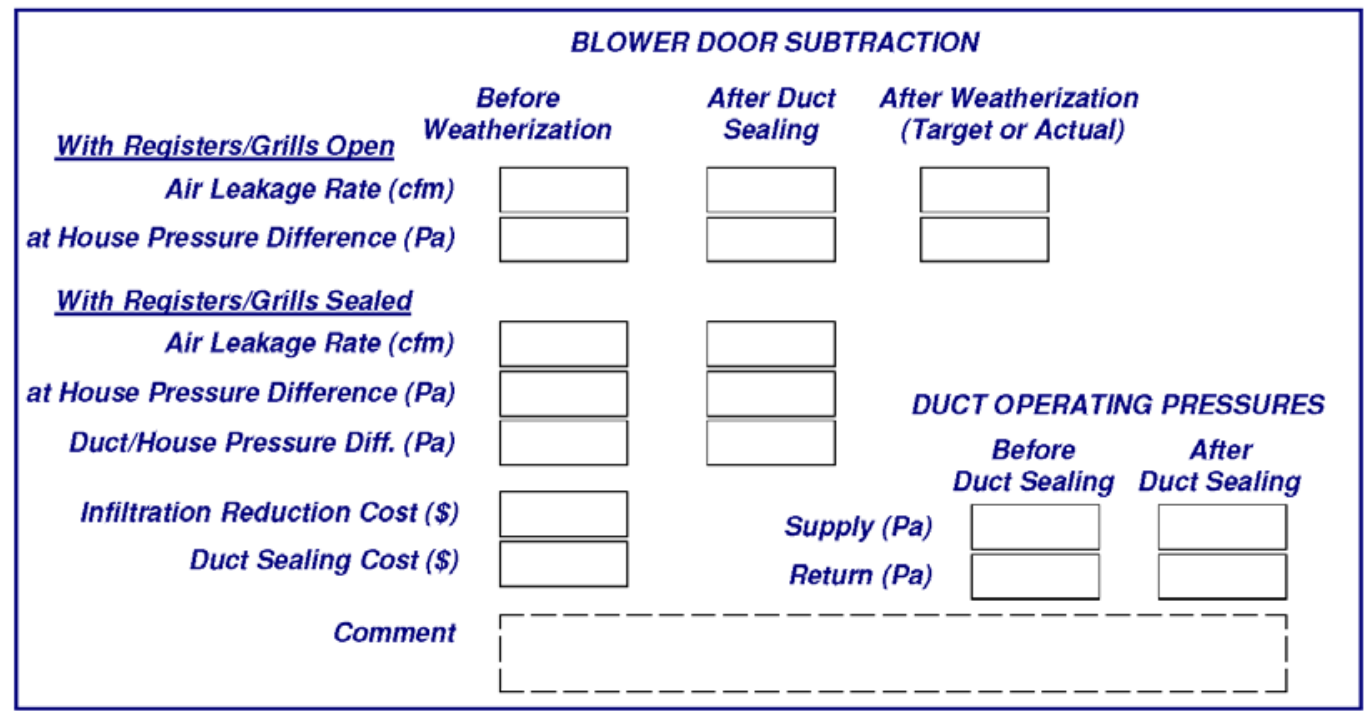

WHOLE HOUSE BLOWER DOOR MEASUREMENT

Before Weatherizatio
DUCT OPERATING PRESSURES Before Duct Sealing Duct Sealing

Air Leakage Rate (CFM)

at House Pressure Difference $(\mathrm{Pa})$
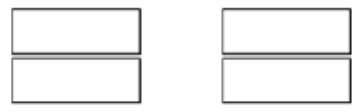

Supply $(\mathrm{Pa})$

Return (Pa)

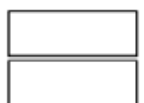

DUCT BLOWER MEASUREMENTS

\section{Before Duct Sealing}

\section{After Duct Sealing}

" 'Outside' readings are taken while Total Outside *

Total Outside * differential provided by a blower door is maintained at the same

Fan Flow (CFM) at Duct Pressure $(\mathrm{Pa})$ House Pressure $(\mathrm{Pa})$ wrt outside
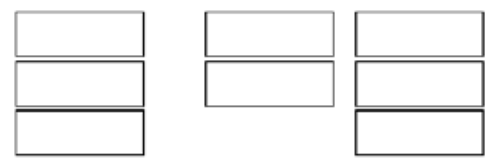
level as the duct / outdoor pressure differential created by the ductblower. Thus the 'Duct Pressure' and the 'House Pressure wrt outside' should be equal.

Infiltration Reduction Cost (\$) Duct Sealing Cost (\$)
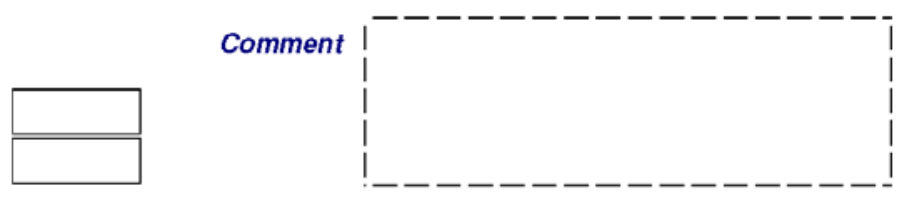

MHEA Data Collection Form

Form Run On: 11/3/2011
DOE Weatherization Assistant

Version 8.9 .0

Page 27 of 41 


\section{Appendix C: Reports}

Ducts / Infiltration (Continued)

\section{AIR and DUCT LEAKAGES (Continued)}

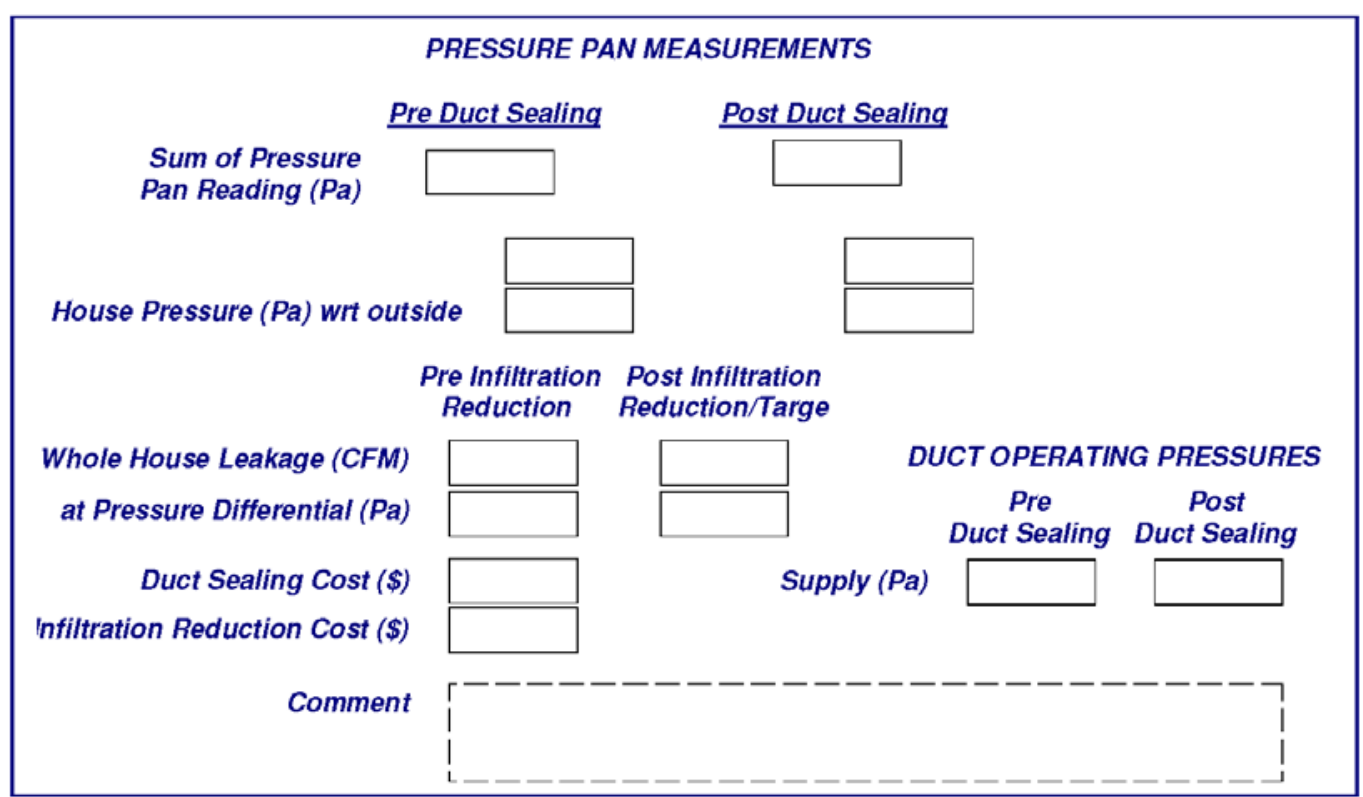

Notes:

Client Name:
Client ID:
Att. Client ID:


Ducts / Infiltration (Continued)

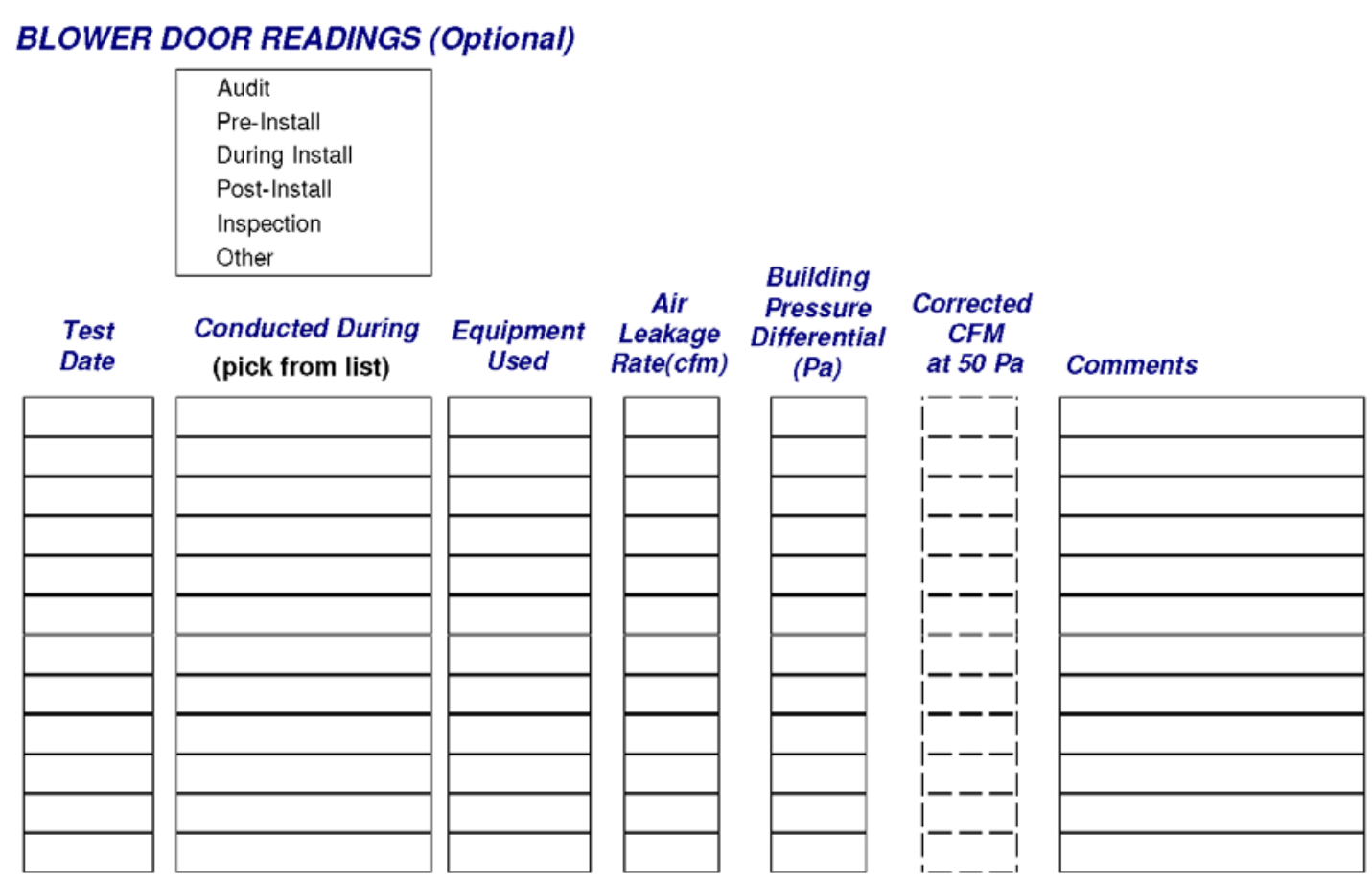

Notes :

Client Name:
Client ID:
Alt. Client ID:




\section{Appendix C: Reports}

Ducts / Infiltration (Continued)

\section{ZONAL PRESSURES (Optional)}

\begin{tabular}{l|ll|}
\hline Audit & Attic & Exterior Wall \\
Pre-Install & Side Attic & Interior Wall \\
During Install & Kneewall & Basement \\
Post-Install & Ceiling Joist Space & Crawl Space \\
Inspection & Attached Garage & Mobile Home Belly \\
Other & Unheated Addition & Other \\
\hline
\end{tabular}

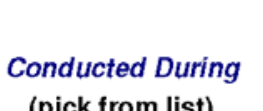

Building
Pressure
Differential (pick from list)

(Pa)

Zone Pressure

Location

(pick from list or describe)

Zone
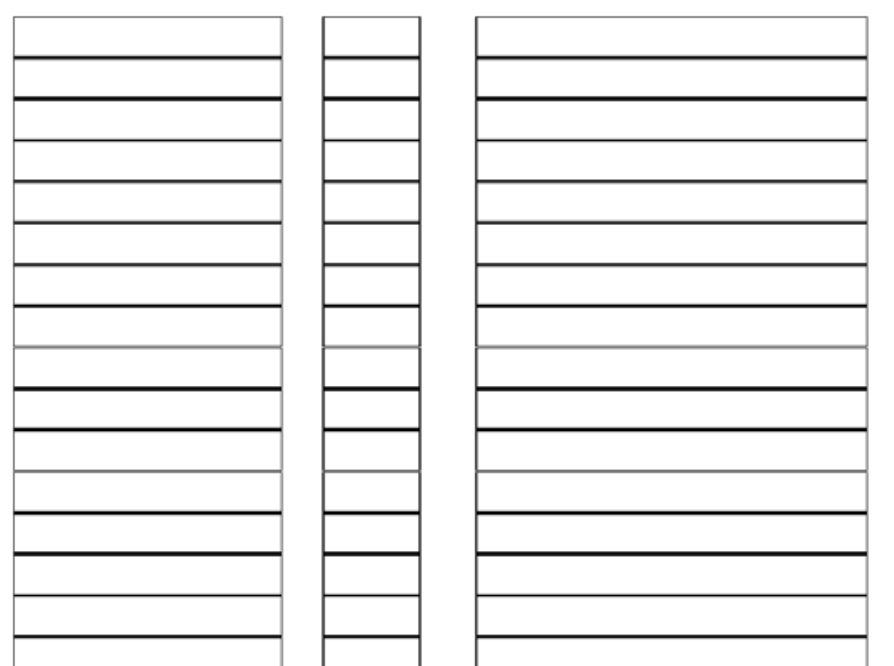

(Pa)

\section{Ducts}

Present Comments
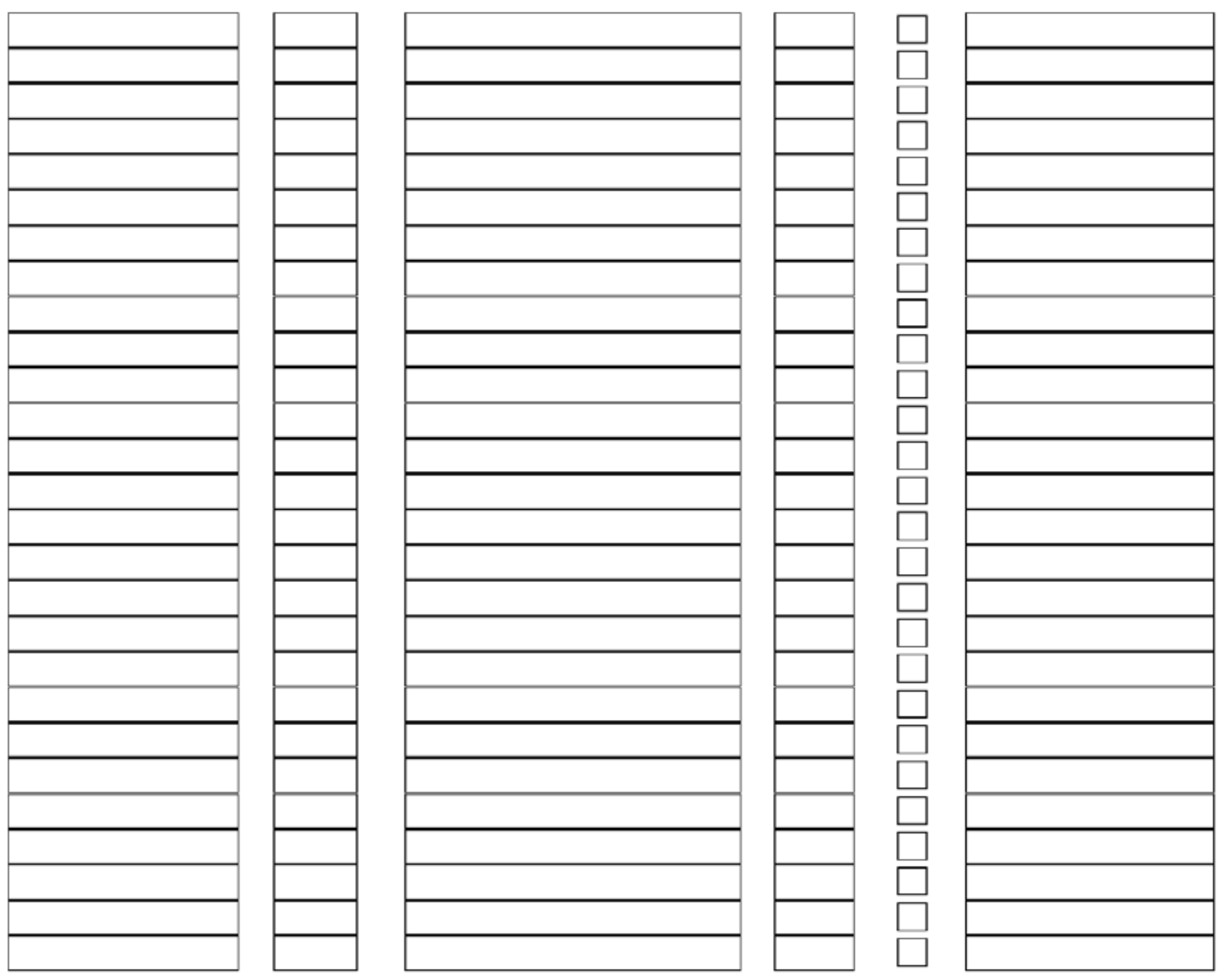

Notes :

Client Name:
Client ID:
Att. Client ID:

MHEA Data Collection Form

Form Run On: 11/3/2011
DOE Weatherization Assistant

Version 8.9.0

Page 30 of 41 
Ducts / Infiltration (Continued)

\section{PRESSURE BALANCE READINGS (Optional)}

\begin{tabular}{|ll|}
\hline Family Room & Bdrm1 \\
Living Room & Bdrm2 \\
Dining Room & Bdrm3 \\
Kitchen & Bdrm4 \\
Bath1 & Basement \\
Bath2 & Addition \\
Bath3 & Other \\
\hline
\end{tabular}

Location Initial Final

(pick one or describe) Pressure $(\mathrm{Pa}) \quad$ Pressure $(\mathrm{Pa})$
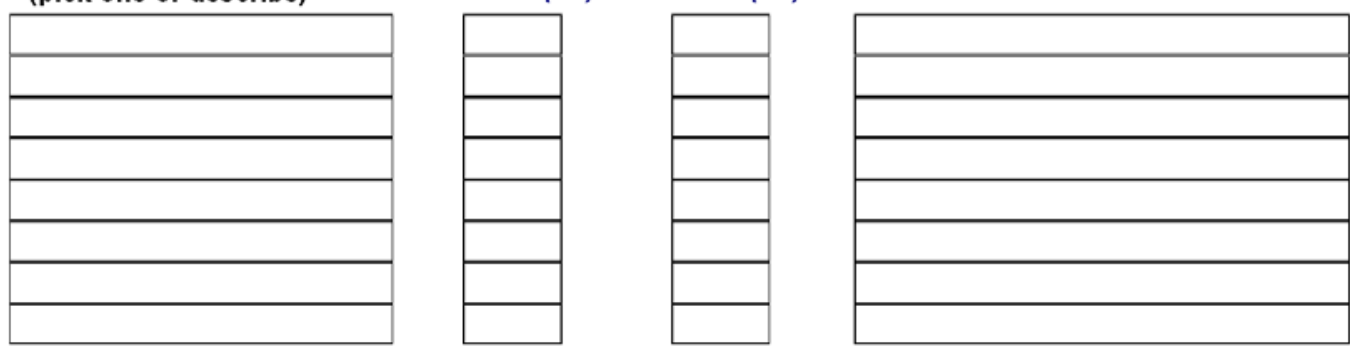

Notes :

Client Name:
Client ID:
Att. Client ID:




\section{Appendix C: Reports}

Ducts / Infiltration (Continued)

\section{PRESSURE PAN READINGS (Optional)}

\begin{tabular}{|ll|}
\hline Family Room & Bdrm1 \\
Living Room & Bdrm2 \\
Dining Room & Bdrm3 \\
Kitchen & Bdrm4 \\
Bath1 & Basement \\
Bath2 & Foyer \\
Bath3 & Hallway \\
& Other \\
\hline
\end{tabular}

Location

Register \# (pick one or describe)
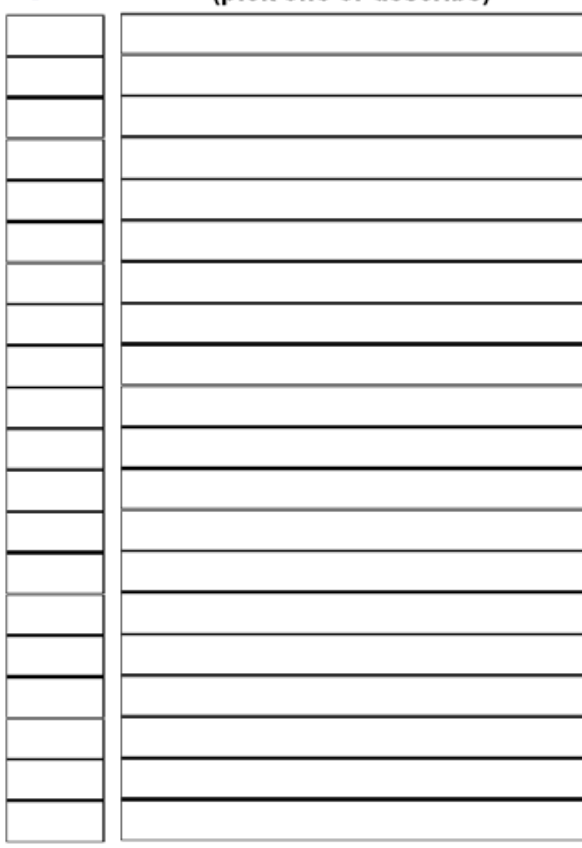

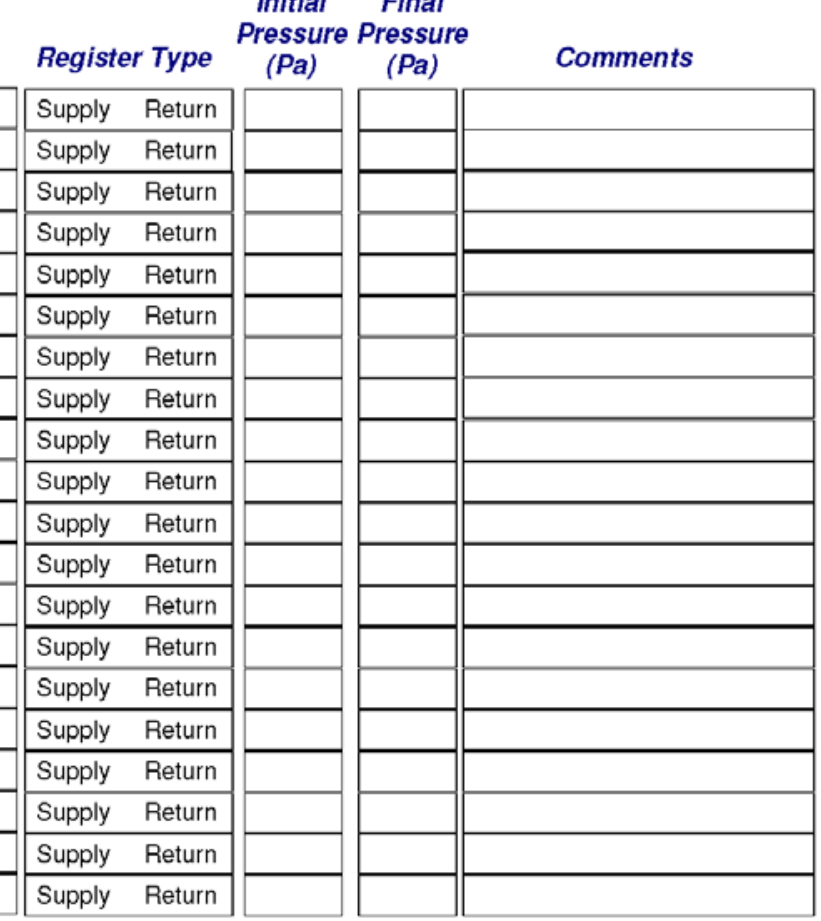

\section{Notes:}

Client Name:
Client ID:
Att. Client ID:


Base Load - Water Heater

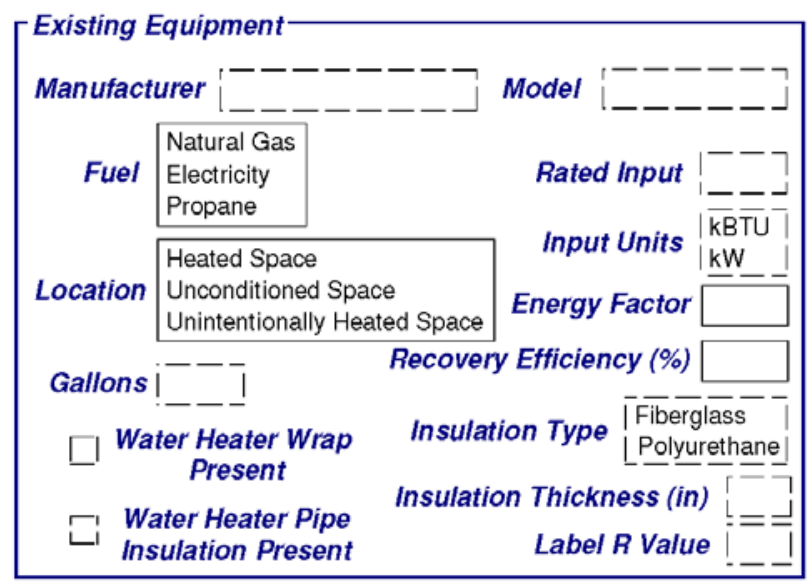

Shower Heads

Number of Showerheads $\left.\right|^{-}-$

Minutes of Shower Use Per Day

Average GPM $\left.\right|_{--}$ $-1$

Comment

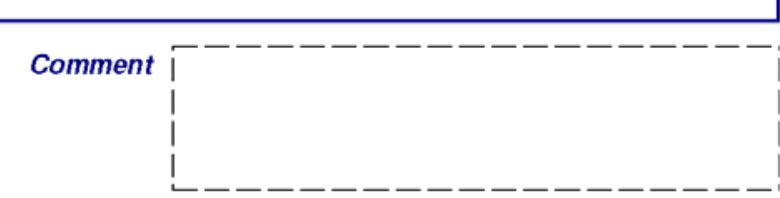

Notes:

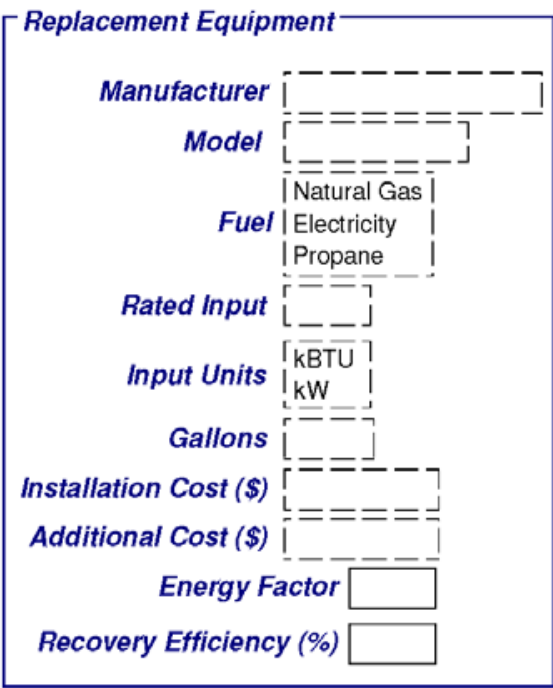

Replacement Equipment

acturer

- - - -$\lrcorner$

Natural Gas |

$\mathrm{L}_{--} \mathrm{J}$

$\lceil\overline{\mathrm{kBT}} \overline{\mathrm{B}}$

:

$[= \pm--$

Installation Cost (\$)

Cost (\$)

Client Name:
Client ID:
Att. Client ID:




\section{Appendix C: Reports}

\section{Base Load - Water Heater (Continued)}

OPTIONAL WATER HEATING SYSTEM DETAIL

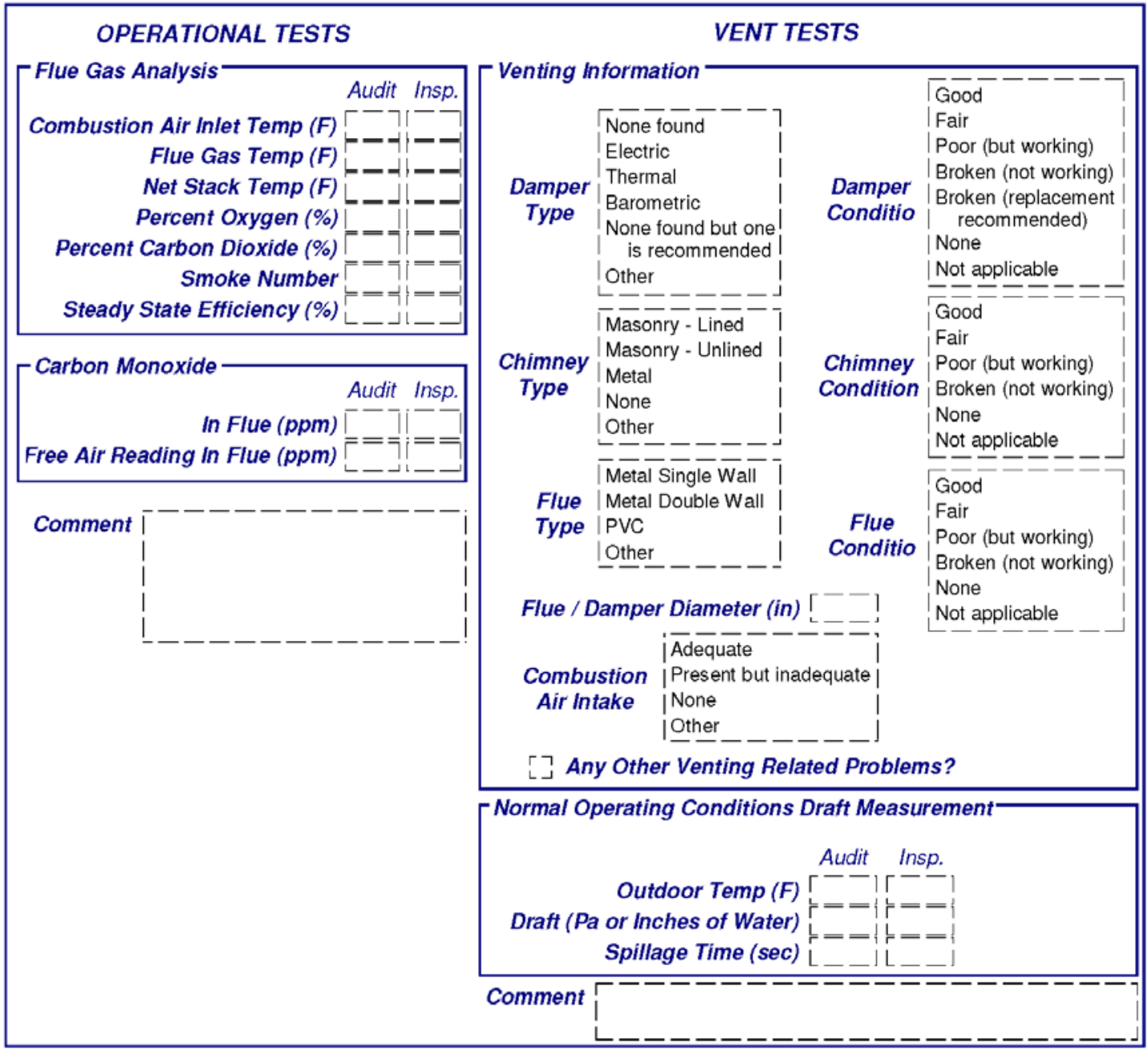

\section{Notes :}

Client Name:
Client ID:
Att. Client ID:

MHEA Data Collection Form

Form Run On: 11/3/2011
DOE Weatherization Assistant

Version 8.9.0

Page 34 of 41 
Base Load - Water Heater (Continued)

\section{OPTIONAL WATER HEATING SYSTEM DETAILS (Continued)}

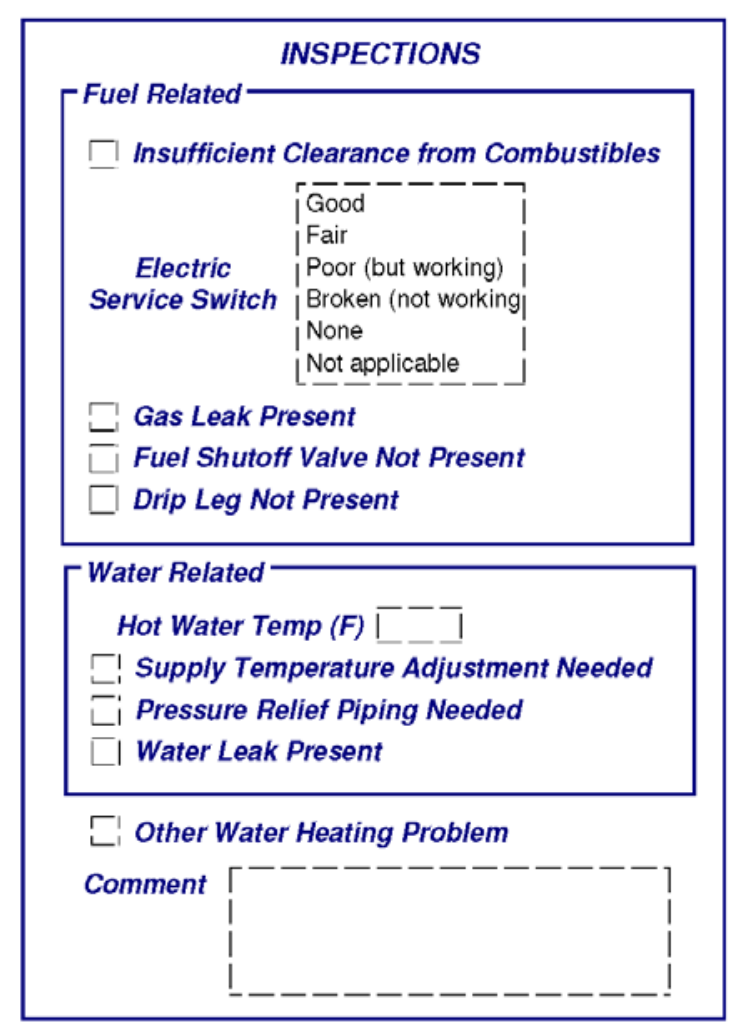

Notes :

Client Name:
Client ID:
Att. Client ID:




\section{Appendix C: Reports}

\section{Base Load - Refrigerator}

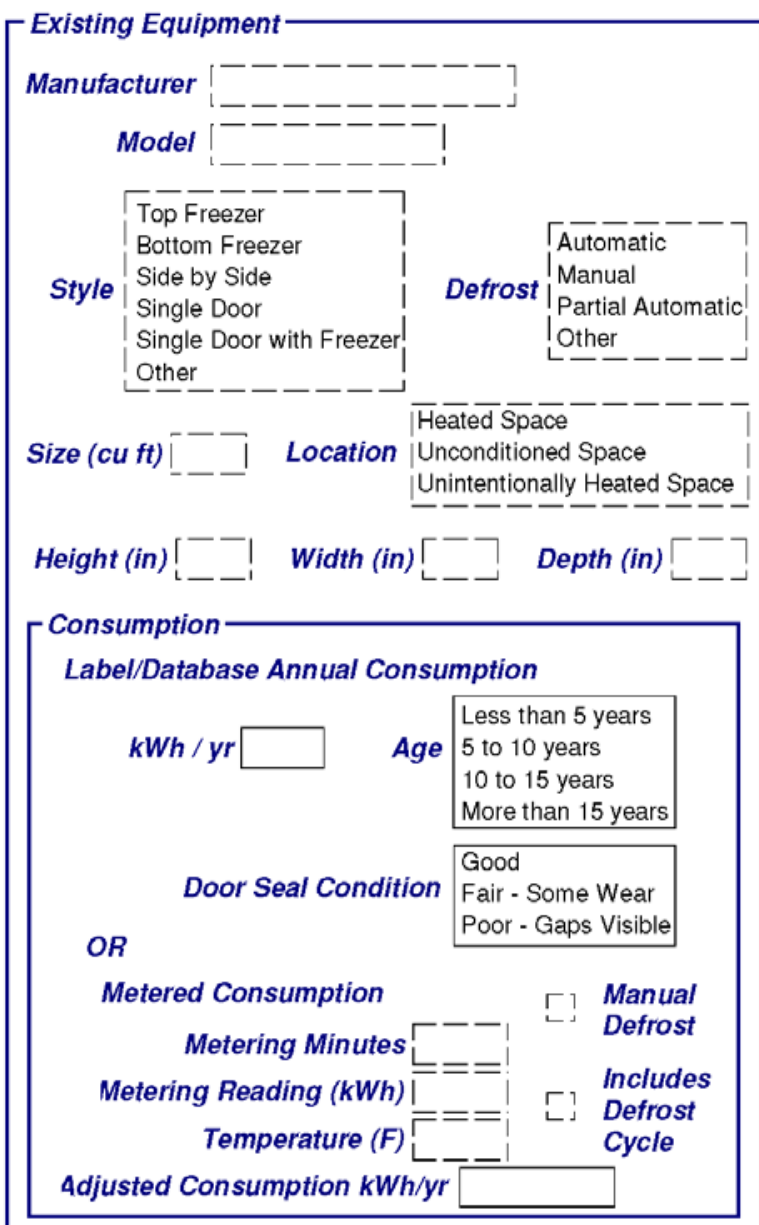

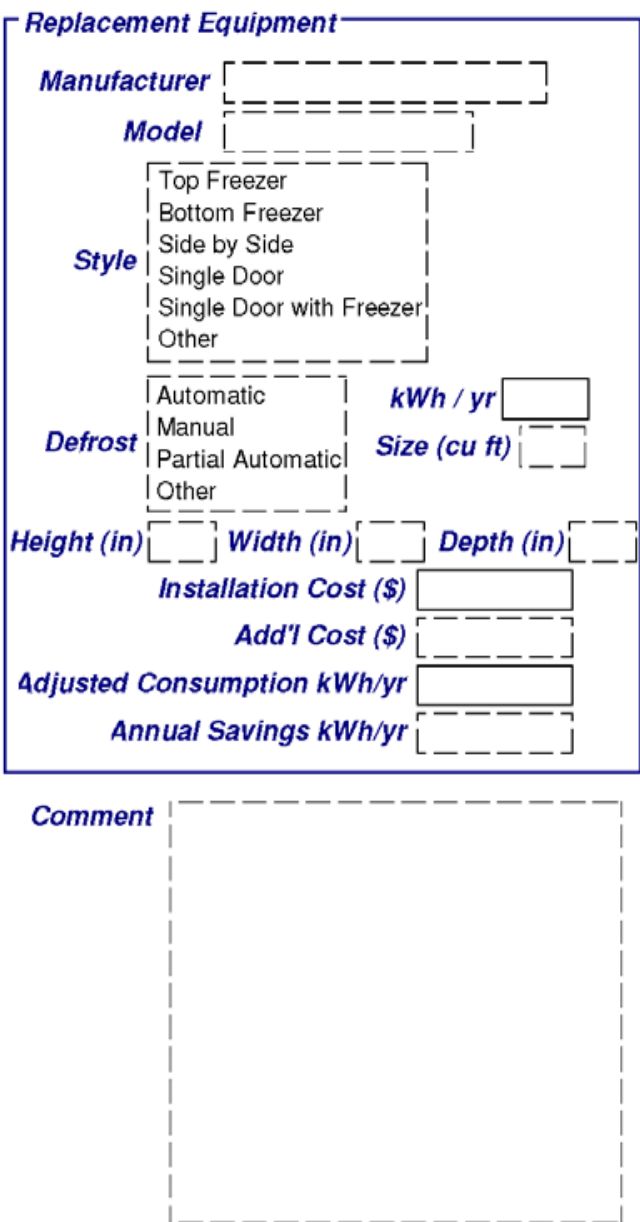

\section{Notes:}

\begin{tabular}{|c|c|c|}
\hline Client Name: & MHEA Data Collection Form & DOE Weatherization Assistant \\
\hline Client ID: & Form Run On: 11/3/2011 & Version 8.9 .0 \\
\hline Alt. Client ID: & & Page 36 of 41 \\
\hline
\end{tabular}


Baseload - Lighting Systems

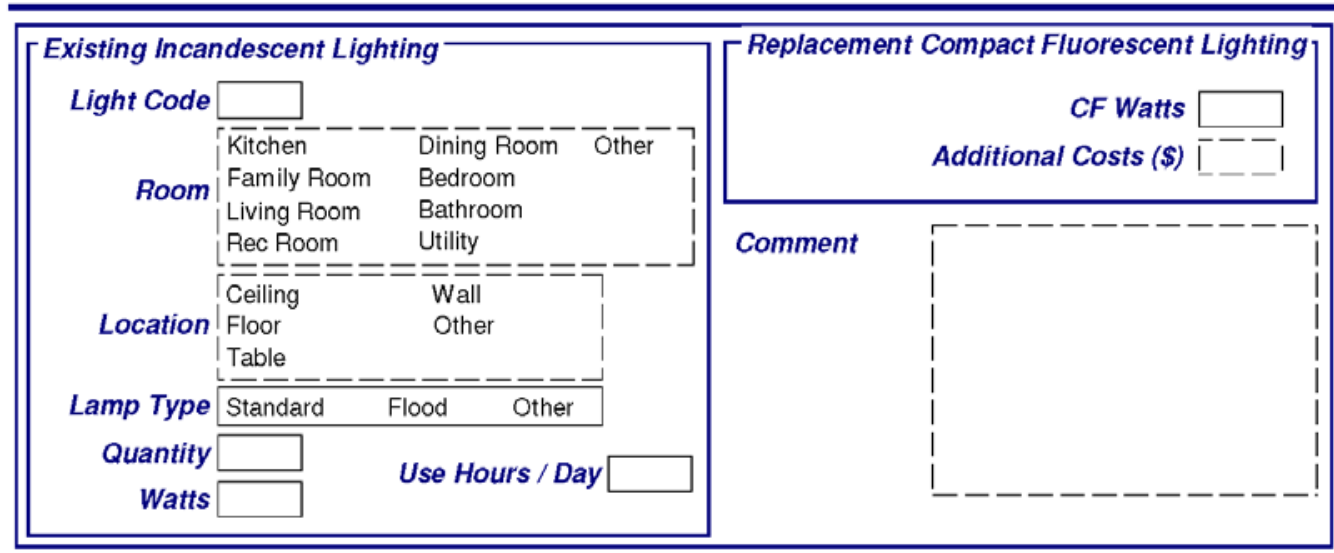

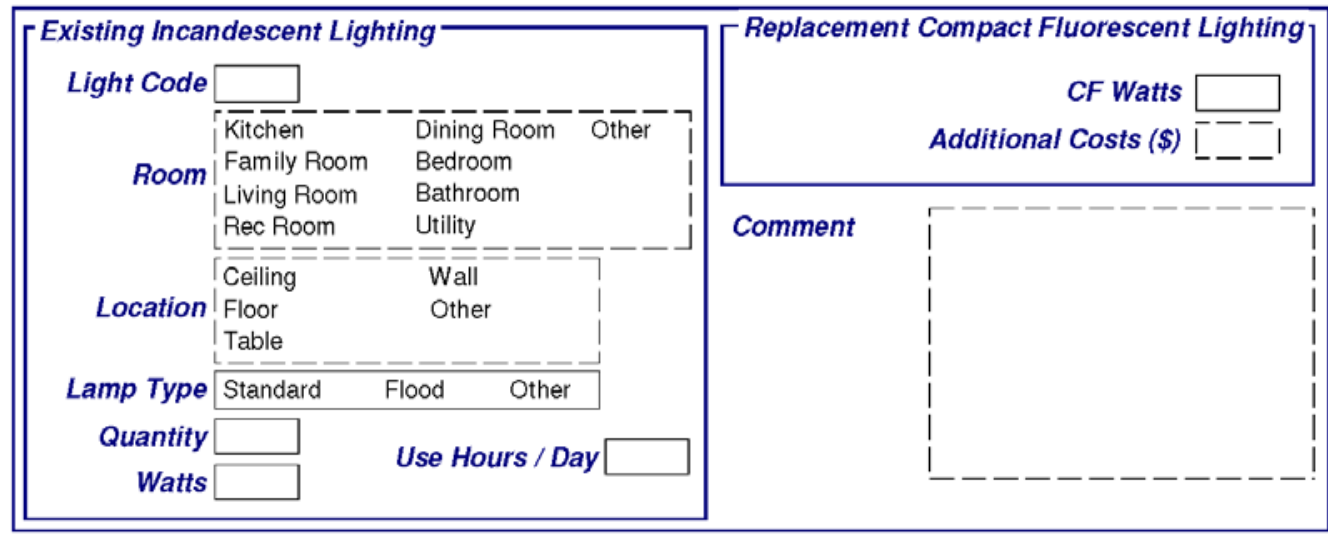

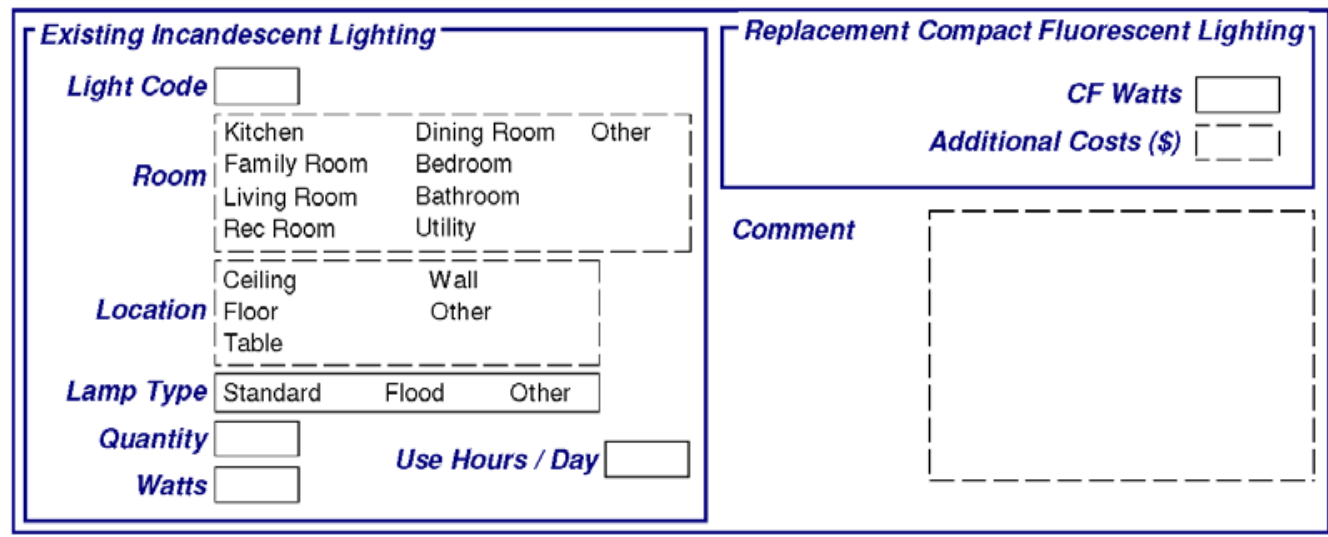

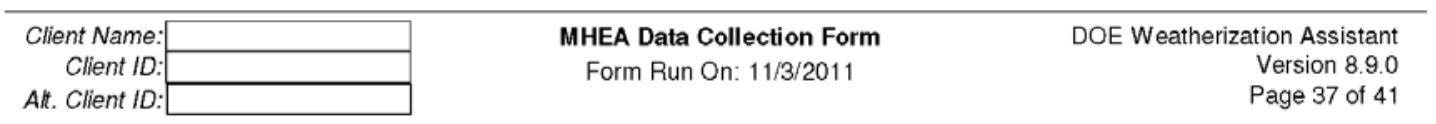




\section{Appendix C: Reports}

\section{Health \& Safety}

WHOLE HOUSE

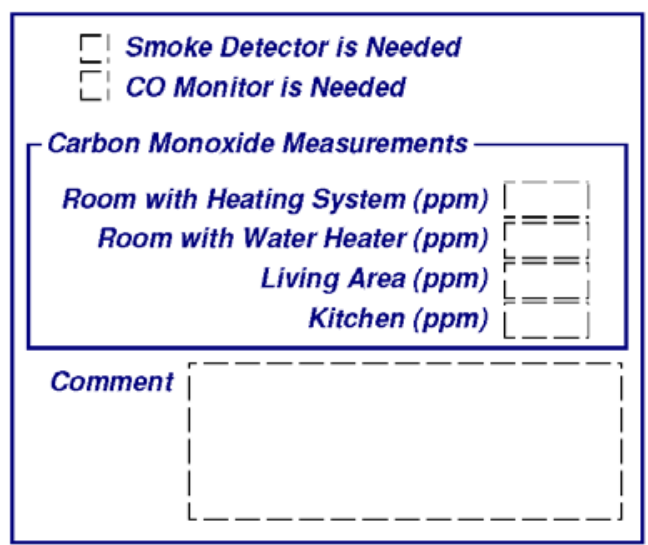

\section{BUILDING SHELL}

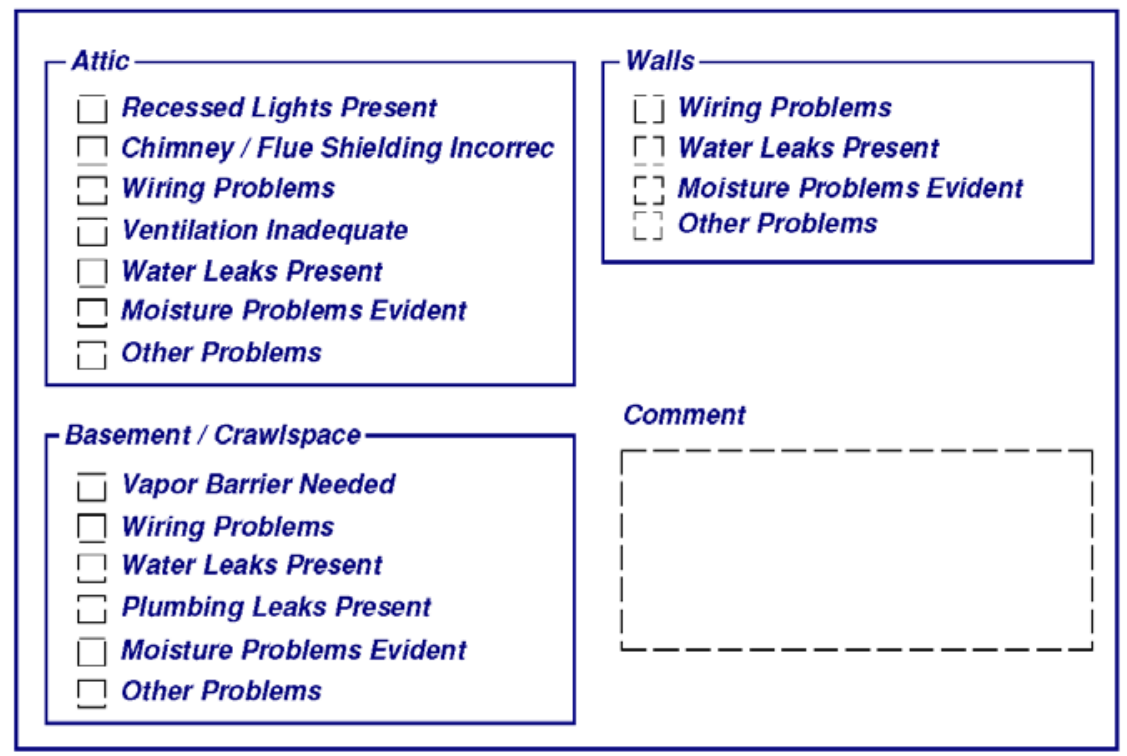

\section{Notes :}

Client Name:
Client ID:
Att. Client ID:




\section{Health \& Safety (Continued)}

\section{EQUIPMENT}

[Worst Case Condition Draft Measurements - Space Heating System(

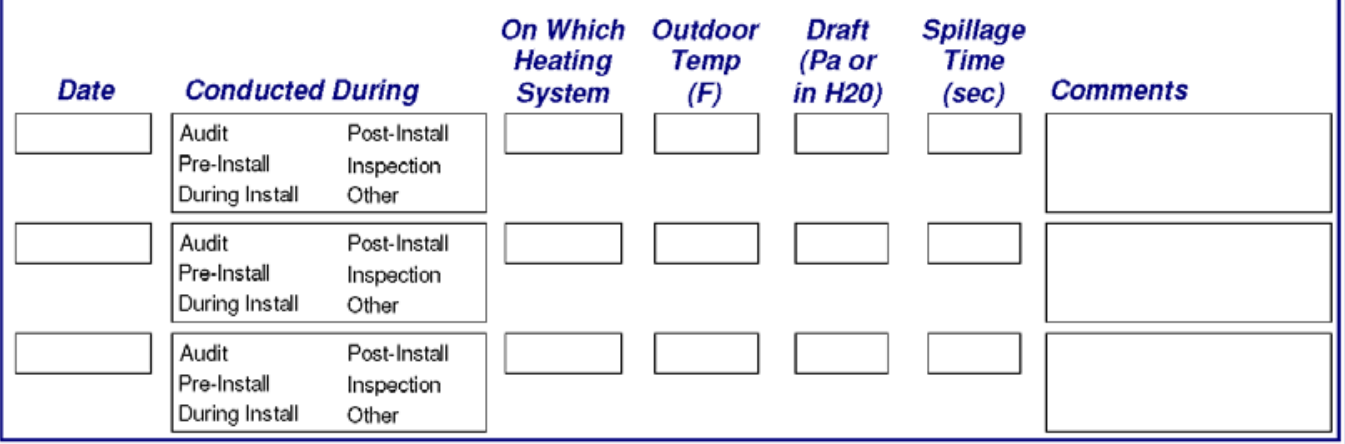

[Worst Case Condition Draft Measurements - Water Heating

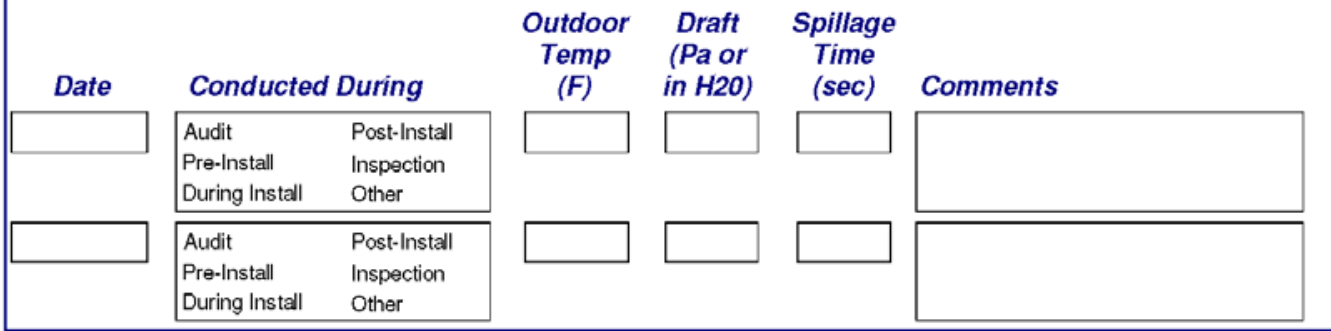

Wood Stove / Fireplace

\ulcorner\urcorner Wood Stove / Fireplace is Present

¿ᄃ] Improper Venting

[.] Combustion Air is Inadequate

Clothes Dryer

$[\neg$ Improper Venting

$\left[\begin{array}{c}\text { Cook Stove } \\ \text { Co Measurement Oven (ppm) } \\ \text { co Measurement Burner } 1(\mathrm{ppm}) \\ \text { co Measurement Burner } 2(\mathrm{ppm}) \\ \text { co Measurement Burner } 3(\mathrm{ppm}) \\ \text { co Measurement Burner } 4(\mathrm{ppm})\end{array}\right.$

[j] Gas Leak Present

\section{[ Exhaust Fans \\ Bathrooms \\ Б) Missing \\ ¿| Not Operational \\ $\Pi$ Improper Venting \\ Kitchen \\ Missing \\ Not Operational \\ Improper Venting}

Comment

Client Name:
Client ID:
Att. Client ID:

MHEA Data Collection Form

Form Run On: 11/3/2011
DOE Weatherization Assistant

Version 8.9 .0

Page 39 of 41 


\section{Appendix C: Reports}

\section{Itemized Costs}

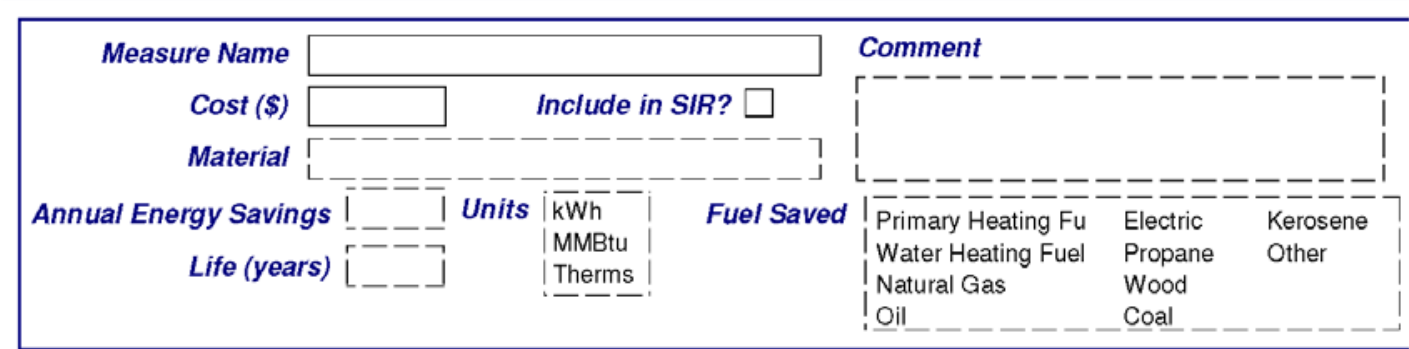

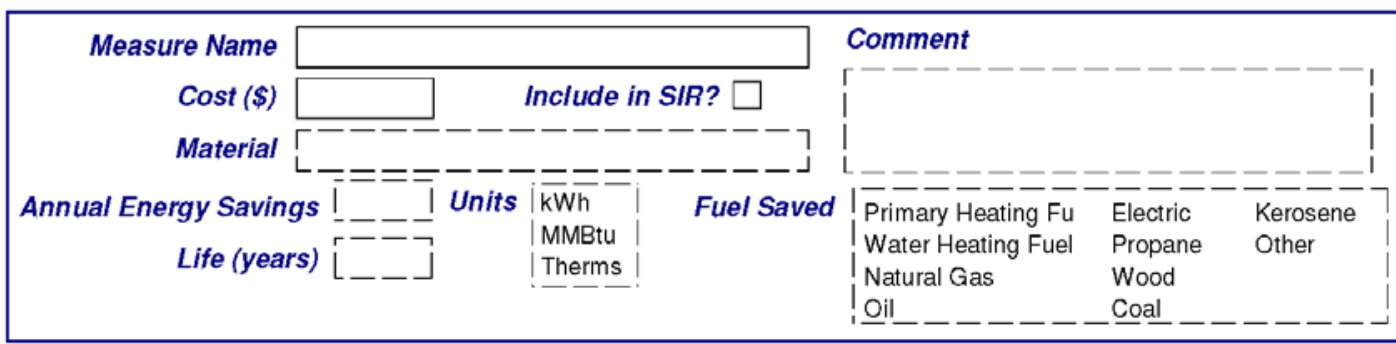

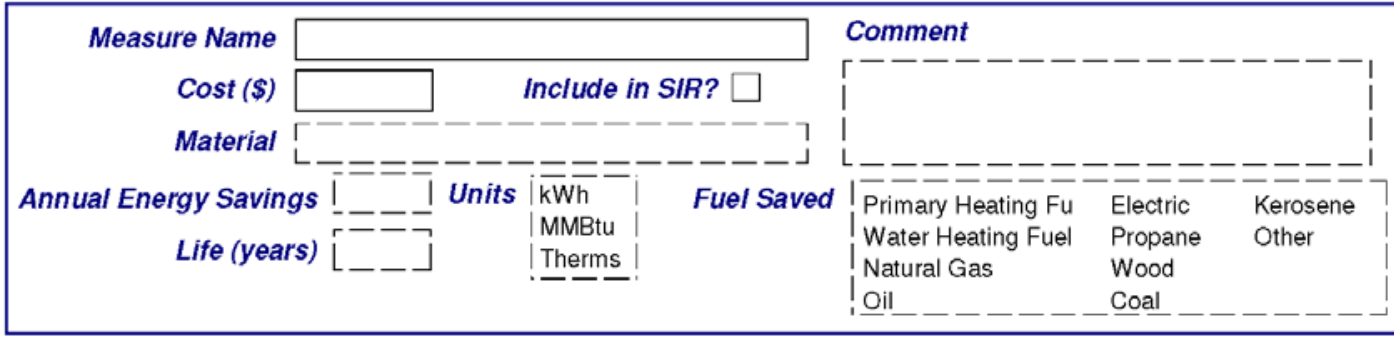

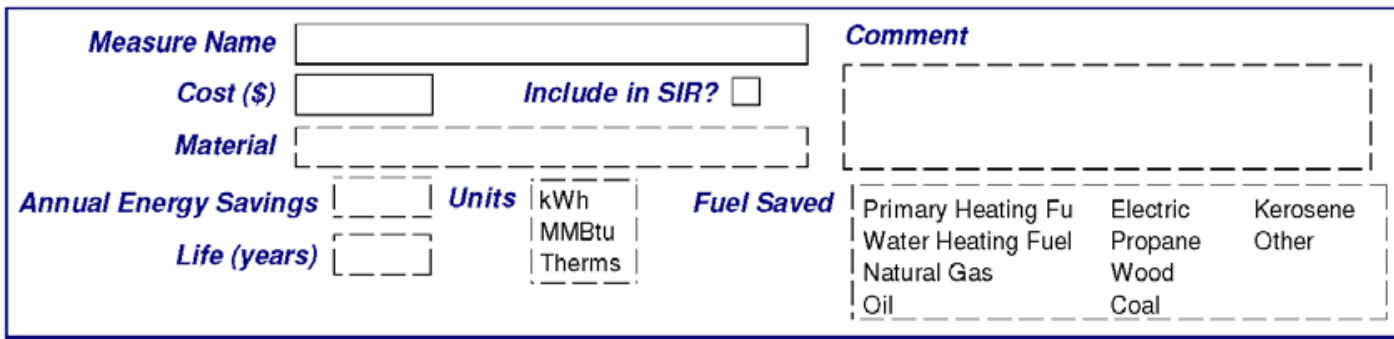

\section{Notes :}

\section{Itemized Costs (Continued)}

\begin{tabular}{|c|c|c|}
\hline Client Name: & MHEA Data Collection Form & DOE Weatherization Assistant \\
\hline Client ID: & Form Run On: 11/3/2011 & Version 8.9 .0 \\
\hline Alt. Client ID: & & Page 40 of 41 \\
\hline
\end{tabular}




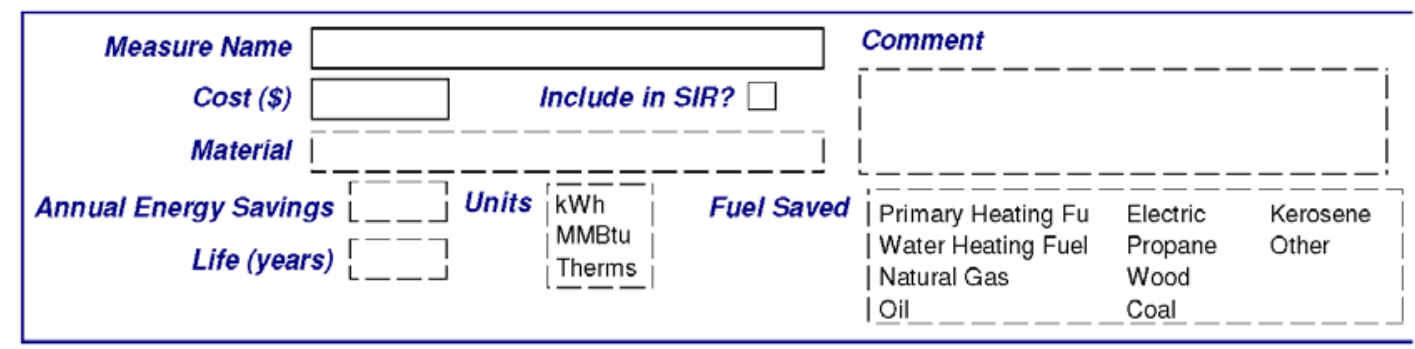

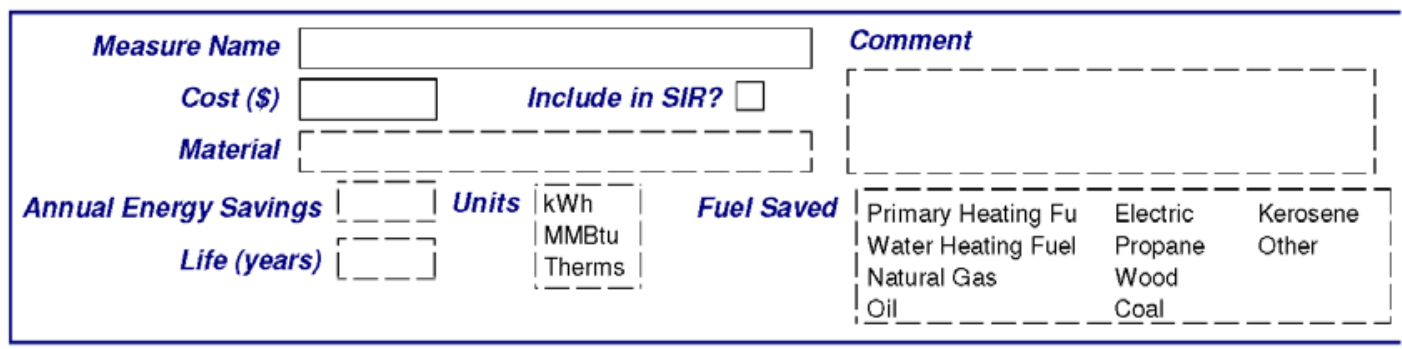

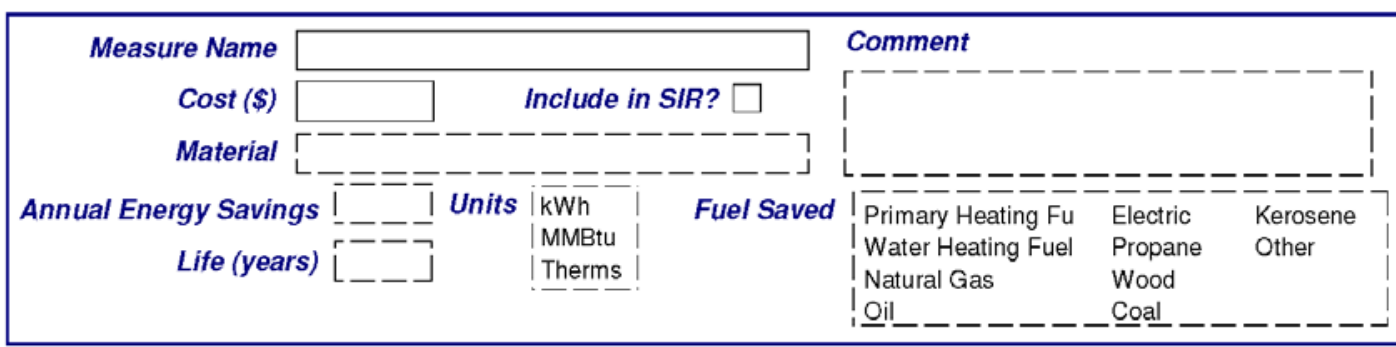

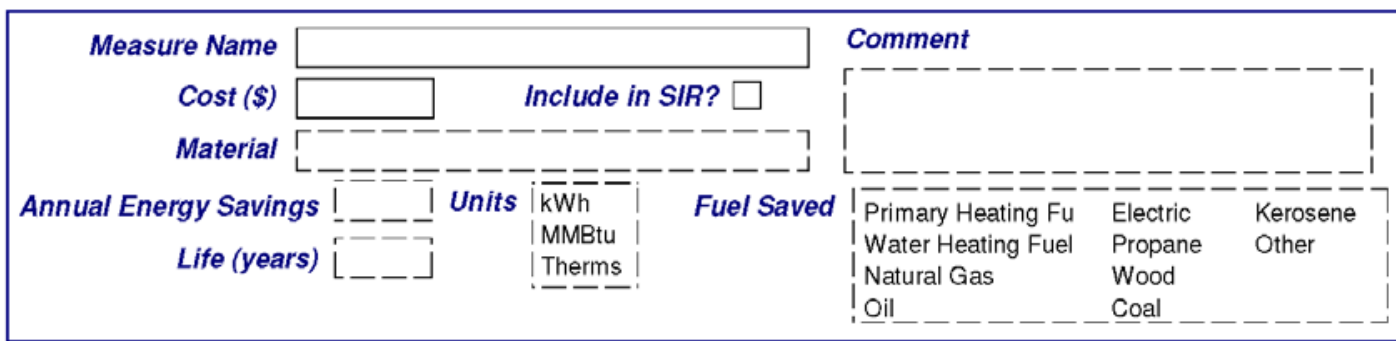

\section{Notes :}

Client Name:
Client ID:
Alt. Client ID:

MHEA Data Collection Form

Form Run On: 11/3/2011
DOE Weatherization Assistant

Version 8.9 .0

Page 41 of 41 


\section{Appendix C: Reports}

\section{Client Surveys}

Assistance

Program

CLIENT INFORMATION

Client ID: 11348

Client Name: Tanner, David

Alt. Client ID:

Address: 114 Athens

Anytown

US 01234

\section{AGENCY INFORMATION}

Agency Name: Demonstration Agency

Address: 725 Jefferson St.

Any City

US 11111

Office Phone: (123) 456-7890

Office Email: agencyemail@localisp.net

$\begin{array}{lcccccc}\text { Contact Name } & \text { Home Ph } & \text { Work Ph } & \text { Cell Ph } & \text { Contact Type Applican Comment } & \begin{array}{c}\text { Primary } \\ \text { Tanner, David }\end{array} \\ \text { Tanner, John } & (111) 764-5687 & (111) 764-3789 & \text { (111) } 764-9902 & \begin{array}{c}\text { Applicant/Person of } \\ \text { Record }\end{array} \\ & & (254) 567-8908 & \text { Applicant/Person of } \\ \text { Record } & \square & \text { Son of primary applicant }\end{array}$

Client Name: Tanner, David

Client ID: $11 \_348$

Att. Client ID:
Client Surveys

Report Run On: 11/3/2011
DOE Weatherization Assistant

Version 8.9.0

Page 1 of 2 


\section{Client Surveys}

\section{Survey Name Intake Survey}

\section{Question}

1 Age of dwelling (year built)

2 Thermostat setting - Day

3 Thermostat setting - Night

4 Existing setback thermostat?

5 Setback thermostat properly used?

6 Install setback thermostat?

7 Client comfort at temperature settings (specify location of drafts, warm rooms, cold rooms)

8 Suuply/returns in cold rooms? Specify.

9 Basement used as living space? If yes, describe.

10 Basement temperature during winter?

11 Attic use (storage, other)

12 How will attic use affect attic insulating?

13 Rooms closed off during winter (locate and explain)?

14 Age (years)

15 Describe repairs in last 3 years

16 Routine maintenance (Yes or No)?

17 Describe routine maintenance

18 Does the dwelling have icicles or ice dams (Yes or No)? Explain if Yes.

19 Does the dwelling have moisture problems, mold or mildew (Yes or No)? Explain if Yes

20 Does the dwelling have freezing pipes (Yes or No)? Explain if Yes.

21 Does the client have recurrent headaches, itching or burning eyes while at home (Yes or No)? Explain if Yes.

22 Other (specify)

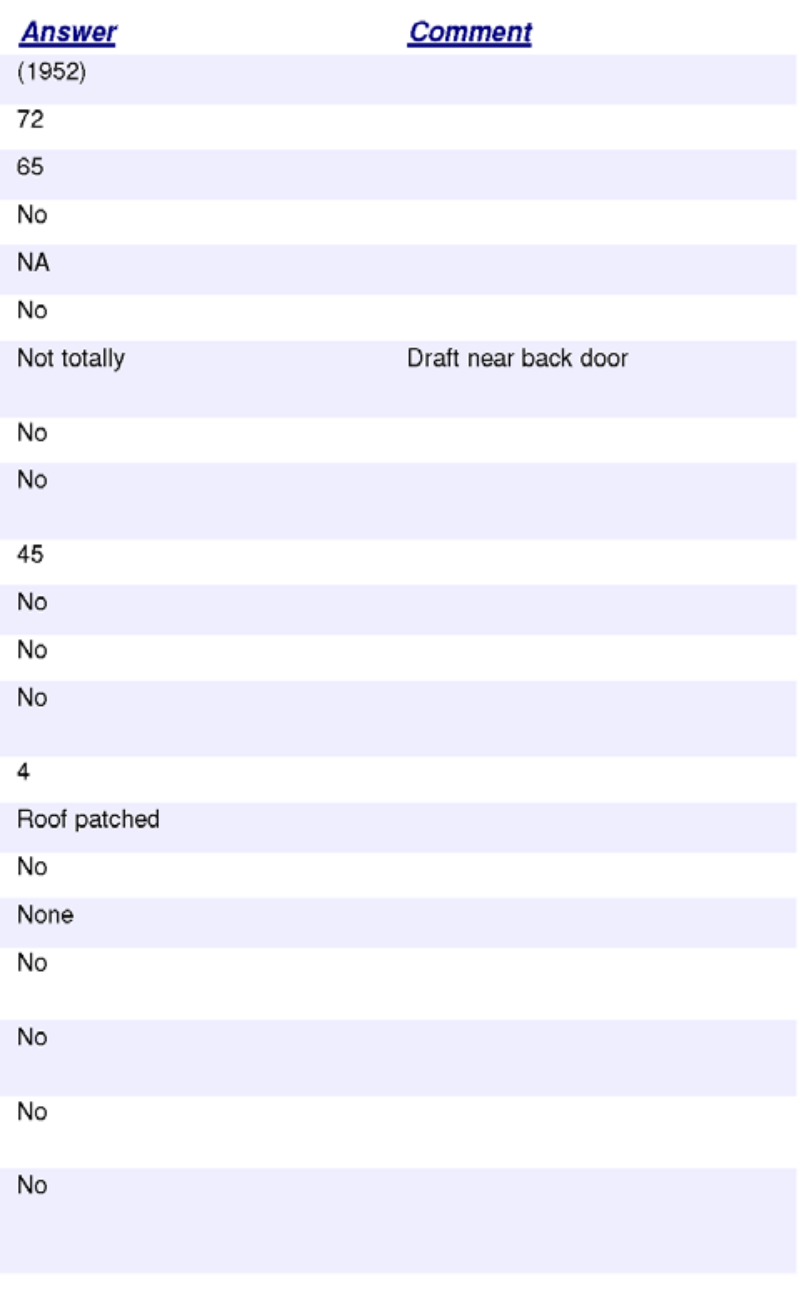

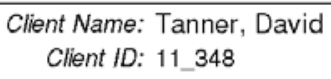

DOE Weatherization Assistant

Version 8.9 .0

Page 2 of 2 


\section{NEAT Recommended Measures}

Agency Demonstration Agency

Client ID 11 348
State US Run On 8/24/20112:51:53P RunID 1314211913 Version $8.9 .0 .5(2 / 10 / 2012)$ AuditID 267501792 Audit Date 8/24/2011

Auditor AT

Setup Library Name Demonstration Setup Library

Comment

\section{Annual Energy and Cost Savings}

\begin{tabular}{|c|c|c|c|c|c|c|c|c|c|c|}
\hline \multirow[t]{2}{*}{ Index } & \multirow{2}{*}{$\begin{array}{l}\text { Recommended } \\
\text { Measure }\end{array}$} & \multirow[t]{2}{*}{ Components } & \multicolumn{2}{|c|}{ Heating } & \multicolumn{2}{|c|}{ Cooling } & \multicolumn{2}{|c|}{ BaseLoad } & \multirow{2}{*}{\multicolumn{2}{|c|}{$\begin{array}{r}\text { Total } \\
\text { (MMBtu) }\end{array}$}} \\
\hline & & & (MMBtu) & $(\$)$ & $(k W h)$ & $(\$)$ & $(k W h)$ & $(\$)$ & & \\
\hline 1 & Infiltration Redctn & & 3.0 & 30 & 23 & $\overline{2}$ & $\overline{0}$ & & $\overline{0}$ & 3.1 \\
\hline 2 & Low Flow Showerheads & & 0.0 & 0 & 0 & 0 & 259 & & 18 & 0.9 \\
\hline 3 & DWH Pipe Insulation & & 0.0 & 0 & 0 & 0 & 197 & & 14 & 0.7 \\
\hline 4 & Smart Thermostat & & 5.9 & 59 & 0 & 0 & 0 & & 0 & 5.9 \\
\hline 5 & Attic Ins. R-30 & UA1 & 12.3 & 123 & 158 & 11 & 0 & & 0 & 12.9 \\
\hline 6 & DWH Tank Insulation & & 0.0 & 0 & 0 & 0 & 411 & & 29 & 1.4 \\
\hline 7 & Fill Ceiling Cavity & FA3 & 7.3 & 73 & 102 & 7 & 0 & & 0 & 7.7 \\
\hline 8 & Insulate and seal attic access & & 0.0 & 0 & 0 & 0 & 0 & & 0 & 0.7 \\
\hline 9 & Attic Ins. R-30 & FA1 & 7.7 & 77 & 93 & 7 & 0 & & 0 & 8.0 \\
\hline 10 & Attic Ins. R-30 & FA4 & 1.1 & 11 & 13 & 1 & 0 & & 0 & 1.1 \\
\hline 11 & Lighting Retrofits & LT1 & 0.0 & 0 & 0 & 0 & 686 & & 48 & 2.3 \\
\hline 12 & Kneewall Insulation & $\mathrm{FA} 2$ & 1.6 & 16 & 3 & 0 & 0 & & 0 & 1.6 \\
\hline 13 & Refrigerator Riplomnt & & 0.0 & 0 & 0 & 0 & 1778 & & 24 & 6.1 \\
\hline 14 & Wall Insulation & $\begin{array}{l}\text { WLE-1,WLN-1,WLN- } \\
2, W L S-2, W L W-1\end{array}$ & 6.8 & 68 & 56 & 4 & 0 & & 0 & 7.0 \\
\hline 15 & Window Replacement & WD4 & 1.3 & 13 & -7 & 0 & 0 & & 0 & 1.2 \\
\hline
\end{tabular}

\section{Energy Saving Measure Economics}

\begin{tabular}{|c|c|c|c|c|c|c|c|}
\hline Index & $\begin{array}{l}\text { Recommended } \\
\text { Measure }\end{array}$ & Components & $\begin{array}{r}\text { Measure } \\
\text { Savings }(\$ / y r)\end{array}$ & $\begin{array}{r}\text { Measure } \\
\text { Cost }(\$)\end{array}$ & $\begin{array}{r}\text { Measure } \\
\text { SIR }\end{array}$ & $\begin{array}{r}\text { Cumulative } \\
\text { Cost }(\$)\end{array}$ & $\begin{array}{r}\text { Cumulative } \\
\text { SIR }\end{array}$ \\
\hline 1 & $\begin{array}{l}\text { Anticipator Adjus tment } \\
\text { Needed }\end{array}$ & & 0 & 20 & 0.0 & 20 & 0.0 \\
\hline 2 & Repair Roof & & 0 & 80 & 0.0 & 100 & 0.0 \\
\hline 3 & Infiltration Redctn & & 32 & 250 & 1.1 & 350 & 0.8 \\
\hline 4 & Low Flow Showerheads & & 18 & 20 & 10.7 & 370 & 1.3 \\
\hline 5 & DWH Pipe Insulation & & 14 & 15 & 9.6 & 385 & 1.6 \\
\hline 6 & Smart Thermostat & & 59 & 75 & 9.5 & 460 & 2.9 \\
\hline 7 & Attic Ins. R-30 & UA1 & 134 & 270 & 7.8 & 730 & 4.7 \\
\hline 8 & DWH Tank Insulation & & 29 & 40 & 7.5 & 770 & 4.9 \\
\hline
\end{tabular}




\begin{tabular}{|c|c|c|c|c|c|c|c|}
\hline Index & $\begin{array}{l}\text { Recommended } \\
\text { Measure }\end{array}$ & Components & $\begin{array}{r}\text { Measure } \\
\text { Savings }(\$ / y r)\end{array}$ & $\begin{array}{r}\text { Measure } \\
\text { Cost }(\$)\end{array}$ & $\begin{array}{r}\text { Measure } \\
\text { SIR }\end{array}$ & $\begin{array}{r}\text { Cumulative } \\
\text { Cost }(\$)\end{array}$ & $\begin{array}{r}\text { Cumulative } \\
\text { SIR }\end{array}$ \\
\hline 9 & Fill Ceiling Cavity & $\mathrm{FA3}$ & 81 & 221 & 5.7 & 991 & 5.0 \\
\hline 10 & $\begin{array}{l}\text { Insulate and seal attic } \\
\text { access }\end{array}$ & & 7 & 30 & 3.7 & 1021 & 5.0 \\
\hline 11 & Attic Ins. R-30 & FA1 & 83 & 353 & 3.7 & 1374 & 4.7 \\
\hline 12 & Attic Ins. R-30 & FA4 & 12 & 50 & 3.6 & 1424 & 4.6 \\
\hline 13 & Lighting Retrofits & LT1 & 48 & 38 & 3.3 & 1462 & 4.6 \\
\hline 14 & Kneewall Insulation & $\mathrm{FA} 2$ & 16 & 91 & 2.8 & 1553 & 4.5 \\
\hline 15 & Refrigerator Rplcmnt & & 124 & 800 & 1.8 & 2353 & 3.6 \\
\hline 16 & Wall Insulation & $\begin{array}{l}\text { WLE-1,WLN- } \\
\text { 1,WLN-2,WLS- } \\
2, \text { WLW-1 }\end{array}$ & 72 & 854 & 1.3 & 3208 & 3.0 \\
\hline 17 & Window Replacement & WD4 & 12 & 346 & 0.6 & 3554 & 0.0 \\
\hline 18 & CO Monitor is Needed & & 0 & 70 & 0.0 & 3624 & 0.0 \\
\hline 19 & $\begin{array}{l}\text { Install Bathroom Exhaust } \\
\text { Fan }\end{array}$ & & 0 & 270 & 0.0 & 3894 & 0.0 \\
\hline
\end{tabular}

\section{Materials}

\begin{tabular}{|c|c|c|c|c|}
\hline Index & Material & Type & Quantity & Units \\
\hline 1 & Wall Insulation & Blown Cellulose - $2 \times 4$ Filled & 846 & $\mathrm{SqFt}$ \\
\hline 2 & Attic Insulation & Blown Cellulose - R-30 & 748 & $\mathrm{SqFt}$ \\
\hline 3 & Kneewall Insulation & Fiberglass Batts - R-13 & 120 & $\mathrm{SqFt}$ \\
\hline 4 & Smart Thermostat & & 1 & Each \\
\hline 5 & Window Replacement & & 2 & Each \\
\hline 6 & Compact FI. & 13 Watt & 4 & Each Lamp \\
\hline 7 & DHW Tank Insulation & & 1 & Each \\
\hline 8 & DHW Pipe Insulation & & 1 & Each \\
\hline 9 & Low Flow Shower Heads & & 1 & Each \\
\hline 10 & Attic Insulation & Blown Cellulose - 5 in. & 437 & $\mathrm{SqFt}$ \\
\hline 11 & New Refrigerator & AS22M8* & 1 & Each \\
\hline 12 & $\mathrm{CO}$ monitor $(+)$ & & 1 & Each \\
\hline 13 & Bathroom exhaust fan (+) & & 1 & Each \\
\hline 14 & R-30 faced batt insulation (+) & & 1 & Each \\
\hline
\end{tabular}

\section{Pre/Post Retrofit Energy and Loads}

\begin{tabular}{lrrrr}
\hline & \multicolumn{2}{c}{ Pre Retrofit } & \multicolumn{2}{c}{ Post Retrofit } \\
& Heating & Cooling & Heating & Cooling \\
\hline Annual load (MBtu/yr) & 64.0 & 18.6 & 29.2 & 13.9 \\
Annual Energy (MBtu/yr) & 86.4 & 5.9 & 39.4 & 4.4 \\
Heat loss/gain (kBtu/hr) & 52.5 & 20.3 & 27.0 & 9.5 \\
Output required (kBtu/hr)(ton) & 63.0 & 2.0 & 31.0 & 1.0
\end{tabular}

\section{Annual Energy and Cost Savings (Adjusted)}

\begin{tabular}{|c|c|c|c|c|c|c|c|c|c|}
\hline \multirow[t]{2}{*}{ Index } & \multirow{2}{*}{$\begin{array}{l}\text { Recommended } \\
\text { Measure }\end{array}$} & \multirow{2}{*}{ Components } & \multicolumn{2}{|c|}{ Heating } & \multicolumn{2}{|c|}{ Cooling } & \multicolumn{2}{|c|}{ BaseLoad } & \multirow{2}{*}{$\begin{array}{r}\text { Total } \\
(M M B t u) \\
\end{array}$} \\
\hline & & & (MMBtu) & $(\$)$ & $(k W h)$ & $(\$)$ & $(k W h)$ & (\$) & \\
\hline$\overline{1}$ & Infiltration Redctn & & 2.8 & 28 & 23 & 2 & 0 & & 2.9 \\
\hline Audit & Name: 11_348SB & Client: & 348 & & Date: & $4 / 2011$ & & & Page 2 of 6 \\
\hline
\end{tabular}




\begin{tabular}{|c|c|c|c|c|c|c|c|c|c|}
\hline \multirow[t]{2}{*}{ Index } & \multirow{2}{*}{$\begin{array}{l}\text { Recommended } \\
\text { Measure }\end{array}$} & \multirow[t]{2}{*}{ Components } & \multicolumn{2}{|c|}{ Heating } & \multicolumn{2}{|c|}{ Cooling } & \multicolumn{2}{|c|}{ BaseLoad } & \multirow{2}{*}{$\begin{array}{r}\text { Total } \\
(M M B t u)\end{array}$} \\
\hline & & & (MMBtu) & $(\$)$ & $(k W h)$ & $(\$)$ & $(k W h)$ & $(\$)$ & \\
\hline 2 & Low Flow Showerheads & & 0.0 & 0 & 0 & 0 & 259 & 18 & 0.9 \\
\hline 3 & DWH Pipe Insulation & & 0.0 & 0 & 0 & 0 & 197 & 14 & 0.7 \\
\hline 4 & Smart Thermostat & & 5.5 & 55 & 0 & 0 & 0 & 0 & 5.5 \\
\hline 5 & DWH Tank Insulation & & 0.0 & 0 & 0 & 0 & 411 & 29 & 1.4 \\
\hline 6 & Attic Ins. R-30 & UA1 & 11.5 & 115 & 158 & 11 & 0 & 0 & 12.0 \\
\hline 7 & Fill Ceiling Cavity & FA3 & 6.8 & 68 & 102 & 7 & 0 & 0 & 7.2 \\
\hline 8 & Insulate and seal attic access & & 0.0 & 0 & 0 & 0 & 0 & 0 & 0.7 \\
\hline 9 & Attic Ins. R-30 & FA1 & 7.2 & 72 & 93 & 7 & 0 & 0 & 7.5 \\
\hline 10 & Attic Ins. R-30 & FA4 & 1.0 & 10 & 13 & 1 & 0 & 0 & 1.0 \\
\hline 11 & Lighting Retrofits & LT1 & 0.0 & 0 & 0 & 0 & 686 & 48 & 2.3 \\
\hline 12 & Kneewall Insulation & FA2 & 1.5 & 15 & 3 & 0 & 0 & 0 & 1.5 \\
\hline 13 & Refrigerator Rplcmnt & & 0.0 & 0 & 0 & 0 & 1778 & 124 & 6.1 \\
\hline 14 & Wall Insulation & $\begin{array}{l}\text { WLE-1,WLN-1,WLN- } \\
2, \text { WLS-2,WLW-1 }\end{array}$ & 6.3 & 63 & 56 & 4 & 0 & 0 & 6.5 \\
\hline 15 & Window Replacement & WD4 & 1.2 & 12 & -7 & 0 & 0 & 0 & 1.2 \\
\hline
\end{tabular}

\section{Energy Saving Measure Economics (Adjusted)}

\begin{tabular}{|c|c|c|c|c|c|c|c|}
\hline Index & $\begin{array}{l}\text { Recommended } \\
\text { Measure }\end{array}$ & Components & $\begin{array}{r}\text { Measure } \\
\text { Savings }(\$ / y r)\end{array}$ & $\begin{array}{r}\text { Measure } \\
\text { Cost }(\$)\end{array}$ & $\begin{array}{r}\text { Measure } \\
\text { SIR }\end{array}$ & $\begin{array}{r}\text { Cumulative } \\
\text { Cost (\$) }\end{array}$ & $\begin{array}{r}\text { Cumulative } \\
\text { SIR }\end{array}$ \\
\hline 1 & $\begin{array}{l}\text { Anticipator Adjustment } \\
\text { Needed }\end{array}$ & & 0 & 20 & 0.0 & 20 & 0.0 \\
\hline 2 & Repair Roof & & 0 & 80 & 0.0 & 100 & 0.0 \\
\hline 3 & Infiltration Redctn & & 30 & 250 & 1.0 & 350 & 0.7 \\
\hline 4 & Low Flow Showerheads & & 18 & 20 & 10.7 & 370 & 1.3 \\
\hline 5 & DWH Pipe Insulation & & 14 & 15 & 9.6 & 385 & 1.6 \\
\hline 6 & Smart Thermostat & & 55 & 75 & 8.9 & 460 & 2.8 \\
\hline 7 & DWH Tank Insulation & & 29 & 40 & 7.5 & 500 & 3.2 \\
\hline 8 & Attic Ins. R-30 & UA1 & 126 & 270 & 7.3 & 770 & 4.6 \\
\hline 9 & Fill Ceiling Cavity & FA3 & 76 & 221 & 5.3 & 991 & 4.8 \\
\hline 10 & $\begin{array}{l}\text { Insulate and seal attic } \\
\text { access }\end{array}$ & & 7 & 30 & 3.7 & 1021 & 4.7 \\
\hline 11 & Attic Ins. R-30 & FA1 & 78 & 353 & 3.5 & 1374 & 4.4 \\
\hline 12 & Attic Ins. R-30 & FA4 & 11 & 50 & 3.3 & 1424 & 4.4 \\
\hline 13 & Lighting Retrofits & LT1 & 48 & 38 & 3.3 & 1462 & 4.3 \\
\hline 14 & Kneewall Insulation & FA2 & 15 & 91 & 2.6 & 1553 & 4.2 \\
\hline 15 & Refrigerator Rplcmnt & & 124 & 800 & 1.8 & 2353 & 3.4 \\
\hline 16 & Wall Insulation & $\begin{array}{l}\text { WLE-1,WLN- } \\
1, W L N-2, W L S- \\
2, W L W-1\end{array}$ & 67 & 854 & 1.2 & 3208 & 2.8 \\
\hline 17 & Window Replacement & WD4 & 11 & 346 & 0.5 & 3554 & 0.0 \\
\hline 18 & CO Monitor is Needed & & 0 & 70 & 0.0 & 3624 & 0.0 \\
\hline 19 & $\begin{array}{l}\text { Install Bathroom Exhaust } \\
\text { Fan }\end{array}$ & & 0 & 270 & 0.0 & 3894 & 0.0 \\
\hline
\end{tabular}

\section{Materials (Adjusted)}

\begin{tabular}{|c|c|c|c|}
\hline Index & Material & Type & Quantity Units \\
\hline 1 & Wall Insulation & Blown Cellulose - $2 \times 4$ Filled & $846 \mathrm{SqFt}$ \\
\hline 2 & Attic Insulation & Blown Cellulose - R-30 & $748 \mathrm{SqFt}$ \\
\hline 3 & Kneewall Insulation & Fiberglass Batts - R-13 & $120 \mathrm{SqFt}$ \\
\hline
\end{tabular}

Audit Name: 11_348SB

Client: $11 \_348$

Date: $8 / 24 / 2011$

Page 3 of 6 


\begin{aligned} \hline Index & Material & Type & Quantity Units \\ \hline 4 & Smart Thermostat & & 1 Each \\ 5 & Window Replacement & & 2 Each \\ 6 & Compact Fl. & 13 Watt & 4 Each Lamp \\ 7 & DHW Tank Insulation & & 1 Each \\ 8 & DHW Pipe Insulation & & 1 Each \\ 9 & Low Flow Shower Heads & & 1 Each \\ 10 & Attic Insulation & Blown Cellulose - 5 in. & 437 SqFt \\ 11 & New Refrigerator & AS22M8* & 1 Each \\ 12 & CO monitor (+) & & 1 Each \\ 13 & Bathroom exhaust fan (+) & & 1 Each \\ 14 & R-30 faced batt insulation (+) & & 1 Each \end{aligned}

\section{Heating Energy Consumption Comparison}

\begin{tabular}{|c|c|c|c|c|c|c|}
\hline \multirow[t]{2}{*}{ Month } & \multirow[t]{2}{*}{ Day } & \multirow{2}{*}{$\begin{array}{c}\text { Days in } \\
\text { Period }\end{array}$} & \multicolumn{2}{|c|}{ Consumption } & \multicolumn{2}{|c|}{ Degree Days } \\
\hline & & & Actual & Predicted & Actual & Predicted \\
\hline 1 & 15 & 30 & 161 & 179 & 815 & 913 \\
\hline 2 & 17 & 33 & 180 & 213 & 690 & 1090 \\
\hline 3 & 14 & 25 & 114 & 123 & 585 & 669 \\
\hline 4 & 16 & 33 & 33 & 90 & 312 & 549 \\
\hline 5 & 13 & 27 & 55 & 29 & 144 & 215 \\
\hline 6 & 15 & 33 & 17 & 14 & 42 & 117 \\
\hline 7 & 15 & 30 & 0 & 2 & 11 & 19 \\
\hline 8 & 14 & 30 & 0 & 1 & 15 & 9 \\
\hline 9 & 16 & 33 & 11 & 3 & 95 & 36 \\
\hline 10 & 17 & 31 & 44 & 24 & 253 & 182 \\
\hline 11 & 13 & 27 & 71 & 64 & 507 & 401 \\
\hline 12 & 12 & 29 & 121 & 125 & 726 & 688 \\
\hline \multirow{2}{*}{\multicolumn{2}{|c|}{$\begin{array}{l}\text { Total } \\
\text { ODifference }\end{array}$}} & 361 & 807 & 867 & 4195 & 4888 \\
\hline & & & & 4 & & 16.5 \\
\hline
\end{tabular}

\section{Approximate Component Contributions} to Peak HEATING Load

\begin{tabular}{llrrr}
\hline Component Type & Component Name & $\begin{array}{r}\text { Area or } \\
\text { Volume }(\text { Inf) }\end{array}$ & $\begin{array}{r}\text { Pre Retrofit } \\
\text { Load }(\text { Btu/h })\end{array}$ & $\begin{array}{r}\text { Post Retrofit } \\
\text { Load }(\boldsymbol{B T U} / \boldsymbol{h})\end{array}$ \\
\hline Wall & WLE-1 & 224 & 1949.5 & 1266.0 \\
Wall & WLN-1 & 234 & 2036.5 & 1322.6 \\
Wall & WLN-2 & 90 & 783.3 & 508.7 \\
Wall & WLS-1 & 234 & 2036.5 & 2036.5 \\
Wall & WLS-2 & 90 & 783.3 & 508.7 \\
Wall & WLW-1 & 208 & 1810.2 & 1175.6 \\
Window & WD1 & 16 & 509.2 & 509.2 \\
Window & WD2 & 16 & 509.2 & 509.2 \\
Window & WD4 & 16 & 509.2 & 590.7 \\
Window & WD5 & 8 & 254.6 & 254.6 \\
Window & WD6 & 8 & 254.6 & 254.6 \\
Door & DR1 & 20 & 408.7 & 408.7
\end{tabular}

Audit Name: 11_348SB

Client: $11 \_348$

Date: 8/24/2011

Page 4 of 6 


\section{Appendix C: Reports}

\begin{tabular}{llrrr}
\hline Component Type & Component Name & $\begin{array}{r}\text { Area or } \\
\text { Volume }(\text { Inf })\end{array}$ & $\begin{array}{r}\text { Pre Retrofit } \\
\text { Load }(\text { Btu/h) }\end{array}$ & $\begin{array}{r}\text { Post Retrofit } \\
\text { Load }(\boldsymbol{B T U} / \mathbf{h})\end{array}$ \\
\hline Door & DR2 & 20 & 408.7 & 408.7 \\
Attic & UA1 & 300 & 12039.9 & 660.4 \\
Attic & FA1 & 392 & 5366.1 & 778.2 \\
Attic & FA2 & 120 & 1642.7 & 485.2 \\
Attic & FA3 & 437 & 5287.1 & 1416.8 \\
Attic & FA4 & 56 & 766.6 & 111.2 \\
Foundation & F1 & 840 & 6895.4 & 6895.4 \\
Infiltration & Inf & 10320 & 8250.7 & 6875.5 \\
Total heat loss & Tot & 0 & 52501.7 & 26976.4 \\
Duct loss & Duct & 319 & 10500.3 & 4046.5 \\
Output required & Output & 0 & 63002.1 & 31022.8
\end{tabular}

\section{Approximate Component Contributions}

to Peak COOLING Load

\begin{tabular}{|c|c|c|c|c|}
\hline Component Type & Component Name & $\begin{array}{r}\text { Area or } \\
\text { Volume }(\text { Inf })\end{array}$ & $\begin{array}{r}\text { Pre Retrofit } \\
\text { Load }(\text { Btu/h) }\end{array}$ & $\begin{array}{r}\text { Post Retrofit } \\
\text { Load }(\text { BTU/h) }\end{array}$ \\
\hline Wall & WLE-1 & 224 & 541.2 & 351.5 \\
\hline Wall & WLN-1 & 234 & 565.4 & 367.2 \\
\hline Wall & WLN-2 & 90 & 217.4 & 141.2 \\
\hline Wall & WLS-1 & 234 & 304.0 & 304.0 \\
\hline Wall & WLS-2 & 90 & 217.4 & 141.2 \\
\hline Wall & WLW-1 & 208 & 502.5 & 326.4 \\
\hline Window & WD1 & 16 & 336.0 & 336.0 \\
\hline Window & WD2 & 16 & 576.0 & 576.0 \\
\hline Window & WD4 & 16 & 963.2 & 963.2 \\
\hline Window & WD5 & 8 & 168.0 & 168.0 \\
\hline Window & WD6 & 8 & 288.0 & 288.0 \\
\hline Door & DR1 & 20 & 113.5 & 113.5 \\
\hline Door & DR2 & 20 & 113.5 & 113.5 \\
\hline Attic & UA1 & 300 & 5112.9 & 386.8 \\
\hline Attic & FA1 & 392 & 2779.9 & 454.3 \\
\hline Attic & FA2 & 120 & 851.0 & 272.7 \\
\hline Attic & FA3 & 437 & 2540.5 & 771.8 \\
\hline Attic & FA4 & 56 & 397.1 & 64.9 \\
\hline Foundation & $\mathrm{F} 1$ & 840 & 0.0 & 0.0 \\
\hline Infiltration & $\operatorname{lnf}$ & 10320 & 1918.5 & 1646.1 \\
\hline People & People & 2 & 552.0 & 552.0 \\
\hline Appliances & Appl & 1 & 1200.0 & 1200.0 \\
\hline Total Sensible & TotS & 0 & 20258.1 & 9538.1 \\
\hline Ducts & Ducts & 0 & 2025.8 & 953.8 \\
\hline Total (with ducts) & TotW & 0 & 22283.9 & 10491.9 \\
\hline Size (tons) & Size & 0 & 1.9 & 0.9 \\
\hline Latent Load (inf) & Latentl & 0 & 1312.8 & 1126.4 \\
\hline Latent Load (occ) & Latento & 0 & 460.0 & 460.0 \\
\hline Latent Load (tot) & Latent T & 0 & 1772.8 & 1586.4 \\
\hline Total Load & Total & 0 & 24056.7 & 12078.3 \\
\hline Size (tons) & Size & 0 & 2.0 & 1.0 \\
\hline
\end{tabular}




\section{Special Notes}

NOTE: Heat loss and Output required are only guides to sizing equipment.

NOTE: See NEAT User's Manual for further sizing details.

NOTE: Read cautions in NEAT User's Manual related to sizing results.

NOTE: $(+)$ in the Materials list indicates there are more related User Defined Materials.

\section{Comments}

$\begin{array}{ll}\text { Type } & \text { Code } \\ \text { Wall } & \text { WLE-1 } \\ \text { Wall } & \text { WLN-1 } \\ \text { Wall } & \text { WLN-2 } \\ \text { Wall } & \text { WLS-1 } \\ \text { Wall } & \text { WLS-2 } \\ \text { Wall } & \text { WLW-1 } \\ \text { Infiltration } & \end{array}$

\section{Comment}

1st story west wall.

1st story north wall. Height of 9 ' includes joist space.

2nd story north wall.

1st story south wall. Height of 9 ' includes joist space.

2nd story south wall.

1 st story west wall.

Target (post weatherization) blower door reading estimated assuming that wall insulation would not be installed because there is already a 1" batt installed. Infiltration reduction cost is a typical value.

\section{Retrofit Measures NOT Considered}

Attic insulation $\mathrm{R} 38$

Duct insulation

Water heater replacement 


\section{NEAT Input Report}

Since adding the capability of specifying "NEAT Insulation Types" in the setup library (see Section 14.7, NEAT Insulation Types), the NEAT Input Report has lost its ability to specify the "Added Insulation Type" entries for those components whose added types can be so specified. The entries will remain blank. For example, see page C-105 following. If the user frequently specifies multiple insulation types for any of these building components and relies on the information in the NEAT Input Report, he/she could use the Comment field on the component form to designate the added insulation type being specified. This will then be visible in the Comment field on the NEAT Input Report for that component. 


\section{NEAT Input Report}

\section{Client Information}

\begin{tabular}{|c|c|c|c|c|}
\hline Client ID & $11 \_348$ & & & \\
\hline \multicolumn{5}{|l|}{ Alt. Client ID } \\
\hline \multirow{2}{*}{$\begin{array}{r}\text { Client Name } \\
\text { Address }\end{array}$} & \multicolumn{4}{|c|}{ Tanner, David } \\
\hline & \multicolumn{4}{|l|}{114 Athens } \\
\hline \multicolumn{5}{|l|}{ Unit No. } \\
\hline City & \multicolumn{2}{|l|}{ Oak Ridge } & \multicolumn{2}{|c|}{ State TN Zip 37830} \\
\hline County & & & \multicolumn{2}{|l|}{ Other Geo. Ident. } \\
\hline \multicolumn{5}{|l|}{-Dwelling - } \\
\hline \multicolumn{2}{|c|}{ Dwelling Type } & Site Built & \multicolumn{2}{|c|}{ Ownership Owned } \\
\hline \multicolumn{2}{|c|}{ Primary Heat. Fuel } & Natural Gas & \multirow{2}{*}{\multicolumn{2}{|c|}{$\begin{array}{l}\square \text { High Energy Use } \\
\square \text { High Energy Burden } \\
\square \text { Low Cost/No Cost }\end{array}$}} \\
\hline \multicolumn{2}{|c|}{ Secondary Heat. Fuel } & & & \\
\hline \multicolumn{2}{|c|}{$\square$ Previously Weatherized } & & \\
\hline Year & & & Year Built & 1952 \\
\hline \multicolumn{5}{|c|}{ Energy Index } \\
\hline \multicolumn{2}{|c|}{ Floor Area $(s q f t)$} & 1290 & \multirow{2}{*}{$\begin{array}{c}\text { Total Heating } \\
(\text { BTU/HDD/sq ft })\end{array}$} & 22.3 \\
\hline \multirow{2}{*}{\multicolumn{2}{|c|}{$\begin{array}{l}\text { Heating Degree Days } \\
\quad \text { (base } 65 \text { F) }\end{array}$}} & 3400 & & Estim. \% \\
\hline & & Fuel Type & Annual Cost & for heating \\
\hline \multirow{2}{*}{\multicolumn{2}{|c|}{$\begin{array}{l}\text { Primary Heating Fuel } \\
\text { Secondary Heating Fuel }\end{array}$}} & Natural Gas & $\$ 978.00$ & 100 \\
\hline & & & & \\
\hline
\end{tabular}

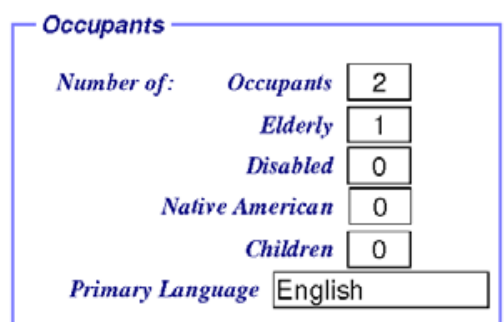

\section{Contact Information}

\begin{tabular}{lllll}
\hline Contact Name & Home Ph & Work Ph & Cell Ph & Contact Type Applican Comment \\
\hline Tanner, David & & $\begin{array}{c}\text { Applicant/Person of } \\
\text { Record }\end{array}$
\end{tabular}

\section{Audit Information}

\begin{tabular}{lrr}
\hline Client Name: Tanner, David & NEAT Input Report & DOE Weatherization Assistant \\
Client ID: $11 \_348$ & Audit Name: $11 \_348 \mathrm{SB}$ & Version 8.9 .0 \\
Ait. Client ID: & Report Run On: $8 / 28 / 2011$ & Page 1 of 16
\end{tabular}




\section{Appendix C: Reports}

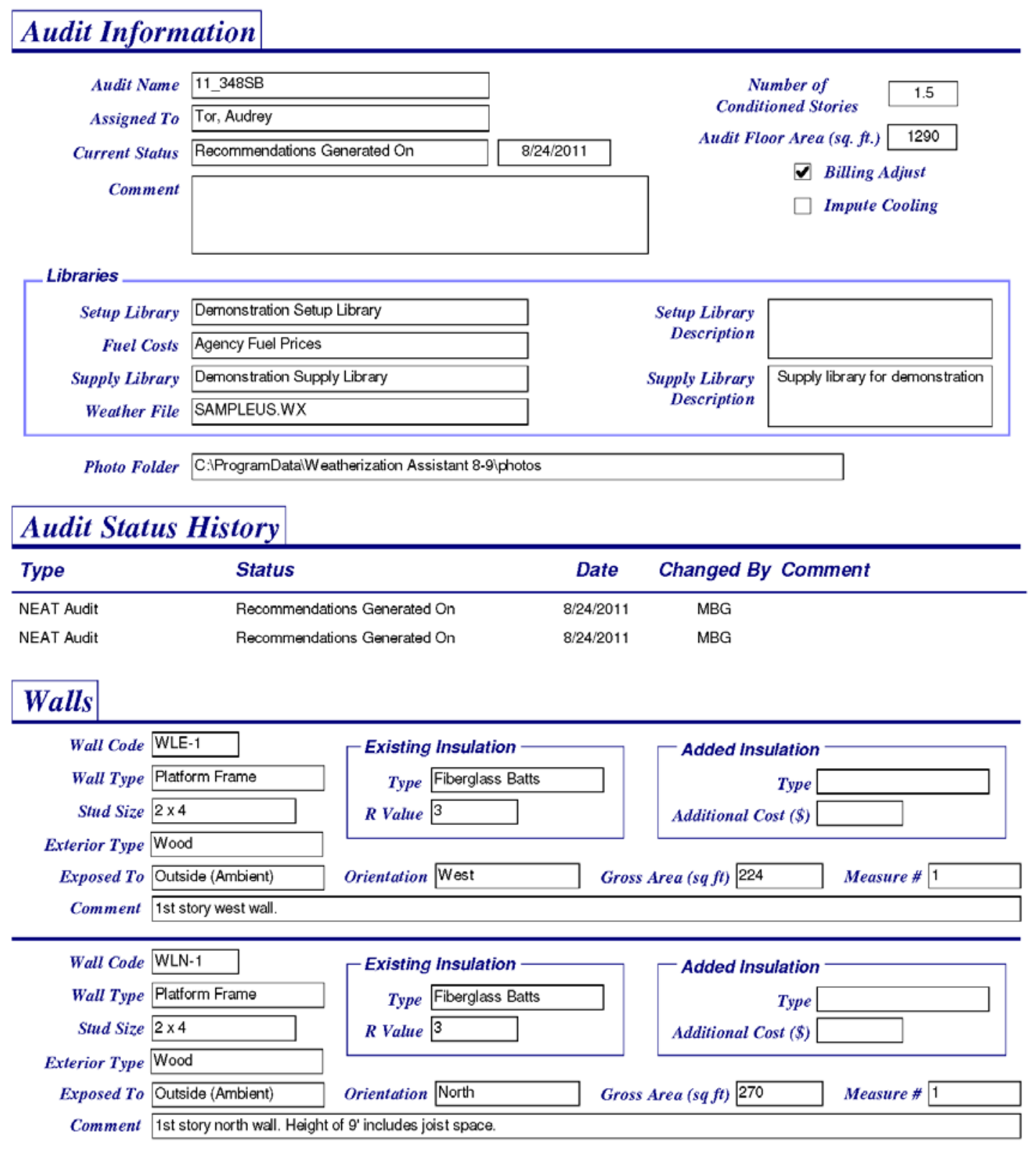

\begin{tabular}{lrr}
\hline Client Name: Tanner, David & NEAT Input Report & DOE Weatherization Assistant \\
Client ID: $11 \_348$ & Audit Name: $11 \_348$ SB & Version 8.9 .0 \\
Alt. Client ID: & Report Run On: 8/28/2011 & Page 2 of 16
\end{tabular}




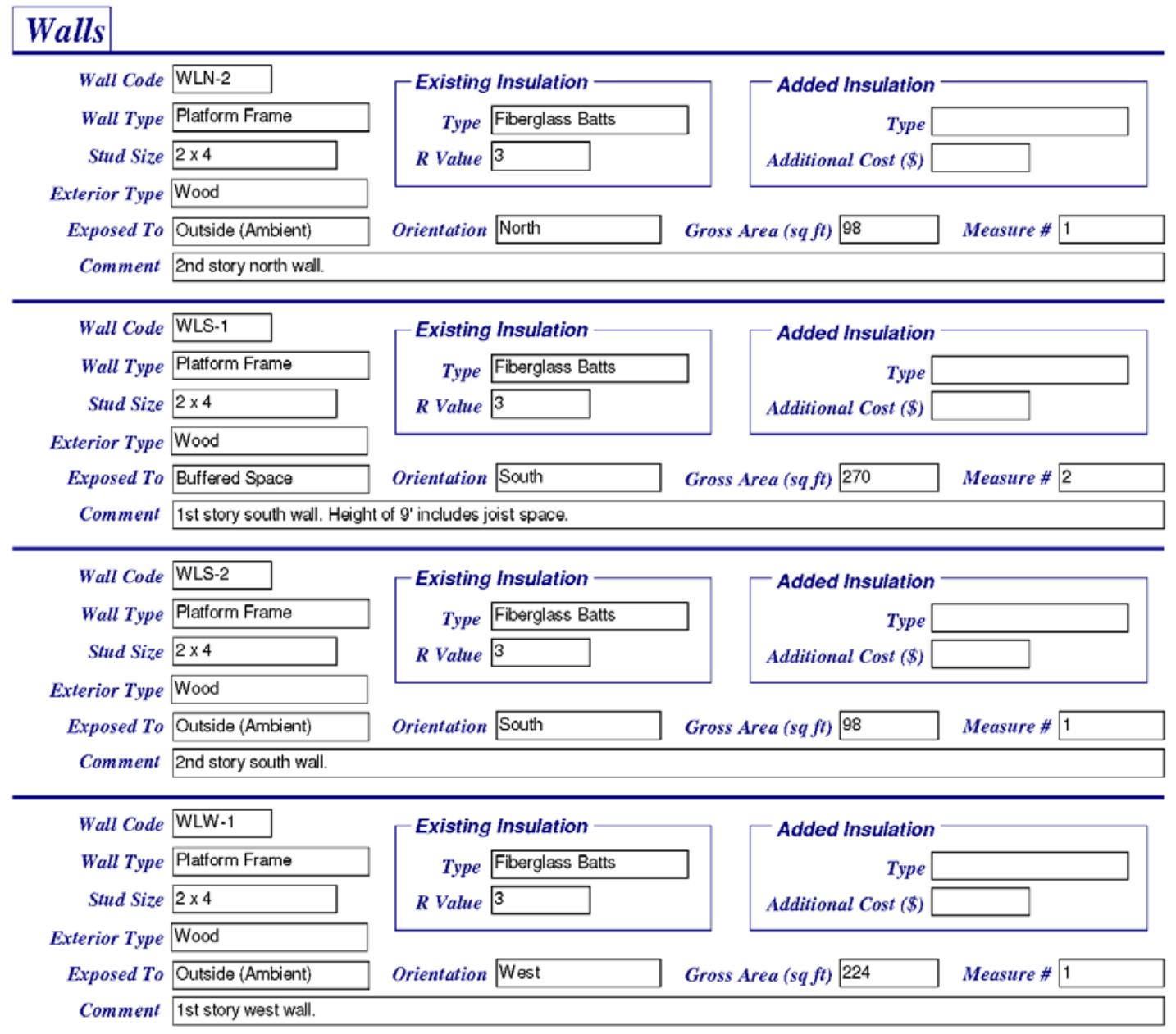

\section{Windows}

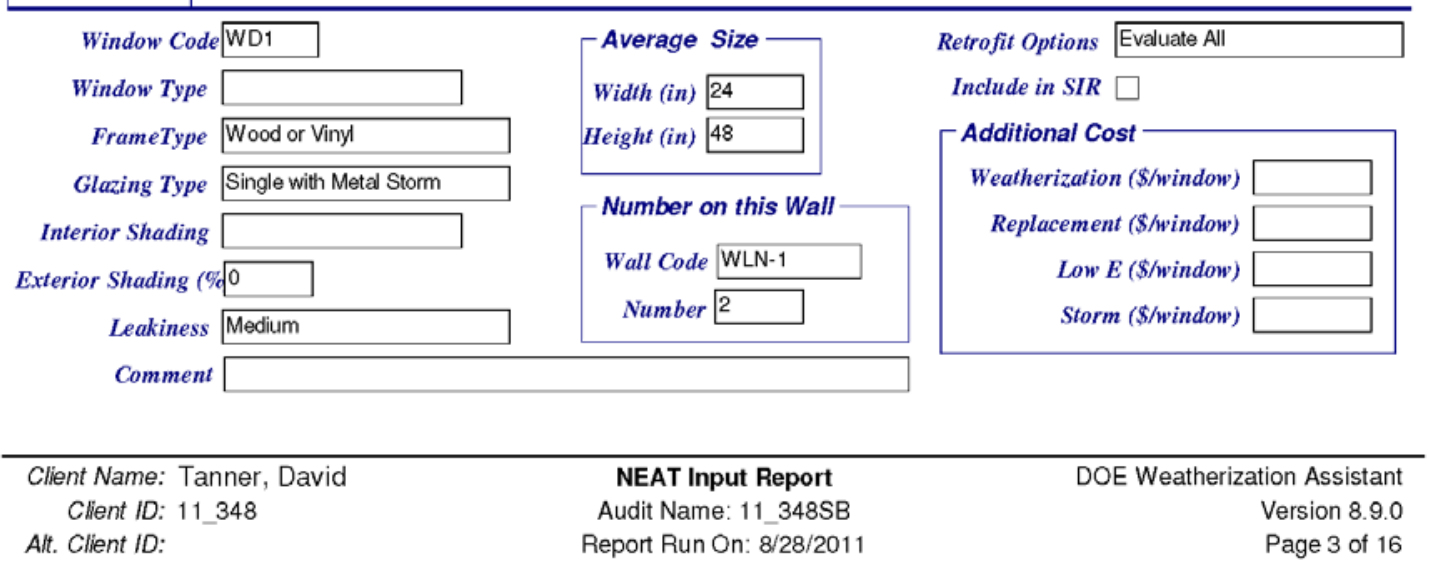




\section{Appendix C: Reports}

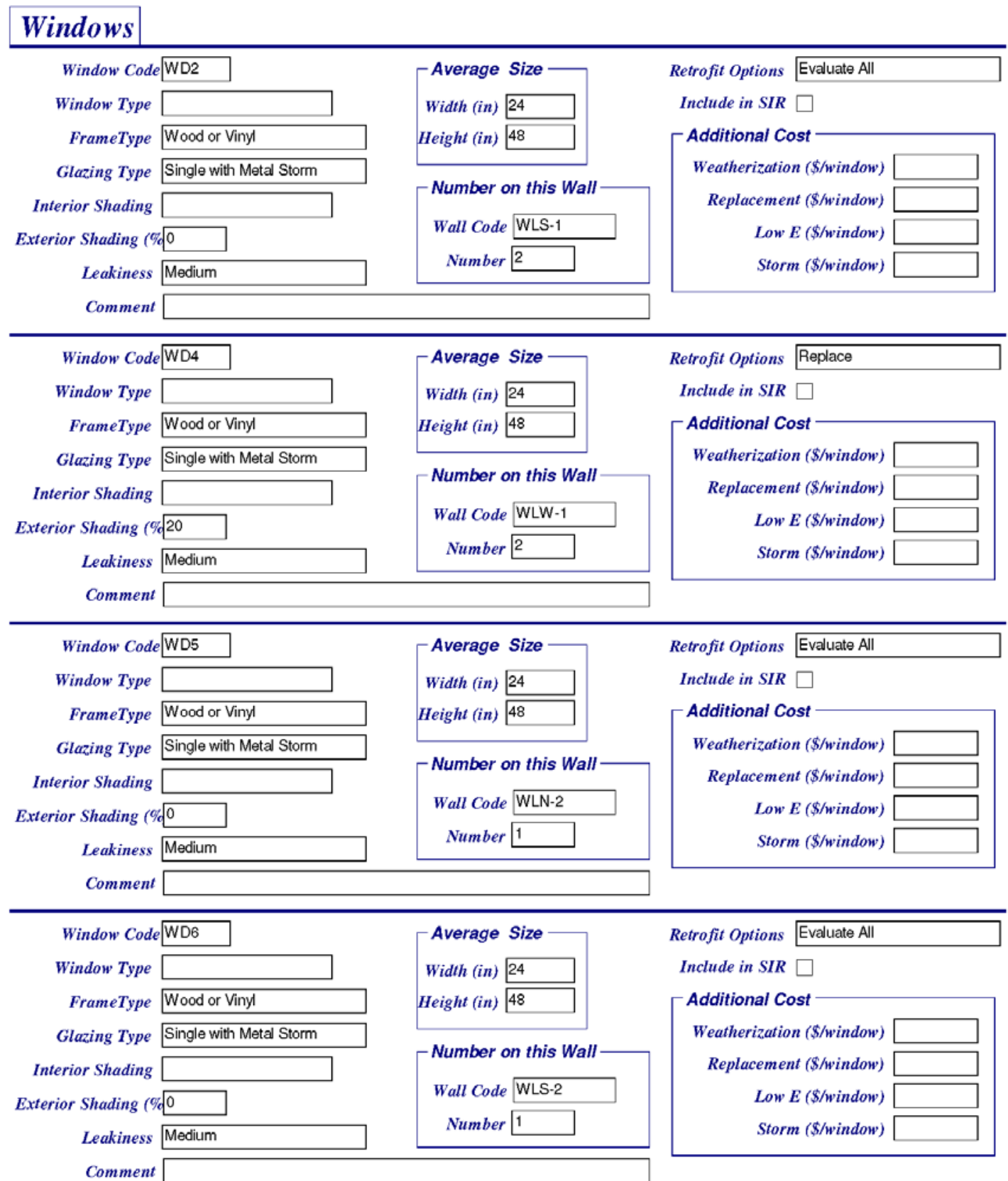

\section{Doors}

\begin{tabular}{lrr}
\hline Client Name: Tanner, David & NEAT Input Report & DOE Weatherization Assistant \\
Client ID: $11 \_348$ & Audit Name: $11 \_348$ SB & Version 8.9 .0 \\
Alt. Client ID: & Report Run On: $8 / 28 / 2011$ & Page 4 of 16
\end{tabular}




\section{Doors}

\begin{aligned} \hline Door Code & DR1 \\ Door Type & Solid Core Wood \\ Area $(s q$ ft $t) & 20 \\$ Storm Door Condition & Adequate \\ Leakiness & Medium \\ Comment & \end{aligned}

$\left[\begin{array}{c}\text { Optional Dimensions } \\ \text { Width (in) } \square \\ \text { Height (in) }\end{array}\right] \begin{array}{r}\text { Replacement Door Required } \\ \text { Include in SIR } \square \\ \text { Additional Cost (\$/door) }\end{array}$

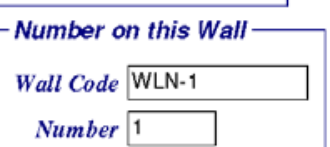

\begin{aligned} \hline Door Code & DR2 \\ Door Type & Solid Core Wood \\ Area $(s q$ f $t) & 20 \\$ Storm Door Condition & Adequate \\ Leakiness & Medium \\ Comment & \end{aligned}

$\left[\begin{array}{c}\text { Optional Dimensions } \\ \text { Width (in) } \square \\ \text { Height (in) }\end{array} \quad \begin{array}{r}\text { Replacement Door Required } \square \\ \text { Include in SIR } \square \\ \text { Additional Cost (\$/door) } \square\end{array}\right.$

Number on this Wall
Wall Code WLS-1
Number 1

\section{Unfinished Attics}

\begin{tabular}{rlr|} 
Attic Code & UA1 \\
Attic Type & Unfloored & Existing Insulation \\
Joist Spacing (in) $)$ & 24 \\
Area (sq ft) & 300 \\
Roof Color & Normal or Weathered \\
Comment &
\end{tabular}

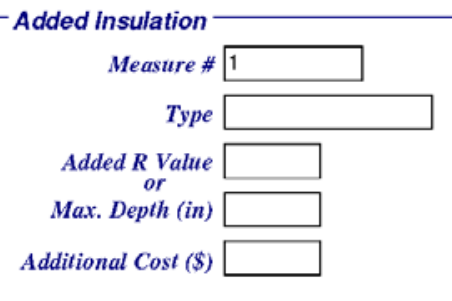

\section{Finished Attics}

\begin{tabular}{|c|c|c|}
\hline Attic Code & FA1 & \multirow{3}{*}{$\begin{aligned} & \text { Existing } \text { Insulation - } \\
& \text { Type } \text { Fiberglass Batt: } \\
& \text { Depth (in) } 1\end{aligned}$} \\
\hline Attic Area Type & Outer Ceiling Joist & \\
\hline Attic Floor Type & Unfloored & \\
\hline Area $(s q f t)$ & 392 & \\
\hline Roof Color & Normal or Weathered & \\
\hline Comment & & \\
\hline
\end{tabular}
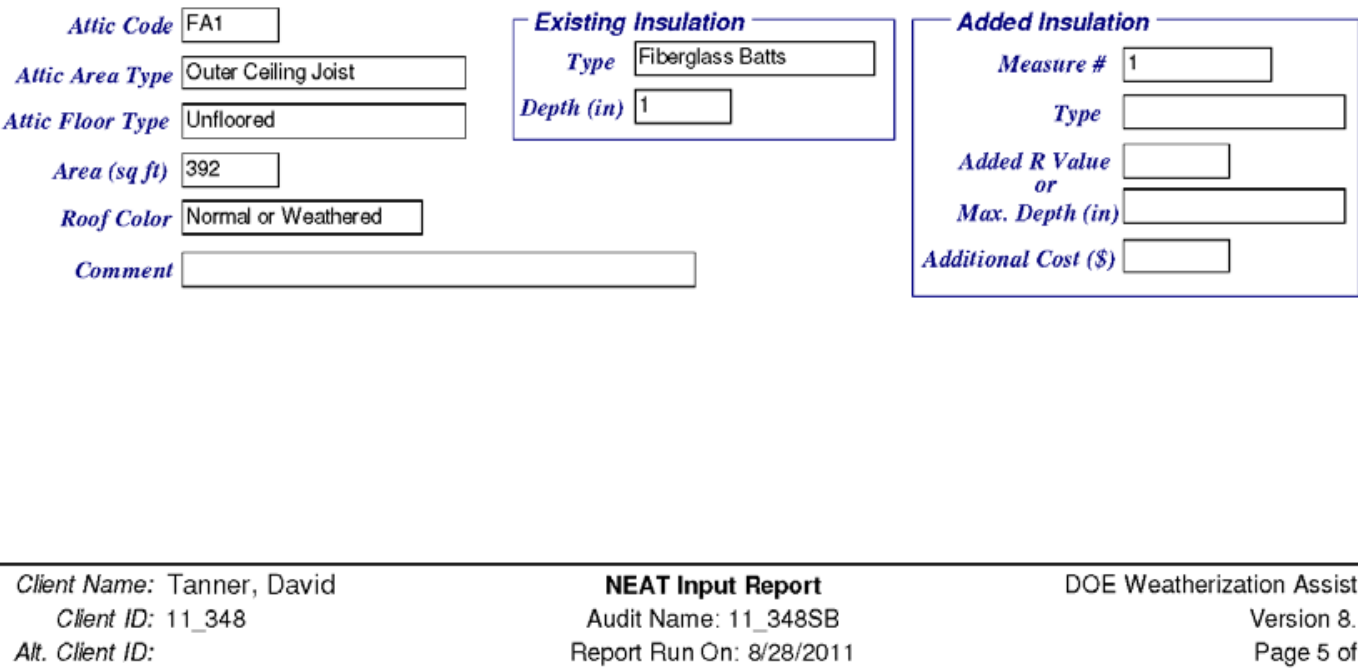

Alt. Client ID:

NEAT Input Report

Audit Name: 11 348SB

Report Run On: 8/28/2011
DOE Weatherization Assistant

Version 8.9 .0

Page 5 of 16 


\section{Appendix C: Reports}

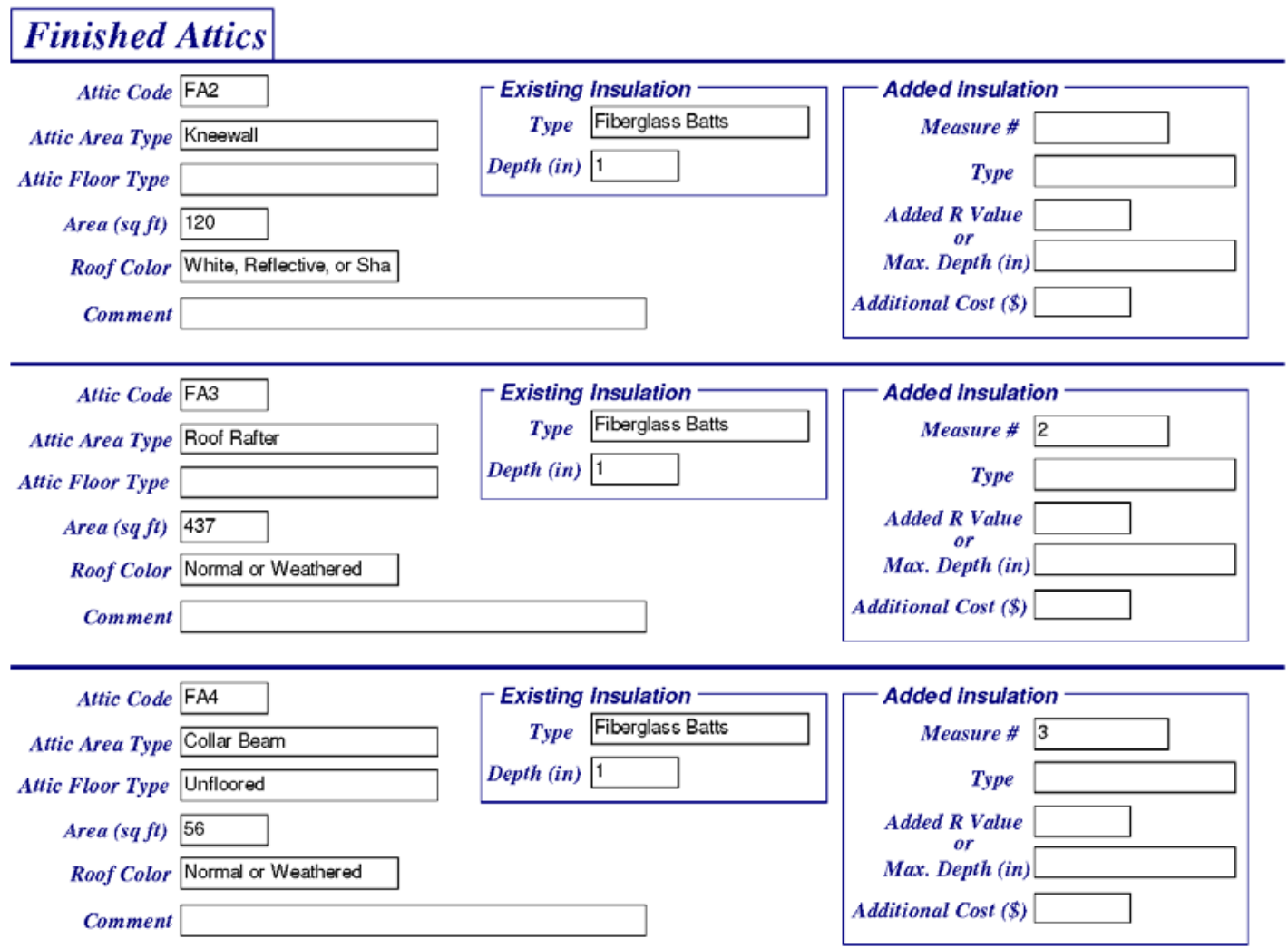

\section{Foundations}

\begin{tabular}{|c|c|c|}
\hline Foundation Code $\mathrm{F} 1$ & Foundation Type Unintentionally Conditioned & Measure \# 1 \\
\hline Area $(s q f t) 8$ & Added Insulation Type & \\
\hline Existing Insulation $R$ Value 0 & Additional $\operatorname{Cos} t(\$)$ & \\
\hline
\end{tabular}

\begin{tabular}{|c|c|}
\hline Floor Joist Size (in) 8 & Added Insulation Type \\
\hline Perimeter to Insulate (ft) 116 & Additional Cost (\$) \\
\hline
\end{tabular}

\begin{tabular}{|c|c|c|c|}
\hline Height $(f t) 8$ & Perimeter $(f t)$ & 116 & Added Insulation Type \\
\hline Height Exposed (\%) 25 & Existing Insulation $\boldsymbol{R}$ Value & 0 & Additional Cost (\$) \\
\hline
\end{tabular}

\begin{tabular}{lrr}
\hline Client Name: Tanner, David & NEAT Input Report & DOE Weatherization Assistant \\
Client ID: $11 \_348$ & Audit Name: 11_348SB & Version 8.9.0 \\
Alt. Client ID: & Report Run On: 8/28/2011 & Page 6 of 16
\end{tabular}




\section{Heating Systems}

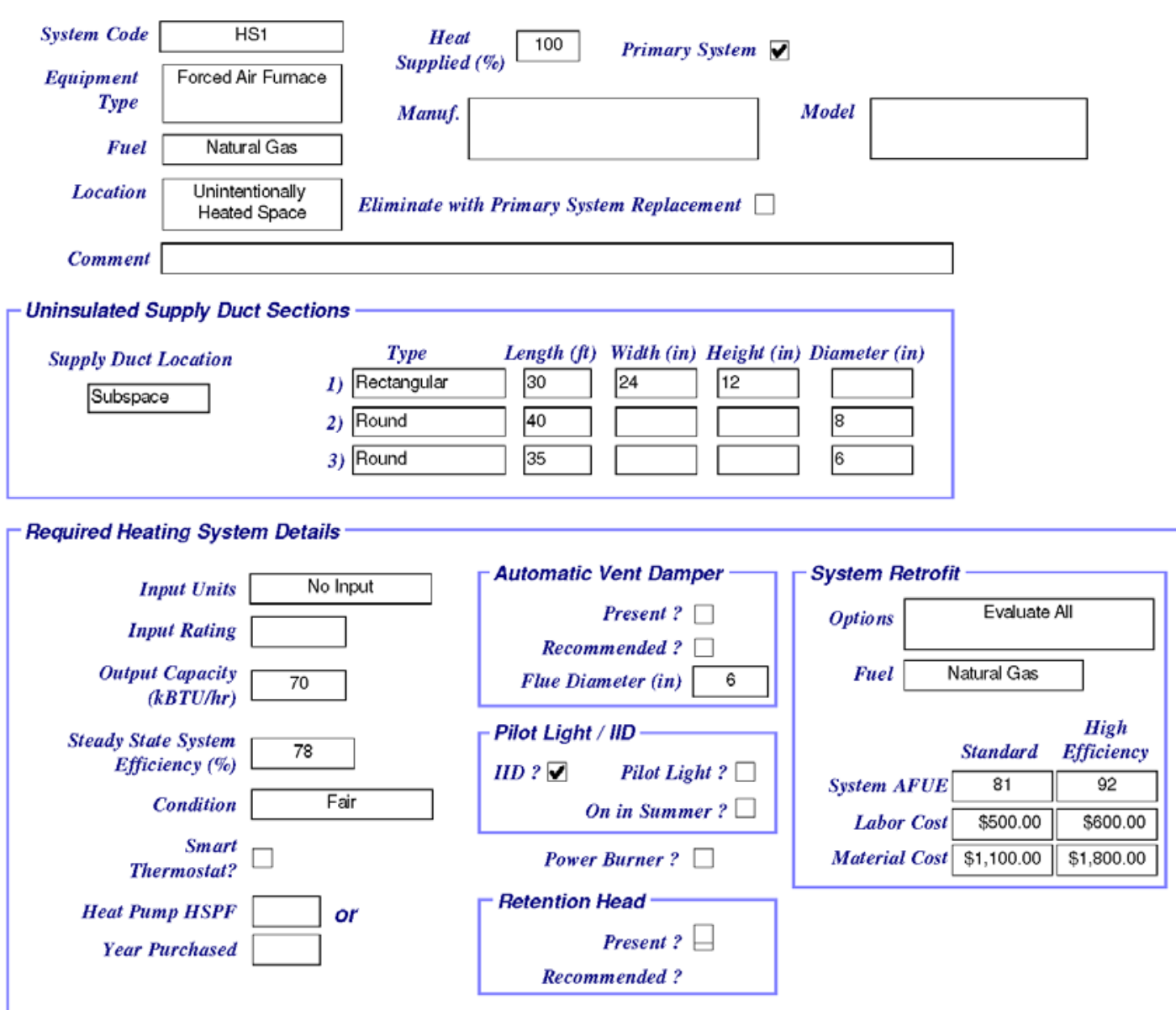

\section{Heating Systems (Continued}

\begin{tabular}{lrr}
\hline Client Name: Tanner, David & NEAT Input Report & DOE Weatherization Assistant \\
Client ID: $11 \_348$ & Audit Name: 11_348SB & Version 8.9 .0 \\
Alt. Client ID: & Report Run On: 8/28/2011 & Page 7 of 16
\end{tabular}




\section{Appendix C: Reports}

Heating Systems (Continued

OPTIONAL HEATING SYSTEM DETAILS

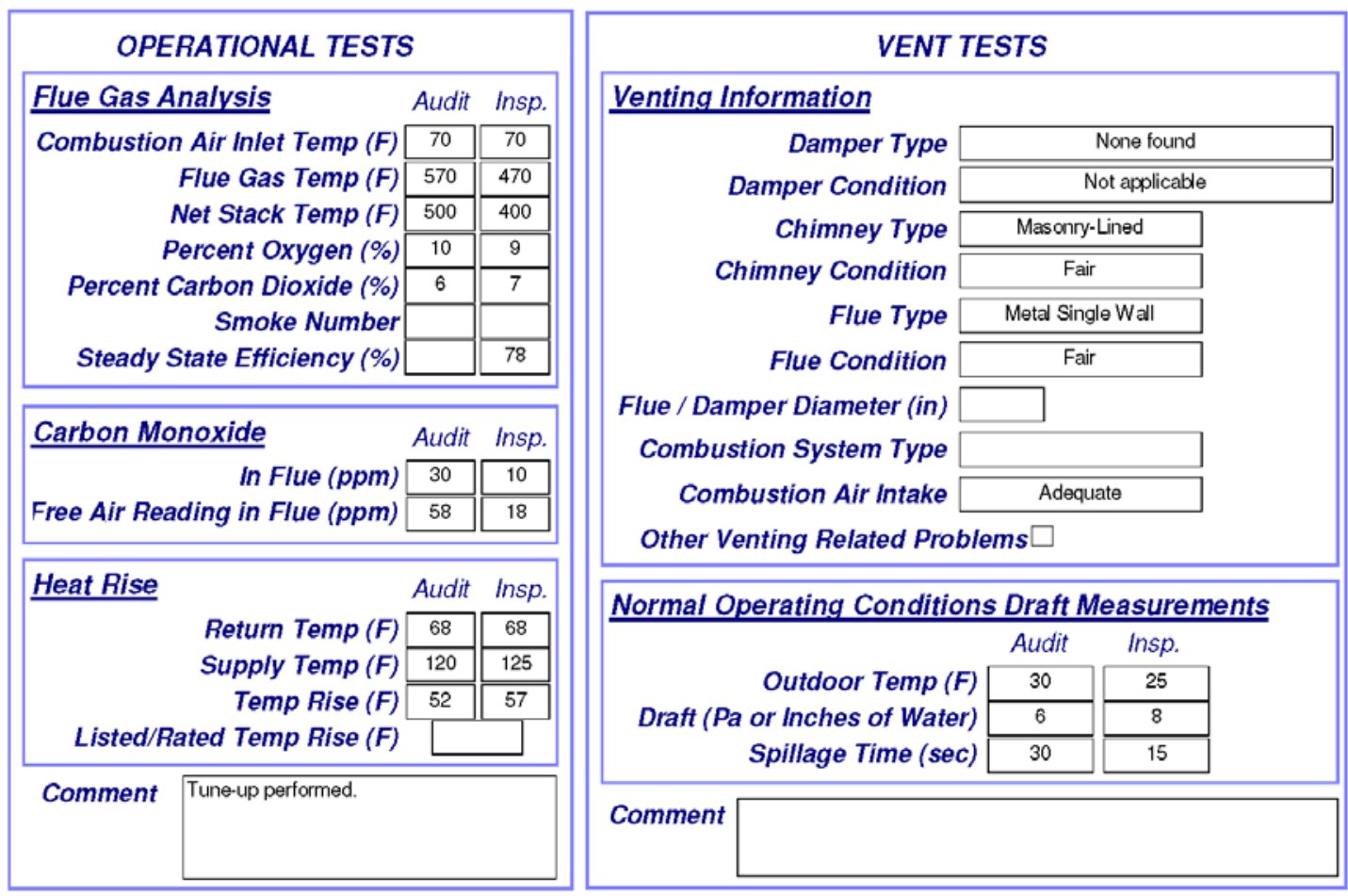

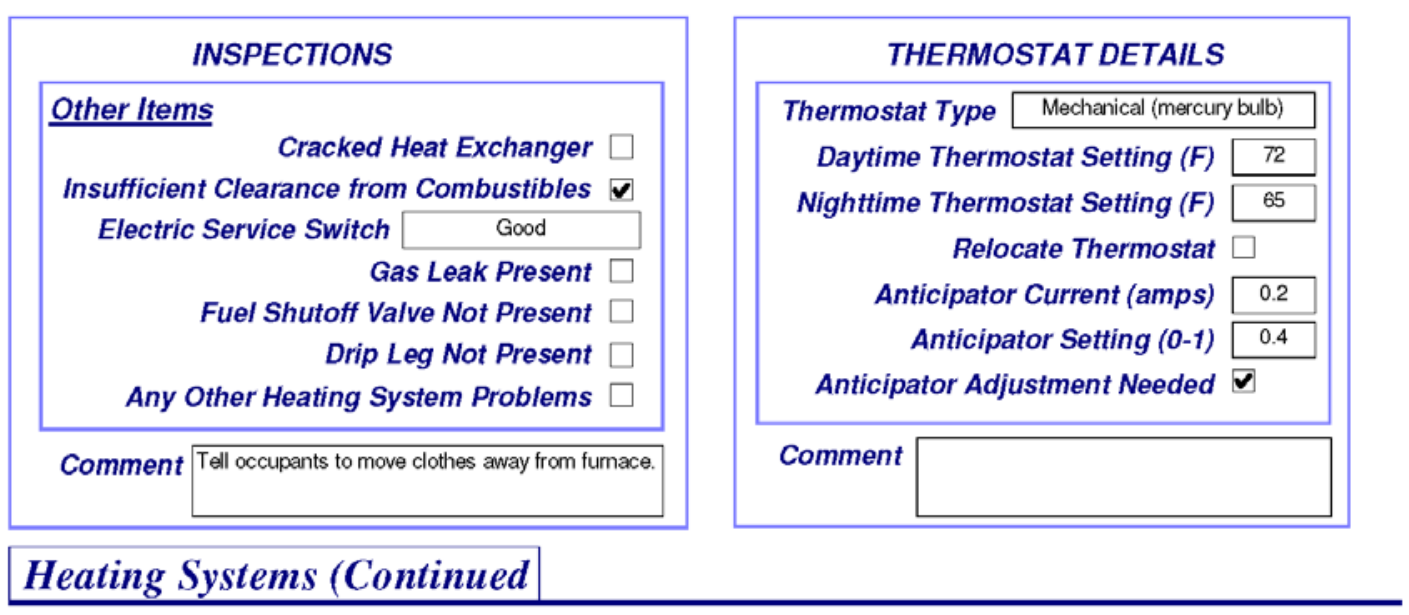

\begin{tabular}{lrr}
\hline Client Name: Tanner, David & NEAT Input Report & DOE Weatherization Assistant \\
Client ID: $11 \_348$ & Audit Name: $11 \_348$ SB & Version 8.9 .0 \\
Alt. Client ID: & Report Run On: $8 / 28 / 2011$ & Page 8 of 16
\end{tabular}




\section{Heating Systems (Continued}

OPTIONAL HEATING SYSTEM DETAILS (Continued)

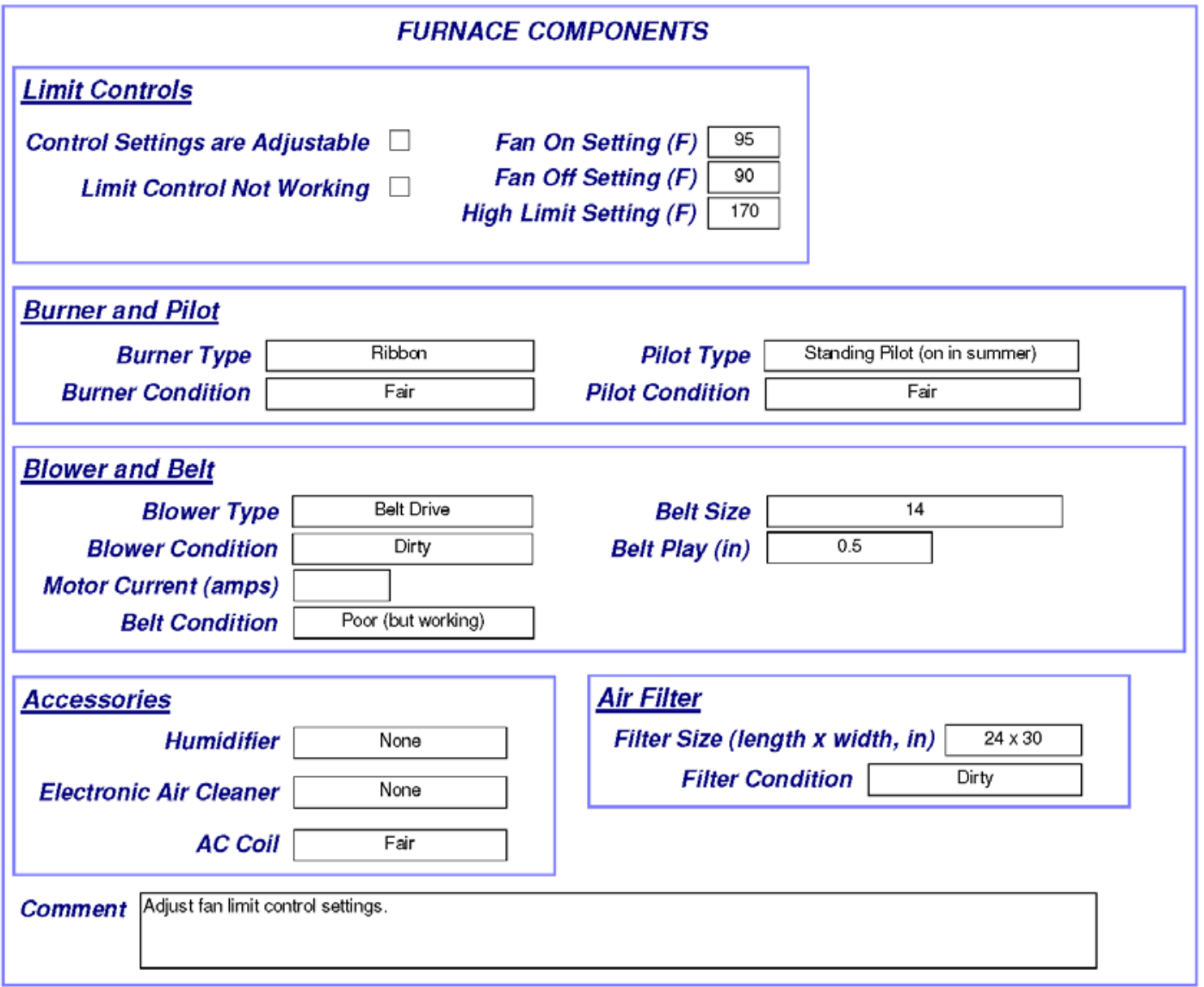

\section{Cooling Systems}

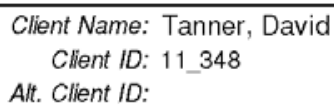

Report Run On: 8/28/2011
DOE Weatherization Assistant

Version 8.9.0

Page 9 of 16 


\section{Cooling Systems}

\begin{tabular}{|c|c|c|c|}
\hline AC Code & $A C 1$ & & \multirow{5}{*}{$\begin{array}{c}\text { Required Retrofits } \\
\text { Replacement Required } \boldsymbol{\nabla} \\
\text { Tune-up Mandatory } \square \\
\text { Include in SIR } \square\end{array}$} \\
\hline Equipment Type & \multicolumn{2}{|l|}{ Heat Pump } & \\
\hline Manufacturer & & \multirow{6}{*}{ Comment } & \\
\hline Model & & & \\
\hline Floor Area Cooled (sq ft) & 1290 & & \\
\hline Capacity (kBtu/hr) & 30 & & \\
\hline SEER & & & \\
\hline Year Manufactured & 2001 & & \\
\hline
\end{tabular}

\section{Ducts / Infiltration - Air and Duct Leakage}

Evaluate Duct Sealing ? $\square \quad$ Duct Leakage Method

\begin{tabular}{|c|c|}
\hline $\begin{array}{c}\text { WHOLE HOUSE BLOWER DOOR MEASUREMENTS } \\
\text { Before } \\
\text { Weatherizatio } \\
\text { Air Leakage Rate }(\mathrm{cfm})\end{array}$ & $\begin{array}{c}\text { After } \\
\text { Weatherization } \\
\text { (Target or }\end{array}$ \\
at House Pressure Difference (Pa) & 3000 \\
Infiltration Reduction Cost (\$) & 50 \\
Comment & $\begin{array}{l}\text { Target (post weatherization) blower door reading estimated } \\
\text { assuming that wall insulation would not be installed because } \\
\text { there is already a 1" batt installed. Infiltration reduction cost is } \\
\text { a typical value. }\end{array}$ \\
\hline
\end{tabular}

\section{Duct / Infiltration - Blower Door Readings (Optional)}

No data were entered for this audit.

\section{Ducts / Infiltration Zonal Pressure Readings (Optional)}

No data were entered for this audit.

\section{Ducts / Infiltration Pressure Balance Readings (Optional)}

No data were entered for this audit.

\begin{tabular}{lrr}
\hline Client Name: Tanner, David & NEAT Input Report & DOE Weatherization Assistant \\
Client ID: $11 \_348$ & Audit Name: 11_348SB & Version 8.9 .0 \\
Alt. Client ID: & Report Run On: $8 / 28 / 2011$ & Page 10 of 16
\end{tabular}




\section{Ducts / Infiltration Pressure Pan Readings (Optional)}

No data were entered for this audit.

\section{Base Load - Water Heater}

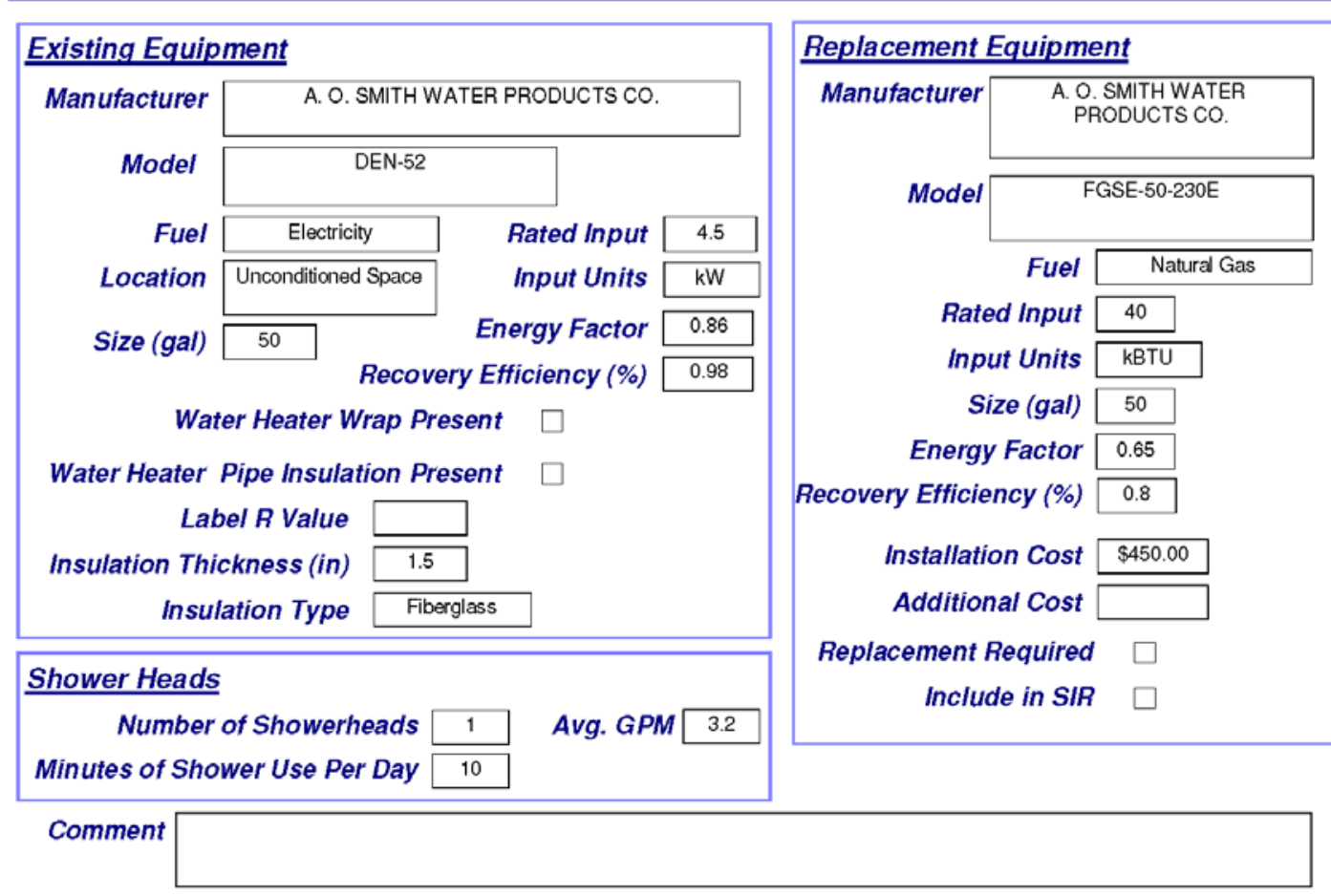

\section{Base Load - Water Heater (Continued)}

\begin{tabular}{lrr}
\hline Client Name: Tanner, David & NEAT Input Report & DOE Weatherization Assistant \\
Client ID: $11 \_348$ & Audit Name: 11348 SB & Version 8.9 .0 \\
Alt. Client ID: & Report Run On: $8 / 28 / 2011$ & Page 11 of 16
\end{tabular}




\section{Appendix C: Reports}

\section{Base Load - Water Heater (Continued)}

OPTIONAL WATER HEATING SYSTEM DETAILS

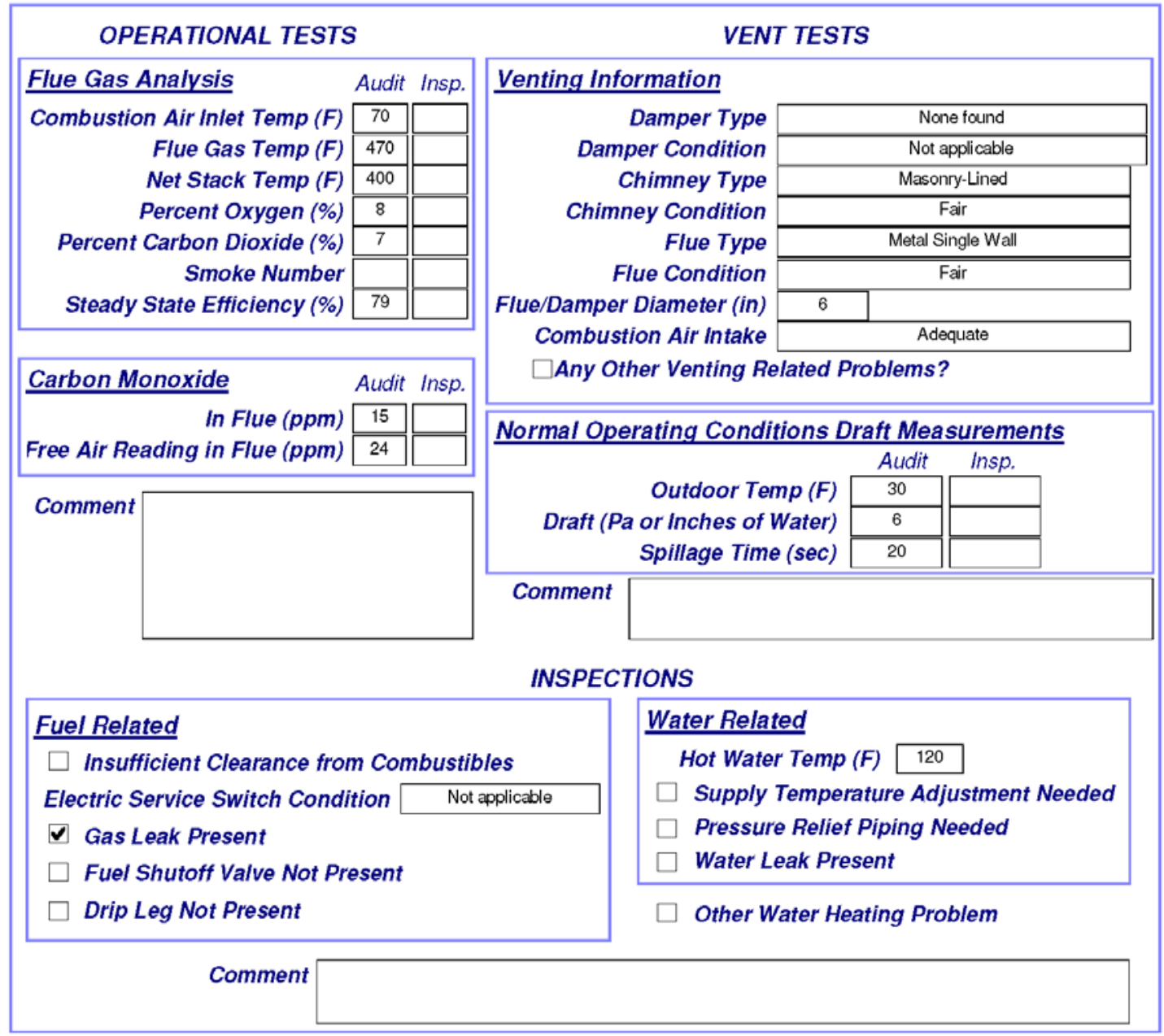

\section{Base Load - Refrigerator}

\begin{tabular}{lrr}
\hline Client Name: Tanner, David & NEAT Input Report & DOE Weatherization Assistant \\
Client ID: $11 \_348$ & Audit Name: $11 \_348$ SB & Version 8.9 .0 \\
Alt. Client ID: & Report Run On: $8 / 28 / 2011$ & Page 12 of 16
\end{tabular}




\section{Base Load - Refrigerator}

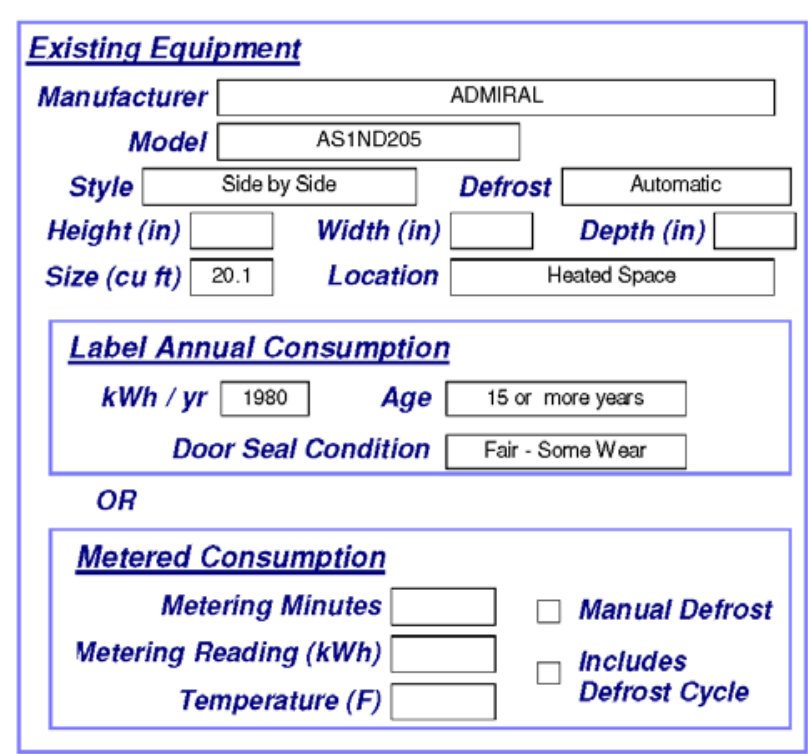

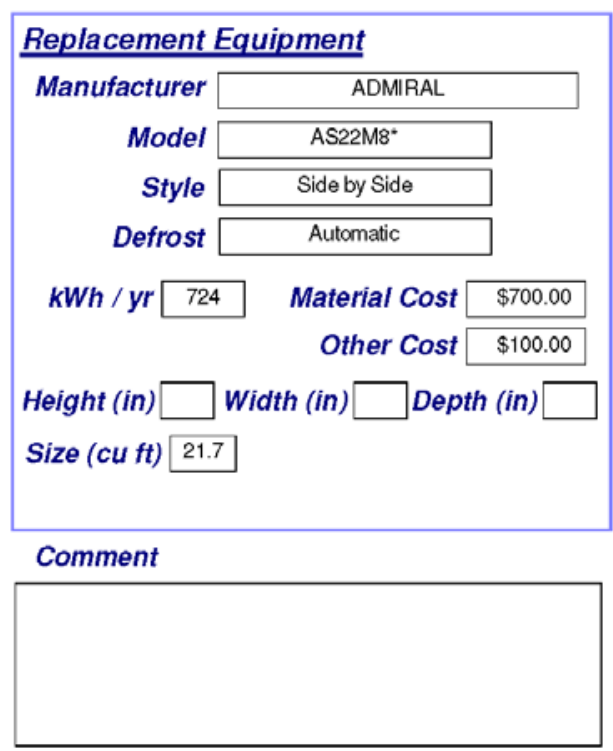

Base Load - Lighting System

\begin{tabular}{|c|c|c|c|c|c|c|c|c|c|}
\hline \multirow[b]{2}{*}{$\begin{array}{l}\text { Light } \\
\text { Code }\end{array}$} & \multirow[b]{2}{*}{ Room } & \multirow[b]{2}{*}{ Location } & \multicolumn{4}{|c|}{ Existing Incandescent } & \multicolumn{2}{|c|}{ Replacement CFL } & \multirow[b]{2}{*}{ Comment } \\
\hline & & & $\begin{array}{c}\text { Lamp } \\
\text { Type }\end{array}$ & Quantity & Watts & $\begin{array}{c}\text { Hours } \\
\text { per Day }\end{array}$ & $\begin{array}{c}\text { CF } \\
\text { Watts }\end{array}$ & $\begin{array}{c}\text { AddI. } \\
\text { Cost }\end{array}$ & \\
\hline LT1 & Dining Room & Ceiling & Standard & 4 & 60 & 10 & 13 & & \\
\hline
\end{tabular}

\section{Health \& Safety}

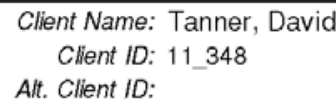

Alt. Client ID:

Report Run On: 8/28/2011
DOE Weatherization Assistant

Version 8.9 .0

Page 13 of 16 


\section{Appendix C: Reports}

\section{Health \& Safety}

\section{WHOLE HOUSE}

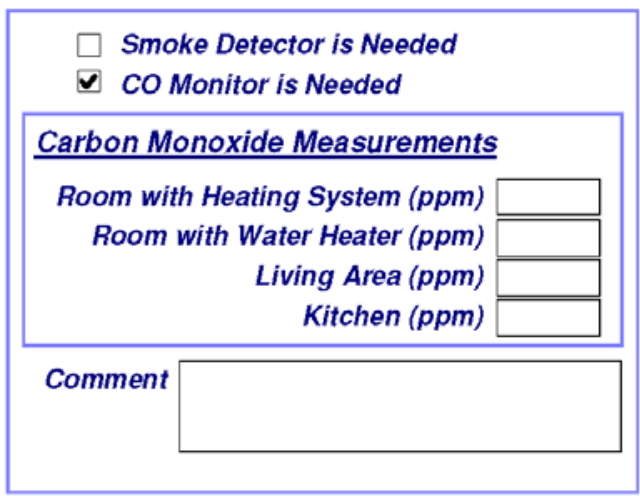

\section{BUILDING SHELL}

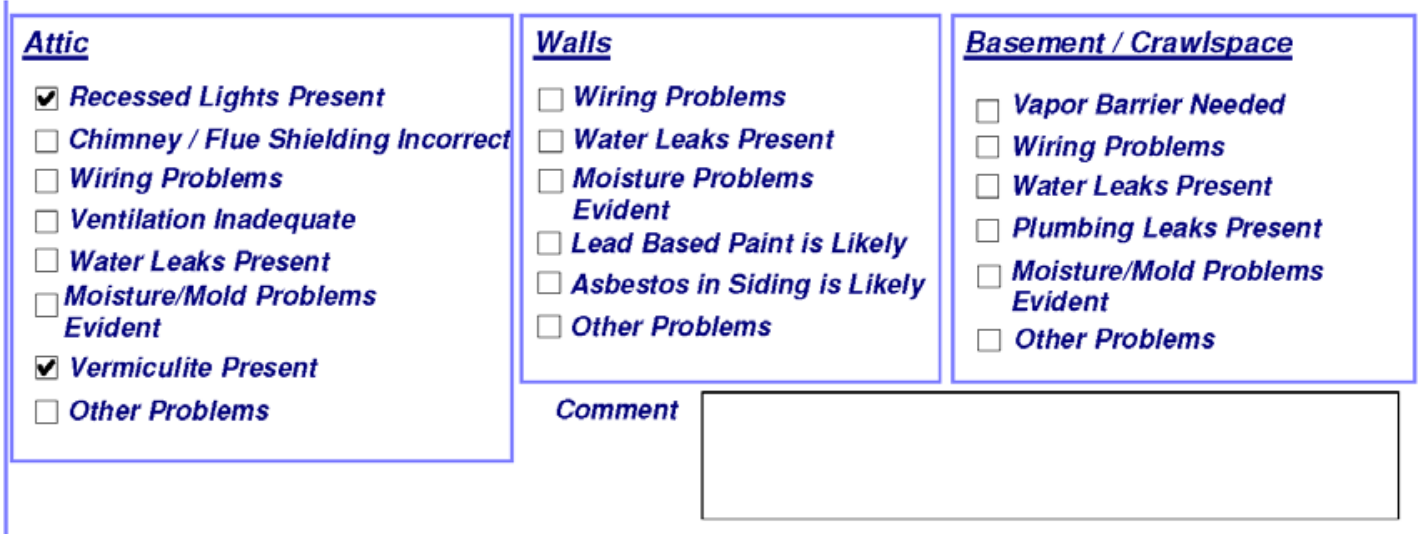

\section{Health \& Safety (Continued)}

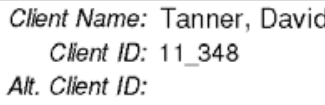

NEAT Input Report

Audit Name: 11_348SB

Report Run On: 8/28/2011
DOE Weatherization Assistant

Version 8.9.0

Page 14 of 16 


\section{Health \& Safety (Continued)}

\section{EQUIPMENT}

\begin{tabular}{|l|}
\hline Wood Stove / Fireplace \\
$\square$ Wood Stove / Fireplace is Present \\
$\square$ Improper Venting \\
$\square$ Combustion Air is Inadequate
\end{tabular}

\section{Clothes Dryer}

Improper Venting

\section{Cook Stove}

CO Measurement Oven (ppm) co Measurement Burner 1 (ppm) CO Measurement Burner 2 (ppm) CO Measurement Burner 3 (ppm) cO Measurement Burner 4 (ppm)

\section{Gas Leak Present}

\begin{tabular}{|cll}
\hline Exhaust Fans & \\
$\underline{\text { Bathrooms }}$ & $\underline{\text { Kitchen }}$ & $\underline{\text { Air-to-Air Heat Exchanger }}$ \\
$\square$ Missing & $\square$ Missing & $\square$ Exists \\
$\square$ Not Operational & $\square$ Not Operational & $\square$ Not Operational \\
$\square$ Improper Venting & $\square$ Improper Venting & \\
\hline
\end{tabular}

Comment

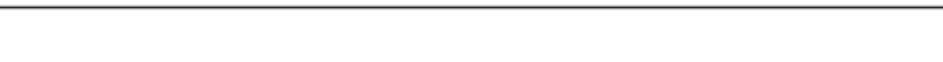

\begin{tabular}{|c|c|c|c|c|c|c|c|c|}
\hline \multicolumn{9}{|c|}{ Itemized Costs } \\
\hline Description & Cost & $\begin{array}{l}\text { Include } \\
\text { in SIR? }\end{array}$ & Material & $\begin{array}{c}\text { Energy } \\
\text { Savings } \\
\text { (mBTU/yr) }\end{array}$ & $\begin{array}{c}\text { Units } \\
\text { (of energy } \\
\text { saved) }\end{array}$ & $\begin{array}{c}\text { Life of } \\
\text { measure } \\
\text { (years) }\end{array}$ & $\begin{array}{l}\text { Fuel } \\
\text { Type } \\
\text { Saved }\end{array}$ & Comment \\
\hline $\begin{array}{l}\text { Install Bathroom } \\
\text { Exhaust Fan }\end{array}$ & $\$ 270.0$ & $\square$ & $\begin{array}{l}\text { Bathroom exhaust } \\
\text { fan }(+)\end{array}$ & & & & & \\
\hline CO Monitor is Needed & $\$ 70.00$ & $\square$ & $\mathrm{CO}$ monitor $(+)$ & & & & & \\
\hline $\begin{array}{l}\text { Anticipator } \\
\text { Adjustment Needed }\end{array}$ & $\$ 20.00$ & $\nabla$ & & & & & & \\
\hline Repair Roof & $\$ 80.00$ & $\nabla$ & & & & & & \\
\hline $\begin{array}{l}\text { Insulate and seal attic } \\
\text { access }\end{array}$ & $\$ 29.60$ & $\nabla$ & $\begin{array}{l}\text { R-30 faced batt } \\
\text { insulation }(+)\end{array}$ & 0.7 & MBtu & 20 & & \\
\hline Utility Bills & & & & & & & & \\
\hline
\end{tabular}

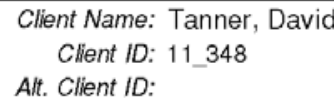

Alt. Client ID:

Report Run On: 8/28/2011
DOE Weatherization Assistant

Version 8.9.0

Page 15 of 16 


\section{Appendix C: Reports}

\begin{tabular}{|c|c|c|c|c|c|c|c|}
\hline \multicolumn{8}{|c|}{ Utility Bills } \\
\hline $\begin{array}{l}\text { Billing } \\
\text { Type }\end{array}$ & \multicolumn{2}{|c|}{$\begin{array}{l}\text { Billing } \\
\text { Period }\end{array}$} & $\begin{array}{l}\text { Billing } \\
\text { Units }\end{array}$ & $\begin{array}{r}\text { First } \\
\text { Period Days }\end{array}$ & $\begin{array}{l}\text { Base } \\
\text { Temp }\end{array}$ & $\begin{array}{l}\text { Base } \\
\text { Load }\end{array}$ & Comment \\
\hline \multirow[t]{14}{*}{ Heating } & \multicolumn{2}{|c|}{ Pre-Retrofit } & Therms & 30 & 65 & 28.9 & \\
\hline & $\#$ & Month & Day & Usage & $D e$ & ays & \\
\hline & 1 & 1 & 15 & 190 & & 815 & \\
\hline & 2 & 2 & 17 & 212 & & 690 & \\
\hline & 3 & 3 & 14 & 138 & & 585 & \\
\hline & 4 & 4 & 16 & 65 & & 312 & \\
\hline & 5 & 5 & 13 & 81 & & 144 & \\
\hline & 6 & 6 & 15 & 49 & & 42 & \\
\hline & 7 & 7 & 15 & 15 & & 11 & \\
\hline & 8 & 8 & 14 & 14 & & 15 & \\
\hline & 9 & 9 & 16 & 43 & & 95 & \\
\hline & 10 & 10 & 17 & 74 & & 253 & \\
\hline & 11 & 11 & 13 & 97 & & 507 & \\
\hline & 12 & 12 & 12 & 149 & & 726 & \\
\hline
\end{tabular}

\begin{tabular}{lcr}
\hline Client Name: Tanner, David & NEAT Input Report & DOE Weatherization Assistant \\
Client ID: $11 \_348$ & Audit Name: $11 \_348$ SB & Version 8.9 .0 \\
Alt. Client ID: & Report Run On: 8/28/2011 & Page 16 of 16
\end{tabular}




\section{NEAT Heating System Summary}

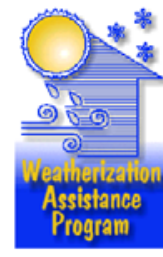

$\begin{aligned} \text { Client ID } & 11 \text { 348 Alt. Client ID }[----- \\ \text { Client Name } & \text { Tanner, David }\end{aligned}$

\section{Heating Systems}
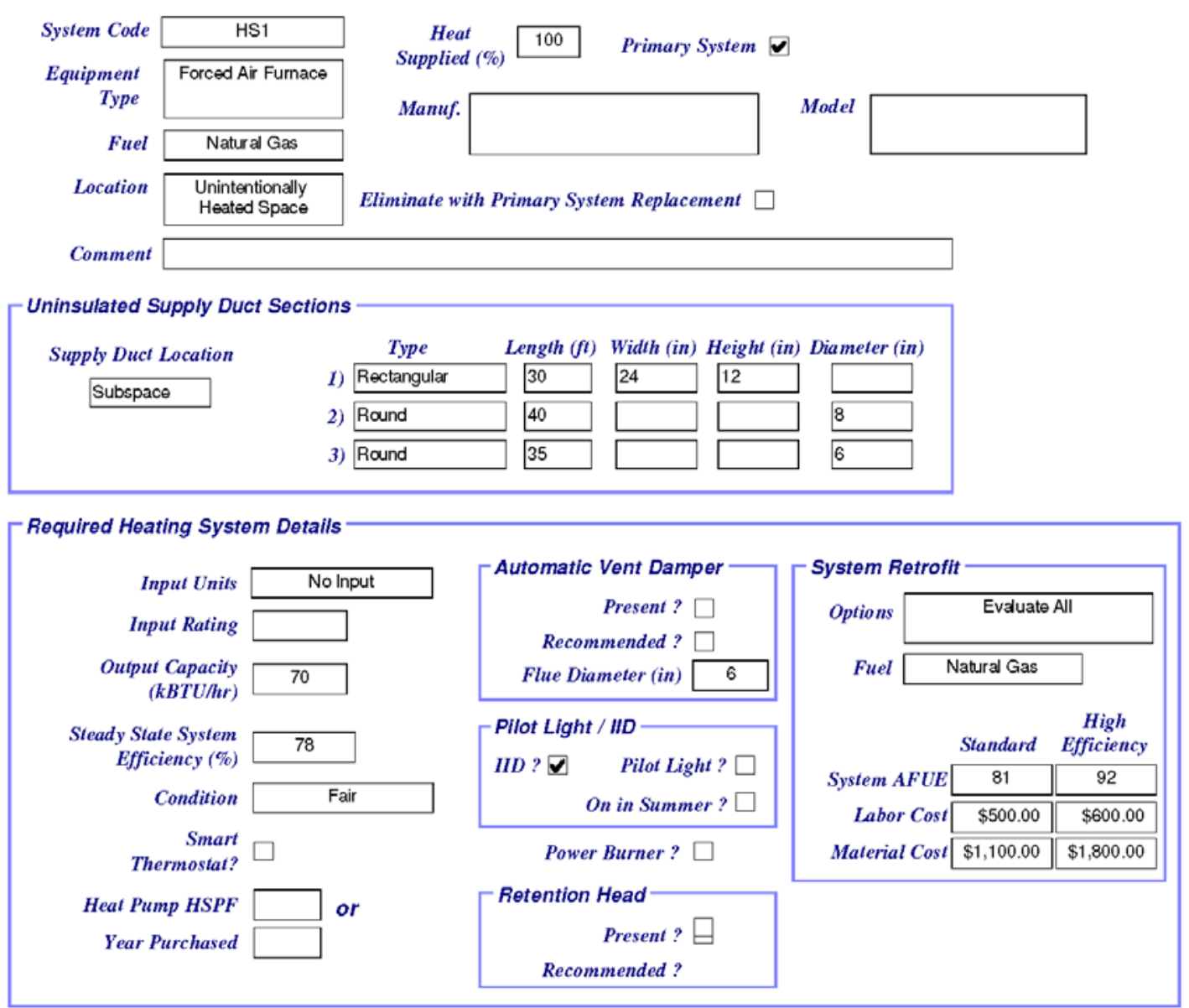

\section{Heating Systems (Continued}

\begin{tabular}{lrr}
\hline Client Name: Tanner, David & NEAT Heating System Summary & DOE Weatherization Assistant \\
Client ID: $11 \_348$ & Audit Name: $11 \_348 S B$ & Version 8.9 .0 \\
Aft. Client ID: & Report Run On: $8 / 27 / 2011$ & Page 1 of 3
\end{tabular}




\section{Appendix C: Reports}

\section{Heating Systems (Continued}

OPTIONAL HEATING SYSTEM DETAILS

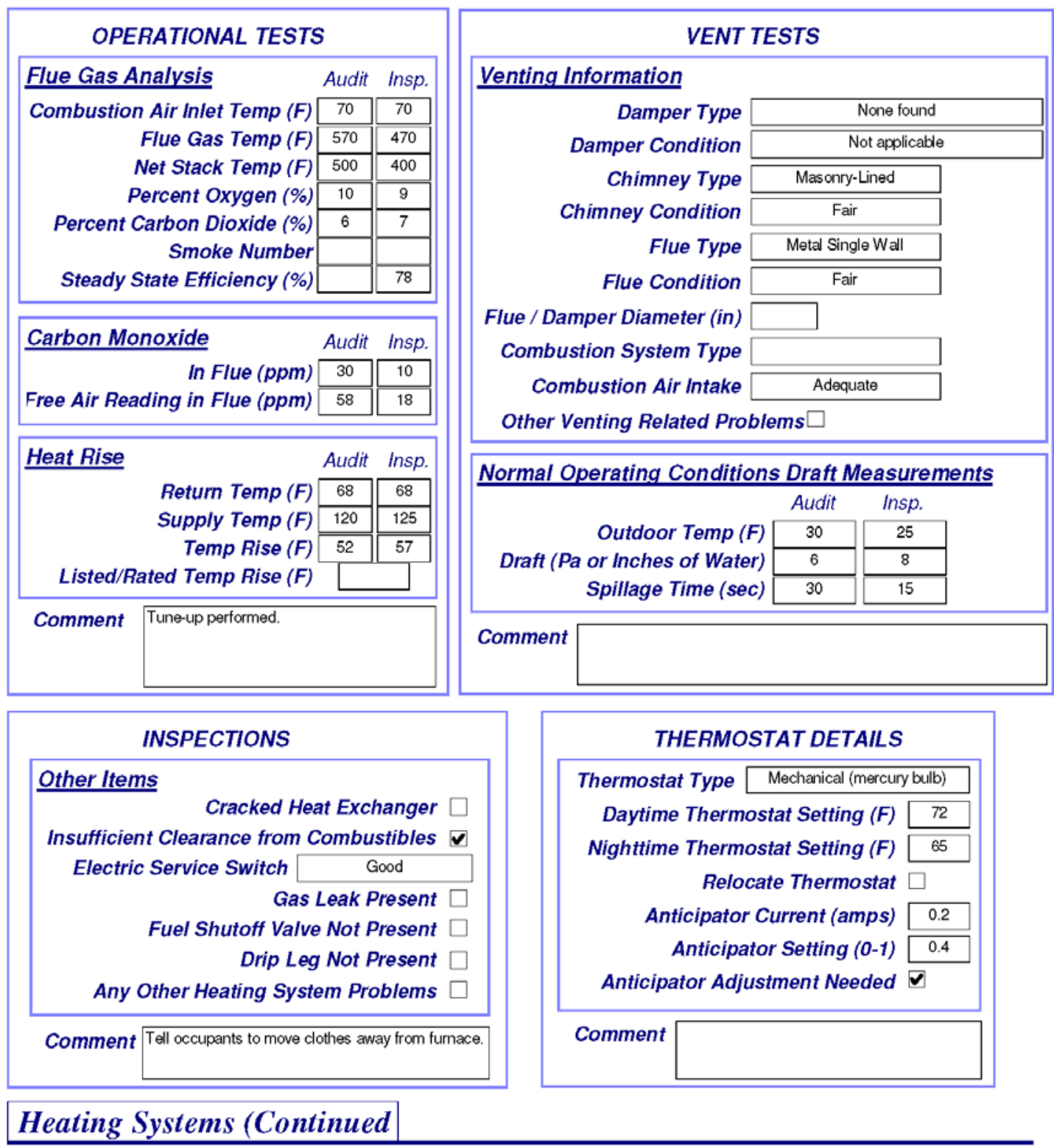

$\begin{array}{lrr}\text { Client Name: Tanner, David } & \text { NEAT Heating System Summary } & \text { DOE Weatherization Assistant } \\ \text { Client ID: } 11 \_348 & \text { Audit Name: } 11 \_348 \text { SB } & \text { Version } 8.9 .0 \\ \text { Alt. Client ID: } & \text { Report Run On: } 8 / 27 / 2011 & \text { Page } 2 \text { of } 3\end{array}$


Heating Systems (Continued

OPTIONAL HEATING SYSTEM DETAILS (Continued)

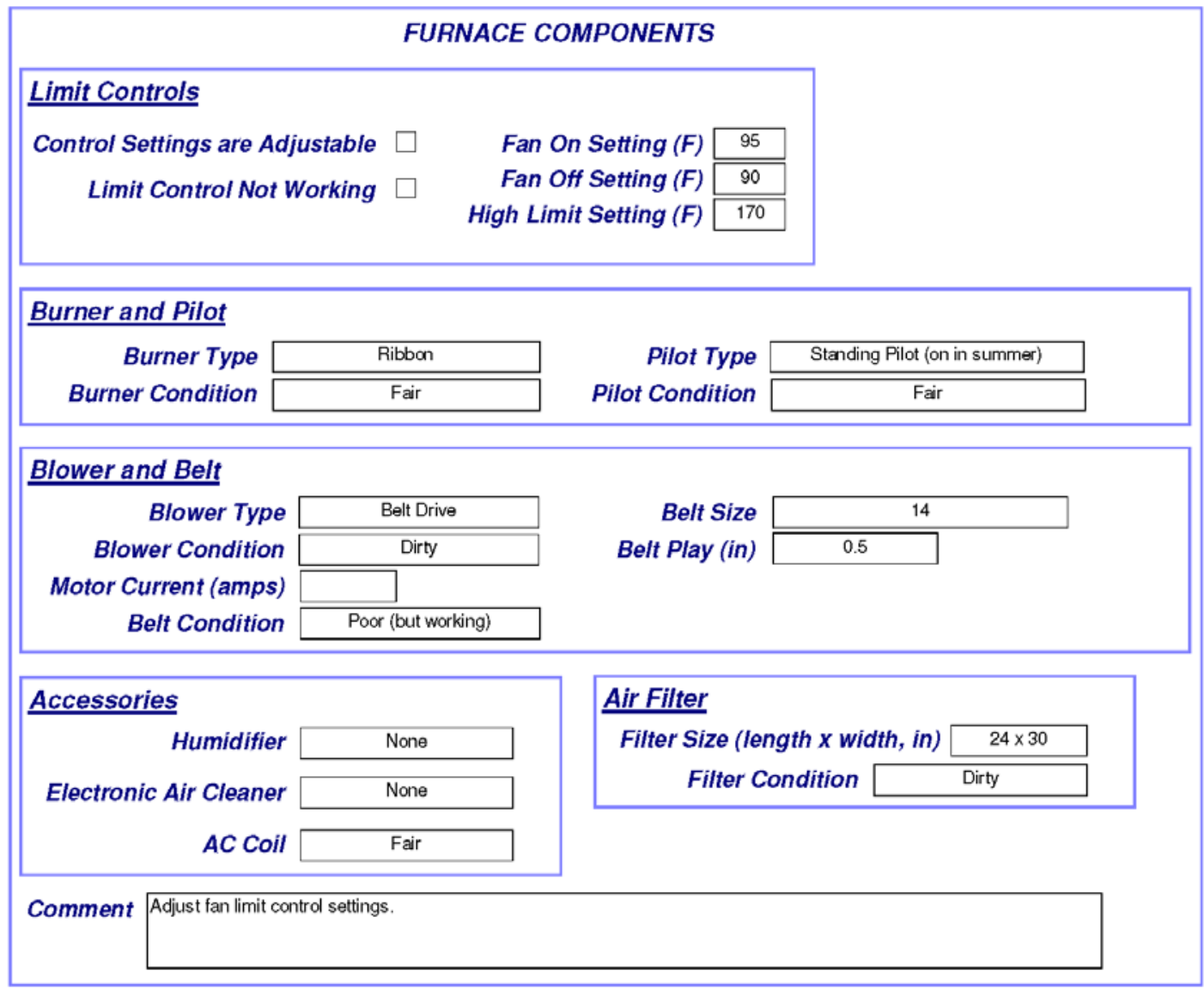

\begin{tabular}{lrr}
\hline Client Name: Tanner, David & NEAT Heating System Summary & DOE Weatherization Assistant \\
Client ID: $11 \_348$ & Audit Name: $11 \_348$ SB & Version 8.9 .0 \\
Alt. Client ID: & Report Run On: $8 / 27 / 2011$ & Page 3 of 3
\end{tabular}




\section{Appendix C: Reports}

\section{NEAT Pressure Diagnostics}

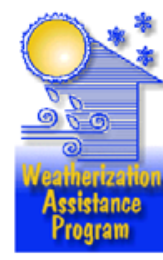

Alt. Client ID

Client ID 11_348

Client Name Tanner, David

$[----\urcorner$

\section{Blower Door Readings (Existing)}

No data were entered for this audit.

\section{Blower Door Readings (New)}
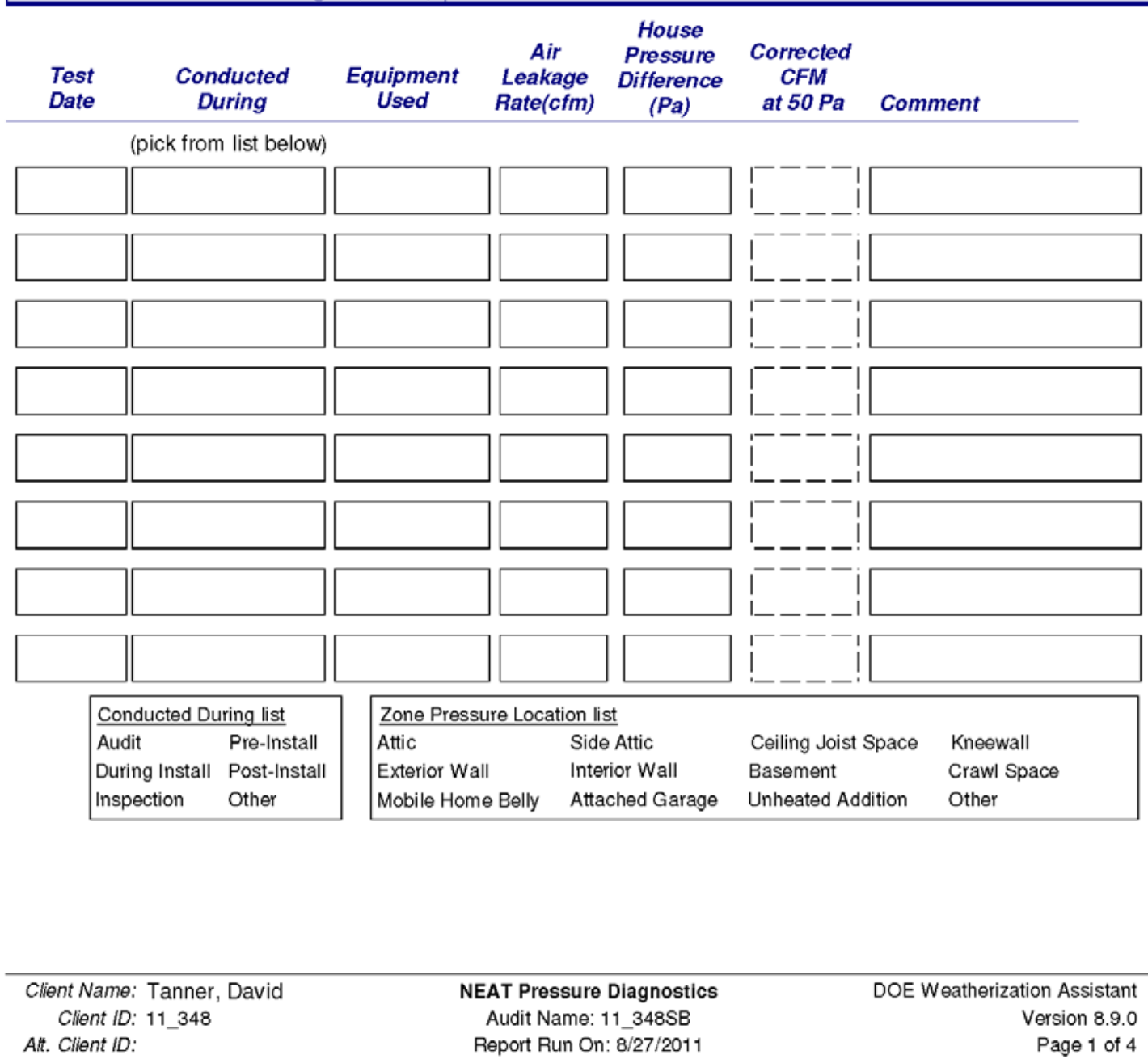
Zonal Pressure Readings (Existing)

No data were entered for this audit.

\section{Zonal Pressure Readings (New)}

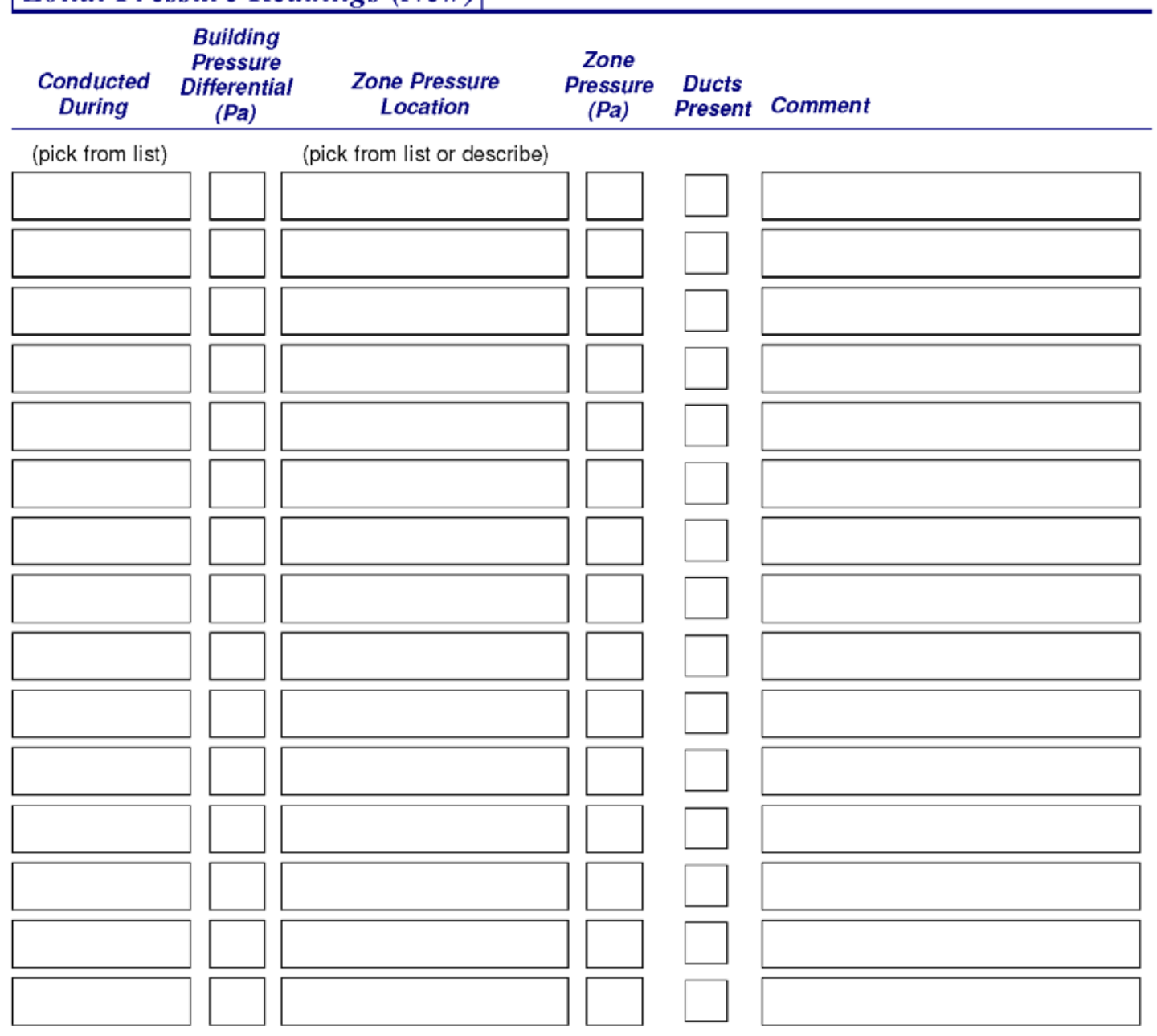

Client Name: Tanner, David

Client ID: 11348

Alt. Client ID:
NEAT Pressure Diagnostics

Audit Name: 11 348SB

Report Run On: 8/27/2011
DOE Weatherization Assistant

Version 8.9 .0

Page 2 of 4 


\section{Appendix C: Reports}

\section{Pressure Balance Readings (Existing)}

No data were entered for this audit.

\section{Pressure Balance Readings (New)}

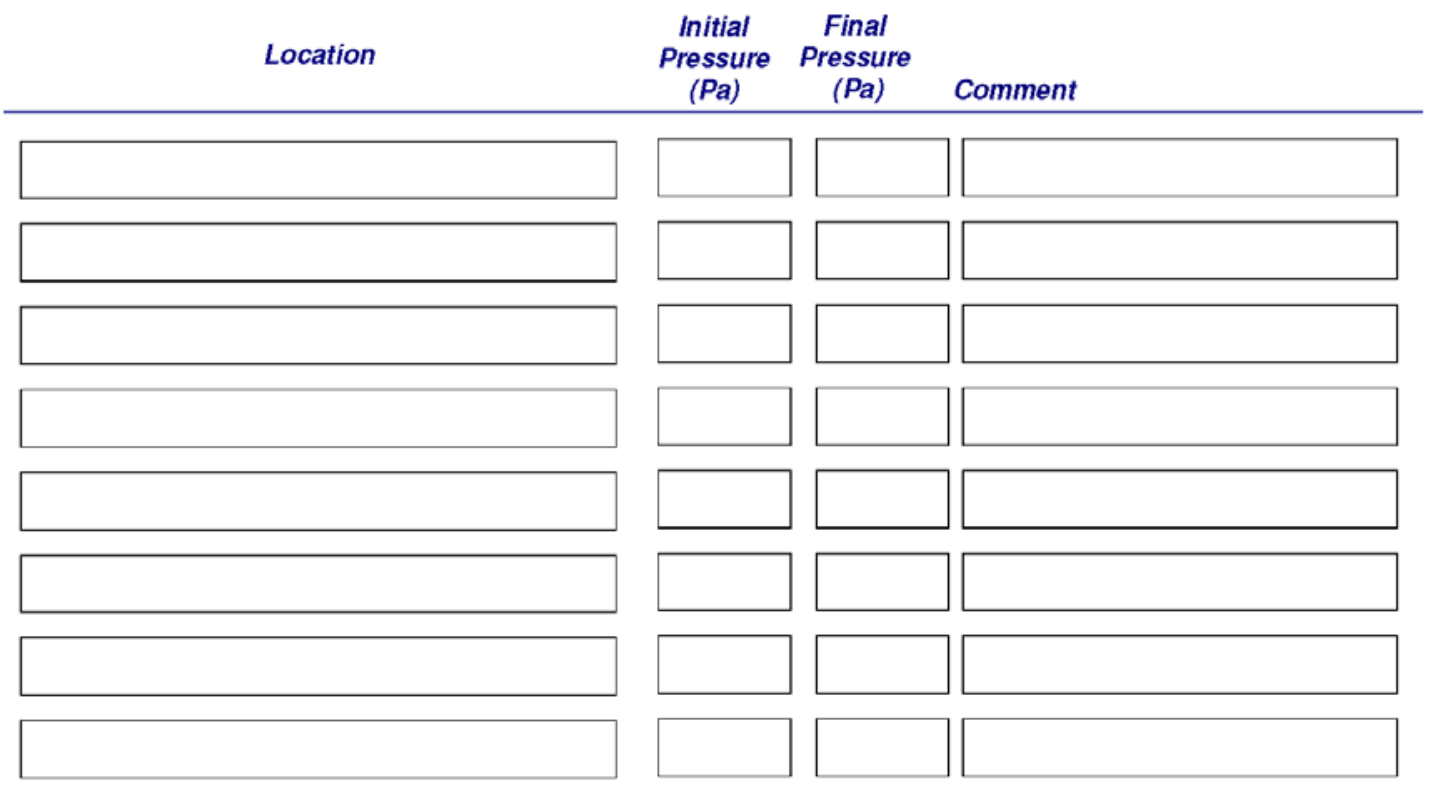




\section{Pressure Pan Readings (Existing)}

No data were entered for this audit.

\section{Pressure Pan Readings (New)}

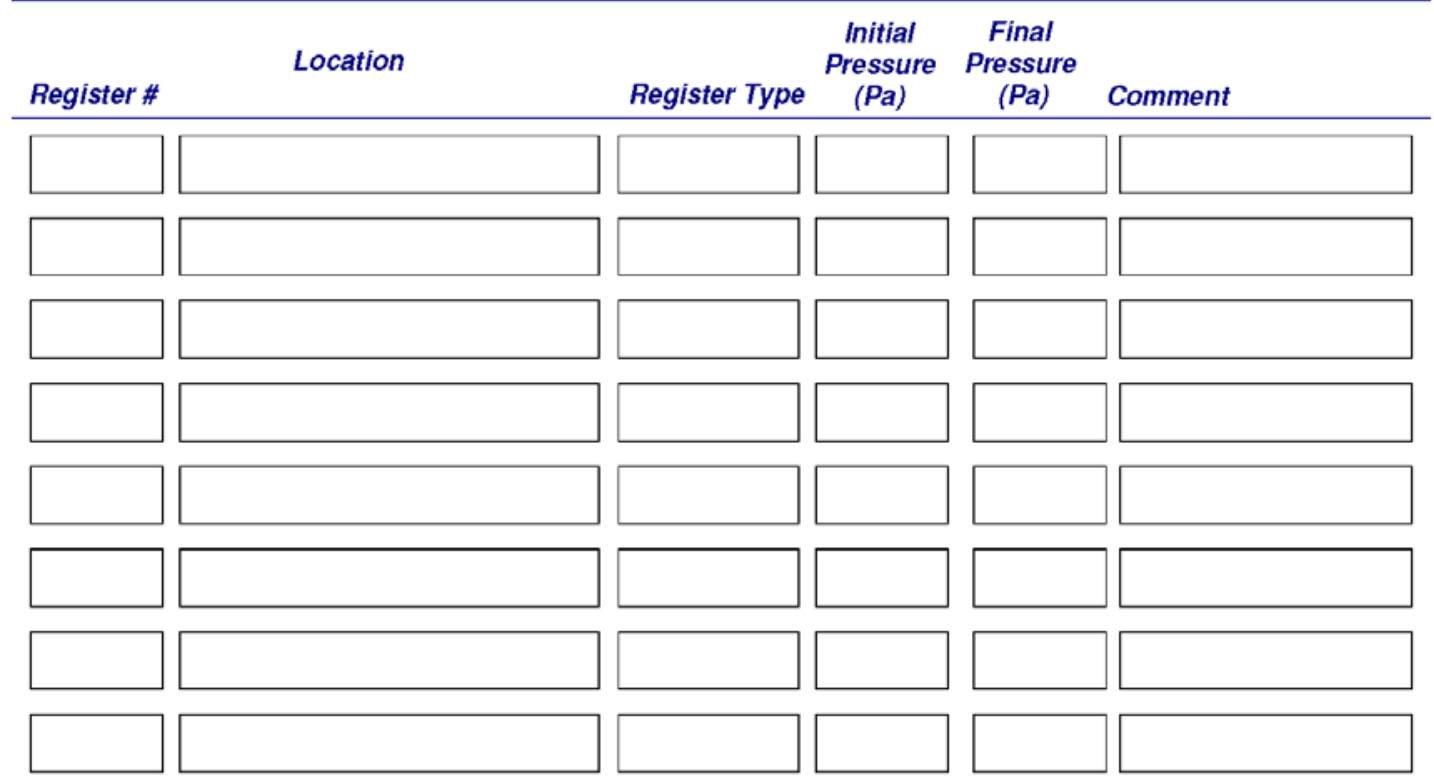




\section{Appendix C: Reports}

\section{NEAT Health and Safety Summary}

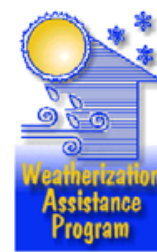

Alt. Client ID $\left[\begin{array}{c}----\cdots \\ \mathrm{L}\end{array}\right.$

Client ID 11348 alt.

Client Name Tanner, David

\section{Health \& Safety}

\section{WHOLE HOUSE}

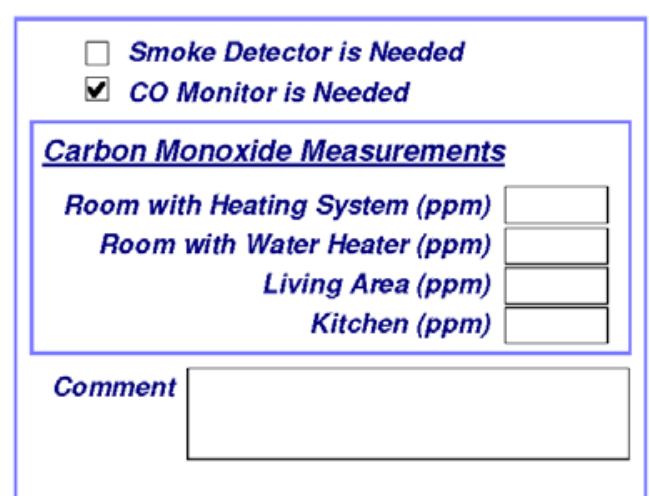

\section{BUILDING SHELL}

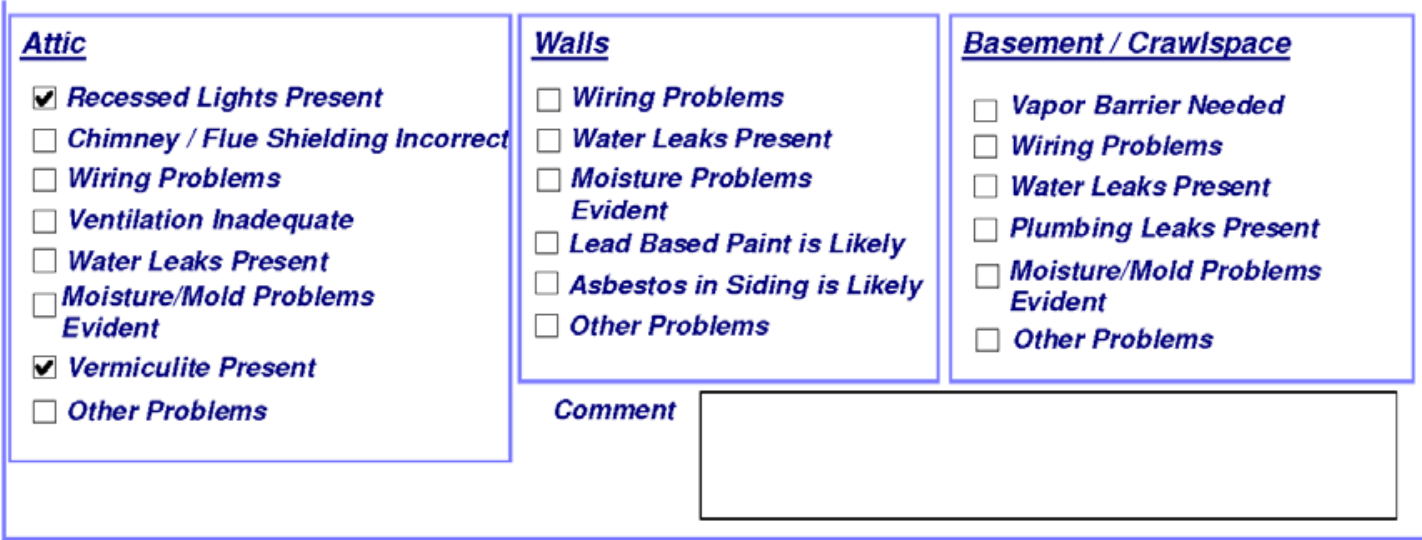

\footnotetext{
Client Name: Tanner, David Client ID: 11_348

Att. Client ID:
}

NEAT Health and Safety Summary

Audit Name: 11_348SB

Report Run On: 8/28/2011
DOE Weatherization Assistant Version 8.9.0

Page 1 of 5 


\section{Health \& Safety (Continued)}

EQUIPMENT

\section{Wood Stove / Fireplace}

$\square$ Wood Stove / Fireplace is Present

Improper Venting

Combustion Air is Inadequate

\section{Clothes Dryer}

Improper Venting

\section{Cook Stove}

CO Measurement Oven (ppm)

CO Measurement Burner 1 ( $p p m)$

CO Measurement Burner 2 ( $\mathrm{ppm}$ )

CO Measurement Burner 3 (ppm)

co Measurement Burner 4 (ppm)

$\square$ Gas Leak Present

\begin{tabular}{|lll}
\hline Exhaust Fans & \\
Bathrooms & $\underline{\text { Kitchen }}$ & $\underline{\text { Air-to-Air Heat Exchanger }}$ \\
$\square$ Missing & $\square$ Missing & $\square$ Exists \\
$\square$ Not Operational & $\square$ Not Operational & $\square$ Not Operational \\
$\square$ Improper Venting & $\square$ Improper Venting &
\end{tabular}

Comment 


\section{Appendix C: Reports}

\section{Heating Systems}

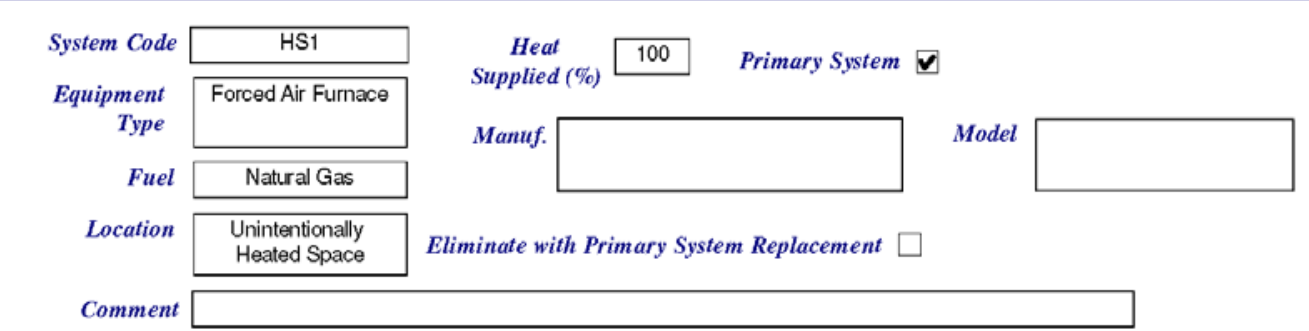

-Uninsulated Supply Duct Sections

Supply Duct Location
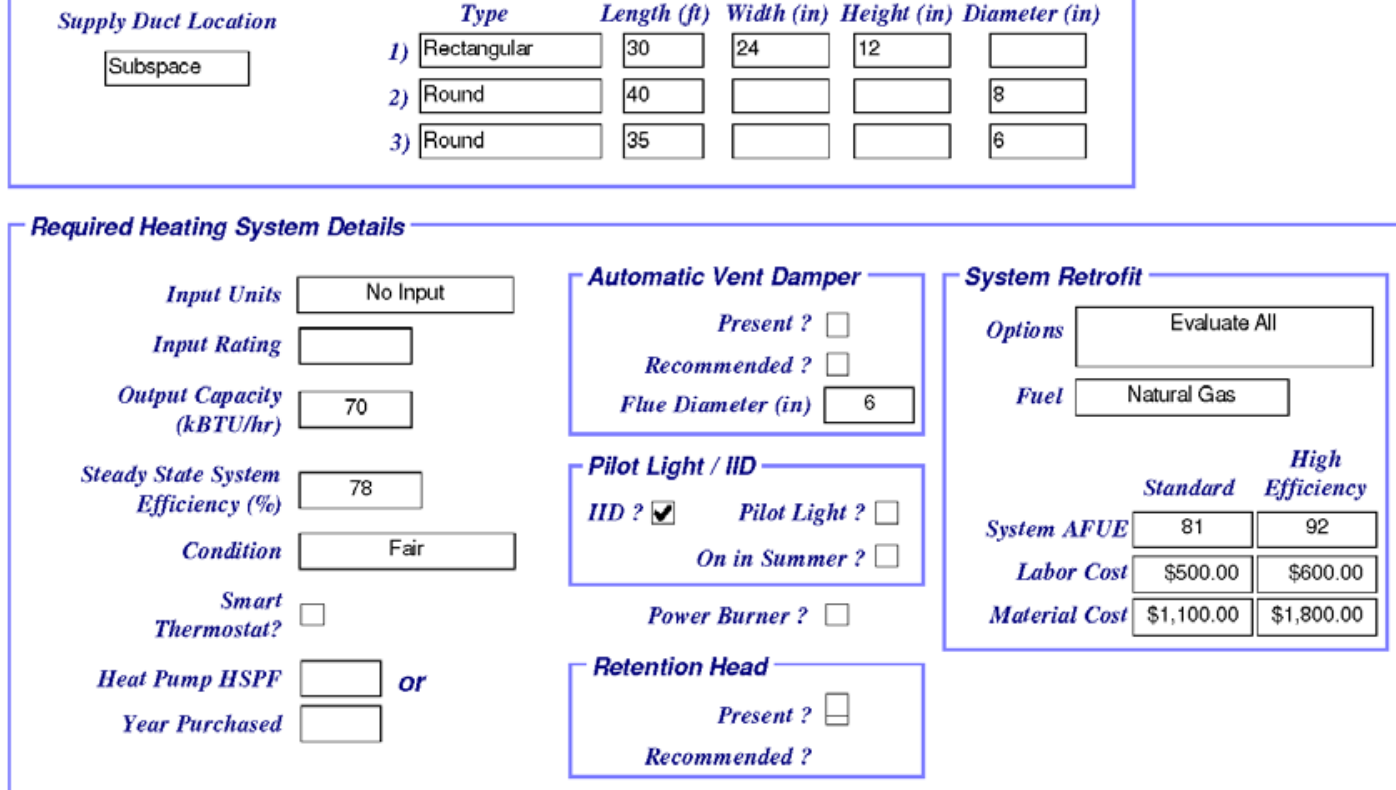

\section{Heating Systems (Continued}

\begin{tabular}{lrr}
\hline Client Name: Tanner, David & NEAT Health and Safety Summary & DOE Weatherization Assistant \\
Client ID: $11 \_348$ & Audit Name: $11 \_348$ SB & Version 8.9 .0 \\
Alt. Client ID: & Report Run On: $8 / 28 / 2011$ & Page 3 of 5
\end{tabular}


Heating Systems (Continued

OPTIONAL HEATING SYSTEM DETAILS

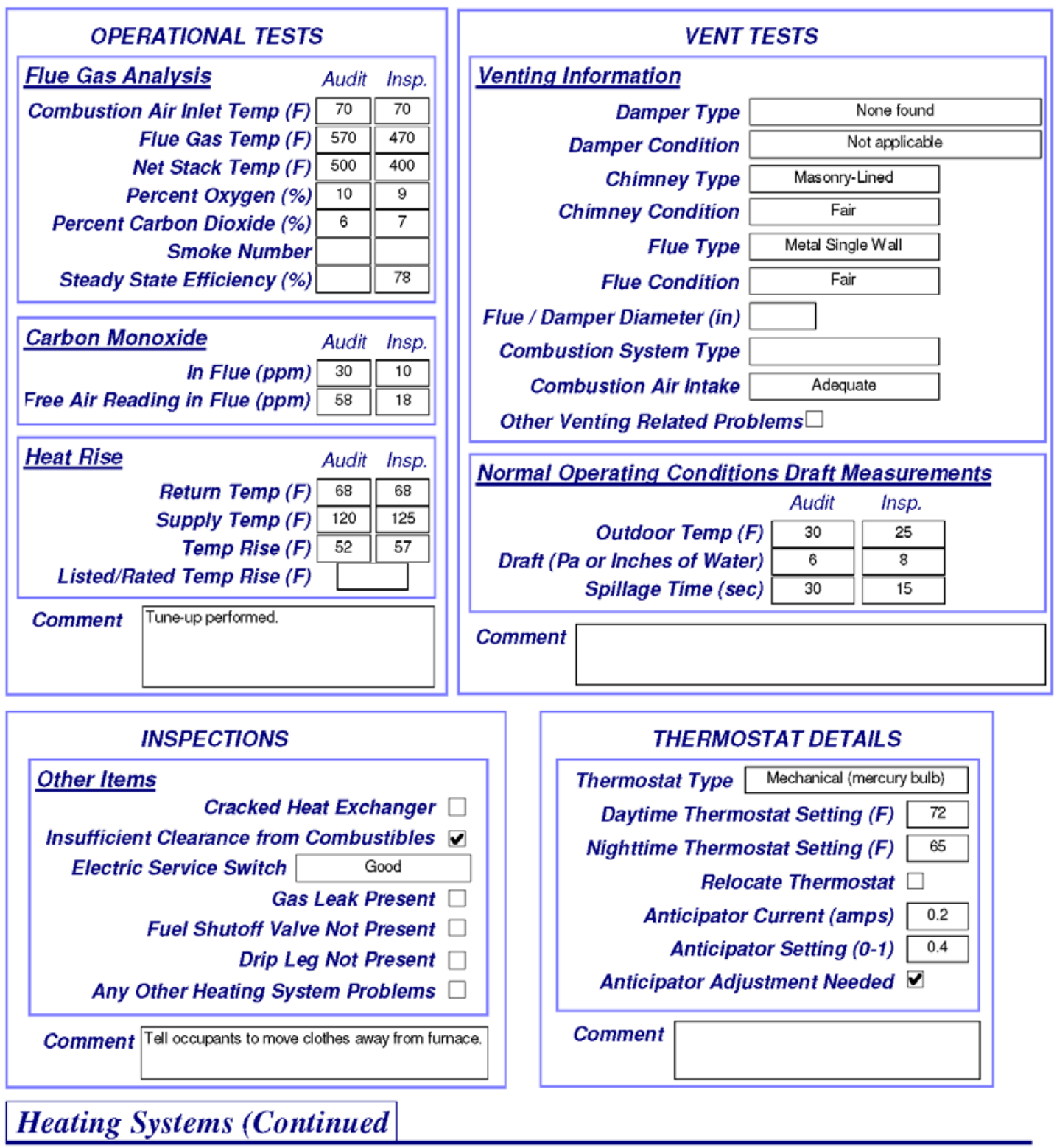

\begin{tabular}{lrr}
\hline Client Name: Tanner, David & NEAT Health and Safety Summary & DOE Weatherization Assistant \\
Client ID: $11 \_348$ & Audit Name: $11 \_348$ SB & Version 8.9 .0 \\
Alt. Client ID: & Report Run On: $8 / 28 / 2011$ & Page 4 of 5
\end{tabular}




\section{Appendix C: Reports}

Heating Systems (Continued

OPTIONAL HEATING SYSTEM DETAILS (Continued)

\begin{tabular}{|c|c|c|c|}
\hline & \multicolumn{3}{|c|}{ FURNACE COMPONENTS } \\
\hline \multicolumn{4}{|l|}{ Limit Controls } \\
\hline Control Settings are Adjustable & $\square$ & Fan On Setting $(F)$ & 95 \\
\hline \multirow{2}{*}{ Limit Control Not Working } & $\square$ & Fan Off Setting $(F)$ & 90 \\
\hline & & High Limit Setting $(F)$ & 1770 \\
\hline
\end{tabular}

Burner and Pilot

\begin{tabular}{llll|} 
Burner Type & Pilot Type & Standing Pilot (on in summer) \\
Burner Condition & Pilot Condition & Fair \\
& Fair & &
\end{tabular}

Blower and Belt

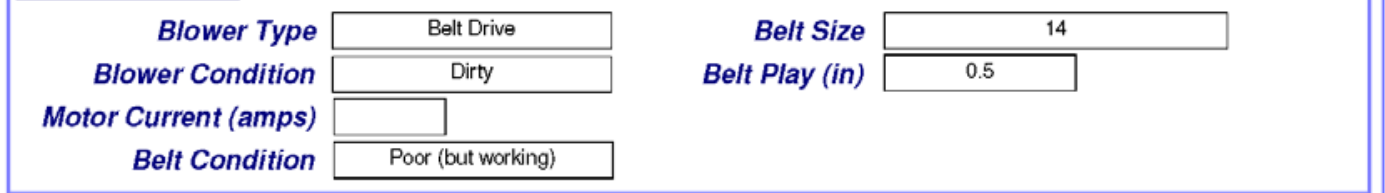

Accessories

Humidifier $\quad$ None

Air Filter

Filter Size (length $x$ width, in) $24 \times 30$

Filter Condition

Dirt

$A C$ Coil $\quad$ Fair

Comment Adjust fan limit control settings.

\begin{tabular}{lrr}
\hline Client Name: Tanner, David & NEAT Health and Safety Summary & DOE Weatherization Assistant \\
Client ID: $11 \_348$ & Audit Name: $11 \_348 S B$ & Version 8.9 .0 \\
Alt. Client ID: & Report Run On: $8 / 28 / 2011$ & Page 5 of 5
\end{tabular}




\section{MHEA Recommended Measures}

\section{Annual Energy and Cost Savings}

\begin{tabular}{|c|c|c|c|c|c|c|c|c|c|}
\hline \multirow[b]{2}{*}{$\#$} & \multirow{2}{*}{$\begin{array}{l}\text { Recommended } \\
\text { Measure }\end{array}$} & \multirow[b]{2}{*}{ Components } & \multicolumn{2}{|c|}{ Heating } & \multicolumn{2}{|c|}{ Cooling } & \multicolumn{2}{|c|}{ BaseLoad } & \multirow{2}{*}{$\begin{array}{r}\text { Total } \\
(M M B t u)\end{array}$} \\
\hline & & & (MMBtu) & $(\$)$ & $(k W h)$ & $(\$)$ & $(k W h)$ & $(\$)$ & \\
\hline 1 & Seal Ducts & & 3.7 & 37 & 135 & 9 & 0 & 0 & 4.1 \\
\hline 2 & General Air Sealing & & 5.6 & 56 & 0 & 0 & 0 & 0 & 5.6 \\
\hline 3 & DWH Pipe Insulation & & 0.0 & 0 & 0 & 0 & 223 & 8 & 0.8 \\
\hline 4 & DWH Tank Insulation & & 0.0 & 0 & 0 & 0 & 531 & 18 & 1.8 \\
\hline 5 & Setback [heating] & & 2.3 & 23 & 0 & 0 & 0 & 0 & 2.3 \\
\hline 6 & Roof Cellulose Loose & & 4.2 & 42 & 368 & 26 & 0 & 0 & 5.4 \\
\hline 7 & Belly Cellulose Loose & & 4.6 & 46 & -5 & 0 & 0 & 0 & 4.6 \\
\hline 8 & Refrigerator Replacement & & 0.0 & 0 & 0 & 0 & 922 & 65 & 3.1 \\
\hline 9 & Glass Storm Windows & WD4 & 0.3 & 3 & -1 & 0 & 0 & 0 & 0.3 \\
\hline
\end{tabular}

\section{Energy Saving Measure Economics}

\begin{tabular}{|c|c|c|c|c|c|c|c|c|}
\hline$\#$ & $\begin{array}{l}\text { Recommended } \\
\text { Measure }\end{array}$ & Components & $\begin{array}{r}\text { Measure } \\
\text { Savings } \\
(\$ / y r)\end{array}$ & $\begin{array}{r}\text { Measure } \\
\text { Cost } \\
(\$)\end{array}$ & $\begin{array}{r}\text { Measure } \\
\text { SIR }\end{array}$ & $\begin{array}{r}\text { Cost } \\
(\$)\end{array}$ & $\begin{array}{c}\text { Cumul } \\
\text { Savings } \\
(\$ / y r)\end{array}$ & $\begin{array}{l}\text { ative } \\
\text { SIR }\end{array}$ \\
\hline 1 & Seal Ducts & & 46 & 320 & 1.2 & 320 & 46 & 12 \\
\hline 2 & General Air Sealing & & 56 & 250 & 1.9 & 570 & 103 & 1.5 \\
\hline 3 & DWH Pipe Insulation & & 8 & 15 & 5.5 & 585 & 110 & 1.6 \\
\hline 4 & DWH Tank Insulation & & 18 & 40 & 4.9 & 625 & 128 & 1.8 \\
\hline 5 & Setback [heating] & & 23 & 75 & 3.7 & 700 & 151 & 2.0 \\
\hline 6 & Roof Cellulose Loose & & 67 & 680 & 1.5 & 1380 & 219 & 1.8 \\
\hline 7 & Belly Cellulose Loose & & 45 & 573 & 1.2 & 1953 & 264 & 1.6 \\
\hline 8 & Refrigerator Replacement & & 65 & 620 & 1.2 & 2573 & 329 & 1.5 \\
\hline 9 & Glass Storm Windows & WD4 & 3 & 32 & 1.1 & 2605 & 331 & 1.5 \\
\hline 10 & Fix Wiring Problems (Attic) & & 0 & 120 & 0.0 & 2725 & 331 & 0.0 \\
\hline
\end{tabular}

\section{Materials}

\begin{tabular}{lccc}
\hline Index Material & Quantity & Units \\
\hline Audit Name: 11_353MH & Client: $11 \_353$ & Date: $9 / 26 / 2011$ & Page 1 of 2
\end{tabular}




\section{Appendix C: Reports}

\begin{tabular}{clcl}
\hline Index & Material & Quantity & Units \\
\hline 1 & Duct sealing (setup cost) & 1 & Each \\
2 & General air sealing (setup cost) & 1 & Each \\
3 & DWH Pipe Insulation & 1 & Each \\
4 & DWH Tank Insulation & 1 & Each \\
5 & Setback thermostat & 1 & Each \\
6 & Roof Insulation & 40 & Bag \\
7 & Floor Insulation & 39 & Bag \\
8 & Refrigerator & 1 & Ea \\
9 & Glass storm windows & 1 & Each
\end{tabular}

\section{Pre/Post Retrofit Energy Consumption}

\begin{tabular}{cccccc}
\hline & Pre Retrofit & & \multicolumn{3}{c}{ Post Retrofit } \\
Heating (MMBtu) & Cooling(kWh) & BaseLoad(kWh) & Heating(MMBtu) & Cooling(kWh) & BaseLoad(kWh) \\
\hline 74.5 & 2180.9 & 2817.9 & 53.8 & 1684.3 & 1141.7
\end{tabular}

\section{Approximate Component Contributions}

to Peak Heating Load

\begin{tabular}{lrr}
\hline Component Type & $\begin{array}{r}\text { Pre Retrofit } \\
\text { Load }(\text { Btu/h })\end{array}$ & $\begin{array}{r}\text { Post Retrofit } \\
\text { Load }(\boldsymbol{B T U} / \boldsymbol{h})\end{array}$ \\
\hline Wall & 5764.1 & 5764.1 \\
Floor & 6089.9 & 3209.5 \\
Roof & 5355.5 & 1714.3 \\
Windows & 7394.4 & 5545.4 \\
Doors & 586.4 & 586.4 \\
Infiltration & 7984.5 & 2875.5 \\
Duct Loss & 3317.5 & 1969.5 \\
Total & 36492.2 & 21664.8
\end{tabular}

\section{Special Notes}

Manuall sizing based on 70F indoor and $3 \mathrm{~F}$ outdoor temp

10 Base case duct loss fraction

10 Retrofit case duct loss fraction

Sizing estimate are general guidelines only

Sizing estimate should be review by qualified heating contractor

$(+)$ in the Materials list indicates there are more related User Defined Materials

Cumulative Expenditure Exceeds Limit of 2500 Dollars

\section{Comments}

$\begin{array}{lll}\text { Type } & \text { Code } & \text { Comment } \\ \text { Itemized } & \text { Fix Wiring } & \text { In kitchen dropdown ceiling at lights. } \\ & \text { Problems } & \end{array}$




\section{MHEA Input Report}

\section{Client Information}

\begin{tabular}{|l|l|} 
Client ID 11 353 \\
Alt. Client ID \\
Client Name \\
Address 210 North Illinois Ave \\
Unit No. \\
City Oak Ridge \\
County & State TN Zip 37830 \\
\hline & Other Geo. Ident. \\
\hline
\end{tabular}

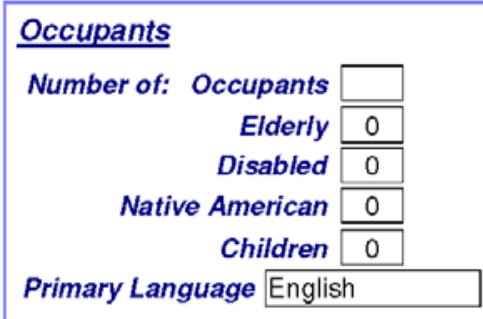

\begin{tabular}{|c|c|c|}
\hline \multicolumn{3}{|l|}{ Dwelling } \\
\hline Dwelling Type & Mobile Home & Ownership \\
\hline Primary Heat. Fuel & & $\square$ High Energy Use \\
\hline Secondary Heat. Fuei & & $\square$ High Energy Burden \\
\hline \multicolumn{2}{|c|}{ Previously Weatherized } & Year Built [ \\
\hline Year & & \\
\hline
\end{tabular}

\section{Comment}

\section{Energy Index}

Floor Area (sq ft)

Heating Degree Days

(base 65 F)

Total Heating

(BTU/HDD/sq ft)

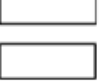

Primary Heating Fuel

Secondary Heating Fuel

Estim. \%

Annual Cost for heating

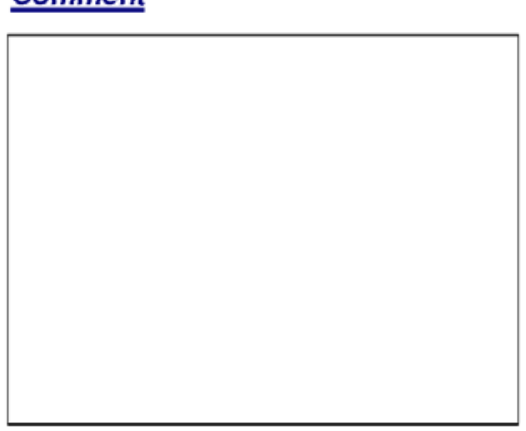

el

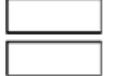

\section{Contact Information}

\begin{tabular}{lllll}
\hline Contact Name & Home Ph & Work Ph & Cell Ph & Contact Type Applicant Comment \\
\hline Anderson, Grace & & $\begin{array}{c}\text { Applicant/Person of } \\
\text { Record }\end{array}$ \\
\multicolumn{1}{|l|}{ Audit Information } & & \\
\hline
\end{tabular}

\footnotetext{
Client Name: Anderson, Grace

Client ID: 11_353

Alt. Client ID:
} 


\section{Appendix C: Reports}

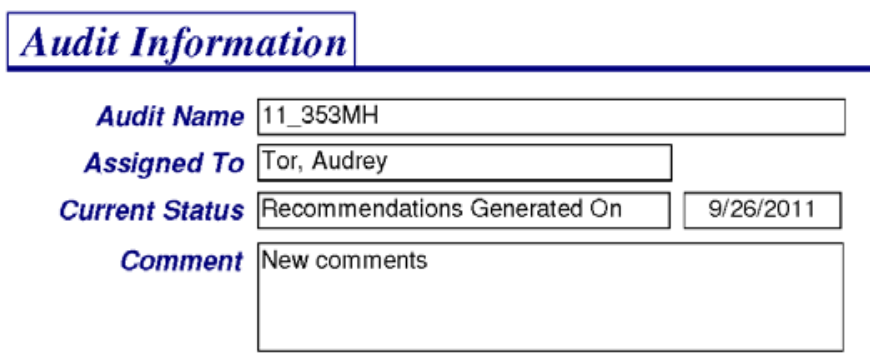

$\begin{aligned} \text { Length } & 60 \\ \text { Width } & 15 \\ \text { Height } & 7\end{aligned}$

Wind Shielding Normal Shielding

Home Leakiness Medium

Billing Adjust

$\square$ Water Heater Closet

\begin{tabular}{|c|c|c|}
\hline Libraries & \multirow{5}{*}{$\begin{array}{r}\text { Setup Library } \\
\text { Description } \\
\text { Supply Library } \\
\text { Description }\end{array}$} & \\
\hline Setup Library Demonstration Setup Library & & \multirow[t]{2}{*}{ Original Demonstration Library } \\
\hline Fuel Costs Agency Fuel Prices & & \\
\hline Supply Library Demonstration Supply Library & & \multirow[t]{2}{*}{ Supply library for demonstration } \\
\hline Weather File SAMPLEUS.WX & & \\
\hline
\end{tabular}

Photo Foldet

\section{Audit Status History}

\begin{tabular}{llcc}
\hline Type & Status & Date & Changed By Comment \\
\hline & Recommendations Generated On & $11 / 4 / 2014$ & MBG \\
& Recommendations Generated On & $9 / 26 / 2011$ & MBG
\end{tabular}

\section{Walls}

Wall Stud Size $2 \times 4$

Orientation of Long Wall South

Type of Wall Ventilation Not Vented

-Insulation Type Thickness

Batt/Blanket (in) 3.5

Loose Fill (in) 0

Foam Core (in)

UninsulatableArea (sqft)

Added Cost 0
- Carport/Porch Roof

Length $(f t)$

Width $(f t) 12$

Orientation South

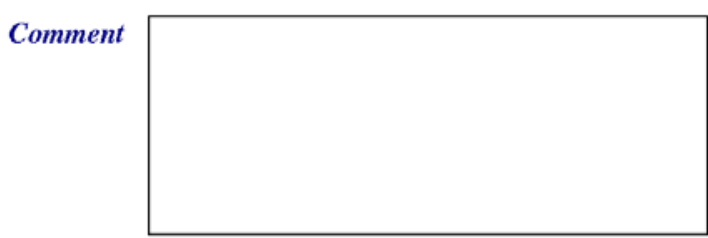

\section{Windows}

Client Name: Anderson, Grace
Client ID: 11 353

Att. Client ID:
MHEA Input Report

Audit Name: 11_353MH

Report Run On: 9/26/2011
DOE Weatherization Assistant

Version 8.9.0

Page 2 of 17 


\section{Windows}

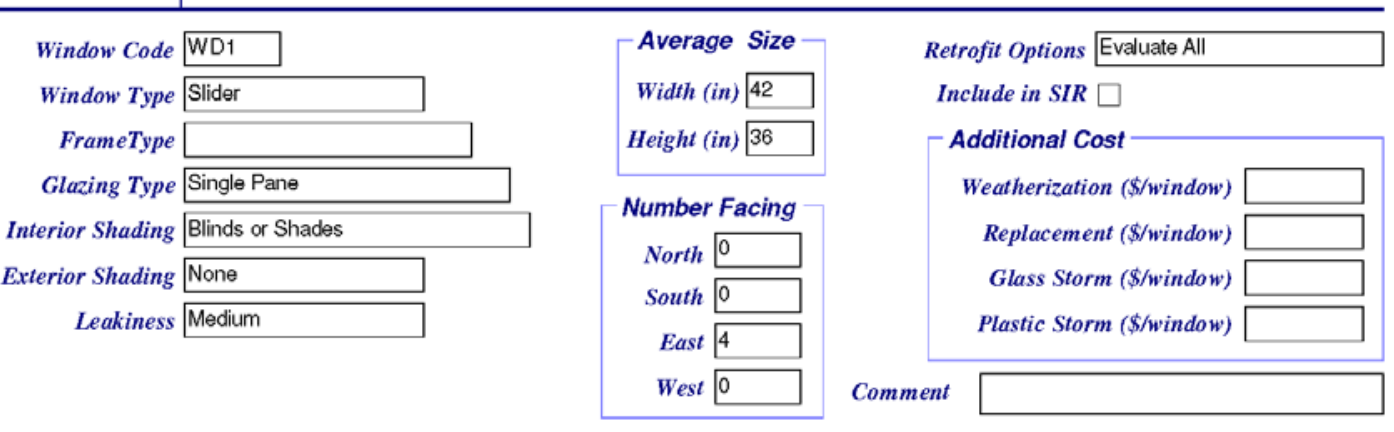

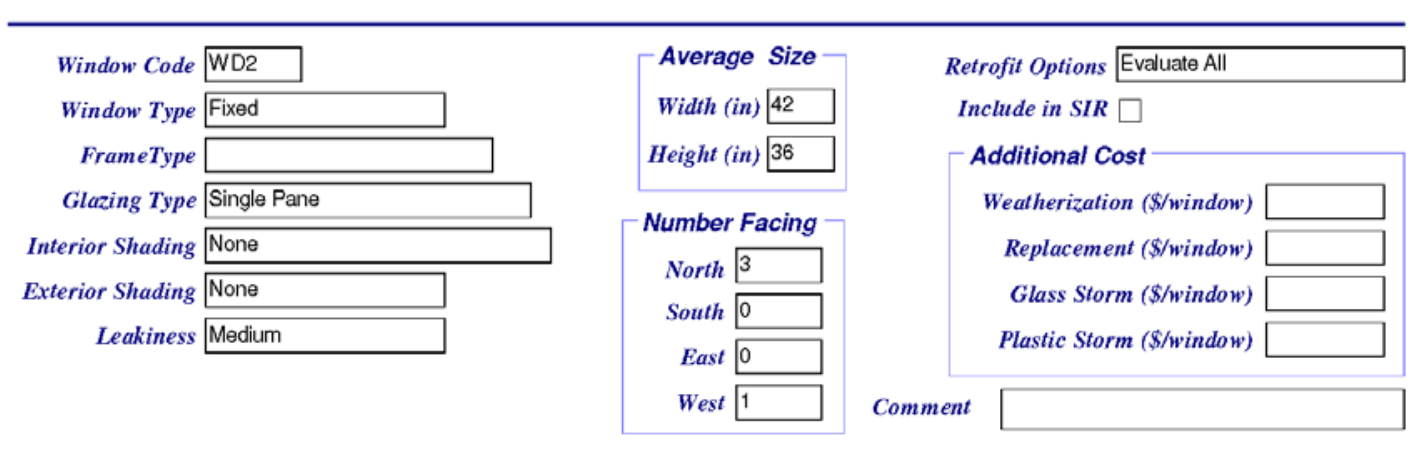

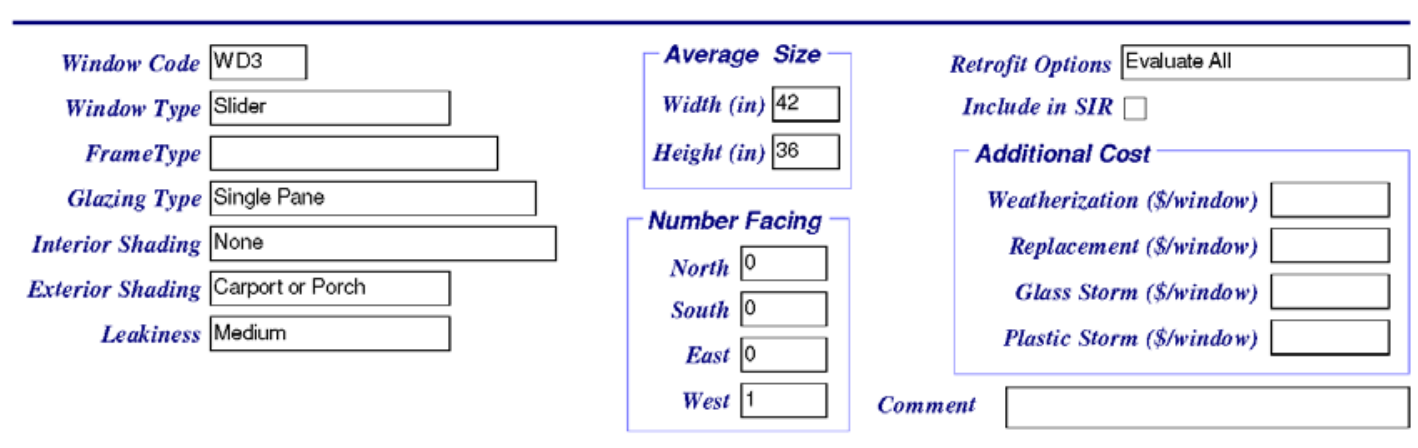

\begin{tabular}{lrr}
\hline Client Name: Anderson, Grace & MHEA Input Report & DOE Weatherization Assistant \\
Client ID: $11 \_353$ & Audit Name: $11 \_353 \mathrm{MH}$ & Version 8.9 .0 \\
Att. Client ID: & Report Run On: $9 / 26 / 2011$ & Page 3 of 17
\end{tabular}




\section{Appendix C: Reports}

\begin{tabular}{|c|c|c|c|}
\hline Windows & & & \\
\hline Window Code & WD4 & \multirow{3}{*}{$\begin{array}{l}\text { Average Size }- \\
\text { Width (in) } 24 \\
\text { Height (in) } 24\end{array}$} & \multirow{2}{*}{$\begin{array}{l}\text { Retrofit Options } \text { Evaluate All } \\
\text { Include in SIR }\end{array}$} \\
\hline Window Type & Door Window & & \\
\hline FrameType & & & - Additional Cost \\
\hline Glazing Type & Single Pane & \multirow{2}{*}{$\begin{array}{c}\text { Number Facing } \\
\text { North } 0\end{array}$} & Weatherization $(\$ /$ window $)$ \\
\hline Interior Shading & None & & Replacement $(\$ /$ window $)$ \\
\hline Exterior Shading & None & North & Glass Storm (\$/window) \\
\hline \multirow[t]{2}{*}{ Leakiness } & Medium & East & Plastic Storm $(\$ /$ window) \\
\hline & & West 0 & Comment \\
\hline
\end{tabular}

\section{Doors}

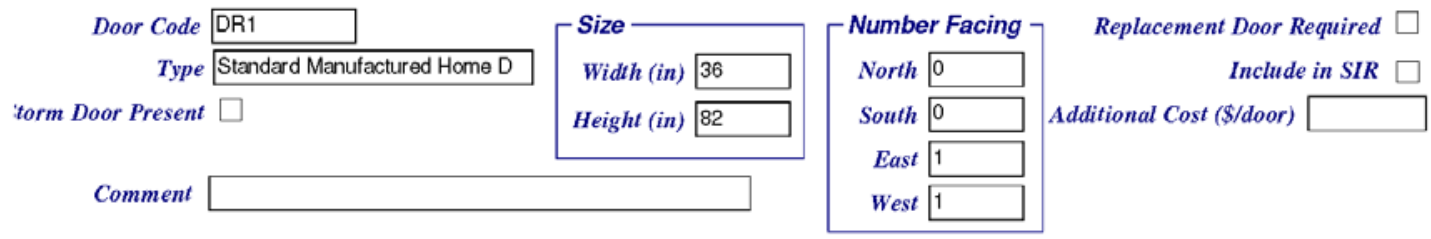

\section{Ceiling}

$\begin{array}{ll}\text { Roof Type } & \text { Pitched } \\ \text { Roof Color } & \text { Normal or Weathered }\end{array}$

Bowstring Height of Roof (in) 8

Pitched Roof Added Insul. (in) 12

Flat Roof Joist Size

$\lceil$ Insulation Type Thickness

Batt/Blanket (in)

Loose Fill (in)

Foam Core (in) 0.5

\section{Addded Cost 0}

Cathedral Ceiling (\%) 20

Step Wall Orientation East

Comment

Floor

\begin{tabular}{lrr}
\hline Client Name: Anderson, Grace & MHEA Input Report & DOE Weatherization Assistant \\
Client ID: $11 \_353$ & Audit Name: $11 \_353 \mathrm{MH}$ & Version 8.9 .0 \\
Att. Client ID: & Report Run On: $9 / 26 / 2011$ & Page 4 of 17
\end{tabular}




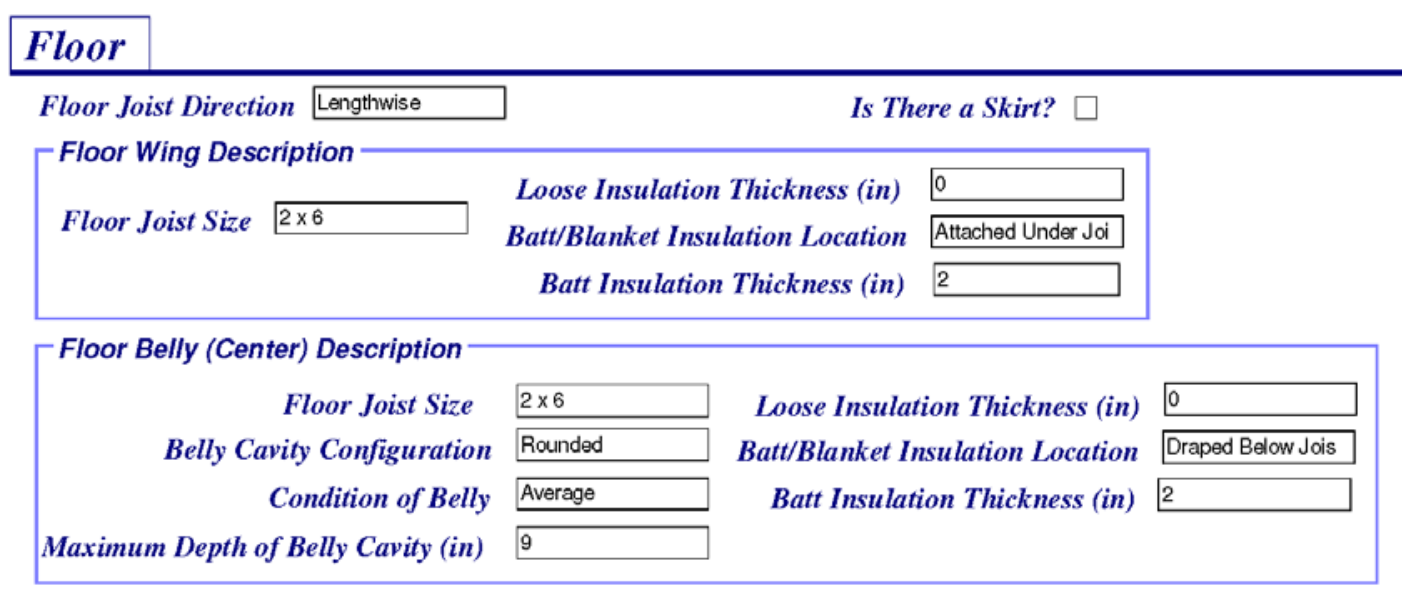

Comment

\section{Walls (Addition)}

$\begin{gathered}\text { Wall Stud Size } 2 \times 4 \\ \text { Addition Orientation } \\ \text { Wall Ventilation } \\ \text { Not Vented }\end{gathered}$
$\begin{gathered}\text { Insulation Type Thickness } \\ \text { Batt/Blanket (in) }\end{gathered}$
Loose Fill (in)
Foam Core (in)

\section{Windows (Addition)}

Client Name: Anderson, Grace
Client ID: 11353

Att. Client ID:
MHEA Input Report

Audit Name: 11 353MH

Report Run On: 9/26/2011
DOE Weatherization Assistant

Version 8.9.0

Page 5 of 17 


\section{Appendix C: Reports}

\begin{tabular}{|c|c|c|}
\hline Windows (Addition) & & \\
\hline Window Code AWD1 & \multirow{3}{*}{$\begin{array}{l}\text { Average Size }- \\
\text { Width (in) } 60 \\
\text { Height (in) } 24\end{array}$} & \multirow{2}{*}{$\begin{array}{l}\text { Retrofit Options Evaluate None } \\
\text { Include in SIR }\end{array}$} \\
\hline Window Type Slider & & \\
\hline FrameType Wood or Vinyl & & - Additional Cost \\
\hline Glazing Type Double Pane & \multirow{2}{*}{$\begin{array}{c}\text { Number Facing } \\
\text { North } 1\end{array}$} & Weatherization $(\$ /$ window $)$ \\
\hline Interior Shading Blinds or Shades & & Replacement (\$/window) \\
\hline Exterior Shading None & North & Glass Storm (\$/window) \\
\hline \multirow[t]{2}{*}{ Leakiness Tight } & $\begin{aligned} \text { South } & 0 \\
\text { East } 1 & \end{aligned}$ & Plastic Storm $(\$ /$ window $)$ \\
\hline & & Comment \\
\hline
\end{tabular}

\section{Doors (Addition}

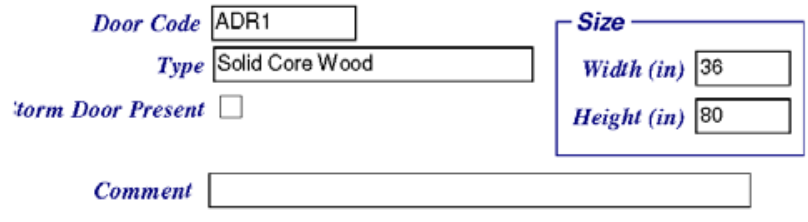

Comment

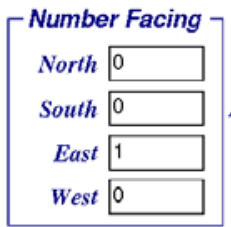

Replacement Door Required

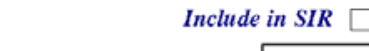
Additional Cost (\$/door)

\section{Ceiling (Addition)}

Joist Size $2 \times 6$

Roof Color Normal or Weathered

Addded Cost 0

Insulation Type Thickness
Batt/Blanket (in) 0
Loose Fill (in)
Foam Core (in)

Comment

\section{Floor (Addition)}

Client Name: Anderson, Grace
Client ID: $11 \_353$

Att. Client ID:
MHEA Input Report

Audit Name: 11_353MH

Report Run On: 9/26/2011
DOE Weatherization Assistant

Version 8.9.0

Page 6 of 17 


\section{Floor (Addition)}

Floor Type Crawl Space

Joist Size $2 \times 6$

Floor Dimensions

Length (ft) 15

Width (ft) 10
Batt/Blanket Location Between Joists

-Insulation Type Thickness

Batt/Blanket (in)

Loose Fill (in) 0

Depth Available for Added Insulation (in)

Comment

\section{Heating - Primary}

Equipment Type Furnace

Fuel Type Natura Gas

Capacity (kBtu/hr)

Efficiency (\%)

Efficiency Units

Duct Location

Duct Insulation Location

Percent Total Heat Supplied (\%)

Programmable Thermosta

\begin{tabular}{|l|}
\hline 80 \\
\hline 75 \\
\hline Steady State \\
\hline Floor \\
\hline Below Duct \\
\hline
\end{tabular}

Tune-up Mandator Average Indoor Temperature ( $F$

\begin{tabular}{|rc|}
\hline Day & 68 \\
Night & 68 \\
\hline
\end{tabular}

Comment

\section{Heating - Primary (Continued)}

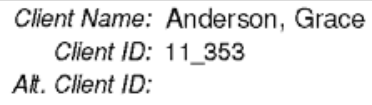

Att. Client ID:

Report Run On: 9/26/2011
DOE Weatherization Assistant Version 8.9.0

Page 7 of 17 


\section{Appendix C: Reports}

\section{Heating - Primary (Continued)}

OPTIONAL HEATING SYSTEM DETAILS

\begin{tabular}{|r|r|r|}
\hline \multicolumn{3}{|c|}{ OPERATIONAL TESTS } \\
\hline Flue Gas Analysis & Audit Insp. \\
\hline Combustion Air Inlet Temp $(F)$ & & \\
Flue Gas Temp $(F)$ & & \\
Net Stack Temp (F) & & \\
Percent Oxygen (\%) & & \\
\hline Percent Carbon Dioxide (\%) & & \\
Smoke Number & & \\
\hline Steady State Efficiency $(\%)$ & & \\
\hline
\end{tabular}

\begin{tabular}{|l|l|}
\hline Carbon Monoxide \\
In Flue (ppm) \\
Free Air Reading in Flue (ppm, Insp.
\end{tabular}

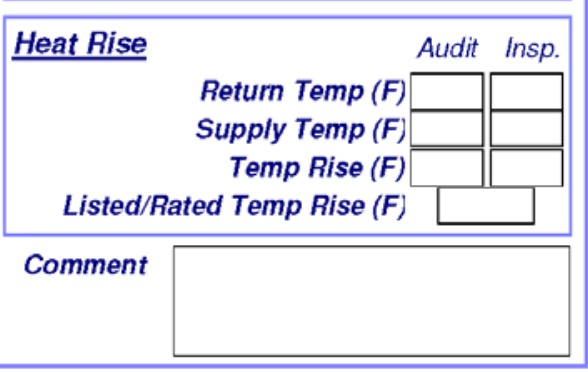

\begin{tabular}{|c|}
\hline \multicolumn{1}{|c|}{ VENT TESTS } \\
\hline Venting Information \\
Damper Type \\
Damper Condition \\
Chimney Type \\
Chimney Condition \\
Flue Type \\
Flue Condition \\
\\
Flue / Damper Diameter (in) \\
Combustion System Type \\
Combustion Air Intake \\
\\
Other Venting Related Problems $\square$ \\
\hline
\end{tabular}

\section{Normal Operating Conditions Draft Measurements}

$\frac{\text { Normal Operating Conditions Draft Measureme }}{\text { Audit Insp. }}$

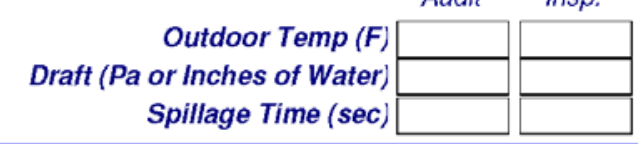

Comment

\section{INSPECTIONS}

\section{Other Items \\ Cracked Heat Exchanget}

Insufficient Clearance from Combustibles

Electric Service Switch

Gas Leak Present

Fuel Shutoff Valve Not Presen

Drip Leg Not Present

Any Other Heating System Problems

Comment

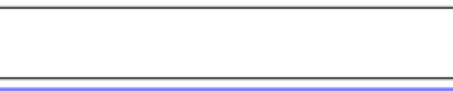

Comt

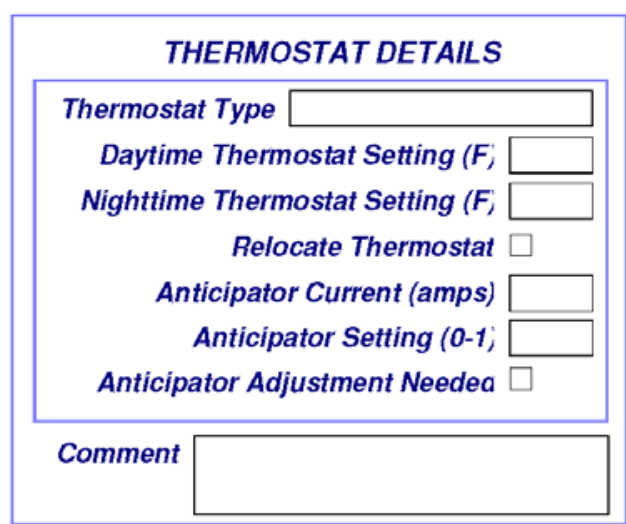

\section{Heating Primary (Continued)}

\begin{tabular}{lrr}
\hline Client Name: Anderson, Grace & MHEA Input Report & DOE Weatherization Assistant \\
Client ID: $11 \_353$ & Audit Name: 11 353MH & Version 8.9 .0 \\
Alt. Client ID: & Report Run On: $9 / 26 / 2011$ & Page 8 of 17
\end{tabular}


Heating Primary (Continued)

OPTIONAL HEATING SYSTEM DETAILS (Continued)

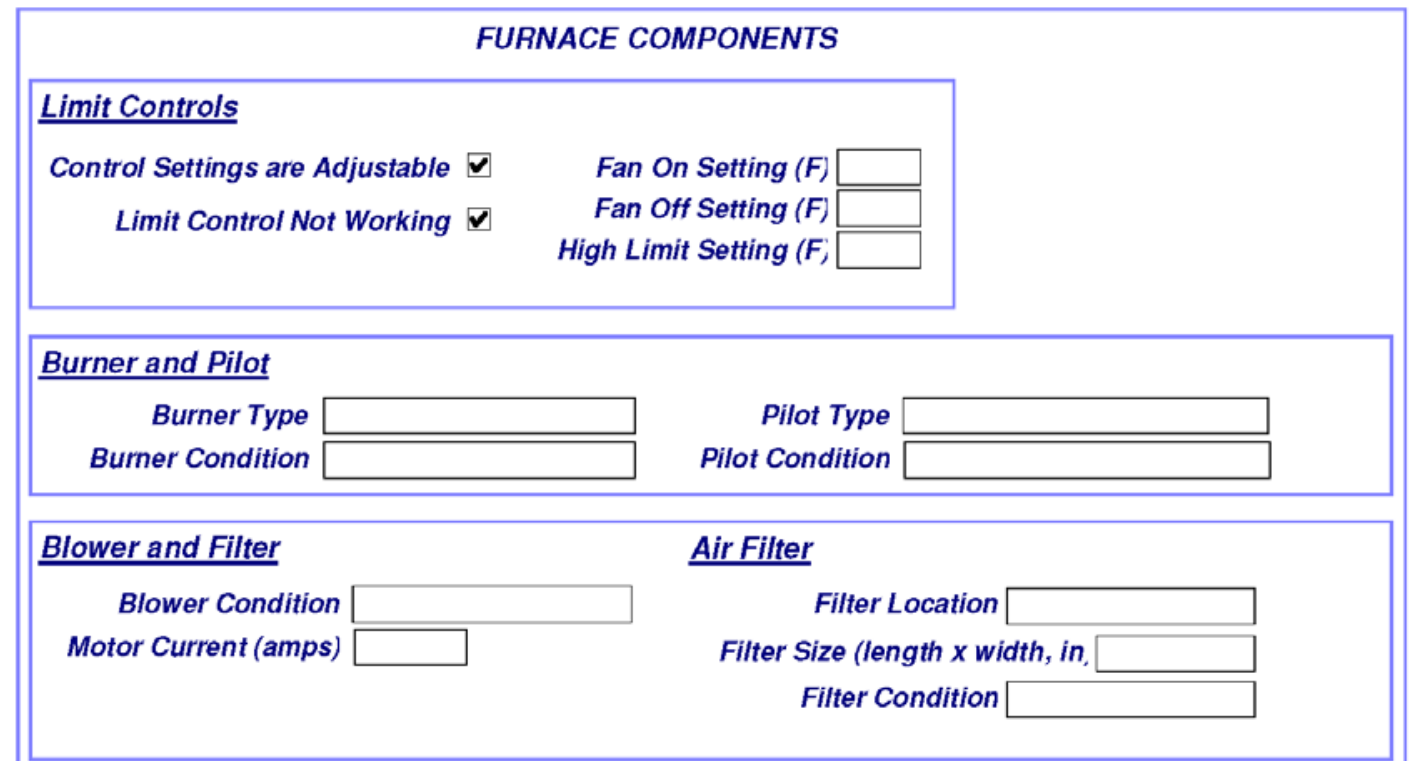

\section{Accessories}

Humidifier

Electronic Air Cleanet

AC Coil

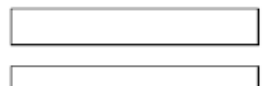

Comment

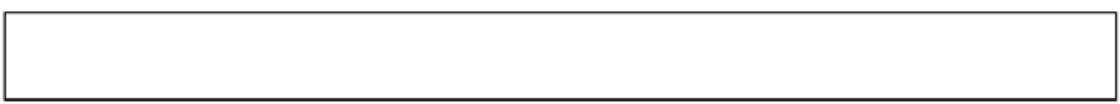

\section{Heating - Secondary}

No data were entered for this audit.

\section{Heating - Replacement}

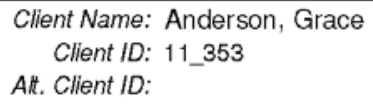

Att. Client ID: 


\section{Appendix C: Reports}

\begin{tabular}{|c|c|c|c|c|}
\hline Heating - Replacement & & & & \\
\hline Equipment Type & Furnace & & & \\
\hline Fuel Type & Natural Gas & & Cost & \\
\hline Capacity $(k B t u / h r)$ & 80 & & & \\
\hline Efficiency $(\%)$ & 92 & & Labor & 1000 \\
\hline Efficiency Units & AFUE & & & \\
\hline Duct Location & Floor & & & \\
\hline Duct Insulation Location & Below Duct & & & \\
\hline Replacement Required $\square$ & $\begin{array}{l}\text { Include Replacement } \\
\text { Costs in Home Retrofit }\end{array}$ & $\square$ & & \\
\hline
\end{tabular}

\section{Cooling - Primary}

$\begin{aligned} \text { AC Unit Type } & \text { Central Air Conditioner } \\ \text { Capacity (kBtu/hr) } & 24 \\ \text { Efficiency } & 9 \\ \text { Efficiency Units } & \text { SEER } \\ \text { Duct Location } & \text { Floor }\end{aligned}$

Duct Insulation Location Below Duct

Floor Area Cooled (\% 80

Comment

\section{Cooling - Secondary}

AC Unit Type Window or Room Air Conditioner

Capacity $(\mathrm{kBtu} / \mathrm{hr})$

Efficiency 9.7

Efficiency Units EER

Floor Area Cooled (\%) 20

Comment

\section{Cooling - Replacement}

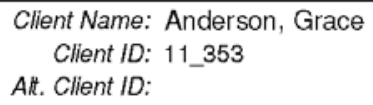

At. Client ID:

MHEA Input Report

Audit Name: 11_353MH

Report Run On: 9/26/2011
DOE Weatherization Assistant

Version 8.9.0

Page 10 of 17 


\section{Cooling - Replacement}

AC Unit Type Central Air Conditioner

Capacity (kBtu/hr) 24

Efficiency 16

Efficiency Units SEER

Duct Locatio Floor

Duct InsulationLocation Below Duct

Replacement Required

Comment

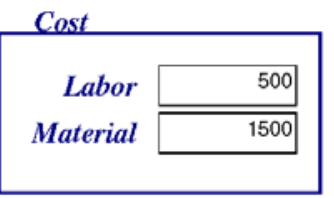

\section{Ducts / Infiltration - Air and Duct Leakages}

Evaluate Duct Sealing ? $\quad$ Duct Leakage Methoo Pressure Pan Measurements

\begin{tabular}{|c|c|c|c|c|}
\hline & RESSURE PAN & I MEASUREMENTS & & \\
\hline Before & Duct Sealing & After Duct Sealing & & \\
\hline Sum of Pressure Pan & 18 & 2 & & \\
\hline & $\begin{array}{c}\text { Before } \\
\text { Weatherizatio } \\
n \text { (Existing) }\end{array}$ & $\begin{array}{c}\text { After } \\
\text { Weatherization } \\
\text { (Target or Actual) }\end{array}$ & & \\
\hline Air Leakage Rate (cfm) & 3000 & 1500 & UCT OPERATIN & VG PRESSURES \\
\hline at House Pressure Difference $(\mathrm{Pa})$ & 50 & 50 & $\begin{array}{c}\text { Before } \\
\text { Duct Sealing }\end{array}$ & $\begin{array}{c}\text { After } \\
\text { Duct Sealing }\end{array}$ \\
\hline Infiltration Reduction Cost (\$) & $\$ 250.00$ & Supply (Pa) & 35 & 40 \\
\hline Duct Sealing Cost (\$) & $\$ 320.00$ & Return (Pa) & & \\
\hline Comment & & & & \\
\hline
\end{tabular}

\section{Ducts / Infiltration Blower Door}

No data were entered for this audit.

\section{Ducts / Infiltration Zonal Press}

\begin{tabular}{lrr}
\hline Client Name: Anderson, Grace & MHEA Input Report & DOE Weatherization Assistant \\
Client ID: $11 \_353$ & Audit Name: $11 \_353 \mathrm{MH}$ & Version 8.9 .0 \\
Att. Client ID: & Report Run On: $9 / 26 / 2011$ & Page 11 of 17
\end{tabular}




\section{Appendix C: Reports}

No data were entered for this audit.

\section{Ducts / Infiltration Pressure Balance Readings (Optional)}

No data were entered for this audit.

\section{Ducts / Infiltration Pressure Pan Readings (Optional)}

No data were entered for this audit.

\section{Base Load - Water Heater}

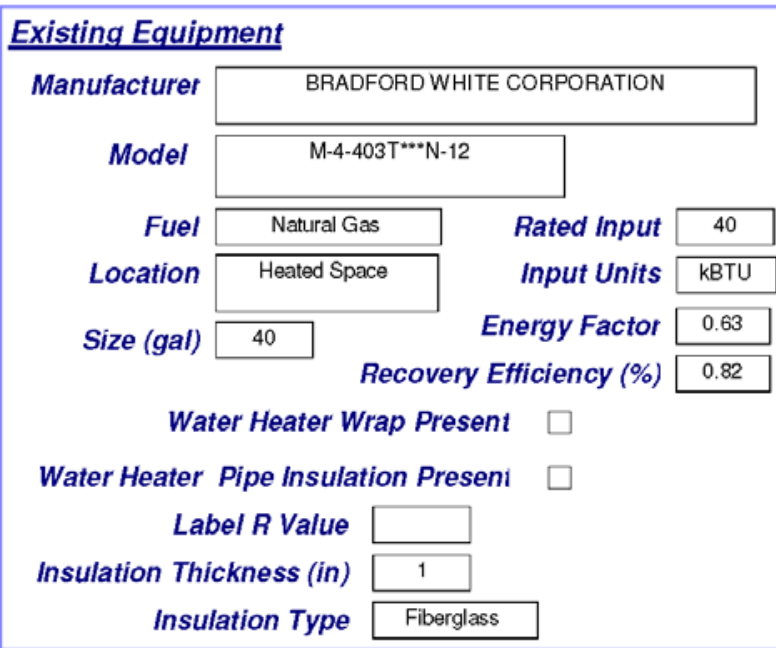

\section{Shower Heads}

Number of Showerheads

Avg. GPM

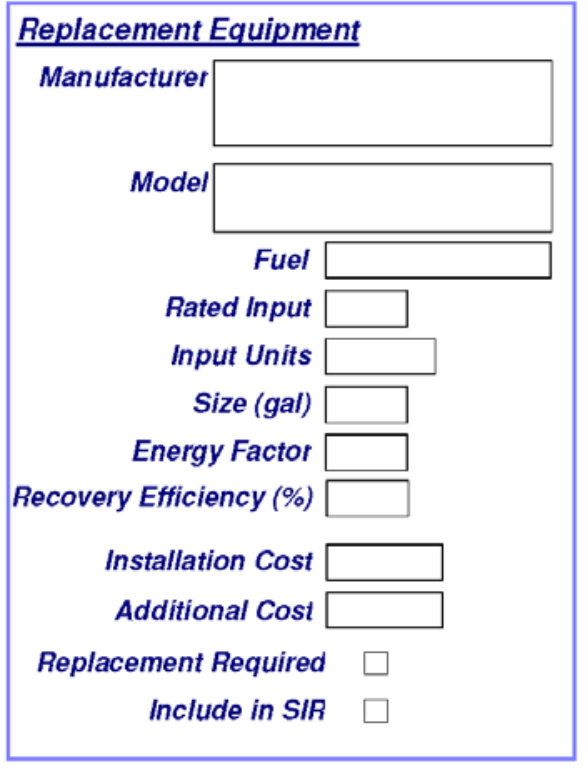

Minutes of Shower Use Per Day

Comment

Base Load - Water Heater (Continued)

Client Name: Anderson, Grace

Client ID: $11 \_353$

Att. Client ID:
MHEA Input Report

Audit Name: 11 353MH

Report Run On: 9/26/2011
DOE Weatherization Assistant

Version 8.9 .0

Page 12 of 17 


\section{Base Load - Water Heater (Continued)}

\section{OPTIONAL WATER HEATING SYSTEM DETAILS}

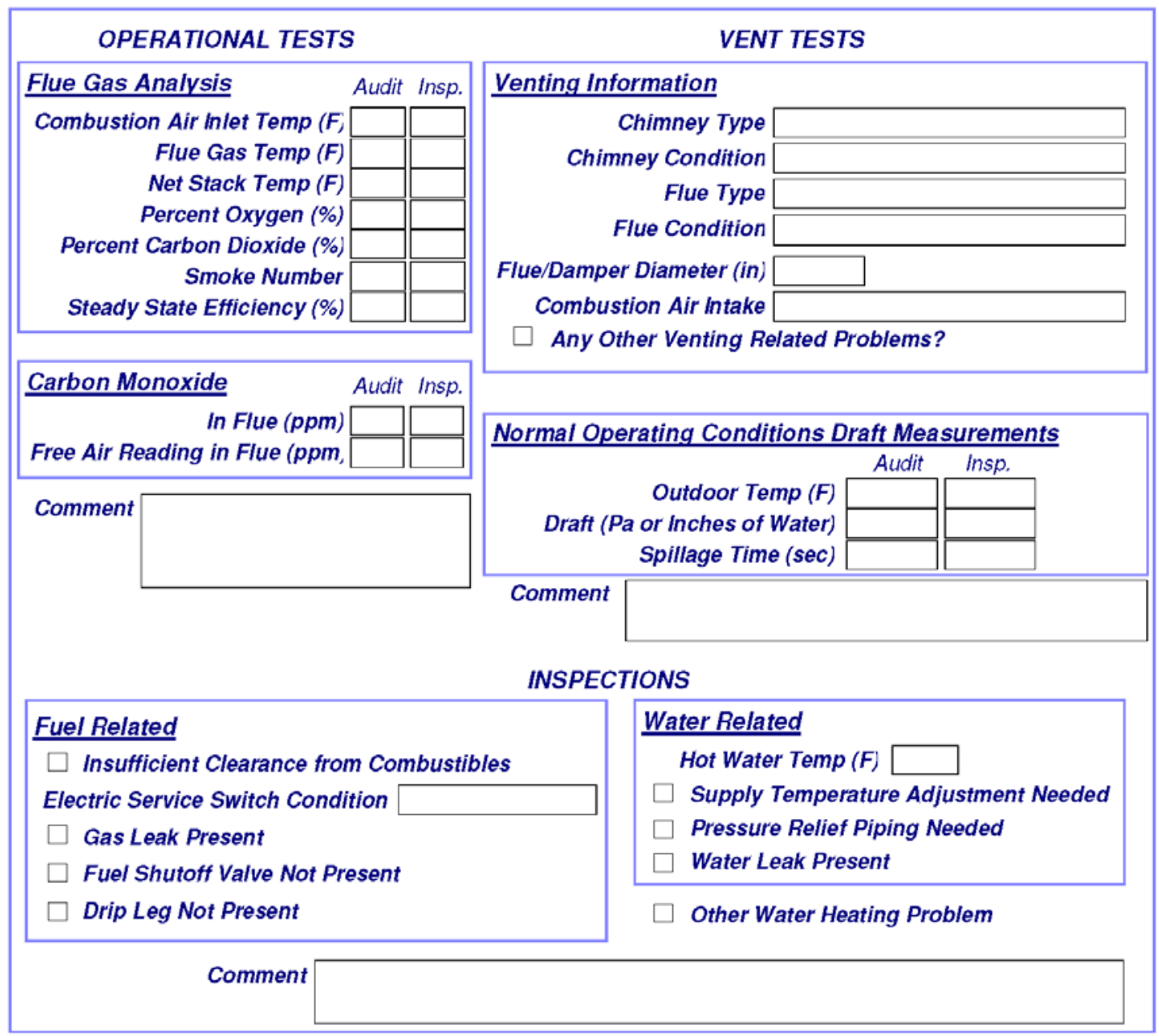

\section{Base Load - Refrigerator}

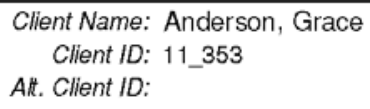

Att. Client ID:

Report Run On: 9/26/2011
DOE Weatherization Assistant

Version 8.9 .0

Page 13 of 17 


\section{Appendix C: Reports}

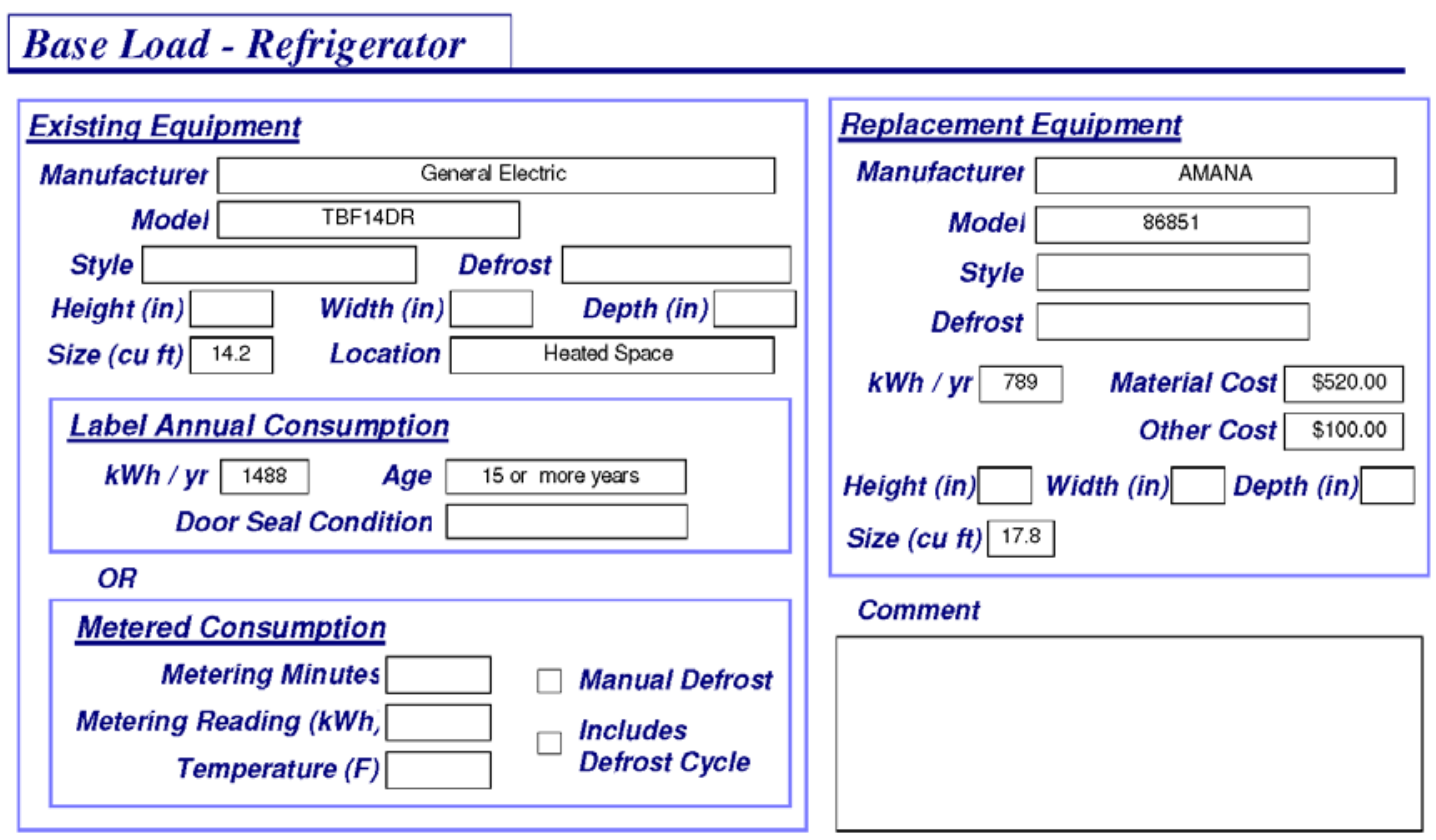

Base Load - Lighting Systems

No data were entered for this audit.

\section{Health \& Safety}

Client Name: Anderson, Grace
Client ID: $11 \_353$

Att. Client ID:
MHEA Input Report

Audit Name: 11_353MH

Report Run On: 9/26/2011
DOE Weatherization Assistant

Version 8.9 .0

Page 14 of 17 


\section{Health \& Safety}

\section{WHOLE HOUSE}

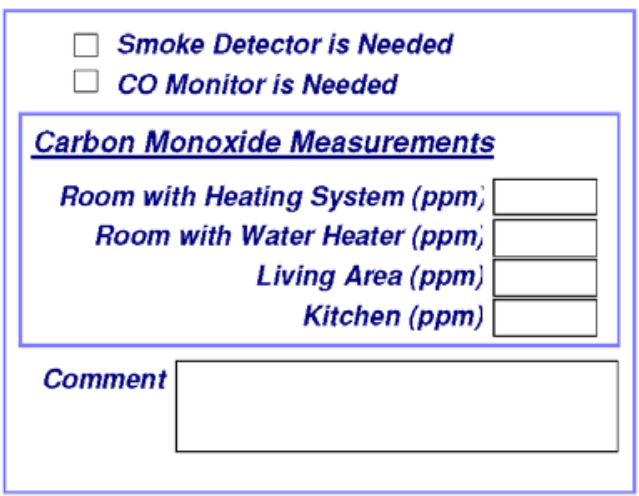

\section{BUILDING SHELL}

\begin{tabular}{|c|c|c|}
\hline $\begin{array}{l}\text { Attic } \\
\square \text { Recessed Lights Present } \\
\square \text { Chimney / Flue Shielding Incorrect } \\
\checkmark \text { Wiring Problems } \\
\square \text { Ventilation Inadequate } \\
\square \text { Water Leaks Present } \\
\square \text { Moisture/Mold Problems Eviden } \\
\square \text { Other Problems }\end{array}$ & $\begin{array}{l}\text { Walls } \\
\square \text { Wiring Problems } \\
\square \text { Water Leaks Present } \\
\square \text { Moisture/Mold Problems } \\
\text { Evident } \\
\square \text { Other Problems }\end{array}$ & $\begin{array}{l}\text { Crawlspace } \\
\square \text { Vapor Barrier Needed } \\
\square \text { Wiring Problems } \\
\square \text { Water Leaks Present } \\
\square \text { Plumbing Leaks Present } \\
\square \text { Moisture/Mold Problems } \\
\text { Evident } \\
\square \text { Other Problems }\end{array}$ \\
\hline & Comment & \\
\hline
\end{tabular}

Health \& Safety (Continued)

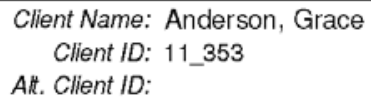

Att. Client ID:

Report Run On: 9/26/2011
DOE Weatherization Assistant

Version 8.9 .0

Page 15 of 17 


\section{Appendix C: Reports}

\section{Health \& Safety (Continued)}

EQUIPMENT

\section{Wood Stove / Fireplace \\ Wood Stove / Fireplace is Present \\ Venting is Incorrect \\ Combustion Air is Inadequate}

\section{Clothes Dryer}

Improper Venting

\section{Cook Stove}

co Measurement Oven (ppm) cO Measurement Burner 1 ( $p p m$ ) CO Measurement Burner 2 (ppm) co Measurement Burner 3 (ppm), co Measurement Burner 4 ( $p p m$ )

\section{Gas Leak Present}

\section{Exhaust Fans}

Bathrooms

\section{Kitchen}

$\square$ Missing

Not Operational

Missing

Improper Venting

Not Operational

Improper Venting

Comment

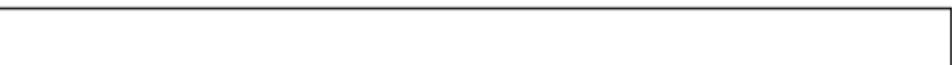

\section{Itemized Costs}

\begin{tabular}{|c|c|c|c|c|c|c|c|c|}
\hline Description & Cost & $\begin{array}{l}\text { Include } \\
\text { in SIR? }\end{array}$ & Material & $\begin{array}{c}\text { Energy } \\
\text { Savings } \\
\text { (mBTU/yr) }\end{array}$ & $\begin{array}{c}\text { Units } \\
\text { (of energy } \\
\text { saved) }\end{array}$ & $\begin{array}{c}\text { Life of } \\
\text { measure } \\
\text { (years) }\end{array}$ & $\begin{array}{l}\text { Fuel } \\
\text { Type } \\
\text { Saved }\end{array}$ & Comment \\
\hline $\begin{array}{l}\text { Fix Wiring Problems } \\
\text { (Attic) }\end{array}$ & $\$ 120.0$ & $\square$ & & & & & & $\begin{array}{l}\text { In kitchen dropdom } \\
\text { ceiling at lights. }\end{array}$ \\
\hline
\end{tabular}

\section{Utility Bills}

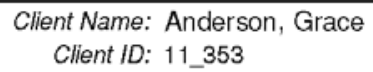

Att. Client ID:
MHEA Input Report

Audit Name: $11353 \mathrm{MH}$

Report Run On: 9/26/2011
DOE Weatherization Assistant

Version 8.9.0

Page 16 of 17 


\section{Utility Bills}

\begin{tabular}{|c|c|c|c|c|c|c|}
\hline $\begin{array}{l}\text { Billing } \\
\text { Type }\end{array}$ & $\begin{array}{l}\text { Billi } \\
\text { Peric }\end{array}$ & & $\begin{array}{l}\text { Billing } \\
\text { Units }\end{array}$ & $\begin{array}{r}\text { First } \\
\text { Period Days }\end{array}$ & $\begin{array}{l}\text { Base } \\
\text { Temp }\end{array}$ & $\begin{array}{l}\text { Base } \\
\text { Load }\end{array}$ \\
\hline \multirow[t]{14}{*}{ Heating } & \multicolumn{2}{|c|}{ Pre-Retrofit } & Therms & 30 & 65 & \\
\hline & \# & Month & Day & Usage & Deg & ays \\
\hline & 1 & 1 & 29 & 250 & & 1108 \\
\hline & 2 & 2 & 27 & 293 & & 968 \\
\hline & 3 & 3 & 30 & 182 & & 715 \\
\hline & 4 & 4 & 28 & 141 & & 350 \\
\hline & 5 & 5 & 31 & 65 & & 238 \\
\hline & 6 & 6 & 29 & 47 & & 96 \\
\hline & 7 & 7 & 30 & 36 & & 38 \\
\hline & 8 & 8 & 31 & 35 & & 0 \\
\hline & 9 & 9 & 28 & 36 & & 32 \\
\hline & 10 & 10 & 30 & 57 & & 246 \\
\hline & 11 & 11 & 29 & 106 & & 680 \\
\hline & 12 & 12 & 31 & 181 & & 905 \\
\hline
\end{tabular}

Client Name: Anderson, Grace Client ID: 11_353

Att. Client ID:
Report Run On: 9/26/2011
DOE Weatherization Assistant

Version 8.9.0

Page 17 of 17 


\section{Appendix C: Reports}

\section{MHEA Heating System Summary}

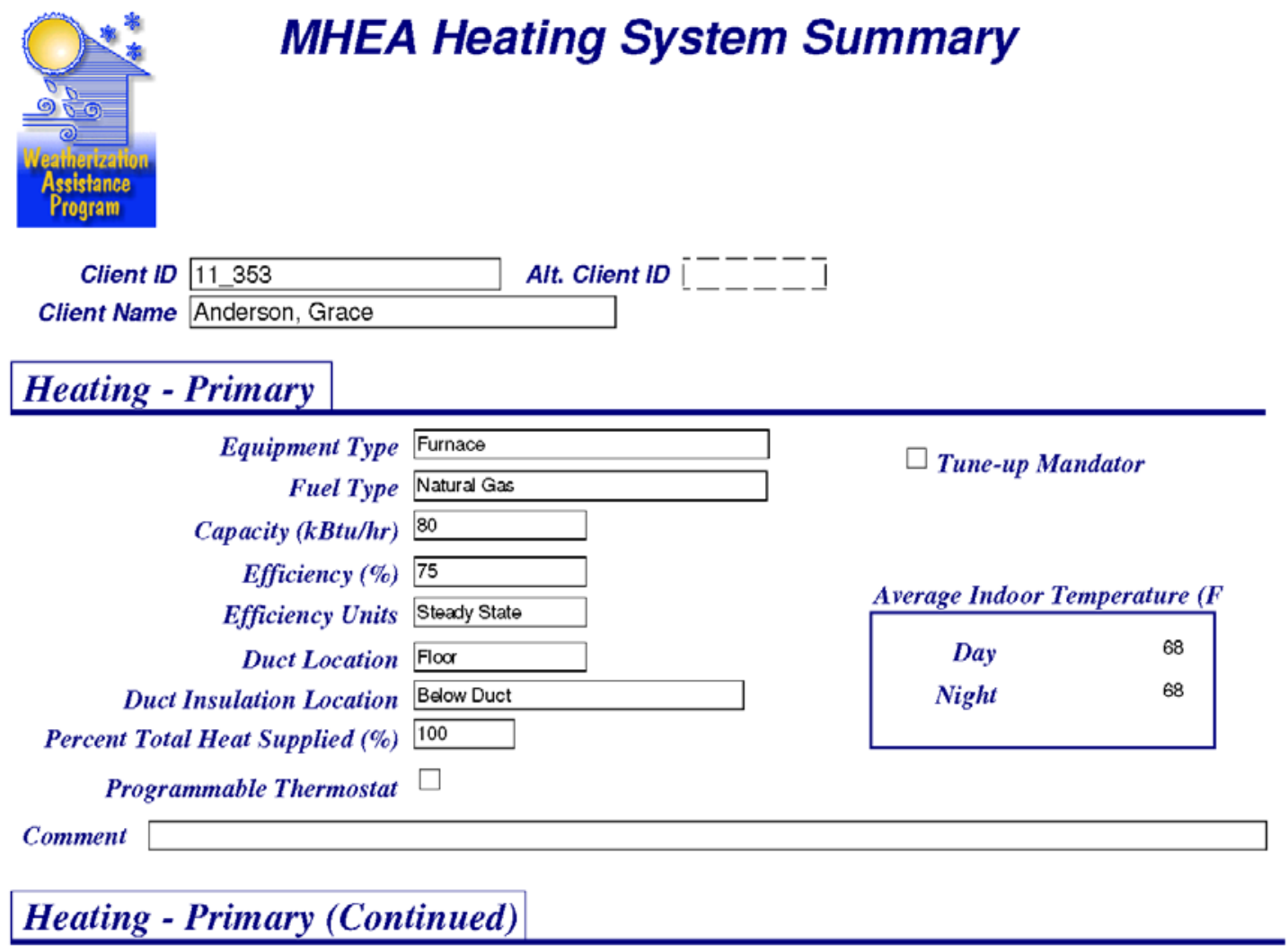

Client Name: Anderson, Grace Client ID: 11_353

Ait. Client ID:
MHEA Heating System Summary

Audit Name: 11_353MH

Report Run On: $9 / 26 / 2011$
DOE Weatherization Assistant Version 8.9.0

Page 1 of 4 
Heating - Primary (Continued)

OPTIONAL HEATING SYSTEM DETAILS

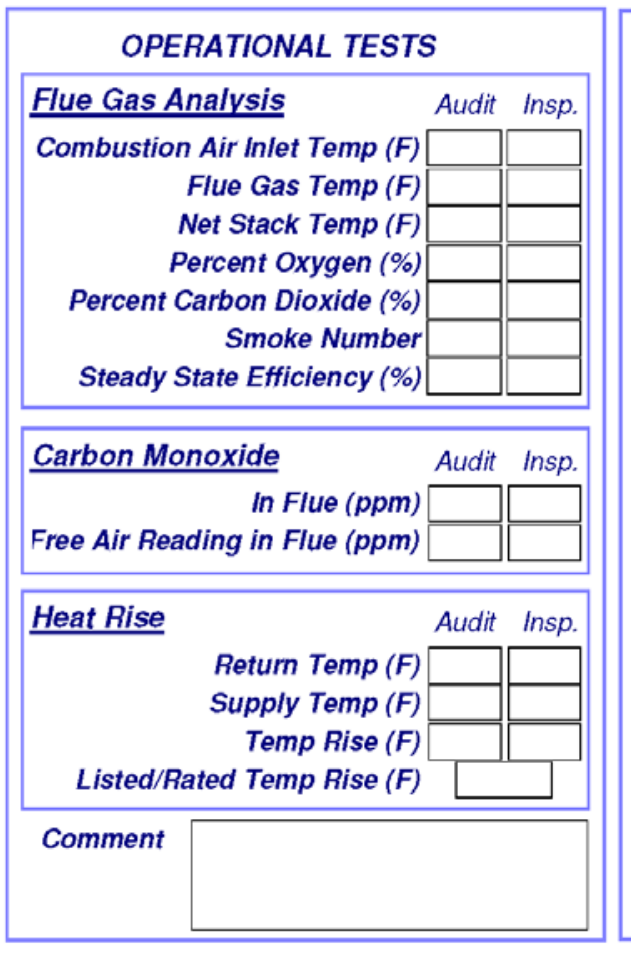

INSPECTIONS

\section{Other Items}

Cracked Heat Exchanger

Insufficient Clearance from Combustibles

Electric Service Switch

Gas Leak Present

Fuel Shutoff Valve Not Present

Drip Leg Not Present

Any Other Heating System Problems

Comment

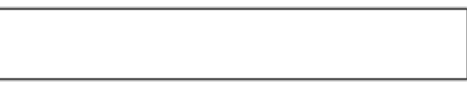

\section{Heating Primary (Continued)}

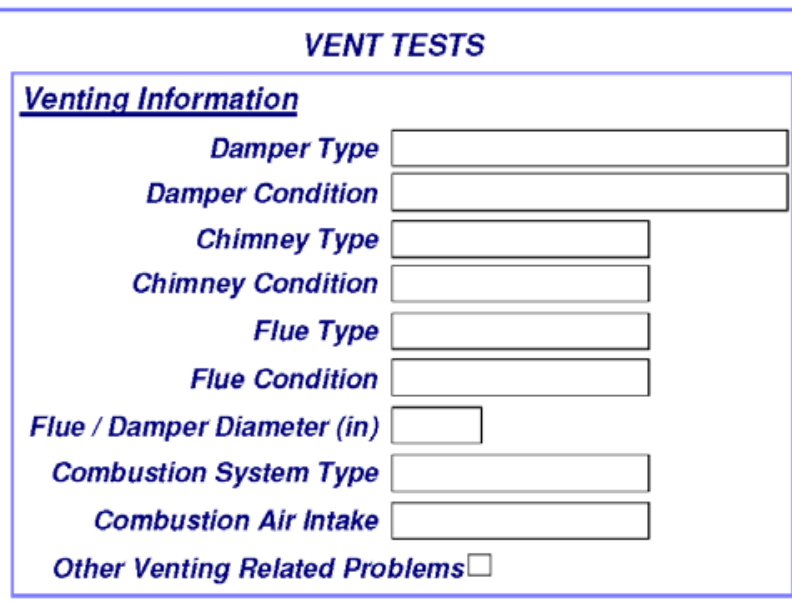

Normal Operating Conditions Draft Measurements

Audit Insp.

Outdoor Temp (F)

Draft (Pa or Inches of Water)

Spillage Time (sec)

Comment

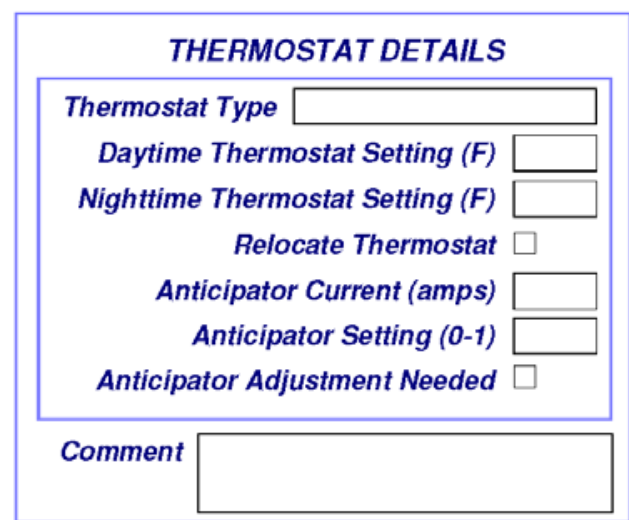

\begin{tabular}{lrr}
\hline Client Name: Anderson, Grace & MHEA Heating System Summary & DOE Weatherization Assistant \\
Client ID: $11 \_353$ & Audit Name: $11 \_353 \mathrm{MH}$ & Version 8.9 .0 \\
Alt. Client ID: & Report Run On: $9 / 26 / 2011$ & Page 2 of 4
\end{tabular}




\section{Appendix C: Reports}

Heating Primary (Continued)

OPTIONAL HEATING SYSTEM DETAILS (Continued)

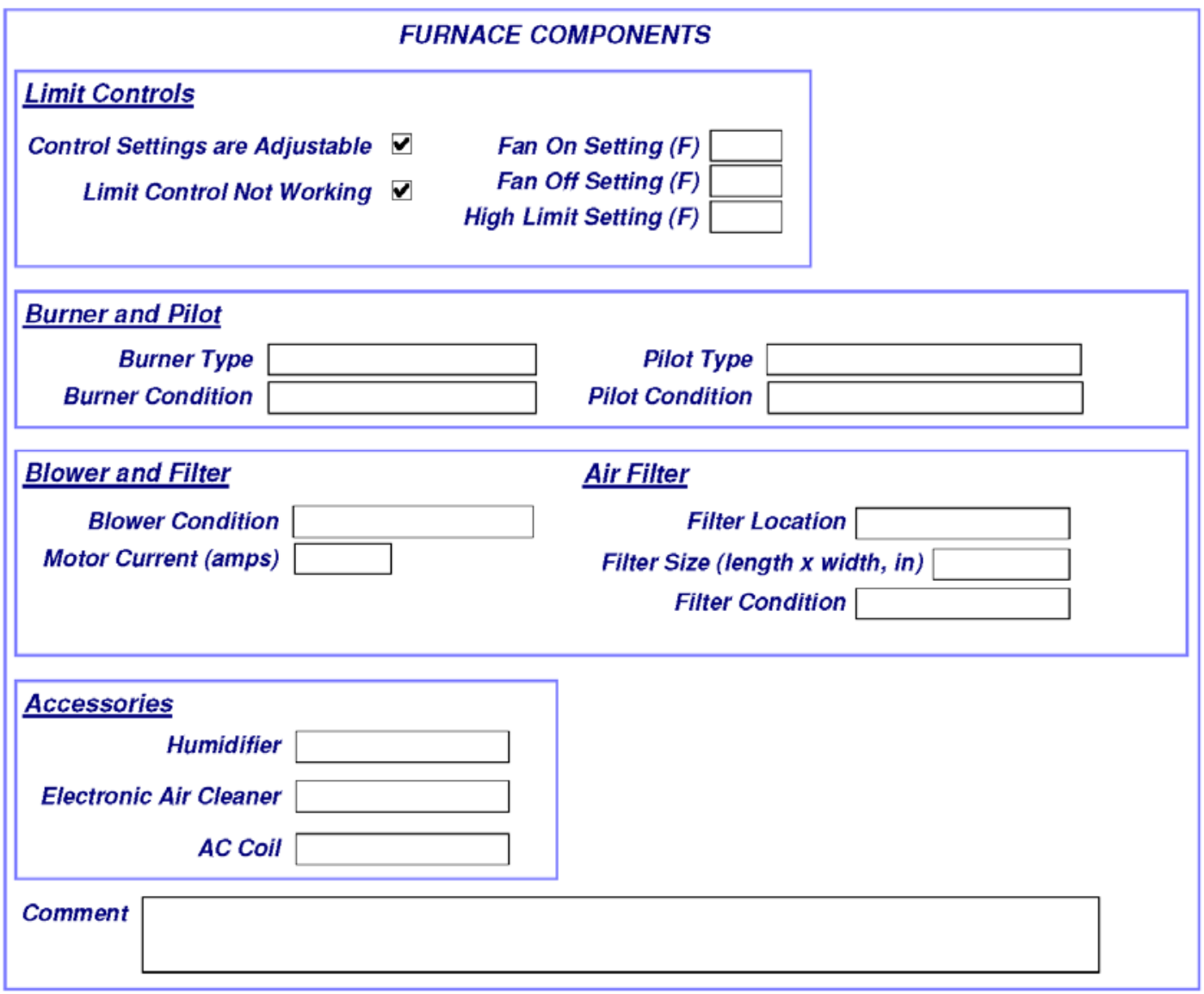

Heating - Secondary

No data were entered for this audit.

\section{Heating - Replacement}

$\begin{array}{lrr}\text { Client Name: Anderson, Grace } & \text { MHEA Heating System Summary } & \text { DOE Weatherization Assistant } \\ \text { Client ID: } 11 \_353 & \text { Audit Name: } 11 \_353 \mathrm{MH} & \text { Version } 8.9 .0 \\ \text { Alt. Client ID: } & \text { Report Run On: } 9 / 26 / 2011 & \text { Page } 3 \text { of } 4\end{array}$




\section{Heating - Replacement}

Equipment Type Furnace

Fuel Type Natural Gas

Capacity $(\mathrm{kBtu} / \mathrm{hr}) 80$

Efficiency (\%) 92

Efficiency Units AFUE

80

Duct Location Floor

Duct Insulation Location Below Duct

$$
\begin{aligned}
& \text { Replacement Required } \square \quad \begin{array}{l}
\text { Include Replacement } \\
\text { Costs in Home Retrofit }
\end{array}
\end{aligned}
$$

Comment

Client Name: Anderson, Grace

Client ID: 11_353

Alt. Client ID:
MHEA Heating System Summary

Audit Name: $11353 \mathrm{MH}$

Report Run On: 9/26/2011
600

1200 


\section{Appendix C: Reports}

\section{MHEA Pressure Diagnostics}

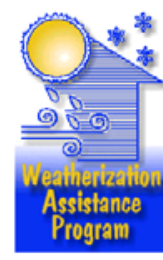

Alt. Client ID $\left[\begin{array}{c}----\urcorner \\ \mathrm{L}\end{array}\right.$

Client ID 11353

Client Name Anderson, Grace

\section{Blower Door Readings (Existing)}

No data were entered for this audit.

\section{Blower Door Readings (New)}
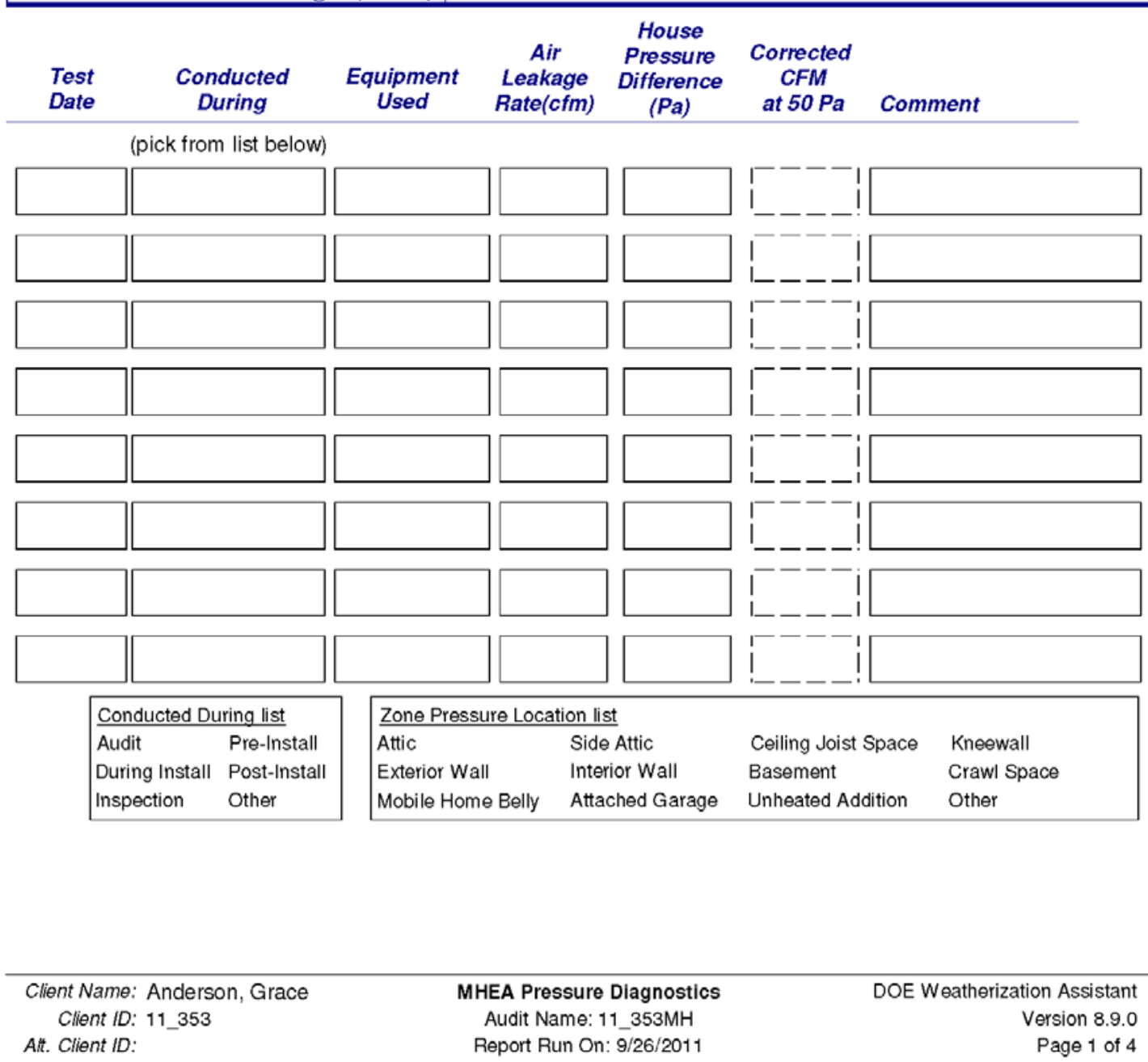
Zonal Pressure Readings (Existing)

No data were entered for this audit.

\section{Zonal Pressure Readings (New)}

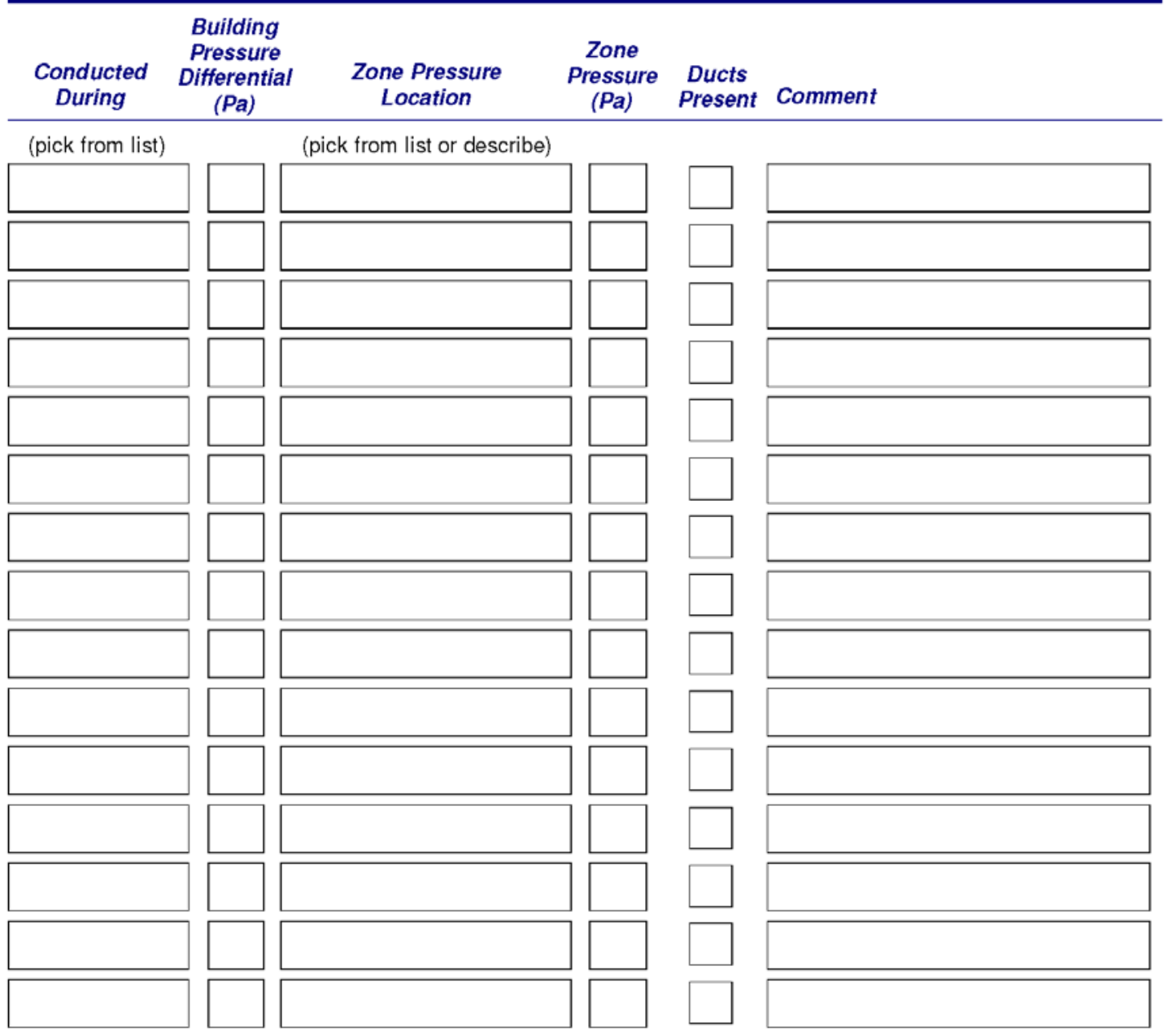

Client Name: Anderson, Grace

Client ID: 11353

Alt. Client ID:
MHEA Pressure Diagnostics

Audit Name: 11 353MH

Report Run On: 9/26/2011
DOE Weatherization Assistant

Version 8.9.0

Page 2 of 4 


\section{Appendix C: Reports}

\section{Pressure Balance Readings (Existing)}

No data were entered for this audit.

\section{Pressure Balance Readings (New)}

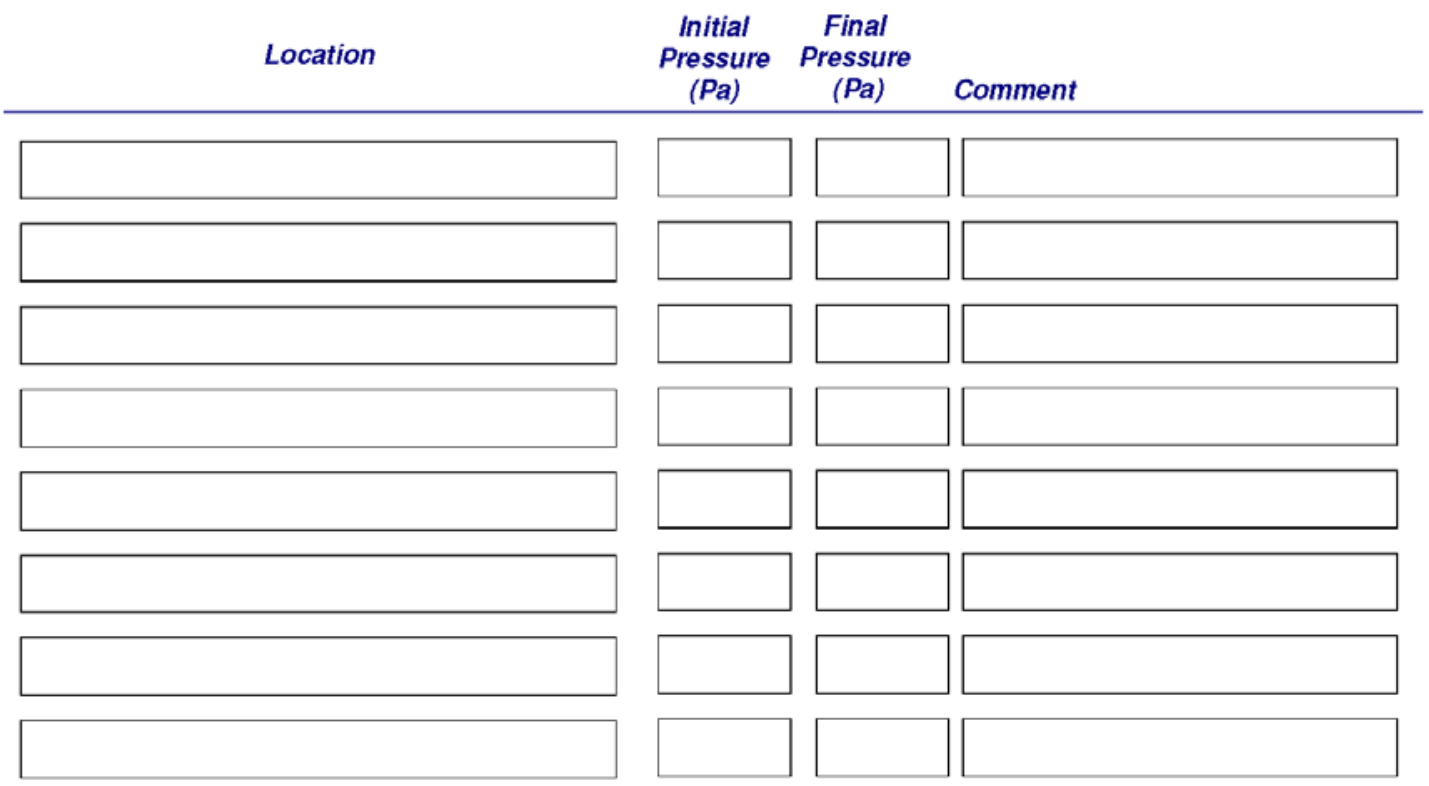




\section{Pressure Pan Readings (Existing)}

No data were entered for this audit.

\section{Pressure Pan Readings (New)}

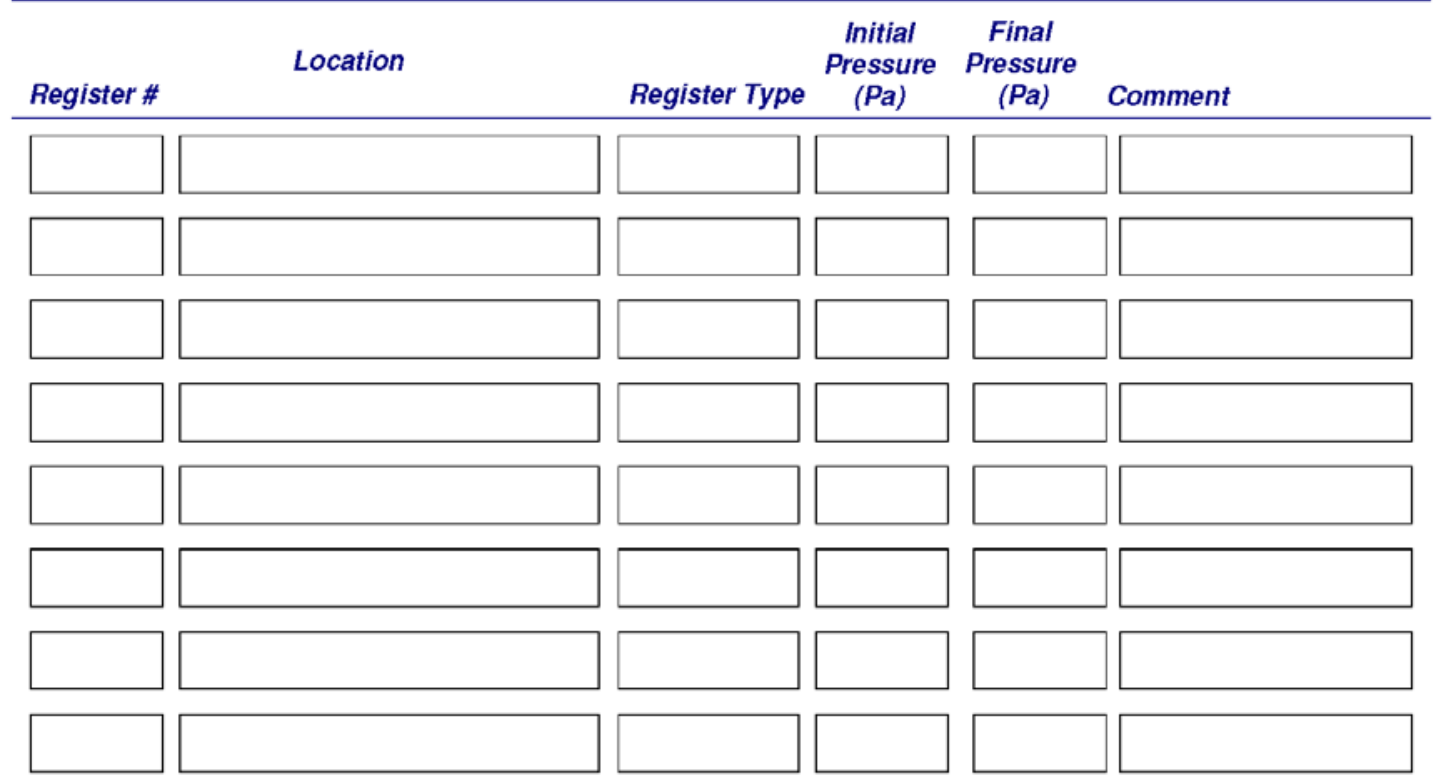




\section{Appendix C: Reports}

\section{MHEA Health and Safety Summary}

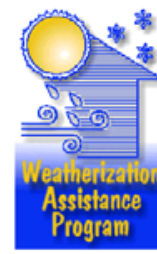

$\begin{array}{rll}\text { Client ID } & 11 \_353 & \text { Alt. Client ID } \\ \text { Client Name } & \text { Anderson, Grace }\end{array}$

\section{Health \& Safety}

\section{WHOLE HOUSE}

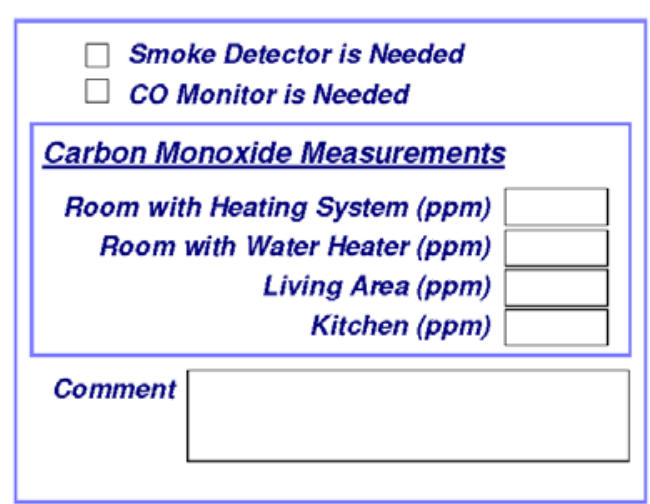

\section{BUILDING SHELL}

\begin{tabular}{|c|c|c|}
\hline $\begin{array}{l}\text { Attic } \\
\square \text { Recessed Lights Present } \\
\square \text { Chimney/Flue Shielding Incorrect } \\
\nabla \text { Wiring Problems } \\
\square \text { Ventilation Inadequate } \\
\square \text { Water Leaks Present } \\
\square \text { Moisture/Mold Problems Evide } \\
\square \text { Other Problems }\end{array}$ & $\begin{array}{l}\text { Walls } \\
\square \text { Wiring Problems } \\
\square \text { Water Leaks Present } \\
\square \text { Moisture/Mold } \\
\text { Problems } \\
\square \text { Other Problems }\end{array}$ & $\begin{array}{l}\text { Crawlspace } \\
\square \text { Vapor Barrier Needed } \\
\square \text { Wiring Problems } \\
\square \text { Water Leaks Present } \\
\square \text { Plumbing Leaks Present } \\
\square \text { Moisture/Mold Problems } \\
\text { Evident } \\
\square \text { Other Problems }\end{array}$ \\
\hline & Comment & \\
\hline
\end{tabular}

$\begin{array}{lrr}\text { Client Name: Anderson, Grace } & \text { MHEA Health and Safety Summary } & \text { DOE Weatherization Assistant } \\ \text { Client ID: } 11 \_353 & \text { Audit Name: } 11 \_353 \mathrm{MH} & \text { Version } 8.9 .0 \\ \text { Aft. Client ID: } & \text { Report Run On: } 9 / 26 / 2011 & \text { Page } 1 \text { of } 5\end{array}$


Health \& Safety (Continued)

EQUIPMENT

\section{Wood Stove / Fireplace}

$\square$ Wood Stove / Fireplace is Present

Venting is Incorrect

Combustion Air is Inadequate

\section{Clothes Dryer}

Improper Venting

\section{Cook Stove}

co Measurement Oven (ppm)

CO Measurement Burner 1 ( $p p m)$

cO Measurement Burner 2 (ppm)

CO Measurement Burner 3 (ppm)

co Measurement Burner 4 (ppm)

Gas Leak Present
Exhaust Fans

Bathrooms

$\square$ Missing

$\square$ Not Operational

Improper Venting

Comment $\underline{\text { Kitchen }}$

$\square$ Missing

$\square$ Not Operational

$\square$ Improper Venting
Client Name: Anderson, Grace

Client ID: 11353

Alt. Client ID:
MHEA Health and Safety Summary

Audit Name: 11 353MH

Report Run On: 9/26/2011
DOE Weatherization Assistant

Version 8.9.0

Page 2 of 5 


\section{Appendix C: Reports}

\section{Heating - Primary}

Equipment Type Furnace

Fuel Type Natural Gas

$\square$ Tune-up Mandator

Capacity (kBtu/hr)

80

Efficiency (\%)

Efficiency Units Steady State

Duct Location

Duct Insulation Location Below Duct

Percent Total Heat Supplied (\%)

100

Average Indoor Temperature (F

Programmable Thermostat

Comment

\section{Heating - Primary (Continued)}

Client Name: Anderson, Grace

Client ID: 11353

Alt. Client ID:
MHEA Health and Safety Summary

Audit Name: 11_353MH

Report Run On: 9/26/2011
DOE Weatherization Assistant

Version 8.9 .0

Page 3 of 5 


\section{Heating - Primary (Continued)}

OPTIONAL HEATING SYSTEM DETAILS

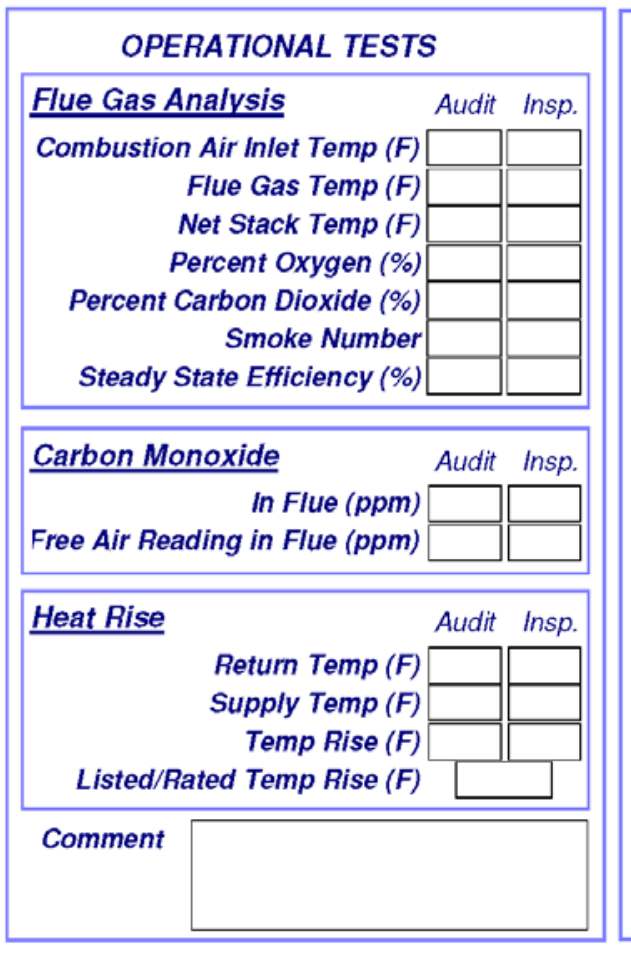

INSPECTIONS

\section{Other Items}

Cracked Heat Exchanger Insufficient Clearance from Combustibles Electric Service Switch

Gas Leak Present Fuel Shutoff Valve Not Present Drip Leg Not Present Any Other Heating System Problems

Comment

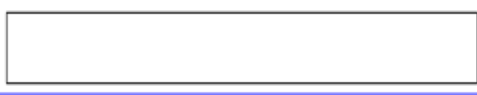

\section{Heating Primary (Continued)}

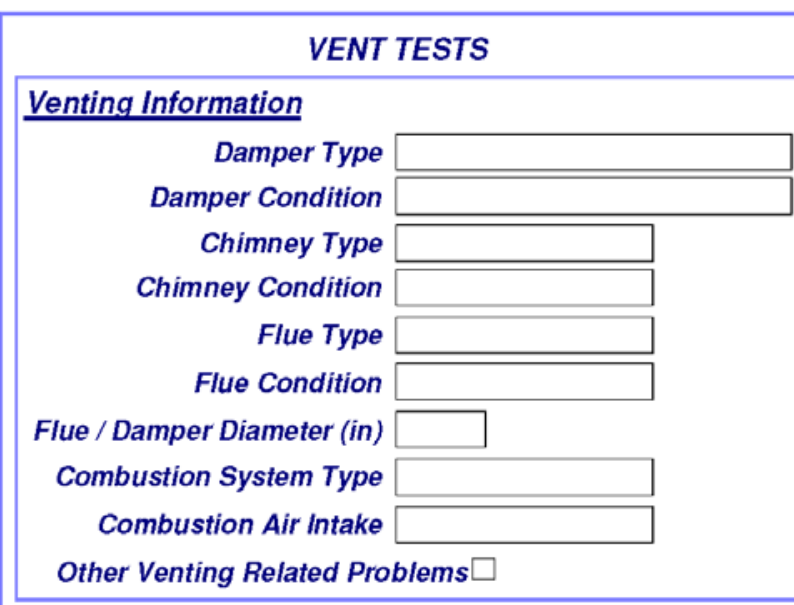

Normal Operating Conditions Draft Measurements

Audit Insp.

Outdoor Temp (F)

Draft (Pa or Inches of Water)

Spillage Time (sec)

Comment

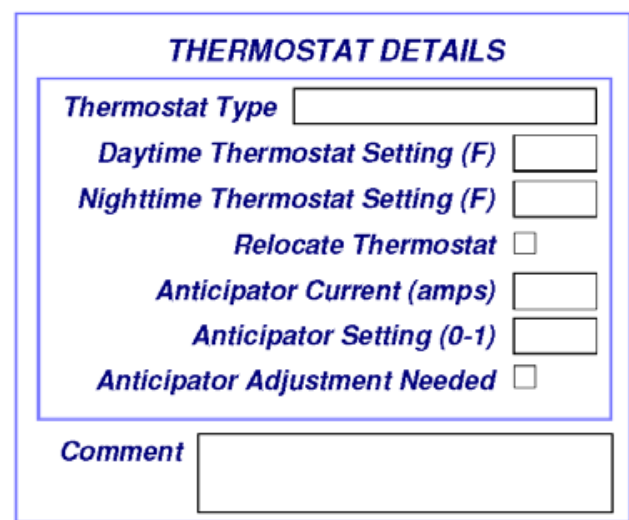

\begin{tabular}{lrr}
\hline Client Name: Anderson, Grace & MHEA Health and Safety Summary & DOE Weatherization Assistant \\
Client ID: $11 \_353$ & Audit Name: $11 \_353 \mathrm{MH}$ & Version 8.9 .0 \\
Alt. Client ID: & Report Run On: $9 / 26 / 2011$ & Page 4 of 5
\end{tabular}




\section{Appendix C: Reports}

Heating Primary (Continued)

OPTIONAL HEATING SYSTEM DETAILS (Continued)

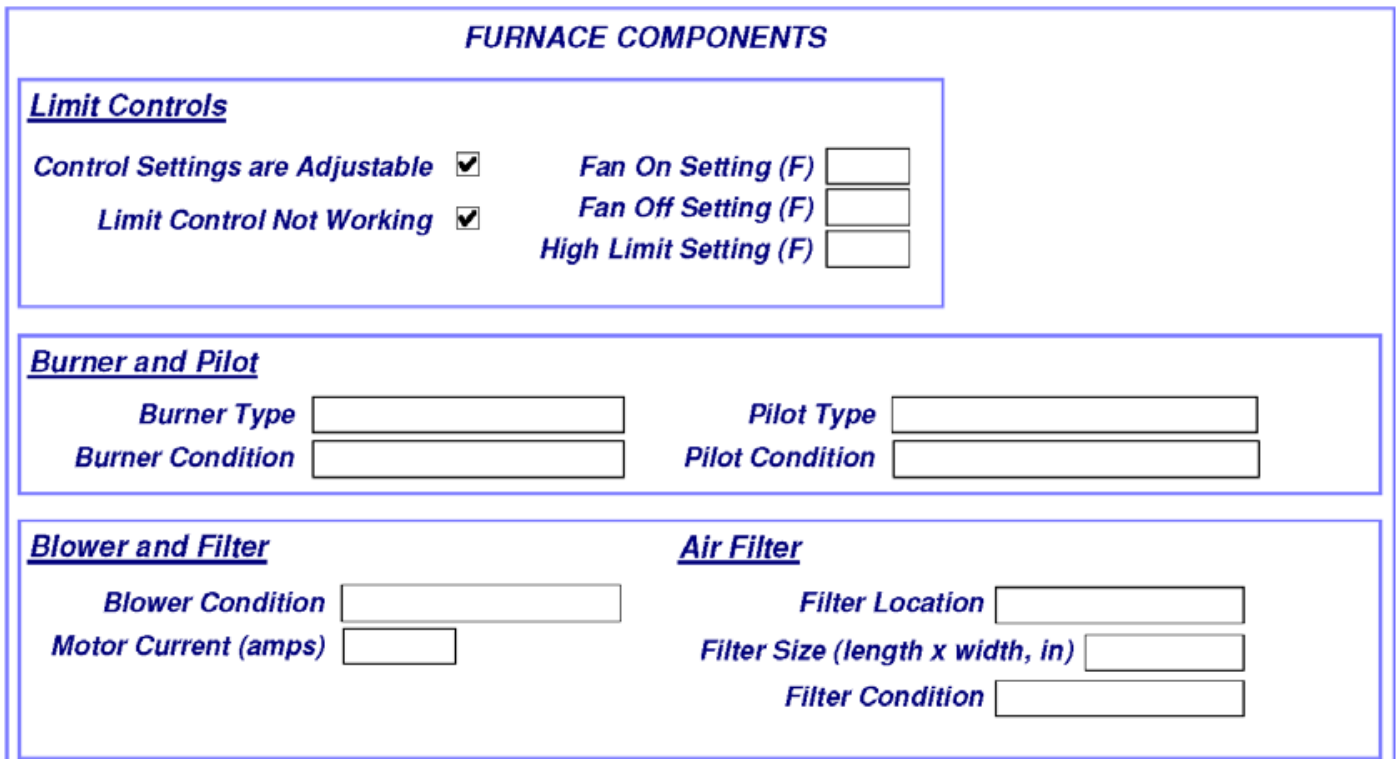

Accessories

Humidifier

Electronic Air Cleaner

AC Coil

Comment

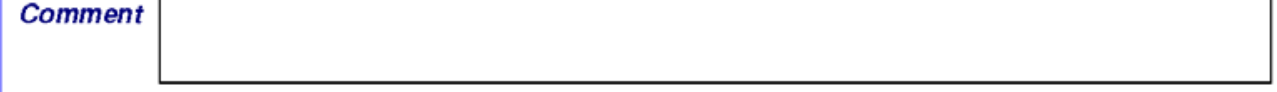

Client Name: Anderson, Grace

Client ID: 11353

Alt. Client ID:
MHEA Health and Safety Summary

Audit Name: 11_353MH

Report Run On: 9/26/2011
DOE Weatherization Assistant

Version 8.9 .0

Page 5 of 5 


\title{
Work Order
}

\author{
Address: 114 Athens \\ Oak Ridge, TN 37830
}

\section{WORK ORDER INFORMATION}

Work Order Name: WO/11_348/JT/1

Work Order Type: Weatherization

Audit Name: 11_348SB

\section{CLIENT INFORMATION}

Client Name: Tanner, David

Client ID: 11348

Alt. Client ID:

\section{CLIENT CONTACT INFORMATION}

Tanner, David

Applicant Person of

Record

\section{AGENCY INFORMATION}

Agency: Demonstration Agency

Address: 725 Jefferson St.

Any City, US 11111

Agency Contact: Tor, Audrey

\section{Cell Phone:}

Email Address:

\begin{abstract}
Agency Phone: (123) 456-7890
\end{abstract}
Fax: (234) 567-8901

Email Address: agencyemail@localisp.net

\section{CONTRACTOR / CREW INFORMATION}

Company:

Work Phone:

Address:

Cell Phone:

Email Address:

Contact: Contractor, John

Company Name \& License Number:

Contractor's Signature:

\section{COMMENT}

Client Name: Tanner, David
Client ID: 11 348

Alt. Client ID:
Work Order

Work Order Name: WO/11_348/JT/1

Report Run On: 9/27/2011
DOE Weatherization Assistant

Version 8.9 .0

Page 1 of 8 


\section{Appendix C: Reports}

\section{Measures}

\begin{tabular}{|c|c|c|c|c|c|c|c|c|}
\hline \multirow{3}{*}{$\begin{array}{l}\text { Measure } 2 \mathrm{R} \\
\text { Comment }\end{array}$} & Repair Roof & & \multicolumn{3}{|c|}{ Components } & & & \multirow[t]{2}{*}{ Inspected } \\
\hline & & & & & & & & \\
\hline & & & \multicolumn{3}{|c|}{ Estimated } & \multicolumn{3}{|c|}{ Actual } \\
\hline \# Material / Labor & Description /Comment & Units & Oty & Unit Cost & Total & Oty & Unit Cost & Total \\
\hline 10 Unspecified & Misc Material & Each & 1 & $\$ 80.00$ & $\$ 80.00$ & 1 & $\$ 135.00$ & $\$ 135.00$ \\
\hline
\end{tabular}

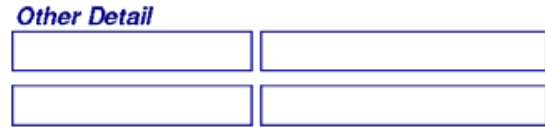

Measure Sub Total: $\quad \$ 80.00$

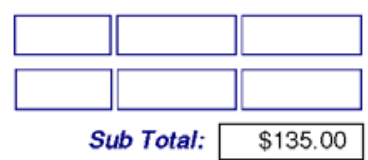

Field Notes:

Measure 3 Infiltration Redctn Comment

\# Material / Labor Description /Comment

10 Miscellaneous Su Infiltration Reduction

Other Detail

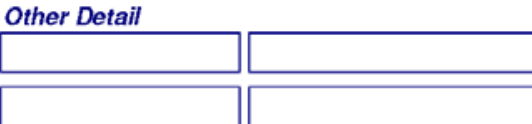

\section{Components}

\begin{tabular}{cccc} 
& \multicolumn{3}{c}{ Estimated } \\
\cline { 2 - 4 } Units & Qty & Unit Cost & Total \\
Each & 1 & $\$ 250.00$ & $\$ 250.00$
\end{tabular}

Measure Sub Total:

$\$ 250.00$

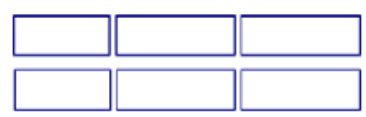

Sub Total:

$\$ 250.00$

Field Notes:

Client Name: Tanner, David

Client ID: 11_348

Att. Client ID:
Work Order

Work Order Name: WO/11_348/JT/1

Report Run On: 9/27/2011
DOE Weatherization Assistant

Version 8.9.0

Page 2 of 8 


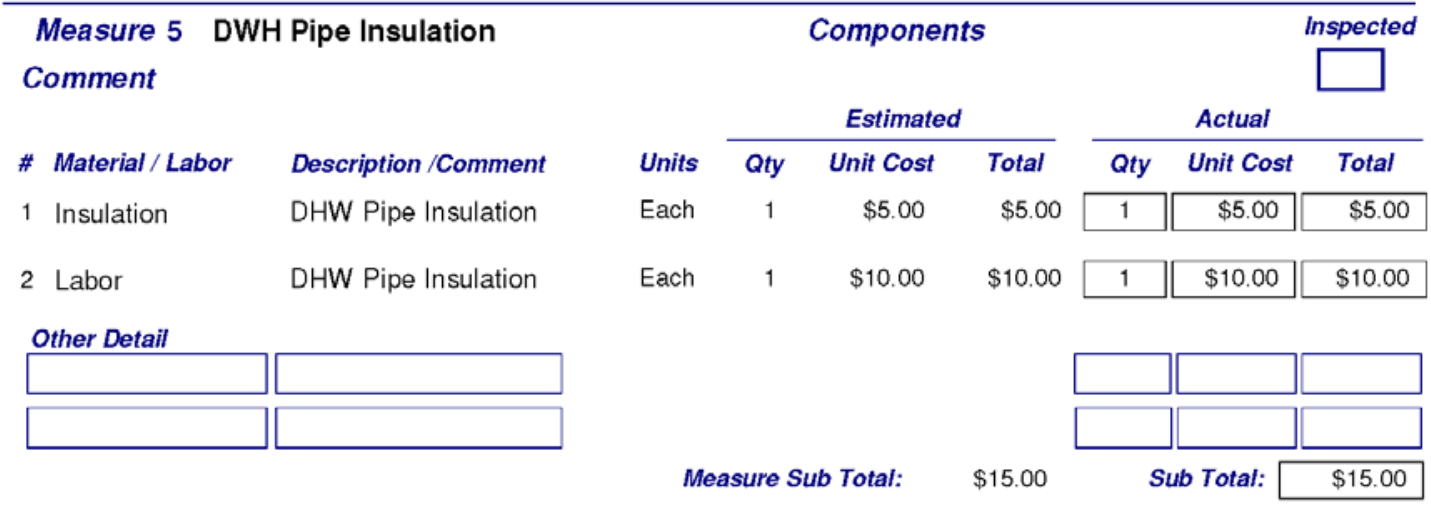

\section{Field Notes:}

Measure 7 DWH Tank Insulation

Comment

\begin{tabular}{ll} 
\# Material / Labor & Description /Comment \\
1 Hot Water Equip & DHW Tank Insulation \\
2 Labor & DHW Tank Insulation \\
\hline & \\
\hline
\end{tabular}

\# Material / Labor

1 Hot Water Equip

Other Detail

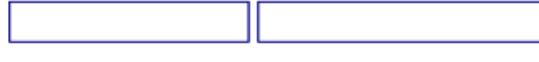

\section{Components}

Inspected

\begin{tabular}{lccl} 
& \multicolumn{3}{c}{ Estimated } \\
\cline { 2 - 4 } Units & Qty & Unit Cost & Total \\
Each & 1 & $\$ 15.00$ & $\$ 15.00$ \\
Each & 1 & $\$ 25.00$ & $\$ 25.00$
\end{tabular}

\begin{tabular}{ll}
\multicolumn{3}{c}{ Actual } \\
\hline Qty Unit Cost Total
\end{tabular}

\begin{tabular}{|l|l|l|}
\hline 1 & $\$ 15.00$ & $\$ 15.00$ \\
\hline
\end{tabular}

\begin{tabular}{|l|l|l|}
\hline 1 & $\$ 25.00$ & $\$ 25.00$ \\
\hline
\end{tabular}

\section{Field Notes:}

Measure Sub Total:

$\$ 40.00$

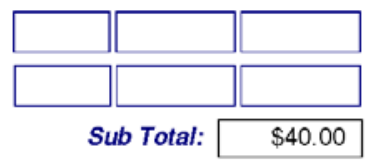

Field Notes:

Client Name: Tanner, David Client ID: 11_348

Att. Client ID:
Report Run On: 9/27/2011
DOE Weatherization Assistant Version 8.9.0

Page 3 of 8 


\section{Appendix C: Reports}

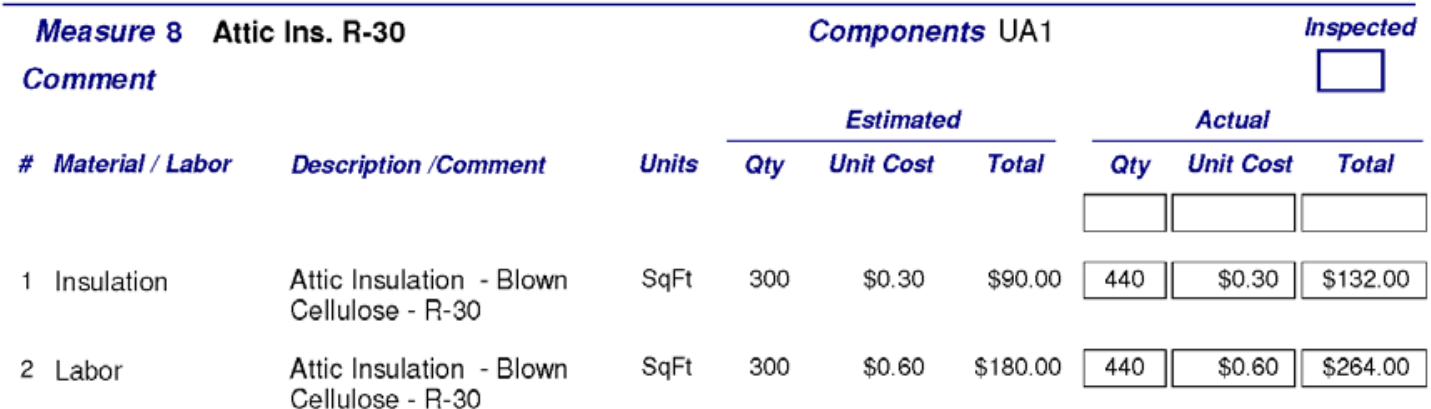

Other Detail

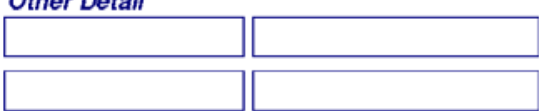

Measure Sub Total: $\quad \$ 270.00$

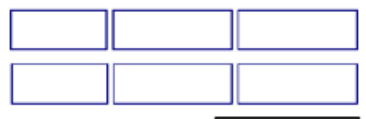

Field Notes:

Measure 9 Fill Ceiling Cavity

\section{Comment}

\section{\# Material / Labor}

1 Insulation

2 Labor

Other Detail

\begin{tabular}{|l|}
\hline \\
\hline
\end{tabular}

Attic Insulation - Blown Cellulose -5 in.

Attic Insulation - Blown Cellulose -5 in.
Components FA3

Sub Total: $\$ 396.00$

Measure sub tota:

\begin{tabular}{|c|c|c|c|c|c|c|}
\hline \multirow[b]{3}{*}{ Units } & \multicolumn{3}{|c|}{ Components FA3 } & \multicolumn{3}{|c|}{ Inspected } \\
\hline & \multicolumn{3}{|c|}{ Estimated } & \multicolumn{3}{|c|}{ Actual } \\
\hline & Qty & Unit Cost & Total & Qty & Unit Cost & Total \\
\hline $\mathrm{SqFt}$ & 437 & $\$ 0.17$ & $\$ 73.77$ & 437 & $\$ 0.17$ & $\$ 73.77$ \\
\hline $\mathrm{SqFt}$ & 437 & $\$ 0.34$ & $\$ 147.49$ & 437 & $\$ 0.34$ & $\$ 147.49$ \\
\hline
\end{tabular}

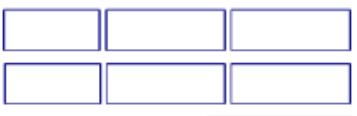

Measure Sub Total:

$\$ 221.26$

\section{Field Notes:}

Client Name: Tanner, David Client ID: 11_348

Att. Client ID:
Work Order

Work Order Name: WO/11_348/JT/1

Report Run On: 9/27/2011
DOE Weatherization Assistant

Version 8.9.0

Page 4 of 8 


\section{Measure 10 Insulate and seal attic access}

\section{Comment}

\# Material / Labor

10 Unspecified

Description /Comment

$\mathrm{R}-30$ faced batt insulation $(+)$

Other Detail

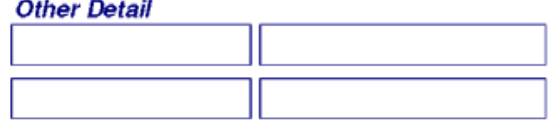

Measure Sub Total:

\begin{tabular}{lll} 
& \multicolumn{3}{c}{ Estimated } \\
\cline { 2 - 3 } Units & Qty & Unit Cost Total
\end{tabular}

Each $\quad 1 \quad \$ 29.60 \quad \$ 29.60$

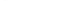

\begin{tabular}{|c|c|c|}
\hline \multicolumn{3}{|c|}{ Actual } \\
\hline Qty & Unit Cost & Total \\
\hline 1 & $\$ 29.60$ & $\$ 29.60$ \\
\hline
\end{tabular}

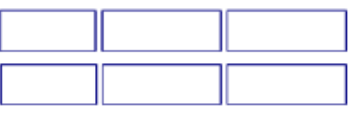

Sub Total:

$\$ 29.60$

Field Notes:

Measure 11 Attic Ins. R-30

Components FA1

Inspected

Comment

\begin{tabular}{|c|c|c|c|c|c|c|c|c|c|}
\hline \multirow[b]{2}{*}{ \# } & \multirow[b]{2}{*}{ Material / Labor } & \multirow[b]{2}{*}{ Description /Comment } & \multirow[b]{2}{*}{ Units } & \multicolumn{3}{|c|}{ Estimated } & \multicolumn{3}{|c|}{ Actual } \\
\hline & & & & Qty & Unit Cost & Total & Qty & Unit Cost & Total \\
\hline 1 & Insulation & $\begin{array}{l}\text { Attic Insulation - Blown } \\
\text { Cellulose - R-30 }\end{array}$ & $\mathrm{SqFt}$ & 392 & $\$ 0.30$ & $\$ 117.60$ & 392 & $\$ 0.30$ & $\$ 117.60$ \\
\hline 2 & Labor & $\begin{array}{l}\text { Attic Insulation - Blown } \\
\text { Cellulose - R-30 }\end{array}$ & $\mathrm{SqFt}$ & 392 & $\$ 0.60$ & $\$ 235.20$ & 392 & $\$ 0.60$ & $\$ 235.20$ \\
\hline
\end{tabular}

Other Detail

\begin{tabular}{|l|l|}
\hline \\
\hline \\
\hline
\end{tabular}

Measure Sub Total:

$\$ 352.80$

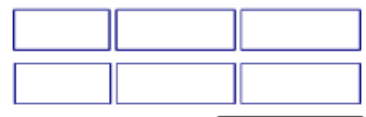

Sub Total:

$\$ 352.80$
Client Name: Tanner, David

Client ID: 11_348

Att. Client ID:
Work Order

Work Order Name: WO/11_348/JT/1

Report Run On: 9/27/2011
DOE Weatherization Assistant

Version 8.9.0

Page 5 of 8 


\section{Appendix C: Reports}

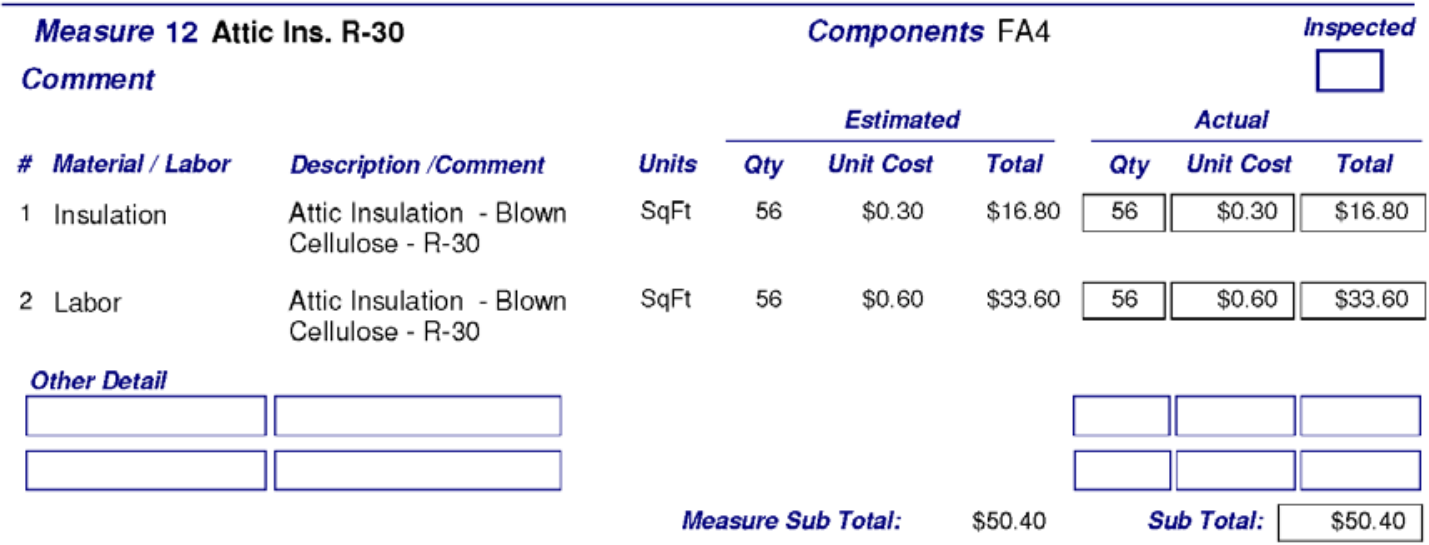

\section{Field Notes:}

Measure 14 Kneewall Insulation

Comment

\begin{tabular}{lll} 
\# Material / Labor & $\begin{array}{l}\text { Description /Comment } \\
\text { Kneewall Insulation - } \\
1 \text { Insulation }\end{array}$ \\
& $\begin{array}{l}\text { Fiberglass Batts - R-13 } \\
2 \text { Labor }\end{array}$ \\
& Kneewall Insulation - \\
& Fiberglass Batts - R-13 \\
\hline
\end{tabular}

\section{Components FA2}

\begin{tabular}{llllllll} 
& \multicolumn{3}{c}{ Estimated } & & \multicolumn{3}{c}{ Actual } \\
\cline { 2 - 3 } \cline { 5 - 7 } Units & Qty & Unit Cost & Total & & Qty & Unit Cost & Total \\
SqFt & 120 & $\$ 0.26$ & $\$ 31.20$ & 120 & $\$ 0.26$ & $\$ 31.20$ \\
\cline { 5 - 7 } & & & & & &
\end{tabular}

$\begin{array}{llll}\mathrm{SqFt} & 120 & \$ 0.50 & \$ 60.00\end{array}$

$\$ 60.00 \quad 120$

$\$ 0.50$

$\$ 60.00$

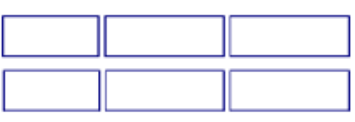

Measure Sub Total: $\quad \$ 91.20$

$\$ 91.20$

Field Notes:

Client Name: Tanner, David Client ID: 11_348

Att. Client ID:
Work Order

Work Order Name: WO/11_348/JT/1

Report Run On: 9/27/2011
DOE Weatherization Assistant

Version 8.9.0

Page 6 of 8 


\section{Measure 16 Wall Insulation}

Comment

\# Material / Labor
1 Insulation
2 Labor

Other Detail

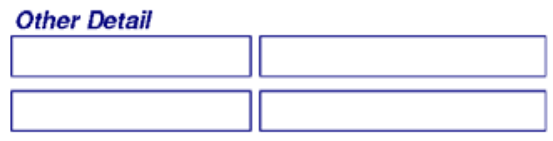

\section{Description /Comment}

Wall Insulation - Blown

Cellulose - 2x4 Filled Cellulose - 2x4 Filled
Components WLE-1,WLN-1,WLN- Inspected 2,WLS-2,WLW-1

\begin{tabular}{|c|c|c|c|c|c|}
\hline \multicolumn{3}{|c|}{ Estimated } & \multicolumn{3}{|c|}{ Actual } \\
\hline Qty & Unit Cost & Total & Qty & Unit Cost & Total \\
\hline 846 & $\$ 0.26$ & $\$ 219.96$ & 710 & $\$ 0.26$ & $\$ 184.60$ \\
\hline 846 & $\$ 0.75$ & $\$ 634.50$ & 710 & $\$ 0.75$ & $\$ 532.50$ \\
\hline
\end{tabular}

Wall Insulation - Blown

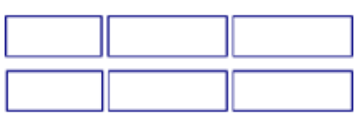

Measure Sub Total: $\quad \$ 854.46$

Sub Total:

$\$ 717.10$

\section{Field Notes:}

\section{Comment}

\# Material / Labor
1 Windows
2 Labor
3 Other

Other Detail

\begin{tabular}{|l|}
\hline \\
\hline
\end{tabular}

Components WD4

Inspected

\begin{tabular}{lcccc} 
& & \multicolumn{3}{c}{ Estimated } \\
\cline { 3 - 5 } Description /Comment & Units & Qty & Unit Cost & Total \\
Window Replacement & SqFt & 16 & $\$ 6.00$ & $\$ 96.00$ \\
Window Replacement & SqFt & 16 & $\$ 0.00$ & $\$ 0.00$ \\
Window Replacement & $\begin{array}{c}\text { Each } \\
\text { Window }\end{array}$ & 2 & $\$ 125.00$ & $\$ 250.00$
\end{tabular}

\begin{tabular}{l|r|r|}
\multicolumn{3}{c}{ Actual } \\
\hline Qty & Unit Cost & Total \\
\hline 16 & $\$ 6.00$ & $\$ 96.00$ \\
\hline
\end{tabular}
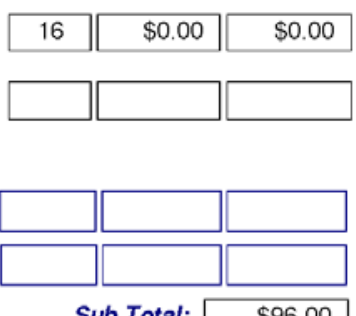

Measure Sub Total:

$\$ 346.00$

Sub Total:

$\$ 96.00$

Field Notes:

Client Name: Tanner, David Client ID: 11_348

Att. Client ID:

\section{Work Order}

Work Order Name: WO/11_348/JT/1

Report Run On: 9/27/2011
DOE Weatherization Assistant Version 8.9.0

Page 7 of 8 


\section{Appendix C: Reports}

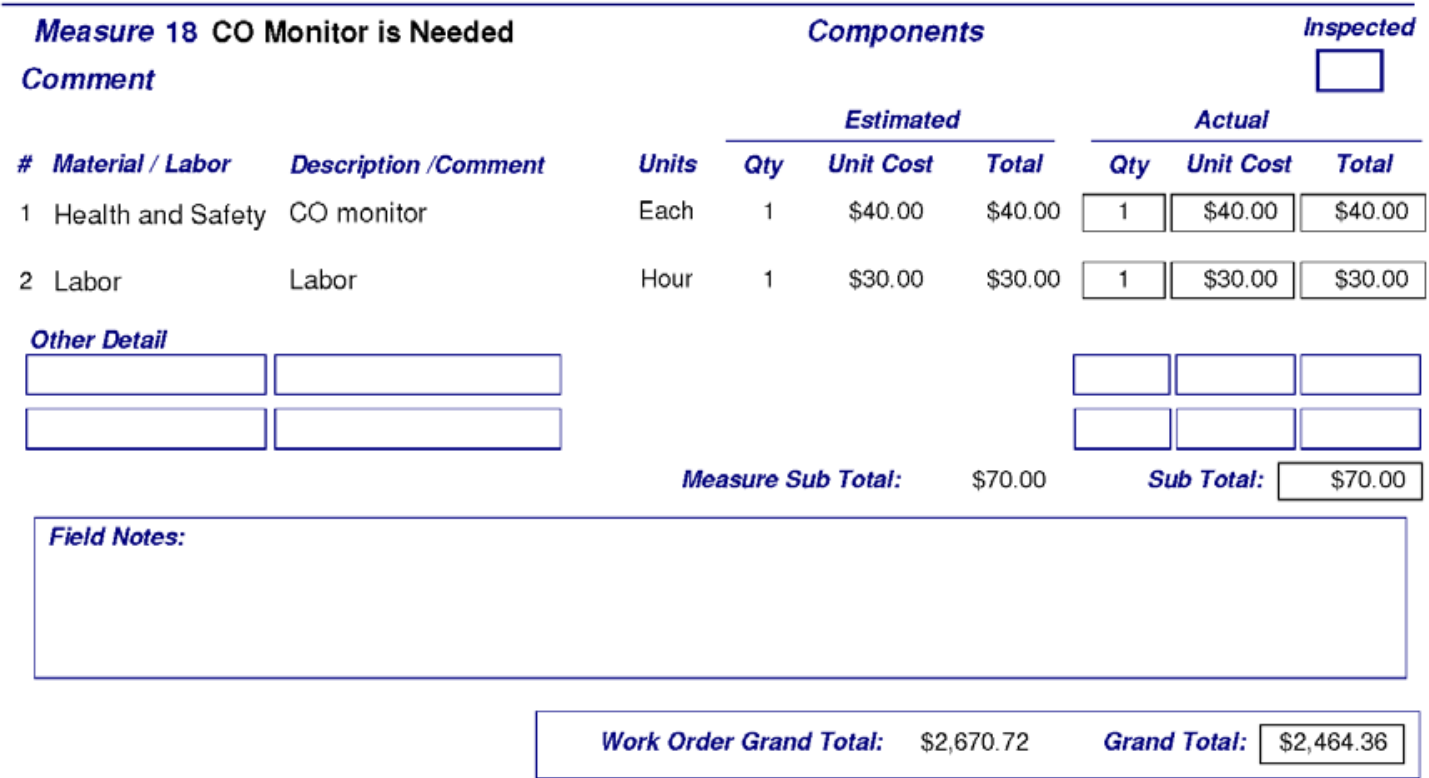

Client Name: Tanner, David Client ID: 11_348

Att. Client ID:
Report Run On: 9/27/2011
DOE Weatherization Assistant Version 8.9 .0

Page 8 of 8 


\section{Work Order Bid (ID)}

Asulerizarifon

Assistance

Program

\section{WORK ORDER INFORMATION}

Work Order Name: WO/11_348/JT/1

Work Order Type: Weatherization

Audit Name: 11_348SB

\section{CLIENT INFORMATION}

Client ID: 11348

\section{AGENCY INFORMATION}

Agency: Demonstration Agency

Address: 725 Jefferson St.

Any City, US 11111

Agency Contact: Tor, Audrey

Agency Phone: (123) 456-7890

Fax: (234) 567-8901

Email Address: agencyemail@localisp.net

Work Phone:

Cell Phone:

Email Address:

\section{CONTRACTOR / CREW INFORMATION}

Company:

Work Phone:

Address:

Cell Phone:

Email Address:

Contact: Contractor, John

Company Name \& License Number:

Contractor's Signature:

\section{COMMENT}




\section{Appendix C: Reports}

\section{Measures}

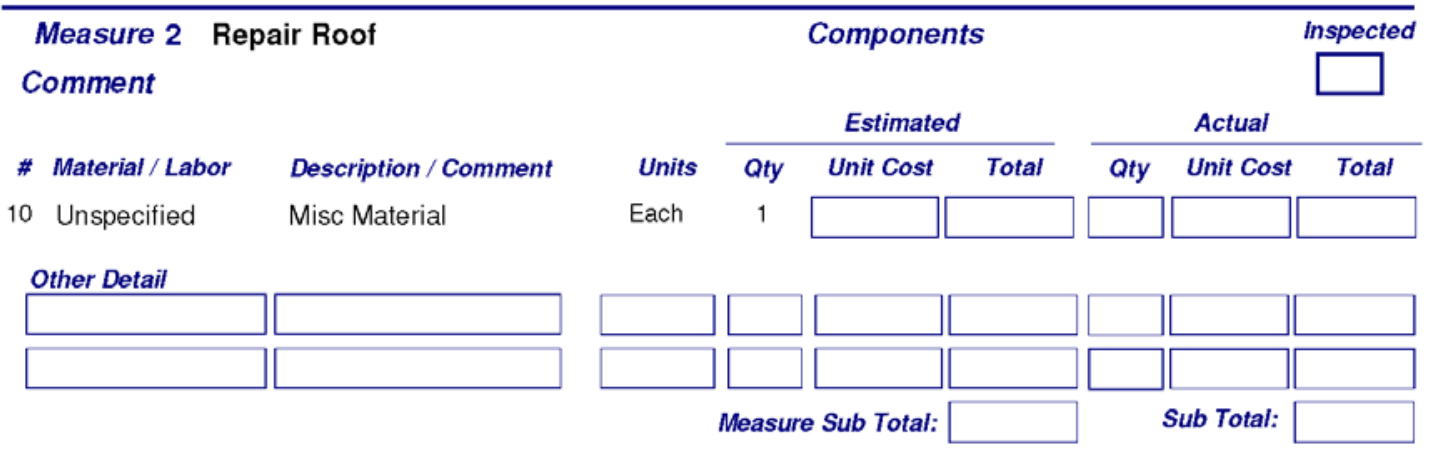

Field Notes:

Measure 3 Infiltration Redctn

Components

Inspected Comment

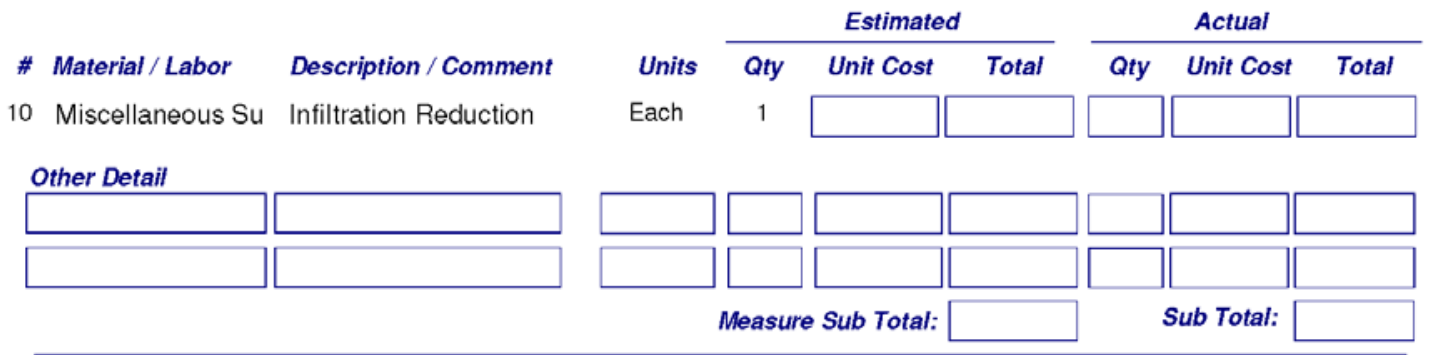

Field Notes: 

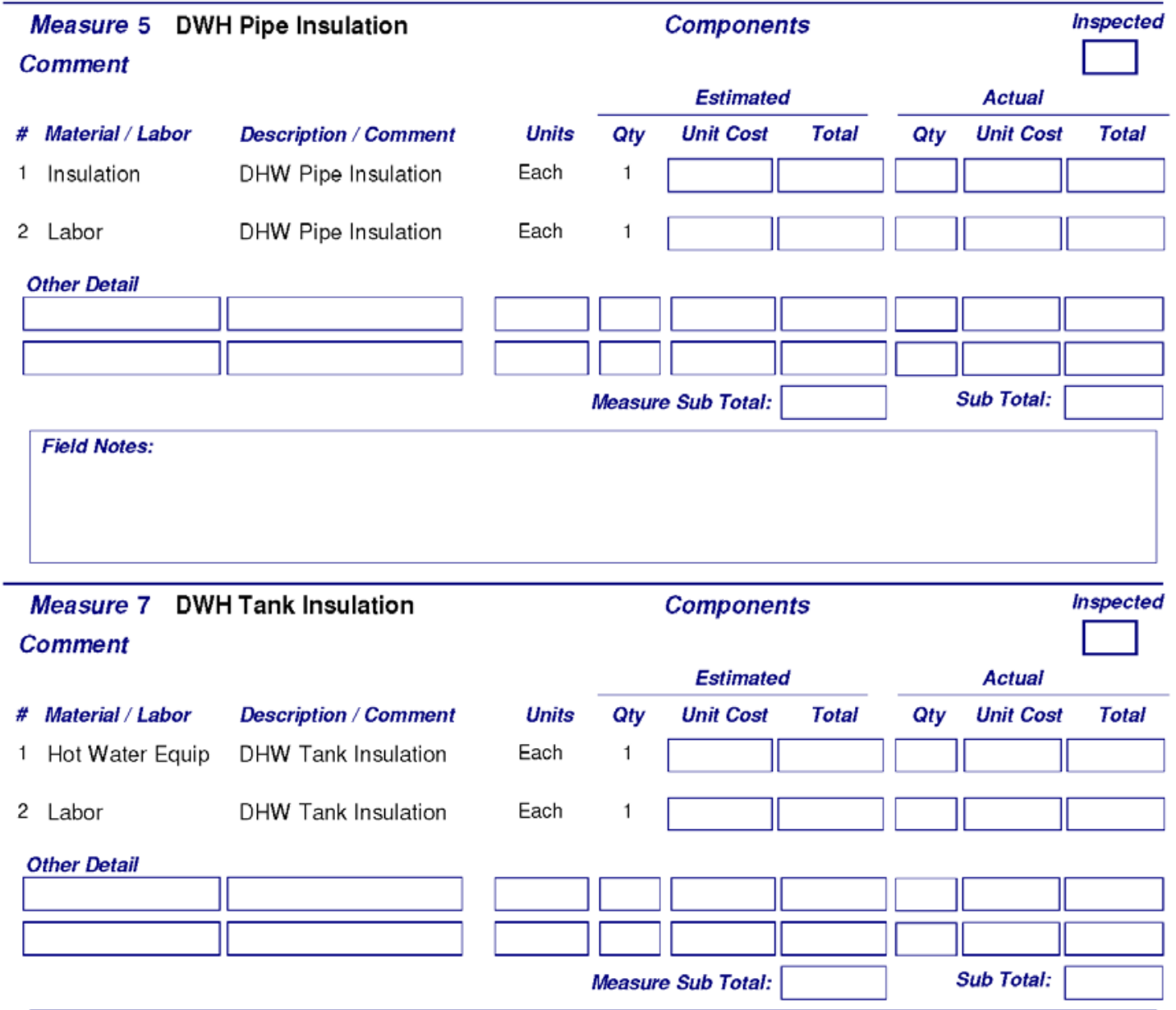

Field Notes: 


\section{Appendix C: Reports}

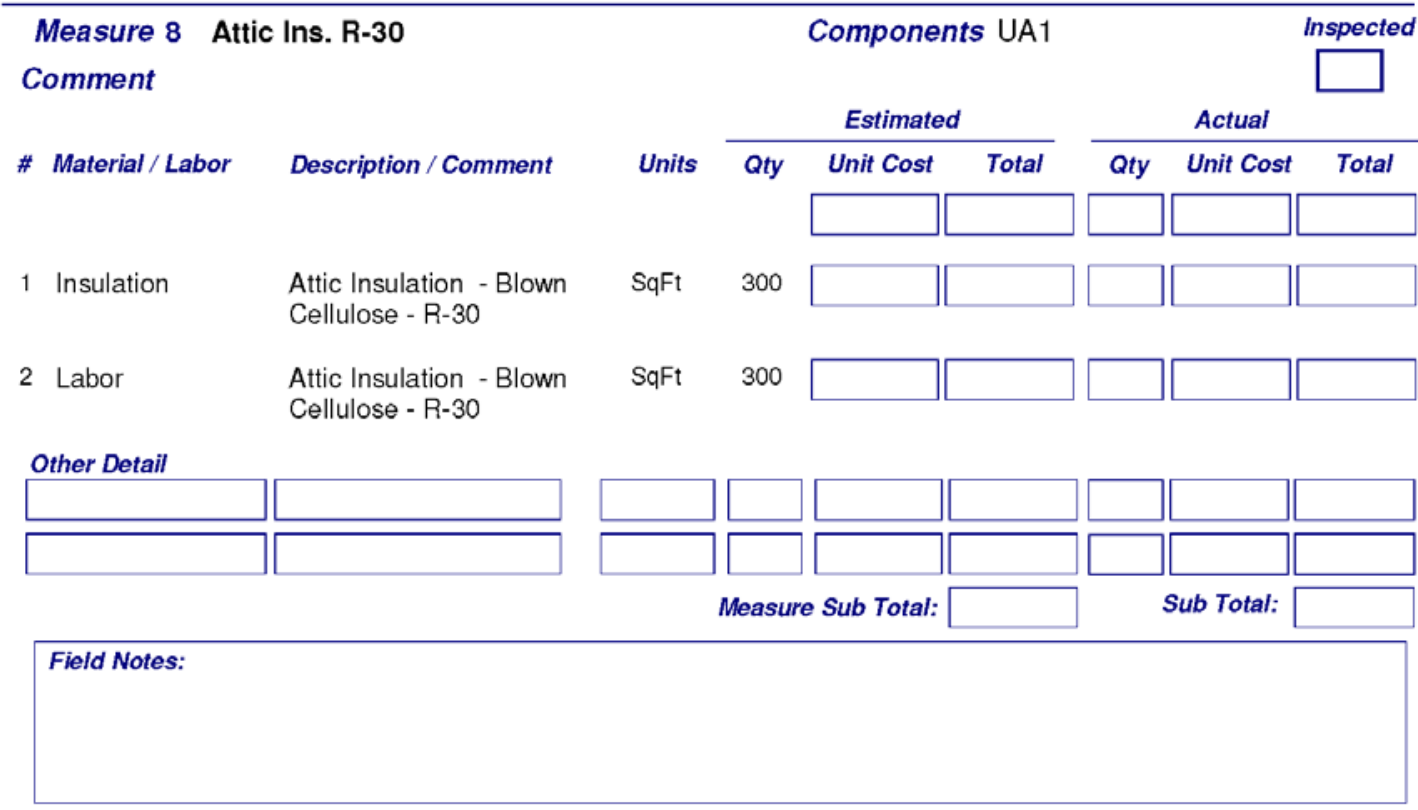

Measure 9 Fill Ceiling Cavity Comment

\begin{tabular}{lll} 
\# Material / Labor & $\begin{array}{l}\text { Description / Comment } \\
1 \text { Insulation }\end{array}$ \\
& $\begin{array}{l}\text { Attic Insulation - Blown } \\
\text { Cellulose - } 5 \text { in. }\end{array}$ \\
2 Labor & $\begin{array}{l}\text { Attic Insulation - Blown } \\
\text { Cellulose - } 5 \text { in. }\end{array}$ \\
\hline & \\
\hline
\end{tabular}

\section{Field Notes:}

\section{Components $\mathrm{FA} 3$}

Inspected

\begin{tabular}{|c|c|c|c|c|c|c|}
\hline \multirow[b]{2}{*}{ Units } & \multicolumn{3}{|c|}{ Estimated } & \multicolumn{3}{|c|}{ Actual } \\
\hline & Qty & Unit Cost & Total & Qty & Unit Cost & Total \\
\hline $\mathrm{SqFt}$ & 437 & & & & & \\
\hline $\mathrm{SqFt}$ & 437 & & & & & \\
\hline
\end{tabular}

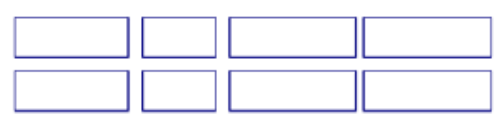

Measure Sub Total:

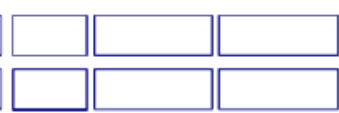

Sub Total: 
Measure 10 Insulate and seal attic access Comment

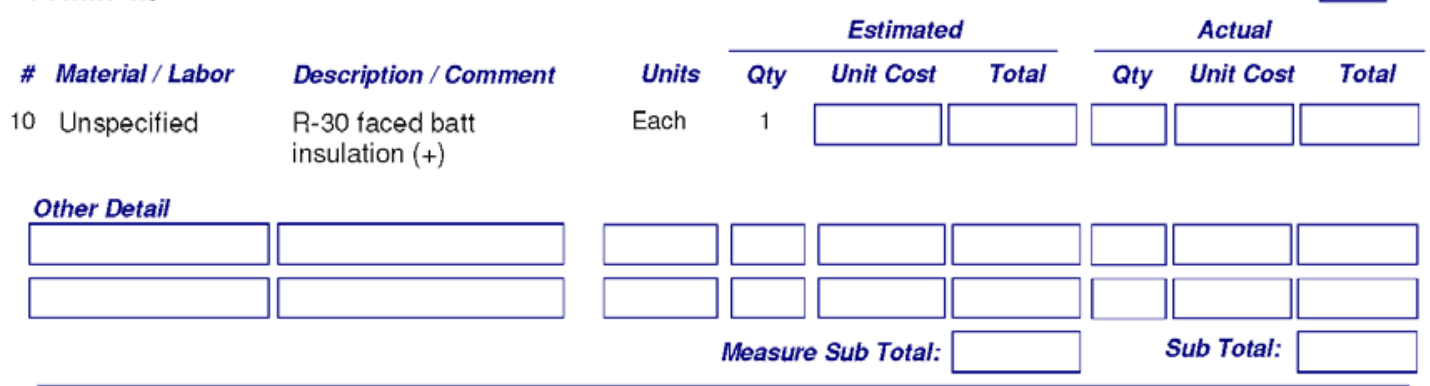

\section{Field Notes:}

\section{Measure 11 Attic Ins. R-30}

\section{Comment}

\begin{tabular}{ll} 
\# Material / Labor & $\begin{array}{l}\text { Description / Comment } \\
\text { Attic Insulation - Blown } \\
\text { Cellulose - R-30 }\end{array}$ \\
2 Insulation & $\begin{array}{l}\text { Attic Insulation - Blown } \\
\text { Cellulose - R-30 }\end{array}$ \\
\hline
\end{tabular}

Field Notes:

\section{Components FA1}

Inspected

\begin{tabular}{lll} 
& \multicolumn{3}{c}{ Estimated } \\
\cline { 2 - 3 } Units & Qty & \multicolumn{1}{c}{ Unit Cost Total } \\
SqFt & 392 & \\
& &
\end{tabular}

\begin{tabular}{lll}
\multicolumn{2}{c}{ Actual } \\
\hline Qty Unit Cost Total \\
\hline
\end{tabular}

$\mathrm{SqFt} \quad 392$
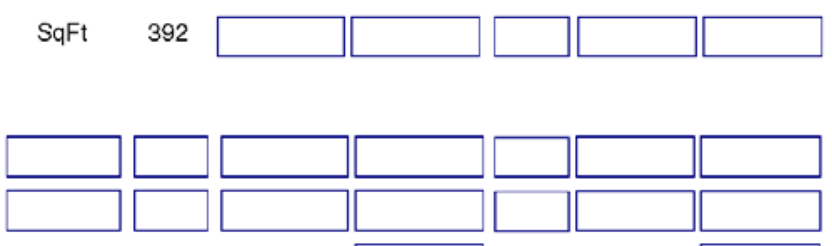

Measure Sub Total:

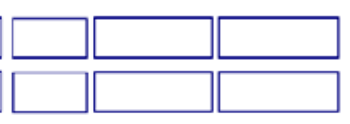

Sub Total: 


\section{Appendix C: Reports}

\begin{tabular}{|c|c|c|c|c|c|c|c|c|}
\hline Measure 12 Att & Ins. R-30 & & & Compone & S FA4 & & & spected \\
\hline Comment & & & & & & & & \\
\hline & & & & Estimate & & & Actual & \\
\hline \# Material / Labor & Description / Comment & Units & Qty & Unit Cost & Total & Qty & Unit Cost & Total \\
\hline 1 Insulation & Attic Insulation - Blown & $\mathrm{SqFt}$ & 56 & & & & & \\
\hline 2 Labor & Attic Insulation - Blown & $\mathrm{SqFt}$ & 56 & & & & & \\
\hline Other Detail & & & & & & & & \\
\hline & & & & & & & & \\
\hline & & & & & & & & \\
\hline & & & ba & $u b$ Tota & & & Sub Total: & \\
\hline
\end{tabular}

\begin{tabular}{|l|}
\hline Field Notes: \\
\end{tabular}

Measure 14 Kneewall Insulation Comment

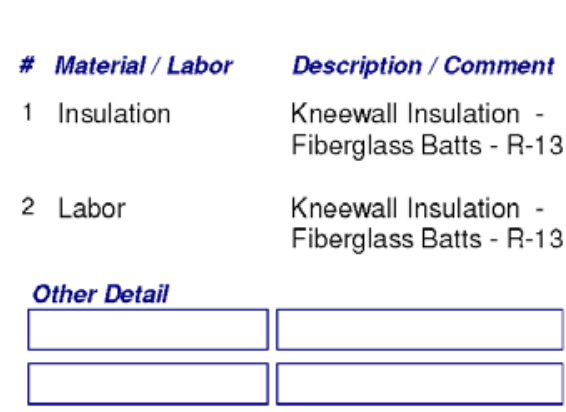

\section{Field Notes:}

\section{Components FA2}

Inspected
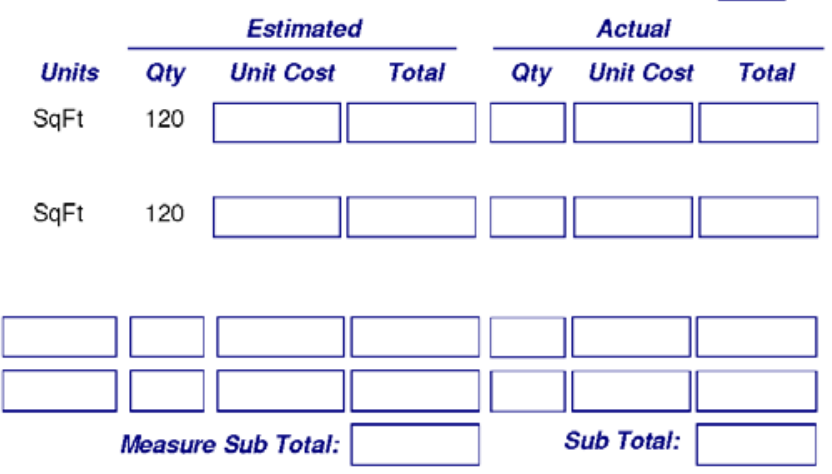

Client ID: 11348

Work Order Bid (ID)

WO/11_348/JT/1

DOE Weatherization Assistant

Report Run On: 9/27/2011 


\section{Measure 16 Wall Insulation}

Comment

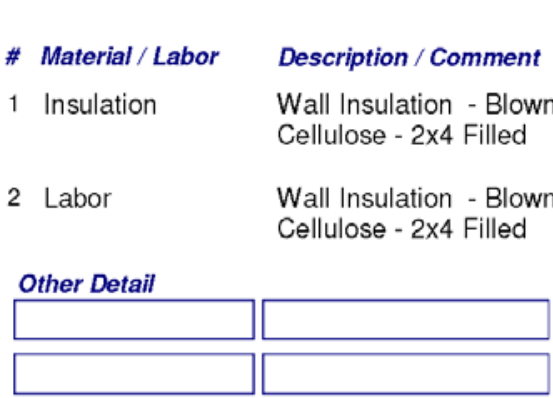

\section{Field Notes:}

Components WLE-1,WLN-1,WLN- Inspected 2, WLS-2,WLW-1
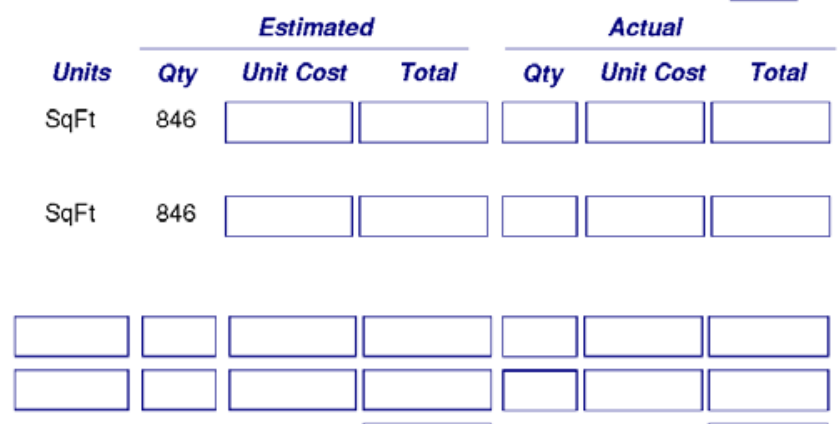

Measure Sub Total

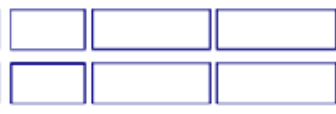

Sub Total:

\section{Field Notes:}

\section{Measure 17 Window Replacement}

\section{Comment}

\# Material / Labor
1 Windows
2 Labor
3 Other

Other Detail

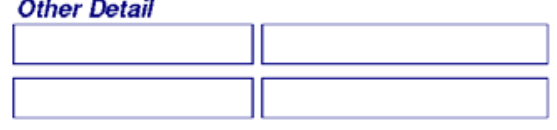

Field Notes:

Description / Comment

Window Replacement

Window Replacement

Window Replacement

\section{Components WD4}
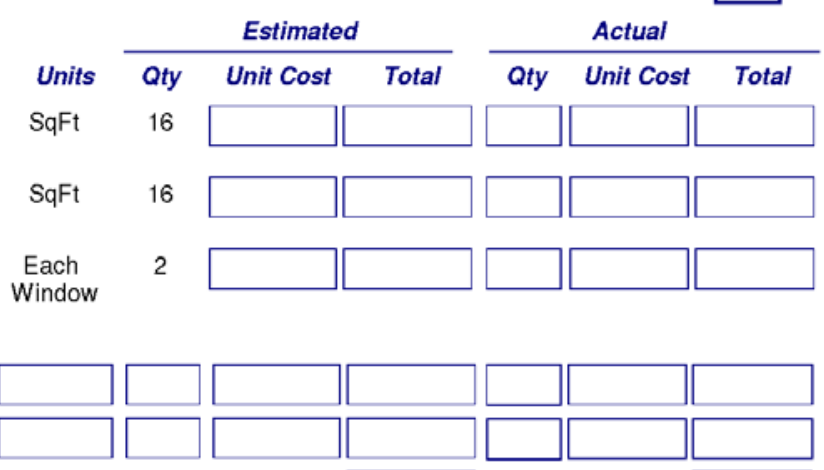

Measure Sub Total:
Sub Total:

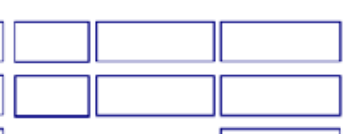




\section{Appendix C: Reports}

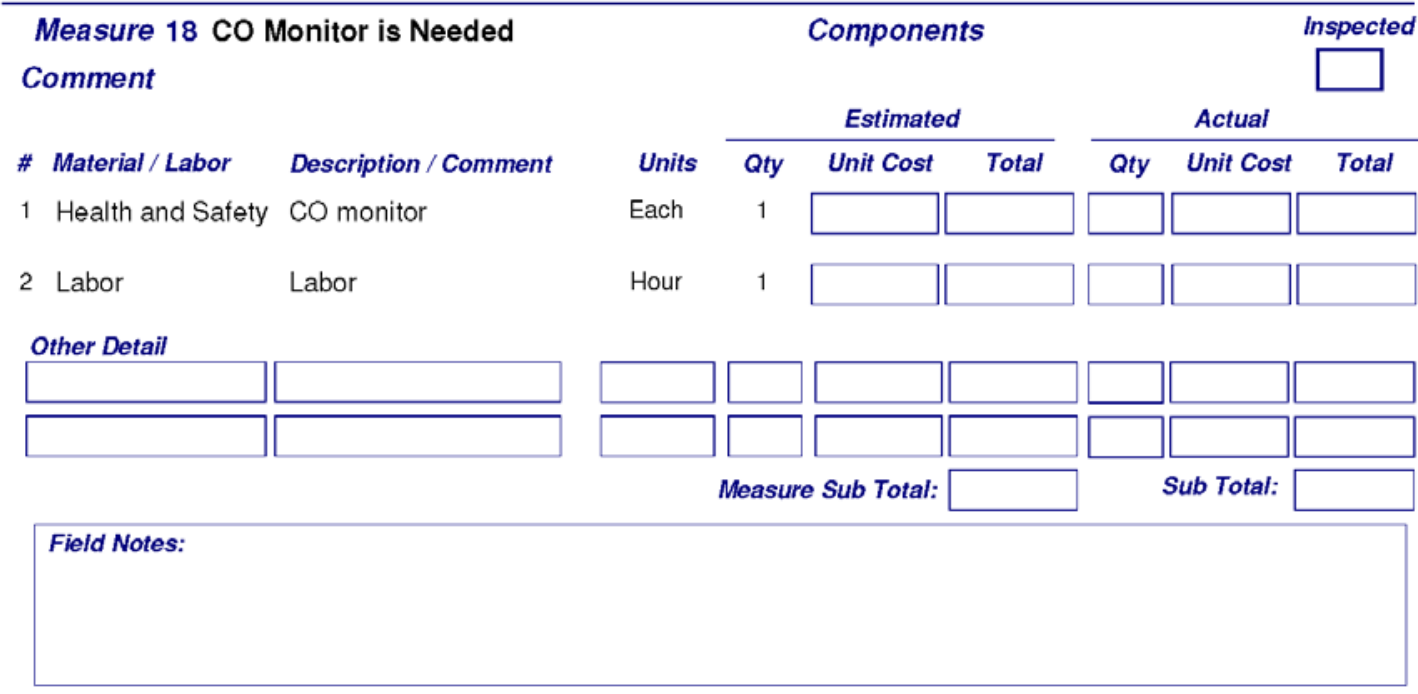




\section{Appendix D. Development of Customized Reports}

\section{D.1 Introduction}

Versions of the Weatherization Assistance database software prior to 8.2.6 contained a set of static, predefined reports in the wa.mde database front-end file. The format of those existing reports and the number of available reports could not be changed by users of the Weatherization Assistant. The desire for customized and user-specific reports has been a stated objective since the first versions of Weatherization Assistant that stored data in a standard database file format. For this reason, and because the system used for development (MS Access) has useful tools for visual query and report development, it was decided to make the Weatherization Assistant reporting module open source.

Starting with Version 8.2.6, all of the report objects, the queries used by those reports, and the Visual Basic source code modules used to generate reports were moved to a separate database file that can be modified by users. The only requirement to modify existing reports or create new reports is:

1. A copy of the Microsoft Access database program (Access 2002/2003 or later)

2. A working knowledge of query and report development in that environment (a somewhat specialized skill that is becoming more common with time)

This document is written for Weatherization Assistant users who wish to modify existing reports or develop new ones. Note that all new or modified reports in the customized reporting database file can be selected and run from the main Weatherization Assistant user interface. This document explains the inner workings of the customized reporting database file that is available for download in source code form with the current Weatherization Assistant release. Included here are descriptions of the tables and other objects that can be copied/edited, or extended along with some detailed examples. There is not much hand holding when it comes to describing the basic features and operations of MS Access, so you may need to refer back to the help material for that application depending on your experience. 


\section{Appendix D: Development of Customized Reports}

\section{D.2 Conventions}

This document contains a number of references to various systems, files, or objects that need to be clarified. So this is really a brief glossary.

wa.mde This refers to the main front-end database file for the Weatherization Assistant application. This is a compiled MS Access 2002/2003 application that is linked to the Weatherization Assistant Backend database file as well as the Customized Reporting database file.

Backend This refers to the database file containing all of the actual data collected on forms in wa.mde. It is stored in MS Access 2002/2003 MDB format for easy access but direct manipulation of the table data is discouraged due to the number of data cross checks built into the wa.mde forms. Each of the tables containing data is linked to the wa.mde front end and the waReport.mdb Customized Reporting database. Changes in the existing structures in the file can damage it, making it unusable. However, it is possible to make additive changes like adding new tables, new relationships, or new fields to existing tables.

waReport.mdb This refers to the open source customized reporting database file that is the subject of this document. In the normal distribution, this file is named waReport.mde since it is distributed in compiled format. It is in MS Access 2002/2003 format and contains linkages to the same Backend tables as wa.mde. The wa.mde front end is linked to the customized reporting database in either open source (mdb) or compiled (mde) format. The links to the backend tables in the reporting module are managed automatically by the wa.mde front end. The open source version of the customized reporting database file (mdb format) is available as a separate download from the Weatherization Assistance Program sponsored site on which the main program is posted. 


\section{D.3 Download Instructions}

The main distribution of Weatherization Assistant is a self-extracting executable file that includes a copy of the current reporting database file in MDE format (compiled). Weatherization Assistant is distributed as a single file that follows the naming convention:

waX-X-X-XSS.exe where $\mathrm{X}-\mathrm{X}-\mathrm{X}-\mathrm{X}$ is the version number and SS an optional code for distributions to a specific state.

In the same directory on the web where this distribution file is located you should also find another file named:

waReportXXXX.zip This is the corresponding waReport.mdb file (zipped). It is an Access 2002/2003 database file that was used to compile the waReport.mde file contained in the main installation file. It is necessary to download this file only if you plan to modify or add reports yourself.

\section{D.4 Help Available}

This document is the main help provided for knowledgeable users wishing to develop their own reports. Some technical assistance may be available on a caseby-case basis. Contact the technical assistance for the Weatherization Assistant program for details. Please read Appendix D.12, Handling Upgrades, to learn about limitations and cautionary notes.

\section{D.5 Requirements}

The Weatherization Assistant was developed in MS Access 2002/2003. Most of the instructions in this document are specific to that version. This is an older version of the software that may only be available from second hand sources (eBay). However, it is possible to use more recent versions of the program for report development. Refer to Appendix D.11, Using More Recent Versions of Access, for specific instructions in using other versions of Access to develop reports. 


\section{Appendix D: Development of Customized Reports}

\section{D.6 Operations}

This section contains a description of the operation of the wa.mde Weatherization Assistant front end reporting. When the wa.mde front-end makes a list of available reports or calls for a specific report, it opens the customized reporting database file as another task on the Windows task bar. The report is then opened, previewed, or printed from the customized reporting database. In this way, all the reports defined in the customized reporting file are immediately visible and can be called from the regular wa.mde user interface.

The wa.mde file contains a link (Main Menu/Link Form) that stores the complete path name of the reporting database file. The reporting database can be in either MDE (compiled) or MDB (uncompiled/open) file formats. This last point is important as it gives you the ability to update and distribute reports in either format. The MDE format is compiled and cannot be altered whereas the MDB file is open and can be altered by anyone with a copy of MS Access installed on their computer.

Because the full path to the file is stored, the naming convention and location of the reporting database is not fixed. The reporting database file is referred to as waReport.mdb by convention in this document, but really it can be any file in either MDB or MDE format. It is recommended that the reporting database file be located on the same disk as the Weatherization Assistant front end file (wa.mde) on the local machine for best performance. It is not recommended that the waReport.mdb/mde file be shared over a network. The waReport.mdb (or mde) MUST be in Access 2002/2003 file format. See Appendix D.11, Using More Recent Versions of Access, for details.

The waReport.mdb file contains all the report, query, and Visual Basic modules necessary to create the reports. It also has access to the same backend data as the wa.mde file through dynamically adjusted table linkages. When you use wa.mde to link to a new backend file, the table linkages are refreshed in BOTH the wa.mde file and the currently linked waReport.mdb file. This ensures that waReport.mdb is linked to the same data as the wa.mde and it allows the waReport.mdb to be run independent of wa.mde for testing purposes.

When wa.mde calls for a report, it first tests to see if the DatesRequired field in tblzReport is checked. If so, the date range pop-up form is displayed and start/end 
dates are collected. Then tblzReportSetup in waReport.mdb is filled in by wa.mde. This table in the reporting database is how ALL parameters are passed from wa.mde to waReport.mdb. See Appendix D.7, Reference for tblzReport, for details for each field. The configuration of tblzReport is crucial if you are adding a new report and want that report visible in the Weatherization Assistant user interface.

Calling for a report from wa.mde starts a new MS Access task on the Windows task bar so users see a 'Report' entry on the task bar for each open report. Multiple reports can be open at the same time limited only by the memory resources on the host computer. When any report is closed, the associated Reporting task (instance of Access) is also closed. If the report includes external file references, then instances of the associated display application remain open and must be closed manually. Printing reports to paper leaves no extra tasks opened.

\section{D.7 Reference for tblzReport}

(where waReport.mdb makes reports visible to wa.mde)

This section contains a complete listing of all of the fields in tblzReport. The first thing to realize is that new user developed report objects are ONLY visible to wa.mde IF there is an active record pointing to that report in this table. This way you can have several reports in various stages of development stored in the waReport.mdb file without having to expose them in the wa.mde user interface. You can also insert records and use the Active field to turn off those reports that you don't want visible to users.

\section{Field Name Description}

UI The User Interface code in which this report is visible. Note that this is typically set to the string 'ALL' indicating that the visibility of the report should not be restricted to a certain UI code.

Group This is a drop-down selector indicating in which wa.mde form the report should be listed. The choices are as follows.

Agency: $\quad$ Agency form

Client: $\quad$ The Client form 


\section{Appendix D: Development of Customized Reports}

Field Name Description

NAudit:

MAudit:

FAudit:

WorkOrder:

Library:

Supply:
NEAT Audit form (site built)

MHEA Audit form (mobile home)

MFEA Audit form (multi-family

The Work Order form

The Setup Library form

The Supply Library form

SortOrder Controls the order of appearance in the drop-down list of reports in wa.mde. The list of report entries gets sorted on this value for display only.

ReportNum A report grouping can be made up of several individual Access report objects and/or external files. In this way you can create aggregate reports made from several objects. A unique index on the combination of the ReportID and ReportNum is defined for tblzReport, thus the combination of the ReportID and ReportNum fields must be unique. See Appendix D.9, Aggregate Reports, for more details.

ReportID Each report grouping in tblzReport must have a unique ReportID index. This is the index used internally by the program to reference this particular group of reports By convention ReportIDs 1 through 100 are reserved for use by the wa.mde program. IMPORTANT: When adding custom reports (new records to tblzReport) be sure to use ReportIDs > 100. Most reports are single Access reports so each report has its own record in tblzReport. It is only in cases where several Access reports are combined into a single Aggregate report where the same ReportID is shared with several tblzReport records. See Appendix D.9, Aggregate Reports, for more details.

Active This is a checkbox field indicating if a report record is active. You can selectively make reports visible in wa.mde using this flag. 
Field Name Description

Default Within each Group, one record can be identified as the default report. This is the report that is automatically selected in the report selection drop-down list when the form identified by the Group field is opened in wa.mde.

Description This is the description of the report that is displayed in the dropdown list. For aggregate reports, only the first record for the ReportID is used. This is a separate field from the title of the report although they are likely to be similar.

Title $\quad$ This is the string used at the title area of the MS Access report. This allows the use of a standard header where the title string at the top of the report gets replace with the string you enter here.

DatesNeeded This checkbox determines if the date range dialog box should be displayed prior to opening the report. This date range dialog fills in the ReportDateRangeStart and the ReportDateRangeEnd fields in tblzReportSetup. See Appendix D.10, Reference for tblzReportSetup, for more information.

Type This drop-down selection should be set to 'report' for MS Access report objects and to 'file' for external files. External file types supported include any file types for which Window has a default viewer and printer defined.

Name This field contains the object name for MS Access report objects (Type $=$ report $)$ or the pathname of the external file $($ Type $=$ file). If the Type = 'file' then this name can be a absolute pathname (e.g., c:lyourpathlyourfile.txt) or a are relative pathname (e.g., yourfile.txt). Relative pathnames are relative to the location of the waReport.mdb file.

OutFilePrefix Snapshot is one of the output options for MS Access reports. This string provides the file name prefix used in the generation of the 


\section{Appendix D: Development of Customized Reports}

\section{Field Name Description}

snapshot (.snp) output files. Microsoft provides a free viewer for snapshot files.

PreviewPages This is the number of pages displayed in the report Preview window. It only applies to Preview type output. Good choices are 2 for portrait type reports and 1 for landscape.

PreviewMax If checked, the preview window size is maximized and the report takes up the whole application client area. It is a good choice to Preview reports maximized.

\section{D.8 External Files}

You can specify the pathname to an external file as a report object. The only limitation is that the computer generating the report must have an application registered for the file extension you list. For example, if you can assume that every computer has software to handle MSWord files with the .doc extension, you can list the name of a .doc file as a report. In that case the Type field is set to 'file' and the Name field contains the path name to the .doc file. The path name can be an absolute path name or a name relative to the path where the waReport.mdb is installed. This may be the best way to include certain boiler-plate type information before or after a normal Access report.

\section{D.9 Aggregate Reports}

Most reports will be comprised of a single MS Access report object. In those cases a single record in tblzReport corresponds with a single MS Access report object. In that case the record would have a unique ReportID and ReportNum $=1$.

However, there may be instances where you would like several report objects and perhaps some external files (like a boiler-plate in MSWord for instance) previewed or printed as a group in a certain order. You can accomplish this by creating a group of records in tblzReport all sharing the same ReportID. In that case, the ReportNum controls the order of display and printing of the report elements. See the Appendix D.8, External Files, for more information about incorporating external files as a report or in a report group. 
The table tblzReport contains a sample aggregate report that by default is turned off (InActive). It demonstrates how two Access reports and one external text file can be combined into a single aggregate report.

Note that if multiple Access reports are opened in preview mode, the Windows main task menu at the bottom of the screen is used to switch between the different preview windows for the different. When any of the reports are closed, the reporting database closes and you return to the wa.mde front end.

\section{D.10 Reference for tblzReportSetup}

(how wa.mde passes values to waReport.mdb)

This table contains just a single record that includes all of the parameters last passed between wa.mde and the waReport.mdb reporting database. These values are normally only written by wa.mde but they can be manipulated manually in cases where waReport.mdb is being tested independent of wa.mde. In some instances fields in this table need to be used in record selection criteria of named queries. In each of those cases, a public Visual Basic function has been provided in the basReportCalc module to simplify query development. Refer to existing queries in waReport.mdb for examples of how the functions are used to simplify query selection criteria. The reference of fields below shows the name of the public function where applicable.

Field Name

Title

Period

\section{Description}

This is the string to be used as the title for the report being generated. It is copied from the tblzReport. Title field at the time the report is generated.

basReportCalc.ReportTitle() as String

String description of period ie. "6/1/04 to 6/31/04". This is used in the report header. This is non-null only if the ReportDateRangeStart or ReportDateRangeEnd fields are non-null.

ReportDateRangeStart If the DatesNeeded checkbox is marked in tblzReport, then wa.mde will prompt for the start date and fill in the 


\section{Appendix D: Development of Customized Reports}

Field Name

ReportDateRangeEnd

ReportSubLabel

ReportSubLabelLeft

\section{Description}

date here.

basReportCalc.ReportStart() As Date
If the DatesNeeded checkbox is marked in tblzReport, then wa.mde will prompt for the end date and fill in the date here.

basReportCalc.ReportEnd() As Date

This is the the Report Center Label copied from wa.mde MainMenu/Preferences providing a general user configurable report header string. This overlaps the ReportSubLabelLeft and Right, so typically only one of the two is used.

This is the the Report Left Label copied from wa.mde MainMenu/Preferences providing a general user configurable report header string. This overlaps the ReportSubLabel, so typically only one of the two is used.

ReportSubLabelRight

This is the the Report Right Label copied from wa.mde MainMenu/Preferences providing a general user configurable report header string. This overlaps the ReportSubLabel, so typically only one of the two is used.

ProgramLogo1 This is the full path name to a bitmap (.bmp) file used for the graphic in the upper LEFT hand corner of the report header. It is also used as the LEFT hand side graphic for the wa.mde Main Menu and is set using the Main Menu/Preferences form in wa.mde.

ProgramLogo2
This is the full path name to a bitmap (.bmp) file used for the graphic in the upper RIGHT hand corner of the report header. It is also used as the RIGHT hand side graphic for the wa.mde Main Menu and is set using the Main 
Field Name

RecordID

ReportAgencyName

ReportAgencyState

\section{Description}

Menu/Preferences form in wa.mde.

This is the long integer identifier for the current record for the current form in wa.mde. Which record is used depends on the Group field in the tblzReport table. Here is the name of the table and the long integer ID associated

Group RecordID is

Agency Null, See ReportAgencyName below

Client tblClient.ClientID

NAudit tblNJob.JobID

MAudit tblMJob.JobID

FAudit tblFJob.JobID

WorkOrder tblWorkOrder.WorkOrderID

Library tblLib.LibID

Supply tblSupply.SupplyID

basReportCalc.RecordID() as Long

The name of the associated tblAgency.AgencyName field regardless of the Group.

basReportCalc ReportAgencyName() as String

The name of the associated tblAgency.AgencyState field 


\section{Appendix D: Development of Customized Reports}

Field Name

ReportID

OutputType

LinkPath

\section{Description}

regardless of the Group.

basReportCalc ReportAgencyState() as String

The ReportID field from tblzReport for the report currently being generated

What type of output is being called for:

Preview $=$ Access Report Preview window

Print $=$ Hard Copy to the Default Windows Printer

Snap = An MS Access snapshot file

The full pathname to the backend database file currently linked to the wa.mde AND the waReport.mdb database files. This pathname is used to dynamically maintain these links.

\section{Example 1: How to Alter an Existing Report}

Here is a step-by-step example of how to modify an existing report. The first step is to make copies of all the query and report objects. Avoid editing existing object since those object may change on the next upgrade and you would have difficulty merging the changes into existing objects. By creating new objects, upgrading to the next version is made much simpler because you simply copy your custom/new objects into the new waReport.mdb file.

Suppose you would like to add the Work Phone for the contractor assigned to the Open Work Order report visible from the Agency form. Here is an outline of steps to perform:

1. Examine tblzReport and notice that rptAgencyOpenWorkOrders contains the report of interest 
2. Make a copy of that report object. NOTE: pick a simple prefix for all of your copies (makes it easier to spot your customized objects when it comes time to migrate your changes to the next version). Suppose your prefix is "abc_" so copy

3. rptAgencyOpenWorkOrders $\rightarrow$ abc_rptAgencyOpenWorkOrders

4. Notice that the report is based (Record Source) on the named query qry_rptAgencyOpenWorkOrders. So make a copy of that as well.

5. qry_rptAgencyOpenWorkOrders $\rightarrow$ abc_qry_rptAgencyOpenWorkOrders

6. Now we can just work on the copies leaving the original objects unchanged. First, change the Record Source property of your copy of the report to your new copy of the query.

7. Now modify your copy of the query with the MS Access query design tool. Add the WorkPhone field from tblContact to the list of fields reported by the query. Save your changes.

8. Modify the details section of your copy of the abc_rptAgencyOpenWorkOrders report object. Add a new text control linked to the new WorkPhone field in the query. Save your changes.

9. Test your new report manually. See Appendix D.13, Testing During Development, for hints for testing. When your changes are working to your satisfaction, it is time to register the report in tblzReport.

10.The way to update tblzReport is to make a copy of the existing record for the work order report (ReportID $=21)$. Copy this to a new record and make the following changes:

11. ReportID $=101($ or some number $>100)$

12.Description = "New Open Work Orders" (some unique description)

13.Name = abc_qry_rptAgencyOpenWorkOrders (the name of your new report object) 


\section{Appendix D: Development of Customized Reports}

14.You might optionally disable the old copy of this report by turning off the Active flag for the previous version in tblzReport. That makes it invisible in the wa.mde front end.

15.Now the new report should show up in the wa.mde Agency form. If you don't see the new report, check to be sure you are linked to the correct reporting file using the wa.mde Link form. Test run it from there. If everything is working, you are ready to distribute. If you are working with a version of Access other than Access 2002/2003, you will need to save your work as an Access 2002/2003 file for wa.mde to reference it correctly.

\section{Example 2: How to Create a New Report}

The best way to create a new report is to start from an existing one. This way you get all the standard header controls and code to manage report open, close, and NoData events. Look at the code behind one of the reports to see the standard (fairly simple code) to hand the Open, Close, and NoData events. Let say you want to develop a new report based on the Client table to perform some economic summaries.

1. First create a query that contains the records you are interested in. In this case an existing query (qry_MeasureCost) shows the economics for each measure in each work order associated with each client. We can define a new query with this query as its source. The new query joins two tables and set the criteria including a date range and does the summary across work order measures. The new query has been left in the waReport.mdb for example purposes and is named abc_qrySampleClientSummary. This query summarizes the estimated and actual initial costs as well as the estimated and actual savings to investment ratios (SIR). NOTE: the query does not limit the work orders or client records considered by any status settings but it does limit the records with criteria for the Agency Name, State, and start/end dates.

2. Now develop the report object based (Record Source) on that query. In this case I started with a copy of the Open Work Order report, then deleted all the objects in the detail section leaving the standard report header and page footer unchanged. That is the quickest way to get a new report. Then I changed the Record Source property to the new query and created a simple page header and detail section. Report generation is a fairly involved process well beyond the 
scope of this document but the MS Access help material may help you. The resulting report is saved as an example in the waReport.mdb database named abc_rptSampleClientSummary.

3. The final step is to register the new report in tblzReport so it is visible from wa.mde. Again the quickest method is to copy an existing record then make changes. In this case I copied the record for the Open Work Order report and modified the following:

4. ReportID $=102$ (or something $>100$ )

5. SortOrder $=40$ (so it shows up last in the list)

6. Description

7. Title

8. DatesNeeded (yes, checked)

The tlbzReport record is not checked Active since this record is in the table only for example purposes. Change the record to Active to test the report from the wa.mde Agency form.

\section{D.11 Using More Recent Versions of Access}

Although a copy of Access 2002/2003 is recommended, you can use more recent versions of the MS Access database software for report development with a little extra effort.

If using Access 2007 to develop your customized reports, opening the waReport.mdb file should automatically prompt you to convert the file to this later Access file format. Allow the conversion to occur. Perform your development. You should be able to manually open reports using the backend data that you were linked to at the time you did the conversion. When finished, choose "Save As" “Access 2002-2003 Database” using a file name that retains "waReport” and then adds characters to identify the report file as a modification of the original. 


\section{Appendix D: Development of Customized Reports}

Access 2010 will automatically recognize the file format as Access 2002-2003. The software allows you to make your changes, then automatically saves the file back into this same format.

You are restricted to releasing your report updates in MDB (open source) format unless you have a full copy of MS Access 2002/2003, which allows you to create an MDE (compiled) file in that format.

\section{D.12 Handling Upgrades}

There are a number of potential pit falls associated with updates that can be anticipated and accounted for. The basic problem is that development of the Weatherization Assistant will continue with likely changes to existing objects and new objects in waReport.mdb. Each distribution will have a waReport.mdb file available but it is up to users to migrate their changes to these new versions.

A potential conflict arises if you modify an existing object in your copy of waReport.mdb while that same object is updated between versions. The best way to avoid such conflicts is to follow the following principles:

1. Never modify an existing object, always make a copy and edit just the copy

2. Use a unique prefix for all new object names

An example might help to illustrate. Suppose you want to modify the Quarterly report (rptAgencyQuarterlyUnits) to suit a particular reporting requirement. First, notice that this report is based on a set of queries.

qry_QuarterlyUnit

qry_QuarterlyUnitDetail

qry_QuarterlyUnitDetailOther

qry_QuarterlyUnitDetailOtherSum

qry_QuarterlyUnitDetailSum 
The first order of business is to make copies of all these objects. To make it easier to spot the custom object you create, pick a simple prefix to use consistenty. Let's assume that your prefix is $\mathrm{ABC}$, so do the following copies:

rptAgencyQuarterlyUnits $\rightarrow$ abc_rptAgencyQuarterlyUnits

qry_QuarterlyUnit $\rightarrow$ abc_qry_QuarterlyUnit

qry_QuarterlyUnitDetail $\rightarrow$ abc_qry_QuarterlyUnit

qry_QuarterlyUnitDetailOther $\rightarrow$ abc_qry_QuarterlyUnitDetailOther

qry_QuarterlyUnitDetailOtherSum $\rightarrow$ abc_qry_QuarterlyUnitDetailOtherSum

qry_QuarterlyUnitDetailSum $\rightarrow$ abc_qry_QuarterlyUnitDetailSum

Now change only the new copies. First make sure the references within the new objects consistently point to just the new copies of the queries and reports. Next you can make the custom changes to your copied objects. The main idea is that the original objects remain unchanged and all new objects have a consistent naming convention with your prefix. To complete this example you would also update tblzReport to enable the new report and perhaps disable the previous version. See Appendix D.7, Reference for tblzReport, for details.

Now comes the important part relative to upgrades. When a new Weatherization Schema is release, obtain a copy of the new waReport.mdb then import (File/Get External Data/Import) your customized objects with the easily identified prefix FROM the existing waReport.mdb TO the new version. Providing that no table objects or fields have been renamed or removed (should normally be the case), then all of your custom objects should import and run in the new version. The final step is to update tblzReport in the new version to reflect the changes you made in the previous version. Using ReportIDs > 100 for any records you add or modify will make the process simpler. Appendix D.7, Reference for tblzReport, contains some hints on making that upgrade process easier.

\section{D.13 Testing During Development}

Here are some suggestions that may help during the development of new reports. 


\section{Appendix D: Development of Customized Reports}

1. Use wa.mde to enter data into the backend database to provide your sample database. When you link to your waReport.mdb file in development, the table links are automatically refreshed. You can optionally use the Tools/Add Ins/Linked Table Manger to manage the links manually in the customized reporting database file.

2. Use wa.mde to call up your new report. Once the record is entered into tblzReport, the new report should show up in the appropriate drop-down list of available reports. You may have to close and re-open the form where the dropdown list of reports is displayed (to refresh the drop-down list) if you keep wa.mde open.

3. An important point to remember is that you do not have to use wa.mde to open the report. If you open the waReport.mdb file directly you can open reports manually. The database window is not automatically displayed so press F11 on start-up if you open waReport.mdb manually. The entries in tblzReportSetup from the last call using wa.mde are saved and can be reused. This may be a more convenient method for opening the report for testing numerous small changes.

\section{D.14 Relationships View}

To better understand the existing queries and reports, it may help to look at the relationships view of the Weatherization Assistant backend database. The database has a hierarchical structure with defined relationships between the various tables containing the raw data. It is necessary to have a working understanding of these relationships when you design new queries and reports. The database relationships view is available in either the Backend MDB file or the Customized Reporting database MDB file. Here is the basic outline of the relationships at the highest level of the database starting with the Agency table. Many of the sub-tables are not shown in this view for the sake of clarity. 


\section{Appendix D: Development of Customized Reports}

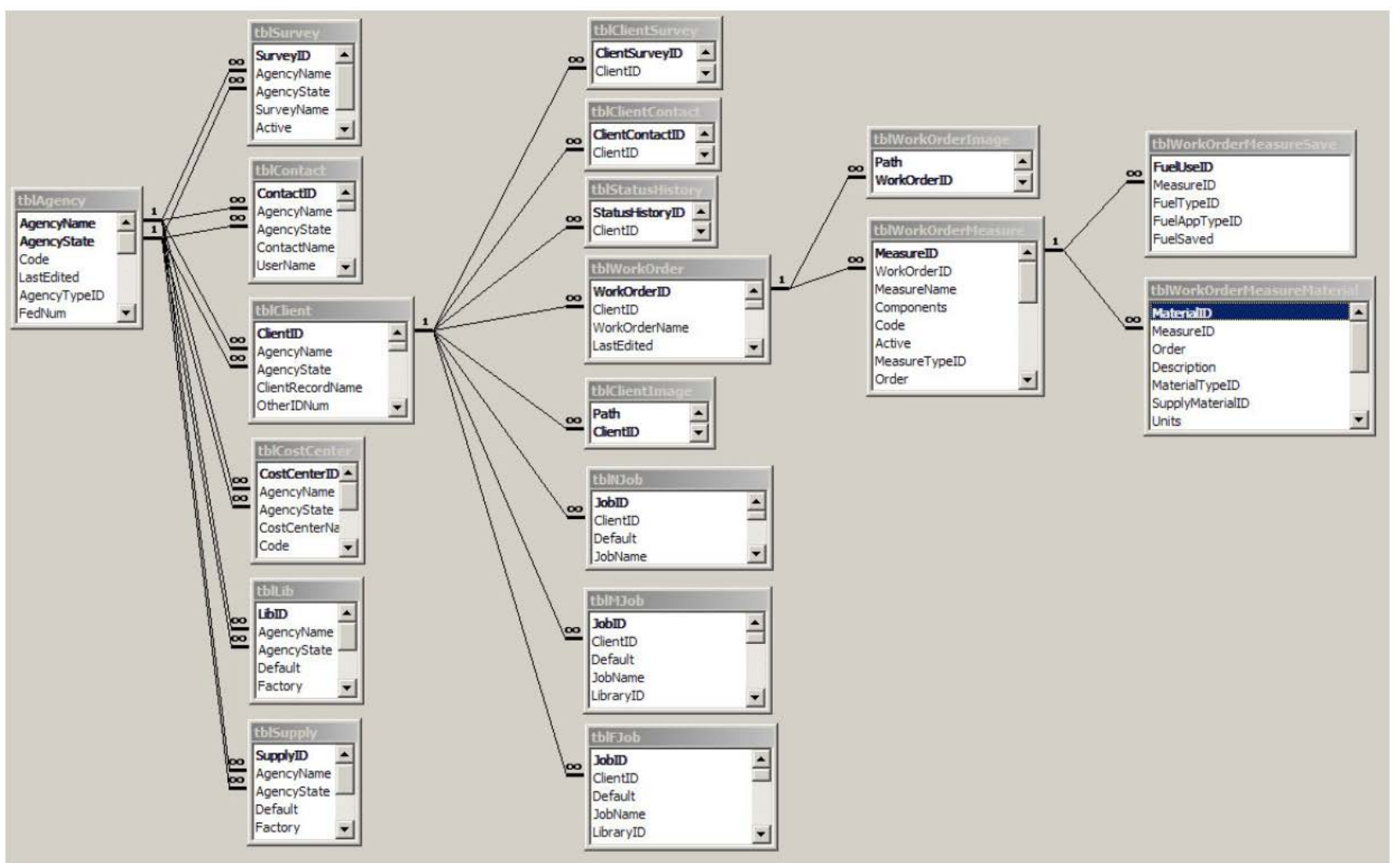

Figure D.1. The relationships view of the Weatherization Assistant backend database. 


\section{Appendix E. Window Leakiness}

\section{E.1 Introduction}

The "Leakiness" data field in the Weatherization Assistant is found under the "Windows" tab of both NEAT and MHEA. The "Leakiness" field allows the user to describe the existing air leakage characteristics of each window entered. This input is used to calculate the energy savings due to reduced air infiltration for three window retrofit measures: window replacement, storms windows, and window weatherization (i.e., sealing). For each window retrofit measure, NEAT and MHEA add the energy savings due to reduced air infiltration to other energy savings associated with the measure to obtain the total energy savings.

Five options are allowed under the "Leakiness" data field: Very Tight, Tight, Medium, Loose, and Very Loose. Guidance on the applicability of these options is provided below for each of the various window types that can be specified in NEAT and MHEA: Jalousie, Awning, Slider, Fixed, Door Window, Sliding Glass Door, and Skylight. The options that are typical for windows encountered in homes served by the Weatherization Assistance Program are also identified.

The guidance provided below is based primarily on the condition of the frame, sashes, and weatherstripping. Once a leakiness level is selected using the guidance below, it should be modified as follows to take into account the condition of the window panes and the presence of a storm window:

- Condition of window panes

o No adjustment should be made if the window pane is cracked or if less than 2 sq. in. of glass is missing in the window (e.g., up to about a 1.5 in. diameter hole or a 1.5 in. $\times 1.5$ in. glass section).

o Degrade the leakiness one level if 2 to 9 sq. in. of glass is missing in the window (e.g., about a 1.5 in. to 3.5 in. diameter hole or a 1.5 in. $\times 1.5$ in. to 3 in. $\times 3$ in. glass section).

o Degrade the leakiness two levels if 9 to 25 sq. in. of glass is missing in the window (e.g., about a 3.5 in. to 5.5 in. diameter hole or a 3 in. $\times 3$ in. to 5 in. $\times 5$ in. glass section). 


\section{Appendix E: Window Leakiness}

o Specify the window to be Very Loose if more than 25 sq. in. of glass is missing in the window (e.g., a hole bigger than about a 5.5 in. diameter or a 5 in. $\times 5$ in. square).

- Presence of storm window - Upgrade the leakiness one level if a storm window in average or better condition is installed.

\section{E.2 Fixed Window, Door Window, and Skylight}

Fixed-type windows are sealed in their frames and cannot be opened. Fixed-type windows include large picture windows, decorative windows in doors, and most skylights (i.e., windows in the ceiling). The leakiness of a typical fixed window, door window, or skylight is Very Tight.

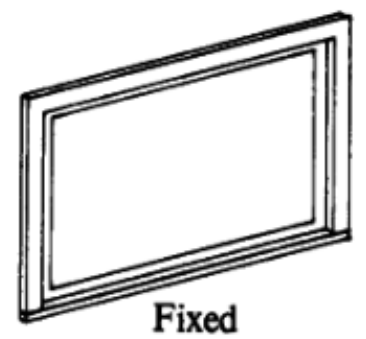

\section{E.3 Awning Window (Including Hopper and Casement Window)}

The awning window type used in NEAT and MHEA includes hopper and casement windows. Most awning, hopper, and casement windows have just a single sash, although casement windows can have two sashes and awning windows can have two or three sashes. The sash of an awning window is hinged at the top of the window frame and opens outward and upward. The sash of a hopper window is hinged at the bottom of the window frame so that the window tilts open at the top. The sash of a casement window is hinged on the side of the window frame and swings out right or left. On a casement window with two sashes, a vertical framing member is often present in the middle of the window that houses a locking mechanism (not shown in the figure below).

These types of windows are often operated by a cranking mechanism. In awning and hopper windows with two or three sashes, a common crank is usually present so that the sashes open and close together at the same angle. When closed, the sashes press against the window frame and any installed weatherstripping to form a seal. In windows with multiple sashes, the sashes are usually designed to fit together at their interface when closed to form a tight seal. A lock or latch is usually present that further helps seal the window by drawing the sashes tightly against the frame, each other, and/or any installed weatherstripping. 


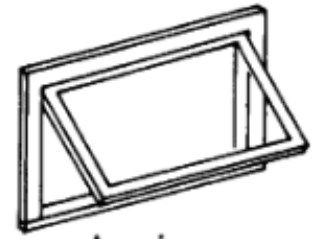

Awning

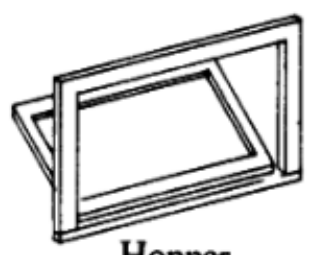

Hopper

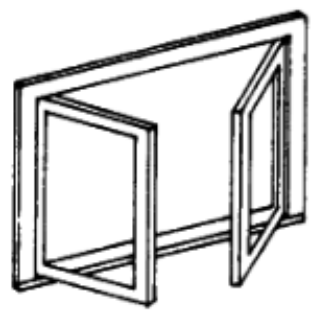

Casement

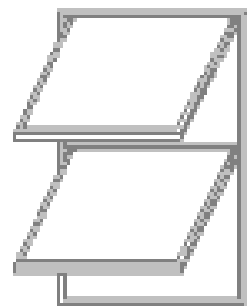

Àning

Awning, hopper, and casement windows with a single sash are generally tighter than other types of moveable windows. The leakiness of a typical single-sash awning, hopper, or casement window is Tight, while the leakiness of a typical multiple-sash awning, hopper, or casement window is Medium.

- Very Tight - The sashes and window frame fit together snuggly to form a complete seal when the window is closed. The sashes and frame are in excellent condition, or they can be in average condition if weatherstripping in good condition is also present. The cranking and locking mechanisms are typically operable and assist in securely pulling the sashes and window frame together. Typical of a new window.

- Tight (typical of a window with a single sash) — No visible gaps are observed between the sashes or between the sashes and the window frame when the window is closed. The sashes and frame are in average condition. Weatherstripping can be absent or deteriorated. The cranking and locking mechanisms are typically operable and assist in securely pulling the sashes and window frame together.

- Medium (typical of a window with multiple sashes) - Small gaps up to 1/8 in. are observable between the sashes and/or between the sashes and the window frame when the window is closed, even with the aid of a locking mechanism. The sashes and frame are in average to poor condition. Weatherstripping is usually absent or deteriorated.

- Loose - Gaps up to 1/4 in. are observable between the sashes at their interface when the window is closed as much as the cranking and locking mechanism allow, and/or gaps up to 1/2 in. are observable between the sashes and the window frame when the window is closed. The sashes and frame are in poor 


\section{Appendix E: Window Leakiness}

condition, and may be warped or not square. Weatherstripping is absent or ineffective.

- Very Loose - Gaps 1/4 in. or greater are observable between the sashes at their interface when the window is closed, and/or gaps $1 / 2$ in. or more are observable between the sashes and the window frame when the window is closes. The sashes and frame are in very poor condition and are likely warped or not square. Weatherstripping is absent or ineffective. The locking mechanism may not be able to be engaged.

\section{E.4 Slider Window and Sliding Glass Door}

A vertical slider window has at least one sash that slides up and down within the window frame. In a double-hung slider window, both sashes slide vertically past one another. Only the bottom sash slides up and down in a single-hung slider window. A horizontal slider window (designated as "Sliding" in the figures) or sliding glass door has at least one sash that slides horizontally within the window or door frame. A locking mechanism is often present on a slider window that draws the two sashes together at their interface and helps press each moveable sash into the window frame.

Horizontal slider windows and sliding glass doors are usually in poorer condition and, thus, leakier than comparable vertical slider windows. The typical leakiness

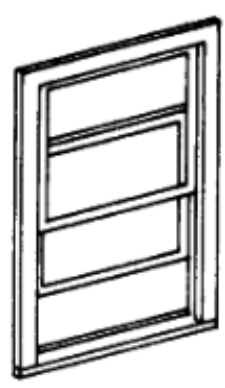
of an original double- or single-hung vertical slider window is Medium if installed in a house built in the 1960s and before, and Tight if installed in a house built in the 1970s and after. The leakiness of a typical horizontal slider window or sliding glass door is Medium.

- Very Tight - The moveable sashes and window frame fit together tightly as designed when the window is closed such that no gaps are present. The sashes and frame are in excellent condition. Each moveable sash is secure and tight in its track. The moveable sashes are able to be closed such that the window locking mechanism can be fully engaged. Weatherstripping is present and in good condition. Typical of a new window. 
- Tight (typical of an original double- or single-hung vertical slider window installed in a home built in the 1970 s or later) - No visible gaps are observed between the sashes or between the moveable sashes and the window frame when the window is closed. The sashes and frame are in average condition. Each moveable sash is secure in its track although some play may be present. The moveable sashes are able to be closed such that the window locking mechanism can be engaged, although perhaps not fully. Weatherstripping is present and in good to fair condition.

- Medium (typical of an original double- or single-hung vertical slider window installed in a home built in the 1960s or earlier, a horizontal slider window, or a sliding glass door) - Small gaps up to 1/8 in. are observable between the sashes at their interface and/or between the moveable sashes and the window frame when the window is closed. The sashes and frame are in average condition. Each moveable sash is operable in its track although some play is likely. The moveable sashes may not sit perfectly horizontal or vertical when closed. The locking mechanism may not be able to be engaged. Weatherstripping is absent or deteriorated.

- Loose - Gaps up to 1/4 in. are observable between the sashes at their interface when the window is closed, and/or gaps up to $1 / 2 \mathrm{in}$. are observable between the moveable sashes and the window frame. The sashes and frame are in poor condition. Each moveable sash may be loose in its track. The moveable sashes likely do not sit horizontal or vertical when closed. The locking mechanism may not be able to be engaged. Weatherstripping is absent or ineffective.

- Very Loose — Gaps 1/4 in. or greater are observable between the sashes at their interface when the window is closed, and/or gaps $1 / 2$ in. or more are observable between the moveable sashes and the window frame. The sashes and frame are in poor condition. Each moveable sash may no longer fit in its track. The moveable sashes likely do not sit horizontal or vertical when closed. There may be considerable movement (rattling) between sashes. The locking mechanism is likely to be inoperative. Weatherstripping is absent or ineffective. 


\section{Appendix E: Window Leakiness}

\section{E.5 Jalousie Window}

A jalousie window is made up of multiple horizontallymounted glass louvers or slats. The glass louvers are usually 3 in. to 5 in. wide and are mounted in a metal panel. A crank typically rotates the glass louvers outward like a shutter when open. The glass louvers overlap each other slightly when closed. The leakiness of a typical jalousie window is Loose.

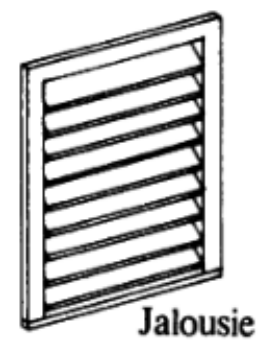

- Very Tight — Generally not applicable to jalousie windows.

- Tight - Generally not applicable to jalousie windows.

- Medium - A tight glass-to-glass seal is visually obtained at the overlap of all the glass louvers when the window is closed. The cranking mechanism is in good working order. All window panes are securely attached to the cranking mechanism. Typical of a new window.

- Loose (typical) — Small gaps up to 1/8 in. are observable between the glass louvers when the window is closed. One or two of the glass louvers may not be securely attached to the cranking mechanism. The cranking mechanism may not be able to fully rotate the glass louvers to their fully closed position.

- Very Loose - Gaps 1/8 in. or greater are observable between the glass louvers when the window is closed. Multiple glass louvers may not be securely attached to the cranking mechanism. The cranking mechanism is likely not able to fully rotate the glass louvers to their fully closed position. 